Comparative Effectiveness Review

Number 189

\title{
Medical Therapies for Children With Autism Spectrum Disorder -An Update
}




\section{Comparative Effectiveness Review}

Number 189

\section{Medical Therapies for Children With Autism Spectrum Disorder-An Update}

Prepared for:

Agency for Healthcare Research and Quality

U.S. Department of Health and Human Services

5600 Fishers Lane

Rockville, MD 20857

www.ahrq.gov

Contract No. 290-2015-00003-I

Prepared by:

Vanderbilt Evidence-based Practice Center

Nashville, TN

Investigators:

Edwin Williamson, M.D.

Nila A. Sathe, M.A., M.L.I.S.

Jeffrey C. Andrews, M.D.

Shanthi Krishnaswami, M.P.H., M.B.B.S.

Melissa L. McPheeters, Ph.D., M.P.H.

Christopher Fonnesbeck, Ph.D.

Kevin Sanders, M.D.

Amy Weitlauf, Ph.D.

Zachary Warren, Ph.D.

AHRQ Publication No. 17-EHC009-EF

May 2017 
This report is based on research conducted by the Vanderbilt Evidence-based Practice Center (EPC) under contract to the Agency for Healthcare Research and Quality (AHRQ), Rockville, MD (Contract No. 290-2015-00003-I). The findings and conclusions in this document are those of the authors, who are responsible for its contents; the findings and conclusions do not necessarily represent the views of AHRQ. Therefore, no statement in this report should be construed as an official position of AHRQ or of the U.S. Department of Health and Human Services.

\section{None of the investigators have any affiliations or financial involvement that conflicts with the material presented in this report.}

The information in this report is intended to help health care decisionmakers—patients and clinicians, health system leaders, and policymakers, among others-make well-informed decisions and thereby improve the quality of health care services. This report is not intended to be a substitute for the application of clinical judgment. Anyone who makes decisions concerning the provision of clinical care should consider this report in the same way as any medical reference and in conjunction with all other pertinent information, i.e., in the context of available resources and circumstances presented by individual patients.

This report is made available to the public under the terms of a licensing agreement between the author and the Agency for Healthcare Research and Quality. This report may be used and reprinted without permission except those copyrighted materials that are clearly noted in the report. Further reproduction of those copyrighted materials is prohibited without the express permission of copyright holders.

AHRQ or U.S. Department of Health and Human Services endorsement of any derivative products that may be developed from this report, such as clinical practice guidelines, other quality enhancement tools, or reimbursement or coverage policies, may not be stated or implied.

This report may periodically be assessed for the currency of conclusions. If an assessment is done, the resulting surveillance report describing the methodology and findings will be found on the Effective Health Care Program Web site at www.effectivehealthcare.ahrq.gov. Search on the title of the report.

Persons using assistive technology may not be able to fully access information in this report. For assistance contact EffectiveHealthCare@ahrq.hhs.gov.

Suggested citation: Williamson E, Sathe NA, Andrews JC, Krishnaswami S, McPheeters ML, Fonnesbeck C, Sanders K, Weitlauf A, Warren Z. Medical Therapies for Children With Autism Spectrum Disorder-An Update. Comparative Effectiveness Review No. 189. (Prepared by the Vanderbilt Evidence-based Practice Center under Contract No. 290-2015-00003-I.) AHRQ Publication No. 17-EHC009-EF. Rockville, MD: Agency for Healthcare Research and Quality; May 2017. www.effectivehealthcare.ahrq.gov/reports/final.cfm. doi: https://doi.org/10.23970/AHRQEPCCER189. 
The Agency for Healthcare Research and Quality (AHRQ), through its Evidence-based Practice Centers (EPCs), sponsors the development of systematic reviews to assist public- and private-sector organizations in their efforts to improve the quality of health care in the United States. These reviews provide comprehensive, science-based information on common, costly medical conditions, and new health care technologies and strategies.

Systematic reviews are the building blocks underlying evidence-based practice; they focus attention on the strength and limits of evidence from research studies about the effectiveness and safety of a clinical intervention. In the context of developing recommendations for practice, systematic reviews can help clarify whether assertions about the value of the intervention are based on strong evidence from clinical studies. For more information about AHRQ EPC systematic reviews, see www.effectivehealthcare.ahrq.gov/reference/purpose.cfm.

AHRQ expects that these systematic reviews will be helpful to health plans, providers, purchasers, government programs, and the health care system as a whole. Transparency and stakeholder input are essential to the Effective Health Care Program. Please visit the Web site (www.effectivehealthcare.ahrq.gov) to see draft research questions and reports or to join an email list to learn about new program products and opportunities for input.

If you have comments on this systematic review, they may be sent by mail to the Task Order Officers named below at: Agency for Healthcare Research and Quality, 5600 Fishers Lane, Rockville, MD 20857, or by email to epc@ahrq.hhs.gov.

Gopal Khanna, M.B.A.

Director

Agency for Healthcare Research and Quality

Stephanie Chang, M.D., M.P.H.

Director

Evidence-based Practice Center Program

Center for Evidence and Practice Improvement Agency for Healthcare Research and Quality

Elisabeth U. Kato, M.D., M.R.P.

Task Order Officer

Center for Evidence and Practice Improvement Agency for Healthcare Research and Quality
Arlene S. Bierman, M.D., M.S.

Director

Center for Evidence and Practice Improvement

Agency for Healthcare Research and Quality

Laura Pincock, Pharm.D., M.P.H.

Task Order Officer

Center for Evidence and Practice

Improvement

Agency for Healthcare Research and Quality 


\section{Acknowledgments}

The authors gratefully acknowledge the following individuals for their contributions to this project: Ms. Jessica Kimber was an invaluable resource for assistance with data extraction and checking, and helped to locate studies and track data. Ms. Katie Worley helped to extract data and create tables. Dr. Mamata Raj assisted with screening studies and risk of bias assessment. We sincerely appreciate their dedicated work and the input of our Task Order Officers; Associate Editor, Margaret Maglione; Key Informants; and Technical Experts.

\section{Key Informants}

In designing the study questions, the EPC consulted several Key Informants who represent the end-users of research. The EPC sought the Key Informant input on the priority areas for research and synthesis. Key Informants are not involved in the analysis of the evidence or the writing of the report. Therefore, in the end, study questions, design, methodological approaches, and/or conclusions do not necessarily represent the views of individual Key Informants.

Key Informants must disclose any financial conflicts of interest greater than $\$ 10,000$ and any other relevant business or professional conflicts of interest. Because of their role as end-users, individuals with potential conflicts may be retained. The TOO and the EPC work to balance, manage, or mitigate any conflicts of interest.

The list of Key Informants who provided input to this report follows:

Daniel L. Coury, M.D.*

Nationwide Children's Hospital

Columbus, $\mathrm{OH}$

Jennifer Frost, M.D.

American Academy of Family Physicians

Leawood, KS

Susan Levy, M.D., M.P.H.*

Children's Hospital of Philadelphia

Philadelphia, PA

Tristram Smith, Ph.D.*

University of Rochester

Rochester, NY

Larry Wexler, Ph.D.

Oak Brook Psychology

Chicago, IL

*Provided input on Draft Report. 


\section{Technical Expert Panel}

In designing the study questions and methodology at the outset of this report, the EPC consulted several technical and content experts. Broad expertise and perspectives were sought. Divergent and conflicted opinions are common and perceived as healthy scientific discourse that results in a thoughtful, relevant systematic review. Therefore, in the end, study questions, design, methodologic approaches, and/or conclusions do not necessarily represent the views of individual technical and content experts.

Technical Experts must disclose any financial conflicts of interest greater than $\$ 10,000$ and any other relevant business or professional conflicts of interest. Because of their unique clinical or content expertise, individuals with potential conflicts may be retained. The TOO and the EPC work to balance, manage, or mitigate any potential conflicts of interest identified.

The list of Technical Experts who provided input to this report follows:

Grace Baranek, Ph.D.

University of North Carolina

Chapel Hill, NC

Susan Levy, M.D., M.P.H.*

Daniel L. Coury, M.D.*

Nationwide Children's Hospital

Columbus, $\mathrm{OH}$

Children's Hospital of Philadelphia

Philadelphia, PA

Jennifer Frost, M.D.

American Academy of Family Physicians

Leawood, KS

Jeremy Veenstra-VanderWeele, M.D.*

Columbia University Medical Center

New York, NY

Paul Wang, M.D. $\neq$

Simons Foundation

New York, NY

Susan Hyman, M.D.*

University of Rochester

Rochester, NY

*Provided input on Draft Report.

$\neq$ Dr. Wang was affiliated with Autism Speaks at the time he served as a Technical Expert Panel member and when he provided input on the Draft Report.

\section{Peer Reviewers}

Prior to publication of the final evidence report, EPCs sought input from independent Peer Reviewers without financial conflicts of interest. However, the conclusions and synthesis of the scientific literature presented in this report do not necessarily represent the views of individual reviewers.

Peer Reviewers must disclose any financial conflicts of interest greater than $\$ 10,000$ and any other relevant business or professional conflicts of interest. Because of their unique clinical or 
content expertise, individuals with potential nonfinancial conflicts may be retained. The TOO and the EPC work to balance, manage, or mitigate any potential nonfinancial conflicts of interest identified.

The list of Peer Reviewers follows:

Christian Gold, Ph.D.

GAMUT-The Grieg Academy Music Therapy Research Centre, Uni Research Health Bergen, Norway

Patricia Howlin, Ph.D.

Institute of Psychiatry, London

London, England, UK 


\section{Medical Therapies for Children With Autism Spectrum Disorder-An Update}

\section{Structured Abstract}

Objectives. To evaluate the comparative effectiveness and safety of medical interventions (defined broadly as interventions involving the administration of external substances to the body or use of external nonbehavioral procedures to treat symptoms of autism spectrum disorder [ASD]) for children with ASD.

Data sources. We searched MEDLINE ${ }^{\circledR}$, Embase ${ }^{\circledR}$, the Cumulative Index of Nursing and Allied Health Literature, and PsycINFO ${ }^{\circledR}$ from January 2010 through September 2016.

Review methods. We included comparative studies of medical interventions that included at least 10 children with ASD. Two investigators independently screened studies and rated risk of bias. We extracted and summarized data qualitatively given significant heterogeneity. We also assessed strength of the evidence (SOE) and considered cumulative data from eligible studies included in our 2011 review of medical therapies and newly published studies.

Results. The 76 unique comparative studies (including 12 comparative studies addressed in the 2011 review) meeting our criteria included 72 randomized controlled trials (RCTs), 2 nonrandomized trials, and 2 retrospective cohort studies. Thirty-nine studies had low, 29 had moderate, and 8 had high risk of bias. Populations, treatment approaches, and outcomes assessed varied across studies. Relative to placebo, seven studies addressing risperidone or aripiprazole reported statistically significant improvements in challenging behavior in the short term $(<6$ months) but also clinically significant harms. Longer term effectiveness was reported in uncontrolled extensions. Three studies comparing risperidone and aripiprazole reported few significant differences in effects on weight gain between agents. RCTs addressing methylphenidate $(n=2)$, atomoxetine $(n=2)$, and guanfacine $(n=1)$ reported significant improvements in hyperactivity, with frequent harms. Omega-3 fatty acids (4 RCTs) were not associated with changes in challenging behavior. $\mathrm{N}$-acetylcysteine and tetrahydrobiopterin were not associated with improvements in social skills and symptom severity, respectively. Despite the number of RCTs with low or moderate risk of bias addressing nutritional supplements or specialized diets, evidence is insufficient for all clinical efficacy and harms outcomes because few, small studies addressed each diet or supplement. Similarly, although 14 RCTs with low or moderate risk of bias compared risperidone plus an adjunct medication with risperidone plus placebo, few addressed the same adjunct agents. Studies of hyperbaric oxygen therapy versus sham treatment using differing protocols reported conflicting results. Fourteen studies addressed other interventions, most evaluated in only one study, and typically reported some positive treatment effects on sleep, ASD symptoms, or language.

Conclusions. Risperidone and aripiprazole ameliorated challenging behaviors in the short term, but with clinically significant side effects (high SOE). Methylphenidate and atomoxetine were also associated with improvements in hyperactivity in small short-term RCTs (low SOE), with improvements maintained over 6 months for atomoxetine (low SOE for longer term effects). Methylphenidate was associated with clinically significant harms (low SOE), while atomoxetine 
was associated with clinically moderate harms (low SOE). Omega-3 fatty acid supplementation, $\mathrm{N}$-acetylcysteine, and tetrahydrobiopterin failed to show benefits (low SOE). Evidence for other interventions and outcomes studied was insufficient. While the conduct of studies has improved considerably over time (i.e., growing number of RCTs and use of standardized measures), data on longer term ( $\geq 6$ months) results and harms of most interventions are lacking. Similarly, more research is needed to understand characteristics of the child or treatment that modify outcomes, whether effectiveness of interventions generalizes across different settings such as the home or school, and how components of interventions may drive effects. 


\section{Contents}

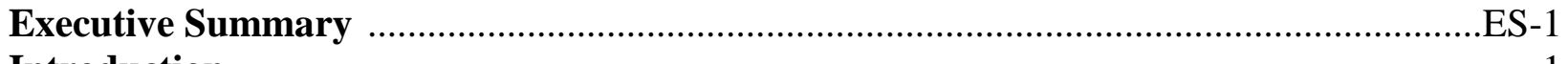

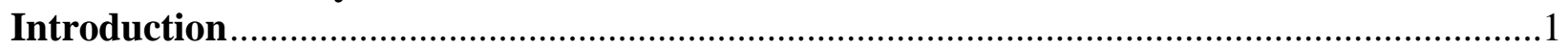

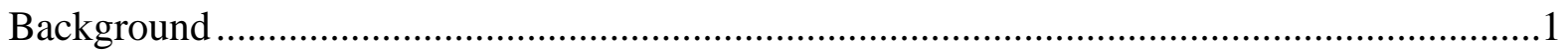

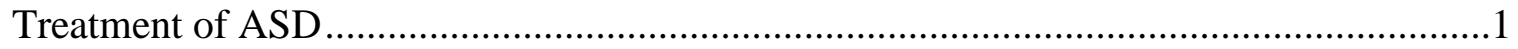

Scope and Key Questions ..........................................................................................

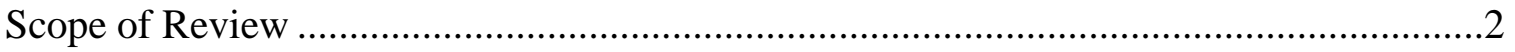

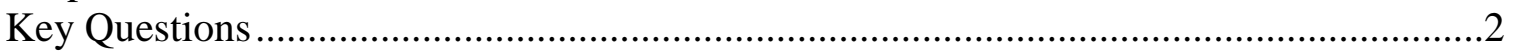

Analytic Framework …………………….................................................................

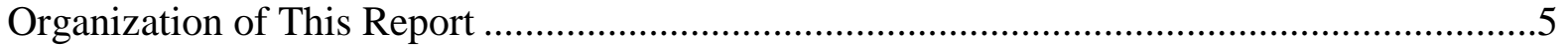

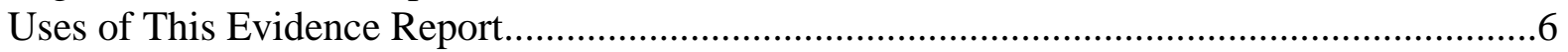

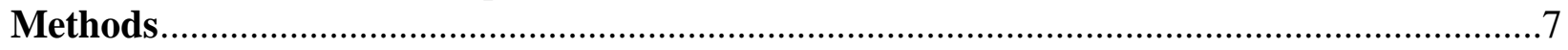

Topic Surveillance and Review Protocol............................................................................

Literature Search Strategy ..............................................................................................

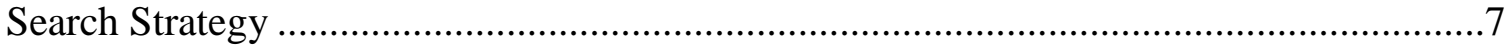

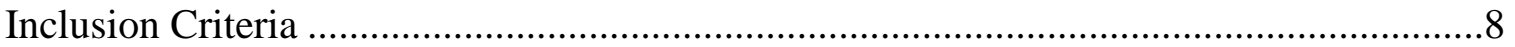

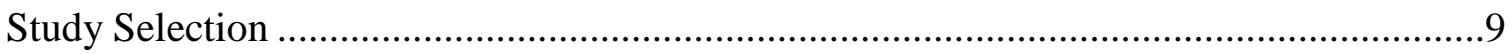

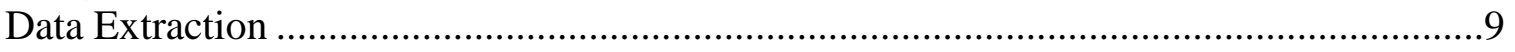

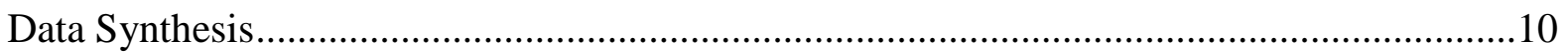

Risk of Bias Assessment of Individual Studies ...................................................................10

Determining Overall Risk of Bias Ratings …………......................................................10

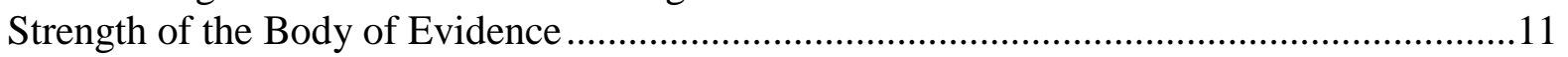

Applicability .................................................................................................................12

Peer Review and Public Commentary ……………….....................................................12

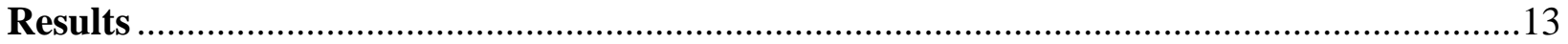

Results of Literature Searches for Key Questions …………..................................................13

Description of Included Studies....................................................................................14

Gray Literature.............................................................................................................15

KQ1. Benefits and Harms of Medical Treatments................................................................16

Studies of Antipsychotics ........................................................................................16

Studies of Medications Used To Treat ADHD ..............................................................38

Studies of Combined Medical and Behavioral Treatments ................................................50

Studies of Nutritional Supplements or Specialized Diets ...................................................52

Studies of Risperidone Adjuncts.........................................................................................5

Studies of Hyperbaric Oxygen Therapy …………………............................................60

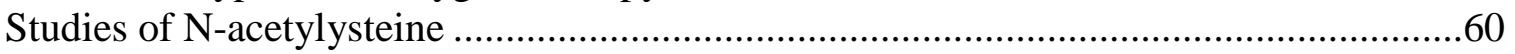

Studies of Tetrahydrobiopterin ......................................................................................65

Studies of Other Medical Interventions ........................................................................68

KQ2. Modifiers of Treatment Outcomes ................................................................................71

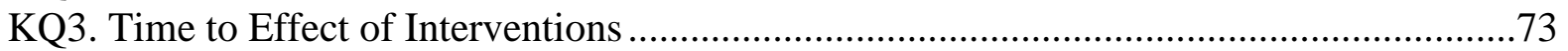

KQ4. Evidence That Effects Measured at the End of Treatment Predict Long-Term

Functional Outcomes ..............................................................................................................

KQ5. Effectiveness Across Environments or Contexts ...........................................................73

KQ6. Drivers of Treatment Outcomes.............................................................................74

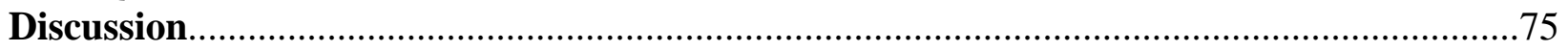




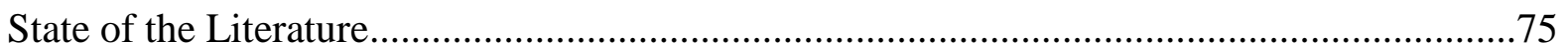

Summary of Key Findings and Strength of the Evidence....................................................76

Key Question (KQ) 1. Benefits and Harms of Medical Treatments...................................76

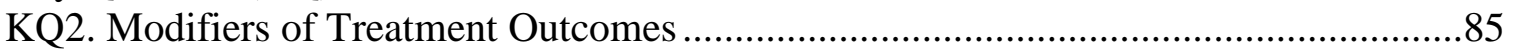

KQ3. Time to Effect of Interventions ………………….................................................

KQ4. Evidence That Effects Measured at the End of Treatment Predict Long-Term

Functional Outcomes ………………………………….............................................85

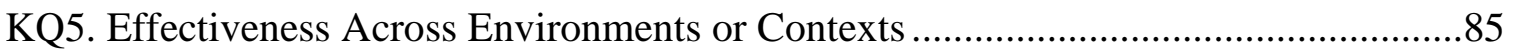

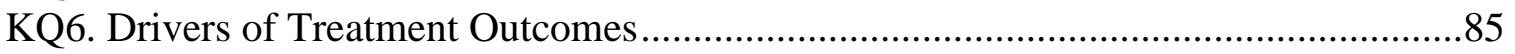

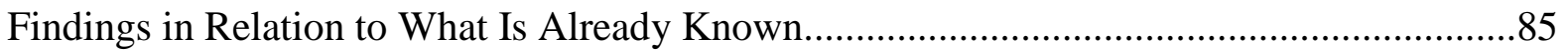

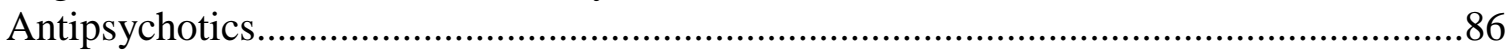

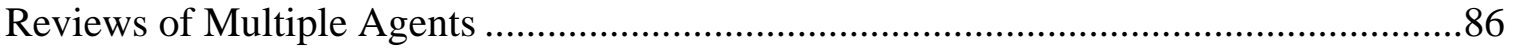

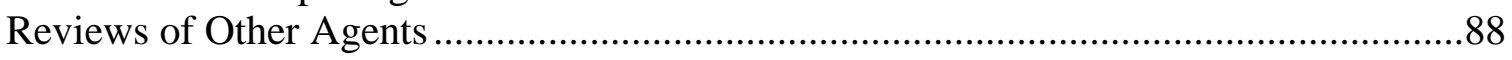

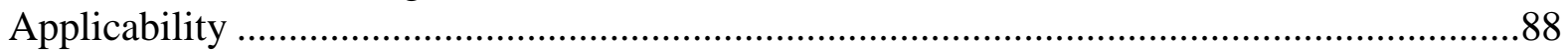

Implications for Clinical and Policy Decisionmaking ..............................................................90

Limitations of the Comparative Effectiveness Review Process ...............................................90

Limitations of the Evidence Base ........................................................................................

Research Gaps and Areas for Future Research....................................................................91

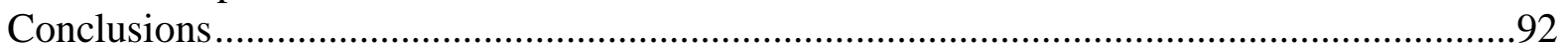

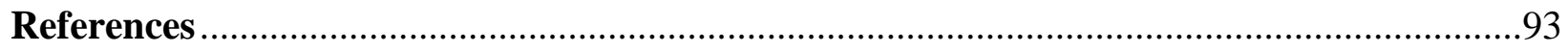

Acronyms and Abbreviations ......................................................................................105

\section{Tables}

Table A. Inclusion criteria

Table B. Summary of evidence in studies addressing medical interventions for children with ASD.

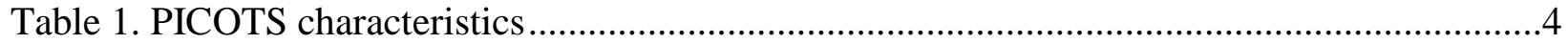

Table 2. Inclusion criteria .....................................................................................................

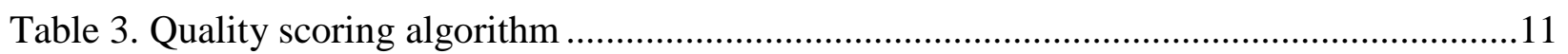

Table 4. Domains used to assess strength of evidence ................................................................12

Table 5. Overview of studies ................................................................................................14

Table 6. Key outcomes targeted in studies of medical interventions ............................................15

Table 7. Key outcomes in studies comparing risperidone and placebo ..........................................20

Table 8. Key outcomes in studies comparing aripiprazole and placebo .........................................26

Table 9. Key outcomes in studies comparing risperidone and aripiprazole ..................................30

Table 10. Harms/adverse events reported by study phase in RUPP risperidone studies ................33

Table 11. Harms/adverse effects in other studies of risperidone ......................................................33

Table 12. Harms/adverse events reported by study phase in aripiprazole "family" studies...........36

Table 13. Harms/adverse effects in other studies of aripiprazole......................................................37

Table 14. Key outcomes in studies of ADHD medications ........................................................41

Table 15. Harms/adverse effects in studies of methylphenidate.....................................................46

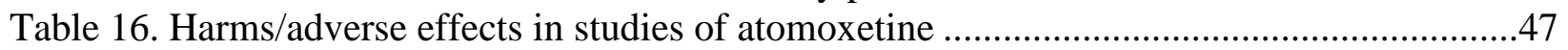

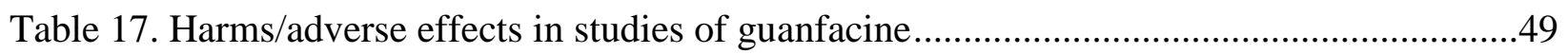

Table 18. Key outcomes in studies of omega-3 fatty acids ..........................................................53

Table 19. Key outcomes in studies of $\mathrm{N}$-acetylcysteine ..................................................................62

Table 20. Harms/adverse effects reported in studies of $\mathrm{N}$-acetylcysteine .....................................65 
Table 21. Key outcomes in studies of tetrahydrobiopterin .66

Table 22. Harms/adverse effects reported in studies of tetrahydrobiopterin ..................................68

Table 23. Strength of evidence for effectiveness of antipsychotics versus placebo ........................77

Table 24. Strength of evidence for effects of medications to treat ADHD......................................79

Table 25. Strength of evidence for effects of omega-3 supplementation ........................................82

Table 26. Strength of evidence for effects of $\mathrm{N}$-acetylcysteine.....................................................83

Table 27. Strength of evidence for tetrahydrobiopterin .................................................................84

\section{Figures}

Figure A. Analytic framework ............................................................................................

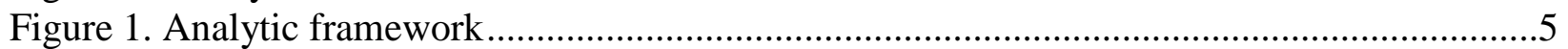

Figure 2. Disposition of studies identified for this review …………............................................13

\section{Appendixes}

Appendix A. Search Strategies

Appendix B. Screening and Risk of Bias Assessment Forms

Appendix C. Excluded Studies

Appendix D. Risk of Bias Ratings

Appendix E. Applicability Summary Tables

Appendix F. Detailed Tables of Findings 


\section{Executive Summary}

\section{Introduction}

Autism spectrum disorder (ASD) is a neurodevelopmental disorder broadly defined by impaired social communication as well as restricted or repetitive patterns of behavior and interest. As defined by the Diagnostic and Statistical Manual of Mental Disorders, Fifth edition (DSM-5), specific features of ASD include deficits in social and emotional reciprocity (e.g., atypical social approaches, conversational impairment, atypical sharing of interests, attention, and affect); deficits in nonverbal communication (e.g., poorly integrated verbal and nonverbal communication, atypical body-language and gesture use, deficits in use and understanding of nonverbal communication), and deficits in maintaining appropriate relationships (e.g., challenges with peer interest, vulnerabilities forming friendships, difficulties adjusting behavior to suit social contexts) as well as restricted and repetitive patterns of behavior such as stereotyped speech, motor movements, or use of objects; excessive adherence to routine or insistence on sameness; intense interest patterns; and atypical sensory interests or responses. Symptoms of the disorder impair and limit everyday functioning and are thought to be evident in early childhood; although they may not be fully evident until later ages. Although not core symptoms, many children with ASD may also have significant cognitive and language impairments.

\section{Treatment of ASD}

The manifestation and severity of symptoms of ASD differ widely, and treatments include a range of behavioral, psychosocial, educational, medical, and complementary approaches ${ }^{1-4}$ that vary by a child's age and developmental status. The goals of treatment for ASD typically focus on improving core deficits in communication, social interactions, or restricted behaviors, as changing these fundamental deficits may help children develop greater functional skills and independence. ${ }^{5}$ Treatment frequently is complicated by symptoms or comorbidities that may warrant targeted intervention. Individual goals for treatment vary for different children and may include combinations of approaches such as behavioral and medical therapies; parents may also pursue complementary and alternative medicine therapies.

The antipsychotics risperidone (Risperdal) and aripiprazole (Abilify) have been specifically approved by the U.S. Food and Drug Administration (FDA) for treatment of irritability and challenging behaviors in ASD. Many other medications are used off -label to manage behavioral symptoms such as anxiety and hyperactivity. In addition, devices such as hyperbaric oxygen chambers may be used to treat symptoms of ASD.

\section{Scope and Key Questions (KQs)}

\section{Scope of the Review}

This review updates findings reported in the 2011 AHRQ review Therapies for Children with $\mathrm{ASD}^{6}$ with a focus on studies of medical interventions. We defined medical interventions broadly as interventions involving the administration of external substances to the body or use of external, nonbehavioral procedures to treat symptoms of ASD, which includes pharmacologic agents, diet therapies, vitamins and supplements, chelating agents, electroconvulsive therapy, transcranial magnetic stimulation and hyperbaric oxygen, among other modalities. We used this broad definition, developed with input from our clinical experts, in order to capture the landscape 
of medically-related interventions used to treat children with ASD. A companion review updating findings related to interventions targeting sensory challenges is available on the AHRQ Effective Health Care Web site.

\section{Key Questions}

We developed KQs in consultation with Key Informants and Task Order Officers. KQs were posted for review to the AHRQ Effective Health Care Web site.

KQs were as follows:

KQ1: Among children ages 2-12 with ASD, what is the comparative effectiveness (benefits and harms) of medical treatments?

a. What are the effects on core symptoms (e.g., deficits in social communication and interaction; restricted, repetitive patterns of behavior, interests, or activities including hyper- or hypo- reactivity to sensory input or unusual interest in sensory aspects of the environment) in the short term (<6 months)?

b. What are the effects on commonly associated symptoms (e.g., motor, medical, mood/anxiety, irritability, and hyperactivity) in the short term (<6 months)?

c. What are the longer term effects ( $\geq 6$ months) on core symptoms (e.g., social deficits, communication deficits, and repetitive behaviors)?

d. What are the longer term effects ( $\geq 6$ months) on commonly associated symptoms (e.g., motor, medical, mood/anxiety, irritability, and hyperactivity)?

KQ2: Among children ages 2-12 with ASD, what are the modifiers of outcome for different medical treatments?

a. Is the effectiveness of the therapies reviewed affected by the frequency, duration, intensity, or dose of the intervention?

b. Is the effectiveness of the therapies reviewed affected by cointerventions or prior treatment, or the training and/or experience of the individual providing the therapy?

c. What characteristics (e.g., age, symptom severity), if any, of the child modify the effectiveness of the therapies reviewed?

d. What characteristics, if any, of the family modify the effectiveness of the therapies reviewed?

KQ3: What is the time to effect of medical interventions? 
KQ4: What is the evidence that effects measured at the end of the treatment phase predict long-term functional outcomes of medical interventions?

KQ5: Is the effectiveness of medical interventions maintained across environments or contexts (e.g., people, places, materials)?

KQ6: What evidence supports specific components of treatment with medical interventions as driving outcomes, either within a single treatment or across treatments?

\section{Analytic Framework}

The analytic framework (Figure A) illustrates the population, interventions, and outcomes that guided the literature search and synthesis.

Figure A. Analytic framework

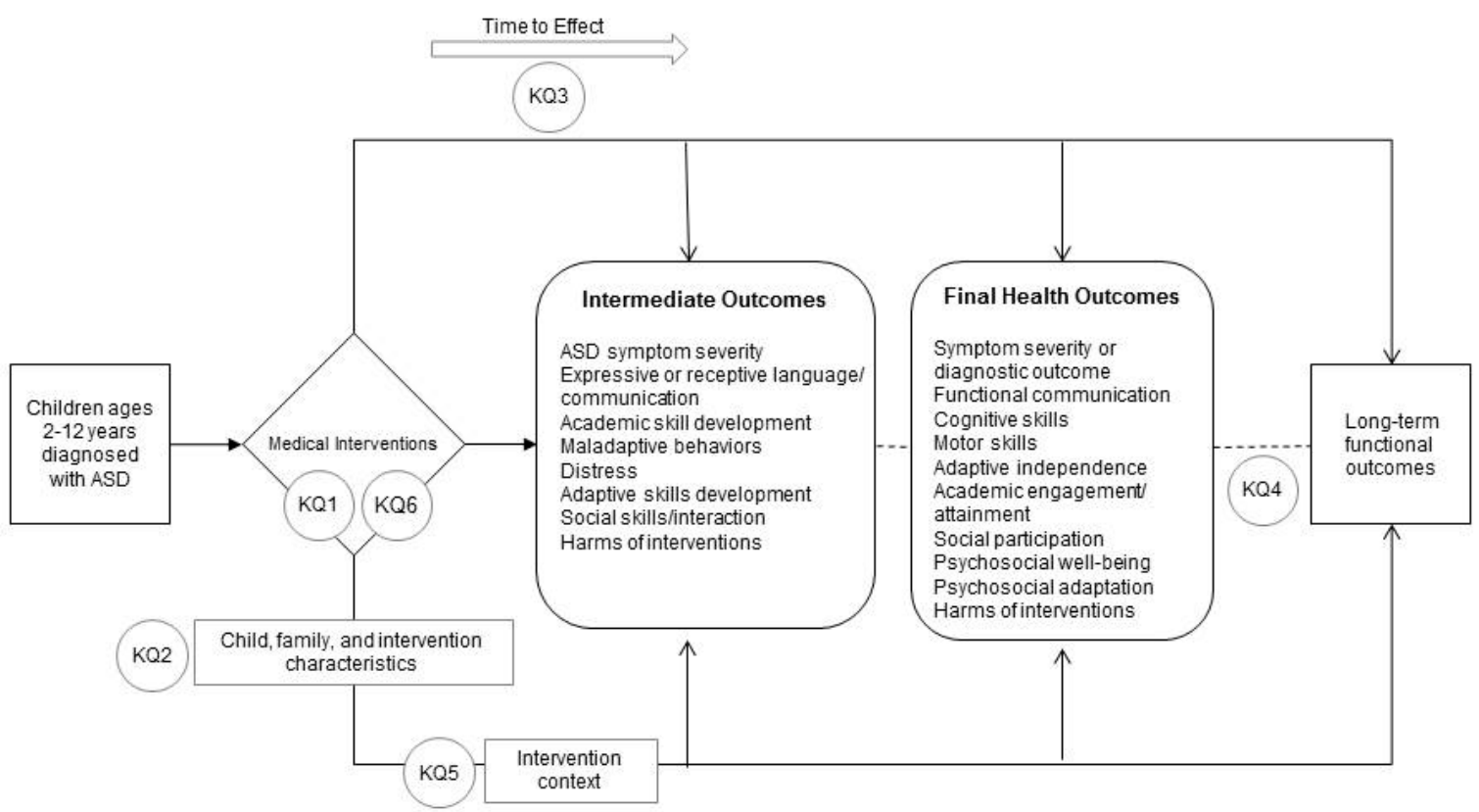

ASD=autism spectrum disorder; KQ=Key Question 


\section{Methods}

\section{Topic Surveillance}

The topic for a 2011 report on therapies for children with $\mathrm{ASD}^{6}$ was nominated by Autism Speaks in a public process using the Effective Health Care Web site. AHRQ published an update addressing behavioral interventions in $2014 .^{7}$ We conducted a surveillance process to assess the need to update the earlier report by contacting topic experts about the relevance of the KQs and new evidence that may address them. In consultation with clinical experts and stakeholders, and based on our preliminary scan of the literature and surveillance findings, we focused the review update on medical approaches and approaches to address sensory challenges (reported in a separate update). These areas reflect both areas of clinical relevance and sufficient newly published literature for a review update.

\section{Literature Search Strategy}

To ensure comprehensive retrieval of relevant studies of medical therapies for children with ASD, we used four key databases: the MEDLINE ${ }^{\circledR}$ medical literature database via the PubMed ${ }^{\circledR}$ interface; EMBASE (Excerpta Medica Database); the Cumulative Index of Nursing and Allied Health Literature (CINAHL); and PsycINFO ${ }^{\circledR}$. Search strategies applied a combination of controlled terms and key words. We last conducted searches for the review in September 2016.

We hand searched the reference lists of recent systematic reviews or meta-analyses of studies addressing therapies for ASD. The investigative team also scanned the reference lists of studies included after the full-text review phase for additional potentially relevant studies.

\section{Inclusion Criteria}

Table A lists our inclusion criteria. We focused the review on children between 2 and 12 years of age. We chose to limit the age range to this span because a) diagnosis of ASD earlier than age 2 is less established and b) adolescents likely have substantially different challenges and would warrant different interventions than children in the preschool, elementary, and middle school age groups.

Table A. Inclusion criteria

\begin{tabular}{|l|l|}
\hline Category & Criteria \\
\hline Study population & $\begin{array}{l}\text { Children ages 2-12 with ASD (mean age plus standard deviation is } \leq 12 \text { years and } 11 \\
\text { months) }\end{array}$ \\
\hline Publication languages & English only \\
\hline $\begin{array}{l}\text { Admissible evidence } \\
\text { (study design and other } \\
\text { criteria) }\end{array}$ & $\begin{array}{l}\text { Admissible designs } \\
\text { Randomized controlled trials, prospective and retrospective cohort studies with } \\
\text { comparison groups, and nonrandomized controlled trials }\end{array}$ \\
& $\begin{array}{l}\text { Other criteria } \\
\text { Original research studies published from 2010-present and not addressed in prior } \\
\text { reviews } \\
\text { Studies must have relevant population and } \geq 20 \text { participants with ASD (non-RCTs) or at } \\
\text { least 10 total participants (RCTs) } \\
\text { Studies must address one or more of the following for ASD: } \\
\text {-Outcomes of interest } \\
\text {-Treatment modality of interest }\end{array}$ \\
\hline
\end{tabular}




\begin{tabular}{|l|l|}
\hline Category & Criteria \\
\hline & -Predictors or drivers of treatment outcomes (e.g., biomarkers, clinical changes) \\
& -Maintenance of outcomes across environments or contexts \\
& -Sufficiently detailed methods and results to enable data extraction \\
& -Reporting of outcome data by target population or intervention \\
\hline
\end{tabular}

ASD = autism spectrum disorder; RCT = randomized controlled trial

\section{Study Selection}

Two reviewers independently assessed each abstract and the full text of studies proceeding to full text review. A senior reviewer adjudicated disagreements in full text review .

\section{Data Extraction and Synthesis}

Data were initially extracted by one team member and reviewed for accuracy by a second. We summarized data for KQs qualitatively using summary tables as studies were too heterogeneous to allow for meta-analyses.

\section{Risk-of-Bias Assessment of Individual Studies}

We evaluated the overall methodologic risk of bias of individual studies using the ASDspecific assessment approach developed and used in our prior reviews of interventions for ASD and informed by the Methods Guide for Effectiveness and Comparative Effectiveness Reviews. ${ }^{8}$ Two senior investigators assessed each included study independently with disagreements resolved through discussion. Appendix D of the main report includes ratings for each study.

\section{Strength of the Body of Evidence}

Two senior investigators graded the strength of the evidence (SOE) for key intervention/outcome pairs using methods based on the Methods Guide for Effectiveness and Comparative Effectiveness Reviews. ${ }^{8}$ We assessed the domains of study limitations (low, medium, high level of limitation), consistency (inconsistency not present, inconsistency present, unknown), directness (direct, indirect), precision (precise, imprecise), and reporting bias (detected, unsuspected). The full team reviewed the final SOE designations. The possible grades were:

- High: High confidence that the evidence reflects the true effect. Further research is unlikely to change estimates.

- Moderate: Moderate confidence that the evidence reflects the true effect. Further research may change our confidence in the estimate of effect and may change the estimate.

- Low: Low confidence that the evidence reflects the true effect. Further research is likely to change confidence in the estimate of effect and is also likely to change the estimate.

- Insufficient: Evidence is either unavailable or does not permit a conclusion. ${ }^{9}$

\section{Applicability}

We assessed the applicability of findings reported in the included literature addressing our KQs to the general population of children with ASD by determining the population, intervention, comparator, and setting in each study and developing an overview of these elements for each intervention category. We anticipated that areas in which applicability would be especially important to describe would include ASD severity, comorbidities, age at treatment, and 
intervention characteristics such provider, dosing/intensity, and setting. Applicability tables are in Appendix E of the full report.

\section{Results}

We identified 6583 nonduplicative titles or abstracts with potential relevance, with 554 proceeding to full text review. We excluded 469 studies at full text review. We included 68 unique studies (85 publications) in the review. In addition to these 68 studies published since the completion of our original review of therapies for children with ASD in 2011, we include 12 comparative studies addressed in the 2011 review that also addressed an agent reported on in the current review. Four studies included in the 2011 review now include followup analyses published since the completion of that report; thus we describe a total of 76 studies in the review.

The 76 studies included in the review comprised 72 randomized controlled trials (RCTs), 2 nonrandomized trials, and 2 retrospective cohort studies. Studies addressed the following categories:

- Antipsychotics: 11 RCTs and one retrospective cohort study (n=1055 children) with low $(n=7)$ and moderate $(n=5)$ risk of bias.

- Medications to treat attention deficit hyperactivity disorder (ADHD): Five RCTs ( $\mathrm{n}=265$ children) with low ( $n=4)$ and moderate $(n=1)$ risk of bias.

- Combination medical and behavioral treatments: Three RCTs and two nonrandomized trials ( $n=419$ children) with low $(n=2)$, moderate $(n=1)$ and high $(n=2)$ risk of bias.

- Nutritional supplements and dietary interventions: 19 RCTs ( $n=732$ children) with low $(n=4)$, moderate $(n=10)$, and high $(n=5)$ risk of bias.

- Risperidone adjuncts: 14 RCTs ( $n=561$ children) with low $(n=12)$ and moderate $(n=2)$ risk of bias.

- Hyperbaric oxygen therapy: Three RCTs ( $n=150$ children) with low ( $n=2)$ and moderate $(\mathrm{n}=1)$ risk of bias.

- N-acetylcysteine: Two RCTs ( $\mathrm{n}=123$ children) with low and moderate risk of bias.

- Tetrahydrobiopterin: Two RCTs ( $n=56$ children) with low and moderate risk of bias.

- Other interventions: 13 RCTs and 1 retrospective cohort study ( $\mathrm{n}=829$ children) with low $(n=6)$, moderate $(n=7)$, and high $(n=1)$ risk of bias. We categorized studies as "other" if we could not assess strength of evidence for interventions and outcomes reported (i.e., insufficient strength of evidence) and the studies did not fall under a broader category of intervention such as diet or nutritional supplements.

Overall, 39 studies had low, 29 had moderate, and 8 had high risk of bias. Despite the high number of low and moderate risk of bias studies, few studies addressed the same interventions or outcomes, and most studies included few participants, evaluated only in the short term $(<6$ months). Thus, evidence for many agents remains insufficient. Because few studies addressed subquestions under Key Questions (KQ) 1 and 2, we present results in the aggregate under each of these KQ.

\section{KQ1. Benefits and Harms of Medical Treatments}

Antipsychotics. Studies of antipsychotics addressed either risperidone or aripiprazole and reported significant improvements in measures of challenging behavior in the short term $(<6$ months) in children receiving the medications compared with those receiving placebo. Harms of 
these agents, including extrapyramidal symptoms and weight gain, were also clinically significant. Studies reporting longer term followup (up to 21 months for risperidone) reported continued effectiveness in most children but did not include control groups.

Medications to treat ADHD. RCTs of methylphenidate, atomoxetine, and guanfacine reported improvements in hyperactivity and other challenging behaviors with treatment compared with placebo. Clinically significant side effects were associated with methylphenidate including aggressive behavior and appetite changes. Harms reported with atomoxetine and guanfacine included irritability, gastrointestinal symptoms, drowsiness, and decreased appetite.

Studies of combined medical and behavioral treatments. In three of the five studies of combined medical and behavioral treatments, the addition of a behavioral therapy (e.g., cognitive behavioral therapy, parent training) did not increase effectiveness over medical therapy alone. In two small trials, bumetanide plus applied behavior analysis improved symptom severity more than applied behavioral analysis alone and stem cell transplantation plus rehabilitation therapy improved symptom severity, lethargy, and stereotypy more than umbilical cord blood cell transplant plus rehabilitation therapy or rehabilitation therapy alone.

Diet and nutritional supplements. Omega-3 fatty acid supplementation did not affect challenging behaviors and was not associated with clinically significant harms. Seven studies addressed variations of the gluten-free diet, but studies addressed different outcomes and different approaches to restricted and control diets. Similarly, a number of RCTs with low or moderate risk of bias addressed other agents, but studies were small and few addressed the same agent or outcomes.

Risperidone adjuncts. Study medications added to risperidone included celecoxib, minocycline, Ginkgo biloba, memantine, topiramate, riluzole, buspirone, $\mathrm{N}$-acetylcysteine (addressed in 2 studies), amantadine , pioglitazone, pentoxifylline, galantamine, and piracetam. Most studies (12 of 14) reported improvements in irritability measured on the Aberrant Behavior Checklist $(\mathrm{ABC})$ in the adjunct groups compared with placebo plus risperidone.

Hyperbaric oxygen therapy. Three RCTs of hyperbaric oxygen used different doses and reported inconsistent outcomes and harms.

$\mathbf{N}$-acetylcysteine. N-acetylcysteine had no effect on social skills outcomes in two small RCTs. Harms of this agent were not clinically significant.

Tetrahydrobiopterin. Tetrahydrobiopterin had no effect on symptom severity and was not associated with clinically significant harms.

Other medical interventions. Few studies addressed the same agent or outcomes. Studies of donepezil, melatonin, bumetanide, citalopram, amantadine, divalproex, prednisolone, and transcranial stimulation reported some positive effects on outcomes including symptom severity, language, and sleep. Studies of oxytocin and mecamylamine reported no statistically significant effects. Harms reported in studies comparing these interventions were diverse, and their clinical significance is difficult to determine. 


\section{KQ2. Modifiers of Treatment Outcomes}

Few studies reported modifiers, and few were likely adequately powered to detect effects. In one subanalysis, higher baseline irritability was associated with greater improvement in irritability than was low severity in improvement with risperidone. Greater weight gain was associated with less irritability improvement in the risperidone group. In another study of risperidone, younger age and better communication skills were associated with greater gains in communication but not with gains in daily living skills or socialization.

Studies of stimulants identified no significant phenotypic predictors of effects (e.g., baseline cognitive skills, age, IQ), but one genetic analysis identified seven genetic variations that predicted response to methylphenidate. Modifiers reported in studies of other agents were varied and included cognitive skills, age, and symptom severity. No characteristics had consistent effects.

\section{KQ3. Time to Effect of Interventions}

While several studies reported changes in the number of children responding to a given agent over time, studies typically did not provide data to determine the initiation of effects.

\section{KQ4. Evidence that Effects Measured at the End of Treatment Predict Long-Term Functional Outcomes}

Few studies had longer term followup and those with more than 6 months of treatment or followup typically did not report functional outcomes. In one study, risperidone use was not associated with changes in IQ: changes from baseline to the end of study in class assignment (e.g., special education, regular classroom) were not significant.

\section{KQ5. Effectiveness Across Environments or Contexts}

Seven studies reported teacher ratings of outcome measures that provide some information to address this KQ, but the limited results preclude conclusions. One RCT of omega-3 fatty acids reported no significant group differences in teacher ratings of challenging behaviors (parents also rated few measures as improved), while another RCT of docosahexaenoic acid (DHA) supplementation versus placebo reported improvement in parent-rated social skills in children receiving placebo vs. those receiving DHA, while teachers rated communication as more improved in the treatment group compared with placebo. An RCT of challenge foods introduced to a gluten-free diet reported no statistically significant changes in behavior as rated by parents or teachers on the Connors scale. An RCT of levetiracetam vs. placebo reported no significant group differences on any parent- or teacher- rated measures but also noted that teachers, but not parents, rated children in the placebo arm as more improved on irritability compared with the levetiracetam group.

RCTs of methylphenidate reported general agreement between parent and teacher ratings of hyperactivity. In one RCT, both parents and teachers considered hyperactivity and impulsive behavior to be significantly improved in the treatment group compared with placebo, but teachers (vs. parents) reported no significant group differences in inattention or oppositional behavior. Finally, one RCT of atomoxetine reported significant teacher-rated improvements in hyperactivity in the atomoxetine group compared with placebo but teacher ratings of cognitive problems/inattention, oppositional behavior, or overall ADHD symptoms did not differ between 
groups. In another RCT comparing atomoxetine alone, atomoxetine + parent training , placebo alone, and placebo + parent training, parents, but not teachers, rated children in active treatment groups as significantly improved on measures of ADHD, inattention, hyperactivity, and oppositional behavior.

\section{KQ6. Drivers of Treatment Outcomes}

We did not identify any studies that provided data to address this KQ.

\section{Discussion}

\section{State of the Literature}

We identified a total of 76 unique comparative studies, primarily ( $n=72)$ RCTs, addressing medical interventions. Most studies were small (median 40 total participants/study) and addressed variable agents. Most studies had placebo comparators, while five compared a pharmaceutical agent to behavioral treatment or combined pharmaceutical and behavioral treatment. Studies were typically of short duration ( $<6$ months, range 4 days to 24 months), with few studies reporting longer term followup after the immediate intervention period.

The methodologic rigor of studies has increased substantially compared with those studies reported in our 2011 review of therapies for children with autism spectrum disorder (ASD). ${ }^{6}$ However, while studies were generally well conducted, evidence remains insufficient for most interventions due to small sample sizes, lack of long term followup, and heterogeneous agents and populations.

Despite the number of new studies, we can make few conclusions beyond those reached in our 2011 review. Evidence supports the effectiveness of antipsychotics in improving challenging behaviors, but with significant harms. Methylphenidate also improves hyperactivity but with significant harms. Evidence is promising for the ADHD medication atomoxetine. More studies have addressed combination approaches, but data are inadequate to draw conclusions. Data were limited and inconsistent for other interventions.

\section{Strength of Evidence}

\section{KQ1. Benefits and Harms of Medical Treatments}

Antipsychotics. Our confidence in the conclusion that risperidone and aripiprazole improve challenging behaviors in the short term ( $<6$ months), with clinically significant harms, is high (high strength of evidence). Behaviors improved in the longer term ( $\geq 6$ months) with these agents compared with placebo, but our confidence in this conclusion is low (low strength of evidence) as only five studies had $\geq 6$ months followup. In studies comparing risperidone and aripiprazole, BMI increased with both drugs over treatment durations of 6 months to more than 2 years, but group differences were not significant. We have low confidence that effects on BMI do not differ between agents given the few studies addressing this outcome (low strength of evidence). Other outcomes (e.g., challenging behaviors, attention) were not consistently addressed; thus we considered strength of evidence insufficient for all other intervention/outcome pairs. Table B outlines findings for all comparisons with greater than insufficient strength of evidence. 
Medications to treat ADHD. Methylphenidate versus placebo improved hyperactivity and was associated with clinically significant harms (Table B). Our confidence in these conclusions is low as studies were small and short term (low strength of evidence). Data were inadequate to assess effects on social communication and oppositional behavior (insufficient strength of evidence). Findings for oppositional behavior were inconsistent in two studies; thus, we could not assess the strength of evidence (insufficient). We considered the evidence inadequate to comment on potential effects on social communication or oppositional behavior (insufficient strength of evidence).

We found positive effects of atomoxetine compared with placebo on hyperactivity in children with ASD and ADHD in the short term ( $<6$ months), with effects maintained over the longer term ( $\geq 6$ months) (Table B). Our confidence in this conclusion is low (low strength of evidence). Atomoxetine was associated with harms considered to be clinically moderate, and our confidence in this conclusion is low (low strength of evidence). Data were inadequate to assess effects on inattention as studies reported inconsistent findings (insufficient strength of evidence).

Data were inadequate in a small study of guanfacine to draw conclusions about effects on any outcomes (insufficient strength of evidence).

Studies of combination medical and behavioral treatments. Given that combination therapies were investigated in single studies, we could not make conclusions about their effects on any outcomes (insufficient strength of evidence).

Nutritional supplements and dietary interventions. Omega-3 fatty acid supplementation and placebo did not affect challenging behaviors. Our confidence in this conclusion is low (low strength of evidence for no effect) (Table B). We also have low confidence in the conclusion that omega-3 supplementation was associated with minimal harms (low strength of evidence).

Despite the number of RCTs with low or moderate risk of bias addressing other agents, evidence was inadequate to make conclusions about all clinical efficacy and harms outcomes because few, small, underpowered studies addressed each diet or supplement (insufficient strength of evidence). Data in two small studies of methyl-B12 were inadequate to draw conclusions (insufficient strength of evidence). While seven studies addressed variations of the gluten-free diet, studies addressed different outcomes and different approaches to restricted and control diets; thus, data were inadequate to make conclusions about the body of evidence (insufficient strength of evidence). Data were inadequate to allow conclusions about the relative effectiveness of other dietary interventions (e.g., camels' milk, challenge foods containing gluten) compared with placebo (insufficient strength of evidence).

Risperidone adjuncts. Data were inadequate to assess effects of risperidone plus adjunctive agents including amantadine, buspirone, celecoxib, memantine, riluzole, Gingko biloba, pioglitazone, or topiramate on any outcome assessed as no study addressed the same adjunctive agent (insufficient strength of evidence). While two RCTs addressed risperidone plus Nacetylcysteine, data are inadequate to comment on effects given the small number of participants and high attrition (insufficient SOE).

Hyperbaric oxygen therapy. Three RCTs of hyperbaric oxygen used different doses and reported inconsistent results. We considered SOE to be insufficient to assess effects. 
N-acetylcysteine. N-acetylcysteine had no effect on social skills outcomes in two small RCTs; harms of this agent were not clinically significant. Our confidence in these conclusions is low (low strength of evidence) (Table B). Data were inadequate to assess effects on other outcomes given inconsistent findings in these two studies (insufficient strength of evidence).

Tetrahydrobiopterin. Tetrahydrobiopterin had no effect on symptom severity and was not associated with clinically significant harms. Our confidence in these conclusions is low (low strength of evidence) (Table B). Data were inadequate to assess effects on other outcomes (insufficient strength of evidence).

Studies of other medical interventions. Data were inadequate to make conclusions about the effects of amantadine, bumetanide, divalproex, oxytocin, mecamylamine, prednisolone, citalopram, melatonin, and neurostimulation vs. placebo as few studies addressed the same agents or outcomes (insufficient strength of evidence).

Table B. Summary of evidence in studies addressing medical interventions for children with ASD

\begin{tabular}{|c|c|c|c|c|}
\hline $\begin{array}{l}\text { Intervention and } \\
\text { comparator }\end{array}$ & $\begin{array}{l}\text { Number/Type } \\
\text { of Studies } \\
\text { (Total N } \\
\text { Participants) }\end{array}$ & $\begin{array}{l}\text { Key } \\
\text { Outcome(s) }\end{array}$ & $\begin{array}{l}\text { Strength of } \\
\text { Evidence (SOE) } \\
\text { Grade }\end{array}$ & Findings \\
\hline \multicolumn{5}{|l|}{ Antipsychotics } \\
\hline \multirow[t]{3}{*}{$\begin{array}{l}\text { Risperidone vs. } \\
\text { placebo }\end{array}$} & 3 RCT (274) & $\begin{array}{l}\text { Challenging } \\
\text { behavior (<6 } \\
\text { months) }\end{array}$ & High SOE & $\begin{array}{l}\text { Significant improvement in treatment } \\
\text { group vs. placebo in } 3 \text { RCTs with } 6-8 \\
\text { week treatment phases; improvement } \\
\text { maintained in } 2 \text { RCTs with } 6 \text { months } \\
\text { of treatment }\end{array}$ \\
\hline & 3 RCT (118) & $\begin{array}{l}\text { Challenging } \\
\text { behavior ( } \geq 6 \\
\text { months) }\end{array}$ & Low SOE & $\begin{array}{l}\text { Improvement maintained in } 1 \mathrm{RCT} \\
\text { with } 6 \text { months of treatment and in one } \\
\text { open label extension with no } \\
\text { comparison group with mean } 21 \\
\text { months treatment duration; in another } \\
\text { open label extension, more children } \\
\text { relapsed with placebo vs. risperidone }\end{array}$ \\
\hline & $\begin{array}{l}9 \text { RCT (262) } \\
1 \\
\text { Retrospective } \\
\text { cohort (72) }\end{array}$ & Harms & $\begin{array}{l}\text { High SOE for } \\
\text { clinically } \\
\text { significant harms } \\
\text { associated with } \\
\text { risperidone }\end{array}$ & $\begin{array}{l}\text { Harms including weight gain, appetite } \\
\text { changes, drowsiness, fatigue, } \\
\text { extrapyramidal symptoms, } \\
\text { drooling/hypersalivation, and } \\
\text { gastrointestinal symptoms } \\
\text { consistently reported }\end{array}$ \\
\hline \multirow[t]{3}{*}{$\begin{array}{l}\text { Aripiprazole vs. } \\
\text { placebo }\end{array}$} & 2 RCT (316) & $\begin{array}{l}\text { Challenging } \\
\text { behavior }(<6 \\
\text { months) }\end{array}$ & High SOE & $\begin{array}{l}\text { Significant improvements in } 2 \text { short- } \\
\text { term RCTs in treatment groups }\end{array}$ \\
\hline & 2 RCT (415) & $\begin{array}{l}\text { Challenging } \\
\text { behavior ( } \geq 6 \\
\text { months) }\end{array}$ & Low SOE & $\begin{array}{l}\text { In longer term followup, no } \\
\text { differences in time to relapse of } \\
\text { symptoms between aripiprazole and } \\
\text { placebo groups in one } 16 \text { week RCT } \\
\text { and continued improvements in ABC } \\
\text { in one } 52 \text {-week open label } \\
\text { continuation with no control arm }\end{array}$ \\
\hline & $\begin{array}{l}4 \text { RCT (422) } \\
1 \text { Retropective } \\
\text { chort (70) }\end{array}$ & Harms & $\begin{array}{l}\text { High SOE for } \\
\text { clinically } \\
\text { significant harms } \\
\text { associated with } \\
\text { aripiprazole }\end{array}$ & $\begin{array}{l}\text { Harms including weight gain, appetite } \\
\text { changes, somnolence, } \\
\text { extrapyramidal symptoms, } \\
\text { drooling/hypersalivation, infection, } \\
\text { and gastrointestinal symptoms } \\
\text { consistently reported }\end{array}$ \\
\hline
\end{tabular}




\begin{tabular}{|c|c|c|c|c|}
\hline $\begin{array}{l}\text { Intervention and } \\
\text { comparator }\end{array}$ & $\begin{array}{l}\text { Number/Type } \\
\text { of Studies } \\
\text { (Total N } \\
\text { Participants) }\end{array}$ & $\begin{array}{l}\text { Key } \\
\text { Outcome(s) }\end{array}$ & $\begin{array}{l}\text { Strength of } \\
\text { Evidence (SOE) } \\
\text { Grade }\end{array}$ & Findings \\
\hline $\begin{array}{l}\text { Risperidone vs. } \\
\text { aripirazole }\end{array}$ & $\begin{array}{l}1 \mathrm{RCT}(37) \\
1 \text { Retropective } \\
\text { cohort }(142)\end{array}$ & BMI change & $\begin{array}{l}\text { Low SOE for no } \\
\text { difference in } \\
\text { effects }\end{array}$ & $\begin{array}{l}\text { BMI increased with both drugs over } \\
\text { treatment durations of } 6 \text { months to } \\
\text { more than } 2 \text { years, but group } \\
\text { differences were not significant }\end{array}$ \\
\hline \multicolumn{5}{|l|}{$\begin{array}{l}\text { Medications to } \\
\text { treat ADHD }\end{array}$} \\
\hline \multirow[t]{3}{*}{ MPH vs. placebo } & 2 RCT (90) & Hyperactivity & Low SOE & $\begin{array}{l}\text { Significant improvement with MPH } \\
\text { compared with placebo on parent and } \\
\text { teacher-rated measures; differential } \\
\text { effect of dose not clear (little effect on } \\
1 \text { study and linear effect in another); } \\
\text { SOE is low given small sample size } \\
\text { and lack of long-term followup }\end{array}$ \\
\hline & 2 RCT (90) & $\begin{array}{l}\text { Oppositional } \\
\text { behavior }\end{array}$ & $\begin{array}{l}\text { Low SOE for no } \\
\text { effect }\end{array}$ & $\begin{array}{l}\text { Significant improvement with MPH on } \\
\text { parent-rated measure at medium } \\
\text { dose level only in } 1 \mathrm{RCT} \text {; no } \\
\text { differences on teacher-rated } \\
\text { measures. No differences in teacher-, } \\
\text { parent-, or clinician-rated measures in } \\
\text { another RCT }\end{array}$ \\
\hline & 2 RCT (90) & Harms & $\begin{array}{l}\text { Low SOE for } \\
\text { association of } \\
\text { MPH with clinically } \\
\text { significant harms }\end{array}$ & $\begin{array}{l}\text { Rates of children experiencing harms } \\
\text { ranged from } 0-75 \% \text {; higher rates } \\
\text { reported for repetitive behaviors or } \\
\text { speech, loss of appetite, and } \\
\text { irritability. Irritability responsible for } \\
\text { withdrawals }(n=6) \text { in one RCT; SOE } \\
\text { is low given small sample size }\end{array}$ \\
\hline \multirow[t]{2}{*}{$\begin{array}{l}\text { Atomoxetine vs. } \\
\text { placebo }\end{array}$} & 2 RCT (113) & $\begin{array}{l}\text { Hyperactivity ( } \leq \\
3 \text { months) }\end{array}$ & $\begin{array}{l}\text { Low SOE for } \\
\text { improvements in } \\
\text { the short-term }\end{array}$ & $\begin{array}{l}\text { Significant improvements in rating of } \\
\text { hyperactivity in treatment group } \\
\text { compared with placebo in both } \\
\text { studies }\end{array}$ \\
\hline & 3 RCT (241) & Harms & $\begin{array}{l}\text { Low SOE for } \\
\text { clinically moderate } \\
\text { harms associated } \\
\text { with atomoxetine }\end{array}$ & $\begin{array}{l}\text { No serious adverse events reported; } \\
\text { most harms attenuated over open } \\
\text { label extension phase }\end{array}$ \\
\hline \multicolumn{5}{|l|}{ Other agents } \\
\hline \multirow[t]{2}{*}{$\begin{array}{l}\text { Omega-3 } \\
\text { supplementation } \\
\text { vs. placebo }\end{array}$} & \multirow[t]{2}{*}{3 RCT (119) } & $\begin{array}{l}\text { Challenging } \\
\text { behaviors }\end{array}$ & $\begin{array}{l}\text { Low SOE for no } \\
\text { effect }\end{array}$ & $\begin{array}{l}\text { No significant differences between } \\
\text { groups in three small, short-term } \\
\text { RCTs }\end{array}$ \\
\hline & & Harms & $\begin{array}{l}\text { Low SOE for } \\
\text { minimal harms }\end{array}$ & $\begin{array}{l}\text { No clinically significant harms } \\
\text { reported in any study }\end{array}$ \\
\hline \multirow[t]{2}{*}{$\begin{array}{l}\mathrm{N} \text {-acetylcysteine } \\
\text { vs. placebo }\end{array}$} & \multirow[t]{2}{*}{2 RCT (127) } & Social skills & $\begin{array}{l}\text { Low SOE for lack } \\
\text { of effect }\end{array}$ & $\begin{array}{l}\text { No significant effects in either small, } \\
\text { short-term RCT }\end{array}$ \\
\hline & & Harms & $\begin{array}{l}\text { Low SOE for } \\
\text { minimal harms }\end{array}$ & $\begin{array}{l}\text { No study reported harms considered } \\
\text { clinically important }\end{array}$ \\
\hline \multirow{2}{*}{$\begin{array}{l}\text { Tetra- } \\
\text { hydrobiopterin } \\
\text { vs. placebo }\end{array}$} & \multirow[t]{2}{*}{2 RCT (54) } & $\begin{array}{l}\text { Symptom } \\
\text { severity }\end{array}$ & $\begin{array}{l}\text { Low SOE for lack } \\
\text { of effect }\end{array}$ & $\begin{array}{l}\text { No significant effects in either small, } \\
\text { short-term RCT }\end{array}$ \\
\hline & & Harms & $\begin{array}{l}\text { Low SOE for } \\
\text { minimal harms }\end{array}$ & $\begin{array}{l}\text { No study reported harms considered } \\
\text { clinically important }\end{array}$ \\
\hline
\end{tabular}

ADHD = attention deficit hyperactivity disorder; ASD = autism spectrum disorder; BMI = body mass index; $\mathrm{MPH}=$ methylphenidate; $\mathrm{RCT}=$ randomized controlled trial; $\mathrm{SOE}=$ strength of evidence

\section{Other Key Questions}

Few studies reported modifying characteristics, and no characteristics were consistent modifiers. Few studies reported data to assess time to effect of interventions. Few studies had 
longer-term followup and those few with 6 months or more of treatment or followup typically did not report functional outcomes; thus our understanding of whether effects at the end of treatment predict functional outcomes is limited. Four studies reported teacher ratings of outcome measures that provide some information to address effectiveness of treatments across environments or contexts, but the limited results preclude conclusions. Finally, we did not identify studies that provided data to address drivers of treatment outcomes.

\section{Applicability}

Study participants were generally recruited from specialty clinical service programs and represent non-primary care populations. As such, families of these children may be seeking a higher level of care than those of the broader population of children with ASD based upon more severe or acute symptoms, including aggression or other challenging behaviors. Most studies of medical interventions targeted elementary school aged and older children with autism, with little data on the treatment of younger children. Most studies included majority male populations (consistent with the male prevalence of ASD).

Studies also included children with highly variable severity of challenging behaviors, ASD symptom severity, and cognitive impairment. Studies of pharmacological agents often sampled children with high levels of specific symptom patterns (e.g., children with severe challenging behavior at baseline where parents may be willing to pursue pharmacologic intervention and trial participation) who may not reflect the wider population of children with ASD in whom these challenges may not be present. Most of the studies reported including children with at least moderate level of severity of ASD. Studies of stimulants included children with cognitive impairment and with comorbidities including attention deficit hyperactivity disorder, oppositional defiant disorder, and obsessive compulsive disorder. Studies of other approaches had similarly heterogeneous populations. Dietary and nutritional studies included some younger children, with severity of autism not well described or the degree of intellectual functioning not well characterized in most studies. This heterogeneity in population characteristics may limit the generalizability of findings to children with differing levels of symptom expression or comorbidities but likely reflects the heterogeneity of the broader population of children with ASD.

Studies addressed a variety of agents and typically reported use of concurrent medications or other therapies. Most agents studied are accessible in the United States albeit with few receiving FDA approval for use. Comparators among nonplacebo controlled studies varied, and few studies assessed the effect of concomitant behavioral or other therapies, though many children with ASD receive multiple interventions. The treatments studied may not adequately reflect the broad range of treatment combinations used in the general population of children with ASD.

As noted, few studies evaluated longer term treatment ( $\geq 6$ months); short treatment and followup periods limit our ability to understand potential longer term outcomes such as academic achievement or longer term harms.

Overall, the heterogeneity of these studies parallels the heterogeneity of children with ASD, and some findings may be more applicable to children with specific levels of baseline severity or comorbidities. These limitations to generalizability likely reflect both the significant heterogeneity of ASD itself as well as its associated features, such as irritability. Thus, while there is a growing evidence base for treating certain symptoms in certain populations, these findings underscore the continued need for individualized treatment approaches that are informed by the emerging evidence base for benefits as well as harms of medical intervention, 
with careful consideration of symptom presentation and functioning level relative to study populations and applicability of the known literature.

\section{Limitations of the Comparative Effectiveness Review Process}

We included studies published in English only and did not include unpublished data. In our scan of a random sample of 150 non-English abstracts retrieved by our MEDLINE search, only two studies appeared to meet inclusion criteria; thus, given the high percentage of ineligible items in this scan (99\%), we concluded that excluding non-English studies would not introduce significant bias into the review. We recognize that this preliminary scan did not address the entire corpus of ASD literature in other languages.

We also included only comparative studies of medical interventions with at least 10 children with ASD. To ensure comprehensive coverage of the literature, we included comparative studies with a smaller sample size that would have been excluded in our 2011 review (which required a sample size of 30) in the present report. We did not conduct a de novo search for such studies but re-examined the excluded studies from the prior review. This approach may have overlooked relevant studies.

Given heterogeneity in treatment regimens, outcomes addressed in each study, and patient populations, we were limited in our ability to meta-analyze findings or identify potential subgroups that may respond more favorably to specific treatments. Finally, we used a nonvalidated tool to assess risk of bias, though we note that the tool evaluates similar constructs to those assessed in tools such as that used by the Cochrane Collaboration, with the addition of ASD-specific domains.

\section{Limitations of the Evidence Base}

As noted, studies in the review had small sample sizes and typically limited duration of intervention and followup after intervention, despite significant improvements in study design and execution over time. Populations across studies were heterogeneous in terms of challenging behaviors, ASD symptom severity, age, and comorbidities. Few studies addressed the same agent and outcomes, and few assessed potential factors that may modify effectiveness or drive effects of interventions. Many ( $n=63)$ studies also explicitly noted that concomitant interventions were held steady during the study treatment period; however, few studies reported specific analyses to control for or assess the effects of additional treatments.

Despite these limitations, investigators have made significant improvements in incorporating commonly used measures of symptom severity and behavior to facilitate comparisons across studies. Studies also typically described interventions fully, used standardized diagnostic processes and blinded assessors, and reported on the use or restriction of concomitant interventions.

\section{Implications for Clinical and Policy Decisionmaking}

This review provides some evidence for decisionmaking about medical interventions for children with ASD. The clearest evidence favors the use of the antipsychotics risperidone and aripiprazole to address challenging behaviors in the short-term ( $<6$ months); however, clinicians and caregivers must balance the significant harms of these agents. The significant side effect profiles make it clear that although these drugs are efficacious, caution is warranted regarding their use in patients without severe impairments or risk of injury. Few studies addressed longer 
term effects of these agents; thus, our confidence in longer term ( $\geq 6$ months) effectiveness is low. Studies of adjuncts to risperidone typically reported positive effects on challenging behaviors, but few studies addressed the same agents, precluding our ability to draw conclusions about their effectiveness.

Some evidence supports the use of methylphenidate and atomoxetine for hyperactivity, but few comparative studies addressed each agent, so our confidence in effects is limited. Given that many children with ASD are currently treated with medical interventions, strikingly little evidence exists to support clear benefit for most medical interventions, especially in the realm of interventions such as restrictive diets and supplements. Studies of nutritional supplements or specialized diets were typically underpowered and provided little evidence of effects of these approaches. Several agents were addressed in single studies, which limits conclusions about their effects.

Decisional dilemmas remain regarding characteristics of the child, family, or intervention that may modify effectiveness or predict which children may be most likely to benefit from a given approach. Similarly, the literature base is currently insufficient to inform our understanding of the time to effect of interventions, longer term effectiveness of interventions, generalizability of effects outside the treatment context, effectiveness and applicability to broader ASD populations, and components that may drive effectiveness.

\section{Research Gaps and Areas for Future Research}

Improving research in this area should include methodologic considerations of power and sample size and durability of effects. Sample size and participant followup were frequently insufficient to allow firm conclusions. Duration of treatment and followup were generally short ( $<6$ months); those studies with longer duration of treatment were typically open label extensions of RCTs and lacked control arms. While duration was typically short, retaining participants in studies, especially in placebo arms, is difficult when parents or children perceive little improvement in symptoms. Longer duration of treatment, however, is also important to rule out meaningful improvements in placebo groups and help inform our understanding of the placebo effect.

Few studies provided data on long-term outcomes after cessation of treatment. Future studies should extend the followup period and assess the degree to which outcomes are durable in "real world" situations. The literature includes many single studies of various agents. Studies of adjuncts to risperidone, for example, examined different adjunct agents, with some positive effects on challenging behaviors reported with most. Understanding which agents should be examined further is lacking. Another critical area for further research is identifying which children are likely to benefit from particular interventions. To date, studies have provided limited characterization of the subpopulation of children who experience positive response to medical interventions and limited characterization of the extent or type of behavioral challenges children experience at baseline.

Children with ASD also typically receive multiple types of therapies, but few studies addressed combinations of medical and behavioral or other categories of interventions or a medical treatment compared with a nonmedical treatment. Few attempted to account for potential effects on ongoing interventions. This not only limited our ability to interpret the effects of medical treatments in isolation but represents a significant gap for families and providers in choosing additional treatments that may bolster (or impair) the effects of behavioral, medication, or other therapies. Few studies $(n=10)$ compared active treatments, and future 
research to assess comparative effectiveness of antipsychotics, ADHD medications, and other medications is necessary.

In addition, much of the medical intervention literature relies on baseline and outcome measures that have specific limits in understanding individualized response. Future research attempting to elucidate potential biobehavioral markers of response may prove useful. Research in understanding outcomes of importance to patients and caregivers, such as quality of life, is also lacking.

Harms reporting varied across studies; some studies amply described how harms were tracked, while others listed harms with no indication of how they were assessed (e.g., parent recall, checklist, clinician assessment during followup). This lack of reporting makes comparing harms across studies difficult. For instance, while studies of atomoxetine generally reported fewer harms than did studies of methylphenidate in children with ADHD symptoms, exploring differences in safety profiles is an important area for additional research.

Harms reporting varied across studies; some studies amply described how harms were tracked, while others listed harms with no indication of how they were assessed (e.g., parent recall, checklist, clinician assessment during followup). This lack of reporting makes comparing harms across studies difficult. For instance, while studies of atomoxetine generally reported fewer harms than did studies of methylphenidate in children with ADHD symptoms, exploring differences in safety profiles is an important area for additional research.

\section{Conclusions}

Risperidone and aripiprazole ameliorated challenging behaviors in the short term $(<6$ months), but had clinically significant side effects. Methylphenidate and atomoxetine were also associated with improvements in hyperactivity in small, short-term RCTs (with uncontrolled open label extensions). Atomoxetine plus parent training was not more effective for hyperactivity than atomoxetine alone. Omega-3 fatty acid supplementation was not associated with improvements in challenging behaviors, and $\mathrm{N}$-acetylcysteine and tetrahydrobiopterin were not associated with improvements in social skills and symptom severity, respectively. Some positive effects were reported with other agents studied (risperidone adjuncts, melatonin), but few studies addressed the same agent or outcomes. Data on longer term ( $\geq 6$ months) results and harms of interventions are lacking. Similarly, more research is needed to understand characteristics of the child or treatment that modify outcomes and whether effectiveness of interventions generalizes across different settings such as the home or school. Current evidence also does not inform our understanding of components of interventions that may drive effects. Some therapies hold promise and warrant further study, and the conduct of studies has improved considerably over time (i.e., growing number of RCTs and use of standardized measures). However, additional studies with larger, well-characterized populations over longer time frames, and that utilize transparent and rigorous methods that permit comparison across studies, would further inform decisionmaking. 


\section{References}

1. Ospina MB, Krebs Seida J, Clark B, et al. Behavioural and developmental interventions for autism spectrum disorder: a clinical systematic review. PLoS One.

2008;3(11):e3755. doi:

10.1371/journal.pone.0003755 [doi]. PMID: 19015734.

2. Seida JK, Ospina MB, Karkhaneh M, et al. Systematic reviews of psychosocial interventions for autism: an umbrella review. Dev Med Child Neurol. 2009 Feb;51(2):95-104. doi: DMCN3211 [pii] conco10.1111/j.1469-8749.2008.03211.x [doi]. PMID: 19191842.

3. Parr J. Autism. BMJ Clin Evid. 2010;2010 PMID: 21729335.

4. Wan Yunus F, Liu KP, Bissett M, et al. Sensory-Based Intervention for Children with Behavioral Problems: A Systematic Review. J Autism Dev Disord. 2015 Nov;45(11):3565-79. doi: 10.1007/s10803015-2503-9. PMID: 26092640.

5. Myers SM, Johnson CP. Management of children with autism spectrum disorders. Pediatrics. 2007 Nov;120(5):1162-82. doi: peds.2007-2362 [pii]

10.1542/peds.2007-2362 [doi]. PMID: 17967921.

6. Warren Z, Veenstra-VanderWeele J, Stone W, Bruzek JL, Nahmias AS, Foss-Feig JH, Jerome RN, Krishnaswami S, Sathe NA, Glasser AM, Surawicz T, McPheeters ML. Therapies for Children With Autism Spectrum Disorders. Comparative Effectiveness Review No. 26. (Prepared by the Vanderbilt Evidence-based Practice Center under Contract No. 290-2007-10065-
I.) AHRQ Publication No. 11-EHC029-EF. Rockville, MD: Agency for Healthcare Research and Quality. April 2011. Available at:

www.effectivehealthcare.ahrq.gov/reports/fi nal.cfm.

7. Weitlauf AS, McPheeters ML, Peters B, et al. Therapies for Children With Autism Spectrum Disorder: Behavioral Interventions Update. Rockville (MD); 2014. Weitlauf AS, McPheeters ML, Peters B, Sathe N, Travis R, Aiello R, Williamson E, Veenstra-VanderWeele J, Krishnaswami $\mathrm{S}$, Jerome R, Warren Z. Therapies for Children With Autism Spectrum Disorder: Behavioral Interventions Update. Comparative Effectiveness Review No. 137. (Prepared by the Vanderbilt Evidence-based Practice Center under Contract No. 2902012-00009-I.) AHRQ Publication No. 14EHC036-EF. Rockville, MD: Agency for Healthcare Research and Quality; August 2014.

www.effectivehealthcare.ahrq.gov/reports/fi nal.cfm.

8. Methods Guide for Effectiveness and Comparative Effectiveness Reviews. AHRQ Publication No. 10(14)-EHC063-EF. Rockville, MD: Agency for Healthcare Research and Quality. January 2014. Chapters available at: www.effectivehealthcare.ahrq.gov

9. Berkman ND, Lohr KN, Ansari MT, et al. Grading the strength of a body of evidence when assessing health care interventions: an EPC update. J Clin Epidemiol. 2014 Dec 20doi: 10.1016/j.jclinepi.2014.11.023. PMID: 25721570. 


\section{Introduction}

\section{Background}

Autism spectrum disorder (ASD) is a neurodevelopmental disorder broadly defined by impaired social communication as well as restricted or repetitive patterns of behavior and interest. As defined by the Diagnostic and Statistical Manual of Mental Disorders, Fifth edition (DSM-5), specific features of ASD include deficits in social and emotional reciprocity (e.g., atypical social approaches, conversational impairment, atypical sharing of interests, attention, and affect); deficits in nonverbal communication (e.g., poorly integrated verbal and nonverbal communication, atypical body-language and gesture use, deficits in use and understanding of nonverbal communication), and deficits in maintaining appropriate relationships (e.g., challenges with peer interest, vulnerabilities forming friendships, difficulties adjusting behavior to suit social contexts) as well as restricted and repetitive patterns of behavior such as stereotyped speech, motor movements, or use of objects; excessive adherence to routine or insistence on sameness; intense interest patterns; and atypical sensory interests or responses. Symptoms of the disorder impair and limit everyday functioning and are thought to be evident in early childhood; although they may not be fully evident until later ages. Although not core symptoms, many children with ASD may also have significant cognitive impairment and language impairments.

The prevalence of ASD in the United States is 14.7 cases per 1,000 children living in the communities surveyed, or 1 in 68, with rate estimates varying widely by region of the country, sex, and race/ethnicity. ${ }^{1}$ Considerably more males ( 1 in 42$)$ than females (1 in 189) are affected. For some individuals, symptoms of ASD may improve with intervention and maturation; however, core deficits typically translate into varying developmental presentations that persist throughout the lifespan. ${ }^{2}$

\section{Treatment of ASD}

The manifestation and severity of symptoms of ASD differ widely, and treatments include a range of behavioral, psychosocial, educational, medical, and complementary approaches ${ }^{3-6}$ that vary by a child's age and developmental status. The goals of treatment for ASD typically focus on improving core deficits in communication, social interactions, or restricted behaviors, as changing these fundamental deficits may help children develop greater functional skills and independence. ${ }^{7}$ Treatment frequently is complicated by symptoms or comorbidities that may warrant targeted intervention (e.g., significant challenging behavior, attention and hyperactivity concerns, depression, anxiety). There is no cure for ASD and no global consensus on which intervention is most effective. ${ }^{8,9}$ Individual goals for treatment vary for different children and may include combinations of behavioral therapies, educational therapies, medical and related therapies, approaches targeting sensory issues, and allied health therapies; parents may also pursue complementary and alternative medicine therapies.

The antipsychotics risperidone (Risperdal) and aripiprazole (Abilify) have been specifically approved by the U.S. Food and Drug Administration (FDA) for treatment of the comorbid symptoms of irritability and challenging behaviors in ASD. No medications have been approved specifically to treat core ASD symptoms such as communication impairments. Many medications are used off-label to manage behavioral symptoms such as anxiety and hyperactivity. In addition, other treatments such as nutritional supplements or devices such as 
hyperbaric oxygen chambers have been used to treat symptoms of ASD, though neither supplements or hyperbaric oxygen have been approved by the FDA for ASD treatment. ${ }^{10}$

\section{Scope and Key Questions}

\section{Scope of Review}

This review updates findings reported in the 2011 Agency for Healthcare Research and Quality (AHRQ) review of Therapies for Children with ASD ${ }^{11}$ with a focus on studies of medical interventions. We defined medical interventions broadly as interventions involving the administration of external substances to the body or use of external, nonbehavioral procedures to treat symptoms of ASD, which includes pharmacologic agents, diet therapies, vitamins and supplements, chelating agents, electroconvulsive therapy, transcranial magnetic stimulation and hyperbaric oxygen, among other modalities. We used this broad definition, developed with input from our clinical experts, in order to capture the landscape of medically-related interventions used to treat children with ASD.

We focused the review on children between 2 and 12 years of age. We chose to limit the age range to this span because a) diagnosis of ASD earlier than age 2 is less established and $b$ ) adolescents likely have substantially different challenges and would warrant different interventions than children in the preschool, elementary, and middle school age groups.

We integrate syntheses of comparative studies evaluating medical interventions addressed in our 2011 review of therapies for children with ASD ${ }^{11}$ if they addressed an agent evaluated in a study identified for the current review. To ensure comprehensive coverage of the medical literature in the current update, we also included studies that had originally been excluded in the 2011 review because of sample size. We set a lower sample size inclusion criterion in the current update.

A companion review updating findings related to interventions targeting sensory challenges is available on the AHRQ Effective Health Care Web site.

\section{Key Questions}

We developed Key Questions (KQs) in consultation with Key Informants and the Task Order Officer. KQs were posted for review to the AHRQ Effective Health Care Web site.

KQs were as follows:

KQ1: Among children ages 2-12 with ASD, what is the comparative effectiveness (benefits and harms) of medical treatments?

a. What are the effects on core symptoms (e.g., deficits in social communication and interaction; restricted, repetitive patterns of behavior, interests, or activities including hyper- or hypo- reactivity to sensory input or unusual interest in sensory aspects of the environment) in the short term ( $<6$ months)?

b. What are the effects on commonly associated symptoms (e.g., motor, medical, mood/anxiety, irritability, and hyperactivity) in the short term (<6 months)? 
c. What are the longer term effects ( $\geq 6$ months) on core symptoms (e.g., social deficits, communication deficits, and repetitive behaviors)?

d. What are the longer term effects ( $\geq 6$ months) on commonly associated symptoms (e.g., motor, medical, mood/anxiety, irritability, and hyperactivity)?

KQ2: Among children ages 2-12 with ASD, what are the modifiers of outcome for different medical treatments?

a. Is the effectiveness of the therapies reviewed affected by the frequency, duration, intensity, or dose of the intervention?

b. Is the effectiveness of the therapies reviewed affected by cointerventions or prior treatment, or the training and/or experience of the individual providing the therapy?

c. What characteristics (e.g., age, symptom severity), if any, of the child modify the effectiveness of the therapies reviewed?

d. What characteristics, if any, of the family modify the effectiveness of the therapies reviewed?

KQ3: What is the time to effect of medical interventions?

KQ4: What is the evidence that effects measured at the end of the treatment phase predict long-term functional outcomes of medical interventions?

KQ5: Is the effectiveness of medical interventions maintained across environments or contexts (e.g., people, places, materials)?

KQ6: What evidence supports specific components of treatment with medical interventions as driving outcomes, either within a single treatment or across treatments?

Table 1 outlines population, intervention, comparator, outcomes, timing, and setting (PICOTS) characteristics for each KQ. 
Table 1. PICOTS characteristics

\begin{tabular}{|c|c|}
\hline PICOTS & Criteria \\
\hline Population & $\begin{array}{l}\text { Children with ASD between the ages of } 2 \text { and } 12 \text { years (mean age plus standard deviation is } \leq \\
12 \text { years and } 11 \text { months) }\end{array}$ \\
\hline Intervention(s) & Medical interventions (pharmaceutical agents, supplements and diets, hyperbaric oxygen, etc.) \\
\hline Comparator & $\begin{array}{l}\text { - Inactive control (e.g., no treatment, watchful waiting, waitlist control, placebo) } \\
\text { - Alternate intervention }\end{array}$ \\
\hline Outcomes & $\begin{array}{l}\text { Intermediate outcomes } \\
\text { - ASD symptom severity } \\
\text { - } \text { Expressive or receptive language/communication } \\
\text { - Academic skill development } \\
\text { - Maladaptive behaviors } \\
\text { - Distress } \\
\text { - Adaptive skills development } \\
\text { - Social skills/interaction } \\
\text { - Harms of interventions } \\
\text { Final health outcomes } \\
\text { - Symptom severity or diagnostic outcome } \\
\text { - } \text { Counctional communication } \\
\text { - Motor skills } \\
\text { - Adaptive independence } \\
\text { - } \text { Academic engagement/attainment (e.g., mainstream school placement or integration) } \\
\text { - Psychosocial well-being } \\
\text { - Psychosocial adaptation } \\
\text { - Harms of interventions }\end{array}$ \\
\hline Timing & Any (i.e., short and long term outcomes as reported in eligible study publications) \\
\hline Setting & Any primary, specialty, community, or educational setting \\
\hline
\end{tabular}

ASD = autism spectrum disorder; PICOTS = population, intervention, comparator, outcome, timing, setting

\section{Analytic Framework}

The analytic framework (Figure 1) illustrates the population, interventions, outcomes, and adverse effects that guided the literature search and synthesis. 


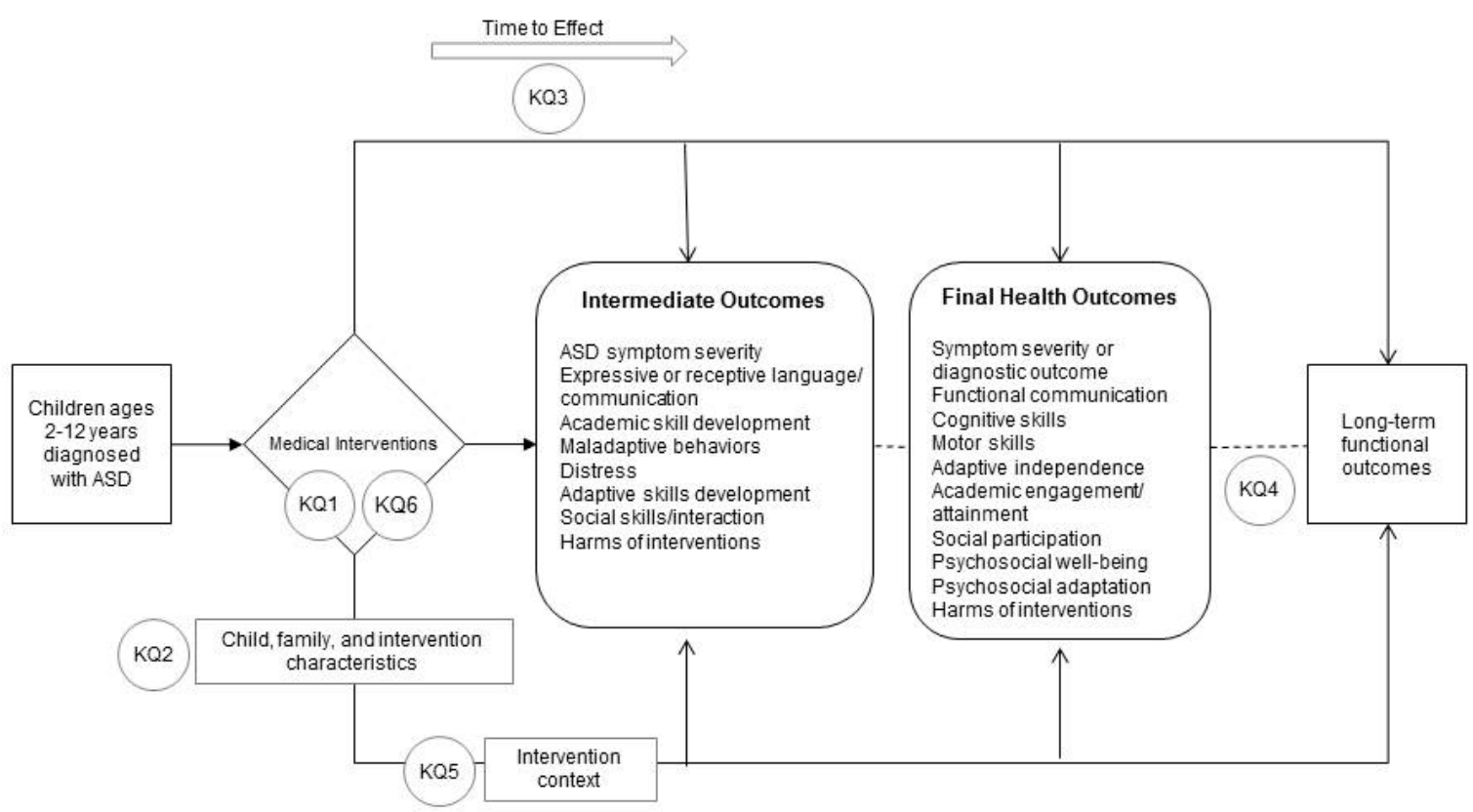

ASD=autism spectrum disorder; KQ=Key Question

\section{Organization of This Report}

The Methods section describes the review processes including search strategy, inclusion and exclusion criteria, approach to review of abstracts and full publications, methods for extraction of data, and compiling evidence. We also describe our approach to grading the risk of bias of the literature and describing the strength of the body of evidence.

The Results section presents the findings of the literature search and the review of the evidence by KQ, synthesizing the findings across strategies. We present findings for each $\mathrm{KQ}$ organized by intervention and outcome area. We include summary tables in the Results section for those intervention areas for which we could assess the strength of evidence for effectiveness outcomes. All other summary tables are in Appendix F. Because few studies addressed subquestions under KQ1 and 2, we present results in the aggregate under each of these KQ.

The Discussion section of the report discusses the results and expands on methodologic considerations relevant to each KQ. We also outline the current state of the literature and challenges for future research in the field. The report includes a number of appendixes to provide further detail on our methods and the studies assessed. The appendixes are as follows:

- Appendix A: Search Strategies

- Appendix B: Screening and Risk of Bias Assessment Forms

- Appendix C: Excluded Studies

- Appendix D: Risk of Bias Ratings

- Appendix E: Applicability Summary Tables

- Appendix F: Detailed Tables of Findings 


\section{Uses of This Evidence Report}

We anticipate that the report will be of value to clinicians who treat children with ASD, who can use the report to assess the evidence for different treatment strategies. In addition, this review will be of use to the National Institutes of Health, U.S. Centers for Disease Control and Prevention, Centers for Medicare \& Medicaid Services, and the Health Resources and Services Administration-all of which have offices or bureaus devoted to child health issues and who may use the report to compare treatments and determine priorities for funding. This report can bring practitioners up to date about the current state of evidence related to medical interventions, and it provides an assessment of the quality of studies that aim to determine the outcomes of medical options for the management of ASD. It will be of interest to families affected by ASD because of the recurring need for families and their health care providers to make the best possible decisions among numerous options. We also anticipate it will be of use to private sector organizations concerned with ASD; the report can inform such organizations' understanding of the effectiveness of treatments and the amount and quality of evidence available. Researchers can obtain a concise analysis of the current state of knowledge and future research needs related to medical interventions for ASD. 


\section{Methods}

In this chapter, we document the procedures that we used to produce a comparative effectiveness review update addressing medical interventions for children with autism spectrum disorder (ASD). These procedures follow the methods outlined in the Agency for Healthcare Research and Quality (AHRQ) Effective Health Care Program Methods Guide for Effectiveness and Comparative Effectiveness Reviews. ${ }^{12}$

\section{Topic Surveillance and Review Protocol}

The topic for the original report $\left(2011^{11}\right)$ was nominated by Autism Speaks in a public process using the Effective Health Care Web site. AHRQ published an update addressing behavioral interventions in 2014. ${ }^{13}$ We conducted a surveillance process to assess the need to update the report by contacting topic experts about the relevance of the Key Questions (KQs) and new evidence that may address them. All members of the research team were required to submit information about potential conflicts of interest before initiation of the work. No members of the review team had any conflicts.

In consultation with clinical experts and stakeholders, and based on our preliminary scan of the literature and surveillance findings, we focused the review update on medical approaches and approaches to address sensory challenges (reported in a separate update). These areas reflect both areas of clinical relevance and sufficient newly published literature for a review update. Based also on the surveillance process and discussions with stakeholders, we revised the Key Questions (KQs) addressed in the 2011 report $^{11}$ to reflect the focus on medical and sensory approaches specifically. We also eliminated a question on approaches for children at risk for ASD as such children are unlikely to be included in studies in the target areas for this review update.

After review from AHRQ, the questions and framework were posted online for public comment. No changes to the questions or framework were recommended. We identified technical experts on the topic to provide assistance during the project. The Technical Expert Panel (TEP), representing the fields of pediatrics and developmental pediatrics, psychiatry, family medicine, and occupational therapy and allied health, contributed to the AHRQ's broader goals of (1) creating and maintaining science partnerships as well as public-private partnerships and (2) meeting the needs of an array of potential users of its products. Thus, the TEP was both an additional resource and a sounding board during the project. The TEP included seven members serving as technical or clinical experts. To ensure robust, scientifically relevant work, TEP members participated in conference calls to:

- Help to refine the analytic framework and KQ at the beginning of the project;

- Discuss inclusion/exclusion criteria; and

- Assist with determining key interventions and outcomes of interest.

The final protocol was posted to the AHRQ Effective Health Care Web site and registered in the PROSPERO international register of systematic reviews (ID\#: CRD42016033941).

\section{Literature Search Strategy}

\section{Search Strategy}

To ensure comprehensive retrieval of relevant studies of medical therapies for children with ASD, we used four key databases: the MEDLINE ${ }^{\circledR}$ medical literature database via the PubMed ${ }^{\circledR}$ interface; EMBASE (Excerpta Medica Database), an international biomedical and 
pharmacological literature database via the Ovid ${ }^{\circledR}$ interface; the Cumulative Index of Nursing and Allied Health Literature (CINAHL), and PsycINFO ${ }^{\circledR}$. Search strategies for KQs applied a combination of controlled vocabulary (Medical Subject Headings [MeSH] and Emtree headings) and key words to focus specifically on medical interventions for ASD and harms of interventions (Appendix A). We restricted literature searches for KQs to studies published from 2010 to the present to reflect literature available since the publication of the 2011 review. ${ }^{11}$ We last conducted searches for the review in September 2016.

\section{Gray Literature}

We searched Web sites of organizations likely to conduct research, issue guidance, or generate policies for ASD (e.g., Autism Speaks, the American Academy of Child and Adolescent Psychiatry) to inform the review's background and discussion sections. We searched government and regulatory agency Web sites for contextual information on benefits and harms of ASD interventions. We searched ClinicalTrials.gov, the International Standard Randomized Controlled Trials Number (ISRCTN) registry, and other trial registries for information about relevant ongoing trials and to confirm that we had obtained available publications of results from completed trials.

\section{Inclusion Criteria}

Table 2 outlines inclusion criteria. We required that eligible randomized controlled trials (RCTs) have a total minimum sample size of 10 . We required a higher minimum sample size $(n=20)$ for other comparative studies as they typically have fewer controls for bias than RCTs.

We included studies published in English only. In the opinion of our content experts, much of the relevant literature on ASD is published in English; however, we scanned a sample of 150 non-English abstracts to gauge the number of anticipated non-English studies that would meet inclusion criteria. Two non-English studies appeared to meet our criteria. Given this small proportion of potentially eligible studies, we feel that excluding these publications is unlikely to introduce significant bias.

Eligible studies also reported one or more outcomes of interest and included children at least 2 years of age and up to and including age 12. As noted, we focused the review on children in this age range given greater diagnostic stability and differences in behaviors and challenges across age ranges. Studies also included only children with a diagnosis of ASD (or data reported separately for children with ASD). 
Table 2. Inclusion criteria

\begin{tabular}{|l|l|}
\hline Category & Criteria \\
\hline Study Population & $\begin{array}{l}\text { Children ages 2-12 with ASD (mean age plus standard deviation is } \leq 12 \text { years and } 11 \\
\text { months) }\end{array}$ \\
\hline Publication Languages & English only \\
\hline $\begin{array}{l}\text { Admissible Evidence } \\
\text { Other Criteria) }\end{array}$ & $\begin{array}{l}\text { Admissible designs } \\
\text { Randomized controlled trials, prospective and retrospective cohort studies with } \\
\text { comparison groups, and nonrandomized controlled trials }\end{array}$ \\
& $\begin{array}{l}\text { Other criteria } \\
\text { Original research studies published from 2010-present and not addressed in prior } \\
\text { reviews (except for those otherwise eligible studies with a sample size of <30 that } \\
\text { excluded them from the 2011 review) } \\
\text { Studies must have relevant population and } \geq 20 \text { participants with ASD (nonRCTs) or at } \\
\text { least 10 total participants (RCTs) } \\
\text { Studies must address one or more of the following for ASD: } \\
\text {-Outcomes of interest } \\
\text {-Treatment modality of interest } \\
\text {-Predictors or drivers of treatment outcomes (e.g., biomarkers, clinical changes) } \\
\text {-Maintenance of outcomes across environments or contexts } \\
\text {-Sufficiently detailed methods and results to enable data extraction } \\
\text {-Reporting of outcome data by target population or intervention }\end{array}$ \\
\hline
\end{tabular}

ASD = autism spectrum disorder; RCT = randomized controlled trial

\section{Study Selection}

Once we identified articles through the electronic database searches and hand-searching, we examined abstracts of articles to determine whether studies met our criteria. Two reviewers separately evaluated the abstracts of studies identified in our searches for KQs for inclusion or exclusion, using an Abstract Review Form (Appendix B). If one reviewer concluded that the article could be eligible for the review based on the abstract, we retained it. Following abstract review, two reviewers independently assessed the full text of each included study using a standardized form (Appendix B) that included questions stemming from our inclusion and exclusion criteria. A senior reviewer resolved disagreements between reviewers.

We conducted all abstract and full text reviews using the DistillerSR online screening application (Evidence Partners Incorporated, Ottawa, Ontario). Appendix C includes a list of excluded studies and the reasons for exclusion. Data extracted for each study are available via the Systematic Review Data Repository (http://srdr.ahrq.gov/).

\section{Data Extraction}

The staff members and clinical experts (including two psychiatrists, two psychologists, and three epidemiologists/systematic reviewers) who conducted this review jointly developed the data extraction forms for the KQs. We designed forms to provide sufficient information to enable readers to understand the studies and to determine their quality; we gave particular emphasis to essential information related to the KQs. The team was trained to extract data by extracting several articles into the template and then reconvening as a group to discuss the utility of the template. We repeated this process through several iterations until we decided that the templates included the appropriate categories for gathering the information contained in the articles and for potential meta-analyses. Team data extractors shared the task of initially entering information into the evidence tables. A second team member also reviewed the articles and edited all initial 
entries for accuracy, completeness, and consistency. A senior reviewer reconciled disagreements concerning the information reported.

The full research team met regularly during the article extraction period and discussed issues related to the data extraction process. In addition to outcomes related to the effectiveness of treatment (e.g., changes in ASD severity), we extracted all data available on harms. Harms encompass the full range of specific negative effects, including the narrower definition of adverse events.

\section{Data Synthesis}

We summarized data for KQs qualitatively using summary tables. We integrate syntheses of comparative studies evaluating medical interventions addressed in our 2011 review of therapies for children with $\mathrm{ASD}^{11}$ if they addressed an agent evaluated in a study identified for the current review.

We attempted to perform a quantitative meta-analysis for the effects of risperidone on outcomes related to challenging behaviors using a multivariate normal response to simultaneously model four outcome scales. However, only four studies satisfied the criteria for inclusion, which included reporting baseline and end-of-treatment (or change from baseline) means and standard deviations. This number of studies limited use of a random effects metaanalysis, which was warranted to account for the variation in outcomes. We fit a prototype model using a fixed effects meta-analysis, but the goodness-of-fit evaluation was very poor, so we elected not continue the meta-analysis. We summarize prior meta-analyses and systematic reviews addressing many of the same agents in the Findings in Relation to What Is Already Known section of the report.

\section{Risk of Bias Assessment of Individual Studies}

We evaluated the overall methodologic risk of bias of individual studies using the ASDspecific assessment approach we developed and used in our prior reviews of interventions for ASD and informed by the Methods Guide for Effectiveness and Comparative Effectiveness Reviews. ${ }^{12}$ We developed this tool (Appendix B) because standard risk of bias assessment tools (e.g., Cochrane risk of bias assessment) do not fully account for the complexity of interventions and populations represented in the ASD literature. Specifically, the tool includes questions to address diagnostic approaches and measures of treatment fidelity that may affect outcomes. The tool has not been formally validated.

Two senior investigators assessed each included study independently with disagreements resolved through discussion or by an independent senior investigator/methodologist. Appendix D includes ratings for each study.

\section{Determining Overall Risk of Bias Ratings}

We used the thresholds we establish in prior reviews to assess overall high, medium or low risk of bias. We assessed the risk of bias based upon the study-defined primary outcome(s). We assessed each domain evaluated in the tool (i.e., study design, participant ascertainment/inclusion, intervention description, outcome measurement, statistical analysis). We considered the individual ratings to determine an overall quality assessment of low, moderate, or high risk of bias. 
We required that studies receive positive scores questions related to randomization and diagnostic approach to be considered low risk of bias. We summed and weighted scores as described in Table 3 to determine overall study risk of bias. Studies could receive up to two points on the domains of study design, diagnostic approach, participant ascertainment, and intervention, and up to one point on the domains of outcome measurement and statistical analysis.

Table 3. Quality scoring algorithm

\begin{tabular}{|l|l|}
\hline Definition and Scoring Algorithm & Rating \\
\hline $\begin{array}{l}\geq 8 / 10 \text { points, including a }++ \text { on study design and }++ \text { on diagnostic } \\
\text { approach }\end{array}$ & Low risk of bias \\
\hline$\bullet \quad \geq 6 / 10$ points, including at least $\mathrm{a}+$ on intervention & Moderate risk of bias \\
\hline$\bullet \quad \leq 5 / 10$ points & High risk of bias \\
\hline
\end{tabular}

\section{Strength of the Body of Evidence}

The assessment of the literature is done by considering both the observed effectiveness of interventions and the confidence that we have in the stability of those effects in the face of future research. The degree of confidence that the observed effect of an intervention is unlikely to change is presented as strength of evidence, and it can be regarded as insufficient, low, moderate, or high. Strength of evidence describes the adequacy of the current research, both in terms of quantity and quality, as well as the degree to which the entire body of current research provides a consistent and precise estimate of effect. Interventions that have demonstrated benefit in a small number of studies but have not yet been replicated using the most rigorous study designs will therefore have insufficient or low strength of evidence to describe the body of research. Future research may find that the intervention is either effective or ineffective. Strength of the evidence is assessed for a limited set of critical outcomes, typically those related to effectiveness of an intervention.

Methods for applying strength of evidence assessments are established in the Methods Guide for Effectiveness and Comparative Effectiveness Reviews ${ }^{12}$ and are based on consideration of five domains (Table 4): study limitations, consistency in direction of the effect, directness in measuring intended outcomes, precision of effect, and reporting bias. Strength of evidence is assessed separately for major intervention-outcome pairs and incorporates data from the entire body of reviewed evidence on behavioral interventions (i.e., comparative studies-both RCTs and prospective and retrospective cohort studies - reported in the 2011 review $^{11}$ and studies reported in the current review). We required at least one low risk of bias study for moderate strength of evidence and two low risk studies for high strength of evidence. In addition, to be considered "moderate" or higher, intervention-outcome pairs needed a positive response on two out of the three domains other than study limitations.

Once we had established the maximum strength of evidence possible based upon these criteria, we assessed the number of studies and range of study designs for a given interventionoutcome pair, and downgraded the rating when the cumulative evidence was not sufficient to justify the higher rating. The possible grades were:

- High: High confidence that the evidence reflects the true effect. Further research is unlikely to change estimates.

- Moderate: Moderate confidence that the evidence reflects the true effect. Further research may change our confidence in the estimate of effect and may change the estimate. 
- Low: Low confidence that the evidence reflects the true effect. Further research is likely to change confidence in the estimate of effect and is also likely to change the estimate.

- Insufficient: Evidence is either unavailable or does not permit a conclusion.

Table 4. Domains used to assess strength of evidence ${ }^{a}$

\begin{tabular}{|c|c|}
\hline Domain & Explanation \\
\hline $\begin{array}{l}\text { Study } \\
\text { Limitations }\end{array}$ & $\begin{array}{l}\text { Degree to which included studies for a given outcome have a high likelihood of adequate protection } \\
\text { against bias (i.e., good internal validity), assessed through study design and study conduct. }\end{array}$ \\
\hline Consistency & $\begin{array}{l}\text { Degree to which included studies find either the same direction or similar magnitude of effect. } \\
\text { Assessed through two main elements: } \\
\text { - Direction of effect: Effect sizes have the same sign (that is, are on the same side of no } \\
\text { effect or a minimally important difference). } \\
\text { - Magnitude of effect: The range of effect sizes is similar. }\end{array}$ \\
\hline Directness & $\begin{array}{l}\text { Extent to which evidence links interventions directly to a health outcome of specific importance for } \\
\text { the review, and for comparative studies, whether the comparisons are based on head-to-head } \\
\text { studies. Evidence may be indirect in several situations such as: } \\
\text { - Outcome being graded is considered intermediate in a review that is focused on clinical } \\
\text { - health outcomes (such as morbidity, mortality). } \\
\text { Data do not come from head-to-head comparisons but rather from two or more bodies of } \\
\text { evidence to compare. } \\
\text { Data are available only for proxy respondents instead of directly from patients for situations } \\
\text { in which patients are capable of self-reporting and self-report is more reliable. }\end{array}$ \\
\hline Precision & $\begin{array}{l}\text { Degree of certainty surrounding an effect estimate with respect to a given outcome, based on the } \\
\text { sufficiency of sample size and number of events. A body of evidence will generally be imprecise if } \\
\text { the optimal information size (OIS) is not met. OIS refers to the minimum number of patients (and } \\
\text { events when assessing dichotomous outcomes) needed for an evidence base to be considered } \\
\text { adequately powered. }\end{array}$ \\
\hline $\begin{array}{l}\text { Reporting } \\
\text { bias }\end{array}$ & $\begin{array}{l}\text { Degree of selective publishing or reporting of research findings based on the favorability of direction } \\
\text { or magnitude of effect. }\end{array}$ \\
\hline
\end{tabular}

${ }^{\mathrm{a}}$ Excerpted from Berkman et al. $2013^{14}$

\section{Applicability}

We assessed the applicability of findings reported in the included literature addressing our KQs to the general population of children with ASD by determining the population, intervention, comparator, and setting in each study and developing an overview of these elements for each intervention category. We anticipated that areas in which applicability would be especially important to describe would include ASD severity, comorbidities, age at treatment, and intervention characteristics such provider, dosing/intensity, and setting. Applicability tables for each KQ are in Appendix E.

\section{Peer Review and Public Commentary}

Researchers and clinicians with expertise in treating children with ASD and individuals representing stakeholder and user communities provided external peer review of this report. The draft report was posted on the AHRQ Web site for 4 weeks to elicit public comment. We addressed all reviewer comments, revised the text as appropriate, and documented changes and revisions to the report in a disposition of comments report that will be made available 3 months after AHRQ posts the final review on the AHRQ Web site. 


\section{Results}

\section{Results of Literature Searches for Key Questions}

We identified 6583 nonduplicative titles or abstracts with potential relevance, with 554 proceeding to full text review (Figure 2). We excluded 469 studies at full text review. We included 68 unique studies (85 publications) in the review. In addition to these 68 studies included since the completion of our original review of therapies for children with autism spectrum disorder (ASD) in 2011, ${ }^{11}$ we include 12 comparative studies addressed in the 2011 review that also addressed an agent used in the current review. Four studies (reported in multiple publications) included in the 2011 review now include followup analyses published since the completion of that report. ${ }^{15-39}$ We outline findings from all 76 studies below.

\section{Figure 2. Disposition of studies identified for this review}
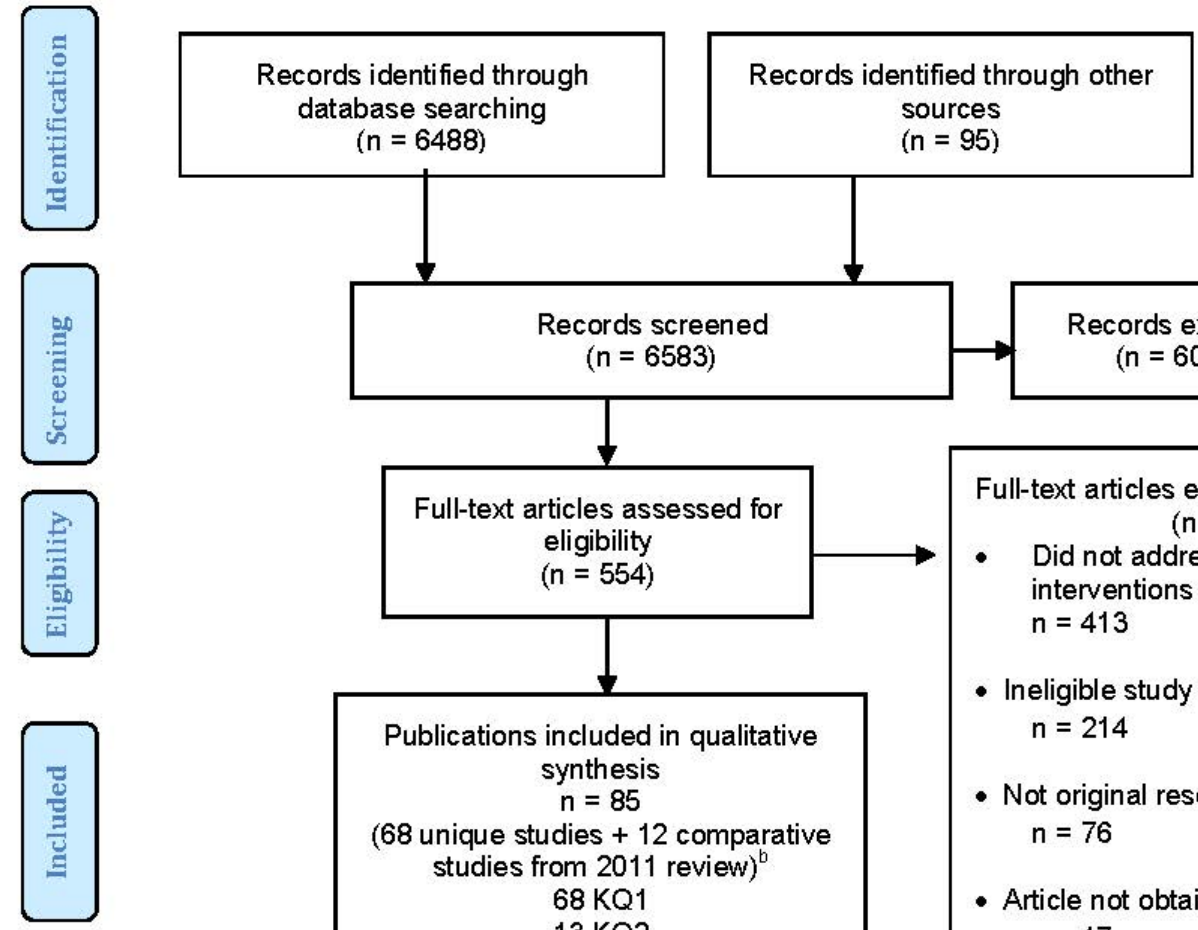

Publications included in qualitative synthesis $\mathrm{n}=85$

(68 unique studies +12 comparative studies from 2011 review) ${ }^{b}$

$68 \mathrm{KQ1}$

$13 \mathrm{KQ} 2$

$2 \mathrm{KQ3}$

$1 \mathrm{KQ4}$

$8 \mathrm{KQ5}$

$0 \mathrm{KQ6}$

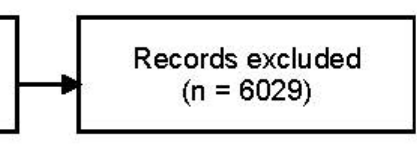

Full-text articles excluded, with reasons ${ }^{a}$ $(n=469)$

- Did not address a KQ (outcomes or interventions of interest) $n=413$

- Ineligible study design or size $n=214$

- Not original research $n=76$

- Article not obtainable or not in English $\mathrm{n}=17$

Numbers next to each Key Question indicate number of unique studies addressing the question. Studies could address more than one Key Question.

${ }^{2}$ Numbers do not tally as studies could be excluded for multiple reasons.

${ }^{b}$ We also include analysis of 12 comparative studi es reported in our 2011 review of therapies for children with ASD, four of which include new sub-analyses or longer term analyses published since the completion of the 2011 review; thus, we describe a total of $\underline{76}$ studies. Abbreviations: $\mathrm{KQ}=$ key question; $\mathrm{n}=$ number.

\section{Description of Included Studies}

The 76 studies included in the review comprised 72 randomized controlled trials (RCTs) reported in multiple publications, ${ }^{15-119}$ two nonrandomized trials, ${ }^{120,121}$ and two retrospective 
cohort studies. ${ }^{122,123}$ Among the 65 studies clearly reporting a funding source, 12 were industrysponsored, ${ }^{15-21,40-44,48,51-53,67,75,101,108,112,113,115}$ and the remainder were funded by government health agencies, foundations, or universities.

We considered 39 studies to have low risk of bias, $15-39,41-46,48-55,57,58,60,67,73-76,78,81,83,89-100$, 102, 108, 110, 111, 11429 to have moderate, ${ }^{40,47,59,63,66,68-72,77,79,80,82,84-87,101,103,106,107,109,112,113,115-}$ 118, 121, 122, 124 and eight to have high risk. ${ }^{56,61,62,64,65,88,104,105,120,123}$ Despite the high number of low and moderate risk of bias studies, few studies addressed the same interventions or outcomes, and most studies included few participants, evaluated only in the short term ( $<6$ months); thus, evidence for many agents remains insufficient. Table 5 outlines key study characteristics. Because few studies addressed sub-questions under Key Questions (KQ) 1 and 2, we present results in the aggregate under each of these KQ.

Table 5. Overview of studies

\begin{tabular}{|c|c|c|c|c|}
\hline Characteristic & 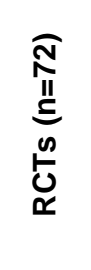 & 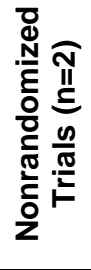 & 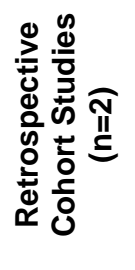 & 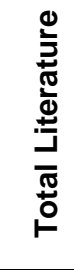 \\
\hline \multicolumn{5}{|l|}{ Intervention category } \\
\hline Antipsychotics & 11 & 0 & 1 & 12 \\
\hline Medications used to treat ADHD & 5 & 0 & 0 & 5 \\
\hline Combined medical and behavioral approaches & 3 & 2 & 0 & 5 \\
\hline Nutrition and diet & 19 & 0 & 0 & 19 \\
\hline Risperidone adjuncts & 14 & 0 & 0 & 14 \\
\hline Hyperbaric oxygen therapy & 3 & 0 & 0 & 3 \\
\hline N-acetylcysteine & 2 & 0 & 0 & 2 \\
\hline Tetrahydrobiopterin & 2 & 0 & 0 & 2 \\
\hline Other medical approaches $^{a}$ & 13 & 0 & 1 & 14 \\
\hline \multicolumn{5}{|l|}{ Treatment duration } \\
\hline$<1-4$ weeks & 7 & 1 & 0 & 8 \\
\hline 5-8 weeks & 15 & 0 & 0 & 15 \\
\hline 9-12 weeks & 28 & 1 & 0 & 29 \\
\hline 13-20 weeks & 5 & 0 & 0 & 5 \\
\hline 21-36 weeks & 13 & 0 & 1 & 14 \\
\hline$>52$ weeks & 4 & 0 & 1 & 5 \\
\hline \multicolumn{5}{|l|}{ Region of study conduct } \\
\hline Africa & 2 & 0 & 0 & 2 \\
\hline Asia & 23 & 2 & 0 & 25 \\
\hline Australia & 3 & 0 & 0 & 3 \\
\hline Europe & 11 & 0 & 0 & 11 \\
\hline North America & 33 & 0 & 2 & 35 \\
\hline \multicolumn{5}{|l|}{ Risk of bias } \\
\hline Low & 39 & 0 & 0 & 39 \\
\hline Moderate & 27 & 1 & 1 & 29 \\
\hline High & 6 & 1 & 1 & 8 \\
\hline Total N participants & 3902 & 102 & 186 & 4190 \\
\hline
\end{tabular}

${ }^{\mathrm{a}}$ Includes two of donepezil and one each of neurostimulation, amantadine, divalproex, stem cell transplantation, melatonin, bumetanide, oxytocin, mecamylamine, prednisolone, and citalopram. ADHD = attention deficit hyperactivity disorder; $\mathrm{N}=$ number; RCT = randomized controlled trial 
Table 6 outlines key outcomes addressed by studies evaluating each intervention class. Challenging behaviors and symptom severity were frequently targeted. All studies reported harms.

Table 6. Key outcomes targeted in studies of medical interventions

\begin{tabular}{|c|c|c|c|c|c|c|c|c|c|c|c|}
\hline $\begin{array}{l}\text { Intervention } \\
\text { Categoryl } \\
\text { Outcome }\end{array}$ & 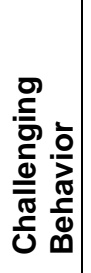 & 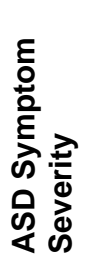 & 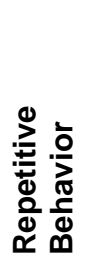 & 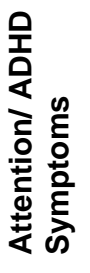 & 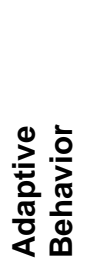 & 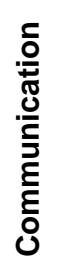 & 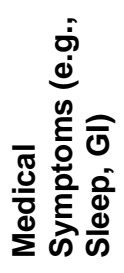 & 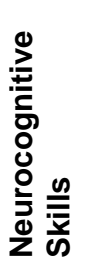 & 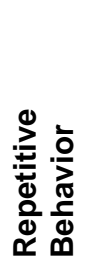 & $\begin{array}{l}\frac{n}{\bar{y}} \\
\frac{\pi}{n} \\
\frac{\pi}{0} \\
\dot{0}\end{array}$ & $\underset{\frac{c}{5}}{\stackrel{n}{I}}$ \\
\hline $\begin{array}{l}\text { Antipsychotics }{ }^{15-21,} \\
\text { 28-39, 47-52, 101, 102, 109, } \\
112,113,122\end{array}$ & $X$ & $X$ & $X$ & $X$ & $X$ & & & & $X$ & & $X$ \\
\hline $\begin{array}{l}\text { ADHD } \\
\text { medications } \\
\text { 24-27, 40-46 }\end{array}$ & $X$ & $X$ & $X$ & $X$ & $X$ & $X$ & & $X$ & $X$ & & $X$ \\
\hline $\begin{array}{l}\text { Combination } \\
\text { medical and } \\
\text { behavioral } \\
\text { treatments }^{54,55,57,120,} \\
121\end{array}$ & $X$ & $X$ & & $X$ & & & & $X$ & & & $X$ \\
\hline $\begin{array}{l}\text { Omega-3 fatty } \\
\text { acids }^{80-82,84}\end{array}$ & $X$ & $X$ & & & $X$ & $X$ & & & & $X$ & $X$ \\
\hline $\begin{array}{l}\text { Specialized diets } \\
65,104-107\end{array}$ & $X$ & $X$ & & $X$ & & $X$ & $X$ & $X$ & & & $X$ \\
\hline $\begin{array}{l}\text { Nutritional } \\
\text { supplements } \\
117\end{array}$ & $X$ & $X$ & & $X$ & & $X$ & & $X$ & & & $X$ \\
\hline $\begin{array}{l}\text { Risperidone } \\
\text { adjuncts }{ }^{47,89-100,118,} \\
124\end{array}$ & $X$ & $X$ & & & & & & & & & $X$ \\
\hline $\begin{array}{l}\text { Hyperbaric } \\
\text { oxygen }^{66,67,114}\end{array}$ & $X$ & $x$ & & & $x$ & $X$ & & $x$ & & $X$ & $X$ \\
\hline $\begin{array}{l}\mathrm{N} \text {-acetylcysteine } \\
79\end{array}$ & $x$ & $X$ & $X$ & & & $X$ & & & $X$ & $X$ & $X$ \\
\hline $\begin{array}{l}\text { Tetrahydrobiopterin } \\
53,103\end{array}$ & $X$ & $X$ & & $X$ & $X$ & $X$ & & & & $X$ & $X$ \\
\hline $\begin{array}{l}\text { Other medical } \\
\text { treatments } 22,23,56,68- \\
75,77,101,108,115,116,123\end{array}$ & $X$ & $X$ & $X$ & $X$ & $X$ & $X$ & $X$ & $X$ & $X$ & $X$ & $X$ \\
\hline
\end{tabular}

ADHD = attention deficit hyperactivity disorder; ASD = autism spectrum disorder

\section{Gray Literature}

Our searches of ClinicalTrials.gov and other trial registers did not yield additional eligible studies for the review. We did not receive information in response to requests for scientific information from manufacturers or device makers. We used information from organization web sites searched to provide additional context for the discussion section of the report. 


\section{KQ1. Benefits and Harms of Medical Treatments}

\section{Studies of Antipsychotics}

\section{Key Points}

- $\quad$ Five RCTs addressing risperidone reported significant improvements in measures of irritability and other challenging behaviors in the treatment group compared with placebo in the short-term ( $\leq 6$ months), with continued positive effects over a mean 21 -month treatment period in an uncontrolled extension study. Side effects including somnolence and weight gain were clinically significant.

- Two RCTs of aripiprazole reported statistically significant improvements in irritability and challenging behavior in the treatment groups compared with placebo over 8 weeks of treatment and maintenance of improvements in a 52-week uncontrolled extension. Harms were also clinically significant. Another RCT reported no differences in time to relapse (return of significant negative symptoms) between children taking aripiprazole versus placebo; quality of life measures also did not differ between groups.

- Three small studies comparing risperidone with aripiprazole reported no significant differences in effects on challenging behaviors or general improvement; one study noted no significant differences in weight gain associated with each agent.

- One small RCT reported greater improvements in challenging behavior with risperidone vs. haloperidol, and one comparing risperidone and memantine and reported no group differences on any outcomes.

- Risperidone and aripiprazole improved challenging behaviors in the short-term (<6 months), with clinically significant harms. Our confidence in these conclusions is high (high strength of evidence). Behaviors improved in the longer term ( $\geq 6$ months) with these agents compared with placebo, but our confidence in this conclusion is low (low strength of evidence) as few studies had longer-term followup.

- In studies comparing risperidone and aripiprazole, BMI increased with both drugs over treatment durations of 6 months to more than 2 years, but group differences were not significant. We have low confidence in this conclusion given the few studies addressing this outcome (low strength of evidence).

- Other outcomes (e.g., challenging behaviors, attention) were not consistently addressed; thus we considered strength of evidence insufficient for all other intervention/outcome pairs.

\section{Overview of the Literature}

We identified 11 unique RCTs (reported in multiple publications and including comparative studies identified for the current review and those reported in our 2011 review $^{11}$ ) addressing antipsychotics ${ }^{15-21,28-39,47-52,101,102,109,111-113}$ and one retrospective cohort that reported harms data only. ${ }^{122}$ Seven studies had low risk of bias, ${ }^{15-21,28-39,48-52,102,112,113}$ and five had moderate risk. ${ }^{47,101,109,112,113,122}$ Studies included a total of 1055 children ranging in age from 2 to 20 years and were conducted in the United States $(n=5)$, Iran $(n=2)$, and one each in Canada, Turkey, Italy, the Netherlands, and India.

Two RCTs addressed aripiprazole compared with placebo; ${ }^{15-21,48}$ five addressed risperidone compared with placebo; ${ }^{28-39,51,52,102,109,111-113}$ and two compared risperidone and aripiprazole. ${ }^{49}$,

${ }^{50}$ A retrospective cohort compared these agents and reported differences in weight gain. ${ }^{122}$ One 
RCT compared risperidone and haloperidol, ${ }^{101}$ while another compared risperidone and memantine. ${ }^{47}$

Five of these eight RCTs were also included in our 2011 review, ${ }^{15-21,28-39,52,109,111-113,125}$ and investigators of four of these studies have published additional analyses of participants included in the original trials. These studies included two "families" of papers that report post-hoc and additional or combined analyses of participants in the initial trials. The first family of studies, conducted by Research Units on Pediatric Psychopharmacology (RUPP) Autism Network investigators, assessed risperidone and included an initial 8-week trial comparing risperidone and placebo in 101 children; ${ }^{34}$ one paper reporting potential moderators of effect in the 8-week trial; ${ }^{29}$ one paper reporting parent concerns assessed during the initial 8-week trial; ${ }^{35}$ one paper reporting social interaction measures in these participants ${ }^{39}$ and including data from a study (included in our 2011 review) that compared risperidone alone with risperidone plus parent training; ${ }^{126}$; one reporting measures of repetitive behavior ${ }^{38}$ and also including data from the risperidone plus parent training study ${ }^{126}$ and one paper that reported cognitive changes in a subset of children in the original 8-week trial. ${ }^{30}$ This family also includes another paper that assessed longer term (16 weeks) effects in children who responded to risperidone in the original 8-week trial plus children who originally received placebo and were considered placebo nonresponders but had subsequent positive response to risperidone (total $n=63$ ); ${ }^{36} 32$ of these children went on to enroll in an RCT comparing either continued risperidone or risperidone with gradual placebo replacement. ${ }^{31}$ The investigators followed up these reports with a paper reporting additional social interaction and stereotypy analyses of the 101 original participants in the 8-week trial and the 63 participants in the extension trial, ${ }^{32}$ analyses of weight changes in the 63 children in the extension trial, ${ }^{33}$ and analysis of adaptive behavior measures in 48 of these 63 children for whom such data were available. ${ }^{37}$ Finally, the family includes a paper reporting longer term effects (mean 21 months) in 84 of the original 101 trial participants (38 of whom participated in the extension trial); among these 84 individuals, 53 continued to receive risperidone in the month before followup. ${ }^{28}$ Another paper reports changes in prolactin levels in these children after 8 weeks, 6 months, and roughly 22 months of risperidone treatment. ${ }^{111}$

The second family of papers assessed aripiprazole and includes one 8-week trial comparing fixed doses of aripiprazole with placebo; ${ }^{20}$ another 8-week trial comparing titrated doses of aripiprazole with placebo; ${ }^{21}$ one paper reporting safety data in these two original trials; ${ }^{19}$ another reporting health-related quality of life measures assessed in the two original trials; ${ }^{17}$ and another outlining Aberrant Behavior Checklist (ABC) data in the two trials. ${ }^{16}$ The family also includes as open label extension that combined children from the original RCTs (both treatment and placebo groups) and added children who had not participated in the prior studies (de novo subjects) and in which all 330 participants received 52 weeks of aripiprazole. ${ }^{15}$ Finally, this family includes a paper reporting adverse events/safety data for children in the 52-week open label extension. ${ }^{18}$

Across studies, treatment duration ranged from 8 weeks to over 2 years, with followup immediately post-treatment in all studies.

\section{Detailed Analysis}

The literature on antipsychotic effects in children with ASD reports a variety of outcomes but converges on the ABC, a rating scale completed by caregivers of individuals with ASD. Studies also typically assessed potential side effects or harms, including assessment of weight gain, somnolence, and gastrointestinal symptoms and used the Clinical Global Impression (CGI) rating scale. Studies of antipsychotics addressed either risperidone or aripiprazole and reported 
significant improvements in measures of challenging behavior in the short term ( $<6$ months) in children receiving the medications compared with those receiving placebo. Harms of these agents, including extrapyramidal symptoms and weight gain, were also significant. Studies reporting longer term followup (up to 21 months for risperidone) reported continued effectiveness in most children but did not include control groups. We report brief summaries of outcomes reported in each study below and end of treatment outcomes on the ABC and CGI in Tables 7-10. Appendix F includes detailed summary tables outlining other outcomes.

\section{Studies of Risperidone}

The four RCTs comparing risperidone and placebo included one study (reported in multiple publications) conducted by RUPP investigators (low risk of bias). ${ }^{28-39,112,113}$ In the initial 8-week trial including 101 children, ${ }^{34}$ baseline ratings of irritability were similar across risperidone and placebo arms. The risperidone arm had significantly greater decreases (improvement) in ABCIrritability scores compared with the placebo arm (improvements of 15.1 vs. 3.6 points, $\mathrm{p}<0.001)$. Clinician ratings of outcomes considered by parents to be chief concerns paralleled these findings of significant improvement in challenging behavior in the risperidone group. ${ }^{35} \mathrm{In}$ other sub-analyses of participants in the original 8-week trial, ABC-Social Withdrawal scores were significantly improved in the treatment group compared with placebo (mean difference of 3.82, $\mathrm{p}=0.05$, effect size: 0.42 ) as were scores on the Children's Yale-Brown Obsessive Compulsive Scale (CYBOCS) ( $\mathrm{p}=0.005$, effect size: 0.74 ), but scores on cognitive measures in a subset of 38 participants who were able to complete the assessments typically did not differ between groups, though no decline in cognitive skills was associated with treatment. ${ }^{30,38,39}$

In another series of followup papers from this original RCT, investigators randomized responders to risperidone from the original trial and children who originally received placebo and were considered placebo nonresponders but had subsequent positive response to risperidone to either risperidone or risperidone with gradual placebo replacement for 16 weeks. ${ }^{31,36}$ More children in the gradual replacement arm $(n=10,62.5 \%)$ compared with risperidone $(n=2,12.5 \%$, $\mathrm{p}=0.01$ ) experienced relapse (25\% increase in ABC-Irritability score and CGI-Improvement rating of much or very much worse compared with baseline). In followup analyses of these participants plus children from the initial 101 in the 8-week trial, children receiving risperidone for up to 16 weeks had significant improvements in parent-rated measures of affect, repetitive and restricted behaviors, and sensory responses compared with children receiving placebo, but measures of social relatedness or language did not differ between groups. ${ }^{32}$ In a report of 48 children participating in the 16-week extension and receiving risperidone, scores on Vineland Adaptive Behavior Scales (VABS) measures of communication and daily living skills improved significantly over the treatment period; however, this analysis lacked a control arm. ${ }^{37}$

In an extension of these analyses, investigators followed 84 of the initial 101 children in the 8-week trial (38 also participated in the extension trials). ${ }^{28}$ These 84 children received risperidone for some portion of the mean 21-month uncontrolled followup; children could have received another antipsychotic or other medication, but 96 percent received risperidone over the followup period, and 68 percent were taking an antipsychotic in the month prior to followup. CGI-Severity scores improved significantly from baseline, regardless of treatment in the original trial (effect size: -0.75) with risperidone. ABC-Irritability similarly improved significantly from baseline (effect size -1.01) as did the ABC-Social Withdrawal (effect size: -0.85), ABC-

Stereotypy (effect size: -0.82), ABC-Hyperactivity (effect size: -1.07), and ABC-Inappropriate Speech (-0.41) scales. Scores on the CYBOCS also improved significantly from baseline (effect 
size: -0.79), while scores on the VABS typically improved, but not significantly. IQ did not change significantly with risperidone.

Finally, in post-hoc analyses reporting data from the original RUPP triall ${ }^{34}$ and an RUPP trial of risperidone compared with risperidone plus parent training ${ }^{126}$ (reported in detail in our 2011 review), effect sizes on the ABC-Social withdrawal subscale were 0.65 in the risperidone only group and 0.65 in the combination group; ${ }^{39}$ scores were significantly better than those of the placebo arm in the original 8-week trial or risperidone vs. placebo. ${ }^{34}$ In another subanalysis, ${ }^{38}$ effect sizes on the CYBOCS were 0.88 in the risperidone only group and 0.86 in the combination group, both significantly improved compared with the placebo arm in the original RUPP risperidone trial.

In another RCT (low risk of bias) comparing a low dose risperidone group, a high dose risperidone group, and a placebo group (total $n=96$ ), baseline scores for ABC-Irritability and the CGI scales were similar across all groups. ${ }^{51,52}$ The high dose risperidone arm had the greatest improvement in ABC-Irritability scores, followed by the low-dose group and placebo. The difference between high dose risperidone and placebo alone was statistically significant $(\mathrm{p}<0.001)$, but differences between low dose and placebo were not. The study reported similar improvements in CGI, with the greatest decrease in CGI scores for high dose risperidone and statistically significant differences between only the high dose group and placebo $(\mathrm{p}<0.001)$. In a 6-month open label extension of risperidone including 79 (56 completers) of the 96 children originally enrolled in the RCT, children received either fixed dose or flexibly dose risperidone with a median dose of $0.875 \mathrm{mg} /$ day in the open-label phase. All groups improved from baseline on the ABC-Irritability scale, with no significant differences between groups. Other measures taken at the end of the study included ABC-Hyperactivity, ABC-Stereotypic Behavior, ABCInappropriate Speech, ABC-Social Withdrawal, CYBOCS, CGI-Severity and CGI-Improvement, all of which showed improvement from baseline with no significant group differences.

One moderate risk of bias RCT reported in the 2011 review reported statistically significant improvements on the ABC-Irritability, Stereotypy, and Hyperactivity subscales in children receiving risperidone compared with the placebo group. ${ }^{112,113}$ The final RCT (moderate risk of bias) assessed outcomes after 6 months of risperidone treatment using a variety of general rating scales but provided quantitative data on only some of these scales. ${ }^{109}$ The primary outcome measures were parent ratings on the Childhood Autism Rating Scale (CARS) and clinician ratings on the Children's Global Assessment Scale (CGAS). The study only reported CARS median ratings for those participants with at least a 20 percent response; more children receiving risperidone achieved this goal compared with placebo (12 vs. $0, \mathrm{p}<0.001$ ). Average ratings on the CGAS were similar in the risperidone (29.8) and placebo (32.7) arms, with more improvement in the risperidone vs. placebo arms $(\mathrm{p}=0.04)$. Parent-rated scores did not differ between groups.

In one discontinuation study, investigators randomized children who had responded to risperidone in an initial 24-week trial to discontinuation (taper for three weeks and 5 weeks off of risperidone) or continuation for eight weeks. ${ }^{102}$ For the primary outcome measure of relapse, 8 of 12 patients relapsed in the placebo group and 3 of 12 patients relapsed in the risperidone group $(p=0.49)$. ABC-Irritably scores increased by 60 percent in the placebo group 14 percent in the risperidone group $(\mathrm{p}=0.43$ ). Differences in other ABC scales (Social Withdrawal, Stereotypy, Hyperactivity and Inappropriate Speech) were not statistically significant. Table 7 outlines key outcomes. 
Table 7. Key outcomes in studies comparing risperidone and placebo

\begin{tabular}{|c|c|c|}
\hline $\begin{array}{l}\text { Author, Year, Study Design } \\
\text { Groups (Dose), N Enrollment / N } \\
\text { Final } \\
\text { Treatment Duration/Follow-Up } \\
\text { Time Point Post-Treatment } \\
\text { Risk Of Bias }\end{array}$ & Baseline Scores, Mean \pm SD & Post-Treatment Scores, Mean \pm SD \\
\hline $\begin{array}{l}\text { Scahill } 2015^{34} \text { RCT } \\
\text { G1: Risperidone (up to } 2.5 \mathrm{mg} / \mathrm{kg} \text { ), } \\
49 / 49 \\
\text { G2: Placebo (NA), } 52 / 52 \\
8 \text { weeks/EOT } \\
\text { Moderate ROB }\end{array}$ & $\begin{array}{l}\text { ABC-Irritability } \\
\text { G1: } 26.2 \pm 7.9 \\
\text { G2: } 25.5 \pm 6.6 \\
\text { ABC-Lethargy/Social Withdrawal } \\
\text { G1: } 16.4 \pm 8.2 \\
\text { G2: } 16.1 \pm 8.7 \\
\text { ABC-Stereotypic Behavior } \\
\text { G1: } 10.6 \pm 4.9 \\
\text { G2: } 9 \pm 4.4 \\
\text { ABC-Hyperactivity } \\
\text { G1: } 31.8 \pm 9.6 \\
\text { G2: } 32.3 \pm 8.5 \\
\text { ABC-Inappropriate Speech } \\
\text { G1: } 4.8 \pm 4.1 \\
\text { G2: } 6.5 \pm 3.6 \\
\text { CGI-S - Moderate } \\
\text { G1: } 9 \text { (18) } \\
\text { G2: } 9 \text { (18) } \\
\text { CGI-S - Marked } \\
\text { G1: } 27 \text { (55) } \\
\text { G2: } 28 \text { (57) } \\
\text { CGI-S - Severe } \\
\text { G1: } 12 \text { (24) } \\
\text { G2: } 12 \text { (24) } \\
\text { CGI-S - Extreme } \\
\text { G1: } 1 \text { (2) } \\
\text { G2: } 0 \text { (0) }\end{array}$ & $\begin{array}{l}\text { ABC-Irritability } \\
\text { G1: } 11.3 \pm 7.4 \\
\text { G2: } 21.9 \pm 9.5 \\
\text { G1 vs. G2: } p<0.001 ; \mathrm{ES}=1.2 \\
\text { ABC-Lethargy/Social Withdrawal } \\
\text { G1: } 8.9 \pm 6.4 \\
\text { G2: } 12 \pm 8.3 \\
\text { G1 vs. G2: } p=0.03, \mathrm{ES}=0.4 \\
\text { ABC-Stereotypic Behavior } \\
\text { G1: } 5.8 \pm 4.6 \\
\text { G2: } 7.3 \pm 4.8 \\
\text { G1 vs. G2: } p<0.001 \\
\text { ES=0.8 } \\
\text { ABC-Hyperactivity } \\
\text { G1: } 17 \pm 9.7 \\
\text { G2: } 27.6 \pm 10.6 \\
\text { G1 vs. G2: } p<0.001 \\
\text { ABC-Inappropriate Speech } \\
\text { G1: } 3 \pm 3.1 \\
\text { G2: } 5.9 \pm 3.8 \\
\text { G1 vs. G2: } p=0.03 \text {. ES=0.3 } \\
\text { CGI-I - Much Improved or very much } \\
\text { improved }+25 \% \text { reduction on ABI-I } \\
\text { G1: } 34(69) \\
\text { G2: } 6 \text { (12) }\end{array}$ \\
\hline $\begin{array}{l}\text { Scahill } 2015^{36} \text { RCT } \\
\text { G1: Risperidone ( } 2.5 \mathrm{mg} / \text { day), } \\
63 / 63 \\
\text { G2: Placebo-Substitution (NA), NA } \\
4 \text { weeks during open label } \\
\text { extension/EOT } \\
\text { Moderate ROB }\end{array}$ & $\begin{array}{l}\text { End of initial } 8 \text { wks of treatment } \\
\text { exposure } \\
\text { CGI-I - Very much improved } \\
\text { G1: } 19(30.2) \\
\text { G2: ND } \\
\text { CGI-I - Much Improved } \\
\text { G1: } 42 \text { (66.7) } \\
\text { G2: ND } \\
\text { CGI-I Minimally Improved } \\
\text { G1: O (0) } \\
\text { G2: ND } \\
\text { CGI-I - No Change } \\
\text { G1: } 2 \text { (3.2) } \\
\text { G2: ND }\end{array}$ & $\begin{array}{l}\text { ABC-Irritability } \\
\text { G1: } 11.7 \pm 8 \\
\text { G2: ND } \\
\text { ABC-Social Withdrawal/Lethargy } \\
\text { G1: } 6.8 \pm 5.9 \\
\text { G2: ND } \\
\text { ABC-Stereotypy } \\
\text { G1: } 5.8 \pm 4.7 \\
\text { G2: ND } \\
\text { ABC-Hyperactivity } \\
\text { G1: } 15.8 \pm 10.2 \\
\text { G2: ND } \\
\text { ABC-Inappropriate Speech } \\
\text { G1: } 3.4 \pm 3.2\end{array}$ \\
\hline
\end{tabular}




\begin{tabular}{|c|c|c|}
\hline $\begin{array}{c}\text { Author, Year, Study Design } \\
\text { Groups (Dose), N Enrollment I N } \\
\text { Final } \\
\text { Treatment Duration/Follow-Up } \\
\text { Time Point Post-Treatment } \\
\text { Risk Of Bias }\end{array}$ & Baseline Scores, Mean \pm SD & Post-Treatment Scores, Mean \pm SD \\
\hline & $\begin{array}{l}\text { CGI-I - Worse } \\
\text { G1: } 0 \text { (0) } \\
\text { G2: ND } \\
\text { CGI-I - Much Worse } \\
\text { G1: } 0 \text { (0) } \\
\text { G2: ND } \\
\text { ABC-Irritability } \\
\text { G1: } 9.5 \pm 6.8 \\
\text { G2: ND } \\
\text { ABC-Social Withdrawal/Lethargy } \\
\text { G1: } 7.3 \pm 5.4 \\
\text { G2: ND } \\
\text { ABC-Stereotypy } \\
\text { G1: } 4.9 \pm 4.3 \\
\text { G2: ND } \\
\text { ABC-Hyperactivity } \\
\text { G1: } 15.1 \pm 10 \\
\text { G2: ND } \\
\text { ABC-Inappropriate Speech } \\
\text { G1: } 3.4 \pm 3.6 \\
\text { G2: ND }\end{array}$ & G2: ND \\
\hline $\begin{array}{l}\text { Scahill } 2015^{28} \mathrm{RCT} \\
\text { G1: Risperidone ( } 2.5 \mathrm{mg} / \text { day), } \\
\text { 57/55 } \\
\text { G2: Placebo (NA), } 27 / 26 \\
\text { Mean } 21 \text { months/2 Years post- } \\
\text { treatment } \\
\text { Moderate ROB }\end{array}$ & $\begin{array}{l}\text { ABC-Irritability } \\
\text { G1: } 27.22 \pm 7.28 \\
\text { G2: } 23.44 \pm 7.24 \\
\text { ABC-Social Withdrawal } \\
\text { G1: } 16.05 \pm 8.55 \\
\text { G2: } 18.52 \pm 9.72 \\
\text { ABC-Stereotypic Behavior } \\
\text { G1: } 10.5 \pm 4.43 \\
\text { G2: } 8.84 \pm 5.22 \\
\text { ABC-Hyperactivity/Noncompliance } \\
\text { G1: } 34.3 \pm 7.95 \\
\text { G2: } 28.58 \pm 10.4 \\
\text { ABC-Inappropriate Speech } \\
\text { G1: } 5.71 \pm 3.93 \\
\text { G2: } 5.59 \pm 4.03 \\
\text { CGI-Severity } \\
\text { G1: } 5.090 .7 \\
\text { G2: } 5.230 .65\end{array}$ & $\begin{array}{l}2 \text { years post-treatment } \\
\text { ABC-Irritability } \\
\text { G1: } 14.82 \pm 8.4 \\
\text { G2: } 17.78 \pm 10.82 \\
\text { G1 vs. G2: } p=0.0147 \\
\text { ABC-Social Withdrawal } \\
\text { G1: } 8.43 \pm 6.77 \\
\text { G2: } 13.33 \pm 8.73 \\
\text { G1 vs. G2: } p=0.0130 \\
\text { ABC-Stereotypic Behavior } \\
\text { G1: } 6.02 \pm 4.4 \\
\text { G2: } 6.76 \pm 5.37 \\
\text { G1 vs. } G 2: p=0.0866 \\
\text { ABC-Hyperactivity/Noncompliance } \\
\text { G1: } 17.68 \pm 10.16 \\
\text { G2: } 23.38 \pm 12.06 \\
\text { G1 vs. G2: } p=0.0020 \\
\text { ABC-Inappropriate Speech } \\
\text { G1: } 3.86 \pm 3.01 \\
\text { G2: } 5.15 \pm 4.24 \\
\text { G1 vs. G2: } p=0.0433\end{array}$ \\
\hline
\end{tabular}




\begin{tabular}{|c|c|c|}
\hline $\begin{array}{c}\text { Author, Year, Study Design } \\
\text { Groups (Dose), N Enrollment / N } \\
\text { Final } \\
\text { Treatment Duration/Follow-Up } \\
\text { Time Point Post-Treatment } \\
\text { Risk Of Bias }\end{array}$ & Baseline Scores, Mean \pm SD & Post-Treatment Scores, Mean \pm SD \\
\hline & & $\begin{array}{l}\text { CGI-Severity } \\
\text { G1: } 4.4 \pm 0.89 \\
\text { G2: } 4.65 \pm 1.09 \\
\text { G1 vs. G2: } p=0.3004\end{array}$ \\
\hline $\begin{array}{l}\text { Kent } 2013^{51,52} \text { RCT } \\
\text { G1: Risperidone }(0.125-0.175 \\
\text { mg/day; low dose), 30/25 } \\
\text { G2: Risperidone }(1.25-1.75 \text { mg/day; } \\
\text { high dose), 31/25 } \\
\text { G3: Placebo (NA), 35/27 } \\
6 \text { weeks/EOT } \\
\text { Low ROB }\end{array}$ & 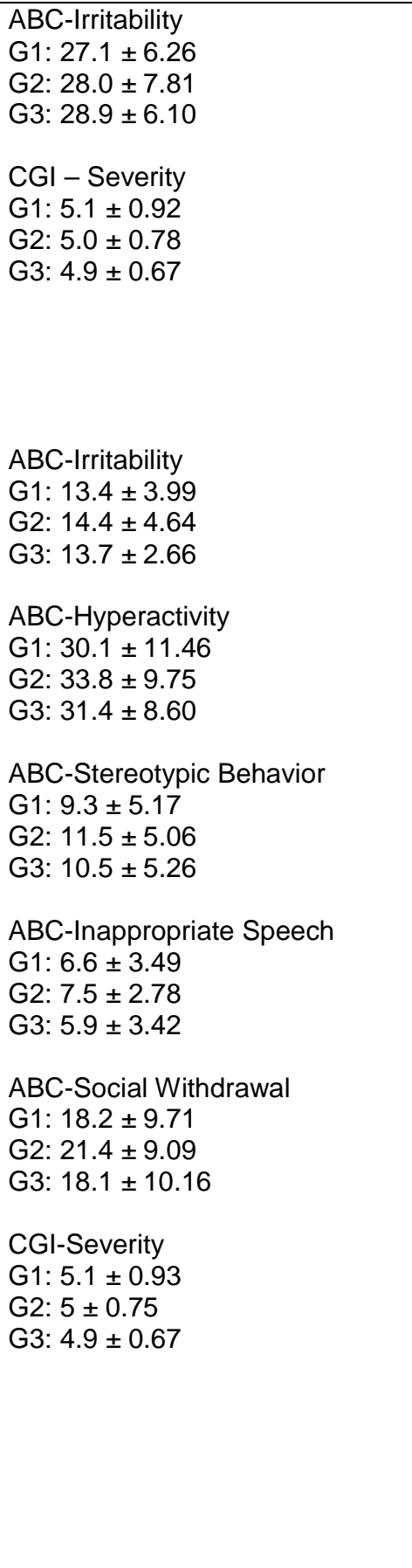 & 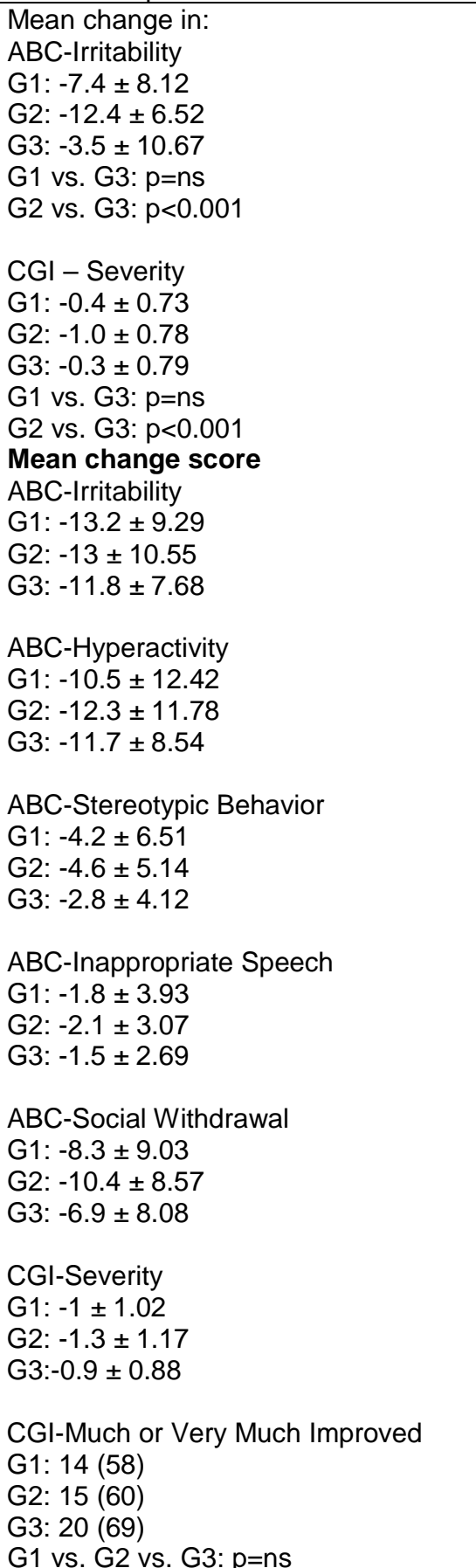 \\
\hline
\end{tabular}




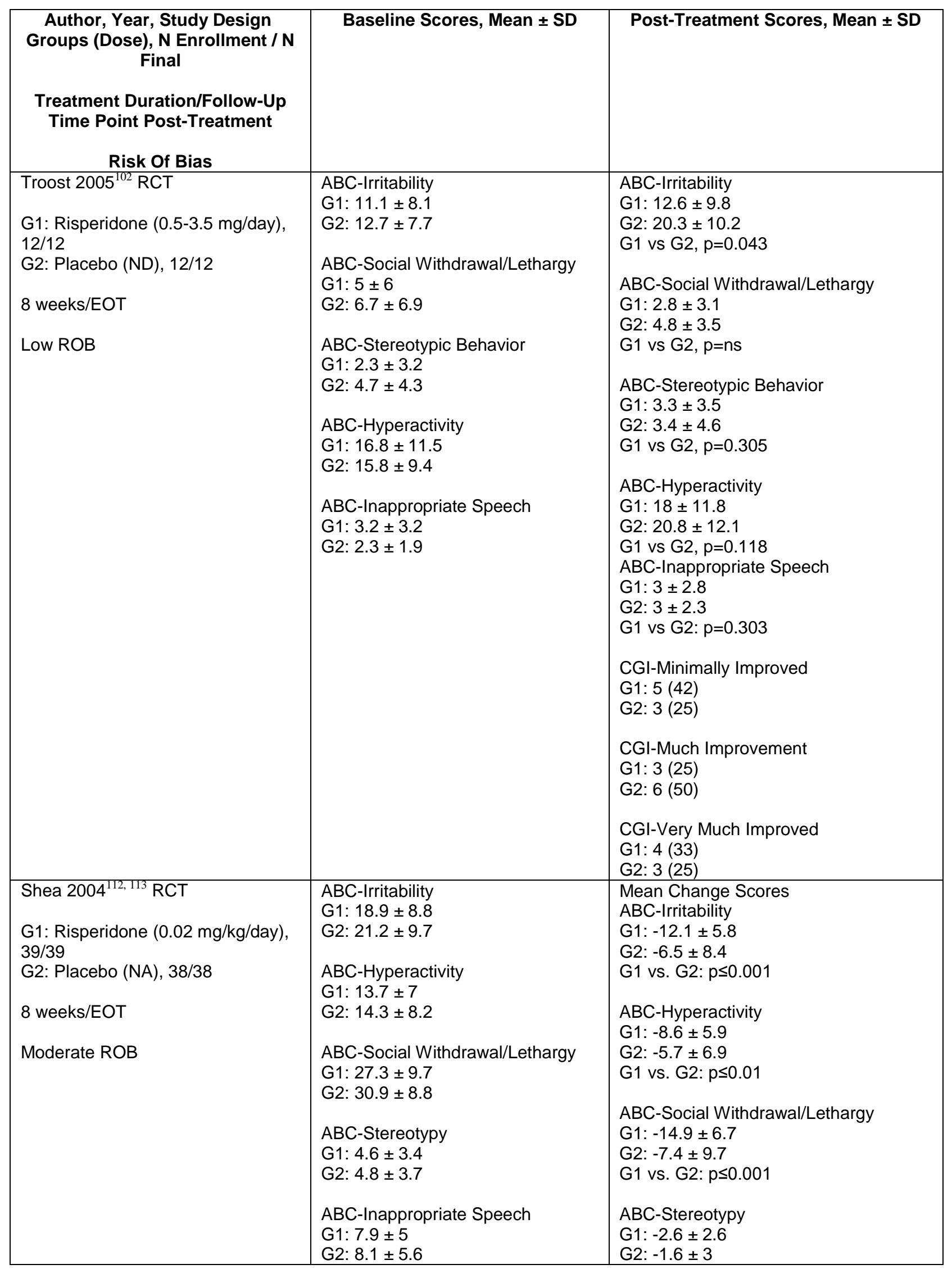




\begin{tabular}{|c|l|l|}
\hline $\begin{array}{c}\text { Author, Year, Study Design } \\
\text { Groups (Dose), N Enrollment } / \mathbf{N} \\
\text { Final }\end{array}$ & Baseline Scores, Mean \pm SD & Post-Treatment Scores, Mean \pm SD \\
$\begin{array}{c}\text { Treatment Duration/Follow-Up } \\
\text { Time Point Post-Treatment }\end{array}$ & & \\
Risk Of Bias & & \\
\hline & & G1 vs. G2: $p \leq 0.05$ \\
& & ABC-Inappropriate Speech \\
& & G1: $-4.3 \pm 3.8$ \\
& & G2: $-2.4 \pm 4$ \\
& & G1 vs. G2: $p \leq 0.05$ \\
\hline
\end{tabular}

ABC = Aberrant Behavior Checklist; CGI-I = Clinical Global Impression Scale Improvement, EOT = end of treatment; ES = effect size; $\mathrm{G}=$ group; $\mathrm{kg}=$ kilograms; $\mathrm{mg}=$ milligrams; $\mathrm{mL}=$ milliliters; $\mathrm{NA}=$ not applicable; $\mathrm{ND}=$ no data; $\mathrm{RCT}=$ randomized controlled trial; $\mathrm{ROB}$ = risk of bias; $\mathrm{SD}=$ standard deviation

\section{Studies of Aripiprazole}

Two 8-week RCTs (low risk of bias) of aripiprazole compared with placebo were reported in our 2011 review. $^{20,21}$ In these two studies, baseline ratings of irritability were similar across aripiprazole and placebo arms (Table 8). Decreases in ABC-Irritability were significantly greater for the aripiprazole arms in both studies, with improvements of 12.4 to14.4, in comparison with the placebo arms, with improvements of 5.0 to 8.4. The trial with differing set doses of aripiprazole demonstrated increasing response with increasing dose. ${ }^{20}$ Overall, the results of the trial that used titration following clinical judgment were more pronounced. ${ }^{21}$ Decreases (improvements) in ABC-Hyperactivity and Stereotypy subscales were significantly greater in children receiving aripiprazole compared with placebo arms. Scores in the ABC-Inappropriate Speech subscale were also significantly improved in the treatment group vs. placebo in the flexibly dosed study but not in the fixed dose RCT. Both studies also used the CYBOCS scale to assess repetitive behavior, finding no baseline differences between the groups but a greater decrease in the aripiprazole compared with placebo arms (2.4 to 3.8 vs. 0.8 to 1.7$)$. A number of other outcomes were measured in these two studies, but none outside of challenging behavior and repetitive behavior yielded statistically significant findings once corrected for multiple comparisons.

Post-hoc analyses of these RCTs analyzed changes in ABC scores ${ }^{16}$ and quality of life measures. ${ }^{17}$ The first post-hoc analysis ${ }^{16}$ analyzed the following changes in ABC subscales. Scores on the ABC-Irritability, Stereotypy, Hyperactivity, and Inappropriate Speech subscales were significantly improved in the aripiprazole arms compared with placebo (all p values $<0.05$ ), typically with greater decreases in the flexibly dosed group compared with the fixed dose group. Scores on the Social Withdrawal subscale did not differ significantly between treatment and placebo groups. Compared with placebo, the pooled aripiprazole groups had greater improvements in total health-related quality of life scores and emotional, social, and cognitive functioning scores measured using the Pediatric Quality of Life Inventory (all p values < 0.05). ${ }^{17}$ Children who received aripiprazole were also more likely to have clinically meaningful improvement on all these scales compared with those receiving placebo (odds ratios ranging from 1.2 to 2.2 , p values $<0.05$ ).

Investigators extended these 8-week studies with an uncontrolled, 52-week open label analysis including 70 children who had received placebo in the original RCTs, 174 who had received aripiprazole, and 86 "de novo" subjects. ${ }^{15}$ Primary outcomes included the ABCIrritability and CGI-Severity scales. ABC-Irritability mean scores at baseline were higher for the 
de novo (23.2 \pm 8.9$)$ and prior placebo (21.5 \pm 9.8$)$ groups compared with the prior aripiprazole group (15.0 \pm 9.2 ). Mean change from baseline in the de novo group was $-8.0 \pm-10.1$ and in the prior placebo group was $-6.1 \pm 11.9$. Improvements in scores in the de novo and prior aripiprazole groups occurred in first 8 weeks of the open label phase. The CGI decreased in the same manner, with greater reductions (improvements) in the de novo and prior placebo groups than in the prior aripiprazole group, in which improvements reported in the prior RCTs were maintained. Scores in the ABC-Hyperactivity subscale and CYBOCS followed similar patterns.

An additional low risk of bias RCT randomized 85 children who had shown a stable improvement ( $\geq 25 \%$ decrease in ABC-Irritability scores for 12 weeks) in an initial 13-26 week open label phase to continued, flexibly dosed aripiprazole or placebo until relapse (defined as $\geq 25 \%$ increase in ABC-Irritability score, CGI ratings of worse or much worse, loss-to-followup plus elevated scores, elevated scores plus initiation of other medication to treat symptoms, or discontinuation due to worsening symptoms) or 16 weeks. ${ }^{48}$ The difference between the two groups in time to relapse was not statistically significant (35\% in the aripiprazole arm at 16 weeks vs. $52 \%$ in placebo, hazard ratio $=0.57,95 \%$ confidence interval [CI]: 0.28 to 1.12 , number needed to treat=6). Mean change in the ABC-Irritability and Social Withdrawal scores or CGIImprovement score from baseline to week 16 did not differ between groups ( $\mathrm{p} \geq 0.05)$, but children in the placebo group had greater increases (i.e., worsening behavior) on the ABCHyperactivity and Inappropriate Speech subscales than did children in the treatment group (p values $<0.05$ ); pediatric quality of life measures also did not differ between groups. 
Table 8. Key outcomes in studies comparing aripiprazole and placebo

\begin{tabular}{|c|c|c|}
\hline $\begin{array}{c}\text { Author, Year, Study Design } \\
\text { Groups (Dose), N Enrollment I N } \\
\text { Final } \\
\text { Treatment Duration/Follow-Up Time } \\
\text { Point Post-Treatment } \\
\text { Risk Of Bias }\end{array}$ & Baseline Scores, Mean \pm SD & $\begin{array}{c}\text { Post-Treatment Scores, Mean } \\
\pm \text { SD }\end{array}$ \\
\hline $\begin{array}{l}\text { Marcus } 2011^{20} \mathrm{RCT} \\
\text { G1: Aripiprazole (5 mg/kg), 53/44 } \\
\text { G2: Aripiprazole (10 mg/kg), 59/49 } \\
\text { G3: Aripiprazole }(15 \mathrm{mg} / \mathrm{kg}), 54 / 47 \\
\text { G4: Placebo (NA), } 52 / 38 \\
8 \text { weeks/EOT } \\
\text { Moderate ROB }\end{array}$ & $\begin{array}{l}\text { ABC-Irritability } \\
\text { G1: } 28.6 \pm 7.6 \\
\text { G2: } 28.2 \pm 7.4 \\
\text { G3: } 28.9 \pm 6.4 \\
\text { G4: } 28 \pm 6.9 \\
\text { ABC-Hyperactivity/Noncompliance } \\
\text { G1: } 33.1 \pm 1.4 \\
\text { G2: } 33.7 \pm 1.3 \\
\text { G3: } 32.2 \pm 1.4 \\
\text { G4: } 31 \pm 1.4 \\
\text { ABC-Stereotypic Behavior } \\
\text { G1: } 11.4 \pm 0.8 \\
\text { G2: } 11.6 \pm 0.8 \\
\text { G3: } 11.6 \pm 0.8 \\
\text { G4:10.7 } 0.8 \\
\text { ABC-Social Withdrawal/Lethargy } \\
\text { G1: } 17.7 \pm 1.4 \\
\text { G2: } 16.8 \pm 1.3 \\
\text { G3: } 18.9 \pm 1.4 \\
\text { G4: } 18 \pm 1.5 \\
\text { ABC-Inappropriate Speech } \\
\text { G1: } 5.8 \pm 0.6 \\
\text { G2: } 6.8 \pm 0.5 \\
\text { G3: } 6.3 \pm 0.5 \\
\text { G4: } 5.9 \pm 0.6 \\
\text { CGI-S } \\
\text { G1: } 5 \pm 0.1 \\
\text { G2: } 4.9 \pm 0.1 \\
\text { G3: } 5.1 \pm 0.1 \\
\text { G4: } 4.7 \pm 0.1 \\
\end{array}$ & 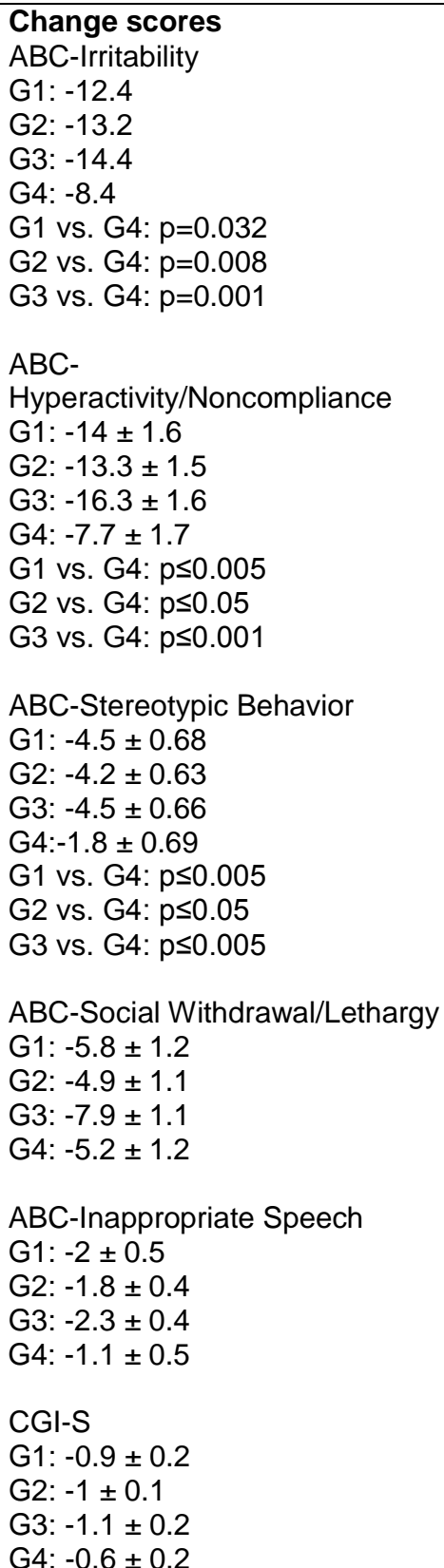 \\
\hline $\begin{array}{l}\text { Marcus } 2011^{21} \mathrm{RCT} \\
\text { G1: Aripiprazole (2-15 mg/kg), 47/39 } \\
\text { G2: Placebo (NA), } 51 / 36 \\
8 \text { weeks/EOT }\end{array}$ & $\begin{array}{l}\text { ABC-Irritability } \\
\text { G1: } 29.6 \pm 6.4 \\
\text { G2: } 30.2 \pm 6.5 \\
\text { ABC-Hyperactivity/Noncompliance } \\
\text { G1: } 34.1 \\
\text { G2: } 34.7\end{array}$ & $\begin{array}{l}\text { Change Scores } \\
\text { ABC-Irritability } \\
\text { G1: }-12.9 \\
\text { G2: }-5 \\
\text { G1 vs. G2: } p<0.001 \\
\text { ABC- }\end{array}$ \\
\hline
\end{tabular}




\begin{tabular}{|c|c|c|}
\hline $\begin{array}{c}\text { Author, Year, Study Design } \\
\text { Groups (Dose), N Enrollment / N } \\
\text { Final } \\
\text { Treatment Duration/Follow-Up Time } \\
\text { Point Post-Treatment } \\
\text { Risk Of Bias }\end{array}$ & Baseline Scores, Mean \pm SD & $\begin{array}{c}\text { Post-Treatment Scores, Mean } \\
\pm \text { SD }\end{array}$ \\
\hline Moderate ROB & $\begin{array}{l}\text { ABC-Stereotypic Behavior } \\
\text { G1: } 11.9 \\
\text { G2: } 10.7 \\
\text { ABC-Inappropriate Speech } \\
\text { G1: } 7 \\
\text { G2: } 7\end{array}$ & $\begin{array}{l}\text { Hyperactivity/Noncompliance } \\
\text { G1: -12.7 } \\
\text { G2: }-2.8 \\
\text { G1 vs. G2: p<0.001 } \\
\text { ABC-Stereotypic Behavior } \\
\text { G1: -4.8 } \\
\text { G2: -2 } \\
\text { G1 vs. G2: p<0.001 } \\
\text { ABC-Inappropriate Speech } \\
\text { G1: -2.5 } \\
\text { G2: }-0.4 \\
\text { G1 vs. G2: p<0.001 } \\
\text { CGI-Severity } \\
\text { G1: -1.2 } \\
\text { G2: -0.4 } \\
\text { CGI-I - Very much improved or } \\
\text { much improved } \\
\text { G1: } 31 \text { (67) } \\
\text { G2: } 8 \text { (16) } \\
\text { CGI-I - Minimally improved } \\
\text { G1: } 7 \text { (15) } \\
\text { G2: } 10 \text { (20) } \\
\text { CGI-I - No change } \\
\text { G1: } 6 \text { (13) } \\
\text { G2: } 22 \text { (45) } \\
\text { CGI-I - Minimally worse } \\
\text { G1: } 2 \text { (4) } \\
\text { G2: } 5 \text { (10) } \\
\text { CGI-I - Much or very much } \\
\text { worse } \\
\text { G1: } 0 \text { (0) } \\
\text { G2: } 4 \text { (8) }\end{array}$ \\
\hline $\begin{array}{l}\text { Marcus } 2011^{15} \mathrm{RCT} \\
\text { G1: De Novo Subjects }(2-15 \mathrm{mg} / \mathrm{kg}) \\
\text { 84/55 } \\
\text { G2: Prior Placebo }(2-15 \mathrm{mg} / \mathrm{kg}), 69 / 37 \\
\text { G3: Prior Aripiprazole }(2-15 \mathrm{mg} / \mathrm{kg}) \\
\text { 169/107 } \\
52 \text { weeks }\end{array}$ & $\begin{array}{l}\text { CGI-Severity } \\
\text { G1: } 4.8 \pm 1 \\
\text { G2: } 4.2 \pm 1 \\
\text { G3: } 3.9 \pm 1.1 \\
\text { ABC-Irritability } \\
\text { G1: } 23.2 \pm 8.9 \\
\text { G2: } 21.5 \pm 9.8 \\
\text { G3: } 15 \pm 9.2\end{array}$ & $\begin{array}{l}\text { CGI-Severity } \\
\text { G1: }-1 \pm 0.8 \\
\text { G2: }-0.6 \pm 1.2 \\
\text { G3: }-0.1 \pm 1 \\
\text { ABC-Irritability } \\
\text { G1: }-8 \pm 10.1 \\
\text { G2: }-6.1 \pm 1.9 \\
\text { G3: } 0.7 \pm 10.2\end{array}$ \\
\hline Moderate ROB & $\begin{array}{l}\text { ABC-Lethargy/Social Withdrawal } \\
\text { G1: } 14.6 \pm 8.6 \\
\text { G2: } 11.3 \pm 9.2 \\
\text { G3: } 10.4 \pm 8.9\end{array}$ & $\begin{array}{l}\text { ABC-Lethargy/Social Withdrawal } \\
\text { G1: }-6.4 \pm 7.9 \\
\text { G2: }-4.1 \pm 7.2 \\
\text { G3: }-2.3 \pm 6.4\end{array}$ \\
\hline
\end{tabular}




\begin{tabular}{|c|c|c|}
\hline $\begin{array}{c}\text { Author, Year, Study Design } \\
\text { Groups (Dose), N Enrollment / N } \\
\text { Final } \\
\text { Treatment Duration/Follow-Up Time } \\
\text { Point Post-Treatment } \\
\text { Risk Of Bias }\end{array}$ & Baseline Scores, Mean \pm SD & $\begin{array}{c}\text { Post-Treatment Scores, Mean } \\
\pm \text { SD }\end{array}$ \\
\hline & $\begin{array}{l}\text { ABC-Stereotypic Behavior } \\
\text { G1: } 8.1 \pm 5.2 \\
\text { G2: } 9.1 \pm 5.6 \\
\text { G3: } 6.4 \pm 5.5 \\
\text { ABC-Hyperactivity } \\
\text { G1: } 28.4 \pm 10.9 \\
\text { G2: } 25.8 \pm 13.2 \\
\text { G3: } 18.4 \pm 12 \\
\text { ABC-Inappropriate Speech } \\
\text { G1: } 5.8 \pm 3.2 \\
\text { G2: } 5.7 \pm 4.2 \\
\text { G3: } 4.2 \pm 3.6\end{array}$ & $\begin{array}{l}\text { ABC-Stereotypic Behavior } \\
\text { G1: }-2.7 \pm 3.1 \\
\text { G2: }-1.9 \pm 4.1 \\
\text { G3: }-0.5 \pm 4.4 \\
\text { ABC-Hyperactivity } \\
\text { G1: }-12.3 \pm 8.5 \\
\text { G2: }-9.1 \pm 11.5 \\
\text { G3: } 0.6 \pm 10.3 \\
\text { ABC-Inappropriate Speech } \\
\text { G1: }-2 \pm 2.5 \\
\text { G2: }-1.8 \pm 3 \\
\text { G3: }-0.3 \pm 2.4\end{array}$ \\
\hline $\begin{array}{l}\text { Marcus } 2011^{16} \mathrm{RCT} \\
\text { G1: Aripiprazole (2-15 mg/kg; flexibly } \\
\text { dosed study), } 46 / 46 \\
\text { G2: Aripiprazole ( } 5 \mathrm{mg} / \mathrm{kg}), 52 / 52 \\
\text { G3: Aripiprazole }(10 \mathrm{mg} / \mathrm{kg}), 59 / 59 \\
\text { G4: Aripiprazole }(15 \mathrm{mg} / \mathrm{kg}), 53 / 53 \\
\text { G5: Placebo (flexibly dosed study) } \\
\text { 49/49 } \\
\text { G6: Placebo (fixed-dose study) 49/49 } \\
8 \text { weeks/EOT } \\
\text { Moderate ROB }\end{array}$ & 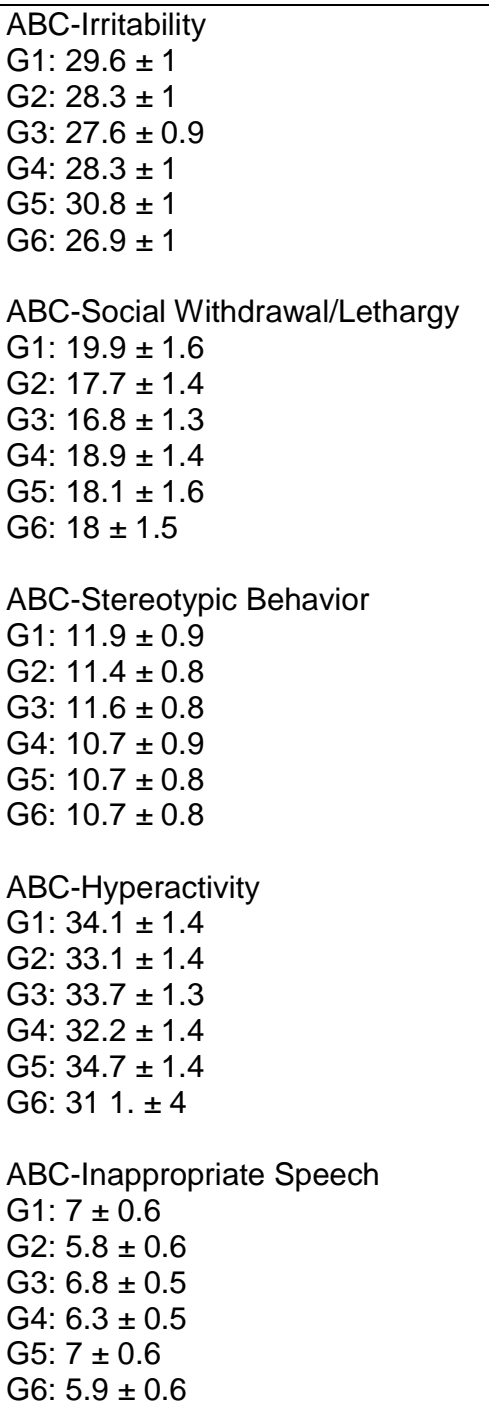 & 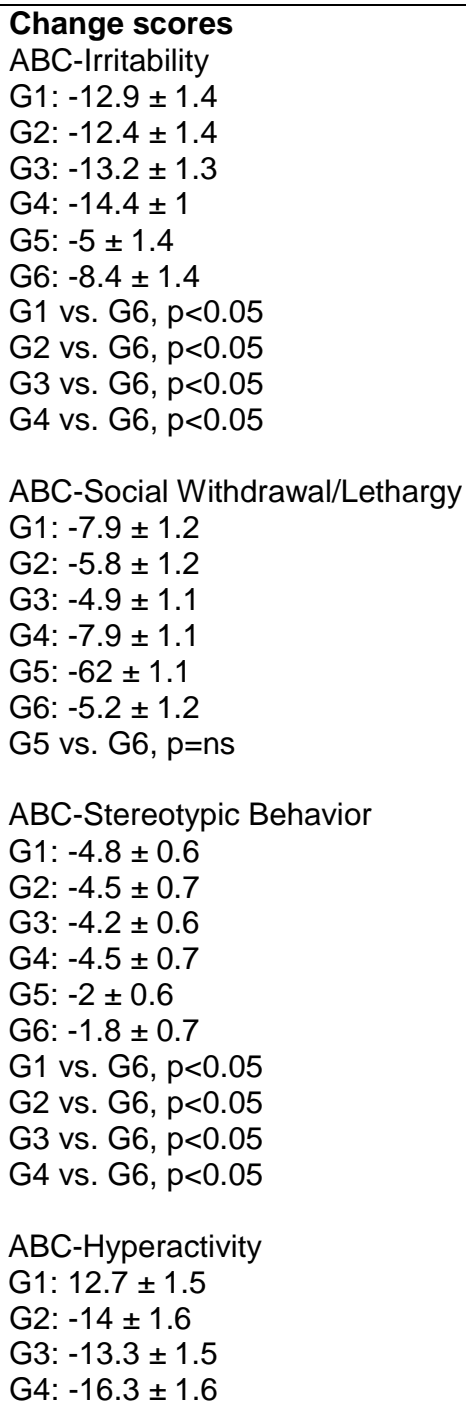 \\
\hline
\end{tabular}




\begin{tabular}{|c|c|c|}
\hline $\begin{array}{c}\text { Author, Year, Study Design } \\
\text { Groups (Dose), N Enrollment / N } \\
\text { Final } \\
\text { Treatment Duration/Follow-Up Time } \\
\text { Point Post-Treatment } \\
\text { Risk Of Bias }\end{array}$ & Baseline Scores, Mean \pm SD & $\begin{array}{c}\text { Post-Treatment Scores, Mean } \\
\pm \text { SD }\end{array}$ \\
\hline & & $\begin{array}{l}\text { G5: }-2.8 \pm 1.5 \\
\text { G6: }-7.7 \pm 1.7 \\
\text { G1 vs. } G 6, p<0.05 \\
\text { G2 vs. G6, } p<0.05 \\
\text { G3 vs. G6, } p<0.05 \\
\text { G4 vs. G6, } p<0.05 \\
\text { ABC-Inappropriate Speech } \\
\text { G1: }-2.5 \pm 0.4 \\
\text { G2: }-2 \pm 0.5 \\
\text { G3: }-1.8 \pm 0.4 \\
\text { G4: }-2.3 \pm 0.4 \\
\text { G5: }-0.4 \pm 0.4 \\
\text { G6: }-1.1 \pm 5 \\
\text { G4 vs. G6: } p<0.05\end{array}$ \\
\hline $\begin{array}{l}\text { Findling } 2014^{48} \mathrm{RCT} \\
\text { G1: Aripiprazole (2-15 mg/day), 41/22 } \\
\text { G2: Placebo (NA), } 44 / 19 \\
16 \text { weeks/EOT } \\
\text { Low ROB }\end{array}$ & $\begin{array}{l}\text { ABC-I - adjusted mean score } \\
\text { NR } \\
\text { CGI - improvement scale } \\
\text { NR }\end{array}$ & $\begin{array}{l}\text { Change scores } \\
\text { ABC- I } \\
\text { G1: } 5.2 \\
\text { G2: } 9.6 \\
\text { G1 vs. G2: } p=n s \\
\text { ABC- Hyperactivity } \\
\text { G1: } 5.0 \\
\text { G2: } 10.3 \\
\text { G1 vs. G2: } p=0.041 \\
\text { ABC- Stereotypy } \\
\text { G1: } 0.8 \\
\text { G2: } 2.8 \\
\text { G1 vs. G2: } p=0.018 \\
\text { ABC- Inappropriate speech } \\
\text { G1: } 0.6 \\
\text { G2: } 2.1 \\
\text { G1 vs. G2: } p=0.013 \\
\text { ABC- Social withdrawal } \\
\text { G1: } 0 \\
\text { G2: } 1.5 \\
\text { G1 vs. G2: } p=n s \\
\text { CGI- I } \\
\text { G1: } 4.2 \\
\text { G2: } 4.8 \\
\text { G1 vs. G2: } p=n s\end{array}$ \\
\hline
\end{tabular}

ABC = Aberrant Behavior Checklist; CGI-I = Clinical Global Impression Scale Improvement, EOT = end of treatment; $\mathrm{G}=$ group; kg = kilograms; mg = milligrams; NR = not reported; NS = not significant; RCT = randomized controlled trial; ROB = risk of bias; $\mathrm{SD}=$ standard deviation

\section{Studies Comparing Risperidone and Aripiprazole}

Three small studies comparing these agents reported no significant group differences in measures of challenging behavior or general improvement (Table 9). In one 24-week RCT (low 
risk of bias) comparing two groups of patients with ASD and attention deficit hyperactivity disorder (ADHD), both groups improved on measures of symptom severity including the ADHD Rating Scale and CGI (all p=NS) Of note, improvement with aripiprazole was evident in the first 12 weeks of treatment without additional improvements at 24 weeks, while scores on measures in children taking risperidone improved at 12 weeks and continued to improve over the 24 week period. $^{50}$ Another 8-week RCT (low risk of bias) reported improvements in ABC-Irritability, Hyperactivity, Lethargy, Stereotypy, and Inappropriate speech scores in both risperidone and aripiprazole arms. ${ }^{49}$ Most patients were much or minimally improved on the CGI-Improvement scale, but differences between the groups on all of these scales were not statistically significant. Another retrospective cohort study (moderate risk of bias), which primarily reports weight change (see Harms section below), noted no significant group differences in mean CGIImprovement scores (risperidone $=3.2 \pm 1.2$, aripiprazole $=2.9 \pm 1.2, \mathrm{p}=0.32$ ) after treatment with risperidone (mean treatment duration $=2.37 \pm 2.55$ years) or aripiprazole (mean treatment duration=1.47 \pm 1.21 years). ${ }^{122}$ The study did not report CGI scores at baseline so the magnitude of change cannot be assessed.

Table 9. Key outcomes in studies comparing risperidone and aripiprazole

\begin{tabular}{|c|c|c|}
\hline $\begin{array}{c}\text { Author, Year, Study Design } \\
\text { Groups (Dose), N Enrollment I N Final } \\
\text { Treatment Duration/Follow-Up Time } \\
\text { Point Post-Treatment } \\
\text { Risk Of Bias }\end{array}$ & Baseline Scores, Mean \pm SD & Post-Treatment Scores, Mean \pm SD \\
\hline $\begin{array}{l}\text { Lamberti } 2016^{50} \text { RCT } \\
\text { G1: Aripirazole (up to } 15 \mathrm{mg} / \text { day), 22/19 } \\
\text { G2: Risperidone (up to } 3 \text { mg/day), 22/18 } \\
24 \text { weeks/EOT } \\
\text { Low ROB }\end{array}$ & $\begin{array}{l}\text { CGI - Severity } \\
\text { G1: } 5.4 \pm 0.5 \\
\text { G2: } 5.5 \pm 0.6 \\
\text { CGAS - Total Score } \\
\text { G1: } 38 \pm 8.3 \\
\text { G2: } 31.42 \pm 12.4 \\
\text { ADHD Rating Scale - Total } \\
\text { Score } \\
\text { G1: } 39.4 \pm 2.8 \\
\text { G2: } 37.4 \pm 3.9 \\
\text { CPRS - Hyperactivity } \\
\text { G1: } 5.7 \pm 0.7 \\
\text { G2: } 5.4 \pm 0.7 \\
\text { CPRS - Inattention } \\
\text { G1: } 5.4 \pm 0.8 \\
\text { G2: } 4.9 \pm 0.9\end{array}$ & $\begin{array}{l}\text { CGI - Severity } \\
\text { G1: } 5.4 \pm 0.5 \\
\text { G2: } 5.5 \pm 0.6 \\
\text { CGAS - Total Score } \\
\text { G1: } 38 \pm 8.3 \\
\text { G2: } 31.42 \pm 12.4 \\
\text { ADHD Rating Scale - Total Score } \\
\text { G1: } 39.4 \pm 2.8 \\
\text { G2: } 37.4 \pm 3.9 \\
\text { CPRS - Hyperactivity } \\
\text { G1: } 5.7 \pm 0.7 \\
\text { G2: } 5.4 \pm 0.7 \\
\text { CPRS - Inattention } \\
\text { G1: } 5.4 \pm 0.8 \\
\text { G2: } 4.9 \pm 0.9\end{array}$ \\
\hline $\begin{array}{l}\text { Ghanizadeh } 2014^{49} \mathrm{RCT} \\
\text { G1: Aripiprazole ( } 1.25-10 \mathrm{mg} / \text { day), } \\
\text { 29/29 } \\
\text { G2: Risperidone (0.25-3 mg/day), 30/30 } \\
2 \text { months/EOT } \\
\text { Low ROB }\end{array}$ & $\begin{array}{l}\text { ABC-Irritability } \\
\text { G1: } 26.2 \pm 4.1 \\
\text { G2: } 21.5 \pm 7.4 \\
\text { ABC-Hyperactivity } \\
\text { G1: } 37.1 \pm 7 \\
\text { G2: } 36 \pm 6.2 \\
\text { ABC-Lethargy } \\
\text { G1: } 27.5 \pm 8.4 \\
\text { G2: } 25.3 \pm 8.9 \\
\text { ABC-Stereotypy }\end{array}$ & $\begin{array}{l}\text { EOT } \\
\text { ABC-Irritability } \\
\text { G1: } 14.6 \pm 5.5 \\
\text { G2: } 12.5 \pm 5.4 \\
\text { G1 vs. G2: } p=n s \\
\text { ABC-Hyperactivity } \\
\text { G1: } 21.1 \pm 9 \\
\text { G2: } 19.1 \pm 6.1 \\
\text { G1 vs. G2: p=ns } \\
\text { ABC-Lethargy } \\
\text { G1: } 17.3 \pm 7.4\end{array}$ \\
\hline
\end{tabular}




\begin{tabular}{|c|c|c|}
\hline $\begin{array}{c}\text { Author, Year, Study Design } \\
\text { Groups (Dose), N Enrollment / N Final } \\
\text { Treatment Duration/Follow-Up Time } \\
\text { Point Post-Treatment } \\
\text { Risk Of Bias }\end{array}$ & Baseline Scores, Mean \pm SD & Post-Treatment Scores, Mean \pm SD \\
\hline & $\begin{array}{l}\text { G1: } 13.6 \pm 5.7 \\
\text { G2: } 13.2 \pm 4.2 \\
\text { ABC-Speech } \\
\text { G1: } 8.6 \pm 3.1 \\
\text { G2: } 8.9 \pm 3.6\end{array}$ & $\begin{array}{l}\text { G2: } 16.1 \pm 6.9 \\
\text { G1 vs. } G 2: p=n s \\
\text { ABC-Stereotypy } \\
\text { G1: } 8.2 \pm 5 \\
\text { G2: } 7.4 \pm 3.9 \\
\text { G1 vs. G2: } p=n s \\
\text { ABC-Speech } \\
\text { G1: } 4.9 \pm 2.3 \\
\text { G2: } 5.7 \pm 3.1 \\
\text { G1 vs. } \mathrm{G} 2: \mathrm{p}=\mathrm{ns} \\
\text { CGI-Improvement, } \mathrm{n} \\
\text { Much improved } \\
\text { G1: } 9 \\
\text { G2: } 5 \\
\text { Minimally improved } \\
\text { G1: } 7 \\
\text { G2: } 12 \\
\text { No change } \\
\text { G1: } 5 \\
\text { G2: } 8 \\
\text { Minimally worse } \\
\text { G1: } 3 \\
\text { G2: } 2 \\
\text { G1 vs. G2: } p=n s\end{array}$ \\
\hline
\end{tabular}

ABC = Aberrant Behavior Checklist; CGI = Clinical Global Impression Scale; EOT = end of treatment; ES = effect size; $\mathrm{G}=$ group; $\mathrm{mg}$ = milligrams; $\mathrm{N}$ = number; $\mathrm{NS}=$ not significant; $\mathrm{RCT}=$ randomized controlled trial; $\mathrm{ROB}=$ risk of bias; $\mathrm{SD}=$ standard deviation

\section{Studies Comparing Risperidone and Other Agents}

Haloperidol. In one small RCT (moderate risk of bias) comparing risperidone with haloperidol, both groups improved from baseline on the Ritvo-Freeman scale, ABC, and Turgay DSM-IV Pervasive Development Disorder scale, with significantly greater improvements in the risperidone group on the ABC and Turgay scales ( $\mathrm{p}$ values $<0.01$ ). ${ }^{101}$ Appendix F outlines key data from this study.

Memantine. In one small RCT (moderate risk of bias) comparing risperidone with memantine, children in both groups improved significantly from baseline on all ABC subscales except stereotypy and on most CARS subscales (no improvements in either group in fear/nervousness, nonverbal communication, and intellectual response. Improvements in memantine arm on taste, smell, touch response and imitation), but differences between groups were not significant. ${ }^{47}$ CGI ratings were not significantly different between groups. Appendix F outlines key data from this study. 


\section{Harms of Antipsychotics}

Risperidone. Table 10 outlines harms reported in study arms addressing risperidone. Among clinically important harms, appetite increase occurred in over 70 percent of children and extrapyramidal symptoms in 36 percent. Gastrointestinal symptoms (including constipation, diarrhea, and abdominal pain) occurred in nearly all children in the 8 week RCT, and more than 25 percent across phases experienced anxiety and hypersalivation. Across studies, six children withdrew due to adverse events.

Some studies reported comparative analyses of harms data: In the RUPP extension study urinary problems occurred in 19.6 percent of patients who took risperidone, and $0 \%$ of patients that did not $(\mathrm{p}=0.01)$. Excessive appetite occurred in 42.1 percent of patients who took risperidone and 20 percent of those who did not $(\mathrm{p}=\mathrm{ns})$. In another RCT comparing risperidone doses and placebo, the incidence of treatment-emergent adverse events was higher in the high dose group compared with the low dose group and placebo. ${ }^{125,}{ }^{127}$ In combining high and low dose groups, the most common events included increased appetite (26\%), sedation (15\%), somnolence (11\%) and weight increase (11\%). Sedation, somnolence and increased appetite occurred with twice the frequency in the high dose group than in the low dose group.

In an analysis of prolactin changes in children participating in the RUPP trial, serum prolactin increased significantly from baseline in children taking risperidone at 8 weeks and 6 months, though concentrations were lower at 6 months than at weeks. ${ }^{111}$ Levels were higher in treated children compared with those in the placebo group. In the 20 treated children with measurements at 8 weeks, 6 months, and $\sim 22$ months, levels were significantly elevated at each time point compared with baseline, but levels at 22 months were significantly lower than those at 6 months $(\mathrm{p}=0.016)$. While these elevations represented 2 to 4 fold increases in prolactin, no children reported clinical complaints such as galactorrhea, gynecomastia, or menstrual changes. Another study reported no significant differences in weight change between risperidone and placebo groups (increase of $17 \%$ in the risperidone group and $9.3 \%$ in placebo). ${ }^{109}$

One analysis of side effects in a discontinuation study ${ }^{102}$ reported adverse events that occurred in at least 10 percent of patients taking risperidone, including increased appetite (mild$49 \%$, moderate $8 \%$ ), anxiety (mild $31 \%$, moderate $7 \%$ ), fatigue (mild $32 \%$, moderate $3 \%$ ), increased thirst (26\%, enuresis (19\%), insomnia, (17\%), headache (13\%), rhinitis $(11 \%)$, stomachache (10\%), nausea $(10 \%)$ and drooling $(10 \%)$. The authors did not report differences in groups during the discontinuation period. 
Table 10. Harms/adverse events reported by study phase in RUPP risperidone studies

\begin{tabular}{|c|c|c|c|c|}
\hline $\begin{array}{l}\text { Treatment Duration } \\
\text { Adverse Event }\end{array}$ & $\begin{array}{c}\text { Risperidone: } 8 \\
\text { weeks }^{34}(n=49) \\
N(\%)\end{array}$ & $\begin{array}{c}\text { Risperidone: } 6 \\
\text { months }^{36} \\
(n=95) \\
N(\%) \\
\end{array}$ & $\begin{array}{c}\text { Risperidone: } 21.4 \\
\text { months (mean) } \\
(n=84) \\
N(\%)\end{array}$ & $\begin{array}{c}\text { Placebo: } 8 \\
\text { weeks }^{34} \\
(n=51) \\
\text { N (\%) } \\
\end{array}$ \\
\hline Accidental injury & NR & $2(3.2)$ & NR & NR \\
\hline $\begin{array}{l}\text { Agitation/nervousness/ } \\
\text { restlessness }\end{array}$ & $3(6)$ & $1(1.6)$ & NR & $3(6)$ \\
\hline Anxiety & $12(24)$ & $3(4.8)$ & $7(12.3)$ & $10(20)$ \\
\hline Appetite change & NR & NR & $4(7.1)$ & \\
\hline Appetite increase & $36(73)$ & $5(7.9)$ & $24(42.1)$ & $15(29)$ \\
\hline Appetite decrease & $3(6)$ & NR & NR & $5(10)$ \\
\hline Depression/sadness & NR & $1(1.6)$ & NR & NR \\
\hline Dizziness & $8(16)$ & NR & NR & $2(4)$ \\
\hline Drooling/increased saliva & $13(27)$ & $2(3.2)$ & $10(17.5)$ & $3(6)$ \\
\hline Dry mouth & $9(18)$ & NR & $6(10.6)$ & $5(10)$ \\
\hline EPS/impaired movement & $18(36)$ & $3(4.8)$ & NR & $5(10)$ \\
\hline Gastrointestinal symptoms & $48(98)$ & $7(11.1)$ & $25(44.2)$ & $43(86)$ \\
\hline Headache & $9(18)$ & $2(3.2)$ & NR & $6(12)$ \\
\hline Heart rate changes & $6(12)$ & NR & NR & $1(2)$ \\
\hline $\begin{array}{l}\text { Infection/fever/cold/ } \\
\text { congestion symptoms }\end{array}$ & $35(71)$ & $18(28.6)$ & $7(12.5)$ & $23(45)$ \\
\hline Insomnia & $7(14)$ & $3(4.8)$ & NR & $15(29)$ \\
\hline Skin changes & $11(22)$ & $3(4.8)$ & $3(5.4)$ & $7(14)$ \\
\hline Sleep changes & $11(22)$ & NR & $6(10.5)$ & $9(18)$ \\
\hline Fatigue & $23(47)$ & $1(1.1)$ & NR & $6(12)$ \\
\hline Drowsiness & $16(32.6)$ & $2(4.1)$ & $13(15.5)$ & $\mathrm{NR}$ \\
\hline Thirst & $6(12)$ & $\mathrm{NR}$ & NR & $5(10)$ \\
\hline Urinary changes & $15(31)$ & $3(4.8)$ & $11(19.6)$ & $15(29)$ \\
\hline
\end{tabular}

EPS = Extrapyramidal Symptoms; N = number; NR = Not Reported; RUPP = Research Units on Pediatric Psychopharmacology

Harms reported in other risperidone studies were similar (Table 11).

Table 11. Harms/adverse effects in other studies of risperidone

\begin{tabular}{|c|c|c|}
\hline Harm/Adverse Event $^{\mathrm{a}}$ & $\begin{array}{c}\text { N Studies Reporting } \\
\text { Harm (\# Participants With } \\
\text { Harm/Total Participants) }\end{array}$ & $\begin{array}{l}\text { Reported Rates Across } \\
\text { Studies }\end{array}$ \\
\hline \multicolumn{3}{|l|}{ Risperidone, 1-1.17mg/day } \\
\hline Appetite increase ${ }^{49,112,113}$ & $2(21 / 70)$ & $22.5 \%-40 \%$ \\
\hline Insomnia ${ }^{112,113}$ & $1(6 / 40)$ & $15 \%$ \\
\hline Challenging behavior $^{112,113}$ & $1(5 / 40)$ & $12.5 \%$ \\
\hline Headache ${ }^{112,113}$ & $1(5 / 40)$ & $12.5 \%$ \\
\hline Somnolence $^{49,109,112,113}$ & $3(46 / 89)$ & $10 \%-72.5 \%$ \\
\hline Drooling/increased saliva ${ }^{49,112,113}$ & $2(16 / 70)$ & $10 \%-40 \%$ \\
\hline${ }_{113}^{\text {Infection/fever/cold/congestion symptoms }}{ }^{112}$ & $1(44 / 40)$ & $10 \%-37.5 \%$ \\
\hline Appetite decrease $^{49,112,113}$ & $2(8 / 70)$ & $10 \%-13.3 \%$ \\
\hline Gastrointestinal symptoms ${ }^{49,112,113}$ & $2(25 / 70)$ & $3.3 \%-20 \%$ \\
\hline EPS/impaired movement ${ }^{49,109,112,113}$ & $3(24 / 89)$ & $3.3 \%-16 \%$ \\
\hline Heart rate changes ${ }^{49,112,113}$ & $2(6 / 70)$ & $3.3 \%-12.5 \%$ \\
\hline Agitation/nervousness/restlessness ${ }^{49}$ & $1(3 / 30)$ & $10 \%$ \\
\hline Dizziness $^{49}$ & $1(3 / 30)$ & $10 \%$ \\
\hline Weight gain $^{112,113}$ & $1(4 / 40)$ & $10 \%$ \\
\hline Urinary changes ${ }^{49}$ & $1(2 / 30)$ & $6.7 \%$ \\
\hline \multicolumn{3}{|l|}{ Risperidone, $1.25-1.75 \mathrm{mg} /$ day } \\
\hline Somnolence $^{51,52}$ & $1(20 / 31)$ & $64.5 \%$ \\
\hline Infection/fever/cold/congestion symptoms ${ }^{51,52}$ & $1(7 / 25)$ & $28 \%$ \\
\hline EPS/impaired movement ${ }^{51,52}$ & $1(7 / 31)$ & $22.6 \%$ \\
\hline Gastrointestinal symptoms $^{51,52}$ & $1(4 / 25)$ & $16 \%$ \\
\hline
\end{tabular}




\begin{tabular}{|c|c|c|}
\hline Harm/Adverse Event ${ }^{a}$ & $\begin{array}{c}\text { N Studies Reporting } \\
\text { Harm (\# Participants With } \\
\text { Harm/Total Participants) }\end{array}$ & $\begin{array}{c}\text { Reported Rates Across } \\
\text { Studies }\end{array}$ \\
\hline Pain $^{51,52}$ & $1(2 / 25)$ & $8 \%$ \\
\hline \multicolumn{3}{|l|}{ Risperidone, 0.125-0.175mg/day } \\
\hline Infection/fever/cold/congestion symptoms ${ }^{51,52}$ & $1(8 / 24)$ & $33.3 \%$ \\
\hline Gastrointestinal symptoms ${ }^{51,52}$ & $1(4 / 24)$ & $16.7 \%$ \\
\hline Somnolence $^{51,52}$ & $1(3 / 30)$ & $10 \%$ \\
\hline Appetite increase ${ }^{51,52}$ & $1(2 / 24)$ & $8 \%$ \\
\hline EPS/impaired movement ${ }^{51,52}$ & $1(2 / 24)$ & $8 \%$ \\
\hline Insomnia ${ }^{51,52}$ & $1(2 / 24)$ & $8 \%$ \\
\hline \multicolumn{3}{|l|}{ Risperidone (0.01 - $0.08 \mathrm{mg} / \mathrm{kg} /$ day) } \\
\hline Gastrointestinal symptoms $^{101}$ & $1(3 / 13)$ & $23.1 \%$ \\
\hline Urinary changes $^{101}$ & $1(3 / 13)$ & $23.1 \%$ \\
\hline Infection/fever/cold/congestion symptoms ${ }^{101}$ & $1(7 / 13)$ & $53.8 \%$ \\
\hline \multicolumn{3}{|l|}{ Risperidone (0.5 - $3.5 \mathrm{mg} / \mathrm{kg} /$ day) } \\
\hline Appetite increase (mild) ${ }^{102}$ & $1(13 / 26)$ & $49 \%$ \\
\hline Appetite increase (moderate) ${ }^{102}$ & $1(3 / 12)$ & $8 \%$ \\
\hline Anxiety (mild) ${ }^{102}$ & $1(8 / 12)$ & $7 \%-1 \%$ \\
\hline Anxiety (moderate) ${ }^{102}$ & $1(1 / 12)$ & $7 \%$ \\
\hline Fatigue (mild) ${ }^{102}$ & $1(8 / 12)$ & $32 \%$ \\
\hline Fatigue (moderate) ${ }^{102}$ & $1(1 / 12)$ & $3 \%$ \\
\hline Thirst $^{102}$ & $1(7 / 12)$ & $26 \%$ \\
\hline Urinary changes $^{102}$ & $1(5 / 12)$ & $19 \%$ \\
\hline Insomnia ${ }^{102}$ & $1(4 / 12)$ & $17 \%$ \\
\hline Headache $^{102}$ & $1(3 / 12)$ & $13 \%$ \\
\hline Infection/fever/cold/congestion symptoms ${ }^{102}$ & $1(3 / 12)$ & $11 \%$ \\
\hline Gastrointestinal symptoms ${ }^{102}$ & $1(6 / 12)$ & $10 \%$ \\
\hline Drooling/increased saliva ${ }^{102}$ & $1(3 / 12)$ & $10 \%$ \\
\hline \multicolumn{3}{|l|}{ Risperidone (up to $3 \mathrm{mg} / \mathrm{kg} /$ day) } \\
\hline Appetite increase ${ }^{47,50}$ & $2(19 / 34)$ & $50 \%-53 \%$ \\
\hline Abnormal behavior ${ }^{47}$ & $1(3 / 15)$ & $20 \%$ \\
\hline Agitation/nervousness/restlessness ${ }^{50}$ & $1(1 / 19)$ & $4 \%$ \\
\hline Anxiety $^{50}$ & $1(1 / 19)$ & $4 \%$ \\
\hline Drooling/increased saliva ${ }^{47,50}$ & $2(3 / 34)$ & $6.7 \%-9 \%$ \\
\hline Dry mouth $^{50}$ & $1(3 / 19)$ & $13 \%$ \\
\hline Enuresis $^{47,50}$ & $2(6 / 34)$ & $13.3 \%-18 \%$ \\
\hline EPS/impaired movement ${ }^{50}$ & $1(1 / 19)$ & $4 \%$ \\
\hline Fatigue $^{47}$ & $1(1 / 15)$ & $6.7 \%$ \\
\hline Gastrointestinal symptoms $^{50}$ & $1(1 / 19)$ & $4 \%$ \\
\hline Infection/fever/cold/congestion symptoms ${ }^{47}$ & $1(5 / 15)$ & $6.7 \%-26.7 \%$ \\
\hline Somnolence 47,50 & $2(11 / 34)$ & $27 \%-33.3 \%$ \\
\hline Weight gain ${ }^{50}$ & $1(8 / 19)$ & $42 \%$ \\
\hline \multicolumn{3}{|l|}{ Placebo } \\
\hline Agitation/nervousness/restlessness ${ }^{51,52}$ & $1(2 / 30)$ & $7 \%$ \\
\hline Appetite increase ${ }^{51,52,112,113}$ & $2(11 / 69)$ & $15.9 \%$ \\
\hline Challenging behavior ${ }^{51,52}$ & $1(3 / 35)$ & $9 \%$ \\
\hline EPS/impaired movement ${ }^{51,52,112,113}$ & $2(4 / 58)$ & $6.9 \%$ \\
\hline Gastrointestinal $^{48,51,52,112,113}$ & $3(20 / 117)$ & $17.1 \%$ \\
\hline Headache $^{51,52,112,113}$ & $2(9 / 74)$ & $12.2 \%$ \\
\hline $\begin{array}{l}\text { Infection/fever/cold/congestion symptoms }{ }^{48,51} \text {, } \\
52,112,113\end{array}$ & $3(35 / 112)$ & $31.3 \%$ \\
\hline Insomnia ${ }^{51,52,112,113}$ & $2(9 / 74)$ & $12.2 \%$ \\
\hline Somnolence ${ }^{51,52,112,113}$ & $2(15 / 74)$ & $20.3 \%$ \\
\hline Urinary changes $^{51,52}$ & $1(2 / 30)$ & $7 \%$ \\
\hline
\end{tabular}

${ }^{\mathrm{a}}$ Harms reported by more than one participant; EPS = Extrapyramidal Symptoms; kg = kilograms; $\mathrm{mg}$ = milligrams; $\mathrm{N}=$ number

Aripiprazole. Table 12 outlines harms reported in study arms addressing aripiprazole. Harms reported across studies included weight gain, appetite changes, lethargy, and extrapyramidal 
symptoms. Across studies 34 children withdrew due to adverse events. Some studies reported comparative analyses of harms data: in an analysis of harms reported in two 8-week trials ${ }^{20,21}$ of aripiprazole vs. placebo, the percentage of patients who discontinued due to at least adverse event with aripiprazole was 10.4 percent compared with 6.9 percent in the placebo groups. ${ }^{19}$ The most common adverse events reported in the aripiprazole groups compared with placebo were: sedation (10.4\% vs. 4.0\%), fatigue (16.5\% vs. 2.0\%), vomiting (13.7\% vs 6.9\%), increased appetite $12.7 \%$ vs. $6.9 \%$ ), somnolence (10.4\% vs. $4.0 \%$ ), and tremor (9.9\% vs. $0.0 \%)$. Younger children (6-12 years) had a higher group rate of salivary hypersecretion in the aripiprazole group (6.6\% vs $0 \%$ in the placebo group), but older children (13-17 years) did not. The majority of adverse events had a peak incidence of onset at week 1 or 2, except for nasopharyngitis and tremor, which had a peak incidence at week 3, extrapyramidal disorder and aggression at week 4, drooling at week 5, diarrhea at weeks 2 and 5, enuresis and upper respiratory tract infection at week 6 and cough at week 7. Investigators rated most adverse events as mild or moderate. Most of the adverse events rated as severe were in the younger (6-12 years) subgroup, with one event of severe fatigue in the 13-17 age group. Fatigue was the only adverse event that showed a statistically significant dose-response relationship, with 3.8 percent reporting fatigue at $5 \mathrm{mg} / \mathrm{d}$, 22 percent at $10 \mathrm{mg} / \mathrm{d}$ and 18.5 percent at $15 \mathrm{mg} / \mathrm{d}$. The adjusted mean change (last observation carried forward) in body weight was higher in the aripiprazole group (1.6 kg) than the placebo group $(0.4 \mathrm{~kg})(\mathrm{p}<0.001)$. The median change in BMI $(0.7 \mathrm{vs} .0 .2 \mathrm{~kg} / \mathrm{m} 2)$ was also higher in the aripiprazole group than the placebo group. Extrapyramidal-related adverse events were also more frequent in the aripiprazole group (20.8\% vs. 9.9\%). The most common extrapyramidal-related adverse events were tremor (9.9\% vs $0 \%$ in placebo) and extrapyramidal disorder (6.1\% vs $0 \%$ in placebo).

In the 52-week open label extension of these trials, 286 of 330 patients (86.7\%) reported adverse events. ${ }^{18}$ Discontinuations were significant in the three groups: 36 percent of de novo patients discontinued, 47.1 percent of prior placebo patients discontinued, and 38.5 percent of prior aripiprazole patients discontinued. Common reasons for discontinuation were adverse events, withdrawal of consent, lost to follow up and lack of efficacy. The most common adverse events reported from the three groups combined were weight increased (23\%), vomiting (18.8\%), nasopharyngitis (13.3\%), increased appetite (13.0\%), pyrexia (11.8\%), upper respiratory tract infection (11.5\%), insomnia (10.0\%), headache $(9.7 \%)$, cough $(9.4 \%)$, diarrhea (9.1\%), aggression (8.8\%), sedation (8.2\%) and fatigue (7.0\%). Drooling, agitation, epistaxis, ear infection, nasal congestion, sinusitis, and constipation were also reported in $>5 \%$ of patients.

In another RCT, 56.4 percent of children in the aripiprazole group reported a treatmentemergent adverse event compared with 32.6 percent of the placebo group. Adverse events reported by at least 5 percent of participants and at least twice the rate of placebo included upper respiratory tract infections (10.3\% for aripiprazole vs. $2.3 \%$ for placebo), constipation (5.1\% for aripiprazole vs. $0 \%$ for placebo), and movement disorder (5.1\% for aripiprazole vs. $0 \%$ for placebo, $\mathrm{p}=\mathrm{NR}$ ). Weight change was 0.15 standard deviations greater in the aripiprazole group compared with placebo (mean gain of $2.2 \mathrm{~kg}$ vs. $0.6 \mathrm{~kg}, \mathrm{p}=0.001$ ). Fasting metabolic measurements were not different between groups, but the mean change in prolactin showed a difference of -4.8 (95\% CI: -6.8 to $-2.9 ;-0.2 \mathrm{ng} / \mathrm{ml}$ for aripiprazole and $4.6 \mathrm{ng} / \mathrm{ml}$ for placebo). ${ }^{48}$ 
Table 12. Harms/adverse events reported by study phase in aripiprazole "family" studies

\begin{tabular}{|c|c|c|c|}
\hline $\begin{array}{l}\text { Treatment Duration } \\
\text { Adverse Event }\end{array}$ & $\begin{array}{c}\text { Aripiprazole: Fixed } \\
\text { Dose+Flexible Dose, } 8 \\
\text { weeks }^{19}\left(n=212^{\mathrm{a}}\right) \\
\mathrm{N}(\%)\end{array}$ & $\begin{array}{c}\text { Aripiprazole: } 52 \text { week } \\
\text { open label extension }{ }^{18} \\
(n=330) \\
N(\%)\end{array}$ & 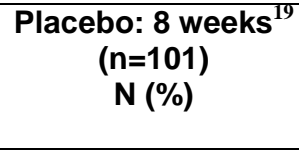 \\
\hline $\begin{array}{l}\text { Agitation/nervousness/ } \\
\text { restlessness }\end{array}$ & NR & $21(6.4)$ & NR \\
\hline Anxiety & NR & $13(3.9)$ & NR \\
\hline Appetite increase & $27(12.7)$ & $43(13.0)$ & $7(6.9)$ \\
\hline Appetite decrease & $14(6.6)$ & $15(4.5)$ & $2(2.0)$ \\
\hline Challenging behavior & $6(2.8)$ & $44(13.3)$ & $7(6.9)$ \\
\hline Drooling/increased saliva & $31(14.7)$ & $22(6.7)$ & $1(1.0)$ \\
\hline Epistaxis & NR & $21(6.4)$ & NR \\
\hline EPS/impaired movement & $34(16.0)$ & $16(4.8)$ & 0 \\
\hline Gastrointestinal symptoms & $56(26.4)$ & $132(40.0)$ & $20(19.8)$ \\
\hline Headache & $16(7.5)$ & $32(9.7)$ & $10(9.9)$ \\
\hline $\begin{array}{l}\text { Infection/fever/cold/congestion } \\
\text { symptoms }\end{array}$ & $56(26.4)$ & $220(70.8)$ & $16(16.0)$ \\
\hline Insomnia & $11(5.2)$ & $33(10.0)$ & $11(10.9)$ \\
\hline Lethargy & $10(4.7)$ & $10(3.0)$ & 0 \\
\hline Menstrual & NR & $1(2.3)$ & NR \\
\hline Skin changes & NR & $15(4.5)$ & NR \\
\hline Somnolence & $101(47.7)$ & $63(19.1)$ & $10(10.0)$ \\
\hline Urinary changes & $7(3.3)$ & $15(4.5)$ & $5(5.0)$ \\
\hline Weight gain & NR & $76(23.0)$ & NR \\
\hline
\end{tabular}

${ }^{\mathrm{a}}$ Study reported only events occurring in at least $5 \%$ of participants. EPS = extrapyramidal symptoms; $\mathrm{N}=$ number; $\mathrm{NR}=$ not reported

Harms reported in other studies of aripiprazole were similar (Table 13). 
Table 13. Harms/adverse effects in other studies of aripiprazole

\begin{tabular}{|c|c|c|}
\hline Harm/Adverse Event $^{\mathrm{a}}$ & $\begin{array}{l}\text { N Studies Reporting Harm (\# } \\
\text { Participants With Harm/Total } \\
\text { Participants) }\end{array}$ & $\begin{array}{c}\text { Reported Rates Across } \\
\text { Studies }\end{array}$ \\
\hline Appetite decrease ${ }^{49}$ & $1(6 / 29)$ & $20.7 \%$ \\
\hline EPS/impaired movement ${ }^{48,49}$ & $2(14 / 68)$ & $20.6 \%$ \\
\hline Weight gain ${ }^{50}$ & $1(4 / 18)$ & $18 \%$ \\
\hline Skin changes $^{49}$ & $1(4 / 29)$ & $13.8 \%$ \\
\hline Infection/fever/cold/congestion symptoms ${ }^{48}$ & $1(4 / 39)$ & $10.3 \%$ \\
\hline Enuresis $^{50}$ & $1(2 / 18)$ & $9 \%$ \\
\hline Somnolence $^{49,50}$ & $2(15 / 47)$ & $18 \%-37.9 \%$ \\
\hline Appetite increase ${ }^{49,50}$ & $2(15 / 47)$ & $22 \%-34.5 \%$ \\
\hline Drooling/increased saliva ${ }^{49,50}$ & $2(10 / 47)$ & $4 \%-31 \%$ \\
\hline Gastrointestinal symptoms $^{48-50}$ & $3(16 / 86)$ & $9 \%-20.6 \%$ \\
\hline Agitation/nervousness/restlessness ${ }^{49,50}$ & $2(4 / 47)$ & $4 \%-10.3 \%$ \\
\hline Dry mouth ${ }^{49,50}$ & $2(4 / 47)$ & $6.9 \%-9 \%$ \\
\hline Heart rate changes $^{49}$ & $1(2 / 29)$ & $6.9 \%$ \\
\hline Anxiety $^{50}$ & $1(1 / 18)$ & $4 \%$ \\
\hline Dizziness $^{50}$ & $1(1 / 18)$ & $4 \%$ \\
\hline Insomnia ${ }^{50}$ & $1(1 / 18)$ & $4 \%$ \\
\hline
\end{tabular}

${ }^{\mathrm{a}}$ Harms reported by more than one participant. EPS = extrapyramidal symptoms; $\mathrm{N}$ = number

Risperidone Versus Aripiprazole. In one RCT the three most common side effects in both groups were increased appetite, weight gain and drowsiness. ${ }^{50}$ Differences in changes in height, weight gain, BMI and blood glucose between the two groups were not statistically significant, while prolactin increased in the risperidone arm compared with aripiprazole (mean increase from $167.5 \pm 80$ to $464 \pm 190$ in the risperidone group, mean decrease from $158.2 \pm 70.2$ to $127.5 \pm 75.1$ in the aripiprazole group, $\mathrm{p}<0.001)$. One retrospective cohort study compared these agents in 142 children, all of whom were relatively overweight at baseline (BMI Z scores of $0.67 \pm 1.44$ in children receiving risperidone and $0.64 \pm 1.94$ in those receiving aripiprazole, $p=n s) .{ }^{122}$ Children taking aripiprazole were older than those taking risperidone at baseline $(9.74 \pm 3.46$ years vs. $8.41 \pm 3.59, \mathrm{p}=0.03$ ), and children taking risperidone had a longer mean treatment duration ( $2.37 \pm 2.55$ vs. $1.47 \pm 1.21$ years, $p=0.01$ ). Other demographic characteristics and use of concomitant medications did not differ significantly between groups. Both groups gained weight over time (significant increases in BMI and BMI Z-scores in each group), with no significant group differences in BMI change per year of treatment (2.36 \pm 3.80 in risperidone group vs. $2.05 \pm 5.02$ for aripiprazole, $\mathrm{p}=0.68)$ or BMI Z-score change per year of treatment $(0.53 \pm 1.21$ for risperidone vs. $0.56 \pm 2.21$ for aripiprazole, $\mathrm{p}=0.91$ ). Investigators note that the study was underpowered to detect differences in Z-scores. In another RCT, four children withdrew from the study because of adverse events. ${ }^{49}$

Risperidone Versus Haloperidol. Alanine amino transferase (ALT) was increased in the haloperidol group and decreased in the risperidone group $(p=0.04)$ and prolactin increased more in the risperidone group vs. the haloperidol group $(\mathrm{p}=0.01)$. Both groups experienced weight gain (risperidone increase from $33.3 \mathrm{~kg} \pm 9.1$ to $37.6 \pm 9.8$, haloperidol $42.1 \pm 17.9$ to $46.7 \pm 18$, $\mathrm{p}=\mathrm{NS}$ ), constipation, enuresis, and upper respiratory tract infections. 


\section{Studies of Medications Used To Treat ADHD}

\section{Key Points}

- Both RCTs addressing methylphenidate reported significant improvements in hyperactivity in children treated with medium to high doses compared with placebo. One RCT also noted significant treatment effects on inattention. Harms were frequent and included challenging behavior, anxiety, and appetite changes.

- Methylphenidate improved hyperactivity vs. placebo and was associated with clinically significant harms. Our confidence in these conclusions is low as studies were small and short term (low strength of evidence). Data were inadequate to assess effects on social communication and oppositional behavior (insufficient strength of evidence).

- Studies of atomoxetine compared with placebo reported promising findings related to improvements in hyperactivity in children with ASD and ADHD with clinically moderate adverse effects.

- We found positive effects of atomoxetine compared with placebo on hyperactivity in children with ASD and ADHD and moderate harms. Our confidence in these conclusions is low (low strength of evidence). Data were inadequate to assess effects on inattention as studies reported inconsistent findings (insufficient strength of evidence).

- One RCT of guanfacine reported significant improvements in hyperactivity in treated participants compared with the placebo group and no significant group differences in measures of cognitive skills. Harms included drowsiness and fatigue.

- Data were inadequate to assess effects of guanfacine given the small sample size and shortterm assessment in one RCT (insufficient strength of evidence).

\section{Overview of the Literature}

We identified five RCTs (reported in multiple publications) addressing medications for ADHD. ${ }^{24-27,40-46}$ Two RCTs addressed methylphenidate ${ }^{24-27,45}$ One study, conducted by the RUPP network, was included in our 2011 review. ${ }^{24-27}$ Two RCTs assessed atomoxetine. ${ }^{40-44}$ We describe effectiveness outcomes reported in another RCT comparing atomoxetine with and without parent training and placebo with and without parent training ${ }^{54,55}$ in the section on Studies Addressing Combined Medical and Behavioral Treatments but report harms data from this study with other atomoxetine studies. Finally, one RCT evaluated guanfacine, ${ }^{46}$

Studies included a total of 265 children between the ages of 5 and 17. Four RCTs had low risk of bias, ${ }^{24-27,41-45}$ and one had moderate. ${ }^{40}$ Studies were conducted in the United States ${ }^{24-27,40 \text {, }}$ 45, 46 Treatment duration ranged from 4 to 28 weeks, with one trial including a 4-week randomized phase and an 8-week open label continuation phase ${ }^{24-27}$ and another including 8 weeks of treatment followed by a 20 -week open label phase. ${ }^{41-44}$

\section{Detailed Analysis}

Studies of methylphenidate, atomoxetine, and guanfacine reported improvements in hyperactivity and other challenging behaviors with treatment compared with placebo (Table 14). Side effects were associated with all agents, including aggressive behavior and appetite changes (methylphenidate); irritability and gastrointestinal symptoms (atomoxetine); and gastrointestinal symptoms and somnolence (guanfacine). 
Methylphenidate. The RUPP Autism Network's double-blind cross-over trial of methylphenidate compared a one-day placebo followed by two days at each of three (low, medium, high) test doses of methylphenidate; ${ }^{25-27}$ doses ranged from $7.5 \mathrm{mg}$ /day to $50.0 \mathrm{mg} / \mathrm{day}$. Children tolerating methylphenidate $(\mathrm{n}=66)$ moved on to a 4-week, double-blind crossover phase. Children with a positive response in the double blind phase $(n=34)$ completed an 8-week open-label continuation phase at their best dose. The primary outcome measure was hyperactivity as assessed by the $\mathrm{ABC}$ teacher-rated hyperactivity subscale; secondary measures included the ABC parent-rated hyperactivity subscale. Blinded clinicians also assessed participants using the Clinical Global Impression (CGI)-Irritability scale; investigators combined this subscale and the $\mathrm{ABC}$ parent and teacher rated hyperactivity subscales to assess response (Table 14).

In the double-blind crossover phase, all methylphenidate doses demonstrated effects that were statistically superior to placebo, and effect sizes favored the medium dose for parent ratings and high dose for teacher ratings. Parent-rated lethargy/ social withdrawal significantly worsened during the high dose of methylphenidate compared with placebo. Parent-rated stereotypy and inappropriate speech scores improved significantly at the medium dose of methylphenidate compared with placebo. Hyperactivity/impulsivity also improved more with the medium and high methylphenidate doses than at the low dose. Significantly more joint attention behaviors occurred in the intervention group both at the best methylphenidate dose and at the low dose compared with placebo. Self-regulation, as assessed in a "competing demands" task, improved in low dose as well as in medium dose methylphenidate compared with placebo, and neutral affect significantly increased at the medium and high dose, which could be either beneficial, in the case of children with a labile mood, or damaging, in the case of children with flattened affect due to a medication side effect. ${ }^{25-27}$

In another 4-week RCT comparing extended release methylphenidate and placebo in children with ASD and significant ADHD symptoms with higher IQs (mean $=85 \pm 16.8$ ), children in the treatment group received extended release methylphenidate (low, medium, or high doses) in the morning and immediate release methylphenidate in the afternoon. ${ }^{45}$ Parent-rated measures of attention, ASD symptoms, hyperactivity and impulsivity improved significantly more at higher doses compared with lower doses and with placebo, and teachers reported significant improvements in hyperactivity, inattention and impulsivity at higher doses compared with lower doses and with placebo. Teachers (but not parents) also reported significant improvements in oppositional behavior with high dose methylphenidate compared with placebo. Neither teachers nor parents reported significant group differences in measures of emotional lability or social communication. Clinician-rated severity was significantly improved at all methylphenidate doses compared with placebo.

Atomoxetine. Two RCTs of atomoxetine reported significant treatment-related improvements compared with placebo that were maintained over 20 weeks of open label, uncontrolled treatment in one study; inattention was significantly improved in one study, and side effects were generally moderate (Table 16). In one low risk of bias RCT (reported in multiple publications), the initial study phase included participants with ASD and ADHD and compared atomoxetine with placebo. ${ }^{41-44}$ After 8 weeks of treatment, the atomoxetine group improved significantly more on the clinician-rated ADHD Rating Scale total score and on the inattention and hyperactivity/impulsivity subscales ( $p$ values $\leq 0.003$ ). Nine of 48 treatment group participants and 4/49 placebo group were considered very much or much improved in the CGI-ADHD-I 
( $\mathrm{p}=\mathrm{ns}$ ). Scores on only the hyperactivity subscale of the Conners Teacher Rating Scale were significantly different between groups (difference in least square means: -2.0 [95\% CI: -3.7 to 0.3 ], $\mathrm{p}=0.02$ ). Children in the atomoxetine arm also had greater improvements on the ABC Hyperactivity, Inappropriate Speech, and Stereotypic Behavior (but not other subscales) than did children receiving placebo ( $p$ values $<0.05$, effect sizes of 0.4 to 0.6 ). Scores on the Children's Social Behavior Questionnaire did not differ between groups. Response inhibition (as measured using a go-no go task) improved significantly in the atomoxetine group versus placebo but distractibility did not. ${ }^{41}$

In a 20-week open label extension including 88 children from the 8-week study (42 from the treatment group and 46 from placebo), overall scores on the ADHD Rating Scale improved from the 8-week baseline to the 28 week followup ( $p=0.015)$; changes on the inattention subscale were not significant.

An earlier 6-week crossover RCT (moderate risk of bias) including children with ASD and ADHD reported significant improvements in hyperactivity associated with atomoxetine compared with placebo (effect size $=0.90, \mathrm{p}=0.04$ ), but changes in inattention measures were not significantly different between groups. ${ }^{40}$ Investigators considered seven children (43\%) to be responders to atomoxetine (25\% improvement in ABC-Hyperactivity scale and CGI rating of very much improved or improved). Table 14 outlines outcomes.

Guanfacine. One RCT evaluating extended release guanfacine included mostly males with ASD and hyperactivity, impulsiveness, and distractibility and reported significant improvements in hyperactivity (effect size=1.67, $\mathrm{p}<0.001$ ) in the treated group compared with placebo. ${ }^{46}$ Cognitive tests of working memory did not differ between groups (Table 14). 
Table 14. Key outcomes in studies of ADHD medications

\begin{tabular}{|c|c|c|}
\hline $\begin{array}{c}\text { Author, Year, Study Design } \\
\text { Groups (dose), N enrollment / N } \\
\text { Final } \\
\text { Treatment Duration } \\
\text { Risk of Bias }\end{array}$ & Baseline Scores, Mean \pm SD & Post-Treatment Scores, Mean \pm SD \\
\hline Methylphenidate & & \\
\hline $\begin{array}{l}\text { McCracken } 2014^{24-27} \mathrm{RCT} \\
\text { (crossover) } \\
\text { Total N=66 } \\
\text { G1: MPH - low dose }(0.125 \mathrm{mg} / \mathrm{kg} \text { ), } \\
\text { 66/45 } \\
\text { G2: Methylphenidate - medium } \\
\text { dose }(0.250 \mathrm{mg} / \mathrm{kg}), 66 / 52 \\
\text { G3: MPH - high dose } 0.500 \mathrm{mg} / \mathrm{kg} \\
\text { (), } 66 / 33 \\
\text { G4: MPH - optimal dose (NR), } \\
66 / 58 \\
\text { G5: Placebo (NA), 66/46 } \\
\text { 13 weeks/EOT } \\
\text { Low ROB }\end{array}$ & $\begin{array}{l}\text { Overall ratings: } \\
\text { CGI severity sub-scale rating, } n \\
\text { (\%): } \\
\text { Moderately ill: } 20 \text { (30.3) } \\
\text { Markedly ill: } 35 \text { (52.0) } \\
\text { Severely ill: } 11 \text { (16.7) } \\
\text { ABC score, parent-rated, mean } \pm \\
\text { SD (range): } † \\
\text { Irritability: } \\
16.9 \pm 10.1(0-41) \\
\text { Lethargy/social withdrawal: } \\
12.1 \pm 8.9(0-33) \\
\text { Stereotypy: } \\
7.6 \pm 5.9(0-21) \\
\text { Hyperactivity: } \\
33.2 \pm 8.7(2-47) \\
\text { Inappropriate speech: } \\
6.0 \pm 4.1(0-12) \\
\text { ABC score, teacher-rated, mean } \\
\pm \text { SD (range): } \dagger \\
\text { Irritability: } \\
16.1 \pm 9.4(0-43) \\
\text { Lethargy/social withdrawal: } \\
15.5 \pm 10.9(0-42) \\
\text { Stereotypy: } \\
7.6 \pm 5.1(0-19) \\
\text { Hyperactivity: } \\
30.9 \pm 7.9(16-45) \\
\text { Inappropriate speech: } \\
5.8 \pm 3.6(0-12)\end{array}$ & $\begin{array}{l}\text { ABC-hyperactivity subscale score, mean } \pm \\
\text { SD: } \\
\text { Parent-rated: } \\
\text { G1: } 23.0 \pm 11.29 \\
\text { G2: } 20.6 \pm 10.27 \\
\text { G3: } 22.1 \pm 9.67 \\
\text { G4: } 17.2 \pm 9.87 \\
\text { G5: } 26.0 \pm 9.90 \\
\text { G1 vs. G5: } p=0.03(E S=0.29) \\
\text { G2 vs. G5: } p<0.001(E S=0.54) \\
\text { G3 vs. G5: } p=0.003(E S=0.40) \\
\text { G4 vs.G5: } p<0.001(E S=0.89) \\
\text { Teacher-rated: } \\
\text { G1: } 22.9 \pm 12.84 \\
\text { G2: } 23.6 \pm 12.53 \\
\text { G3: } 20.3 \pm 11.94 \\
\text { G4: } 20.1 \pm 12.40 \\
\text { G5: } 26.0 \pm 11.66 \\
\text { G1 vs. G5: } p=0.03(E S=0.25) \\
\text { G2 vs.G5: } p=0.008(E S=0.20) \\
\text { G3 vs.G5: } p=0.002(E S=0.48) \\
\text { G4 vs.G5: } p<0.001(E S=0.48)\end{array}$ \\
\hline $\begin{array}{l}\text { Pearson } 2013^{45} \text { RCT (crossover) } \\
\text { G1: MPH - low dose }(0.125 \mathrm{mg} / \mathrm{kg}) \text {, } \\
\text { 24/24 } \\
\text { G2: MPH - medium dose }(0.250 \\
\mathrm{mg} / \mathrm{kg}), 24 / 24 \\
\text { G3: MPH - high dose }(0.500 \\
\mathrm{mg} / \mathrm{kg}), 24 / 24 \\
\text { G4: Placebo, } 24 / 24 \\
4 \text { weeks/EOT } \\
\text { Low ROB }\end{array}$ & $\begin{array}{l}\mathrm{ABC} \\
\mathrm{NR}\end{array}$ & $\begin{array}{l}\text { EOT } \\
\text { Parent Ratings } \\
\text { ABC-Irritability } \\
\text { G1: } 10 \pm 9.2 \\
\text { G2: } 8.2 \pm 8.1 \\
\text { G3: } 7.2 \pm 6.9 \\
\text { G4: } 12.6 \pm 10.4 \\
\text { ABC-Social Withdrawal/Lethargy } \\
\text { G1: } 7.3 \pm 5.6 \\
\text { G2: } 8.1 \pm 5.9 \\
\text { G3: } 8.5 \pm 6.6 \\
\text { G4: } 9.3 \pm 8.1 \\
\text { ABC-Stereotypy } \\
\text { G1: } 4.3 \pm 4.5 \\
\text { G2: } 4 \pm 3.8 \\
\text { G3: } 3.5 \pm 3.8 \\
\text { G4: } 4.9 \pm 5.4\end{array}$ \\
\hline
\end{tabular}




\begin{tabular}{|c|c|c|}
\hline $\begin{array}{c}\text { Author, Year, Study Design } \\
\text { Groups (dose), N enrollment / N } \\
\text { Final } \\
\text { Treatment Duration } \\
\text { Risk of Bias }\end{array}$ & Baseline Scores, Mean \pm SD & Post-Treatment Scores, Mean \pm SD \\
\hline & & $\begin{array}{l}\text { ABC-Hyperactivity } \\
\text { G1: } 18.1 \pm 10.5 \\
\text { G2: } 14.5 \pm 7.7 \\
\text { G3: } 14.5 \pm 9.2 \\
\text { G4: } 24.1 \pm 13 \\
\\
\text { ABC-Inappropriate Speech } \\
\text { G1: } 4.3 \pm 3.2 \\
\text { G2: } 4 \pm 3.1 \\
\text { G3: } 3.9 \pm 3.1 \\
\text { G4: } 5.2 \pm 3.1 \\
\text { CGI-Severity (clinician 1) } \\
\text { G1: } 4 \pm 0.81 \\
\text { G2: } 3.8 \pm 0.82 \\
\text { G3: } 3.8 \pm 0.74 \\
\text { G4: } 4.8 \pm 0.61 \\
\text { CGI-Improvement (clinician 1) } \\
\text { G1: } 2.8 \pm 1.3 \\
\text { G2: } 2.4 \pm 1.3 \\
\text { G3: } 2.1 \pm 1.2 \\
\text { G4: } 4 \pm 0.81 \\
\text { CGI-Severity (clinician 2) } \\
\text { G1: } 4 \pm 0.72 \\
\text { G2: } 4 \pm 0.62 \\
\text { G3: } 3.9 \pm 0.74 \\
\text { G4: } 4.7 \pm 0.76 \\
\text { CGI-Improvement (clinician } 2) \\
\text { G1: } 2.8 \pm 1.4 \\
\text { G2: } 2.6 \pm 1.3 \\
\text { G3: } 2 \pm 1 \\
\text { G4: } 4.1 \pm 0.95\end{array}$ \\
\hline \multicolumn{3}{|l|}{ Atomoxetine } \\
\hline $\begin{array}{l}\text { Van der Meer } 2013^{41-44} \\
\text { RCT } \\
\text { G1: Atomoxetine (1.2mg/kg/day), } \\
\text { 48/43 } \\
\text { G2: Placebo (NA), 49/46 } \\
8 \text { weeks/EOT } \\
\text { Low ROB }\end{array}$ & $\begin{array}{l}\text { ADHD Rating Scale - Total } \\
\text { Score } \\
\text { G1: } 40.7 \pm 7.5 \\
\text { G2: } 38.6 \pm 8.4 \\
\text { ADHD Rating Scale - Inattention } \\
\text { G1: } 20.7 \pm 3.9 \\
\text { G2: } 20.6 \pm 4.6 \\
\text { ADHD Rating Scale - } \\
\text { Hyperactivity/limpulsivity } \\
\text { G1: } 20.0 \pm 5.3 \\
\text { G2: } 17.9 \pm 6.1 \\
\text { CTRS-R:S - Oppositional } \\
\text { G1: } 4.1 \pm 3.5 \\
\text { G2: } 3.6 \pm 3.5\end{array}$ & $\begin{array}{l}\text { EOT (8 wks) } \\
\text { ADHD Rating Scale - Total Score } \\
\text { G1: } 31.2(29.2-33.9) \\
\text { G2: } 38.3(36.0-40.5) \\
\text { G1 vs. G2: } p<0.001 \\
\text { ADHD Rating Scale - Inattention } \\
\text { G1: } 17.0(15.7-18.4) \\
\text { G2: } 19.9(18.7-21.1) \\
\text { G1 vs. G2: } p=0.002 \\
\text { ADHD Rating Scale - } \\
\text { Hyperactivity/impulsivity } \\
\text { G1: } 14.2(12.8-15.7) \\
\text { G2: } 18.4(17.0-19.7) \\
\text { G1 vs. G2: } p=0.001 \\
\text { CTRS-R:S - Oppositional }\end{array}$ \\
\hline
\end{tabular}




\begin{tabular}{|c|c|c|}
\hline $\begin{array}{c}\text { Author, Year, Study Design } \\
\text { Groups (dose), N enrollment / N } \\
\text { Final } \\
\text { Treatment Duration } \\
\text { Risk of Bias }\end{array}$ & Baseline Scores, Mean \pm SD & Post-Treatment Scores, Mean \pm SD \\
\hline & $\begin{array}{l}\text { CTRS-R:S - Hyperactivity } \\
\text { G1: } 8.8 \pm 5.5 \\
\text { G2: } 8.2 \pm 5.1 \\
\text { CTRS-R:S - Cognitive/Attention } \\
\text { G1: } 6.8 \pm 4.5 \\
\text { G2: } 4.8 \pm 3.7 \\
\text { CTRS-R:S - ADHD } \\
\text { G1: } 18.5 \pm 9.3 \\
\text { G2: } 18.1 \pm 7.5 \\
\text { Go/No-Go Task - Proportion } \\
\text { false alarms } \\
\text { G1: } 0.07 \pm 0.1 \\
\text { G2: } 0.06 \pm 0.08 \\
\text { Missed Go Signals - Proportion } \\
\text { misses } \\
\text { G1: } 0.02 \pm 0.06 \\
\text { G2: } 0.02 \pm 0.05 \\
\text { Response Time } \\
\text { G1: } 532.7 \pm 144.9 \\
\text { G2: } 485 \pm 120.7 \\
\text { Response Time Variability } \\
\text { G1: } 125.1 \pm 64 \\
\text { G2: } 120 \pm 77.2 \\
\text { ABC - Irritability } \\
\text { G1: } 17.3 \pm 9.1 \\
\text { G2: } 16.2 \pm 9.5 \\
\text { ABC - Lethargy/Social } \\
\text { Withdrawal } \\
\text { G1: } 12.5 \pm 8.4 \\
\text { G2: } 12.5 \pm 8 \\
\text { ABC }- \text { Stereotypic Behavior } \\
\text { G1: } 6.5 \pm 5.1 \\
\text { G2: } 4.1 \pm 4.5 \\
\text { ABC - Hyperactivity } \\
\text { G1: } 28.4 \pm 9.3 \\
\text { G2: } 25.4 \pm 11.5 \\
\text { ABC - Inappropriate Speech } \\
\text { G1: } 4.7 \pm 3.2 \\
\text { G2: } 4.6 \pm 3.4 \\
\text { CSBQ - Total Score } \\
\text { G1: } 53.6 \pm 14.8 \\
\text { G2: } 53.1 \pm 15.7\end{array}$ & 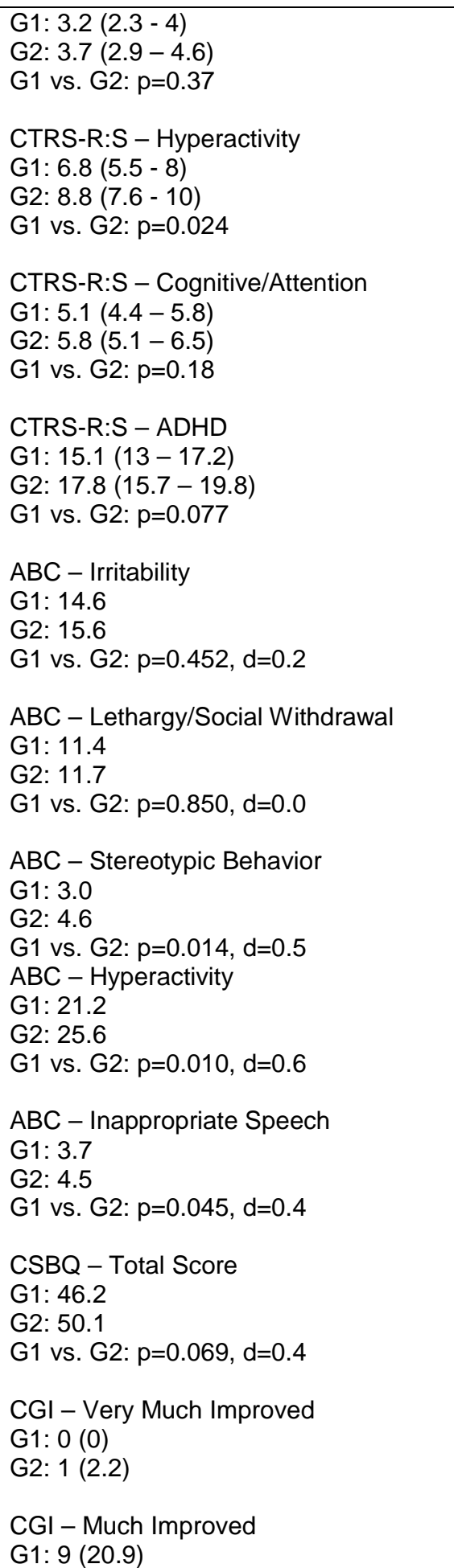 \\
\hline
\end{tabular}




\begin{tabular}{|c|c|c|}
\hline $\begin{array}{c}\text { Author, Year, Study Design } \\
\text { Groups (dose), N enrollment / N } \\
\text { Final } \\
\text { Treatment Duration } \\
\text { Risk of Bias }\end{array}$ & Baseline Scores, Mean \pm SD & Post-Treatment Scores, Mean \pm SD \\
\hline & & $\begin{array}{l}\text { G2: } 3 \text { (6.5) } \\
\text { G1 vs. G2: p=ns } \\
\text { CGI - Minimally Improved } \\
\text { G1: } 12(27.9) \\
\text { G2: } 6 \text { (13) } \\
\text { CGI - No Change } \\
\text { G1: } 16 \text { (37.2) } \\
\text { G2: } 30 \text { (65.2) } \\
\text { CGI - Minimally Worse } \\
\text { G1: } 4 \text { (9.3) } \\
\text { G2: } 3(6.5) \\
\text { CGI - Much Worse } \\
\text { G1: } 2 \text { (4.7) } \\
\text { G2: } 3(6.5) \\
\text { CGI - Very Much Worse } \\
\text { G1: } 0(0) \\
\text { G2: } 0(0)\end{array}$ \\
\hline $\begin{array}{l}\text { Arnold } 2006^{40} \\
\text { RCT } \\
\text { G1: Atomoxetine (up to } \\
\text { 1.4mg/kg/day), } 16 / 16 \\
\text { G2: Placebo ( } 1.2 \mathrm{mg} / \mathrm{kg} / \text { day), 16/16 } \\
6 \text { weeks/EOT (crossover) } \\
\text { Moderate ROB }\end{array}$ & $\begin{array}{l}\text { ABC - Hyperactivity } \\
\text { G1: } 24.69 \pm 13.08 \\
\text { G2: } 22.5 \pm 12.87 \\
\text { ABC - Irritability } \\
\text { G1: } 16 \pm 9.28 \\
\text { G2: } 14.13 \pm 9.89 \\
\text { ABC-Lethargy/social withdrawal } \\
\text { G1: } 8.69 \pm 9.24 \\
\text { G2: } 6.62 \pm 8.36 \\
\text { ABC - Stereotypic behavior } \\
\text { G1: } 7.37 \pm 6.20 \\
\text { G2: } 6.19 \pm 5.86 \\
\text { ABC - Inappropriate speech } \\
\text { G1: } 5.75 \pm 3.38 \\
\text { G2: } 5.43 \pm 3.16 \\
\text { CGI-Severity } \\
\text { G1 }+ \text { G2: } 4.69 \pm 0.60 \\
\\
\text { Repetitive behavior scales }- \\
\text { Total score } \\
\text { G1: } 53.12 \pm 22.2 \\
\text { G2: } 49.06 \pm 21.54\end{array}$ & 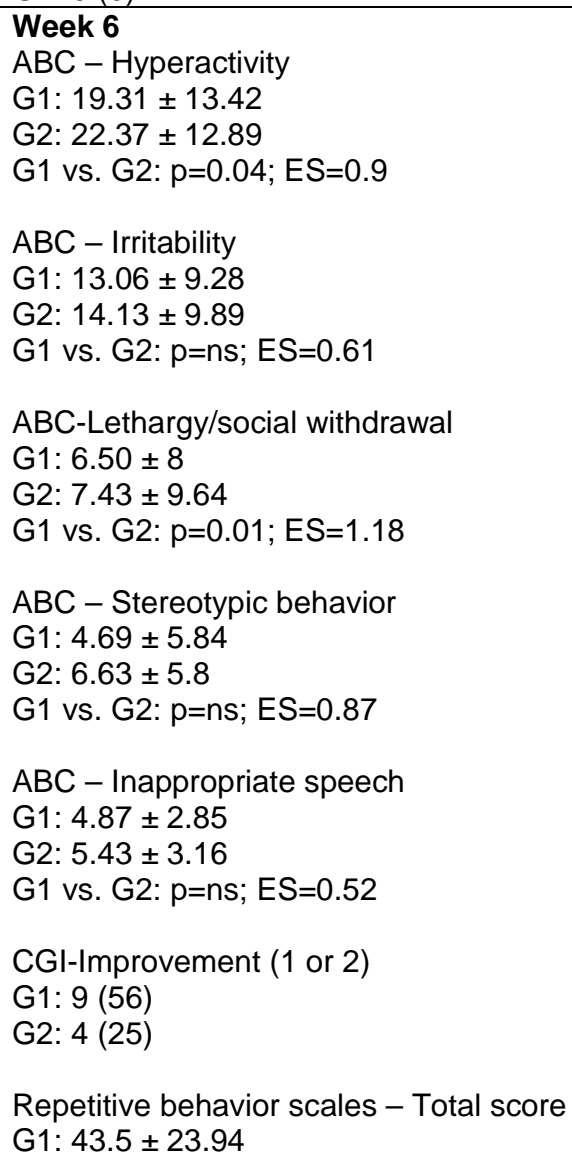 \\
\hline
\end{tabular}




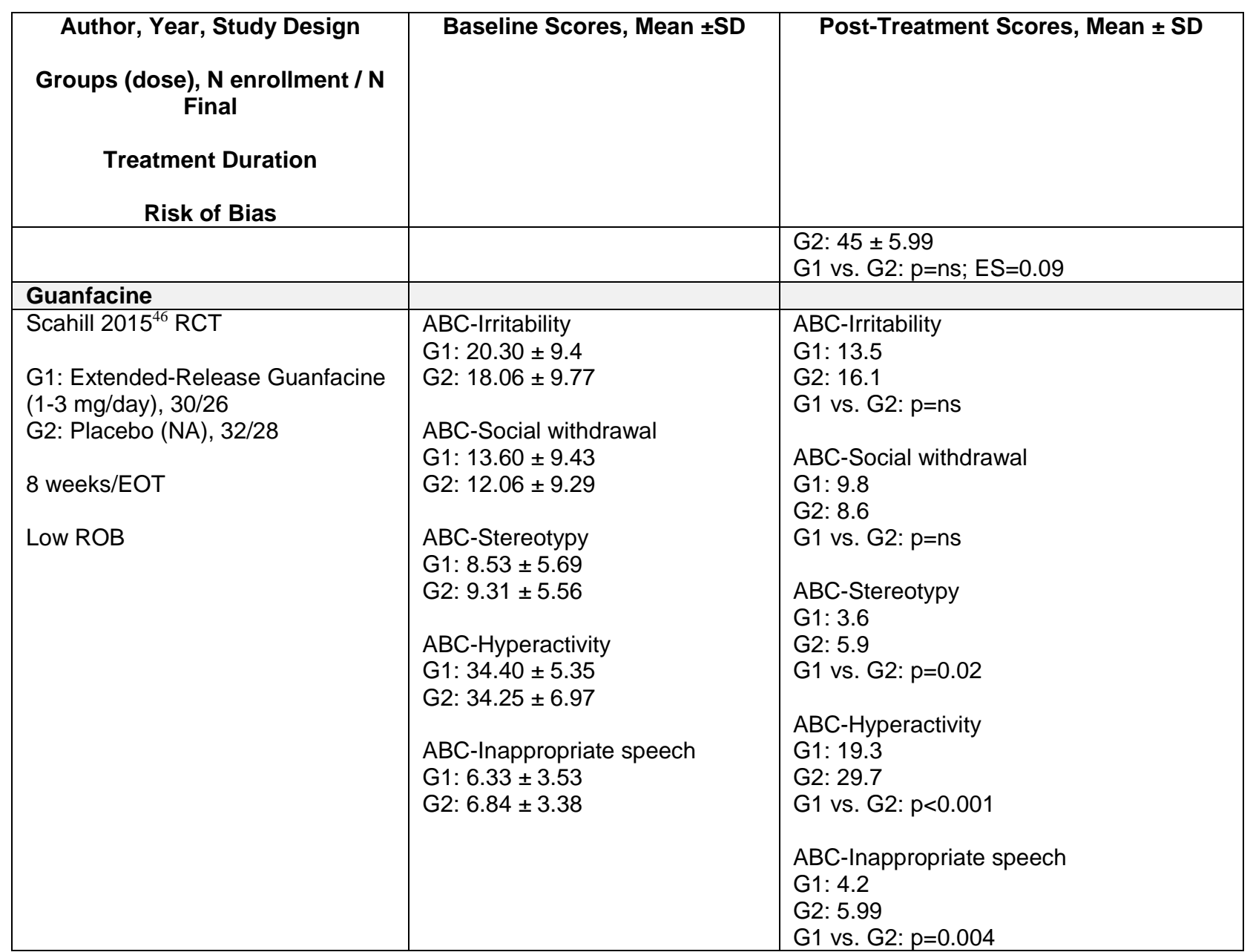

$\mathrm{ABC}=$ Aberrant Behavior Checklist; ADHD = attention deficit hyperactivity disorder; CGI = Clinical Global Impressions Scale; CSBQ = Children's Social Behavior Questionnaire; CTRS = Connor's Teacher Rating Scales; EOT = end of treatment; ES = effect size; G = groups; kg = Kilograms; $\mathrm{mg}$ = milligrams; NA = not applicable; $\mathrm{NS}=$ not significant; $\mathrm{RCT}=$ randomized controlled trial; $\mathrm{ROB}=$ risk of bias; $\mathrm{SD}=$ standard deviation

\section{Harms of ADHD Medications}

Methylphenidate. Methylphenidate was associated with changes in appetite and challenging and repetitive behavior at all doses (rates ranging from 5\% to 50\%) and with anxiety and insomnia (at lower doses). Five (out of 24) children discontinued an afternoon dose of medication because of irritability in one study, ${ }^{45}$ and 13 of 72 discontinued a second RCT due to irritability ( $\mathrm{n}=6$ ) or other adverse effects. ${ }^{24-27}$ Table 15 outlines harms. 
Table 15. Harms/adverse effects in studies of methylphenidate

\begin{tabular}{|c|c|c|}
\hline Harm/Adverse Event $^{a}$ & $\begin{array}{l}\text { N Studies Reporting Harm (\# } \\
\text { Participants With Harm/Total } \\
\text { Participants) }\end{array}$ & $\begin{array}{c}\text { Reported Rates Across } \\
\text { Studies }\end{array}$ \\
\hline \multicolumn{3}{|l|}{$\begin{array}{l}\text { Methylphenidate (0.125-0.21 } \\
\mathrm{mg} / \mathrm{kg} / \mathrm{day})\end{array}$} \\
\hline Repetitive language $^{45}$ & $1(13 / 24)$ & $54.2 \%$ \\
\hline EPS/impaired movement ${ }^{24-27,45}$ & $2(6 / 90)$ & $4.6 \%-8 \%$ \\
\hline Appetite decrease ${ }^{24-27,45}$ & $2(10 / 90)$ & $4.6 \%-29 \%$ \\
\hline Anxiety $^{24-27,45}$ & $2(7 / 90)$ & $4.6 \%-17 \%$ \\
\hline Gastrointestinal symptoms $^{24-27,45}$ & $2(6 / 90)$ & $4 \%-7.6 \%$ \\
\hline Heart rate changes ${ }^{24-27,45}$ & $2(3 / 90)$ & $4 \%-4.6 \%$ \\
\hline Headache ${ }^{24-27,45}$ & $2(4 / 90)$ & $3.0 \%-8 \%$ \\
\hline Withdrawal $^{24-27}$ & $1(2 / 66)$ & $3.0 \%$ \\
\hline Repetitive behavior ${ }^{24-27,45}$ & $2(20 / 90)$ & $3 \%-46 \%$ \\
\hline Challenging behavior ${ }^{24-27,45}$ & $2(18 / 90)$ & $15.2 \%-33 \%$ \\
\hline Insomnia ${ }^{24-27,45}$ & $2(14 / 90)$ & $10.6 \%-50 \%$ \\
\hline Depression/sadness ${ }^{24-27,45}$ & $2(3 / 90)$ & $1.5 \%-8 \%$ \\
\hline Somnolence ${ }^{24-27,45}$ & $2(2 / 90)$ & $1.5 \%-4 \%$ \\
\hline \multicolumn{3}{|c|}{ Methylphenidate $(0.24-0.35 \mathrm{mg} / \mathrm{kg} /$ day $)$} \\
\hline Repetitive language $^{45}$ & $1(12 / 24)$ & $50 \%$ \\
\hline Hair/skin pulling ${ }^{45}$ & $1(3 / 24)$ & $12.5 \%$ \\
\hline Dry mouth ${ }^{45}$ & $1(2 / 24)$ & $8 \%$ \\
\hline Staring $^{45}$ & $1(2 / 24)$ & $8 \%$ \\
\hline Withdrawal $^{24-27}$ & $1(4 / 66)$ & $6.1 \%$ \\
\hline Self-injury behavior ${ }^{24-27}$ & $1(3 / 66)$ & $4.6 \%$ \\
\hline Somnolence ${ }^{24-27,45}$ & $2(5 / 90)$ & $4 \%-6.1 \%$ \\
\hline EPS/impaired movement ${ }^{24-27,45}$ & $2(3 / 90)$ & $1.5 \%-8 \%$ \\
\hline Challenging behavior ${ }^{24-27,45}$ & $2(25 / 90)$ & $25.7 \%-33 \%$ \\
\hline Gastrointestinal symptoms ${ }^{24-27,45}$ & $2(9 / 90)$ & $7.6 \%-17 \%$ \\
\hline Depression/sadness ${ }^{24-27,45}$ & $2(7 / 90)$ & $4.6 \%-17 \%$ \\
\hline Appetite decrease ${ }^{24-27,45}$ & $2(25 / 90)$ & $24.2 \%-38 \%$ \\
\hline Headache $^{24-27,45}$ & $2(5 / 90)$ & $1.5 \%-17 \%$ \\
\hline Anxiety $^{24-27,45}$ & $2(7 / 90)$ & $1.5 \%-25 \%$ \\
\hline Insomnia ${ }^{24-27,45}$ & $2(14 / 90)$ & $18.2 \%-50 \%$ \\
\hline Repetitive behavior ${ }^{24-27,45}$ & $2(16 / 90)$ & $6 \%-50 \%$ \\
\hline \multicolumn{3}{|c|}{ Methylphenidate (0.27-0.50 mg/kg/day) } \\
\hline Repetitive language $^{45}$ & $1(9 / 24)$ & $37.5 \%$ \\
\hline Appetite decrease ${ }^{24-27,45}$ & $2(21 / 74)$ & $24 \%-38 \%$ \\
\hline Insomnia ${ }^{24-27,45}$ & $2(21 / 74)$ & $24 \%-38 \%$ \\
\hline Challenging behavior ${ }^{24-27,45}$ & $2(17 / 74)$ & $20 \%-29 \%$ \\
\hline Gastrointestinal symptoms $^{24-27,45}$ & $2(10 / 74)$ & $12 \%-12.2 \%$ \\
\hline Hair/skin pulling ${ }^{45}$ & $1(2 / 24)$ & $8.3 \%$ \\
\hline Anxiety $^{24-27,45}$ & $2(6 / 74)$ & $8 \%$ \\
\hline Self-injury behavior ${ }^{24-27}$ & $1(3 / 50)$ & $6.0 \%$ \\
\hline Repetitive behavior ${ }^{24-27,45}$ & $2(12 / 74)$ & $6 \%-37 \%$ \\
\hline Depression/sadness ${ }^{24-27,45}$ & $2(5 / 74)$ & $4 \%-8 \%$ \\
\hline Headache $^{24-27,45}$ & $2(4 / 74)$ & $4 \%-6 \%$ \\
\hline Withdrawal $^{24-27}$ & $1(2 / 50)$ & $4 \%$ \\
\hline EPS/impaired movement ${ }^{24-27,45}$ & $2(3 / 74)$ & $2 \%-8 \%$ \\
\hline Somnolence ${ }^{24-27,45}$ & $2(2 / 74)$ & $1.5 \%-8 \%$ \\
\hline
\end{tabular}

${ }^{\mathrm{a}}$ Harms reported by more than one participant. EPS = extrapyramidal symptoms; $\mathrm{N}=$ number 
Atomoxetine. During the 8-week treatment versus placebo phase in one RCT, 81.3 percent of children receiving atomoxetine compared with 65.3 percent of placebo participants reported at least one adverse event ( $p=n s$ ) in one study; one child in the atomoxetine group discontinued due to fatigue. ${ }^{41-44}$ Significantly more treatment than placebo participants reported nausea (14 vs. 4), decreased appetite (13 vs. 3), fatigue (11 vs. 3), and early morning awakening (5 vs. 0, all p values $<0.05$ ). In the open-label extension phase, 11 of 88 participants withdrew from the study due to adverse effects. Adverse effects occurring in more than 10 percent of participants in the first 8 weeks of treatment included upper abdominal pain (12.5\%), decreased appetite (18.2\%), fatigue (18.2\%), headache (20.5\%), and nausea (13.6\%). Most harms attenuated over time: during the open label phase, only headache continued to occur in more than 10 percent of participants (14.8\%).

In another RCT, more children in the atomoxetine group had gastrointestinal symptoms, fatigue, and racing heart rate ( $p$ values $<0.05$ ) than did children in the placebo group. ${ }^{40}$ Four children receiving placebo had severe adverse events including restlessness, mood swings, decreased appetite; among those receiving atomoxetine severe events included rage requiring hospitalization in one child (leading to study withdrawal) and tiredness in another.

In an RCT comparing atomoxetine plus parent training with atomoxetine alone, placebo, and placebo plus parent training (see effectiveness outcomes reported in the section on Combination Medical and Behavioral Treatment), abdominal pain and decreased appetite occurred more frequently in the atomoxetine groups than in placebo groups ( $p$ values $\leq 0.07$ ) as did greater issues with sleep onset ( $\mathrm{p}=\mathrm{NS}$ ). One child in the atomoxetine group who received levetiracetam for seizures was hospitalized for a seizure, but investigators did not consider the event related to atomoxetine. Fifteen participants withdrew from the study due to adverse events, five of whom were receiving atomoxetine ( $8 \%$ of children receiving active medication). Table 16 outlines harms.

Table 16. Harms/adverse effects in studies of atomoxetine

\begin{tabular}{|c|c|c|}
\hline Harm/Adverse Event $^{\mathrm{a}}$ & $\begin{array}{l}\text { N Studies Reporting Harm (\# } \\
\text { Participants With Harm/Total } \\
\text { Participants) }\end{array}$ & $\begin{array}{c}\text { Reported Rates Across } \\
\text { Studies }\end{array}$ \\
\hline \multicolumn{3}{|l|}{ Atomoxetine } \\
\hline Somnolence $^{40}$ & $1(12 / 16)$ & $75 \%$ \\
\hline Skin changes ${ }^{40}$ & $1(8 / 16)$ & $67 \%$ \\
\hline EPS/impaired movement ${ }^{40}$ & $1(7 / 16)$ & $43.8 \%$ \\
\hline Constipation $^{54,55}$ & $1(20 / 64)$ & $31 \%$ \\
\hline Abdominal pain ${ }^{54,55}$ & $1(19 / 64)$ & $30 \%$ \\
\hline Vomiting $^{54,55}$ & $1(19 / 64)$ & $28 \%$ \\
\hline Dry mouth $^{40}$ & $1(4 / 16)$ & $25 \%$ \\
\hline Headache $^{40}$ & $1(4 / 16)$ & $25 \%$ \\
\hline Heart rate changes ${ }^{40}$ & $1(4 / 16)$ & $25 \%$ \\
\hline Gastrointestinal symptoms $^{40-44}$ & $2(59 / 64)$ & $25 \%-64.6 \%$ \\
\hline Appetite decrease ${ }^{40-44,54,55}$ & $3(54 / 128)$ & $27.1 \%-75 \%$ \\
\hline Insomnia ${ }^{40,54,55}$ & $2(26 / 80)$ & $22 \%-75 \%$ \\
\hline $\begin{array}{l}\text { Agitation/nervousness/restlessness }{ }^{40,54,} \text {, } \\
55\end{array}$ & $2(43 / 80)$ & $42 \%-100 \%$ \\
\hline Challenging behavior ${ }^{41-44,54,55}$ & $3(64 / 128)$ & $4.2 \%-88 \%$ \\
\hline Diarrhea $^{54,55}$ & $1(11 / 64)$ & $17 \%$ \\
\hline Dizziness $^{40-44}$ & $1(3 / 48)$ & $6.3 \%$ \\
\hline \multicolumn{3}{|l|}{ Placebo } \\
\hline Gastrointestinal symptoms $^{40}$ & $1(14 / 16)$ & $87.5 \%$ \\
\hline$\underset{55}{\text { Agitation/nervousness/restlessness }}{ }^{40,54,}$ & $2(26 / 80)$ & $16 \%-100$ \\
\hline
\end{tabular}




\begin{tabular}{|l|c|c|}
\hline EPS/impaired movement $^{40-44}$ & $2(11 / 65)$ & $8.2 \%-31 \%$ \\
\hline Challenging behavior $^{40-44,54,55}$ & $3(35 / 129)$ & $6.1 \%-81 \%$ \\
\hline Appetite decrease $^{40-44,54,55}$ & $3(18 / 129)$ & $6.1 \%-52 \%$ \\
\hline Insomnia $^{40,54,55}$ & $2(11 / 80)$ & $6 \%-44 \%$ \\
\hline Abdominal pain $^{54,55}$ & $1(4 / 64)$ & $5 \%$ \\
\hline Vomiting $^{5,55}$ & $1(3 / 64)$ & $5 \%$ \\
\hline Constipation $^{54,55}$ & $1(3 / 64)$ & $44 \%$ \\
\hline Headache $^{40}$ & $1(7 / 16)$ & $44 \%$ \\
\hline Somnolence $^{40}$ & $1(7 / 16)$ & $38 \%$ \\
\hline Skin changes $^{40}$ & $1(6 / 16)$ & $3 \%$ \\
\hline Diarrhea $^{54,55}$ & $1(2 / 64)$ & $25 \%$ \\
\hline Dry mouth $^{40}$ & $1(4 / 16)$ & \\
\hline
\end{tabular}

Note: The Handen study ${ }^{54,55}$ collapsed harms of atomoxetine and atomoxetine plus parent training under atomoxetine and harms in the placebo and placebo plus parent training groups under placebo.

${ }^{\mathrm{a}}$ Harms reported by more than one participant. EPS = extrapyramidal symptoms; $\mathrm{n}=$ number

Guanfacine. Guanfacine was fairly well tolerated: four children withdrew from the trial (2 for adverse events and 2 for lack of efficacy), and one child had severe aggressive behavior that resulted in patient psychiatric hospitalization. Other side effects included fatigue, decreased appetite, drowsiness, dry mouth, emotional lability, and anxiety. Heart rate and blood pressure decreased in treated patients with attenuation over time. Table 17 outlines harms. 
Table 17. Harms/adverse effects in studies of guanfacine

\begin{tabular}{|c|c|c|}
\hline Harm/Adverse Event $^{\mathrm{a}}$ & $\begin{array}{l}\text { N Studies Reporting Harm (\# } \\
\text { Participants With Harm/Total } \\
\text { Participants) }\end{array}$ & $\begin{array}{l}\text { Reported Rates Across } \\
\text { Studies }\end{array}$ \\
\hline \multicolumn{3}{|l|}{$\begin{array}{l}\text { Guanfacine-extended release (1-3 } \\
\text { mg/day) }\end{array}$} \\
\hline Gastrointestinal symptoms $^{46}$ & $1(24 / 30)$ & $80 \%$ \\
\hline Somnolence $^{46}$ & $1(19 / 30)$ & $63.3 \%$ \\
\hline Challenging behavior $^{46}$ & $1(18 / 30)$ & $60 \%$ \\
\hline Appetite Decrease $^{46}$ & $1(13 / 30)$ & $43.3 \%$ \\
\hline Dry mouth ${ }^{46}$ & $1(12 / 30)$ & $40 \%$ \\
\hline Anxiety $^{46}$ & $1(9 / 30)$ & $30 \%$ \\
\hline Energy level changes $^{46}$ & $1(9 / 30)$ & $30 \%$ \\
\hline Headache $^{46}$ & $1(9 / 30)$ & $30 \%$ \\
\hline Insomnia ${ }^{46}$ & $1(9 / 30)$ & $30 \%$ \\
\hline Repetitive behaviors or language $^{46}$ & $1(6 / 30)$ & $20 \%$ \\
\hline $\begin{array}{l}\text { Infection/fever/cold/congestion } \\
\text { symptoms }^{46}\end{array}$ & $1(5 / 30)$ & $16.7 \%$ \\
\hline Depression/sadness ${ }^{46}$ & $1(4 / 30)$ & $13.3 \%$ \\
\hline EPS/impaired movement ${ }^{46}$ & $1(4 / 30)$ & $13.3 \%$ \\
\hline Dizziness $^{46}$ & $1(3 / 30)$ & $10 \%$ \\
\hline Self-injury behavior ${ }^{46}$ & $1(3 / 30)$ & $10 \%$ \\
\hline Silliness $^{46}$ & $1(3 / 30)$ & $10 \%$ \\
\hline Sleep changes $^{46}$ & $1(3 / 30)$ & $10 \%$ \\
\hline Appetite Increase $^{46}$ & $1(2 / 30)$ & $6.7 \%$ \\
\hline Eye/vision changes $^{46}$ & $1(2 / 30)$ & $6.7 \%$ \\
\hline Nightmares $^{46}$ & $1(2 / 30)$ & $6.7 \%$ \\
\hline Skin changes ${ }^{46}$ & $\frac{1(2 / 30)}{1(2 / 30)}$ & $6.7 \%$ \\
\hline Urinary changes $^{46}$ & $1(2 / 30)$ & $6.7 \%$ \\
\hline \multicolumn{3}{|l|}{ Placebo } \\
\hline Repetitive language $^{45}$ & $1(12 / 24)$ & $50 \%$ \\
\hline $\begin{array}{l}\text { Infection/fever/cold/congestion } \\
\text { symptoms }^{46}\end{array}$ & $1(7 / 32)$ & $21.9 \%$ \\
\hline Energy level changes ${ }^{46}$ & $1(6 / 32)$ & $18.8 \%$ \\
\hline Staring $^{45}$ & $1(4 / 24)$ & $17 \%$ \\
\hline Silliness $^{46}$ & $1(5 / 32)$ & $15.6 \%$ \\
\hline Headache $^{45,46}$ & $2(7 / 56)$ & $12.5 \%$ \\
\hline Skin changes ${ }^{46}$ & $1(4 / 32)$ & $12.5 \%$ \\
\hline Somnolence ${ }^{45,46}$ & $1(7 / 56)$ & $12.5 \%$ \\
\hline Appetite Increase $^{46}$ & $1(2 / 32)$ & $6.3 \%$ \\
\hline Dizziness $^{46}$ & $1(2 / 32)$ & $6.3 \%$ \\
\hline Sleep changes ${ }^{46}$ & $1(2 / 32)$ & $6.3 \%$ \\
\hline Urinary changes $^{46}$ & $1(2 / 32)$ & $6.3 \%$ \\
\hline Self-injury behavior ${ }^{24-27}$ & $1(2 / 66)$ & $3 \%$ \\
\hline Appetite decrease ${ }^{24-27,45,46}$ & $3(5 / 122)$ & $3.0 \%-6.3 \%$ \\
\hline Hair/skin pulling ${ }^{45,46}$ & $2(6 / 56)$ & $4 \%-9.4 \%$ \\
\hline Depression $^{45,46}$ & $2(7 / 56)$ & $3.1 \%-13 \%$ \\
\hline EPS/impaired movement ${ }^{24-27,45,46}$ & $3(4 / 122)$ & $1.5 \%-13 \%$ \\
\hline Anxiety $^{24-27,45,46}$ & $3(7 / 122)$ & $3.0 \%-17.0 \%$ \\
\hline Insomnia ${ }^{24-27,45,46}$ & $3(10 / 122)$ & $1.5 \%-21 \%$ \\
\hline Gastrointestinal $^{24-27,45,46}$ & $3(18 / 122)$ & $7.6 \%-34.4 \%$ \\
\hline Repetitive behavior $^{24-27,45,46}$ & $3(25 / 122)$ & $3 \%-50 \%$ \\
\hline Challenging behavior ${ }^{24-27,45,46}$ & $3(30 / 122)$ & $3.0 \%-53.1 \%$ \\
\hline
\end{tabular}

${ }^{\mathrm{a}}$ Harms reported by more than one participant. EPS = extrapyramidal symptoms; $\mathrm{mg}=$ milligrams; $\mathrm{n}=$ number 


\section{Studies of Combined Medical and Behavioral Treatments}

\section{Key Points}

- Atomoxetine plus parent training or atomoxetine alone were both associated with improvements in ADHD, inattention, hyperactivity, noncompliance, and overall symptom severity compared with placebo, with improvements maintained over 24 weeks for most treatment responders. Differences between atomoxetine groups were not statistically significant for any outcome.

- Melatonin and melatonin plus cognitive behavioral therapy (CBT) both improved sleeprelated outcomes.

- Folic acid plus Treatment and Education of Autistic and Related Communication Handicapped Children (TEACCH) treatment and folic acid alone both improved behaviors, with no statistically significant group differences.

- Bumetanide plus applied behavior analysis improved symptom severity and behavior more than applied behavior analysis alone.

- Stem cell transplantation plus rehabilitation therapy improved symptom severity, lethargy, and stereotypy more than umbilical cord blood cell transplant plus rehabilitation therapy or rehabilitation therapy alone.

- Given that combination therapies were investigated in single studies, we could not make conclusions about their effects on any outcomes (insufficient strength of evidence).

\section{Overview of the Literature}

Three RCTs ${ }^{54-57}$ and two nonrandomized trials ${ }^{120,121}$ evaluated different medical treatments in combination with behavioral compared with another treatment or placebo. Studies had low, ${ }^{54,}$ 55,57 moderate, ${ }^{121}$ and high risk of bias ${ }^{56,120}$ and included a total of 419 children (median=66/study) ranging in age from 3 to 14 years. One RCT comparing risperidone plus parent training with risperidone alone was included in our 2011 review but not in the present medical treatment update as it evaluates the effect of parent training; ${ }^{126}$ however, it is one of the few studies to compare a combination medical and behavioral treatment.

\section{Detailed Analysis}

Atomoxetine. A 10-week trial with a 24-week extension randomized children with ASD and ADHD symptoms to one of 4 arms: atomoxetine, atomoxetine plus parent training, placebo, or

placebo plus parent training. ${ }^{54,55}$ Families assigned to parent training received nine $60-90$ minute training sessions addressing preventing behavior problems and behavioral reinforcement. After 10 weeks of treatment over 45 percent of children in each of the atomoxetine groups met the definition of response ( $>30 \%$ decrease [improvement] on measures of ADHD and symptom severity) compared with 29 percent in the placebo plus parent training group and 19 percent in the placebo group ( $\mathrm{p}=0.015$ for atomoxetine vs. placebo). More children in the atomoxetine groups improved on measures of noncompliance but differences compared with the placebo group were not statistically significant. Parent ratings of inattention, hyperactivity, and ADHD were significantly improved in active treatment groups compared with placebo alone (p values $\leq 0.05$ ); teacher ratings improved in all groups from baseline to followup but group differences were not significant. Both parents and teachers rated children in the atomoxetine groups as significantly improved compared with the placebo group on noncompliance (Home Situations 
Questionnaire) (p values $\leq 0.05$ ). Parents also rated children in the placebo plus parent training group as significantly improved on the ABC-Hyperactivity and Inappropriate Speech scales compared with placebo alone ( $\mathrm{p}$ values $\leq 0.05$ ). Differences between the atomoxetine plus parent training and atomoxetine alone groups were not statistically significant for any outcomes.

After 10 weeks of treatment, 117 of the 128 children in the 10-week trial entered a 24-week extension. Atomoxetine responders and nonresponders who originally been assigned to a placebo group continued or began atomoxetine and maintained parent training if originally assigned to it in the 10-week trial. At followup, analyses of the effects of parent training vs. no parent training showed no significant group differences on measures of symptom severity, ADHD, inattention, hyperactivity, and parent-rated irritability and hyperactivity, though improvements were typically greater with parent training. Twenty-six of 40 children (60\%) who were responders to atomoxetine maintained response; 37 percent of placebo nonresponders had favorable ADHD outcomes and 34 percent had favorable noncompliance outcomes. Harms reported in this study are described in Table 16.

Melatonin. One 12-week RCT with low risk of bias compared cognitive behavioral therapy (CBT) alone, melatonin alone, CBT plus melatonin, and placebo in 160 children. ${ }^{57} \mathrm{CBT}$ consisted of four 50-minute sessions focused on recognizing dysfunctional attitudes about sleep, parent-management of children's sleep, and replacing poor sleep habits with appropriate behavior. All active treatment groups improved in most measures of sleep quality compared with the control group $(\mathrm{p}<0.01)$. In general, the combination group improved more than the others, followed by melatonin, and CBT. Scores for children who received melatonin alone improved on bedtime resistance, sleep onset delay, sleep duration, and night waking compared with the CBT group ( $<<0.001)$. Effect sizes ranged from medium to high. Sleep onset latency (time to fall asleep) and sleep efficiency (ratio of total sleep time to total time in bed) were reduced by 50 percent (sleep latency) or 85 percent (efficiency) in 85 and 63 percent of children in the combination group and 39 and 46 percent of children in the melatonin group, respectively. In the CBT arm, 10 percent of children met each criterion, and no children in the control arm achieved these percentages of reduced latency or improved efficiency.

Folic Acid. One 12-week nonrandomized trial (moderate risk of bias) compared folic acid supplementation plus the TEACCH program to the TEACCH program alone and reported no statistically significant differences between groups on measures of challenging behavior, symptom severity, and teacher-rated communication, though both groups typically improved from baseline. ${ }^{121}$

Bumetanide. In a high risk of bias, 12-week RCT comparing bumetanide plus daily applied behavior analysis and applied behavior analysis alone, children in the combination arm had significantly improved ABC, CARS, and CGI scores (p values <0.05). ${ }^{56}$

Stem Cell Transplantation. In one 24-week nonrandomized trial (high risk of bias) including 37 children received either rehabilitation therapy plus umbilical cord blood cell transplant, rehabilitation therapy plus stem cell transplant (4 intravenous or intrathecal transplants at 5-7 day intervals), or rehabilitation therapy alone. ${ }^{120}$ All children also received sensory integration and behavioral treatment. Symptom severity improved over time in all groups, with significantly greater improvements in the stem cell group compared with each of the other arms $(\mathrm{p}<0.05)$. CGI 
and total ABC scores also improved more in the stem cell group compared with the other groups. Lethargy/social withdrawal and stereotypy scales improved significantly more in the stem cell group compared with the other arms $(\mathrm{p}<0.05)$, but scores on the other individual ABC scales did not differ significantly.

\section{Studies of Nutritional Supplements or Specialized Diets}

\section{Key Points}

- Four RCTs compared omega-3 fatty acid supplementation and placebo and three addressing the outcome reported no significant effects on challenging behaviors. Our confidence in this conclusion is low (low strength of evidence for no effect). We also have low confidence in the conclusion that omega-3 supplementation was associated with minimal harms (low strength of evidence).

- Despite the number of RCTs with low or moderate risk of bias addressing other agents, evidence was inadequate to make conclusions about all clinical efficacy and harms outcomes because few, small, underpowered studies addressed each diet or supplement (insufficient strength of evidence).

- While seven studies addressed variations of the gluten-free casein-free (GFCF) diet, studies addressed different outcomes and different approaches to restricted and control diets; thus, data were inadequate to make conclusions about the body of evidence (insufficient strength of evidence).

- Data were inadequate to allow conclusions about the relative effectiveness of the other dietary interventions (e.g., camels’ milk, gluten- or casein-containing challenge foods) compared with placebo (insufficient strength of evidence).

\section{Overview of the Literature}

We identified 19 RCTs (three reported in multiple publications) ${ }^{58-65,80-88,104-107,117,128}$ that evaluated the use of supplements or dietary manipulation to treat ASD symptoms. Two of these studies ${ }^{117,128}$ were included in our 2011 review. Studies addressed nutritional supplements including omega-3 long-chain free fatty acid (FFA) supplementation, ${ }^{80-82,84}$ methyl-B12 supplement, ${ }^{83,87}$ digestive enzymes, ${ }^{85}, 117$ and L-carnitine. ${ }^{86,88}$ Dietary interventions addressed in studies included GFCF diets, ${ }^{58,62-65,}{ }^{104-107}$ gluten/casein challenge foods, ${ }^{59,60}$ and camels' milk. ${ }^{61}$

Studies were conducted in the United States, ${ }^{58,60,63,80-83,87,88,106,107,128}$ Australia, ${ }^{117}$ Belgium, ${ }^{59}$ Denmark, ${ }^{64,65}$ Egypt, ${ }^{85,}{ }^{86}$ Iran, ${ }^{62}$ Holland, ${ }^{84}$ Norway, ${ }^{104,}, 105$ and Saudi Arabia. ${ }^{61}$ Study treatment durations ranged from 7 days to 2 years, and sample sizes ranged from 12 to 92 (total $\mathrm{N}=732$ ). Followup occurred immediately post-treatment in all studies. Four RCTs had low risk of bias, ${ }^{58,60,81,83} 10$ had moderate, ${ }^{59,63,80,82,84-87,106,107,117,128}$ and five had high risk. ${ }^{61,62,64,65,88 \text {, }}$ 104,105

\section{Detailed Analysis}

Despite the number of RCTs with low or moderate risk of bias addressing supplements or diets, evidence is insufficient to determine their effects on any outcome in the short- or longterm. Most studies were small (median 30 total participants), short-term (ranging from 1 week to 7 months, with 24 months of treatment in one study). We provide brief summaries of reported outcomes by agent below. Appendix F includes summary tables with detailed findings. 


\section{Studies of Nutritional Supplements}

\section{Free Fatty Acid (Omega-3) Supplementation}

Little evidence supports the effectiveness of FFA supplementation to improve core or associated ASD symptoms (Table 18). Three RCTs of omega-3 FFA versus placebo (low ${ }^{81}$ and moderate ${ }^{80,84}$ risk of bias) reported no significant group differences on most measures of challenging behavior, communication, language, and adaptive behavior including the ABC, CGI, Peabody Picture Vocabulary Test, Pervasive Development Disorder Behavioral Inventory (PDDBI), VABS, Behavior Assessment System for Children (BASC), and Social Responsiveness Scale ${ }^{80,81,84}$ One study reported significantly improved scores in the placebo group compared with the omega-3 group on the BASC externalizing problems scale after 6 months of treatment, ${ }^{84}$ and another reported significant improvement in parent ratings of stereotypy and lethargy in children receiving omega-3 supplements compared with those receiving placebo, but teacher ratings were not significantly different. ${ }^{80}$

Another moderate risk of bias RCT of dietary docosahexanoic acid (DHA) supplementation versus placebo reported improvement in parent-rated social skills on the BASC in children receiving placebo vs. those receiving DHA, while teachers rated communication as more improved in the treatment group compared with placebo. ${ }^{82}$ Scores on other measures including the CGI, ABC, and Child Development Inventory did not differ significantly between groups.

Table 18. Key outcomes in studies of omega-3 fatty acids

\begin{tabular}{|c|c|c|}
\hline $\begin{array}{l}\text { Author, Year, Study Design } \\
\text { Groups (dose), N Enrollment / N } \\
\text { Final } \\
\text { Treatment Duration/Follow-up } \\
\text { Timepoint Post-Treatment } \\
\text { Risk of Bias }\end{array}$ & $\begin{array}{l}\text { Outcome } \\
\text { Measure/Baseline } \\
\text { Scores, Mean } \pm S D\end{array}$ & $\begin{array}{l}\text { Outcome Measure/Post-Treatment Scores, } \\
\text { Mean } \pm \text { SD }\end{array}$ \\
\hline $\begin{array}{l}\text { Mankad } 2015^{84} \mathrm{RCT} \\
\text { G1: Omega-3 fatty acid (3.5 } \\
\mathrm{mL} / \text { day), } 19 / 18 \\
\mathrm{G} 2 \text { : Placebo, } 19 / 19 \\
6 \text { months/EOT } \\
\text { Moderate RoB }\end{array}$ & $\begin{array}{l}\text { Baseline scores } \\
\text { NR }\end{array}$ & $\begin{array}{l}\text { Change from Baseline } \\
\text { PDDBI - Autism Composite } \\
\text { G1: }-4.5 \\
\text { G2: }-6.4 \\
\text { G1 vs G2: } p=n s \\
\text { BASC }- \text { Externalizing } \\
\text { G1: } 3.2 \\
\text { G2: }-3.0 \\
\text { G1 vs G2: } p=0.02 \\
\text { CGI } \\
\text { G1: NR } \\
\text { G2: NR } \\
\text { G1 vs G2:p=ns } \\
\text { VABS } \\
\text { G1: } 2.8 \\
\text { G2: }-0.2 \\
\text { G1 vs G2: } p=n s \\
\text { Preschool Language Scale (PLS4) } \\
\text { G1: } 0.7 \\
\text { G2: }-0.6 \\
\text { G1 vs G2: } p=n s\end{array}$ \\
\hline $\begin{array}{l}\text { Voigt } 2014^{82} \mathrm{RCT} \\
\text { G1: Docosahexaenoic acid (DHA) }\end{array}$ & $\begin{array}{l}\text { CGI-I Parent } \\
\text { NR }\end{array}$ & $\begin{array}{l}\text { CGI-I Parent } \\
3 \text { months } \\
\text { G1: } 4 / 21 \text { (19) }\end{array}$ \\
\hline
\end{tabular}




\begin{tabular}{|c|c|c|}
\hline $\begin{array}{l}\text { (500 mg/day), 24/19 } \\
\text { G2: Placebo, 24/15 } \\
6 \text { months/EOT } \\
\text { Moderate ROB }\end{array}$ & $\begin{array}{l}\text { BASC-Parent } \\
\text { G1: } 26.5 \pm 7.1 \\
\text { G2: } 30.3 \pm 9.1 \\
\text { BASC- Teacher } \\
\text { G1: } 32.2 \pm 7.6 \\
\text { G2: } 38.5 \pm 4.3\end{array}$ & $\begin{array}{l}\text { G2: } 5 / 16(31) \\
\text { G1 vs } G 2: p=n s \\
6 \text { months } \\
\text { G1: } 5 / 18(28) \\
\text { G2: } 2 / 13(15) \text { G1 vs } G 2: p=n s \\
\text { CGI-I Investigator } \\
3 \text { months } \\
\text { G1: } 1 / 17(6)) \\
\text { G2: } 0 / 13(0) \\
\text { G1 vs } \mathrm{G} 2: p=n s \\
6 \text { months } \\
\text { G1: } 0 / 18(0) \\
\text { G2: } 1 / 14(7) \\
\text { G1 vs } G 2: p=n s \\
\text { EOT } \\
\text { BASC }- \text { Parent } \\
\text { G1: } 26.3 \pm 6.8 \\
\text { G2: } 33.3 \pm 9.7 \\
\text { G1 vs } G 2: p=0.04 \\
\text { BASC }- \text { Teacher } \\
\text { G1: } 33.6 \pm 9.3 \\
\text { G2: } 34.0 \pm 5.6 \\
\text { G1 vs } G 2: p=0.02\end{array}$ \\
\hline $\begin{array}{l}\text { Bent } 2011^{81} \\
\text { RCT } \\
\text { G1: Omega-3 fatty acid (1.3 g/day), } \\
\text { 14/13 } \\
\text { G2: Placebo, 13/12 } \\
12 \text { weeks/EOT } \\
\text { Low RoB }\end{array}$ & $\begin{array}{l}\text { ABC-Hyperactivity } \\
\text { G1: } 16.8 \pm 13 \\
\text { G2: } 20.3 \pm 8 \\
\text { PPVT } \\
\text { G1: } 72.2 \pm 28 \\
\text { G2: } 85.8 \pm 12 \\
\text { EVT } \\
\text { G1: } 70.8 \pm 33 \\
\text { G2: } 86.4 \pm 14 \\
\text { SRS } \\
\text { G1: } 76.9 \pm 11 \\
\text { G2: } 79.0 \pm \\
\text { BASC-Externalizing } \\
\text { G1: } 53.8 \pm 13 \\
\text { G2: } 66.3 \pm 25 \\
\text { BASC- Internalizing } \\
\text { G1: } 43.3 \pm 10 \\
\text { G2: } 50.1 \pm 9 \\
\text { BASC-Behavioral } \\
\text { G1: } 60.9 \pm 14 \\
\text { G2: } 65.4 \pm 3 \\
\text { BASC-Adaptive skill } \\
\text { G1: } 29.8 \pm 9 \\
\text { G2: } 31.9 \pm 9 \\
\text { BASC-Hyperactivity } \\
\text { G1: } 61.8 \pm 17\end{array}$ & $\begin{array}{l}\text { Mean change score } \\
\text { ABC }- \text { Hyperactivity } \\
\text { G1: } 2.7 \pm 4.8 \\
\text { G2: } 0.3 \pm 7.2 \\
\text { G1 vs G2: } p=n s \\
\text { PPVT } \\
\text { G1: } 2.7 \pm 11.6 \\
\text { G2: } 1.9 \pm 12.4 \\
\text { G1 vs G2: } p=n s \\
\text { EVT } \\
\text { G1: } 2.2 \pm 7.6 \\
\text { G2: } 5.8 \pm 5.7 \\
\text { G1 vs G2: } p=n s \\
\text { SRS } \\
\text { G1: }-0.9 \pm .5 \\
\text { G2: } 1.7 \pm 7.2 \\
\text { G1 vs G2: } p=n s \\
\text { BASC-Externalizing } \\
\text { G1: } 0.1 \pm 6.7 \\
\text { G2: } 6.6 \pm 30.4 \\
\text { G1 vs G2: } p=n s \\
\text { BASC-Internalizing } \\
\text { G1: } 0.3 \pm 6.6 \\
\text { G2: }-2.9 \pm 7.6 \\
\text { G1 vs G2: } p=n s \\
\text { BASC-Behavioral } \\
\text { G1: }-1.1 \pm 6.1 \\
\text { G2: }-2.0 \pm 4.9\end{array}$ \\
\hline
\end{tabular}




\begin{tabular}{|l|l|l|}
\hline & G2: $64.6 \pm 7$ & G1 vs $G 2: p=n s$ \\
& & BASC-Adaptive skill \\
& & G1: $1.8 \pm 6.8$ \\
& G2: $0.8 \pm 7.1$ \\
& G1 vs G2: $p=n s$ \\
& BASC-Hyperactivity \\
& G1: $2.1 \pm 6.3$ \\
& G2: $1.2 \pm 5.8$ \\
& G1 vs G2: $p=n s$ \\
\hline
\end{tabular}

ABC = Aberrant Behavior Checklist; BASC = Behavior Assessment System for Children; CGI = Clinical Global Impression; EOT = end of treatment; EVT = Expressive Vocabulary Test; PPVT = Peabody Picture Vocabulary Test; ROB = risk of bias; SRS $=$ Social Responsiveness Scale

\section{Digestive Enzyme Supplementation}

Evidence is insufficient to determine if short-term digestive enzyme supplements affect ASD core or associated symptoms. Two RCTs with moderate risk of bias addressed digestive enzyme supplements compared with placebo: one evaluated a proteolytic enzyme supplement (Peptizyde ${ }^{117}$ and the other a digestive enzyme supplement (Neo-Digestin). ${ }^{85}$ The Peptizyde RCT reported no significant differences in measures of behavior, sleep quality, or gastrointestinal symptoms, and no significant differences in adverse effects. ${ }^{117}$ In a 3-month trial of Neo-Digestin versus placebo, CARS scores improved significantly in the treatment group compared with placebo. ${ }^{85}$

\section{Other Supplements}

Two RCTs with low ${ }^{83}$ and moderate ${ }^{87}$ risk of bias addressed methyl B12 supplementation. CGI scores improved significantly in the methyl B12 group in one RCT (effect size $=0.84$, $\mathrm{p}=0.005$ ), but studies reported few other significant group differences in measures of behavior or communication. ${ }^{87}$ In two RCTs addressing L-carnitine (moderate ${ }^{86}$ and high ${ }^{88}$ risk of bias), ASD severity scores improved significantly in the L-Carnitine group compared with placebo in one, but scores on other behavioral measures or measures of adverse effects did not differ between groups ${ }^{88}$ In the second RCT, symptom severity did not differ between groups after 6 months of treatment. ${ }^{86}$

\section{Studies of Dietary Manipulation}

\section{Gluten-Free Casein-Free Diets (GFCF)}

Data to assess effects of GFCF diets are limited as dietary approaches and outcome measures varied among studies as did control diets and monitoring of adherence to GFCF diets. Four RCTs (in multiple publications) compared GFCF diets to either an unaltered diet, ${ }^{64,65,104-107}$ or a low sugar diet (Total $\mathrm{N}$ across studies $=82$ ). ${ }^{63}$ One 12-week RCT (moderate risk of bias) reported no significant differences between groups on measures of development or behavior (Mullen Scales of Early Learning, Child Behavior Checklist), though the control group improved significantly from baseline on visual reception, withdrawal, aggression, and attention subscales ( $\mathrm{p}$ values $<0.05$ ) Another 12-week crossover RCT (moderate risk of bias) similarly reported no significant differences between groups on measures of symptom severity or language, though parents of 7 of the 15 children participating in the study reported improvements in language. ${ }^{106,107}$ In a retrospective analysis of videotapes recorded during the study period, investigators found no significant group differences in verbal communication between children in the diet or control 
groups or between children whose parents reported language improvements after the study period and those whose parents did not.

A 12-month RCT (high risk of bias) including children with urinary peptide abnormalities reported significant improvements(unblinded parent-rated questions from the Diagnosis of Psychotic Behavior in Children questionnaire) in communication, resistance to communication, social isolation, repetitive or challenging behavior, and overall impairment in children on a GFCF diet compared with those on a usual diet (p values $\leq 0.007$ ). ${ }^{104,105}$ Children in the GFCF diet also improved significantly on tests of cognitive skills, motor skills, verbal and social communication, anxiety, and reaction to changes in environment and routine compared with control children ( $p$ values $<0.05$ ). Another high risk of bias RCT with 24-month followup of participants reported few differences in behavioral measures between children on a GFCF diet and those with no dietary restrictions; ${ }^{64,65}$ scores on the Autism Diagnostic Observation Schedule (ADOS) and Gilliam Autism Rating Scale improved significantly in participants in the GFCF group vs. no diet group at 12 months, but scores were not different on any measure in a subset of participants followed for 24 months.

\section{Gluten-Free Diets}

One 6-week, high risk of bias RCT compared a gluten-free diet to a usual diet and reported significant improvements in gastrointestinal symptoms (stomachache, bloating, constipation) from baseline in the gluten-free diet group but not in the control group. Diarrhea did not improve significantly in either group. Stereotyped behavior, communication, social interaction, and ASD symptoms also improved from baseline in the gluten-free group (p values $<0.02$ ) but not in the control arm; differences in stereotyped behaviors and communication, but not social interaction, were significant between groups ( $p$ values $\leq 0.005$ ). The study did not report other between-group comparisons.

\section{Gluten-Free and Dairy-Free Diets}

In a small, low risk of bias trial comparing a gluten and dairy free diet with a diet including both gluten and dairy, scores on measures of challenging behavior (hyperactivity, irritability, inattention) did not differ between groups after 4 weeks of therapy. ${ }^{58}$ Children in both groups had gastrointestinal symptoms (loose or hard stools, abdominal pain, $\mathrm{p}=\mathrm{NR}$ ).

\section{Gluten and Casein Challenge Foods or Supplementation}

Two small RCTs (low ${ }^{129}$ and moderate ${ }^{59}$ risk of bias) evaluated "challenges" of gluten or casein containing foods, but evidence is inadequate to determine if short-term gluten- casein containing foods affect ASD symptoms or gastrointestinal function. One RCT randomized children who were maintaining GFCF diets to foods with gluten, gluten and casein, or placebo foods. ${ }^{60}$ The study reported no significant group differences in measures of challenging behaviors or measures of sleep quality and stool frequency at any time point over the 30-week trial. Another RCT assessing effects of introducing gluten-casein containing foods versus placebo foods similarly reported no significant effects of added gluten or casein on behavior or gastrointestinal symptoms. ${ }^{59}$

\section{Camel Milk}

A single RCT (high risk of bias) compared boiled or raw camel's milk with cow's milk and reported no significant differences in ASD severity between groups after 2 weeks of treatment. ${ }^{61}$ 


\section{Harms of Nutritional or Dietary Interventions}

Studies that reported harms either reported no significant difference between the intervention group and the control group, or reported zero harms for each group. Appendix F includes detailed harms tables.

\section{Studies of Risperidone Adjuncts}

\section{Key Points}

- Though 14 RCTs with low or moderate risk of bias compared risperidone plus an adjunct medication with risperidone plus placebo, few compared the same adjunct agents. Studies thus provide little evidence to inform treatment decisions and can be considered primarily as pilot efficacy trials.

- Most studies reported improvements in irritability with combined treatment compared with placebo plus risperidone, but data were inadequate to assess effects for all comparisons and outcomes given the heterogeneity of agents (insufficient strength of evidence).

\section{Overview of the Literature}

We identified 14 placebo-controlled RCTs addressing risperidone plus an adjunct medication (titrated to 0.5 to $3 \mathrm{mg}$ /day based on body weight). ${ }^{89-100,118,124}$ Two of these studies were included in our 2011 review. ${ }^{118,124}$ Study medications added to risperidone included celecoxib, ${ }^{94}$ Ginkgo biloba, ${ }^{97}$ memantine, ${ }^{95}$ topiramate, ${ }^{98}$ riluzole, ${ }^{91}$ buspirone, ${ }^{92}$ N-acetylcysteine, ${ }^{90,93}$ amantadine, ${ }^{96}$ pioglitazone, ${ }^{99}$ pentoxifylline, ${ }^{118}$ galantamine, ${ }^{100}$ minocycline, ${ }^{89}$ and piracetam. ${ }^{124}$ Studies were short-term ( $\leq 10$ weeks of treatment) with no longer term followup evaluation once treatment ended.

Studies included a total of 561 children ranging in age from 3 to 17 years. All studies were conducted in Iran. $^{90-100,118,124}$ We considered 11 studies to have low risk of bias ${ }^{90-100}$ and two to have moderate risk of bias. ${ }^{118,124}$

\section{Detailed Analysis}

Despite the number of RCTs with low or moderate risk of bias, studies present little evidence to inform treatment decisions and can be considered primarily as pilot efficacy trials. As noted, all studies were short-term and lacked followup past the end of treatment. Studies included few participants (median 40 total/study) and few examined the same adjunct agent or outcomes besides the ABC Irritability subscale. Only two studies ${ }^{90,93}$ addressed the same outcomes with different doses of the same agent (N-acetylcysteine). All studies except one of gingko biloba added to risperidone reported significant improvements on the ABC-Irritability subscale in the adjunct groups compared with placebo plus risperidone; one study reporting only total ABC scores reported significant improvements in the adjunct group compared with placebo. ${ }^{124} \mathrm{We}$ present a brief summary of key outcomes in each study below; Appendix F includes detailed tables of outcomes and harms. Harms typically did not differ between groups.

N-acetylcysteine. Two studies ${ }^{90,93}$ compared the effect of different doses of N-acetylcysteine as an adjunctive therapy to risperidone vs. placebo plus risperidone in a total of 80 children with autistic disorder. By the end of treatment, the $\mathrm{N}$-acetylcysteine groups had significantly greater reduction in irritability scores $(\mathrm{p}<0.035)$ than the placebo group in both the trials; in one RCT scores on the hyperactivity/noncompliance $(\mathrm{p}<0.05)$ subscales were also significantly improved 
in the N-acetylcysteine group. ${ }^{90}$ Other subscale scores did not differ between groups in either RCT. Adverse events were mild and transient, with a similar incidence in both trials.

Celecoxib. One RCT explored the effectiveness of adding celecoxib as an adjunct to risperidone vs. placebo plus risperidone in 40 children reported significant improvements on the ABC irritability, lethargy/social withdrawal, and stereotypy scales in the adjunct group compared with placebo plus risperidone. ${ }^{94}$ Hyperactivity/noncompliance or inappropriate speech did not differ between groups. The frequency of adverse effects as reported by parents was similar between the two groups. By week 10, complete response (50\% reduction in irritability subscale) was achieved by 11 of the children in the celecoxib group compared with four (20\%) in the placebo group $(\mathrm{p}=0.02)$.

Ginkgo Biloba. In one RCT comparing Ginkgo biloba plus risperidone with risperidone plus placebo, investigators found no significant differences between groups on any of the ABC subscales. Side effects were similar between groups.

Memantine. One RCT reported significant reduction in ABC subscale scores for irritability, stereotypic behavior, and hyperactivity in the memantine adjunct group compared with risperidone plus placebo (all $\mathrm{p}<0.01$ ). ${ }^{95}$ No significant effects were found on the lethargy or inappropriate speech subscales. Frequency of side effects including extrapyramidal symptoms was similar between the two groups.

Riluzole. In one RCT including children with autistic disorder who responded suboptimally to previous medication, children treated with riluzole and risperidone had significantly greater improvement in four of the five ABC subscales $(\mathrm{p}<0.01)$ than those receiving placebo plus risperidone. ${ }^{91}$ Based on CGI-I scores, complete response was achieved by 11 children (55\%) in the riluzole group compared with five $(25 \%)$ in the placebo group $(p=0.05)$. Among the 16 side effects observed, increased appetite and weight gain were more frequently reported in the riluzole group versus the placebo group $(\mathrm{p} \leq 0.03)$. All other side effects occurred at a similar frequency in both groups.

Buspirone. More children receiving buspirone plus risperidone had $\mathrm{a} \geq 30 \%$ reduction in irritability score ( $81.2 \%$ vs. $38.9 \%, \mathrm{p}<0.01, \mathrm{RR}=2.1$ ) than those receiving placebo and risperidone. Investigators reported no serious adverse events in either group, but the odds ratio for increased appetite was 2.61 (61.1\% with buspirone vs. 35.3\% with placebo). Other commonly reported adverse events were drowsiness (11.1\%) and fatigue $(11.1 \%)$ in the buspirone group and dry mouth (5.9\%) by the placebo group.

Topiramate. In one RCT children in the topiramate adjunct group had significantly greater reduction in ABC-C subscale scores for irritability, stereotypic behavior, and hyperactivity/noncompliance (all $\mathrm{p}=0.04$ ) than those receiving placebo. ${ }^{98}$ Frequencies of most side effects including extrapyramidal symptoms were similar, though somnolence (35\% vs. 5\%, $\mathrm{p}=0.04$ ) and decreased appetite (35\% vs. $5 \%, \mathrm{p}=0.04$ ) occurred more frequently in the topiramate-treated group than the placebo group. 
Amantadine. In one RCT including children with severe disruptive symptoms, ABC-Irritability and Hyperactivity/Noncompliance scores were significantly improved in amantadine adjunct group compared with the placebo group $(p \leq 0.03){ }^{96}$ By week 10,17 (85\%) of the children in the amantadine group compared with $13(65 \%)$ in the placebo group had partial response (25\% reduction in irritability subscale) $(\mathrm{p}=0.14)$, while complete response ( $50 \%$ reduction in irritability score) was achieved by 7 (35\%) vs. 3 (15\%) in the amantadine and placebo groups respectively $(\mathrm{p}=\mathrm{ns})$. None of the other ABC subscale scores differed between the groups by the end of treatment. Frequency of adverse effects did not differ between groups. Based on the improvement measured by CGI scale, a higher proportion of children in the amantadine group responded to treatment than those in the placebo group ( $50 \%$ vs. $20 \%, \mathrm{p}=0.047)$, with two and eight children judged to have very much improved and much improved respectively in the amantadine group compared with one and three children in the placebo group.

Pioglitazone. In one RCT children receiving pioglitazone adjunct had significant improvement in the ABC Irritability $(p=0.03)$, Lethargy/social withdrawal $(p=0.04)$ and Hyperactivity/noncompliance $(\mathrm{p}=0.04)$ subscale scores compared with the placebo group; ${ }^{99}$ scores on other measures did not differ between groups. More children in the adjunct group also had a partial response ( $\geq 25 \%$ reduction in irritability score) than did children receiving placebo ( $45 \%$ vs. $15 \%$, $\mathrm{p}=0.04$ ). Nine children ( $45 \%$ ) in the pioglitazone group had complete response $(\geq$ $50 \%$ reduction in irritability score) compared with 7 (35\%) in the placebo group (p=ns). Adverse events were mild and transient with no group differences in the frequency.

Galantamine. In a trial evaluating galantamine or placebo in addition to risperidone, children in the galantamine adjunct group showed significantly greater improvement in ABC-Irritability $(p=0.017)$ and Lethargy/social withdrawal $(p=0.005)$ subscales than the placebo group. ${ }^{100}$ Reduction in other ABC subscale scores after treatment was similar between the groups. Sixteen children $(80 \%)$ in the galantamine group had a complete response $(\geq 50 \%$ reduction in the irritability subscale score) compared with $10(50 \%)$ in the placebo group $(\mathrm{p}=0.047)$, while partial response ( $\geq 25 \%$ reduction in the ABC-I subscale) was reported in $90 \%$ in the galantamine and $65 \%$ in the placebo group, $\mathrm{p}=0.058$. The investigators noted no serious adverse events. Both groups reported weight gain by the end of the trial, with no significant group difference.

Pentoxifylline. Scores on the ABC-Irritability, Lethargy/Social Withdrawal, Stereotypic behavior, Hyperactivity/Noncompliance, and Inappropriate Speech subscales were significantly better for the pentoxifylline adjunct group compared with placebo $(\mathrm{p} \leq 0.0001)$ in one RCT. ${ }^{118}$ Frequency of side effects including extrapyramidal symptoms did not differ between groups.

Piracetam. Risperidone plus piracetem was associated with more improvement on the ABC-C total score than risperidone given with placebo in one RCT, with similar incidence of extrapyramidal symptoms and other adverse events. ${ }^{124}$

Minocycline. Improvements in ABC-Irritability and Hyperactivity scales were greater in children receiving minocycline+risperidone than in children receiving placebo+risperidone ( $\mathrm{p}$ values $\leq 0.003$ ), but scores on the other subscales did not differ significantly between groups in one RCT (low risk of bias). ${ }^{89}$ Adverse events (including sedation, appetite changes, and gastrointestinal issues) did not differ between groups ( $\mathrm{p}$ values=NS) 


\section{Studies of Hyperbaric Oxygen Therapy}

\section{Key Points}

- Studies of hyperbaric oxygen using differing protocols reported conflicting results: no treatment effects in two RCTs and significant improvements in ASD symptoms in another.

- Data were inadequate to assess the effects of hyperbaric oxygen compared with placebo on ASD symptoms, language, or harms (insufficient strength of evidence).

\section{Overview of the Literature}

We identified three RCTs with low ${ }^{67,114}$ and moderate ${ }^{66}$ risk of bias addressing hyperbaric oxygen compared with a sham treatment. Studies were conducted in the United States ${ }^{67,114}$ and Thailand $^{66}$ and included a total of 150 children between 2 and 14 years old. Treatment duration ranged from 20 days to 15 weeks with followup immediately post-treatment.

\section{Detailed Analysis}

Three RCTs of hyperbaric oxygen used different doses and reported inconsistent results (favorable effects associated with treatment in one and no significant effects in two). The RCTs included children with diagnoses of autistic disorder ${ }^{67,114}$ or autism. ${ }^{66}$ Two studies used a 24 percent oxygen treatment ${ }^{67,114}$ and a third used 100 percent oxygen; ${ }^{66}$ children continued concomitant treatments including behavioral and medical interventions instituted prior to the studies. Two studies of $80^{67}$ or $20^{66}$ hourly treatments reported no significant differences between groups on measures of symptom severity, language, and adaptive behavior. ${ }^{66,67}$ In a third RCT including 40 treatment sessions, clinician-rated overall CGI scores, parent-rated language and eye contact measures, the ABC-Irritability subscale, and the Autism Treatment Evaluation Checklist (ATEC) sensory scale improved significantly in the hyperbaric oxygen group compared with the placebo group. Other ABC or ATEC subscale scores did not differ significantly between groups. ${ }^{114}$

\section{Harms of Hyperbaric Oxygen}

Studies typically noted that no significant harms occurred. One child had worsening asthma symptoms and discontinued treatment in one study, ${ }^{114}$ while 11 children in another experienced middle ear barotrauma that did not lead to discontinuation of the treatment session or the study. ${ }^{66}$ Across studies, one child withdrew due to seizures and one due to worsening asthma.

\section{Studies of $\mathbf{N}$-acetylcysteine}

\section{Key Points}

- Two small RCTs of N-acetylcysteine reported few significant treatment effects and few clinically significant harms.

- N-acetylcysteine had no effect on social skills outcomes in two small RCTs; harms of this agent were not clinically significant. Our confidence in these conclusions is low (low strength of evidence). Data were inadequate to assess effects on other outcomes (insufficient strength of evidence). 


\section{Overview of the Literature}

Two RCTs with low ${ }^{78}$ and moderate ${ }^{79}$ risk of bias assessed $\mathrm{N}$-acetylcysteine compared with placebo. Studies were conducted in the United States and Australia over 12 to 24 weeks and included a total of 123 children between 3 and 10 years old. Followup in both studies was immediately post-treatment.

\section{Detailed Analysis}

In one 12-week RCT (low risk of bias) comparing the utility of N-acetylcysteine with placebo, children treated with $\mathrm{N}$-acetylcysteine had significant improvement on the ABCIrritability subscale as compared with placebo-treated children $(\mathrm{p}<0.001) ;{ }^{78}$ but effects on other behavioral and social measures did not differ. A second 24-week RCT (moderate risk of bias) included 98 children randomized to $\mathrm{N}$-acetylcysteine or placebo and reported no group differences on any outcome assessed including social communication, adaptive behavior, symptom severity, or repetitive behavior. ${ }^{79}$ Table 19 outlines key outcomes. 
Table 19. Key outcomes in studies of $\mathrm{N}$-acetylcysteine

\begin{tabular}{|c|c|c|}
\hline $\begin{array}{l}\text { Author, Year Study Design } \\
\text { Groups (Dose), N Enrollment I } \\
\text { N Final } \\
\text { Treatment Duration/Follow-Up } \\
\text { Timepoint Post-Treatment } \\
\text { Risk of Bias }\end{array}$ & $\begin{array}{l}\text { Outcome Measure/Baseline } \\
\text { Scores, Mean } \pm \text { SD }\end{array}$ & $\begin{array}{l}\text { Outcome Measure/Post-Treatment } \\
\text { Scores, Mean } \pm \text { SD }\end{array}$ \\
\hline $\begin{array}{l}\text { Dean } 2016^{79} \mathrm{RCT} \\
\text { G1: N-acetylcysteine (500 } \\
\text { mg/day), } 48 / 34 \\
\text { G2: Placebo (ND),50 /37 } \\
6 \text { months/EOT } \\
\text { Moderate ROB }\end{array}$ & $\begin{array}{l}\text { SRS-Total Score } \\
\text { G1: } 102.9 \pm 26.4 \\
\text { G2: } 99.7 \pm 24.8 \\
\text { CCC-General Communication } \\
\text { G1: } 33.6 \pm 13.1 \\
\text { G2: } 34.7 \pm 14.6 \\
\text { CCC-Social Interaction Deviance } \\
\text { G1: } 3.1 \pm 8.5 \\
\text { G2: } 3.3 \pm 6.7 \\
\text { Repetitive Behavior Scale-Total } \\
\text { Score } \\
\text { G1: } 28.5 \pm 19.4 \\
\text { G2: } 24.8 \pm 14.1 \\
\text { CGI-Severity } \\
\text { G1: } 4.1 \pm 1 \\
\text { G2: } 4.2 \pm 1 \\
\text { Developmental Behavior Checklist- } \\
\text { Total Score } \\
\text { G1: } 57 \pm 23.2 \\
\text { G2: } 59 \pm 27.6\end{array}$ & $\begin{array}{l}\text { SRS-Total Score } \\
\text { G1: } 92.1 \pm 29.3 \\
\text { G2: } 88.7 \pm 27.7 \\
\text { G1 vs } G 2, p=n s \\
\text { CCC-General Communication } \\
\text { G1: } 34.7 \pm 11.9 \\
\text { G2: } 41.5 \pm 16.5 \\
\text { G1 vs } G 2, p=n s \\
\text { CCC-Social Interaction Deviance } \\
\text { Change Score } \\
\text { G1: } 0.05 \pm 8.4 \\
\text { G2: }-2.2 \pm 8.2 \\
\text { G1 vs } G 2, p=n s \\
\text { Repetitive Behavior Scale-Total Score } \\
\text { G1: } 24.3 \pm 24.3 \\
\text { G2: } 20.8 \pm 16.3 \\
\text { G1 vs } G 2, p=n s \\
\text { CGI-Severity } \\
\text { G1: } 3.9 \pm 1 \\
\text { G2: } 3.6 \pm 1 \\
\text { G1 vs } G 2, p=n s \\
\text { Developmental Behavior Checklist-Total } \\
\text { Score } \\
\text { G1: } 43.9 \pm 25.2 \\
\text { G2: } 48.2 \pm 18 \\
\text { G1 vs } G 2, p=n s\end{array}$ \\
\hline $\begin{array}{l}\text { Hardan } 2012^{78} \mathrm{RCT} \\
\text { G1: N-acetylcysteine (900 mg up } \\
\text { to } 3 \text { times/day), } 15 / 134 \\
\text { G2: Placebo, } 18 / 15 \\
12 \text { weeks/EOT } \\
\text { Low ROB }\end{array}$ & $\begin{array}{l}\text { ABC Irritability } \\
\text { G1: } 16.9 \pm 7.9 \\
\text { G2: } 14.8 \pm 9.6 \\
\text { ABC Lethargy } \\
\text { G1: } 15.2 \pm 9.5 \\
\text { G2: } 12.1 \pm 7.8 \\
\text { ABC Stereotypy } \\
\text { G1: } 9.1 \pm 5.5 \\
\text { G2: } 8.9 \pm 6.5 \\
\text { ABC Hyperactivity } \\
\text { G1: } 23.4 \pm 9.0 \\
\text { G2: } 23.8 \pm 9.3 \\
\text { ABC Inappropriate Speech } \\
\text { G1: } 4.9 \pm 3.2 \\
\text { G2: } 4.1 \pm 3.7\end{array}$ & $\begin{array}{l}\text { EOT } \\
\text { ABC Irritability } \\
\text { G1: } 7.2 \pm 5.7 \\
\text { G2: } 13.1 \pm 9.9 \\
p<.001 \\
\text { ABC Lethargy } \\
\text { G1: } 11 \pm 9.4 \\
\text { G2: } 8.3 \pm 7.7 \\
p=. n s \\
\text { ABC Stereotypy } \\
\text { G1: } 5.6 \pm 5.7 \\
\text { G2: } 8.0 \pm 7.0 \\
\text { p=ns } \\
\text { ABC Hyperactivity } \\
\text { G1: } 12.4 \pm 11.4 \\
\text { G2: } 21.0 \pm 11.5 \\
\text { p=ns }\end{array}$ \\
\hline
\end{tabular}




\begin{tabular}{|c|c|c|}
\hline $\begin{array}{l}\text { Author, Year Study Design } \\
\text { Groups (Dose), N Enrollment I } \\
\text { N Final } \\
\text { Treatment Duration/Follow-Up } \\
\text { Timepoint Post-Treatment } \\
\text { Risk of Bias }\end{array}$ & $\begin{array}{l}\text { Outcome Measure/Baseline } \\
\text { Scores, Mean } \pm \text { SD }\end{array}$ & $\begin{array}{l}\text { Outcome Measure/Post-Treatment } \\
\text { Scores, Mean } \pm \text { SD }\end{array}$ \\
\hline & 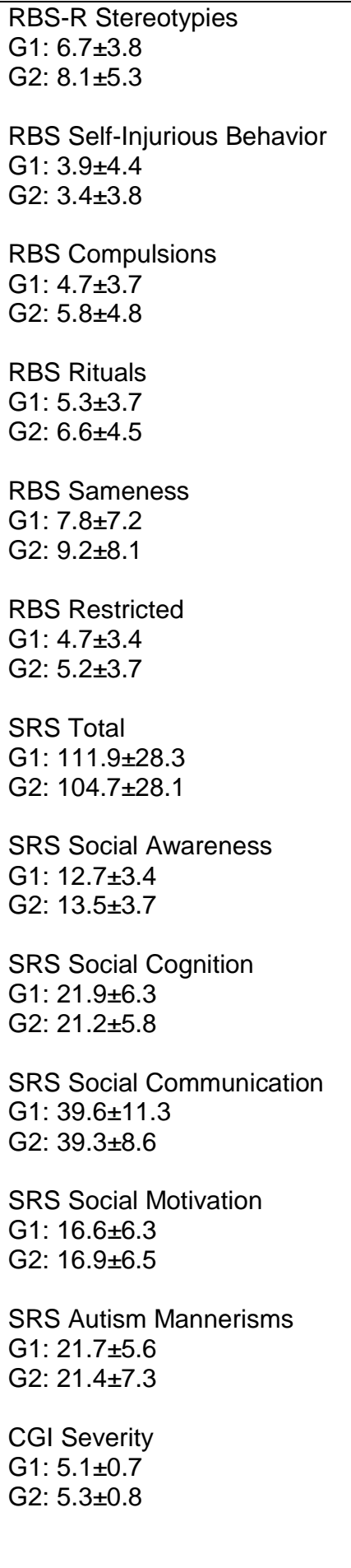 & 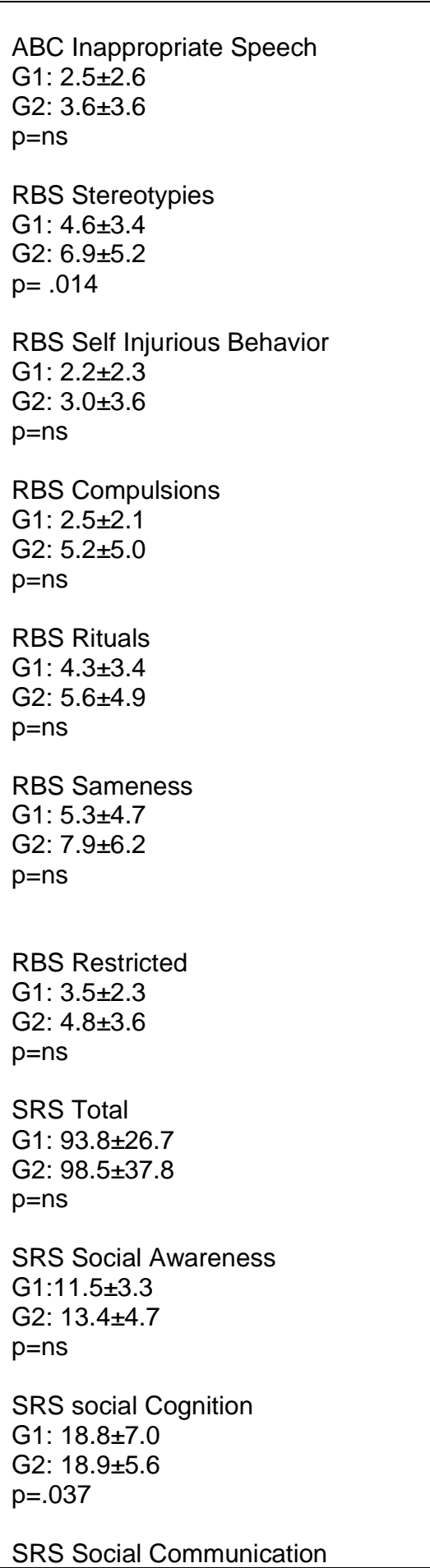 \\
\hline
\end{tabular}




\begin{tabular}{|l|l|l|}
\hline $\begin{array}{l}\text { Author, Year Study Design } \\
\text { Groups (Dose), N Enrollment I } \\
\text { N Final }\end{array}$ & $\begin{array}{l}\text { Outcome Measure/Baseline } \\
\text { Scores, Mean } \pm \text { SD }\end{array}$ & $\begin{array}{l}\text { Outcome Measure/Post-Treatment } \\
\text { Scores, Mean } \pm \text { SD }\end{array}$ \\
$\begin{array}{l}\text { Treatment Duration/Follow-Up } \\
\text { Timepoint Post-Treatment }\end{array}$ & \\
Risk of Bias & \\
\hline & \\
& & G1: $33.3 \pm 10.9$ \\
& G2: $34.5 \pm 14.5$ \\
& p=.ns \\
& SRS Social Motivation \\
& G1: $13.0 \pm 4.7$ \\
& G2: $14.5 \pm 7.0$ \\
& $p=n s$ \\
& SRS Autism Mannerisms \\
& G1: $16.0 \pm 6.1$ \\
& G2: $20.3 \pm 6.9$ \\
& p=.045 \\
& CGI Severity \\
& G1: $4.5 \pm 0.8$ \\
& G2: $4.9 \pm 0.9$ \\
& p=ns \\
& CGI Improvement \\
& G1: $2.9 \pm 1.1$ \\
& G2: $3.2 \pm .09$ \\
& p=ns \\
\hline
\end{tabular}

ABC = Aberrant Behavior Checklist; CCC = Children’s Communication Checklist; CGI = Clinical Global Impression; EOT = end of treatment; RBS = Repetitive Behavior Scale; ROB = risk of bias; SRS = Social Responsiveness Scale

\section{Harms of N-acetylcysteine}

Adverse events also did not differ between groups in either study, though one noted treatment discontinuation of one participant related to worsening of irritability. ${ }^{78}$ Table 20 outlines harms. 
Table 20. Harms/adverse effects reported in studies of $\mathrm{N}$-acetylcysteine

\begin{tabular}{|c|c|c|}
\hline Harm/Adverse Event & $\begin{array}{l}\text { N Studies Reporting Harm (\# } \\
\text { Participants With Harm/Total } \\
\text { Participants) }\end{array}$ & $\begin{array}{c}\text { Reported Rates Across } \\
\text { Studies }\end{array}$ \\
\hline \multicolumn{3}{|l|}{ N-acetylcysteine } \\
\hline Gastrointestinal symptoms $^{78,79}$ & $2(23 / 48)$ & $26.5 \%-100 \%$ \\
\hline Agitation/nervousness/restlessness ${ }^{78}$ & $1(3 / 14)$ & $21.4 \%$ \\
\hline EPS/impaired movement ${ }^{78}$ & $1(3 / 14)$ & $21.4 \%$ \\
\hline Appetite decrease $^{78}$ & $1(2 / 14)$ & $14.3 \%$ \\
\hline Appetite increase ${ }^{78}$ & $1(2 / 14)$ & $14.3 \%$ \\
\hline Skin changes $^{79}$ & $1(2 / 34)$ & $5.9 \%$ \\
\hline Asthma ${ }^{79}$ & $1(1 / 34)$ & $2.9 \%$ \\
\hline Cysts - Unspecified ${ }^{79}$ & $1(1 / 34)$ & $2.9 \%$ \\
\hline $\begin{array}{l}\text { Infection/fever/cold/congestion } \\
\text { symptoms }{ }^{78,79}\end{array}$ & $2(20 / 48)$ & $2.9 \%-28.6 \%$ \\
\hline \multicolumn{3}{|l|}{ Placebo } \\
\hline Appetite decrease ${ }^{78}$ & $1(3 / 15)$ & $20 \%$ \\
\hline Drooling/increased saliva $^{78}$ & $1(2 / 15)$ & $13.3 \%$ \\
\hline Skin changes $^{79}$ & $1(2 / 37)$ & $5.4 \%$ \\
\hline Cysts - Unspecified ${ }^{79}$ & $11(/ 37)$ & $2.7 \%$ \\
\hline Agitation/nervousness/restlessness ${ }^{78,79}$ & $1(3 / 15)$ & $6 \%-13 \%$ \\
\hline EPS/impaired movement ${ }^{78}$ & $1(3 / 15)$ & $6 \%-13 \%$ \\
\hline Gastrointestinal symptoms $^{78,79}$ & $2(17 / 52)$ & $6 \%-21 \%$ \\
\hline $\begin{array}{l}\text { Infection/fever/cold/congestion } \\
\text { symptoms }^{78,79}\end{array}$ & $2(21 / 52)$ & $2.7 \%-40 \%$ \\
\hline
\end{tabular}

EPS = extrapyramidal symptoms; $\mathrm{N}$ = number

\section{Studies of Tetrahydrobiopterin}

\section{Key Points}

- Two small RCTs reported inconsistent effects of tetrahydrobiopterin on symptom severity and few clinically significant harms.

- Tetrahydrobiopterin had no effect on symptom severity and was not associated with clinically significant harms. Our confidence in these conclusions is low (low strength of evidence). Data were inadequate to assess effects on other outcomes (insufficient strength of evidence).

\section{Overview of the Literature}

Two RCTs with low ${ }^{53}$ and moderate ${ }^{103}$ risk of bias compared tetrahydrobiopterin and placebo. The studies included 56 children between 3 and 7 years old and ranged from 16 to 24 weeks duration with followup immediately post-treatment. Studies were conducted in the United States and Sweden.

\section{Detailed Analysis}

In one 16-week RCT (low risk of bias) comparing tetrahydrobiopterin and including children with VABS developmental quotients of at least 50, children in the treatment arm had better language, adaptive behavior, and social interaction skills at baseline compared with placebo participants. ${ }^{53}$ At followup immediately post-treatment, scores on the primary outcome, the CGI, were not significantly different between groups. Secondary outcomes favored the treatment group: children in the treatment group improved significantly more on the ABC Irritability, Lethargy, Stereotypy, Hyperactive and Inappropriate Speech scales; Social Responsiveness Scale 
(SRS) total and subscale scores; and VABS composite and subscale measures than did children in the placebo group ( $\mathrm{p}$ values $<0.00$ ).

In another 24-week crossover RCT (moderate risk of bias) including 12 boys, overall CARS scores did not differ significantly between group. In a post-hoc analysis grouping CARS variables into those assessing social interaction, communication, and stereotypy, children in the treatment group had significant improvement in social interaction $(\mathrm{p}=0.04)$ but not in the other categories. $^{103}$ Table 21 outlines key outcomes.

Table 21. Key outcomes in studies of tetrahydrobiopterin

\begin{tabular}{|c|c|c|}
\hline $\begin{array}{l}\text { Author, Year Study Design } \\
\text { Groups (Dose), N Enrollment I N } \\
\text { Final } \\
\text { Treatment Duration/Follow-Up } \\
\text { Timepoint Post-Treatment } \\
\text { Risk of Bias }\end{array}$ & $\begin{array}{l}\text { Outcome Measure/Baseline } \\
\text { Scores, Mean } \pm \text { SD }\end{array}$ & $\begin{array}{l}\text { Outcome Measure/Post-Treatment } \\
\text { Scores, Mean } \pm \text { SD }\end{array}$ \\
\hline $\begin{array}{l}\text { Klaiman } 2013^{53} \text { RCT } \\
\text { G1: Tetrahydrobiopterin } \\
\text { (20mg/kg/day), 23/23 } \\
\text { G2: Placebo (NA), 23/23 }\end{array}$ & $\begin{array}{l}\text { CGI-Severity [N (\%)] } \\
\text { Frequency of markedly, severely, } \\
\text { or extremely ill } \\
\text { G1: } 10(48) \\
\text { G2: } 15(68)\end{array}$ & $\begin{array}{l}16 \text { wks follow-up } \\
\text { CGI-Severity [N (\%)] } \\
\text { Frequency of markedly, severely, or } \\
\text { extremely ill } \\
\text { G1: } 7(35) \\
\text { G2: } 14(64)\end{array}$ \\
\hline 16 weeks/EOT & $\begin{array}{l}\text { ABC-Irritability } \\
\text { G1: } 11.1 \pm 7.7\end{array}$ & G1 vs $G 2: p=n s$ \\
\hline Low RoB & $\begin{array}{l}\text { G2: } 11.9 \pm 7.8 \\
\text { ABC-Social withdrawal/lethargy } \\
\text { G1: } 9.5 \pm 7.5 \\
\text { G2: } 16.2 \pm 10.0 \\
\text { ABC-Stereotypy } \\
\text { G1: } 6.1 \pm 3.9 \\
\text { G2: } 6.1 \pm 3.6 \\
\text { ABC-Hyperactivity } \\
\text { G1: } 21.5 \pm 10.3 \\
\text { G2: } 22.9 \pm 11.6 \\
\text { ABC-Inappropriate speech } \\
\text { G1: } 3.7 \pm 2.4 \\
\text { G2: } 3.4 \pm 4.1 \\
\text { SRS } \\
\text { G1: } 81.4 \pm 10.3 \\
\text { G2: } 83.6 \pm 9.2 \\
\text { PLS } \\
\text { G1: } 77.8 \pm 29.2 \\
\text { G2: } 57.1 \pm 25.7 \\
\text { VABS } \\
\text { G1: } 320.5 \pm 47.9 \\
\text { G2: } 274.4 \pm 51.4\end{array}$ & $\begin{array}{l}\text { Improvement, frequency of very } \\
\text { much or much improved } \\
\text { G1: } 5 \text { (25) } \\
\text { G2: } 3(14) \\
\text { G1 vs G2: } p=n s \\
\text { ABC-Irritability } \\
\text { G1: } 10.0 \pm 7.8 \\
\text { G2: } 10.8 \pm 7.8 \\
\text { G1 vs G2: } p=n s \\
\text { ABC-Social withdrawal/lethargy } \\
\text { G1: } 5.2 \pm 4.4 \\
\text { G2: } 13.6 \pm 7.5 \\
\text { G1 vs G2: } p<0.01 \\
\text { ABC-Stereotypy } \\
\text { G1: } 5.4 \pm 3.8 \\
\text { G2: } 6.7 \pm 4.5 \\
\text { G1 vs } G 2: p=n s \\
\text { ABC-Hyperactivity } \\
\text { G1: } 18.2 \pm 8.5 \\
\text { G2: } 22.8 \pm 10.3 \\
\text { G1 vs G2: p=ns } \\
\text { ABC-Inappropriate speech } \\
\text { G1: } 2.6 \pm 1.9 \\
\text { G2: } 3.9 \pm 3.6 \\
\text { G1 vs G2: } p=n s\end{array}$ \\
\hline $\begin{array}{l}\text { Klaiman } 2013^{53} \text { RCT, continued } \\
\text { G1: Tetrahydrobiopterin } \\
\text { (20mg/kg/day), } 23 / 23 \\
\text { G2: Placebo (NA), } 23 / 23\end{array}$ & & $\begin{array}{l}\text { SRS } \\
\text { G1: } 76.7 \pm 10.9 \\
\text { G2: } 83.2 \pm 10.4 \\
\text { G1 vs } G 2: p=n s \\
\text { PLS }\end{array}$ \\
\hline
\end{tabular}




\begin{tabular}{|c|c|c|}
\hline $\begin{array}{l}\text { Author, Year Study Design } \\
\text { Groups (Dose), N Enrollment / N } \\
\text { Final } \\
\text { Treatment Duration/Follow-Up } \\
\text { Timepoint Post-Treatment } \\
\text { Risk of Bias }\end{array}$ & $\begin{array}{l}\text { Outcome Measure/Baseline } \\
\text { Scores, Mean } \pm \text { SD }\end{array}$ & $\begin{array}{l}\text { Outcome Measure/Post-Treatment } \\
\text { Scores, Mean } \pm \text { SD }\end{array}$ \\
\hline $\begin{array}{l}16 \text { weeks/EOT } \\
\text { Low RoB }\end{array}$ & & $\begin{array}{l}\text { G1: } 84.0 \pm 28.8 \\
\text { G2: } 60.4 \pm 25.4 \\
\text { G1 vs } G 2: p=0.01 \\
\text { VABS } \\
\text { G1: } 344.76 \pm 50.0 \\
\text { G2: } 294.9 \pm 70.1 \\
\text { G1 vs } G 2: p=0.02\end{array}$ \\
\hline $\begin{array}{l}\text { Danfors } 2005^{103} \text { RCT } \\
\text { G1: Tetrahydrobiopterin/Placebo } \\
\text { (3mg/kg), } 12 \\
\text { G2: Placebo/ Tetrahydrobiopterin } \\
\text { (ND), } 12 \\
12 \text { months/EOT } \\
\text { Moderate ROB }\end{array}$ & $\begin{array}{l}\text { CARS-Total Score } \\
\text { G1: } 35.4 \pm 2.6 \\
\text { G2: } 37.4 \pm 7.1 \\
\text { Social Interaction } \\
\text { G1: } 9 \pm 0.6 \\
\text { G2: } 10.8 \pm 1.6\end{array}$ & $\begin{array}{l}\text { Points Decreased } \\
\text { CARS-Total Score } \\
\text { G1: } 2.1 \pm 2.1 \\
\text { G2: } 2.1 \pm 4.3 \\
\text { G1 vs } \mathrm{G} 2, \mathrm{p}=\mathrm{ns} \\
\text { Social Interaction } \\
\text { G1: } 1.6 \pm 1.1 \\
\text { G2: } 0.3 \pm 1.4 \\
\text { G1 vs } \mathrm{G} 2, \mathrm{p}=0.04\end{array}$ \\
\hline
\end{tabular}

\section{Harms of Tetrahydrobiopterin}

Several children in both groups reported sleeping problems and behavioral issues, but investigators did not consider any harms to be clinically significant, and no child withdrew from either study due to adverse events. Table 22 outlines harms. 
Table 22. Harms/adverse effects reported in studies of tetrahydrobiopterin

\begin{tabular}{|c|c|c|}
\hline Harm/Adverse Event & $\begin{array}{l}\text { N Studies Reporting Harm (\# } \\
\text { Participants With Harm/Total } \\
\text { Participants) }\end{array}$ & $\begin{array}{c}\text { Reported Rates Across } \\
\text { Studies }\end{array}$ \\
\hline \multicolumn{3}{|l|}{ Tetrahydrobiopterin } \\
\hline Sleeping problems ${ }^{103}$ & $1(4 / 5)$ & $80 \%$ \\
\hline Agitation/nervousness/restlessness ${ }^{103}$ & $1(3 / 5)$ & $60 \%$ \\
\hline Challenging behavior ${ }^{53}$ & $1(4 / 20)$ & $22 \%$ \\
\hline Hyperactivity $^{53}$ & $1(2 / 20)$ & $9 \%$ \\
\hline Insomnia ${ }^{53}$ & $1(2 / 20)$ & $9 \%$ \\
\hline Skin changes ${ }^{53}$ & $1(2 / 20)$ & $9 \%$ \\
\hline \multicolumn{3}{|l|}{ Placebo } \\
\hline Sleeping problems ${ }^{103}$ & $1(5 / 6)$ & $83.3 \%$ \\
\hline Agitation/nervousness/restlessness ${ }^{103}$ & $1(3 / 6)$ & $50 \%$ \\
\hline Challenging behavior ${ }^{53}$ & $1(4 / 22)$ & $17 \%$ \\
\hline Insomnia ${ }^{53}$ & $1(4 / 22)$ & $17 \%$ \\
\hline Gl symptoms $^{53}$ & $1(4 / 22)$ & $17 \%$ \\
\hline Repetitive behavior or language ${ }^{53}$ & $12(/ 22)$ & $9 \%$ \\
\hline Seizure ${ }^{53}$ & $1(1 / 22)$ & $4.5 \%$ \\
\hline Hyperactivity $^{53}$ & $1(1 / 22)$ & $4 \%$ \\
\hline
\end{tabular}

$\mathrm{N}=$ number

\section{Studies of Other Medical Interventions}

\section{Key Points}

- Most agents or interventions were addressed in only one study.

- While most studies reported some positive treatment effects on sleep, ASD symptoms, or language, data were inadequate to assess any comparisons given the heterogeneity of interventions (insufficient strength of evidence).

\section{Overview of the Literature}

We categorized studies as "other" if we could not assess strength of evidence for interventions and outcomes reported (i.e., insufficient strength of evidence) and the studies did not fall under a broader category of intervention such as diet or nutritional supplements. Fourteen

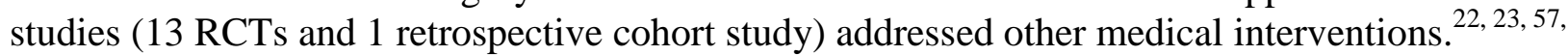
68-75, 77, 100, 108, 115, 116, 123 Most agents or interventions were addressed in only one study. Two studies evaluated donepezil. ${ }^{77,116}$ Agents or interventions addressed in single studies included melatonin, ${ }^{68}$ bumetanide, $^{72}$ transcranial stimulation (addressed in one multi-publication $\mathrm{RCT}^{69}$, ${ }^{70}$ ), amantadine, ${ }^{115}$ citalopram, ${ }^{22,23}$ divalproex, ${ }^{74}$ oxytocin, ${ }^{71}$ mecamylamine, ${ }^{73}$ memantine, ${ }^{75}$ prednisolone, ${ }^{123}$ and levetiracetam. ${ }^{108}$

Three studies were included in our prior review, ${ }^{23,115,116}$ including one RCT that now

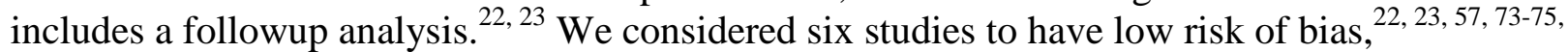

${ }^{108}$ seven to have moderate risk, ${ }^{68-72,77,115,116}$ and one to have high risk. ${ }^{123}$

Studies included a total of 829 children (median 39 total children/study) between the ages of 2 and 19 years receiving treatment for 4 days to 9 months. Two studies reported followup after the end of treatment (1-3 months post-treatment). ${ }^{71,72}$

\section{Detailed Analysis}

Despite the number of RCTs with low or moderate risk of bias, studies present little evidence to inform treatment decisions and can be considered primarily as pilot efficacy trials. Most 
studies were short-term and lacked followup past the end of treatment. Studies included few participants and few examined the same agents. Two studies addressed donepezil but examined different outcomes. Harms reported in studies comparing these interventions were diverse, and their clinical significance is difficult to determine given the short-term nature of the studies and the typically low numbers of participants. Gastrointestinal symptoms including constipation, diarrhea, and abdominal pain occurred frequently as did agitation, nervousness, or restlessness and appetite changes. We present a brief summary of key effectiveness outcomes in each study below; Appendix F includes detailed tables of outcomes and harms.

Donepezil. Two RCTs (moderate risk of bias) of donepezil assessed differing outcomes and reported no effects on executive function and treatment-associated improvements in language. In an RCT comparing donepezil and placebo, changes between groups on measures of executive function did not differ significantly after 10 weeks of treatment, though each group generally improved on each measure over time. ${ }^{77}$ In a 10 -week open label extension, participants generally improved slightly on most measures (differences between baseline and $5 \mathrm{mg}$ or $10 \mathrm{mg}$ doses not significant). Scores on verbal and nonverbal problem solving skills and flexibility of thinking worsened over time (baseline vs. 10 -mg dose, $\mathrm{p} \leq 0.004$ ). In another crossover RCT addressed in our prior review, ${ }^{11}$ children receiving donepezil versus placebo improved on clinician-rated measures of receptive and expressive language and symptom severity after 6 weeks of treatment (p values <0.05). ${ }^{116}$

Citalopram. One low risk of bias RCT (reported in multiple publications) addressed the serotonin reuptake inhibitor citalopram and reported no significant effects on repetitive behavior and some positive effects on challenging behaviors compared with placebo. ${ }^{22,23}$ The original study was included in our 2011 review and now includes a followup study examining potential predictors of response to citalopram (see KQ2). ${ }^{22,23}$ The study (low risk of bias) focused on repetitive behavior outcomes in children with PDD and significant repetitive behavior. Investigators reported no significant differences between citalopram and placebo arms on measures of repetitive behavior, with similar baseline scores on the CYBOCS and similar improvements in each arm. The other measures of repetitive behavior, including the Repetitive Behavior Scale-Revised, also had similar improvements in each arm with no evidence for an effect of citalopram. The CGI-Improvement scale similarly showed no significant difference between the citalopram and the placebo arm. On the other hand, the primary measure of challenging behavior reported in this trial, the ABC-Irritability subscale, showed an advantage for citalopram with more improvement in the citalopram arm than for placebo.

Memantine. One low risk of bias RCT included a 12-week, placebo-controlled phase to determine safety and tolerability of memantine and a 48 week extension (6 weeks dose titration, with placebo participants moving to memantine followed by 42 weeks of maintenance treatment). ${ }^{75}$ Both groups improved from baseline on caregiver-rated social skills (Social Responsiveness Scale) and on an unvalidated scale that measured symptom severity (Core Autism Treatment Scale-Improvement). Improvements were maintained over the extension period, but differences between groups at 12 weeks were not significant. Group differences on other measures of behavior and language (e.g., ABC, Children's Communication Checklist) were not statistically different between groups. 
Levetiracetam. One 10-week, low risk of bias RCT reported no significant group differences in parent- or teacher-rated ABC scores, overall symptoms (CGI), impulsivity/hyperactivity, or repetitive behavior, though repetitive behavior improved in both groups from baseline. ${ }^{108}$

Mecamylamine. One low risk of bias, 14-week RCT randomized children to either mecamylamine in ascending doses (up to a maximum $5 \mathrm{mg} /$ day) or placebo and reported no significant differences between groups on any of the outcome measures. ${ }^{73}$

Divalproex Sodium. In a 12-week RCT with low risk of bias including children with ASD and significant irritability or aggression, children receiving divalproex had greater improvements in the CGI-Irritability and ABC-Irritability scales ( $p$ values <0.05), but scores on measures of aggression and repetitive behavior (Overt Aggression Scale-Irritability, CYBOCS) did not differ between groups. ${ }^{74}$ In exploratory analyses changes in adaptive behavior or manic symptoms also did not differ between groups.

Melatonin. A crossover RCT with moderate risk of bias compared melatonin or placebo for 3 months followed by a 1-month washout and 3 months of either melatonin or placebo. ${ }^{68}$ Sleep latency and total sleep were significantly reduced in the melatonin group compared with placebo ( $\mathrm{p} \leq .004)$, but night wakings did not differ between groups. Similarly, scores on the dysomnia scale of the Sleep Difficulties Questionnaire, but not the other sub-scales, were significantly lower for the treatment group compared with placebo $(\mathrm{p}=.041)$. Scores on the Developmental Behavior Checklist were significantly different between groups (mean difference $=6.0, p=0.05$ ), with lower scores (improved behavior) in the melatonin group.

Bumetanide. One RCT with moderate risk of bias compared bumetanide or placebo for 3 months followed by repeat evaluations 1 month after the end of treatment. ${ }^{72}$ After the 3 month treatment period, CARS scores for participants in the treatment group declined from the severe range to medium or mild severity. At the 120 day followup, scores had shifted back toward pretreatment values in both groups ( $\mathrm{p}=\mathrm{ns})$. CGI scores were significantly improved in the treatment group compared with placebo at 120 days $(p=0.02)$, but ADOS total scores did not differ between groups. In analyses removing children with the most severe symptoms, ADOS scores improved significantly in the treatment group $(\mathrm{p}=0.03)$.

Amantadine. One moderate risk of bias RCT of amantadine reported no significant effect of daily amantadine over 4 weeks on parent-rated ABC behavior scores and clinician-rated CGI rating of overall improvement compared with placebo. ${ }^{115}$ However, children in the amantadine arm improved significantly more than those receiving placebo in clinician-rated ABC Hyperactivity and Inappropriate Speech subscales.

Oxytocin. One RCT with moderate risk of bias including 38 boys with IQs of at least 80 and comorbid ADHD, oppositional defiant disorder, or anxiety compared four doses of nasal oxytocin or placebo over 5 days. ${ }^{71}$ Children also received an emotion recognition training program and completed family interaction tasks both before and after oxytocin administration. No outcomes (social interaction, repetitive behavior, emotion recognition, ASD severity) differed between groups on blinded investigator-, parent-, and observation coder-ratings. 
Prednisolone. In one retrospective study (high risk of bias) including children considered to have "regressive" autism (defined as clinically determined loss of age-appropriate language, communication, cognitive abilities and behavior), children who received prednisolone (mean treatment duration of $9.13 \pm 3.26$ months, range $=4-14$ months) improved significantly more on a parent- and clinician-rated measure of receptive and expressive language developed for the study ${ }^{123}$ In followup of treated participants approximately 12 months after the end of treatment, participants with improved language $(n=17)$ maintained or increased their improvements; three nonresponders continued to have no change in language in parent reports.

Transcranial Stimulation. One RCT with moderate risk of bias assessed transcranial direct current stimulation using electrodes attached to the scalp to provide positive and negative electrical currents to putatively affect activity in regions of the brain that may play a role in ASD

symptoms. ${ }^{69,70}$ Investigators allocated study participants (all males) with mild to moderate ASD to either active stimulation (2 sessions of roughly 20 minutes) or sham simulation. At 7 days post-treatment, mean clinician-rated CARS scores and parent-rated ATEC total, social, sensory, and health and behavioral problem (but not language) scores were significantly improved in the treatment group compared with placebo ( $\mathrm{p}$ values $<0.05$ ) Scores on the clinician-rated CGAS were also more improved in the treatment group $(p<0.05)$ but CGI-Severity scores did not differ between groups. Investigators rated 45 percent of children receiving active treatment and 15 percent receiving sham treatment as "much improved" $(\mathrm{p}<0.05)$ and 10 percent in each arm as "much worse" ( $\mathrm{p}=\mathrm{NS})$.

\section{KQ2. Modifiers of Treatment Outcomes}

Understanding the degree to which child characteristics (i.e., age, specific ASD-related difficulties and skills), treatment factors (e.g., type, duration, intensity), and systems (e.g., family, community) influence response to treatments could improve targeting of treatments to the appropriate children and circumstances. While we sought modifying effects of child, provider, or intervention characteristics, few studies reported modifiers, and few were likely adequately powered to detect effects. We report modifying variables addressed in studies meeting our criteria as an indication of potential characteristics that may affect findings.

Antipsychotics. A sub-analysis of an 8-week RCT of risperidone vs. placebo ${ }^{34}$ analyzed mediators and moderators of the decrease in irritability. ${ }^{29}$ Baseline ABC-Irritability subscale score severity was the only significant moderator found. High severity was associated with greater improvement in irritability than was low severity in improvement with risperidone. Weight gain was the only significant mediator of response to risperidone. Greater weight gain was associated with less irritability improvement in the risperidone group. In an analysis of dose and compliance, better compliance was found to be associated with more improvement in the risperidone group and greater dose was associated with greater improvement.

In another post-hoc analysis of data from an extension of the 8-week trial of risperidone, younger age and better communication skills were associated with greater gains in communication but not with gains in daily living skills or socialization as measured on the VABS ${ }^{37}$ No child characteristics were associated with gains in adaptive behavior and gains in each domain of adaptive behavior (e.g., communication, socialization) appeared to contribute equally to gains in the overall adaptive behavior score. Reductions in aggression were also not associated with the magnitude of gains in adaptive behavior. 
An additional analysis of this study analyzed the effect of initial severity of ASD on the efficacy of risperidone. ${ }^{110}$ This study found that parent-, but not clinician-, efficacy of risperidone on irritability and lethargy was greater with higher baseline measurements of severity (moderate through severity) of challenging behaviors. Effects of baseline severity on clinicianrated $\mathrm{ABC}$ or CGI scores or parent ratings of other ABC scales (stereotypy, hyperactivity, inappropriate speech) were not statistically significant.

In secondary analyses of one RCT comparing aripiprazole and placebo, Caucasian children receiving aripiprazole had a relapse rate of 25.8 percent compared with 60.7 percent in the placebo group (HR=0.33, 95\% CI: 0.14 to 0.78 , $\mathrm{p}=0.01$ ). Among non-White patients, the difference was not statistically significant. Age also did not interact significantly with relapse. ${ }^{48}$ Finally, in one retrospective cohort study primarily assessing BMI change in children taking either risperidone or aripiprazole, investigators found no variables (baseline BMI or age, race, gender, intellectual disability, concomitant drug use, treatment duration) to be significant covariates of BMI Z-score change per year of treatment. ${ }^{122}$

ADHD Medications. In a double-blind cross-over trial of methylphenidate in 66 children, ${ }^{24-27}$ authors found no effect of age, IQ, weight, or diagnosis on teacher- or parent-rated hyperactivity subscale scores, Swanson Nolan and Pelham rating scale (SNAP-IV), or CYBOCS scores. Children with Asperger syndrome/PDD-NOS $(n=19)$ showed a trend of being more likely to be classified as responders to both placebo and methylphenidate than those with autism. Response to each dose of methylphenidate was significantly superior to placebo in the autism subgroup but not for the Asperger / PDD-NOS subgroup. In a later analysis assessing gene variants potentially associated with response, variations in seven genes (SLC6A4, SLC6A3, DRD1, DRD3, DRD4, $C O M T$ and $A D R A 2 A$ ) that influence monoaminergic signaling were significant predictors, though the study was not powered to correct for multiple comparisons. ${ }^{24}$ In another RCT comparing guanfacine and placebo, cognitive skills were not significantly associated with treatment effects. ${ }^{46}$

Other Agents. Studies of other agents reported various potential modifiers of effects: Response to placebo (but not citalopram) was predicted by severity of disruptive behaviors, particularly hyperactivity, ASD severity and mood, and caregiver strain ( $p$ values $\leq .012$ ) in one study. ${ }^{22,23}$ Children with higher baseline scores on these measures exhibited less response to placebo. In a trial of atomoxetine, scores on tests of inhibition control (go-no go task) and degree of distractibility by irrelevant information (focused-attention task) were not significantly correlated and did not correlate with changes in measures of ADHD symptoms. ${ }^{41-44}$

In a trial of divalproex, analyses suggested that children with abnormal epileptiform electroencephalogram (EEG) results were more likely to respond to divalproex than those with normal EEGs. ${ }^{74}$ In analyses of treated children in this trial $(n=16)$, children with higher blood levels of valproate (87-100 mcg/ml) had a better response rate, and higher dose was associated with a moderate effect on improvement scores (p values=NR).

Higher IQ was significantly correlated with improvements in social interaction in a post-hoc analysis in a crossover RCT comparing tetrahydrobiopterin and placebo. ${ }^{103}$ Age or total CARS scores were not significantly associated with improvements. In a trial of buspirone, children with fewer brain abnormalities in positron emission tomography scans had significantly more behavioral improvement than those with fewer, as children with normal (not elevated) blood serotonin levels ( values $\leq 0.04$ ); the serotonin association was not significant in multiple regression analysis. ${ }^{76}$ In one RCT comparing hyperbaric oxygen and sham treatment, older age 
(> 5 years) and lower baseline symptom severity as measured on the ADOS were associated with better outcomes. $^{114}$

An RCT comparing active and sham transcranial stimulation reported that increased left frontal lobe activity as indicated by increases in peak alpha frequencies was associated with improvements in ATEC measures of social problems and health and behavior problems. ${ }^{69,70} \mathrm{Few}$ of the included trials of diet or supplement interventions: one trial of gluten-free diet compared with a regular diet reported no significant correlations between baseline behavioral or gastrointestinal measures and outcomes. ${ }^{62}$ In a trial of methyl-B12, improvement was positively correlated with increases in plasma levels of methionine an decreases in S-adenosylmethionine, and the ratio of these two levels ( $p$ values $\leq 0.05$ ). ${ }^{87}$

No studies of risperidone adjuncts reported modifiers of effectiveness.

\section{KQ3. Time to Effect of Interventions}

Information about early response to treatment, or lack thereof, could guide treatment selection, implementation, and modification; however, no studies reported data to assess time to effect of interventions. While several studies reported changes in the number of children responding to a given agent over time, studies did not provide data to determine the initiation of effects. One study of aripiprazole noted that clinically important improvements were seen within 8 weeks of treatment, but treatment was not associated with delayed relapse (return of significant symptoms by 16 weeks of treatment/placebo) compared with placebo. ${ }^{48}$ In one study comparing risperidone and aripiprazole, improvement with aripiprazole was evident in the first 12 weeks of treatment without additional improvements at 24 weeks, while scores on measures in children taking risperidone improved at 12 weeks and continued to improve over the 24 week period. ${ }^{50}$ The study does not indicate, however, when effects began to be observed. Another study assessing transcranial stimulation reported changes in peak alpha frequency immediately posttreatment in children receiving active versus sham stimulation (significant change from baseline in active treatment group and significant between group differences at some electrode sites), but the clinical effects of such changes are not clear. ${ }^{69,70}$

\section{KQ4. Evidence that Effects Measured at the End of Treatment Predict Long-Term Functional Outcomes}

Few studies provided data to address this KQ. Few studies had longer-term followup and those with more than 6 months of treatment or followup typically did not report functional outcomes. In one study, risperidone use was not associated with changes in IQ. ${ }^{28}$ Changes from baseline to the end of study in class assignment (e.g., special education, regular classroom) were not significant.

\section{KQ5. Effectiveness Across Environments or Contexts}

Seven studies reported teacher ratings of outcome measures that provide some information to address this KQ, but the limited results preclude conclusions. One RCT of omega-3 fatty acids reported no significant group differences in teacher ratings of challenging behaviors (parents also rated few measures as improved), ${ }^{80}$ while another RCT of DHA supplementation versus placebo reported improvement in parent-rated social skills in children receiving placebo vs. those receiving DHA, while teachers rated communication as more improved in the treatment group compared with placebo. ${ }^{82}$ An RCT of gluten- or casein- containing challenge foods introduced to 
a gluten-free diet reported no statistically significant changes in behavior as rated by parents or teachers on the Connors scale. ${ }^{60}$ An RCT of levetiracetam vs. placebo reported no significant group differences on any parent- or teacher- rated measures but also noted that teachers, but not parents, rated children in the placebo arm as more improved on irritability compared with the levetiracetam group $(\mathrm{p}=0.003){ }^{108}$

RCTs of methylphenidate reported general agreement between parent and teacher ratings of hyperactivity. ${ }^{24-27,45}$ In one RCT, both parents and teachers considered hyperactivity and impulsive behavior to be significantly improved in the treatment group compared with placebo, but teachers (vs. parents) reported no significant group differences in inattention or oppositional behavior. Finally, one RCT of atomoxetine reported significant teacher-rated improvements in hyperactivity in the atomoxetine group compared with placebo, but teacher ratings of cognitive problems/inattention, oppositional behavior, or overall ADHD symptoms did not differ between groups. ${ }^{41-44}$ In another RCT comparing atomoxetine alone, atomoxetine + parent training , placebo alone, and placebo + parent training, parents, but not teachers, rated children in active treatment groups as significantly improved on measures of ADHD, inattention, hyperactivity, and oppositional behavior. ${ }^{54,55}$

\section{KQ6. Drivers of Treatment Outcomes}

We did not identify studies that provided data to address this KQ. 


\section{Discussion}

\section{State of the Literature}

We identified a total of 76 unique comparative studies, primarily $(n=72)$ randomized controlled trials (RCTs), addressing medical interventions. Most studies were small (median 40 total participants/study) and addressed variable agents. Most studies had placebo comparators, while five (reported in multiple publications) compared a pharmaceutical agent to behavioral treatment or combined pharmaceutical and behavioral treatment. ${ }^{54-57,120,121}$ Treatment length varied from four days to 24 months, with few studies $(n=3)$ reporting longer term followup after the immediate intervention period. ${ }^{71,72,120}$

The methodologic rigor of studies increased substantially over those studies reported in our 2011 review of therapies for children with autism spectrum disorder (ASD). ${ }^{11}$ Thirty-three studies in the current review have low risk of bias and 20 have moderate risk. While studies were generally well-conducted, evidence remains insufficient for most interventions given small sample sizes, lack of longer term followup, and heterogeneous agents and populations.

While most studies targeted challenging behaviors, only four (reported in multiple publications) explicitly included children with diagnosed comorbidities such as attention deficit hyperactivity disorder (ADHD). ${ }^{40-44,71,80}$ Twenty-eight studies used variable criteria to define challenging behaviors, including specific cut-off scores on subscales of the Aberrant Behavior Checklist (ABC); parent-reported irritability; clinician observations of irritability or hyperactivity; or the presence of undefined "severe" behavioral symptoms. Other studies reported no specific indications.

Despite the limitations of the literature, some interventions have high strength of evidence. Specifically, the strength of evidence for the antipsychotics risperidone and aripiprazole is high for the amelioration of irritability in the short term ( $\leq 6$ months of treatment) for children with significant challenging behaviors at baseline. However, the strength of evidence is also high for significant side effects (e.g., extrapyramidal symptoms, weight gain). Longer term effectiveness is not as well studied, but uncontrolled open-label analyses have suggested some continued efficacy. In studies (reported in multiple publications), children receiving the psychostimulant methylphenidate had improvements in hyperactivity, but small sample sizes preclude firm conclusions about durability of effects. ${ }^{24-27,45}$ Two studies (in multiple publications) of atomoxetine also reported positive effects on hyperactivity, potentially with fewer adverse effects than methylphenidate. ${ }^{40-44}$ Other studies of agents such as adjuncts to risperidone reported some positive effects but studies were small, often underpowered, and typically not replicated. Studies of nutritional supplements or specialized diets reported few positive effects as did studies of hyperbaric oxygen.

Despite the number of new studies, we can make few conclusions beyond those reached in our 2011 review. Evidence supports the effectiveness of antipsychotics in improving challenging behaviors, but with significant harms. Methylphenidate also improves hyperactivity but with significant harms. Evidence is promising for the ADHD medication atomoxetine. More studies have addressed combination approaches, but data are inadequate to draw conclusions. Data were limited and inconsistent for other interventions. 


\section{Summary of Key Findings and Strength of the Evidence}

\section{Key Question (KQ) 1. Benefits and Harms of Medical Treatments}

\section{Studies of Antipsychotics}

\section{Key Findings}

Studies of antipsychotics addressed either risperidone or aripiprazole and reported significant improvements in measures of challenging behavior in the short term ( $<6$ months) in children receiving the medications compared with those receiving placebo. Harms of these agents, including extrapyramidal symptoms and weight gain, were also significant. Studies reporting longer term followup (up to 21 months for risperidone) suggest continued potential efficacy in many children but did not include control groups that would permit stronger conclusions. Studies comparing risperidone and aripiprazole reported few differences in effects on outcomes or harms.

\section{Strength of the Evidence}

Our confidence in the conclusion that risperidone and aripiprazole improve challenging behaviors in the short term ( $<6$ months), with clinically significant harms. is high (high strength of evidence). Behaviors improved in the longer term ( $\geq 6$ months) with these agents compared with placebo, but our confidence in this conclusion is low (low strength of evidence) as few studies had longer-term followup.

In studies comparing risperidone and aripiprazole, BMI increased with both drugs over treatment durations of 6 months to more than 2 years, but group differences were not significant. We have low confidence in this conclusion given the few studies addressing this outcome (low strength of evidence). Other outcomes (e.g., challenging behaviors, attention) were not consistently addressed; thus we considered strength of evidence insufficient for all other intervention/outcome pairs. Table 23 outlines these findings. 
Table 23. Strength of evidence for effectiveness of antipsychotics versus placebo

\begin{tabular}{|c|c|c|c|c|c|c|}
\hline $\begin{array}{l}\text { Intervention/ } \\
\text { Outcome } \\
\text { Study Design } \\
\text { Risk of Bias and } \\
\text { Number of } \\
\text { Studies (N } \\
\text { Total) }\end{array}$ & 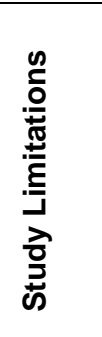 & 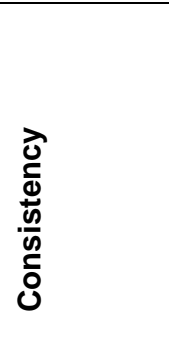 & 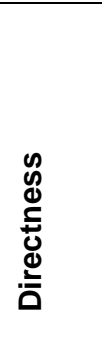 & $\frac{\check{0}}{\frac{0}{0}}$ & 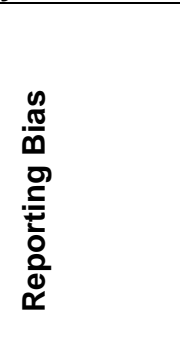 & $\begin{array}{l}\text { Finding } \\
\text { Strength of Evidence Grade }\end{array}$ \\
\hline \multicolumn{7}{|l|}{$\begin{array}{l}\text { Risperidone vs. } \\
\text { placebo }\end{array}$} \\
\hline $\begin{array}{l}\text { Challenging } \\
\text { behavior ( } \leq 6 \\
\text { months) } \\
\text { RCT: } 2 \text { low, }{ }^{34,51,} \\
52 \\
1 \text { moderate }{ }^{112,113} \\
(\mathrm{~N}=274)\end{array}$ & Low & Consistent & Direct & Imprecise & Undetected & $\begin{array}{l}\text { High SOE for short-term } \\
\text { effectiveness of risperidone in } \\
\text { improving challenging } \\
\text { behavior compared with } \\
\text { placebo } \\
\text { Significant improvement in } \\
\text { treatment group vs. placebo in } 3 \\
\text { RCTs with } 6-8 \text { week treatment } \\
\text { phases; improvement } \\
\text { maintained in } 2 \text { RCTs with } 6 \\
\text { months of treatment }\end{array}$ \\
\hline $\begin{array}{l}\text { Challenging } \\
\text { behavior ( }>6 \\
\text { months) } \\
\text { RCT: } 2 \text { low, }{ }^{28,102} \\
1 \text { moderate } \\
(\mathrm{N}=118)\end{array}$ & Low & Consistent & Direct & Imprecise & Undetected & $\begin{array}{l}\text { Low SOE for effectiveness in } \\
\text { the longer term } \\
\text { Improvement maintained in } 1 \\
\text { RCT with } 6 \text { months of treatment } \\
\text { and in one open label extension } \\
\text { with no comparison group with } \\
\text { mean } 21 \text { months treatment } \\
\text { duration; in another open label } \\
\text { extension, more children } \\
\text { relapsed with placebo vs. } \\
\text { risperidone }\end{array}$ \\
\hline $\begin{array}{l}\text { Harms } \\
\text { RCT: } 5 \text { low, }{ }^{34,49} \\
51,52,102,130 \\
4 \text { moderate }^{47,101,} \\
109,112,113 \\
\text { Retrospecitve } \\
\text { cohort: } 1 \\
\text { moderate } \\
(\mathrm{N}=334)\end{array}$ & Low & Consistent & $\begin{array}{l}\text { Dir } \\
\text { ect }\end{array}$ & $\begin{array}{l}\text { Impreci } \\
\text { se }\end{array}$ & $\begin{array}{l}\text { Undetec } \\
\text { ted }\end{array}$ & $\begin{array}{l}\text { High SOE for clinically } \\
\text { significant harms associated } \\
\text { with risperidone } \\
\text { Harms including weight gain, } \\
\text { appetite changes, drowsiness, } \\
\text { fatigue, extrapyramidal } \\
\text { symptoms, } \\
\text { drooling/hypersalivation, and } \\
\text { gastrointestinal symptoms } \\
\text { consistently reported }\end{array}$ \\
\hline \multicolumn{7}{|l|}{$\begin{array}{l}\text { Aripiprazole vs. } \\
\text { Placebo }\end{array}$} \\
\hline $\begin{array}{l}\text { Challenging } \\
\text { behavior ( } \leq 6 \\
\text { months) } \\
\text { RCT: } 2 \text { low }^{20,21} \\
(\mathrm{~N}=316)\end{array}$ & Low & Consistent & Direct & Precise & Undetected & $\begin{array}{l}\text { High SOE for short-term } \\
\text { effectiveness of aripiprazole in } \\
\text { improving challenging } \\
\text { behavior compared with } \\
\text { placebo } \\
\text { Significant improvements in } 2 \\
\text { short-term RCTs in treatment } \\
\text { groups vs. placebo }\end{array}$ \\
\hline
\end{tabular}




\begin{tabular}{|c|c|c|c|c|c|c|}
\hline $\begin{array}{l}\text { Intervention/ } \\
\text { Outcome } \\
\text { Study Design } \\
\text { Risk of Bias and } \\
\text { Number of } \\
\text { Studies (N } \\
\text { Total) }\end{array}$ & 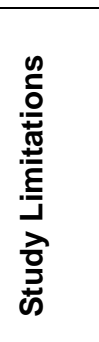 & 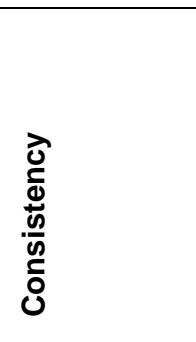 & 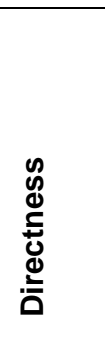 & $\begin{array}{l}\frac{\sigma}{0} \\
\frac{\mathscr{N}}{\mathrm{d}} \\
\frac{\mathrm{d}}{\alpha}\end{array}$ & 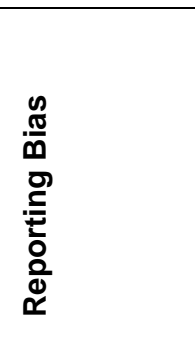 & $\begin{array}{l}\text { Finding } \\
\text { Strength of Evidence Grade }\end{array}$ \\
\hline $\begin{array}{l}\text { Challenging } \\
\text { behavior ( } \geq 6 \\
\text { months) } \\
\text { RCT: } 2 \text { low }^{15,20,21,} \\
(\mathrm{~N}=415)\end{array}$ & Low & Inconsistent & Direct & Precise & Undetected & $\begin{array}{l}\text { Low SOE for longer term } \\
\text { effectiveness in improving } \\
\text { challenging behaviors } \\
\text { In longer term followup, no } \\
\text { differences in time to relapse of } \\
\text { symptoms between aripiprazole } \\
\text { and placebo groups in one } 16 \\
\text { week RCT and continued } \\
\text { improvements in ABC in one 52- } \\
\text { week open label continuation } \\
\text { with no control arm }\end{array}$ \\
\hline $\begin{array}{l}\text { Harms } \\
\text { RCT: } 4 \text { low, }{ }^{18,20,}, 48-50 \\
\text { Retrospective } \\
\text { cohort: } 1 \\
\text { moderate } \\
(\mathrm{N}=492)\end{array}$ & Low & Consistent & Direct & Precise & Undetected & $\begin{array}{l}\text { High SOE for clinically } \\
\text { significant harms associated } \\
\text { with aripiprazole } \\
\text { Harms including weight gain, } \\
\text { appetite changes, somnolence, } \\
\text { extrapyramidal symptoms, } \\
\text { drooling/hypersalivation, } \\
\text { infection, and gastrointestinal } \\
\text { symptoms consistently reported }\end{array}$ \\
\hline \multicolumn{7}{|l|}{$\begin{array}{l}\text { Risperidone vs. } \\
\text { Aripirazole }\end{array}$} \\
\hline $\begin{array}{l}\text { BMI } \\
\text { RCT: } 1 \text { low }^{50} \\
(\mathrm{~N}=37) \\
\text { Retrospective } \\
\text { cohort: } 1 \\
\text { moderate } \\
(\mathrm{N}=142)\end{array}$ & $\begin{array}{l}\text { Me- } \\
\text { dium }\end{array}$ & Consistent & Direct & Imprecise & Undetected & $\begin{array}{l}\text { Low SOE for no difference in } \\
\text { effects on BMI } \\
\text { BMI increased with both drugs } \\
\text { over treatment durations of } 6 \\
\text { months to more than } 2 \text { years, but } \\
\text { group differences were not } \\
\text { significant }\end{array}$ \\
\hline
\end{tabular}

$\mathrm{N}$ = number; RCT = randomized controlled trial; SOE = strength of evidence

\section{Studies of ADHD Medications}

\section{Key Findings}

Studies of methylphenidate , atomoxetine, and guanfacine reported improvements in hyperactivity and other challenging behaviors with treatment compared with placebo. Both RCTs addressing methylphenidate reported statistically significant improvements in hyperactivity in children treated with medium to high doses compared with placebo. ${ }^{24-27,45}$ Findings for oppositional behavior and social communication were inconsistent.

RCTs addressing atomoxetine reported statistically significant treatment-related improvements compared with placebo that were maintained over 20 weeks of open label, uncontrolled treatment in one study; inattention was statistically significantly improved in one study. ${ }^{40-44}$ One small RCT of guanfacine reported improvements in hyperactivity, impulsiveness, 
and attention. ${ }^{46}$ Side effects were associated with all agents including aggressive behavior, gastrointestinal symptoms, irritability, and appetite changes.

\section{Strength of the Evidence}

Methylphenidate. Methylphenidate vs. placebo improved hyperactivity and was associated with clinically significant harms (Table 24). Our confidence in these conclusions is low as studies were small and short term (low strength of evidence). Data were inadequate to assess effects on social communication and oppositional behavior (insufficient strength of evidence). Findings for oppositional behavior were inconsistent in two studies; ${ }^{25,45}$ thus, we could not assess the strength of evidence (insufficient). We considered the evidence inadequate to comment on potential effects on social communication or oppositional behavior (insufficient strength of evidence).

Atomoxetine. We found positive effects of atomoxetine compared with placebo on hyperactivity in children with ASD and ADHD in the short term ( $<6$ months), with effects maintained over the longer term ( $\geq 6$ months) (Table 24). Our confidence in this conclusion is low (low strength of evidence). Atomoxetine was associated with harms considered to be clinically moderate, and our confidence in this conclusion is low (low strength of evidence). Data were inadequate to assess effects on inattention as studies reported inconsistent findings (insufficient strength of evidence).

Guanfacine. Data were inadequate in this small study to draw conclusions about effects on any outcomes (insufficient strength of evidence).

Table 24. Strength of evidence for effects of medications to treat ADHD

\begin{tabular}{|c|c|c|c|c|c|c|}
\hline $\begin{array}{l}\text { Intervention/ } \\
\text { Outcome } \\
\text { Study Design } \\
\text { Risk of Bias and } \\
\text { Number of } \\
\text { Studies (N } \\
\text { Total) }\end{array}$ & 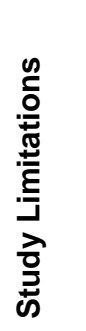 & $\begin{array}{l}\text { Jे } \\
\frac{c}{0} \\
\frac{n}{0} \\
\frac{n}{n} \\
0 \\
0\end{array}$ & 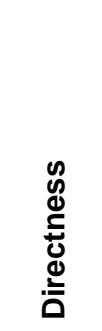 & $\begin{array}{l}\frac{0}{0} \\
\frac{0}{0} \\
\frac{d}{2}\end{array}$ & 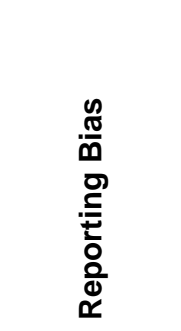 & $\begin{array}{l}\text { Finding } \\
\text { Strength of Evidence Grade }\end{array}$ \\
\hline \multicolumn{7}{|l|}{$\begin{array}{l}\text { Methyl- } \\
\text { phenidate vs. } \\
\text { Placebo }\end{array}$} \\
\hline $\begin{array}{l}\text { Hyperactivity } \\
\text { RCT: } 2 \text { low }^{24-27,45} \\
(\mathrm{~N}=90)\end{array}$ & $\begin{array}{l}\text { Me- } \\
\text { dium }\end{array}$ & Consistent & Direct & Imprecise & Undetected & $\begin{array}{l}\text { Low SOE for short-term (<6 } \\
\text { months) improvements in } \\
\text { hyperactivity with } \\
\text { methylphenidate compared } \\
\text { with placebo } \\
\text { Significant improvement with } \\
\text { MPH compared with placebo on } \\
\text { parent and teacher-rated } \\
\text { measures; differential effect of } \\
\text { dose not clear (little effect on } 1 \\
\text { study and linear effect in } \\
\text { another); SOE is low given small } \\
\text { sample size and lack of long- } \\
\text { term followup }\end{array}$ \\
\hline
\end{tabular}




\begin{tabular}{|c|c|c|c|c|c|c|}
\hline $\begin{array}{l}\text { Harms } \\
\text { RCT: } 2 \text { low } 24-27,45 \\
(\mathrm{~N}=90)\end{array}$ & $\begin{array}{l}\text { Me- } \\
\text { dium }\end{array}$ & Consistent & Direct & Imprecise & Undetected & $\begin{array}{l}\text { Low SOE for association of } \\
\text { methylphenidate with } \\
\text { clinically significant harms } \\
\text { Rates of children experiencing } \\
\text { harms ranged from } 0-75 \% \text {; } \\
\text { higher rates reported for } \\
\text { repetitive behaviors or speech, } \\
\text { loss of appetite, and irritability. } \\
\text { Irritability responsible for } \\
\text { withdrawals (n=6) in one RCT; } \\
\text { SOE is low given small sample } \\
\text { size }\end{array}$ \\
\hline \multicolumn{7}{|l|}{$\begin{array}{l}\text { Atomoxetine vs. } \\
\text { Placebo }\end{array}$} \\
\hline $\begin{array}{l}\text { Hyperactivity } \\
\text { RCT: } 2 \text { low, } 1 \\
\text { moderate } \\
(\mathrm{N}=163)\end{array}$ & $\begin{array}{l}\text { Me- } \\
\text { dium }\end{array}$ & Consistent & Direct & Imprecise & Undetected & $\begin{array}{l}\text { Low SOE for improvements in } \\
\text { hyperactivity in the short-term } \\
<6 \text { months) with atomoxetine } \\
\text { vs. placebo } \\
\text { Significant improvements in } \\
\text { rating of hyperactivity in } \\
\text { treatment group compared with } \\
\text { placebo in both studies }\end{array}$ \\
\hline $\begin{array}{l}\text { Hyperactivity } \\
\text { RCT: } 2 \text { low } 42-44 \text {, } \\
54,55,131 \text { (N=106) }\end{array}$ & $\begin{array}{l}\text { Me- } \\
\text { dium }\end{array}$ & Consistent & Direct & Imprecise & Undetected & $\begin{array}{l}\text { Low SOE for improvements in } \\
\text { hyperactivity in the longer- } \\
\text { term } \geq 6 \text { months) with } \\
\text { atomoxetine vs. placebo } \\
\text { Improvements in hyperactivity } \\
\text { maintained in open label } \\
\text { extensions }\end{array}$ \\
\hline $\begin{array}{l}\text { Harms } \\
\text { RCT: } 2 \text { low, } 1 \\
\text { moderate } \\
54,55,131(\mathrm{~N}=241)\end{array}$ & $\begin{array}{l}\text { Me- } \\
\text { dium }\end{array}$ & Consistent & Direct & Imprecise & Undetected & $\begin{array}{l}\text { Low SOE for clinically } \\
\text { moderate harms associated } \\
\text { with atomoxetine } \\
\text { No serious adverse events } \\
\text { reported; most harms attenuated } \\
\text { over open label extension phase }\end{array}$ \\
\hline
\end{tabular}

ADHD = attention deficit hyperactivity disorder; $\mathrm{MPH}$ = methylphenidate; $\mathrm{N}$ = number; $\mathrm{RCT}$ = randomized controlled trial; SOE

$=$ strength of evidence

\section{Studies of Combined Medical and Behavioral Treatments}

\section{Key Findings}

Three RCTs ( 2 low and 1 high risk of bias) and two nonrandomized trials (1 moderate and 1 high risk of bias) addressed different medical agents in combination with behavioral approaches. Atomoxetine plus parent training or atomoxetine alone were both associated with improvements in ADHD, inattention, hyperactivity, noncompliance, and overall symptom severity compared with placebo, with improvements maintained over 24 weeks for most treatment responders in one RCT. ${ }^{55,132}$ Differences between atomoxetine groups were not statistically significant for any outcome. Melatonin and melatonin plus cognitive behavioral therapy (CBT) both improved sleep-related outcomes in a second RCT. ${ }^{57}$

Folic acid plus Treatment and Education of Autistic and Related Communication Handicapped Children (TEACCH) treatment and folic acid alone both improved behaviors, with 
no significant group differences in one nonrandomized trial. ${ }^{121}$ In another RCT, bumetanide plus applied behavior analysis improved symptom severity and behavior more than applied behavior analysis alone. ${ }^{56}$ In a final nonrandomized trial, stem cell transplantation plus rehabilitation therapy improved symptom severity, lethargy, and stereotypy more than umbilical cord blood cell transplant plus rehabilitation therapy or rehabilitation therapy alone. ${ }^{120}$

\section{Strength of the Evidence}

Given that combination therapies were investigated in single studies, we could not make conclusions about their effects on any outcomes (insufficient strength of evidence).

\section{Studies of Nutritional Supplements and Dietary Interventions}

\section{Key Findings}

Three RCTs comparing omega-3 fatty acid supplementation with placebo reported no significant group differences in measures of challenging behavior; these studies did not consistently assess language and adaptive behavior outcomes, and no study reported clinically significant harms. While two RCTs addressed methyl B12 supplementation, results were inconsistent, with one study reporting positive effects on symptom severity ${ }^{87}$ and another reporting no significant effects. ${ }^{83}$

Four RCTs (multiple publications) compared GFCF diets to either an unaltered diet or a diet that contained gluten and dairy and reported few differences in behavioral measures between children on restricted or unrestricted diets. ${ }^{63-65,104-107}$ One RCT comparing a gluten-free diet and regular diet reported significant improvements in gastrointestinal symptoms and behavior in the gluten-free group, ${ }^{62}$ while another comparing a gluten and dairy-free diet to usual diet reported no significant effects. ${ }^{58}$ Two RCTs evaluating "challenges" of gluten- or casein-containing foods reported no significant group differences in measures of challenging behavior, ${ }^{59,60}$ while a high risk of bias study evaluating antioxidant-rich camel's milk reported no significant differences in ASD severity between children receiving boiled or raw camel's milk or cow's milk. ${ }^{61}$

Despite the number of RCTs with low or moderate risk of bias addressing other supplements or diets, data are also inadequate to determine effects on any outcome in the short- or long-term. Most studies were small and short-term (ranging from 1 week to 7 months, with 24 months of treatment in one study).

\section{Strength of the Evidence}

Omega-3 fatty acid supplementation and placebo did not affect challenging behaviors. Our confidence in this conclusion is low (low strength of evidence for no effect) (Table 25). We also have low confidence in the conclusion that omega-3 supplementation was associated with minimal harms (low strength of evidence).

Data in two small studies of methyl-B12 were inadequate to draw conclusions (insufficient strength of evidence). Despite the number of RCTs with low or moderate risk of bias addressing other agents, evidence was inadequate to make conclusions about all clinical efficacy and harms outcomes because few, small, underpowered studies addressed each diet or supplement (insufficient strength of evidence).

While seven studies addressed variations of the GFCF diet, studies addressed different outcomes and different approaches to restricted and control diets; thus, data were inadequate to make conclusions about the body of evidence (insufficient strength of evidence). Data were 
inadequate to allow conclusions about the relative effectiveness of other dietary interventions (e.g., camels' milk, gluten-containing challenge foods) compared with placebo (insufficient strength of evidence).

Table 25. Strength of evidence for effects of omega-3 supplementation

\begin{tabular}{|c|c|c|c|c|c|c|}
\hline $\begin{array}{l}\text { Intervention/ } \\
\text { Outcome } \\
\text { Study Design } \\
\text { Risk of Bias and } \\
\text { Number of } \\
\text { Studies (N } \\
\text { Total) }\end{array}$ & 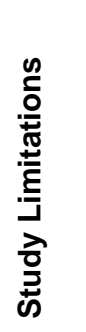 & $\begin{array}{l}\text { dे } \\
\text { d } \\
\frac{n}{0} \\
\frac{0}{0} \\
0 \\
0\end{array}$ & 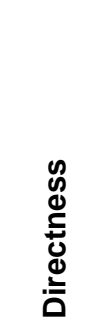 & $\frac{c}{\frac{0}{n}}$ & 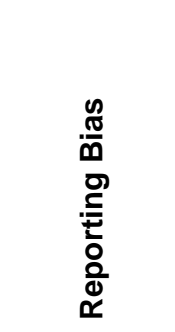 & $\begin{array}{l}\text { Finding } \\
\text { Strength of Evidence Grade }\end{array}$ \\
\hline \multicolumn{7}{|l|}{$\begin{array}{l}\text { Omega-3 fatty } \\
\text { acids vs. } \\
\text { Placebo }\end{array}$} \\
\hline $\begin{array}{l}\text { Challenging } \\
\text { behaviors } \\
\text { RCT: } 1 \text { low, }{ }^{81} 2 \\
\text { moderate }{ }^{80,84} \\
\mathrm{~N}=119\end{array}$ & $\begin{array}{l}\text { Me- } \\
\text { dium }\end{array}$ & Consistent & Direct & Imprecise & Undetected & $\begin{array}{l}\text { Low SOE for no effect on } \\
\text { challenging behavior } \\
\text { No significant differences } \\
\text { between groups in three small, } \\
\text { short-term RCTs }\end{array}$ \\
\hline $\begin{array}{l}\text { Harms } \\
\text { RCT: } 1 \text { low, }{ }^{81} 2 \\
\text { moderate }{ }^{80,84} \\
\text { N=119 }\end{array}$ & $\begin{array}{l}\text { Me- } \\
\text { dium }\end{array}$ & Consistent & Direct & Imprecise & Undetected & $\begin{array}{l}\text { Low SOE for minimal harms } \\
\text { associated with } \\
\text { supplementation } \\
\text { No clinically significant harms } \\
\text { reported in any study }\end{array}$ \\
\hline
\end{tabular}

$\mathrm{N}=$ number; RCT = randomized controlled trial; $\mathrm{SOE}=$ strength of evidence

\section{Studies of Risperidone Adjuncts}

\section{Key Findings}

Despite the number of RCTs with low or moderate risk of bias, studies present little evidence to inform treatment decisions and can be considered primarily as pilot efficacy trials. All studies were short-term and lacked followup past the end of treatment. Studies included few participants (median 40 total/study) and few examined the same adjunct agent or outcomes besides the ABC Irritability subscale. Only two studies ${ }^{90,93}$ addressed the same outcomes with different doses of the same agent (N-acetylcysteine). All studies except one of Gingko biloba added to risperidone reported significant improvements on the ABC-Irritability subscale in the adjunct groups compared with placebo or placebo plus risperidone; one study reporting only total ABC scores reported significant improvements in the adjunct group compared with placebo. ${ }^{124}$

\section{Strength of the Evidence}

Data were inadequate to assess effects of risperidone plus adjunctive agents including amantadine, buspirone, celecoxib, memantine, riluzole, Gingko biloba, pioglitazone, or topiramate on any outcome assessed as no study addressed the same adjunctive agent (insufficient strength of evidence). Studies were also small ( $<50$ children in any study) and shortterm (8-10 weeks of treatment). While two RCTs addressing risperidone plus N-acetylcysteine reported improvements in irritability with the combination vs. risperidone plus placebo, evidence 
is inadequate to comment on effects given the small number of participants ( $\mathrm{n}=71$ total), high attrition (15\% to $30 \%$ across groups) and short-term nature (10 weeks each) of the studies (insufficient strength of evidence).

\section{Studies of Hyperbaric Oxygen Therapy}

\section{Key Findings}

Three RCTs of hyperbaric oxygen used different doses and reported inconsistent results (favorable effects associated with treatment in only one ${ }^{114}$ and no significant effects in two ${ }^{66,67}$ ).

\section{Strength of the Evidence}

Data were inadequate to assess effects on outcomes given inconsistencies in outcome reporting (insufficient strength of evidence).

\section{Studies of $\mathrm{N}$-acetylcysteine}

\section{Key Findings}

One RCT assessing N-acetylcysteine reported significant improvements in irritability (ABCI) in treated children vs. those receiving placebo and no statistically significant effects on other measures of social skills or challenging behaviors. ${ }^{78}$ Another RCT reported no significant treatment effects on any outcomes (symptom severity, social skills, repetitive behaviors). ${ }^{79}$ Harms were not statistically significantly different between treatment and placebo groups.

\section{Strength of the Evidence}

N-acetylcysteine had no effect on social skills outcomes in two small RCTs; harms of this agent were not clinically significant. Our confidence in these conclusions is low (low strength of evidence) (Table 26). Data were inadequate to assess effects on other outcomes including symptom severity given inconsistent findings in these two studies (insufficient strength of evidence).

Table 26. Strength of evidence for effects of $\mathrm{N}$-acetylcysteine

\begin{tabular}{|c|c|c|c|c|c|c|}
\hline $\begin{array}{l}\text { Intervention/ } \\
\text { Outcome } \\
\text { Study Design } \\
\text { Risk of Bias and } \\
\text { Number of } \\
\text { Studies (N } \\
\text { Total) }\end{array}$ & 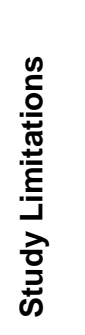 & 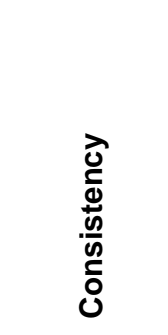 & 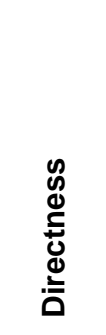 & $\frac{\frac{0}{0}}{\frac{0}{0}}$ & 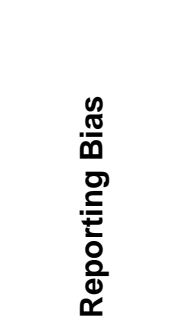 & $\begin{array}{l}\text { Finding } \\
\text { Strength of Evidence Grade }\end{array}$ \\
\hline \multicolumn{7}{|l|}{$\begin{array}{l}\mathrm{N}- \\
\text { acetylcysteine } \\
\text { vs. Placebo }\end{array}$} \\
\hline $\begin{array}{l}\text { Social skills } \\
\text { RCT: } 1 \text { low, }{ }^{78} 1 \\
\text { moderate }{ }^{79} \\
(\mathrm{~N}=127)\end{array}$ & High & Consistent & Direct & Imprecise & Undetected & $\begin{array}{l}\text { Low SOE for lack of effect on } \\
\text { social skills } \\
\text { No significant effects in either } \\
\text { small, short-term RCT }\end{array}$ \\
\hline
\end{tabular}




\begin{tabular}{|l|l|l|l|l|l|l|}
\hline Harms & High & Consistent & Direct & Imprecise & Undetected & Low SOE for minimal harms \\
$\begin{array}{l}\text { RCT: } 1 \text { low, }^{78} 1 \\
\text { moderate } \\
(\mathrm{N}=127)\end{array}$ & & & & & $\begin{array}{l}\text { No study reported harms } \\
\text { considered clinically important }\end{array}$ \\
\hline
\end{tabular}

$\mathrm{N}=$ number; $\mathrm{RCT}=$ randomized controlled trial; $\mathrm{SOE}=$ strength of evidence

\section{Studies of Tetrahydrobiopterin}

\section{Key Findings}

Outcomes were not consistent in two small RCTs of tetrahydrobiopterin. ${ }^{53,103}$ Changes in symptom severity were not significantly different between treatment and placebo groups; scores on other measures of challenging and adaptive behavior improved significantly in one study. ${ }^{53}$ Investigators did not consider harms to be clinically significant.

\section{Strength of the Evidence}

Tetrahydrobiopterin had no effect on symptom severity and was not associated with significant harms. Our confidence in these conclusions is low (low strength of evidence). Data were inadequate to assess effects on other outcomes including adaptive and challenging behavior (insufficient strength of evidence). Table 27 outlines findings.

Table 27. Strength of evidence for tetrahydrobiopterin

\begin{tabular}{|c|c|c|c|c|c|c|}
\hline $\begin{array}{l}\text { Intervention/ } \\
\text { Outcome } \\
\text { Study Design } \\
\text { Risk of Bias and } \\
\text { Number of } \\
\text { Studies (N } \\
\text { Total) }\end{array}$ & 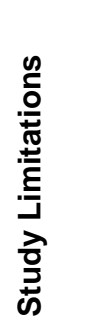 & $\begin{array}{l}\text { dे } \\
\frac{c}{0} \\
\frac{0}{0} \\
\frac{0}{n} \\
0 \\
0\end{array}$ & 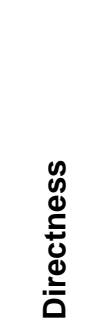 & $\frac{\frac{0}{0}}{\frac{0}{0}}$ & 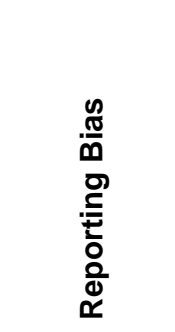 & $\begin{array}{l}\text { Finding } \\
\text { Strength of Evidence Grade }\end{array}$ \\
\hline \multicolumn{7}{|l|}{$\begin{array}{l}\text { Tetra- } \\
\text { hydrobiopterin } \\
\text { vs. Placebo }\end{array}$} \\
\hline $\begin{array}{l}\text { Symptom } \\
\text { severity } \\
\text { RCT: } 1 \text { low }^{53}, 1 \\
\text { moderate } \\
(\mathrm{N}=54)\end{array}$ & High & Consistent & Direct & Imprecise & Undetected & $\begin{array}{l}\text { Low SOE for lack of effect } \\
\text { No significant effects in either } \\
\text { small, short-term RCT }\end{array}$ \\
\hline $\begin{array}{l}\text { Harms } \\
\text { RCT: } 1 \text { low }^{53}, 1 \\
\text { moderate }{ }^{103} \\
(\mathrm{~N}=54)\end{array}$ & High & Consistent & Direct & Imprecise & Undetected & $\begin{array}{l}\text { Low SOE for minimal harms } \\
\text { No study reported harms } \\
\text { considered clinically important }\end{array}$ \\
\hline
\end{tabular}

$\mathrm{N}=$ number; $\mathrm{RCT}$ = randomized controlled trial; SOE = strength of evidence

\section{Other Medical Interventions}

\section{Key Findings}


Donepezil. Two RCTs of donepezil assessed differing outcomes and reported no effects on executive function and treatment-associated improvements in language. ${ }^{11,77}$

Agents Addressed in Single Studies. Studies of citalopram, ${ }^{22,23}$ and divalproex ${ }^{74}$ reported positive effects on measures of challenging behavior associated with treatment compared with placebo. Measures of language or symptom severity improved more in treatment arms versus control arms in studies of prednisolone, ${ }^{123}$ and transcranial stimulation, ${ }^{69,70}$ One study of amantadine reported significant improvements in hyperactivity relative to placebo. ${ }^{115}$ One RCT reported improvements in time to fall asleep and sleep time with melatonin versus placebo, ${ }^{68}$ and one of bumetanide compared with placebo reported positive effects of bumetanide on symptom severity. ${ }^{72}$ Single studies of oxytocin ${ }^{71}$ and mecamylamine ${ }^{73}$ reported no significant treatment effects.

Harms reported in studies comparing these interventions were diverse, and their clinical significance is difficult to determine given the short-term nature of the studies and the typically low numbers of participants.

\section{Strength of the Evidence}

Data were inadequate to make conclusions about the effects amantadine, bumetanide, divalproex, oxytocin, mecamylamine, prednisolone, citalopram, melatonin, and neurostimulation vs. placebo as no studies addressed the same agents (insufficient strength of evidence).

\section{KQ2. Modifiers of Treatment Outcomes}

Few studies reported modifying characteristics, and no characteristics were consistent modifiers.

\section{KQ3. Time to Effect of Interventions}

Few studies reported data to assess time to effect of interventions, and the lack of data precludes conclusions.

\section{KQ4. Evidence That Effects Measured at the End of Treatment Predict Long-Term Functional Outcomes}

Few studies had longer-term followup and those few with 6 months or more of treatment or followup typically did not report functional outcomes.

\section{KQ5. Effectiveness Across Environments or Contexts}

Five studies reported teacher ratings of outcome measures that provide some information to address this KQ, but the limited results preclude conclusions.

\section{KQ6. Drivers of Treatment Outcomes}

We did not identify studies that provided data to address this KQ.

\section{Findings in Relation to What Is Already Known}

We identified 16 recent (2010-present) systematic reviews or meta-analyses addressing medical interventions for children with ASD. Three reviews evaluated the antipsychotics 
aripiprazole and risperidone; six evaluated multiple agents including antipsychotics; and seven evaluated agents including SSRIs, atomoxetine, gluten-free casein-free diets, omega-3 fatty acids, melatonin, and hyperbaric oxygen.

Overall, findings in these reviews generally aligned with the findings presented here, with high strength of evidence for short-term effectiveness of aripiprazole and risperidone to ameliorate challenging behaviors and high strength of evidence for adverse effects of each agent. Several reviews commented on atomoxetine as a promising agent, and one commented on melatonin to improve sleep problems. Reviews noted little evidence for gluten-free casein-free (GFCF) diets, omega-3 fatty acids, hyperbaric oxygen, antiepileptic medications, selective serotonin reuptake inhibitors (SSRIs), and chelating agents.

Reviews generally considered studies of antipsychotics to have high quality and the quality of studies addressing stimulants and atomoxetine as moderate to high. Reviews generally considered studies addressing nutritional supplements, diets and hyperbaric oxygen as moderate to low quality. Reviews consistently commented on a lack of long term data and typically small sample sizes as limitations of the strength of the evidence.

\section{Antipsychotics}

One Cochrane review of aripiprazole included two RCTs and reported significant short-term treatment-related improvements on irritability, hyperactivity, and repetitive movement with weight gain, sedation, drooling, and neurological side effects. ${ }^{133}$ Another review including children with ASD ( $n=637)$, developmental disorders $(n=68)$, or intellectual disability $(n=50)$ noted that all of the 20 included randomized and observational studies reported significant treatment-related improvements in problem behaviors measured on the Clinical Global Impression (CGI) scale or ABC. ${ }^{134}$ Most studies reported adverse effects including weight gain, sedation, and tremor. Both reviews noted a need for longer term studies to address durability of effects. One meta-analysis of risperidone ( $\mathrm{n}=608$ children) reported a mean effect size for risperidone of 1.09 across studies (1.14 for open label studies and 1.12 for placebo-controlled) using global outcome measures such as the Childhood Autism Rating Scale (CARS) and CGI. ${ }^{135}$ The effect size for outcomes related to maladaptive behaviors such as irritability and aggression was 1.20 .

\section{Reviews of Multiple Agents}

Agents To Treat Irritability and Problem Behaviors. One review and meta-analysis addressed treatment of severe irritability and problem behaviors and included 11 RCTs that used the ABCIrritability subscale in quantitative analyses. The review reported significant treatment effects for aripiprazole (effect size $=0.9$ ), risperidone (effect size $=0.8$ ), and $\mathrm{N}$-acetylcysteine (effect size $=0.7$ ) compared with placebo. ${ }^{136}$ Harms occurring with risperidone and aripiprazole included somnolence or sedation, and extrapyramidal symptoms occurred with aripiprazole and haloperidol. Aripiprazole, risperidone, and valproate caused greater weight gain compared with placebo (effect sizes of 3.1, 0.8, and 0.3, respectively). Investigators also noted smaller effects for clonidine, methylphenidate, tianeptine, venlafaxine, and naltrexone in studies that did not specifically target irritability and that atomoxetine and dextromethorphan were associated with improvements in hyperactivity and impulsivity on the ABC-Hyperactivity subscale.

Psychotropic Medications. One review of 17 different medications reported addressed in 33 RCTS reported little established evidence to support any medication. ${ }^{137}$ Investigators considered 
aripiprazole, risperidone, haloperidol to be have established evidence for treating irritability and hyperactivity (risperidone); irritability, hyperactivity, and stereotypy (aripiprazole); and unspecified behavioral symptoms (haloperidol). The review noted promising evidence for methylphenidate's effects on hyperactivity. Investigators considered evidence preliminary for the effects of risperidone on repetitive behavior and stereotypy, for the effects of atomoxetine and naltrexone on hyperactivity, and for pentoxifylline on irritability and social withdrawal. Investigators reported evidence as insufficient for all other agents (clonidine, guanfacine, olanzapine, divalproex, lamotrigine, levetiracetam, citalopram, fluoxetine, clomipramine, amantadine, naltrexone) on outcomes including social behavior, hyperactivity, and repetitive behavior.

Agents To Treat ADHD Symptoms. One review identified seven placebo-controlled trials of medications targeting ADHD symptoms in children with Pervasive Development Disorders ( $n=225$ children) and reported that methylphenidate was significantly more effective than placebo in treating ADHD symptoms (effect size 0.67) and hyperactivity specifically (effect size=0.66). ${ }^{138}$ Appetite decrease, insomnia, depressive symptoms, irritability, and social withdrawal occurred significantly more frequently in the treatment group versus placebo. The review reported no significant effects for clonidine versus placebo and significant improvements in ADHD symptoms and hyperactivity in children taking atomoxetine compared with placebo. Harms included nausea, decreased appetite, and sleep changes.

Antiepileptic Medications. One meta-analysis evaluated valproate, lamotrigine, levetiracetam, and topiramate in children with ASD. ${ }^{139}$ In meta-analyses, single agents studied were not significantly different between treatment and placebo arms. One study of topiramate combined with risperidone reported improvements in irritability in the combination group compared with risperidone plus placebo. Two studies of valproate and one of levetiracetam reported no response to treatment as defined by CGI ratings. Discontinuation due to adverse effects did not differ between groups nor did the rate of total adverse events.

Alzheimer's Medications. One review addressed use of these medications for children and adults with ASD and included case reports and other observational studies and controlled trials. ${ }^{140}$ Drugs assessed in children included donepezil, galantamine, rivastigmine, and memantine. Four uncontrolled and one controlled studies of donepezil reported treatmentassociated improvements in ASD symptoms. All three studies of galantamine (2 RCTs and 1 case series) reported positive effects on core and associated ASD symptoms. One small case series addressing rivastigmine reported improvements in expressive language and ASD symptoms, and three studies (1 RCT, 2 case series) of memantine use in children also reported positive effects on irritability and associated symptoms with side effects including gastrointestinal symptoms, worsening behavior, and sedation. The review concluded that evidence is inconsistent and inconclusive but further study of some agents such as rivastigmine may be warranted.

Predictors of Placebo Response. One meta-analysis included data from 25 RCTs measuring outcomes with either the ABC, CGI, VABS, CARS, or Children's Yale-Brown Obsessive Compulsive Scale (CYBOCS) and reported a moderate but significant placebo response across studies (effect size $=0.45$, 95\% CI: 0.34 to $0.56, \mathrm{p}<0.001$ ). ${ }^{141}$ Investigators identified clinician- 
completed outcome measures, level of response to active intervention, a pharmacologic active intervention (vs. diet, etc.), use of adjunctive treatment, and geographical location of the trial (greater response in Iran vs. United States) as significant moderators of the placebo response, with each factor associated with an increased response.

\section{Reviews of Other Agents}

Atomoxetine. One review of atomoxetine included six studies (1 RCT) evaluating the agent for treatment of hyperactivity in children with ADHD $(n=90) .{ }^{142}$ All studies except one (which included children with "severe autistic disorder") reported significant improvements in behavior in parent, teacher, and clinician ratings with treatment. Harms reported included gastrointestinal symptoms, somnolence, irritability, and weight loss; the review concluded that evidence suggests potential efficacy but small sample sizes and short-term studies limit conclusions.

Hyperbaric Oxygen Therapy. One review including eight studies (2 RCTs) reported little evidence for effects of hyperbaric oxygen in controlled trials, and some promising evidence in small case series. ${ }^{143}$ Few studies reported any adverse effects at the pressure levels studied.

SSRIs. One Cochrane review included nine RCTs (5 including only children) evaluating ( $\mathrm{n}=239$ children) evaluating the SSRIs fluoxetine, fluvoxamine, fenfluramine, and citalopram. ${ }^{144}$ The review reported no evidence for the effectiveness of SSRIs and potential evidence for harms.

Omega-3 Fatty Acids. One Cochrane review included two RCTs ( $n=37$ children, predominately male with moderate to severe ASD symptoms) evaluating omega-3 fatty acids and reported no evidence for effects on social interaction, communication, hyperactivity, or stereotypy. ${ }^{145}$

GFCF Diets. One review included 32 studies, typically with high risk of bias, and noted scarce evidence for GFCF diets, with positive effects reported only in lower quality studies. ${ }^{146}$ The review concluded that evidence for the effectiveness or harms of GFCF diets is limited and weak.

Melatonin. One review included 18 studies addressing melatonin for the treatment of sleep problems in ASD. ${ }^{147}$ Thirteen observational studies reported improvements in sleep duration, night awakenings, or sleep onset latency, as did five RCTs. Investigators meta-analyzed RCT data and reported significant improvements on these measures with melatonin versus placebo. Harms associated with melatonin included drowsiness, gastrointestinal symptoms, and worsening behavior. No study reported serious adverse events.

Chelation. Though we did not identify studies addressing chelation agents in the current review, one Cochrane review addressed chelation therapy and included one RCT ( $n=49$ children). ${ }^{148}$ Investigators considered the study to have high risk of bias and noted that no evidence suggests efficacy for ASD symptoms.

\section{Applicability}

By definition, ASD is heterogeneous. Characterizing a "typical" child with an ASD is not possible, although certain symptoms are central to the range of children within the autism spectrum. Individual therapies are developed and tested to ameliorate specific symptoms or 
groups of symptoms, often in a fairly circumscribed subset of children. We provide details on the population, intervention, comparator, outcomes, and setting (PICOS) for each intervention addressed in more than one study in Appendix E to support translation of our findings and assessment of the applicability of each for differing circumstances and children.

Overall, study participants were generally recruited from specialty clinical service programs and represent nonprimary care populations. As such, families of these children may be seeking a higher level of care than those of the broader population of children with ASD based upon more severe or acute symptoms, including aggression or other challenging behaviors. Most studies of medical interventions targeted elementary school aged and older children with autism, with little data on the treatment of younger children. Most studies included majority male populations (consistent with the male prevalence of ASD).

Studies also included children with highly variable severity of challenging behaviors, ASD symptom severity, and cognitive impairment. Studies of pharmacological agents often sampled children with high levels of specific symptom patterns (e.g., children with severe challenging behavior at baseline where parents may be willing to pursue pharmacologic intervention and trial participation) who may not reflect the wider population of children with ASD in whom these challenges may not be present. Most of the studies reported including children with at least moderate level of severity of ASD. Studies of stimulants included children with cognitive impairment and with comorbidities including attention deficit hyperactivity disorder, oppositional defiant disorder, and obsessive compulsive disorder. Studies of other approaches had similarly heterogeneous populations. Dietary and nutritional studies included some younger children, with severity of autism not well described or the degree of intellectual functioning not well characterized in most studies. This heterogeneity in population characteristics may limit the generalizability of findings to children with differing levels of symptom expression or comorbidities but likely reflects the heterogeneity of the broader population of children with ASD

Studies addressed a variety of agents and typically reported use of concurrent medications or other therapies. Most agents studied are accessible in the United States, albeit with few receiving FDA approval for use. Comparators among non-placebo controlled studies varied, and few studies assessed the effect of concomitant behavioral or other therapies, though many children with ASD receive multiple interventions. The treatments studied may not adequately reflect the broad range of treatment combinations used in the general population of children with ASD.

As noted, few studies evaluated longer term treatment (>6 months); short treatment and followup periods limit our ability to understand potential longer term outcomes such as academic achievement or longer term harms. Overall, the heterogeneity of these studies parallels the heterogeneity of children with ASD, and some findings may be more applicable to children with specific levels of baseline severity or comorbidities. These limitations to generalizability likely reflect both the significant heterogeneity of ASD itself as well as its associated features, such as irritability. Thus, while there is a growing evidence base for treating certain symptoms in certain populations, these findings underscore the continued need for individualized treatment approaches that are informed by the emerging evidence base for benefits as well as harms of medical intervention, with careful consideration of symptom presentation and functioning level relative to study populations and applicability of the known literature. 


\section{Implications for Clinical and Policy Decisionmaking}

This review provides some evidence for decisionmaking about medical interventions for children with ASD. The clearest evidence favors the use of the antipsychotics risperidone and aripiprazole to address challenging behaviors in the short-term ( $<6$ months); however, clinicians and caregivers must balance the significant harms of these agents. The significant side effect profiles make it clear that although these drugs are efficacious, caution is warranted regarding their use in patients without severe impairments or risk of injury. Few studies addressed longer term effects of these agents; thus, our confidence in longer term ( $>6$ months) effectiveness is low. Studies of adjuncts to risperidone typically reported positive effects on challenging behaviors, but few studies addressed the same agents, precluding our ability to draw conclusions about their effectiveness.

Some evidence supports the use of methylphenidate and atomoxetine for hyperactivity, but few comparative studies addressed each agent, so our confidence in effects is limited. Given that many children with ASD are currently treated with medical interventions, strikingly little evidence exists to support clear benefit for most medical interventions, especially in the realm of interventions such as restrictive diets and supplements. Studies of nutritional supplements or specialized diets were typically underpowered and provided little evidence of effects of these approaches. Several agents were addressed in single studies, which limits conclusions about their effects.

Decisional dilemmas remain regarding characteristics of the child, family, or intervention that may modify effectiveness or predict which children may be most likely to benefit from a given approach. Similarly, the literature base is currently insufficient to inform our understanding of the time to effect of interventions, longer term effectiveness of interventions, generalizability of effects outside the treatment context, effectiveness and applicability to broader ASD populations, and components that may drive effectiveness.

\section{Limitations of the Comparative Effectiveness Review Process}

We included studies published in English only and did not include unpublished data. We scanned a random sample of 150 non-English abstracts retrieved by our MEDLINE search. Most studies appeared to be case series, narrative reviews, basic science studies, or studies assessing etiology. Only two studies appeared to meet inclusion criteria; thus, given the high percentage of ineligible items in this scan (99\%), we concluded that excluding non-English studies would not introduce significant bias into the review. We recognize that this preliminary scan did not address the entire corpus of ASD literature in other languages.

We also included only comparative studies of medical interventions with at least 10 children with ASD. To ensure comprehensive coverage of the literature, we included comparative studies with smaller sample size that would have been excluded in our 2011 review (which required a sample size of 30) in the present report. We did not conduct a de novo search for such studies but re-examined the excluded studies from the prior review. This approach may have overlooked relevant studies.

Given heterogeneity in treatment regimens, outcomes addressed in each study, and patient populations, we were limited in our ability to meta-analyze findings or identify potential subgroups that may respond more favorably to specific treatments. Finally, we used a nonvalidated tool to assess risk of bias, though we note that the tool evaluates similar constructs 
to those assessed in tools such as that used by the Cochrane Collaboration, with the addition of ASD-specific domains.

\section{Limitations of the Evidence Base}

As noted, studies in the review had small sample sizes and typically limited duration of intervention and followup after intervention, despite significant improvements in study design and execution over time. Populations across studies were heterogeneous in terms of challenging behaviors, ASD symptom severity, age, and comorbidities. Few studies addressed the same agent and outcomes, and few assessed potential factors that may modify effectiveness or drive effects of interventions. Many ( $n=63)$ studies also explicitly noted that concomitant interventions were held steady during the study treatment period; however, few studies reported specific analyses to control for or assess the effects of additional treatments.

Despite these limitations, investigators have made significant improvements in incorporating commonly used measures of symptom severity and behavior to facilitate comparisons across studies. Studies also typically described interventions fully, used standardized diagnostic processes and blinded assessors, and reported on the use or restriction of concomitant interventions.

\section{Research Gaps and Areas for Future Research}

Improving research in this area should include methodologic considerations of power and sample size and durability of effects. Sample size and participant followup were frequently insufficient to allow firm conclusions. Duration of treatment and followup were generally short ( $<6$ months); those studies with longer duration of treatment were typically open label extensions of RCTs and lacked control arms. While duration was typically short, retaining participants in studies, especially in placebo arms, is difficult when parents or children perceive little improvement in symptoms. Longer duration of treatment, however, is also important to rule out meaningful improvements in placebo groups and help inform our understanding of the placebo effect.

Few studies provided data on long-term outcomes after cessation of treatment. Future studies should extend the followup period and assess the degree to which outcomes are durable in "real world" situations. The literature includes many single studies of various agents. Studies of adjuncts to risperidone, for example, examined different adjunct agents, with some positive effects on challenging behaviors reported with most. Understanding which agents should be examined further is lacking. Another critical area for further research is identifying which children are likely to benefit from particular interventions. To date, studies have provided limited characterization of the subpopulation of children who experience positive response to medical interventions and limited characterization of the extent or type of behavioral challenges children experience at baseline.

Children with ASD also typically receive multiple types of therapies, but few studies addressed combinations of medical and behavioral or other categories of interventions or a medical treatment compared with a nonmedical treatment. Few attempted to account for potential effects on ongoing interventions. This not only limited our ability to interpret the effects of medical treatments in isolation but represents a significant gap for families and providers in choosing additional treatments that may bolster (or impair) the effects of behavioral, medication, or other therapies. Few studies $(n=10)$ compared active treatments, and future 
research to assess the comparative effectiveness of antipsychotics, ADHD medications, and other medications is necessary.

In addition, much of the medical intervention literature relies on baseline and outcome measures that have specific limits in understanding individualized response. Future research attempting to elucidate potential biobehavioral markers of response may prove useful. Research in understanding outcomes of importance to patients and caregivers, such as quality of life, is also lacking.

Harms reporting varied across studies; some studies amply described how harms were tracked, while others listed harms with no indication of how they were assessed (e.g., parent recall, checklist, clinician assessment during followup). This lack of reporting makes comparing harms across studies difficult. For instance, while studies of atomoxetine generally reported fewer harms than did studies of methylphenidate in children with ADHD symptoms, exploring differences in safety profiles is an important area for additional research.

\section{Conclusions}

Risperidone and aripiprazole ameliorated challenging behaviors in the short term $(<6$ months), but had significant side effects. Methylphenidate and atomoxetine were also associated with improvements in hyperactivity in small, short-term RCTs (with uncontrolled open label extensions). Atomoxetine plus parent training was not more effective for hyperactivity than atomoxetine alone. Omega-3 fatty acid supplementation was not associated with improvements in challenging behaviors, and $\mathrm{N}$-acetylcysteine and tetrahydrobiopterin were not associated with improvements in social skills and symptom severity, respectively. Some positive effects were reported with other agents studied (risperidone adjuncts, melatonin), but few studies addressed the same agent or outcomes. Data on longer term ( $>6$ months) results and harms of interventions are lacking. Similarly, more research is needed to understand characteristics of the child or treatment that modify outcomes and whether effectiveness of interventions generalizes across different settings such as the home or school. Current evidence also does not inform our understanding of components of interventions that may drive effects. Some therapies hold promise and warrant further study, and the conduct of studies has improved considerably over time (i.e., growing number of randomized controlled trials and use of standardized measures). However, additional studies with larger, well-characterized populations over longer time frames, and that utilize transparent and rigorous methods that permit comparison across studies, would further inform decisionmaking. 


\section{References}

1. Christensen DL, Baio J, Braun KV, et al. Prevalence and Characteristics of Autism Spectrum Disorder Among Children Aged 8 Years - Autism and Developmental Disabilities Monitoring Network, 11 Sites, United States, 2012. MMWR Surveill Summ. 2016;65(3):1-23. doi: 10.15585/mmwr.ss6503a1. PMID: 27031587.

2. Seltzer MM, Shattuck P, Abbeduto L, et al. Trajectory of development in adolescents and adults with autism. Ment Retard Dev Disabil Res Rev. 2004;10(4):234-47. doi: 10.1002/mrdd.20038. PMID: 15666341.

3. Ospina MB, Krebs Seida J, Clark B, et al. Behavioural and developmental interventions for autism spectrum disorder: a clinical systematic review. PLoS One. 2008;3(11):e3755. doi: 10.1371/journal.pone.0003755 [doi]. PMID: 19015734 .

4. Seida JK, Ospina MB, Karkhaneh M, et al. Systematic reviews of psychosocial interventions for autism: an umbrella review. Dev Med Child Neurol. 2009 Feb;51(2):95-104. doi: DMCN3211 [pii] 10.1111/j.1469-8749.2008.03211.x [doi]. PMID: 19191842.

5. Parr J. Autism. BMJ Clin Evid. 2010;2010 PMID: 21729335.

6. Wan Yunus F, Liu KP, Bissett M, et al. Sensory-Based Intervention for Children with Behavioral Problems: A Systematic Review. J Autism Dev Disord. 2015 Nov;45(11):3565-79. doi: 10.1007/s10803015-2503-9. PMID: 26092640.

7. Myers SM, Johnson CP. Management of children with autism spectrum disorders. Pediatrics. 2007 Nov;120(5):1162-82. doi: peds.2007-2362 [pii] 10.1542/peds.2007-2362 [doi]. PMID: 17967921.
8. National Autism Center. National Standards Report National Autism Center. Randolph, MA: National Autism Center; 2009.

Available from http://www.nationalautismcenter.org/pdf/N AC\%20Standards\%20Report.pdf

9. National Research Council. Committee on Educational Interventions for Children with Autism. Educating Children with Autism Washington DC: National Academies Press; 2001. Available from http://www.nap.edu/openbook.php?record_i $\underline{\mathrm{d}=10017 \& \text { page }=\mathrm{R} 2}$

10. US Food and Drug Administration. Beware of False or Misleading Claims for Treating Autism. Available from http://www.fda.gov/ForConsumers/Consum erUpdates/ucm394757.htm.

11. Warren Z, Veenstra-VanderWeele J, Stone W, Bruzek JL, Nahmias AS, Foss-Feig JH, Jerome RN, Krishnaswami S, Sathe NA, Glasser AM, Surawicz T, McPheeters ML. Therapies for Children With Autism Spectrum Disorders. Comparative Effectiveness Review No. 26. (Prepared by the Vanderbilt Evidence-based Practice Center under Contract No. 290-2007-10065I.) AHRQ Publication No. 11-EHC029-EF. Rockville, MD: Agency for Healthcare Research and Quality. April 2011. Available at:

www.effectivehealthcare.ahrq.gov/reports/fi nal.cfm.

12. Methods Guide for Effectiveness and Comparative Effectiveness Reviews. AHRQ Publication No. 10(14)-EHC063-EF. Rockville, MD: Agency for Healthcare Research and Quality. January 2014. Chapters available at: www.effectivehealthcare.ahrq.gov

13. Weitlauf AS, McPheeters ML, Peters B, Sathe N, Travis R, Aiello R, Williamson E, Veenstra-VanderWeele J, Krishnaswami S, Jerome R, Warren Z. Therapies for Children With Autism Spectrum Disorder: Behavioral 
Interventions Update. Comparative Effectiveness Review No. 137. (Prepared by the Vanderbilt Evidence-based Practice Center under Contract No. 290-2012-00009I.) AHRQ Publication No. 14-EHC036-EF. Rockville, MD: Agency for Healthcare Research and Quality; August 2014. www.effectivehealthcare.ahrq.gov/reports/fi nal.cfm.

14. Berkman ND, Lohr KN, Ansari M, McDonagh M, Balk E, Whitlock E, Reston J, Bass E, Butler M, Gartlehner G, Hartling L, Kane R, McPheeters M, Morgan L, Morton SC, Sista P, Viswanathan M, Chang S. Grading the Strength of a Body of Evidence When Assessing Health Care Interventions for the Effective Health Care Program of the Agency for Healthcare Research and Quality: An Update RTI-UNC Evidence-based Practice Center, Contract No. HHSA-290-2007-10056-I-EPC3, Task Order \#5.

15. Marcus RN, Owen R, Manos G, et al. Aripiprazole in the treatment of irritability in pediatric patients (aged 6-17 years) with autistic disorder: results from a 52-week, open-label study. J Child Adolesc Psychopharmacol. 2011 Jun;21(3):229-36. doi: 10.1089/cap.2009.0121. PMID: 21663425 .

16. Aman MG, Kasper W, Manos G, et al. Lineitem analysis of the Aberrant Behavior Checklist: results from two studies of aripiprazole in the treatment of irritability associated with autistic disorder. J Child Adolesc Psychopharmacol. 2010 Oct;20(5):415-22. doi: 10.1089/cap.2009.0120. PMID: 20973712.

17. Varni JW, Handen BL, Corey-Lisle PK, et al. Effect of aripiprazole 2 to $15 \mathrm{mg} / \mathrm{d}$ on health-related quality of life in the treatment of irritability associated with autistic disorder in children: a post hoc analysis of two controlled trials. Clin Ther. 2012 Apr;34(4):980-92. doi: 10.1016/j.clinthera.2012.02.023. PMID: 22444782.

18. Marcus RN, Owen R, Manos G, et al. Safety and tolerability of aripiprazole for irritability in pediatric patients with autistic disorder: a 52-week, open-label, multicenter study. J Clin Psychiatry. 2011 Sep;72(9):1270-6. doi: 10.4088/JCP.09m05933. PMID: 21813076.

19. Robb AS, Andersson C, Bellocchio EE, et al. Safety and tolerability of aripiprazole in the treatment of irritability associated with autistic disorder in pediatric subjects (6-17 years old):results from a pooled analysis of 2 studies. Prim Care Companion CNS Disord. 2011;13(1)doi: 10.4088/PCC.10m01008gry. PMID: 21731831.

20. Marcus RN, Owen R, Kamen L, et al. A placebo-controlled, fixed-dose study of aripiprazole in children and adolescents with irritability associated with autistic disorder. J Am Acad Child Adolesc Psychiatry. 2009 Nov;48(11):1110-9. doi: 10.1097/CHI.0b013e3181b76658 [doi]. PMID: 19797985.

21. Owen R, Sikich L, Marcus RN, et al. Aripiprazole in the treatment of irritability in children and adolescents with autistic disorder. Pediatrics. 2009 Dec;124(6):153340. doi: 124/6/1533 [pii] 10.1542/peds.2008-3782 [doi]. PMID: 19948625.

22. King BH, Dukes K, Donnelly CL, et al. Baseline factors predicting placebo response to treatment in children and adolescents with autism spectrum disorders: a multisite randomized clinical trial. JAMA Pediatr. 2013 Nov;167(11):1045-52. doi: 10.1001/jamapediatrics.2013.2698. PMID: 24061784.

23. King BH, Hollander E, Sikich L, et al. Lack of efficacy of citalopram in children with autism spectrum disorders and high levels of repetitive behavior: citalopram ineffective in children with autism. Arch Gen Psychiatry. 2009 Jun;66(6):583-90. doi: 10.1001/archgenpsychiatry.2009.30. PMID: 19487623.

24. McCracken JT, Badashova KK, Posey DJ, et al. Positive effects of methylphenidate on hyperactivity are moderated by monoaminergic gene variants in children with autism spectrum disorders. 
Pharmacogenomics J. 2014 Jun;14(3):295302. doi: 10.1038/tpj.2013.23. PMID: 23856854.

25. Posey DJ, Aman MG, McCracken JT, et al. Positive effects of methylphenidate on inattention and hyperactivity in pervasive developmental disorders: an analysis of secondary measures. Biol Psychiatry. 2007 Feb 15;61(4):538-44. doi: S00063223(06)01215-7 [pii] 10.1016/j.biopsych.2006.09.028 [doi]. PMID: 17276750.

26. Jahromi LB, Kasari CL, McCracken JT, et al. Positive Effects of Methylphenidate on Social Communication and Self-Regulation in Children with Pervasive Developmental Disorders and Hyperactivity. Journal of Autism and Developmental Disorders. 2009 Mar;39(3):395-404. PMID: EJ827753.

27. Randomized, controlled, crossover trial of methylphenidate in pervasive developmental disorders with hyperactivity. Arch Gen Psychiatry. 2005 Nov;62(11):1266-74. doi: 62/11/1266 [pii] 10.1001/archpsyc.62.11.1266 [doi]. PMID: 16275814 .

28. Aman M, Rettiganti M, Nagaraja HN, et al. Tolerability, Safety, and Benefits of Risperidone in Children and Adolescents with Autism: 21-Month Follow-up After 8Week Placebo-Controlled Trial. J Child Adolesc Psychopharmacol. 2015 Aug;25(6):482-93. doi: 10.1089/cap.2015.0005. PMID: 26262903.

29. Arnold LE, Farmer C, Kraemer HC, et al. Moderators, mediators, and other predictors of risperidone response in children with autistic disorder and irritability. J Child Adolesc Psychopharmacol. 2010 Apr;20(2):83-93. doi: 10.1089/cap.2009.0022. PMID: 20415603.

30. Aman MG, Hollway JA, McDougle CJ, et al. Cognitive effects of risperidone in children with autism and irritable behavior. J Child Adolesc Psychopharmacol. 2008 Jun;18(3):227-36. doi: 10.1089/cap.2007.0133 [doi]. PMID: 18582177.
31. Aman MG, Arnold LE, McDougle CJ, et al. Acute and long-term safety and tolerability of risperidone in children with autism. J Child Adolesc Psychopharmacol. 2005 Dec;15(6):869-84. doi: 10.1089/cap.2005.15.869 [doi]. PMID: 16379507.

32. McDougle CJ, Scahill L, Aman MG, et al. Risperidone for the core symptom domains of autism: results from the study by the autism network of the research units on pediatric psychopharmacology. Am J Psychiatry. 2005 Jun;162(6):1142-8. doi: 162/6/1142 [pii] 10.1176/appi.ajp.162.6.1142 [doi]. PMID: 15930063.

33. Martin A, Scahill L, Anderson GM, et al. Weight and leptin changes among risperidone-treated youths with autism: 6month prospective data. Am J Psychiatry. 2004 Jun;161(6):1125-7. PMID: 15169706.

34. McCracken JT, McGough J, Shah B, et al. Risperidone in children with autism and serious behavioral problems. N Engl J Med. 2002 Aug 1;347(5):314-21. doi: 10.1056/NEJMoa013171 [doi] 347/5/314 [pii]. PMID: 12151468.

35. Arnold LE, Vitiello B, McDougle C, et al. Parent-defined target symptoms respond to risperidone in RUPP autism study: customer approach to clinical trials. J Am Acad Child Adolesc Psychiatry. 2003 Dec;42(12):144350. doi:

10.1097/01.chi.0000091946.28938.54 [doi]. PMID: 14627879.

36. Risperidone treatment of autistic disorder: longer-term benefits and blinded discontinuation after 6 months. Am J Psychiatry. 2005 Jul;162(7):1361-9. doi: 162/7/1361 [pii] 10.1176/appi.ajp.162.7.1361 [doi]. PMID: 15994720.

37. Williams SK, Scahill L, Vitiello B, et al. Risperidone and adaptive behavior in children with autism. J Am Acad Child Adolesc Psychiatry. 2006 Apr;45(4):431-9. doi: 10.1097/01.chi.0000196423.80717.32 
[doi] 00004583-200604000-00010 [pii].

PMID: 16601648.

38. Scahill L, Sukhodolsky DG, Anderberg E, et al. Sensitivity of the modified Children's Yale-Brown Obsessive Compulsive Scale to detect change: Results from two multi-site trials. Autism. 2015 Apr 16doi: 10.1177/1362361315574889. PMID: 25882391.

39. Scahill L, Hallett V, Aman MG, et al. Brief Report: social disability in autism spectrum disorder: results from Research Units on Pediatric Psychopharmacology (RUPP) Autism Network trials. J Autism Dev Disord. 2013 Mar;43(3):739-46. doi: 10.1007/s10803-012-1689-3. PMID: 23104617.

40. Arnold LE, Aman MG, Cook AM, et al. Atomoxetine for hyperactivity in autism spectrum disorders: placebo-controlled crossover pilot trial. J Am Acad Child Adolesc Psychiatry. 2006 Oct;45(10):1196205. doi: 10.1097/01.chi.0000231976.28719.2a [doi] 00004583-200610000-00007 [pii]. PMID: 17003665.

41. van der Meer JM, Harfterkamp M, van de Loo-Neus G, et al. A randomized, doubleblind comparison of atomoxetine and placebo on response inhibition and interference control in children and adolescents with autism spectrum disorder and comorbid attention-deficit/hyperactivity disorder symptoms. J Clin

Psychopharmacol. 2013 Dec;33(6):824-7. doi: 10.1097/JCP.0b013e31829c764f. PMID: 24018545.

42. Harfterkamp M, Buitelaar JK, Minderaa RB, et al. Atomoxetine in autism spectrum disorder: no effects on social functioning; some beneficial effects on stereotyped behaviors, inappropriate speech, and fear of change. J Child Adolesc Psychopharmacol. 2014 Nov;24(9):481-5. doi: 10.1089/cap.2014.0026. PMID: 25369243.

43. Harfterkamp M, van de Loo-Neus G, Minderaa RB, et al. A randomized doubleblind study of atomoxetine versus placebo for attention-deficit/hyperactivity disorder symptoms in children with autism spectrum disorder. J Am Acad Child Adolesc Psychiatry. 2012 Jul;51(7):733-41. doi: 10.1016/j.jaac.2012.04.011. PMID: 22721596.

44. Harfterkamp M, Buitelaar JK, Minderaa RB, et al. Long-term treatment with atomoxetine for attention-deficit/hyperactivity disorder symptoms in children and adolescents with autism spectrum disorder: an open-label extension study. J Child Adolesc Psychopharmacol. 2013 Apr;23(3):194-9. doi: 10.1089/cap.2012.0012. PMID: 23578015.

45. Pearson DA, Santos CW, Aman MG, et al. Effects of extended release methylphenidate treatment on ratings of attentiondeficit/hyperactivity disorder (ADHD) and associated behavior in children with autism spectrum disorders and ADHD symptoms. J Child Adolesc Psychopharmacol. 2013 Jun;23(5):337-51. doi: 10.1089/cap.2012.0096. PMID: 23782128.

46. Scahill L, McCracken JT, King BH, et al. Extended-Release Guanfacine for Hyperactivity in Children With Autism Spectrum Disorder. Am J Psychiatry. 2015 Aug 28:appiajp201515010055. doi: 10.1176/appi.ajp.2015.15010055. PMID: 26315981.

47. Nikvarz N, Alaghband-Rad J, Tehrani-Doost $\mathrm{M}$, et al. Comparing Efficacy and Side Effects of Memantine vs. Risperidone in the Treatment of Autistic Disorder. Pharmacopsychiatry. 2016 Jun 14doi: 10.1055/s-0042-108449. PMID: 27299475.

48. Findling RL, Mankoski R, Timko K, et al. A randomized controlled trial investigating the safety and efficacy of aripiprazole in the long-term maintenance treatment of pediatric patients with irritability associated with autistic disorder. J Clin Psychiatry. 2014 Jan;75(1):22-30. doi:

10.4088/JCP.13m08500 10.4088/JCP.13m8500. PMID: 24502859.

49. Ghanizadeh A, Sahraeizadeh A, Berk M. A head-to-head comparison of aripiprazole and risperidone for safety and treating autistic disorders, a randomized double blind 
clinical trial. Child Psychiatry Hum Dev. 2014;45(2):185-92. doi: 10.1007/s10578013-0390-x. PMID: 23801256.

50. Lamberti M, Siracusano R, Italiano D, et al. Head-to-Head Comparison of Aripiprazole and Risperidone in the Treatment of ADHD Symptoms in Children with Autistic Spectrum Disorder and ADHD: A Pilot, Open-Label, Randomized Controlled Study. Paediatr Drugs. 2016 Aug;18(4):319-29. doi: 10.1007/s40272-016-0183-3. PMID: 27278054.

51. Kent JM, Kushner S, Ning X, et al. Risperidone dosing in children and adolescents with autistic disorder: a doubleblind, placebo-controlled study. J Autism Dev Disord. 2013 Aug;43(8):1773-83. doi: 10.1007/s10803-012-1723-5. PMID: 23212807.

52. Kent JM, Hough D, Singh J, et al. An openlabel extension study of the safety and efficacy of risperidone in children and adolescents with autistic disorder. J Child Adolesc Psychopharmacol. 2013 Dec;23(10):676-86. doi: 10.1089/cap.2012.0058. PMID: 24350813.

53. Klaiman C, Huffman L, Masaki L, et al. Tetrahydrobiopterin as a treatment for autism spectrum disorders: a double-blind, placebo-controlled trial. J Child Adolesc Psychopharmacol. 2013 Jun;23(5):320-8. doi: 10.1089/cap.2012.0127. PMID: 23782126.

54. Handen BL, Aman MG, Arnold LE, et al. Atomoxetine, Parent Training, and Their Combination in Children With Autism Spectrum Disorder and AttentionDeficit/Hyperactivity Disorder. J Am Acad Child Adolesc Psychiatry. 2015

Nov;54(11):905-15. doi: 10.1016/j.jaac.2015.08.013. PMID: 26506581.

55. Smith T, Aman MG, Arnold LE, et al. Atomoxetine and Parent Training for Children With Autism and AttentionDeficit/Hyperactivity Disorder: A 24-Week Extension Study. J Am Acad Child Adolesc Psychiatry. 2016 Oct;55(10):868-76.e2. doi: 10.1016/j.jaac.2016.06.015. PMID: 27663942.

56. Du L, Shan L, Wang B, et al. A Pilot Study on the Combination of Applied Behavior Analysis and Bumetanide Treatment for Children with Autism. J Child Adolesc Psychopharmacol. 2015 Sep;25(7):585-8. doi: 10.1089/cap.2015.0045. PMID: 26258842.

57. Cortesi F, Giannotti F, Sebastiani T, et al. Controlled-release melatonin, singly and combined with cognitive behavioural therapy, for persistent insomnia in children with autism spectrum disorders: a randomized placebo-controlled trial. J Sleep Res. 2012 Dec;21(6):700-9. doi: 10.1111/j.1365-2869.2012.01021.x. PMID: 22616853.

58. Navarro F, Pearson DA, Fatheree N, et al. Are 'leaky gut' and behavior associated with gluten and dairy containing diet in children with autism spectrum disorders? Nutr Neurosci. 2015 May;18(4):177-85. doi: 10.1179/1476830514y.0000000110. PMID: 24564346.

59. Pusponegoro HD, Ismael S, Firmansyah A, et al. Gluten and casein supplementation does not increase symptoms in children with autism spectrum disorder. Acta Paediatr. 2015 Nov;104(11):e500-e5. doi: 10.1111/apa.13108. PMID: 26148018.

60. Hyman SL, Stewart PA, Foley J, et al. The Gluten-Free/Casein-Free Diet: A DoubleBlind Challenge Trial in Children with Autism. J Autism Dev Disord. 2015 Sep 5doi: 10.1007/s10803-015-2564-9. PMID: 26343026.

61. Al-Ayadhi LY, Elamin NE. Camel Milk as a Potential Therapy as an Antioxidant in Autism Spectrum Disorder (ASD). Evid Based Complement Alternat Med. 2013;2013:602834. doi: 10.1155/2013/602834. PMID: 24069051.

62. Ghalichi F, Ghaemmaghami J, Malek A, et al. Effect of gluten free diet on gastrointestinal and behavioral indices for children with autism spectrum disorders: a 
randomized clinical trial. World J Pediatr. 2016 Jun 10doi: 10.1007/s12519-016-0040z. PMID: 27286693.

63. Johnson CR, Handen BL, Zimmer M, et al. Effects of gluten free / casein free diet in young children with autism: A pilot study. Journal of Developmental and Physical Disabilities. 2011 2015-12-09;23(3):213-25. doi: http://dx.doi.org/10.1007/s10882-0109217-x. PMID: 888750170; 2011-08445004.

64. Whiteley P, Haracopos D, Knivsberg AM, et al. The ScanBrit randomised, controlled, single-blind study of a gluten- and caseinfree dietary intervention for children with autism spectrum disorders. Nutr Neurosci. 2010 Apr;13(2):87-100. doi: 10.1179/147683010x12611460763922. PMID: 20406576.

65. Pedersen L, Parlar S, Kvist K, et al. Data mining the ScanBrit study of a gluten- and casein-free dietary intervention for children with autism spectrum disorders: behavioural and psychometric measures of dietary response. Nutr Neurosci. 2014

Sep;17(5):207-13. doi:

10.1179/1476830513y.0000000082. PMID: 24075141.

66. Sampanthavivat M, Singkhwa W, Chaiyakul $\mathrm{T}$, et al. Hyperbaric oxygen in the treatment of childhood autism: a randomised controlled trial. Diving Hyperb Med. 2012 Sep;42(3):128-33. PMID: 22987458.

67. Granpeesheh D, Tarbox J, Dixon DR, et al. Randomized trial of hyperbaric oxygen therapy for children with autism. Research in Autism Spectrum Disorders. 2010 April 2010/June;4(2):268-75. doi: http://dx.doi.org/10.1016/j.rasd.2009.09.014. PMID: 2010025300.

68. Wright B, Sims D, Smart S, et al. Melatonin versus placebo in children with autism spectrum conditions and severe sleep problems not amenable to behaviour management strategies: a randomised controlled crossover trial. J Autism Dev Disord. 2011 Feb;41(2):175-84. doi: 10.1007/s10803-010-1036-5. PMID: 20535539.
69. Amatachaya A, Jensen MP, Patjanasoontorn $\mathrm{N}$, et al. The short-term effects of transcranial direct current stimulation on electroencephalography in children with autism: a randomized crossover controlled trial. Behav Neurol. 2015;2015:928631. doi: 10.1155/2015/928631. PMID: 25861158.

70. Amatachaya A, Auvichayapat N, Patjanasoontorn N, et al. Effect of anodal transcranial direct current stimulation on autism: a randomized double-blind crossover trial. Behav Neurol. 2014;2014:173073. doi: 10.1155/2014/173073. PMID: 25530675.

71. Dadds MR, MacDonald E, Cauchi A, et al. Nasal oxytocin for social deficits in childhood autism: a randomized controlled trial. J Autism Dev Disord. 2014 Mar;44(3):521-31. doi: 10.1007/s10803013-1899-3. PMID: 23888359.

72. Lemonnier E, Degrez C, Phelep M, et al. A randomised controlled trial of bumetanide in the treatment of autism in children. Transl Psychiatry. 2012;2:e202. doi: 10.1038/tp.2012.124. PMID: 23233021.

73. Arnold LE, Aman MG, Hollway J, et al. Placebo-controlled pilot trial of mecamylamine for treatment of autism spectrum disorders. J Child Adolesc Psychopharmacol. 2012 Jun;22(3):198-205. doi: 10.1089/cap.2011.0056. PMID: 22537359.

74. Hollander E, Chaplin W, Soorya L, et al. Divalproex sodium vs placebo for the treatment of irritability in children and adolescents with autism spectrum disorders. Neuropsychopharmacology. 2010 Mar;35(4):990-8. doi: 10.1038/npp.2009.202. PMID: 20010551.

75. Aman MG, Findling RL, Hardan AY, et al. Safety and Efficacy of Memantine in Children with Autism: Randomized, Placebo-Controlled Study and Open-Label Extension. J Child Adolesc Psychopharmacol. 2016 Mar 15doi: 10.1089/cap.2015.0146. PMID: 26978327. 
76. Chugani DC, Chugani HT, Wiznitzer M, et al. Efficacy of Low-Dose Buspirone for Restricted and Repetitive Behavior in Young Children with Autism Spectrum Disorder: A Randomized Trial. J Pediatr. 2016 Mar;170:45-53.e1-4. doi: 10.1016/j.jpeds.2015.11.033. PMID: 26746121.

77. Handen BL, Johnson CR, McAuliffe-Bellin $\mathrm{S}$, et al. Safety and efficacy of donepezil in children and adolescents with autism: neuropsychological measures. J Child Adolesc Psychopharmacol. 2011 Feb;21(1):43-50. doi: 10.1089/cap.2010.0024. PMID: 21309696.

78. Hardan AY, Fung LK, Libove RA, et al. A randomized controlled pilot trial of oral $\mathrm{N}$ acetylcysteine in children with autism. Biol Psychiatry. 2012 Jun 1;71(11):956-61. doi: 10.1016/j.biopsych.2012.01.014. PMID: 22342106.

79. Dean OM, Gray KM, Villagonzalo KA, et al. A randomised, double blind, placebocontrolled trial of a fixed dose of $\mathrm{N}$-acetyl cysteine in children with autistic disorder. Aust N Z J Psychiatry. 2016 Jun 17doi: 10.1177/0004867416652735. PMID: 27316706.

80. Bent S, Hendren RL, Zandi T, et al. Internetbased, randomized, controlled trial of omega-3 fatty acids for hyperactivity in autism. J Am Acad Child Adolesc Psychiatry. 2014 Jun;53(6):658-66. doi: 10.1016/j.jaac.2014.01.018. PMID: 24839884.

81. Bent S, Bertoglio K, Ashwood P, et al. A pilot randomized controlled trial of omega-3 fatty acids for autism spectrum disorder. $\mathrm{J}$ Autism Dev Disord. 2011 May;41(5):54554. doi: 10.1007/s10803-010-1078-8. PMID: 20683766.

82. Voigt RG, Mellon MW, Katusic SK, et al. Dietary docosahexaenoic acid supplementation in children with autism. J Pediatr Gastroenterol Nutr. 2014 Jun;58(6):715-22. doi: 10.1097/mpg.0000000000000260. PMID: 24345834.
83. Bertoglio K, Jill James S, Deprey L, et al. Pilot study of the effect of methyl B12 treatment on behavioral and biomarker measures in children with autism. J Altern Complement Med. 2010 May;16(5):555-60. doi: 10.1089/acm.2009.0177. PMID: 20804367.

84. Mankad D, Dupuis A, Smile S, et al. A randomized, placebo controlled trial of omega-3 fatty acids in the treatment of young children with autism. Mol Autism. 2015;6:18. doi: 10.1186/s13229-015-00107. PMID: 25798215.

85. Saad K, Eltayeb AA, Mohamad IL, et al. A Randomized, Placebo-controlled Trial of Digestive Enzymes in Children with Autism Spectrum Disorders. Clin Psychopharmacol Neurosci. 2015 Aug 31;13(2):188-93. doi: 10.9758/cpn.2015.13.2.188. PMID: 26243847.

86. Fahmy SF, El-hamamsy MH, Zaki OK, et al. L-Carnitine supplementation improves the behavioral symptoms in autistic children. Research in Autism Spectrum Disorders. 2013;7(1):159-66. doi: http://dx.doi.org/10.1016/j.rasd.2012.07.006. PMID: 1267033812; 2012-29769-018.

87. Hendren RL, James SJ, Widjaja F, et al. Randomized, Placebo-Controlled Trial of Methyl B12 for Children with Autism. J Child Adolesc Psychopharmacol. 2016 Feb 18doi: 10.1089/cap.2015.0159. PMID: 26889605.

88. Geier DA, Kern JK, Davis G, et al. A prospective double-blind, randomized clinical trial of levocarnitine to treat autism spectrum disorders. Med Sci Monit. 2011 Jun;17(6):Pi15-23. PMID: 21629200.

89. Ghaleiha A, Alikhani R, Kazemi MR, et al. Minocycline as Adjunctive Treatment to Risperidone in Children with Autistic Disorder: A Randomized, Double-Blind Placebo-Controlled Trial. J Child Adolesc Psychopharmacol. 2016 Apr 29doi: 10.1089/cap.2015.0175. PMID: 27128958. 
90. Nikoo M, Radnia H, Farokhnia M, et al. Nacetylcysteine as an adjunctive therapy to risperidone for treatment of irritability in autism: a randomized, double-blind, placebo-controlled clinical trial of efficacy and safety. Clin Neuropharmacol. 2015 JanFeb;38(1):11-7. doi: 10.1097/wnf.0000000000000063. PMID: 25580916.

91. Ghaleiha A, Mohammadi E, Mohammadi $\mathrm{MR}$, et al. Riluzole as an adjunctive therapy to risperidone for the treatment of irritability in children with autistic disorder: a doubleblind, placebo-controlled, randomized trial. Paediatr Drugs. 2013 Dec;15(6):505-14. doi: 10.1007/s40272-013-0036-2. PMID: 23821414.

92. Ghanizadeh A, Ayoobzadehshirazi A. A randomized double-blind placebo-controlled clinical trial of adjuvant buspirone for irritability in autism. Pediatr Neurol. 2015 Jan;52(1):77-81. doi: 10.1016/j.pediatrneurol.2014.09.017. PMID: 25451017.

93. Ghanizadeh A, Moghimi-Sarani E. A randomized double blind placebo controlled clinical trial of N-Acetylcysteine added to risperidone for treating autistic disorders. BMC Psychiatry. 2013;13:196. doi: 10.1186/1471-244x-13-196. PMID: 23886027.

94. Asadabadi M, Mohammadi MR, Ghanizadeh A, et al. Celecoxib as adjunctive treatment to risperidone in children with autistic disorder: a randomized, doubleblind, placebo-controlled trial.

Psychopharmacology (Berl). 2013 Jan;225(1):51-9. doi: 10.1007/s00213-0122796-8. PMID: 22782459.

95. Ghaleiha A, Asadabadi M, Mohammadi $\mathrm{MR}$, et al. Memantine as adjunctive treatment to risperidone in children with autistic disorder: a randomized, doubleblind, placebo-controlled trial. Int J Neuropsychopharmacol. 2013 May;16(4):783-9. doi: 10.1017/s1461145712000880. PMID: 22999292.
96. Mohammadi MR, Yadegari N, Hassanzadeh E, et al. Double-blind, placebo-controlled trial of risperidone plus amantadine in children with autism: a 10-week randomized study. Clin Neuropharmacol. 2013 NovDec;36(6):179-84. doi: 10.1097/WNF.0b013e3182a9339d. PMID: 24201232.

97. Hasanzadeh E, Mohammadi MR, Ghanizadeh A, et al. A double-blind placebo controlled trial of Ginkgo biloba added to risperidone in patients with autistic disorders. Child Psychiatry Hum Dev. 2012 Oct;43(5):674-82. doi: 10.1007/s10578-0120292-3. PMID: 22392415.

98. Rezaei V, Mohammadi MR, Ghanizadeh A, et al. Double-blind, placebo-controlled trial of risperidone plus topiramate in children with autistic disorder. Prog Neuropsychopharmacol Biol Psychiatry. 2010 Oct 1;34(7):1269-72. doi: 10.1016/j.pnpbp.2010.07.005. PMID: 20637249.

99. Ghaleiha A, Rasa SM, Nikoo M, et al. A pilot double-blind placebo-controlled trial of pioglitazone as adjunctive treatment to risperidone: Effects on aberrant behavior in children with autism. Psychiatry Res. 2015 Sep 30;229(1-2):181-7. doi: 10.1016/j.psychres.2015.07.043. PMID: 26208985.

100. Ghaleiha A, Ghyasvand M, Mohammadi MR, et al. Galantamine efficacy and tolerability as an augmentative therapy in autistic children: A randomized, doubleblind, placebo-controlled trial. J Psychopharmacol. 2013 Oct 15;28(7):67785. doi: $10.1177 / 0269881113508830$. PMID: 24132248.

101. Miral S, Gencer O, Inal-Emiroglu FN, et al. Risperidone versus haloperidol in children and adolescents with AD : a randomized, controlled, double-blind trial. Eur Child Adolesc Psychiatry. 2008 Feb;17(1):1-8. doi: 10.1007/s00787-007-0620-5 [doi]. PMID: 18080171.

102. Troost PW, Lahuis BE, Steenhuis MP, et al. Long-term effects of risperidone in children with autism spectrum disorders: a placebo 
discontinuation study. J Am Acad Child Adolesc Psychiatry. 2005 Nov;44(11):113744. doi: 00004583-200511000-00009 [pii]. PMID: 16239862.

103. Danfors T, von Knorring AL, Hartvig P, et al. Tetrahydrobiopterin in the treatment of children with autistic disorder: a doubleblind placebo-controlled crossover study. J Clin Psychopharmacol. 2005 Oct;25(5):4859. doi: 00004714-200510000-00015 [pii]. PMID: 16160627.

104. Knivsberg AM, Reichelt KL, Hoien T, et al. A randomised, controlled study of dietary intervention in autistic syndromes. Nutr Neurosci. 2002 Sep;5(4):251-61. PMID: 12168688 .

105. Knivsberg A-M, Reichelt K-L, HÃ ,ien T, et al. Effect of a Dietary Intervention on Autistic Behavior. Focus on Autism and Other Developmental Disabilities. 2003 Win;18(4):247-56.

106. Harrison Elder J, Shankar M, Shuster J, et al. The Gluten-Free, Casein-Free Diet In Autism: Results of A Preliminary Double Blind Clinical Trial. Journal of Autism and Developmental Disorders. 2006 Apr;36(3):413-20. PMID: 2006-07517-011.

107. Seung H, Rogalski Y, Shankar M, et al. The gluten- and casein-free diet and autism: Communication outcomes from a preliminary double-blind clinical trial. Journal of Medical Speech-Language Pathology. 2007 Dec;15(4):337-45.

108. Wasserman S, Iyengar R, Chaplin WF, et al. Levetiracetam versus placebo in childhood and adolescent autism: a double-blind placebo-controlled study. Int Clin Psychopharmacol. 2006 Nov;21(6):363-7. doi: 10.1097/01.yic.0000224787.13782.0f [doi] 00004850-200611000-00006 [pii]. PMID: 17012983.

109. Nagaraj R, Singhi P, Malhi P. Risperidone in children with autism: randomized, placebo-controlled, double-blind study. J Child Neurol. 2006 Jun;21(6):450-5. PMID: 16948927.
110. Levine SZ, Kodesh A, Goldberg Y, et al. Initial severity and efficacy of risperidone in autism: Results from the RUPP trial. Eur Psychiatry. 2016 Feb;32:16-20. doi: 10.1016/j.eurpsy.2015.11.004. PMID: 26802979.

111. Anderson GM, Scahill L, McCracken JT, et al. Effects of short- and long-term risperidone treatment on prolactin levels in children with autism. Biol Psychiatry. 2007 Feb 15;61(4):545-50. doi: S00063223(06)00384-2 [pii] 10.1016/j.biopsych.2006.02.032 [doi]. PMID: 16730335.

112. Shea S, Turgay A, Carroll A, et al. Risperidone in the treatment of disruptive behavioral symptoms in children with autistic and other pervasive developmental disorders. Pediatrics. 2004

Nov;114(5):e634-41. doi: peds.2003-0264-F [pii] 10.1542/peds.2003-0264-F [doi]. PMID: 15492353.

113. Pandina GJ, Bossie CA, Youssef E, et al. Risperidone improves behavioral symptoms in children with autism in a randomized, double-blind, placebo-controlled trial. J Autism Dev Disord. 2007 Feb;37(2):367-73. doi: 10.1007/s10803-006-0234-7 [doi]. PMID: 17019624.

114. Rossignol DA, Rossignol LW, Smith S, et al. Hyperbaric treatment for children with autism: a multicenter, randomized, doubleblind, controlled trial. BMC Pediatr. 2009;9:21. doi: 1471-2431-9-21 [pii] 10.1186/1471-2431-9-21 [doi]. PMID: 19284641.

115. King BH, Wright DM, Handen BL, et al. Double-blind, placebo-controlled study of amantadine hydrochloride in the treatment of children with autistic disorder. J Am Acad Child Adolesc Psychiatry. 2001 Jun;40(6):658-65. PMID: 11392343.

116. Chez MG, Buchanan TM, Becker M, et al. Donepezil hydrochloride: A double-blind study in autistic children. Journal of Pediatric Neurology. 2003 Oct-Dec;1(2):838. PMID: 2004-12732-002. 
117. Munasinghe SA, Oliff C, Finn J, et al. Digestive enzyme supplementation for autism spectrum disorders: a double-blind randomized controlled trial. J Autism Dev Disord. 2010 Sep;40(9):1131-8. doi: 10.1007/s10803-010-0974-2. PMID: 20204691.

118. Akhondzadeh S, Fallah J, Mohammadi MR, et al. Double-blind placebo-controlled trial of pentoxifylline added to risperidone: effects on aberrant behavior in children with autism. Prog Neuropsychopharmacol Biol Psychiatry. 2010 Feb 1;34(1):32-6. doi: 10.1016/j.pnpbp.2009.09.012. PMID: 19772883.

119. Selim ME, Al-Ayadhi LY. Possible ameliorative effect of breastfeeding and the uptake of human colostrum against coeliac disease in autistic rats. World $\mathrm{J}$ Gastroenterol. 2013 Jun 7;19(21):3281-90. doi: 10.3748/wjg.v19.i21.3281. PMID: 23745030 .

120. Lv YT, Zhang Y, Liu M, et al. Transplantation of human cord blood mononuclear cells and umbilical cordderived mesenchymal stem cells in autism. $\mathrm{J}$ Transl Med. 2013;11:196. doi: 10.1186/1479-5876-11-196. PMID: 23978163.

121. Sun C, Zou M, Zhao D, et al. Efficacy of Folic Acid Supplementation in Autistic Children Participating in Structured Teaching: An Open-Label Trial. Nutrients. 2016;8(6)doi: 10.3390/nu8060337. PMID: 27338456.

122. Wink LK, Early M, Schaefer T, et al. Body mass index change in autism spectrum disorders: comparison of treatment with risperidone and aripiprazole. J Child Adolesc Psychopharmacol. 2014 Mar;24(2):78-82. doi: 10.1089/cap.2013.0099. PMID: 24564519.

123. Duffy FH, Shankardass A, McAnulty GB, et al. Corticosteroid therapy in regressive autism: a retrospective study of effects on the Frequency Modulated Auditory Evoked Response (FMAER), language, and behavior. BMC Neurol. 2014;14:70. doi:
10.1186/1471-2377-14-70. PMID: 24885033.

124. Akhondzadeh S, Tajdar H, Mohammadi MR, et al. A double-blind placebo controlled trial of piracetam added to risperidone in patients with autistic disorder. Child Psychiatry Hum Dev. 2008 Sep;39(3):23745. doi: 10.1007/s10578-007-0084-3 [doi]. PMID: 17929164.

125. Scarpa A, Reyes NM. Improving emotion regulation with $\mathrm{CBT}$ in young children with high functioning autism spectrum disorders: a pilot study. Behav Cogn Psychother. 2011 Jul;39(4):495-500. doi: 10.1017/s1352465811000063. PMID: 21457605.

126. Aman MG, McDougle CJ, Scahill L, et al. Medication and Parent Training in Children With Pervasive Developmental Disorders and Serious Behavior Problems: Results From a Randomized Clinical Trial. J Am Acad Child Adolesc Psychiatry. 2009 Oct 23doi: 10.1097/CHI.0b013e3181bfd669 [doi]. PMID: 19858761.

127. Cunningham AB. Measuring change in social interaction skills of young children with autism. J Autism Dev Disord. 2012 Apr;42(4):593-605. doi: 10.1007/s10803011-1280-3. PMID: 21638109.

128. Kern JK, Miller VS, Cauller PL, et al. Effectiveness of N,N-dimethylglycine in autism and pervasive developmental disorder. J Child Neurol. 2001 Mar;16(3):169-73. PMID: 11305684.

129. Hong ER, Neely L, Rispoli MJ, et al. A comparison of general and explicit delay cues to reinforcement for tangiblemaintained challenging behaviour. Dev Neurorehabil. 2015 Aug 24:1-7. doi: 10.3109/17518423.2013.874378. PMID: 24392663.

130. Barnard-Brak L, Thompson S, Wei T, et al. Assistive technology as a predictor of general or alternate assessment among elementary-aged students with autism spectrum disorders. Assist Technol. 2014 Summer;26(2):81-7. doi: 
10.1080/10400435.2013.833557. PMID: 25112052.

131. Goldman S, DeNigris D. Parents' strategies to elicit autobiographical memories in autism spectrum disorders, developmental language disorders and typically developing children. J Autism Dev Disord. 2015 May;45(5):1464-73. doi: 10.1007/s10803014-2271-y. PMID: 25312278.

132. Zeedyk SM, Cohen SR, Eisenhower A, et al. Perceived social competence and loneliness among young children with ASD: Child, parent and teacher reports. Journal of Autism and Developmental Disorders. 2016;46(2):436-49. doi: http://dx.doi.org/10.1007/s10803-015-25756.

133. Ching H, Pringsheim T. Aripiprazole for autism spectrum disorders (ASD). Cochrane Database of Systematic Reviews. 2012(5).

134. Deb S, Farmah BK, Arshad E, et al. The effectiveness of aripiprazole in the management of problem behaviour in people with intellectual disabilities, developmental disabilities and/or autistic spectrum disorder--a systematic review. Res Dev Disabil. 2014 Mar;35(3):711-25. doi: 10.1016/j.ridd.2013.12.004. PMID: 24405794.

135. Sharma A, Shaw SR. Efficacy of Risperidone in Managing Maladaptive Behaviors for Children With Autistic Spectrum Disorder: A Meta-Analysis. Journal of Pediatric Healthcare.

2012;26(4):291-9 9p. doi:

10.1016/j.pedhc.2011.02.008.

136. Fung LK, Mahajan R, Nozzolillo A, et al. Pharmacologic Treatment of Severe Irritability and Problem Behaviors in Autism: A Systematic Review and Metaanalysis. Pediatrics. 2016 Feb;137 Suppl 2:S124-35. doi: 10.1542/peds.2015-2851K. PMID: 26908468.

137. Siegel M, Beaulieu A. Psychotropic Medications in Children with Autism Spectrum Disorders: A Systematic Review and Synthesis for Evidence-Based Practice.
Journal of Autism \& Developmental Disorders. 2012;42(8):1592-605 14p. doi: 10.1007/s10803-011-1399-2.

138. Reichow B, Volkmar FR, Bloch MH. Systematic review and meta-analysis of pharmacological treatment of the symptoms of attention-deficit/hyperactivity disorder in children with pervasive developmental disorders. J Autism Dev Disord. 2013 Oct;43(10):2435-41. doi: 10.1007/s10803013-1793-z. PMID: 23468071.

139. Hirota T, Veenstra-VanderWeele J, Hollander E, et al. Antiepileptic Medications in Autism Spectrum Disorder: A Systematic Review and Meta-Analysis. Journal of Autism \& Developmental Disorders. 2014;44(4):948-57 10p. doi: 10.1007/s10803-013-1952-2.

140. Rossignol DA, Frye RE. The use of medications approved for Alzheimer's disease in autism spectrum disorder: a systematic review. Front Pediatr. 2014;2:87. doi: 10.3389/fped.2014.00087. PMID: 25202686.

141. Masi A, Lampit A, Glozier N, et al. Predictors of placebo response in pharmacological and dietary supplement treatment trials in pediatric autism spectrum disorder: a meta-analysis. Transl Psychiatry. 2015;5:e640. doi: 10.1038/tp.2015.143. PMID: 26393486.

142. Ghanizadeh A. Atomoxetine for treating ADHD symptoms in autism: a systematic review. J Atten Disord. 2013

Nov;17(8):635-40. doi: 10.1177/1087054712443154. PMID: 22544388.

143. Ghanizadeh A. Hyperbaric oxygen therapy for treatment of children with autism: a systematic review of randomized trials. Med Gas Res. 2012;2:13. doi: 10.1186/20459912-2-13. PMID: 22577817.

144. Williams K, Brignell A, Randall M, et al. Selective serotonin reuptake inhibitors (SSRIs) for autism spectrum disorders (ASD). Cochrane Database of Systematic Reviews. 2013(8). 
145. James S, Montgomery P, Williams K. Omega-3 fatty acids supplementation for autism spectrum disorders (ASD). Cochrane Database Syst Rev. 2011(11):Cd007992. doi: 10.1002/14651858.CD007992.pub2. PMID: 22071839.

146. Mari-Bauset S, Zazpe I, Mari-Sanchis A, et al. Evidence of the gluten-free and caseinfree diet in autism spectrum disorders: a systematic review. J Child Neurol. 2014 Dec;29(12):1718-27. doi: 10.1177/0883073814531330. PMID: 24789114 .
147. Rossignol DA, Frye RE. Melatonin in autism spectrum disorders: a systematic review and meta-analysis. Dev Med Child Neurol. 2011 Sep;53(9):783-92. doi: 10.1111/j.1469-8749.2011.03980.x. PMID: 21518346.

148. James S, Stevenson SW, Silove N, et al. Chelation for autism spectrum disorder (ASD). Cochrane Database Syst Rev. 2015 May 11;5:Cd010766. doi: 10.1002/14651858.CD010766.pub2. PMID: 26114777. 


\section{Acronyms and Abbreviations}

\begin{tabular}{|c|c|}
\hline$A B C$ & Aberrant Behavior Checklist \\
\hline ADHD & attention deficit hyperactivity disorder \\
\hline ADOS & Autism Diagnostic Observation Schedule \\
\hline AHRQ & Agency For Healthcare Research And Quality \\
\hline ASD & Autism Spectrum Disorder \\
\hline ATEC & Autism Treatment Evaluation Checklist \\
\hline BASC & Behavior Assessment System for Children \\
\hline BMI & Body Mass Index \\
\hline CARS & Childhood Autism Rating Scale \\
\hline CBT & Cognitive Behavioral Therapy \\
\hline $\mathrm{CCC}$ & Children's Communication Checklist \\
\hline CER & Comparative Effectiveness Review \\
\hline CGAS & Children's Global Assessment Scale \\
\hline CGI & Clinical Global Impression \\
\hline $\mathrm{Cl}$ & Confidence Interval \\
\hline CSBQ & Children's Social Behavior Questionnaire \\
\hline CTRS & Connor's Teacher Rating Scales \\
\hline CYBOCS & Children's Yale-Brown Obsessive Compulsive Scale \\
\hline DHA & Docosahexanoic Acid \\
\hline DSM-IV & Diagnostic Statistical Manual - IV \\
\hline EEG & Electroencephalogram \\
\hline EOT & End of Treatment \\
\hline EPS & Extrapyramidal symptoms \\
\hline EVT & Expressive Vocabulary Test \\
\hline ES & Effect Size \\
\hline FDA & Food and Drug Administration \\
\hline FFA & Free Fatty Acid \\
\hline G & Group \\
\hline GFCF & Gluten Free, Casein Free (Diet) \\
\hline IQ & Intelligence Quotient \\
\hline $\mathrm{kg}$ & Kilograms \\
\hline $\mathrm{KQ}$ & Key Question \\
\hline $\mathrm{mg}$ & Milligram \\
\hline MPH & Methylphenidate \\
\hline $\mathrm{n}$ & Number \\
\hline NA & Not Applicable \\
\hline ND & No Data \\
\hline NR & Not Reported \\
\hline ns & Not Significant \\
\hline ODD & Oppositional Defiant Disorder \\
\hline $\mathrm{OT} / \mathrm{SI}$ & Occupational Therapy With Sensory Integration \\
\hline PDD & Pervasive Developmental Disorder \\
\hline PDD-BI & Pervasive Development Disorder Behavioral Inventory \\
\hline PDD-NOS & Pervasive Developmental Disorder - Not Otherwise Specified \\
\hline PICOTS & Population, Intervention, Comparator, Outcome, Timing, Setting \\
\hline PLS & Preschool Language Scale \\
\hline PPVT & Peabody Picture Vocabulary Test \\
\hline RBS & Repetitive Behavior Scale \\
\hline RCT & Randomized, Controlled Trial \\
\hline ROB & Risk of Bias \\
\hline $\mathrm{RR}$ & Risk Ratio \\
\hline RUPP & Research Units on Pediatric Psychopharmacology \\
\hline SD & Standard Deviation \\
\hline SNAP-IV & Swanson Nolan and Pelham rating scale \\
\hline SOE & Strength of Evidence \\
\hline
\end{tabular}




\begin{tabular}{|l|l|}
\hline SRS & Social Responsiveness Scale \\
\hline SSRI & Selective Serotonin Reuptake Inhibitors \\
\hline TEACCH & $\begin{array}{l}\text { Treatment and Education of Autistic and Communication related handicapped } \\
\text { CHildren }\end{array}$ \\
\hline TEP & Technical Expert Panel \\
\hline VABS & Vineland Adaptive Behavior Scales \\
\hline
\end{tabular}




\section{Appendix A. Search Strategies}

Table A-1. Treatment/intervention

Interface: PubMed; Database: Medline

\begin{tabular}{|l|l|r|}
\hline & Search & Records \\
\hline 1 & "Child Development Disorders, Pervasive"[Mesh] & 22690 \\
\hline 2 & $\begin{array}{l}\text { (autistic[tiab] OR autism[tiab] OR asperger[tiab] OR asperger's[tiab] OR aspergers[tiab] OR } \\
\text { pervasive development[tiab] OR pervasive developmental[tiab] OR pdd[tiab]) NOT medline[sb] }\end{array}$ & 5613 \\
\hline 3 & \#1 OR \#2 & 28303 \\
\hline 4 & therapy[sh] OR therapeutics[mh] OR psychotherapy[mh] OR treatment outcome[mh] & 7271756 \\
\hline 5 & $\begin{array}{l}\text { (treatment[tiab] OR therapy[tiab] OR intervention[tiab] OR "control group"[tiab] OR } \\
\text { randomized[tiab] OR outcome[tiab] OR randomized[tiab] OR efficacy[tiab] OR effectiveness[tiab] } \\
\text { OR comparison[tiab] OR compared[tiab] OR trial[tiab] OR "pilot study"[tiab] ) NOT medline[sb] }\end{array}$ & 794459 \\
\hline 6 & \#4 OR \#5 & 8058741 \\
\hline 7 & \#3 AND \#6 & 9859 \\
\hline 8 & $\begin{array}{l}\text { (newspaper article[pt] OR comment[pt] OR case reports[pt] OR review[pt] OR practice guideline[pt] } \\
\text { published erratum[pt] OR congresses[pt]) }\end{array}$ & 5128288 \\
\hline 9 & \#7 NOT \#8 & 6801 \\
\hline 10 & \#9 limited to ("2010/01/01"[Date - Publication] : "3000"[Date - Publication]) \\
\hline $\begin{array}{l}\text { Key: [mh] Medical Subject Heading; [tiab] title/abstract word; [pt] publication type; [sh] subheading; [dp] publication date; [la] } \\
\text { language; [pt] publication type. }\end{array}$ & 3894 \\
\hline
\end{tabular}

Table A-2. Medication

Interface: EBSCOhost; Database: CINAHL

\begin{tabular}{|l|l|r|}
\hline & Search & Records \\
\hline 1 & (MH "Autistic Disorder") & 10,856 \\
\hline 2 & (MH "Child Development Disorders") OR (MH "Child Development Disorders, Pervasive") & 2,067 \\
\hline 3 & "autism" & 8,856 \\
\hline 4 & \#2 AND \#3 & 511 \\
\hline 5 & \#1 OR \#4 & 11,134 \\
\hline 6 & (MH "Psychopharmacology") OR (MH "Central Nervous System Agents+") & 92,105 \\
\hline 7 & (MM "Autistic Disorder/DT") & 237 \\
\hline 8 & \#6 OR \#7 & 92,210 \\
\hline 9 & \#5 AND \#8 & 493 \\
\hline 10 & \#9 limited to 2010-2015 & 215 \\
\hline 11 & \#10 limited to English language & 214 \\
\hline
\end{tabular}


Table A-3. Medication

Interface: OVID; Database: EMBASE

\begin{tabular}{|r|l|r|}
\hline & Search & Results \\
\hline 1 & exp autism/ & 43068 \\
\hline 2 & exp autism/dt [Drug Therapy] & 2497 \\
\hline 3 & exp *autism/dt [Drug Therapy] & 1766 \\
\hline 4 & exp central nervous system agents/ & 1386129 \\
\hline 5 & exp *entral nervous system agents/ & 734471 \\
\hline 6 & exp *autism/ & 28323 \\
\hline 7 & 5 and 6 & 1213 \\
\hline 8 & 3 or 7 & 2307 \\
\hline 9 & limit 8 to (english language and yr="2010") & 116 \\
\hline
\end{tabular}




\section{Appendix B. Screening and Risk of Bias Assessment Forms}

\section{Medical and Sensory-Related Therapies for Children with Autism Spectrum Disorder Abstract Review Form}

1. Addresses intervention approach and outcomes for young children (2-12 years) with ASD.

$\square$ Yes $\square$ No $\square$ Cannot Determine

If answer to question 1 is $\mathrm{NO}$, this form is complete. Please submit and proceed to next abstract.

2. Original research (does not include systematic reviews and meta-analyses)

$\square$ Yes $\square$ No $\square$ Cannot Determine

3. Is this a comparative study (includes a treatment and comparison group)?

$\square$ Yes $\square$ No $\square$ Cannot Determine

\section{Addresses one of the following:}

$\square$ Behavioral intervention involving training parents

$\square$ Sensory or auditory-focused intervention (e.g., sensory or auditory integration, weighted vest, therapeutic swinging, snoezelen room)

$\square$ Medical/pharmacologic intervention, including vitamins/supplements, hyperbaric oxygen, electroconvulsive therapy, transcranial magnetic stimulation

$\square$ Music therapy

$\square$ Educational intervention

$\square$ Complementary and alternative medicine (acupuncture, massage, etc.)

$\square$ Allied health intervention (non-sensory/auditory-related such as language, exercise, animalassisted)

$\square$ Other behavioral intervention (e.g., social skills, CBT, early intensive intervention)

$\square$ Other

$\square$ Severe/challenging behavior (e.g., elopement, property destruction, self/other injury, severe aggression)

$\square$ Cannot determine

5. Eligible study size (at least 10 total participants in target population)

$\square$ Yes $\square$ No $\square$ Cannot Determine

5a. Record total N with ASD: 


\section{Medical and Sensory-Related Therapies for Children with Autism Spectrum Disorder Full Text Review Form}

1. Study population is children with autism between the ages of 2 and 12 years (mean+SD $\leq 12$ yrs, 11 months)

$\square$ Yes $\square$ No $\square$ Cannot Determine

2. Original research (does not include systematic reviews and meta-analyses)

$\square$ Yes $\square$ No $\square$ Cannot Determine

3. Is this a comparative study (includes a treatment and comparison group)?

$\square$ Yes $\square$ No $\square$ Cannot Determine

4. Does this study address:

$\square$ Medical intervention

$\square$ Sensory intervention

$\square$ Other intervention

$\square$ Not an intervention study

5. Eligible study size (at least 10 total participants in RCT; 20 total participants in target population for observational studies)

$\square$ Yes $\square$ No $\square$ Cannot Determine

5a. Record total N with ASD:

6. Reports an outcome of interest for individuals with ASD:

$\square$ Yes $\square$ No $\square$ Cannot Determine

Comments:

If excluded, retain for review of references or background/contextual questions?

$\square$ Background $\quad \square$ Review of References $\quad \square$ Other 


\section{Medical and Sensory-Related Therapies for Children with Autism Spectrum Disorder Risk of Bias Form}

1. Did the study employ a group design?

$\square$ Yes $\square$ No

2. Were the groups randomly assigned?

$\square$ Yes $\square$ No $\square$ Comments

3. Was there an appropriate comparison group?

$\square$ Yes $\square$ No or NR $\square$ Comments

4. If an RCT, was randomization done correctly?

$\square$ Yes $\square$ No $\square$ NR $\square$ NA (non-RCT)

$\square$ Comments

5. Was a valid diagnostic approach for ASD used within the study, or were referred participants diagnosed using a valid approach?

$\square$ A. clinical DSM-IV/5-based diagnosis + ADI-R and/or ADOS

$\square$ B. [clinical DSM-IV/5-based diagnosis + other] OR [ADOS + other, such as SRS, CARS, SCQ, CAST, ASSQ, OR STAT, MCHAT for under 30 months]

$\square$ C. Only clinical DSM-IV/5-based diagnosis OR Only ADOS

$\square$ D. Neither clinical DSM-IV/5-based diagnosis NOR ADOS

$\square$ Comments

6. Was the sample clearly characterized (e.g., information provided to characterize participants in terms of impairments associated with their ASD, such as cognitive or developmental level)?

$\square$ Yes $\square$ No or NR $\square$ Comments

7. Were inclusion and exclusion criteria clearly stated?

$\square$ Yes $\square$ No or NR $\square$ Comments

8. Do the authors report attrition?

$\square$ Yes $\square$ No $\square$ Comments

9. Were characteristics of drop-out group evaluated for differences with the participant group as a whole? 
$\square$ Yes $\square$ No or NR $\square$ NA or minimal attrition

$\square$ Comments

10. Was the intervention fully described?

$\square$ Yes $\square$ No or NR $\square$ Comments

11. For behavioral/non-medical studies, was treatment fidelity monitored in a systematic way?

$\square$ Yes $\square$ No or NR $\square$ NA

$\square$ Comments

12. Did the authors measure and report adherence to the intended treatment process?

$\square$ Yes $\square$ No or NR $\square$ Comments

13. Did the authors report differences in or hold steady all concomitant interventions?

$\square$ Yes $\square$ No or NR $\square$ Comments

14. Did outcome measures demonstrate adequate reliability and validity (including interobserver reliability for behavior observation coding)?

$\square$ Yes $\square$ No or NR $\square$ Comments

15. Were the primary \& secondary outcomes clearly specified a priori?

$\square$ Yes $\square$ No or NR $\square$ Comments

16. Were outcome data collected from sources appropriate to the target outcome (e.g. parent report, teacher report, direct behavior observation)?

$\square$ Yes $\square$ No or NR $\square$ Comments

17. Were outcomes coded by individuals blinded to the intervention status of the participants?

$\square$ Yes $\square$ No or NR $\square$ Comments

18. Was an appropriate statistical analysis used?

$\square$ Yes $\square$ No $\square$ Comments

19. a. For RCTs, was there an intent-to-treat analysis? 
$\square$ Yes $\square$ No $\square$ NA

$\square$ Comments

20. b. For negative studies, was a power calculation provided?

$\square$ Yes $\square$ No $\square$ NA

$\square$ Comments

21. c. Did the study correct for multiple testing?

$\square$ Yes $\square$ No $\square$ NA

$\square$ Comments

22. d. For observational studies, were potential confounders and effect measure modifiers captured?

$\square$ Yes $\square$ No $\square$ NA

$\square$ Comments

23. e. For observational studies, were potential confounders and effect measure modifiers handled appropriately?

$\square$ Yes $\square$ No $\square$ NA

$\square$ Comments

24. Were outcomes measured in at least one context outside of the treatment setting?

$\square$ Yes $\square$ No or NR $\square$ Comments

25. Were outcomes measured in natural environments to assess generalization?

$\square$ Yes $\square$ No or NR $\square$ Comments

26. Were follow-up measures of outcome conducted to assess maintenance of skills at least 3 months after the end of treatment?

$\square$ Yes $\square$ No or NR $\square$ NA

$\square$ Comments

27. Comments 


\title{
Appendix C. Excluded Studies
}

\author{
Reasons for Exclusion \\ X-1 Does not address interventions or outcomes of interest \\ $\mathrm{X}-2 \quad$ Not original research \\ $\mathrm{X}-3 \quad$ Does not include an appropriate comparison group \\ X-4 Does not meet sample size criterion \\ X-5 Not in English or not obtainable
}

1. Finucane BM, Lusk L, Arkilo D, et al. 15q Duplication Syndrome and Related 2Disorders. In: Pagon RA, Adam MP, Ardinger $\mathrm{HH}$, Wallace SE, Amemiya A, Bean LJH, et al., eds. GeneReviews(R). Seattle (WA): University of Washington, Seattle University of Washington, Seattle. All rights reserved.; 1993.

2. Humphries TW, Snider L, McDougall B. Clinical evaluation of the effectiveness of sensory integrative and perceptual motor therapy in improving sensory integrative function in children with learning disabilities. Occupational Therapy Journal of Research. 1993 2015-04-15;13(3):163-82. PMID: 618426376; 1994-03315-001.X-1

3. Mefford H, Mitchell E, Hodge J. 17q12 Recurrent Duplication. In: Pagon RA, Adam MP, Ardinger HH, Wallace SE, Amemiya A, Bean LJH, et al., eds. GeneReviews(R). Seattle (WA): University of Washington, Seattle University of Washington, Seattle. All rights reserved.; 1993.

4. Morgan A, Fisher SE, Scheffer I, et al. FOXP2-Related Speech and Language Disorders. In: Pagon RA, Adam MP, Ardinger HH, Wallace SE, Amemiya A, Bean LJH, et al., eds. GeneReviews(R). Seattle (WA): University of Washington, Seattle University of Washington, Seattle. All rights reserved.; 1993.

5. Van Dijck A, Helsmoortel C, Vandeweyer G, et al. ADNP-Related Intellectual Disability and Autism Spectrum Disorder. In: Pagon RA, Adam MP, Ardinger HH, Wallace SE, Amemiya A, Bean LJH, et al., eds. GeneReviews(R). Seattle (WA): University of Washington, Seattle University of Washington, Seattle. All rights reserved.; 1993.

6. Buday EM. The effects of signed and spoken words taught with music on sign and speech imitation by children with autism. Journal of Music Therapy. 1995 2016-0429;32(3):189-202. doi: http://dx.doi.org/10.1093/jmt/32.3.189. PMID: 618913263; 1996-14715-001.X-3, X-4

7. Davidson T, Williams B. Occupational therapy for children with developmental coordination disorder: A study of the effectiveness of a combined sensory integration and perceptual-motor intervention. The British Journal of Occupational Therapy. 2000 201605-11;63(10):495-9. doi: http://dx.doi.org/10.1177/030802260006301007 . PMID: 619548016; 2000-16436-006.X-1

8. Adams JB, Holloway C. Pilot study of a moderate dose multivitamin/mineral supplement for children with autistic spectrum disorder. J Altern Complement Med. 2004 Dec;10(6):1033-9. doi: 10.1089/acm.2004.10 .1033 [doi]. PMID: 15673999.X-1

9. Niederhofer H. Venlafaxine has modest effects in autistic children. Therapy. 2004 2004/09/01;1(1):87-90. doi: 10.2217/14750708.1.1.87.X-1

10. Seltzer MM, Shattuck P, Abbeduto L, et al. Trajectory of development in adolescents and adults with autism. Ment Retard Dev Disabil Res Rev. 2004;10(4):234-47. doi: 10.1002/mrdd.20038. PMID: 15666341.X-4 BACKGROUND REFERENCES-checked 08192011

11. John MECfCD, Communications S. AHRQ Comparative Effectiveness Reviews Therapies for Children With Autism Spectrum Disorder: A Review of the Research for Parents and Caregivers. Comparative Effectiveness Review Summary Guides for Consumers. 
Rockville (MD): Agency for Healthcare Research and Quality (US); 2005.

12. John MECfCD, Communications S. AHRQ Comparative Effectiveness Reviews Therapies for Children With Autism Spectrum Disorders: A Review of the Research for Parents and Caregivers. Comparative Effectiveness Review Summary Guides for Consumers. Rockville (MD): Agency for Healthcare Research and Quality (US); 2005.

13. Solomons S. Using aromatherapy massage to increase shared attention behaviours in children with autistic spectrum disorders and severe learning difficulties. British Journal of Special Education. 2005;32(3):127-37. doi: 10.1111/j.0952-3383.2005.00385.x.X-1, X-3, $\mathrm{X}-4$

14. Hellings JA, Zarcone JR, Reese RM, et al. A crossover study of risperidone in children, adolescents and adults with mental retardation. J Autism Dev Disord. 2006 Apr;36(3):401-11. doi: 10.1007/s10803-006-0078-1 [doi]. PMID: 16596465.X-1

15. Williams TI. Evaluating effects of aromatherapy massage on sleep in children with autism: a pilot study. Evid Based Complement Alternat Med. 2006 Sep;3(3):373-7. doi: 10.1093/ecam/nel017. PMID: 16951722.X-1, $\mathrm{X}-3$

16. Boso M, Emanuele E, Minazzi V, et al. Effect of long-term interactive music therapy on behavior profile and musical skills in young adults with severe autism. J Altern Complement Med. 2007 Sep;13(7):709-12. doi: 10.1089/acm.2006.6334. PMID: 17931062.X-1

17. Hilton C, Graver K, LaVesser P. Relationship between social competence and sensory processing in children with high functioning autism spectrum disorders. Research in Autism Spectrum Disorders. 2007 2015-12-09;1(2):164-73. doi: http://dx.doi.org/10.1016/j.rasd.2006.10.002. PMID: 621837115; 2007-12217-004.X-1

18. John MECfCD, Communications S. AHRQ Comparative Effectiveness Reviews Comparative Effectiveness of Therapies for Children With Autism Spectrum Disorder. Comparative Effectiveness Review Summary Guides for Clinicians. Rockville (MD): Agency for Healthcare Research and Quality (US); 2007.

19. John MECfCD, Communications S. AHRQ Comparative Effectiveness Reviews Comparative Effectiveness of Therapies for Children With Autism Spectrum Disorders. Comparative Effectiveness Review Summary Guides for Clinicians. Rockville (MD): Agency for Healthcare Research and Quality (US); 2007.

20. Kern P, Wolery M, Aldridge D. Use of songs to promote independence in morning greeting routines for young children with autism. J Autism Dev Disord. 2007 Aug;37(7):1264-71. doi: 10.1007/s10803-0060272-1. PMID: 17120150.X-1

21. McNally P, McNicholas F, Oslizlok P. The QT interval and psychotropic medications in children: recommendations for clinicians. Eur Child Adolesc Psychiatry. 2007 Feb;16(1):33-47. doi: 10.1007/s00787-0060573-0. PMID: 16944043.X-1

22. Myers SM, Johnson CP. Management of children with autism spectrum disorders. Pediatrics. 2007 Nov;120(5):1162-82. doi: peds.2007-2362 [pii] 10.1542/peds.2007-2362 [doi]. PMID: 17967921.X-1

$\mathrm{X}-2$

$\mathrm{X}-3$

$\mathrm{X}-4$

REFERENCES

23. Watling RL, Dietz J. Immediate effect of Ayres's sensory integration-based occupational therapy intervention on children with autism spectrum disorders. Am J Occup Ther. 2007 Sep-Oct;61(5):574-83. PMID: 17944295.X-3, X-4

24. Woodard C, Groden J, Goodwin M, et al. A placebo double-blind pilot study of dextromethorphan for problematic behaviors in children with autism. Autism. 2007

Jan;11(1):29-41. doi: 10.1177/1362361307070989. PMID: 17175572.X-1, X-4

25. Chungpaibulpatana J, Sumpatanarax T, Thadakul N, et al. Hyperbaric oxygen therapy in Thai autistic children. J Med Assoc Thai. 
2008 Aug;91(8):1232-8. PMID: 18788696.X3, X-4

26. Clark DL, Arnold LE, Crowl L, et al. Vestibular Stimulation for ADHD: randomized controlled trial of Comprehensive Motion Apparatus. J Atten Disord. 2008 Mar;11(5):599-611. doi: 10.1177/1087054707311042. PMID: 18198165.X-1

27. Lee HK. Effects of Massage and Attachment Promotion Program on Social Maturity, Child Autism and Attachment of Children with Autism and Their Mothers. J Korean Acad Child Health Nurs. 2008 1;14(1):14-21. INCLUDE

28. Ospina MB, Krebs Seida J, Clark B, et al. Behavioural and developmental interventions for autism spectrum disorder: a clinical systematic review. PLoS One. 2008;3(11):e3755. doi: 10.1371/journal.pone.0003755 [doi]. PMID: 19015734.X-2

29. Petrus C, Adamson SR, Block L, et al. Effects of exercise interventions on stereotypic behaviours in children with autism spectrum disorder. Physiother Can. 2008

Spring;60(2):134-45. doi: 10.3138/physio.60.2.134. PMID: 20145777.X2, $\mathrm{X}-3$

30. Rodger S, Ireland S, Vun M. Can Cognitive Orientation to daily Occupational Performance (CO-OP) help Children with Asperger's Syndrome to Master Social and Organisational Goals? The British Journal of Occupational Therapy. 2008 January 1, 2008;71(1):23-32. doi:

10.1177/030802260807100105.X-1, X-2, X-3

31. Silva LM, Ayres R, Schalock M. Outcomes of a pilot training program in a qigong massage intervention for young children with autism. Am J Occup Ther. 2008 SepOct;62(5):538-46. PMID: 18826014.X-3

32. Correll CU, Manu P, Olshanskiy V, et al. Cardiometabolic risk of second-generation antipsychotic medications during first-time use in children and adolescents. Jama. 2009 Oct 28;302(16):1765-73. doi: 10.1001/jama.2009.1549. PMID: 19861668.X-1
33. Cox AL, Gast DL, Luscre D, et al. The effects of weighted vests on appropriate in-seat behaviors of elementary-age students with autism and severe to profound intellectual disabilities. Focus on Autism and Other Developmental Disabilities. 2009 2015-1209;24(1):17-26. doi: http://dx.doi.org/10.1177/1088357608330753. PMID: 621855692; 2009-02961-002.X-3, X-4

34. Keino H, Funahashi A, Keino H, et al. Psycho-educational Horseback Riding to Facilitate Communication Ability of Children with Pervasive Developmental Disorders. J Equine Sci. 2009;20(4):79-88. doi: 10.1294/jes.20.79. PMID: 24833971.X-1, X-3

35. Kim J, Wigram T, Gold C. Emotional, motivational and interpersonal responsiveness of children with autism in improvisational music therapy. Autism. 2009 Jul;13(4):389-409. doi: 10.1177/1362361309105660. PMID: 19535468.X-1, X-4

36. Lundqvist L-O, Andersson G, Viding J. Effects of vibroacoustic music on challenging behaviors in individuals with Autism and developmental disabilities. Research in Autism Spectrum Disorders. 2009 2015-1209;3(2):390-400. doi: http://dx.doi.org/10.1016/j.rasd.2008.08.005. PMID: 622172722; 2010-06021-009.X-1

37. Seida JK, Ospina MB, Karkhaneh M, et al. Systematic reviews of psychosocial interventions for autism: an umbrella review. Dev Med Child Neurol. 2009 Feb;51(2):95-104. doi: DMCN3211 [pii] 10.1111/j.1469-8749.2008.03211.x [doi]. PMID: 19191842.X-2

38. Stephenson J, Carter M. The use of weighted vests with children with autism spectrum disorders and other disabilities. J Autism Dev Disord. 2009 Jan;39(1):105-14. doi: 10.1007/s10803-008-0605-3. PMID: 18592366.X-2

39. Van Rie GL, Heflin LJ. The effect of sensory activities on correct responding for children with autism spectrum disorders. Research in Autism Spectrum Disorders. 2009 2015-12-09;3(3):783-96. doi: http://dx.doi.org/10.1016/j.rasd.2009.03.001. PMID: 622173765; 2010-06022-019.X-1, X-3, $\mathrm{X}-4$ 
40. . Autism spectrum disorders. Diagnosis and management involve time and patience. Harv Ment Health Lett. 2010 Sep;27(3):4-5. PMID: 20941863.X-1

41. . Autism spectrum disorders and the gut. Consensus recommendations for evaluation and treatment. Harv Ment Health Lett. 2010 Apr;26(10):4. PMID: 20499455.X-1, X-2, X-3

42. . Massachusetts \& New Hampshire. Insurance; autism spectrum disorders. Ment Phys Disabil Law Rep. 2010 SepOct;34(5):812. PMID: 21197720.X-1

43. . Current world literature. Curr Opin Neurol. 2010 Apr;23(2):194-201. doi: 10.1097/WCO.0b013e328338cade. PMID: 20216346.X-1, X-2

44. . Mental health. Nursing Times. 2010;106(31):7- 1p. PMID: 105077539. Language: English. Entry Date: 20101001. Revision Date: 20150711. Publication Type: Journal Article.X-1, X-2

45. . Medication plus behavioral intervention for aggression in autism. Brown University Child \& Adolescent Psychopharmacology Update. 2010;12(8):4-5 2p. PMID: 105068473. Language: English. Entry Date: 20101001. Revision Date: 20150711. Publication Type: Journal Article. Journal Subset: Biomedical.X-1, X-2, X-3

46. . FDA warns maker of product used as alternative autism treatment. Contemporary Rehab. 2010;66(4):7- 1p. PMID: 105111455. Language: English. Entry Date: 20101126. Revision Date: 20150711. Publication Type: Journal Article.X-1, X-2

47. . Lack of evidence for antidepressant use in autism. Contemporary Rehab. 2010;66(4):11- 1p. PMID: 105111459. Language: English. Entry Date: 20101126. Revision Date: 20150711. Publication Type: Journal Article.X-1, X-2

48. . Divalproex sodium for irritability in autism. Brown University Child \& Adolescent Psychopharmacology Update. 2010;12(7):7- 1p. PMID: 105045860. Language: English. Entry Date: 20100827. Revision Date: 20150711. Publication Type: Journal Article.X-1, X-2
49. . NAC effective for self-injurious behavior in 4-year-old with ASD. Brown University Child \& Adolescent Psychopharmacology Update. 2010;12(5):7-8 2p. PMID: 103840426. Language: English. Entry Date: 20150227. Revision Date: 20150710. Publication Type: Journal Article. Journal Subset: Biomedical.X-1, X-2, X-3, X-4

50. . Clozapine for aggression in adolescent autism. Brown University Child \& Adolescent Psychopharmacology Update. 2010;12(5):8- 1p. PMID: 105193002. Language: English. Entry Date: 20150227. Revision Date: 20150711. Publication Type: Journal Article.X-1, X-2

51. . Drug \& device news. Medical Malpractice Law \& Strategy. 2010;27(8):5- 1p. PMID: 105201156. Language: English. Entry Date: 20100709. Revision Date: 20150711. Publication Type: Journal Article. Journal Subset: Biomedical.X-1, X-2

52. . Keep your eye on. Brown University Child \& Adolescent Behavior Letter. 2010;26(4):2- 1p. PMID: 105155628. Language: English. Entry Date: 20100507. Revision Date: 20150711. Publication Type: Journal Article. Journal Subset: Biomedical.X$1, \mathrm{X}-2$

53. . Oxytocin improves social interaction in autistic patients. Brown University Child \& Adolescent Psychopharmacology Update. 2010;12(4):7-8 2p. PMID: 105162352. Language: English. Entry Date: 20100521. Revision Date: 20150711. Publication Type: Journal Article. Journal Subset: Biomedical.X$1, \mathrm{X}-2$

54. . Briefly noted. Mental Health Weekly. 2010;20(7):7-8 2p. PMID: 105120514. Language: English. Entry Date: 20100402. Revision Date: 20150711. Publication Type: Journal Article.X-1, X-2

55. . Aripiprazole effective in treating irritability in autistic youth. Brown University Child \& Adolescent Psychopharmacology Update. 2010;12(2):4-5 2p. PMID: 105306069. Language: English. Entry Date: 20100312. Revision Date: 20150711. Publication Type: Journal Article. Journal Subset: Biomedical.X2, X-6 
56. . For the record. Journal of Reproductive Medicine. 2010;55(1-2):2A-A 1p. PMID: 105160564. Language: English. Entry Date: 20100409. Revision Date: 20150711. Publication Type: Journal Article.X-1, X-2

57. . Extending trust. Nature

Neuroscience. 2010 August;13(8):905. doi: http://dx.doi.org/10.1038/nn0810-905. PMID: 2010410948.X-1

58. Abdala APL, Dutschmann M, Bissonnette JM, et al. Correction of respiratory disorders in a mouse model of Rett syndrome. Proceedings of the National Academy of Sciences of the United States of America. 2010 19 Oct;107(42):18208-13. doi: http://dx.doi.org/10.1073/pnas.1012104107. PMID: 2010609918.X-1

59. Abraham E, Shah S, Parray T. Combination of oral clonidine and midazolam as premedication when ketamine is not an option in an uncooperative autistic patient. Internet Journal of Anesthesiology. 2010;24(1):8p-p 1p. PMID: 105034318. Language: English. Entry Date: 20100820. Revision Date: 20150820. Publication Type: Journal Article.X-1

60. Acef S, Aubrun P. [Somatic care and autism: removal of barriers to reducing inequalities]. Sante Publique. 2010 SepOct;22(5):529-39. PMID: 21360862.X-1

61. Agam Y, Joseph RM, Barton JJ, et al. Reduced cognitive control of response inhibition by the anterior cingulate cortex in autism spectrum disorders. Neuroimage. 2010 Aug 1;52(1):336-47. doi: 10.1016/j.neuroimage.2010.04.010. PMID: 20394829.X-1

62. Ahearn WH. What Every Behavior Analyst Should Know About the "MMR Causes Autism" Hypothesis. Behav Anal Pract. 2010 Spring;3(1):46-50. PMID: 22479671.X-1

63. Akhondzadeh S. Adjunctive pentoxifylline for disruptive symptoms in autistic children. Brown University Child \& Adolescent Psychopharmacology Update. 2010;12(1):4-5 2p. PMID: 105281074. Language: English. Entry Date: 20100212.
Revision Date: 20150711. Publication Type: Journal Article. Journal Subset: Biomedical.X-2

64. Akins RS, Angkustsiri K, Hansen RL. Complementary and alternative medicine in autism: An evidence-based approach to negotiating safe and efficacious interventions with families. Neurotherapeutics. 2010 July;7(3):307-19. doi: http://dx.doi.org/10.1016/j.nurt.2010.05.002. PMID: 2010406864.X-2, X-3

65. Al Anbar NN, Dardennes RM, PradoNetto A, et al. Treatment choices in autism spectrum disorder: the role of parental illness perceptions. Res Dev Disabil. 2010 MayJun;31(3):817-28. doi: 10.1016/j.ridd.2010.02.007. PMID: 20299185.X-1

66. Alvarez A. Levels of analytic work and levels of pathology: the work of calibration. Int J Psychoanal. 2010 Aug;91(4):859-78. doi: 10.1111/j.1745-8315.2010.00284.x. PMID: 20840643.X-1

67. Aman MG, Arnold LE, Lecavalier L, et al. Dr. Aman et al. reply. Journal of the American Academy of Child and Adolescent Psychiatry. 2010 April;49(4):407-8. doi: http://dx.doi.org/10.1097/00004583201004000-00016. PMID: 2010180130.X-1

68. Aman MG, McDougle CJ, Scahill L. Lower dose risperidone combined with parent training improves PDD outcomes...pervasive developmental disorder. Brown University Child \& Adolescent Psychopharmacology Update. 2010;12(1):1-3 p. PMID: 105281082. Language: English. Entry Date: 20100212. Revision Date: 20150711. Publication Type: Journal Article. Journal Subset: Biomedical.X$1, \mathrm{X}-2$

69. Amiet C, Gourfinkel-An I, Consoli A, et al. [Epilepsy and autism: a complex issue]. Arch Pediatr. 2010 Jun;17(6):650-1. doi: 10.1016/s0929-693x(10)70043-9. PMID: 20654825.X-2, X-3

70. Andari E, Duhamel JR, Zalla T, et al. Promoting social behavior with oxytocin in high-functioning autism spectrum disorders. Proc Natl Acad Sci U S A. 2010 Mar 2;107(9):4389-94. doi: 
10.1073/pnas.0910249107. PMID:

20160081.X-1, X-4

71. Arieff Z, Kaur M, Gameeldien H, et al. 5-HTTLPR polymorphism: analysis in South African autistic individuals. Hum Biol. 2010 Jun;82(3):291-300. doi: 10.3378/027.082.0303. PMID: 20649385.X-1

72. Arunkumar T, Supe SS, Ravikumar M, et al. Electron beam characteristics at extended source-to-surface distances for irregular cutouts. J Med Phys. 2010 Oct;35(4):207-14. doi: 10.4103/0971-6203.71763. PMID: 21170185.X-1

73. Assouline B. [Autism announcement at consultation is a therapeutic step]. Arch Pediatr. 2010 Jun;17(6):647-8. doi: 10.1016/s0929693x(10)70041-5. PMID: 20654823.X-5

74. Athens ES, Vollmer TR. An investigation of differential reinforcement of alternative behavior without extinction. J Appl Behav Anal. 2010 Winter;43(4):569-89. doi: 10.1901/jaba.2010.43-569. PMID: 21541145.X-1, X-3

75. Atladottir HO, Thorsen P, Ostergaard $\mathrm{L}$, et al. Maternal infection requiring hospitalization during pregnancy and autism spectrum disorders. J Autism Dev Disord. 2010 Dec;40(12):1423-30. doi: 10.1007/s10803-0101006-y. PMID: 20414802.X-1

76. Atladottir HO, Thorsen P, Schendel DE, et al. Association of hospitalization for infection in childhood with diagnosis of autism spectrum disorders: a Danish cohort study. Arch Pediatr Adolesc Med. 2010 May;164(5):470-7. doi: 10.1001/archpediatrics.2010.9. PMID: 20439799.X-1

77. Auyeung B, Taylor K, Hackett G, et al. Foetal testosterone and autistic traits in 18 to 24-month-old children. Mol Autism. 2010;1(1):11. doi: 10.1186/2040-2392-1-11. PMID: 20678186.X-1

78. Axe JB, Sainato DM. Matrix training of preliteracy skills with preschoolers with autism. J Appl Behav Anal. 2010

Winter;43(4):635-52. doi: 10.1901/jaba.2010.43-635. PMID: 21541149.X-1, X-3, X-4
79. Bagatell N, Mirigliani G, Patterson C, et al. Effectiveness of therapy ball chairs on classroom participation in children with autism spectrum disorders. Am J Occup Ther. 2010 Nov-Dec;64(6):895-903. PMID: 21218680.X3, X-4

80. Baharav E, Reiser C. Using telepractice in parent training in early autism. Telemed J E Health. 2010 Jul-Aug;16(6):72731. doi: 10.1089/tmj.2010.0029. PMID: 20583950.X-3, X-4

81. Baker N. Exploring the mental health nurse practitioner scope of practice in youth early psychosis: an anecdotal account. Contemp Nurse. 2010 Feb-Mar;34(2):211-20. doi: 10.5172/conu.2010.34.2.211. PMID: 20509805.X-1, X-2

82. Bakhtiari M, Malhotra H, Jones MD, et al. Applying graphics processor units to Monte Carlo dose calculation in radiation therapy. J Med Phys. 2010 Apr;35(2):120-2. doi: 10.4103/0971-6203.62198. PMID: 20589122.X-1

83. Banda DR, Copple KS, Koul RK, et al. Video modelling interventions to teach spontaneous requesting using AAC devices to individuals with autism: a preliminary investigation. Disabil Rehabil. 2010;32(16):1364-72. doi: 10.3109/09638280903551525. PMID: 20465397.X-1

84. Barba L. A one-stop shop for autism services. Behav Healthc. 2010 Jun;30(6):28, 301. PMID: 20666201.X-1

85. Baruth JM, Casanova MF, El-Baz A, et al. Low-Frequency Repetitive Transcranial Magnetic Stimulation (rTMS) Modulates Evoked-Gamma Frequency Oscillations in Autism Spectrum Disorder (ASD). J Neurother. 2010 Jul 1;14(3):179-94. doi: 10.1080/10874208.2010.501500. PMID: 21116441.X-1

86. Baruth JM, Casanova MF, Sears L, et al. Early-stage visual processing abnormalities in high-functioning autism spectrum disorder (ASD). Transl Neurosci. 2010 Jun;1(2):177-87. doi: 10.2478/v10134-010-0024-9. PMID: 22563527.X-1 
87. Bathla M, Chandna S, Bathla JC. Rett's syndrome: Diagnostic and therapeutic dilemma. German Journal of Psychiatry. 2010;13(3):157-60. PMID: 2010520442.X-1

88. Bennett M, Mitchell S, Neuman T, et al. Hyperbaric oxygen therapy and neurological disease. Undersea \& hyperbaric medicine : journal of the Undersea and Hyperbaric Medical Society, Inc. 2010 2010;37(5):371-3; author reply 3-4. PMID: 20929186.X-5

89. Bent S, Hendren RL. Improving the prediction of response to therapy in autism. Neurotherapeutics. 2010 July;7(3):232-40. doi: http://dx.doi.org/10.1016/j.nurt.2010.05.011. PMID: 2010406855.X-1

90. Bhatara A, Quintin EM, Levy B, et al. Perception of emotion in musical performance in adolescents with autism spectrum disorders. Autism Res. 2010 Oct;3(5):214-25. doi: 10.1002/aur.147. PMID: 20717952.X-1

91. Biemans B, Aubert L, Jagasia R, et al. The effects of prenatal valproic acid on adult neurogenesis in a mouse model for autism. Behavioural Pharmacology. 2010 September;21 (5-6):587. doi:

http://dx.doi.org/10.1097/FBP.0b013e32833dbe 35. PMID: 70448404.X-1

92. Bigham S, Boucher J, Mayes A, et al. Assessing recollection and familiarity in autistic spectrum disorders: methods and findings. Journal of Autism \& Developmental Disorders. 2010;40(7):878-89 12p. doi: 10.1007/s10803010-0937-7. PMID: 105038411. Language: English. Entry Date: 20100820. Revision Date: 20150711. Publication Type: Journal Article.X1

93. Blake KE. Spectrum disorders: a new generation of complex patients. Pa Nurse. 2010 Dec;65(4):9-11, 5. PMID: 21329281.X-5

94. Blankenship K, Erickson CA, Stigler $\mathrm{KA}$, et al. Aripiprazole for irritability associated with autistic disorder in children and adolescents aged 6-17 years. Ped Health. 2010 Sep 29;4(4):375-81. PMID: 21359119.X-1

95. Blankenship K, Minshawi NF. Behavioral Therapy with an Individual with Asperger's Disorder. Psychiatry (Edgmont).
2010 Aug;7(8):38-41. PMID: 20877532.X-2, $\mathrm{X}-3, \mathrm{X}-4$

96. Blardi P, de Lalla A, Ceccatelli L, et al. Variations of plasma leptin and adiponectin levels in autistic patients. Neurosci Lett. 2010 Jul 19;479(1):54-7. doi:

10.1016/j.neulet.2010.05.027. PMID: 20478355.X-1

97. Blundell J, Blaiss CA, Etherton MR, et al. Genetic animal models of Autism. Annals of Neurology. 2010;68:S31. doi: http://dx.doi.org/10.1002/ana.22175. PMID: 70368706.X-1

98. Boggio EM, Lonetti G, Pizzorusso T, et al. Synaptic determinants of Rett syndrome. Frontiers in Synaptic Neuroscience. 2010;(AUG)(Article 28)doi: http://dx.doi.org/10.3389/fnsyn.2010.00028. PMID: 2012338577.X-1

99. Bonnel A, McAdams S, Smith B, et al. Enhanced pure-tone pitch discrimination among persons with autism but not Asperger syndrome. Neuropsychologia. 2010 Jul;48(9):2465-75. doi: 10.1016/j.neuropsychologia.2010.04.020. PMID: 20433857.X-1

100. Bonnet-Brilhault F. [Managing an autistic child]. Rev Prat. 2010 Mar 20;60(3):385-8. PMID: 20402134.X-1

101. Boso M, Comelli M, Emanuele E, et al. Seasonal fluctuations in problem behaviors among young adults with autism and intellectual disability. Med Sci Monit. 2010 May;16(5):Cr213-6. PMID: 20424547.X-1

102. Boyd BA, Baranek GT, Sideris J, et al. Sensory features and repetitive behaviors in children with autism and developmental delays. Autism Res. 2010 Apr;3(2):78-87. doi: 10.1002/aur.124. PMID: 20437603.X-1

103. Bozdagi O, Sakurai T, Papapetrou D, et al. Haploinsufficiency of the autismassociated Shank3 gene leads to deficits in synaptic function, social interaction, and social communication. Mol Autism. 2010;1(1):15. doi: 10.1186/2040-2392-1-15. PMID: 21167025.X-1 
104. Bradford K. Brief education about autism spectrum disorders for family therapists. Journal of Family Psychotherapy. 2010 July;21(3):161-79. doi: http://dx.doi.org/10.1080/08975353.2010.50552 9. PMID: 2010497839.X-1, X-2

105. Brambring M, Asbrock D. Validity of False Belief Tasks in Blind Children. Journal of Autism \& Developmental Disorders. 2010;40(12):1471-84 14p. doi: 10.1007/s10803-010-1002-2. PMID: 104942420. Language: English. Entry Date: 20101129. Revision Date: 20150711. Publication Type: Journal Article.X-1

106. Brookman-Frazee LI, Taylor R, Garland AF. Characterizing community-based mental health services for children with autism spectrum disorders and disruptive behavior problems. J Autism Dev Disord. 2010 Oct;40(10):1188-201. doi: 10.1007/s10803010-0976-0. PMID: 20204690.X-1

107. Brownlow C. Presenting the self: negotiating a label of autism. J Intellect Dev Disabil. 2010 Mar;35(1):14-21. doi: 10.3109/13668250903496336. PMID: 20121662.X-1

108. Burke LM, Kalpakjian CZ, Smith YR, et al. Gynecologic issues of adolescents with Down syndrome, autism, and cerebral palsy. J Pediatr Adolesc Gynecol. 2010 Feb;23(1):11-5. doi: 10.1016/j.jpag.2009.04.005. PMID: 19643648.X-1

109. Burke RV, Andersen MN, Bowen SL, et al. Evaluation of two instruction methods to increase employment options for young adults with autism spectrum disorders. Res Dev Disabil. 2010 Nov-Dec;31(6):1223-33. doi: 10.1016/j.ridd.2010.07.023. PMID: 20800988.X-1

110. Bussing R, Siddiqi S, Janicke DM, et al. Late onset autistic symptoms and other fluctuating behaviors. J Dev Behav Pediatr. 2010 Apr;31(3 Suppl):S38-41. doi: 10.1097/DBP.0b013e3181db78e3. PMID: 20414073.X-1

111. Calhoun V, Wu L, Kiehl K, et al. Aberrant Processing of Deviant Stimuli in Schizophrenia Revealed by Fusion of FMRI and EEG Data. Acta Neuropsychiatr. 2010
Jun;22(3):127-38. doi: 10.1111/j.16015215.2010.00467.x. PMID: 21331320.X-1

112. Callahan K, Shukla-Mehta S, Magee $S$, et al. ABA versus TEACCH: the case for defining and validating comprehensive treatment models in autism. J Autism Dev Disord. 2010 Jan;40(1):74-88. doi: 10.1007/s10803-009-0834-0. PMID: 19649699.X-1

113. Cannon DS, Miller JS, Robison RJ, et al. Genome-wide linkage analyses of two repetitive behavior phenotypes in Utah pedigrees with autism spectrum disorders. Mol Autism. 2010;1(1):3. doi: 10.1186/2040-23921-3. PMID: 20678246.X-1

114. Capp PL, de Faria ME, Siqueira SR, et al. Special care dentistry: Midazolam conscious sedation for patients with neurological diseases. Eur J Paediatr Dent. 2010 Dec;11(4):162-4. PMID: 21250764.X-1

115. Carayol J, Schellenberg GD, Tores F, et al. Assessing the impact of a combined analysis of four common low-risk genetic variants on autism risk. Mol Autism. 2010;1(1):4. doi: 10.1186/2040-2392-1-4. PMID: 20678243.X-1

116. Carbone PS, Behl DD, Azor V, et al. The medical home for children with autism spectrum disorders: parent and pediatrician perspectives. J Autism Dev Disord. 2010 Mar;40(3):317-24. doi: 10.1007/s10803-0090874-5. PMID: 19768528.X-1

117. Carbone PS, Farley M, Davis T. Primary care for children with autism. Am Fam Physician. 2010 Feb 15;81(4):453-60. PMID: 20148499.X-1

118. Carbone VJ, Sweeney-Kerwin EJ, Attanasio V, et al. Increasing the vocal responses of children with autism and developmental disabilities using manual sign mand training and prompt delay. J Appl Behav Anal. 2010 Winter;43(4):705-9. doi: 10.1901/jaba.2010.43-705. PMID: 21541153.X-3, X-4

119. Careaga M, Van de Water J, Ashwood P. Immune dysfunction in autism: A pathway to treatment. Neurotherapeutics. 2010 July;7(3):283-92. doi: 
http://dx.doi.org/10.1016/j.nurt.2010.05.003. PMID: 2010406861.X-1, X-2, X-3

120. Carroll VS. Newsworthy neuroscience. Journal of Neuroscience Nursing. 2010;42(2):59-60 2p. PMID: 105157050. Language: English. Entry Date: 20100521. Revision Date: 20150819. Publication Type: Journal Article.X-1, X-2

121. Cascio CJ. Somatosensory processing in neurodevelopmental disorders. J Neurodev Disord. 2010 Jun;2(2):62-9. doi: 10.1007/s11689-010-9046-3. PMID: 22127855.X-1

122. Casella SE, Wilder DA, Neidert P, et al. The effects of response effort on safe performance by therapists at an autism treatment facility. J Appl Behav Anal. 2010 Winter;43(4):729-34. doi: 10.1901/jaba.2010.43-729. PMID: 21541157.X-1

123. Charania SM, LeBlanc LA, Sabanathan N, et al. Teaching effective hand raising to children with autism during group instruction. J Appl Behav Anal. 2010 Fall;43(3):493-7. doi: 10.1901/jaba.2010.43493. PMID: 21358908.X-1, X-3, X-4

124. Charman T. Developmental approaches to understanding and treating autism. Folia Phoniatr Logop. 2010;62(4):16677. doi: 10.1159/000314032. PMID: 20460929.X-1

125. Chez MG, Guido-Estrada N. Immune therapy in autism: Historical experience and future directions with immunomodulatory therapy. Neurotherapeutics. 2010 July;7(3):293301. doi: http://dx.doi.org/10.1016/j.nurt.2010.05.008. PMID: 2010406862.X-1, X-2, X-3

126. Chisholm K, Gibbons A, Psarros C, et al. Longitudinal outcomes of early implantation in children with auditory neuropathy spectrum disorder (ANSD). Cochlear Implants Int. 2010 Jun;11 Suppl 1:169-75. doi: 10.1179/146701010x12671177818588. PMID: 21756606.X-1

127. Chok JT, Reed DD, Kennedy A, et al. A single-case experimental analysis of the effects of ambient prism lenses for an adolescent with developmental disabilities. Behav Anal Pract. 2010 Fall;3(2):42-51. PMID: 22532893.X-1

128. Christon LM, Mackintosh VH, Myers BJ. Use of complementary and alternative medicine (CAM) treatments by parents of children with autism spectrum disorders. Research in Autism Spectrum Disorders. 2010 April 2010/June;4(2):249-59. doi: http://dx.doi.org/10.1016/j.rasd.2009.09.013. PMID: 2010025299.X-1, X-3

129. Clark B, Vandermeer B, Simonetti A, et al. Is lead a concern in Canadian autistic children? Paediatr Child Health. 2010 Jan;15(1):17-22. PMID: 21197164.X-1

130. Clark M, Harris R, Jolleff N, et al. Worster-Drought syndrome: poorly recognized despite severe and persistent difficulties with feeding and speech. Dev Med Child Neurol. 2010 Jan;52(1):27-32. doi: 10.1111/j.14698749.2009.03475.x. PMID: 19824895.X-1

131. Coben R, Myers TE. The relative efficacy of connectivity guided and symptom based EEG biofeedback for autistic disorders. Appl Psychophysiol Biofeedback. 2010 Mar;35(1):13-23. doi: 10.1007/s10484-0099102-5. PMID: 19649702.X-2, X-3

132. Coghlan A. Autism drug aims to balance brain signals. New Scientist. 2010;207(2778):01- 1p. PMID: 104935308. Language: English. Entry Date: 20110208. Revision Date: 20150711. Publication Type: Journal Article. Journal Subset: Consumer Health.X-1, X-2

133. Consoli A, Gheorghiev C, Jutard C, et al. Lorazepam, fluoxetine and packing therapy in an adolescent with pervasive developmental disorder and catatonia. Journal of Physiology Paris. 2010 December;104(6):309-14. doi: http://dx.doi.org/10.1016/j.jphysparis.2010.09.0 02. PMID: 2010672610.X-1, X-3, X-4

134. Constantino JN, Majmudar P, Bottini A, et al. Infant head growth in male siblings of children with and without autism spectrum disorders. J Neurodev Disord. 2010 Mar;2(1):39-46. doi: 10.1007/s11689-0099036-5. PMID: 20651949.X-1 
135. Cook Jr EH. Reduction of increased repetitive self-grooming in ASD mouse model by metabotropic 5 glutamate receptor antagonism; randomized controlled trial of Early Start Denver Model. Autism research : official journal of the International Society for Autism Research. 2010 Feb;3(1):40-2. doi: http://dx.doi.org/10.1002/aur.118. PMID: 20175131.X-1

136. Coolican J, Smith IM, Bryson SE. Brief parent training in pivotal response treatment for preschoolers with autism. J Child Psychol Psychiatry. 2010 Dec;51(12):1321-30. doi: 10.1111/j.1469-7610.2010.02326.x. PMID: 21073457.X-3, X-4

137. Correia CT, Almeida JP, Santos PE, et al. Pharmacogenetics of risperidone therapy in autism: association analysis of eight candidate genes with drug efficacy and adverse drug reactions. Pharmacogenomics J. 2010 Oct;10(5):418-30. doi: 10.1038/tpj.2009.63. PMID: $19997080 . \mathrm{X}-3$

138. Coury D. Medical treatment of autism spectrum disorders. Current Opinion in Neurology. 2010 April;23(2):131-6. doi: http://dx.doi.org/10.1097/WCO.0b013e3283372 2fa. PMID: 2010168656.X-1, X-2

139. Cuvo AJ, Godard A, Huckfeldt R, et al. Training children with autism spectrum disorders to be compliant with an oral assessment. Research in Autism Spectrum Disorders. 2010 October-December;4(4):68196. doi: http://dx.doi.org/10.1016/j.rasd.2010.01.007. PMID: 2010552030.X-1

140. Czapanskiy KS. Chalimony: seeking equity between parents of children with disabilities and chronic illnesses. Rev Law Soc Change. 2010;34(2):253-98. PMID: 20722202.X-1

141. Darrou C, Pry R, Pernon E, et al. Outcome of young children with autism: does the amount of intervention influence developmental trajectories? Autism. 2010 Nov;14(6):663-77. doi: 10.1177/1362361310374156. PMID: 21149421.X-1, X-3

142. Davidson J. 'It cuts both ways': a relational approach to access and accommodation for autism. Soc Sci Med. 2010 Jan;70(2):305-12. doi:

10.1016/j.socscimed.2009.10.017. PMID: 19883964.X-1

143. Davies PL, Tucker R. Evidence Review to Investigate the Support for Subtypes of Children With Difficulty Processing and Integrating Sensory Information. American Journal of Occupational Therapy. 2010;64(3):391-402 12p. doi: 10.5014/ajot.2010.09070. PMID: 105032109. Language: English. Entry Date: 20130221. Revision Date: 20150711. Publication Type: Journal Article.X-1

144. Dawson G, Rogers S, Munson J, et al. Randomized, controlled trial of an intervention for toddlers with autism: the Early Start Denver Model. Pediatrics. 2010 Jan;125(1):e17-23. doi: 10.1542/peds.2009-0958. PMID: 19948568.X-1

145. de Bruijn DR, van Dijk AH, Pfundt R, et al. Severe Progressive Autism Associated with Two de novo Changes: A 2.6-Mb 2q31.1 Deletion and a Balanced t(14;21)(q21.1;p11.2) Translocation with Long-Range Epigenetic Silencing of LRFN5 Expression. Mol Syndromol. 2010 Feb;1(1):46-57. doi: 10.1159/000280290. PMID: 20648246.X-1

146. De Filippis B, Ricceri L, Laviola G. Investigating rett syndrome through genetic mouse models: Presymptomatic, clearly symptomatic phases, and innovative therapeutic approaches. In: Kalueff AV, Bergner CL, eds. Transgenic and Mutant Tools to Model Brain Disorders. 999 Riverview Drive, Suite 208, Totowa NJ 07512-1165, United States: Humana Press; 2010:151-78.

147. de Los Reyes EC. Autism and immunizations: separating fact from fiction. Arch Neurol. 2010 Apr;67(4):490-2. doi: 10.1001/archneurol.2010.57. PMID: 20385917.X-1, X-2

148. De Schipper JC, Schuengel C. Attachment behaviour towards support staff in young people with intellectual disabilities: associations with challenging behaviour. J Intellect Disabil Res. 2010 Jul;54(7):584-96. doi: 10.1111/j.1365-2788.2010.01288.x. PMID: 20492348.X-1 
149. Desousa A. An Open-label Trial of Risperidone and Fluoxetine in Children with Autistic Disorder. Indian J Psychol Med. 2010 Jan;32(1):17-21. doi: 10.4103/02537176.70522. PMID: 21799554.X-1

150. Desruisseaux MS, Iacobas DA, Iacobas $\mathrm{S}$, et al. Alterations in the Brain Transcriptome in ANKA Infected Mice. J Neuroparasitology. 2010 Oct;1 PMID: 23467761.X-1

151. Deutsch SI, Urbano MR, Neumann SA, et al. Cholinergic abnormalities in autism: Is there a rationale for selective nicotinic agonist interventions? Clinical

Neuropharmacology. 2010 May;33(3):114-20.

doi:

http://dx.doi.org/10.1097/WNF.0b013e3181d6f

7ad. PMID: 2010315521.X-1

152. Dias GG, Prado EF, Vadasz E, et al. Evaluation of the efficacy of a dental plaque control program in autistic patients. J Autism Dev Disord. 2010 Jun;40(6):704-8. doi: 10.1007/s10803-009-0918-x. PMID: 20052608.X-1

153. Dichter GS, Benning SD, Holtzclaw $\mathrm{TN}$, et al. Affective modulation of the startle eyeblink and postauricular reflexes in autism spectrum disorder. J Autism Dev Disord. 2010 Jul;40(7):858-69. doi: 10.1007/s10803-0090925-y. PMID: 20049632.X-1

154. Dichter GS, Radonovich KJ, TurnerBrown LM, et al. Performance of children with autism spectrum disorders on the DimensionChange Card Sort task. Journal of Autism \& Developmental Disorders. 2010;40(4):448-56 9p. doi: 10.1007/s10803-009-0886-1. PMID: 105140299. Language: English. Entry Date: 20100430. Revision Date: 20150711. Publication Type: Journal Article.X-1

155. Dichter GS, Sikich L, Mahorney S, et al. fMRI tracks reductions in repetitive behaviors in autism: two case studies. Neurocase (Psychology Press). 2010;16(4):30716 10p. doi: 10.1080/13554790903559671. PMID: 105061556. Language: English. Entry Date: 20101022. Revision Date: 20150711. Publication Type: Journal Article.X-1, X-3, X-4

156. Dickstein DP. In this issue/abstract thinking: the new normal. J Am Acad Child
Adolesc Psychiatry. 2010 Nov;49(11):1087-8. doi: 10.1016/j.jaac.2010.08.010. PMID: 20970695.X-1

157. Dillon SR, Casebolt KM, Tindall DW, et al. Digest. Adapted Physical Activity Quarterly. 2010;27(3):261-5 5p. PMID: 105034839. Language: English. Entry Date: 20100813. Revision Date: 20150711. Publication Type: Journal Article.X-1

158. Dowd AM, Rinehart NJ, McGinley J. Motor function in children with autism: Why is this relevant to psychologists? Clinical Psychologist. 2010;14(3):90-6 7p. doi: 10.1080/13284207.2010.525532. PMID: 104938830. Language: English. Entry Date: 20101216. Revision Date: 20150711. Publication Type: Journal Article.X-1

159. Drahota A, Chavira DA, Stein MT. Ian: a 7-year old with prenatal drug exposure and early exposure to family violence. J Dev Behav Pediatr. 2010 Apr;31(3 Suppl):S3-6. doi: 10.1097/DBP.0b013e3181d82fa7. PMID: 20414070.X-1

160. Dufour-Rainfray D, Vourc'h P, Le Guisquet AM, et al. Behavior and serotonergic disorders in rats exposed prenatally to valproate: A model for autism. Neuroscience Letters. 201005 Feb;470(1):55-9. doi: http://dx.doi.org/10.1016/j.neulet.2009.12.054. PMID: 2010042341.X-1

161. Dunst CJ, Trivette CM, Masiello T. Influence of the interests of children with autism on everyday learning opportunities. Psychol Rep. 2010 Aug;107(1):281-8. doi: 10.2466/04.10.11.15.21.pr0.107.4.281-288. PMID: 20923073.X-1

162. Eby CM, Greer RD, Tullo LD, et al. Effects of multiple exemplar instruction on transformation of stimulus function across written and vocal spelling responses by students with autism. The Journal of Speech and Language Pathology - Applied Behavior Analysis. 2010 2015-12-09;5(1):20-31. doi: http://dx.doi.org/10.1037/h0100262. PMID: 1640008797; 2014-51868-002.X-1

163. Einspieler C, Marschik PB, Prechtl HF. [What do early childhood movements tell us?]. Kinderkrankenschwester. 2010 Sep;29(9):389-92. PMID: 20942313.X-1 
164. Ekinci O, Arman AR, Isik U, et al. EEG abnormalities and epilepsy in autistic spectrum disorders: Clinical and familial correlates. Epilepsy and Behavior. 2010 February;17(2):178-82. doi: http://dx.doi.org/10.1016/j.yebeh.2009.11.014. PMID: 2010079168.X-1

165. Eklund M, Ostman M. Belonging and doing: important factors for satisfaction with sexual relations as perceived by people with persistent mental illness. Int J Soc Psychiatry. 2010 Jul;56(4):336-47. doi:

10.1177/0020764008101635. PMID: 19617283.X-1

166. Elvins R, Green J. Pharmacological management of core and comorbid symptoms in autism-spectrum disorder. Advances in Psychiatric Treatment. 2010 September;16(5):349-60. doi: http://dx.doi.org/10.1192/apt.bp.108.005538. PMID: 2010482184.X-1, X-2, X-3

167. Emanuele E, Colombo R, Martinelli V, et al. Elevated urine levels of bufotenine in patients with autistic spectrum disorders and schizophrenia. Neuroendocrinology Letters. 2010;31(1):117-21. PMID: 2010157086.X-1

168. Enticott PG, Rinehart NJ, Tonge BJ, et al. A preliminary transcranial magnetic stimulation study of cortical inhibition and excitability in high-functioning autism and Asperger disorder. Dev Med Child Neurol. 2010 Aug;52(8):e179-83. doi: 10.1111/j.14698749.2010.03665.x. PMID: 20370810.X-1

169. Erickson CA, Mullett JE, McDougle CJ. Brief report: Acamprosate in fragile X syndrome. Journal of Autism and Developmental Disorders. 2010

November;40(11):1412-6. doi: http://dx.doi.org/10.1007/s10803-010-0988-9. PMID: 2010636651.X-1

170. Erickson CA, Stigler KA, Posey DJ, et al. Aripiprazole in autism spectrum disorders and fragile $\mathrm{X}$ syndrome. Neurotherapeutics. 2010 July;7(3):258-63. doi: http://dx.doi.org/10.1016/j.nurt.2010.04.001. PMID: 2010406858.X-1, X-2, X-3

171. Esch JW, Esch BE, McCart JD, et al. An assessment of self-echoic behavior in young children. Anal Verbal Behav. 2010;26(1):3-13. PMID: 22477458.X-1

172. Faber A, Kalverdijk LJ, de Jong-van den Berg LT, et al. Co-morbidity and patterns of care in stimulant-treated children with ADHD in the Netherlands. Eur Child Adolesc Psychiatry. 2010 Feb;19(2):159-66. doi: 10.1007/s00787-009-0075-y. PMID: 19894075.X-1

173. Falcomata TS, Roane HS, Feeney BJ, et al. Assessment and treatment of elopement maintained by access to stereotypy. J Appl Behav Anal. 2010 Fall;43(3):513-7. doi: 10.1901/jaba.2010.43-513. PMID: 21358912.X-1, X-3

174. Farr W, Yuill N, Raffle H. Social benefits of a tangible user interface for children with Autistic Spectrum Conditions. Autism. 2010 May;14(3):237-52. doi: 10.1177/1362361310363280. PMID: 20484323.X-1

175. Fernandes FD, Santos TH, Amato CA, et al. Computerized resources in language therapy with children of the autistic spectrum. Pro Fono. 2010 Oct-Dec;22(4):415-20. PMID: 21271092.X-3

176. Fernell E, Gillberg C. Autism spectrum disorder diagnoses in Stockholm preschoolers. Res Dev Disabil. 2010 MayJun;31(3):680-5. doi: 10.1016/j.ridd.2010.01.007. PMID: 20149593.X-1

177. Finnigan E, Starr E. Increasing social responsiveness in a child with autism. A comparison of music and non-music interventions. Autism. 2010 Jul;14(4):321-48. doi: 10.1177/1362361309357747. PMID: 20591958.X-3, X-4

178. Forbes F, Merriman H, Sparrow N. SIGN guideline on ADHD/HKD updates treatment options. Guidelines in Practice. 2010;13(2):31-41 9p. PMID: 105199214. Language: English. Entry Date: 20100618. Revision Date: 20150711. Publication Type: Journal Article.X-1, X-2

179. Foss-Feig JH, Kwakye LD, Cascio CJ, et al. An extended multisensory temporal binding window in autism spectrum disorders. 
Exp Brain Res. 2010 Jun;203(2):381-9. doi: 10.1007/s00221-010-2240-4. PMID: 20390256.X-1

180. Fournier KA, Hass CJ, Naik SK, et al. Motor coordination in autism spectrum disorders: a synthesis and meta-analysis. Journal of Autism \& Developmental Disorders. 2010;40(10):1227-40 14p. doi: 10.1007/s10803-010-0981-3. PMID: 105106241. Language: English. Entry Date: 20101119. Revision Date: 20150711. Publication Type: Journal Article.X-1, X-2

181. Frankel F, Myatt R, Sugar C, et al. A randomized controlled study of parent-assisted Children's Friendship Training with children having autism spectrum disorders. J Autism Dev Disord. 2010 Jul;40(7):827-42. doi: 10.1007/s10803-009-0932-z. PMID: 20058059.X-1, X-3

182. Frazier TW, Youngstrom EA, Haycook T, et al. Effectiveness of medication combined with intensive behavioral intervention for reducing aggression in youth with autism spectrum disorder. J Child Adolesc Psychopharmacol. 2010 Jun;20(3):167-77. doi: 10.1089/cap.2009.0048. PMID: 20578929.X-1, $\mathrm{X}-3$

183. Fries A. [Adult psychiatry makes wrong assessment of autism spectrum conditions. Correct diagnosis can protect against serious therapeutic errors]. Lakartidningen. 2010 Oct 27-Nov 2;107(43):2660-1. PMID: 21137538.X-1

184. Frye RE, Butler I, Strickland D, et al. Electroencephalogram discharges in atypical cognitive development. J Child Neurol. 2010 May;25(5):556-66. doi: 10.1177/0883073809344743. PMID: 20299700.X-1

185. Frye RE, Huffman LC, Elliott GR. Tetrahydrobiopterin as a novel therapeutic intervention for autism. Neurotherapeutics. 2010 July;7(3):241-9. doi: http://dx.doi.org/10.1016/j.nurt.2010.05.004. PMID: 2010406856.X-1, X-2, X-3

186. Fuentes CT, Mostofsky SH, Bastian AJ. Perceptual reasoning predicts handwriting impairments in adolescents with autism. Neurology. 2010;75(20):1825-9 5p. doi:
10.1212/WNL.0b013e3181fd633d. PMID: 104945920. Language: English. Entry Date: 20110107. Revision Date: 20150711. Publication Type: Journal Article.X-1

187. Fujikawa-Brooks S, Isenberg AL, Osann K, et al. The effect of rate stress on the auditory brainstem response in autism: a preliminary report. Int J Audiol. 2010 Feb;49(2):129-40. doi: 10.3109/14992020903289790. PMID: 20151887.X-1

188. Gal E, Dyck MJ, Passmore A. Relationships Between Stereotyped Movements and Sensory Processing Disorders in Children With and Without Developmental or Sensory Disorders. American Journal of Occupational Therapy. 2010;64(3):453-61 9p. doi: 10.5014/ajot.2010.09075. PMID: 105032111. Language: English. Entry Date: 20130221. Revision Date: 20150711. Publication Type: Journal Article.X-1

189. Gallagher CM, Goodman MS. Hepatitis B vaccination of male neonates and autism diagnosis, NHIS 1997-2002. J Toxicol Environ Health A. 2010;73(24):1665-77. doi: 10.1080/15287394.2010.519317. PMID: 21058170.X-1

190. Ganz JB, Lashley E, Rispoli MJ. Nonresponsiveness to intervention: children with autism spectrum disorders who do not rapidly respond to communication interventions. Dev Neurorehabil. 2010;13(6):399-407. doi: 10.3109/17518423.2010.508298. PMID: 21034281.X-3, X-4

191. Garcia-Villamisar DA, Dattilo J. Effects of a leisure programme on quality of life and stress of individuals with ASD. J Intellect Disabil Res. 2010 Jul;54(7):611-9. doi: 10.1111/j.1365-2788.2010.01289.x. PMID: 20500784.X-1

192. Gentry T, Wallace J, Kvarfordt C, et al. Personal digital assistants as cognitive aids for high school students with autism: results of a community-based trial. Journal of Vocational Rehabilitation. 2010;32(2):101-7 7p. doi: 10.3233/JVR-2010-0499. PMID: 105184442. Language: English. Entry Date: 20100611. Revision Date: 20150819. Publication Type: Journal Article.X-1, X-3 
193. Ghaffari M, West SH, Malone RP, et al. The effects of olanzapine on QTc in children with autistic disorder. Journal of Child and Adolescent Psychopharmacology. 2010 December;20 (6):532. doi: http://dx.doi.org/10.1089/cap.2010.2064. PMID: 70323780.X-2

194. Ghanizadeh A. Transplantation of GABAergic cell line as a novel hypothesized treatment for autism. Epilepsy Behav. 2010 Dec;19(4):664. doi: 10.1016/j.yebeh.2010.09.009. PMID: 20934920.X-1

195. Ghanizadeh A. Methionine sulfoximine may improve inflammation in autism, a novel hypothesized treatment for autism. Arch Med Res. 2010 Nov;41(8):651-2. doi: 10.1016/j.arcmed.2010.10.012. PMID: 21199736.X-2, X-3

196. Ghanizadeh A. Possible role of caffeine in autism spectrum disorders, a new testable hypothesis. J Food Sci. 2010 Aug 1;75(6):ix. doi: 10.1111/j.17503841.2010.01760.x. PMID: 20722962.X-1

197. Ghanizadeh A. Clinical approach to motor stereotypies in autistic children. Iran J Pediatr. 2010 Jun;20(2):149-59. PMID: 23056697.X-1

198. Ghanizadeh A. Targeting neurotensin as a potential novel approach for the treatment of autism. Journal of Neuroinflammation. 2010 01 Oct;7(58)doi: http://dx.doi.org/10.1186/1742-2094-7-58. PMID: 2010562395.X-1, X-2

199. Ghanizadeh A. A novel explanation for potential toxic effects of valproic acid on creatine: Implications for autism. Molecular Genetics and Metabolism. 2010 October;101(23):304. doi:

http://dx.doi.org/10.1016/j.ymgme.2010.07.019. PMID: 2010540656.X-1

200. Gika AD, Hughes E, Goyal S, et al. Trihexyphenidyl for acute life-threatening episodes due to a dystonic movement disorder in Rett syndrome. Movement Disorders. 2010 15 Feb;25(3):385-9. doi: http://dx.doi.org/10.1002/mds.22926. PMID: 2010139061.X-1
201. Gjaerum RG, Ineland J, Sauer L. The story about theater organizations, the public's approval, and the actors' identity formation in Nordic disability theater. J Soc Work Disabil Rehabil. 2010;9(4):254-73. doi: 10.1080/1536710x.2010.523648. PMID: 21104515.X-1

202. Glance JM, Maniloff DJ. Oral motor and articulation therapy for the ASD population...CASPLA Conference 2010, Whitehorse, Yukon, May 19 to 22, 2010. Canadian Journal of Speech-Language Pathology \& Audiology. 2010 Spring2010;34(1):59-1p. PMID: 105171141. Language: English. Entry Date: 20100514. Revision Date: 20150711. Publication Type: Journal Article.X-1

203. Golan O, Ashwin E, Granader Y, et al. Enhancing emotion recognition in children with autism spectrum conditions: an intervention using animated vehicles with real emotional faces. J Autism Dev Disord. 2010 Mar;40(3):269-79. doi: 10.1007/s10803-0090862-9. PMID: 19763807.X-1, X-3

204. Green J, Charman T, McConachie H, et al. Parent-mediated communication-focused treatment in children with autism (PACT): a randomised controlled trial. Lancet. 2010 Jun 19;375(9732):2152-60. doi: 10.1016/s01406736(10)60587-9. PMID: 20494434.X-1

205. Green JJ, Hollander E. Autism and oxytocin: New developments in translational approaches to therapeutics. Neurotherapeutics. 2010 July;7(3):250-7. doi: http://dx.doi.org/10.1016/j.nurt.2010.05.006. PMID: 2010406857.X-1, X-2, X-3

206. Green SA, Ben-Sasson A. Anxiety disorders and sensory over-responsivity in children with autism spectrum disorders: Is there a causal relationship? Journal of Autism and Developmental Disorders. 2010 2015-1209;40(12):1495-504. doi: http://dx.doi.org/10.1007/s10803-010-1007-x. PMID: 819632013; 2010-23786-007.X-1

207. Greenberger C, Serper MR. Examination of clinical and cognitive insight in acute schizophrenia patients. J Nerv Ment Dis. 2010 Jul;198(7):465-9. doi: 10.1097/NMD.0b013e3181e4f35d. PMID: 20611048.X-1 
208. Grether JK, Li SX, Yoshida CK, et al. Antenatal ultrasound and risk of autism spectrum disorders. J Autism Dev Disord. 2010 Feb;40(2):238-45. doi: 10.1007/s10803-0090859-4. PMID: 19728066.X-1

209. Groskreutz NC, Karsina A, Miguel $\mathrm{CF}$, et al. Using complex auditory-visual samples to produce emergent relations in children with autism. J Appl Behav Anal. 2010 Mar;43(1):131-6. doi: 10.1901/jaba.2010.43131. PMID: 20808504.X-1, X-3, X-4

210. Guastella AJ, Einfeld SL, Gray KM, et al. Intranasal oxytocin improves emotion recognition for youth with autism spectrum disorders. Biol Psychiatry. 2010 Apr 1;67(7):692-4. doi: 10.1016/j.biopsych.2009.09.020. PMID: 19897177.X-1, X-4

211. Gulsrud AC, Jahromi LB, Kasari C. The co-regulation of emotions between mothers and their children with autism. J Autism Dev Disord. 2010 Feb;40(2):227-37. doi: 10.1007/s10803-009-0861-X. PMID: 19714458.X-1, X-3

212. Gupta S. Antibodies: basic mechanisms and emerging concepts. J Clin Immunol. 2010 May;30 Suppl 1:S1-3. doi: 10.1007/s10875-010-9395-4. PMID: 20387105.X-1

213. Gupta S, Samra D, Agrawal S. Adaptive and innate immune responses in autism: Rationale for therapeutic use of intravenous immunoglobulin. Journal of Clinical Immunology. 2010 May;30(SUPPL. 1):S90-S6. doi: http://dx.doi.org/10.1007/s10875-010-9402-9. PMID: 2010471828.X-1, X-2

214. Hadjikhani N. Serotonin, pregnancy and increased autism prevalence: is there a link? Med Hypotheses. 2010 May;74(5):880-3. doi: 10.1016/j.mehy.2009.11.015. PMID: 20018455.X-1

215. Hagerman R, Hoem G, Hagerman P. Fragile $\mathrm{X}$ and autism: Intertwined at the molecular level leading to targeted treatments. Molecular Autism. 2010;1(1)doi: http://dx.doi.org/10.1186/2040-2392-1-12. PMID: 2012438020.X-1
216. Hamad CD, Serna RW, Morrison L, et al. Extending the Reach of Early Intervention Training for Practitioners: A Preliminary Investigation of an Online Curriculum for Teaching Behavioral Intervention Knowledge in Autism to Families and Service Providers. Infants Young Child. 2010 Jul 1;23(3):195-208. doi: 10.1097/IYC.0b013e3181e32d5e. PMID: 23504540.X-1

217. Hampel OA, Schaadt AK, Hasmann SE, et al. [Evaluation of Stepping Stones Triple P: Interims analysis of the Stepping-StonesSPC-Multicentric Study]. Klin Padiatr. 2010 Jan-Feb;222(1):18-25. doi: 10.1055/s-00291243161. PMID: 20084587.X-5

218. Hamre HJ, Witt CM, Kienle GS, et al. Anthroposophic therapy for attention deficit hyperactivity: a two-year prospective study in outpatients. Int J Gen Med. 2010;3:239-53. PMID: 20830200.X-1

219. Harony H, Wagner S. The contribution of oxytocin and vasopressin to mammalian social behavior: Potential role in autism spectrum disorder. NeuroSignals. 2010 February;18(2):82-97. doi: http://dx.doi.org/10.1159/000321035. PMID: 2011133478.X-1

220. Hartley-McAndrew M, Weinstock A. Autism Spectrum Disorder: Correlation between aberrant behaviors, EEG abnormalities and seizures. Neurol Int. 2010;2(1):e10. doi: 10.4081/ni.2010.e10. PMID: 21577334.X-1

221. Heinicke MR, Carr JE, Leblanc LA, et al. On the use of fluency training in the behavioral treatment of autism: a commentary. Behav Anal. 2010 Fall;33(2):223-9. PMID: 22532717.X-1

222. Hendy HM, Williams KE, Riegel K, et al. Parent mealtime actions that mediate associations between children's fussy-eating and their weight and diet. Appetite. 2010

Feb;54(1):191-5. doi: 10.1016/j.appet.2009.10.006. PMID: 19887094.X-1

223. Henning OJ, Nakken KO. Psychiatric comorbidity and use of psychotropic drugs in epilepsy patients. Acta Neurol Scand Suppl. 
2010(190):18-22. doi: 10.1111/j.1600-

0404.2010.01370.x. PMID: 20586730.X-1

224. Herbert MR. Contributions of the environment and environmentally vulnerable physiology to autism spectrum disorders. Current Opinion in Neurology. 2010 April;23(2):103-10. doi: http://dx.doi.org/10.1097/WCO.0b013e328336a 01f. PMID: 2010168652.X-1

225. Herguner S. Excessive masturbation associated with olanzapine in a pediatric case. Progress in Neuro-Psychopharmacology and Biological Psychiatry. 2010 October;34(7):1349-50. doi: http://dx.doi.org/10.1016/j.pnpbp.2010.06.017. PMID: 2010489827.X-1

226. Hintzen A, Delespaul P, van Os J, et al. Social needs in daily life in adults with Pervasive Developmental Disorders. Psychiatry Res. 2010 Aug 30;179(1):75-80. doi: 10.1016/j.psychres.2010.06.014. PMID: 20638732.X-1

227. Hobson RP. Explaining autism: Ten reasons to focus on the developing self. Autism. 2010 Sep;14(5):391-407. doi: 10.1177/1362361310364142. PMID: 20926456.X-1

228. Hochhauser M, Engel-Yeger B. Sensory processing abilities and their relation to participation in leisure activities among children with high-functioning autism spectrum disorder (HFASD). Research in Autism Spectrum Disorders. 2010 2015-1209;4(4):746-54. doi: 10.5014/ajot.62.5.554 http://dx.doi.org/10.1016/j.rasd.2010.01.015. PMID: 754048474; 2010-11895-023.X-1

229. Hoekstra PJ, Troost PW, Lahuis BE, et al. Risperidone-induced weight gain in referred children with autism spectrum disorders is associated with a common polymorphism in the 5-hydroxytryptamine 2C receptor gene. J Child Adolesc Psychopharmacol. 2010

Dec;20(6):473-7. doi: 10.1089/cap.2009.0071. PMID: 21186965.X-1, X-3

230. Hoglund Carlsson L, Gillberg C, Lannero E, et al. Autism: screening toddlers with CHAT in a child health care programme did not improve early identification. Acta Paediatr. 2010 Dec;99(12):1897-9. doi: 10.1111/j.1651-2227.2010.01958.x. PMID: 20670307.X-1

231. Holland CD. Autism, insurance, and the idea: providing a comprehensive legal framework. Cornell Law Rev. 2010 Sep;95(6):1253-82. PMID: 20939148.X-1

232. Hollenweger J. MHADIE's matrix to analyse the functioning of education systems. Disabil Rehabil. 2010;32 Suppl 1:S116-24. doi: 10.3109/09638288.2010.520809. PMID: 20874661.X-1

233. Huang SC, Tsai SJ, Yang HJ. Aripiprazole improves social interaction in taiwanese children with pervasive developmental disorder. Chang Gung Medical Journal. 2010 March-April;33(2):211-5. PMID: 2010257149.X-3, X-4

234. Huemer SV, Mann V. A

Comprehensive Profile of Decoding and Comprehension in Autism Spectrum Disorders. J Autism Dev Disord. 2010 Apr;40(4):485-93. doi: 10.1007/s10803-009-0892-3. PMID: 19915968.X-1

235. Huhtanen S. Helping your child build strength: looking outside the box. Exceptional Parent. 2010;40(5):60- 1p. PMID: 105203205. Language: English. Entry Date: 20100618. Revision Date: 20150711. Publication Type: Journal Article. Journal Subset: Consumer Health.X-1

236. Hurwitz R, Blackmore R, Woolfenden $\mathrm{S}$, et al. Tricyclic antidepressants in autism - A cochrane systematic review. Journal of Paediatrics and Child Health. 2010 March;46:7. doi: http://dx.doi.org/10.1111/j.14401754.2010.01722.x. PMID: 70128302.X-1, X-2

237. Hwang SJ, Chen YS. Congenital Rubella Syndrome With Autistic Disorder. Journal of the Chinese Medical Association. 2010 February;73(2):104-7. doi: http://dx.doi.org/10.1016/S17264901\%2810\%2970011-3. PMID: 2010228807.X-1

238. Iarocci G, Rombough A, Yager J, et al. Visual influences on speech perception in children with autism. Autism. 2010 Jul;14(4):305-20. doi: 
10.1177/1362361309353615. PMID: 20591957.X-1, X-3

239. Ingersoll B. Pilot randomized controlled trial of Reciprocal Imitation Training for teaching elicited and spontaneous imitation to children with autism. J Autism Dev Disord. 2010 Sep;40(9):1154-60. doi: 10.1007/s10803010-0966-2. PMID: 20155309.X-1

240. Ingersoll B, Lalonde K. The impact of object and gesture imitation training on language use in children with autism spectrum disorder. J Speech Lang Hear Res. 2010 Aug;53(4):1040-51. doi: 10.1044/10924388(2009/09-0043). PMID: 20631228.X-3, X4

241. Jackett JM. Transition and beyond for individuals with autism spectrum disorders (ASD): a New Jersey case study of the adult service sector, its inherent shortcomings, and hope for the future. Seton Hall Law Rev. 2010;40(4):1733-74. PMID: 21280391.X-1

242. Jafferany M, Shireen F, Ibrahim A. An open-label trial of topiramate in the treatment of skin picking in pervasive developmental disorder not otherwise specified. Prim Care Companion J Clin Psychiatry. 2010;12(2)doi: 10.4088/PCC.09100829yel. PMID: 20694122.X-1

243. James WH. Behavioural and biological determinants of human sex ratio at birth. J Biosoc Sci. 2010 Sep;42(5):587-99. doi: 10.1017/s002193201000012x. PMID: 20519063.X-1

244. Janusis GM, Weyandt LL. An exploratory study of substance use and misuse among college students with and without ADHD and other disabilities. J Atten Disord. 2010 Nov;14(3):205-15. doi: 10.1177/1087054710367600. PMID: 20479474.X-1

245. Jarrold C, Mansergh R, Whiting C. The representational status of pretence: evidence from typical development and autism. British Journal of Developmental Psychology. 2010;28(2):239-54 16p. doi: 10.1348/026151008X400085. PMID: 105019798. Language: English. Entry Date: 20100709. Revision Date: 20150711. Publication Type: Journal Article.X-1
246. Johnson SA, Blaha LM, Houpt JW, et al. Systems Factorial Technology provides new insights on global-local information processing in autism spectrum disorders. J Math Psychol. 2010 Feb;54(1):53-72. doi: 10.1016/j.jmp.2009.06.006. PMID: 23750050.X-1

247. Jones AT, Kildea S, Hunt J, et al. Book reviews. Clinical Child Psychology \& Psychiatry. 2010;15(1):129-43 15p. doi: 10.1177/1359104509348671. PMID: 105166609. Language: English. Entry Date: 20100416. Revision Date: 20150711. Publication Type: Journal Article.X-1

248. Jordan CJ. Evolution of autism support and understanding via the World Wide Web. Intellect Dev Disabil. 2010 Jun;48(3):220-7. doi: 10.1352/1934-9556-48.3.220. PMID: 20597733.X-1

249. Joshi G, Petty C, Wozniak J, et al. The heavy burden of psychiatric comorbidity in youth with autism spectrum disorders: a large comparative study of a psychiatrically referred population. J Autism Dev Disord. 2010 Nov;40(11):1361-70. doi: 10.1007/s10803-0100996-9. PMID: 20309621.X-1

250. Jyonouchi H. Autism spectrum disorders and allergy: observation from a pediatric allergy/immunology clinic. Expert Rev Clin Immunol. 2010 May;6(3):397-411. doi: 10.1586/eci.10.18. PMID: 20441426.X-1

251. Kallstrand J, Olsson O, Nehlstedt SF, et al. Abnormal auditory forward masking pattern in the brainstem response of individuals with Asperger syndrome. Neuropsychiatr Dis Treat. 2010;6:289-96. PMID: 20628629.X-1

252. Kamp-Becker I, Schroder J, Remschmidt $\mathrm{H}$, et al. Health-related quality of life in adolescents and young adults with high functioning autism-spectrum disorder.

Psychosoc Med. 2010;7doi: 10.3205/psm000065. PMID: 20930927.X-1

253. Karakaya P, Yis U, Kurul SH, et al. Rhabdomyolysis associated with olanzapine treatment in a child with Autism. Pediatric Emergency Care. 2010;26(1):41-2 2p. doi: 10.1097/PEC.0b013e3181c39a22. PMID: 105286086. Language: English. Entry Date: 
20100416. Revision Date: 20150711.

Publication Type: Journal Article.X-1, X-3, X-4

254. Karanth P, Shaista S, Srikanth N. Efficacy of communication DEALL--an indigenous early intervention program for children with autism spectrum disorders. Indian J Pediatr. 2010 Sep;77(9):957-62. doi: 10.1007/s12098-010-0144-8. PMID: 20821283.X-3

255. Kasari C, Gulsrud AC, Wong C, et al. Randomized controlled caregiver mediated joint engagement intervention for toddlers with autism. J Autism Dev Disord. 2010 Sep;40(9):1045-56. doi: 10.1007/s10803-0100955-5. PMID: 20145986.X-1

256. Keane BP, Rosenthal O, Chun NH, et al. Audiovisual integration in high functioning adults with autism. Research in Autism Spectrum Disorders. 2010 2015-1209;4(2):276-89. doi: http://dx.doi.org/10.1016/j.rasd.2009.09.015. PMID: 856410148; 2011-03277-014.X-1

257. Ketelaars MP, Cuperus J, Jansonius K, et al. Pragmatic language impairment and associated behavioural problems. Int J Lang Commun Disord. 2010 Mar-Apr;45(2):204-14. doi: 10.3109/13682820902863090. PMID: 22748032.X-1

258. Khetrapal N. Overlap of autism and seizures: understanding cognitive comorbidity. Mens Sana Monogr. 2010 Jan;8(1):122-8. doi: 10.4103/0973-1229.58823. PMID: 21327174.X-1

259. Kim Y, Cho SC, Shin MS, et al. Retrospective case series of aripiprazole augmentation in pervasive developmental disorders. Psychiatry Investig. 2010 Sep;7(3):220-3. doi: 10.4306/pi.2010.7.3.220. PMID: 20927312.X-1, X-3

260. Koegel LK, Singh AK, Koegel RL. Improving motivation for academics in children with autism. J Autism Dev Disord. 2010 Sep;40(9):1057-66. doi: 10.1007/s10803-0100962-6. PMID: 20221791.X-1

261. Koenig K, White SW, Pachler M, et al. Promoting social skill development in children with pervasive developmental disorders: a feasibility and efficacy study. J Autism Dev
Disord. 2010 Oct;40(10):1209-18. doi: 10.1007/s10803-010-0979-x. PMID: 20204689.X-1

262. Kolar D. Efficacy of risperidone on the core symptoms of autistic disorder. European Neuropsychopharmacology. 2010

August;20:S621. doi: http://dx.doi.org/10.1016/S0924977X\%2810\%2970951-2. PMID: 70311951.X$1, \mathrm{X}-3$

263. Koldewyn K, Whitney D, Rivera SM. The psychophysics of visual motion and global form processing in autism. Brain: A Journal of Neurology. 2010;133(2):599-610 12p. doi: brain/awp272. PMID: 105128108. Language: English. Entry Date: 20100326. Revision Date: 20150711. Publication Type: Journal Article. Journal Subset: Allied Health.X-1

264. Kopp S, Beckung E, Gillberg C. Developmental coordination disorder and other motor control problems in girls with autism spectrum disorder and/or attentiondeficit/hyperactivity disorder. Res Dev Disabil. 2010 Mar-Apr;31(2):350-61. doi: 10.1016/j.ridd.2009.09.017. PMID: 19910158.X-1

265. Koshelev M, Lohrenz T, Vannucci M, et al. Biosensor approach to psychopathology classification. PLoS Comput Biol. 2010;6(10):e1000966. doi: 10.1371/journal.pcbi.1000966. PMID: 20975934.X-1

266. Kozulin A, Lebeer J, Madella-Noja A, et al. Cognitive modifiability of children with developmental disabilities: a multicentre study using Feuerstein's Instrumental Enrichment-Basic program. Res Dev Disabil. 2010 MarApr;31(2):551-9. doi: 10.1016/j.ridd.2009.12.001. PMID: 20056377.X-1

267. Kream RM, Stefano GB, Ptacek R. Psychiatric implications of endogenous morphine: Up-to-date review. Folia Biologica. 2010;56(6):231-41. PMID: 2011027328.X-1, $\mathrm{X}-2$

268. Kring SR, Greenberg JS, Seltzer MM. The Impact of Health Problems on Behavior Problems in Adolescents and Adults With Autism Spectrum Disorders: Implications for 
Maternal Burden. Soc Work Ment Health. 2010

Jan;8(1):54-71. doi:

10.1080/15332980902932441. PMID:

20657702.X-1

269. Kroeger K, Sorensen R. A parent training model for toilet training children with autism. J Intellect Disabil Res. 2010

Jun;54(6):556-67. doi: 10.1111/j.13652788.2010.01286.x. PMID: 20576064.X-3, X-4

270. Kuhn G, Kourkoulou A, Leekam SR. How magic changes our expectations about autism. Psychol Sci. 2010 Oct;21(10):1487-93. doi: 10.1177/0956797610383435. PMID: 20855904.X-1

271. Kujala T, Kuuluvainen S, Saalasti S, et al. Speech-feature discrimination in children with Asperger syndrome as determined with the multi-feature mismatch negativity paradigm. Clin Neurophysiol. 2010 Sep;121(9):1410-9. doi: 10.1016/j.clinph.2010.03.017. PMID: 20382070.X-1

272. Kuwagata M, Ogawa T, Shioda S, et al. Development of offspring brain in a rat valproate-induced autism model. Congenital Anomalies. 2010 December;50 (4):A17. doi: http://dx.doi.org/10.1111/j.17414520.2010.00297.x. PMID: 70434145.X-1

273. LaCava PG, Rankin A, Mahlios E, et al. A single case design evaluation of a software and tutor intervention addressing emotion recognition and social interaction in four boys with ASD. Autism. 2010 May;14(3):161-78. doi: 10.1177/1362361310362085. PMID: 20488823.X-3, X-4

274. Lai DK, Barnes G, Fu C, et al. Autism spectrum disorders and epilepsy in patients referred for electroencephalography. Annals of Neurology. 2010;68:S40-S1. doi: http://dx.doi.org/10.1002/ana.22175. PMID: 70368747.X-1

275. Lam SF, Wong BP, Leung D, et al. How parents perceive and feel about participation in community activities. The comparison between parents of preschoolers with and without Autism Spectrum Disorders. Autism. 2010 Jul;14(4):359-77. doi: 10.1177/1362361309346558. PMID: 20591960.X-1
276. Lambrey S, Falissard B, Martin-

Barrero M, et al. Effectiveness of clozapine for the treatment of aggression in an adolescent with autistic disorder. Journal of Child and Adolescent Psychopharmacology. 201001 Feb;20(1):79-80. doi: http://dx.doi.org/10.1089/cap.2009.0057. PMID: 2010129311.X-3, X-4

277. Lang R, Koegel LK, Ashbaugh K, et al. Physical exercise and individuals with autism spectrum disorders: A systematic review. Research in Autism Spectrum Disorders. 2010 October-December;4(4):56576. doi: http://dx.doi.org/10.1016/j.rasd.2010.01.006. PMID: 2010552029.X-1, X-2

278. Larson E. Ever vigilant: maternal support of participation in daily life for boys with autism. Phys Occup Ther Pediatr. 2010 Feb;30(1):16-27. doi: 10.3109/01942630903297227. PMID: 20170429.X-1

279. Lawrence DH, Alleckson DA, Bjorklund P. Beyond the Roadblocks: Transitioning to Adulthood With Asperger's Disorder. Archives of Psychiatric Nursing. 2010 August;24(4):227-38. doi: http://dx.doi.org/10.1016/j.apnu.2009.07.004. PMID: 20650368.X-1

280. Lee-Mosier J, Waldrop JB. Advisor forum. Alternatives to stimulants for patients with autism. Clinical Advisor. 2010;13(5):481p. PMID: 105209947. Language: English. Entry Date: 20100702. Revision Date: 20150711. Publication Type: Journal Article.X$1, \mathrm{X}-2$

281. Leew SV, Stein NG, Gibbard WB. Weighted vests' effect on social attention for toddlers with Autism Spectrum Disorders. Can J Occup Ther. 2010 Apr;77(2):113-24. PMID: 20464896.X-1, X-3

282. Lemonnier E, Ben-Ari Y. The diuretic bumetanide decreases autistic behaviour in five infants treated during 3 months with no side effects. Acta Paediatr. 2010 Dec;99(12):1885-8. doi: 10.1111/j.1651-2227.2010.01933.x. PMID: 20608900.X-3, X-4

283. Leonard H, Downs J, Jian L, et al. Valproate and risk of fracture in Rett syndrome. 
Archives of Disease in Childhood. 2010

June;95(6):444-8. doi:

http://dx.doi.org/10.1136/adc.2008.148932.

PMID: 2010342729.X-1

284. Lepach AC, Lehmkuhl G, Petermann

F. [Neuropsychological issues in child

psychology and child psychiatry]. Prax

Kinderpsychol Kinderpsychiatr.

2010;59(7):576-87. doi:

10.13109/prkk.2010.59.7.576. PMID:

20957778.X-1

285. Levy ML, Levy KM, Hoff D, et al. Vagus nerve stimulation therapy in patients with autism spectrum disorder and intractable epilepsy: results from the vagus nerve stimulation therapy patient outcome registry. $\mathrm{J}$ Neurosurg Pediatr. 2010 Jun;5(6):595-602. doi: 10.3171/2010.3.peds09153. PMID: 20515333.X-1, X-3

286. Lim HA. Effect of "developmental speech and language training through music" on speech production in children with autism spectrum disorders. J Music Ther. 2010 Spring;47(1):2-26. PMID: 20635521.X-1

287. Litras S, Moore DW, Anderson A. Using video self-modelled social stories to teach social skills to a young child with autism. Autism Res Treat. 2010;2010:834979. doi: 10.1155/2010/834979. PMID: 22937239.X-3, $\mathrm{X}-4$

288. Lomas JE, Fisher WW, Kelley ME. The effects of variable-time delivery of food items and praise on problem behavior reinforced by escape. J Appl Behav Anal. 2010 Fall;43(3):425-35. doi: 10.1901/jaba.2010.43425. PMID: 21358903.X-3, X-4

289. Lombardo MV, Chakrabarti B, Bullmore ET, et al. Atypical neural selfrepresentation in autism. Brain: A Journal of Neurology. 2010;133(2):611-24 14p. doi: brain/awp306. PMID: 105128113. Corporate Author: MRC AIMS Consortium. Language: English. Entry Date: 20100326. Revision Date: 20150711. Publication Type: Journal Article. Journal Subset: Allied Health.X-1

290. Lopata C, Thomeer ML, Volker MA, et al. RCT of a manualized social treatment for high-functioning autism spectrum disorders. J Autism Dev Disord. 2010 Nov;40(11):1297-
310. doi: 10.1007/s10803-010-0989-8. PMID: 20232240.X-1

291. Loth E, Happe F, Gomez JC. Variety is not the spice of life for people with autism spectrum disorders: frequency ratings of central, variable and inappropriate aspects of common real-life events. J Autism Dev Disord. 2010 Jun;40(6):730-42. doi: 10.1007/s10803009-0929-7. PMID: 20066484.X-1

292. Luppanapornlarp S, Leelataweewud P, Putongkam P, et al. Periodontal status and orthodontic treatment need of autistic children. World J Orthod. 2010 Fall;11(3):256-61. PMID: 20877735.X-1

293. Luthy KE, Beckstrand RL, Callister LC. Parental hesitation in immunizing children in Utah. Public Health Nurs. 2010 JanFeb;27(1):25-31. doi: 10.1111/j.15251446.2009.00823.x. PMID: 20055965.X-1

294. Lynch E. Making sense of autism. Nurs Stand. 2010 Sep 15-21;25(2):18-9. PMID: 20949817.X-1

295. Maenner MJ, Durkin MS. Trends in the prevalence of autism on the basis of special education data. Pediatrics. 2010

Nov;126(5):e1018-25. doi: 10.1542/peds.20101023. PMID: 20974790.X-1

296. Majewska MD, Urbanowicz E, RokBujko P, et al. Age-dependent lower or higher levels of hair mercury in autistic children than in healthy controls. Acta Neurobiol Exp (Wars). 2010;70(2):196-208. PMID: 20628443.X-1

297. Malhotra S, Rajender G, Bhatia MS, et al. Effects of picture exchange communication system on communication and behavioral anomalies in autism. Indian J Psychol Med. 2010 Jul;32(2):141-3. doi: 10.4103/02537176.78513. PMID: 21716776.X-3, X-4

298. Malone RP, West SH, Ghaffari M, et al. Metabolic effects of olanzapine in children with autistic disorder. Journal of Child and Adolescent Psychopharmacology. 2010 December;20 (6):531-2. doi: http://dx.doi.org/10.1089/cap.2010.2064. PMID: 70323779.X-2

299. Mandell DS, Morales KH, Xie M, et al. Age of diagnosis among Medicaid-enrolled 
children with autism, 2001-2004. Psychiatr

Serv. 2010 Aug;61(8):822-9. doi:

10.1176/appi.ps.61.8.822. PMID: 20675842.X-

1

300. Markram K, Markram H. The intense world theory - a unifying theory of the neurobiology of autism. Front Hum Neurosci. 2010;4:224. doi: 10.3389/fnhum.2010.00224. PMID: 21191475.X-1

301. Marmon P. The map: an innocent interaction with far-reaching outcomes. School Nurse News. 2010 May;27(3):15-6. PMID: 20476529.X-1

302. Marr D, Nackley V. Using social stories \& sensory stories in autism intervention. OT Practice. 2010;15(10):17-20 4p. PMID: 105022860. Language: English. Entry Date: 20100716. Revision Date: 20150820.

Publication Type: Journal Article.X-1

303. Marshall J, Sheller B, Mancl L. Cariesrisk assessment and caries status of children with autism. Pediatr Dent. 2010 JanFeb;32(1):69-75. PMID: 20298657.X-1

304. Martsenkovsky I, Bikshaieva I, Vashenko O, et al. Memantine therapy of cognitive, behavioral, and social dysfunction in children witch autism spectrum disorders (ASD). International Journal of Neuropsychopharmacology. 2010 June;13:138. doi:

http://dx.doi.org/10.1017/S1461145710000635. PMID: 70220667.X-2

305. Matson JL, Boisjoli JA, Hess JA, et al. Factor structure and diagnostic fidelity of the Baby and Infant Screen for Children with aUtIsm Traits-Part 1 (BISCUIT-part 1). Dev Neurorehabil. 2010;13(2):72-9. doi: 10.3109/17518420903213576. PMID: 20222767.X-1

306. Matsuo M, Maeda T, Sasaki K, et al. Frequent association of autism spectrum disorder in patients with childhood onset epilepsy. Brain Dev. 2010 Oct;32(9):759-63. doi: 10.1016/j.braindev.2010.05.005. PMID: 20542395.X-1

307. May-Benson TA, Koomar JA. Systematic review of the research evidence examining the effectiveness of interventions using a sensory integrative approach for children. Am J Occup Ther. 2010 MayJun;64(3):403-14. PMID: 20608272.X-1, X-2

308. McCleery JP, Ceponiene R, Burner $\mathrm{KM}$, et al. Neural correlates of verbal and nonverbal semantic integration in children with autism spectrum disorders. J Child Psychol Psychiatry. 2010 Mar;51(3):277-86. doi: 10.1111/j.1469-7610.2009.02157.x. PMID: 20025622.X-1

309. McCracken JT, Aman MG, McDougle $\mathrm{CJ}$, et al. Possible influence of variant of the Pglycoprotein gene (MDR1/ABCB1) on clinical response to guanfacine in children with pervasive developmental disorders and hyperactivity. J Child Adolesc Psychopharmacol. 2010 Feb;20(1):1-5. doi: 10.1089/cap.2009.0059. PMID: 20166790.X-1, $\mathrm{X}-3$

310. McCravy S, Johnson A, Wetsel MA, et al. Speak the language of autism. Nurse Practitioner. 2010;35(4):26-34 9p. doi: 10.1097/01.NPR.0000369939.77975.54. PMID: 105163758. Language: English. Entry Date: 20100528. Revision Date: 20150819. Publication Type: Journal Article.X-1, X-2

311. McDuffie A, Yoder P. Types of parent verbal responsiveness that predict language in young children with autism spectrum disorder. J Speech Lang Hear Res. 2010 Aug;53(4):102639. doi: 10.1044/1092-4388(2009/09-0023). PMID: 20605942.X-1

312. McGonigle-Chalmers M, AldersonDay B. Free classification as a window on executive functioning in autism spectrum disorders. Journal of Autism \& Developmental Disorders. 2010;40(7):844-57 14p. doi: 10.1007/s10803-010-0947-5. PMID: 105038413. Language: English. Entry Date: 20100820. Revision Date: 20150711. Publication Type: Journal Article.X-1

313. McGuinness TM, Lewis S. Update on autism and vaccines. J Psychosoc Nurs Ment Health Serv. 2010 Jun;48(6):15-8. doi: 10.3928/02793695-20100506-02. PMID: 20506968.X-1

314. Mehl-Madrona L, Leung B, Kennedy $\mathrm{C}$, et al. Micronutrients versus standard medication management in autism: a 
naturalistic case-control study. J Child Adolesc Psychopharmacol. 2010 Apr;20(2):95-103. doi: 10.1089/cap.2009.0011. PMID: 20415604.X-1

315. Mesibov GB, Shea V. The TEACCH program in the era of evidence-based practice. $\mathrm{J}$ Autism Dev Disord. 2010 May;40(5):570-9. doi: 10.1007/s10803-009-0901-6. PMID: 19937103.X-1

316. Michel TM, Sheldrick AJ, Frentzel TG, et al. [Evaluation of diagnostic and therapeutic services in German university hospitals for adults with autism spectrum disorder (ASD)]. Fortschr Neurol Psychiatr. 2010 Jul;78(7):402-13. doi: 10.1055/s-00291245494. PMID: 20607640.X-1

317. Molinari JA. Vaccination: science versus perception. Dent Today. 2010 Dec;29(12):84, 6. PMID: 21229925.X-1

318. Monk CS, Weng SJ, Wiggins JL, et al. Neural circuitry of emotional face processing in autism spectrum disorders. Journal of Psychiatry and Neuroscience. 2010 March;35(2):105-14. doi: http://dx.doi.org/10.1503/jpn.090085. PMID: 2010152931.X-1

319. Moon M. Can parents of a child with autism refuse treatment for him? Virtual Mentor. 2010;12(11):844-8. doi: 10.1001/virtualmentor.2010.12.11.ccas1-1011. PMID: 23186788.X-1

320. Mothander PR, Moe RG. Self-reported depressive symptoms and parental stress in mothers and fathers who bring their infants to an infant mental health clinic. Nord $\mathrm{J}$ Psychiatry. 2010 Oct;64(5):310-6. doi: 10.3109/08039480903528138. PMID: 20184497.X-1

321. Mrozek-Budzyn D, Kieltyka A, Majewska R. Lack of association between measles-mumps-rubella vaccination and autism in children: a case-control study. Pediatr Infect Dis J. 2010 May;29(5):397-400. doi: 10.1097/INF.0b013e3181c40a8a. PMID: 19952979.X-1

322. Mulloy A, Lang R, O'Reilly M, et al. Gluten-free and casein-free diets in the treatment of autism spectrum disorders: A systematic review. Research in Autism
Spectrum Disorders. 2010 July 2010/September;4(3):328-39. doi: http://dx.doi.org/10.1016/j.rasd.2009.10.008. PMID: 2010176368.X-1, X-2

323. Murphy C, Barnes-Holmes D. Establishing five derived mands in three adolescent boys with autism. J Appl Behav Anal. 2010 Fall;43(3):537-41. doi: 10.1901/jaba.2010.43-537. PMID: 21358916.X-1

324. Murray MJ. Attentiondeficit/hyperactivity disorder in the context of autism spectrum disorders. Current Psychiatry Reports. 2010 October;12(5):382-8. doi: http://dx.doi.org/10.1007/s11920-010-0145-3. PMID: 2010584756.X-1

325. Murza KA, Pavelko SL, Malani MD, et al. Vitamin B6-magnesium treatment for autism: the current status of the research. Magnesium research : official organ of the International Society for the Development of Research on Magnesium. 2010 Jun;23(2):115-7. PMID: 20562088.X-2

326. Myers SM. Citalopram not effective for repetitive behaviour in autistic spectrum disorders. Evidence Based Mental Health. 2010;13(1):22- 1p. doi: 10.1136/ebmh.13.1.22. PMID: 105126637. Language: English. Entry Date: 20100423. Revision Date: 20150711. Publication Type: Journal Article.X-2

327. Narayanan A, White CA, Saklayen S, et al. Effect of propranolol on functional connectivity in autism spectrum disorder--a pilot study. Brain Imaging Behav. 2010 Jun;4(2):189-97. doi: 10.1007/s11682-0109098-8. PMID: 20502989.X-1, X-4

328. Narita M, Oyabu A, Imura Y, et al. Nonexploratory movement and behavioral alterations in a thalidomide or valproic acidinduced autism model rat. Neuroscience Research. 2010 January;66(1):2-6. doi: http://dx.doi.org/10.1016/j.neures.2009.09.001. PMID: 2010009308.X-1

329. Nigro-Bruzzi D, Sturmey P. The effects of behavioral skills training on mand training by staff and unprompted vocal mands by children. J Appl Behav Anal. 2010 Winter;43(4):757-61. doi: 
10.1901/jaba.2010.43-757. PMID:

21541162.X-1, X-3, X-4

330. Nizamie A, Sengupta U, Mishra BR, et al. Role of early multimodal interventions in a case with autistic regression. Acta Neurologica Taiwanica. 2010 March;19(1):51-6. PMID: 2010216450.X-1, X-3, X-4

331. Noterdaeme M, Hutzelmeyer-Nickels A. Early symptoms and recognition of pervasive developmental disorders in Germany. Autism. 2010 Nov;14(6):575-88. doi: 10.1177/1362361310371951. PMID: 20923894.X-1

332. Noterdaeme M, Wriedt E, Höhne C. Asperger's syndrome and high-functioning autism: language, motor and cognitive profiles. European Child \& Adolescent Psychiatry. 2010;19(6):475-81 7p. doi: 10.1007/s00787009-0057-0. PMID: 105023319. Language: English. Entry Date: 20100813. Revision Date: 20150711. Publication Type: Journal Article.X1

333. Oberman LM, Horvath JC, PascualLeone A. TMS: using the theta-burst protocol to explore mechanism of plasticity in individuals with Fragile X syndrome and autism. J Vis Exp. 2010(46)doi: 10.3791/2272. PMID:

21248685.X-1, X-2, X-3

334. Olfson M, Crystal S, Huang C, et al. Trends in antipsychotic drug use by very young, privately insured children. J Am Acad Child Adolesc Psychiatry. 2010 Jan;49(1):13-23. PMID: 20215922.X-1

335. Olin AR, Reichle J, Johnson L, et al. Examining dynamic visual scene displays: implications for arranging and teaching symbol selection. Am J Speech Lang Pathol. 2010 Nov;19(4):284-97. doi: 10.1044/10580360(2010/09-0001). PMID: 20581110.X-1

336. Ooga K, Abe Y, Okudaira T, et al. Lamotrigine adjunctive therapy and possible sleep regulation in adult autism spectrum disorder: A case report. European

Neuropsychopharmacology. 2010

August;20:S414-S5. doi: http://dx.doi.org/10.1016/S0924977X\%2810\%2970595-2. PMID: 70311595.X1
337. Oosterling I, Visser J, Swinkels S, et al. Randomized controlled trial of the focus parent training for toddlers with autism: 1-year outcome. J Autism Dev Disord. 2010 Dec;40(12):1447-58. doi: 10.1007/s10803-0101004-0. PMID: 20440639.X-1

338. Owley T, Brune CW, Salt J, et al. A pharmacogenetic study of escitalopram in autism spectrum disorders. Autism Res. 2010 Feb;3(1):1-7. doi: 10.1002/aur.109. PMID: 20020537.X-3

339. Ozguven HD, Oner O, Baskak B, et al. Theory of Mind in Schizophrenia and Asperger's Syndrome: Relationship with Negative Symptoms. Klinik Psikofarmakol Bulteni. 2010;20(1):5-13. PMID: 25584026.X1

340. Palmer RF, Walker T, Mandell D, et al. Explaining low rates of autism among Hispanic schoolchildren in Texas. Am J Public Health. 2010 Feb;100(2):270-2. doi: 10.2105/ajph.2008.150565. PMID: 20019320.X-1

341. Pan CY. Effects of water exercise swimming program on aquatic skills and social behaviors in children with autism spectrum disorders. Autism. 2010 Jan;14(1):9-28. doi: 10.1177/1362361309339496. PMID: 20124502.X-4

342. Panagiotopoulos C, Ronsley R, Elbe D, et al. First do no harm: promoting an evidence-based approach to atypical antipsychotic use in children and adolescents. J Can Acad Child Adolesc Psychiatry. 2010 May;19(2):124-37. PMID: 20467549.X-1, X-2

343. Pardini M, Guida S, Gialloreti LE. Aripiprazole treatment for coprophagia in autistic disorder. Journal of Neuropsychiatry and Clinical Neurosciences. 2010 Fall;22(4):E33. doi: http://dx.doi.org/10.1176/appi.neuropsych.22.4. 451-s.e33. PMID: 2010612213.X-1, X-2

344. Parr J. Autism. BMJ Clin Evid. 2010;2010 PMID: 21729335.X-2

345. Paul LK, Corsello C, Tranel D, et al. Does bilateral damage to the human amygdala produce autistic symptoms? J Neurodev Disord. 
2010 Sep;2(3):165-73. doi: 10.1007/s11689010-9056-1. PMID: 20700516.X-1

346. Pedrosa E, Shah A, Tenore C, et al. beta-catenin promoter ChIP-chip reveals potential schizophrenia and bipolar disorder gene network. J Neurogenet. 2010

Dec;24(4):182-93. doi: 10.3109/01677063.2010.495182. PMID: 20615089.X-1

347. Peters-Scheffer N, Didden R, Mulders $\mathrm{M}$, et al. Low intensity behavioral treatment supplementing preschool services for young children with autism spectrum disorders and severe to mild intellectual disability. Res Dev Disabil. 2010 Nov-Dec;31(6):1678-84. doi: 10.1016/j.ridd.2010.04.008. PMID: 20627451.X-1

348. Petrovic B, Grzadziel A, Rutonjski L, et al. Linear array measurements of enhanced dynamic wedge and treatment planning system (TPS) calculation for $15 \mathrm{MV}$ photon beam and comparison with electronic portal imaging device (EPID) measurements. Radiol Oncol. 2010 Sep;44(3):199-206. doi: 10.2478/v10019010-0037-5. PMID: 22933916.X-1

349. Philippe P, Scholl JM, Jacques J. Comorbidity in autism spectrum. Psychiatr Danub. 2010 Nov;22 Suppl 1:S158-60. PMID: 21057429.X-1

350. Polanczyk G, Bigarella MP, Hutz MH, et al. Pharmacogenetic approach for a better drug treatment in children. Current Pharmaceutical Design. 2010;16(22):2462-73. doi:

http://dx.doi.org/10.2174/138161210791959872 . PMID: 2010444956.X-1, X-2, X-3

351. Polatajko HJ, Cantin N. Exploring the effectiveness of occupational therapy interventions, other than the sensory integration approach, with children and adolescents experiencing difficulty processing and integrating sensory information. Am J Occup Ther. 2010 May-Jun;64(3):415-29. PMID: 20608273.X-2

352. Pop-Jordanova N, Zorcec T, Demerdzieva A, et al. QEEG characteristics and spectrum weighted frequency for children diagnosed as autistic spectrum disorder.
Nonlinear Biomed Phys. 2010;4(1):4. doi: 10.1186/1753-4631-4-4. PMID: 20920283.X-1

353. Portoghese C, Buttiglione M, De Giacomo A, et al. Leiter-R versus developmental quotient for estimating cognitive function in preschoolers with pervasive developmental disorders. Neuropsychiatr Dis Treat. 2010;6:337-42. PMID: 20856598.X-1

354. Radhakrishna S. Application of integrated yoga therapy to increase imitation skills in children with autism spectrum disorder. Int J Yoga. 2010 Jan;3(1):26-30. doi: 10.4103/0973-6131.66775. PMID: 20948898.X-3, X-4

355. Radhakrishna S, Nagarathna R, Nagendra HR. Integrated approach to yoga therapy and autism spectrum disorders. J Ayurveda Integr Med. 2010 Apr;1(2):120-4. doi: 10.4103/0975-9476.65089. PMID: 21836799.X-1, X-3

356. Rajapakse T, Pringsheim T. Pharmacotherapeutics of tourette syndrome and stereotypies in autism. Seminars in Pediatric Neurology. 2010;17(4):254-60 7p. doi: 10.1016/j.spen.2010.10.008. PMID: 104972997. Language: English. Entry Date: 20110512. Revision Date: 20150711. Publication Type: Journal Article.X-1, X-2

357. Rao S, Salmon G. Autism spectrum disorders. Br J Hosp Med (Lond). 2010 Dec;71(12):699-703. PMID: 21135768.X-1

358. Read N, Schofield A. Autism: are mental health services failing children and parents? J Fam Health Care. 2010;20(4):120-4. PMID: 21053660.X-1, X-2

359. Reed P, Osborne LA, Corness M. Effectiveness of special nursery provision for children with autism spectrum disorders. Autism. 2010 Jan;14(1):67-82. doi: 10.1177/1362361309340030. PMID: 20124504.X-1

360. Reichow B, Barton EE, Sewell JN, et al. Effects of weighted vests on the engagement of children with developmental delays and autism. Focus on Autism and Other Developmental Disabilities. 2010 2015-1209;25(1):3-11. doi: 
http://dx.doi.org/10.1177/1088357609353751. PMID: 622209770; 2010-02848-001.X-3, X-4

361. Riquet A, Cuisset JM, Cuvellier JC, et al. [Characteristics of tuberous sclerosis in children]. Arch Pediatr. 2010 Sep;17(9):133845. doi: 10.1016/j.arcped.2010.06.019. PMID: 20709508.X-1

362. Rivera-Baltanas T, Romay-Tallon R, Dopeso-Reyes IG, et al. Serotonin transporter clustering in blood lymphocytes of reeler mice. Cardiovasc Psychiatry Neurol.

2010;2010:396282. doi: 10.1155/2010/396282. PMID: 20414372.X-1

363. Robb AS. Managing irritability and aggression in autism spectrum disorders in children and adolescents. Developmental Disabilities Research Reviews. 2010;16(3):25864. doi: http://dx.doi.org/10.1002/ddrr.118. PMID: 2011190147.X-1, X-2

364. Robel L. [Medical care organizations for the management of autism in France]. Rev Prat. 2010 Mar 20;60(3):389-90. PMID: 20402135.X-2, X-3

365. Roberts D, Pickering N. Parent training programme for autism spectrum disorders: an evaluation. Community Pract. 2010 Oct;83(10):27-30. PMID: 21049753.X-3

366. Roberts TP, Khan SY, Rey M, et al. MEG detection of delayed auditory evoked responses in autism spectrum disorders: towards an imaging biomarker for autism. Autism Res. 2010 Feb;3(1):8-18. doi: 10.1002/aur.111. PMID: 20063319.X-1

367. Rodger S, Ashburner J, Cartmill L, et al. Helping children with autism spectrum disorders and their families: are we losing our occupation-centred focus? Aust Occup Ther J. 2010 Aug;57(4):276-80. doi: 10.1111/j.14401630.2010.00877.x. PMID: 20854603.X-1, X-2

368. Rodger S, Vishram A. Mastering social and organization goals: strategy use by two children with Asperger syndrome during cognitive orientation to daily occupational performance. Phys Occup Ther Pediatr. 2010 Nov;30(4):264-76. doi: 10.3109/01942638.2010.500893. PMID: 20822395.X-1
369. Romano E, Adriani W, Marco-Lopez EM, et al. Cannabinoid-induced modulation of behavioural stereotypies induced by amphetamine in a putative mouse model of autism. Behavioural Pharmacology. 2010 September;21 (5-6):592-3. doi: http://dx.doi.org/10.1097/FBP.0b013e32833dbe 35. PMID: 70448425.X-1

370. Rosenberg RE, Mandell DS, Farmer JE, et al. Psychotropic medication use among children with autism spectrum disorders enrolled in a national registry, 2007-2008. J Autism Dev Disord. 2010 Mar;40(3):342-51. doi: 10.1007/s10803-009-0878-1. PMID: 19806445.X-1, X-3

371. Roullet FI, Wollaston L, deCatanzaro $\mathrm{D}$, et al. Behavioral and molecular changes in the mouse in response to prenatal exposure to the anti-epileptic drug valproic acid.

Neuroscience. 201013 Oct;170(2):514-22. doi: http://dx.doi.org/10.1016/j.neuroscience.2010.0 6.069. PMID: 2010473848.X-1

372. Roux JC, Villard L. Biogenic amines in rett syndrome: The usual suspects. Behavior Genetics. 2010 January;40(1):59-75. doi: http://dx.doi.org/10.1007/s10519-009-9303-y. PMID: 2010163163.X-1

373. Ruble LA, Dalrymple NJ, McGrew JH. The Effects of Consultation on Individualized Education Program Outcomes for Young Children With Autism: The Collaborative Model for Promoting Competence and Success. J Early Interv. 2010 Sep;32(4):286-301. doi: 10.1177/1053815110382973. PMID: 21691449.X-1, X-3

374. Russo N, Foxe JJ, Brandwein AB, et al. Multisensory processing in children with autism: high-density electrical mapping of auditory-somatosensory integration. Autism Res. 2010 Oct;3(5):253-67. doi: 10.1002/aur.152. PMID: 20730775.X-1

375. Russo NM, Hornickel J, Nicol T, et al. Biological changes in auditory function following training in children with autism spectrum disorders. Behav Brain Funct. 2010;6:60. doi: 10.1186/1744-9081-6-60. PMID: 20950487.X-4 
376. Salamanca AA. Psychotherapy treatment program in a group of adults with high-functioning autism spectrum disorder. Actas Esp Psiquiatr. 2010 Mar-Apr;38(2):94100. PMID: 20976638.X-1

377. Sappok T, Bergmann T, Kaiser H, et al. [Autism in adults with intellectual disabilities]. Nervenarzt. 2010

Nov;81(11):1333-45. doi: 10.1007/s00115-0103098-1. PMID: 20857275.X-1

378. Sardari D, Maleki R, Samavat H, et al. Measurement of depth-dose of linear accelerator and simulation by use of Geant 4 computer code. Rep Pract Oncol Radiother. 2010;15(3):64-8. doi: 10.1016/j.rpor.2010.03.001. PMID: 24376926.X-1

379. Sawyer MG, Bittman M, La Greca AM, et al. Time demands of caring for children with autism: what are the implications for maternal mental health? J Autism Dev Disord. 2010 May;40(5):620-8. doi: 10.1007/s10803009-0912-3. PMID: 19949845.X-1

380. Sbacchi S, Acquadro F, Calo I, et al. Functional annotation of genes overlapping copy number variants in autistic patients: focus on axon pathfinding. Curr Genomics. 2010 Apr;11(2):136-45. doi: 10.2174/138920210790886880. PMID: 20885821.X-1

381. Scheeren AM, Begeer S, Banerjee R, et al. Can you tell me something about yourself?: Self-presentation in children and adolescents with high functioning autism spectrum disorder in hypothetical and real life situations. Autism. 2010 Sep;14(5):457-73. doi: 10.1177/1362361310366568. PMID: 20841344.X-1

382. Schultz ST. Can autism be triggered by acetaminophen activation of the endocannabinoid system? Acta Neurobiol Exp (Wars). 2010;70(2):227-31. PMID: 20628445.X-1

383. Self TL, Hale LS, Crumrine D. Pharmacotherapy and children with autism spectrum disorder: a tutorial for speechlanguage pathologists. Lang Speech Hear Serv Sch. 2010 Jul;41(3):367-75. doi: 10.1044/0161-
1461(2009/08-0106). PMID: 20543026.X-1, X2, X-3

384. Senel HG. Parents' views and experiences about complementary and alternative medicine treatments for their children with autistic spectrum disorder. $\mathrm{J}$ Autism Dev Disord. 2010 Apr;40(4):494-503. doi: 10.1007/s10803-009-0891-4. PMID: 19904598.X-1

385. Shapiro JR, Bibat G, Hiremath G, et al. Bone mass in rett syndrome: Association with clinical parameters and MECP2 mutations. Pediatric Research. 2010 November;68(5):44651. doi: http://dx.doi.org/10.1203/PDR.0b013e3181f2ed d2. PMID: 2010586586.X-1

386. Shaw W. Increased urinary excretion of a 3-(3-hydroxyphenyl)-3-hydroxypropionic acid (HPHPA), an abnormal phenylalanine metabolite of Clostridia spp. in the gastrointestinal tract, in urine samples from patients with autism and schizophrenia. Nutr Neurosci. 2010 Jun;13(3):135-43. doi: 10.1179/147683010x12611460763968. PMID: 20423563.X-1

387. Sheldrick RC, Perrin EC. Medical home services for children with behavioral health conditions. J Dev Behav Pediatr. 2010 Feb-Mar;31(2):92-9. doi: 10.1097/DBP.0b013e3181cdabda. PMID: 20110825.X-1

388. Sheng L, Ding X, Ferguson M, et al. Prenatal polycyclic aromatic hydrocarbon exposure leads to behavioral deficits and downregulation of receptor tyrosine kinase, MET. Toxicol Sci. 2010 Dec;118(2):625-34. doi: 10.1093/toxsci/kfq304. PMID: 20889680.X-1

389. Shute N. Desperate for an autism cure. Sci Am. 2010 Oct;303(4):80-5. PMID: 20923134.X-1

390. Shyu YI, Tsai JL, Tsai WC. Explaining and selecting treatments for autism: parental explanatory models in Taiwan. $\mathrm{J}$ Autism Dev Disord. 2010 Nov;40(11):1323-31. doi: 10.1007/s10803-010-0991-1. PMID: 20224997.X-1, X-4 
391. Siegel B, Ihie E, Marco E, et al. Update on Autism. Psychiatric Times. 2010;27(10):33-9 7p. PMID: 108234602. Language: English. Entry Date: 20110719. Revision Date: 20150712. Publication Type: Journal Article.X-1

392. Silverman JL, Tolu SS, Barkan CL, et al. Repetitive self-grooming behavior in the BTBR mouse model of autism is blocked by the mGluR5 antagonist MPEP.

Neuropsychopharmacology. 2010

March;35(4):976-89. doi:

http://dx.doi.org/10.1038/npp.2009.201. PMID: 2010112991.X-1

393. Smith IM, Koegel RL, Koegel LK, et al. Effectiveness of a novel community-based early intervention model for children with autistic spectrum disorder. Am J Intellect Dev Disabil. 2010 Nov;115(6):504-23. doi: 10.1352/1944-7558-115.6.504. PMID: 20946003.X-1, X-3

394. Smyth C, Slevin E. Experiences of family life with an autism assistance dog. Learning Disability Practice. 2010;13(4):12-7 6p. PMID: 105201556. Language: English. Entry Date: 20100625. Revision Date: 20150820. Publication Type: Journal Article.X$1, \mathrm{X}-2$

395. Sokhadze E, Baruth J, El-Baz A, et al. Impaired Error Monitoring and Correction Function in Autism. J Neurother. 2010 Apr 1;14(2):79-95. doi: 10.1080/10874201003771561. PMID: 20523752.X-1

396. Sokhadze E, Baruth J, Tasman A, et al. Low-frequency repetitive transcranial magnetic stimulation (rTMS) affects event-related potential measures of novelty processing in autism. Appl Psychophysiol Biofeedback. 2010 Jun;35(2):147-61. doi: 10.1007/s10484-0099121-2. PMID: 19941058.X-3, X-4

397. Specchio N, Balestri M, Striano P, et al. Efficacy of levetiracetam in the treatment of drug-resistant Rett syndrome. Epilepsy Research. 2010 February;88(2-3):112-7. doi: http://dx.doi.org/10.1016/j.eplepsyres.2009.10.0 05. PMID: 2010088877.X-1

398. Sroka M, Regula J, Lobodziec W. The influence of the bolus-surface distance on the dose distribution in the build-up region. Rep Pract Oncol Radiother. 2010;15(6):161-4. doi: 10.1016/j.rpor.2010.09.003. PMID: 24376943.X-1

399. St Pourcain B, Wang K, Glessner JT, et al. Association between a high-risk autism locus on 5p14 and social communication spectrum phenotypes in the general population. Am J Psychiatry. 2010 Nov;167(11):1364-72. doi: 10.1176/appi.ajp.2010.09121789. PMID: 20634369.X-1

400. Staines R. School nurses can help identify children with undiagnosed autism. Paediatr Nurs. 2010 Mar;22(2):7. PMID: 20373658.X-1

401. Staples K, Reid G. Fundamental movement skills and autism spectrum disorders. Journal of Autism \& Developmental Disorders. 2010;40(2):209-17 9p. doi: 10.1007/s10803009-0854-9. PMID: 105302485. Language: English. Entry Date: 20100319. Revision Date: 20150711. Publication Type: Journal Article.X1

402. Steward R. On the record: Robyn Steward. Interview by Alita Howe. Ment Health Today. 2010 Dec-2011 Jan:38. PMID: 21235057.X-1, X-2

403. Stichter JP, Herzog MJ, Visovsky K, et al. Social competence intervention for youth with Asperger Syndrome and high-functioning autism: an initial investigation. J Autism Dev Disord. 2010 Sep;40(9):1067-79. doi: 10.1007/s10803-010-0959-1. PMID: 20162344.X-1, X-3

404. Stieglitz Ham H, Bartolo A, Corley M, et al. Case report: Selective deficit in the production of intransitive gestures in an individual with autism. Cortex: A Journal Devoted to the Study of the Nervous System \& Behavior. 2010;46(3):407-9 3p. doi: 10.1016/j.cortex.2009.06.005. PMID: 105119188. Language: English. Entry Date: 20100514. Revision Date: 20150711. Publication Type: Journal Article.X-1

405. Stigler K, Wang Y, McDonald B, et al. Effects of aripiprazole on brain circuitry in youth with pervasive developmental disorders. Neuropsychopharmacology. 2010 December;35:S367. doi: 
http://dx.doi.org/10.1038/npp.2010.218. PMID: 70807855.X-3, X-4

406. Stigler KA, Erickson CA, Mullett JE, et al. Paliperidone for irritability in autistic disorder. Journal of Child and Adolescent Psychopharmacology. 201001 Feb;20(1):75-8. doi: http://dx.doi.org/10.1089/cap.2009.0019. PMID: 2010129310.X-1, X-2

407. Su H, Dickstein-Fischer L, Harrington $\mathrm{K}$, et al. Cable-driven elastic parallel humanoid head with face tracking for Autism Spectrum Disorder interventions. Conf Proc IEEE Eng Med Biol Soc. 2010;2010:467-70. doi: 10.1109/iembs.2010.5626186. PMID: 21095653.X-1

408. Sung M, Fung DSS, Cai Y, et al. Pharmacological management in children and adolescents with pervasive developmental disorder. Australian \& New Zealand Journal of Psychiatry. 2010;44(5):410-28 19p. doi: 10.3109/00048670903493330. PMID: 105176411. Language: English. Entry Date: 20100521. Revision Date: 20150711. Publication Type: Journal Article.X-1, X-2

409. Sutherland A, Crewther DP. Magnocellular visual evoked potential delay with high autism spectrum quotient yields a neural mechanism for altered perception. Brain: A Journal of Neurology. 2010;133(7):2089-97 9p. doi: brain/awq122. PMID: 105040490. Language: English. Entry Date: 20100806. Revision Date: 20150711. Publication Type: Journal Article.X-1

410. Tanaka JW, Wolf JM, Klaiman C, et al. Using computerized games to teach face recognition skills to children with autism spectrum disorder: the Let's Face It! program. J Child Psychol Psychiatry. 2010 Aug;51(8):94452. doi: 10.1111/j.1469-7610.2010.02258.x. PMID: 20646129.X-1

411. Taurines R, Thome J, Duvigneau JC, et al. Expression analyses of the mitochondrial complex i 75-kDa subunit in early onset schizophrenia and autism spectrum disorder: Increased levels as a potential biomarker for early onset schizophrenia. European Child and Adolescent Psychiatry. 2010 May;19(5):441-8. doi: http://dx.doi.org/10.1007/s00787-0090074-z. PMID: 2010340479.X-1
412. Taylor N, Isaac C, Milne E. A comparison of the development of audiovisual integration in children with autism spectrum disorders and typically developing children. J Autism Dev Disord. 2010 Nov;40(11):1403-11. doi: 10.1007/s10803-010-1000-4. PMID: 20354776.X-1

413. Thiemann-Bourque KS. Instruction using the Picture Exchange Communication Systems (PECS) appears to enhance generalization of communication skills among children with autism in comparison to Responsive Education and Prelinguistic Milieu Teaching (RPMT). Evid Based Commun Assess Interv. 2010;4(4)doi: 10.1080/17489539.2011.554004. PMID: 24391678.X-1

414. Thomas RH, Foley KA, Mepham JR, et al. Altered brain phospholipid and acylcarnitine profiles in propionic acid infused rodents: further development of a potential model of autism spectrum disorders. J Neurochem. 2010 Apr;113(2):515-29. PMID: 20405543.X-1

415. Thompson L, Thompson M, Reid A. Neurofeedback outcomes in clients with Asperger's syndrome. Appl Psychophysiol Biofeedback. 2010 Mar;35(1):63-81. doi: 10.1007/s10484-009-9120-3. PMID: 19908142.X-1, X-2, X-3

416. Todd T, Reid G, Butler-Kisber L. Cycling for students with ASD: self-regulation promotes sustained physical activity. Adapt Phys Activ Q. 2010 Jul;27(3):226-41. PMID: 20571157.X-1, X-3, X-4

417. Tom NC, Assinder SJ. Oxytocin: recent developments. Biomol Concepts. 2010 Dec 1;1(5-6):367-80. doi: 10.1515/bmc.2010.036. PMID: 25962010.X-1

418. Torii M, Shimoyama I, Sugita K. Phonemic and semantic working memory in information processing in children with high function pervasive developmental disorders. International Medical Journal. 2010;17(1):35-9 5p. PMID: 105135946. Language: English. Entry Date: 20100409. Revision Date: 20150711. Publication Type: Journal Article.X1 
419. Tsakanikos E, McCarthy J, Kravariti $E$, et al. The role of ethnicity in clinical psychopathology and care pathways of adults with intellectual disabilities. Res Dev Disabil. 2010 Mar-Apr;31(2):410-5. doi: 10.1016/j.ridd.2009.10.007. PMID: 19932597.X-1

420. Tsiouris JA. Pharmacotherapy for aggressive behaviours in persons with intellectual disabilities: treatment or mistreatment? Journal of Intellectual Disability Research. 2010;54(1):1-16 p. doi: 10.1111/j.1365-2788.2009.01232.x. PMID: 105264757. Language: English. Entry Date: 20100205. Revision Date: 20150711. Publication Type: Journal Article.X-1, X-2

421. Tsouderos T. Industrial chemical as autism treatment: the FDA hasn't declared OSR\#1 safe, but a company is selling it as a supplement for children anyway. Los Angeles Times -- Southern California Edition (Front Page). 2010:E1-E5 2p. PMID: 105132696. Language: English. Entry Date: 20100312. Revision Date: 20150711. Publication Type: Journal Article.X-1, X-2

422. Tuchman R, Alessandri M, Cuccaro M. Autism spectrum disorders and epilepsy: Moving towards a comprehensive approach to treatment. Brain and Development. 2010 October;32(9):719-30. doi: http://dx.doi.org/10.1016/j.braindev.2010.05.00 7. PMID: 2010476501.X-1, X-2

423. Tunson J, Candler C. Behavioral States of children with severe disabilities in the multisensory environment. Phys Occup Ther Pediatr. 2010 May;30(2):101-10. doi: 10.3109/01942630903546651. PMID: 20367515.X-1

424. Ulke-Kurkcuoglu B, Kircaali-Iftar G. A comparison of the effects of providing activity and material choice to children with autism spectrum disorders. J Appl Behav Anal. 2010 Winter;43(4):717-21. doi: 10.1901/jaba.2010.43-717. PMID: 21541155.X-1, X-4

425. Umeda T, Takashima N, Nakagawa R, et al. Evaluation of pax6 mutant rat as a model for autism. PLoS ONE. 2010;5(12)doi: http://dx.doi.org/10.1371/journal.pone.0015500. PMID: 2011018828.X-1
426. Valdovinos MG, Bailey L, Taylor SL. Examining risperidone use in those diagnosed with autism 1 year after FDA approval. J Clin Psychiatry. 2010 May;71(5):651-2. doi: 10.4088/JCP.09105665yel. PMID: 20492856.X2

427. Valenti M, Cerbo R, Masedu F, et al. Intensive intervention for children and adolescents with autism in a community setting in Italy: a single-group longitudinal study. Child Adolesc Psychiatry Ment Health. 2010;4:23. doi: 10.1186/1753-2000-4-23. PMID: 20809976.X-3

428. Valentine K. A consideration of medicalisation: choice, engagement and other responsibilities of parents of children with autism spectrum disorder. Soc Sci Med. 2010 Sep;71(5):950-7. doi: 10.1016/j.socscimed.2010.06.010. PMID: 20619521.X-1

429. van Haaren F. Risperidone and parent training in pervasive developmental disorders. J Am Acad Child Adolesc Psychiatry. 2010 Apr;49(4):406-7; author reply 7-8. PMID: 20410733.X-1, X-2

430. Van Santen JP, Prud'hommeaux ET, Black LM, et al. Computational prosodic markers for autism. Autism: The International Journal of Research \& Practice. 2010;14(3):215-36 22p. doi: 10.1177/1362361309363281. PMID: 105059961. Language: English. Entry Date: 20100910. Revision Date: 20150711. Publication Type: Journal Article.X-1

431. Veenstra-Vanderweele J. Increase in valproic acid levels during riluzole treatment in an adolescent with autism. Journal of Child and Adolescent Psychopharmacology. 201001 Apr;20(2):163-5. doi: http://dx.doi.org/10.1089/cap.2009.0087. PMID: 2010252297.X-1, X-2, X-3, X-4

432. Vets TL, Green G. Three important things to consider when starting intervention for a child diagnosed with autism. Behav Anal Pract. 2010 Fall;3(2):56-7. PMID: 22532896.X-1

433. Viau R, Arsenault-Lapierre G, Fecteau $\mathrm{S}$, et al. Effect of service dogs on salivary 
cortisol secretion in autistic children.

Psychoneuroendocrinology. 2010

Sep;35(8):1187-93. doi:

10.1016/j.psyneuen.2010.02.004. PMID: 20189722.X-3

434. Villa S, Micheli E, Villa L, et al. Further empirical data on the psychoeducational profile-revised (PEP-R): reliability and validation with the Vineland adaptive behavior scales. J Autism Dev Disord. 2010

Mar;40(3):334-41. doi: 10.1007/s10803-009-

0877-2. PMID: 19777332.X-1

435. Villagonzalo KA, Dodd S, Dean O, et al. Oxidative pathways as a drug target for the treatment of autism. Expert Opinion on

Therapeutic Targets. 2010

December;14(12):1301-10. doi:

http://dx.doi.org/10.1517/14728222.2010.52839

4. PMID: 2010621556.X-1, X-2

436. Vlassopoulos M, Rotsika V, Mela L, et al. Increased frequency and service delivery for children with pervasive developmental disorders. Psychiatriki. 2010 Oct-

Dec;21(4):294-303. PMID: 21914612.X-1

437. Voelker R. FDA warning targets OTC chelation products. JAMA - Journal of the American Medical Association. 201017 Nov;304(19):2112. doi: http://dx.doi.org/10.1001/jama.2010.1654. PMID: 2010628766.X-1

438. Volden J, Phillips L. Measuring pragmatic language in speakers with autism spectrum disorders: Comparing the children's communication checklist--2 and the test of pragmatic language. Am J Speech Lang Pathol. 2010 Aug;19(3):204-12. doi: 10.1044/10580360(2010/09-0011). PMID: 20220047.X-1

439. Wachtel LE, Dhossche DM. Selfinjury in autism as an alternate sign of catatonia: implications for electroconvulsive therapy. Med Hypotheses. 2010 Jul;75(1):1114. doi: 10.1016/j.mehy.2010.02.001. PMID: 20202760.X-1, X-2, X-3

440. Walker AN, Barry TD, Bader SH. Therapist and parent ratings of changes in adaptive social skills following a summer treatment camp for children with autism spectrum disorders: A preliminary study. Child \& Youth Care Forum. 2010 2015-12-
09;39(5):305-22. doi:

http://dx.doi.org/10.1007/s10566-010-9110-x. PMID: 763172198; 2010-20371-001.X-1, X-3

441. Wallace GL, Dankner N, Kenworthy L, et al. Age-related temporal and parietal cortical thinning in autism spectrum disorders. Brain. 2010 Dec;133(Pt 12):3745-54. doi: 10.1093/brain/awq279. PMID: 20926367.X-1

442. Wallace S, Parsons S, Westbury A, et al. Sense of presence and atypical social judgments in immersive virtual environments. Responses of adolescents with Autism Spectrum Disorders. Autism. 2010

May;14(3):199-213. doi: 10.1177/1362361310363283. PMID: 20484000.X-1

443. Wang L, Leslie DL. Health care expenditures for children with autism spectrum disorders in Medicaid. J Am Acad Child Adolesc Psychiatry. 2010 Nov;49(11):1165-71. doi: 10.1016/j.jaac.2010.08.003. PMID: 20970704.X-1

444. Wang LW, Berry-Kravis E, Hagerman RJ. Fragile X: Leading the way for targeted treatments in autism. Neurotherapeutics. 2010 July;7(3):264-74. doi: http://dx.doi.org/10.1016/j.nurt.2010.05.005. PMID: 2010406859.X-1

445. Warren SF, Brady N, Sterling A, et al. Maternal responsivity predicts language development in young children with fragile $\mathrm{X}$ syndrome. Am J Intellect Dev Disabil. 2010 Jan;115(1):54-75. doi: 10.1352/1944-7558115.1.54. PMID: 20025359.X-1

446. Warren ZE, Sanders KB, VeenstraVanderweele J. Identity crisis involving body image in a young man with autism. American Journal of Psychiatry. 2010;167(11):1299-303 5p. doi: 10.1176/appi.ajp.2010.09091281. PMID: 104937205. Language: English. Entry Date: 20110107. Revision Date: 20150711. Publication Type: Journal Article.X-1

447. Watson LR, Baranek GT, Roberts JE, et al. Behavioral and physiological responses to child-directed speech as predictors of communication outcomes in children with autism spectrum disorders. J Speech Lang Hear Res. 2010 Aug;53(4):1052-64. doi: 
10.1044/1092-4388(2009/09-0096). PMID: 20631229.X-1

448. Webb S. Drugmakers dance with autism. Nature Biotechnology. 2010

August;28(8):772-4. doi: http://dx.doi.org/10.1038/nbt0810-772. PMID: 2010432690.X-1

449. Wei R, Li Q, Chua SE, et al. Prenatal exposure to valproic acid induces a dose dependent impairment in sensorimotor gating in a mouse model of autism. International Journal of Neuropsychopharmacology. 2010 June;13:68. doi: http://dx.doi.org/10.1017/S1461145710000635. PMID: 70220411.X-1

450. Weil TN, Inglehart MR. Dental education and dentists' attitudes and behavior concerning patients with autism. J Dent Educ. 2010 Dec;74(12):1294-307. PMID: 21123497.X-1

451. Weng SJ, Wiggins JL, Peltier SJ, et al. Alterations of resting state functional connectivity in the default network in adolescents with autism spectrum disorders. Brain Res. 2010 Feb 8;1313:202-14. doi: 10.1016/j.brainres.2009.11.057. PMID: 20004180.X-1

452. Whalen C, Moss D, Ilan AB, et al. Efficacy of TeachTown: Basics computerassisted intervention for the Intensive Comprehensive Autism Program in Los Angeles Unified School District. Autism. 2010 May;14(3):179-97. doi:

10.1177/1362361310363282. PMID: 20484002.X-1

453. Wheelwright S, Auyeung B, Allison C, et al. Defining the broader, medium and narrow autism phenotype among parents using the Autism Spectrum Quotient (AQ). Mol Autism. 2010;1(1):10. doi: 10.1186/2040-2392-1-10. PMID: 20678260.X-1

454. White SW, Albano AM, Johnson CR, et al. Development of a cognitive-behavioral intervention program to treat anxiety and social deficits in teens with high-functioning autism. Clin Child Fam Psychol Rev. 2010 Mar;13(1):77-90. doi: 10.1007/s10567-0090062-3. PMID: 20091348.X-1
455. Wilder DA, Allison J, Nicholson K, et al. Further evaluation of antecedent interventions on compliance: the effects of rationales to increase compliance among preschoolers. J Appl Behav Anal. 2010 Winter;43(4):601-13. doi: 10.1901/jaba.2010.43-601. PMID: 21541147.X-1, X-3, X-4

456. Williams K, Wheeler DM, Silove N, et al. Selective serotonin reuptake inhibitors (SSRIs) for autism spectrum disorders (ASD). Cochrane database of systematic reviews (Online). 2010;8:CD004677. PMID: 20687077.X-2

457. Wimmer MC, Doherty MJ. Children with autism's perception and understanding of ambiguous figures: evidence for pictorial metarepresentation, a research note. British Journal of Developmental Psychology. 2010;28(3):627-41 15p. doi: 64110.1348/026151009X465362. PMID: 105100794. Language: English. Entry Date: 20101012. Revision Date: 20150711. Publication Type: Journal Article.X-1

458. Wink LK, Erickson CA, McDougle CJ. Pharmacologic treatment of behavioral symptoms associated with autism and other pervasive developmental disorders. Curr Treat Options Neurol. 2010 Nov;12(6):529-38. doi: 10.1007/s11940-010-0091-8. PMID: 20848330.X-2, X-3, X-4

459. Wink LK, Plawecki MH, Erickson $\mathrm{CA}$, et al. Emerging drugs for the treatment of symptoms associated with autism spectrum disorders. Expert Opinion on Emerging Drugs. 2010 September;15(3):481-94. doi: http://dx.doi.org/10.1517/14728214.2010.48786 0. PMID: 2010457371.X-2, X-3

460. Wise MD, Little AA, Holliman JB, et al. Can state early intervention programs meet the increased demand of children suspected of having autism spectrum disorders? J Dev Behav Pediatr. 2010 Jul-Aug;31(6):469-76. doi: 10.1097/DBP.0b013e3181e56db2. PMID: 20585267.X-1

461. Wong P. Selective mutism: a review of etiology, comorbidities, and treatment. Psychiatry (Edgmont). 2010 Mar;7(3):23-31. PMID: 20436772.X-1 
462. Wong VC, Chen WX. Randomized controlled trial of electro-acupuncture for autism spectrum disorder. Altern Med Rev. 2010 Jul;15(2):136-46. PMID: 20806998.X-1

463. Wong VC, Kwan QK. Randomized controlled trial for early intervention for autism: a pilot study of the Autism 1-2-3 Project. J Autism Dev Disord. 2010 Jun;40(6):677-88. doi: 10.1007/s10803-009-0916-z. PMID: 20020319.X-4

464. Wong VC, Sun JG. Randomized controlled trial of acupuncture versus sham acupuncture in autism spectrum disorder. J Altern Complement Med. 2010 May;16(5):54553. doi: 10.1089/acm.2007.0768. PMID: 20804366.X-1

465. Wu YW, Bi YP, Kou XX, et al. 17Beta-estradiol enhanced allodynia of inflammatory temporomandibular joint through upregulation of hippocampal TRPV1 in ovariectomized rats. J Neurosci. 2010 Jun 30;30(26):8710-9. doi: 10.1523/jneurosci.632309.2010. PMID: 20592193.X-1

466. Wuang YP, Wang CC, Huang MH, et al. The effectiveness of simulated developmental horse-riding program in children with autism. Adapt Phys Activ Q. 2010 Apr;27(2):113-26. PMID: 20440023.X-1

467. Wynn T, Rawlings K. Sleepless in america: compounding with melatonin. Int $\mathrm{J}$ Pharm Compd. 2010 Jan-Feb;14(1):10. PMID: 23965363.X-1

468. Yochum CL, Bhattacharya P, Patti L, et al. Animal model of autism using GSTM1 knockout mice and early post-natal sodium valproate treatment. Behav Brain Res. $2010 \mathrm{Jul}$ 11;210(2):202-10. doi: 10.1016/j.bbr.2010.02.032. PMID: 20178820.X-1

469. Yoder PJ, Lieberman RG. Brief Report: Randomized test of the efficacy of picture exchange communication system on highly generalized picture exchanges in children with ASD. J Autism Dev Disord. 2010 May;40(5):629-32. doi: 10.1007/s10803-0090897-y. PMID: 19904596.X-1

470. Yokotani K. Educational level signals unobserved abilities of people with high functioning autism spectrum disorders. Psychol Rep. 2010 Aug;107(1):227-35. doi: 10.2466/11.13.15.pr0.107.4.227-235. PMID: 20923067.X-1

471. Zaidman-Zait A, Mirenda P, Zumbo $\mathrm{BD}$, et al. An item response theory analysis of the Parenting Stress Index-Short Form with parents of children with autism spectrum disorders. J Child Psychol Psychiatry. 2010

Nov;51(11):1269-77. doi: 10.1111/j.14697610.2010.02266.x. PMID: 20546082.X-1

472. Zappella M. Autistic regression with and without EEG abnormalities followed by favourable outcome. Brain and Development. 2010 October;32(9):739-45. doi: http://dx.doi.org/10.1016/j.braindev.2010.05.00 4. PMID: 2010476503.X-1

473. Zhang J. A review of Autism Spectrum Disorders (ASD) from a perspective of Classical Chinese Medicine (CCM). Journal of Traditional Chinese Medicine. 2010 March;30(1):53-9. PMID: 2010240071.X-1

474. . No "magic pill" for autism spectrum disorders. Although medication prescriptions are common, there is little evidence they do any good. Harv Ment Health Lett. 2011 Aug;28(2):4. PMID: 21980632.X-1

475. . Autism spectrum disorders revisited. Several recent studies raise new questions about cause and prevention. Harv Ment Health Lett. 2011 Oct;28(4):1-3. PMID: 22125823.x-1

476. . Fear and its consequences. Sci Am. 2011 Feb;304(2):14. PMID: 21319529.X-1

477. . Motor training can advance social development in infants with autism. AACN Bold Voices. 2011;3(12):8- 1p. PMID: 108209686. Language: English. Entry Date: 20120120. Revision Date: 20150712. Publication Type: Journal Article.X-1, X-2

478. . DIGEST. Adapted Physical Activity Quarterly. 2011;28(2):176-9 4p. PMID: 104894751. Language: English. Entry Date: 20110603. Revision Date: 20150711. Publication Type: Journal Article. Journal Subset: Allied Health.X-1, X-2

479. . Autism and aquatics: activity manual for families. Journal of Aquatic Physical 
Therapy. 20112011 Spring;19(1):30- 1p. PMID: 108160887. Language: English. Entry Date: 20120302. Revision Date: 20150712. Publication Type: Journal Article.X-1

480. . Motor characteristics of young children with autism spectrum disorders are much lower than normal references. Pediatric Physical Therapy. 20112011 Spring;23(1):1161p. PMID: 108231010. Language: English. Entry Date: 20110701. Revision Date: 20150712. Publication Type: Journal Article.X$1, \mathrm{X}-2$

481. . A systematic review of motor impairments in children with autism spectrum disorder. Pediatric Physical Therapy. 20112011 Spring;23(1):132- 1p. PMID: 108231039. Language: English. Entry Date: 20110701. Revision Date: 20150712. Publication Type: Journal Article.X-1, X-2

482. . BRIEFLY NOTED. Mental Health Weekly. 2011;21(46):8- 1p. PMID: 104141268. Language: English. Entry Date: 20131008. Revision Date: 20150710. Publication Type: Journal Article.X-1, X-2

483. . Long-term risperidone effects on body weight in autism. Brown University Child \& Adolescent Psychopharmacology Update. 2011;13(12):7- 1p. PMID: 104404399. Language: English. Entry Date: 20130206. Revision Date: 20150711. Publication Type: Journal Article.X-1, X-2

484. . Oculogyric crises induced by lowdose risperidone. Brown University Child \& Adolescent Psychopharmacology Update. 2011;13(11):8- 1p. PMID: 104639505. Language: English. Entry Date: 20111110. Revision Date: 20150711. Publication Type: Journal Article.X-1, X-2

485. . Favorable atomoxetine response in autism and ADHD. Brown University Child \& Adolescent Psychopharmacology Update. 2011;13(10):7-8 2p. PMID: 104693581. Language: English. Entry Date: 20111005. Revision Date: 20150711. Publication Type: Journal Article.X-1, X-2

486. . Aripiprazole safety and tolerability for irritability in autism. Brown University Child \& Adolescent Psychopharmacology Update. 2011;13(9):4-5 2p. PMID: 104685810.
Language: English. Entry Date: 20110911. Revision Date: 20150711. Publication Type: Journal Article. Journal Subset: Biomedical.X$1, \mathrm{X}-2$

487. . NEWS IN BRIEF. ASHA Leader. 2011;16(10):3- 1p. PMID: 108253968. Language: English. Entry Date: 20110906. Revision Date: 20150712. Publication Type: Journal Article.X-1, X-2

488. . Antidepressant use during pregnancy may have link to autism. Mental Health Weekly. 2011;21(32):3-4 2p. PMID: 104681268. Language: English. Entry Date: 20110831. Revision Date: 20150711. Publication Type: Journal Article. Journal Subset: Biomedical.X-1, X-2

489. . Patient-oriented evidence that matters: POEMs. JAAPA: Journal of the American Academy of Physician Assistants (Haymarket Media, Inc.). 2011;24(8):59-60 2p. PMID: 108261947. Language: English. Entry Date: 20111014 . Revision Date: 20150712. Publication Type: Journal Article.X-1, X-2

490. . . Product update. Journal of Psychosocial Nursing \& Mental Health Services. 2011;49(6):5- 1p. doi: 10.3928/02793695-20110428-01. PMID: 104649846. Language: English. Entry Date: 20110812. Revision Date: 20150711. Publication Type: Journal Article.X-1, X-2

491. . Donepezil shows limited effect on cognitive functioning in autism. Brown University Child \& Adolescent Psychopharmacology Update. 2011;13(4):1-3 p. doi: 10.1002/cpu.20137. PMID: 104643438. Language: English. Entry Date: 20110713. Revision Date: 20150711. Publication Type: Journal Article. Journal Subset: Biomedical.X$1, \mathrm{X}-2$

492. . Aripiprazole treatment of autistic disorder. Brown University Child \& Adolescent Psychopharmacology Update. 2011;13(1):4-5 2p. PMID: 104643430. Language: English. Entry Date: 20110713. Revision Date: 20150711. Publication Type: Journal Article. Journal Subset: Biomedical.X-1, X-2

493. . Weight gain during aripiprazole treatment of irritability. Brown University Child \& Adolescent Psychopharmacology Update. 
2011;13(1):5-6 2p. PMID: 104643432. Language: English. Entry Date: 20110713. Revision Date: 20150711. Publication Type: Journal Article. Journal Subset: Biomedical.X$1, \mathrm{X}-2$

494. Adamou M, Bowers S. Dose of Methylphenidate during Service Transition for Adults with ADHD. Ther Adv Psychopharmacol. 2011 Jun;1(3):71-5. doi: 10.1177/2045125311411603. PMID: 23983928.X-1

495. Adams JB, Audhya T, McDonoughMeans S, et al. Effect of a vitamin/mineral supplement on children and adults with autism. BMC Pediatr. 2011;11:111. doi: 10.1186/14712431-11-111. PMID: 22151477.X-3

496. Adams JB, Audhya T, McDonoughMeans S, et al. Nutritional and metabolic status of children with autism vs. neurotypical children, and the association with autism severity. Nutr Metab (Lond). 2011;8(1):34. doi: 10.1186/1743-7075-8-34. PMID: 21651783.X1

497. Adams JB, Johansen LJ, Powell LD, et al. Gastrointestinal flora and gastrointestinal status in children with autism--comparisons to typical children and correlation with autism severity. BMC Gastroenterol. 2011;11:22. doi: 10.1186/1471-230x-11-22. PMID: 21410934.X-1

498. Ahrens EN, Lerman DC, Kodak T, et al. Further evaluation of response interruption and redirection as treatment for stereotypy. J Appl Behav Anal. 2011 Spring;44(1):95-108. doi: 10.1901/jaba.2011.44-95. PMID: 21541130.X-3, X-4

499. Al Abdulmohsen T, Kruger TH. The contribution of muscular and auditory pathologies to the symptomatology of autism. Med Hypotheses. 2011 Dec;77(6):1038-47. doi: 10.1016/j.mehy.2011.08.044. PMID: 21925796.X-1, X-2, X-3, X-4

500. Aldridge K, George ID, Cole KK, et al. Facial phenotypes in subgroups of prepubertal boys with autism spectrum disorders are correlated with clinical phenotypes. Mol Autism. 2011;2(1):15. doi: 10.1186/2040-2392-2-15. PMID: 21999758.X1
501. Amaral D, Rogers SJ, Baron-Cohen S, et al. Against le packing: a consensus statement. J Am Acad Child Adolesc Psychiatry. 2011

Feb;50(2):191-2. doi: 10.1016/j.jaac.2010.11.018. PMID: 21241956.X-2

502. Amirabdollahian F, Robins B, Dautenhahn K, et al. Investigating tactile event recognition in child-robot interaction for use in autism therapy. Conf Proc IEEE Eng Med Biol Soc. 2011;2011:5347-51. doi: 10.1109/iembs.2011.6091323. PMID: 22255546.X-1, X-2, X-3

503. Anagnostou E, Hansen R. Medical treatment overview: traditional and novel psycho-pharmacological and complementary and alternative medications. Current Opinion in Pediatrics. 2011;23(6):621-7 7p. PMID: 108215402. Language: English. Entry Date: 20120127. Revision Date: 20150712. Publication Type: Journal Article.X-2

504. Andanson J, Pourre F, Maffre T, et al. [Social skills training groups for children and adolescents with Asperger syndrome: A review]. Arch Pediatr. 2011 May;18(5):589-96. doi: 10.1016/j.arcped.2011.02.019. PMID: 21458972.X-1, X-2, X-3

505. Anderberg D, Chevalier A, Wadsworth J. Anatomy of a health scare: education, income and the MMR controversy in the UK. J Health Econ. 2011 May;30(3):515-30. doi: 10.1016/j.jhealeco.2011.01.009. PMID: 21439663.X-1

506. Anderson-Hanley C, Tureck K, Schneiderman RL. Autism and exergaming: effects on repetitive behaviors and cognition. Psychol Res Behav Manag. 2011;4:129-37. doi: 10.2147/prbm.s24016. PMID: 22114543.X-1

507. Antshel KM, Polacek C, McMahon M, et al. Comorbid ADHD and anxiety affect social skills group intervention treatment efficacy in children with autism spectrum disorders. J Dev Behav Pediatr. 2011 JulAug;32(6):439-46. doi: 10.1097/DBP.0b013e318222355d. PMID: 21654508.X-1, X-3

508. Asokan S, Ajit P. Mirror magic. Br Dent J. 2011 Jun;210(12):559. doi: 
10.1038/sj.bdj.2011.491. PMID: 21701456.X-1, $\mathrm{X}-2$

509. Bagshaw M. Anaesthesia and the autistic child. J Perioper Pract. 2011 Sep;21(9):313-7. PMID: 22474776.X-1

510. Bakare MO, Munir KM. Excess of non-verbal cases of autism spectrum disorders presenting to orthodox clinical practice in Africa - a trend possibly resulting from late diagnosis and intervention. S Afr J Psychiatr. 2011 Dec;17(4):118-20. PMID: 22229024.X-1

511. Bakare MO, Munir KM, Kinney DK. Association of hypomelanotic skin disorders with autism: links to possible etiologic role of vitamin-D levels in autism? Hypothesis (Tor). 2011 Sep;9(1) PMID: 21949515.X-1

512. Bakare MO, Munir KM, Kinney DK. Associations of hypomelanotic skin disorders with autism: Do they reflect the effects of genetic mutations and epigenetic factors on vitamin-D metabolism in individuals at risk for autism? Hypothesis (Macon). 2011 Apr 16;9(1):e2. PMID: 25067901.X-1

513. Bakken TL. Review of My sensory book: Working together to explore sensory issues and the big feelings they can cause: A workbook for parents, professionals, and children. Journal of Autism and Developmental Disorders. 2011 2015-12-09;41(1):132-3. doi: http://dx.doi.org/10.1007/s10803-009-0934-x. PMID: 853490483; 2010-26385-016.X-1, X-2, $\mathrm{X}-3$

514. Bakouie F, Gharibzadeh S, Towhidkhah F. Does the "dynamic core" approach help to manage autistic behavioral disorders? J Neuropsychiatry Clin Neurosci. 2011 Summer;23(3):E1. doi: 10.1176/appi.neuropsych.23.3.E1. PMID: 21948902.X-2

515. Bancroft SL, Weiss JS, Libby ME, et al. A comparison of procedural variations in teaching behavior chains: manual guidance, trainer completion, and no completion of untrained steps. J Appl Behav Anal. 2011 Fall;44(3):559-69. doi: 10.1901/jaba.2011.44559. PMID: 21941385.X-3, X-4

516. Banji D, Banji OJ, Abbagoni S, et al. Amelioration of behavioral aberrations and oxidative markers by green tea extract in valproate induced autism in animals. Brain Res. 2011 Sep 2;1410:141-51. doi:

10.1016/j.brainres.2011.06.063. PMID: 21820650.X-1

517. Barbaro J, Ridgway L, Dissanayake C. Developmental surveillance of infants and toddlers by maternal and child health nurses in an Australian community-based setting: promoting the early identification of autism spectrum disorders. J Pediatr Nurs. 2011 Aug;26(4):334-47. doi: 10.1016/j.pedn.2010.04.007. PMID: 21726784.X-1

518. Barnea-Goraly N, Hardan A. Advances in clinical neuroimaging: implications for autism spectrum disorders. Expert Opin Med Diagn. 2011 Nov;5(6):47582. doi: $10.1517 / 17530059.2011 .595785$. PMID: 23484746.X-1

519. Beherec L, Lambrey S, Quilici G, et al. Retrospective review of clozapine in the treatment of patients with autism spectrum disorder and severe disruptive behaviors. J Clin Psychopharmacol. 2011 Jun;31(3):341-4. doi: 10.1097/JCP.0b013e318218f4a1. PMID: 21508854.X-1, X-3

520. Ben Said M, Robel L, Vion E, et al. Implementation and experimentation of TEDIS: an information system dedicated to patients with pervasive developmental disorders. Stud Health Technol Inform. 2011;169:270-4. PMID: 21893755.X-1

521. Bendixen RM, Elder JH, Donaldson S, et al. Effects of a father-based in-home intervention on perceived stress and family dynamics in parents of children with autism. Am J Occup Ther. 2011 Nov-Dec;65(6):67987. PMID: 22214112.X-3

522. Benton TD. Aripiprazole to treat irritability associated with autism: a placebocontrolled, fixed-dose trial. Curr Psychiatry Rep. 2011 Apr;13(2):77-9. doi: 10.1007/s11920-010-0172-0. PMID: 21286868.X-1, X-2

523. Berger BE, Navar-Boggan AM, Omer $\mathrm{SB}$. Congenital rubella syndrome and autism spectrum disorder prevented by rubella vaccination--United States, 2001-2010. BMC 
Public Health. 2011;11:340. doi: 10.1186/14712458-11-340. PMID: 21592401.X-1

524. Bernardi L, Tuzzi A. Analyzing written communication in AAC contexts: a statistical perspective. Augment Altern Commun. 2011 Sep;27(3):183-94. doi: 10.3109/07434618.2011.610353. PMID: 22008031.X-1

525. Berry-Kravis E, Knox A, Hervey C. Targeted treatments for fragile $\mathrm{X}$ syndrome. J Neurodev Disord. 2011 Sep;3(3):193-210. doi: 10.1007/s11689-011-9074-7. PMID: 21484200.X-1

526. Betz AM, Higbee TS, Kelley KN, et al. Increasing response variability of mand frames with script training and extinction. J Appl Behav Anal. 2011 Summer;44(2):357-62. doi: 10.1901/jaba.2011.44-357. PMID: 21709793.X-3, X-4

527. Beversdorf DQ, Saklayen S, Higgins KF, et al. Effect of propranolol on word fluency in autism. Cogn Behav Neurol. 2011 Mar;24(1):11-7. doi: 10.1097/WNN.0b013e318204d20e. PMID: 21487259.X-1

528. Bhandari A, Sandlow JI, Brannigan RE. Risks to offspring associated with advanced paternal age. J Androl. 2011 MarApr;32(2):121-2. doi: 10.2164/jandrol.110.010595. PMID: 20467047.X-1

529. Bhat AN, Landa RJ, Galloway JC. Current perspectives on motor functioning in infants, children, and adults with autism spectrum disorders. Phys Ther. 2011 Jul;91(7):1116-29. doi: 10.2522/ptj.20100294. PMID: 21546566.X-1

530. Bishop DV, Jacobs PA, Lachlan K, et al. Autism, language and communication in children with sex chromosome trisomies. Arch Dis Child. 2011 Oct;96(10):954-9. doi: 10.1136/adc.2009.179747. PMID: 20656736.X1

531. Blaurock-Busch E, Amin OR, Rabah T. Heavy metals and trace elements in hair and urine of a sample of arab children with autistic spectrum disorder. Maedica (Buchar). 2011 Oct;6(4):247-57. PMID: 22879836.X-1
532. Bolton PF, Carcani-Rathwell I, Hutton J, et al. Epilepsy in autism: features and correlates. Br J Psychiatry. 2011

Apr;198(4):289-94. doi:

10.1192/bjp.bp.109.076877. PMID: 21972278.X-1

533. Bowker A, D'Angelo NM, Hicks R, et al. Treatments for autism: parental choices and perceptions of change. J Autism Dev Disord. 2011 Oct;41(10):1373-82. doi: 10.1007/s10803010-1164-y. PMID: 21161676.X-1

534. Boyd BA, McDonough SG, Rupp B, et al. Effects of a family-implemented treatment on the repetitive behaviors of children with autism. J Autism Dev Disord. 2011 Oct;41(10):1330-41. doi: 10.1007/s10803-0101156-y. PMID: 21161576.X-3, X-4

535. Boyd BA, Woodard CR, Bodfish JW. Modified exposure and response prevention to treat the repetitive behaviors of a child with autism: a case report. Case Rep Psychiatry. 2011;2011:241095. doi: 10.1155/2011/241095. PMID: 22937399.X-1, X-3, X-4

536. Bragesjo F, Hallberg M. Dilemmas of a vitalizing vaccine market: lessons from the MMR vaccine/autism debate. Sci Context. 2011 Mar;24(1):107-25. PMID: 21560548.X-1

537. Breslin CM, Rudisill ME. The Effect of Visual Supports on Performance of the TGMD-2 for Children With Autism Spectrum Disorder. Adapted Physical Activity Quarterly. 2011;28(4):342-53 12p. PMID: 104698353. Language: English. Entry Date: 20111025. Revision Date: 20150819. Publication Type: Journal Article.X-1

538. Brown T. Right treatment, right patient? Am J Nurs. 2011 Jun;111(6):72. doi: 10.1097/01.NAJ.0000398546.90401.4b. PMID: 21613926.X-1

539. Buckley AW, Sassower K, Rodriguez AJ, et al. An open label trial of donepezil for enhancement of rapid eye movement sleep in young children with autism spectrum disorders. J Child Adolesc Psychopharmacol. 2011 Aug;21(4):353-7. doi: 10.1089/cap.2010.0121. PMID: 21851192.X-1, X-3 
540. Burket JA, Herndon AL, Winebarger EE, et al. Complex effects of mGluR5 antagonism on sociability and stereotypic behaviors in mice: possible implications for the pharmacotherapy of autism spectrum disorders. Brain Res Bull. 2011 Oct 10;86(3-4):152-8. doi: 10.1016/j.brainresbull.2011.08.001. PMID: 21840381.X-1

541. Calarge CA, Miller del D. Predictors of risperidone and 9-hydroxyrisperidone serum concentration in children and adolescents. J Child Adolesc Psychopharmacol. 2011 Apr;21(2):163-9. doi: 10.1089/cap.2010.0038. PMID: 21486167.X-1

542. Call NA, Pabico RS, Findley AJ, et al. Differential reinforcement with and without blocking as treatment for elopement. J Appl Behav Anal. 2011 Winter;44(4):903-7. doi: 10.1901/jaba.2011.44-903. PMID: 22219538.X-1, X-3

543. Cardon TA, Wilcox MJ. Promoting imitation in young children with autism: a comparison of reciprocal imitation training and video modeling. J Autism Dev Disord. 2011 May;41(5):654-66. doi: 10.1007/s10803-0101086-8. PMID: 20697791.X-3, X-4

544. Carter AS, Messinger DS, Stone WL, et al. A randomized controlled trial of Hanen's 'More Than Words' in toddlers with early autism symptoms. J Child Psychol Psychiatry. 2011 Jul;52(7):741-52. doi: 10.1111/j.14697610.2011.02395.x. PMID: 21418212.X-1

545. Cassella MD, Sidener TM, Sidener DW, et al. Response interruption and redirection for vocal stereotypy in children with autism: a systematic replication. J Appl Behav Anal. 2011 Spring;44(1):169-73. doi: 10.1901/jaba.2011.44-169. PMID: 21541114.X-1, X-3, X-4

546. Casselyn M. [Early detection of autism: a questionnaire for parents. Interview with Hilary Wood]. Rev Med Suisse. 2011 Sep 14;7(308):1776. PMID: 21954820.X-1

547. Castorina LL, Negri LM. The inclusion of siblings in social skills training groups for boys with Asperger syndrome. J Autism Dev Disord. 2011 Jan;41(1):73-81. doi: 10.1007/s10803-010-1023-X. PMID: 20461452.X-1
548. Celik G, Tahiroglu AY, Firat S, et al. Aripiprazole Improved Obsessive Compulsive Symptoms in Asperger's Disorder. Clin Psychopharmacol Neurosci. 2011 Dec;9(3):134-6. doi: 10.9758/cpn.2011.9.3.134. PMID: 23429759.X-3, X-4

549. Cezar GG. Profile of Gabriela G. Cezar. Interview by Kristie Nybo. Biotechniques. 2011 May;50(5):281. doi: 10.2144/000113662. PMID: 21548887.X-1

550. Chan AS, Sze SL, Cheung MC, et al. Dejian mind-body intervention improves the cognitive functions of a child with autism. Evid Based Complement Alternat Med. 2011;2011:549254. doi: 10.1155/2011/549254. PMID: 21584249.X-1

551. Charles J, Harrison C, Britt H. Autism spectrum disorders. Aust Fam Physician. 2011 Sep;40(9):665. PMID: 21894271.X-1, X-2

552. Charles J, Harrison CM, Britt H. Management of children's psychological problems in general practice 1970-1971, 19901991 and 2008-2009. Australian \& New Zealand Journal of Psychiatry. 2011;45(11):976-84 9p. doi: 10.3109/00048674.2011.610743. PMID: 104637252. Language: English. Entry Date: 20111118. Revision Date: 20150711. Publication Type: Journal Article.X-1

553. Charnsil C. Efficacy of atomoxetine in children with severe autistic disorders and symptoms of ADHD: an open-label study. J Atten Disord. 2011 Nov;15(8):684-9. doi: 10.1177/1087054710376907. PMID: 20686100.X-3, X-4

554. Chen B, Girgis S, Elmasry M, et al. Abnormal Gastrointestinal Histopathology in Children With Autism Spectrum Disorders. J Pediatr Gastroenterol Nutr. 2011 Feb 2doi: 10.1097/MPG.0b013e3181f54f05. PMID: 21297509.X-1

555. Chevallier C, Noveck I, Happe F, et al. What's in a voice? Prosody as a test case for the Theory of Mind account of autism. Neuropsychologia. 2011 Feb;49(3):507-17. doi: 10.1016/j.neuropsychologia.2010.11.042.

PMID: 21134386.X-1 
556. Chien IC, Lin CH, Chou YJ, et al. Prevalence and incidence of autism spectrum disorders among national health insurance enrollees in Taiwan from 1996 to 2005. J Child Neurol. 2011 Jul;26(7):830-4. doi: 10.1177/0883073810393964. PMID: 21460178.X-1

557. Chow JC, Grigorov GN. Effect of the bone heterogeneity on the dose prescription in orthovoltage radiotherapy: A Monte Carlo study. Rep Pract Oncol Radiother.

2011;17(1):38-43. doi:

10.1016/j.rpor.2011.09.001. PMID: 24376995.X-1

558. Chung SY, Yoon HJ. A framework for treatment of autism using affective computing. Stud Health Technol Inform. 2011;163:132-4. PMID: 21335775.X-1

559. Chung TK, Lynch ER, Fiser CJ, et al. Psychiatric comorbidity and treatment response in patients with tuberous sclerosis complex. Ann Clin Psychiatry. 2011 Nov;23(4):263-9. PMID: 22073383.X-1

560. Collins A, Dworkin RJ. Pilot study of the effectiveness of weighted vests. Am J Occup Ther. 2011 Nov-Dec;65(6):688-94. PMID: 22214113.X-1

561. Corbett BA, Gunther JR, Comins D, et al. Brief report: theatre as therapy for children with autism spectrum disorder. J Autism Dev Disord. 2011 Apr;41(4):505-11. doi: 10.1007/s10803-010-1064-1. PMID: 20640592.X-3, X-4

562. Cordeiro L, Ballinger E, Hagerman R, et al. Clinical assessment of DSM-IV anxiety disorders in fragile $\mathrm{X}$ syndrome: prevalence and characterization. J Neurodev Disord. 2011 Mar;3(1):57-67. doi: 10.1007/s11689-0109067-y. PMID: 21475730.X-1

563. Coulter ME, Miller DT, Harris DJ, et al. Chromosomal microarray testing influences medical management. Genet Med. 2011 Sep;13(9):770-6. doi: 10.1097/GIM.0b013e31821dd54a. PMID: 21716121.X-1

564. Coury DL. [Commentary on] Review: little evidence of clear benefit for most medical treatments for children with autism spectrum disorders. Evidence Based Mental Health. 2011;14(4):105-1p. doi: 10.1136/ebmh.2011.100112. PMID: 104641030. Language: English. Entry Date: 20111116. Revision Date: 20150711. Publication Type: Journal Article.X-1, X-2

565. Crighton P. Vaccines need a shot in the arm. Aust Nurs J. 2011 May;18(10):40-1. PMID: 21667703.X-1

566. Critchfield JW, van Hemert S, Ash M, et al. The potential role of probiotics in the management of childhood autism spectrum disorders. Gastroenterol Res Pract. 2011;2011:161358. doi: 10.1155/2011/161358. PMID: 22114588.X-1

567. Croen LA, Grether JK, Yoshida CK, et al. Antidepressant use during pregnancy and childhood autism spectrum disorders. Arch Gen Psychiatry. 2011 Nov;68(11):1104-12. doi: 10.1001/archgenpsychiatry.2011.73. PMID: 21727247.X-1

568. Cukier SH, Wahlberg E. [Autism spectrum disorders. Functional-emotional development hypotheses and their relationship with therapeutic interventions]. Vertex. 2011 Mar-Apr;22(96):135-46. PMID: 21977608.X-1

569. Curran MP. Aripiprazole in the treatment of irritability associated with autistic disorder in paediatric patients: profile report. CNS Drugs. 2011 Sep 1;25(9):801-2. doi: 10.2165/11208280-000000000-00000. PMID: 21870890.X-2, X-3

570. Daley MF, Glanz JM. Straight talk about vaccination. Sci Am. 2011 Sep;305(3):32, 4. PMID: 21870438.X-1

571. Dardennes RM, Al Anbar NN, PradoNetto A, et al. Treating the cause of illness rather than the symptoms: parental causal beliefs and treatment choices in autism spectrum disorder. Res Dev Disabil. 2011 MayJun;32(3):1137-46. doi: 10.1016/j.ridd.2011.01.010. PMID: 21316189.X-1

572. David N, Schneider TR, Vogeley K, et al. Impairments in multisensory processing are not universal to the autism spectrum: No evidence for crossmodal priming deficits in Asperger syndrome. Autism Research. 2011 
2015-12-09;4(5):383-8. doi: 10.1007/s 10803010-1000-4

http://dx.doi.org/10.1002/aur.210. PMID: 964198553; 2011-23487-007.X-1

573. David N, T RS, Vogeley K, et al. Impairments in multisensory processing are not universal to the autism spectrum: no evidence for crossmodal priming deficits in Asperger syndrome. Autism Res. 2011 Oct;4(5):383-8. doi: 10.1002/aur.210. PMID: 21882310.X-1

574. Davis TN, Durand S, Chan JM. The effects of a brushing procedure on stereotypical behavior. Research in Autism Spectrum Disorders. 2011 2015-02-24;5(3):1053-8. doi: http://dx.doi.org/10.1016/j.rasd.2010.11.011. PMID: 867318762; 2011-04787-013.X-3, X-4

575. Davit CJ, Hundley RJ, Bacic JD, et al. A pilot study to improve venipuncture compliance in children and adolescents with autism spectrum disorders. J Dev Behav Pediatr. 2011 Sep;32(7):521-5. doi: 10.1097/DBP.0b013e3182245b09. PMID: 21694630.X-1, X-3

576. De Leo G, Gonzales CH, Battagiri P, et al. A smart-phone application and a companion website for the improvement of the communication skills of children with autism: clinical rationale, technical development and preliminary results. J Med Syst. 2011 Aug;35(4):703-11. doi: 10.1007/s10916-0099407-1. PMID: 20703781.X-1, X-2

577. Defensor EB, Pearson BL, Pobbe RL, et al. A novel social proximity test suggests patterns of social avoidance and gaze aversionlike behavior in BTBR T+tf/J mice. Behav Brain Res. 2011 Mar 1;217(2):302-8. doi: 10.1016/j.bbr.2010.10.033. PMID: 21055421.X-1

578. Delong G. A positive association found between autism prevalence and childhood vaccination uptake across the U.S. population. J Toxicol Environ Health A. 2011;74(14):903-16. doi: 10.1080/15287394.2011.573736. PMID: 21623535.X-1

579. Demb H, Valicenti-McDermott M, Navarro A, et al. The effect of long-term use of risperidone on body weight of children with an autism spectrum disorder. J Clin
Psychopharmacol. 2011 Oct;31(5):669-70. doi: 10.1097/JCP.0b013e31822befa9. PMID: 21881456.X-2

580. DeRosier ME, Swick DC, Davis NO, et al. The efficacy of a Social Skills Group Intervention for improving social behaviors in children with High Functioning Autism Spectrum disorders. J Autism Dev Disord. 2011 Aug;41(8):1033-43. doi: 10.1007/s10803-0101128-2. PMID: 21042870.X-1

581. Devlin S, Healy O, Leader G, et al. Comparison of behavioral intervention and sensory-integration therapy in the treatment of challenging behavior. J Autism Dev Disord. 2011 Oct;41(10):1303-20. doi: 10.1007/s10803010-1149-X. PMID: 21161577.X-4

582. Dickstein-Fischer L, Alexander E, Yan $\mathrm{X}$, et al. An affordable compact humanoid robot for Autism Spectrum Disorder interventions in children. Conf Proc IEEE Eng Med Biol Soc. 2011;2011:5319-22. doi:

10.1109/iembs.2011.6091316. PMID: 22255539.X-1

583. DiGennaro Reed FD, Reed DD, Baez $\mathrm{CN}$, et al. A parametric analysis of errors of commission during discrete-trial training. $\mathrm{J}$ Appl Behav Anal. 2011 Fall;44(3):611-5. doi: 10.1901/jaba.2011.44-611. PMID: 21941391.X-1, X-4

584. Dingfelder HE, Mandell DS. Bridging the research-to-practice gap in autism intervention: an application of diffusion of innovation theory. J Autism Dev Disord. 2011 May;41(5):597-609. doi: 10.1007/s10803-0101081-0. PMID: 20717714.X-1, X-2, X-3

585. Dittlinger LH, Lerman DC. Further analysis of picture interference when teaching word recognition to children with autism. J Appl Behav Anal. 2011 Summer;44(2):341-9. doi: 10.1901/jaba.2011.44-341. PMID: 21709789.X-1

586. Dodds L, Fell DB, Shea S, et al. The role of prenatal, obstetric and neonatal factors in the development of autism. J Autism Dev Disord. 2011 Jul;41(7):891-902. doi: 10.1007/s10803-010-1114-8. PMID: 20922473.X-1 
587. Donaldson SO, Elder JH, Self EH, et al. Fathers' perceptions of their roles during inhome training for children with autism. J Child Adolesc Psychiatr Nurs. 2011 Nov;24(4):200-7. doi: 10.1111/j.1744-6171.2011.00300.x. PMID: 22044567.X-1, X-3, X-4

588. Donnachie P. CHILDREN BEHAVING BADLY. Health \& Homeopathy. 2011:9-11 3p. PMID: 104552243. Language: English. Entry Date: 20120810. Revision Date: 20150711. Publication Type: Journal Article.X1

589. Donoghue K, Stallard P, Kucia J. The clinical practice of Cognitive Behavioural Therapy for children and young people with a diagnosis of Asperger's Syndrome. Clin Child Psychol Psychiatry. 2011 Jan;16(1):89-102. doi: 10.1177/1359104509355019. PMID: 20516059.X-1

590. Doty KJ. Student perceptions of progress in a postsecondary residential program for adults with learning disabilities. J Soc Work Disabil Rehabil. 2011;10(3):150-65. doi: 10.1080/1536710x.2011.596431. PMID: 21827300.X-1

591. Doughty AH, Hopkins MN. Reducing stimulus overselectivity through an increased observing-response requirement. J Appl Behav Anal. 2011 Fall;44(3):653-7. doi: 10.1901/jaba.2011.44-653. PMID: 21941399.X-1

592. Douglas-Hall P, Curran S, Bird V, et al. Aripiprazole: a review of its use in the treatment of irritability associated with autistic disorder patients aged 6-17. J Cent Nerv Syst Dis. 2011;3:143-53. doi: 10.4137/jcnsd.s4140. PMID: 23861644.X-2

593. Dowben JS, Grant JS, Keltner NL. Clonidine: Diverse Use in Pharmacologic Management. Perspectives in Psychiatric Care. 2011;47(2):105-8 4p. doi: 10.1111/j.17446163.2011.00302.x. PMID: 104850512. Language: English. Entry Date: 20110330. Revision Date: 20150818. Publication Type: Journal Article.X-1, X-2

594. Drahota A, Wood JJ, Sze KM, et al. Effects of cognitive behavioral therapy on daily living skills in children with high-functioning autism and concurrent anxiety disorders. $\mathrm{J}$
Autism Dev Disord. 2011 Mar;41(3):257-65. doi: 10.1007/s10803-010-1037-4. PMID: 20508979.X-1

595. Dunst CJ, Trivette CM, Masiello T. Exploratory investigation of the effects of interest-based learning on the development of young children with autism. Autism. 2011 May;15(3):295-305. doi: 10.1177/1362361310370971. PMID: 21430019.X-3, X-4

596. Dykens EM, Lee E, Roof E. PraderWilli syndrome and autism spectrum disorders: an evolving story. J Neurodev Disord. 2011 Sep;3(3):225-37. doi: 10.1007/s11689-0119092-5. PMID: 21858456.X-1

597. Edrisinha C, O'Reilly MF, Choi HY, et al. "Say Cheese": teaching photography skills to adults with developmental disabilities. Res Dev Disabil. 2011 Mar-Apr;32(2):636-42. doi: 10.1016/j.ridd.2010.12.006. PMID: 21227636.X-1

598. Ekas NV, Whitman TL. Adaptation to daily stress among mothers of children with an autism spectrum disorder: the role of daily positive affect. J Autism Dev Disord. 2011 Sep;41(9):1202-13. doi: 10.1007/s10803-0101142-4. PMID: 21125322.X-1

599. Erdmann J. Broad collaborations bring new energy to autism therapeutics. Chem Biol. 2011 Feb 25;18(2):142-3. doi: 10.1016/j.chembiol.2011.02.006. PMID: 21338911.X-1, X-2, X-3

600. Erickson CA, Early M, Stigler KA, et al. An open-label naturalistic pilot study of acamprosate in youth with autistic disorder. J Child Adolesc Psychopharmacol. 2011 Dec;21(6):565-9. doi: 10.1089/cap.2011.0034. PMID: 22136091.X-3, X-4

601. Erickson CA, Weng N, Weiler IJ, et al. Open-label riluzole in fragile $\mathrm{X}$ syndrome. Brain Res. 2011 Mar 22;1380:264-70. doi: 10.1016/j.brainres.2010.10.108. PMID: 21059347.X-1

602. Esposito G, Venuti P, Bornstein MH. ASSESSMENT OF DISTRESS IN YOUNG CHILDREN: A COMPARISON OF AUTISTIC DISORDER, DEVELOPMENTAL DELAY, AND TYPICAL DEVELOPMENT. 
Res Autism Spectr Disord. 2011;5(4):1510-6. doi: 10.1016/j.rasd.2011.02.013. PMID: 21647245.X-1, X-3

603. Estigarribia B, Martin GE, Roberts JE, et al. Narrative Skill in Boys with Fragile $\mathrm{X}$ Syndrome with and without Autism Spectrum Disorder. Appl Psycholinguist. 2011;32(2):35988. doi: 10.1017/s0142716410000445. PMID: 21516264.X-1

604. Farmer CA, Aman MG. Aripiprazole for the treatment of irritability associated with autism. Expert Opin Pharmacother. 2011 Mar;12(4):635-40. doi: 10.1517/14656566.2011.557661. PMID: 21294670.X-2, X-3, X-4

605. Farook S, Subudhi C, Syed A. MINERVA. BMJ: British Medical Journal (Overseas \& Retired Doctors Edition). 2011;343(7824):646- 1p. PMID: 104639578. Language: English. Entry Date: 20111108. Revision Date: 20150711. Publication Type: Journal Article.X-1

606. Fecteau S, Agosta S, Oberman L, et al. Brain stimulation over Broca's area differentially modulates naming skills in neurotypical adults and individuals with Asperger's syndrome. Eur J Neurosci. 2011 Jul;34(1):158-64. doi: 10.1111/j.14609568.2011.07726.x. PMID: 21676037.X-1

607. Fernandes FD, Amato CA, Balestro JI, et al. Orientation to mothers of children of the autistic spectrum about language and communication. J Soc Bras Fonoaudiol. 2011 Mar;23(1):1-7. PMID: 21552725.X-1

608. Fernell E, Hedvall A, Westerlund J, et al. Early intervention in 208 Swedish preschoolers with autism spectrum disorder. A prospective naturalistic study. Res Dev Disabil. 2011 Nov-Dec;32(6):2092-101. doi: 10.1016/j.ridd.2011.08.002. PMID: 21985993.X-1

609. Ferreri SJ, Plavnick JB. A functional analysis of gestural behaviors emitted by young children with severe developmental disabilities. Anal Verbal Behav. 2011;27(1):185-90. PMID: 22532763.X-1

610. Finegold SM. State of the art; microbiology in health and disease. Intestinal bacterial flora in autism. Anaerobe. 2011 Dec;17(6):367-8. doi: 10.1016/j.anaerobe.2011.03.007. PMID: 21524713.X-1

611. Fitzpatrick M. Warehousing. Br J Gen Pract. 2011 Jul;61(588):466. doi:

10.3399/bjgp11X583443. PMID: 21722473.X2, X-3

612. Flores CG, Valcante G, Guter S, et al. Repetitive behavior profiles: Consistency across autism spectrum disorder cohorts and divergence from Prader-Willi syndrome. J Neurodev Disord. 2011 Dec;3(4):316-24. doi: 10.1007/s11689-011-9094-3. PMID: 21881965.X-1

613. Fong SS, Lee VY, Chan NN, et al. Motor ability and weight status are determinants of out-of-school activity participation for children with developmental coordination disorder. Res Dev Disabil. 2011 Nov-Dec;32(6):2614-23. doi: 10.1016/j.ridd.2011.06.013. PMID: 21767931.X-1

614. Fragala-Pinkham MA, Haley SM, O'Neil ME. Group swimming and aquatic exercise programme for children with autism spectrum disorders: a pilot study. Dev Neurorehabil. 2011;14(4):230-41. doi: 10.3109/17518423.2011.575438. PMID: 21732807.X-4

615. Frazier TW, Shattuck PT, Narendorf SC, et al. Prevalence and correlates of psychotropic medication use in adolescents with an autism spectrum disorder with and without caregiver-reported attentiondeficit/hyperactivity disorder. J Child Adolesc Psychopharmacol. 2011 Dec;21(6):571-9. doi: 10.1089/cap.2011.0057. PMID: 22166171.X-1

616. Freed J, Adams C, Lockton E. Literacy skills in primary school-aged children with pragmatic language impairment: a comparison with children with specific language impairment. Int J Lang Commun Disord. 2011 May-Jun;46(3):334-47. doi: 10.3109/13682822.2010.500316. PMID: 21575074.X-1

617. Freitag C, Herpertz-Dahlmann B, Dose $\mathrm{M}$, et al. [Statement on a letter by $<<$ Pyramid Educational Consultants Germany UG $\gg>$ of 
May 2010]. Z Kinder Jugendpsychiatr Psychother. 2011 Nov;39(6):417-9. doi: 10.1024/1422-4917/a000140. PMID: 22031014.X-1

618. Froehlich W. Making a case to continue considering treatment with selective serotonin reuptake inhibitors for children with autism spectrum disorders. Curr Psychiatry

Rep. 2011 Jun;13(3):170-3. doi: 10.1007/s11920-011-0196-0. PMID: 21404127.X-1, X-2

619. Frye RE, Sreenivasula S, Adams JB. Traditional and non-traditional treatments for autism spectrum disorder with seizures: an online survey. BMC Pediatr. 2011;11:37. doi: 10.1186/1471-2431-11-37. PMID: 21592359.X-1

620. Fuentes C, Mostofsky S, Bastian A. No Proprioceptive Deficits in Autism Despite Movement-Related Sensory and Execution Impairments. Journal of Autism \& Developmental Disorders. 2011;41(10):1352-61 10p. doi: 10.1007/s10803-010-1161-1. PMID: 104685458. Language: English. Entry Date: 20110930. Revision Date: 20150711. Publication Type: Journal Article.X-1

621. Gadberry AL. A survey of the use of aided augmentative and alternative communication during music therapy sessions with persons with autism spectrum disorders. J Music Ther. 2011 Spring;48(1):74-89. PMID: 21866714.X-1

622. Gale CM, Eikeseth S, Rudrud E. Functional assessment and behavioural intervention for eating difficulties in children with autism: a study conducted in the natural environment using parents and $\mathrm{ABA}$ tutors as therapists. J Autism Dev Disord. 2011 Oct;41(10):1383-96. doi: 10.1007/s10803-0101167-8. PMID: 21181250.X-3, X-4

623. Gallup GG, Jr., Hobbs DR. Evolutionary medicine: bottle feeding, birth spacing, and autism. Med Hypotheses. 2011 Sep;77(3):345-6. doi: 10.1016/j.mehy.2011.05.010. PMID: 21641730.X-1

624. Gargaro BA, Rinehart NJ, Bradshaw JL, et al. Autism and ADHD: how far have we come in the comorbidity debate? Neurosci
Biobehav Rev. 2011 Apr;35(5):1081-8. doi: 10.1016/j.neubiorev.2010.11.002. PMID: 21093480.X-1

625. Gastgeb H, Wilkinson D, Minshew N, et al. Can Individuals with Autism Abstract Prototypes of Natural Faces? Journal of Autism \& Developmental Disorders. 2011;41(12):160918 10p. doi: 10.1007/s10803-011-1190-4. PMID: 104599067. Language: English. Entry Date: 20111212. Revision Date: 20150711. Publication Type: Journal Article.X-1

626. Gattino GS, dos Santos Riesgo R, Longo D, et al. Effects of relational music therapy on communication of children with autism: A randomized controlled study. Nordisk tidskrift for musikkterapi - Nordic Journal of Music Therapy. 2011 2015-12-09;20(2):142-54. doi:

http://dx.doi.org/10.1080/08098131.2011.56693 3. PMID: 941008755; 2011-14788-003. INCLUDE

627. Gawronski A, Kuzmanovic B, Georgescu A, et al. [Expectations concerning psychotherapy of high-functioning adults with autism spectrum disorders]. Fortschr Neurol Psychiatr. 2011 Nov;79(11):647-54. doi: 10.1055/s-0031-1281734. PMID: 22006206.X1

628. Gerber F, Bessero S, Robbiani B, et al. Comparing residential programmes for adults with autism spectrum disorders and intellectual disability: outcomes of challenging behaviour and quality of life. J Intellect Disabil Res. 2011 Sep;55(9):918-32. doi: 10.1111/j.13652788.2011.01455.x. PMID: 21806693.X-1

629. Gerdts J, Bernier R. The broader autism phenotype and its implications on the etiology and treatment of autism spectrum disorders. Autism Res Treat. 2011;2011:545901. doi: 10.1155/2011/545901. PMID: 22937250.X-1

630. Ghanizadeh A. c-Kit+ cells transplantation as a new treatment for autism, a novel hypothesis with important research and clinical implication. J Autism Dev Disord. 2011 Nov;41(11):1591-2. doi: 10.1007/s10803-0101174-9. PMID: 21225454.X-2

631. Ghanizadeh A. Targeting of glycine site on NMDA receptor as a possible new 
strategy for autism treatment. Neurochem Res. 2011 May;36(5):922-3. doi: 10.1007/s11064010-0381-2. PMID: 21210221.X-1, X-2, X-3

632. Ghanizadeh A. May lovastatin target both autism and epilepsy? A novel hypothesized treatment. Epilepsy Behav. 2011 Feb;20(2):422. doi: 10.1016/j.yebeh.2010.12.027. PMID: 21269887.X-2

633. Ghanizadeh A. Can ziconotide as a Ntype voltage-sensitive calcium channel blocker open a new mode for treatment of autism? A hypothesis. Neurosciences (Riyadh). 2011 Jan;16(1):83. PMID: 21206454.X-1, X-2

634. Ghanizadeh A. Gold implants and increased expression of metallothionein-I/II as a novel hypothesized therapeutic approach for autism. Toxicology. 2011 Apr 28;283(1):63-4. doi: 10.1016/j.tox.2011.02.002. PMID: 21335051.X-2

635. Ghanizadeh A. Ghrelin as a promising therapeutic target for co-occurring autism and epilepsy. Epilepsy Behav. 2011 Feb;20(2):4201. doi: 10.1016/j.yebeh.2010.12.009. PMID: 21220213.X-1

636. Ghanizadeh A. Can tactile sensory processing differentiate between children with autistic disorder and asperger's disorder? Innov Clin Neurosci. 2011 May;8(5):25-30. PMID: 21686145.X-1

637. Ghanizadeh A. A novel hypothesized clinical implication of zonisamide for autism...Ann Neurol. 2010 Feb;67(2):239-49. Annals of Neurology. 2011;69(2):426- 1p. doi: 10.1002/ana.22153. PMID: 104841194. Language: English. Entry Date: 20110527. Revision Date: 20150711. Publication Type: Journal Article.X-1, X-2

638. Ghoneim OM, Ibrahim DA, El-Deeb IM, et al. A novel potential therapeutic avenue for autism: design, synthesis and pharmacophore generation of SSRIs with dual action. Bioorg Med Chem Lett. 2011 Nov 15;21(22):6714-23. doi: 10.1016/j.bmcl.2011.09.046. PMID: 21982496.X-1

639. Gibbs V, Toth-Cohen S. Familycentered occupational therapy and telerehabilitation for children with autism spectrum disorders. Occup Ther Health Care. 2011 Oct;25(4):298-314. doi: 10.3109/07380577.2011.606460. PMID: 23899082.X-1, X-3, X-4

640. Gillesen JC, Barakova EI, Huskens $\mathrm{BE}$, et al. From training to robot behavior: towards custom scenarios for robotics in training programs for ASD. IEEE Int Conf Rehabil Robot. 2011;2011:5975381. doi: 10.1109/icorr.2011.5975381. PMID: 22275585.X-1

641. Golubchik P, Sever J, Weizman A. Low-dose quetiapine for adolescents with autistic spectrum disorder and aggressive behavior: open-label trial. Clin Neuropharmacol. 2011 Nov-Dec;34(6):216-9. doi: 10.1097/WNF.0b013e31823349ac. PMID: 21996644.X-1

642. Gomot M, Blanc R, Clery H, et al. Candidate electrophysiological endophenotypes of hyper-reactivity to change in autism. J Autism Dev Disord. 2011 Jun;41(6):705-14. doi: 10.1007/s10803-010-1091-y. PMID: 20827502.X-1

643. Gooding LF. The effect of a music therapy social skills training program on improving social competence in children and adolescents with social skills deficits. J Music Ther. 2011 Winter;48(4):440-62. PMID: 22506299.X-1, X-3, X-4

644. Gordon K, Pasco G, McElduff F, et al. A communication-based intervention for nonverbal children with autism: what changes? Who benefits? J Consult Clin Psychol. 2011 Aug;79(4):447-57. doi: 10.1037/a0024379. PMID: 21787048.X-1

645. Gray K, Jenkins AC, Heberlein AS, et al. Distortions of mind perception in psychopathology. Proc Natl Acad Sci U S A. 2011 Jan 11;108(2):477-9. doi: 10.1073/pnas.1015493108. PMID: 21187372.X-1

646. Greco CM, Navarro CS, Hunsaker MR, et al. Neuropathologic features in the hippocampus and cerebellum of three older men with fragile X syndrome. Mol Autism. 2011;2(1):2. doi: 10.1186/2040-2392-2-2. PMID: 21303513.X-1 
647. Greer RD, Pistoljevic N, Cahill C, et al. Effects of conditioning voices as reinforcers for listener responses on rate of learning, awareness, and preferences for listening to stories in preschoolers with autism. Anal Verbal Behav. 2011;27(1):103-24. PMID: 22532758.X-1, X-3, X-4

648. Griffith GM, Hastings RP, Oliver C, et al. Psychological well-being in parents of children with Angelman, Cornelia de Lange and Cri du Chat syndromes. J Intellect Disabil Res. 2011 Apr;55(4):397-410. doi: 10.1111/j.13652788.2011.01386.x. PMID: 21323782.X-1

649. Grow LL, Carr JE, Kodak TM, et al. A comparison of methods for teaching receptive labeling to children with autism spectrum disorders. J Appl Behav Anal. 2011 Fall;44(3):475-98. doi: 10.1901/jaba.2011.44475. PMID: 21941380.X-3, X-4

650. Guénolé F, Baleyte J-M. Melatonin for sleep-disturbed children with autism spectrum disorders: can we really speak of a substitution treatment? European Child \& Adolescent Psychiatry. 2011;20(9):493-4 2p. doi: 10.1007/s00787-011-0196-y. PMID: 104687664. Language: English. Entry Date: 20111004. Revision Date: 20150711. Publication Type: Journal Article.X-1, X-2

651. Guerra DJ. The molecular genetics of autism spectrum disorders: genomic mechanisms, neuroimmunopathology, and clinical implications. Autism Res Treat. 2011;2011:398636. doi: 10.1155/2011/398636. PMID: 22937247.X-1

652. Guex P, Halfon O. [Hegemonic model of autism: epistemological crossroads between medicine, family and politics]. Rev Med Suisse. 2011 Sep 21;7(309):1813-6. PMID: 22016936.X-1

653. Guiraud JA, Kushnerenko E, Tomalski $\mathrm{P}$, et al. Differential habituation to repeated sounds in infants at high risk for autism. Neuroreport. 2011 Nov 16;22(16):845-9. doi: 10.1097/WNR.0b013e32834c0bec. PMID: 21934535.X-1

654. Hall HR, Graff JC. The relationships among adaptive behaviors of children with autism, family support, parenting stress, and coping. Issues Compr Pediatr Nurs.

2011;34(1):4-25. doi:

10.3109/01460862.2011.555270. PMID: 21341964.X-1

655. Ham HS, Bartolo A, Corley M, et al. Exploring the relationship between gestural recognition and imitation: evidence of dyspraxia in autism spectrum disorders. Journal of Autism \& Developmental Disorders. 2011;41(1):1-12 p. doi: 10.1007/s10803-0101011-1. PMID: 104970838. Language: English. Entry Date: 20110228. Revision Date:

20150711. Publication Type: Journal Article.X1

656. Hameury L, Delavous P, Leroy C. [Hippotherapy in the paedopsychiatric care project]. Soins Pediatr Pueric. 2011 JanFeb(258):37-40. PMID: 21328838.X-1, X-3

657. Hamilton A, Marshal MP, Murray PJ. Autism spectrum disorders and menstruation. J Adolesc Health. 2011 Oct;49(4):443-5. doi: 10.1016/j.jadohealth.2011.01.015. PMID: 21939879.X-1

658. Hampson DR, Adusei DC, Pacey LK. The neurochemical basis for the treatment of autism spectrum disorders and Fragile $X$ Syndrome. Biochem Pharmacol. 2011 May 1;81(9):1078-86. doi: 10.1016/j.bcp.2011.02.005. PMID: 21333634.X-1

659. Hayakawa K, Kobayashi K. Physical and motor skill training for children with intellectual disabilities. Percept Mot Skills. 2011 Apr;112(2):573-80. doi: 10.2466/06.13.15.pms.112.2.573-580. PMID: 21667765.X-1

660. Healy O, Hughes B, Leader G, et al. Response to a Letter to the Editors re: 'Comparison of Behavioral Intervention and Sensory-Integration Therapy in the Treatment of Challenging Behavior'...Devlin, S., Healy, O., Leader, G., \& Hughes, B. M. (2010). Journal of Autism and Developmental Disorders, 1-18. doi:10.1007/ s10803-0101149-x. Journal of Autism \& Developmental Disorders. 2011;41(10):1439-41 3p. doi: 10.1007/s10803-011-1295-9. PMID: 104685451. Language: English. Entry Date: 20110930. Revision Date: 20150711. Publication Type: Journal Article.X-1, X-2 
661. Hellings JA, Boehm D, Yeh HW, et al. Long-term aripiprazole in youth with developmental disabilities including autism. Journal of Mental Health Research in Intellectual Disabilities. 2011 2015-1209;4(1):40-52. doi: http://dx.doi.org/10.1080/19315864.2010.54227 4. PMID: 872590596; 2011-05041-003.X-1, X3

662. Hepburn SL, Moody EJ. Diagnosing Autism in Individuals with Known Genetic Syndromes: Clinical Considerations and Implications for Intervention. Int Rev Res Dev Disabil. 2011;40:229-59. doi: 10.1016/b978-012-374478-4.00009-5. PMID: 26269783.X-1

663. Hernandez P, Ikkanda Z. Applied behavior analysis: behavior management of children with autism spectrum disorders in dental environments. J Am Dent Assoc. 2011 Mar;142(3):281-7. PMID: 21357862.X-1

664. Hines M, Balandin S, Togher L. Communication and AAC in the lives of adults with autism: the stories of their older parents. Augment Altern Commun. 2011

Dec;27(4):256-66. doi: 10.3109/07434618.2011.587830. PMID: 22136364.X-1

665. Hodgetts S, Magill-Evans J, Misiaszek J. Effects of weighted vests on classroom behavior for children with autism and cognitive impairments. Research in Autism Spectrum Disorders. 2011 2015-12-09;5(1):495-505. doi: 10.1016/j.rasd.2006.08.006 http://dx.doi.org/10.1016/j.rasd.2010.06.015. PMID: 815571560; 2010-22960-053.X-1, X-3

666. Hodgetts S, Magill-Evans J, Misiaszek JE. Weighted vests, stereotyped behaviors and arousal in children with autism. J Autism Dev Disord. 2011 Jun;41(6):805-14. doi: 10.1007/s10803-010-1104-X. PMID: 20839040.X-3, X-4

667. Hodgson ES, McGonigle-Chalmers M. Partial occlusion depiction and its relationship with field independence in children with ASD. Autism: The International Journal of Research \& Practice. 2011;15(4):473-95 23p. doi: 10.1177/1362361310363279. PMID: 104663575. Language: English. Entry Date:
20110901. Revision Date: 20150711. Publication Type: Journal Article.X-1

668. Hopkins IM, Gower MW, Perez TA, et al. Avatar assistant: improving social skills in students with an ASD through a computerbased intervention. J Autism Dev Disord. 2011 Nov;41(11):1543-55. doi: 10.1007/s10803-0111179-z. PMID: 21287255.X-1, X-3

669. Howlett MA, Sidener TM, Progar PR, et al. Manipulation of motivating operations and use of a script-fading procedure to teach mands for location to children with language delays. J Appl Behav Anal. 2011 Winter;44(4):943-7. doi: 10.1901/jaba.2011.44943. PMID: 22219545.X-3, X-4

670. Hussein H, Taha GR, Almanasef A. Characteristics of autism spectrum disorders in a sample of egyptian and saudi patients: transcultural cross sectional study. Child Adolesc Psychiatry Ment Health. 2011;5:34. doi: 10.1186/1753-2000-5-34. PMID: 22051160.X-1

671. Hvidtjorn D, Grove J, Schendel D, et al. Risk of autism spectrum disorders in children born after assisted conception: a population-based follow-up study. J Epidemiol Community Health. 2011 Jun;65(6):497-502. doi: 10.1136/jech.2009.093823. PMID: 20584728.X-1

672. Ingvarsson ET, Hollobaugh T. A comparison of prompting tactics to establish intraverbals in children with autism. J Appl Behav Anal. 2011 Fall;44(3):659-64. doi: 10.1901/jaba.2011.44-659. PMID: 21941400.X-3, X-4

673. Ingvarsson ET, Le DD. Further evaluation of prompting tactics for establishing intraverbal responding in children with autism. Anal Verbal Behav. 2011;27(1):75-93. PMID: 22532756.X-1

674. Irwin JR, Tornatore LA, Brancazio L, et al. Can children with autism spectrum disorders "hear" a speaking face? Child Dev. 2011 Sep-Oct;82(5):1397-403. doi: 10.1111/j.1467-8624.2011.01619.x. PMID: 21790542.X-1

675. Ishikawa T, Takahashi K, Ikeda N, et al. Transporter-Mediated Drug Interaction 
Strategy for 5-Aminolevulinic Acid (ALA)Based Photodynamic Diagnosis of Malignant Brain Tumor: Molecular Design of ABCG2 Inhibitors. Pharmaceutics. 2011;3(3):615-35. doi: 10.3390/pharmaceutics3030615. PMID: 24310600.X-1

676. Jaber MA. Dental caries experience, oral health status and treatment needs of dental patients with autism. J Appl Oral Sci. 2011

May-Jun;19(3):212-7. PMID: 21625735.X-1

677. Jepson B, Granpeesheh D, Tarbox J, et al. Controlled evaluation of the effects of hyperbaric oxygen therapy on the behavior of 16 children with autism spectrum disorders. J Autism Dev Disord. 2011 May;41(5):575-88. doi: 10.1007/s10803-010-1075-y. PMID: 20680427.X-3, X-4

678. Jeste SS. The neurology of autism spectrum disorders. Current Opinion in Neurology. 2011;24(2):132-9 8p. doi: 10.1097/WCO.0b013e3283446450. PMID: 104865655. Language: English. Entry Date: 20110527. Revision Date: 20150711. Publication Type: Journal Article.X-1

679. Joosten A. Evidence-based occupational therapy with children with autism: using information about sensory processing to increase the child's participation in daily life...Occupational Therapy Australia, 24th National Conference and Exhibition, 29 June 1 July 2011. Australian Occupational Therapy Journal. 2011;58:93- 1p. doi: 10.1111/j.14401630.2011.00938.x. PMID: 104690138. Language: English. Entry Date: 20111209. Revision Date: 20150711. Publication Type: Journal Article.X-5

680. Kaluzna-Czaplinska J, Michalska M, Rynkowski J. Vitamin supplementation reduces the level of homocysteine in the urine of autistic children. Nutr Res. 2011 Apr;31(4):318-21. doi: 10.1016/j.nutres.2011.03.009. PMID: 21530806.X-3

681. Kaluzna-Czaplinska J, Socha E, Rynkowski J. B vitamin supplementation reduces excretion of urinary dicarboxylic acids in autistic children. Nutr Res. 2011 Jul;31(7):497-502. doi: 10.1016/j.nutres.2011.06.002. PMID: 21840465.X-1, X-3
682. Kamp-Becker I, Schroder J, Muehlan $\mathrm{H}$, et al. Health-related quality of life in children and adolescents with autism spectrum disorder. Z Kinder Jugendpsychiatr Psychother. 2011 Mar;39(2):123-31. doi: 10.1024/14224917/a000098. PMID: 21442600.X-1

683. Karsten AM, Carr JE, Lepper TL. Description of a practitioner model for identifying preferred stimuli with individuals with autism spectrum disorders. Behav Modif. 2011 Jul;35(4):347-69. doi: 10.1177/0145445511405184. PMID: 21613240.X-1

684. Kern JK, Fletcher CL, Garver CR, et al. Prospective trial of equine-assisted activities in autism spectrum disorder. Altern Ther Health Med. 2011 May-Jun;17(3):14-20. PMID: 22164808.X-3

685. Kern JK, Geier DA, Adams JB, et al. A clinical trial of glutathione supplementation in autism spectrum disorders. Med Sci Monit. 2011 Dec;17(12):Cr677-82. PMID: 22129897.X-1, X-3

686. Khanna R, Madhavan SS, Smith MJ, et al. Assessment of health-related quality of life among primary caregivers of children with autism spectrum disorders. J Autism Dev Disord. 2011 Sep;41(9):1214-27. doi: 10.1007/s10803-010-1140-6. PMID: 21103917.X-1

687. Kim HJ, Lee JE, Shin SJ, et al. Analysis of the substantia innominata volume in patients with Parkinson's disease with dementia, dementia with lewy bodies, and Alzheimer's disease. J Mov Disord. 2011 Oct;4(2):68-72. doi: 10.14802/jmd.11014. PMID: 24868398.X1

688. Kim WS. Mammalian target of rapamycin inhibitors for treatment in tuberous sclerosis. Korean J Pediatr. 2011 Jun;54(6):2415. doi: 10.3345/kjp.2011.54.6.241. PMID: 21949518.X-1

689. King CR. A novel embryological theory of autism causation involving endogenous biochemicals capable of initiating cellular gene transcription: a possible link between twelve autism risk factors and the autism 'epidemic'. Med Hypotheses. 2011 May;76(5):653-60. doi: 
10.1016/j.mehy.2011.01.024. PMID: 21388746.X-1

690. Kliebert ML, Tiger JH. Direct and distal effects of noncontingent juice on rumination exhibited by a child with autism. J Appl Behav Anal. 2011 Winter;44(4):955-9. doi: 10.1901/jaba.2011.44-955. PMID: 22219547.X-3, X-4

691. Klintwall L, Holm A, Eriksson M, et al. Sensory abnormalities in autism. A brief report. Res Dev Disabil. 2011 MarApr;32(2):795-800. doi: 10.1016/j.ridd.2010.10.021. PMID: 21111574.X-1

692. Knickmeyer RC, Davenport M. Turner syndrome and sexual differentiation of the brain: implications for understanding malebiased neurodevelopmental disorders. J Neurodev Disord. 2011 Dec;3(4):293-306. doi: 10.1007/s11689-011-9089-0. PMID: 21818630.X-1

693. Kohls G, Peltzer J, Schulte-Rüther M, et al. Atypical Brain Responses to Reward Cues in Autism as Revealed by Event-Related Potentials. Journal of Autism \& Developmental Disorders. 2011;41(11):1523-33 11p. doi: 10.1007/s10803-011-1177-1. PMID: 104703794. Language: English. Entry Date: 20111109. Revision Date: 20150711. Publication Type: Journal Article.X-1

694. Komoto Y, Hashimoto N, Ikegami Y. [Effects of autism features on alcoholism-especially from the aspect of recovery promoting factors]. Nihon Arukoru Yakubutsu Igakkai Zasshi. 2011 Oct;46(5):454-68; discussion 69. PMID: 22256594.X-1

695. Kott KM, Nervik D. Book reviews. Pediatric Physical Therapy. 20112011 Winter;23(4):408-10 3p. PMID: 108203873. Language: English. Entry Date: 20120210. Revision Date: 20150712. Publication Type: Journal Article.X-1

696. Kovshoff H, Hastings RP, Remington B. Two-year outcomes for children with autism after the cessation of early intensive behavioral intervention. Behav Modif. 2011

Sep;35(5):427-50. doi: 10.1177/0145445511405513. PMID: 21586502.X-1
697. Kuehn BM. Scientists probe oxytocin therapy for social deficits in autism, schizophrenia. JAMA: Journal of the American Medical Association. 2011;305(7):659-61 3p. doi: 10.1001/jama.2011.117. PMID: 104825484. Language: English. Entry Date: 20110304. Revision Date: 20150711.

Publication Type: Journal Article. Journal Subset: Biomedical.X-1, X-2

698. Kunda M, Goel AK. Thinking in Pictures as a Cognitive Account of Autism. Journal of Autism \& Developmental Disorders. 2011;41(9):1157-77 21p. doi: 10.1007/s10803010-1137-1. PMID: 104673815. Language: English. Entry Date: 20110831. Revision Date: 20150711. Publication Type: Journal Article.X1

699. Kurtz AL, Kaufer DI. Dementia in Parkinson's disease. Curr Treat Options Neurol. 2011 Jun;13(3):242-54. doi: 10.1007/s11940011-0121-1. PMID: 21461668.X-1

700. Kushki A, Chau T, Anagnostou E. Handwriting difficulties in children with autism spectrum disorders: A scoping review. Journal of Autism and Developmental Disorders. 2011 2015-12-09;41(12):1706-16. doi: http://dx.doi.org/10.1007/s10803-011-1206-0. PMID: 910378995; 2011-26942-013.X-1, X-2

701. Kwakye LD, Foss-Feig JH, Cascio CJ, et al. Altered auditory and multisensory temporal processing in autism spectrum disorders. Front Integr Neurosci. 2011;4:129. doi: 10.3389/fnint.2010.00129. PMID: 21258617.X-1

702. Lahiri U, Trewyn A, Warren Z, et al. Dynamic Eye gaze and its Potential in Virtual Reality Based Applications for Children with Autism Spectrum Disorders. Autism Open Access. 2011;1(1)doi: 10.4172/21657890.1000101. PMID: 23504265.X-1, X-3, X-4

703. Lahiri U, Warren Z, Sarkar N. Design of a gaze-sensitive virtual social interactive system for children with autism. IEEE Trans Neural Syst Rehabil Eng. 2011 Aug;19(4):44352. doi: 10.1109/tnsre.2011.2153874. PMID: 21609889.X-1

704. Lampi KM, Banerjee PN, Gissler M, et al. Finnish Prenatal Study of Autism and 
Autism Spectrum Disorders (FIPS-A): overview and design. J Autism Dev Disord. 2011 Aug;41(8):1090-6. doi: 10.1007/s10803010-1132-6. PMID: 21082229.X-1

705. Landa RJ, Holman KC, O'Neill AH, et al. Intervention targeting development of socially synchronous engagement in toddlers with autism spectrum disorder: a randomized controlled trial. J Child Psychol Psychiatry. 2011 Jan;52(1):13-21. doi: 10.1111/j.14697610.2010.02288.x. PMID: 21126245.X-1

706. Lang R, Mahoney R, El Zein F, et al. Evidence to practice: treatment of anxiety in individuals with autism spectrum disorders. Neuropsychiatr Dis Treat. 2011;7:27-30. doi: 10.2147/ndt.s10327. PMID: 21326652.X-1, X-2

707. Lanovaz MJ, Sladeczek IE, Rapp JT. Effects of music on vocal stereotypy in children with autism. J Appl Behav Anal. 2011 Fall;44(3):647-51. doi: 10.1901/jaba.2011.44647. PMID: 21941398.X-3, X-4

708. Larson HJ, Cooper LZ, Eskola J, et al. Addressing the vaccine confidence gap. Lancet. 2011 Aug 6;378(9790):526-35. doi: 10.1016/s0140-6736(11)60678-8. PMID: 21664679.X-1

709. LaRue RH, Sloman KN, Weiss MJ, et al. Correspondence between traditional models of functional analysis and a functional analysis of manding behavior. Res Dev Disabil. 2011 Nov-Dec;32(6):2449-57. doi: 10.1016/j.ridd.2011.07.015. PMID: 21821393.X-1

710. Lehtimaki KA, Liimatainen S, Peltola $\mathrm{J}$, et al. The serum level of interleukin-6 in patients with intellectual disability and refractory epilepsy. Epilepsy Res. 2011 Jun;95(1-2):184-7. doi: 10.1016/j.eplepsyres.2011.03.004. PMID: 21530175.X-1

711. Lemmon ME, Gregas M, Jeste SS. Risperidone use in autism spectrum disorders: a retrospective review of a clinic-referred patient population. J Child Neurol. 2011 Apr;26(4):428-32. doi: 10.1177/0883073810382143. PMID: 20929907.X-3
712. Lemon J, Gargaro B, Enticott P, et al. Brief report: executive functioning in autism spectrum disorders: a gender comparison of response inhibition. Journal of Autism \& Developmental Disorders. 2011;41(3):352-6 5p. doi: 10.1007/s10803-010-1039-2. PMID: 104815772. Language: English. Entry Date: 20110419. Revision Date: 20150711. Publication Type: Journal Article.X-1

713. Lerman DC, Dittlinger LH, Fentress $\mathrm{G}$, et al. A comparison of methods for collecting data on performance during discrete trial teaching. Behav Anal Pract. 2011 Summer;4(1):53-62. PMID: 22532904.X-1

714. Lerner MD, Mikami AY, Levine K. Socio-dramatic affective-relational intervention for adolescents with asperger syndrome \& high functioning autism: pilot study. Autism. 2011 Jan;15(1):21-42. doi: 10.1177/1362361309353613. PMID: 20923890.X-1

715. Leupen A. Helium and autism. Homoeopathic Links. 20112011 Summer;24(2):106- 1p. doi: 10.1055/s-00301271051. PMID: 108231917. Language: English. Entry Date: 20110729. Revision Date: 20150712. Publication Type: Journal Article. Journal Subset: Alternative/Complementary Therapies.X-1, X-2

716. Li N, Jin BX, Li JL, et al. [Treatment of autism with scalp acupunctur]. Zhongguo Zhen Jiu. 2011 Aug;31(8):692-6. PMID: 21894689.X-1

717. Lim HA, Draper E. The effects of music therapy incorporated with applied behavior analysis verbal behavior approach for children with autism spectrum disorders. J Music Ther. 2011 Winter;48(4):532-50. PMID: 22506303.X-3

718. Long C, Gurka MJ, Blackman J. Cognitive Skills of Young Children with and without Autism Spectrum Disorder Using the BSID-III. Autism Res Treat. 2011;2011:759289. doi: 10.1155/2011/759289. PMID: 22937256.X-1

719. Lonsdale D, Shamberger RJ, Obrenovich ME. Dysautonomia in autism spectrum disorder: case reports of a family with review of the literature. Autism Res Treat. 
2011;2011:129795. doi: 10.1155/2011/129795. PMID: 22937241.X-1, X-3, X-4

720. Lopez Moratalla N, Palacios Ortega S. [Delay the age of procreation, decline in fertility and increased use of assisted reproduction: risk of birth defects]. Cuad Bioet. 2011 May-Aug;22(75):259-72. PMID: 22040138.X-1

721. Macdonald M, Esposito P, Ulrich D. The physical activity patterns of children with autism. BMC Res Notes. 2011;4:422. doi:

10.1186/1756-0500-4-422. PMID: 22008607.X-1

722. Mace FC, Pratt JL, Prager KL, et al. An evaluation of three methods of saying "no" to avoid an escalating response class hierarchy. J Appl Behav Anal. 2011 Spring;44(1):83-94. doi: 10.1901/jaba.2011.44-83. PMID: 21541139.X-1

723. MacFabe DF, Cain NE, Boon F, et al. Effects of the enteric bacterial metabolic product propionic acid on object-directed behavior, social behavior, cognition, and neuroinflammation in adolescent rats: Relevance to autism spectrum disorder. Behav Brain Res. 2011 Feb 2;217(1):47-54. doi: 10.1016/j.bbr.2010.10.005. PMID: 20937326.X-1

724. Magiati I, Moss J, Yates R, et al. Is the Autism Treatment Evaluation Checklist a useful tool for monitoring progress in children with autism spectrum disorders? J Intellect Disabil Res. 2011 Mar;55(3):302-12. doi: 10.1111/j.1365-2788.2010.01359.x. PMID: 21199043.X-1, X-3

725. Magnee MJ, de Gelder B, van Engeland $\mathrm{H}$, et al. Multisensory integration and attention in autism spectrum disorder: evidence from event-related potentials. PLoS One. 2011;6(8):e24196. doi: 10.1371/journal.pone.0024196. PMID: 21887382.X-1

726. Magnée MJCM, de Gelder B, van Engeland $\mathrm{H}$, et al. Multisensory integration and attention in autism spectrum disorder: Evidence from event-related potentials. PLoS ONE. 2011 2015-12-09;6(8) PMID: 900619714; 201120677-001.X-1
727. Makkonen I, Kokki H, Kuikka J, et al. Effects of fluoxetine treatment on striatal dopamine transporter binding and cerebrospinal fluid insulin-like growth factor-1 in children with autism. Neuropediatrics. 2011 Oct;42(5):207-9. doi: 10.1055/s-0031-1291242. PMID: 22015434.X-1, X-3

728. Malisza KL, Clancy C, Shiloff D, et al. Functional evaluation of hidden figures object analysis in children with autistic disorder. Journal of Autism \& Developmental Disorders. 2011;41(1):13-22 10p. doi: 10.1007/s10803010-1013-z. PMID: 104970837. Language: English. Entry Date: 20110228. Revision Date: 20150711. Publication Type: Journal Article.X1

729. Maljaars JPW, Noens ILJ, Scholte EM, et al. Visual local and global processing in low-functioning deaf individuals with and without autism spectrum disorder. Journal of Intellectual Disability Research. 2011;55(1):95105 11p. doi: 10.1111/j.13652788.2010.01351.x. PMID: 104864861. Language: English. Entry Date: 20110407. Revision Date: 20150711. Publication Type: Journal Article.X-1

730. Margoob MA, Mushtaq D. Serotonin transporter gene polymorphism and psychiatric disorders: is there a link? Indian J Psychiatry. 2011 Oct;53(4):289-99. doi: 10.4103/00195545.91901. PMID: 22303036.X-1

731. Marquenie K, Rodger S, Mangohig K, et al. Dinnertime and bedtime routines and rituals in families with a young child with an autism spectrum disorder. Aust Occup Ther J. 2011 Jun;58(3):145-54. doi: 10.1111/j.14401630.2010.00896.x. PMID: 21599679.X-1

732. Marzullo-Kerth D, Reeve SA, Reeve $\mathrm{KF}$, et al. Using multiple-exemplar training to teach a generalized repertoire of sharing to children with autism. J Appl Behav Anal. 2011 Summer;44(2):279-94. doi: 10.1901/jaba.2011.44-279. PMID: 21709784.X-1, X-3

733. Mashal N, Kasirer A. Thinking maps enhance metaphoric competence in children with autism and learning disabilities. Res Dev Disabil. 2011 Nov-Dec;32(6):2045-54. doi: 10.1016/j.ridd.2011.08.012. PMID: 21985987.X-1, X-3 
734. Masliyah T, Ad-Dab'bagh Y. LowDose Risperidone-Induced Oculogyric Crises in an Adolescent Male with Autism, Tourette's and Developmental Delay. J Can Acad Child Adolesc Psychiatry. 2011 Aug;20(3):214-6. PMID: 21804851.X-1

735. Mason MC. Head for bed. Nurs Stand. 2011 Jan 26-Feb 1;25(21):20. PMID: 21329128.X-1

736. Mathewson KJ, Drmic IE, Jetha MK, et al. Behavioral and cardiac responses to emotional stroop in adults with autism spectrum disorders: influence of medication. Autism Res. 2011 Apr;4(2):98-108. doi: 10.1002/aur.176. PMID: 21360828.X-1

737. Matisz CE, McDougall JJ, Sharkey KA, et al. Helminth parasites and the modulation of joint inflammation. J Parasitol Res. 2011;2011:942616. doi: 10.1155/2011/942616. PMID: 21584243.X-1

738. Matson JL, Wilkins J, Fodstad JC. The Validity of the Baby and Infant Screen for Children with aUtIsm Traits: Part 1 (BISCUIT: Part 1). Journal of Autism \& Developmental Disorders. 2011;41(9):1139-46 8p. doi: 10.1007/s10803-010-0973-3. PMID: 104673819. Language: English. Entry Date: 20110831. Revision Date: 20150711. Publication Type: Journal Article.X-1

739. Mavropoulou S, Papadopoulou E, Kakana D. Effects of task organization on the independent play of students with autism spectrum disorders. J Autism Dev Disord. 2011 Jul;41(7):913-25. doi: 10.1007/s10803-0101116-6. PMID: 20960042.X-1, X-3, X-4

740. Mazefsky CA, Kao J, Oswald DP. Preliminary evidence suggesting caution in the use of psychiatric self-report measures with adolescents with high-functioning autism spectrum disorders. Res Autism Spectr Disord. 2011 Jan;5(1):164-74. doi: 10.1016/j.rasd.2010.03.006. PMID: 24013401.X-1

741. Mazzei D, Lazzeri N, Billeci L, et al. Development and evaluation of a social robot platform for therapy in autism. Conf Proc IEEE Eng Med Biol Soc. 2011;2011:4515-8. doi: 10.1109/iembs.2011.6091119. PMID: 22255342.X-1

742. McLennan Y, Polussa J, Tassone F, et al. Fragile x syndrome. Curr Genomics. 2011 May;12(3):216-24. doi: 10.2174/138920211795677886. PMID: 22043169.X-1

743. Mechling L, Savidge E. Using a Personal Digital Assistant to Increase Completion of Novel Tasks and Independent Transitioning by Students with Autism Spectrum Disorder. Journal of Autism \& Developmental Disorders. 2011;41(6):687-704 18p. doi: 10.1007/s10803-010-1088-6. PMID: 104896825. Language: English. Entry Date: 20110719. Revision Date: 20150711. Publication Type: Journal Article.X-3, X-4

744. Mehta MV, Gandal MJ, Siegel SJ. mGluR5-antagonist mediated reversal of elevated stereotyped, repetitive behaviors in the VPA model of autism. PLoS One. 2011;6(10):e26077. doi: 10.1371/journal.pone.0026077. PMID: 22016815.X-1

745. Miller JS, Gabrielsen T, Villalobos M, et al. The each child study: systematic screening for autism spectrum disorders in a pediatric setting. Pediatrics. 2011 May;127(5):866-71. doi: 10.1542/peds.2010-0136. PMID: 21482605.X-1

746. Mills R, Marchant S. Feature: Intervention in autism: a brief review of the literature. Tizard Learning Disability Review. 2011;16(4):20-35 16p. doi: 10.1108/13595471111172822. PMID: 104687522. Language: English. Entry Date: 20111016. Revision Date: 20150711. Publication Type: Journal Article.X-1, X-2

747. Milne E. Increased intra-participant variability in children with autistic spectrum disorders: evidence from single-trial analysis of evoked EEG. Front Psychol. 2011;2:51. doi: 10.3389/fpsyg.2011.00051. PMID: 21716921.X-1

748. Minchella L, Preti L. Autism spectrum disorder: clinical considerations for the school nurse. NASN Sch Nurse. 2011 May;26(3):1435. PMID: 21675293.X-1 
749. Mines MA, Jope RS. Glycogen synthase kinase-3: a promising therapeutic target for fragile x syndrome. Front Mol Neurosci. 2011;4:35. doi: 10.3389/fnmol.2011.00035. PMID: 22053151.X-1

750. Ming X, Hashim A, Fleishman S, et al. Access to specialty care in autism spectrum disorders-a pilot study of referral source. BMC Health Serv Res. 2011;11:99. doi: 10.1186/1472-6963-11-99. PMID: 21569571.X-1

751. Minjarez MB, Williams SE, Mercier EM, et al. Pivotal response group treatment program for parents of children with autism. J Autism Dev Disord. 2011 Jan;41(1):92-101. doi: 10.1007/s10803-010-1027-6. PMID: 20440638.X-1, X-3

752. Montes G, Halterman JS. White-black disparities in family-centered care among children with autism in the United States: evidence from the NS-CSHCN 2005-2006. Acad Pediatr. 2011 Jul-Aug;11(4):297-304. doi: 10.1016/j.acap.2011.02.002. PMID: 21622042.X-1

753. Montgomery J, Storey K, Post M, et al. The use of auditory prompting systems for increasing independent performance of students with autism in employment training. Int $\mathrm{J}$ Rehabil Res. 2011 Dec;34(4):330-5. doi: 10.1097/MRR.0b013e32834a8fa8. PMID: 21885987.X-3, X-4

754. Moore TR, Symons FJ. Adherence to treatment in a behavioral intervention curriculum for parents of children with autism spectrum disorder. Behav Modif. 2011

Nov;35(6):570-94. doi:

10.1177/0145445511418103. PMID:

21885470.X-1, X-3

755. Morrison H, Roscoe EM, Atwell A. An evaluation of antecedent exercise on behavior maintained by automatic reinforcement using a three-component multiple schedule. J Appl Behav Anal. 2011 Fall;44(3):523-41. doi: 10.1901/jaba.2011.44523. PMID: 21941383.X-1

756. Mrozek-Budzyn D, Majewska R, Kieltyka A, et al. [Lack of association between thimerosal-containing vaccines and autism].
Przegl Epidemiol. 2011;65(3):491-5. PMID: 22184954.X-1

757. Myck-Wayne J, Robinson S, Henson E. Serving and supporting young children with a dual diagnosis of hearing loss and autism: the stories of four families. Am Ann Deaf. 2011 Fall;156(4):379-90. PMID: 22256539.X-1

758. Nadeau J, Sulkowski ML, Ung D, et al. Treatment of comorbid anxiety and autism spectrum disorders. Neuropsychiatry (London). 2011 Dec;1(6):567-78. PMID: 24174992.X-1, $\mathrm{X}-2$

759. Nadel J, Aouka N, Coulon N, et al. Yes they can! Autism: The International Journal of Research \& Practice. 2011;15(4):421-35 15p. doi: 10.1177/1362361310386508. PMID: 104663578. Language: English. Entry Date: 20110901. Revision Date: 20150711.

Publication Type: Journal Article.X-1

760. Nagel JD, Rudick J. Abstracts from the NIH Office of Research on Women's Health Eighth Annual Interdisciplinary Women's Health Research Symposium November 17, 2011. Journal of Women's Health (15409996). 2011;20(10):1382-413 32p. doi: 10.1089/jwh.2011.Ab02. PMID: 104698736. Language: English. Entry Date: 20111025. Revision Date: 20150711. Publication Type: Journal Article.X-1

761. Narendorf SC, Shattuck PT, Sterzing PR. Mental health service use among adolescents with an autism spectrum disorder. Psychiatr Serv. 2011 Aug;62(8):975-8. doi: 10.1176/appi.ps.62.8.975. PMID: 21807842.X1

762. Nazeer A. Psychopharmacology of autistic spectrum disorders in children and adolescents. Pediatric Clinics of North America. 2011;58(1):85-97 13p. doi: 10.1016/j.pcl.2010.10.011. PMID: 104810866. Language: English. Entry Date: 20110401. Revision Date: 20150711. Publication Type: Journal Article.X-1, X-2

763. Niederhofer H. Efficacy of duloxetine and agomelatine does not exceed that of other antidepressants in patients with autistic disorder: preliminary results in 3 patients. Prim Care Companion CNS Disord. 2011;13(1)doi: 
10.4088/PCC.10l01038blu. PMID:

21731837.X-1, X-2

764. Normand MP, Beaulieu L. Further evaluation of response-independent delivery of preferred stimuli and child compliance. J Appl Behav Anal. 2011 Fall;44(3):665-9. doi: 10.1901/jaba.2011.44-665. PMID: 21941401.X-1, X-3, X-4

765. O'Brien K, Slaughter V, Peterson CC. Sibling influences on theory of mind development for children with ASD. J Child Psychol Psychiatry. 2011 Jun;52(6):713-9. doi: 10.1111/j.1469-7610.2011.02389.x. PMID: 21418062.X-1

766. Obrusnikova I, Dillon SR. Challenging situations when teaching children with autism spectrum disorders in general physical education. Adapt Phys Activ Q. 2011 Apr;28(2):113-31. PMID: 21757784.X-1

767. O'Keeffe N, McNicholas F. Paediatricians' views on their role in the assessment and management of ADHD and autism. Ir Med J. 2011 Oct;104(9):282-4. PMID: 22132601.X-1

768. Oldershaw A, Treasure J, Hambrook $\mathrm{D}$, et al. Is anorexia nervosa a version of autism spectrum disorders? European Eating Disorders Review. 2011;19(6):462-74 13p. doi: 10.1002/erv.1069. PMID: 104590160. Language: English. Entry Date: 20120112. Revision Date: 20150711. Publication Type: Journal Article.X-1

769. Olivar-Parra JS, De-La-IglesiaGutierrez M, Forns M. Training referential communicative skills to individuals with autism spectrum disorder: a pilot study. Psychol Rep. 2011 Dec;109(3):921-39. doi: 10.2466/10.11.15.28.pr0.109.6.921-939. PMID: 22420121.X-1

770. Oriel KN, George CL, Peckus R, et al. The effects of aerobic exercise on academic engagement in young children with autism spectrum disorder. Pediatr Phys Ther. 2011 Summer;23(2):187-93. doi: 10.1097/PEP.0b013e318218f149. PMID: 21552085.X-1

771. Orsmond GI, Kuo HY. The daily lives of adolescents with an autism spectrum disorder: discretionary time use and activity partners. Autism. 2011 Sep;15(5):579-99. doi: 10.1177/1362361310386503. PMID: 21697194.X-1

772. Pajareya K, Nopmaneejumruslers K. A pilot randomized controlled trial of DIR/Floortime parent training intervention for pre-school children with autistic spectrum disorders. Autism. 2011 Sep;15(5):563-77. doi: 10.1177/1362361310386502. PMID: 21690083.X-1

773. Palmen A, Didden R, Korzilius H. An outpatient group training programme for improving leisure lifestyle in high-functioning young adults with ASD: a pilot study. Dev Neurorehabil. 2011;14(5):297-309. doi: 10.3109/17518423.2011.595433. PMID: 21870954.X-1

774. Pang KH, Croaker GD. Constipation in children with autism and autistic spectrum disorder. Pediatr Surg Int. 2011 Apr;27(4):3538. doi: 10.1007/s00383-010-2680-8. PMID: 20697898.X-1

775. Papavasiliou AS, Nikaina I, Rizou J, et al. The effect of a psycho-educational program on CARS scores and short sensory profile in autistic children. Eur J Paediatr Neurol. 2011 Jul;15(4):338-44. doi: 10.1016/j.ejpn.2011.02.004. PMID: 21354837.X-3

776. Park JY, Zhang HY, Oh MC. New technical tip for anterior cervical plating : make hole first and choose the proper plate size later. J Korean Neurosurg Soc. 2011 Apr;49(4):2126. doi: 10.3340/jkns.2011.49.4.212. PMID: 21607178.X-1

777. Park KW, Kim HS, Cheon SM, et al. Dementia with Lewy Bodies versus Alzheimer's Disease and Parkinson's Disease Dementia: A Comparison of Cognitive Profiles. J Clin Neurol. 2011 Mar;7(1):19-24. doi: 10.3988/jcn.2011.7.1.19. PMID: 21519522.X-1

778. Pasco G, Tohill C. Predicting progress in Picture Exchange Communication System (PECS) use by children with autism. Int J Lang Commun Disord. 2011 Jan-Feb;46(1):120-5. doi: 10.3109/13682822.2010.484851. PMID: 20536353.X-1 
779. Patil RR. MMR vaccination and autism: learnings and implications. Hum Vaccin. 2011 Feb;7(2):281-2. PMID: 21343697.X-1

780. Patten E, Watson LR. Interventions targeting attention in young children with autism. Am J Speech Lang Pathol. 2011 Feb;20(1):60-9. doi: 10.1044/10580360(2010/09-0081). PMID: 20739632.X-1, X2, $\mathrm{X}-3$

781. Petursdottir AI, Carp CL, Matthies DW, et al. Analyzing stimulus-stimulus pairing effects on preferences for speech sounds. Anal Verbal Behav. 2011;27(1):45-60. PMID: 22532754.X-1

782. Pfeiffer BA, Koenig K, Kinnealey M, et al. Effectiveness of sensory integration interventions in children with autism spectrum disorders: a pilot study. Am J Occup Ther. 2011 Jan-Feb;65(1):76-85. PMID: 21309374. INCLUDE

783. Pierpont EI, Richmond EK, Abbeduto L, et al. Contributions of phonological and verbal working memory to language development in adolescents with fragile $\mathrm{X}$ syndrome. J Neurodev Disord. 2011 Dec;3(4):335-47. doi: 10.1007/s11689-0119095-2. PMID: 21993552.X-1

784. Pillay M, Alderson-Day B, Wright B, et al. Autism Spectrum Conditions--enhancing Nurture and Development (ASCEND): an evaluation of intervention support groups for parents. Clin Child Psychol Psychiatry. 2011 Jan;16(1):5-20. doi:

10.1177/1359104509340945. PMID: 20223793.X-3

785. Plavnick JB, Ferreri SJ. Establishing verbal repertoires in children with autism using function-based video modeling. J Appl Behav Anal. 2011 Winter;44(4):747-66. doi: 10.1901/jaba.2011.44-747. PMID: 22219527.X-1, X-4

786. Polsek D, Jagatic T, Cepanec M, et al. RECENT DEVELOPMENTS IN NEUROPATHOLOGY OF AUTISM SPECTRUM DISORDERS. Transl Neurosci. 2011;2(3):256-64. PMID: 22180840.X-1
787. Poon KK. The activities and participation of adolescents with autism spectrum disorders in Singapore: findings from an ICF-based instrument. J Intellect Disabil Res. 2011 Aug;55(8):790-800. doi: 10.1111/j.1365-2788.2011.01397.x. PMID: 21375640.X-1

788. Powell SG, Thomsen PH, Frydenberg $\mathrm{M}$, et al. Long-term treatment of ADHD with stimulants: a large observational study of reallife patients. J Atten Disord. 2011

Aug;15(6):439-51. doi: 10.1177/1087054710368486. PMID: 20631198.X-1

789. Puleo CM, Kendall PC. Anxiety disorders in typically developing youth: autism spectrum symptoms as a predictor of cognitivebehavioral treatment. J Autism Dev Disord. 2011 Mar;41(3):275-86. doi: 10.1007/s10803010-1047-2. PMID: 20694508.X-1

790. Qian N, Lipkin RM. A learning-style theory for understanding autistic behaviors. Front Hum Neurosci. 2011;5:77. doi: 10.3389/fnhum.2011.00077. PMID: 21886617.X-1

791. Quigley SP, Peterson L, Frieder JE, et al. Effects of a weighted vest on problem behaviors during functional analyses in children with Pervasive Developmental Disorders. Research in Autism Spectrum Disorders. 2011 2015-12-09;5(1):529-38. doi: doi: 10.1901/jaba.1994.27-197. (Reprinted from Analysis and Intervention in Developmental Disabilities, 1982, Vol. 2, pp. 3-20) http://dx.doi.org/10.1016/j.rasd.2010.06.019. PMID: 815570608; 2010-22960-057.X-3, X-4

792. Ramachandran VS, Seckel EL. Synchronized dance therapy to stimulate mirror neurons in autism. Med Hypotheses. 2011 Jan;76(1):150-1. doi: 10.1016/j.mehy.2010.10.047. PMID: 21095065.X-2

793. Ray-Subramanian CE, Huai N, Ellis Weismer S. Brief report: adaptive behavior and cognitive skills for toddlers on the autism spectrum. J Autism Dev Disord. 2011 May;41(5):679-84. doi: 10.1007/s10803-0101083-y. PMID: 20697794.X-1 
794. Reed P, Staytom L, Stott S, et al. Comparison of conditioning impairments in children with Down syndrome, autistic spectrum disorders and mental age-matched controls. J Intellect Disabil Res. 2011 Oct;55(10):988-97. doi: 10.1111/j.13652788.2011.01454.x. PMID: 21790825.X-1

795. Reynolds KE. Autism spectrum disorders in childhood: a clinical update. Community Pract. 2011 Jul;84(7):36-8. PMID: 21941709.X-1, X-2, X-3

796. Rezendes DL, Scarpa A. Associations between Parental Anxiety/Depression and Child Behavior Problems Related to Autism Spectrum Disorders: The Roles of Parenting Stress and Parenting Self-Efficacy. Autism Res Treat. 2011;2011:395190. doi: 10.1155/2011/395190. PMID: 22937246.X-1

797. Richling SM, Rapp JT, Carroll RA, et al. Using noncontingent reinforcement to increase compliance with wearing prescription prostheses. J Appl Behav Anal. 2011

Summer;44(2):375-9. doi: 10.1901/jaba.2011.44-375. PMID: 21709796.X-1, X-3, X-4

798. Ridge K, Guerin S. Irish clinicians' views of interventions for children with autistic spectrum disorders. Autism. 2011 Mar;15(2):239-52. doi: 10.1177/1362361310364141. PMID: 21325370.X-1

799. Riviere V, Becquet M, Peltret E, et al. Increasing compliance with medical examination requests directed to children with autism: effects of a high-probability request procedure. J Appl Behav Anal. 2011 Spring;44(1):193-7. doi: 10.1901/jaba.2011.44193. PMID: 21541109.X-1, X-3

800. Roberts JE, Miranda M, Boccia M, et al. Treatment effects of stimulant medication in young boys with fragile $\mathrm{X}$ syndrome. $\mathrm{J}$ Neurodev Disord. 2011 Sep;3(3):175-84. doi: 10.1007/s11689-011-9085-4. PMID: 21671049.X-1

801. Roberts TP, Cannon KM, Tavabi K, et al. Auditory magnetic mismatch field latency: a biomarker for language impairment in autism. Biol Psychiatry. 2011 Aug 1;70(3):263-9. doi: 10.1016/j.biopsych.2011.01.015. PMID: 21392733.X-1

802. Robinson BH. Understanding ADHD in youth. Acupuncture Today. 2011;12(2):12-24 3p. PMID: 104819772. Language: English. Entry Date: 20110225. Revision Date: 20150711. Publication Type: Journal Article. Journal Subset: Alternative/Complementary Therapies.X-1

803. Rosenberg RE, Kaufmann WE, Law JK, et al. Parent report of community psychiatric comorbid diagnoses in autism spectrum disorders. Autism Res Treat. 2011;2011:405849. doi: 10.1155/2011/405849. PMID: 22937248.X-1

804. Rosenberg RE, Landa R, Law JK, et al. Factors affecting age at initial autism spectrum disorder diagnosis in a national survey. Autism Res Treat. 2011;2011:874619. doi: 10.1155/2011/874619. PMID: 22937257.X-1, X-3

805. Rosenblatt LE, Gorantla S, Torres JA, et al. Relaxation response-based yoga improves functioning in young children with autism: a pilot study. J Altern Complement Med. 2011 Nov;17(11):1029-35. doi: 10.1089/acm.2010.0834. PMID: 21992466.X-3

806. Rothman SM. Health advocacy organizations and evidence-based medicine. Jama. 2011 Jun 22;305(24):2569-70. doi: 10.1001/jama.2011.866. PMID: 21693747.X-1

807. Ruble L, Birdwhistell J, Toland MD, et al. Analysis of Parent, Teacher, and Consultant Speech Exchanges and Educational Outcomes of Students With Autism During COMPASS Consultation. J Educ Psychol Consult. 2011;21(4):259-83. doi: 10.1080/10474412.2011.620818. PMID: 22639523.X-1, X-3

808. Ruble LA, Usher EL, McGrew JH. Preliminary Investigation of the Sources of Self-Efficacy Among Teachers of Students with Autism. Focus Autism Other Dev Disabl. 2011 Jun;26(2):67-74. doi: 10.1177/1088357610397345. PMID: 21691453.X-1

809. Russo AJ. Increased Copper in Individuals with Autism Normalizes Post Zinc 
Therapy More Efficiently in Individuals with Concurrent GI Disease. Nutr Metab Insights. 2011;4:49-54. doi: 10.4137/nmi.s6827. PMID: 23946661.X-1

810. Russo AJ, Devito R. Analysis of Copper and Zinc Plasma Concentration and the Efficacy of Zinc Therapy in Individuals with Asperger's Syndrome, Pervasive Developmental Disorder Not Otherwise Specified (PDD-NOS) and Autism. Biomark Insights. 2011;6:127-33. doi: 10.4137/bmi.s7286. PMID: 22174567.X-1

811. Saalasti S, Tiippana K, Katsyri J, et al. The effect of visual spatial attention on audiovisual speech perception in adults with Asperger syndrome. Exp Brain Res. 2011 Sep;213(2-3):283-90. doi: 10.1007/s00221-0112751-7. PMID: 21660467.X-1

812. Saalasti S, Tiippana K, Kätsyri J, et al. The effect of visual spatial attention on audiovisual speech perception in adults with Asperger syndrome. Experimental Brain Research. 2011 2015-12-09;213(2-3):283-90. doi: http://dx.doi.org/10.1007/s00221-0112751-7. PMID: 888874716; 2011-18088015.X-1

813. Saint-Andre S, Neira Zalentein W, Robin D, et al. [Telepsychiatry at the service of autism]. Encephale. 2011 Feb;37(1):18-24. doi: 10.1016/j.encep.2010.03.010. PMID: 21349370.X-1, X-2

814. Samson F, Hyde KL, Bertone A, et al. Atypical processing of auditory temporal complexity in autistics. Neuropsychologia. 2011 Feb;49(3):546-55. doi: 10.1016/j.neuropsychologia.2010.12.033. PMID: 21192958.X-1

815. Samyn V, Roeyers H, Bijttebier P. Effortful control in typically developing boys and in boys with ADHD or autism spectrum disorder. Res Dev Disabil. 2011 MarApr;32(2):483-90. doi: 10.1016/j.ridd.2010.12.038. PMID: 21255973.X-1

816. Sanchez-Barcelo EJ, Mediavilla MD, Reiter RJ. Clinical uses of melatonin in pediatrics. Int J Pediatr. 2011;2011:892624. doi: 10.1155/2011/892624. PMID: 21760817.X-1
817. Sansa G, Carlson C, Doyle W, et al. Medically refractory epilepsy in autism. Epilepsia. 2011 Jun;52(6):1071-5. doi: 10.1111/j.1528-1167.2011.03069.x. PMID: 21671922.X-1, X-3

818. Santesso DL, Drmic IE, Jetha MK, et al. An event-related source localization study of response monitoring and social impairments in autism spectrum disorder. Psychophysiology. 2011 Feb;48(2):241-51. doi: 10.1111/j.14698986.2010.01056.x. PMID: 20557481.X-1

819. Sasson NJ, Pinkham AE, Carpenter $\mathrm{KL}$, et al. The benefit of directly comparing autism and schizophrenia for revealing mechanisms of social cognitive impairment. J Neurodev Disord. 2011 Jun;3(2):87-100. doi: 10.1007/s11689-010-9068-X. PMID: 21484194.X-1

820. Scarpa A, Reyes NM. Improving emotion regulation with CBT in young children with high functioning autism spectrum disorders: a pilot study. Behav Cogn Psychother. 2011 Jul;39(4):495-500. doi: 10.1017/s1352465811000063. PMID: 21457605.X-4

821. Schaaf R, Blanche E. Comparison of Behavioral Intervention and SensoryIntegration Therapy in the Treatment of Challenging Behavior...Devlin, S., Healy, O., Leader, G., \& Hughes, B. M. (2010). Journal of Autism and Developmental Disorders. Advance online publication. doi:10.1007/s10803-0101149-x. Journal of Autism \& Developmental Disorders. 2011;41(10):1436-8 3p. doi: 10.1007/s10803-011-1303-0. PMID: 104685452. Language: English. Entry Date: 20110930. Revision Date: 20150711. Publication Type: Journal Article.X-1, X-2

822. Schaaf RC. Interventions that address sensory dysfunction for individuals with autism spectrum disorders: Preliminary evidence for the superiority of sensory integration compared to other sensory approaches. Evidence-based practices and treatments for children with autism.: Springer Science + Business Media, New York, NY; 2011:245-73.

823. Schaaf RC, Toth-Cohen S, Johnson SL, et al. The everyday routines of families of children with autism: examining the impact of sensory processing difficulties on the family. 
Autism. 2011 May;15(3):373-89. doi: 10.1177/1362361310386505. PMID: 21430016.X-1

824. Schieve LA, Rice C, Devine O, et al. Have secular changes in perinatal risk factors contributed to the recent autism prevalence increase? Development and application of a mathematical assessment model. Ann Epidemiol. 2011 Dec;21(12):930-45. doi: 10.1016/j.annepidem.2011.08.009. PMID: 22000328.X-1

825. Schiff A, Tarbox J, Lanagan T, et al. Establishing compliance with liquid medication administration in a child with autism. J Appl Behav Anal. 2011 Summer;44(2):381-5. doi: 10.1901/jaba.2011.44-381. PMID: 21709797.X-1

826. Schlosser RW, Shane H, Sorce J, et al. Identifying performing and under performing graphic symbols for verbs and prepositions in animated and static formats: a research note. Augment Altern Commun. 2011 Sep;27(3):20514. doi: 10.3109/07434618.2011.592217. PMID: 22008033.X-1

827. Schmid AM, Truog AW, Damian FJ. Care of the suicidal pediatric patient in the ED: a case study. Am J Nurs. 2011 Sep;111(9):3443; quiz 4-5. doi: 10.1097/01.NAJ.0000405060.13022.b7. PMID: 21865931.X-1

828. Schmidt C, Stichter JP, Lierheimer K, et al. An initial investigation of the generalization of a school-based social competence intervention for youth with highfunctioning autism. Autism Res Treat. 2011;2011:589539. doi: 10.1155/2011/589539. PMID: 22937252.X-3, X-4

829. Schneider HD, Hopp JP. The use of the Bilingual Aphasia Test for assessment and transcranial direct current stimulation to modulate language acquisition in minimally verbal children with autism. Clin Linguist Phon. 2011 Jun;25(6-7):640-54. doi:

10.3109/02699206.2011.570852. PMID:

21631313.X-1, X-3, X-4

830. Schoen E, Paul R, Chawarska K. Phonology and vocal behavior in toddlers with autism spectrum disorders. Autism Res. 2011
Jun;4(3):177-88. doi: 10.1002/aur.183. PMID: 21308998.X-1

831. Schumacher BI, Rapp JT. Evaluation of the immediate and subsequent effects of response interruption and redirection on vocal stereotypy. J Appl Behav Anal. 2011 Fall;44(3):681-5. doi: 10.1901/jaba.2011.44681. PMID: 21941405.X-3, X-4

832. Scoles HA, Urraca N, Chadwick SW, et al. Increased copy number for methylated maternal $15 q$ duplications leads to changes in gene and protein expression in human cortical samples. Mol Autism. 2011;2(1):19. doi: 10.1186/2040-2392-2-19. PMID: 22152151.X1

833. Seiverling L, Hendy HM, Williams K. The Screening Tool of Feeding Problems applied to children (STEP-CHILD): psychometric characteristics and associations with child and parent variables. Res Dev Disabil. 2011 May-Jun;32(3):1122-9. doi: 10.1016/j.ridd.2011.01.012. PMID: 21316919.X-1

834. Semansky RM, Xie M, Mandell DS. Medicaid's increasing role in treating youths with autism spectrum disorders. Psychiatr Serv. 2011 Jun;62(6):588. doi:

10.1176/appi.ps.62.6.588. PMID: 21632723.X1

835. Senechal C, Fontaine C, Larivee S, et al. [Integration in the workforce of Quebec of adults living with Autism Spectrum Disorder or Asperger Syndrome]. Sante Ment Que. 2011 Spring;36(1):181-99. PMID: 21983910.X-1

836. Shattuck PT, Wagner M, Narendorf S, et al. Post-high school service use among young adults with an autism spectrum disorder. Arch Pediatr Adolesc Med. 2011 Feb;165(2):141-6. doi: 10.1001/archpediatrics.2010.279. PMID: 21300654.X-1

837. Shriberg L, Paul R, Black L, et al. The Hypothesis of Apraxia of Speech in Children with Autism Spectrum Disorder. Journal of Autism \& Developmental Disorders. 2011;41(4):405-26 22p. doi: 10.1007/s10803010-1117-5. PMID: 104843334. Language: English. Entry Date: 20110427. Revision Date: 20150711. Publication Type: Journal Article.X1 
838. Sihvonen J. [Neuropsychiatric coaching of an adult with Asperger syndrome]. Duodecim. 2011;127(2):118-25. PMID: 21442862.X-1

839. Silva LM, Schalock M, Ayres R. A model and treatment for autism at the convergence of Chinese medicine and Western science: first 130 cases. Chin J Integr Med. 2011 Jun;17(6):421-9. doi: 10.1007/s11655011-0635-0. PMID: 21660676.X-1, X-2, X-3

840. Silva LM, Schalock M, Gabrielsen K. Early intervention for autism with a parentdelivered Qigong massage program: a randomized controlled trial. Am J Occup Ther. 2011 Sep-Oct;65(5):550-9. PMID: 22026323. INCLUDE

841. Sofronoff K, Dark E, Stone V. Social vulnerability and bullying in children with Asperger syndrome. Autism. 2011

May;15(3):355-72. doi:

10.1177/1362361310365070. PMID: 21430018.X-1

842. Sofronoff K, Eloff J, Sheffield J, et al. Increasing the understanding and demonstration of appropriate affection in children with asperger syndrome: a pilot trial. Autism Res Treat. 2011;2011:214317. doi: 10.1155/2011/214317. PMID: 22937243.X-3

843. Song G, Tin C, Giacometti E, et al. Habituation without NMDA ReceptorDependent Desensitization of Hering-Breuer Apnea Reflex in a Mecp2 Mutant Mouse Model of Rett Syndrome. Front Integr Neurosci. 2011;5:6. doi: 10.3389/fnint.2011.00006. PMID: 21629824.X-1

844. Spencer L, Lyketsos CG, Samstad E, et al. A suicidal adult in crisis: an unexpected diagnosis of autism spectrum disorder. Am J Psychiatry. 2011 Sep;168(9):890-2. doi: 10.1176/appi.ajp.2011.10091261. PMID: 21890803.X-1

845. Srivastava RK, Agarwal M, Pundhir A. Role of donepezil in autism: its conduciveness in psychopharmacotherapy. Case Rep Psychiatry. 2011;2011:563204. doi: 10.1155/2011/563204. PMID: 22937405.X-3, $\mathrm{X}-4$
846. Stahmer AC, Akshoomoff N, Cunningham AB. Inclusion for toddlers with autism spectrum disorders: the first ten years of a community program. Autism. 2011 Sep;15(5):625-41. doi: 10.1177/1362361310392253. PMID: 21486899.X-1, X-3

847. Stahmer AC, Brookman-Frazee L, Lee E, et al. Parent and Multidisciplinary Provider Perspectives on Earliest Intervention for Children at Risk for Autism Spectrum Disorders. Infants Young Child. 2011 Oct;24(4):344-63. PMID: 24058266.X-1

848. Stein LI, Polido JC, Mailloux Z, et al. Oral care and sensory sensitivities in children with autism spectrum disorders. Spec Care Dentist. 2011 May-Jun;31(3):102-10. doi: 10.1111/j.1754-4505.2011.00187.x. PMID: 21592164.X-1

849. Stephenson DT, O'Neill SM, Narayan $\mathrm{S}$, et al. Histopathologic characterization of the BTBR mouse model of autistic-like behavior reveals selective changes in neurodevelopmental proteins and adult hippocampal neurogenesis. Mol Autism. 2011;2(1):7. doi: 10.1186/2040-2392-2-7. PMID: 21575186.X-1

850. Stevenson RA, VanDerKlok RM, Pisoni DB, et al. Discrete neural substrates underlie complementary audiovisual speech integration processes. NeuroImage. 2011 201502-24;55(3):1339-45. doi: http://dx.doi.org/10.1016/j.neuroimage.2010.12. 063. PMID: 851237669; 2011-02101-001.X-1

851. Stocco CS, Thompson RH, Rodriguez NM. Restricted interests and teacher presentation of items. J Appl Behav Anal. 2011 Fall;44(3):499-512. doi: 10.1901/jaba.2011.44499. PMID: 21941381.X-1

852. Stone WS, Iguchi L. Do Apparent Overlaps between Schizophrenia and Autistic Spectrum Disorders Reflect Superficial Similarities or Etiological Commonalities? N Am J Med Sci (Boston). 2011 Jul 25;4(3):12433. PMID: 22563520.X-1

853. Strehle EM. Dysmorphological and pharmacological studies in 4q- syndrome. Genet Couns. 2011;22(2):173-85. PMID: 21848010.X-1 
854. Suda S, Iwata K, Shimmura C, et al. Decreased expression of axon-guidance receptors in the anterior cingulate cortex in autism. Mol Autism. 2011;2(1):14. doi: 10.1186/2040-2392-2-14. PMID: 21859478.X1

855. Sumiyoshi C, Kawakubo Y, Suga M, et al. Impaired ability to organize information in individuals with autism spectrum disorders and their siblings. Neurosci Res. 2011

Mar;69(3):252-7. doi:

10.1016/j.neures.2010.11.007. PMID: 21129422.X-1

856. Sundberg ML, Sundberg CA. Intraverbal behavior and verbal conditional discriminations in typically developing children and children with autism. Anal Verbal Behav. 2011;27(1):23-43. PMID: 22532753.X-1

857. Sung M, Ooi YP, Goh TJ, et al. Effects of cognitive-behavioral therapy on anxiety in children with autism spectrum disorders: a randomized controlled trial. Child Psychiatry Hum Dev. 2011 Dec;42(6):634-49. doi: 10.1007/s10578-011-0238-1. PMID: 21660428.X-1

858. Tallberg T, Dabek J, Hallamaa R, et al. Lipidomics: the function of vital lipids in embryogenesis preventing autism spectrum disorders, treating sterile inflammatory diatheses with a lymphopoietic central nervous system component. J Lipids.

2011;2011:137175. doi: 10.1155/2011/137175. PMID: 21490796.X-1

859. Tamanaha AC, Perissinoto J. Comparison of the evolutional process of children with autism spectrum disorders in different language therapeutic interventions. J Soc Bras Fonoaudiol. 2011 Mar;23(1):8-12. PMID: 21552726.X-4

860. Taylor JL, Seltzer MM. Employment and post-secondary educational activities for young adults with autism spectrum disorders during the transition to adulthood. J Autism Dev Disord. 2011 May;41(5):566-74. doi: 10.1007/s10803-010-1070-3. PMID: 20640591.X-1

861. Thomas M, Hunt A, Hurley M, et al. Time-use diaries are acceptable to parents with a disabled preschool child and are helpful in understanding families' daily lives. Child Care Health Dev. 2011 Mar;37(2):168-74. doi: 10.1111/j.1365-2214.2010.01156.x. PMID: 20854450.X-1

862. Tiinanen S, Maatta A, Silfverhuth M, et al. HRV and EEG based indicators of stress in children with Asperger syndrome in audiovisual stimulus test. Conf Proc IEEE Eng Med Biol Soc. 2011;2011:2021-4. doi: 10.1109/iembs.2011.6090371. PMID: 22254732.X-1

863. Tobiasova Z, van der Lingen $\mathrm{KH}$, Scahill L, et al. Risperidone-related improvement of irritability in children with autism is not associated with changes in serum of epidermal growth factor and interleukin-13. J Child Adolesc Psychopharmacol. 2011 Dec;21(6):555-64. doi: 10.1089/cap.2010.0134. PMID: 22070180.X-3

864. Tops M, van Ijzendoorn MH, Riem $\mathrm{MM}$, et al. Oxytocin receptor gene associated with the efficiency of social auditory processing. Front Psychiatry. 2011;2:60. doi: 10.3389/fpsyt.2011.00060. PMID: 22069391.X-1

865. Trepagnier CY, Olsen DE, Boteler L, et al. Virtual conversation partner for adults with autism. Cyberpsychol Behav Soc Netw. 2011 Jan-Feb;14(1-2):21-7. doi: 10.1089/cyber.2009.0255. PMID: 21329439.X1

866. Trottier N, Kamp L, Mirenda P. Effects of peer-mediated instruction to teach use of speech-generating devices to students with autism in social game routines. Augment Altern Commun. 2011 Mar;27(1):26-39. doi: 10.3109/07434618.2010.546810. PMID: 21284561.X-3, X-4

867. Turner-Brown LM, Lam KS, Holtzclaw TN, et al. Phenomenology and measurement of circumscribed interests in autism spectrum disorders. Autism. 2011 Jul;15(4):437-56. doi: 10.1177/1362361310386507. PMID: 21454386.X-1

868. Umeda C, Deitz J. Effects of therapy cushions on classroom behaviors of children with autism spectrum disorder. Am J Occup 
Ther. 2011 Mar-Apr;65(2):152-9. PMID: 21476362.X-3, X-4

869. Vaidya CJ, Foss-Feig J, Shook D, et al. Controlling attention to gaze and arrows in childhood: an fMRI study of typical development and Autism Spectrum Disorders. Dev Sci. 2011 Jul;14(4):911-24. doi: 10.1111/j.1467-7687.2011.01041.x. PMID: 21676110.X-1

870. Van Adel JM, Geier JD, Perry A, et al. Credible knowledge: a pilot evaluation of a modified GRADE method using parentimplemented interventions for children with autism. BMC Health Serv Res. 2011;11:60. doi: 10.1186/1472-6963-11-60. PMID: 21426564.X-1, X-2, X-3

871. Velasques B, Machado S, Paes F, et al. Sensorimotor integration and psychopathology: Motor control abnormalities related to psychiatric disorders. The World Journal of Biological Psychiatry. 2011 2015-12-09;12(78):560-73. doi: 10.1093/schbul/sbq027. http://dx.doi.org/10.3109/15622975.2010.55140 5. PMID: 923958196; 2012-00584-010.X-1

872. Vyas AK, White NH. Case of hypercalcemia secondary to hypervitaminosis a in a 6-year-old boy with autism. Case Rep Endocrinol. 2011;2011:424712. doi: 10.1155/2011/424712. PMID: 22937283.X-1

873. Wachtel LE, Reti IM, Dhossche DM, et al. Stability of neuropsychological testing during two years of maintenance electroconvulsive therapy in an autistic man. Prog Neuropsychopharmacol Biol Psychiatry. 2011 Jan 15;35(1):301-2. doi: 10.1016/j.pnpbp.2010.11.013. PMID: 21111019.X-1, X-3, X-4

874. Walsh MB. The top 10 reasons children with autism deserve ABA. Behav Anal Pract. 2011 Summer;4(1):72-9. PMID: 22532906.X-1

875. Wan CY, Bazen L, Baars R, et al. Auditory-motor mapping training as an intervention to facilitate speech output in nonverbal children with autism: a proof of concept study. PLoS One. 2011;6(9):e25505. doi: 10.1371/journal.pone.0025505. PMID: 21980480.X-3, X-4
876. Wei BY, Huang F, Qin XT, et al. [Treatment of behavioral disorders by risperidone in children with autism]. Zhongguo Dang Dai Er Ke Za Zhi. 2011 Mar;13(3):216-8. PMID: 21426640.X-3

877. Welch MG. [Commentary on] Review: secretin is not effective for the treatment of children with autism spectrum disorders. Evidence Based Mental Health.

2011;14(4):104- 1p. doi: 10.1136/ebmh.2011.100131. PMID: 104641029. Language: English. Entry Date: 20111116. Revision Date: 20150711. Publication Type: Journal Article.X-1, X-2

878. Westendorp M, Houwen S, Hartman $\mathrm{E}$, et al. Are gross motor skills and sports participation related in children with intellectual disabilities? Res Dev Disabil. 2011 MayJun;32(3):1147-53. doi: 10.1016/j.ridd.2011.01.009. PMID: 21310587.X-1

879. Westmark CJ, Hervey CM, BerryKravis EM, et al. Effect of Anticoagulants on Amyloid beta-Protein Precursor and Amyloid Beta Levels in Plasma. J Alzheimers Dis Parkinsonism. 2011 Jul 24;1:101. doi: 10.4172/2161-0460.1000101. PMID: 23459194.X-1

880. White ER, Hoffmann B, Hoch H, et al. Teaching teamwork to adolescents with autism: the cooperative use of activity schedules. Behav Anal Pract. 2011 Summer;4(1):27-35. PMID: 22532901.X-1

881. Whitehouse AJ, Maybery M, Wray JA, et al. No association between early gastrointestinal problems and autistic-like traits in the general population. Dev Med Child Neurol. 2011 May;53(5):457-62. doi: 10.1111/j.1469-8749.2011.03915.x. PMID: 21418197.X-1

882. Woodard CR, Van Reet J. Object identification and imagination: an alternative to the meta-representational explanation of autism. J Autism Dev Disord. 2011 Feb;41(2):213-26. doi: 10.1007/s10803-010-1044-5. PMID: 20532603.X-1

883. Yang M, Perry K, Weber MD, et al. Social peers rescue autism-relevant sociability deficits in adolescent mice. Autism Res. 2011 
Feb;4(1):17-27. doi: 10.1002/aur.163. PMID: 20928844.X-1

884. Yasuda Y, Hashimoto R, Yamamori $\mathrm{H}$, et al. Gene expression analysis in lymphoblasts derived from patients with autism spectrum disorder. Mol Autism. 2011;2(1):9. doi: 10.1186/2040-2392-2-9. PMID: 21615902.X-1

885. Yazawa M, Hsueh B, Jia X, et al. Using induced pluripotent stem cells to investigate cardiac phenotypes in Timothy syndrome. Nature. 2011 Mar 10;471(7337):230-4. doi: 10.1038/nature09855. PMID: 21307850.X-1

886. Zachor DA, Ben Itzchak E. Assisted reproductive technology and risk for autism spectrum disorder. Res Dev Disabil. 2011 NovDec;32(6):2950-6. doi: 10.1016/j.ridd.2011.05.007. PMID: 21658904.X-1

887. Zayat M, Kalb L, Wodka E. Brief Report: Performance Pattern Differences Between Children with Autism Spectrum Disorders and Attention Deficit-Hyperactivity Disorder on Measures of Verbal Intelligence. Journal of Autism \& Developmental Disorders. 2011;41(12):1743-7 5p. doi: 10.1007/s10803011-1207-z. PMID: 104599053. Language: English. Entry Date: 20111212. Revision Date: 20150711. Publication Type: Journal Article.X1

888. Zeiner P, Gjevik E, Weidle B. Response to atomoxetine in boys with highfunctioning autism spectrum disorders and attention deficit/hyperactivity disorder. Acta Paediatr. 2011 Sep;100(9):1258-61. doi: 10.1111/j.1651-2227.2011.02263.x. PMID: 21392103.X-3, X-4

889. Zhu H, Sun Y, Zeng J, et al. Mirror neural training induced by virtual reality in brain-computer interfaces may provide a promising approach for the autism therapy. Med Hypotheses. 2011 May;76(5):646-7. doi: 10.1016/j.mehy.2011.01.022. PMID: 21300442.X-1, X-2, X-3, X-4

890. . Trialing targeted therapies for autism. Nat Med. 2012 Dec;18(12):1746-7. doi: 10.1038/nm.3027. PMID: 23223062.X-1
891. . Improving support for staff and patients with Asperger's syndrome. Nurs Stand. 2012 Oct 10-16;27(6):63. doi: 10.7748/ns2012.10.27.6.63.p9616. PMID: 23189584.X-1

892. . DIGEST. Adapted Physical Activity Quarterly. 2012;29(3):281-5 5p. PMID: 104460992. Language: English. Entry Date: 20120705. Revision Date: 20150819. Publication Type: Journal Article. Journal Subset: Allied Health.X-1

893. . NEWS IN BRIEF. ASHA Leader. 2012;17(6):3- 1p. PMID: 108113131. Language: English. Entry Date: 20120520. Revision Date: 20150712. Publication Type: Journal Article.X-1, X-2

894. . Abstracts 16/3. Autism: The International Journal of Research \& Practice. 2012;16(3):328-31 4p. doi: 10.1177/1362361312441427. PMID: 104456648. Language: English. Entry Date: 20120705. Revision Date: 20150711. Publication Type: Journal Article.X-1, X-2

895. . Standing while learning: a clinical case presentation utilizing a standing desk for a student with educational and postural challenges. Pediatric Physical Therapy. 2012 2012 Spring;24(1):109- 1p. PMID: 108158644. Language: English. Entry Date: 20120406. Revision Date: 20150712. Publication Type: Journal Article.X-1, X-2

896. . PATIENT INFORMATION SHEET: Risperidone (generic) Risperdal (brand). Brown University Child \& Adolescent Psychopharmacology Update. 2012;14:1-2 p. PMID: 104502995. Language: English. Entry Date: 20120904. Revision Date: 20150711. Publication Type: Journal Article.X-1, X-2

897. . Significant publication bias found in autism research on repetitive behaviors. Brown University Child \& Adolescent Behavior Letter. 2012;28(9):4-5 2p. PMID: 104496840. Language: English. Entry Date: 20120824. Revision Date: 20150711. Publication Type: Journal Article. Journal Subset: Biomedical.X$1, \mathrm{X}-2$

898. . Risperidone may improve maladaptive behaviors in autism. Brown University Child \& Adolescent 
Psychopharmacology Update. 2012;14(9):8- 1p. PMID: 104502993. Language: English. Entry Date: 20120904. Revision Date: 20150711. Publication Type: Journal Article.X-1, X-2

899. . Celecoxib added to risperidone effective for autism. Brown University Child \& Adolescent Psychopharmacology Update. 2012;14(8):8- 1p. PMID: 104481244. Language: English. Entry Date: 20120827. Revision Date: 20150711. Publication Type: Journal Article.X-1, X-2

900. . Autism: Fatty acids may improve social interaction. Brown University Child \& Adolescent Psychopharmacology Update. 2012;14(6):7- 1p. PMID: 104464495. Language: English. Entry Date: 20120625. Revision Date: 20150711. Publication Type: Journal Article.X-1, X-2

901. . Parent training in combination with risperidone shows benefit. Brown University Child \& Adolescent Psychopharmacology Update. 2012;14(3):5- 1p. PMID: 104544494. Language: English. Entry Date: 20120404. Revision Date: 20150711. Publication Type: Journal Article. Journal Subset: Biomedical.X$1, \mathrm{X}-2$

902. . Risperidone and parent training for PDD. Brown University Child \& Adolescent Psychopharmacology Update. 2012;14(3):7-8 2p. PMID: 104544497. Language: English. Entry Date: 20120404. Revision Date: 20150711. Publication Type: Journal Article. Journal Subset: Biomedical.X-1, X-2

903. . Risperidone linked to incontinence in a child with autism. Brown University Child \& Adolescent Psychopharmacology Update. 2012;14(3):8- 1p. PMID: 104544499. Language: English. Entry Date: 20120404. Revision Date: 20150711. Publication Type: Journal Article.X-2, X-3

904. . News. Journal of Psychosocial Nursing \& Mental Health Services. 2012;50(2):6-43 3p. doi: 10.3928/0279369520120113-99. PMID: 104524412. Language: English. Entry Date: 20120413. Revision Date: 20150711. Publication Type: Journal Article.X$1, \mathrm{X}-2$

905. . DRUG NEWS. Nursing. 2012;42(2):21- 1p. doi:
10.1097/01.NURSE.0000410316.69228.e9. PMID: 108160396. Language: English. Entry Date: 20120227. Revision Date: 20150712. Publication Type: Journal Article.X-1, X-2

906. . Intramuscular paliperidone in a young autistic boy with aggression. Brown University Child \& Adolescent Psychopharmacology Update. 2012;14(1):8- 1p. PMID: 104549140. Language: English. Entry Date: 20120410. Revision Date: 20150711. Publication Type: Journal Article.X-1, X-2, X3, X-4

907. Abdallah MW, Hougaard DM, Norgaard-Pedersen B, et al. Infections during pregnancy and after birth, and the risk of autism spectrum disorders: a register-based study utilizing a Danish historic birth cohort. Turk Psikiyatri Derg. 2012 Winter;23(4):229-35. PMID: 23225123.X-1

908. Abu Shmais GA, Al-Ayadhi LY, AlDbass AM, et al. Mechanism of nitrogen metabolism-related parameters and enzyme activities in the pathophysiology of autism. J Neurodev Disord. 2012;4(1):4. doi: 10.1186/1866-1955-4-4. PMID: 22958401.X-1

909. Ackerman S, Reilly B, Bernier R. Tympanostomy tube placement in children with autism. J Dev Behav Pediatr. 2012 Apr;33(3):252-8. doi: 10.1097/DBP.0b013e31824b9f57. PMID: 22343482.X-1

910. Adams C, Lockton E, Freed J, et al. The Social Communication Intervention Project: a randomized controlled trial of the effectiveness of speech and language therapy for school-age children who have pragmatic and social communication problems with or without autism spectrum disorder. Int J Lang Commun Disord. 2012 May-Jun;47(3):233-44. doi: 10.1111/j.1460-6984.2011.00146.x. PMID: 22512510.X-1

911. Adkins KW, Molloy C, Weiss SK, et al. Effects of a standardized pamphlet on insomnia in children with autism spectrum disorders. Pediatrics. 2012 Nov;130 Suppl 2:S139-44. doi: 10.1542/peds.2012-0900K. PMID: 23118244.X-1

912. Adluru N, Ennis CM, Davidson RJ, et al. Max Margin General Linear Modeling for 
Neuroimage Analyses. Proc Workshop Math Methods Biomed Image Analysis. 2012 Jan 10;2012doi: 10.1109/mmbia.2012.6164735. PMID: 24163827.X-1

913. Ahmedani BK, Hock RM. Health care access and treatment for children with comorbid autism and psychiatric conditions. Soc Psychiatry Psychiatr Epidemiol. 2012

Nov;47(11):1807-14. doi: 10.1007/s00127-0120482-0. PMID: 22322982.X-1

914. Albores-Gallo L, Roldan-Ceballos O, Villarreal-Valdes G, et al. M-CHAT Mexican Version Validity and Reliability and Some Cultural Considerations. ISRN Neurol. 2012;2012:408694. doi: 10.5402/2012/408694. PMID: 22811934.X-1

915. Alcantara JI, Cope TE, Cope W, et al. Auditory temporal-envelope processing in highfunctioning children with Autism Spectrum Disorder. Neuropsychologia. 2012

Jun;50(7):1235-51. doi:

10.1016/j.neuropsychologia.2012.01.034.

PMID: 22349444.X-1

916. Aldred C, Green J, Emsley R, et al. Brief report: mediation of treatment effect in a communication intervention for pre-school children with autism. J Autism Dev Disord. 2012 Mar;42(3):447-54. doi: 10.1007/s10803011-1248-3. PMID: 21512834.X-1, X-2

917. Allison J, Wilder DA, Chong I, et al. A comparison of differential reinforcement and noncontingent reinforcement to treat food selectivity in a child with autism. J Appl Behav Anal. 2012 Fall;45(3):613-7. doi: 10.1901/jaba.2012.45-613. PMID: 23060675.X-1, X-3, X-4

918. Allman MJ, Meck WH.

Pathophysiological distortions in time perception and timed performance. Brain: A Journal of Neurology. 2012;135(3):656-77 22p. PMID: 104531935. Language: English. Entry Date: 20120420. Revision Date: 20150711. Publication Type: Journal Article.X-1

919. Altenmuller E, Demorest SM, Fujioka $\mathrm{T}$, et al. Introduction to The neurosciences and music IV: learning and memory. Ann N Y Acad Sci. 2012 Apr;1252:1-16. doi: 10.1111/j.17496632.2012.06474.x. PMID: 22524334.X-1
920. Ameis SH, Szatmari P. Imaginggenetics in autism spectrum disorder: advances, translational impact, and future directions. Front Psychiatry. 2012;3:46. doi: 10.3389/fpsyt.2012.00046. PMID: 22615702.X-1

921. Amodeo DA, Jones JH, Sweeney JA, et al. Differences in BTBR T+ tf $/ \mathrm{J}$ and C57BL/6J mice on probabilistic reversal learning and stereotyped behaviors. Behav Brain Res. 2012 Feb 1;227(1):64-72. doi: 10.1016/j.bbr.2011.10.032. PMID: 22056750.X-1

922. Anagnostou E, Soorya L, Chaplin W, et al. Intranasal oxytocin versus placebo in the treatment of adults with autism spectrum disorders: a randomized controlled trial. Mol Autism. 2012;3(1):16. doi: 10.1186/2040-23923-16. PMID: 23216716.X-1

923. Anitha A, Nakamura K, Thanseem I, et al. Brain region-specific altered expression and association of mitochondria-related genes in autism. Mol Autism. 2012;3(1):12. doi: 10.1186/2040-2392-3-12. PMID: 23116158.X1

924. Anonymous F. Sam's transition story. Narrat Inq Bioeth. 2012 Winter;2(3):179-81. doi: 10.1353/nib.2012.0075. PMID: 24406885.X-1

925. Anonymous O. My son's life with autistic spectrum disorder. Narrat Inq Bioeth. 2012 Winter;2(3):151-3. doi: 10.1353/nib.2012.0063. PMID: 24406874.X-1

926. Anonymous T. "Call me 'he"': on the relationship between autism, gender, and authenticity. Narrat Inq Bioeth. 2012 Winter;2(3):153-6. doi: 10.1353/nib.2012.0066. PMID: 24406875.X-1

927. Anonymous T. Life: one step at a time. Narrat Inq Bioeth. 2012 Winter;2(3):159-61. doi: 10.1353/nib.2012.0072. PMID: 24406877.X-1

928. Arnold LE. [Commentary on] Atomoxetine reduces ADHD symptoms in children with autism spectrum disorder. Evidence Based Mental Health. 2012;15(4):961p. PMID: 104434421. Language: English. Entry Date: 20121107. Revision Date: 
20150711. Publication Type: Journal Article.X$1, \mathrm{X}-2$

929. Arnold LE, Aman MG, Li X. Effect of parent training with meds for autism weakens over time. Brown University Child \& Adolescent Psychopharmacology Update. 2012;14(12):1-3 p. PMID: 104445081. Language: English. Entry Date: 20121203. Revision Date: 20150711. Publication Type: Journal Article. Journal Subset: Biomedical.X$1, \mathrm{X}-2$

930. Arnold LE, Aman MG, Li X, et al. Research Units of Pediatric Psychopharmacology (RUPP) autism network randomized clinical trial of parent training and medication: one-year follow-up. J Am Acad Child Adolesc Psychiatry. 2012

Nov;51(11):1173-84. doi: 10.1016/j.jaac.2012.08.028. PMID: 23101743.X-1

931. Arnold LE, Farmer CA, Aman M, et al. Points for practitioners. Brown University Child \& Adolescent Psychopharmacology Update. 2012;14(1):3- 1p. PMID: 104549135. Language: English. Entry Date: 20120410. Revision Date: 20150711. Publication Type: Journal Article.X-1, X-2

932. Asada K, Itakura S. Social phenotypes of autism spectrum disorders and williams syndrome: similarities and differences. Front Psychol. 2012;3:247. doi: 10.3389/fpsyg.2012.00247. PMID: 22866045.X-1

933. Assouline S, Foley Nicpon M, Dockery L. Predicting the Academic Achievement of Gifted Students with Autism Spectrum Disorder. Journal of Autism \& Developmental Disorders. 2012;42(9):1781-9 9p. doi: 10.1007/s10803-011-1403-x. PMID: 104496836. Language: English. Entry Date: 20120824. Revision Date: 20150711.

Publication Type: Journal Article.X-1

934. Atladottir HO, Henriksen TB, Schendel DE, et al. Autism after infection, febrile episodes, and antibiotic use during pregnancy: an exploratory study. Pediatrics. 2012 Dec;130(6):e1447-54. doi: 10.1542/peds.2012-1107. PMID: 23147969.X-1
935. Atzori M, Garcia-Oscos F, Mendez JA. Role of IL-6 in the etiology of hyperexcitable neuropsychiatric conditions: experimental evidence and therapeutic implications. Future Med Chem. 2012 Nov;4(17):2177-92. doi: 10.4155/fmc.12.156. PMID: 23190106.X-1

936. Auert EJ, Trembath D, Arciuli J, et al. Parents' expectations, awareness, and experiences of accessing evidence-based speech-language pathology services for their children with autism. Int J Speech Lang Pathol. 2012 Apr;14(2):109-18. doi:

10.3109/17549507.2011.652673. PMID: 22390744.X-1

937. Ayaz M, Ayaz AB, Basgul SS, et al. Prevelance of mental disorders and associated factors in institutionalized 3-5 year old children. Turk Psikiyatri Derg. 2012 Summer;23(2):82-8. PMID: 22648870.X-1

938. Ayuda-Pascual R, Llorente-Comi M, Martos-Perez J, et al. [Measurements of stress and family impact in the parents of children with autism spectrum disorders before and after taking part in a training programme]. Rev Neurol. 2012 Feb 29;54 Suppl 1:S73-80. PMID: 22374775.X-1, X-3

939. Babineau BA, Yang M, Crawley JN. Mainstreaming mice.

Neuropsychopharmacology. 2012

Jan;37(1):300-1. doi: 10.1038/npp.2011.168. PMID: 22157866.X-1

940. Baez S, Rattazzi A, Gonzalez-Gadea ML, et al. Integrating intention and context: assessing social cognition in adults with Asperger syndrome. Front Hum Neurosci. 2012;6:302. doi: 10.3389/fnhum.2012.00302. PMID: 23162450.X-1

941. Bagni C, Tassone F, Neri G, et al. Fragile $\mathrm{X}$ syndrome: causes, diagnosis, mechanisms, and therapeutics. J Clin Invest. 2012 Dec;122(12):4314-22. doi: 10.1172/jci63141. PMID: 23202739.X-1

942. Bahrami F, Movahedi A, Marandi SM, et al. Kata techniques training consistently decreases stereotypy in children with autism spectrum disorder. Res Dev Disabil. 2012 JulAug;33(4):1183-93. doi: 
10.1016/j.ridd.2012.01.018. PMID: 22502844.X-1

943. Barnes RE, McCabe H. Should we welcome a cure for autism? A survey of the arguments. Med Health Care Philos. 2012 Aug;15(3):255-69. doi: 10.1007/s11019-0119339-7. PMID: 21837546.X-1

944. Bazzano A, Zeldin A, Schuster E, et al. Vaccine-related beliefs and practices of parents of children with autism spectrum disorders. Am J Intellect Dev Disabil. 2012 May;117(3):23342. doi: 10.1352/1944-7558-117.3.233. PMID: 22716265.X-2

945. Ben Said M, Robel L, Pellegrin-Touati M, et al. TEDIS, Pervasive Developmental Disorder' patients information system, preliminary results. Stud Health Technol Inform. 2012;180:285-9. PMID: 22874197.X-1

946. Bent S, Bertoglio K, Ashwood P, et al. Brief report: Hyperbaric oxygen therapy (HBOT) in children with autism spectrum disorder: a clinical trial. J Autism Dev Disord. 2012 Jun;42(6):1127-32. doi: 10.1007/s10803011-1337-3. PMID: 21818676.X-3, X-4

947. Bergstrom R, Najdowski AC, Tarbox $\mathrm{J}$. Teaching children with autism to seek help when lost in public. J Appl Behav Anal. 2012 Spring;45(1):191-5. doi: 10.1901/jaba.2012.45191. PMID: 22403465.X-1, X-3, X-4

948. Bett GC, Lis A, Wersinger SR, et al. A Mouse Model of Timothy Syndrome: a Complex Autistic Disorder Resulting from a Point Mutation in Cav1.2. N Am J Med Sci (Boston). 2012 Jul 25;5(3):135-40. PMID: 24371506.X-1

949. Bevan Jones R, Thapar A, Lewis G, et al. The association between early autistic traits and psychotic experiences in adolescence. Schizophr Res. 2012 Mar;135(1-3):164-9. doi: 10.1016/j.schres.2011.11.037. PMID:

22245185.X-1

950. Bilbo SD, Jones JP, Parker W. Is autism a member of a family of diseases resulting from genetic/cultural mismatches? Implications for treatment and prevention. Autism Res Treat. 2012;2012:910946. doi: 10.1155/2012/910946. PMID: 22928103.X-1
951. Blanche EI, Reinoso G, Chang MC, et al. Proprioceptive processing difficulties among children with autism spectrum disorders and developmental disabilities. American Journal of Occupational Therapy. 2012 2015-1209;66(5):621-4. doi: http://dx.doi.org/10.5014/ajot.2012.004234. PMID: 1428013992; 2012-25996-013.X-1

952. Blaucok-Busch E, Amin OR, Dessoki $\mathrm{HH}$, et al. Efficacy of DMSA Therapy in a Sample of Arab Children with Autistic Spectrum Disorder. Maedica (Buchar). 2012 Sep;7(3):214-21. PMID: 23400264.X-1

953. Bodner KE, Beversdorf DQ, Saklayen SS, et al. Noradrenergic moderation of working memory impairments in adults with autism spectrum disorder. J Int Neuropsychol Soc. 2012 May;18(3):556-64. doi: 10.1017/s1355617712000070. PMID: 22414705.X-1

954. Boisvert M, Hall N, Andrianopoulos $\mathrm{M}$, et al. The Multi-faceted Implementation of Telepractice to Service Individuals with Autism. Int J Telerehabil. 2012 Fall;4(2):11-24. doi: 10.5195/ijt.2012.6104. PMID: 25945200.X-1

955. Bolduc ME, du Plessis AJ, Sullivan N, et al. Regional cerebellar volumes predict functional outcome in children with cerebellar malformations. Cerebellum. 2012 Jun;11(2):531-42. doi: 10.1007/s12311-0110312-z. PMID: 21901523.X-1

956. Borthwick L. Adulthood: Life lessons. Nature. 2012 Nov 1;491(7422):S10-1. PMID: 23136653.X-1

957. Bou Khalil R. Would some cannabinoids ameliorate symptoms of autism? Eur Child Adolesc Psychiatry. 2012 Apr;21(4):237-8. doi: 10.1007/s00787-0120255-z. PMID: 22350104.X-2

958. Bourret JC, Iwata BA, Harper JM, et al. Elimination of position-biased responding in individuals with autism and intellectual disabilities. J Appl Behav Anal. 2012 Summer;45(2):241-50. doi: 10.1901/jaba.2012.45-241. PMID: 22844134.X-1 
959. Bourzac K. Child development: The first steps. Nature. 2012 Nov 1;491(7422):S7-9. PMID: 23136652.X-1

960. Brady NC, Anderson CJ, Hahn LJ, et al. Eye Tracking as a Measure of Receptive Vocabulary in Children with Autism Spectrum Disorders. AAC: Augmentative \& Alternative Communication. 2012;30(2):147-59 13p. doi: 10.3109/07434618.2014.904923. PMID: 107854294. Language: English. Entry Date: 20140620. Revision Date: 20150819. Publication Type: Journal Article.X-1

961. Bransfield RC. The psychoimmunology of lyme/tick-borne diseases and its association with neuropsychiatric symptoms. Open Neurol J. 2012;6:88-93. doi: 10.2174/1874205x01206010088. PMID: 23091569.X-1

962. Brookman-Frazee L, Baker-Ericzen M, Stadnick N, et al. Parent Perspectives on Community Mental Health Services for Children with Autism Spectrum Disorders. J Child Fam Stud. 2012 Aug 1;21(4)doi: 10.1007/s10826-011-9506-8. PMID: 24244083.X-1

963. Brookman-Frazee L, Drahota A, Stadnick N, et al. Therapist perspectives on community mental health services for children with autism spectrum disorders. Adm Policy Ment Health. 2012 Sep;39(5):365-73. doi: 10.1007/s10488-011-0355-y. PMID: 21533846.X-1

964. Brookman-Frazee LI, Drahota A, Stadnick N. Training community mental health therapists to deliver a package of evidencebased practice strategies for school-age children with autism spectrum disorders: a pilot study. J Autism Dev Disord. 2012 Aug;42(8):1651-61. doi: 10.1007/s10803-011-1406-7. PMID: 22102293.X-3, X-4

965. Brown HK, Ouellette-Kuntz H, Hunter $\mathrm{D}$, et al. Unmet needs of families of schoolaged children with an autism spectrum disorder. J Appl Res Intellect Disabil. 2012 Nov;25(6):497-508. doi: 10.1111/j.14683148.2012.00692.x. PMID: 23055284.X-1

966. Brown KF, Long SJ, Ramsay M, et al. U.K. parents' decision-making about measlesmumps-rubella (MMR) vaccine 10 years after the MMR-autism controversy: a qualitative analysis. Vaccine. 2012 Feb 27;30(10):1855-64. doi: 10.1016/j.vaccine.2011.12.127. PMID: 22230590.X-1

967. Buchbinder M. "Sticky" brains and sticky encounters in a U.S. pediatric pain clinic. Cult Med Psychiatry. 2012 Mar;36(1):102-23. doi: 10.1007/s11013-011-9237-8. PMID: 22006075.X-1

968. Busnelli M, Sauliere A, Manning M, et al. Functional selective oxytocin-derived agonists discriminate between individual $G$ protein family subtypes. J Biol Chem. 2012 Feb 3;287(6):3617-29. doi:

10.1074/jbc.M111.277178. PMID: 22069312.X-1

969. Butler MG, Youngs EL, Roberts JL, et al. Assessment and treatment in autism spectrum disorders: a focus on genetics and psychiatry. Autism Res Treat.

2012;2012:242537. doi: 10.1155/2012/242537. PMID: 22934170.X-1

970. Buxbaum JD, Betancur C, Bozdagi O, et al. Optimizing the phenotyping of rodent ASD models: enrichment analysis of mouse and human neurobiological phenotypes associated with high-risk autism genes identifies morphological, electrophysiological, neurological, and behavioral features. Mol Autism. 2012;3(1):1. doi: 10.1186/2040-23923-1. PMID: 22348382.X-1

971. Campbell D, Love E, Crum J, et al. PP142. Independent effects of pregnancy induced hypertension on childhood development: A retrospective cohort study. Pregnancy Hypertens. 2012 Jul;2(3):316. doi: 10.1016/j.preghy.2012.04.253. PMID: 26105463.X-1

972. Capozza LE, Bimstein E. Preferences of parents of children with autism spectrum disorders concerning oral health and dental treatment. Pediatr Dent. 2012 NovDec;34(7):480-4. PMID: 23265165.X-1

973. Carp CL, Peterson SP, Arkel AJ, et al. A further evaluation of picture prompts during auditory-visual conditional discrimination training. J Appl Behav Anal. 2012 Winter;45(4):737-51. doi: 
10.1901/jaba.2012.45-737. PMID: 23322929.X-3, X-4

974. Carrasco M, Volkmar FR. Significant publication bias found in research on repetitive behaviors. Brown University Child \& Adolescent Psychopharmacology Update. 2012;14(6):1-4 p. doi: 10.1542/peds.2011-3285. PMID: 104464490. Language: English. Entry Date: 20120625. Revision Date: 20150711. Publication Type: Journal Article. Journal Subset: Biomedical.X-1

975. Casanova MF, Baruth JM, El-Baz A, et al. Repetitive Transcranial Magnetic Stimulation (rTMS) Modulates Event-Related Potential (ERP) Indices of Attention in Autism. Transl Neurosci. 2012 Jun 1;3(2):170-80. doi: 10.2478/s13380-012-0022-0. PMID: 24683490.X-1

976. Cascio CJ, Foss-Feig JH, Heacock JL, et al. Response of neural reward regions to food cues in autism spectrum disorders. J Neurodev Disord. 2012;4(1):9. doi: 10.1186/1866-1955-49. PMID: 22958533.X-1

977. Cavalari RN, Romanczyk RG. Caregiver perspectives on unintentional injury risk in children with an autism spectrum disorder. J Pediatr Nurs. 2012 Dec;27(6):63241. doi: 10.1016/j.pedn.2011.07.013. PMID: 23101727.X-1

978. Cebula KR. Applied behavior analysis programs for autism: sibling psychosocial adjustment during and following intervention use. J Autism Dev Disord. 2012 May;42(5):847-62. doi: 10.1007/s10803-0111322-x. PMID: 21725720.X-1

979. Centineo L. Above the noise and confusion. Narrat Inq Bioeth. 2012 Winter;2(3):156-9. doi: 10.1353/nib.2012.0069. PMID: 24406876.X-1

980. Chan AS, Sze SL, Han YM, et al. A chan dietary intervention enhances executive functions and anterior cingulate activity in autism spectrum disorders: a randomized controlled trial. Evid Based Complement Alternat Med. 2012;2012:262136. doi: 10.1155/2012/262136. PMID: 22666288.X-1

981. Chan AW, Kocerha J. The Path to microRNA Therapeutics in Psychiatric and
Neurodegenerative Disorders. Front Genet. 2012;3:82. doi: 10.3389/fgene.2012.00082. PMID: 22629284.X-1

982. Chang MC, Parham LD, Blanche EI, et al. Autonomic and behavioral responses of children with autism to auditory stimuli. Am J Occup Ther. 2012 Sep-Oct;66(5):567-76. doi: 10.5014/ajot.2012.004242. PMID: 22917123.X-1, X-3

983. Chen GM, Yoder KJ, Ganzel BL, et al. Harnessing repetitive behaviours to engage attention and learning in a novel therapy for autism: an exploratory analysis. Front Psychol. 2012;3:12. doi: 10.3389/fpsyg.2012.00012. PMID: 22355292.X-3, X-4

984. Cheng Y, Huang R. Using virtual reality environment to improve joint attention associated with pervasive developmental disorder. Res Dev Disabil. 2012 NovDec;33(6):2141-52. doi: 10.1016/j.ridd.2012.05.023. PMID: 22776822.X-1, X-3

985. Chester VL, Calhoun M. Gait symmetry in children with autism. Autism Res Treat. 2012;2012:576478. doi: 10.1155/2012/576478. PMID: 22934175.X-1

986. Chez M, Low R, Parise C, et al. Safety and observations in a pilot study of lenalidomide for treatment in autism. Autism Res Treat. 2012;2012:291601. doi: 10.1155/2012/291601. PMID: 22997574.X-3

987. Ching H, Pringsheim T. Aripiprazole for autism spectrum disorders (ASD). Cochrane Database of Systematic Reviews. 2012(5):N.PAG-N.PAG 1p. PMID: 108115260. Language: English. Entry Date: 20101029. Revision Date: 20150712. Publication Type: Journal Article.X-2

988. Chiri G, Warfield ME. Unmet need and problems accessing core health care services for children with autism spectrum disorder. Matern Child Health J. 2012 Jul;16(5):1081-91. doi: 10.1007/s10995-0110833-6. PMID: 21667201.X-1

989. Christophersen OA. Should autism be considered a canary bird telling that Homo sapiens may be on its way to extinction? Microb Ecol Health Dis. 2012;23doi: 
10.3402/mehd.v23i0.19008. PMID: 23990819.X-1

990. Cihak DF, Kildare LK, Smith CC, et al. Using video Social Stories to increase task engagement for middle school students with autism spectrum disorders. Behav Modif. 2012 May;36(3):399-425. doi:

10.1177/0145445512442683. PMID:

22609764.X-3, X-4

991. Cimera RE, Wehman P, West M, et al. Do sheltered workshops enhance employment outcomes for adults with autism spectrum disorder? Autism. 2012 Jan;16(1):87-94. doi: 10.1177/1362361311408129. PMID: 21610189.X-1

992. Cocchiola MA, Jr., Martino GM, Dwyer LJ, et al. Toilet training children with autism and developmental delays: an effective program for school settings. Behav Anal Pract. 2012 Winter;5(2):60-4. PMID: 23730467.X-1, $\mathrm{X}-3, \mathrm{X}-4$

993. Cohen-Ophir M, Castel-Deutsh T, Tirosh E. Autism in early childhood: an unusual developmental course-three case reports. Case Rep Psychiatry. 2012;2012:946109. doi: 10.1155/2012/946109. PMID: 22937419.X-1, $\mathrm{X}-3$

994. Colon CL, Ahearn WH, Clark KM, et al. The effects of verbal operant training and response interruption and redirection on appropriate and inappropriate vocalizations. J Appl Behav Anal. 2012 Spring;45(1):107-20. doi: 10.1901/jaba.2012.45-107. PMID: 22403453.X-3, X-4

995. Constable PA, Gaigg SB, Bowler DM, et al. Motion and pattern cortical potentials in adults with high-functioning autism spectrum disorder. Doc Ophthalmol. 2012 Aug 25doi: 10.1007/s10633-012-9349-7. PMID: 22918709.X-1

996. Constantine RJ, Jentz S, Bengtson M, et al. Exposure to antipsychotic medications over a 4-year period among children who initiated antipsychotic treatment before their sixth birthday. Pharmacoepidemiol Drug Saf. 2012 Feb;21(2):152-60. doi: 10.1002/pds.2189. PMID: 21793097.X-1, X-3
997. Coo H, Ouellette-Kuntz H, Lam M, et al. Correlates of age at diagnosis of autism spectrum disorders in six Canadian regions. Chronic Dis Inj Can. 2012 Mar;32(2):90-100. PMID: 22414306.X-1

998. Corbett BA, Schupp CW, Lanni KE. Comparing biobehavioral profiles across two social stress paradigms in children with and without autism spectrum disorders. Mol Autism. 2012;3(1):13. doi: 10.1186/2040-23923-13. PMID: 23158965.X-1

999. Cornell C. Health care disparities: not just for the physically disabled. Narrat Inq Bioeth. 2012 Winter;2(3):163-5. doi: 10.1353/nib.2012.0076. PMID: 24406879.X-1

1000. Cornell C. Transition years: from learning, living and loving to maintenance and mediocrity. Narrat Inq Bioeth. 2012

Winter;2(3):E1-3. doi: 10.1353/nib.2012.0077. PMID: 24406892.X-1

1001. Cornish K, Cole V, Longhi E, et al. Does attention constrain developmental trajectories in fragile x syndrome? A 3-year prospective longitudinal study. Am J Intellect Dev Disabil. 2012 Mar;117(2):103-20. doi: 10.1352/1944-7558-117.2.103. PMID: 22515826.X-1

1002. Costigan FA, Light JC, Newell KM. Factors affecting computer mouse use for young children: implications for AAC. Augment Altern Commun. 2012 Jun;28(2):8595. doi: 10.3109/07434618.2012.679235. PMID: 22670726.X-1

1003. Coury DL, Anagnostou E, ManningCourtney P, et al. Use of psychotropic medication in children and adolescents with autism spectrum disorders. Pediatrics. 2012 Nov;130 Suppl 2:S69-76. doi: 10.1542/peds.2012-0900D. PMID: 23118256.X-1, X-3

1004. Cox DJ. From interdisciplinary to integrated care of the child with autism: the essential role for a code of ethics. J Autism Dev Disord. 2012 Dec;42(12):2729-38. doi: 10.1007/s10803-012-1530-z. PMID: 22527703.X-1

1005. Cox N, Reeve R, Cox S, et al. Brief Report: Driving and Young Adults with ASD: 
Parents' Experiences. Journal of Autism \& Developmental Disorders. 2012;42(10):2257-62 6p. doi: 10.1007/s10803-012-1470-7. PMID: 104419551. Language: English. Entry Date: 20120925. Revision Date: 20150711. Publication Type: Journal Article.X-1

1006. Crespi BJ, Crofts HJ. Association testing of copy number variants in schizophrenia and autism spectrum disorders. J Neurodev Disord. 2012;4(1):15. doi: 10.1186/1866-1955-4-15. PMID: 22958593.X1

1007. Cummings C. Melatonin for the management of sleep disorders in children and adolescents. Paediatr Child Health. 2012 Jun;17(6):331-6. PMID: 23730172.X-1

1008. Cunningham AB. Measuring change in social interaction skills of young children with autism. J Autism Dev Disord. 2012 Apr;42(4):593-605. doi: 10.1007/s10803-0111280-3. PMID: 21638109.X-1

1009. D'Alessandro TMM. Low-dose AbilifyRTM and the treatment of disruptive behaviors in children with autism spectrum disorders: University of Florida; 2012.

1010. Damiano CR, Aloi J, Treadway M, et al. Adults with autism spectrum disorders exhibit decreased sensitivity to reward parameters when making effort-based decisions. J Neurodev Disord. 2012;4(1):13. doi: 10.1186/1866-1955-4-13. PMID: 22958545.X-1

1011. Danesh AA, Kaf WA. DPOAEs and contralateral acoustic stimulation and their link to sound hypersensitivity in children with autism. Int J Audiol. 2012 Apr;51(4):345-52. doi: 10.3109/14992027.2011.626202. PMID: 22299666.X-1

1012. Dannemiller L, LaVesser P, Kinsey E, et al. Timing and rhythmicity intervention for motor impairments in children with autism spectrum disorder. Pediatric Physical Therapy. 20122012 Winter;24(4):372- 1p. PMID: 108103870. Language: English. Entry Date: 20121207. Revision Date: 20150712.

Publication Type: Journal Article.X-5

1013. David FJ, Baranek GT, Wiesen C, et al. Coordination of precision grip in 2-6 years- old children with autism spectrum disorders compared to children developing typically and children with developmental disabilities. Front Integr Neurosci. 2012;6:122. doi:

10.3389/fnint.2012.00122. PMID: 23293589.X1

1014. Dawson G, Bernier R, Ring RH. Social attention: a possible early indicator of efficacy in autism clinical trials. J Neurodev Disord. 2012;4(1):11. doi: 10.1186/1866-1955-4-11. PMID: 22958480.X-1, X-2

1015. Dawson G, Jones EJ, Merkle K, et al. Early behavioral intervention is associated with normalized brain activity in young children with autism. J Am Acad Child Adolesc Psychiatry. 2012 Nov;51(11):1150-9. doi: 10.1016/j.jaac.2012.08.018. PMID: 23101741.X-1

1016. De Andres-Garcia S, Moya-Albiol L, Gonzalez-Bono E. Salivary cortisol and immunoglobulin A: responses to stress as predictors of health complaints reported by caregivers of offspring with autistic spectrum disorder. Horm Behav. 2012 Sep;62(4):464-74. doi: 10.1016/j.yhbeh.2012.08.003. PMID: 22981424.X-1

1017. de Araujo AN, de Sena EP, de Oliveira IR, et al. Antipsychotic agents: efficacy and safety in schizophrenia. Drug Healthc Patient Saf. 2012;4:173-80. doi: 10.2147/dhps.s37429. PMID: 23236256.X-1

1018. de Bruin EI, Verheij F. Social skills training in children with PDD-NOS: an exploratory study. Int J Psychiatry Clin Pract. 2012 Mar;16(1):60-7. doi: 10.3109/13651501.2011.617455. PMID: 22122657.X-3, X-4

1019. Delmonte S, Balsters JH, McGrath J, et al. Social and monetary reward processing in autism spectrum disorders. Mol Autism. 2012;3(1):7. doi: 10.1186/2040-2392-3-7. PMID: 23014171.X-1, X-3

1020. Depape AM, Chen A, Hall GB, et al. Use of prosody and information structure in high functioning adults with autism in relation to language ability. Front Psychol. 2012;3:72. doi: 10.3389/fpsyg.2012.00072. PMID: 22470358.X-1 
1021. DePape AM, Hall GB, Tillmann B, et al. Auditory processing in high-functioning adolescents with Autism Spectrum Disorder. PLoS One. 2012;7(9):e44084. doi: 10.1371/journal.pone.0044084. PMID: 22984462.X-1

1022. Desai MU, Divan G, Wertz FJ, et al. The discovery of autism: Indian parents' experiences of caring for their child with an autism spectrum disorder. Transcult Psychiatry. 2012 Jul;49(3-4):613-37. doi: 10.1177/1363461512447139. PMID: 22722980.X-1

1023. Deutsch SI, Pepe GJ, Burket JA, et al. D-cycloserine improves sociability and spontaneous stereotypic behaviors in 4-week old mice. Brain Res. 2012 Feb 23;1439:96-107. doi: 10.1016/j.brainres.2011.12.040. PMID: 22261249.X-1

1024. Dichter GS, Damiano CA, Allen JA. Reward circuitry dysfunction in psychiatric and neurodevelopmental disorders and genetic syndromes: animal models and clinical findings. J Neurodev Disord. 2012;4(1):19. doi: 10.1186/1866-1955-4-19. PMID: 22958744.X1

1025. Diehl JJ, Paul R. Acoustic Differences In The Imitation Of Prosodic Patterns In Children With Autism Spectrum Disorders. Res Autism Spectr Disord. 2012 Jan;6(1):123-34. doi: 10.1016/j.rasd.2011.03.012. PMID: 22125576.X-1

1026. Diehl JJ, Schmitt LM, Villano M, et al. The Clinical Use of Robots for Individuals with Autism Spectrum Disorders: A Critical Review. Res Autism Spectr Disord. 2012 Jan;6(1):24962. doi: 10.1016/j.rasd.2011.05.006. PMID: 22125579.X-1, X-2

1027. Dineen K, Bultas M. Introduction: parenting children with autism spectrum disorders during the transition to adulthood. Narrat Inq Bioeth. 2012 Winter;2(3):147-9. doi: 10.1353/nib.2012.0061. PMID: 24406872.X-1

1028. Divan G, Vajaratkar V, Desai MU, et al. Challenges, coping strategies, and unmet needs of families with a child with autism spectrum disorder in Goa, India. Autism Res. 2012 Jun;5(3):190-200. doi: 10.1002/aur.1225. PMID: 22473816.X-1
1029. Donohue SE, Darling EF, Mitroff SR. Links between multisensory processing and autism. Exp Brain Res. 2012 Oct;222(4):37787. doi: 10.1007/s00221-012-3223-4. PMID: 22923209.X-1

1030. Dove D, Warren Z, McPheeters ML. Evidence on medication treatment for autism lacking. Brown University Child \& Adolescent Psychopharmacology Update. 2012;14(12):51p. PMID: 104445077. Language: English. Entry Date: 20121203. Revision Date: 20150711. Publication Type: Journal Article.X$1, \mathrm{X}-2$

1031. Dove D, Warren Z, McPheeters ML, et al. Medications for Adolescents and Young Adults With Autism Spectrum Disorders: A Systematic Review. Pediatrics. 2012;130(4):717-26 10p. doi: 10.1542/Deds.2012-0683. PMID: 108100574. Language: English. Entry Date: 20121004. Revision Date: 20150712. Publication Type: Journal Article.X-1, X-2

1032. Dowd A, McGinley J, Taffe J, et al. Do Planning and Visual Integration Difficulties Underpin Motor Dysfunction in Autism? A Kinematic Study of Young Children with Autism. Journal of Autism \& Developmental Disorders. 2012;42(8):1539-48 10p. doi: 10.1007/s10803-011-1385-8. PMID: 104482198. Language: English. Entry Date: 20120724. Revision Date: 20150711. Publication Type: Journal Article.X-1

1033. Downey R, Rapport MJ. Motor activity in children with autism: a review of current literature. Pediatric Physical Therapy. 20122012 Spring;24(1):2-20 19p. PMID: 108222317. Language: English. Entry Date: 20120406. Revision Date: 20150820. Publication Type: Journal Article.X-1, X-2

1034. Drahota A, Aarons GA, Stahmer AC. Developing the Autism Model of Implementation for autism spectrum disorder community providers: study protocol. Implement Sci. 2012;7:85. doi: 10.1186/17485908-7-85. PMID: 22963616.X-2

1035. Ducharme JM, Ng O. Errorless academic compliance training: a school-based application for young students with autism. Behav Modif. 2012 Sep;36(5):650-69. doi: 
10.1177/0145445511436006. PMID: 22421393.X-3, X-4

1036. Dugger BN, Serrano GE, Sue LI, et al. Presence of Striatal Amyloid Plaques in Parkinson's Disease Dementia Predicts Concomitant Alzheimer's Disease: Usefulness for Amyloid Imaging. J Parkinsons Dis. 2012 Jan 1;2(1):57-65. doi: 10.3233/jpd-2012-11073. PMID: 22924088.X-1

1037. Dunbar SB, Carr-Hertesl J, Lieberman HA, et al. A Pilot Study Comparison of Sensory Integration Treatment and Integrated Preschool Activities for Children with Autism. Internet Journal of Allied Health Sciences \& Practice. 2012;10(3):8p-p 1p. PMID: 108141757. Language: English. Entry Date: 20120928. Revision Date: 20150818. Publication Type: Journal Article.X-3

1038. Dunn W, Cox J, Foster L, et al. Impact of a contextual intervention on child participation and parent competence among children with autism spectrum disorders: a pretest-posttest repeated-measures design. Am J Occup Ther. 2012 Sep-Oct;66(5):520-8. doi: 10.5014/ajot.2012.004119. PMID: 22917118.X-3

1039. Dunphy-Lelii S, Wellman HM. Delayed Self Recognition in Autism: A Unique Difficulty? Res Autism Spectr Disord. 2012 Jan;6(1):212-23. doi: 10.1016/j.rasd.2011.05.002. PMID: 22125578.X-1

1040. Dunst CJ, Trivette CM, Hamby DW. Meta-analysis of studies incorporating the interests of young children with autism spectrum disorders into early intervention practices. Autism Res Treat. 2012;2012:462531. doi: 10.1155/2012/462531. PMID: 22934173.X-2

1041. Dworzynski K, Ronald A, Bolton P, et al. How different are girls and boys above and below the diagnostic threshold for autism spectrum disorders? J Am Acad Child Adolesc Psychiatry. 2012 Aug;51(8):788-97. doi: 10.1016/j.jaac.2012.05.018. PMID: 22840550.X-1

1042. Dykstra JR, Boyd BA, Watson LR, et al. The impact of the Advancing Socialcommunication And Play (ASAP) intervention on preschoolers with autism spectrum disorder. Autism. 2012 Jan;16(1):27-44. doi: 10.1177/1362361311408933. PMID: 21788256.X-3, X-4

1043. Eisenberg E. Long-term outcomes in children born after assisted conception. Semin Reprod Med. 2012 Apr;30(2):123-30. doi: 10.1055/s-0032-1307420. PMID: 22549712.X1

1044. Eisenstein M. Treatments: In the waiting room. Nature. 2012 Nov 1;491(7422):S14-6. PMID: 23136658.X-2

1045. Elbe D, Lalani Z. Review of the pharmacotherapy of irritability of autism. J Can Acad Child Adolesc Psychiatry. 2012 May;21(2):130-46. PMID: 22548111.X-2, X-3

1046. Eldevik S, Hastings RP, Jahr E, et al. Outcomes of behavioral intervention for children with autism in mainstream pre-school settings. J Autism Dev Disord. 2012 Feb;42(2):210-20. doi: 10.1007/s10803-0111234-9. PMID: 21472360.X-1

1047. Ellegood J, Henkelman RM, Lerch JP. Neuroanatomical Assessment of the Integrin beta3 Mouse Model Related to Autism and the Serotonin System Using High Resolution MRI. Front Psychiatry. 2012;3:37. doi: 10.3389/fpsyt.2012.00037. PMID: 22557981.X-1, X-2

1048. Emck C, Bosscher RJ, van Wieringen PC, et al. Psychiatric symptoms in children with gross motor problems. Adapt Phys Activ Q. 2012 Apr;29(2):161-78. PMID: 22467835.X-1

1049. Enticott PG, Kennedy HA, Rinehart NJ, et al. Mirror neuron activity associated with social impairments but not age in autism spectrum disorder. Biol Psychiatry. $2012 \mathrm{Mar}$ 1;71(5):427-33. doi:

10.1016/j.biopsych.2011.09.001. PMID: 21974786.X-1

1050. Enticott PG, Rinehart NJ, Tonge BJ, et al. Repetitive transcranial magnetic stimulation (rTMS) improves movement-related cortical potentials in autism spectrum disorders. Brain Stimul. 2012 Jan;5(1):30-7. doi: 10.1016/j.brs.2011.02.001. PMID: 22037133.X-1, X-3, X-4 
1051. Eriksson MA, Westerlund J, Anderlid BM, et al. First-degree relatives of young children with autism spectrum disorders: some gender aspects. Res Dev Disabil. 2012 Sep-

Oct;33(5):1642-8. doi:

10.1016/j.ridd.2012.03.025. PMID:

22554810.X-1

1052. Eyler LT, Pierce K, Courchesne E. A failure of left temporal cortex to specialize for language is an early emerging and fundamental property of autism. Brain. 2012 Mar;135(Pt 3):949-60. doi: 10.1093/brain/awr364. PMID: 22350062.X-1

1053. Faja S, Webb SJ, Jones E, et al. The effects of face expertise training on the behavioral performance and brain activity of adults with high functioning autism spectrum disorders. J Autism Dev Disord. 2012 Feb;42(2):278-93. doi: 10.1007/s10803-0111243-8. PMID: 21484517.X-1

1054. Falter CM, Noreika V, Wearden JH, et al. More consistent, yet less sensitive: interval timing in autism spectrum disorders. Q J Exp Psychol (Hove). 2012;65(11):2093-107. doi: 10.1080/17470218.2012.690770. PMID: 22800511.X-1

1055. Fan J, Bernardi S, Van Dam NT, et al. Functional deficits of the attentional networks in autism. Brain Behav. 2012 Sep;2(5):647-60. doi: 10.1002/brb3.90. PMID: 23139910.X-1

1056. Farmer C, Lecavalier L, Yu S, et al. Predictors and moderators of parent training efficacy in a sample of children with autism spectrum disorders and serious behavioral problems. J Autism Dev Disord. 2012 Jun;42(6):1037-44. doi: 10.1007/s10803-0111338-2. PMID: 21822762.X-1

1057. Farrell L, Waters A, Milliner E, et al. Comorbidity and treatment response in pediatric obsessive-compulsive disorder: a pilot study of group cognitive-behavioral treatment. Psychiatry Res. 2012 Sep 30;199(2):115-23. doi: 10.1016/j.psychres.2012.04.035. PMID: 22633155.X-1, X-3, X-4

1058. Feehan K, O'Neil ME, Abdalla D, et al. Factors influencing physical activity in children and youth with special health care needs: a pilot study. Int J Pediatr.
2012;2012:583249. doi: 10.1155/2012/583249. PMID: 22611411.X-1

1059. Felce D, Perry J. Diagnostic grouping among adults with intellectual disabilities and autistic spectrum disorders in staffed housing. J Intellect Disabil Res. 2012 Dec;56(12):1187-93. doi: 10.1111/j.1365-2788.2011.01496.x. PMID: 22044559.X-1

1060. Fengler S. [The long way to an emotionally closed off girl].

Kinderkrankenschwester. 2012 Sep;31(9):390-

1. PMID: 23016230.X-1

1061. Fiksdal BL, Houlihan D, Barnes AC. Dolphin-Assisted Therapy: Claims versus Evidence. Autism Res Treat. 2012;2012:839792. doi: 10.1155/2012/839792. PMID: 22928101.X-2

1062. Finn HE, Miguel CF, Ahearn WH. The emergence of untrained mands and tacts in children with autism. J Appl Behav Anal. 2012 Summer;45(2):265-80. doi: 10.1901/jaba.2012.45-265. PMID: 22844136.X-1, X-4

1063. Fleischer AS. Support to students with Asperger syndrome in higher education--the perspectives of three relatives and three coordinators. Int J Rehabil Res. 2012 Mar;35(1):54-61. doi: 10.1097/MRR.0b013e32834f4d3b. PMID: 22315142.X-1

1064. Fletcher PC, Markoulakis R, Bryden PJ. The costs of caring for a child with an autism spectrum disorder. Issues Compr Pediatr Nurs. 2012;35(1):45-69. doi: 10.3109/01460862.2012.645407. PMID: 22250966.X-1

1065. Flores M, Musgrove K, Renner S, et al. A comparison of communication using the Apple iPad and a picture-based system. Augment Altern Commun. 2012 Jun;28(2):7484. doi: 10.3109/07434618.2011.644579. PMID: 22263895.X-1, X-3, X-4

1066. Fogleman CD. Therapies for children with autism spectrum disorders. Am Fam Physician. 2012 May 1;85(9):878-80. PMID: 22612182.X-2 
1067. Foley AG, Gannon S, RombachMullan N, et al. Class I histone deacetylase inhibition ameliorates social cognition and cell adhesion molecule plasticity deficits in a rodent model of autism spectrum disorder.

Neuropharmacology. 2012 Sep;63(4):750-60. doi: 10.1016/j.neuropharm.2012.05.042. PMID: 22683514.X-1

1068. Forbes D, Parsons H. Essential fatty acids: food for mind and body. Acta Paediatr. 2012 Aug;101(8):808-10. doi: 10.1111/j.16512227.2012.02748.x. PMID: 22646925.X-1

1069. Foss-Feig JH, Heacock JL, Cascio CJ. TACTILE RESPONSIVENESS PATTERNS AND THEIR ASSOCIATION WITH CORE FEATURES IN AUTISM SPECTRUM DISORDERS. Res Autism Spectr Disord. 2012 Winter;6(1):337-44. doi: 10.1016/j.rasd.2011.06.007. PMID: 22059092.X-1

1070. Foster EM, Pearson E. Is inclusivity an indicator of quality of care for children with autism in special education? Pediatrics. 2012 Nov;130 Suppl 2:S179-85. doi:

10.1542/peds.2012-0900P. PMID: 23118249.X$1, \mathrm{X}-3$

1071. Fragale CL, O'Reilly MF, Aguilar J, et al. The influence of motivating operations on generalization probes of specific mands by children with autism. J Appl Behav Anal. 2012 Fall;45(3):565-77. doi: 10.1901/jaba.2012.45565. PMID: 23060669.X-1, X-3, X-4

1072. François M, Teissier N, Barthod G, et al. Sedation for children 2 to 5 years of age undergoing auditory brainstem response and auditory steady state responses recordings. International Journal of Audiology. 2012;51(4):282-6 5p. doi: 10.3109/14992027.2011.601469. PMID: 104528630. Language: English. Entry Date: 20120322. Revision Date: 20150820.

Publication Type: Journal Article.X-1

1073. Freitag CM, Feineis-Matthews S, Valerian J, et al. The Frankfurt early intervention program FFIP for preschool aged children with autism spectrum disorder: a pilot study. J Neural Transm (Vienna). 2012

Sep;119(9):1011-21. doi: 10.1007/s00702-0120792-0. PMID: 22460295.X-3, X-4
1074. Freuler A, Baranek GT, Watson LR, et al. Precursors and Trajectories of Sensory Features: Qualitative Analysis of Infant Home Videos. American Journal of Occupational Therapy. 2012;66(5):e81-4 1p. doi: 10.5014/ajot.2012.004465. PMID: 104439439. Language: English. Entry Date: 20121121. Revision Date: 20150819. Publication Type: Journal Article.X-1

1075. Fritz JN, Iwata BA, Rolider NU, et al. Analysis of self-recording in self-management interventions for stereotypy. J Appl Behav Anal. 2012 Spring;45(1):55-68. doi: 10.1901/jaba.2012.45-55. PMID: 22403449.X1

1076. Froehlich AL, Anderson JS, Bigler ED, et al. Intact Prototype Formation but Impaired Generalization in Autism. Res Autism Spectr Disord. 2012 Spring;6(2):921-30. doi: 10.1016/j.rasd.2011.12.006. PMID: 22291857.X-1

1077. Fung LK, Chahal L, Libove RA, et al. A retrospective review of the effectiveness of aripiprazole in the treatment of sensory abnormalities in autism. J Child Adolesc Psychopharmacol. 2012 Jun;22(3):245-8. doi: 10.1089/cap.2010.0103. PMID: 22537360.X-3, $\mathrm{X}-4$

1078. Gabriels RL, Agnew JA, Beresford C, et al. Improving psychiatric hospital care for pediatric patients with autism spectrum disorders and intellectual disabilities. Autism Res Treat. 2012;2012:685053. doi: 10.1155/2012/685053. PMID: 22934179.X-1

1079. Gaesser GA, Angadi SS. Gluten-free diet: imprudent dietary advice for the general population? J Acad Nutr Diet. 2012

Sep;112(9):1330-3. doi: 10.1016/j.jand.2012.06.009. PMID: 22939437.X-1

1080. Gallagher S, Whiteley J. Social support is associated with blood pressure responses in parents caring for children with developmental disabilities. Res Dev Disabil. 2012 Nov-Dec;33(6):2099-105. doi: 10.1016/j.ridd.2012.06.007. PMID: 22771985.X-1

1081. Gantman A, Kapp SK, Orenski K, et al. Social skills training for young adults with 
high-functioning autism spectrum disorders: a randomized controlled pilot study. J Autism Dev Disord. 2012 Jun;42(6):1094-103. doi: 10.1007/s10803-011-1350-6. PMID: 21915740.X-1

1082. Ganz JB, Heath AK, Lund EM, et al. Effects of peer-mediated implementation of visual scripts in middle school. Behav Modif. 2012 May;36(3):378-98. doi: 10.1177/0145445512442214. PMID: 22582326.X-3, X-4

1083. Gardner J, Mulry CM, Chalik S. Considering college? Adolescents with autism and learning disorders participate in an oncampus service-learning program. Occup Ther Health Care. 2012 Oct;26(4):257-69. doi: 10.3109/07380577.2012.720052. PMID: 23899200.X-1

1084. Gebregziabher M, Shotwell MS, Charles JM, et al. Comparison of Methods for Identifying Phenotype Subgroups Using Categorical Features Data With Application to Autism Spectrum Disorder. Comput Stat Data Anal. 2012 Jan 1;56(1):114-25. doi: 10.1016/j.csda.2011.06.014. PMID: 21927523.X-1

1085. Geier DA, Kern JK, Geier MR. A prospective Cross-sectional Cohort Assessment of Health, Physical, and Behavioral Problems in Autism Spectrum Disorders. Maedica (Buchar). 2012 Sep;7(3):193-200. PMID: 23400046.X-1

1086. Geiger KB, Carr JE, Leblanc LA, et al. Teaching receptive discriminations to children with autism: a comparison of traditional and embedded discrete trial teaching. Behav Anal Pract. 2012 Winter;5(2):49-59. PMID: 23730466.X-4

1087. Geretsegger M, Holck U, Gold C. Randomised controlled trial of improvisational music therapy's effectiveness for children with autism spectrum disorders (TIME-A): study protocol. BMC Pediatr. 2012;12:2. doi: 10.1186/1471-2431-12-2. PMID: 22221670.X$1, \mathrm{X}-2$

1088. Getchell N, Miccinello D, Blom M, et al. Comparing Energy Expenditure in Adolescents With and Without Autism While Playing Nintendo((R)) Wii() Games. Games
Health J. 2012 Feb;1(1):58-61. doi: 10.1089/g4h.2011.0019. PMID: 26196433.X-1

1089. Geurts HM, Jansen MD. A retrospective chart study: the pathway to a diagnosis for adults referred for ASD assessment. Autism. 2012 May;16(3):299-305. doi: 10.1177/1362361311421775. PMID: 21949003.X-1

1090. Ghanizadeh A. Hydrogen as a novel hypothesized emerging treatment for oxidative stress in autism. Eur Rev Med Pharmacol Sci. 2012 Sep;16(9):1313-4. PMID: 23047522.X-1, $\mathrm{X}-2$

1091. Ghanizadeh A. Hyperbaric oxygen therapy for treatment of children with autism: a systematic review of randomized trials. Med Gas Res. 2012;2:13. doi: 10.1186/2045-9912-213. PMID: 22577817.X-2

1092. Ghanizadeh A. Atomoxetine for comorbid autism and ADHD symptoms. Brown University Child \& Adolescent

Psychopharmacology Update. 2012;14(6):6- 1p. PMID: 104464494. Language: English. Entry Date: 20120625. Revision Date: 20150711. Publication Type: Journal Article. Journal Subset: Biomedical.X-1, X-2

1093. Ghanizadeh A, Derakhshan N. Nacetylcysteine for treatment of autism, a case report. J Res Med Sci. 2012 Oct;17(10):985-7. PMID: 23826003.X-3, X-4

1094. Giarelli E, Ruttenberg J, Segal A. Continuing education for nurses in the clinical management of autism spectrum disorders: results of a pilot evaluation. J Contin Educ Nurs. 2012 Apr;43(4):169-76. doi: 10.3928/00220124-20111115-01. PMID: 22106877.X-1

1095. Giles AF, St Peter CC, Pence ST, et al. Preference for blocking or response redirection during stereotypy treatment. Res Dev Disabil. 2012 Nov-Dec;33(6):1691-700. doi: 10.1016/j.ridd.2012.05.008. PMID: 22695072.X-1, X-3, X-4

1096. Gleberzon BJ, Arts J, Mei A, et al. The use of spinal manipulative therapy for pediatric health conditions: a systematic review of the literature. J Can Chiropr Assoc. 2012 Jun;56(2):128-41. PMID: 22675226.X-1 
1097. Goldman S, Greene PE. Stereotypies in autism: a video demonstration of their clinical variability. Front Integr Neurosci. 2012;6:121. doi: 10.3389/fnint.2012.00121. PMID: 23316144.X-1

1098. Golnik A, Maccabee-Ryaboy N, Scal $\mathrm{P}$, et al. Shared decision making: improving care for children with autism. Intellect Dev Disabil. 2012 Aug;50(4):322-31. doi: 10.1352/1934-9556-50.4.322. PMID: 22861133.X-1

1099. Golnik A, Scal P, Wey A, et al. Autism-specific primary care medical home intervention. J Autism Dev Disord. 2012 Jun;42(6):1087-93. doi: 10.1007/s10803-0111351-5. PMID: 21853373.X-1

1100. Gor RA, Fuhrer J, Schober JM. A retrospective observational study of enuresis, daytime voiding symptoms, and response to medical therapy in children with attention deficit hyperactivity disorder and autism spectrum disorder. J Pediatr Urol. 2012 Jun;8(3):314-7. doi: 10.1016/j.jpurol.2010.10.009. PMID: 21131234.X-3, X-4

1101. Grabrucker AM. Environmental factors in autism. Front Psychiatry. 2012;3:118. doi: 10.3389/fpsyt.2012.00118. PMID: 23346059.X-1

1102. Graff RB, Karsten AM. Evaluation of a self-instruction package for conducting stimulus preference assessments. J Appl Behav Anal. 2012 Spring;45(1):69-82. doi: 10.1901/jaba.2012.45-69. PMID: 22403450.X1

1103. Grandgeorge M, Tordjman S, Lazartigues A, et al. Does pet arrival trigger prosocial behaviors in individuals with autism? PLoS One. 2012;7(8):e41739. doi: 10.1371/journal.pone.0041739. PMID: 22870246.X-1

1104. Grannan L, Rehfeldt RA. Emergent intraverbal responses via tact and match-tosample instruction. J Appl Behav Anal. 2012 Fall;45(3):601-5. doi: 10.1901/jaba.2012.45601. PMID: 23060673.X-3, X-4
1105. Green D. Limitations of MetaAnalyses...Fournier, K. A., Hass, C. J., Naik, S. $\mathrm{K}$., et al (2010). Motor coordination in autism spectrum disorders: A synthesis and metaanalysis. Journal of Autism and Developmental Disorders, 40, 1227-1240. Journal of Autism \& Developmental Disorders. 2012;42(8):1774-5 2p. doi: 10.1007/s10803-011-1405-8. PMID: 104482204. Language: English. Entry Date: 20120724. Revision Date: 20150711. Publication Type: Journal Article.X-1, X-2

1106. Greffou S, Bertone A, Hahler E-M, et al. Postural Hypo-Reactivity in Autism is Contingent on Development and Visual Environment: A Fully Immersive Virtual Reality Study. Journal of Autism \& Developmental Disorders. 2012;42(6):961-70 10p. doi: 10.1007/s10803-011-1326-6. PMID: 104451838. Language: English. Entry Date: 20120604. Revision Date: 20150711. Publication Type: Journal Article.X-1

1107. Griffith GM, Totsika V, Nash S, et al. 'I just don't fit anywhere': support experiences and future support needs of individuals with Asperger syndrome in middle adulthood. Autism. 2012 Sep;16(5):532-46. doi: 10.1177/1362361311405223. PMID: 21610188.X-1

1108. Grindle CF, Hastings RP, Saville M, et al. Outcomes of a behavioral education model for children with autism in a mainstream school setting. Behav Modif. 2012 May;36(3):298319. doi: 10.1177/0145445512441199. PMID: 22569577.X-4

1109. Grinker RR, Chambers N, Njongwe N, et al. "Communities" in community engagement: lessons learned from autism research in South Korea and South Africa. Autism Res. 2012 Jun;5(3):201-10. doi: 10.1002/aur.1229. PMID: 22566396.X-1

1110. Gross E, El-Baz AS, Sokhadze GE, et al. INDUCED EEG GAMMA OSCILLATION ALIGNMENT IMPROVES DIFFERENTIATION BETWEEN AUTISM AND ADHD GROUP RESPONSES IN A FACIAL CATEGORIZATION TASK. J Neurother. 2012 Jan 1;16(2):78-91. doi: 10.1080/10874208.2012.677631. PMID: 22754277.X-1 
1111. Grossman RB, Tager-Flusberg H. "Who said that?" Matching of low- and highintensity emotional prosody to facial expressions by adolescents with ASD. J Autism Dev Disord. 2012 Dec;42(12):2546-57. doi: 10.1007/s10803-012-1511-2. PMID: 22450703.X-1

1112. Grynszpan O, Nadel J, Martin JC, et al. Self-monitoring of gaze in high functioning autism. J Autism Dev Disord. 2012 Aug;42(8):1642-50. doi: 10.1007/s10803-0111404-9. PMID: 22102292.X-1

1113. Gucev Z, Tasic V, Polenakovic M. Meeting Report: Rare disease in south-eastern Europe, 15-17 November 2012, Skopje, Republic of Macedonia. Prilozi. 2012 Dec;33(2):279-81. PMID: 23425886.X-1

1114. Gurkan CK, Hagerman RJ. TARGETED TREATMENTS IN AUTISM AND FRAGILE X SYNDROME. Res Autism Spectr Disord. 2012 Oct 1;6(4):1311-20. doi: 10.1016/j.rasd.2012.05.007. PMID: 23162607.X-1, X-2, X-3, X-4

1115. Gutman SA, Raphael-Greenfield EI, Rao AK. Effect of a motor-based role-play intervention on the social behaviors of adolescents with high-functioning autism: multiple-baseline single-subject design. Am J Occup Ther. 2012 Sep-Oct;66(5):529-37. doi: 10.5014/ajot.2012.003756. PMID: 22917119.X-1

1116. Guzinski EM, Cihon TM, Eshleman J. The effects of tact training on stereotypic vocalizations in children with autism. Anal Verbal Behav. 2012;28(1):101-10. PMID: 22754107.X-1, X-3, X-4

1117. Hagenmuller F, Rossler W, Endrass J, et al. [Impaired resonance in offenders with psychopathic traits]. Neuropsychiatr. 2012;26(2):65-71. doi: 10.1007/s40211-0120015-9. PMID: 22836550.X-1

1118. Hahn S. Environments and autistic spectrum conditions. Nurs Times. 2012 Dec 410;108(49):23-5. PMID: 23342838.X-1

1119. Hall HR, Graff JC. Maladaptive behaviors of children with autism: parent support, stress, and coping. Issues Compr Pediatr Nurs. 2012;35(3-4):194-214. doi:
10.3109/01460862.2012.734210. PMID: 23140414.X-1

1120. Hall SE, Riccio CA. Complementary and alternative treatment use for autism spectrum disorders. Complement Ther Clin Pract. 2012 Aug;18(3):159-63. doi: 10.1016/j.ctcp.2012.03.004. PMID: 22789791.X-1

1121. Halpert J. Altering the primal environment: health effects associated with assisted reproductive technologies. Environ Health Perspect. 2012 Oct;120(10):a390-5. doi: 10.1289/ehp.120-a390. PMID: 23026346.X-1

1122. Han S, Tai C, Westenbroek RE, et al. Autistic-like behaviour in Scn1a+/- mice and rescue by enhanced GABA-mediated neurotransmission. Nature. 2012 Sep 20;489(7416):385-90. doi: 10.1038/nature11356. PMID: 22914087.X-1

1123. Hanley GP. Functional assessment of problem behavior: dispelling myths, overcoming implementation obstacles, and developing new lore. Behav Anal Pract. 2012 Summer;5(1):54-72. PMID: 23326630.X-1

1124. Harris C, Card B. A pilot study to evaluate nutritional influences on gastrointestinal symptoms and behavior patterns in children with Autism Spectrum Disorder. Complement Ther Med. 2012 Dec;20(6):43740. doi: 10.1016/j.ctim.2012.08.004. PMID: 23131376.X-1, X-3, X-4

1125. Hellwig JP. Birth Trends. Nursing for Women's Health. 2012;16(3):192-7 6p. doi: 10.1111/j.1751-486X.2012.01729.x. PMID: 104461710. Language: English. Entry Date: 20120619. Revision Date: 20150711.

Publication Type: Journal Article.X-1

1126. Herren J. Unbound April. Narrat Inq Bioeth. 2012 Winter;2(3):E3-5. doi: 10.1353/nib.2012.0064. PMID: 24406894.X-1, $\mathrm{X}-2$

1127. Higashida H, Yokoyama S, Huang JJ, et al. Social memory, amnesia, and autism: brain oxytocin secretion is regulated by NAD+ metabolites and single nucleotide polymorphisms of CD38. Neurochem Int. 2012 Nov;61(6):828-38. doi: 
10.1016/j.neuint.2012.01.030. PMID: 22366648.X-1

1128. Hill EL, Crane L, Bremner AJ. Developmental disorders and multisensory perception. Multisensory development.: Oxford University Press, New York, NY; 2012:273300.

1129. Ho JD, Nystrom PC, Calvo DV, et al. Prehospital chemical restraint of a noncommunicative autistic minor by law enforcement. Prehospital Emergency Care. 2012;16(3):407-11 5p. doi: 10.3109/10903127.2011.640767. PMID: 104453987. Language: English. Entry Date: 20120803. Revision Date: 20150820.

Publication Type: Journal Article.X-1

1130. Ho JG, Caldwell RL, McDougle CJ, et al. The effects of aripiprazole on electrocardiography in children with pervasive developmental disorders. J Child Adolesc Psychopharmacol. 2012 Aug;22(4):277-83. doi: 10.1089/cap.2011.0129. PMID: 22849533.X-1, $\mathrm{X}-3$

1131. Hoddenbach E, Koot HM, Clifford P, et al. Individual differences in the efficacy of a short theory of mind intervention for children with autism spectrum disorder: a randomized controlled trial. Trials. 2012;13:206. doi: 10.1186/1745-6215-13-206. PMID: 23140338.X-2

1132. Hoglund LT, Scott BW. Automobilization intervention and exercise for temporomandibular joint open lock. J Man Manip Ther. 2012 Nov;20(4):182-91. doi: 10.1179/2042618612y.0000000008. PMID: 24179326.X-1

1133. Hollander E, Soorya L, Chaplin W, et al. A double-blind placebo-controlled trial of fluoxetine for repetitive behaviors and global severity in adult autism spectrum disorders. Am J Psychiatry. 2012 Mar;169(3):292-9. doi: 10.1176/appi.ajp.2011.10050764. PMID: 22193531.X-1

1134. Holler K, Scalzo A. "I've heard some things that scare me". Responding with empathy to parents' fears of vaccinations. Mo Med. 2012 Jan-Feb;109(1):10-3, 6-8. PMID: 22428439.X-1
1135. Honaker D, Rosello SS, Candler C. Test-retest reliability of Family L.I.F.E. (Looking Into Family Experiences): an occupation-based assessment. Am J Occup Ther. 2012 Sep-Oct;66(5):617-20. doi: 10.5014/ajot.2012.004002. PMID: 22917128.X-1

1136. Hosenbocus S, Chahal R. A review of executive function deficits and pharmacological management in children and adolescents. J Can Acad Child Adolesc Psychiatry. 2012 Aug;21(3):223-9. PMID: 22876270.X-1

1137. Howell DM, Wittman P, Bundy MB. Interprofessional clinical education for occupational therapy and psychology students: a social skills training program for children with autism spectrum disorders. J Interprof Care. 2012 Jan;26(1):49-55. doi: 10.3109/13561820.2011.620186. PMID: 22233368.X-1

1138. Hugdahl K, Beyer MK, Brix M, et al. Autism spectrum disorder, functional MRI and MR spectroscopy: possibilities and challenges. Microb Ecol Health Dis. 2012;23doi: 10.3402/mehd.v23i0.18960. PMID: 23990828.X-1

1139. Hume K, Plavnick J, Odom S. Promoting Task Accuracy and Independence in Students with Autism Across Educational Setting Through the Use of Individual Work Systems. Journal of Autism \& Developmental Disorders. 2012;42(10):2084-99 16p. doi: 10.1007/s10803-012-1457-4. PMID: 104419563. Language: English. Entry Date: 20120925. Revision Date: 20150711. Publication Type: Journal Article.X-1, X-3

1140. Hurwitz R, Blackmore R, Hazell P, et al. Tricyclic antidepressants for autism spectrum disorders (ASD) in children and adolescents. Cochrane Database of Systematic Reviews. 2012(3):N.PAG-N.PAG 1p. PMID: 108170370. Language: English. Entry Date: 20091023. Revision Date: 20150712. Publication Type: Journal Article.X-2

1141. Hyman SE. Revolution stalled. Sci Transl Med. 2012 Oct 10;4(155):155cm11. doi: 10.1126/scitranslmed.3003142. PMID: 23052291.X-1 
1142. Imperatore Blanche E, Reinoso G, Chang MC, et al. Proprioceptive Processing Difficulties Among Children With Autism Spectrum Disorders and Developmental Disabilities. American Journal of Occupational Therapy. 2012;66(5):621-4 4p. doi: 10.5014/ajot.2012.004234. PMID: 104439435. Language: English. Entry Date: 20121121. Revision Date: 20150819. Publication Type: Journal Article.X-1

1143. Indah Winarni T, Chonchaiya W, Adams E, et al. Sertraline may improve language developmental trajectory in young children with fragile $\mathrm{x}$ syndrome: a retrospective chart review. Autism Res Treat. 2012;2012:104317. doi: 10.1155/2012/104317. PMID: 22934167.X-1

1144. Ingersoll B. Brief report: effect of a focused imitation intervention on social functioning in children with autism. J Autism Dev Disord. 2012 Aug;42(8):1768-73. doi: 10.1007/s10803-011-1423-6. PMID: 22146934.X-1

1145. Ingersoll B, Meyer K, Bonter N, et al. A comparison of developmental socialpragmatic and naturalistic behavioral interventions on language use and social engagement in children with autism. J Speech Lang Hear Res. 2012 Oct;55(5):1301-13. doi: 10.1044/1092-4388(2012/10-0345). PMID: 22361104.X-3, X-4

1146. Irby MB, Kolbash S, Garner-Edwards $\mathrm{D}$, et al. Pediatric Obesity Treatment in Children With Neurodevelopmental Disabilities: A Case Series and Review of the Literature. Infant Child Adolesc Nutr. 2012

Aug 1;4(4):215-21. doi: 10.1177/1941406412448527. PMID: 24723990.X-1

1147. Ishitobi M, Hiratani M, Kosaka H, et al. Switching to aripiprazole in subjects with pervasive developmental disorders showing tolerability issues with risperidone. Prog Neuropsychopharmacol Biol Psychiatry. 2012 Apr 27;37(1):128-31. doi: 10.1016/j.pnpbp.2011.12.015. PMID: 22245026.X-1, X-3

1148. Izawa J, Pekny SE, Marko MK, et al. Motor learning relies on integrated sensory inputs in ADHD, but over-selectively on proprioception in autism spectrum conditions. Autism Res. 2012 Apr;5(2):124-36. doi: 10.1002/aur.1222. PMID: 22359275.X-1

1149. Janssen R, Maes B. Psychometric evaluation of a Dutch version of the Mini PASADD for assessing psychiatric disorders in adults with different levels of intellectual disability. J Intellect Disabil Res. 2012 Mar 28doi: 10.1111/j.1365-2788.2012.01544.x. PMID: 22463729.X-1

1150. Jordan I, Robertson D, Catani M, et al. Aripiprazole in the treatment of challenging behaviour in adults with autism spectrum disorder. Psychopharmacology (Berl). 2012 Oct;223(3):357-60. doi: 10.1007/s00213-0122723-z. PMID: 22535309.X-1

1151. Joshi G, Biederman J, Wozniak J, et al. Response to second generation antipsychotics in youth with comorbid bipolar disorder and autism spectrum disorder. CNS Neurosci Ther. 2012 Jan;18(1):28-33. doi: 10.1111/j.17555949.2010.00219.x. PMID: 21114638.X-1, X-3

1152. Juneja M, Mukherjee SB, Sharma S, et al. Evaluation of a parent-based behavioral intervention program for children with autism in a low-resource setting. J Pediatr Neurosci. 2012 Jan;7(1):16-8. doi: 10.4103/18171745.97612. PMID: 22837770.X-3, X-4

1153. Jurgens A, Anderson A, Moore DW. Parent-implemented picture exchange communication system (PECS) training: an analysis of YouTube videos. Dev Neurorehabil. 2012;15(5):351-60. doi: 10.3109/17518423.2012.692125. PMID: 22712574.X-1, X-3

1154. Kaale A, Smith L, Sponheim E. A randomized controlled trial of preschool-based joint attention intervention for children with autism. J Child Psychol Psychiatry. 2012 Jan;53(1):97-105. doi: 10.1111/j.14697610.2011.02450.x. PMID: 21883204.X-1

1155. Kadar M, McDonald R, Lentin P. Evidence-based practice in occupational therapy services for children with autism spectrum disorders in Victoria, Australia. Aust Occup Ther J. 2012 Aug;59(4):284-93. doi: 10.1111/j.1440-1630.2012.01015.x. PMID: 22934901.X-1 
1156. Kalas A. Joint attention responses of children with autism spectrum disorder to simple versus complex music. J Music Ther. 2012 Winter;49(4):430-52. PMID: 23705346.X-3

1157. Kalb LG, Stuart EA, Freedman B, et al. Psychiatric-related emergency department visits among children with an autism spectrum disorder. Pediatr Emerg Care. 2012

Dec;28(12):1269-76. doi: 10.1097/PEC.0b013e3182767d96. PMID: 23187983.X-1

1158. Kalnak N, Peyrard-Janvid M, Sahlen B, et al. Family history interview of a broad phenotype in specific language impairment and matched controls. Genes Brain Behav. 2012 Nov;11(8):921-7. doi: 10.1111/j.1601183X.2012.00841.x. PMID: 22928858.X-1

1159. Kaluzna-Czaplinska J, Blaszczyk S. The level of arabinitol in autistic children after probiotic therapy. Nutrition. 2012

Feb;28(2):124-6. doi:

10.1016/j.nut.2011.08.002. PMID: 22079796.X-3

1160. Kamp-Becker I, Baumann K, Sprenger $\mathrm{L}$, et al. [The concept of $<<$ multiple complex developmental disorder $>>$ - a disorder of social interaction, paranoid thinking and social anxiety in a 17-year-old boy]. Z Kinder Jugendpsychiatr Psychother. 2012 Sep;40(5):341-9. doi: 10.1024/14224917/a000191. PMID: 22869228.X-1

1161. Kanda Y, Kumagami H, Hara M, et al. What factors are associated with good performance in children with cochlear implants? From the outcome of various language development tests, research on sensory and communicative disorders project in Japan: nagasaki experience. Clin Exp Otorhinolaryngol. 2012 Apr;5 Suppl 1:S59-64. doi: 10.3342/ceo.2012.5.S1.S59. PMID: 22701149.X-1

1162. Kaplan G, McCracken JT. Psychopharmacology of autism spectrum disorders. Pediatric Clinics of North America. 2012;59(1):175-87 13p. PMID: 108153900. Language: English. Entry Date: 20120413. Revision Date: 20150712. Publication Type: Journal Article.X-1, X-2
1163. Kasari C, Gulsrud A, Freeman S, et al. Longitudinal follow-up of children with autism receiving targeted interventions on joint attention and play. J Am Acad Child Adolesc Psychiatry. 2012 May;51(5):487-95. doi: 10.1016/j.jaac.2012.02.019. PMID: 22525955.X-1, X-3

1164. Kasari C, Rotheram-Fuller E, Locke J, et al. Making the connection: randomized controlled trial of social skills at school for children with autism spectrum disorders. J Child Psychol Psychiatry. 2012 Apr;53(4):4319. doi: 10.1111/j.1469-7610.2011.02493.x. PMID: 22118062.X-1

1165. Katsnelson A. The autism pill. Sci Am. 2012 Nov;307(5):16. PMID: 23120883.X-1, X2

1166. Khanna R, Jariwala K. Awareness and knowledge of autism among pharmacists. Res Social Adm Pharm. 2012 Sep-Oct;8(5):464-71. doi: 10.1016/j.sapharm.2011.11.002. PMID: 22222344.X-1

1167. Khanna R, Madhavan SS, Smith MJ, et al. Psychometric properties of the Caregiver Strain Questionnaire (CGSQ) among caregivers of children with autism. Autism. 2012

Mar;16(2):179-99. doi:

10.1177/1362361311406143. PMID: 21715548.X-1

1168. Kheir NM, Ghoneim OM, Sandridge AL, et al. Concerns and considerations among caregivers of a child with autism in Qatar. BMC Res Notes. 2012;5:290. doi: 10.1186/17560500-5-290. PMID: 22695064.X-1

1169. Kinnealey M, Pfeiffer B, Miller J, et al. Effect of classroom modification on attention and engagement of students with autism or dyspraxia. Am J Occup Ther. 2012 Sep-Oct;66(5):511-9. doi: 10.5014/ajot.2012.004010. PMID: 22917117.X-1

1170. Klintwall L, Gillberg C, Bolte S, et al. The efficacy of intensive behavioral intervention for children with autism: a matter of allegiance? J Autism Dev Disord. 2012 Jan;42(1):139-40. doi: 10.1007/s10803-0111223-z. PMID: 21424234.X-1, X-3 
1171. Knoth IS, Lippe S. Event-related potential alterations in fragile $\mathrm{X}$ syndrome. Front Hum Neurosci. 2012;6:264. doi: 10.3389/fnhum.2012.00264. PMID: 23015788.X-1

1172. Kobayashi T, Inagaki M, Kaga M. Professional Caregiver's View on Mental Health in Parents of Children with Developmental Disabilities: A Nationwide Study of Institutions and Consultation Centers in Japan. ISRN Pediatr. 2012;2012:121898. doi: 10.5402/2012/121898. PMID: 22389791.X-1

1173. Kodak T, Fuchtman R, Paden A. A comparison of intraverbal training procedures for children with autism. J Appl Behav Anal. 2012 Spring;45(1):155-60. doi:

10.1901/jaba.2012.45-155. PMID: 22403459.X-1, X-3, X-4

1174. Kodak T, Paden A, Dickes N. Training and Generalization of Peer-Directed Mands With Non-vocal Children With Autism. Anal Verbal Behav. 2012;28(1):119-24. PMID: 22754109.X-1, X-3, X-4

1175. Koegel LK, Kuriakose S, Singh AK, et al. Improving generalization of peer socialization gains in inclusive school settings using initiations training. Behav Modif. 2012

May;36(3):361-77. doi:

10.1177/0145445512445609. PMID:

22645399.X-3, X-4

1176. Koegel LK, Vernon T, Koegel RL, et al. Improving Social Engagement and Initiations between Children with Autism Spectrum Disorder and Their Peers in Inclusive Settings. J Posit Behav Interv. 2012

Oct;14(4):220-7. doi:

10.1177/1098300712437042. PMID: 25328380.X-1, X-4

1177. Koegel R, Fredeen R, Kim S, et al. Using Perseverative Interests to Improve Interactions Between Adolescents with Autism and their Typical Peers in School Settings. J Posit Behav Interv. 2012 Jul 1;14(3):133-41. doi: 10.1177/1098300712437043. PMID: 24163577.X-1

1178. Koegel RL, Bharoocha AA, Ribnick $\mathrm{CB}$, et al. Using individualized reinforcers and hierarchical exposure to increase food flexibility in children with autism spectrum disorders. J Autism Dev Disord. 2012

Aug;42(8):1574-81. doi: 10.1007/s10803-0111392-9. PMID: 22042309.X-1, X-3, X-4

1179. Koenig KP, Buckley-Reen A, Garg S. Efficacy of the Get Ready to Learn yoga program among children with autism spectrum disorders: a pretest-posttest control group design. Am J Occup Ther. 2012 SepOct;66(5):538-46. doi: 10.5014/ajot.2012.004390. PMID: 22917120.X-1, X-3

1180. Kohannim O, Hibar DP, Stein JL, et al. Discovery and Replication of Gene Influences on Brain Structure Using LASSO Regression. Front Neurosci. 2012;6:115. doi: 10.3389/fnins.2012.00115. PMID: 22888310.X-1

1181. Kohls G, Chevallier C, Troiani V, et al. Social 'wanting' dysfunction in autism: neurobiological underpinnings and treatment implications. J Neurodev Disord. 2012;4(1):10. doi: 10.1186/1866-1955-4-10. PMID: 22958468.X-1

1182. Kotagal S. Treatment of dyssomnias and parasomnias in childhood. Curr Treat Options Neurol. 2012 Dec;14(6):630-49. doi: 10.1007/s11940-012-0199-0. PMID: 23011807.X-1

1183. Kramer JM, Coster WJ, Kao YC, et al. A new approach to the measurement of adaptive behavior: development of the PEDICAT for children and youth with autism spectrum disorders. Phys Occup Ther Pediatr. 2012 Feb;32(1):34-47. doi:

10.3109/01942638.2011.606260. PMID: 21846290.X-1

1184. Krieger B, Kinebanian A, Prodinger B, et al. Becoming a member of the work force: perceptions of adults with Asperger Syndrome. Work. 2012;43(2):141-57. doi: 10.3233/wor2012-1392. PMID: 22927626.X-1

1185. Lajonchere C, Jones N, Coury DL, et al. Leadership in health care, research, and quality improvement for children and adolescents with autism spectrum disorders: Autism Treatment Network and Autism Intervention Research Network on Physical Health. Pediatrics. 2012 Nov;130 Suppl 2:S62- 
8. doi: 10.1542/peds.2012-0900C. PMID: 23118255.X-1

1186. Landa RJ, Kalb LG. Long-term outcomes of toddlers with autism spectrum disorders exposed to short-term intervention. Pediatrics. 2012 Nov;130 Suppl 2:S186-90. doi: 10.1542/peds.2012-0900Q. PMID: 23118250.X-1, X-3

1187. Lane A, Harpster K, Heathcock J. Motor characteristics of young children referred for possible autism spectrum disorder. Pediatric Physical Therapy. 20122012 Spring;24(1):21-9 9p. PMID: 108222318. Language: English. Entry Date: 20120406. Revision Date: 20150820. Publication Type: Journal Article.X1

1188. Lane SJ, Reynolds S, Dumenci L. Sensory overresponsivity and anxiety in typically developing children and children with autism and attention deficit hyperactivity disorder: cause or coexistence? Am J Occup Ther. 2012 Sep-Oct;66(5):595-603. doi: 10.5014/ajot.2012.004523. PMID: 22917126.X-1

1189. Lang R, O’Reilly M, Healy O, et al. Sensory integration therapy for autism spectrum disorders: A systematic review. Research in Autism Spectrum Disorders. 2012 2015-0224;6(3):1004-18. doi: http://dx.doi.org/10.1016/j.rasd.2012.01.006. PMID: 1022253260; 2012-13564-007.X-1, X-2

1190. Laugeson EA, Frankel F, Gantman A, et al. Evidence-based social skills training for adolescents with autism spectrum disorders: the UCLA PEERS program. J Autism Dev Disord. 2012 Jun;42(6):1025-36. doi: 10.1007/s10803011-1339-1. PMID: 21858588.X-1

1191. Lawton K, Kasari C. Teacherimplemented joint attention intervention: pilot randomized controlled study for preschoolers with autism. J Consult Clin Psychol. 2012 Aug;80(4):687-93. doi: 10.1037/a0028506. PMID: 22582764.X-1

1192. Leaf JB, Oppenheim-Leaf ML, Call NA, et al. Comparing the teaching interaction procedure to social stories for people with autism. J Appl Behav Anal. 2012 Summer;45(2):281-98. doi: 10.1901/jaba.2012.45-281. PMID: 22844137.X-3, X-4

1193. Lemonda BC, Holtzer R, Goldman S. Relationship between executive functions and motor stereotypies in children with Autistic Disorder. Res Autism Spectr Disord. 2012 Jul 1;6(3):1099-106. doi: 10.1016/j.rasd.2012.03.001. PMID: 23637708.X-1

1194. Lerna A, Esposito D, Conson M, et al. Social-communicative effects of the Picture Exchange Communication System (PECS) in autism spectrum disorders. Int J Lang Commun Disord. 2012 Sep-Oct;47(5):609-17. doi: 10.1111/j.1460-6984.2012.00172.x. PMID: 22938071.X-4

1195. Leupen A. Proteus for autistic children with temper tantrums. Homoeopathic Links. 2012;25(2):120- 1p. PMID: 104482643. Language: English. Entry Date: 20120907. Revision Date: 20150711. Publication Type: Journal Article.X-1, X-2

1196. Levine TP, Sheinkopf SJ, Pescosolido M, et al. Physiologic Arousal to Social Stress in Children with Autism Spectrum Disorders: A Pilot Study. Res Autism Spectr Disord. 2012 Winter;6(1):177-83. doi: 10.1016/j.rasd.2011.04.003. PMID: 22081773.X-1

1197. Levy-Osborne S. Thinking about the past hoping for the future. Narrat Inq Bioeth. 2012 Winter;2(3):E5-7. doi: 10.1353/nib.2012.0067. PMID: 24406895.X-1

1198. Li K-H, Lou S-J, Tsai H-Y, et al. The effects of applying game-based learning to Webcam motion sensor games for autistic students' sensory integration training. TOJET: The Turkish Online Journal of Educational Technology. 2012 2015-02-24;11(4):451-9. doi: 10.6223/qcpe.2601.201203.2005. PMID: 1477173807; 2013-28852-044.X-3, X-4

1199. Lian WB, Ho SK, Choo SH, et al. Children with developmental and behavioural concerns in Singapore. Singapore Med J. 2012 Jul;53(7):439-45. PMID: 22815011.X-1

1200. Lickel A, MacLean WE, Jr., BlakeleySmith A, et al. Assessment of the prerequisite skills for cognitive behavioral therapy in 
children with and without autism spectrum disorders. J Autism Dev Disord. 2012 Jun;42(6):992-1000. doi: 10.1007/s10803-0111330-x. PMID: 21818677.X-1, X-3

1201. Lin A, Rangel A, Adolphs R. Impaired learning of social compared to monetary rewards in autism. Front Neurosci. 2012;6:143. doi: 10.3389/fnins.2012.00143. PMID: 23060743.X-1

1202. Lin A, Tsai K, Rangel A, et al. Reduced social preferences in autism: evidence from charitable donations. J Neurodev Disord. 2012;4(1):8. doi: 10.1186/1866-1955-4-8. PMID: 22958506.X-1

1203. Lin LY, Yu SN, Yu YT. A study of activities of daily living and employment in adults with autism spectrum disorders in Taiwan. Int J Rehabil Res. 2012 Jun;35(2):10915. doi: 10.1097/MRR.0b013e32835108b1. PMID: 22334003.X-1

1204. Lindberg S, von Post I, Eriksson K. The experiences of parents of children with severe autism in connection with their children's anaesthetics, in the presence and absence of the perioperative dialogue: a hermeneutic study. Scandinavian Journal of Caring Sciences. 2012;26(4):627-34 8p. doi: 10.1111/j.14716712.2012.00971.x. PMID: 104434433. Language: English. Entry Date: 20121109. Revision Date: 20150819. Publication Type: Journal Article.X-1

1205. Ling CY, Mak WW. Coping with challenging behaviours of children with autism: effectiveness of brief training workshop for frontline staff in special education settings. J Intellect Disabil Res. 2012 Mar;56(3):258-69. doi: 10.1111/j.1365-2788.2011.01469.x. PMID: 21955252.X-1

1206. Lit L, Sharp FR, Bertoglio K, et al. Gene expression in blood is associated with risperidone response in children with autism spectrum disorders. Pharmacogenomics J. 2012 Oct;12(5):368-71. doi: 10.1038/tpj.2011.23. PMID: 21647175.X-3

1207. Litresh W, Lei G, Shirley A, et al. Gross motor skills of children with autism. American Journal of Recreation Therapy. 2012;11(3):39-47 9p. PMID: 108082482. Language: English. Entry Date: 20130322.
Revision Date: 20150818. Publication Type: Journal Article.X-1

1208. Lo SY. Diagnosis, treatment and prevention of autism via meridian theory. Am J Chin Med. 2012;40(1):39-56. doi: 10.1142/s0192415x12500048. PMID: 22298447.X-1, X-3

1209. Lofthouse N, Hendren R, Hurt E, et al. A review of complementary and alternative treatments for autism spectrum disorders. Autism Res Treat. 2012;2012:870391. doi: 10.1155/2012/870391. PMID: 23243505.X-1, $\mathrm{X}-2$

1210. Logan SL, Nicholas JS, Carpenter LA, et al. High prescription drug use and associated costs among Medicaid-eligible children with autism spectrum disorders identified by a population-based surveillance network. Ann Epidemiol. 2012 Jan;22(1):1-8. doi: 10.1016/j.annepidem.2011.10.007. PMID: 22153288.X-1

1211. Lokhandwala T, Khanna R, WestStrum D. Hospitalization burden among individuals with autism. J Autism Dev Disord. 2012 Jan;42(1):95-104. doi: 10.1007/s10803011-1217-x. PMID: 21404084.X-1

1212. Lombardo MV, Chakrabarti B, Lai MC, et al. Self-referential and social cognition in a case of autism and agenesis of the corpus callosum. Mol Autism. 2012;3(1):14. doi: 10.1186/2040-2392-3-14. PMID: 23171505.X1

1213. Lopatina O, Inzhutova A, Salmina AB, et al. The roles of oxytocin and CD38 in social or parental behaviors. Front Neurosci. 2012;6:182. doi: 10.3389/fnins.2012.00182. PMID: 23335873.X-1

1214. Lopez M, Bellando J. An informal survey on family/caregiver wishes for individuals with autism in Arkansas. J Ark Med Soc. 2012 Dec;109(7):137-9. PMID: 23304848.X-1

1215. Lopez M, Schulz EG, Baroud T, et al. The Arkansas Autism Developmental Disabilities Monitoring (AR ADDM) project: statewide autism surveillance in a rural state. $\mathrm{J}$ Ark Med Soc. 2012 Mar;108(10):220, 2-4. PMID: 22479981.X-1 
1216. Lorange M, Kristmundsdottir K, Skarphedinsson G, et al. [Relationship between pre-adoptive risk factors and psychopathological difficulties of internationally adopted children in Iceland]. Laeknabladid. 2012 Jan;98(1):19-23. PMID: 22253083.X-1

1217. Lough CL, Rice MS, Lough LG. Choice as a strategy to enhance engagement in a colouring task in children with autism spectrum disorders. Occup Ther Int. 2012 Dec;19(4):204-11. doi: 10.1002/oti.1337. PMID: 23019122.X-1, X-3

1218. Love JJ, Miguel CF, Fernand JK, et al. The effects of matched stimulation and response interruption and redirection on vocal stereotypy. J Appl Behav Anal. 2012 Fall;45(3):549-64. doi: 10.1901/jaba.2012.45549. PMID: 23060668.X-3, X-4

1219. Lovell B, Moss M, Wetherell M. The psychosocial, endocrine and immune consequences of caring for a child with autism or ADHD. Psychoneuroendocrinology. 2012 Apr;37(4):534-42. doi: 10.1016/j.psyneuen.2011.08.003. PMID: 21889267.X-1

1220. Lundervold AJ, Stickert M, Hysing M, et al. Attention Deficits in Children With Combined Autism and ADHD: A CPT Study. J Atten Disord. 2012 Aug 31doi: 10.1177/1087054712453168. PMID: 22940852.X-1

1221. Lutz HR, Patterson BJ, Klein J. Coping with autism: a journey toward adaptation. J Pediatr Nurs. 2012 Jun;27(3):20613. doi: 10.1016/j.pedn.2011.03.013. PMID: 22525808.X-1

1222. Lyall K, Pauls DL, Spiegelman D, et al. Fertility therapies, infertility and autism spectrum disorders in the Nurses' Health Study II. Paediatr Perinat Epidemiol. 2012 Jul;26(4):361-72. doi: 10.1111/j.13653016.2012.01294.x. PMID: 22686388.X-1

1223. Magana S, Parish SL, Rose RA, et al. Racial and ethnic disparities in quality of health care among children with autism and other developmental disabilities. Intellect Dev Disabil. 2012 Aug;50(4):287-99. doi:
10.1352/1934-9556-50.4.287. PMID: 22861130.X-1

1224. Mahajan R, Pilar Bernal M, Panzer R, et al. Clinical Practice Pathways for Evaluation and Medication Choice for AttentionDeficit/Hyperactivity Disorder Symptoms in Autism Spectrum Disorders. Pediatrics. 2012;130:S125-38 1p. doi: 10.1542/peds.20120900J. PMID: 108107696. Language: English. Entry Date: 20121106. Revision Date: 20150712. Publication Type: Journal Article.X2

1225. Main PA, Angley MT, O'Doherty CE, et al. The potential role of the antioxidant and detoxification properties of glutathione in autism spectrum disorders: a systematic review and meta-analysis. Nutr Metab (Lond). 2012;9:35. doi: 10.1186/1743-7075-9-35. PMID: 22524510.X-2

1226. Mak-Fan K, Taylor M, Roberts W, et al. Measures of Cortical Grey Matter Structure and Development in Children with Autism Spectrum Disorder. Journal of Autism \& Developmental Disorders. 2012;42(3):419-27 9p. doi: 10.1007/s10803-011-1261-6. PMID: 104516628. Language: English. Entry Date: 20120509. Revision Date: 20150711. Publication Type: Journal Article.X-1

1227. Malow B, Adkins KW, McGrew SG, et al. Melatonin for sleep in children with autism: a controlled trial examining dose, tolerability, and outcomes. J Autism Dev Disord. 2012 Aug;42(8):1729-37; author reply 38. doi: 10.1007/s10803-011-1418-3. PMID: 22160300.X-3

1228. Mandell DS. Understanding and addressing the impact of autism on the family. LDI Issue Brief. 2012 Apr-May;17(7):1-4. PMID: 22666896.X-1

1229. Mandell DS, Xie M, Morales KH, et al. The interplay of outpatient services and psychiatric hospitalization among Medicaidenrolled children with autism spectrum disorders. Arch Pediatr Adolesc Med. 2012 Jan;166(1):68-73. doi: 10.1001/archpediatrics.2011.714. PMID: 22213753.X-1

1230. Mangum A, Roane H, Fredrick L, et al. The Role of Context in the Evaluation of 
Reinforcer Efficacy: Implications for the Preference Assessment Outcomes. Res Autism Spectr Disord. 2012 Jan;6(1):158-67. doi: 10.1016/j.rasd.2011.04.001. PMID: 22125577.X-1

1231. Manohara D. Primary care management of tuberous sclerosis complex in children. Journal of the American Academy of Nurse Practitioners. 2012;24(7):391-9 9p. doi: 10.1111/j.1745-7599.2012.00734.x. PMID: 104467949. Language: English. Entry Date: 20120716. Revision Date: 20150819. Publication Type: Journal Article.X-1

1232. Marchese NV, Carr JE, LeBlanc LA, et al. The effects of the question "What is this?" on tact-training outcomes of children with autism. J Appl Behav Anal. 2012 Fall;45(3):539-47. doi: 10.1901/jaba.2012.45539. PMID: 23060667.X-1, X-3, X-4

1233. Marion C, Martin GL, Yu CT, et al. Teaching children with autism spectrum disorder to mand for information using "which?". J Appl Behav Anal. 2012 Winter;45(4):865-70. doi: 10.1901/jaba.2012.45-865. PMID: 23322944.X-3, X-4

1234. Markoulakis R, Scharoun S, Bryden P, et al. An Examination of Handedness and Footedness in Children with High Functioning Autism and Asperger Syndrome. Journal of Autism \& Developmental Disorders. 2012;42(10):2192-201 10p. doi: 10.1007/s10803-012-1469-0. PMID: 104419554. Language: English. Entry Date: 20120925. Revision Date: 20150711. Publication Type: Journal Article.X-1

1235. Martin-Du Pan RC. [Oxytocin: the hormone of love, trust and social bond. Clinical use in autism and social phobia]. Rev Med Suisse. 2012 Mar 21;8(333):627-30. PMID: 22506444.X-1, X-2

1236. Mashal N, Kasirer A. Principal component analysis study of visual and verbal metaphoric comprehension in children with autism and learning disabilities. Res Dev Disabil. 2012 Jan-Feb;33(1):274-82. doi: 10.1016/j.ridd.2011.09.010. PMID: 22001558.X-1
1237. Mason RA, Rispoli M, Ganz JB, et al. Effects of video modeling on communicative social skills of college students with Asperger syndrome. Dev Neurorehabil. 2012;15(6):42534. doi: 10.3109/17518423.2012.704530. PMID: 23030681.X-1

1238. Masterson M. Autism Spectrum Disorder Update. Kansas Nurse. 2012;87(3):146 3p. PMID: 108117510. Language: English. Entry Date: 20120615. Revision Date: 20151015. Publication Type: Journal Article.X$1, \mathrm{X}-2$

1239. Matone M, Localio R, Huang YS, et al. The relationship between mental health diagnosis and treatment with second-generation antipsychotics over time: a national study of U.S. Medicaid-enrolled children. Health Serv Res. 2012 Oct;47(5):1836-60. doi: 10.1111/j.1475-6773.2012.01461.x. PMID: 22946905.X-1

1240. Matsuzaki J, Kagitani-Shimono K, Goto T, et al. Differential responses of primary auditory cortex in autistic spectrum disorder with auditory hypersensitivity. Neuroreport. 2012 Jan 25;23(2):113-8. doi: 10.1097/WNR.0b013e32834ebf44. PMID: 22146579.X-1

1241. Mauron A. [Autism, stem cells, and magical powder]. Rev Med Suisse. 2012 Sep 19;8(354):1795. PMID: 23097920.X-1, X-2

1242. May T, Cornish K, Rinehart NJ. Gender Profiles of Behavioral Attention in Children With Autism Spectrum Disorder. J Atten Disord. 2012 Aug 21doi: 10.1177/1087054712455502. PMID: 22912506.X-1

1243. Mayor S. Advertising watchdog orders website to remove claims linking MMR vaccine with autism. Bmj. 2012;345:e5420. doi: 10.1136/bmj.e5420. PMID: 22879643.X-1

1244. Mazefsky CA, Pelphrey KA, Dahl RE. The Need for a Broader Approach to Emotion Regulation Research in Autism. Child Dev Perspect. 2012 Mar 1;6(1):92-7. doi: 10.1111/j.1750-8606.2011.00229.x. PMID: 22639681.X-1

1245. McBride SM, Holloway SL, Jongens TA. Using as a tool to identify Pharmacological 
Therapies for Fragile X Syndrome. Drug

Discov Today Technol. 2012 Sep

24;10(1):e129-e36. doi:

10.1016/j.ddtec.2012.09.005. PMID:

23730322.X-1

1246. McBride SM, Holloway SL, Jongens

TA. Using Drosophila as a tool to identify

Pharmacological Therapies for Fragile X

Syndrome. Drug Discov Today Technol. 2012

Sep 24;10(1):e129-e36. doi:

10.1016/j.ddtec.2012.09.005. PMID:

23730322.X-1

1247. McClean B, Grey I. An evaluation of an intervention sequence outline in positive behaviour support for people with autism and severe escape-motivated challenging behaviour. J Intellect Dev Disabil. 2012 Sep;37(3):209-20. doi: 10.3109/13668250.2012.704982. PMID: 22873574.X-3, X-4

1248. McDonald D. Much more to come! Narrat Inq Bioeth. 2012 Winter;2(3):167-70. doi: 10.1353/nib.2012.0065. PMID: 24406881.X-1

1249. McDuffie AS, Lieberman RG, Yoder PJ. Object interest in autism spectrum disorder: a treatment comparison. Autism. 2012 Jul;16(4):398-405. doi:

10.1177/1362361309360983. PMID: 22133872.X-1

1250. McFadden KL, Hepburn S, Winterrowd E, et al. Abnormalities in gammaband responses to language stimuli in firstdegree relatives of children with autism spectrum disorder: an MEG study. BMC Psychiatry. 2012;12:213. doi: 10.1186/1471244x-12-213. PMID: 23194079.X-1

1251. McGeown HR, Johnstone EC, McKirdy J, et al. Determinants of adult functional outcome in adolescents receiving special educational assistance. J Intellect Disabil Res. 2012 Feb 28doi: 10.1111/j.13652788.2011.01533.x. PMID: 22369675.X-1

1252. McGuinty E, Armstrong D, Nelson J, et al. Externalizing metaphors: anxiety and high-functioning autism. J Child Adolesc Psychiatr Nurs. 2012 Feb;25(1):9-16. doi: 10.1111/j.1744-6171.2011.00305.x. PMID: 22299802.X-1
1253. McPartland JC, Crowley MJ, Perszyk DR, et al. Preserved reward outcome processing in ASD as revealed by event-related potentials. J Neurodev Disord. 2012;4(1):16. doi: 10.1186/1866-1955-4-16. PMID: 22958616.X1

1254. Mechling L, Ayres K. A Comparative Study: Completion of Fine Motor Office Related Tasks by High School Students with Autism Using Video Models on Large and Small Screen Sizes. Journal of Autism \& Developmental Disorders. 2012;42(11):2364-73 10p. doi: 10.1007/s10803-012-1484-1. PMID: 104427304. Language: English. Entry Date: 20121019. Revision Date: 20150711. Publication Type: Journal Article.X-1

1255. Megargel E, Broder-Fingert S. Autism and hospitals: a difficult match. Acad Pediatr. 2012 Nov-Dec;12(6):469-70. doi: 10.1016/j.acap.2012.10.002. PMID: 23159035.X-1

1256. Meier AE, Fryling MJ, Wallace MD. Using high-probability foods to increase the acceptance of low-probability foods. J Appl Behav Anal. 2012 Spring;45(1):149-53. doi: 10.1901/jaba.2012.45-149. PMID: 22403458.X-1, X-3

1257. Memari AH, Ziaee V, Beygi S, et al. Overuse of psychotropic medications among children and adolescents with autism spectrum disorders: perspective from a developing country. Res Dev Disabil. 2012 MarApr;33(2):563-9. doi: 10.1016/j.ridd.2011.10.001. PMID: 22119705.X-1

1258. Midorikawa A, Kawamura M. The Relationship between Subclinical Asperger's Syndrome and Frontotemporal Lobar Degeneration. Dement Geriatr Cogn Dis Extra. 2012 Jan;2:180-6. doi: 10.1159/000338174. PMID: 22719743.X-1

1259. Midtvedt T. The gut: a triggering place for autism - possibilities and challenges. Microb Ecol Health Dis. 2012;23doi: 10.3402/mehd.v23i0.18982. PMID: 23990818.X-1

1260. Mieres AC, Kirby RS, Armstrong KH, et al. Autism spectrum disorder: an emerging opportunity for physical therapy. Pediatric 
Physical Therapy. 20122012 Spring;24(1):31-7 7p. PMID: 108222320. Language: English. Entry Date: 20120406. Revision Date: 20150820. Publication Type: Journal Article. Journal Subset: Allied Health.X-1

1261. Miliotis A, Sidener TM, Reeve KF, et al. An evaluation of the number of presentations of target sounds during stimulus-stimulus pairing trials. J Appl Behav Anal. 2012 Winter;45(4):809-13. doi: 10.9101/jaba.2012.45-809. PMID: 23322934.X-1

1262. Miller E. Focusing on prevention. Imprint. 2012 Nov-Dec;59(5):12. PMID: 23409627.X-1

1263. Ming X, Chen X, Wang XT, et al. Acupuncture for treatment of autism spectrum disorders. Evid Based Complement Alternat Med. 2012;2012:679845. doi: 10.1155/2012/679845. PMID: 22203876.X-1

1264. Minne EP, Semrud-Clikeman M. A social competence intervention for young children with high functioning autism and Asperger syndrome: a pilot study. Autism. 2012 Nov;16(6):586-602. doi: 10.1177/1362361311423384. PMID: 22087045.X-3, X-4

1265. Miyaoka T, Wake R, Furuya M, et al. Yokukansan (TJ-54) for treatment of pervasive developmental disorder not otherwise specified and Asperger's disorder: a 12-week prospective, open-label study. BMC Psychiatry. 2012;12:215. doi: 10.1186/1471-244x-12-215. PMID: 23194148.X-1, X-3

1266. Mohammadi MR, Zarafshan H, Ghasempour S. Broader Autism Phenotype in Iranian Parents of Children with Autism Spectrum Disorders vs. Normal Children. Iran J Psychiatry. 2012 Fall;7(4):157-63. PMID: 23408558.X-1

1267. Mohinderpal Chadha G, Kakodkar P, Chaugule V, et al. Dental survey of institutionalized children with autistic disorder. Int J Clin Pediatr Dent. 2012 Jan;5(1):29-32. doi: 10.5005/jp-journals-10005-1130. PMID: 25206131.X-1

1268. Moilanen I, Mattila ML, Loukusa S, et al. [Autism spectrum disorders in children and adolescents]. Duodecim. 2012;128(14):145362. PMID: 22937604.X-1

1269. Momeni N, Brudin L, Behnia F, et al. High complement factor I activity in the plasma of children with autism spectrum disorders. Autism Res Treat. 2012;2012:868576. doi: 10.1155/2012/868576. PMID: 22928102.X-1

1270. Monahan M, Classen S, Helsel P. Predriving skills of a teen with attention deficit hyperactivity and autism spectrum disorder. Developmental Disabilities Special Interest Section Quarterly. 2012;35(4):1-4 p. PMID: 108086065. Language: English. Entry Date: 20130104. Revision Date: 20150820. Publication Type: Journal Article.X-1

1271. Motoyama K, Matsuzaka T, Nagaoka $\mathrm{T}$, et al. [The effect of parent training program on children with attention deficit/hyperactivity disorders and/or pervasive developmental disorders]. No To Hattatsu. 2012 Jul;44(4):28994. PMID: 22844759.X-3, X-4

1272. Mulligan S, White BP. Sensory and motor behaviors of infant siblings of children with and without autism. American Journal of Occupational Therapy. 2012 2015-0224;66(5):556-66. doi: http://dx.doi.org/10.5014/ajot.2012.004077. PMID: 1428014768; 2012-25996-006.X-1

1273. Murphy D, Spooren W. EU-AIMS: a boost to autism research. Nat Rev Drug Discov. 2012 Nov;11(11):815-6. doi: 10.1038/nrd3881. PMID: 23123927.X-1

1274. Nau JY. [To dare trying a diuretic in autism treatment]. Rev Med Suisse. 2012 Dec 19;8(367):2458-9. PMID: 23346751.X-1

1275. Nau JY. [Autism: who has a say in treatment?]. Rev Med Suisse. 2012 Mar 21;8(333):658-9. PMID: 22506452.X-1, X-2

1276. Nayate A, Tonge B, Bradshaw J, et al. Differentiation of High-Functioning Autism and Asperger's Disorder Based on Neuromotor Behaviour. Journal of Autism \& Developmental Disorders. 2012;42(5):707-17 11p. doi: 10.1007/s10803-011-1299-5. PMID: 104548472. Language: English. Entry Date: 20120509. Revision Date: 20150711. Publication Type: Journal Article.X-1 
1277. Neely J, Amatea ES, Echevarria-Doan $\mathrm{S}$, et al. Working with families living with autism: potential contributions of marriage and family therapists. J Marital Fam Ther. 2012 Jun;38 Suppl 1:211-26. doi: 10.1111/j.17520606.2011.00265.x. PMID: 22765335.X-1

1278. Nicholson MS, Leask J. Lessons from an online debate about measles-mumps-rubella (MMR) immunization. Vaccine. 2012 May 28;30(25):3806-12. doi: 10.1016/j.vaccine.2011.10.072. PMID: 22063388.X-1

1279. Nicolaidis C. What can physicians learn from the neurodiversity movement? Virtual Mentor. 2012 Jun;14(6):503-10. doi: 10.1001/virtualmentor.2012.14.6.oped1-1206. PMID: 23351267.X-1

1280. Nikki R, Martin B, Gus O, et al. Endolymphatic sac enlargement in a girl with a novel mutation for distal renal tubular acidosis and severe deafness. Case Rep Pediatr. 2012;2012:605053. doi: 10.1155/2012/605053. PMID: 22966473.X-1

1281. Novarino G, El-Fishawy P, Kayserili $\mathrm{H}$, et al. Mutations in BCKD-kinase lead to a potentially treatable form of autism with epilepsy. Science. 2012 Oct 19;338(6105):3947. doi: 10.1126/science.1224631. PMID: 22956686.X-1

1282. Oberman L, Eldaief M, Fecteau S, et al. Abnormal modulation of corticospinal excitability in adults with Asperger's syndrome. Eur J Neurosci. 2012 Sep;36(6):2782-8. doi: 10.1111/j.1460-9568.2012.08172.x. PMID: 22738084.X-1

1283. O'Connor K. Auditory processing in autism spectrum disorder: a review. Neurosci Biobehav Rev. 2012 Feb;36(2):836-54. doi: 10.1016/j.neubiorev.2011.11.008. PMID: 22155284.X-1

1284. O'Donnell S, Deitz J, Kartin D, et al. Sensory processing, problem behavior, adaptive behavior, and cognition in preschool children with autism spectrum disorders. American Journal of Occupational Therapy. 2012 201512-09;66(5):586-94. doi: http://dx.doi.org/10.5014/ajot.2012.004168. PMID: 1428013928; 2012-25996-009.X-1
1285. Olfson M, Blanco C, Liu SM. Study examines office-based antipsychotic prescribing in youth. Brown University Child \& Adolescent Psychopharmacology Update. 2012;14(10):3-4 2p. doi: 10.1001/archgenpsychiatry.2012.647. PMID: 104421952. Language: English. Entry Date: 20121002. Revision Date: 20150711. Publication Type: Journal Article. Journal Subset: Biomedical.X-1

1286. Oner P, Oner O, Cop E, et al. [Reliability and Validity of the Turkish Version of the Social Communication Scale.]. Klinik Psikofarmakol Bulteni. 2012 Jan;22(1):43-50. doi: 10.5455/bcp.20111212091514. PMID: 25580078.X-1

1287. Onore C, Van de Water J, Ashwood P. Decreased levels of EGF in plasma of children with autism spectrum disorder. Autism Res Treat. 2012;2012:205362. doi: 10.1155/2012/205362. PMID: 22937258.X-1

1288. O'Reilly M, Aguilar J, Fragale C, et al. Effects of a motivating operation manipulation on the maintenance of mands. J Appl Behav Anal. 2012 Summer;45(2):443-7. doi: 10.1901/jaba.2012.45-443. PMID: 22844154.X-1, X-3, X-4

1289. O'Reilly M, Cook L, Karim K. Complementary or controversial care? The opinions of professionals on complementary and alternative interventions for Autistic Spectrum Disorder. Clin Child Psychol Psychiatry. 2012 Oct;17(4):602-15. doi: 10.1177/1359104511435340. PMID: 22371629.X-1

1290. O'Reilly M, Fragale C, Gainey S, et al. Examination of an antecedent communication intervention to reduce tangibly maintained challenging behavior: a controlled analog analysis. Res Dev Disabil. 2012 SepOct;33(5):1462-8. doi: 10.1016/j.ridd.2012.03.017. PMID: 22543058.X-3, X-4

1291. Orekhova EV, Tsetlin MM, Butorina AV, et al. Auditory cortex responses to clicks and sensory modulation difficulties in children with autism spectrum disorders (ASD). PLoS One. 2012;7(6):e39906. doi: 10.1371/journal.pone.0039906. PMID: 22768163.X-1 
1292. Orellana LM, Silvestre FJ, MartinezSanchis S, et al. Oral manifestations in a group of adults with autism spectrum disorder. Med Oral Patol Oral Cir Bucal. 2012 May;17(3):e415-9. PMID: 22143726.X-1

1293. Osteen M. Leaving limbo. Narrat Inq Bioeth. 2012 Winter;2(3):170-3. doi: 10.1353/nib.2012.0068. PMID: 24406882.X-1

1294. Ozsivadjian A, Knott F, Magiati I. Parent and child perspectives on the nature of anxiety in children and young people with autism spectrum disorders: a focus group study. Autism. 2012 Mar;16(2):107-21. doi: 10.1177/1362361311431703. PMID: 22297200.X-1

1295. Paden AR, Kodak T, Fisher WW, et al. Teaching children with autism to engage in peer-directed mands using a picture exchange communication system. J Appl Behav Anal. 2012 Summer;45(2):425-9. doi: 10.1901/jaba.2012.45-425. PMID: 22844150.X-3, X-4

1296. Pajareya K, Nopmaneejumruslers K. A one-year prospective follow-up study of a DIR/Floortime parent training intervention for pre-school children with autistic spectrum disorders. J Med Assoc Thai. 2012 Sep;95(9):1184-93. PMID: 23140036.X-3

1297. Palmen A, Didden R, Verhoeven L. A personal digital assistant for improving independent transitioning in adolescents with high-functioning autism spectrum disorder. Dev Neurorehabil. 2012;15(6):401-13. doi: 10.3109/17518423.2012.701240. PMID: 23030628.X-1, X-3, X-4

1298. Palsbo SE, Hood-Szivek P. Effect of robotic-assisted three-dimensional repetitive motion to improve hand motor function and control in children with handwriting deficits: a nonrandomized phase 2 device trial. Am J Occup Ther. 2012 Nov-Dec;66(6):682-90. doi: 10.5014/ajot.2012.004556. PMID:

23106988.X-3, X-4

1299. Pardini M, Elia M, Garaci FG, et al. Long-term cognitive and behavioral therapies, combined with augmentative communication, are related to uncinate fasciculus integrity in autism. J Autism Dev Disord. 2012
Apr;42(4):585-92. doi: 10.1007/s10803-0111281-2. PMID: 21573693.X-1, X-3

1300. Parish S, Thomas K, Rose R, et al. State insurance parity legislation for autism services and family financial burden. Intellect Dev Disabil. 2012 Jun;50(3):190-8. doi: 10.1352/1934-9556-50.3.190. PMID: 22731968.X-1

1301. Park HY, Lin SC, Harwood RL, et al. Autism Intervention Research Programs of the Maternal and Child Health Bureau. Pediatrics. 2012 Nov;130 Suppl 2:S59-61. doi: 10.1542/peds.2012-0900B. PMID: 23118254.X-1, X-2

1302. Park M. Pleasure, Throwing Breaches, and Embodied Metaphors: Tracing

Transformations-in-Participation for a Child With Autism to a Sensory Integration-Based Therapy Session. OTJR (Thorofare N J). 2012 Winter;32(1):S34-47. doi: 10.3928/1539449220110906-05. PMID: 24650787.X-3, X-4

1303. Parsons MB, Reid DH, Bentley E, et al. Identifying indices of happiness and unhappiness among adults with autism: potential targets for behavioral assessment and intervention. Behav Anal Pract. 2012 Summer;5(1):15-25. PMID: 23326627.X-1

1304. Paton B, Hohwy J, Enticott P. The Rubber Hand Illusion Reveals Proprioceptive and Sensorimotor Differences in Autism Spectrum Disorders. Journal of Autism \& Developmental Disorders. 2012;42(9):1870-83 14p. doi: 10.1007/s10803-011-1430-7. PMID: 104496825. Language: English. Entry Date: 20120824. Revision Date: 20150711.

Publication Type: Journal Article.X-1

1305. Peacock G, Lin SC. Enhancing early identification and coordination of intervention services for young children with autism spectrum disorders: report from the Act Early Regional Summit Project. Disabil Health J. 2012 Jan;5(1):55-9. doi: 10.1016/j.dhjo.2011.10.001. PMID: 22226299.X-1

1306. Pearson DA, Aman MG, Arnold LE, et al. High concordance of parent and teacher attention-deficit/hyperactivity disorder ratings in medicated and unmedicated children with autism spectrum disorders. J Child Adolesc 
Psychopharmacol. 2012 Aug;22(4):284-91. doi: 10.1089/cap.2011.0067. PMID: 22849541.X-1

1307. Pearson GS. The transition experience of developmentally impaired young adults living in a structured apartment setting. ANS Adv Nurs Sci. 2012 Jul-Sep;35(3):E73-89. doi: 10.1097/ANS.0b013e31826261b4. PMID: 22869219.X-1

1308. Pellicano E. The development of executive function in autism. Autism Res Treat. 2012;2012:146132. doi: 10.1155/2012/146132. PMID: 22934168.X-1

1309. Pennesi CM, Klein LC. Effectiveness of the gluten-free, casein-free diet for children diagnosed with autism spectrum disorder: based on parental report. Nutr Neurosci. 2012 Mar;15(2):85-91. doi:

10.1179/1476830512y.0000000003. PMID: 22564339.X-3

1310. Penrod B, Gardella L, Fernand J. An evaluation of a progressive high-probability instructional sequence combined with lowprobability demand fading in the treatment of food selectivity. J Appl Behav Anal. 2012 Fall;45(3):527-37. doi: 10.1901/jaba.2012.45527. PMID: 23060666.X-3, X-4

1311. Pepin G, Stagnitti K. Come play with me: an argument to link autism spectrum disorders and anorexia nervosa through early childhood pretend play. Eat Disord. 2012;20(3):254-9. doi: 10.1080/10640266.2012.668488. PMID: 22519903.X-1

1312. Perrin JM, Coury DL, Hyman SL, et al. Complementary and alternative medicine use in a large pediatric autism sample. Pediatrics. 2012 Nov;130 Suppl 2:S77-82. doi: 10.1542/peds.2012-0900E. PMID: 23118257.X-1

1313. Perrin JM, Coury DL, Jones N, et al. The Autism Treatment Network and Autism Intervention Research Network on Physical Health: future directions. Pediatrics. 2012 Nov;130 Suppl 2:S198-201. doi: 10.1542/peds.2012-0900S. PMID: 23118252.X1

1314. Perry A. Autism beyond pediatrics: why bioethicists ought to rethink consent in light of chronicity and genetic identity. Bioethics. 2012 Jun;26(5):236-41. doi: 10.1111/j.1467-8519.2011.01952.x. PMID: 22571426.X-1

1315. Peterson CC, Wellman HM, Slaughter $\mathrm{V}$. The mind behind the message: advancing theory-of-mind scales for typically developing children, and those with deafness, autism, or Asperger syndrome. Child Dev. 2012 MarApr;83(2):469-85. doi: 10.1111/j.14678624.2011.01728.x. PMID: 22304467.X-1

1316. Peters-Scheffer N, Didden R, Korzilius $\mathrm{H}$, et al. Cost comparison of early intensive behavioral intervention and treatment as usual for children with autism spectrum disorder in The Netherlands. Res Dev Disabil. 2012 NovDec;33(6):1763-72. doi: 10.1016/j.ridd.2012.04.006. PMID: 22705454.X-1

1317. Phi L, Ajaj R, Ramchandani MH, et al. Expanding the Grading of Recommendations Assessment, Development, and Evaluation (ExGRADE) for Evidence-Based Clinical Recommendations: Validation Study. Open Dent J. 2012;6:31-40. doi: 10.2174/1874210601206010031. PMID: 22303416.X-1

1318. Pineda JA, Juavinett A, Datko M. Selfregulation of brain oscillations as a treatment for aberrant brain connections in children with autism. Med Hypotheses. 2012 Dec;79(6):7908. doi: 10.1016/j.mehy.2012.08.031. PMID: 22999736.X-1

1319. Polick AS, Carr JE, Hanney NM. A comparison of general and descriptive praise in teaching intraverbal behavior to children with autism. J Appl Behav Anal. 2012 Fall;45(3):593-9. doi: 10.1901/jaba.2012.45593. PMID: 23060672.X-1, X-3, X-4

1320. Pollard JS, Betz AM, Higbee TS. Script fading to promote unscripted bids for joint attention in children with autism. J Appl Behav Anal. 2012 Summer;45(2):387-93. doi: 10.1901/jaba.2012.45-387. PMID: 22844144.X-3, X-4

1321. Ponsen MM, Stam CJ, Bosboom JL, et al. A three dimensional anatomical view of oscillatory resting-state activity and functional connectivity in Parkinson's disease related 
dementia: An MEG study using atlas-based beamforming. Neuroimage Clin. 2012;2:95102. doi: 10.1016/j.nicl.2012.11.007. PMID: 24179762.X-1

1322. Pratt K, Baird G, Gringras P. Ensuring successful admission to hospital for young people with learning difficulties, autism and challenging behaviour: a continuous quality improvement and change management programme. Child Care Health Dev. 2012 Nov;38(6):789-97. doi: 10.1111/j.13652214.2011.01335.x. PMID: 22017703.X-1

1323. Pringle B, Colpe LJ, Blumberg SJ, et al. Diagnostic history and treatment of schoolaged children with autism spectrum disorder and special health care needs. NCHS Data Brief. 2012 May(97):1-8. PMID: 23050521.X1

1324. Randolph-Gips M, Srinivasan P. Modeling autism: a systems biology approach. J Clin Bioinforma. 2012;2(1):17. doi: 10.1186/2043-9113-2-17. PMID: 23043674.X1

1325. Raznahan A, Lenroot R, Thurm A, et al. Mapping cortical anatomy in preschool aged children with autism using surface-based morphometry. Neuroimage Clin. 2012;2:111-9. doi: 10.1016/j.nicl.2012.10.005. PMID: 24179764.X-1

1326. Reaven J, Blakeley-Smith A, CulhaneShelburne K, et al. Group cognitive behavior therapy for children with high-functioning autism spectrum disorders and anxiety: a randomized trial. J Child Psychol Psychiatry. 2012 Apr;53(4):410-9. PMID: 22435114.X-1

1327. Reaven J, Blakeley-Smith A, Leuthe E, et al. Facing your fears in adolescence: cognitive-behavioral therapy for highfunctioning autism spectrum disorders and anxiety. Autism Res Treat. 2012;2012:423905. doi: 10.1155/2012/423905. PMID: 23091719.X-1, X-3

1328. Reed P. Brief Report: The Effect of Delayed Matching to Sample on Stimulus OverSelectivity. Journal of Autism \& Developmental Disorders. 2012;42(7):1515-9 5p. doi: 10.1007/s10803-011-1374-y. PMID: 104461324. Language: English. Entry Date:
20120618. Revision Date: 20150711. Publication Type: Journal Article.X-1

1329. Reed P, McCarthy J. Cross-Modal Attention-Switching is Impaired in Autism Spectrum Disorders. Journal of Autism \& Developmental Disorders. 2012;42(6):947-53 7p. doi: 10.1007/s10803-011-1324-8. PMID: 104451843. Language: English. Entry Date: 20120604. Revision Date: 20150711. Publication Type: Journal Article.X-1

1330. Remington A, Campbell R, Swettenham J. Attentional status of faces for people with autism spectrum disorder. Autism: The International Journal of Research \& Practice. 2012;16(1):59-73 15p. doi: 10.1177/1362361311409257. PMID: 104521305. Language: English. Entry Date: 20120304. Revision Date: 20150711. Publication Type: Journal Article.X-1

1331. Riahi F, Izadi-Mazidi S. Comparison between the mental health of mothers of children with autism and control group. Iran J Psychiatry Behav Sci. 2012 Fall;6(2):91-5. PMID: 24644488.X-1

1332. Rodriguez NM, Thompson RH, Schlichenmeyer K, et al. Functional analysis and treatment of arranging and ordering by individuals with an autism spectrum disorder. J Appl Behav Anal. 2012 Spring;45(1):1-22. doi: 10.1901/jaba.2012.45-1. PMID: 22403446.X-1

1333. Rogers SJ, Estes A, Lord C, et al. Effects of a brief Early Start Denver model (ESDM)-based parent intervention on toddlers at risk for autism spectrum disorders: a randomized controlled trial. J Am Acad Child Adolesc Psychiatry. 2012 Oct;51(10):1052-65. doi: 10.1016/j.jaac.2012.08.003. PMID: 23021480.X-1

1334. Roke Y, Buitelaar JK, Boot AM, et al. Risk of hyperprolactinemia and sexual side effects in males 10-20 years old diagnosed with autism spectrum disorders or disruptive behavior disorder and treated with risperidone. J Child Adolesc Psychopharmacol. 2012 Dec;22(6):432-9. doi: 10.1089/cap.2011.0109. PMID: 23234586.X-1, X-3

1335. Roke Y, van Harten PN, Buitelaar JK, et al. Antipsychotic-induced hyperprolactinemia and testosterone levels in boys. Horm Res 
Paediatr. 2012;77(4):235-40. doi: 10.1159/000337910. PMID: 22538969.X-1

1336. Roke Y, van Harten PN, Buitelaar JK, et al. Bone mineral density in male adolescents with autism spectrum disorders and disruptive behavior disorder with or without antipsychotic treatment. Eur J Endocrinol. 2012

Dec;167(6):855-63. doi: 10.1530/eje-12-0521. PMID: 23011870.X-1, X-3

1337. Rose S, Melnyk S, Trusty TA, et al. Intracellular and extracellular redox status and free radical generation in primary immune cells from children with autism. Autism Res Treat. 2012;2012:986519. doi: 10.1155/2012/986519. PMID: 22928106.X-1

1338. Rosenfeld D. The creation of the self and language: Primitive sensory relations of the child with the outside world. Karnac Books, London; 2012. p. 63-xv, .

1339. Rosenfeld D. The creation of the self and language: Primitive sensory relations of the child with the outside world. 2012:63-Xv, . PMID: 1027510026; 2012-17258-000.X-1

1340. Rossignol DA, Bradstreet JJ, Van Dyke K, et al. Hyperbaric oxygen treatment in autism spectrum disorders. Med Gas Res. 2012;2(1):16. doi: 10.1186/2045-9912-2-16. PMID: 22703610.X-1

1341. Rothenberg KG, Piadlo R, Nagaraj UD, et al. The complex picture test in dementia. Dement Geriatr Cogn Dis Extra. 2012 Jan;2(1):411-7. doi: 10.1159/000342349. PMID: 23139686.X-1

1342. Roy JS, Datta PG. Autism spectrum disorder and detection of autism. Mymensingh Med J. 2012 Jan;21(1):188-9. PMID: 22314481.X-1

1343. Ruble L, McGrew JH, Toland MD. Goal attainment scaling as an outcome measure in randomized controlled trials of psychosocial interventions in autism. J Autism Dev Disord. 2012 Sep;42(9):1974-83. doi: 10.1007/s10803012-1446-7. PMID: 22271197.X-1

1344. Russell B. Reflections on 'autistic integrity'. Bioethics. 2012 Mar;26(3):164-70. doi: 10.1111/j.1467-8519.2010.01827.x. PMID: 20497166.X-1
1345. Russo AJ, Bazin AP, Bigega R, et al. Plasma copper and zinc concentration in individuals with autism correlate with selected symptom severity. Nutr Metab Insights. 2012;5:41-7. doi: 10.4137/nmi.s8761. PMID: 23882147.X-1

1346. Russo N, Mottron L, Burack JA, et al. Parameters of semantic multisensory integration depend on timing and modality order among people on the autism spectrum: evidence from event-related potentials. Neuropsychologia. 2012 Jul;50(9):2131-41. doi:

10.1016/j.neuropsychologia.2012.05.003. PMID: 22613013.X-1

1347. Rutherford M, Troje N. IQ Predicts Biological Motion Perception in Autism Spectrum Disorders. Journal of Autism \& Developmental Disorders. 2012;42(4):557-65 9p. doi: 10.1007/s10803-011-1267-0. PMID: 104529450. Language: English. Entry Date: 20120509. Revision Date: 20150711. Publication Type: Journal Article.X-1

1348. Ryan S, Salisbury H. 'You know what boys are like': pre-diagnosis experiences of parents of children with autism spectrum conditions. Br J Gen Pract. 2012 May;62(598):e378-83. doi: 10.3399/bjgp12X641500. PMID: 22546598.X1

1349. Saalasti S, Katsyri J, Tiippana K, et al. Audiovisual speech perception and eye gaze behavior of adults with asperger syndrome. J Autism Dev Disord. 2012 Aug;42(8):1606-15. doi: 10.1007/s10803-011-1400-0. PMID: 22068821.X-1

1350. Saalasti S, Tiippana K, Kätsyri J, et al. The effect of visual spatial attention on audiovisual speech perception in adults with Asperger syndrome: Erratum. Experimental Brain Research. 2012 2015-12-09;217(2):323. doi: http://dx.doi.org/10.1007/s00221-0123029-4. PMID: 941020577; 2012-04843014.X-1

1351. Sakuma R, Gunji A, Goto T, et al. [Effectiveness of social skills training for children with developmental disorders: behavioral analysis using a two-dimensional motion capture system]. No To Hattatsu. 2012 
Jul;44(4):320-6. PMID: 22844765.X-1, X-3, $\mathrm{X}-4$

1352. Salgueiro E, Nunes L, Barros A, et al. Effects of a dolphin interaction program on children with autism spectrum disorders: an exploratory research. BMC Res Notes. 2012;5:199. doi: 10.1186/1756-0500-5-199. PMID: 22537536.X-1, X-3, X-4

1353. Salmanian M, Tehrani-Doost M, Ghanbari-Motlagh M, et al. Visual memory of meaningless shapes in children and adolescents with autism spectrum disorders. Iran J Psychiatry. 2012 Summer;7(3):104-8. PMID: 23139690.X-1

1354. Sanchez-Ponce R, Wang LQ, Lu W, et al. Metabolic and Pharmacokinetic Differentiation of STX209 and Racemic Baclofen in Humans. Metabolites. 2012;2(3):596-613. doi: 10.3390/metabo2030596. PMID: 24957649.X-1

1355. Sauer C, Montag C, Worner C, et al. Effects of a common variant in the CD38 gene on social processing in an oxytocin challenge study: possible links to autism.

Neuropsychopharmacology. 2012

May;37(6):1474-82. doi:

10.1038/npp.2011.333. PMID: 22278094.X-1

1356. Sawicka K, Zukin RS. Dysregulation of mTOR signaling in neuropsychiatric disorders: therapeutic implications. Neuropsychopharmacology. 2012 Jan;37(1):305-6. doi: 10.1038/npp.2011.210. PMID: 22157871.X-1

1357. Saylor S, Sidener TM, Reeve SA, et al. Effects of three types of noncontingent auditory stimulation on vocal stereotypy in children with autism. J Appl Behav Anal. 2012

Spring;45(1):185-90. doi:

10.1901/jaba.2012.45-185. PMID: 22403464.X-3, X-4

1358. Scahill L, McCracken JT, Bearss K, et al. Design and subject characteristics in the federally-funded citalopram trial in children with pervasive developmental disorders. J Autism Dev Disord. 2012 Mar;42(3):432-40. doi: 10.1007/s10803-011-1251-8. PMID: 21667200.X-1
1359. Scahill L, McDougle CJ, Aman MG, et al. Effects of risperidone and parent training on adaptive functioning in children with pervasive developmental disorders and serious behavioral problems. J Am Acad Child Adolesc Psychiatry. 2012 Feb;51(2):136-46. doi: 10.1016/j.jaac.2011.11.010. PMID: 22265360.X-1

1360. Schaaf RC, Benevides TW, Kelly D, et al. Occupational therapy and sensory integration for children with autism: a feasibility, safety, acceptability and fidelity study. Autism. 2012 May;16(3):321-7. doi: 10.1177/1362361311435157. PMID: 22318118.X-3, X-4

1361. Schaaf RC, Hunt J, Benevides T. Occupational Therapy Using Sensory Integration to Improve Participation of a Child With Autism: A Case Report. American Journal of Occupational Therapy. 2012;66(5):547-55 9p. doi: 10.5014/ajot.2012.004473. PMID: 104439427. Language: English. Entry Date: 20121121. Revision Date: 20150819. Publication Type: Journal Article.X-3, X-4

1362. Schipul SE, Williams DL, Keller TA, et al. Distinctive neural processes during learning in autism. Cereb Cortex. 2012 Apr;22(4):937-50. doi: 10.1093/cercor/bhr162. PMID: 21725037.X-1

1363. Schultz TR, Stichter JP, Herzog MJ, et al. Social Competence Intervention for Parents (SCI-P): Comparing Outcomes for a Parent Education Program Targeting Adolescents with ASD. Autism Res Treat. 2012;2012:681465. doi: 10.1155/2012/681465. PMID: 22934178.X-1

1364. Segura Benedicto A. [The putative link between the MMR vaccine and autism and refusal to vaccinate]. Gac Sanit. 2012 JulAug;26(4):366-71. doi: 10.1016/j.gaceta.2011.11.018. PMID: 22444516.X-1

1365. Seiverling L, Williams K, Sturmey P, et al. Effects of behavioral skills training on parental treatment of children's food selectivity. J Appl Behav Anal. 2012 Spring;45(1):197203. doi: 10.1901/jaba.2012.45-197. PMID: 22403466.X-3, X-4 
1366. Sepeta L, Tsuchiya N, Davies MS, et al. Abnormal social reward processing in autism as indexed by pupillary responses to happy faces. J Neurodev Disord. 2012;4(1):17. doi: 10.1186/1866-1955-4-17. PMID: 22958650.X-1

1367. Sequeira S, Ahmed M. Meditation as a potential therapy for autism: a review. Autism Res Treat. 2012;2012:835847. doi: 10.1155/2012/835847. PMID: 22937260.X-1, $\mathrm{X}-2$

1368. Severtson JM, Carr JE. Training novice instructors to implement errorless discrete-trial teaching: a sequential analysis. Behav Anal Pract. 2012 Winter;5(2):13-23. PMID: 23730463.X-1

1369. Sharma A, Shaw SR. Efficacy of Risperidone in Managing Maladaptive Behaviors for Children With Autistic Spectrum Disorder: A Meta-Analysis. Journal of Pediatric Healthcare. 2012;26(4):291-9 9p. doi: 10.1016/j.pedhc.2011.02.008. PMID: 104467215. Language: English. Entry Date: 20120725. Revision Date: 20150711.

Publication Type: Journal Article.X-3

1370. Shield A, Meier RP. Palm reversal errors in native-signing children with autism. $\mathrm{J}$ Commun Disord. 2012 Nov-Dec;45(6):439-54. doi: 10.1016/j.jcomdis.2012.08.004. PMID: 22981637.X-1

1371. Shields N, Muza R, Kosky C, et al. An Investigation into the Use of Stimulant Therapy during Pregnancy. Sleep Disord. 2012;2012:308952. doi: 10.1155/2012/308952. PMID: 23470841.X-1

1372. Shogren KA, Plotner AJ. Transition planning for students with intellectual disability, autism, or other disabilities: data from the National Longitudinal Transition Study-2. Intellect Dev Disabil. 2012 Feb;50(1):16-30. doi: 10.1352/1934-955650.1.16. PMID: 22316223.X-1

1373. Shumaker L. Love, autism style. Narrat Inq Bioeth. 2012 Winter;2(3):173-6. doi: 10.1353/nib.2012.0071. PMID: 24406883.X-2

1374. Siaperas P, Ring HA, McAllister CJ, et al. Atypical movement performance and sensory integration in Asperger's syndrome.
Journal of Autism and Developmental Disorders. 2012 2015-12-09;42(5):718-25. doi: http://dx.doi.org/10.1007/s10803-011-1301-2. PMID: 1013495786; 2012-09914-007.X-1

1375. Siegel M. Psychopharmacology of autism spectrum disorder: evidence and practice. Child Adolesc Psychiatr Clin N Am. 2012 Oct;21(4):957-73. doi: 10.1016/j.chc.2012.07.006. PMID: 23040909.X-1, X-2

1376. Siegel M, Beaulieu A. Psychotropic Medications in Children with Autism Spectrum Disorders: A Systematic Review and Synthesis for Evidence-Based Practice. Journal of Autism \& Developmental Disorders. 2012;42(8):1592605 14p. doi: 10.1007/s10803-011-1399-2. PMID: 104482206. Language: English. Entry Date: 20120724. Revision Date: 20150711. Publication Type: Journal Article.X-1, X-2

1377. Siegel M, Doyle K, Chemelski B, et al. Specialized inpatient psychiatry units for children with autism and developmental disorders: a United States survey. J Autism Dev Disord. 2012 Sep;42(9):1863-9. doi: 10.1007/s10803-011-1426-3. PMID: 22189962.X-1

1378. Silverman JL, Smith DG, Rizzo SJ, et al. Negative allosteric modulation of the mGluR5 receptor reduces repetitive behaviors and rescues social deficits in mouse models of autism. Sci Transl Med. 2012 Apr 25;4(131):131ra51. doi: 10.1126/scitranslmed.3003501. PMID: 22539775.X-1

1379. Simpson EL. Comorbidity in Atopic Dermatitis. Curr Dermatol Rep. 2012 Mar 1;1(1):29-38. doi: 10.1007/s13671-011-0003-5. PMID: 23745171.X-1

1380. Slocum SK, Miller SJ, Tiger JH. Using a blocked-trials procedure to teach identity matching to a child with autism. J Appl Behav Anal. 2012 Fall;45(3):619-24. doi: 10.1901/jaba.2012.45-619. PMID: 23060677.X-1, X-3, X-4

1381. Smith LE, Maenner MJ, Seltzer MM. Developmental trajectories in adolescents and adults with autism: the case of daily living skills. J Am Acad Child Adolesc Psychiatry. 2012 Jun;51(6):622-31. doi: 
10.1016/j.jaac.2012.03.001. PMID: 22632621.X-1

1382. Smith T. Evolution of research on interventions for individuals with autism spectrum disorder: implications for behavior analysts. Behav Anal. 2012 Spring;35(1):10113. PMID: 22942539.X-1

1383. Snyder K, Higbee TS, Dayton E. Preliminary investigation of a video-based stimulus preference assessment. J Appl Behav Anal. 2012 Summer;45(2):413-8. doi: 10.1901/jaba.2012.45-413. PMID: 22844148.X-1, X-3, X-4

1384. Sokhadze EM, Baruth JM, Sears L, et al. Prefrontal neuromodulation using rTMS improves error monitoring and correction function in autism. Appl Psychophysiol Biofeedback. 2012 Jun;37(2):91-102. doi: 10.1007/s10484-012-9182-5. PMID: 22311204.X-1

1385. Sokhadze EM, Baruth JM, Sears L, et al. EVENT-RELATED POTENTIAL STUDY OF ATTENTION REGULATION DURING ILLUSORY FIGURE CATEGORIZATION TASK IN ADHD, AUTISM SPECTRUM DISORDER, AND TYPICAL CHILDREN. J Neurother. 2012;16(1):12-31. doi: 10.1080/10874208.2012.650119. PMID: 23329879.X-1

1386. South M, Newton T, Chamberlain PD. Delayed reversal learning and association with repetitive behavior in autism spectrum disorders. Autism Res. 2012 Dec;5(6):398-406. doi: 10.1002/aur.1255. PMID: 23097376.X-1

1387. Spencer MD, Chura LR, Holt RJ, et al. Failure to deactivate the default mode network indicates a possible endophenotype of autism. Mol Autism. 2012;3(1):15. doi: 10.1186/20402392-3-15. PMID: 23206727.X-1

1388. Spencer MD, Holt RJ, Chura LR, et al. Atypical activation during the Embedded Figures Task as a functional magnetic resonance imaging endophenotype of autism. Brain: A Journal of Neurology. 2012;135(11):3469-80 12p. doi: brain/aws229. PMID: 104386051. Language: English. Entry Date: 20130215. Revision Date: 20150711. Publication Type: Journal Article.X-1
1389. Stafstrom CE, Rho JM. The ketogenic diet as a treatment paradigm for diverse neurological disorders. Front Pharmacol. 2012;3:59. doi: 10.3389/fphar.2012.00059. PMID: 22509165.X-1, X-2

1390. Stagnitti K, O'Connor C, Sheppard L. Impact of the Learn to Play program on play, social competence and language for children aged 5-8 years who attend a specialist school. Aust Occup Ther J. 2012 Aug;59(4):302-11. doi: 10.1111/j.1440-1630.2012.01018.x. PMID: 22934903.X-3, X-4

1391. Stahmer AC, Suhrheinrich J, Reed S, et al. What works for you? Using teacher feedback to inform adaptations of pivotal response training for classroom use. Autism Res Treat. 2012;2012:709861. doi: 10.1155/2012/709861. PMID: 23209896.X-1, $\mathrm{X}-2$

1392. Stein BD, Sorbero MJ, Goswami U, et al. Impact of a private health insurance mandate on public sector autism service use in Pennsylvania. J Am Acad Child Adolesc Psychiatry. 2012 Aug;51(8):771-9. doi: 10.1016/j.jaac.2012.06.006. PMID: 22840548.X-1

1393. Stein LI, Polido JC, Cermak SA. Oral care and sensory concerns in autism. Am J Occup Ther. 2012 Sep-Oct;66(5):e73-6. doi: 10.5014/ajot.2012.004085. PMID: 22917131.X-1

1394. Stein LI, Polido JC, Najera SO, et al. Oral care experiences and challenges in children with autism spectrum disorders. Pediatr Dent. 2012 Sep-Oct;34(5):387-91. PMID: 23211914.X-1

1395. Steiner A, Goldsmith T, Snow A, et al. Practitioner's Guide to Assessment of Autism Spectrum Disorders in Infants and Toddlers. Journal of Autism \& Developmental Disorders. 2012;42(6):1183-96 14p. doi: 10.1007/s10803011-1376-9. PMID: 104451818. Language: English. Entry Date: 20120604. Revision Date: 20150711. Publication Type: Journal Article.X1

1396. Stigler KA, Mullett JE, Erickson CA, et al. Paliperidone for irritability in adolescents and young adults with autistic disorder. Psychopharmacology (Berl). 2012 
Sep;223(2):237-45. doi: 10.1007/s00213-0122711-3. PMID: 22549762.X-1

1397. Storch EA, Arnold EB, Jones AM, et al. The role of co-occurring disruptive behavior in the clinical presentation of children and adolescents with anxiety in the context of autism spectrum disorders. Child Psychiatry Hum Dev. 2012 Oct;43(5):734-46. doi: 10.1007/s10578-012-0294-1. PMID: 22407279.X-1

1398. Stratta P, de Cataldo S, Bonanni R, et al. Mental health in L'Aquila after the earthquake. Ann Ist Super Sanita. 2012;48(2):132-7. doi: Doi: 10.4415/ann_12_02_05. PMID: 22751555.X-1

1399. Strauss K, Vicari S, Valeri G, et al. Parent inclusion in Early Intensive Behavioral Intervention: the influence of parental stress, parent treatment fidelity and parent-mediated generalization of behavior targets on child outcomes. Res Dev Disabil. 2012 MarApr;33(2):688-703. doi: 10.1016/j.ridd.2011.11.008. PMID: 22188793.X-1

1400. Strauss LV. Comparing a narcissistic and an autistic retreat: 'looking through or at the window'. Int J Psychoanal. 2012 Feb;93(1):97116. doi: 10.1111/j.1745-8315.2011.00488.x. PMID: 22320137.X-1

1401. Sturmey P. Treatment of psychopathology in people with intellectual and other disabilities. Canadian Journal of Psychiatry. 2012;57(10):593-600 8p. PMID: 108072793. Language: English. Entry Date: 20130215. Revision Date: 20150712.

Publication Type: Journal Article.X-1, X-2

1402. Suarez MA. Sensory processing in children with autism spectrum disorders and impact on functioning. Pediatric Clinics of North America. 2012;59(1):203-14 12p. PMID: 108153902. Language: English. Entry Date: 20120413. Revision Date: 20150712. Publication Type: Journal Article. Journal Subset: Biomedical.X-1

1403. Tachibana Y, Green J, Hwang Y, et al. A systematic review with meta-analysis of comprehensive interventions for preschool children with autism spectrum disorder (ASD): study protocol. BMJ Open. 2012;2(2):e000679. doi: 10.1136/bmjopen-2011-000679. PMID: 22396224.X-2

1404. Taheri A, Perry A. Exploring the proposed DSM-5 criteria in a clinical sample. $\mathrm{J}$ Autism Dev Disord. 2012 Sep;42(9):1810-7. doi: 10.1007/s10803-012-1599-4. PMID: 22806000.X-1

1405. Talero-Gutierrez C, Rodriguez M, De La Rosa D, et al. [Profile of children and adolescents with autism spectrum disorders in an institution in Bogota, Colombia]. Neurologia. 2012 Mar;27(2):90-6. doi: 10.1016/j.nrl.2011.03.005. PMID: 21592626.X1

1406. Tanabe HC, Kosaka H, Saito DN, et al. Hard to "tune in": neural mechanisms of live face-to-face interaction with high-functioning autistic spectrum disorder. Front Hum Neurosci. 2012;6:268. doi: 10.3389/fnhum.2012.00268. PMID: 23060772.X-1

1407. Taylor BA, DeQuinzio JA. Observational learning and children with autism. Behav Modif. 2012 May;36(3):341-60. doi: 10.1177/0145445512443981. PMID: 22569578.X-1

1408. Taylor JL, Warren ZE. Maternal depressive symptoms following autism spectrum diagnosis. J Autism Dev Disord. 2012 Jul;42(7):1411-8. doi: 10.1007/s10803-0111375-x. PMID: 21965086.X-1

1409. Tehrani-Doost M, Salmanian M, Ghanbari-Motlagh M, et al. Delayed face recognition in children and adolescents with autism spectrum disorders. Iran J Psychiatry. 2012 Spring;7(2):52-6. PMID: 22952545.X-1

1410. Tellegen CL, Sanders MR. Using primary care parenting interventions to improve outcomes in children with developmental disabilities: a case report. Case Rep Pediatr. 2012;2012:150261. doi: 10.1155/2012/150261. PMID: 22928141.X-2, X-3, X-4

1411. Thomas RH, Meeking MM, Mepham $\mathrm{JR}$, et al. The enteric bacterial metabolite propionic acid alters brain and plasma phospholipid molecular species: further development of a rodent model of autism spectrum disorders. J Neuroinflammation. 
2012;9:153. doi: 10.1186/1742-2094-9-153. PMID: 22747852.X-1

1412. Tilford JM, Payakachat N, Kovacs E, et al. Preference-based health-related qualityof-life outcomes in children with autism spectrum disorders: a comparison of generic instruments. Pharmacoeconomics. 2012 Aug 1;30(8):661-79. doi: 10.2165/11597200000000000-00000. PMID: 22788258.X-1

1413. Tostes MH, Polonini HC, Gattaz WF, et al. Low serum levels of 25-hydroxyvitamin D (25-OHD) in children with autism. Trends Psychiatry Psychother. 2012 Sep;34(3):161-3. PMID: 25923008.X-1

1414. Tsiouri I, Schoen Simmons E, Paul R. Enhancing the application and evaluation of a discrete trial intervention package for eliciting first words in preverbal preschoolers with ASD. J Autism Dev Disord. 2012 Jul;42(7):1281-93. doi: 10.1007/s10803-011-1358-y. PMID: 21918912.X-3, X-4

1415. Turan MK, Moroz L, Croteau NP. Comparing the effectiveness of error-correction strategies in discrete trial training. Behav Modif. 2012 Mar;36(2):218-34. doi: 10.1177/0145445511427973. PMID: 22133991.X-1, X-4

1416. Uno Y, Uchiyama T, Kurosawa M, et al. The combined measles, mumps, and rubella vaccines and the total number of vaccines are not associated with development of autism spectrum disorder: the first case-control study in Asia. Vaccine. 2012 Jun 13;30(28):4292-8. doi: 10.1016/j.vaccine.2012.01.093. PMID: 22521285.X-1

1417. Vajawat M, Deepika PC. Comparative evaluation of oral hygiene practices and oral health status in autistic and normal individuals. J Int Soc Prev Community Dent. 2012 Jul;2(2):58-63. doi: 10.4103/22310762.109369. PMID: 24478969.X-1

1418. Valenti M, Ciprietti T, Egidio C, et al. Adaptive Response of Children and Adolescents with Autism to the 2009 Earthquake in L'Aquila, Italy. Journal of Autism \& Developmental Disorders. 2012;42(6):954-60 7p. doi: 10.1007/s10803011-1323-9. PMID: 104451844. Language: English. Entry Date: 20120604. Revision Date:
20150711. Publication Type: Journal Article.X1

1419. Valentino AL, Shillingsburg MA, Call NA. Comparing the effects of echoic prompts and echoic prompts plus modeled prompts on intraverbal behavior. J Appl Behav Anal. 2012 Summer;45(2):431-5. doi: 10.1901/jaba.2012.45-431. PMID: 22844151.X-1, X-3, X-4

1420. Valicenti-McDermott M, Hottinger K, Seijo R, et al. Age at diagnosis of autism spectrum disorders. J Pediatr. 2012

Sep;161(3):554-6. doi: 10.1016/j.jpeds.2012.05.012. PMID: 22683037.X-1

1421. van der Meer L, Kagohara D, Achmadi $\mathrm{D}$, et al. Speech-generating devices versus manual signing for children with developmental disabilities. Res Dev Disabil. 2012 SepOct;33(5):1658-69. doi: 10.1016/j.ridd.2012.04.004. PMID: 22554812.X-4

1422. van der Net J, Sprong MC.

Commentary on 'motor characteristics of young children referred for possible autism spectrum disorder'. Pediatric Physical Therapy. 2012 2012 Spring;24(1):30- 1p. PMID: 108222319. Language: English. Entry Date: 20120406. Revision Date: 20150820. Publication Type: Journal Article.X-1

1423. Van der Poel J. The many questions of transition. Narrat Inq Bioeth. 2012

Winter;2(3):176-9. doi: 10.1353/nib.2012.0073. PMID: 24406884.X-1

1424. Vandbakk M, Arntzen E, Gisnaas A, et al. Effect of training different classes of verbal behavior to decrease aberrant verbal behavior. Anal Verbal Behav. 2012;28(1):137-44. PMID: 22754112.X-1

1425. Vande Wydeven K, Kwan A, Hardan AY, et al. Underutilization of genetics services for autism: the importance of parental awareness and provider recommendation. J Genet Couns. 2012 Dec;21(6):803-13. doi: 10.1007/s10897-012-9494-x. PMID: 22415587.X-1

1426. Varol E, Gaonkar B, Erus G, et al. FEATURE RANKING BASED NESTED 
SUPPORT VECTOR MACHINE ENSEMBLE FOR MEDICAL IMAGE CLASSIFICATION. Proc IEEE Int Symp Biomed Imaging. 2012:146-9. doi: 10.1109/isbi.2012.6235505. PMID: 23873289.X-1

1427. Veenstra-VanderWeele J. In this issue/abstract thinking: evolving picture of susceptibility factors in autism spectrum disorders. J Am Acad Child Adolesc Psychiatry. 2012 May;51(5):453-4. doi: 10.1016/j.jaac.2012.02.022. PMID: 22525947.X-1

1428. Venker CE, McDuffie A, Ellis Weismer $\mathrm{S}$, et al. Increasing verbal responsiveness in parents of children with autism:a pilot study. Autism. 2012 Nov;16(6):568-85. doi: 10.1177/1362361311413396. PMID: 21846665.X-1, X-3

1429. Verglas M. [Managing unspoken pain]. Soins Psychiatr. 2012 Sep-Oct(282):33-6. PMID: 23050361.X-1

1430. Verhoeven EW, Marijnissen N, Berger HJ, et al. Brief report: relationship between self-awareness of real-world behavior and treatment outcome in autism spectrum disorders. J Autism Dev Disord. 2012 May;42(5):889-94. doi: 10.1007/s10803-0111311-0. PMID: 21698498.X-1

1431. Verhoeven WM, Egger JI, Willemsen $\mathrm{MH}$, et al. Phelan-McDermid syndrome in two adult brothers: atypical bipolar disorder as its psychopathological phenotype? Neuropsychiatr Dis Treat. 2012;8:175-9. doi: 10.2147/ndt.s30506. PMID: 22570549.X-1

1432. Vernon TW, Koegel RL, Dauterman $\mathrm{H}$, et al. An early social engagement intervention for young children with autism and their parents. J Autism Dev Disord. 2012 Dec;42(12):2702-17. doi: 10.1007/s10803-0121535-7. PMID: 22527708.X-3, X-4

1433. Vismara LA, Young GS, Rogers SJ. Telehealth for expanding the reach of early autism training to parents. Autism Res Treat. 2012;2012:121878. doi: 10.1155/2012/121878. PMID: 23227334.X-1, X-3

1434. Vladescu JC, Carroll R, Paden A, et al. The effects of video modeling with voiceover instruction on accurate implementation of discrete-trial instruction. J Appl Behav Anal. 2012 Summer;45(2):419-23. doi: 10.1901/jaba.2012.45-419. PMID: 22844149.X-1

1435. Vlasak E, Ezeani-Antwi R. EPILEPSY \& AUTISM: The IMPORTANCE of MEDICATION MANAGEMENT. Exceptional Parent. 2012;42(8):32-4 3p. PMID: 104408722. Language: English. Entry Date: 20130117. Revision Date: 20150711. Publication Type: Journal Article.X-1

1436. Waligorska A, Pisula E, Waligorski M, et al. AutismPro system in supporting treatment of children with autism in Poland. Pediatr Int. 2012 Oct;54(5):693-700. doi: 10.1111/j.1442200X.2012.03637.x. PMID: 22469462.X-3, X4

1437. Walker HB. A true case story. Microb Ecol Health Dis. 2012;23doi: 10.3402/mehd.v23i0.18985. PMID: 23990822.X-1

1438. Wallace S, Fein D, Rosanoff M, et al. A global public health strategy for autism spectrum disorders. Autism Res. 2012 Jun;5(3):211-7. doi: 10.1002/aur.1236. PMID: 22605577.X-1

1439. Wallen M, Joosten A. Weighted vests did not improve competing behaviours or joint attention of 2 year olds with Autism Spectrum Disorder ( ASD). Australian Occupational Therapy Journal. 2012;59(6):468-70 3p. doi: 10.1111/1440-1630.12014. PMID: 104442203. Language: English. Entry Date: 20121128. Revision Date: 20150820. Publication Type: Journal Article.X-2, X-3

1440. Walton KM, Ingersoll BR. Evaluation of a Sibling-Mediated Imitation Intervention for Young Children with Autism. J Posit Behav Interv. 2012 Oct;14(4)doi: 10.1177/1098300712437044. PMID: 24339726.X-3, X-4

1441. Wang J, Zhou X, Xia W, et al. Autism awareness and attitudes towards treatment in caregivers of children aged 3-6 years in Harbin, China. Soc Psychiatry Psychiatr Epidemiol. 2012 Aug;47(8):1301-8. doi: 10.1007/s00127011-0438-9. PMID: 22009414.X-1 
1442. Wang J, Zhou X, Xia W, et al. Parentreported health care expenditures associated with autism spectrum disorders in Heilongjiang province, China. BMC Health Serv Res. 2012;12:7. doi: 10.1186/1472-6963-12-7. PMID: 22230043.X-1

1443. Warren Z, Taylor JL, McPheeters ML, et al. AHRQ Future Research Needs Papers. Future Research Needs: Interventions for Adolescents and Young Adults With Autism Spectrum Disorders: Identification of Future Research Needs From Comparative Effectiveness Review No. 65. Rockville (MD): Agency for Healthcare Research and Quality (US); 2012.

1444. Wasilewska J, Kaczmarski M, StasiakBarmuta A, et al. Low serum IgA and increased expression of CD23 on B lymphocytes in peripheral blood in children with regressive autism aged 3-6 years old. Arch Med Sci. 2012 May 9;8(2):324-31. doi:

10.5114/aoms.2012.28561. PMID:

22662007.X-1

1445. Wehmeyer ML, Tasse MJ, Davies DK, et al. Support Needs of Adults with Intellectual Disability Across Domains: The Role of Technology. J Spec Educ Technol. 2012 Apr;27(2):11-22. PMID: 25309010.X-1

1446. Wei H, Dobkin C, Sheikh AM, et al. The therapeutic effect of memantine through the stimulation of synapse formation and dendritic spine maturation in autism and fragile X syndrome. PLoS One. 2012;7(5):e36981. doi: 10.1371/journal.pone.0036981. PMID: 22615862.X-1

1447. Weil TN, Inglehart MR. Three- to 21year-old patients with autism spectrum disorders: parents' perceptions of severity of symptoms, oral health, and oral health-related behavior. Pediatr Dent. 2012 Nov-

Dec;34(7):473-9. PMID: 23265164.X-1

1448. Weiss HR, Liu X, Grewal P, et al. Reduced effect of stimulation of AMPA receptors on cerebral $\mathrm{O}(2)$ consumption in a rat model of autism. Neuropharmacology. 2012 Oct;63(5):837-41. doi: 10.1016/j.neuropharm.2012.06.014. PMID: 22722031.X-1
1449. Weiss K. So, what are you doing now? Narrat Inq Bioeth. 2012 Winter;2(3):E7-10. doi: 10.1353/nib.2012.0070. PMID: 24406896.X-1, $\mathrm{X}-2$

1450. Welterlin A, Turner-Brown LM, Harris S, et al. The home TEACCHing program for toddlers with autism. J Autism Dev Disord. 2012 Sep;42(9):1827-35. doi: 10.1007/s10803011-1419-2. PMID: 22160347.X-1, X-3

1451. Wentz E, Nyden A, Krevers B. Development of an internet-based support and coaching model for adolescents and young adults with ADHD and autism spectrum disorders: a pilot study. Eur Child Adolesc Psychiatry. 2012 Nov;21(11):611-22. doi: 10.1007/s00787-012-0297-2. PMID: 22736195.X-1

1452. White H. TRIALS OF ANTIPSYCHOTIC DRUGS NEED TO BE IMPARTIAL. Nursing Standard. 2012;27(1):33- 1p. PMID: 104418199. Language: English. Entry Date: 20120928. Revision Date: 20150711. Publication Type: Journal Article.X-1

1453. Whiteley P, Shattock P, Knivsberg AM, et al. Gluten- and casein-free dietary intervention for autism spectrum conditions. Front Hum Neurosci. 2012;6:344. doi: 10.3389/fnhum.2012.00344. PMID: 23316152.X-2

1454. Whyatt C, Craig C. Motor Skills in Children Aged 7-10 Years, Diagnosed with Autism Spectrum Disorder. Journal of Autism \& Developmental Disorders. 2012;42(9):1799809 11p. doi: 10.1007/s10803-011-1421-8. PMID: 104496829. Language: English. Entry Date: 20120824. Revision Date: 20150711. Publication Type: Journal Article.X-1

1455. Wiggins JL, Peltier SJ, Bedoyan JK, et al. The impact of serotonin transporter genotype on default network connectivity in children and adolescents with autism spectrum disorders.

Neuroimage Clin. 2012;2:17-24. doi: 10.1016/j.nicl.2012.10.008. PMID: 24179754.X-1

1456. Wild K, Poliakoff E, Jerrison A, et al. Goal-Directed and Goal-Less Imitation in Autism Spectrum Disorder. Journal of Autism \& Developmental Disorders. 2012;42(8):1739- 
49 11p. doi: 10.1007/s10803-011-1417-4. PMID: 104482192. Language: English. Entry Date: 20120724. Revision Date: 20150711. Publication Type: Journal Article.X-1

1457. Wilkinson KM, Light J. Preliminary Study of Gaze Toward Humans in Photographs by Individuals with Autism, Down Syndrome, or Other Intellectual Disabilities: Implications for Design of Visual Scene Displays. AAC: Augmentative \& Alternative Communication. 2012;30(2):130-46 17p. doi: 10.3109/07434618.2014.904434. PMID: 107854299. Language: English. Entry Date: 20140620. Revision Date: 20150819. Publication Type: Journal Article.X-1

1458. Williams BT, Gray KM, Tonge BJ. Teaching emotion recognition skills to young children with autism: a randomised controlled trial of an emotion training programme. J Child Psychol Psychiatry. 2012 Dec;53(12):1268-76. doi: 10.1111/j.1469-7610.2012.02593.x. PMID: 22881991.X-1

1459. Williams K, Wray JA, Wheeler DM. Intravenous secretin for autism spectrum disorders (ASD). Cochrane Database of Systematic Reviews. 2012(4):N.PAG-N.PAG 1p. PMID: 105838316. Language: English. Entry Date: 20100514. Revision Date: 20150711. Publication Type: Journal Article.X2

1460. Williams PG, Tomchek S, Grau R, et al. Parent and physician perceptions of medical home care for children with autism spectrum disorders in the state of Kentucky. Clin Pediatr (Phila). 2012 Nov;51(11):1071-8. doi: 10.1177/0009922812460333. PMID: 22984194.X-1

1461. Williams PG, Woods C, Stevenson M, et al. Psychotropic medication use in children with autism in the Kentucky Medicaid population. Clin Pediatr (Phila). 2012 Oct;51(10):923-7. doi: 10.1177/0009922812440837. PMID: 22492834.X-1

1462. Williams SC. Genetics: Searching for answers. Nature. 2012 Nov 1;491(7422):S4-6. PMID: 23136651.X-1

1463. Williamson E, Martin A. Psychotropic Medications in Autism: Practical
Considerations for Parents. Journal of Autism \& Developmental Disorders. 2012;42(6):124955 7p. doi: 10.1007/s10803-010-1144-2. PMID: 104451851. Language: English. Entry Date: 20120604. Revision Date: 20150711. Publication Type: Journal Article.X-1

1464. Wong JD, Seltzer MM, Greenberg JS, et al. Stressful life events and daily stressors affect awakening cortisol level in midlife mothers of individuals with autism spectrum disorders. Aging Ment Health. 2012;16(8):93949. doi: 10.1080/13607863.2012.688191. PMID: 22640177.X-1

1465. Woodward JF, Swigonski NL, Ciccarelli MR. Assessing the health, functional characteristics, and health needs of youth attending a noncategorical transition support program. J Adolesc Health. 2012 Sep;51(3):272-8. doi: 10.1016/j.jadohealth.2011.12.016. PMID: 22921138.X-1

1466. Wright SD, D'Astous V, Wright CA, et al. Grandparents of grandchildren with autism spectrum disorders (ASD): strengthening relationships through technology activities. Int J Aging Hum Dev. 2012;75(2):169-84. PMID: 23185859.X-1

1467. Yamazaki H, Yamamoto R, Moroto S, et al. Cochlear implantation in children with congenital cytomegalovirus infection accompanied by psycho-neurological disorders. Acta Otolaryngol. 2012 Apr;132(4):420-7. doi: 10.3109/00016489.2011.653442. PMID: 22443855.X-1

1468. Yang Y, Shen Y, Wu B. WITHDRAWN: Are there two different neural pathways for gender differences in autism spectrum disorders? - A pilot study. Med Hypotheses. 2012 Sep 11doi: 10.1016/j.mehy.2012.08.015. PMID: 22981007.X-1

1469. Yi L, Liu Y, Li Y, et al. Visual Scanning Patterns during the Dimensional Change Card Sorting Task in Children with Autism Spectrum Disorder. Autism Res Treat. 2012;2012:123053. doi: 10.1155/2012/123053. PMID: 23050145.X-1

1470. Ying KC, Browne G, Hutchinson M, et al. Autism in Vietnam: the case for the 
development and evaluation of an information book to be distributed at the time of diagnosis. Issues Ment Health Nurs. 2012 May;33(5):28892. doi: 10.3109/01612840.2011.653039. PMID: 22545635.X-1

1471. Young RL, Posselt M. Using the transporters DVD as a learning tool for children with Autism Spectrum Disorders (ASD). J Autism Dev Disord. 2012 Jun;42(6):984-91. doi: 10.1007/s10803-011-1328-4. PMID: 21822764.X-1

1472. Yui K. [Useful pharmacologic treatment in impaired social interaction in autism spectrum disorders]. Seishin Shinkeigaku Zasshi. 2012;114(8):934-40. PMID: 23012855.X-1, X-2

1473. Yui K, Koshiba M, Nakamura S, et al. Effects of large doses of arachidonic acid added to docosahexaenoic acid on social impairment in individuals with autism spectrum disorders: a double-blind, placebo-controlled, randomized trial. J Clin Psychopharmacol. 2012 Apr;32(2):200-6. doi: 10.1097/JCP.0b013e3182485791. PMID: 22370992.X-1

1474. Zhang R, Jia MX, Zhang JS, et al. Transcutaneous electrical acupoint stimulation in children with autism and its impact on plasma levels of arginine-vasopressin and oxytocin: a prospective single-blinded controlled study. Res Dev Disabil. 2012 JulAug;33(4):1136-46. doi: 10.1016/j.ridd.2012.02.001. PMID: 22502839.X-1

1475. Zimmer M, Desch L. Sensory Integration Therapies for Children With Developmental and Behavioral Disorders. Pediatrics. 2012;129(6):1186-9 4p. doi: 10.1542/peds.2012-0876. PMID: 108122725. Language: English. Entry Date: 20120614. Revision Date: 20150712. Publication Type: Journal Article.X-1, X-2

1476. . [Meeting minutes of the 1 (st) developmental and behavioral pediatric summit forum in China]. Zhonghua Er Ke Za Zhi. 2013 Sep;51(9):713-4. PMID: 24330999.X-1

1477. . Supplement 2 - SYTAR 2013

Abstracts. Int J Yoga Therap. 2013 Jun;23

Suppl 2:54-62. PMID: 24425502.X-1, X-2
1478. . [Oxidative stress, rRNA genes, and antioxidant enzymes in pathogenesis of schizophrenia and autism: modeling and clinical advices]. Zh Obshch Biol. 2013 SepOct;74(5):340-53. PMID: 25508097.X-1

1479. . Responses to sensory integration therapy challenges. OT Practice. 2013;18(6):231p. PMID: 108004467. Language: English. Entry Date: 20130607. Revision Date: 20150712. Publication Type: Journal Article.X1

1480. . Lay Abstracts. Autism: The International Journal of Research \& Practice. 2013;17(2):242-5 4p. doi: 10.1177/1362361313481408. PMID: 104249025. Language: English. Entry Date: 20130321. Revision Date: 20150711. Publication Type: Journal Article.X-1, X-2

1481. . Noticeboard. Learning Disability Practice. 2013;16(7):14- 1p. PMID: 104223837. Language: English. Entry Date: 20130909. Revision Date: 20150711. Publication Type: Journal Article.X-1

1482. . Methylphenidate dosing improved behavior in children with ASD. Brown University Child \& Adolescent Psychopharmacology Update. 2013;15(8):4-5 2p. PMID: 104202711. Language: English. Entry Date: 20130805. Revision Date: 20150711. Publication Type: Journal Article. Journal Subset: Biomedical.X-1, X-2

1483. . Use of valproate while pregnant increases child's autism risk. Brown University Psychopharmacology Update. 2013;24(8):1-5 p. PMID: 104190957. Language: English. Entry Date: 20130715. Revision Date: 20150711. Publication Type: Journal Article. Journal Subset: Biomedical.X-1, X-2

1484. . Valproate linked to autism spectrum disorders. Nursing. 2013;43(7):10-1 2p. doi: 10.1097/01.NURSE.0000431141.28275.09. PMID: 107956331. Language: English. Entry Date: 20130710. Revision Date: 20150820. Publication Type: Journal Article.X-1, X-2

1485. . Clinical digest. Mothers' prenatal use of antidepressants puts children at risk of autism. Nursing Standard. 2013;27(41):15- 1p. PMID: 104179664. Language: English. Entry 
Date: 20130618. Revision Date: 20150819. Publication Type: Journal Article.X-1

1486. . Clinical digest. Babies exposed to antiepileptic drug valproate in utero are more likely to develop autism. Nursing Standard. 2013;27(40):14-5 2p. PMID: 104175778. Language: English. Entry Date: 20130610. Revision Date: 20150819. Publication Type: Journal Article. Journal Subset: Double Blind Peer Reviewed.X-1, X-2

1487. . Intranasal oxytocin for boys with autism shows promise. Brown University Child \& Adolescent Psychopharmacology Update. 2013;15(5):3-4 2p. PMID: 104278192. Language: English. Entry Date: 20130429. Revision Date: 20150711. Publication Type: Journal Article. Journal Subset: Biomedical.X$1, \mathrm{X}-2$

1488. . Fragile X syndrome study signals potential for acamprosate in autism. Brown University Child \& Adolescent Psychopharmacology Update. 2013;15(4):1-3 p. PMID: 104264228. Language: English. Entry Date: 20130403. Revision Date: 20150711. Publication Type: Journal Article. Journal Subset: Biomedical.X-1

1489. . . Long-term risperidone for young males linked to sexual effects. Brown University Child \& Adolescent Psychopharmacology Update. 2013;15(3):3-4 2p. PMID: 104241901. Language: English. Entry Date: 20130301. Revision Date: 20150711. Publication Type: Journal Article. Journal Subset: Biomedical.X-1, X-2

1490. . News. Journal of Psychosocial Nursing \& Mental Health Services. 2013;51(3):8-10 3p. doi: 10.3928/0279369520130130-89. PMID: 104245479. Language: English. Entry Date: 20130306. Revision Date: 20150711. Publication Type: Journal Article.X$1, \mathrm{X}-2$

1491. . Hyperprolactinemia found in risperidonetreated youth. Brown University Child \& Adolescent Psychopharmacology Update. 2013;15(2):4-5 2p. PMID: 104303200. Language: English. Entry Date: 20130207. Revision Date: 20150711. Publication Type: Journal Article. Journal Subset: Biomedical.X$1, \mathrm{X}-2$
1492. . Medication use in autistic youth examined. Brown University Child \& Adolescent Psychopharmacology Update. 2013;15(1):7- 1p. PMID: 104407624. Language: English. Entry Date: 20130104. Revision Date: 20150711. Publication Type: Journal Article.X-1, X-2

1493. Abisror Nm, Mekinian An, Lachassinne E, et al. Autism spectrum disorders in babies born to mothers with antiphospholipid syndrome. Seminars in Arthritis \& Rheumatism. 2013;43(3):348-51 4p. doi: 10.1016/j.semarthrit.2013.07.001. PMID: 104124696. Language: English. Entry Date: 20131219. Revision Date: 20150710. Publication Type: Journal Article.X-1

1494. Abu-Dahab S, Skidmore E, Holm M, et al. Motor and Tactile-Perceptual Skill Differences Between Individuals with HighFunctioning Autism and Typically Developing Individuals Ages 5-21. Journal of Autism \& Developmental Disorders. 2013;43(10):2241-8 8p. doi: 10.1007/s10803-011-1439-y. PMID: 104226085. Language: English. Entry Date: 20130921. Revision Date: 20150711. Publication Type: Journal Article.X-1

1495. Adluru N, Hanlon BM, Lutz A, et al. Penalized likelihood phenotyping: unifying voxelwise analyses and multi-voxel pattern analyses in neuroimaging: penalized likelihood phenotyping. Neuroinformatics. 2013 Apr;11(2):227-47. doi: 10.1007/s12021-0129175-9. PMID: 23397550.X-1

1496. Ajzenman HF, Standeven JW, Shurtleff TL. Effect of hippotherapy on motor control, adaptive behaviors, and participation in children with autism spectrum disorder: a pilot study. Am J Occup Ther. 2013 Nov-

Dec;67(6):653-63. doi: 10.5014/ajot.2013.008383. PMID: 24195899.X-3, X-4

1497. Aksu F, Baykara B, Ergin C, et al. [Phenotypic features in autistic individuals: the finger length ratio (2D:4D), hair whorl, and hand dominance]. Turk Psikiyatri Derg. 2013 Summer;24(2):94-100. PMID: 23754262.X-1

1498. Alaghband-Rad J, Nikvarz N, TehraniDoost M, et al. Memantine-induced speech problems in two patients with autistic disorder. 
Daru. 2013;21(1):54. doi: 10.1186/2008-223121-54. PMID: 23819879.X-1

1499. Alant E, Zheng W, Harty M, et al. Translucency ratings of Blissymbols over repeated exposures by children with autism. Augment Altern Commun. 2013 Sep;29(3):27283. doi: 10.3109/07434618.2013.813967.

PMID: 23902408.X-1, X-3

1500. Aldbass AM, Bhat RS, El-Ansary A. Protective and therapeutic potency of N-acetylcysteine on propionic acid-induced biochemical autistic features in rats. J Neuroinflammation. 2013;10:42. doi: 10.1186/1742-2094-10-42. PMID: 23537042.X-1

1501. Aldinger KA, Plummer JT, Levitt P. Comparative DNA methylation among females with neurodevelopmental disorders and seizures identifies TAC1 as a MeCP2 target gene. J Neurodev Disord. 2013;5(1):15. doi: 10.1186/1866-1955-5-15. PMID: 23759142.X1

1502. Al-Farsi YM, Waly MI, Deth RC, et al. Impact of nutrition on serum levels of docosahexaenoic acid among Omani children with autism. Nutrition. 2013 Sep;29(9):1142-6. doi: 10.1016/j.nut.2013.03.009. PMID:

23800562.X-1, X-3

1503. Aljunied M, Frederickson N. Does central coherence relate to the cognitive performance of children with autism in dynamic assessments? Autism: The International Journal of Research \& Practice. 2013;17(2):172-83 12p. doi: 10.1177/1362361311409960. PMID: 104249018. Language: English. Entry Date: 20130321. Revision Date: 20150711.

Publication Type: Journal Article.X-1

1504. Amato CA, Santos TH, Barbosa MR, et al. Longitudinal study of language therapy in 142 children and adolescents with autism spectrum disorders. Codas. 2013;25(4):388-90. PMID: 24408489.X-1, X-3

1505. Ameis SH, Corbett-Dick P, Cole L, et al. Decision making and antipsychotic medication treatment for youth with autism spectrum disorders: applying guidelines in the real world. J Clin Psychiatry. 2013

Oct;74(10):1022-4. doi: 10.4088/JCP.13ac08691. PMID: 24229756.X-1
1506. Anderson JS, Nielsen JA, Ferguson MA, et al. Abnormal brain synchrony in Down Syndrome. Neuroimage Clin. 2013;2:703-15. doi: 10.1016/j.nicl.2013.05.006. PMID: 24179822.X-1

1507. Aoki Y, Abe O, Nippashi Y, et al. Comparison of white matter integrity between autism spectrum disorder subjects and typically developing individuals: a meta-analysis of diffusion tensor imaging tractography studies. Mol Autism. 2013;4(1):25. doi: 10.1186/20402392-4-25. PMID: 23876131.X-1

1508. Apicella F, Chericoni N, Costanzo V, et al. Reciprocity in interaction: a window on the first year of life in autism. Autism Res Treat. 2013;2013:705895. doi: 10.1155/2013/705895. PMID: 23762551.X-1

1509. Arber C, Li M. Cortical interneurons from human pluripotent stem cells: prospects for neurological and psychiatric disease. Front Cell Neurosci. 2013;7:10. doi: 10.3389/fncel.2013.00010. PMID: 23493959.X-1

1510. Arvanitis H. Creating a Sensory Integration Gym in your Home. Exceptional Parent. 2013;43(4):30-2 3p. PMID: 104195987. Language: English. Entry Date: 20130723. Revision Date: 20150711. Publication Type: Journal Article.X-1

1511. Asahi Y, Omichi S, Adachi S, et al. Ventilation via cut nasotracheal tube during general anesthesia. Anesth Prog. 2013 Spring;60(1):11-4. doi: 10.2344/11-00031.1. PMID: 23506278.X-1

1512. Assaf M, Hyatt CJ, Wong CG, et al. Mentalizing and motivation neural function during social interactions in autism spectrum disorders. Neuroimage Clin. 2013;3:321-31. doi: 10.1016/j.nicl.2013.09.005. PMID: 24273716.X-1

1513. Bachmann CJ, Manthey T, KampBecker I, et al. Psychopharmacological treatment in children and adolescents with autism spectrum disorders in Germany. Res Dev Disabil. 2013 Sep;34(9):2551-63. doi: 10.1016/j.ridd.2013.05.028. PMID: 23747941.X-1, X-3 
1514. Baghdadli A, Brisot J, Henry V, et al. Social skills improvement in children with high-functioning autism: a pilot randomized controlled trial. Eur Child Adolesc Psychiatry. 2013 Jul;22(7):433-42. doi: 10.1007/s00787013-0388-8. PMID: 23417625.X-4

1515. Bainbridge J. Inducing labour may increase risk of autism. British Journal of Midwifery. 2013;21(11):828- 1p. PMID: 104159058. Language: English. Entry Date: 20131112. Revision Date: 20150711. Publication Type: Journal Article. Journal Subset: Core Nursing.X-1

1516. Baker SM. Scientific narratives in autism spectrum disorder. Glob Adv Health Med. 2013 Nov;2(6):5. doi:

10.7453/gahmj.2013.095. PMID: 24416703.X$1, \mathrm{X}-2$

1517. Baker SM, Milivojevich A. Gender differences among children with autism spectrum disorder: differential symptom patterns. Glob Adv Health Med. 2013 Nov;2(6):8-18. doi: 10.7453/gahmj.2013.003. PMID: 24416704.X-1

1518. Bang J, Burns J, Nadig A. Brief report: Conveying subjective experience in conversation: production of mental state terms and personal narratives in individuals with high functioning autism. J Autism Dev Disord. 2013 Jul;43(7):1732-40. doi: 10.1007/s10803-0121716-4. PMID: 23179342.X-1

1519. Barker RM, Akaba S, Brady NC, et al. Support for AAC use in preschool, and growth in language skills, for young children with developmental disabilities. Augment Altern Commun. 2013 Dec;29(4):334-46. doi: 10.3109/07434618.2013.848933. PMID: 24229337.X-1, X-3

1520. Barlow KE, Tiger JH, Slocum SK, et al. Comparing acquisition of exchange-based and signed mands with children with autism. Anal Verbal Behav. 2013;29(1):59-69. PMID: 23814367.X-1

1521. Baron-Cohen S, Jaffa T, Davies S, et al. Do girls with anorexia nervosa have elevated autistic traits? Mol Autism. 2013;4(1):24. doi: 10.1186/2040-2392-4-24. PMID: 23915495.X1
1522. Bauer AZ, Kriebel D. Prenatal and perinatal analgesic exposure and autism: an ecological link. Environ Health. 2013;12:41. doi: 10.1186/1476-069x-12-41. PMID: 23656698.X-1

1523. Bauman MD, Schumann CM. Is 'bench-to-bedside' realistic for autism? An integrative neuroscience approach. Neuropsychiatry (London). 2013 Apr;3(2):15968. doi: 10.2217/npy.13.18. PMID: 24000295.X-1

1524. Bauminger-Zviely N, Eden S, Zancanaro $\mathrm{M}$, et al. Increasing social engagement in children with high-functioning autism spectrum disorder using collaborative technologies in the school environment. Autism. 2013 May;17(3):317-39. doi: 10.1177/1362361312472989. PMID: 23614935.X-1

1525. Bay B, Mortensen EL, Hvidtjorn D, et al. Fertility treatment and risk of childhood and adolescent mental disorders: register based cohort study. Bmj. 2013;347:f3978. doi: 10.1136/bmj.f3978. PMID: 23833075.X-1

1526. Bearss K, Johnson C, Handen B, et al. A pilot study of parent training in young children with autism spectrum disorders and disruptive behavior. J Autism Dev Disord. 2013 Apr;43(4):829-40. doi: 10.1007/s10803-0121624-7. PMID: 22941342.X-3, X-4

1527. Bearss K, Lecavalier L, Minshawi N, et al. Toward an exportable parent training program for disruptive behaviors in autism spectrum disorders. Neuropsychiatry (London). 2013 Apr;3(2):169-80. doi: 10.2217/npy.13.14. PMID: 23772233.X-1, X-2

1528. Bebbington A, Glasson E, Bourke J, et al. Hospitalisation rates for children with intellectual disability or autism born in Western Australia 1983-1999: a population-based cohort study. BMJ Open. 2013;3(2)doi: 10.1136/bmjopen-2012-002356. PMID: 23449747.X-1

1529. Bedford R, Gliga T, Frame K, et al. Failure to learn from feedback underlies word learning difficulties in toddlers at risk for autism. J Child Lang. 2013 Jan;40(1):29-46. doi: 10.1017/s0305000912000086. PMID: 23217290.X-1 
1530. Begeer S, Mandell D, Wijnker-Holmes $\mathrm{B}$, et al. Sex differences in the timing of identification among children and adults with autism spectrum disorders. J Autism Dev Disord. 2013 May;43(5):1151-6. doi: 10.1007/s10803-012-1656-z. PMID: 23001766.X-1

1531. Bekele ET, Lahiri U, Swanson AR, et al. A step towards developing adaptive robotmediated intervention architecture (ARIA) for children with autism. IEEE Trans Neural Syst Rehabil Eng. 2013 Mar;21(2):289-99. doi: 10.1109/tnsre.2012.2230188. PMID: 23221831.X-1, X-4

1532. Bekhet AK, Zauszniewski JA. Psychometric assessment of the depressive cognition scale among caregivers of persons with autism spectrum disorder. Arch Psychiatr Nurs. 2013 Apr;27(2):96-100. doi: 10.1016/j.apnu.2012.10.004. PMID: 23540520.X-1

1533. Belmonte MK, Saxena-Chandhok T, Cherian R, et al. Oral motor deficits in speechimpaired children with autism. Front Integr Neurosci. 2013;7:47. doi: 10.3389/fnint.2013.00047. PMID: 23847480.X$1, \mathrm{X}-3$

1534. Ben-Cheikh I, Rousseau C. [Autism and social support in recently immigrated families : experience of parents from Maghreb]. Sante Ment Que. 2013 Spring;38(1):189-205. PMID: 24336996.X-1

1535. Bennett KD, Ramasamy R, Honsberger $\mathrm{T}$. The effects of covert audio coaching on teaching clerical skills to adolescents with autism spectrum disorder. J Autism Dev Disord. 2013 Mar;43(3):585-93. doi: 10.1007/s10803-012-1597-6. PMID: 22798051.X-1

1536. Bennett TA, Szatmari P, Bryson S, et al. Theory of Mind, Language and Adaptive Functioning in ASD: A Neuroconstructivist Perspective. J Can Acad Child Adolesc Psychiatry. 2013 Feb;22(1):13-9. PMID: 23390428.X-1

1537. Ben-Sasson A, Lamash L, Gal E. To enforce or not to enforce? The use of collaborative interfaces to promote social skills in children with high functioning autism spectrum disorder. Autism. 2013

Sep;17(5):608-22. doi: 10.1177/1362361312451526. PMID: 22987887.X-4

1538. Benvenuto A, Marciano S, Capuano I, et al. An update on autism spectrum disorders in children. Minerva Pediatr. 2013 Feb;65(1):19-36. PMID: 23422571.X-1

1539. Berger DM. Tests, testing, and tested we need to critically evaluate the meaning of tests in psychiatry. Indian J Psychiatry. 2013 Apr;55(2):200-3. doi: 10.4103/00195545.111466. PMID: 23825862.X-1

1540. Bernier R, Aaronson B, McPartland J. The role of imitation in the observed heterogeneity in EEG mu rhythm in autism and typical development. Brain \& Cognition. 2013;82(1):69-75 7p. doi: 10.1016/j.bandc.2013.02.008. PMID: 104270303. Language: English. Entry Date: 20131101. Revision Date: 20150711. Publication Type: Journal Article. Journal Subset: Biomedical.X-1

1541. Bhatara A, Babikian T, Laugeson E, et al. Impaired Timing and Frequency Discrimination in High-functioning Autism Spectrum Disorders. Journal of Autism \& Developmental Disorders. 2013;43(10):2312-28 17p. doi: 10.1007/s10803-013-1778-y. PMID: 104226077. Language: English. Entry Date: 20130921. Revision Date: 20150711. Publication Type: Journal Article.X-1

1542. Bhatti I, Thome A, Smith PO, et al. A retrospective study of amitriptyline in youth with autism spectrum disorders. J Autism Dev Disord. 2013 May;43(5):1017-27. doi: 10.1007/s10803-012-1647-0. PMID: 23135317.X-3

1543. Bilaver LA, Jordan N. Impact of state mental health parity laws on access to autism services. Psychiatr Serv. 2013 Oct;64(10):96773. doi: 10.1176/appi.ps.201200411. PMID: 23771517.X-1

1544. Billeci L, Sicca F, Maharatna K, et al. On the application of quantitative EEG for characterizing autistic brain: a systematic review. Front Hum Neurosci. 2013;7:442. doi: 
10.3389/fnhum.2013.00442. PMID: 23935579.X-1, X-2

1545. Black K, McCarus C, Collins ML, et al. Ocular manifestations of autism in ophthalmology. Strabismus. 2013 Jun;21(2):98102. doi: 10.3109/09273972.2013.786733. PMID: 23713930.X-1

1546. Blain-Moraes S, Chesser S, Kingsnorth S, et al. Biomusic: a novel technology for revealing the personhood of people with profound multiple disabilities. Augment Altern Commun. 2013 Jun;29(2):15973. doi: 10.3109/07434618.2012.760648. PMID: 23484489.X-1

1547. Bluth K, Roberson PN, Billen RM, et al. A Stress Model for Couples Parenting Children With Autism Spectrum Disorders and the Introduction of a Mindfulness Intervention. J Fam Theory Rev. 2013 Sep;5(3):194-213. doi: 10.1111/jftr.12015. PMID: 24795780.X-1

1548. Bobo WV, Cooper WO, Stein CM, et al. Antipsychotics and the risk of type 2 diabetes mellitus in children and youth. JAMA Psychiatry. 2013 Oct;70(10):1067-75. doi: 10.1001/jamapsychiatry.2013.2053. PMID: 23965896.X-1

1549. Boccuto L, Chen CF, Pittman AR, et al. Decreased tryptophan metabolism in patients with autism spectrum disorders. Mol Autism. 2013;4(1):16. doi: 10.1186/2040-2392-4-16. PMID: 23731516.X-1

1550. Boesch MC, Wendt O, Subramanian A, et al. Comparative efficacy of the picture exchange communication system (PECS) versus a speech-generating device: effects on social-communicative skills and speech development. Augment Altern Commun. 2013 Sep;29(3):197-209. doi: 10.3109/07434618.2013.818059. PMID: 23952565.X-3, X-4

1551. Bolte EE, Diehl JJ. Measurement tools and target symptoms/skills used to assess treatment response for individuals with autism spectrum disorder. J Autism Dev Disord. 2013 Nov;43(11):2491-501. doi: 10.1007/s10803013-1798-7. PMID: 23479074.x-1

1552. Boyd BA, Woodard CR, Bodfish JW. Feasibility of exposure response prevention to treat repetitive behaviors of children with autism and an intellectual disability: a brief report. Autism. 2013 Mar;17(2):196-204. doi: 10.1177/1362361311414066. PMID: 21975037.X-3, X-4

1553. Bradley SJ. The importance of early intervention with children and youth in the autism spectrum. J Can Acad Child Adolesc Psychiatry. 2013 Aug;22(3):197-8. PMID: 23970907.X-1

1554. Brandwein AB, Foxe JJ, Butler JS, et al. The development of multisensory integration in high-functioning autism: high-density electrical mapping and psychophysical measures reveal impairments in the processing of audiovisual inputs. Cereb Cortex. 2013 Jun;23(6):1329-41. doi: 10.1093/cercor/bhs109. PMID: 22628458.X-1

1555. Breivik I, Hemmingsson $\mathrm{H}$. Experiences of handwriting and using a computerized ATD in school: adolescents with Asperger's syndrome. Scand J Occup Ther. 2013 Sep;20(5):349-56. doi: 10.3109/11038128.2012.748822. PMID: 23312011.X-1

1556. Brent J. Commentary on the abuse of metal chelation therapy in patients with autism spectrum disorders. J Med Toxicol. 2013 Dec;9(4):370-2. doi: 10.1007/s13181-0130345-4. PMID: 24113859.X-1

1557. Breslin CM, Rudisill ME. Relationships Among Assessment Time, Time on Task, and Motor Skill Performance in Children With Autism Spectrum Disorder. Adapted Physical Activity Quarterly. 2013;30(4):338-50 13p. PMID: 104234198. Language: English. Entry Date: 20131008. Revision Date: 20150819. Publication Type: Journal Article.X-1

1558. Brock J, Bzishvili S, Reid M, et al. Brief report: atypical neuromagnetic responses to illusory auditory pitch in children with autism spectrum disorders. J Autism Dev Disord. 2013 Nov;43(11):2726-31. doi: 10.1007/s10803-013-1805-z. PMID: 23543291.X-1, X-4

1559. Brockevelt BL, Nissen R, Schweinle WE, et al. A comparison of the Sensory Profile scores of children with autism and an age- and 
gender-matched sample. S D Med. 2013

Nov;66(11):459, 61, 63-5. PMID:

24383262.X-1

1560. Broder-Fingert S, Shui A, Pulcini CD, et al. Racial and ethnic differences in subspecialty service use by children with autism. Pediatrics. 2013 Jul;132(1):94-100. doi: 10.1542/peds.2012-3886. PMID: 23776121.X-1

1561. Brogaard B. Serotonergic hyperactivity as a potential factor in developmental, acquired and drug-induced synesthesia. Frontiers in Human Neuroscience. 2013 2015-12-09;7 PMID: 1492671541; 201341426-001.X-1, X-2

1562. Brondino N, De Silvestri A, Re S, et al. A Systematic Review and Meta-Analysis of Ginkgo biloba in Neuropsychiatric Disorders: From Ancient Tradition to Modern-Day Medicine. Evid Based Complement Alternat Med. 2013;2013:915691. doi: 10.1155/2013/915691. PMID: 23781271.X-1, $\mathrm{X}-2$

1563. Buard I, Rogers SJ, Hepburn S, et al. Altered oscillation patterns and connectivity during picture naming in autism. Front Hum Neurosci. 2013;7:742. doi: 10.3389/fnhum.2013.00742. PMID: 24265611.X-1

1564. Bucci MP, Doyen C, Contenjean Y, et al. The effect of performing a dual task on postural control in children with autism. ISRN Neurosci. 2013;2013:796174. doi: 10.1155/2013/796174. PMID: 24959567.X-1

1565. Buon M, Dupoux E, Jacob P, et al. The Role of Causal and Intentional Judgments in Moral Reasoning in Individuals with High Functioning Autism. Journal of Autism \& Developmental Disorders. 2013;43(2):458-70 13p. doi: 10.1007/s10803-012-1588-7. PMID: 104296937. Language: English. Entry Date: 20130128. Revision Date: 20150711. Publication Type: Journal Article.X-1

1566. Burgess S, Audet L, Harjusola-Webb S. Quantitative and qualitative characteristics of the school and home language environments of preschool-aged children with ASD. J Commun Disord. 2013 Sep-Dec;46(5-6):428-39. doi: 10.1016/j.jcomdis.2013.09.003. PMID: 24209428.X-1
1567. Burke RV, Allen KD, Howard MR, et al. Tablet-based video modeling and prompting in the workplace for individuals with autism. Journal of Vocational Rehabilitation. 2013;38(1):1-14 p. PMID: 104304415. Language: English. Entry Date: 20130201. Revision Date: 20150819. Publication Type: Journal Article.X-1

1568. Burket JA, Benson AD, Tang AH, et al. D-Cycloserine improves sociability in the BTBR T+ Itpr3tf/J mouse model of autism spectrum disorders with altered Ras/Raf/ERK1/2 signaling. Brain Res Bull. 2013 Jul;96:62-70. doi: 10.1016/j.brainresbull.2013.05.003. PMID: 23685206.X-1

1569. Butterly F, Percy C, Ward G. Brief report: do service dog providers placing dogs with children with developmental disabilities use outcome measures and, if so, what are they? J Autism Dev Disord. 2013 Nov;43(11):2720-5. doi: 10.1007/s10803-013-1803-1. PMID: 23479076.X-1, X-3

1570. Buxbaum JD, Baron-Cohen S. DSM-5: the debate continues. Mol Autism.

2013;4(1):11. doi: 10.1186/2040-2392-4-11. PMID: 23676181.X-3

1571. Cai Y, Chia NK, Thalmann D, et al. Design and development of a Virtual Dolphinarium for children with autism. IEEE Trans Neural Syst Rehabil Eng. 2013 Mar;21(2):208-17. doi: 10.1109/tnsre.2013.2240700. PMID: 23362251.X-1, X-3, X-4

1572. Campbell NG, Zhu CB, Lindler KM, et al. Rare coding variants of the adenosine A3 receptor are increased in autism: on the trail of the serotonin transporter regulome. Mol Autism. 2013;4(1):28. doi: 10.1186/2040-23924-28. PMID: 23953133.X-1

1573. Cannell JJ. Autism, will vitamin D treat core symptoms? Med Hypotheses. 2013 Aug;81(2):195-8. doi: 10.1016/j.mehy.2013.05.004. PMID: 23725905.X-1, X-2, X-3, X-4

1574. Carbone PS, Murphy NA, Norlin C, et al. Parent and pediatrician perspectives regarding the primary care of children with 
autism spectrum disorders. J Autism Dev

Disord. 2013 Apr;43(4):964-72. doi:

10.1007/s10803-012-1640-7. PMID:

22948548.X-1

1575. Cascio CJ, Lorenzi J, Baranek GT.

Self-reported Pleasantness Ratings and

Examiner-Coded Defensiveness in Response to

Touch in Children with ASD: Effects of

Stimulus Material and Bodily Location. J

Autism Dev Disord. 2013 Oct 5doi:

10.1007/s10803-013-1961-1. PMID:

24091471.X-1

1576. Casenhiser DM, Shanker SG, Stieben

J. Learning through interaction in children with autism: preliminary data from asocialcommunication-based intervention. Autism.

2013 Mar;17(2):220-41. doi:

10.1177/1362361311422052. PMID:

21949005.X-1

1577. Cashin A, Browne G, Bradbury J, et al. The effectiveness of narrative therapy with young people with autism. J Child Adolesc Psychiatr Nurs. 2013 Feb;26(1):32-41. doi: 10.1111/jcap.12020. PMID: 23351106.X-1, X3, X-4

1578. Castro S, Ferreira T, Dababnah S, et al. Linking autism measures with the ICF-CY: functionality beyond the borders of diagnosis and interrater agreement issues. Dev Neurorehabil. 2013 Oct;16(5):321-31. doi: 10.3109/17518423.2012.733438. PMID: 23869843.X-1

1579. Castro S, Pinto AI. Identification of core functioning features for assessment and intervention in Autism Spectrum Disorders. Disabil Rehabil. 2013 Jan;35(2):125-33. doi: 10.3109/09638288.2012.690494. PMID: 22671037.X-1

1580. Catarino A, Andrade A, Churches O, et al. Task-related functional connectivity in autism spectrum conditions: an EEG study using wavelet transform coherence. Mol Autism. 2013;4(1):1. doi: 10.1186/2040-23924-1. PMID: 23311570.X-1

1581. Cauffield JS. [INFOGRAPHIC] Medication use in autism spectrum disorders: What is the evidence? Formulary. 2013;48(5):110 p. PMID: 103968374 . Language: English. Entry Date: 20140715. Revision Date:
20150710. Publication Type: Journal Article.X$1, \mathrm{X}-2$

1582. Ceritoglu C, Tang X, Chow M, et al. Computational analysis of LDDMM for brain mapping. Front Neurosci. 2013;7:151. doi: 10.3389/fnins.2013.00151. PMID: 23986653.X-1

1583. Chabout J, Cressant A, Hu X, et al. Making choice between competing rewards in uncertain vs. safe social environment: role of neuronal nicotinic receptors of acetylcholine. Front Hum Neurosci. 2013;7:468. doi: 10.3389/fnhum.2013.00468. PMID: 23986674.X-1

1584. Chamberlain PD, Rodgers J, Crowley $\mathrm{MJ}$, et al. A potentiated startle study of uncertainty and contextual anxiety in adolescents diagnosed with autism spectrum disorder. Mol Autism. 2013;4(1):31. doi: 10.1186/2040-2392-4-31. PMID: 24007557.X1

1585. Chan AS, Sze SL, Siu NY, et al. A chinese mind-body exercise improves selfcontrol of children with autism: a randomized controlled trial. PLoS One. 2013;8(7):e68184. doi: 10.1371/journal.pone.0068184. PMID: 23874533.X-1

1586. Charbonneau G, Bertone A, Lepore F, et al. Multilevel alterations in the processing of audio-visual emotion expressions in autism spectrum disorders. Neuropsychologia. 2013 Apr;51(5):1002-10. doi: 10.1016/j.neuropsychologia.2013.02.009. PMID: 23462241.X-1

1587. Cheak-Zamora NC, Yang X, Farmer $\mathrm{JE}$, et al. Disparities in transition planning for youth with autism spectrum disorder.

Pediatrics. 2013 Mar;131(3):447-54. doi: 10.1542/peds.2012-1572. PMID: 23400613.X-1

1588. Chebuhar A, McCarthy AM, Bosch J, et al. Using picture schedules in medical settings for patients with an autism spectrum disorder. J Pediatr Nurs. 2013 Apr;28(2):12534. doi: 10.1016/j.pedn.2012.05.004. PMID: 22742928.X-1, X-3

1589. Cheung G, Trembath D, Arciuli J, et al. The impact of workplace factors on evidence-based speech-language pathology 
practice for children with autism spectrum disorders. Int J Speech Lang Pathol. 2013 Aug;15(4):396-406. doi: 10.3109/17549507.2012.714797. PMID: 22967045.X-1

1590. Chevallier C, Huguet P, Happé F, et al. Salient Social Cues are Prioritized in Autism Spectrum Disorders Despite Overall Decrease in Social Attention. Journal of Autism \& Developmental Disorders. 2013;43(7):1642-51 10p. doi: 10.1007/s10803-012-1710-x. PMID: 104183644. Language: English. Entry Date: 20130620. Revision Date: 20150711. Publication Type: Journal Article.X-1

1591. Chiang HM, Cheung YK, Li H, et al. Factors associated with participation in employment for high school leavers with autism. J Autism Dev Disord. 2013 Aug;43(8):1832-42. doi: 10.1007/s10803-0121734-2. PMID: 23224594.X-1

1592. Chiat S, Roy P. Early predictors of language and social communication impairments at ages 9-11 years: a follow-up study of early-referred children. J Speech Lang Hear Res. 2013 Dec;56(6):1824-36. doi: 10.1044/1092-4388(2013/12-0249). PMID: 23926296.X-1

1593. Chien WH, Gau SS, Chen CH, et al. Increased gene expression of FOXP1 in patients with autism spectrum disorders. Mol Autism. 2013;4(1):23. doi: 10.1186/2040-2392-4-23. PMID: 23815876.X-1

1594. Chien WH, Gau SS, Liao HM, et al. Deep exon resequencing of DLGAP2 as a candidate gene of autism spectrum disorders. Mol Autism. 2013;4(1):26. doi: 10.1186/20402392-4-26. PMID: 23915500.X-1

1595. Christensen J, Gronborg TK, Sorensen $\mathrm{MJ}$, et al. Prenatal valproate exposure and risk of autism spectrum disorders and childhood autism. Jama. 2013 Apr 24;309(16):1696-703. doi: 10.1001/jama.2013.2270. PMID: 23613074.X-1

1596. Cidav Z, Lawer L, Marcus SC, et al. Age-related variation in health service use and associated expenditures among children with autism. J Autism Dev Disord. 2013 Apr;43(4):924-31. doi: 10.1007/s10803-0121637-2. PMID: 22941343.X-1
1597. Cividini-Motta C, Ahearn WH. Effects of two variations of differential reinforcement on prompt dependency. J Appl Behav Anal. 2013 Fall;46(3):640-50. doi: 10.1002/jaba.67. PMID: 24114226.X-1, X-3, X-4

1598. Classen S, Monahan M, Brown KE, et al. Driving indicators in teens with attention deficit hyperactivity and/or autism spectrum disorder: Indicateurs de la conduite automobile chez les jeunes ayant un déficit de l'attention avec hyperactivité ou un trouble du spectre. Canadian Journal of Occupational Therapy. 2013;80(5):274-83 10p. doi: 10.1177/0008417413501072. PMID: 104029842. Language: English. Entry Date: 20140225. Revision Date: 20150820. Publication Type: Journal Article.X-1

1599. Classen S, Monalian M, Wang V. Driving characteristics of teens with attention deficit hyperactivity and autism spectrum disorder. American Journal of Occupational Therapy. 2013 2016-06-17;67(6):664-73. doi: http://dx.doi.org/10.5014/ajot.2013.008821. PMID: 1511554117; 2013-41757-002.X-1

1600. Clay CJ, Samaha AL, Bloom SE, et al. Assessing preference for social interactions.

Res Dev Disabil. 2013 Jan;34(1):362-71. doi: 10.1016/j.ridd.2012.07.028. PMID: 23009945.X-1, X-4

1601. Clery H, Andersson F, BonnetBrilhault F, et al. fMRI investigation of visual change detection in adults with autism. Neuroimage Clin. 2013;2:303-12. doi: 10.1016/j.nicl.2013.01.010. PMID: 24179785.X-1

1602. Clifford T, Minnes P. Logging on: evaluating an online support group for parents of children with autism spectrum disorders. J Autism Dev Disord. 2013 Jul;43(7):1662-75. doi: 10.1007/s10803-012-1714-6. PMID: 23143075.X-1

1603. Clopper CG, Rohrbeck KL, Wagner L. Perception of talker age by young adults with high-functioning autism. J Autism Dev Disord. 2013 Jan;43(1):134-46. doi: 10.1007/s10803012-1553-5. PMID: 22638967.X-1

1604. Cofiel L, Bassi DU, Ray RK, et al. Detecting dissonance in clinical and research 
workflow for translational psychiatric registries. PLoS One. 2013;8(9):e75167. doi:

10.1371/journal.pone.0075167. PMID: 24073246.X-1

1605. Cohen JA, Dickerson TA, Forbes JM. A legal review of autism, a syndrome rapidly gaining wide attention within our society. Albany Law Rev. 2013;77(2):389-423. PMID: 24851321.X-1

1606. Collignon O, Charbonneau G, Peters $\mathrm{F}$, et al. Reduced multisensory facilitation in persons with autism. Cortex: A Journal Devoted to the Study of the Nervous System \& Behavior. 2013;49(6):1704-10 7p. doi: 10.1016/j.cortex.2012.06.001. PMID: 107905473. Language: English. Entry Date: 20140131. Revision Date: 20150712. Publication Type: Journal Article.X-1, X-3

1607. Colver AF, Merrick H, Deverill M, et al. Study protocol: longitudinal study of the transition of young people with complex health needs from child to adult health services. BMC Public Health. 2013;13:675. doi: 10.1186/14712458-13-675. PMID: 23875722.X-1

1608. Conner CM, Maddox BB, White SW. Parents' state and trait anxiety: relationships with anxiety severity and treatment response in adolescents with autism spectrum disorders. J Autism Dev Disord. 2013 Aug;43(8):1811-8. doi: 10.1007/s10803-012-1728-0. PMID: 23224592.X-1

1609. Cook JL, Blakemore S-J, Press C. Atypical basic movement kinematics in autism spectrum conditions. Brain: A Journal of Neurology. 2013;136(9):2816-24 9p. doi: brain/awt208. PMID: 104216029. Language: English. Entry Date: 20131122. Revision Date: 20150711. Publication Type: Journal Article.X1

1610. Corman MK. How mothers talk about placement of their child with autism outside the home. Qual Health Res. 2013 Oct;23(10):132032. doi: 10.1177/1049732313505225. PMID: 24022735.X-1

1611. Coury DL. DSM-5 and autism spectrum disorders: implications for families and clinicians. J Dev Behav Pediatr. 2013 Sep;34(7):494-6. doi:
10.1097/DBP.0b013e31829cac3e. PMID: 24042080.X-1

1612. Crippa A, Forti S, Perego P, et al. EyeHand Coordination in Children with High Functioning Autism and Asperger's Disorder Using a Gap-Overlap Paradigm. Journal of Autism \& Developmental Disorders. 2013;43(4):841-50 10p. doi: 10.1007/s10803012-1623-8. PMID: 104256195. Language: English. Entry Date: 20130321. Revision Date: 20150711. Publication Type: Journal Article.X1

1613. Crowder SA, Merritte K. The possible therapeutic benefits of utilizing motion gaming systems on pediatric patients presenting autism. Tenn Med. 2013 Sep;106(8):41-3. PMID: 24027887.X-5

1614. Curtin S, Vouloumanos A. Speech Preference is Associated with Autistic-Like Behavior in 18-Months-Olds at Risk for Autism Spectrum Disorder. Journal of Autism \& Developmental Disorders. 2013;43(9):2114-20 7p. doi: 10.1007/s10803-013-1759-1. PMID: 104210176. Language: English. Entry Date: 20130820. Revision Date: 20150711. Publication Type: Journal Article.X-1

1615. Daley TC, Singhal N, Krishnamurthy V. Ethical considerations in conducting research on autism spectrum disorders in low and middle income countries. J Autism Dev Disord. 2013 Sep;43(9):2002-14. doi: 10.1007/s10803-012-1750-2. PMID: 23283629.X-1

1616. Dalsgaard S, Nielsen HS, Simonsen M. Five-fold increase in national prevalence rates of attention-deficit/hyperactivity disorder medications for children and adolescents with autism spectrum disorder, attentiondeficit/hyperactivity disorder, and other psychiatric disorders: a Danish register-based study. J Child Adolesc Psychopharmacol. 2013 Sep;23(7):432-9. doi: 10.1089/cap.2012.0111. PMID: 24015896.X-1

1617. Daniels AM, Mandell DS. Children's compliance with American Academy of Pediatrics' well-child care visit guidelines and the early detection of autism. J Autism Dev Disord. 2013 Dec;43(12):2844-54. doi: 10.1007/s10803-013-1831-x. PMID: 23619952.X-1 
1618. Davis TN, Dacus S, Strickland E, et al. Reduction of automatically maintained selfinjurious behavior utilizing noncontingent matched stimuli. Dev Neurorehabil. 2013 Jun;16(3):166-71. doi: 10.3109/17518423.2013.766819. PMID: 23477292.X-3, X-4

1619. de Boer-Schellekens L, Eussen M, Vroomen J. Diminished sensitivity of audiovisual temporal order in autism spectrum disorder. Front Integr Neurosci. 2013;7:8. doi: 10.3389/fnint.2013.00008. PMID: 23450453.X1

1620. de Boer-Schellekens L, Keetels M, Eussen M, et al. No evidence for impaired multisensory integration of low-level audiovisual stimuli in adolescents and young adults with autism spectrum disorders. Neuropsychologia. 2013 Dec;51(14):3004-13. doi: 10.1016/j.neuropsychologia.2013.10.005. PMID: 24157536.X-1

1621. Deshpande G, Libero LE, Sreenivasan $\mathrm{KR}$, et al. Identification of neural connectivity signatures of autism using machine learning. Front Hum Neurosci. 2013;7:670. doi: 10.3389/fnhum.2013.00670. PMID: 24151458.X-1

1622. Dickerson M, Bae Y. Block copolymer nanoassemblies for photodynamic therapy and diagnosis. Ther Deliv. 2013 Nov;4(11):143141. doi: 10.4155/tde.13.105. PMID: 24228992.X-1

1623. Domes G, Heinrichs M, Kumbier E, et al. Effects of intranasal oxytocin on the neural basis of face processing in autism spectrum disorder. Biol Psychiatry. 2013 Aug 1;74(3):164-71. doi: 10.1016/j.biopsych.2013.02.007. PMID: 23510581.X-1, X-3, X-4

1624. Douglas JF, Sanders KB, Benneyworth $\mathrm{MH}$, et al. Brief report: retrospective case series of oxcarbazepine for irritability/agitation symptoms in autism spectrum disorder. $\mathrm{J}$ Autism Dev Disord. 2013 May;43(5):1243-7. doi: 10.1007/s10803-012-1661-2. PMID: 22976374.X-3

1625. Douglas SN, McNaughton D, Light J. Online Training for Paraeducators to Support the Communication of Young Children. Journal of Early Intervention. 2013;35(2):223-42 20p. doi: 10.1177/1053815114526782. PMID: 103986456. Language: English. Entry Date: 20140110. Revision Date: 20150818. Publication Type: Journal Article.X-1

1626. Doyle-Thomas KA, Duerden EG, Taylor MJ, et al. Effects of age and symptomatology on cortical thickness in autism spectrum disorders. Res Autism Spectr Disord. 2013 Jan;7(1):141-50. doi:

10.1016/j.rasd.2012.08.004. PMID: 23678367.X-1

1627. Dratsch T, Schwartz C, Yanev K, et al. Getting a Grip on Social Gaze: Control over Others' Gaze Helps Gaze Detection in HighFunctioning Autism. Journal of Autism \& Developmental Disorders. 2013;43(2):286-300 15p. doi: 10.1007/s10803-012-1569-x. PMID: 104296916. Language: English. Entry Date: 20130128. Revision Date: 20150711. Publication Type: Journal Article.X-1

1628. Dupere S, MacDonald RP, Ahearn WH. Using video modeling with substitutable loops to teach varied play to children with autism. J Appl Behav Anal. 2013 Fall;46(3):662-8. doi: 10.1002/jaba.68. PMID: 24114229.X-1, X-3, X-4

1629. Durrleman S, Pennec X, Trouve A, et al. Toward a comprehensive framework for the spatiotemporal statistical analysis of longitudinal shape data. Int J Comput Vis. 2013 May;103(1):22-59. doi: 10.1007/s11263-0120592-x. PMID: 23956495.X-1

1630. Eack SM, Bahorik AL, Hogarty SS, et al. Brief report: is cognitive rehabilitation needed in verbal adults with autism? Insights from initial enrollment in a trial of cognitive enhancement therapy. J Autism Dev Disord. 2013 Sep;43(9):2233-7. doi: 10.1007/s10803013-1774-2. PMID: 23381484.X-1

1631. Eack SM, Greenwald DP, Hogarty SS, et al. Cognitive enhancement therapy for adults with autism spectrum disorder: results of an 18month feasibility study. J Autism Dev Disord. 2013 Dec;43(12):2866-77. doi: 10.1007/s10803-013-1834-7. PMID: 23619953.X-1 
1632. Eapen V, Crncec R, Walter A. Clinical outcomes of an early intervention program for preschool children with Autism Spectrum Disorder in a community group setting. BMC Pediatr. 2013;13(1):3. doi: 10.1186/1471-243113-3. PMID: 23294523.X-3

1633. Edvinsson D, Lindstrom E, Bingefors $\mathrm{K}$, et al. Gender differences of axis I and II comorbidity in subjects diagnosed with attention-deficit hyperactivity disorder as adults. Acta Neuropsychiatr. 2013 Jun;25(3):165-74. doi: 10.1111/j.16015215.2012.00682.x. PMID: 25287470.X-1

1634. Egan AM, Dreyer ML, Odar CC, et al. Obesity in young children with autism spectrum disorders: prevalence and associated factors. Child Obes. 2013 Apr;9(2):125-31. doi: 10.1089/chi.2012.0028. PMID: 23485020.X-1

1635. Eigsti IM, Fein DA. More is less: pitch discrimination and language delays in children with optimal outcomes from autism. Autism Res. 2013 Dec;6(6):605-13. doi: 10.1002/aur.1324. PMID: 23929787.X-1

1636. Eikeseth S, Smith DP. An analysis of verbal stimulus control in intraverbal behavior: implications for practice and applied research. Anal Verbal Behav. 2013;29(1):125-35. PMID: 23814373.X-1, X-2, X-3

1637. El-Ansary A, Shaker G, Siddiqi NJ, et al. Possible ameliorative effects of antioxidants on propionic acid / clindamycin - induced neurotoxicity in Syrian hamsters. Gut Pathog. 2013;5(1):32. doi: 10.1186/1757-4749-5-32. PMID: 24188374.X-1

1638. El-Ansary A, Shaker GH, El-Gezeery $\mathrm{AR}$, et al. The neurotoxic effect of clindamycin - induced gut bacterial imbalance and orally administered propionic acid on DNA damage assessed by the comet assay: protective potency of carnosine and carnitine. Gut Pathog. 2013;5(1):9. doi: 10.1186/1757-4749-5-9. PMID: 23587115.X-1

1639. Eldevik S, Ondire I, Hughes JC, et al. Effects of computer simulation training on in vivo discrete trial teaching. J Autism Dev Disord. 2013 Mar;43(3):569-78. doi: 10.1007/s10803-012-1593-x. PMID: 22790382.X-1
1640. Ellis-Hervey N. The comparison of sensory integrative therapy (specifically weighted vests) and applied behavioral analysis (specifically a differential schedule of reinforcement) in the treatment of children who have autism spectrum disorder; 2013.

1641. Emerson A, Dearden J. Accommodating to motor difficulties and communication impairments in people with autism: the MORE intervention model. Front Integr Neurosci. 2013;7:45. doi: 10.3389/fnint.2013.00045. PMID: 23785315.X1

1642. Enticott PG, Kennedy HA, Rinehart $\mathrm{NJ}$, et al. GABAergic activity in autism spectrum disorders: an investigation of cortical inhibition via transcranial magnetic stimulation. Neuropharmacology. 2013 May;68:202-9. doi: 10.1016/j.neuropharm.2012.06.017. PMID: 22727823.X-1

1643. Eriksson MA, Westerlund J, Hedvall A, et al. Medical conditions affect the outcome of early intervention in preschool children with autism spectrum disorders. Eur Child Adolesc Psychiatry. 2013 Jan;22(1):23-33. doi: 10.1007/s00787-012-0312-7. PMID: 22836733.X-1

1644. Esch JW, Mahoney AM, Kestner KM, et al. Echoic and self-echoic responses in children. Anal Verbal Behav. 2013;29(1):11723. PMID: 23814372.X-1

1645. Esposito G, Pasca SP. Motor abnormalities as a putative endophenotype for Autism Spectrum Disorders. Front Integr Neurosci. 2013;7:43. doi: 10.3389/fnint.2013.00043. PMID: 23781177.X1

1646. Essa MM, Subash S, Braidy N, et al. Role of $\mathrm{NAD}(+)$, Oxidative Stress, and Tryptophan Metabolism in Autism Spectrum Disorders. Int J Tryptophan Res. 2013;6(Suppl 1):15-28. doi: 10.4137/ijtr.s11355. PMID: 23922500.X-1

1647. Estes A, Olson E, Sullivan K, et al. Parenting-related stress and psychological distress in mothers of toddlers with autism spectrum disorders. Brain Dev. 2013 Feb;35(2):133-8. doi: 
10.1016/j.braindev.2012.10.004. PMID: 23146332.X-1

1648. Fahmie TA, Iwata BA, Harper JM, et al. Evaluation of the divided attention condition during functional analyses. J Appl Behav Anal. 2013 Spring;46(1):71-8. doi: 10.1002/jaba.20. PMID: 24114086.X-1

1649. Falter CM. Impaired and superior mirror symmetry perception in autism...Falter CM and Bailey AJ (2011) Perception of Mirror Symmetry in Autism Spectrum Disorder. Autism, published online first... Perreault A, Gurnsey R, Dawson M, Mottron L and Bertone A (2011) Increased sensitivity to mirror symmetry in autism. PLoS ONE 6(4): e19519. Autism: The International Journal of Research \& Practice. 2013;17(1):117-8 2p. doi: 10.1177/1362361312438618. PMID: 104241915. Language: English. Entry Date: 20130226. Revision Date: 20150711. Publication Type: Journal Article.X-1

1650. Farlow MR, Schmitt F, Aarsland D, et al. Comparing clinical profiles in Alzheimer's disease and Parkinson's disease dementia. Dement Geriatr Cogn Dis Extra. 2013;3(1):28190. doi: 10.1159/000351861. PMID: 24174923.X-1

1651. Farmer C, Thurm A, Grant P. Pharmacotherapy for the Core Symptoms in Autistic Disorder: Current Status of the Research. Drugs. 2013;73(4):303-14 12p. doi: 10.1007/s40265-013-0021-7. PMID: 104268117. Language: English. Entry Date: 20130416. Revision Date: 20150711. Publication Type: Journal Article.X-2

1652. Fatemi SH, Folsom TD, Kneeland RE, et al. Impairment of fragile $\mathrm{X}$ mental retardation protein-metabotropic glutamate receptor 5 signaling and its downstream cognates rasrelated C3 botulinum toxin substrate 1 , amyloid beta A4 precursor protein, striatal-enriched protein tyrosine phosphatase, and homer 1 , in autism: a postmortem study in cerebellar vermis and superior frontal cortex. Mol Autism. 2013;4(1):21. doi: 10.1186/2040-2392-4-21. PMID: 23803181.X-1

1653. Favre MR, Barkat TR, Lamendola D, et al. General developmental health in the VPArat model of autism. Front Behav Neurosci.
2013;7:88. doi: 10.3389/fnbeh.2013.00088. PMID: 23898245.X-1

1654. Feinberg E, Silverstein M, FerreiraCesar Z. Integrating mental health services for mothers of children with autism. Psychiatr Serv. 2013 Sep 1;64(9):930. doi:

10.1176/appi.ps.640603. PMID: 24026841.X-1

1655. Fereshtehnejad SM, Religa D, Westman E, et al. Demography, diagnostics, and medication in dementia with Lewy bodies and Parkinson's disease with dementia: data from the Swedish Dementia Quality Registry (SveDem). Neuropsychiatr Dis Treat. 2013;9:927-35. doi: 10.2147/ndt.s45840. PMID: 23847419.X-1

1656. Fernandez-Jaen A, FernandezMayoralas DM, Calleja-Perez B, et al. Efficacy of atomoxetine for the treatment of ADHD symptoms in patients with pervasive developmental disorders: a prospective, openlabel study. J Atten Disord. 2013

Aug;17(6):497-505. doi: 10.1177/1087054711423626. PMID: 22366240.X-1, X-3

1657. Fernell E, Eriksson MA, Gillberg C. Early diagnosis of autism and impact on prognosis: a narrative review. Clin Epidemiol. 2013;5:33-43. doi: 10.2147/clep.s41714. PMID: 23459124.X-1, X-2

1658. Fernell E, Landgren M, Lindstrom K, et al. [Children and young people with neurodevelopmental problems: Support and efforts must be given even if not all diagnostic criteria are met]. Lakartidningen. 2013 Sep 1824;110(38):1674. PMID: 24199445.X-1

1659. Finucane B, Haas-Givler B, Simon EW. Knowledge and perceptions about fragile $\mathrm{X}$ syndrome: implications for diagnosis, intervention, and research. Intellect Dev Disabil. 2013 Aug;51(4):226-36. doi: 10.1352/1934-9556-51.4.226. PMID: 23909584.X-1

1660. Fisher WW, Rodriguez NM, Owen TM. Functional assessment and treatment of perseverative speech about restricted topics in an adolescent with Asperger syndrome. J Appl Behav Anal. 2013 Spring;46(1):307-11. doi: 10.1002/jaba.19. PMID: 24114104.X-1 
1661. Fitzpatrick P, Diorio R, Richardson $\mathrm{MJ}$, et al. Dynamical methods for evaluating the time-dependent unfolding of social coordination in children with autism. Front Integr Neurosci. 2013;7:21. doi: 10.3389/fnint.2013.00021. PMID: 23580133.X-1

1662. Flechas J. Jorge Flechas, MD: the potential of oxytocin, nitric oxide, and iodine. Altern Ther Health Med. 2013 JulAug;19(4):50-6. PMID: 23981372.X-1, X-2

1663. Foster L, Dunn W, Lawson LM. Coaching mothers of children with autism: a qualitative study for occupational therapy practice. Phys Occup Ther Pediatr. 2013 May;33(2):253-63. doi: 10.3109/01942638.2012.747581. PMID: 23253014.X-1

1664. Foxx RM. The maintenance of behavioral change: the case for long-term follow-ups. Am Psychol. 2013 Nov;68(8):72836. doi: 10.1037/a0033713. PMID: 24320663.X-1, X-2

1665. Frances A, Batstra L. Why so many epidemics of childhood mental disorder? J Dev Behav Pediatr. 2013 May;34(4):291-2. doi: 10.1097/DBP.0b013e31829425f5. PMID: 23669874.X-1

1666. Franco JH, Davis BL, Davis JL. Increasing social interaction using prelinguistic milieu teaching with nonverbal school-age children with autism. Am J Speech Lang Pathol. 2013 Aug;22(3):489-502. doi: 10.1044/1058-0360(2012/10-0103). PMID: 23813208.X-3, X-4

1667. Freitag CM, Cholemkery H, Elsuni L, et al. The group-based social skills training SOSTA-FRA in children and adolescents with high functioning autism spectrum disorder-study protocol of the randomised, multi-centre controlled SOSTA--net trial. Trials. 2013;14:6. doi: 10.1186/1745-6215-14-6. PMID: 23289935.X-2

1668. Frenette P, Dodds L, MacPherson K, et al. Factors affecting the age at diagnosis of autism spectrum disorders in Nova Scotia, Canada. Autism. 2013 Mar;17(2):184-95. doi: 10.1177/1362361311413399. PMID: 21788254.X-1
1669. Frye RE, DeLatorre R, Taylor HB, et al. Metabolic effects of sapropterin treatment in autism spectrum disorder: a preliminary study. Transl Psychiatry. 2013;3:e237. doi: 10.1038/tp.2013.14. PMID: 23462988.X-3, X-4

1670. Frye RE, Melnyk S, Fuchs G, et al. Effectiveness of methylcobalamin and folinic Acid treatment on adaptive behavior in children with autistic disorder is related to glutathione redox status. Autism Res Treat. 2013;2013:609705. doi: 10.1155/2013/609705. PMID: 24224089.X-3

1671. Frye RE, Sequeira JM, Quadros EV, et al. Cerebral folate receptor autoantibodies in autism spectrum disorder. Mol Psychiatry. 2013 Mar;18(3):369-81. doi: 10.1038/mp.2011.175. PMID: 22230883.X-1, X-3

1672. Gal E, Ben Meir A, Katz N. Development and reliability of the Autism Work Skills Questionnaire (AWSQ). Am J Occup Ther. 2013 Jan-Feb;67(1):e1-5. doi: 10.5014/ajot.2013.005066. PMID: 23245792.X-1

1673. Gan SM, Tung LC, Yeh CY, et al. ICF-CY based assessment tool for children with autism. Disabil Rehabil. 2013 Apr;35(8):67885. doi: 10.3109/09638288.2012.705946. PMID: 22897818.X-1, X-3

1674. Ganz JB, Goodwyn FD, Boles MM, et al. Impacts of a PECS instructional coaching intervention on practitioners and children with autism. Augment Altern Commun. 2013 Sep;29(3):210-21. doi: 10.3109/07434618.2013.818058. PMID: 23952566.X-1

1675. Garcia-Penas JJ, Carreras-Saaez I. [Autism, epilepsy and tuberous sclerosis complex: a functional model linked to mTOR pathway]. Rev Neurol. 2013 Feb 22;56 Suppl 1:S153-61. PMID: 23446718.X-1

1676. Gay V, Leijdekkers P, Wong F. Using sensors and facial expression recognition to personalize emotion learning for autistic children. Stud Health Technol Inform. 2013;189:71-6. PMID: 23739360.X-5

1677. Geier DA, Hooker BS, Kern JK, et al. A two-phase study evaluating the relationship between Thimerosal-containing vaccine 
administration and the risk for an autism spectrum disorder diagnosis in the United States. Transl Neurodegener. 2013;2(1):25. doi: 10.1186/2047-9158-2-25. PMID: 24354891.X1

1678. Geier DA, Kern JK, Geier MR. A Comparison of the Autism Treatment Evaluation Checklist (ATEC) and the Childhood Autism Rating Scale (CARS) for the Quantitative Evaluation of Autism. J Ment Health Res Intellect Disabil. 2013 Oct;6(4):25567. doi: 10.1080/19315864.2012.681340.

PMID: 23914277.X-1

1679. Gelder Bd, Stienen BMC, Stock JVd. Emotions by ear and by eye. Integrating face and voice in person perception.: Springer Science + Business Media, New York, NY; 2013:253-68.

1680. Gentile I, Bravaccio C, Bonavolta R, et al. Response to measles-mumps-rubella vaccine in children with autism spectrum disorders. In Vivo. 2013 May-Jun;27(3):377-82. PMID: 23606694.X-1

1681. Georgescu AL, Kuzmanovic B, Schilbach L, et al. Neural correlates of "social gaze" processing in high-functioning autism under systematic variation of gaze duration. Neuroimage Clin. 2013;3:340-51. doi: 10.1016/j.nicl.2013.08.014. PMID: 24273718.X-1

1682. Ghezzo A, Visconti P, Abruzzo PM, et al. Oxidative Stress and Erythrocyte Membrane Alterations in Children with Autism: Correlation with Clinical Features. PLoS One. 2013;8(6):e66418. doi: 10.1371/journal.pone.0066418. PMID: 23840462.X-1

1683. Giallo R, Wood CE, Jellett R, et al. Fatigue, wellbeing and parental self-efficacy in mothers of children with an autism spectrum disorder. Autism. 2013 Jul;17(4):465-80. doi: 10.1177/1362361311416830. PMID: 21788255.X-1

1684. Gibbard CR, Ren J, Seunarine KK, et al. White matter microstructure correlates with autism trait severity in a combined clinicalcontrol sample of high-functioning adults. Neuroimage Clin. 2013;3:106-14. doi: 10.1016/j.nicl.2013.07.007. PMID:

24179854.X-1

1685. Gillies D, Carroll L, Loos M. Commentary on 'Social skills groups for people aged 6 to 21 with autism spectrum disorders (ASD)'. Evid Based Child Health. 2013 Mar 7;8(2):316-7. doi: 10.1002/ebch.1902. PMID: 23877885.X-1

1686. Goldknopf EJ. Atypical resource allocation may contribute to many aspects of autism. Front Integr Neurosci. 2013;7:82. doi: 10.3389/fnint.2013.00082. PMID: 24421760.X1

1687. Golubchik P, Sever J, Weizman A. Reboxetine treatment for autistic spectrum disorder of pediatric patients with depressive and inattentive/hyperactive symptoms: an openlabel trial. Clin Neuropharmacol. 2013 MarApr;36(2):37-41. doi: 10.1097/WNF.0b013e31828003c1. PMID: 23503544.X-3, X-4

1688. Gonzalez C, Martin J, Minshew N, et al. Practice Makes Improvement: How Adults with Autism Out-Perform Others in a Naturalistic Visual Search Task. Journal of Autism \& Developmental Disorders. 2013;43(10):2259-68 10p. doi: 10.1007/s10803-013-1772-4. PMID: 104226063. Language: English. Entry Date: 20130921. Revision Date: 20150711. Publication Type: Journal Article.X-1

1689. Goods KS, Ishijima E, Chang YC, et al. Preschool based JASPER intervention in minimally verbal children with autism: pilot RCT. J Autism Dev Disord. 2013 May;43(5):1050-6. doi: 10.1007/s10803-0121644-3. PMID: 22965298.X-1

1690. Goral M, Conner PS. Language Disorders in Multilingual and Multicultural Populations. Annu Rev Appl Linguist. 2013 Mar;33:128-61. doi: 10.1017/s026719051300010x. PMID: 26257455.X-1

1691. Gordon I, Vander Wyk BC, Bennett $\mathrm{RH}$, et al. Oxytocin enhances brain function in children with autism. Proc Natl Acad Sci U S A. 2013 Dec 24;110(52):20953-8. doi: 10.1073/pnas.1312857110. PMID: 24297883.X-3, X-4 
1692. Gowen E, Hamilton A. Motor Abilities in Autism: A Review Using a Computational Context. Journal of Autism \& Developmental Disorders. 2013;43(2):323-44 22p. doi: 10.1007/s10803-012-1574-0. PMID: 104296934. Language: English. Entry Date: 20130128. Revision Date: 20150711.

Publication Type: Journal Article.X-1

1693. Graf-Myles J, Farmer C, Thurm A, et al. Dietary adequacy of children with autism compared with controls and the impact of restricted diet. J Dev Behav Pediatr. 2013 Sep;34(7):449-59. doi: 10.1097/DBP.0b013e3182a00d17. PMID: 24042076.X-1

1694. Granovetter M. Let's talk therapy: treatments for children with autism. Lancet. 2013 Aug 31;382(9894):753. doi: 10.1016/s0140-6736(13)61677-3. PMID: 23993177.X-1

1695. Grant R, Nozyce M. Proposed changes to the American Psychiatric Association diagnostic criteria for autism spectrum disorder: implications for young children and their families. Matern Child Health J. 2013 May;17(4):586-92. doi: 10.1007/s10995-0131250-9. PMID: 23456348.X-1

1696. Grecucci A, Brambilla P, Siugzdaite $\mathrm{R}$, et al. Emotional Resonance Deficits in Autistic Children. Journal of Autism \& Developmental Disorders. 2013;43(3):616-28 13p. doi: 10.1007/s10803-012-1603-z. PMID: 104318612. Language: English. Entry Date: 20130225. Revision Date: 20150711. Publication Type: Journal Article.X-1

1697. Green J, Wan MW, Guiraud J, et al. Intervention for infants at risk of developing autism: a case series. J Autism Dev Disord. 2013 Nov;43(11):2502-14. doi: 10.1007/s10803-013-1797-8. PMID: 23532347.X-1, X-3

1698. Greener M. Research roundup: June 2013. Nurse Prescribing. 2013;11(6):270-1 2p. PMID: 104204790. Language: English. Entry Date: 20130802. Revision Date: 20150818. Publication Type: Journal Article.X-1

1699. Green-Hennessy S. Coverage of mental health and substance misuse topics in the Cochrane review system. Epidemiol Psychiatr Sci. 2013 Jun;22(2):155-62. doi: 10.1017/s2045796012000455. PMID: 23089060.X-1

1700. Grether JK, Qian Y, Croughan MS, et al. Is infertility associated with childhood autism? J Autism Dev Disord. 2013 Mar;43(3):663-72. doi: 10.1007/s10803-0121598-5. PMID: 22777105.X-1

1701. Grigg-Damberger M, Ralls F. Treatment strategies for complex behavioral insomnia in children with neurodevelopmental disorders. Current Opinion in Pulmonary Medicine. 2013;19(6):616-25 10p. doi: 10.1097/MCP.0b013e328365ab89. PMID: 107913575. Language: English. Entry Date: 20140613. Revision Date: 20150712. Publication Type: Journal Article.X-1

1702. Grossman M, Peskin J, San Juan V. Thinking About a Reader's Mind: Fostering Communicative Clarity in the Compositions of Youth with Autism Spectrum Disorders. Journal of Autism \& Developmental Disorders. 2013;43(10):2376-92 17p. doi: 10.1007/s10803-013-1786-y. PMID: 104226069. Language: English. Entry Date: 20130921. Revision Date: 20150711. Publication Type: Journal Article.X-1, X-2

1703. Grow L, LeBlanc L. Teaching receptive language skills: recommendations for instructors. Behav Anal Pract. 2013 Spring;6(1):56-75. PMID: 25729507.X-1

1704. Gupta N, Saxena RK, Juneja R, et al. Effectiveness of Homeopathy in Four Autism Spectrum Disorder Cases. Homoeopathic Links. 2013 Winter2013;26(4):256-61 6p. PMID: 104171033. Language: English. Entry Date: 20131213. Revision Date: 20150711.

Publication Type: Journal Article.X-1, X-2, X3, X-4

1705. Haddad J. Social skills for teenagers with developmental and autism spectrum disorders: The PEERS treatment manual. J Child Adolesc Ment Health. 2013;25(1):93-4. doi: 10.2989/17280583.2013.802447. PMID: 25860311.X-1

1706. Haines C. SILENTLY SEIZING. Exceptional Parent. 2013;43(3):52-5 4p. PMID: 104195971. Language: English. Entry 
Date: 20130723. Revision Date: 20150711. Publication Type: Journal Article.X-1, X-2

1707. Hall R. ACCOMMODATING. Exceptional Parent. 2013;43(11):49-51 3p. PMID: 103947986. Language: English. Entry Date: 20140530. Revision Date: 20150710. Publication Type: Journal Article.X-1

1708. Hamade A, Salameh P, MedlejHashim M, et al. Autism in children and correlates in Lebanon: a pilot case-control study. J Res Health Sci. 2013 SummerAutumn;13(2):119-24. PMID: 24077467.X-1

1709. Hamlin JC, Pauly M, Melnyk S, et al. Dietary intake and plasma levels of choline and betaine in children with autism spectrum disorders. Autism Res Treat. 2013;2013:578429. doi: 10.1155/2013/578429. PMID: 24396597.X-1

1710. Handen BL, Johnson CR, Butter EM, et al. Use of a Direct Observational Measure in a Trial of Risperidone and Parent Training in Children with Pervasive Developmental Disorders. J Dev Phys Disabil. 2013 Jun 1;25(3):355-71. doi: 10.1007/s10882-012-9316y. PMID: 23730123.X-1

1711. Hansel K. Rethinking insurance coverage of "experimental" Applied Behavioral Analysis therapy and its usefulness in combating autism spectrum disorder. J Leg Med. 2013;34(2):215-33. doi: 10.1080/01947648.2013.800798. PMID: 23980747.X-1

1712. Hardy MW, Lagasse AB. Rhythm, movement, and autism: using rhythmic rehabilitation research as a model for autism. Front Integr Neurosci. 2013;7:19. doi: 10.3389/fnint.2013.00019. PMID: 23543915.X$1, \mathrm{X}-2$

1713. Harper A, Taylor Dyches T, Harper J, et al. Respite care, marital quality, and stress in parents of children with autism spectrum disorders. J Autism Dev Disord. 2013 Nov;43(11):2604-16. doi: 10.1007/s10803-0131812-0. PMID: 23529841.X-1

1714. Harstad E, Huntington N, Bacic J, et al. Disparity of care for children with parentreported autism spectrum disorders. Acad Pediatr. 2013 Jul-Aug;13(4):334-9. doi: 10.1016/j.acap.2013.03.010. PMID: 23830019.X-1

1715. Hasegawa N, Yamamoto Y. [Autism spectrum disorders in elderly]. Nihon Rinsho. 2013 Oct;71(10):1847-52. PMID: 24261218.X1

1716. Hassanpour S, O'Connor MJ, Das AK. A semantic-based method for extracting concept definitions from scientific publications: evaluation in the autism phenotype domain. $\mathrm{J}$ Biomed Semantics. 2013;4(1):14. doi: 10.1186/2041-1480-4-14. PMID: 23937724.X1

1717. Hazen EP, McDougle CJ, Volkmar FR. Changes in the diagnostic criteria for autism in DSM-5: controversies and concerns. J Clin Psychiatry. 2013 Jul;74(7):739-40. doi: 10.4088/JCP.13ac08550. PMID: 23945452.X-1

1718. Hellinckx T, Roeyers H, Van Waelvelde H. Predictors of handwriting in children with autism spectrum disorder. Research in Autism Spectrum Disorders. 2013 2015-12-09;7(1):176-86. doi: 10.5014/ajot.57.5.550 http://dx.doi.org/10.1016/j.rasd.2012.08.009. PMID: 1267033866; 2012-29769-020.X-1

1719. Hetzroni OE, Ne'eman A. Influence of colour on acquisition and generalisation of graphic symbols. J Intellect Disabil Res. 2013 Jul;57(7):669-80. doi: 10.1111/j.13652788.2012.01584.x. PMID: 22676327.X-3, X-4

1720. Hildebrandt H, Fink F, Kastrup A, et al. Cognitive profiles of patients with mild cognitive impairment or dementia in Alzheimer's or Parkinson's disease. Dement Geriatr Cogn Dis Extra. 2013 Jan;3(1):102-12. doi: 10.1159/000348350. PMID: 23637703.X-1

1721. Hill-Yardin EL, Hannan AJ. Translating preclinical environmental enrichment studies for the treatment of autism and other brain disorders: Comment on Woo and Leon (2013). Behavioral Neuroscience. 2013 2015-12-09;127(4):606-9. doi: http://dx.doi.org/10.1037/a0033319. PMID: 1413439757; 2013-26343-005.X-1

1722. Himuro N, Kozuka N, Mori M. Measurement of family-centred care: translation, adaptation and validation of the 
Measure of Processes of Care (MPOC-56 and 20) for use in Japan. Child Care Health Dev. 2013 May;39(3):358-65. doi: 10.1111/j.13652214.2012.01371.x. PMID: 22372945.X-1

1723. Hinckson EA, Dickinson A, Water T, et al. Physical activity, dietary habits and overall health in overweight and obese children and youth with intellectual disability or autism. Res Dev Disabil. 2013 Apr;34(4):1170-8. doi: 10.1016/j.ridd.2012.12.006. PMID: 23400004.X-1

1724. Hinton R, Budimirovic DB, Marschik $\mathrm{PB}$, et al. Parental reports on early language and motor milestones in fragile $\mathrm{X}$ syndrome with and without autism spectrum disorders. Dev Neurorehabil. 2013;16(1):58-66. doi: 10.3109/17518423.2012.704414. PMID: 23249372.X-1

1725. Hirata S, Okuzumi H, Kitajima Y, et al. Speed and accuracy of motor and cognitive control in children with intellectual disabilities. International Journal of Developmental Disabilities. 2013;59(3):166-78 13p. doi: 10.1179/2047387712Y.0000000010. PMID: 104143658. Language: English. Entry Date: 20131025. Revision Date: 20150711. Publication Type: Journal Article.X-1

1726. Hodgetts S, Nicholas D, Zwaigenbaum L, et al. Parents' and professionals' perceptions of family-centered care for children with autism spectrum disorder across service sectors. Soc Sci Med. 2013 Nov;96:138-46. doi: 10.1016/j.socscimed.2013.07.012. PMID: 24034961.X-1

1727. Hodgetts S, Savage A, McConnell D. Experience and outcomes of stepping stones triple P for families of children with autism. Res Dev Disabil. 2013 Sep;34(9):2572-85. doi: 10.1016/j.ridd.2013.05.005. PMID: 23747943.X-3, X-4

1728. Holwerda A, van der Klink JJ, de Boer MR, et al. Predictors of sustainable work participation of young adults with developmental disorders. Res Dev Disabil. 2013 Sep;34(9):2753-63. doi: 10.1016/j.ridd.2013.05.032. PMID: 23792372.X-1

1729. Hong DS. Child and adolescent psychiatrists are often tasked with the challenge of treating patients in various contexts. J Am Acad Child Adolesc Psychiatry. 2013

Sep;52(9):885-6. doi: 10.1016/j.jaac.2013.06.014. PMID: 23972687.X-1

1730. Hosenbocus S, Chahal R. Amantadine: a review of use in child and adolescent psychiatry. J Can Acad Child Adolesc Psychiatry. 2013 Feb;22(1):55-60. PMID: 23390434.X-2, X-3

1731. Hosenbocus S, Chahal R. Memantine: a review of possible uses in child and adolescent psychiatry. J Can Acad Child Adolesc Psychiatry. 2013 May;22(2):166-71. PMID: 23667364.X-2, X-3

1732. Houghton K, Schuchard J, Lewis C, et al. Promoting child-initiated socialcommunication in children with autism: SonRise Program intervention effects. J Commun Disord. 2013 Sep-Dec;46(5-6):495-506. doi: 10.1016/j.jcomdis.2013.09.004. PMID: 24209427.X-3, X-4

1733. Hsieh DT, Jennesson MM, Thiele EA. Epileptic spasms in tuberous sclerosis complex. Epilepsy Res. 2013 Sep;106(1-2):200-10. doi: 10.1016/j.eplepsyres.2013.05.003. PMID: 23796861.X-1

1734. Hsieh YL, Lo JL. Occupational experiences and subjective well-being of mothers of children with ASD in Taiwan. Occup Ther Int. 2013 Mar;20(1):45-53. doi: 10.1002/oti.1339. PMID: 23147991.X-1

1735. Hua X, Thompson PM, Leow AD, et al. Brain growth rate abnormalities visualized in adolescents with autism. Hum Brain Mapp. 2013 Feb;34(2):425-36. doi: 10.1002/hbm.21441. PMID: 22021093.X-1

1736. Huang A, Seshadri K, Matthews TA, et al. Parental perspectives on use, benefits, and physician knowledge of complementary and alternative medicine in children with autistic disorder and attention-deficit/hyperactivity disorder. J Altern Complement Med. 2013 Sep;19(9):746-50. doi: 10.1089/acm.2012.0640. PMID: 23621389.X-1, X-3

1737. Hurt AA, Grist CL, Malesky LA, Jr., et al. Personality traits associated with occupational 'burnout' in ABA therapists. J 
Appl Res Intellect Disabil. 2013 Jul;26(4):299308. doi: 10.1111/jar.12043. PMID:

23505135.X-1

1738. Huskens B, Verschuur R, Gillesen J, et al. Promoting question-asking in school-aged children with autism spectrum disorders: effectiveness of a robot intervention compared to a human-trainer intervention. Dev Neurorehabil. 2013 Oct;16(5):345-56. doi: 10.3109/17518423.2012.739212. PMID: 23586852.x-4

1739. Ichikawa K, Takahashi Y, Ando M, et al. TEACCH-based group social skills training for children with high-functioning autism: a pilot randomized controlled trial. Biopsychosoc Med. 2013;7(1):14. doi: 10.1186/1751-0759-714. PMID: 24083413.X-4

1740. Imbrici P, Camerino DC, Tricarico D. Major channels involved in neuropsychiatric disorders and therapeutic perspectives. Front Genet. 2013;4:76. doi: 10.3389/fgene.2013.00076. PMID: 23675382.X-1

1741. Ingersoll B, Wainer A. Initial efficacy of project ImPACT: a parent-mediated social communication intervention for young children with ASD. J Autism Dev Disord. 2013 Dec;43(12):2943-52. doi: 10.1007/s10803-0131840-9. PMID: 23689760.X-3, X-4

1742. Ingersoll BR, Wainer AL. Pilot study of a school-based parent training program for preschoolers with ASD. Autism. 2013 Jul;17(4):434-48. doi: 10.1177/1362361311427155. PMID: 22087044.X-3

1743. Ishitobi M, Kosaka H, Takahashi T, et al. Effectiveness and tolerability of switching to aripiprazole from risperidone in subjects with autism spectrum disorders: a prospective openlabel study. Clin Neuropharmacol. 2013 SepOct;36(5):151-6. doi: 10.1097/WNF.0b013e3182a31ec0. PMID: 24045605.X-1, X-3, X-4

1744. Jackson BL, Blackwood EM, Blum J, et al. Magno- and Parvocellular Contrast Responses in Varying Degrees of Autistic Trait. PLoS One. 2013;8(6):e66797. doi: 10.1371/journal.pone.0066797. PMID: 23824955.X-1
1745. Jain R, Juneja M, Sairam S. Children with developmental disabilities in India: age of initial concern and referral for rehabilitation services, and reasons for delay in referral. J Child Neurol. 2013 Apr;28(4):455-60. doi: 10.1177/0883073812447685. PMID: 22752480.X-1

1746. Jeans LM, Santos RM, Laxman DJ, et al. Early Predictors of ASD in Young Children Using a Nationally Representative Data Set. Journal of Early Intervention. 2013;35(4):30331 29p. doi: 10.1177/1053815114523319. PMID: 103895897. Language: English. Entry Date: 20140923. Revision Date: 20150818. Publication Type: Journal Article.X-1

1747. Jerrell JM, Hrisko S. Utility of Two PANSS 5-Factor Models for Assessing Psychosocial Outcomes in Clinical Programs for Persons with Schizophrenia. Schizophr Res Treatment. 2013;2013:705631. doi: 10.1155/2013/705631. PMID: 24381761.X-1

1748. Johnson CR, Turner KS, Foldes E, et al. Behavioral parent training to address sleep disturbances in young children with autism spectrum disorder: a pilot trial. Sleep Med. 2013 Oct;14(10):995-1004. doi: 10.1016/j.sleep.2013.05.013. PMID: 23993773.X-1

1749. Johnson NL, Simpson PM. Lack of father involvement in research on children with autism spectrum disorder: maternal parenting stress and family functioning. Issues Ment Health Nurs. 2013 Apr;34(4):220-8. doi: 10.3109/01612840.2012.745177. PMID: 23566184.X-1

1750. Jones RM, Cadby G, Melton PE, et al. Genome-wide association study of autistic-like traits in a general population study of young adults. Front Hum Neurosci. 2013;7:658. doi: 10.3389/fnhum.2013.00658. PMID: 24133439.X-1

1751. Jordan K, King M, Hellersteth S, et al. Feasibility of using a humanoid robot for enhancing attention and social skills in adolescents with autism spectrum disorder. Int J Rehabil Res. 2013 Sep;36(3):221-7. doi: 10.1097/MRR.0b013e32835d0b43. PMID: 23328816.X-1 
1752. Joshi G, Wozniak J, Petty C, et al. Psychiatric comorbidity and functioning in a clinically referred population of adults with autism spectrum disorders: a comparative study. J Autism Dev Disord. 2013 Jun;43(6):1314-25. doi: 10.1007/s10803-012-1679-5. PMID: 23076506.X-1

1753. Jung NH, Janzarik WG, Delvendahl I, et al. Impaired induction of long-term potentiation-like plasticity in patients with highfunctioning autism and Asperger syndrome. Dev Med Child Neurol. 2013 Jan;55(1):83-9. doi: 10.1111/dmcn.12012. PMID: 23157428.X1

1754. Justice MJ, Buchovecky CM, Kyle $\mathrm{SM}$, et al. A role for metabolism in Rett syndrome pathogenesis: New clinical findings and potential treatment targets. Rare Dis. 2013;1:e27265. doi: 10.4161/rdis.27265. PMID: 25003017.X-1

1755. Kadam SD, French BM, Kim ST, et al. Altered postnatal cell proliferation in brains of mouse pups prenatally exposed to IgG from mothers of children with autistic disorder. J Exp Neurosci. 2013;7:93-9. doi:

10.4137/jen.s12979. PMID: 25157212.X-1

1756. Kaf WA, Danesh AA. Distortionproduct otoacoustic emissions and contralateral suppression findings in children with Asperger's Syndrome. Int J Pediatr Otorhinolaryngol. 2013 Jun;77(6):947-54. doi: 10.1016/j.ijporl.2013.03.014. PMID: 23562236.X-1

1757. Kaiser AP, Roberts MY. Parentimplemented enhanced milieu teaching with preschool children who have intellectual disabilities. J Speech Lang Hear Res. 2013 Feb;56(1):295-309. doi: 10.1044/10924388(2012/11-0231). PMID: 22744141.X-1

1758. Kandalaft MR, Didehbani N, Krawczyk DC, et al. Virtual reality social cognition training for young adults with highfunctioning autism. J Autism Dev Disord. 2013 Jan;43(1):34-44. doi: 10.1007/s10803-0121544-6. PMID: 22570145.X-1

1759. Kang S, O'Reilly M, Rojeski L, et al. Effects of tangible and social reinforcers on skill acquisition, stereotyped behavior, and task engagement in three children with autism spectrum disorders. Res Dev Disabil. 2013

Feb;34(2):739-44. doi:

10.1016/j.ridd.2012.10.007. PMID:

23220050.X-1, X-4

1760. Karanth P, Chandhok TS. Impact of early intervention on children with autism spectrum disorders as measured by inclusion and retention in mainstream schools. Indian $\mathrm{J}$ Pediatr. 2013 Nov;80(11):911-9. doi: 10.1007/s12098-013-1014-y. PMID: 23686797.X-1

1761. Karlinsky H. The Horse Boy-attending to the stories our patients tell us. Acad Psychiatry. 2013 Jul 1;37(4):271-5. doi: 10.1176/appi.ap.13040048. PMID: 23820912.X-1

1762. Kasari C, Smith T. Interventions in schools for children with autism spectrum disorder: methods and recommendations. Autism. 2013 May;17(3):254-67. doi: 10.1177/1362361312470496. PMID: 23592848.X-1

1763. Kato K, Mikami K, Akama F, et al. Clinical features of suicide attempts in adults with autism spectrum disorders. Gen Hosp Psychiatry. 2013 Jan-Feb;35(1):50-3. doi: 10.1016/j.genhosppsych.2012.09.006. PMID: 23141028.X-1

1764. Kats D, Payne L, Parlier M, et al. Prevalence of selected clinical problems in older adults with autism and intellectual disability. J Neurodev Disord. 2013;5(1):27. doi: 10.1186/1866-1955-5-27. PMID: 24066979.X-1

1765. Keehn B, Wagner JB, Tager-Flusberg $\mathrm{H}$, et al. Functional connectivity in the first year of life in infants at-risk for autism: a preliminary near-infrared spectroscopy study. Front Hum Neurosci. 2013;7:444. doi: 10.3389/fnhum.2013.00444. PMID: 23964223.X-1

1766. Kelly A, Barnes-Holmes D. Implicit attitudes towards children with autism versus normally developing children as predictors of professional burnout and psychopathology. Res Dev Disabil. 2013 Jan;34(1):17-28. doi: 10.1016/j.ridd.2012.07.018. PMID: 22940155.X-1 
1767. Kenzer AL, Bishop MR, Wilke AE, et al. Including unfamiliar stimuli in preference assessments for young children with autism. J Appl Behav Anal. 2013 Fall;46(3):689-94. doi: 10.1002/jaba.56. PMID: 24114234.X-1, X-3, $\mathrm{X}-4$

1768. Kern JK, Garver CR, Mehta JA, et al. Prospective, blinded exploratory evaluation of the PlayWisely program in children with autism spectrum disorder. Yale J Biol Med. 2013 Jun;86(2):157-67. PMID: 23766737.X-3, X-4

1769. Kern P, Rivera NR, Chandler A, et al. Music therapy services for individuals with autism spectrum disorder: a survey of clinical practices and training needs. J Music Ther. 2013 Winter;50(4):274-303. doi: 10.1093/jmt/50.4.274. PMID: 25014668.X-1

1770. Kerr TM, Muller CL, Miah M, et al. Genetic background modulates phenotypes of serotonin transporter Ala56 knock-in mice. Mol Autism. 2013;4(1):35. doi: 10.1186/2040-23924-35. PMID: 24083388.X-1

1771. Key AP, Jones D, Dykens EM. Social and emotional processing in Prader-Willi syndrome: genetic subtype differences. J Neurodev Disord. 2013;5(1):7. doi: 10.1186/1866-1955-5-7. PMID: 23536992.X-1

1772. Khanna R, Jariwala K, Bentley JP. Health utility assessment using EQ-5D among caregivers of children with autism. Value Health. 2013 Jul-Aug;16(5):778-88. doi: 10.1016/j.jval.2013.04.007. PMID: 23947971.X-1

1773. Khanna R, Jariwala K, West-Strum D. Use and cost of psychotropic drugs among recipients with autism in a state Medicaid feefor-service programme. J Intellect Disabil Res. 2013 Feb;57(2):161-71. doi: 10.1111/j.13652788.2012.01563.x. PMID: 22471524.X-1

1774. Khorrami A, Tehrani-Doost M, Esteky H. Comparison between Face and Object Processing in Youths with Autism Spectrum Disorder: An event related potentials study. Iran J Psychiatry. 2013 Oct;8(4):179-87. PMID: 25628712.X-1

1775. Kim JW, Choi CS, Kim KC, et al. Gastrointestinal tract abnormalities induced by prenatal valproic Acid exposure in rat offspring.
Toxicol Res. 2013 Sep;29(3):173-9. doi: 10.5487/tr.2013.29.3.173. PMID: 24386517.X1

1776. Kim P, Park JH, Kwon KJ, et al. Effects of Korean red ginseng extracts on neural tube defects and impairment of social interaction induced by prenatal exposure to valproic acid. Food Chem Toxicol. 2013 Jan;51:288-96. doi: 10.1016/j.fct.2012.10.011. PMID: 23104247.X-1

1777. King D, Dockrell JE, Stuart M. Event narratives in 11-14 year olds with autistic spectrum disorder. Int J Lang Commun Disord. 2013 Sep-Oct;48(5):522-33. doi: 10.1111/14606984.12025. PMID: 24033651.X-1

1778. Kliemann D, Rosenblau G, Bolte S, et al. Face puzzle-two new video-based tasks for measuring explicit and implicit aspects of facial emotion recognition. Front Psychol. 2013;4:376. doi: 10.3389/fpsyg.2013.00376. PMID: 23805122.X-1

1779. Kodak T, Fisher WW, Paden A, et al. Evaluation of the utility of a discrete-trial functional analysis in early intervention classrooms. J Appl Behav Anal. 2013

Spring;46(1):301-6. doi: 10.1002/jaba.2. PMID: 24114103.X-1

1780. Koehler-Platten K, Grow LL, Schulze $\mathrm{KA}$, et al. Using a lag reinforcement schedule to increase phonemic variability in children with autism spectrum disorders. Anal Verbal Behav. 2013;29(1):71-83. PMID: 23814368.X-1, X-3, $\mathrm{X}-4$

1781. Koldewyn K, Jiang Y, Weigelt S, et al. Global/Local Processing in Autism: Not a Disability, but a Disinclination. Journal of Autism \& Developmental Disorders. 2013;43(10):2329-40 12p. doi: 10.1007/s10803-013-1777-z. PMID: 104226068. Language: English. Entry Date: 20130921. Revision Date: 20150711. Publication Type: Journal Article.X-1

1782. Kouijzer ME, van Schie HT, Gerrits BJ, et al. Is EEG-biofeedback an effective treatment in autism spectrum disorders? A randomized controlled trial. Appl Psychophysiol Biofeedback. 2013 Mar;38(1):17-28. doi: 10.1007/s10484-0129204-3. PMID: 22903518.X-1 
1783. Krieger AE, Saias T, Adrien JL. [Promoting family-professional partnership in institutions for children with autism]. Encephale. 2013 Apr;39(2):130-6. doi: 10.1016/j.encep.2012.06.002. PMID: 23095583.X-1

1784. Kryzak LA, Bauer S, Jones EA, et al. Increasing responding to others' joint attention directives using circumscribed interests. J Appl Behav Anal. 2013 Fall;46(3):674-9. doi: 10.1002/jaba.73. PMID: 24114231.X-3, X-4

1785. Kuroda M, Kawakubo Y, Kuwabara $\mathrm{H}$, et al. A cognitive-behavioral intervention for emotion regulation in adults with highfunctioning autism spectrum disorders: study protocol for a randomized controlled trial. Trials. 2013;14:231. doi: 10.1186/1745-621514-231. PMID: 23880333.X-1

1786. Kutchko KM, Frohlich F. Emergence of metastable state dynamics in interconnected cortical networks with propagation delays. PLoS Comput Biol. 2013 Oct;9(10):e1003304. doi: 10.1371/journal.pcbi.1003304. PMID: 24204238.X-1

1787. Lahav O, Apter A, Ratzon NZ. Psychological adjustment and levels of self esteem in children with visual-motor integration difficulties influences the results of a randomized intervention trial. Res Dev Disabil. 2013 Jan;34(1):56-64. doi: 10.1016/j.ridd.2012.07.024. PMID: 22940159.X-1

1788. Lahiri U, Bekele E, Dohrmann E, et al. Design of a virtual reality based adaptive response technology for children with autism. IEEE Trans Neural Syst Rehabil Eng. 2013 Jan;21(1):55-64. doi: 10.1109/tnsre.2012.2218618. PMID: 23033333.X-1

1789. Lakhan R. The coexistence of psychiatric disorders and intellectual disability in children aged 3-18 years in the barwani district, India. ISRN Psychiatry. 2013;2013:875873. doi: 10.1155/2013/875873. PMID: 23738223.X-1

1790. Landry O, Parker A. A meta-analysis of visual orienting in autism. Front Hum Neurosci. 2013;7:833. doi: 10.3389/fnhum.2013.00833. PMID: 24367314.X-1

1791. Landy SH, Kaniecki RG, Taylor FR. Abstracts and Citations. Headache: The Journal of Head \& Face Pain. 2013;53(9):1504-14 11p. doi: 10.1111/head.12219. PMID: 104234097. Language: English. Entry Date: 20131008. Revision Date: 20150711. Publication Type: Journal Article.X-1

1792. Lane AE, Heathcock JC. Early Sensory-Motor Signs of Autism Spectrum Disorder: Implications for Clinical Practice. Developmental Disabilities Special Interest Section Quarterly. 2013;37(3):1-4 p. PMID: 107818334. Language: English. Entry Date: 20140926. Revision Date: 20150820.

Publication Type: Journal Article. Journal Subset: Allied Health.X-1

1793. Lang R, Davenport K, Britt C, et al. Functional analysis and treatment of diurnal bruxism. J Appl Behav Anal. 2013 Spring;46(1):322-7. doi: 10.1002/jaba.5. PMID: 24114107.X-3, X-4

1794. Langdon PE, Murphy GH, Clare IC, et al. An evaluation of the EQUIP treatment programme with men who have intellectual or other developmental disabilities. J Appl Res Intellect Disabil. 2013 Mar;26(2):167-80. doi: 10.1111/jar.12004. PMID: 23281218.X-1

1795. Langdon PE, Murphy GH, Wilson E, et al. Asperger syndrome and anxiety disorders (PAsSA) treatment trial: a study protocol of a pilot, multicentre, single-blind, randomised crossover trial of group cognitive behavioural therapy. BMJ Open. 2013;3(7)doi: 10.1136/bmjopen-2013-003449. PMID: 23901031.X-1, X-2, X-3

1796. Lange N, McDougle C. Help for the child with autism. Sci Am. 2013 Oct;309(4):727. PMID: 24137859.X-1, X-2

1797. Langone SR, Luiselli JK, Hamill J. Effects of Response Blocking and Programmed Stimulus Control on Motor Stereotypy: A Pilot Study. Child \& Family Behavior Therapy. 2013;35(3):249-55 7p. doi: 10.1080/07317107.2013.818906. PMID: 104222596. Language: English. Entry Date: 20130920. Revision Date: 20150711. Publication Type: Journal Article.X-1, X-3, X-4 
1798. Lanz TA, Guilmette E, Gosink MM, et al. Transcriptomic analysis of genetically defined autism candidate genes reveals common mechanisms of action. Mol Autism. 2013;4(1):45. doi: 10.1186/2040-2392-4-45. PMID: 24238429.X-1

1799. Lau NM, Green PH, Taylor AK, et al. Markers of Celiac Disease and Gluten Sensitivity in Children with Autism. PLoS One. 2013;8(6):e66155. doi:

10.1371/journal.pone.0066155. PMID: 23823064.X-1

1800. Lehti V, Brown AS, Gissler M, et al. Autism spectrum disorders in IVF children: a national case-control study in Finland. Hum Reprod. 2013 Mar;28(3):812-8. doi: 10.1093/humrep/des430. PMID: 23293220.X-1

1801. Lepper TL, Petursdottir AI, Esch BE. Effects of operant discrimination training on the vocalizations of nonverbal children with autism. J Appl Behav Anal. 2013 Fall;46(3):656-61. doi: 10.1002/jaba.55. PMID: 24114228.X-3, X4

1802. Levin-Decanini T, Maltman N, Francis SM, et al. Parental broader autism subphenotypes in ASD affected families: relationship to gender, child's symptoms, SSRI treatment, and platelet serotonin. Autism Res. 2013 Dec;6(6):621-30. doi: 10.1002/aur.1322. PMID: 23956104.X-1

1803. Lipina TV, Roder JC. Co-learning facilitates memory in mice: a new avenue in social neuroscience. Neuropharmacology. 2013 Jan;64:283-93. doi: 10.1016/j.neuropharm.2012.06.054. PMID: 22776545.X-1

1804. Lippman-Bell JJ, Rakhade SN, Klein PM, et al. AMPA receptor antagonist NBQX attenuates later-life epileptic seizures and autistic-like social deficits following neonatal seizures. Epilepsia. 2013 Nov;54(11):1922-32. doi: 10.1111/epi.12378. PMID: 24117347.X-1

1805. Liu KP, Wong D, Chung AC, et al. Effectiveness of a workplace training programme in improving social, communication and emotional skills for adults with autism and intellectual disability in Hong Kong--a pilot study. Occup Ther Int. 2013
Dec;20(4):198-204. doi: 10.1002/oti.1356. PMID: 23861094.X-1

1806. Liu WP, Wang J, Qu SQ, et al. [Transplantation of human neural precursor cells in the treatment of children with pervasive developmental disorder]. Zhongguo Dang Dai Er Ke Za Zhi. 2013 Oct;15(10):860-5. PMID: 24131839.X-1, X-3, X-4

1807. Liyanage VR, Zachariah RM, Rastegar $\mathrm{M}$. Decitabine alters the expression of Mecp2 isoforms via dynamic DNA methylation at the Mecp2 regulatory elements in neural stem cells. Mol Autism. 2013;4(1):46. doi: 10.1186/20402392-4-46. PMID: 24238559.X-1

1808. Lloyd M, MacDonald M, Lord C. Motor skills of toddlers with autism spectrum disorders. Autism: The International Journal of Research \& Practice. 2013;17(2):133-46 14p. doi: $10.1177 / 1362361311402230$. PMID: 104249014. Language: English. Entry Date: 20130321. Revision Date: 20150711. Publication Type: Journal Article.X-1

1809. Lloyd-Fox S, Blasi A, Elwell CE, et al. Reduced neural sensitivity to social stimuli in infants at risk for autism. Proc Biol Sci. 2013 May 7;280(1758):20123026. doi: 10.1098/rspb.2012.3026. PMID: 23486434.X-1

1810. Lopata C, Smith RA, Volker MA, et al. Comparison of Adaptive Behavior Measures for Children with HFASDs. Autism Res Treat. 2013;2013:415989. doi: 10.1155/2013/415989. PMID: 23819048.X-1

1811. Lopata C, Toomey JA, Fox JD, et al. Prevalence and Predictors of Psychotropic Use in Children with High-Functioning ASDs. Autism Res Treat. 2013;2013:384527. doi: 10.1155/2013/384527. PMID: 23762550.X-1

1812. Lord C, Jones RM. New strategies and findings for behavioral interventions in autism spectrum disorders. Ann N Y Acad Sci. 2013 Nov;1304:70-6. doi: 10.1111/nyas.12311. PMID: 24279894.X-1

1813. Louw KA, Bentley J, Sorsdahl K, et al. Prevalence and patterns of medication use in children and adolescents with autism spectrum disorders in the Western Cape, South Africa. J Child Adolesc Ment Health. 2013;25(1):69-79. 
doi: 10.2989/17280583.2013.767265. PMID: 25860309.X-1

1814. Lyalina S, Percha B, Lependu P, et al. Identifying phenotypic signatures of neuropsychiatric disorders from electronic medical records. Journal of the American Medical Informatics Association. 2013;20(e2):e297-305 1p. doi: 10.1136/amiajnl-2013-001933. PMID: 104121838. Language: English. Entry Date: 20140207. Revision Date: 20150710. Publication Type: Journal Article.X-1

1815. Lyall K, Munger KL, O'Reilly EJ, et al. Maternal dietary fat intake in association with autism spectrum disorders. Am J Epidemiol. 2013 Jul 15;178(2):209-20. doi: 10.1093/aje/kws433. PMID: 23813699.X-1

1816. Ma WJ, Hashii M, Munesue T, et al. Non-synonymous single-nucleotide variations of the human oxytocin receptor gene and autism spectrum disorders: a case-control study in a Japanese population and functional analysis. Mol Autism. 2013;4(1):22. doi: 10.1186/20402392-4-22. PMID: 23815867.X-1

1817. MacDonald JM, Ahearn WH, ParryCruwys D, et al. Persistence during extinction: examining the effects of continuous and intermittent reinforcement on problem behavior. J Appl Behav Anal. 2013 Spring;46(1):333-8. doi: 10.1002/jaba.3. PMID: 24114109.X-3, X-4

1818. MacDonald M, Lord C, Ulrich D. The relationship of motor skills and adaptive behavior skills in young children with autism spectrum disorders. Res Autism Spectr Disord. 2013 Nov 1;7(11):1383-90. doi: 10.1016/j.rasd.2013.07.020. PMID: 25774214.X-1

1819. MacDonald M, Lord C, Ulrich DA. The Relationship of Motor Skills and Social Communicative Skills in School-Aged Children With Autism Spectrum Disorder. Adapted Physical Activity Quarterly. 2013;30(3):271-82 12p. PMID: 107955351. Language: English. Entry Date: 20130816. Revision Date: 20150712. Publication Type: Journal Article.X1

1820. Maenner MJ, Smith LE, Hong J, et al. Evaluation of an activities of daily living scale for adolescents and adults with developmental disabilities. Disabil Health J. 2013 Jan;6(1):817. doi: 10.1016/j.dhjo.2012.08.005. PMID: 23260606.X-1

1821. Magana S, Lopez K, Aguinaga A, et al. Access to diagnosis and treatment services among latino children with autism spectrum disorders. Intellect Dev Disabil. 2013 Jun;51(3):141-53. doi: 10.1352/1934-955651.3.141. PMID: 23834211.X-1

1822. Malek-Ahmadi M, Kahlon V, Adler $\mathrm{CH}$, et al. Prevalence of Hippocampal Sclerosis in a Clinicopathologically Characterized Cohort. Clin Exp Med Sci. 2013;1(7):317-27. PMID: 25324686.X-1

1823. Mamidala MP, Polinedi A, Kumar PT, et al. Maternal hormonal interventions as a risk factor for Autism Spectrum Disorder: an epidemiological assessment from India. J Biosci. 2013 Dec;38(5):887-92. PMID: 24296891.X-1

1824. Mankoski R, Stockton G, Manos G, et al. Aripiprazole treatment of irritability associated with autistic disorder and the relationship between prior antipsychotic exposure, adverse events, and weight change. J Child Adolesc Psychopharmacol. 2013 Oct;23(8):572-6. doi: 10.1089/cap.2012.0075. PMID: 24138011.X-2, X-3

1825. Manoach DS, Agam Y. Neural markers of errors as endophenotypes in neuropsychiatric disorders. Front Hum Neurosci. 2013;7:350. doi: 10.3389/fnhum.2013.00350. PMID: 23882201.X-1

1826. Mardh S. Weak central coherence in patients with Alzheimer's disease $(*)$. Neural Regen Res. 2013 Mar 15;8(8):760-6. doi: 10.3969/j.issn.1673-5374.2013.08.011. PMID: 25206723.X-1

1827. Margari L, Matera E, Craig F, et al. Tolerability and safety profile of risperidone in a sample of children and adolescents. Int Clin Psychopharmacol. 2013 Jul;28(4):177-83. doi: 10.1097/YIC.0b013e328362497b. PMID: 23689836.X-1, X-3

1828. Marshall J, Hill RJ, Dodrill P. A survey of practice for clinicians working with 
children with autism spectrum disorders and feeding difficulties. Int J Speech Lang Pathol. 2013 Jun;15(3):279-85. doi:

10.3109/17549507.2013.777972. PMID: 23577744.X-1

1829. Martin GE, Losh M, Estigarribia B, et al. Longitudinal profiles of expressive vocabulary, syntax and pragmatic language in boys with fragile $\mathrm{X}$ syndrome or Down syndrome. Int J Lang Commun Disord. 2013 Jul-Aug;48(4):432-43. doi: 10.1111/14606984.12019. PMID: 23889838.X-1

1830. Martins LZ, Fernandes FD. Short-term speech-language intervention for children with disorders of the autism spectrum. Codas. 2013;25(6):542-7. doi: 10.1590/s231717822014000100007. PMID: 24626980.X-1

1831. Masri AT, Al Suluh N, Nasir R. Diagnostic delay of autism in Jordan: review of 84 cases. Libyan J Med. 2013;8:21725. doi: 10.3402/ljm.v8i0.21725. PMID: 23962635.X-1

1832. Massand E, Bowler D, Mottron L, et al. ERP Correlates of Recognition Memory in Autism Spectrum Disorder...Event-Related Potential. Journal of Autism \& Developmental Disorders. 2013;43(9):2038-47 10p. doi: 10.1007/s10803-012-1755-x. PMID: 104210189. Language: English. Entry Date: 20130820. Revision Date: 20150711. Publication Type: Journal Article.X-1

1833. Mathersul D, McDonald S, Rushby JA. Psychophysiological correlates of social judgement in high-functioning adults with autism spectrum disorder. Int J Psychophysiol. 2013 Jan;87(1):88-94. doi:

10.1016/j.ijpsycho.2012.11.005. PMID: 23183316.X-1

1834. Mattard-Labrecque C, Ben Amor L, Couture MM. Children with Autism and Attention Difficulties: A Pilot Study of the Association between Sensory, Motor, and Adaptive Behaviors. J Can Acad Child Adolesc Psychiatry. 2013 May;22(2):139-46. PMID: 23667360.X-1

1835. Maxwell CR, Parish-Morris J, Hsin O, et al. The broad autism phenotype predicts child functioning in autism spectrum disorders. J Neurodev Disord. 2013;5(1):25. doi:
10.1186/1866-1955-5-25. PMID: 24053506.X1

1836. May T, Rinehart N, Wilding J, et al. The Role of Attention in the Academic Attainment of Children with Autism Spectrum Disorder. Journal of Autism \& Developmental Disorders. 2013;43(9):2147-58 12p. doi: 10.1007/s10803-013-1766-2. PMID: 104210187. Language: English. Entry Date: 20130820. Revision Date: 20150711. Publication Type: Journal Article.X-1

1837. Mazurek MO, Engelhardt CR. Video game use in boys with autism spectrum disorder, ADHD, or typical development. Pediatrics. 2013 Aug;132(2):260-6. doi: 10.1542/peds.2012-3956. PMID: 23897915.X-1

1838. McCabe H. Bamboo shoots after the rain: development and challenges of autism intervention in China. Autism. 2013

Sep;17(5):510-26. doi: 10.1177/1362361312436849. PMID: 23144167.X-1

1839. McCartney M. MMR, measles, and the South Wales Evening Post. Bmj. 2013;346:f2598. doi: 10.1136/bmj.f2598. PMID: 23610382.X-1

1840. McCary LM, Machlin L, Roberts JE. The Development of Adaptive Behavior in Toddlers and Preschoolers with Fragile X versus Autism. Int J Dev Disabil. 2013 Jul 1;59(2):67-79. doi: 10.1179/2047387713y.0000000016. PMID: 25191537.X-1

1841. McCary LM, Machlin L, Roberts JE. The development of adaptive behaviour in toddlers and preschoolers with fragile $\mathrm{X}$ versus autism. International Journal of Developmental Disabilities. 2013;59(2):67-79 13p. doi: 10.1179/2047387713Y.0000000016. PMID: 104172099. Language: English. Entry Date: 20130606. Revision Date: 20150711. Publication Type: Journal Article.X-1

1842. McCleery JP, Elliott NA, Sampanis DS, et al. Motor development and motor resonance difficulties in autism: relevance to early intervention for language and communication skills. Front Integr Neurosci. 2013;7:30. doi: 10.3389/fnint.2013.00030. PMID: 23630476.X-1, X-2 
1843. McGhan AC, Lerman DC. An assessment of error-correction procedures for learners with autism. J Appl Behav Anal. 2013 Fall;46(3):626-39. doi: 10.1002/jaba.65. PMID: 24114225.X-1, X-3, X-4

1844. McGonigle-Chalmers M, AldersonDay B, Fleming J, et al. Profound Expressive Language Impairment in Low Functioning Children with Autism: An Investigation of Syntactic Awareness Using a Computerised Learning Task. Journal of Autism \& Developmental Disorders. 2013;43(9):2062-81 20p. doi: 10.1007/s10803-012-1753-z. PMID: 104210177. Language: English. Entry Date: 20130820. Revision Date: 20150711. Publication Type: Journal Article.X-1

1845. McGregor KK, Rost G, Arenas R, et al. Children with ASD can use gaze in support of word recognition and learning. J Child Psychol Psychiatry. 2013 Jul;54(7):745-53. doi: 10.1111/jcpp.12073. PMID: 23574387.X-1

1846. McGuinness TM, Johnson K. DSM-5 changes in the diagnosis of autism spectrum disorder. J Psychosoc Nurs Ment Health Serv. 2013 Apr;51(4):17-9. doi: 10.3928/0279369520130220-01. PMID: 23445685.X-1

1847. McIntosh CE. A national survey exploring school nurses knowledge and experience when working with children with Autism Spectrum Disorders: Ball State University; 2013.

1848. McMahon CM, Lerner MD, Britton N. Group-based social skills interventions for adolescents with higher-functioning autism spectrum disorder: a review and looking to the future. Adolesc Health Med Ther. 2013 Jan 22;2013(4):23-8. doi: 10.2147/ahmt.s25402. PMID: 23956616.X-1, X-2

1849. McMahon CM, Vismara LA, Solomon M. Measuring changes in social behavior during a social skills intervention for higherfunctioning children and adolescents with autism spectrum disorder. J Autism Dev Disord. 2013 Aug;43(8):1843-56. doi: 10.1007/s10803012-1733-3. PMID: 23239098.X-1

1850. McNally Keehn RH, Lincoln AJ, Brown MZ, et al. The Coping Cat program for children with anxiety and autism spectrum disorder: a pilot randomized controlled trial. J Autism Dev Disord. 2013 Jan;43(1):57-67. doi: 10.1007/s10803-012-1541-9. PMID: 22588377.X-1

1851. Meador KJ, Loring DW. Prenatal valproate exposure is associated with autism spectrum disorder and childhood autism. Journal of Pediatrics. 2013;163(3):922-6 5p. doi: 10.1016/j.jpeds.2013.06.050. PMID: 104215877. Language: English. Entry Date: 20131129. Revision Date: 20150711. Publication Type: Journal Article.X-1

1852. Meador KJ, Loring DW. Risks of in utero exposure to valproate. JAMA: Journal of the American Medical Association. 2013;309(16):1730-1 2p. doi: 10.1001/jama.2013.4001. PMID: 108006671. Language: English. Entry Date: 20130510. Revision Date: 20150712. Publication Type: Journal Article.X-1

1853. Memari AH, Ghaheri B, Ziaee V, et al. Physical activity in children and adolescents with autism assessed by triaxial accelerometry. Pediatr Obes. 2013 Apr;8(2):150-8. doi: 10.1111/j.2047-6310.2012.00101.x. PMID: 23042790.X-1

1854. Memari AH, Shayestehfar M, Mirfazeli FS, et al. Cross-cultural adaptation, reliability, and validity of the autism treatment evaluation checklist in persian. Iran J Pediatr. 2013 Jun;23(3):269-75. PMID: 23795248.X-1

1855. Memari AH, Ziaee V, Shayestehfar M, et al. Cognitive flexibility impairments in children with autism spectrum disorders: links to age, gender and child outcomes. Res Dev Disabil. 2013 Oct;34(10):3218-25. doi: 10.1016/j.ridd.2013.06.033. PMID: 23886763.X-1

1856. Meola D, Huang Z, Petitto JM. Selective Neuronal and Brain Regional Expession of IL-2 in IL2P 8-GFP Transgenic Mice: Relation to Sensorimotor Gating. J Alzheimers Dis Parkinsonism. 2013 Oct 28;3(4):1000127. doi: 10.4172/21610460.1000127. PMID: 24563821.X-1

1857. Mercati O, Danckaert A, AndreLeroux G, et al. Contactin 4, -5 and -6 differentially regulate neuritogenesis while they display identical PTPRG binding sites. Biol 
Open. 2013 Mar 15;2(3):324-34. doi: 10.1242/bio.20133343. PMID: 23519440.X-1

1858. Miguel CF, Kobari-Wright VV. The effects of tact training on the emergence of categorization and listener behavior in children with autism. J Appl Behav Anal. 2013 Fall;46(3):669-73. doi: 10.1002/jaba.62. PMID: 24114230.X-3, X-4

1859. Miyajima T, Kumada T, Saito K, et al. Autism in siblings with autosomal dominant nocturnal frontal lobe epilepsy. Brain Dev. 2013 Feb;35(2):155-7. doi: 10.1016/j.braindev.2012.07.012. PMID: 22883468.X-1

1860. Miyake K, Yang C, Minakuchi Y, et al. Comparison of Genomic and Epigenomic Expression in Monozygotic Twins Discordant for Rett Syndrome. PLoS One.

2013;8(6):e66729. doi:

10.1371/journal.pone.0066729. PMID: 23805272.X-1

1861. Mohiuddin S, Ghaziuddin M. Psychopharmacology of autism spectrum disorders: A selective review. Autism: The International Journal of Research \& Practice. 2013;17(6):645-54 10p. doi: 10.1177/1362361312453776. PMID: 104150148. Language: English. Entry Date: 20131106. Revision Date: 20150711. Publication Type: Journal Article.X-1, X-2

1862. Monahan M, Classen S, Helsel PV. Pre-driving evaluation of a teen with attention deficit hyperactivity disorder and autism spectrum disorder. Can J Occup Ther. 2013 Feb;80(1):35-41. PMID: 23550495.X-1

1863. Monsey MS. Introduction: how dysregulation of mental processes and behaviors lead to pathology. Yale J Biol Med. 2013 Jun;86(2):115-6. PMID: 23923130.X-1

1864. Moran MF, Foley JT, Parker ME, et al. Two-legged hopping in autism spectrum disorders. Front Integr Neurosci. 2013;7:14. doi: 10.3389/fnint.2013.00014. PMID: 23532337.X-1

1865. Moss J, Oliver C, Nelson L, et al. Delineating the profile of autism spectrum disorder characteristics in Cornelia de Lange and Fragile X syndromes. Am J Intellect Dev
Disabil. 2013 Jan;118(1):55-73. doi: 10.1352/1944-7558-118.1.55. PMID: 23301903.X-1

1866. Moy SS, Nonneman RJ, Shafer GO, et al. Disruption of social approach by MK-801, amphetamine, and fluoxetine in adolescent C57BL/6J mice. Neurotoxicol Teratol. 2013 Mar-Apr;36:36-46. doi: 10.1016/j.ntt.2012.07.007. PMID: 22898204.X1

1867. Murdock LC, Ganz J, Crittendon J. Use of an iPad play story to increase play dialogue of preschoolers with Autism Spectrum Disorders. J Autism Dev Disord. 2013 Sep;43(9):2174-89. doi: 10.1007/s10803-0131770-6. PMID: 23371509.X-3, X-4

1868. Muskett T, Body R. The case for multimodal analysis of atypical interaction: questions, answers and gaze in play involving a child with autism. Clin Linguist Phon. 2013 Oct-Nov;27(10-11):837-50. doi: 10.3109/02699206.2013.816780. PMID: 24067142.X-1

1869. Naber FB, Poslawsky IE, van Ijzendoorn $\mathrm{MH}$, et al. Brief report: oxytocin enhances paternal sensitivity to a child with autism: a double-blind within-subject experiment with intranasally administered oxytocin. J Autism Dev Disord. 2013 Jan;43(1):224-9. doi: 10.1007/s10803-0121536-6. PMID: 22544470.X-1, X-4

1870. Narayanan S, Georgiou PG. Behavioral Signal Processing: Deriving Human Behavioral Informatics From Speech and Language: Computational techniques are presented to analyze and model expressed and perceived human behavior-variedly characterized as typical, atypical, distressed, and disordered-from speech and language cues and their applications in health, commerce, education, and beyond. Proc IEEE Inst Electr Electron Eng. 2013 Feb 7;101(5):1203-33. doi: 10.1109/jproc.2012.2236291. PMID: 24039277.X-1

1871. Narcisa V, Discenza M, Vaccari E, et al. Parental interest in a genetic risk assessment test for autism spectrum disorders. Clin Pediatr (Phila). 2013 Feb;52(2):139-46. doi: 10.1177/0009922812466583. PMID: 23193169.X-1 
1872. Naviaux RK, Zolkipli Z, Wang L, et al. Antipurinergic therapy corrects the autismlike features in the poly(IC) mouse model.

PLoS One. 2013;8(3):e57380. doi:

10.1371/journal.pone.0057380. PMID:

23516405.X-1

1873. Neubauer Mde A, Fernandes FD.

Functional Communication Profile and speechlanguage diagnosis in children of the autism spectrum: checklist use. Codas.

2013;25(6):605-9. doi: 10.1590/s2317-

17822014000100013. PMID: 24626986.X-1

1874. Neufeld J, Roy M, Zapf A, et al. Is synesthesia more common in patients with Asperger syndrome? Front Hum Neurosci. 2013;7:847. doi: 10.3389/fnhum.2013.00847. PMID: 24367321.X-1

1875. Nicolaidis C, Raymaker D, McDonald $\mathrm{K}$, et al. Comparison of healthcare experiences in autistic and non-autistic adults: a crosssectional online survey facilitated by an academic-community partnership. J Gen Intern Med. 2013 Jun;28(6):761-9. doi: 10.1007/s11606-012-2262-7. PMID: 23179969.X-1

1876. Nosik MR, Williams WL, Garrido N, et al. Comparison of computer based instruction to behavior skills training for teaching staff implementation of discrete-trial instruction with an adult with autism. Res Dev Disabil. 2013 Jan;34(1):461-8. doi: 10.1016/j.ridd.2012.08.011. PMID: 23041660.x-1

1877. Nurmi EL, Spilman SL, Whelan F, et al. Moderation of antipsychotic-induced weight gain by energy balance gene variants in the RUPP autism network risperidone studies.

Transl Psychiatry. 2013;3:e274. doi: 10.1038/tp.2013.26. PMID: 23799528.X-3

1878. Nylander L, Holmqvist M, Gustafson L, et al. Attention-deficit/hyperactivity disorder (ADHD) and autism spectrum disorder (ASD) in adult psychiatry. A 20-year register study. Nord J Psychiatry. 2013 Oct;67(5):344-50. doi: 10.3109/08039488.2012.748824. PMID: 23234539.X-1

1879. Occelli V, Esposito G, Venuti P, et al. The Takete-Maluma phenomenon in autism spectrum disorders. Perception.

2013;42(2):233-41. PMID: 23700961.X-1

1880. Odeberg H, Martensson B.

[Underlying factors should be examined in prolonged sick leave for depression]. Lakartidningen. 2013 Sep 4-10;110(36):1552-3. PMID: 24163911.X-1

1881. Oerlemans AM, Droste K, van Steijn DJ, et al. Co-segregation of Social Cognition, Executive Function and Local Processing Style in Children with ASD, their Siblings and Normal Controls. Journal of Autism \& Developmental Disorders. 2013;43(12):2764-78 15p. doi: 10.1007/s10803-013-1807-x. PMID: 104158486. Language: English. Entry Date: 20131113. Revision Date: 20150711. Publication Type: Journal Article.X-1

1882. Oguchi-Katayama A, Monma A, Sekino Y, et al. Comparative gene expression analysis of the amygdala in autistic rat models produced by pre- and post-natal exposures to valproic acid. J Toxicol Sci. 2013;38(3):391402. PMID: 23665938.X-1

1883. O'Hearn K, Franconeri S, Wright C, et al. The development of individuation in autism. J Exp Psychol Hum Percept Perform. 2013 Apr;39(2):494-509. doi: 10.1037/a0029400. PMID: 22963232.X-1, X-3

1884. Okuno H, Nagai T, Mohri I, et al. [Effectiveness of a modified parent training of smaller groups and shorter schedules for children with pervasive developmental disorders]. No To Hattatsu. 2013 Jan;45(1):2632. PMID: 23593742.X-3

1885. Olexova L, Senko T, Stefanik P, et al. Habituation of exploratory behaviour in VPA rats: animal model of autism. Interdiscip Toxicol. 2013 Dec;6(4):222-7. doi: 10.2478/intox-2013-0033. PMID: 24678262.X1

1886. Omori M, Yamamoto J. Stimulus pairing training for Kanji reading skills in students with developmental disabilities. Res Dev Disabil. 2013 Apr;34(4):1109-18. doi: 10.1016/j.ridd.2012.12.016. PMID: 23357673.X-1

1887. Onore CE, Careaga M, Babineau BA, et al. Inflammatory macrophage phenotype in 
BTBR T+tf/J mice. Front Neurosci. 2013;7:158. doi: 10.3389/fnins.2013.00158. PMID: 24062633.X-1

1888. Oranje B, Lahuis B, van Engeland H, et al. Sensory and sensorimotor gating in children with multiple complex developmental disorders (MCDD) and autism. Psychiatry Res. 2013 Apr 30;206(2-3):287-92. doi:

10.1016/j.psychres.2012.10.014. PMID: 23164481.X-1

1889. Owen JP, Marco EJ, Desai S, et al. Abnormal white matter microstructure in children with sensory processing disorders. Neuroimage Clin. 2013;2:844-53. doi: 10.1016/j.nicl.2013.06.009. PMID: 24179836.X-1

1890. Palmer CJ, Paton B, Hohwy J, et al. Movement under uncertainty: The effects of the rubber-hand illusion vary along the nonclinical autism spectrum. Neuropsychologia. 2013 2015-12-09;51(10):1942-51. doi: http://dx.doi.org/10.1016/j.neuropsychologia.20 13.06.020. PMID: 1433270056; 2013-29214019.X-1

1891. Pan WD, Yoshida S, Liu Q, et al. Quantitative evaluation of severity of behavioral and psychological symptoms of dementia in patients with vascular dementia. Transl Neurodegener. 2013;2(1):9. doi: 10.1186/2047-9158-2-9. PMID: 23607744.X-1

1892. Panagopoulos VN, Greene DJ, Campbell MC, et al. Towards objectively quantifying sensory hypersensitivity: a pilot study of the "Ariana effect". PeerJ. 2013;1:e121. doi: 10.7717/peerj.121. PMID: 23940834.X-1

1893. Pardo CA, Buckley A, Thurm A, et al. A pilot open-label trial of minocycline in patients with autism and regressive features. J Neurodev Disord. 2013;5(1):9. doi: 10.1186/1866-1955-5-9. PMID: 23566357.X-3

1894. Parellada M, Boada L, Moreno C, et al. Specialty Care Programme for autism spectrum disorders in an urban population: A case-management model for health care delivery in an ASD population. Eur Psychiatry. 2013 Feb;28(2):102-9. doi: 10.1016/j.eurpsy.2011.06.004. PMID: 21907549.X-1
1895. Parenteau RE, Luiselli JK, Keeley M. Direct and collateral effects of staff-worn protective equipment on injury prevention from child aggression. Dev Neurorehabil.

2013;16(1):73-7. doi:

10.3109/17518423.2012.700651. PMID: 23249373.X-1

1896. Parish-Morris J, Chevallier C, Tonge $\mathrm{N}$, et al. Visual attention to dynamic faces and objects is linked to face processing skills: a combined study of children with autism and controls. Front Psychol. 2013;4:185. doi: 10.3389/fpsyg.2013.00185. PMID: 23596436.X-1

1897. Park DH, Lim S, Park ES, et al. A nine-month-old boy with isodicentric chromosome 15: a case report. Ann Rehabil Med. 2013 Apr;37(2):291-4. doi: 10.5535/arm.2013.37.2.291. PMID: 23705128.X-1

1898. Parker W, Ollerton J. Evolutionary biology and anthropology suggest biome reconstitution as a necessary approach toward dealing with immune disorders. Evol Med Public Health. 2013 Jan;2013(1):89-103. doi: 10.1093/emph/eot008. PMID: 24481190.X-1

1899. Pascoe JM. Foreword. Curr Probl Pediatr Adolesc Health Care. 2013 Jan;43(1):1. doi: 10.1016/j.cppeds.2012.09.001. PMID: 23332396.X-1

1900. Pastrana SJ, Rapp JT, Frewing TM. Immediate and Subsequent Effects of Response Interruption and Redirection on Targeted and Untargeted Forms of Stereotypy. Behavior Modification. 2013;37(4):591-610 20p. doi: 10.1177/0145445513485751. PMID: 104178959. Language: English. Entry Date: 20130618. Revision Date: 20150711. Publication Type: Journal Article.X-1, X-3

1901. Patriquin MA, Lorenzi J, Scarpa A. Relationship between respiratory sinus arrhythmia, heart period, and caregiver-reported language and cognitive delays in children with autism spectrum disorders. Appl Psychophysiol Biofeedback. 2013 Sep;38(3):203-7. doi: 10.1007/s10484-013-9225-6. PMID: 23820819.X-1 
1902. Patten E, Baranek GT, Watson LR, et al. Child and family characteristics influencing intervention choices in autism spectrum disorders. Focus Autism Other Dev Disabl. 2013 Sep 1;28(3):138-46. doi: 10.1177/1088357612468028. PMID: 24089593.X-1

1903. Paul R, Campbell D, Gilbert K, et al. Comparing spoken language treatments for minimally verbal preschoolers with autism spectrum disorders. J Autism Dev Disord. 2013 Feb;43(2):418-31. doi: 10.1007/s10803-0121583-z. PMID: 22733301.X-1

1904. Pequegnat B, Sagermann M, Valliani $\mathrm{M}$, et al. A vaccine and diagnostic target for Clostridium bolteae, an autism-associated bacterium. Vaccine. 2013 Jun 10;31(26):278790. doi: 10.1016/j.vaccine.2013.04.018. PMID: 23602537.X-1

1905. Perea RD, Rada RC, Wilson J, et al. A Comparative White Matter Study with Parkinson's disease, Parkinson's Disease with Dementia and Alzheimer's Disease. J Alzheimers Dis Parkinsonism. 2013 Aug 26;3:123. doi: 10.4172/2161-0460.1000123. PMID: 24724042.X-1

1906. Perez Velazquez JL, Galan RF. Information gain in the brain's resting state: A new perspective on autism. Front Neuroinform. 2013;7:37. doi: 10.3389/fninf.2013.00037. PMID: 24399963.X-1

1907. Perou R, Bitsko RH, Blumberg SJ, et al. Mental health surveillance among children-United States, 2005-2011. MMWR Surveill Summ. 2013 May 17;62 Suppl 2:1-35. PMID: 23677130.X-1

1908. Perovic A, Modyanova N, Wexler K. Comparison of Grammar in Neurodevelopmental Disorders: The Case of Binding in Williams Syndrome and Autism With and Without Language Impairment. Lang Acquis. 2013 Apr;20(2):133-54. doi: 10.1080/10489223.2013.766742. PMID: 25170241.X-1

1909. Perumal N, Balan N, Stanfield A. Psychopharmacology in children with intellectual disability and autism - a crosssectional analysis (2010). International Journal of Developmental Disabilities. 2013;59(1):11-9 9p. doi: 10.1179/2047386912Z.00000000016. PMID: 104240475. Language: English. Entry Date: 20130305. Revision Date: 20150711. Publication Type: Journal Article.X-1, X-3

1910. Peters JM, Taquet M, Prohl AK, et al. Diffusion tensor imaging and related techniques in tuberous sclerosis complex: review and future directions. Future Neurol. 2013 Sep;8(5):583-97. doi: 10.2217/fnl.13.37. PMID: 24489482.X-1

1911. Peters LC, Thompson RH. Some indirect effects of positive practice overcorrection. J Appl Behav Anal. 2013 Fall;46(3):613-25. doi: 10.1002/jaba.63. PMID: 24114224.X-1

1912. Peters-Scheffer N, Didden R, Korzilius $\mathrm{H}$, et al. Therapist characteristics predict discrete trial teaching procedural fidelity. Intellect Dev Disabil. 2013 Aug;51(4):263-72. doi: 10.1352/1934-9556-51.4.263. PMID: 23909620.X-1, X-3

1913. Pizzarelli R, Cherubini E. Developmental regulation of GABAergic signalling in the hippocampus of neuroligin 3 R451C knock-in mice: an animal model of Autism. Front Cell Neurosci. 2013;7:85. doi: 10.3389/fncel.2013.00085. PMID: 23761734.X-1

1914. Plumb AM, Plexico LW. Autism spectrum disorders: experience, training, and confidence levels of school-based speechlanguage pathologists. Lang Speech Hear Serv Sch. 2013 Jan;44(1):89-104. doi: 10.1044/01611461(2012/11-0105). PMID: 23087159.X-1

1915. Poinso F, Dubois B, Chatel C, et al. [Prospective assessment of children with pervasive developmental disorder after 2 years of day-hospital treatment]. Arch Pediatr. 2013 Jan;20(1):17-25. doi: 10.1016/j.arcped.2012.10.014. PMID: 23219270.X-3, X-4

1916. Pooragha F, Kafi SM, Sotodeh SO. Comparing response inhibition and flexibility for two components of executive functioning in children with autism spectrum disorder and normal children. Iran J Pediatr. 2013 Jun;23(3):309-14. PMID: 23795254.X-1 
1917. Poot M. Towards identification of individual etiologies by resolving genomic and biological conundrums in patients with autism spectrum disorders. Mol Syndromol. 2013 Jun;4(5):213-26. doi: 10.1159/000350041. PMID: 23885228.X-1

1918. Porges SW, Macellaio M, Stanfill SD, et al. Respiratory sinus arrhythmia and auditory processing in autism: modifiable deficits of an integrated social engagement system? Int J Psychophysiol. 2013 Jun;88(3):261-70. doi: 10.1016/j.ijpsycho.2012.11.009. PMID: 23201146.X-1

1919. Post SG, Pomeroy J, Keirns CC, et al. Brief report: Stony Brook Guidelines on the ethics of the care of people with autism and their families. J Autism Dev Disord. 2013 Jun;43(6):1473-6. doi: 10.1007/s10803-0121680-z. PMID: 23086685.X-1

1920. Pratt DW, Warner JV, Williams MG. Genotyping FOXG1 Mutations in Patients with Clinical Evidence of the FOXG1 Syndrome. Mol Syndromol. 2013 Jan;3(6):284-7. doi: 10.1159/000345845. PMID: 23599699.X-1

1921. Prigge MB, Lange N, Bigler ED, et al. Corpus Callosum Area in Children and Adults with Autism. Res Autism Spectr Disord.

2013;7(2):221-34. doi:

10.1016/j.rasd.2012.09.007. PMID:

23130086.X-1

1922. Prigge MD, Bigler ED, Fletcher PT, et al. Longitudinal Heschl's gyrus growth during childhood and adolescence in typical development and autism. Autism Res. 2013 Apr;6(2):78-90. doi: 10.1002/aur.1265. PMID: 23436773.X-1

1923. Pritchard JK. Skills. Behav Anal Pract. 2013 Spring;6(1):96-100. PMID: 25729511.X1

1924. Puzzo I, Cooper NR, Cantarella S, et al. The effect of rTMS over the inferior parietal lobule on EEG sensorimotor reactivity differs according to self-reported traits of autism in typically developing individuals. Brain Res. 2013 Dec 6;1541:33-41. doi:

10.1016/j.brainres.2013.10.016. PMID:

24161404.X-1
1925. Rada RE. Treatment needs and adverse events related to dental treatment under general anesthesia for individuals with autism. Intellect Dev Disabil. 2013 Aug;51(4):246-52. doi: 10.1352/1934-9556-51.4.246. PMID: 23909586.X-1

1926. Radonovich KJ, Fournier KA, Hass CJ. Relationship between postural control and restricted, repetitive behaviors in autism spectrum disorders. Front Integr Neurosci. 2013;7:28. doi: 10.3389/fnint.2013.00028. PMID: 23675326.X-1

1927. Radulescu E, Minati L, Ganeshan B, et al. Abnormalities in fronto-striatal connectivity within language networks relate to differences in grey-matter heterogeneity in Asperger syndrome. Neuroimage Clin. 2013;2:716-26. doi: 10.1016/j.nicl.2013.05.010. PMID: 24179823.X-1

1928. Rafferty A, Martin J, Strachan D, et al. Cochlear implantation in children with complex needs - outcomes. Cochlear Implants Int. 2013 Mar;14(2):61-6. doi: 10.1179/1754762810y.0000000009. PMID: 22333941.X-1

1929. Rai D, Lee BK, Dalman C, et al. Parental depression, maternal antidepressant use during pregnancy, and risk of autism spectrum disorders: population based casecontrol study. Bmj. 2013;346:f2059. doi: 10.1136/bmj.f2059. PMID: 23604083.X-1

1930. Ramirez PL, Barnhill K, Gutierrez A, et al. Improvements in Behavioral Symptoms following Antibiotic Therapy in a 14-Year-Old Male with Autism. Case Rep Psychiatry. 2013;2013:239034. doi: 10.1155/2013/239034. PMID: 23853732.X-1, X-3, X-4

1931. Ramsey JM, Guest PC, Broek JA, et al. Identification of an age-dependent biomarker signature in children and adolescents with autism spectrum disorders. Mol Autism. 2013;4(1):27. doi: 10.1186/2040-2392-4-27. PMID: 23915542.X-1

1932. Ranft P. Ruminations on Hildegard of Bingen (1098-1179) and autism. J Med Biogr. 2013 Jul 29;22(2):107-15. doi: 10.1177/0967772013479283. PMID: 24585581.X-1 
1933. Rapp JT, Swanson G, Sheridan SM, et al. Immediate and Subsequent Effects of Matched and Unmatched Stimuli on Targeted Vocal Stereotypy and Untargeted Motor Stereotypy. Behavior Modification. 2013;37(4):543-67 25p. doi: 10.1177/0145445512461650. PMID: 104178956. Language: English. Entry Date: 20130618. Revision Date: 20150711. Publication Type: Journal Article.X-1, X-3

1934. Rattaz C, Dubois A, Michelon C, et al. How do children with autism spectrum disorders express pain? A comparison with developmentally delayed and typically developing children. Pain. 2013

Oct;154(10):2007-13. doi: 10.1016/j.pain.2013.06.011. PMID: 24040973.X-1

1935. Reed P, Watts H, Truzoli R. Flexibility in young people with autism spectrum disorders on a card sort task. Autism: The International Journal of Research \& Practice.

2013;17(2):162-71 10p. doi: 10.1177/1362361311409599. PMID: 104249016. Language: English. Entry Date: 20130321. Revision Date: 20150711. Publication Type: Journal Article.X-1

1936. Reed SR, Stahmer AC, Suhrheinrich J, et al. Stimulus overselectivity in typical development: implications for teaching children with autism. J Autism Dev Disord. 2013 Jun;43(6):1249-57. doi: 10.1007/s10803-0121658-x. PMID: 23065100.X-1

1937. Reeves GM, Keeton C, Correll CU, et al. Improving metabolic parameters of antipsychotic child treatment (IMPACT) study: rationale, design, and methods. Child Adolesc Psychiatry Ment Health. 2013;7(1):31. doi: 10.1186/1753-2000-7-31. PMID: 23947389.X$1, \mathrm{X}-2$

1938. Reimer B, Fried R, Mehler B, et al. Brief Report: Examining Driving Behavior in Young Adults with High Functioning Autism Spectrum Disorders: A Pilot Study Using a Driving Simulation Paradigm. Journal of Autism \& Developmental Disorders. 2013;43(9):2211-7 7p. doi: 10.1007/s10803013-1764-4. PMID: 104210185. Language: English. Entry Date: 20130820. Revision Date: 20150711. Publication Type: Journal Article.X1
1939. Reynell C, Harris JJ. The BOLD signal and neurovascular coupling in autism. Dev Cogn Neurosci. 2013 Oct;6:72-9. doi: 10.1016/j.dcn.2013.07.003. PMID: 23917518.X-1

1940. Riby DM, Hancock PJ, Jones N, et al. Spontaneous and cued gaze-following in autism and Williams syndrome. J Neurodev Disord. 2013;5(1):13. doi: 10.1186/1866-1955-5-13. PMID: 23663405.X-1

1941. Rieger A, McGrail JP. Coping humor and family functioning in parents of children with disabilities. Rehabil Psychol. 2013 Feb;58(1):89-97. doi: 10.1037/a0031556. PMID: 23438005.X-1

1942. Rieske RD, Matson JL, Davis TE, 3rd, et al. Examination and validation of a measure of anxiety specific to children with autism spectrum disorders. Dev Neurorehabil. 2013;16(1):9-16. doi: 10.3109/17518423.2012.705909. PMID: 23030738.X-1

1943. Roberts TP, Lanza MR, Dell J, et al. Maturational differences in thalamocortical white matter microstructure and auditory evoked response latencies in autism spectrum disorders. Brain Res. 2013 Nov 6;1537:79-85. doi: 10.1016/j.brainres.2013.09.011. PMID: 24055954.X-1

1944. Robertson RE, Wehby JH, King SM. Increased parent reinforcement of spontaneous requests in children with autism spectrum disorder: effects on problem behavior. Res Dev Disabil. 2013 Mar;34(3):1069-82. doi: 10.1016/j.ridd.2012.12.011. PMID: 23299185.X-3, X-4

1945. Rogers TD, Dickson PE, McKimm E, et al. Reorganization of circuits underlying cerebellar modulation of prefrontal cortical dopamine in mouse models of autism spectrum disorder. Cerebellum. 2013 Aug;12(4):547-56. doi: 10.1007/s12311-013-0462-2. PMID: 23436049.X-1

1946. Roke Y, van Harten PN, Franke B, et al. The effect of the Taq1A variant in the dopamine $\mathrm{D}(2)$ receptor gene and common CYP2D6 alleles on prolactin levels in risperidone-treated boys. Pharmacogenet 
Genomics. 2013 Sep;23(9):487-93. doi: 10.1097/FPC.0b013e3283647c33. PMID: 23851570.X-1, X-3

1947. Ron D. Candida mix? a case study and information about Candidemia. Similia: Journal of the Australian Homoeopathic Association. 2013;25(1):12-4 3p. PMID: 107945886.

Language: English. Entry Date: 20130726. Revision Date: 20150712. Publication Type: Journal Article.X-1

1948. Ronconi L, Gori S, Ruffino M, et al. Zoom-out attentional impairment in children with autism spectrum disorder. Cortex: A Journal Devoted to the Study of the Nervous System \& Behavior. 2013;49(4):1025-33 9p. doi: 10.1016/j.cortex.2012.03.005. PMID: 108002382. Language: English. Entry Date: 20131011. Revision Date: 20150712. Publication Type: Journal Article.X-1

1949. Rosenberg RE, Law JK, Anderson C, et al. Survey of vaccine beliefs and practices among families affected by autism spectrum disorders. Clin Pediatr (Phila). 2013 Sep;52(9):871-4. doi: 10.1177/0009922812438435. PMID: 22387922.X-1

1950. Rossignol DA. My experience learning about autism. Glob Adv Health Med. 2013 Nov;2(6):74-7. doi: 10.7453/gahmj.2013.090. PMID: 24349885.X-1

1951. Roux AM, Shattuck PT, Cooper BP, et al. Postsecondary employment experiences among young adults with an autism spectrum disorder. J Am Acad Child Adolesc Psychiatry. 2013 Sep;52(9):931-9. doi:

10.1016/j.jaac.2013.05.019. PMID:

23972695.X-1

1952. Roux G, Sofronoff K, Sanders M. A randomized controlled trial of group Stepping Stones Triple P: a mixed-disability trial. Fam Process. 2013 Sep;52(3):411-24. doi: 10.1111/famp.12016. PMID: 24033239.X-1

1953. Ruble L, McGrew JH. Teacher and child predictors of achieving IEP goals of children with autism. J Autism Dev Disord. 2013 Dec;43(12):2748-63. doi: 10.1007/s10803-013-1884-X. PMID: 23838728.X-1, X-3
1954. Ruble LA, McGrew JH, Toland MD, et al. A randomized controlled trial of COMPASS web-based and face-to-face teacher coaching in autism. J Consult Clin Psychol. 2013 Jun;81(3):566-72. doi: 10.1037/a0032003. PMID: 23438314.X-1

1955. Ruble LA, Toland MD, Birdwhistell JL, et al. Preliminary Study of the Autism SelfEfficacy Scale for Teachers (ASSET). Res Autism Spectr Disord. 2013 Sep;7(9):1151-9. doi: 10.1016/j.rasd.2013.06.006. PMID: 23976899.X-1

1956. Ruiz-Robledillo N, Moya-Albiol L. Self-reported health and cortisol awakening response in parents of people with asperger syndrome: the role of trait anger and anxiety, coping and burden. Psychol Health. 2013 Nov;28(11):1246-64. doi: 10.1080/08870446.2013.800517. PMID: 23713979.X-1

1957. Ruskin DN, Svedova J, Cote JL, et al. Ketogenic diet improves core symptoms of autism in BTBR mice. PLoS One.

2013;8(6):e65021. doi: 10.1371/journal.pone.0065021. PMID: 23755170.X-1

1958. Russell AJ, Jassi A, Fullana MA, et al. Cognitive behavior therapy for comorbid obsessive-compulsive disorder in highfunctioning autism spectrum disorders: a randomized controlled trial. Depress Anxiety. 2013 Aug;30(8):697-708. doi: 10.1002/da.22053. PMID: 23389964.X-1

1959. Russell-Smith S, Bayliss D, Maybery $\mathrm{M}$, et al. Are the Autism and Positive Schizotypy Spectra Diametrically Opposed in Empathizing and Systemizing? Journal of Autism \& Developmental Disorders. 2013;43(3):695-706 12p. doi: 10.1007/s10803012-1614-9. PMID: 104318605. Language: English. Entry Date: 20130225. Revision Date: 20150711. Publication Type: Journal Article.X1

1960. Russo AJ. Decreased Epidermal Growth Factor (EGF) Associated with HMGB1 and Increased Hyperactivity in Children with Autism. Biomark Insights. 2013;8:35-41. doi: 10.4137/bmi.s11270. PMID: 23645980.X-1 
1961. Russo AJ. Correlation Between Hepatocyte Growth Factor (HGF) and GammaAminobutyric Acid (GABA) Plasma Levels in Autistic Children. Biomark Insights. 2013;8:6975. doi: 10.4137/bmi.s11448. PMID: 23825437.X-1

1962. Rytterstrom P, Unosson M, Arman M. Care culture as a meaning-making process: a study of a mistreatment investigation. Qual Health Res. 2013 Sep;23(9):1179-87. doi: 10.1177/1049732312470760. PMID: 23264536.X-1

1963. Saemundsen E, Magnusson P, Georgsdottir I, et al. Prevalence of autism spectrum disorders in an Icelandic birth cohort. BMJ Open. 2013;3(6)doi: 10.1136/bmjopen2013-002748. PMID: 23788511.X-1

1964. Sahu JK, Gulati S, Sapra S, et al. Effectiveness and safety of donepezil in boys with fragile x syndrome: a double-blind, randomized, controlled pilot study. J Child Neurol. 2013 May;28(5):570-5. doi: 10.1177/0883073812449381. PMID: 22752489.X-1

1965. Sakurai T. [Drug development targeting synaptic molecules - autism mouse models as an example]. Nihon Yakurigaku Zasshi. 2013 Sep;142(3):116-21. PMID: 24025492.X-1

1966. Sallam K, Amr M. The use of the mini-mental state examination and the clockdrawing test for dementia in a tertiary hospital. J Clin Diagn Res. 2013 Mar;7(3):484-8. doi: 10.7860/jcdr/2013/4203.2803. PMID: 23634401.X-1

1967. Salowitz N, Eccarius P, Karst J, et al. Brief Report: Visuo-spatial Guidance of Movement during Gesture Imitation and Mirror Drawing in Children with Autism Spectrum Disorders. Journal of Autism \& Developmental Disorders. 2013;43(4):985-95 11p. doi: 10.1007/s10803-012-1631-8. PMID: 104256179. Language: English. Entry Date: 20130321. Revision Date: 20150711. Publication Type: Journal Article.X-1

1968. Samadi SA, McConkey R, Kelly G. Enhancing parental well-being and coping through a family-centred short course for Iranian parents of children with an autism spectrum disorder. Autism. 2013 Jan;17(1):2743. doi: 10.1177/1362361311435156. PMID: 22297201.X-1

1969. Sandell C, Kjellberg A, Taylor RR. Participating in diagnostic experience: adults with neuropsychiatric disorders. Scand J Occup Ther. 2013 Mar;20(2):136-42. doi: 10.3109/11038128.2012.741621. PMID: 23152985.X-1

1970. Sandiford GA, Mainess KJ, Daher NS. A pilot study on the efficacy of melodic based communication therapy for eliciting speech in nonverbal children with autism. J Autism Dev Disord. 2013 Jun;43(6):1298-307. doi: 10.1007/s10803-012-1672-z. PMID: 23065117.X-4

1971. Sandin S, Nygren KG, Iliadou A, et al. Autism and mental retardation among offspring born after in vitro fertilization. Jama. $2013 \mathrm{Jul}$ 3;310(1):75-84. doi: 10.1001/jama.2013.7222. PMID: 23821091.X-1

1972. Santini E, Huynh TN, MacAskill AF, et al. Exaggerated translation causes synaptic and behavioural aberrations associated with autism. Nature. 2013 Jan 17;493(7432):411-5. doi: 10.1038/nature11782. PMID: 23263185.X1

1973. Sarkamo T, Tervaniemi M, Huotilainen M. Music perception and cognition: development, neural basis, and rehabilitative use of music. Wiley Interdiscip Rev Cogn Sci. 2013 Jul;4(4):441-51. doi: 10.1002/wcs.1237. PMID: 26304229.X-1

1974. Sasson NJ, Lam KS, Parlier M, et al. Autism and the broad autism phenotype: familial patterns and intergenerational transmission. J Neurodev Disord. 2013;5(1):11. doi: 10.1186/1866-1955-5-11. PMID: 23639131.X-1

1975. Saunders JA, Tatard-Leitman VM, Suh J, et al. Knockout of NMDA receptors in parvalbumin interneurons recreates autism-like phenotypes. Autism Res. 2013 Apr;6(2):69-77. doi: 10.1002/aur.1264. PMID: 23441094.X-1

1976. Sawa T, Kodaira M, Oiji A, et al. Dysfunction of orbitofrontal and dorsolateral prefrontal cortices in children and adolescents with high-functioning pervasive developmental 
disorders. Ann Gen Psychiatry. 2013;12(1):31. doi: 10.1186/1744-859x-12-31. PMID: 24103490.X-1

1977. Schafer EC, Mathews L, Mehta S, et al. Personal FM systems for children with autism spectrum disorders (ASD) and/or attention-deficit hyperactivity disorder (ADHD): an initial investigation. J Commun Disord. 2013 Jan-Feb;46(1):30-52. doi: 10.1016/j.jcomdis.2012.09.002. PMID: 23123089.X-3, X-4

1978. Schlosser RW, Laubscher E, Sorce J, et al. Implementing directives that involve prepositions with children with autism: a comparison of spoken cues with two types of augmented input. Augment Altern Commun. 2013 Jun;29(2):132-45. doi: 10.3109/07434618.2013.784928. PMID: 23705815.X-1, X-4

1979. Sears KM, Blair KS, Iovannone R, et al. Using the prevent-teach-reinforce model with families of young children with ASD. J Autism Dev Disord. 2013 May;43(5):1005-16. doi: 10.1007/s10803-012-1646-1. PMID: 22972500.X-3, X-4

1980. Seery AM, Vogel-Farley V, TagerFlusberg H, et al. Atypical lateralization of ERP response to native and non-native speech in infants at risk for autism spectrum disorder. Dev Cogn Neurosci. 2013 Jul;5:10-24. doi: 10.1016/j.dcn.2012.11.007. PMID: 23287023.X-1

1981. Selim ME, Al-Ayadhi LY. Possible ameliorative effect of breastfeeding and the uptake of human colostrum against coeliac disease in autistic rats. World J Gastroenterol. 2013 Jun 7;19(21):3281-90. doi: 10.3748/wjg.v19.i21.3281. PMID: 23745030.X-1

1982. Semansky RM, Xie M, Lawer LJ, et al. How states use Medicaid to fund communitybased services to children with autism spectrum disorders. Psychiatr Serv. 2013

Oct;64(10):1051-5. doi:

10.1176/appi.ps.201200390. PMID:

24081405.X-1

1983. Sgado P, Provenzano G, Dassi E, et al. Transcriptome profiling in engrailed-2 mutant mice reveals common molecular pathways associated with autism spectrum disorders. Mol Autism. 2013;4(1):51. doi: 10.1186/2040-23924-51. PMID: 24355397.X-1

1984. Shadravan F. Sex bias in copy number variation of olfactory receptor gene family depends on ethnicity. Front Genet. 2013;4:32. doi: 10.3389/fgene.2013.00032. PMID: 23503716.X-1

1985. Sharma A, Gokulchandran N, Sane H, et al. Autologous bone marrow mononuclear cell therapy for autism: an open label proof of concept study. Stem Cells Int.

2013;2013:623875. doi: 10.1155/2013/623875. PMID: 24062774.X-3, X-4

1986. Sharpe MA, Gist TL, Baskin DS. Alterations in sensitivity to estrogen, dihydrotestosterone, and xenogens in Blymphocytes from children with autism spectrum disorder and their unaffected twins/siblings. J Toxicol. 2013;2013:159810. doi: 10.1155/2013/159810. PMID: 24363669.X-1

1987. Sharpe MA, Gist TL, Baskin DS. Blymphocytes from a population of children with autism spectrum disorder and their unaffected siblings exhibit hypersensitivity to thimerosal. J Toxicol. 2013;2013:801517. doi: 10.1155/2013/801517. PMID: 23843785.X-1

1988. Shiokawa S, Kitamura K, Fujii T, et al. [Support provided by public health nurses to adolescents with pervasive developmental disorders and their families]. Nihon Koshu Eisei Zasshi. 2013;60(11):705-14. PMID: 24418919.X-1

1989. Siller M, Hutman T, Sigman M. A parent-mediated intervention to increase responsive parental behaviors and child communication in children with ASD: a randomized clinical trial. J Autism Dev Disord. 2013 Mar;43(3):540-55. doi: 10.1007/s10803012-1584-y. PMID: 22825926.X-1

1990. Silva L, Schalock M. Treatment of tactile impairment in young children with autism: results with qigong massage. Int J Ther Massage Bodywork. 2013;6(4):12-20. PMID: 24298297. INCLUDE

1991. Silverman JL, Oliver CF, Karras MN, et al. AMPAKINE enhancement of social 
interaction in the BTBR mouse model of autism. Neuropharmacology. 2013 Jan;64:26882. doi: 10.1016/j.neuropharm.2012.07.013. PMID: 22801296.X-1

1992. Simmeborn Fleischer A, Adolfsson M, Granlund M. Students with disabilities in higher education - perceptions of support needs and received support: a pilot study. Int J Rehabil Res. 2013 Dec;36(4):330-8. doi: 10.1097/MRR.0b013e328362491c. PMID: 23722568.X-1

1993. Simon DM, Corbett BA. Examining associations between anxiety and cortisol in high functioning male children with autism. J Neurodev Disord. 2013;5(1):32. doi: 10.1186/1866-1955-5-32. PMID: 24216056.X1

1994. Singh GK, Kenney MK, Ghandour RM, et al. Mental Health Outcomes in US Children and Adolescents Born Prematurely or with Low Birthweight. Depress Res Treat. 2013;2013:570743. doi: 10.1155/2013/570743. PMID: 24324882.X-1

1995. Singh S. Valproate use during pregnancy was linked to autism spectrum disorder and childhood autism in offspring. ACP Journal Club. 2013;159(4):1- p. PMID: 104218605. Language: English. Entry Date: 20130927. Revision Date: 20150711. Publication Type: Journal Article.X-1

1996. Slotkin TA, Seidler FJ. Terbutaline impairs the development of peripheral noradrenergic projections: potential implications for autism spectrum disorders and pharmacotherapy of preterm labor. Neurotoxicol Teratol. 2013 Mar-Apr;36:91-6. doi: 10.1016/j.ntt.2012.07.003. PMID: 22813780.X-1

1997. Soorya L, Kolevzon A, Zweifach J, et al. Prospective investigation of autism and genotype-phenotype correlations in 22q13 deletion syndrome and SHANK3 deficiency. Mol Autism. 2013;4(1):18. doi: 10.1186/20402392-4-18. PMID: 23758760.X-1

1998. Sorensen MJ, Gronborg TK, Christensen J, et al. Antidepressant exposure in pregnancy and risk of autism spectrum disorders. Clin Epidemiol. 2013;5:449-59. doi: 10.2147/clep.s53009. PMID: 24255601.X-1
1999. Souchay C, Wojcik DZ, Williams HL, et al. Recollection in adolescents with Autism Spectrum Disorder. Cortex: A Journal Devoted to the Study of the Nervous System \& Behavior. 2013;49(6):1598-609 12p. doi: 10.1016/j.cortex.2012.07.011. PMID: 107905487. Language: English. Entry Date: 20140131. Revision Date: 20150712. Publication Type: Journal Article.X-1

2000. Spek AA, van Ham NC, Nyklicek I. Mindfulness-based therapy in adults with an autism spectrum disorder: a randomized controlled trial. Res Dev Disabil. 2013 Jan;34(1):246-53. doi: 10.1016/j.ridd.2012.08.009. PMID: 22964266.X-1

2001. Spencer D, Marshall J, Post B, et al. Psychotropic medication use and polypharmacy in children with autism spectrum disorders. Pediatrics. 2013 Nov;132(5):833-40. doi: 10.1542/peds.2012-3774. PMID: 24144704.X-1

2002. Spencer TD, Kajian M, Petersen DB, et al. Effects of an Individualized Narrative Intervention on Children's Storytelling and Comprehension Skills. Journal of Early Intervention. 2013;35(2):243-69 27p. doi: 10.1177/1053815114540002. PMID: 103986455. Language: English. Entry Date: 20140110. Revision Date: 20150818. Publication Type: Journal Article.X-1, X-3

2003. Spilioti M, Evangeliou AE, Tramma D, et al. Evidence for treatable inborn errors of metabolism in a cohort of 187 Greek patients with autism spectrum disorder (ASD). Front Hum Neurosci. 2013;7:858. doi: 10.3389/fnhum.2013.00858. PMID: 24399946.X-1

2004. Srinivasan SM, Bhat AN. A review of "music and movement" therapies for children with autism: embodied interventions for multisystem development. Front Integr Neurosci. 2013;7:22. doi: 10.3389/fnint.2013.00022. PMID: 23576962.X1, $\mathrm{X}-2$

2005. Srinivasaraghavan R, Mahadevan S, Kattimani S. Impact of Comorbidity on Three Month Follow-up Outcome of Children with ADHD in a Child Guidance Clinic: Preliminary Report. Indian J Psychol Med. 2013 
Oct;35(4):346-51. doi: 10.4103/0253-

7176.122223. PMID: 24379493.X-1

2006. Stadnick NA, Drahota A, BrookmanFrazee L. Parent Perspectives of an EvidenceBased Intervention for Children with Autism Served in Community Mental Health Clinics. J Child Fam Stud. 2013 Apr 1;22(3):414-22. doi: 10.1007/s10826-012-9594-0. PMID: 24019736.X-3

2007. Stamova BS, Tian Y, Nordahl CW, et al. Evidence for differential alternative splicing in blood of young boys with autism spectrum disorders. Mol Autism. 2013;4(1):30. doi: 10.1186/2040-2392-4-30. PMID: 24007566.X1

2008. Staples K. Commentary: The Motor Skills of 7-10 Year Old Children Diagnosed with ASD. Are the Comparison Groups and Assessments Being Used Appropriate for the Research Questions Being Asked?...Whyatt CP, Craig CM. Motor skills in children aged 7-19 years diagnosed with autism spectrum disorder. Journal of Autism and Developmental Disorders 2012; 42:1799-1809. Journal of Autism \& Developmental Disorders. 2013;43(11):2732-6 5p. doi: 10.1007/s10803013-1809-8. PMID: 104145174. Language: English. Entry Date: 20131015. Revision Date: 20150711. Publication Type: Journal Article.X1

2009. Starck T, Nikkinen J, Rahko J, et al. Resting state fMRI reveals a default mode dissociation between retrosplenial and medial prefrontal subnetworks in ASD despite motion scrubbing. Front Hum Neurosci. 2013;7:802. doi: 10.3389/fnhum.2013.00802. PMID: 24319422.X-1

2010. Stein LI, Polido JC, Cermak SA. Oral care and sensory over-responsivity in children with autism spectrum disorders. Pediatr Dent. 2013 May-Jun;35(3):230-5. PMID: 23756306.X-1

2011. Steiner AM, Gengoux GW, Klin A, et al. Pivotal response treatment for infants at-risk for autism spectrum disorders: a pilot study. J Autism Dev Disord. 2013 Jan;43(1):91-102. doi: 10.1007/s10803-012-1542-8. PMID: 22573001.X-3, X-4
2012. Steinman G, Mankuta D.

Breastfeeding as a possible deterrent to autism-a clinical perspective. Med Hypotheses. 2013

Dec;81(6):999-1001. doi:

10.1016/j.mehy.2013.09.013. PMID: 24099931.X-1

2013. Storch EA, Arnold EB, Lewin AB, et al. The effect of cognitive-behavioral therapy versus treatment as usual for anxiety in children with autism spectrum disorders: a randomized, controlled trial. J Am Acad Child Adolesc Psychiatry. 2013 Feb;52(2):132-42.e2. doi: 10.1016/j.jaac.2012.11.007. PMID: 23357440.X-1

2014. Strickland DC, Coles CD, Southern LB. JobTIPS: a transition to employment program for individuals with autism spectrum disorders. J Autism Dev Disord. 2013 Oct;43(10):2472-83. doi: 10.1007/s10803-0131800-4. PMID: 23494559.X-1

2015. Stroganova TA, Kozunov VV, Posikera IN, et al. Abnormal pre-attentive arousal in young children with autism spectrum disorder contributes to their atypical auditory behavior: an ERP study. PLoS One.

2013;8(7):e69100. doi: 10.1371/journal.pone.0069100. PMID: 23935931.X-1, X-3

2016. Sugarman LI, Garrison BL, Williford KL. Symptoms as solutions: hypnosis and biofeedback for autonomic regulation in autism spectrum disorders. Am J Clin Hypn. 2013 Oct;56(2):152-73. PMID: 24665817.X-1

2017. Suhrheinrich J, Stahmer AC, Reed S, et al. Implementation challenges in translating pivotal response training into community settings. J Autism Dev Disord. 2013 Dec;43(12):2970-6. doi: 10.1007/s10803-0131826-7. PMID: 23619949.X-1

2018. Sun C, Xia W, Zhao Y, et al. Nutritional status survey of children with autism and typically developing children aged 4-6 years in Heilongjiang Province, China. J Nutr Sci. 2013;2:e16. doi: 10.1017/jns.2013.9. PMID: 25191564.X-1

2019. Sun X, Allison C, Auyeung B, et al. Service provision for autism in mainland China: preliminary mapping of service pathways. Soc Sci Med. 2013 Dec;98:87-94. doi: 
10.1016/j.socscimed.2013.08.016. PMID: 24331886.X-1

2020. Sun X, Allison C, Auyeung B, et al. Service provision for autism in mainland China: a service providers' perspective. Res Dev Disabil. 2013 Jan;34(1):440-51. doi: 10.1016/j.ridd.2012.08.010. PMID: 23041658.X-1

2021. Sun X, Allison C, Matthews FE, et al. Prevalence of autism in mainland China, Hong Kong and Taiwan: a systematic review and meta-analysis. Mol Autism. 2013;4(1):7. doi: 10.1186/2040-2392-4-7. PMID: 23570419.X-1

2022. Suren P, Roth C, Bresnahan M, et al. Association between maternal use of folic acid supplements and risk of autism spectrum disorders in children. Jama. 2013 Feb 13;309(6):570-7. doi:

10.1001/jama.2012.155925. PMID: 23403681.X-1

2023. Surette S, Vanderjagt L, Vohra S. Surveys of complementary and alternative medicine usage: a scoping study of the paediatric literature. Complement Ther Med. 2013 Apr;21 Suppl 1:S48-53. doi: 10.1016/j.ctim.2011.08.006. PMID: 23578917.X-1

2024. Tachibana M, Kagitani-Shimono K, Mohri I, et al. Long-term administration of intranasal oxytocin is a safe and promising therapy for early adolescent boys with autism spectrum disorders. J Child Adolesc Psychopharmacol. 2013 Mar;23(2):123-7. doi: 10.1089/cap.2012.0048. PMID: 23480321.X-1, $\mathrm{X}-3, \mathrm{X}-4$

2025. Tahmasebi Birgani MJ, Behrouz MA, Aliakbari S, et al. Determination of square equivalent field for rectangular field in electron therapy. J Med Phys. 2013 Apr;38(2):82-6. doi: 10.4103/0971-6203.111317. PMID: 23776311.X-1

2026. Tajeran M, Baghbani F, HassanzadehNazarabadi M. A Case of Autism with Ring Chromosome 14. Iran J Public Health. 2013 Nov;42(11):1316-20. PMID: 26171345.X-1

2027. Takahashi J, Gyoba J, Yamawaki N. Brief Report: Effect of Spatial Complexity on Visual Short-Term Memory and Self-Reported
Autistic-Like Traits in Typically Developed Individuals. Journal of Autism \& Developmental Disorders. 2013;43(7):1725-31 7p. doi: 10.1007/s10803-012-1711-9. PMID: 104183640. Language: English. Entry Date: 20130620. Revision Date: 20150711. Publication Type: Journal Article.X-1

2028. Taliou A, Zintzaras E, Lykouras L, et al. An open-label pilot study of a formulation containing the anti-inflammatory flavonoid luteolin and its effects on behavior in children with autism spectrum disorders. Clin Ther. 2013 May;35(5):592-602. doi: 10.1016/j.clinthera.2013.04.006. PMID: 23688534.X-3

2029. Tamas D, Markovic S, Milankov V. Systemic multimodal approach to speech therapy treatment in autistic children. Med Pregl. 2013 May-Jun;66(5-6):233-9. PMID: 23888732.X-1, X-3

2030. Tchanturia K, Smith E, Weineck F, et al. Exploring autistic traits in anorexia: a clinical study. Mol Autism. 2013;4(1):44. doi: 10.1186/2040-2392-4-44. PMID: 24220604.X1

2031. Teng BL, Nonneman RJ, Agster KL, et al. Prosocial effects of oxytocin in two mouse models of autism spectrum disorders. Neuropharmacology. 2013 Sep;72:187-96. doi: 10.1016/j.neuropharm.2013.04.038. PMID: 23643748.X-1

2032. Thakkar KB, Jain MM, Billa G, et al. A drug utilization study of psychotropic drugs prescribed in the psychiatry outpatient department of a tertiary care hospital. J Clin Diagn Res. 2013 Dec;7(12):2759-64. doi: 10.7860/jcdr/2013/6760.3885. PMID: 24551631.X-1

2033. Thompson RM, Johnston S. Use of social stories to improve self-regulation in children with autism spectrum disorders. Phys Occup Ther Pediatr. 2013 Aug;33(3):271-84. doi: 10.3109/01942638.2013.768322. PMID: 23437903.X-3, X-4

2034. Ting L. SENSORY PROCESSING AND MOTOR SKILL PERFORMANCE IN ELEMENTARY SCHOOL CHILDREN WITH AUTISM SPECTRUM DISORDER. Perceptual \& Motor Skills. 2013;116(1):197-209 13p. doi: 
10.2466/10.25.PMS.116.1.197-209. PMID: 104220816. Language: English. Entry Date: 20130906. Revision Date: 20150711. Publication Type: Journal Article.X-1

2035. Tomizawa Y, Sato Y, Yokoyama H. [Parent training for parents of children with attention deficit/hyperactivity disorder (AD/HD) does not improve the behavior of children with both high-functioning pervasive developmental disorder and AD/HD]. No To Hattatsu. 2013 Jan;45(1):33-7. PMID: 23593743.X-3

2036. Tostes MH, Polonini HC, Mendes R, et al. Fatty acid and phospholipase A2 plasma levels in children with autism. Trends Psychiatry Psychother. 2013;35(1):76-80. PMID: 25923188.X-1

2037. Travers B, Powell P, Mussey J, et al. Spatial and Identity Cues Differentially Affect Implicit Contextual Cueing in Adolescents and Adults with Autism Spectrum Disorder. Journal of Autism \& Developmental Disorders. 2013;43(10):2393-404 12p. doi: 10.1007/s10803-013-1787-x. PMID: 104226070. Language: English. Entry Date: 20130921. Revision Date: 20150711. Publication Type: Journal Article.X-1

2038. Treasure J. Coherence and other autistic spectrum traits and eating disorders: building from mechanism to treatment. The Birgit Olsson lecture. Nord J Psychiatry. 2013 Feb;67(1):38-42. doi: 10.3109/08039488.2012.674554. PMID: 22468644.X-1

2039. Trevarthen C, Delafield-Butt JT. Autism as a developmental disorder in intentional movement and affective engagement. Front Integr Neurosci. 2013;7:49. doi: 10.3389/fnint.2013.00049. PMID: 23882192.X-1

2040. Trontel HG, Duffield TC, Bigler ED, et al. Fusiform correlates of facial memory in autism. Behav Sci (Basel). 2013;3(3):348-71. doi: 10.3390/bs3030348. PMID: 24761228.X-1

2041. Tunc-Ozcan E, Ullmann TM, Shukla PK, et al. Low-dose thyroxine attenuates autism-associated adverse effects of fetal alcohol in male offspring's social behavior and hippocampal gene expression. Alcohol Clin
Exp Res. 2013 Nov;37(11):1986-95. doi: 10.1111/acer.12183. PMID: 23763370.X-1

2042. Uchiyama T. [Case study of 10 subjects diagnosed with autism spectrum disorders in adulthood and currently under long-term follow-up]. Seishin Shinkeigaku Zasshi. 2013;115(6):607-15. PMID: 23944118.X-1

2043. Uddin LQ, Supekar K, Menon V. Reconceptualizing functional brain connectivity in autism from a developmental perspective. Front Hum Neurosci. 2013;7:458. doi: 10.3389/fnhum.2013.00458. PMID: 23966925.X-1

2044. Ulate-Campos A, Benavides-Lara A, Hernandez L. [Characterisation of the paediatric population of Costa Rica with tuberous sclerosis and a description of the behaviour of the associated epilepsy]. Rev Neurol. 2013 Dec 1;57(11):489-94. PMID: 24265142.X-1

2045. Ung D, Wood JJ, Ehrenreich-May J, et al. Clinical characteristics of high-functioning youth with autism spectrum disorder and anxiety. Neuropsychiatry (London). 2013 Apr;3(2)doi: 10.2217/npy.13.9. PMID: 24179485.X-1

2046. van der Meer L, Kagohara D, Roche L, et al. Teaching multi-step requesting and social communication to two children with autism spectrum disorders with three AAC options. Augment Altern Commun. 2013 Sep;29(3):22234. doi: 10.3109/07434618.2013.815801. PMID: 23879660.X-3, X-4

2047. van Gelder MM, Bretveld RW, Roukema J, et al. Rationale and design of the PRegnancy and Infant DEvelopment (PRIDE) Study. Paediatr Perinat Epidemiol. 2013 Jan;27(1):34-43. doi: 10.1111/ppe.12023. PMID: 23215710.X-1

2048. van Steensel FJ, Bogels SM, de Bruin EI. Psychiatric Comorbidity in Children with Autism Spectrum Disorders: A Comparison with Children with ADHD. J Child Fam Stud. 2013 Apr;22(3):368-76. doi: 10.1007/s10826012-9587-z. PMID: 23524401.X-1

2049. Vaz I. Visual symbols in healthcare settings for children with learning disabilities and autism spectrum disorder. Br J Nurs. 2013 
Feb 14-28;22(3):156-9. doi:

10.12968/bjon.2013.22.3.156. PMID: 23411823.X-1

2050. Velmeshev D, Magistri M, Faghihi MA. Expression of non-protein-coding antisense RNAs in genomic regions related to autism spectrum disorders. Mol Autism. 2013;4(1):32. doi: 10.1186/2040-2392-4-32. PMID: 24007600.X-1

2051. Vismara LA, McCormick C, Young GS, et al. Preliminary findings of a telehealth approach to parent training in autism. J Autism Dev Disord. 2013 Dec;43(12):2953-69. doi: 10.1007/s10803-013-1841-8. PMID: 23677382.X-1, X-3

2052. Visser E, Zwiers MP, Kan CC, et al. Atypical vertical sound localization and soundonset sensitivity in people with autism spectrum disorders. J Psychiatry Neurosci. 2013 Nov;38(6):398-406. doi: 10.1503/jpn.120177. PMID: 24148845.X-1

2053. Visser JC, Rommelse N, Vink L, et al. Narrowly versus broadly defined autism spectrum disorders: differences in pre- and perinatal risk factors. J Autism Dev Disord. 2013 Jul;43(7):1505-16. doi: 10.1007/s10803012-1678-6. PMID: 23076505.X-1

2054. Vivanti G, Dissanayake C, Zierhut C, et al. Brief report: Predictors of outcomes in the Early Start Denver Model delivered in a group setting. J Autism Dev Disord. 2013 Jul;43(7):1717-24. doi: 10.1007/s10803-0121705-7. PMID: 23124361.X-1, X-3

2055. Vladescu JC, Kodak TM. Increasing instructional efficiency by presenting additional stimuli in learning trials for children with autism spectrum disorders. J Appl Behav Anal. 2013 Dec;46(4):805-16. doi: 10.1002/jaba.70. PMID: 24114346.X-3, X-4

2056. Wacker DP, Lee JF, Dalmau YC, et al. Conducting functional analyses of problem behavior via telehealth. J Appl Behav Anal. 2013 Spring;46(1):31-46. doi: 10.1002/jaba.29. PMID: 24114083.X-3

2057. Wadell PM, Hagerman RJ, Hessl DR. FRAGILE X SYNDROME: PSYCHIATRIC MANIFESTATIONS, ASSESSMENT AND EMERGING THERAPIES. Curr Psychiatry
Rev. 2013 Feb 1;9(1):53-8. doi: 10.2174/157340013805289644. PMID: 25632275.X-1

2058. Wainer AL, Ingersoll BR.

Disseminating ASD interventions: a pilot study of a distance learning program for parents and professionals. J Autism Dev Disord. 2013 Jan;43(1):11-24. doi: 10.1007/s10803-0121538-4. PMID: 22547028.X-3, X-4

2059. Wake R, Miyaoka T, Inagaki T, et al. Yokukansan (TJ-54) for irritability associated with pervasive developmental disorder in children and adolescents: a 12-week prospective, open-label study. J Child Adolesc Psychopharmacol. 2013 Jun;23(5):329-36. doi: 10.1089/cap.2012.0108. PMID: 23782127.X-3

2060. Walker G, Local J. On the intersection of phonetic detail and the organization of interaction: clinical connections. Clin Linguist Phon. 2013 Oct-Nov;27(10-11):770-83. doi: 10.3109/02699206.2013.813078. PMID: 24067088.X-1

2061. Walters K, Thomson K. The history of behavior analysis in manitoba: a sparsely populated canadian province with an international influence on behavior analysis. Behav Anal. 2013 Spring;36(1):57-72. PMID: 25729132.X-1

2062. Wan Y, Hu Q, Li T, et al. Prevalence of autism spectrum disorders among children in China: a systematic review. Shanghai Arch Psychiatry. 2013 Apr;25(2):70-80. doi: 10.3969/j.issn.1002-0829.2013.02.003. PMID: 24991138.X-1

2063. Wang CC, Lin HC, Chan YH, et al. 5HT1A-receptor agonist modified amygdala activity and amygdala-associated social behavior in a valproate-induced rat autism model. Int J Neuropsychopharmacol. 2013 Oct;16(9):2027-39. doi: 10.1017/s1461145713000473. PMID: 23823694.X-1

2064. Wang L, Mandell DS, Lawer L, et al. Healthcare service use and costs for autism spectrum disorder: a comparison between medicaid and private insurance. J Autism Dev Disord. 2013 May;43(5):1057-64. doi: 10.1007/s10803-012-1649-y. PMID: 22965299.X-1 
2065. Wang M, Reid D. Using the virtual reality-cognitive rehabilitation approach to improve contextual processing in children with autism. ScientificWorldJournal.

2013;2013:716890. doi: 10.1155/2013/716890. PMID: 24324379.X-3, X-4

2066. Ward SC, Whalon K, Rusnak K, et al. The association between therapeutic horseback riding and the social communication and sensory reactions of children with autism. J Autism Dev Disord. 2013 Sep;43(9):2190-8. doi: 10.1007/s10803-013-1773-3. PMID: 23371511.X-3

2067. Waris P, Lindberg N, Kettunen K, et al. The relationship between Asperger's syndrome and schizophrenia in adolescence. Eur Child Adolesc Psychiatry. 2013 Apr;22(4):217-23. doi: 10.1007/s00787-0120338-x. PMID: 23065028.X-1

2068. Warren Z, Vehorn A, Dohrmann E, et al. Brief report: Service implementation and maternal distress surrounding evaluation recommendations for young children diagnosed with autism. Autism. 2013 Nov;17(6):693-700. doi: 10.1177/1362361312453881. PMID: 23045221.X-1

2069. Watts AC, Wilder DA, Gregory MK, et al. The effect of rules on differential reinforcement of other behavior. J Appl Behav Anal. 2013 Fall;46(3):680-4. doi: 10.1002/jaba.53. PMID: 24114232.X-3, X-4

2070. Weiss JA, Viecili MA, Sloman L, et al. Direct and Indirect Psychosocial Outcomes for Children with Autism Spectrum Disorder and their Parents Following a Parent-involved Social Skills Group Intervention. J Can Acad Child Adolesc Psychiatry. 2013 Nov;22(4):3039. PMID: 24223050.X-1, X-3

2071. Weiss MJ, Moran MF, Parker ME, et al. Gait analysis of teenagers and young adults diagnosed with autism and severe verbal communication disorders. Front Integr Neurosci. 2013;7:33. doi: 10.3389/fnint.2013.00033. PMID: 23730274.X1

2072. Werner S, Shulman C. Subjective well-being among family caregivers of individuals with developmental disabilities: the role of affiliate stigma and psychosocial moderating variables. Res Dev Disabil. 2013 Nov;34(11):4103-14. doi: 10.1016/j.ridd.2013.08.029. PMID: 24055712.X-1

2073. White SJ. The Triple I Hypothesis: taking another('s) perspective on executive dysfunction in autism. J Autism Dev Disord. 2013 Jan;43(1):114-21. doi: 10.1007/s10803012-1550-8. PMID: 22584617.X-1

2074. White SW, Ollendick T, Albano AM, et al. Randomized controlled trial: Multimodal Anxiety and Social Skill Intervention for adolescents with autism spectrum disorder. J Autism Dev Disord. 2013 Feb;43(2):382-94. doi: 10.1007/s10803-012-1577-x. PMID: 22735897.X-1, X-3

2075. Whitehouse AJ. Complementary and alternative medicine for autism spectrum disorders: Rationale, safety and efficacy. Journal of Paediatrics \& Child Health. 2013;49(9):E438-42 1p. doi: 10.1111/jpc.12242. PMID: 104095176. Language: English. Entry Date: 20140516. Revision Date: 20150710. Publication Type: Journal Article. Journal Subset: Australia \& New Zealand.X-1, X-2

2076. Whyte EM, Nelson KE, Khan KS. Learning of idiomatic language expressions in a group intervention for children with autism. Autism. 2013 Jul;17(4):449-64. doi: 10.1177/1362361311422530. PMID: 22087041.X-3, X-4

2077. Williams D, Payne H, Marshall C. Non-word Repetition Impairment in Autism and Specific Language Impairment: Evidence for Distinct Underlying Cognitive Causes. Journal of Autism \& Developmental Disorders. 2013;43(2):404-17 14p. doi: 10.1007/s10803012-1579-8. PMID: 104296917. Language: English. Entry Date: 20130128. Revision Date: 20150711. Publication Type: Journal Article.X1

2078. Williams K, Brignell A, Randall M, et al. Selective serotonin reuptake inhibitors (SSRIs) for autism spectrum disorders (ASD). Cochrane Database of Systematic Reviews. 2013(8):N.PAG-N.PAG 1p. PMID: 105008776. Language: English. Entry Date: 
20101029. Revision Date: 20150711.

Publication Type: Journal Article.X-2

2079. Williamson RL, Casey LB, Robertson JS, et al. Video self-modeling in children with autism: a pilot study validating prerequisite skills and extending the utilization of VSM across skill sets. Assist Technol. 2013 Summer;25(2):63-71. doi: 10.1080/10400435.2012.712604. PMID: 23923688.X-1, X-3, X-4

2080. Wilson KP. Incorporating video modeling into a school-based intervention for students with autism spectrum disorders. Lang Speech Hear Serv Sch. 2013 Jan;44(1):105-17. doi: 10.1044/0161-1461(2012/11-0098). PMID: 23087158.X-1, X-2

2081. Wincik S. Learning from Katie.

Nursing. 2013 Jul;43(7):40-1. doi: 10.1097/01.nurse.0000431138.05404.70. PMID: 23778213.X-1

2082. Wolff JJ, Hazlett HC, Lightbody AA, et al. Repetitive and self-injurious behaviors: associations with caudate volume in autism and fragile X syndrome. J Neurodev Disord. 2013;5(1):12. doi: 10.1186/1866-1955-5-12. PMID: 23639144.X-1

2083. Wolff JJ, Hupp SC, Symons FJ. Brief report: Avoidance extinction as treatment for compulsive and ritual behavior in autism. J Autism Dev Disord. 2013 Jul;43(7):1741-6. doi: 10.1007/s10803-012-1721-7. PMID: 23179345.X-1

2084. Won H, Mah W, Kim E. Autism spectrum disorder causes, mechanisms, and treatments: focus on neuronal synapses. Front Mol Neurosci. 2013;6:19. doi: 10.3389/fnmol.2013.00019. PMID: 23935565.X-1, X-2

2085. Woo CC, Leon M. Environmental enrichment as an effective treatment for autism: a randomized controlled trial. Behav Neurosci. 2013 Aug;127(4):487-97. doi: 10.1037/a0033010. PMID: 23688137. INCLUDE

2086. Xie Q, Tang J, Xu Y, et al. [Clinical observation of aripiprazole in the treatment of autism]. Zhongguo Dang Dai Er Ke Za Zhi. 2013 Apr;15(4):294-7. PMID: 23607954.X-3
2087. Yakubova G, Taber-Doughty T. Brief Report: Learning Via the Electronic Interactive Whiteboard for Two Students with Autism and a Student with Moderate Intellectual Disability. Journal of Autism \& Developmental Disorders. 2013;43(6):1465-72 8p. doi: 10.1007/s10803012-1682-x. PMID: 104172357. Language: English. Entry Date: 20130603. Revision Date: 20150711. Publication Type: Journal Article.X$1, \mathrm{X}-3, \mathrm{X}-4$

2088. Yamagata T, Sugie H. [Expert opinion: investigation of drug therapy in children with autistic symptoms]. No To Hattatsu. 2013 May;45(3):223-6. PMID: 23785839.X-2

2089. Yanardag M, Akmanoglu N, Yilmaz I. The effectiveness of video prompting on teaching aquatic play skills for children with autism. Disabil Rehabil. 2013 Jan;35(1):47-56. doi: 10.3109/09638288.2012.687030. PMID: 22624856.X-3, X-4

2090. Yazawa M, Dolmetsch RE. Modeling Timothy syndrome with iPS cells. J Cardiovasc Transl Res. 2013 Feb;6(1):1-9. doi: 10.1007/s12265-012-9444-x. PMID: 23299782.X-1

2091. Yazdani S, Yee CT, Chung PJ. Factors predicting physical activity among children with special needs. Prev Chronic Dis. 2013;10:E119. doi: 10.5888/pcd10.120283. PMID: 23866163.X-1

2092. Yoder PJ, Bottema-Beutel K, Woynaroski T, et al. Social communication intervention effects vary by dependent variable type in preschoolers with autism spectrum disorders. Evid Based Commun Assess Interv. 2013;7(4):150-74. doi: 10.1080/17489539.2014.917780. PMID: 25346776.X-1, X-2

2093. Yoshimura Y, Kikuchi M, Shitamichi $\mathrm{K}$, et al. Atypical brain lateralisation in the auditory cortex and language performance in 3to 7-year-old children with high-functioning autism spectrum disorder: a child-customised magnetoencephalography (MEG) study. Mol Autism. 2013;4(1):38. doi: 10.1186/2040-23924-38. PMID: 24103585.X-1

2094. Yuan Q, Liu YS, Yu YT, et al. [Treatment of autism children: observation on 
efficacy of behavior training with retention of needles on head]. Zhongguo Zhen Jiu. 2013 Jul;33(7):609-13. PMID: 24032193.X-1

2095. Zarafshan H, Mohammadi MR, Ahmadi F, et al. Job Burnout among Iranian Elementary School Teachers of Students with Autism: a Comparative Study. Iran J Psychiatry. 2013 Mar;8(1):20-7. PMID: 23682248.X-1

2096. Zerbo O, Iosif AM, Walker C, et al. Is maternal influenza or fever during pregnancy associated with autism or developmental delays? Results from the CHARGE (CHildhood Autism Risks from Genetics and Environment) study. J Autism Dev Disord. 2013 Jan;43(1):2533. doi: 10.1007/s10803-012-1540-X. PMID: 22562209.X-1, X-3

2097. Zhang XJ, Wu Q. [Effects of electroacupuncture at different acupoints on learning and memory ability and PSD-95 protein expression on hippocampus CA1 in rats with autism]. Zhongguo Zhen Jiu. 2013 Jul;33(7):627-31. PMID: 24032199.X-1

2098. Zhang Y, Gao D, Kluetzman K, et al. The maternal autoimmune environment affects the social behavior of offspring. J Neuroimmunol. 2013 May 15;258(1-2):51-60. doi: 10.1016/j.jneuroim.2013.02.019. PMID: 23537887.X-1

2099. . [Integrated and coordinated measures to manage autistic spectrum disorders]. Vertex. 2014 Jul-Aug;25(116):295-8. PMID:

25558493.X-1, X-2, X-3

2100. . Autism. Nurs Stand. 2014 Mar 1218;28(28):19. doi:

10.7748/ns2014.03.28.28.19.s25. PMID:

24617377.X-2, X-3

2101. . Improving autism care: the NICE quality standard. Perspect Public Health. 2014 Mar;134(2):64. doi: 10.1177/1757913914523909. PMID: 24623750.X-5

2102. . Accepted scientific research works (abstracts). Int J Yoga Therap. 2014 Sep;24:1838. PMID: 25645134.X-1

2103. . e-Pediatrics Perspectives. Pediatrics. 2014;134(2):380-96 17p. PMID: 107870842.
Language: English. Entry Date: 20140807. Revision Date: 20150712. Publication Type: Journal Article.X-1, X-2

2104. . Let's Go Out to Eat! OT Practice. 2014;19(2):10-3 4p. PMID: 107884907. Language: English. Entry Date: 20140210. Revision Date: 20150820. Publication Type: Journal Article.X-1, X-2

2105. . Lay Abstracts. Autism: The International Journal of Research \& Practice. 2014;18(2):203-7 5p. doi: 10.1177/1362361313517447. PMID: 104005354. Language: English. Entry Date: 20140128. Revision Date: 20150710. Publication Type: Journal Article.X-1

2106. . Risperidone doesn't affect core autistic traits, according to 10-year longitudinal study. Brown University Child \& Adolescent Psychopharmacology Update. 2014;16(12):3-4 2p. PMID: 103920901. Language: English. Entry Date: 20141203. Revision Date: 20150710. Publication Type: Journal Article. Journal Subset: Biomedical.X-1, X-2

2107. . ON THE WEB. Nurse.com The Magazine (South). 2014;5(9):12- 1p. PMID: 107828477. Language: English. Entry Date: 20141001. Revision Date: 20150712. Publication Type: Journal Article.X-1, X-2

2108. . Four new Evidence Updates released by NICE. Guidelines in Practice. 2014;17(6):11- 1p. PMID: 107861417. Language: English. Entry Date: 20140707. Revision Date: 20150712. Publication Type: Journal Article.X-1, X-2

2109. . News. Journal of Psychosocial Nursing \& Mental Health Services. 2014;52(6):17-57 41p. doi: 10.3928/0279369520140501-88. PMID: 103757888. Language: English. Entry Date: 20150224. Revision Date: 20150710. Publication Type: Journal Article.X$1, \mathrm{X}-2$

2110. . Adjusted analysis finds no link between exposure to antidepressants, autism. Brown University Psychopharmacology Update. 2014;25(5):4-5 2p. PMID: 104064887. Language: English. Entry Date: 20140414.

Revision Date: 20150710. Publication Type: Journal Article. Journal Subset: Biomedical.X$1, \mathrm{X}-2$ 
2111. . Oxytocin May Boost Social Skills in Children With ASD. ASHA Leader. 2014;19(2):15- 1p. PMID: 107885429. Language: English. Entry Date: 20140206. Revision Date: 20150712. Publication Type: Journal Article.X-1, X-2

2112. . Antispsychotics: 'last option' for people with autism. Nursing Standard. 2014;28(22):10- 1p. doi: 10.7748/ns2014.01.28.22.10.s11. PMID: 104016037. Language: English. Entry Date: 20140131. Revision Date: 20150710. Publication Type: Journal Article.X-1, X-2

2113. . RESEARCH ROUNDUP. Brown University Child \& Adolescent Psychopharmacology Update. 2014;16(1):8- 1p. PMID: 104129511. Language: English. Entry Date: 20131223. Revision Date: 20150710. Publication Type: Journal Article.X-1, X-2

2114. . Research opportunities in the area of mental health promotion, prevention, and intervention for children and youth. Am J Occup Ther. 2014 Sep-Oct;68(5):610-2. doi: 10.5014/ajot.2014.685001. PMID: 25184474.X-1

2115. Aasen G, Naerland T. Enhancing activity by means of tactile symbols: a study of a heterogeneous group of pupils with congenital blindness, intellectual disability and autism spectrum disorder. J Intellect Disabil. 2014 Mar;18(1):61-75. doi: 10.1177/1744629514522142. PMID: 24591428.X-1, X-3

2116. Abbeduto L, McDuffie A, Thurman AJ. The fragile $\mathrm{X}$ syndrome-autism comorbidity: what do we really know? Front Genet. 2014;5:355. doi: 10.3389/fgene.2014.00355. PMID: 25360144.X-1

2117. Abu Kuwaik G, Roberts W, Zwaigenbaum L, et al. Immunization uptake in younger siblings of children with autism spectrum disorder. Autism. 2014

Feb;18(2):148-55. doi: 10.1177/1362361312459111. PMID: 23045216.X-1

2118. Ackerman S, Wenegrat J, Rettew D, et al. No increase in autism-associated genetic events in children conceived by assisted reproduction. Fertil Steril. 2014

Aug;102(2):388-93. doi: 10.1016/j.fertnstert.2014.04.020. PMID: 24842673.X-1

2119. Adamsen D, Ramaekers V, Ho HT, et al. Autism spectrum disorder associated with low serotonin in CSF and mutations in the SLC29A4 plasma membrane monoamine transporter (PMAT) gene. Mol Autism. 2014;5:43. doi: 10.1186/2040-2392-5-43. PMID: 25802735.X-1

2120. Ahmed SS, Unland T, Slaven JE, et al. Successful use of intravenous dexmedetomidine for magnetic resonance imaging sedation in autistic children. South Med J. 2014

Sep;107(9):559-64. doi: 10.14423/smj.0000000000000160. PMID: 25188619.X-1, X-2

2121. Ahn K, An SS, Shugart YY, et al. Common polygenic variation and risk for childhood-onset schizophrenia. Mol Psychiatry. 2014 Dec 16doi: 10.1038/mp.2014.158. PMID: 25510512.X-1

2122. Ahn Y, Narous M, Tobias R, et al. The ketogenic diet modifies social and metabolic alterations identified in the prenatal valproic acid model of autism spectrum disorder. Dev Neurosci. 2014;36(5):371-80. doi: 10.1159/000362645. PMID: 25011527.X-1

2123. Akins RS, Krakowiak P, Angkustsiri $\mathrm{K}$, et al. Utilization patterns of conventional and complementary/alternative treatments in children with autism spectrum disorders and developmental disabilities in a population-based study. J Dev Behav Pediatr. 2014 Jan;35(1):110. doi: $10.1097 / \mathrm{dbp} .0000000000000013$. PMID: 24399100.X-1, X-3

2124. Alashrah S, Kandaiya S, Maalej N, et al. Skin dose measurements using radiochromic films, TLDS and ionisation chamber and comparison with Monte Carlo simulation. Radiat Prot Dosimetry. 2014 Dec;162(3):33844. doi: 10.1093/rpd/nct315. PMID: 24300340.X-1

2125. Alfawaz HA, Bhat RS, Al-Ayadhi L, et al. Protective and restorative potency of Vitamin D on persistent biochemical autistic features induced in propionic acid-intoxicated 
rat pups. BMC Complement Altern Med. 2014;14:416. doi: 10.1186/1472-6882-14-416. PMID: 25344727.X-1

2126. Al-Ghamdi M, Al-Ayadhi L, ElAnsary A. Selected biomarkers as predictive tools in testing efficacy of melatonin and coenzyme Q on propionic acid - induced neurotoxicity in rodent model of autism. BMC Neurosci. 2014;15:34. doi: 10.1186/1471-220215-34. PMID: 24568717.X-1

2127. Al-Hakbany M, Awadallah S, AlAyadhi L. The Relationship of HLA Class I and II Alleles and Haplotypes with Autism: A Case Control Study. Autism Res Treat. 2014;2014:242048. doi: 10.1155/2014/242048. PMID: 24672722.X-1

2128. Allan N, Harden J. Parental decisionmaking in uptake of the MMR vaccination: a systematic review of qualitative literature. $\mathrm{J}$ Public Health (Oxf). 2014 Oct 8doi: 10.1093/pubmed/fdu075. PMID: 25297657.X-1

2129. Allegra M, Genovesi S, Maggia M, et al. Altered GABAergic markers, increased binocularity and reduced plasticity in the visual cortex of Engrailed-2 knockout mice. Front Cell Neurosci. 2014;8:163. doi: 10.3389/fncel.2014.00163. PMID: 24987331.X-1

2130. Allen JL, Liu X, Pelkowski S, et al. Early postnatal exposure to ultrafine particulate matter air pollution: persistent ventriculomegaly, neurochemical disruption, and glial activation preferentially in male mice. Environ Health Perspect. 2014 Sep;122(9):93945. doi: 10.1289/ehp.1307984. PMID: 24901756.X-1

2131. Al-Maweri SA, Halboub ES, AlSoneidar WA, et al. Oral lesions and dental status of autistic children in Yemen: A casecontrol study. J Int Soc Prev Community Dent. 2014 Dec;4(Suppl 3):S199-203. doi: 10.4103/2231-0762.149040. PMID: 25625079.X-1

2132. Al-Sarraj Y, Al-Khair HA, Taha RZ, et al. Distal trisomy 10q syndrome, report of a patient with duplicated q24.31 - qter, autism spectrum disorder and unusual features. Clin Case Rep. 2014 Oct;2(5):201-5. doi: 10.1002/ccr3.94. PMID: 25614812.X-1
2133. Altschuler TS. The developmental trajectory of contour integration in autism spectrum disorders; 2014.

2134. Al-Zaid FS, Alhader AA, Al-Ayadhi LY. Altered ghrelin levels in boys with autism: a novel finding associated with hormonal dysregulation. Sci Rep. 2014;4:6478. doi: 10.1038/srep06478. PMID: 25257829.X-1

2135. Amodeo DA, Yi J, Sweeney JA, et al. Oxotremorine treatment reduces repetitive behaviors in BTBR T+tf/J mice. Front Synaptic Neurosci. 2014;6:17. doi: 10.3389/fnsyn.2014.00017. PMID: 25165445.X-1

2136. Amoros E, Tzvetkovitch A. [Aquatic therapy for children with autism]. Soins Pediatr Pueric. 2014 Jan-Feb(276):29-30. PMID: 24617090.X-1

2137. Andersen AB, Ehrenstein V, Erichsen $\mathrm{R}$, et al. Autism spectrum disorders in children of parents with inflammatory bowel disease - a nationwide cohort study in Denmark. Clin Exp Gastroenterol. 2014;7:105-10. doi: 10.2147/ceg.s59360. PMID: 24855384.X-1

2138. Anderson DK, Liang JW, Lord C. Predicting young adult outcome among more and less cognitively able individuals with autism spectrum disorders. J Child Psychol Psychiatry. 2014 May;55(5):485-94. doi: 10.1111/jcpp.12178. PMID: 24313878.X-1

2139. Anderson G, Maes M. Redox Regulation and the Autistic Spectrum: Role of Tryptophan Catabolites, Immuno-inflammation, Autoimmunity and the Amygdala. Curr Neuropharmacol. 2014 Mar;12(2):148-67. doi: 10.2174/1570159x11666131120223757. PMID: 24669209.X-1

2140. Anderson KA, Shattuck PT, Cooper $\mathrm{BP}$, et al. Prevalence and correlates of postsecondary residential status among young adults with an autism spectrum disorder. Autism. 2014 Jul;18(5):562-70. doi: 10.1177/1362361313481860. PMID: 23996904.X-1

2141. Aoki Y, Yahata N, Watanabe T, et al. Oxytocin improves behavioural and neural deficits in inferring others' social emotions in 
autism. Brain. 2014 Nov;137(Pt 11):3073-86.

doi: 10.1093/brain/awu231. PMID:

25149412.X-1

2142. Armus M, Costa JJ. [Between the exaggerated and the exasperated clinic. Bridges, differences and rupture in child and youth mental care]. Vertex. 2014 Jul-

Aug;25(116):279-89. PMID: 25546643.X-1

2143. Ashburner J, Rodger S, Ziviani J, et al. Occupational therapy services for people with autism spectrum disorders: current state of play, use of evidence and future learning priorities. Aust Occup Ther J. 2014 Apr;61(2):110-20. doi: 10.1111/1440-1630.12083. PMID: 24118044.X-1

2144. Ashburner JK, Rodger SA, Ziviani JM, et al. Optimizing participation of children with autism spectrum disorder experiencing sensory challenges: a clinical reasoning framework. Can J Occup Ther. 2014 Feb;81(1):29-38. PMID: 24783486.X-1

2145. Ashburner JK, Rodger SA, Ziviani JM, et al. Optimizing participation of children with autism spectrum disorder experiencing sensory challenges: A clinical reasoning framework: Optimiser la participation des enfants atteints d'un trouble du spectre autistique ayant des troubles. Canadian Journal of Occupational Therapy. 2014;81(1):29-38 10p. doi: 10.1177/0008417413520440. PMID: 104039562. Language: English. Entry Date: 20140312. Revision Date: 20150820. Publication Type: Journal Article.X-1

2146. Ashburner JK, Rodger SA, Ziviani JM, et al. Comment on: 'An intervention for sensory difficulties in children with autism: A randomized trial' by Schaaf et al. (2013). Journal of Autism and Developmental Disorders. 2014 2015-02-24;44(6):1486-8. doi: 10.1136/bmj.f3755 . 10.1007/s10803-013-1983-8 . http://dx.doi.org/10.1007/s10803-014-2083-0. PMID: 1508428187; 2014-09007-001.X-1, X-2

2147. Ashwin C, Chapman E, Howells J, et al. Enhanced olfactory sensitivity in autism spectrum conditions. Mol Autism. 2014;5:53. doi: 10.1186/2040-2392-5-53. PMID: 25908951.X-1
2148. Ayyangar KM, Rani RA, Kumar A, et al. Monte Carlo study of MLC fields for cobalt therapy machine. J Med Phys. 2014 Apr;39(2):71-84. doi: 10.4103/09716203.131279. PMID: 24872604.X-1

2149. Azuma H, Mohri I, Tachibana M, et al. [An analysis of the developmental trajectory of children with autism spectrum disorders]. No To Hattatsu. 2014 Nov;46(6):429-37. PMID: 25558586.X-1

2150. Bacchelli E, Ceroni F, Pinto D, et al. A CTNNA3 compound heterozygous deletion implicates a role for alphaT-catenin in susceptibility to autism spectrum disorder. J Neurodev Disord. 2014;6(1):17. doi: 10.1186/1866-1955-6-17. PMID: 25050139.X1

2151. Bacon EC, Dufek S, Schreibman L, et al. Measuring outcome in an early intervention program for toddlers with autism spectrum disorder: use of a curriculum-based assessment. Autism Res Treat. 2014;2014:964704. doi: 10.1155/2014/964704. PMID: 24711926.X-1, $\mathrm{X}-3$

2152. Bagatell NJ, Cram M, Alvarez CG, et al. Routines of families with adolescents with autistic disorders: a comparison study. Can J Occup Ther. 2014 Feb;81(1):62-7. PMID: 24783489.X-1

2153. Baghdadli A, Pry R, Michelon C, et al. Impact of autism in adolescents on parental quality of life. Qual Life Res. 2014 Aug;23(6):1859-68. doi: 10.1007/s11136-0140635-6. PMID: 24504623.X-1

2154. Baker SM, Milivojevich A, Kraycar T, et al. Secular trend of sex ratio and symptom patterns among children with autism spectrum disorders. Glob Adv Health Med. 2014 May;3(3):92-101. doi: 10.7453/gahmj.2014.028. PMID: 24944876.X1

2155. Bakri MH, Ismail EA, Elsedfy GO, et al. Behavioral impact of sickle cell disease in young children with repeated hospitalization. Saudi J Anaesth. 2014 Oct;8(4):504-9. doi: 10.4103/1658-354x.140867. PMID: 25422609.X-1 
2156. Balan S, Iwayama Y, Maekawa M, et al. Exon resequencing of H3K9 methyltransferase complex genes, EHMT1, EHTM2 and WIZ, in Japanese autism subjects. Mol Autism. 2014;5(1):49. doi: 10.1186/20402392-5-49. PMID: 25400900.X-1

2157. Bales KL, Solomon M, Jacob S, et al. Long-term exposure to intranasal oxytocin in a mouse autism model. Transl Psychiatry. 2014;4:e480. doi: 10.1038/tp.2014.117. PMID: 25386957.X-1

2158. Bambini-Junior V, Zanatta G, Della Flora Nunes G, et al. Resveratrol prevents social deficits in animal model of autism induced by valproic acid. Neurosci Lett. 2014 Nov 7;583:176-81. doi: 10.1016/j.neulet.2014.09.039. PMID: 25263788.X-1

2159. Banerjee A, Engineer CT, Sauls BL, et al. Abnormal emotional learning in a rat model of autism exposed to valproic acid in utero. Front Behav Neurosci. 2014;8:387. doi: 10.3389/fnbeh.2014.00387. PMID: 25429264.X-1

2160. Barbalat G, Leboyer M, Zalla T. A specific impairment in cognitive control in individuals with high-functioning autism. J Psychiatr Res. 2014 Nov;58:26-35. doi: 10.1016/j.jpsychires.2014.07.013. PMID: 25106070.X-1

2161. Barnard-Brak L, Thompson S, Wei T, et al. Assistive technology as a predictor of general or alternate assessment among elementary-aged students with autism spectrum disorders. Assist Technol. 2014

Summer;26(2):81-7. doi: 10.1080/10400435.2013.833557. PMID: 25112052.X-1

2162. Barry S, O'Sullivan EA, Toumba KJ. Barriers to dental care for children with autism spectrum disorder. Eur Arch Paediatr Dent. 2014 Apr;15(2):127-34. doi: 10.1007/s40368013-0075-y. PMID: 23943360.X-1

2163. Barvalia P. Homeopathic Management of Autism Spectrum Disorder: Illustrative Cases. Homoeopathic Heritage. 2014;39(12):35-42 8p. PMID: 107844824. Language: English. Entry Date: 20140425.
Revision Date: 20150712. Publication Type: Journal Article.X-1

2164. Baxter P. Valproate and folic acid in pregnancy: associations with autism.

Developmental Medicine \& Child Neurology. 2014;56(7):604- 1p. doi: 10.1111/dmcn.12498. PMID: 103958271. Language: English. Entry Date: 20140815 . Revision Date: 20150710. Publication Type: Journal Article.X-1

2165. Bebko JM, Schroeder JH, Weiss JA. The McGurk effect in children with autism and Asperger syndrome. Autism Res. 2014 Feb;7(1):50-9. doi: 10.1002/aur.1343. PMID: 24136870.X-1

2166. Becker JA, Clesse D, Spiegelhalter C, et al. Autistic-like syndrome in mu opioid receptor null mice is relieved by facilitated mGluR4 activity. Neuropsychopharmacology. 2014 Aug;39(9):2049-60. doi:

10.1038/npp.2014.59. PMID: 24619243.X-1

2167. Bedford R, Pickles A, Gliga T, et al. Additive effects of social and non-social attention during infancy relate to later autism spectrum disorder. Dev Sci. 2014 Jul;17(4):612-20. PMID: 25089324.X-1

2168. Bekele E, Crittendon JA, Swanson A, et al. Pilot clinical application of an adaptive robotic system for young children with autism. Autism. 2014 Jul;18(5):598-608. doi: 10.1177/1362361313479454. PMID: 24104517.X-1, X-3

2169. Bekhet AK. Self-assessed health in caregivers of persons with autism spectrum disorder: associations with depressive symptoms, positive cognitions, resourcefulness, and well-being. Perspect Psychiatr Care. 2014 Jul;50(3):210-7. doi: 10.1111/ppc.12046. PMID: 24206628.X-1

2170. Bekhet AK, Zauszniewski JA. Psychometric properties of the resourcefulness scale among caregivers of persons with autism spectrum disorder. West J Nurs Res. 2014 May;36(5):685-702. doi: 10.1177/0193945913509141. PMID: 24151172.X-1

2171. Bellebaum C, Brodmann K, Thoma P. Active and observational reward learning in adults with autism spectrum disorder: 
relationship with empathy in an atypical sample. Cogn Neuropsychiatry.

2014;19(3):205-25. doi:

10.1080/13546805.2013.823860. PMID: 23998722.X-1

2172. Bellusso P, Maumy-Bertrand M, Desnos Y, et al. Intérêts de la psychothérapie à médiation sensorielle dans le cadre de la prise en charge des troubles de la relation et de la communication chez des enfants autistes sévèrement déficitaires II: Illustration clinique. Neuropsychiatrie de l'Enfance et de l'Adolescence. 2014 2015-12-09;62(2):95-101. doi:

http://dx.doi.org/10.1016/j.neurenf.2013.12.009 . PMID: 1642633176; 2014-07522-006.X-1, X3, X-4

2173. Ben Said M, Robel L, Messiaen C, et al. Patient information, consents and privacy protection scheme for an information system dedicated to pervasive developmental disorders. Stud Health Technol Inform. 2014;205:755-9. PMID: 25160288.X-1

2174. Bener A, Khattab AO, Al-Dabbagh MM. Is high prevalence of Vitamin D deficiency evidence for autism disorder?: In a highly endogamous population. J Pediatr Neurosci. 2014 Sep-Dec;9(3):227-33. doi: 10.4103/1817-1745.147574. PMID: 25624924.X-1

2175. Ben-Itzchak E, Watson L, Zachor D. Cognitive Ability is Associated with Different Outcome Trajectories in Autism Spectrum Disorders. Journal of Autism \& Developmental Disorders. 2014;44(9):2221-9 9p. doi: 10.1007/s10803-014-2091-0. PMID: 103986562. Language: English. Entry Date: 20140818. Revision Date: 20150901. Publication Type: Journal Article.X-1, X-3

2176. Benjamin DP, Mastergeorge AM, McDuffie AS, et al. Effects of labeling and pointing on object gaze in boys with fragile $\mathrm{X}$ syndrome: an eye-tracking study. Res Dev Disabil. 2014 Nov;35(11):2658-72. doi: 10.1016/j.ridd.2014.06.021. PMID: 25062097.X-1

2177. Berger N, Ingersoll B. A Further Investigation of Goal-Directed Intention Understanding in Young Children with Autism Spectrum Disorders. Journal of Autism \&
Developmental Disorders. 2014;44(12):3204-14 11p. doi: 10.1007/s10803-014-2181-z. PMID: 103912401. Language: English. Entry Date: 20141111. Revision Date: 20151201. Publication Type: Journal Article.X-1

2178. Bergstrom R, Najdowski AC, Tarbox J. A systematic replication of teaching children with autism to respond appropriately to lures from strangers. J Appl Behav Anal. 2014 Winter;47(4):861-5. doi: 10.1002/jaba.175. PMID: 25327476.X-1, X-3

2179. Blomqvist M, Dahllof G, Bejerot S. Experiences of dental care and dental anxiety in adults with autism spectrum disorder. Autism Res Treat. 2014;2014:238764. doi: 10.1155/2014/238764. PMID: 25530879.X-1

2180. Bodner KE, Williams DL, Engelhardt CR, et al. A Comparison of Measures for Assessing the Level and Nature of Intelligence in Verbal Children and Adults with Autism Spectrum Disorder. Res Autism Spectr Disord. 2014 Nov 1;8(11):1434-42. doi: 10.1016/j.rasd.2014.07.015. PMID: 25180048.X-1

2181. Boon-Yasidhi V, Jearnarongrit $P$, Tulayapichitchock P, et al. Adverse effects of risperidone in children with autism spectrum disorders in a naturalistic clinical setting at siriraj hospital, Thailand. Psychiatry J. 2014;2014:136158. doi: 10.1155/2014/136158. PMID: 24790986.X-1

2182. Bouvet L, Simard-Meilleur AA, Paignon A, et al. Auditory local bias and reduced global interference in autism. Cognition. 2014 Jun;131(3):367-72. doi: 10.1016/j.cognition.2014.02.006. PMID: 24637103.X-1

2183. Boyd BA, Hume K, McBee MT, et al. Comparative efficacy of LEAP, TEACCH and non-model-specific special education programs for preschoolers with autism spectrum disorders. J Autism Dev Disord. 2014 Feb;44(2):366-80. doi: 10.1007/s10803-0131877-9. PMID: 23812661.X-1

2184. Boyle P, Haines D, Lovelock L, et al. Home safety for children with autistic spectrum disorder: local authority occupational therapy intervention. British Journal of Occupational Therapy (College of Occupational Therapists 
Limited). 2014;77(5):243-50 8p. doi: 10.4276/030802214X13990455043485. PMID: 103941582. Language: English. Entry Date: 20140509. Revision Date: 20150820. Publication Type: Journal Article.X-1

2185. Bradstreet JJ, Sych N, Antonucci N, et al. Efficacy of fetal stem cell transplantation in autism spectrum disorders: an open-labeled pilot study. Cell Transplant. 2014;23 Suppl 1:S105-12. doi: 10.3727/096368914x684916. PMID: 25302490.X-3

2186. Braun JM, Froehlich T, Kalkbrenner A, et al. Brief report: are autistic-behaviors in children related to prenatal vitamin use and maternal whole blood folate concentrations? J Autism Dev Disord. 2014 Oct;44(10):2602-7. doi: 10.1007/s10803-014-2114-x. PMID: 24710813.X-1

2187. Brisson J, Martel K, Serres J, et al. Acoustic analysis of oral productions of infants later diagnosed with autism and their mother. Infant Ment Health J. 2014 May-Jun;35(3):28595. doi: 10.1002/imhj.21442. PMID: 25798482.X-1

2188. Broder-Fingert S, Brazauskas K, Lindgren $\mathrm{K}$, et al. Prevalence of overweight and obesity in a large clinical sample of children with autism. Acad Pediatr. 2014 JulAug;14(4):408-14. doi: 10.1016/j.acap.2014.04.004. PMID: 24976353.X-1

2189. Broder-Fingert S, Ferrone CF, Giauque A, et al. Residents' knowledge and comfort with caring for children with autism spectrum disorder. Clin Pediatr (Phila). 2014 Dec;53(14):1390-2. doi: 10.1177/0009922814526982. PMID: 24647693.X-1

2190. Brodeur DA, Gordon Green C, Flores $\mathrm{H}$, et al. Time estimation among lowfunctioning individuals with autism spectrum disorders: evidence of poor sensitivity to variability of short durations. Autism Res. 2014 Apr;7(2):237-44. doi: 10.1002/aur.1364. PMID: 24574256.X-1

2191. Brodhead MT, Higbee TS, Pollard JS, et al. The use of linked activity schedules to teach children with autism to play hide-andseek. J Appl Behav Anal. 2014 Fall;47(3):645-
50. doi: 10.1002/jaba.145. PMID: 24905481.X$3, \mathrm{X}-4$

2192. Brodkin J, Frank D, Grippo R, et al. Validation and implementation of a novel highthroughput behavioral phenotyping instrument for mice. J Neurosci Methods. 2014 Mar 15;224:48-57. doi: 10.1016/j.jneumeth.2013.12.010. PMID: 24384067.X-1

2193. Brown A, Crowe L, Andresen BS, et al. Neurodevelopmental profiles of children with very long chain acyl-CoA dehydrogenase deficiency diagnosed by newborn screening. Mol Genet Metab. 2014 Dec;113(4):278-82. doi: 10.1016/j.ymgme.2014.10.005. PMID: 25456746.X-1

2194. Brown AB, Elder JH. Communication in autism spectrum disorder: a guide for pediatric nurses. Pediatr Nurs. 2014 SepOct;40(5):219-25. PMID: 25929112.X-1

2195. Brown CM, Austin DW, Busija L. Observable essential fatty acid deficiency markers and autism spectrum disorder. Breastfeed Rev. 2014 Jul;22(2):21-6. PMID: 25109097.X-1

2196. Bryson SA, Ostmeyer KF. Increasing the effectiveness of community mental health center social skills groups for children with autism spectrum disorder: a training and consultation example. Adm Policy Ment Health. 2014 Nov;41(6):808-21. doi: 10.1007/s10488-013-0533-1. PMID: 24389835.X-1

2197. Buck TR, Viskochil J, Farley M, et al. Psychiatric comorbidity and medication use in adults with autism spectrum disorder. J Autism Dev Disord. 2014 Dec;44(12):3063-71. doi: 10.1007/s10803-014-2170-2. PMID: 24958436.X-1

2198. Budday S, Raybaud C, Kuhl E. A mechanical model predicts morphological abnormalities in the developing human brain. Sci Rep. 2014;4:5644. doi: 10.1038/srep05644. PMID: 25008163.X-1

2199. Burgess S, Cimera RE. Employment outcomes of transition-aged adults with autism spectrum disorders: a state of the States report. Am J Intellect Dev Disabil. 2014 
Jan;119(1):64-83. doi: 10.1352/1944-7558-

119.1.64. PMID: 24450322.X-1

2200. Burgoyne L, Dowling L, Fitzgerald A, et al. Parents' perspectives on the value of assistance dogs for children with autism spectrum disorder: a cross-sectional study. BMJ Open. 2014;4(6):e004786. doi: 10.1136/bmjopen-2014-004786. PMID: 24928583.X-1

2201. Burke A. Zen shiatsu: a longitudinal case study measuring stress reduction in a child with autism spectrum disorder. Int $\mathrm{J}$ Ther Massage Bodywork. 2014 Dec;7(4):23-8. PMID: 25452821.X-1, X-3, X-4

2202. Butrimaviciute R, Grieve A. Carers' experiences of being exposed to challenging behaviour in services for autism spectrum disorders. Autism. 2014 Nov;18(8):882-90. doi: 10.1177/1362361313508022. PMID: 24142795.X-1

2203. Camacho J, Jones K, Miller E, et al. Embryonic intraventricular exposure to autismspecific maternal autoantibodies produces alterations in autistic-like stereotypical behaviors in offspring mice. Behav Brain Res. 2014 Jun 1;266:46-51. doi: 10.1016/j.bbr.2014.02.045. PMID: 24613242.X-1

2204. Campbell DJ, Chang J, Chawarska K. Early generalized overgrowth in autism spectrum disorder: prevalence rates, gender effects, and clinical outcomes. J Am Acad Child Adolesc Psychiatry. 2014 Oct;53(10):1063-73.e5. doi: 10.1016/j.jaac.2014.07.008. PMID: 25245350.X-1

2205. Campillo C, Herrera G, Remirez de Ganuza C, et al. Using Tic-Tac software to reduce anxiety-related behaviour in adults with autism and learning difficulties during waiting periods: a pilot study. Autism. 2014

Apr;18(3):264-71. doi:

10.1177/1362361312472067. PMID: 24092839.X-1

2206. Canal-Bedia R, Garcia-Primo P, Santos-Borbujo J, et al. [Screening and early care programmes in children with autism spectrum disorders]. Rev Neurol. 2014 Feb
24;58 Suppl 1:S123-7. PMID: 25252658.X-1, $\mathrm{X}-2$

2207. Carroll D, Hallett V, McDougle CJ, et al. Examination of aggression and self-injury in children with autism spectrum disorders and serious behavioral problems. Child Adolesc Psychiatr Clin N Am. 2014 Jan;23(1):57-72. doi: 10.1016/j.chc.2013.08.002. PMID: 24231167.X-1, X-2, X-3

2208. Cassidy S, Bradley P, Robinson J, et al. Suicidal ideation and suicide plans or attempts in adults with Asperger's syndrome attending a specialist diagnostic clinic: a clinical cohort study. Lancet Psychiatry. 2014 Jul;1(2):142-7. doi: 10.1016/s22150366(14)70248-2. PMID: 26360578.X-1

2209. Cattelan C. «Essere un bambino in carne ed ossa»... «diventare come tutti gli altri». Rivista di Psicoanalisi. 2014 2015-0224;60(3):641-55. PMID: 1653144492; 201456459-022.X-1, X-2

2210. Chan GF, Lai KY, Luk ES, et al. Clinical utility of the Chinese Strengths and Weaknesses of ADHD-Symptoms and NormalBehaviors questionnaire (SWAN) when compared with DISC-IV. Neuropsychiatr Dis Treat. 2014;10:1533-42. doi: 10.2147/ndt.s65879. PMID: 25187717.X-1

2211. Chan JS, Naumer MJ. Explaining autism spectrum disorders: Central coherence vs. predictive coding theories. Journal of Neurophysiology. 2014 2015-1209;112(11):2669-71. doi: http://dx.doi.org/10.1152/jn.00242.2014. PMID: 1645208157; 2014-55459-001.X-1

2212. Chandler F, Dissanayake C. An investigation of the security of caregiver attachment during middle childhood in children with high-functioning autistic disorder. Autism. 2014 Jul;18(5):485-92. doi: 10.1177/1362361313486205. PMID: 24072664.X-1

2213. Chang J, Kueon C, Kim J. Influence of lead on repetitive behavior and dopamine metabolism in a mouse model of iron overload. Toxicol Res. 2014 Dec;30(4):267-76. doi: 10.5487/tr.2014.30.4.267. PMID: 25584146.X1 
2214. Chang YC, Laugeson EA, Gantman A, et al. Predicting treatment success in social skills training for adolescents with autism spectrum disorders: the UCLA Program for the Education and Enrichment of Relational Skills. Autism. 2014 May;18(4):467-70. doi: 10.1177/1362361313478995. PMID: 24108192.X-1

2215. Cheak-Zamora NC, Farmer JE, Mayfield WA, et al. Health care transition services for youth with autism spectrum disorders. Rehabil Psychol. 2014 Aug;59(3):340-8. doi: 10.1037/a0036725. PMID: 25019309.X-1

2216. Chen CH, Huang CC, Cheng MC, et al. Genetic analysis of GABRB3 as a candidate gene of autism spectrum disorders. Mol Autism. 2014;5:36. doi: 10.1186/2040-2392-5-36. PMID: 24999380.X-1

2217. Chen CH, Lee IJ, Lin LY. Augmented reality-based self-facial modeling to promote the emotional expression and social skills of adolescents with autism spectrum disorders. Res Dev Disabil. 2014 Nov 8;36c:396-403. doi: 10.1016/j.ridd.2014.10.015. PMID:

25462499.X-1, X-3

2218. Chen R, Li C, Xie B, et al. Clinical and molecular evaluations of siblings with "pure" 11q23.3-qter trisomy or reciprocal monosomy due to a familial translocation $\mathrm{t}(10 ; 11)$ (q26;q23.3). Mol Cytogenet. 2014;7(1):101. doi: 10.1186/s13039-014-0101-8. PMID: 25648949.X-1

2219. Chen YW, Lin HC, Ng MC, et al. Activation of mGluR2/3 underlies the effects of $\mathrm{N}$-acetylcystein on amygdala-associated autism-like phenotypes in a valproate-induced rat model of autism. Front Behav Neurosci. 2014;8:219. doi: 10.3389/fnbeh.2014.00219. PMID: 24987341.X-1

2220. Chi RP, Snyder AW. Treating autism by targeting the temporal lobes. Med Hypotheses. 2014 Nov;83(5):614-8. doi: 10.1016/j.mehy.2014.08.002. PMID: 25227333.X-1

2221. Chilosi AM, Brovedani P, Ferrari AR, et al. Language regression associated with autistic regression and electroencephalographic (EEG) abnormalities: a prospective study. J
Child Neurol. 2014 Jun;29(6):855-9. doi: 10.1177/0883073813482767. PMID: 23562946.X-1, X-3, X-4

2222. Chiocchetti AG, Haslinger D, Boesch $\mathrm{M}$, et al. Protein signatures of oxidative stress response in a patient specific cell line model for autism. Mol Autism. 2014;5(1):10. doi: 10.1186/2040-2392-5-10. PMID: 24512814.X1

2223. Chiu YN, Chou MC, Lee JC, et al. Determinants of maternal satisfaction with diagnosis disclosure of autism. J Formos Med Assoc. 2014 Aug;113(8):540-8. doi: 10.1016/j.jfma.2012.07.040. PMID: 25037759.X-1

2224. Choi CS, Hong M, Kim KC, et al. Effects of atomoxetine on hyper-locomotive activity of the prenatally valproate-exposed rat offspring. Biomol Ther (Seoul). 2014 Sep;22(5):406-13. doi: 10.4062/biomolther.2014.027. PMID: 25414770.X-1

2225. Choi JE, Widjaja F, Careaga M, et al. Change in plasma cytokine levels during risperidone treatment in children with autism. J Child Adolesc Psychopharmacol. 2014 Dec;24(10):586-9. doi: 10.1089/cap.2013.0108. PMID: 24828014.X-3

2226. Chok JT, Koesler B. Distinguishing Obsessive-Compulsive Behavior From Stereotypy: A Preliminary Investigation. Behav Modif. 2014 May;38(3):344-73. doi: 10.1177/0145445513509475. PMID: 24177034.X-1

2227. Chomiak T, Hung J, Cihal A, et al. Auditory-cued sensorimotor task reveals disengagement deficits in rats exposed to the autism-associated teratogen valproic acid. Neuroscience. 2014 May 30;268:212-20. doi: 10.1016/j.neuroscience.2014.02.049. PMID: 24631679.X-1

2228. Chuang HC, Huang TN, Hsueh YP. Neuronal excitation upregulates Tbr1, a highconfidence risk gene of autism, mediating Grin2b expression in the adult brain. Front Cell Neurosci. 2014;8:280. doi: 10.3389/fncel.2014.00280. PMID: 25309323.X-1 
2229. Cidav Z, Marcus SC, Mandell DS. Home- and community-based waivers for children with autism: effects on service use and costs. Intellect Dev Disabil. 2014

Aug;52(4):239-48. doi: 10.1352/1934-9556-

52.4.239. PMID: 25061768.X-1

2230. Cohen IL, Gardner JM, Karmel BZ, et al. Rating scale measures are associated with Noldus EthoVision-XT video tracking of behaviors of children on the autism spectrum. Mol Autism. 2014;5(1):15. doi: 10.1186/20402392-5-15. PMID: 24548743.X-1

2231. Cohen-Silver JH, Muskat B, Ratnapalan S. Autism in the emergency department. Clin Pediatr (Phila). 2014 Oct;53(12):1134-8. doi: 10.1177/0009922814540983. PMID: 25031320.X-1

2232. Corbett BA, Swain DM, Coke C, et al. Improvement in social deficits in autism spectrum disorders using a theatre-based, peermediated intervention. Autism Res. 2014 Feb;7(1):4-16. doi: 10.1002/aur.1341. PMID: 24150989.X-1, X-3

2233. Corejova A, Janosikova D, Pospisilova $\mathrm{V}$, et al. Cessation of Nocturnal Enuresis After Intervention with Methylcobalamin in an 18Year-Old Patient with Autism. J Child Adolesc Psychopharmacol. 2014 Oct 17doi: 10.1089/cap.2014.0023. PMID: 25325786.X-1, $\mathrm{X}-4$

2234. Couper L, van der Meer L, Schafer $\mathrm{MC}$, et al. Comparing acquisition of and preference for manual signs, picture exchange, and speech-generating devices in nine children with autism spectrum disorder. Dev Neurorehabil. 2014 Apr;17(2):99-109. doi: 10.3109/17518423.2013.870244. PMID: 24392652.X-3, X-4

2235. Crewther DP, Crewther DP. Peripheral global neglect in high vs. low autistic tendency. Front Psychol. 2014;5:284. doi: 10.3389/fpsyg.2014.00284. PMID: 24772100.X-1

2236. Crider A, Pandya CD, Peter D, et al. Ubiquitin-proteasome dependent degradation of GABAAalpha1 in autism spectrum disorder. Mol Autism. 2014;5:45. doi: 10.1186/20402392-5-45. PMID: 25392730.X-1
2237. Crider A, Thakkar R, Ahmed AO, et al. Dysregulation of estrogen receptor beta (ERbeta), aromatase (CYP19A1), and ER coactivators in the middle frontal gyrus of autism spectrum disorder subjects. Mol Autism. 2014;5(1):46. doi: 10.1186/2040-2392-5-46. PMID: 25221668.X-1

2238. Cridland EK, Jones SC, Caputi P, et al. Being a girl in a boys' world: investigating the experiences of girls with autism spectrum disorders during adolescence. J Autism Dev Disord. 2014 Jun;44(6):1261-74. doi: 10.1007/s10803-013-1985-6. PMID: 24221816.X-1

2239. Cupples L, Ching TYC, Crowe K, et al. Outcomes of 3-year-old children with hearing loss and different types of additional disabilities. Journal of Deaf Studies and Deaf Education. 2014 2015-12-09;19(1):20-39. doi: http://dx.doi.org/10.1093/deafed/ent039. PMID: 1555974386; 2014-01462-002.X-1

2240. Dahl M, Bergsten C, Hammarberg A, et al. [Autism spectrum disorders in severe cerebral palsy often discovered late. Retrospective review of 10 years of medical records]. Lakartidningen. 2014 Aug 619;111(32-33):1296-8. PMID: 25221821.X-1, $\mathrm{X}-3$

2241. Dalsgaard S, Leckman JF, Nielsen HS, et al. Gender and injuries predict stimulant medication use. J Child Adolesc Psychopharmacol. 2014 Jun;24(5):253-9. doi: 10.1089/cap.2013.0101. PMID: 24813570.X-1

2242. Daly B, Nicholls E, Patrick K, et al. Driving Behaviors in Adults with Autism Spectrum Disorders. Journal of Autism \& Developmental Disorders. 2014;44(12):3119-28 10p. doi: 10.1007/s10803-014-2166-y. PMID: 103912414. Language: English. Entry Date: 20141111. Revision Date: 20151201. Publication Type: Journal Article.X-1

2243. Damiano CR, Aloi J, Dunlap K, et al. Association between the oxytocin receptor (OXTR) gene and mesolimbic responses to rewards. Mol Autism. 2014;5(1):7. doi: 10.1186/2040-2392-5-7. PMID: 24485285.X-1 
2244. Daniel S. [Communicating with a child with autism]. Soins Pediatr Pueric. 2014 JanFeb(276):24-5. PMID: 24617087.X-2

2245. Daniels AM, Mandell DS. Explaining differences in age at autism spectrum disorder diagnosis: a critical review. Autism. 2014 Jul;18(5):583-97. doi: 10.1177/1362361313480277. PMID: 23787411.X-1

2246. David N, Schultz J, Milne E, et al. Right Temporoparietal Gray Matter Predicts Accuracy of Social Perception in the Autism Spectrum. Journal of Autism \& Developmental Disorders. 2014;44(6):1433-46 14p. doi: 10.1007/s10803-013-2008-3. PMID: 103945244. Language: English. Entry Date: 20140520. Revision Date: 20150710. Publication Type: Journal Article.X-1

2247. Davignon MN, Friedlaender E, Cronholm PF, et al. Parent and provider perspectives on procedural care for children with autism spectrum disorders. J Dev Behav Pediatr. 2014 Apr;35(3):207-15. doi: 10.1097/dbp.0000000000000036. PMID: 24662617.X-1

2248. Day-Watkins J, Murray R, Connell JE. Teaching helping to adolescents with autism. J Appl Behav Anal. 2014 Winter;47(4):850-5. doi: 10.1002/jaba.156. PMID: 25168515.X-1

2249. de Bruin EI, Graham JH, Louwerse A, et al. Mild dermatoglyphic deviations in adolescents with autism spectrum disorders and average intellectual abilities as compared to typically developing boys. Autism Res Treat. 2014;2014:968134. doi: 10.1155/2014/968134. PMID: 25478224.X-1

2250. de Rezende MU, Hernandez AJ, Camanho GL. The natural history of the anterior knee instability by stress radiography. Acta Ortop Bras. 2014;22(4):183-7. doi: 10.1590/1413-78522014220400940. PMID: 25246846.X-1

2251. Degenne-Richard C, Wolff M, Fiard $\mathrm{D}$, et al. Les spécificités sensorielles des personnes avec autisme de l'enfance à l'âge adulte. A.N.A.E. Approche Neuropsychologique des Apprentissages chez l'Enfant. 2014 2015-12-09;26(1[128]):69-78.
PMID: 1611624232; 2014-24858-008.X-1, X-2, $\mathrm{X}-3$

2252. Dekker V, Nauta MH, Mulder EJ, et al. A randomized controlled study of a social skills training for preadolescent children with autism spectrum disorders: generalization of skills by training parents and teachers? BMC Psychiatry. 2014;14:189. doi: 10.1186/1471244x-14-189. PMID: 24989854.X-1

2253. Delfs CH, Conine DE, Frampton SE, et al. Evaluation of the efficiency of listener and tact instruction for children with autism. J Appl Behav Anal. 2014 Winter;47(4):793-809. doi: 10.1002/jaba.166. PMID: 25324150.X-3, X-4

2254. D'Elia L, Valeri G, Sonnino F, et al. A longitudinal study of the teacch program in different settings: the potential benefits of low intensity intervention in preschool children with autism spectrum disorder. J Autism Dev Disord. 2014 Mar;44(3):615-26. doi: 10.1007/s10803013-1911-y. PMID: 23949000.X-1

2255. Demoz Z, Legesse B, Teklay G, et al. Medication adherence and its determinants among psychiatric patients in an Ethiopian referral hospital. Patient Prefer Adherence. 2014;8:1329-35. doi: 10.2147/ppa.s69702. PMID: 25284991.X-1

2256. Denmark T, Atkinson J, Campbell R, et al. How do typically developing deaf children and deaf children with autism spectrum disorder use the face when comprehending emotional facial expressions in British sign language? J Autism Dev Disord. 2014 Oct;44(10):2584-92. doi: 10.1007/s10803-014-2130-x. PMID: 24803370.X-1

2257. Dere E, Dahm L, Lu D, et al. Heterozygous ambra1 deficiency in mice: a genetic trait with autism-like behavior restricted to the female gender. Front Behav Neurosci. 2014;8:181. doi: 10.3389/fnbeh.2014.00181. PMID: 24904333.X-1

2258. Dickinson K, Place M. A Randomised Control Trial of the Impact of a ComputerBased Activity Programme upon the Fitness of Children with Autism. Autism Res Treat. 2014;2014:419653. doi: 10.1155/2014/419653. PMID: 25400946.X-1 
2259. Dickstein-Fischer L, Fischer GS. Combining psychological and engineering approaches to utilizing social robots with children with Autism. Conf Proc IEEE Eng Med Biol Soc. 2014 Aug;2014:792-5. doi: 10.1109/embc.2014.6943710. PMID: 25570078.X-1

2260. Diez-Juan M, Schneider A, Phillips T, et al. Parent-delivered touchscreen intervention for children with fragile $\mathrm{X}$ syndrome. Intractable Rare Dis Res. 2014 Nov;3(4):16677. doi: 10.5582/irdr.2014.01026. PMID: 25606367.X-1, X-3

2261. Domes G, Kumbier E, Heinrichs M, et al. Oxytocin promotes facial emotion recognition and amygdala reactivity in adults with asperger syndrome.

Neuropsychopharmacology. 2014

Feb;39(3):698-706. doi: 10.1038/npp.2013.254. PMID: 24067301.X-1

2262. Donaldson AL, Stahmer AC. Team collaboration: the use of behavior principles for serving students with ASD. Lang Speech Hear Serv Sch. 2014 Oct;45(4):261-76. doi: 10.1044/2014_lshss-14-0038. PMID: 25091620.X-1

2263. Donato C, Shane HC, Hemsley B. Exploring the feasibility of the visual language in autism program for children in an early intervention group setting: views of parents, educators, and health professionals. Dev Neurorehabil. 2014 Apr;17(2):115-24. doi: 10.3109/17518423.2014.880526. PMID: 24564265.X-1

2264. Downs R, Perna J, Vitelli A, et al. Model-based hypothesis of gut microbe populations and gut/brain barrier permeabilities in the development of regressive autism. Med Hypotheses. 2014 Dec;83(6):649-55. doi: 10.1016/j.mehy.2014.09.005. PMID: 25288537.X-1

2265. Drahota A, Stadnick N, BrookmanFrazee L. Therapist perspectives on training in a package of evidence-based practice strategies for children with autism spectrum disorders served in community mental health clinics. Adm Policy Ment Health. 2014 Jan;41(1):11425. doi: 10.1007/s10488-012-0441-9. PMID: 23086499.X-1, X-3, X-4
2266. Duan G, Yao M, Ma Y, et al. Perinatal and background risk factors for childhood autism in central China. Psychiatry Res. 2014 Dec 15;220(1-2):410-7. doi: 10.1016/j.psychres.2014.05.057. PMID: 25085792.X-1

2267. Dudova I, Markova D, Kasparova M, et al. Comparison of three screening tests for autism in preterm children with birth weights less than 1,500 grams. Neuropsychiatr Dis Treat. 2014;10:2201-8. doi: 10.2147/ndt.s72921. PMID: 25484588.X-1

2268. Duzyj CM, Paidas MJ, Jebailey L, et al. PreImplantation factor (PIF*) promotes embryotrophic and neuroprotective decidual genes: effect negated by epidermal growth factor. J Neurodev Disord. 2014;6(1):36. doi: 10.1186/1866-1955-6-36. PMID: 26085845.X1

2269. Dvash J, Shamay-Tsoory SG. Theory of Mind and Empathy as Multidimensional Constructs. Topics in Language Disorders. 2014;34(4):282-95 14p. doi: 10.1097/TLD.0000000000000040. PMID: 107838770. Language: English. Entry Date: 20141124. Revision Date: 20150712. Publication Type: Journal Article.X-1

2270. Dykens EM, Fisher MH, Taylor JL, et al. Reducing distress in mothers of children with autism and other disabilities: a randomized trial. Pediatrics. 2014 Aug;134(2):e454-63. doi: 10.1542/peds.2013-3164. PMID: 25049350.X-1

2271. Eapen V, Crncec R, Walter A, et al. Conceptualisation and development of a quality of life measure for parents of children with autism spectrum disorder. Autism Res Treat. 2014;2014:160783. doi: 10.1155/2014/160783. PMID: 24778873.X-1

2272. Eden KE, de Vries PJ, Moss J, et al. Self-injury and aggression in tuberous sclerosis complex: cross syndrome comparison and associated risk markers. J Neurodev Disord. 2014;6(1):10. doi: 10.1186/1866-1955-6-10. PMID: 24822087.X-1

2273. Edmonson C, Ziats MN, Rennert OM. Altered glial marker expression in autistic postmortem prefrontal cortex and cerebellum. Mol Autism. 2014;5(1):3. doi: 10.1186/2040-23925-3. PMID: 24410870.X-1 
2274. Eisenstein M. Public health: An injection of trust. Nature. 2014 Mar 6;507(7490):S17-9. PMID: 24611174.X-1

2275. Eisinger BE, Driessen TM, Zhao C, et al. Medial prefrontal cortex: genes linked to bipolar disorder and schizophrenia have altered expression in the highly social maternal phenotype. Front Behav Neurosci. 2014;8:110. doi: 10.3389/fnbeh.2014.00110. PMID: 24765068.X-1

2276. El Marroun H, White T, Verhulst F, et al. Maternal use of antidepressant or anxiolytic medication during pregnancy and childhood neurodevelopmental outcomes: a systematic review. European Child \& Adolescent Psychiatry. 2014;23(10):973-92 20p. doi: 10.1007/s00787-014-0558-3. PMID: 103902582. Language: English. Entry Date: 20141010. Revision Date: 20151001. Publication Type: Journal Article.X-1, X-2

2277. El-Ansary A, Al-Ayadhi L. GABAergic/glutamatergic imbalance relative to excessive neuroinflammation in autism spectrum disorders. J Neuroinflammation. 2014;11:189. doi: 10.1186/s12974-014-0189-0. PMID: 25407263.X-1

2278. Elberling H, Linneberg A, Olsen EM, et al. Infancy predictors of hyperkinetic and pervasive developmental disorders at ages 5-7 years: results from the Copenhagen Child Cohort CCC2000. J Child Psychol Psychiatry. 2014 Dec;55(12):1328-35. doi: 10.1111/jcpp.12256. PMID: 24889385.X-1

2279. Elder GJ, Taylor JP. Transcranial magnetic stimulation and transcranial direct current stimulation: treatments for cognitive and neuropsychiatric symptoms in the neurodegenerative dementias? Alzheimers Res Ther. 2014;6(9):74. doi: 10.1186/s13195-0140074-1. PMID: 25478032.X-1

2280. Eldridge J, Lane AE, Belkin M, et al. Robust features for the automatic identification of autism spectrum disorder in children. $\mathrm{J}$ Neurodev Disord. 2014;6(1):12. doi: 10.1186/1866-1955-6-12. PMID: 24936212.X1

2281. Elison JT, Wolff JJ, Reznick JS, et al. Repetitive behavior in 12-month-olds later classified with autism spectrum disorder. J Am Acad Child Adolesc Psychiatry. 2014

Nov;53(11):1216-24. doi: 10.1016/j.jaac.2014.08.004. PMID: 25440311.X-1

2282. Engineer CT, Centanni TM, Im KW, et al. Degraded auditory processing in a rat model of autism limits the speech representation in non-primary auditory cortex. Dev Neurobiol. 2014 Oct;74(10):972-86. doi: 10.1002/dneu.22175. PMID: 24639033.X-1

2283. Engineer CT, Centanni TM, Im KW, et al. Speech sound discrimination training improves auditory cortex responses in a rat model of autism. Front Syst Neurosci. 2014;8:137. doi: 10.3389/fnsys.2014.00137. PMID: 25140133.X-1

2284. Enloe KA, Rapp JT. Effects of Noncontingent Social Interaction on Immediate and Subsequent Engagement in Vocal and Motor Stereotypy in Children With Autism. Behav Modif. 2014 May;38(3):374-91. doi: 10.1177/0145445513514081. PMID: 24302122.X-1, X-3, X-4

2285. Enticott PG, Fitzgibbon BM, Kennedy $\mathrm{HA}$, et al. A double-blind, randomized trial of deep repetitive transcranial magnetic stimulation (rTMS) for autism spectrum disorder. Brain Stimul. 2014 Mar-Apr;7(2):20611. doi: 10.1016/j.brs.2013.10.004. PMID: 24280031.X-1

2286. Erickson CA, Ray B, Maloney B, et al. Impact of acamprosate on plasma amyloid-beta precursor protein in youth: a pilot analysis in fragile $\mathrm{X}$ syndrome-associated and idiopathic autism spectrum disorder suggests a pharmacodynamic protein marker. J Psychiatr Res. 2014 Dec;59:220-8. doi: 10.1016/j.jpsychires.2014.07.011. PMID: 25300441.X-1

2287. Erickson CA, Veenstra-Vanderweele JM, Melmed RD, et al. STX209 (arbaclofen) for autism spectrum disorders: an 8-week openlabel study. J Autism Dev Disord. 2014 Apr;44(4):958-64. doi: 10.1007/s10803-0131963-z. PMID: 24272415.X-3

2288. Erickson CA, Wink LK, Early MC, et al. Brief report: Pilot single-blind placebo leadin study of acamprosate in youth with autistic 
disorder. J Autism Dev Disord. 2014

Apr;44(4):981-7. doi: 10.1007/s10803-0131943-3. PMID: 24052275.X-1, X-3, X-4

2289. Estes A, Vismara L, Mercado C, et al. The impact of parent-delivered intervention on parents of very young children with autism. J Autism Dev Disord. 2014 Feb;44(2):353-65. doi: 10.1007/s10803-013-1874-z. PMID: 23838727.X-1

2290. Etzel RA. Foreword: Environmental chemical exposures and autism spectrum disorders: a review of the epidemiological evidence. Curr Probl Pediatr Adolesc Health Care. 2014 Nov;44(10):275-6. doi: 10.1016/j.cppeds.2014.06.002. PMID: 25175915.X-1

2291. Eun JJ, Lee HJ, Kim JK. Developmental profiles of preschool children with delayed language development. Korean J Pediatr. 2014 Aug;57(8):363-9. doi: 10.3345/kjp.2014.57.8.363. PMID: 25210524.X-1

2292. Evers K, de-Wit L, Hallen R, et al. Brief Report: Reduced Grouping Interference in Children with ASD: Evidence from a Multiple Object Tracking Task. Journal of Autism \& Developmental Disorders. 2014;44(7):1779-87 9p. doi: 10.1007/s10803-013-2031-4. PMID: 103963190. Language: English. Entry Date: 20140620. Revision Date: 20150710. Publication Type: Journal Article.X-1

2293. F MC. ["I could see he was not acting like other children"]. Soins Pediatr Pueric. 2014 Jan-Feb(276):34-5. PMID: 24617092.X-1

2294. Faridar A, Jones-Davis D, Rider E, et al. Mapk/Erk activation in an animal model of social deficits shows a possible link to autism. Mol Autism. 2014;5:57. doi: 10.1186/20402392-5-57. PMID: 25874073.X-1

2295. Fauquie-Subrin V. [Early educational intervention for autistic children]. Soins Pediatr Pueric. 2014 Jan-Feb(276):28. PMID: 24617089.X-5

2296. Fayemi AK. Diagnosis of autism, abortion and the ethics of childcare in Yoruba culture. Indian J Med Ethics. 2014 OctDec;11(4):245-8. PMID: 25377038.X-1
2297. Fayyazi A, Salari E, Khajeh A, et al. A comparison of risperidone and buspirone for treatment of behavior disorders in children with phenylketonuria. Iran J Child Neurol. 2014 Fall;8(4):33-8. PMID: 25657768.X-1

2298. Feimann D, Whalberg E, Costa J. [Interview with Debora Feimann]. Vertex. 2014 Jul-Aug;25(116):299-303. PMID: 25558494.X-1

2299. Feinberg E, Augustyn M, Fitzgerald E, et al. Improving maternal mental health after a child's diagnosis of autism spectrum disorder: results from a randomized clinical trial. JAMA Pediatr. 2014 Jan;168(1):40-6. doi: 10.1001/jamapediatrics.2013.3445. PMID: 24217336.X-1

2300. Fellinger J, Holzinger D. Creating innovative clinical and service models for communication: Institut fuer Sinnes- und Sprachneurologie. J Dev Behav Pediatr. 2014 Feb-Mar;35(2):148-53. doi: 10.1097/dbp.0000000000000019. PMID: 24509058.X-1

2301. Feng C, Hackett PD, DeMarco AC, et al. Oxytocin and vasopressin effects on the neural response to social cooperation are modulated by sex in humans. Brain Imaging Behav. 2014 Nov 22doi: 10.1007/s11682-0149333-9. PMID: 25416642.X-1

2302. Fernandez-Jaen A, Cigudosa JC, Martin Fernandez-Mayoralas D, et al. [Genetics applied to clinical practice in neurodevelopmental disorders]. Rev Neurol. 2014 Feb 24;58 Suppl 1:S65-70. PMID: 25252670.X-1

2303. Fernell E, Wilson P, Hadjikhani N, et al. Screening, intervention and outcome in autism and other developmental disorders: the role of randomized controlled trials. J Autism Dev Disord. 2014 Aug;44(8):2074-6. doi: 10.1007/s10803-014-2070-5. PMID: 24554162.X-1

2304. Ferraro FR. No evidence of reaction time slowing in autism spectrum disorder. Autism. 2014 Dec 15doi: 10.1177/1362361314559986. PMID: 25512973.X-1 
2305. Ferreri M. [Pervasive developmental disorders]. Rev Prat. 2014 Apr;64(4):481-6. PMID: 24855782.X-1

2306. Fetner M, Cascio CJ, Essick G. Nonverbal patient with autism spectrum disorder and obstructive sleep apnea: use of desensitization to acclimatize to a dental appliance. Pediatr Dent. 2014 NovDec;36(7):499-501. PMID: 25514080.X-1

2307. Fisher WW, Greer BD, Querim AC, et al. Decreasing excessive functional communication responses while treating destructive behavior using response restriction. Res Dev Disabil. 2014 Nov;35(11):2614-23. doi: 10.1016/j.ridd.2014.06.024. PMID: 25036315.X-1, X-3, X-4

2308. Fitzpatrick EM, Lambert L, Whittingham J, et al. Examination of characteristics and management of children with hearing loss and autism spectrum disorders. Int J Audiol. 2014 Sep;53(9):577-86. doi: 10.3109/14992027.2014.903338. PMID: 24832530.X-1, X-3, X-4

2309. Foley AG, Cassidy AW, Regan CM. Pentyl-4-yn-VPA, a histone deacetylase inhibitor, ameliorates deficits in social behavior and cognition in a rodent model of autism spectrum disorders. Eur J Pharmacol. 2014 Mar 15;727:80-6. doi: 10.1016/j.ejphar.2014.01.050. PMID: 24486700.X-1

2310. Foti F, Mazzone L, Menghini D, et al. Learning by observation in children with autism spectrum disorder. Psychol Med. 2014 Aug;44(11):2437-47. doi: 10.1017/s003329171300322x. PMID: 24433947.X-1

2311. Frazier JA, McDougle CJ. Special issue on autism spectrum disorders. Introduction. Harv Rev Psychiatry. 2014 MarApr;22(2):61-4. doi: 10.1097/hrp.0000000000000028. PMID: 24614761.X-1

2312. Freitag CM. [Autism Spectrum Disorder in DSM-5 - concept, validity, and reliability, impact on clinical care and future research]. Z Kinder Jugendpsychiatr Psychother. 2014 May;42(3):185-92. doi: 10.1024/1422-4917/a000288. PMID: 24846867.X-1
2313. Freuler AC, Baranek GT, Tashjian C, et al. Parent reflections of experiences of participating in a randomized controlled trial of a behavioral intervention for infants at risk of autism spectrum disorders. Autism. 2014 Jul;18(5):519-28. doi:

10.1177/1362361313483928. PMID: 24104508.X-1, X-2

2314. Fujiwara T, Kawachi I. Are maternal social networks and perceptions of trust associated with suspected autism spectrum disorder in offspring? A population-based study in Japan. PLoS One. 2014;9(7):e101359. doi: 10.1371/journal.pone.0101359. PMID: 24983630.X-1

2315. Fulton E, Eapen V, Crncec R, et al. Reducing maladaptive behaviors in preschoolaged children with autism spectrum disorder using the early start denver model. Front Pediatr. 2014;2:40. doi: 10.3389/fped.2014.00040. PMID: 24847474.X1

2316. Funahashi A, Gruebler A, Aoki T, et al. Brief report: the smiles of a child with autism spectrum disorder during an animalassisted activity may facilitate social positive behaviors--quantitative analysis with smiledetecting interface. J Autism Dev Disord. 2014 Mar;44(3):685-93. doi: 10.1007/s10803-0131898-4. PMID: 23893100.X-3, X-4

2317. Funahashi Y, Karashima C, Hoshiyama M. Compensatory postural sway while seated posture during tasks in children with autism spectrum disorder. Occup Ther Int. 2014 Dec;21(4):166-75. doi: 10.1002/oti.1375. PMID: 24956573.X-1

2318. Fung LK, Libove RA, Phillips J, et al. Brief report: an open-label study of the neurosteroid pregnenolone in adults with autism spectrum disorder. J Autism Dev Disord. 2014 Nov;44(11):2971-7. doi: 10.1007/s10803-0142144-4. PMID: 24849255.X-1

2319. Futoo E, Miyawaki D, Goto A, et al. Sensory hypersensitivity in children with highfunctioning pervasive developmental disorder. Osaka City Med J. 2014 Dec;60(2):63-71. PMID: 25803881.X-1 
2320. Gadow KD, Smith RM, Pinsonneault JK. Serotonin 2A receptor gene (HTR2A) regulatory variants: possible association with severity of depression symptoms in children with autism spectrum disorder. Cogn Behav Neurol. 2014 Jun;27(2):107-16. doi: 10.1097/wnn.0000000000000028. PMID: 24968012.X-1

2321. Gandhi RM, Kogan CS, Messier C. 2Methyl-6-(phenylethynyl) pyridine (MPEP) reverses maze learning and PSD-95 deficits in Fmr1 knock-out mice. Front Cell Neurosci. 2014;8:70. doi: 10.3389/fncel.2014.00070. PMID: 24701200.X-1

2322. Garcia-Albea E, Reeve SA, Brothers $\mathrm{KJ}$, et al. Using audio script fading and multiple-exemplar training to increase vocal interactions in children with autism. J Appl Behav Anal. 2014 Summer;47(2):325-43. doi: 10.1002/jaba.125. PMID: 24763913.X-3, X-4

2323. Garcia-Martinez T, Chan JP, PerezCalatayud J, et al. Dosimetric characteristics of a new unit for electronic skin brachytherapy. J Contemp Brachytherapy. 2014 Mar;6(1):45-53. doi: 10.5114/jcb.2014.40770. PMID: 24790622.X-1

2324. Garg P, Lillystone D, Dossetor D, et al. An Exploratory Survey for Understanding Perceptions, Knowledge and Educational Needs of General Practitioners (GSs) Regarding Autistic Disorders in New South Wales (NSW), Australia. J Clin Diagn Res. 2014 Jul;8(7):Pc01-9. doi: 10.7860/jcdr/2014/8243.4527. PMID: 25177611.X-1

2325. Gates C. We are what we eat. Eat right to make sure you don't end up a patient. Jems. 2014 Dec;39(12):66. PMID: 25630188.X-1

2326. Gautam P, Bhatia MS, Rathi A. Intellectual disability and multiple co morbid psychiatric disorders in a child: a case report. J Clin Diagn Res. 2014 Nov;8(11):Wd01-2. doi: 10.7860/jcdr/2014/9336.5110. PMID: 25584292.X-1

2327. Gebauer L, Skewes J, Westphael G, et al. Intact brain processing of musical emotions in autism spectrum disorder, but more cognitive load and arousal in happy vs. sad music. Front Neurosci. 2014;8:192. doi: 10.3389/fnins.2014.00192. PMID: 25076869.X-1

2328. Gee BM, Thompson K, St John H. Efficacy of a sound-based intervention with a child with an autism spectrum disorder and auditory sensory over-responsivity. Occup Ther Int. 2014 Mar;21(1):12-20. doi: 10.1002/oti.1359. PMID: 24532098.X-3, X-4

2329. George B, Padmam MS, Nair MK, et al. CDC Kerala 11: Diagnosis of autism among children between 2 and $6 \mathrm{y}$--comparison of CARS against DSM-IV-TR. Indian J Pediatr. 2014 Dec;81 Suppl 2:S125-8. doi: 10.1007/s12098-014-1625-y. PMID: 25428818.X-1

2330. George B, Padmam MS, Nair MK, et al. CDC Kerala 13: Antenatal, natal and postnatal factors among children (2-6 y) with autism--a case control study. Indian J Pediatr. 2014 Dec;81 Suppl 2:S133-7. doi: 10.1007/s12098-014-1594-1. PMID: 25338492.X-1

2331. George B, Padmam MS, Nair MK, et al. CDC Kerala 14: Early child care practices at home among children (2-6 y) with autism--a case control study. Indian J Pediatr. 2014 Dec;81 Suppl 2:S138-41. doi: 10.1007/s12098014-1602-5. PMID: 25366290.X-1

2332. George B, Padmam MS, Nair MK, et al. CDC Kerala 12: Socio-demographic factors among children (2-6 y) with autism--a case control study. Indian J Pediatr. 2014 Dec;81 Suppl 2:S129-32. doi: 10.1007/s12098-0141593-2. PMID: 25366288.X-1

2333. Gerber A, Morrow E, Sheinkopf SJ, et al. The Rhode Island Consortium for Autism Research and Treatment (RI-CART): a new statewide autism collaborative. R I Med J (2013). 2014 May;97(5):31-4. PMID: 24791265.X-1

2334. Germain ND, Chen PF, Plocik AM, et al. Gene expression analysis of human induced pluripotent stem cell-derived neurons carrying copy number variants of chromosome 15q11q13.1. Mol Autism. 2014;5:44. doi: 10.1186/2040-2392-5-44. PMID: 25694803.X1 
2335. Germani T, Zwaigenbaum L, Bryson S, et al. Brief report: Assessment of early sensory processing in infants at high-risk of autism spectrum disorder. Journal of Autism and Developmental Disorders. 2014 2015-1209;44(12):3264-70. doi: 10.1007/s10803-012$1612-\mathrm{y}$. 10.1007/s10803-013-1876-x . 10.1007/s10802-008-9258-0 . http://dx.doi.org/10.1007/s10803-014-2175-x. PMID: 1560638890; 2014-27047-001.X-1

2336. Ghaeli P, Nikvarz N, Alaghband-Rad $\mathrm{J}$, et al. Effects of risperidone on core symptoms of autistic disorder based on childhood autism rating scale: an open label study. Indian J Psychol Med. 2014 Jan;36(1):66-70. doi: 10.4103/0253-7176.127254. PMID: 24701014.X-3

2337. Giacobini M, Medin E, Ahnemark E, et al. Prevalence, Patient Characteristics, and Pharmacological Treatment of Children, Adolescents, and Adults Diagnosed With ADHD in Sweden. J Atten Disord. 2014 Nov 5doi: 10.1177/1087054714554617. PMID: 25376193.X-1

2338. Giarelli E, Nocera R, Turchi R, et al. Sensory stimuli as obstacles to emergency care for children with autism spectrum disorder. Adv Emerg Nurs J. 2014 Apr-Jun;36(2):145-63. doi: 10.1097/tme.0000000000000013. PMID: 24785668.X-1

2339. Gidaya NB, Lee BK, Burstyn I, et al. In utero exposure to selective serotonin reuptake inhibitors and risk for autism spectrum disorder. J Autism Dev Disord. 2014 Oct;44(10):2558-67. doi: 10.1007/s10803-0142128-4. PMID: 24803368.X-1

2340. Gidley Larson J, Suchy Y. Does Language Guide Behavior in Children with Autism? Journal of Autism \& Developmental Disorders. 2014;44(9):2147-61 15p. doi: 10.1007/s10803-014-2089-7. PMID: 103986563. Language: English. Entry Date: 20140818. Revision Date: 20150901. Publication Type: Journal Article.X-1, X-3

2341. Gigliucci V, Leonzino M, Busnelli M, et al. Region specific up-regulation of oxytocin receptors in the opioid oprm1 (-/-) mouse model of autism. Front Pediatr. 2014;2:91. doi: 10.3389/fped.2014.00091. PMID: 25225634.X1

2342. Gillberg C. [Not Available]. Lakartidningen. 2014 Sep 24-30;111(39) PMID: 25584597.X-1

2343. Gillespie-Smith K, Riby DM, Hancock PJ, et al. Children with autism spectrum disorder (ASD) attend typically to faces and objects presented within their picture communication systems. J Intellect Disabil Res. 2014 May;58(5):459-70. doi: 10.1111/jir.12043. PMID: 23600472.X-1, X-3

2344. Gkogkas CG, Khoutorsky A, Cao R, et al. Pharmacogenetic inhibition of eIF4Edependent Mmp9 mRNA translation reverses fragile X syndrome-like phenotypes. Cell Rep. 2014 Dec 11;9(5):1742-55. doi: 10.1016/j.celrep.2014.10.064. PMID: 25466251.X-1

2345. Goldman SE, Adkins KW, Calcutt MW, et al. Melatonin in children with autism spectrum disorders: endogenous and pharmacokinetic profiles in relation to sleep. $\mathrm{J}$ Autism Dev Disord. 2014 Oct;44(10):2525-35. doi: 10.1007/s10803-014-2123-9. PMID: 24752680.X-1, X-3

2346. Goldstein F, Myers K. Telemental health: a new collaboration for pediatricians and child psychiatrists. Pediatr Ann. 2014 Feb;43(2):79-84. doi: 10.3928/0090448120140127-12. PMID: 24512157.X-1

2347. Gombash SE, Cowley CJ, Fitzgerald JA, et al. Intravenous AAV9 efficiently transduces myenteric neurons in neonate and juvenile mice. Front Mol Neurosci. 2014;7:81. doi: 10.3389/fnmol.2014.00081. PMID: 25360081.X-1

2348. Gomez C, Lizier JT, Schaum M, et al. Reduced predictable information in brain signals in autism spectrum disorder. Front Neuroinform. 2014;8:9. doi: 10.3389/fninf.2014.00009. PMID: 24592235.X1

2349. Gonzalez-Gadea ML, Tripicchio P, Rattazzi A, et al. Inter-individual cognitive variability in children with Asperger's syndrome. Front Hum Neurosci. 2014;8:575. 
doi: 10.3389/fnhum.2014.00575. PMID: 25132817.X-1

2350. Graber S, Liepelt-Scarfone I, Csoti I, et al. Post-cueing deficits with maintained cueing benefits in patients with Parkinson's disease dementia. Front Neurol. 2014;5:236. doi: 10.3389/fneur.2014.00236. PMID: 25477860.X-1

2351. Grainger C, Williams D, Lind S. Online Action Monitoring and Memory for Self-Performed Actions in Autism Spectrum Disorder. Journal of Autism \& Developmental Disorders. 2014;44(5):1193-206 14p. doi: 10.1007/s10803-013-1987-4. PMID: 104063114. Language: English. Entry Date: 20140414. Revision Date: 20150710. Publication Type: Journal Article.X-1

2352. Grant PJ, Joseph LA, Farmer CA, et al. 12-week, placebo-controlled trial of add-on riluzole in the treatment of childhood-onset obsessive-compulsive disorder.

Neuropsychopharmacology. 2014 May;39(6):1453-9. doi: 10.1038/npp.2013.343. PMID: 24356715.X-1, X-4

2353. Gray KM, Keating CM, Taffe JR, et al. Adult outcomes in autism: community inclusion and living skills. J Autism Dev Disord. 2014 Dec;44(12):3006-15. doi: 10.1007/s10803-0142159-x. PMID: 24915930.X-1

2354. Grechanina Iu B, Grechanina E, Beletskaia SV. [Impairments of gastrointestinal tract in autism]. Lik Sprava. 2014 Nov(11):8993. PMID: 25528841.X-1

2355. Green SA, Carter AS. Predictors and course of daily living skills development in toddlers with autism spectrum disorders. $\mathrm{J}$ Autism Dev Disord. 2014 Feb;44(2):256-63. doi: 10.1007/s10803-011-1275-0. PMID: 21598046.X-1

2356. Greenslade KJ, Coggins TE. Assessing young children's intention-reading in authentic communicative contexts: preliminary evidence and clinical utility. Int J Lang Commun Disord. 2014 Jul-Aug;49(4):463-77. doi: 10.1111/14606984.12076. PMID: 24684559.X-1

2357. Gregory G. Too many questions. Aust J Rural Health. 2014 Apr;22(2):87. doi: 10.1111/ajr.12111. PMID: 24731207.X-1
2358. Gringras P, Green D, Wright B, et al. Weighted blankets and sleep in autistic children--a randomized controlled trial. Pediatrics. 2014 Aug;134(2):298-306. doi: 10.1542/peds.2013-4285. PMID: 25022743. INCLUDE

2359. Groskreutz NC, Groskreutz MP, Bloom SE, et al. Generalization of negatively reinforced mands in children with autism. J Appl Behav Anal. 2014 Fall;47(3):560-79. doi: 10.1002/jaba.151. PMID: 25087550.X-3, X-4

2360. Grow LL, Kodak T, Carr JE. A comparison of methods for teaching receptive labeling to children with autism spectrum disorders: a systematic replication. J Appl Behav Anal. 2014 Fall;47(3):600-5. doi: 10.1002/jaba.141. PMID: 24902513.X-1, X-3, $\mathrm{X}-4$

2361. Gu Y, Chen J, Lu Y, et al. Integrative Frequency Power of EEG Correlates with Progression of Mild Cognitive Impairment to Dementia in Parkinson's Disease. Clin EEG Neurosci. 2014 Dec 16doi: 10.1177/1550059414543796. PMID: 25519446.X-1

2362. Gulsrud AC, Hellemann GS, Freeman SF, et al. Two to ten years: developmental trajectories of joint attention in children with ASD who received targeted social communication interventions. Autism Res. 2014 Apr;7(2):207-15. doi: 10.1002/aur.1360. PMID: 24550145.X-1, X-3

2363. Gunby KV, Rapp JT. The use of behavioral skills training and in situ feedback to protect children with autism from abduction lures. J Appl Behav Anal. 2014 Winter;47(4):856-60. doi: 10.1002/jaba.173. PMID: 25311885.X-3, X-4

2364. Gustafsson P, Kerekes N, Anckarsater $\mathrm{H}$, et al. Motor function and perception in children with neuropsychiatric and conduct problems: results from a population based twin study. J Neurodev Disord. 2014;6(1):11. doi: 10.1186/1866-1955-6-11. PMID: 24872861.X1

2365. Gutman SA, Raphael-Greenfield EI, Kerr L, et al. Using Motor-Based Role-Play to Enhance Social Skills in a Nonverbal 
Adolescent With High Functioning Autism: A Case Report. Occupational Therapy in Mental Health. 2014;30(1):12-25 14p. doi: 10.1080/0164212X.2014.878235. PMID: 104043902. Language: English. Entry Date: 20140320. Revision Date: 20150819. Publication Type: Journal Article.X-1, X-3, X-4

2366. Gvozdjakova A, Kucharska J, Ostatnikova D, et al. Ubiquinol improves symptoms in children with autism. Oxid Med Cell Longev. 2014;2014:798957. doi: 10.1155/2014/798957. PMID: 24707344.X-3

2367. Ha VS, Whittaker A, Whittaker M, et al. Living with autism spectrum disorder in Hanoi, Vietnam. Soc Sci Med. 2014 Nov;120:278-85. doi: 10.1016/j.socscimed.2014.09.038. PMID: 25262315.X-1

2368. Halepoto DM, Al-Ayadhi LY, Salam AA. Therapeutic use of hyperbaric oxygen therapy for children with autism spectrum disorder. J Coll Physicians Surg Pak. 2014 Jul;24(7):508-14. doi: 07.2014/jcpsp.508514. PMID: 25052976.X-1, X-2, X-3

2369. Halls G, Cooper PJ, Creswell C. Social communication deficits: Specific associations with Social Anxiety Disorder. J Affect Disord. 2014 Oct 2;172c:38-42. doi:

10.1016/j.jad.2014.09.040. PMID: 25451393.X-1

2370. Han S, Tai C, Jones CJ, et al. Enhancement of inhibitory neurotransmission by GABAA receptors having alpha2,3-subunits ameliorates behavioral deficits in a mouse model of autism. Neuron. 2014 Mar 19;81(6):1282-9. doi: 10.1016/j.neuron.2014.01.016. PMID: 24656250.X-1

2371. Hanaie R, Mohri I, Kagitani-Shimono $\mathrm{K}$, et al. Abnormal Corpus Callosum Connectivity, Socio-communicative Deficits, and Motor Deficits in Children with Autism Spectrum Disorder: A Diffusion Tensor Imaging Study. Journal of Autism \& Developmental Disorders. 2014;44(9):2209-20 12p. doi: 10.1007/s10803-014-2096-8. PMID: 103986558. Language: English. Entry Date: 20140818. Revision Date: 20150901. Publication Type: Journal Article.X-1
2372. Hanley GP, Jin CS, Vanselow NR, et al. Producing meaningful improvements in problem behavior of children with autism via synthesized analyses and treatments. J Appl Behav Anal. 2014 Spring;47(1):16-36. doi: 10.1002/jaba.106. PMID: 24615474.X-3, X-4

2373. Hanna M, Jr. Human vaccines \& immunotherapeutics: news. Hum Vaccin Immunother. 2014;10(7):1773-7. doi: 10.4161/hv.36241. PMID: 25424781.X-1

2374. Hansen BD, Wadsworth JP, Roberts $\mathrm{MR}$, et al. Effects of naturalistic instruction on phonological awareness skills of children with intellectual and developmental disabilities. Res Dev Disabil. 2014 Nov;35(11):2790-801. doi: 10.1016/j.ridd.2014.07.011. PMID: 25086428.X-1

2375. Hansson O, Hall S, Ohrfelt A, et al. Levels of cerebrospinal fluid alpha-synuclein oligomers are increased in Parkinson's disease with dementia and dementia with Lewy bodies compared to Alzheimer's disease. Alzheimers Res Ther. 2014;6(3):25. doi: 10.1186/alzrt255. PMID: 24987465.X-1

2376. Hashemi J, Tepper M, Vallin Spina T, et al. Computer vision tools for low-cost and noninvasive measurement of autism-related behaviors in infants. Autism Res Treat. 2014;2014:935686. doi: 10.1155/2014/935686. PMID: 25045536.X-1

2377. Hawkins BL, Ryan JB, Cory AL, et al. Effects of Equine-Assisted Therapy on Gross

Motor Skills of Two Children With AutismSpectrum Disorder. Therapeutic Recreation Journal. 20142014 2nd Quarter;48(2):135-49 15p. PMID: 103944802. Language: English. Entry Date: 20140618. Revision Date: 20150820. Publication Type: Journal Article.X-1, X-3, X-4

2378. Head AM, McGillivray JA, Stokes MA. Gender differences in emotionality and sociability in children with autism spectrum disorders. Mol Autism. 2014;5(1):19. doi: 10.1186/2040-2392-5-19. PMID: 24576331.X1

2379. Hebert ML, Kehayia E, Prelock P, et al. Does occupational therapy play a role for communication in children with autism spectrum disorders? Int J Speech Lang Pathol. 
2014 Dec;16(6):594-602. doi:

10.3109/17549507.2013.876665. PMID:

24460071.X-1

2380. Hebron J, Humphrey N. Exposure to bullying among students with autism spectrum conditions: a multi-informant analysis of risk and protective factors. Autism. 2014

Aug;18(6):618-30. doi:

10.1177/1362361313495965. PMID: 23886576.X-1

2381. Heitzer AM, Job MA, Pandit NK, et al. Should clinical trial research of psychotropic medication in autism control for gastrointestinal symptoms? J Clin Pharmacol. 2014

Oct;54(10):1093-6. doi: 10.1002/jcph.324.

PMID: 24788353.X-1

2382. Hemmat M, Rumple MJ, Mahon LW, et al. Short stature, digit anomalies and dysmorphic facial features are associated with the duplication of miR-17 92 cluster. Mol Cytogenet. 2014;7:27. doi: 10.1186/1755-81667-27. PMID: 24739087.X-1

2383. Hepburn SL, Stern JA, Blakeley-Smith A, et al. Complex Psychiatric Comorbidity of Treatment-Seeking Youth With Autism Spectrum Disorder and Anxiety Symptoms. J Ment Health Res Intellect Disabil. 2014;7(4):359-78. doi: 10.1080/19315864.2014.932476. PMID: 25960821.X-1

2384. Hesselmark E, Plenty S, Bejerot S. Group cognitive behavioural therapy and group recreational activity for adults with autism spectrum disorders: a preliminary randomized controlled trial. Autism. 2014 Aug;18(6):67283. doi: 10.1177/1362361313493681. PMID: 24089423.X-1

2385. Hill AP, Zuckerman KE, Hagen AD, et al. Aggressive Behavior Problems in Children with Autism Spectrum Disorders: Prevalence and Correlates in a Large Clinical Sample. Res Autism Spectr Disord. 2014 Sep 1;8(9):112133. doi: 10.1016/j.rasd.2014.05.006. PMID: 25221619.X-1

2386. Hilton CL, Cumpata K, Klohr C, et al. Effects of exergaming on executive function and motor skills in children with autism spectrum disorder: a pilot study. Am J Occup Ther. 2014 Jan-Feb;68(1):57-65. doi: 10.5014/ajot.2014.008664. PMID: 24367956.X-1, X-3

2387. Hirjak D, Wolf RC, Koch SC, et al. Neurological abnormalities in recent-onset schizophrenia and Asperger-syndrome. Frontiers in Psychiatry. 2014 2015-12-09;5doi: 10.1016/j.jpsychires.2012.01.015. PMID: 1653145172; 2014-52575-001.X-1

2388. Hirota T, Veenstra-VanderWeele J, Hollander E, et al. Antiepileptic Medications in Autism Spectrum Disorder: A Systematic Review and Meta-Analysis. Journal of Autism \& Developmental Disorders. 2014;44(4):948-57 10p. doi: 10.1007/s10803-013-1952-2. PMID: 104039471. Language: English. Entry Date: 20140311. Revision Date: 20150710. Publication Type: Journal Article.X-2

2389. Hodgetts S, McConnell D, Zwaigenbaum L, et al. The impact of autism services on mothers' occupational balance and participation. OTJR (Thorofare N J). 2014 Spring;34(2):81-92. doi: 10.3928/1539449220130109-01. PMID: 24651599.X-1

2390. Hoefman R, Payakachat N, van Exel J, et al. Caring for a child with autism spectrum disorder and parents' quality of life: application of the CarerQol. J Autism Dev Disord. 2014 Aug;44(8):1933-45. doi: 10.1007/s10803-0142066-1. PMID: 24577786.X-1

2391. Hoffman K, Falcomata TS. An evaluation of resurgence of appropriate communication in individuals with autism who exhibit severe problem behavior. J Appl Behav Anal. 2014 Fall;47(3):651-6. doi: 10.1002/jaba.144. PMID: 24910326.X-1, X-3, $\mathrm{X}-4$

2392. Hogan-Brown AL, Hoedemaker RS, Gordon PC, et al. Eye-voice span during rapid automatized naming: evidence of reduced automaticity in individuals with autism spectrum disorder and their siblings. $\mathrm{J}$ Neurodev Disord. 2014;6(1):33. doi: 10.1186/1866-1955-6-33. PMID: 25177372.X1

2393. Holm MB, Baird JM, Kim YJ, et al. Therapeutic horseback riding outcomes of parent-identified goals for children with autism spectrum disorder: an ABA' multiple case design examining dosing and generalization to 
the home and community. J Autism Dev

Disord. 2014 Apr;44(4):937-47. doi:

10.1007/s10803-013-1949-x. PMID:

24091469.X-3, X-4

2394. Hong YZ, Zhang XJ, Hong L, et al. [Influence of acupuncture of "Changqiang" (GV 1) on learning-memory ability and gap junctionrelated protein expression in the prefrontal cortex in autism rats]. Zhen Ci Yan Jiu. 2014 Jun;39(3):173-9. PMID: 25069191.X-1

2395. Horder J, Wilson CE, Mendez MA, et al. Autistic traits and abnormal sensory experiences in adults. Journal of Autism and Developmental Disorders. 2014 2015-1209;44(6):1461-9. doi: http://dx.doi.org/10.1007/s10803-013-2012-7. PMID: 1466097600; 2013-43128-001.X-1

2396. Horlin C, Falkmer M, Parsons R, et al. The cost of autism spectrum disorders. PLoS One. 2014;9(9):e106552. doi: 10.1371/journal.pone.0106552. PMID: 25191755.X-1

2397. Horstmann M, Banek S, Gakis G, et al. Prospective evaluation of fluorescence-guided cystoscopy to detect bladder cancer in a highrisk population: results from the UroScreenStudy. Springerplus. 2014;3:24. doi: 10.1186/2193-1801-3-24. PMID: 24478941.X1

2398. Hovik KT, Egeland J, Isquith PK, et al. Distinct Patterns of Everyday Executive Function Problems Distinguish Children With Tourette Syndrome From Children With ADHD or Autism Spectrum Disorders. J Atten Disord. 2014 Sep 24doi: 10.1177/1087054714550336. PMID: 25253683.X-1

2399. Howard JS, Stanislaw H, Green G, et al. Comparison of behavior analytic and eclectic early interventions for young children with autism after three years. Res Dev Disabil. 2014 Dec;35(12):3326-44. doi: 10.1016/j.ridd.2014.08.021. PMID: 25190094.X-1

2400. Hsia Y, Wong AY, Murphy DG, et al. Psychopharmacological prescriptions for people with autism spectrum disorder (ASD): a multinational study. Psychopharmacology (Berl). 2014 Mar;231(6):999-1009. doi: 10.1007/s00213-013-3263-x. PMID: 24005531.X-1

2401. Huang CY, Yen HC, Tseng MH, et al. Impacts of autistic behaviors, emotional and behavioral problems on parenting stress in caregivers of children with autism. J Autism Dev Disord. 2014 Jun;44(6):1383-90. doi: 10.1007/s10803-013-2000-y. PMID: 24287878.X-1

2402. Huke V, Turk J, Saeidi S, et al. The clinical implications of high levels of autism spectrum disorder features in anorexia nervosa: a pilot study. Eur Eat Disord Rev. 2014 Mar;22(2):116-21. doi: 10.1002/erv.2269. PMID: 24277715.X-1

2403. Humm LB, Olsen D, Be M, et al. Simulated job interview improves skills for adults with serious mental illnesses. Stud Health Technol Inform. 2014;199:50-4. PMID: 24875689.X-1

2404. Huss E, Sintzel F. [Outpatient and institutional care for the treatment of autism]. Soins Pediatr Pueric. 2014 Jan-Feb(276):20-3. PMID: 24617086.X-1, X-2

2405. Hyvarinen L, Walthes R, Jacob N, et al. Current Understanding of What Infants See. Curr Ophthalmol Rep. 2014;2(4):142-9. doi: 10.1007/s40135-014-0056-2. PMID: 25478306.X-1

2406. Irwin JR, Brancazio L. Seeing to hear? Patterns of gaze to speaking faces in children with autism spectrum disorders. Front Psychol. 2014;5:397. doi: 10.3389/fpsyg.2014.00397. PMID: 24847297.X-1

2407. Ishihara Y, Sawada A, Nakamura M, et al. Development of a dose verification system for Vero4DRT using Monte Carlo method. J Appl Clin Med Phys. 2014;15(6):4961. doi: 10.1120/jacmp.v15i6.4961. PMID: 25493521.X-1

2408. Isong IA, Rao SR, Holifield C, et al. Addressing dental fear in children with autism spectrum disorders: a randomized controlled pilot study using electronic screen media. Clin Pediatr (Phila). 2014 Mar;53(3):230-7. doi: 10.1177/0009922813517169. PMID: 24391123.X-1, X-3 
2409. Isshiki M, Tanaka S, Kuriu T, et al. Enhanced synapse remodelling as a common phenotype in mouse models of autism. Nat Commun. 2014;5:4742. doi:

10.1038/ncomms5742. PMID: 25144834.X-1

2410. Iwanaga R, Honda S, Nakane H, et al. Pilot study: efficacy of sensory integration therapy for Japanese children with highfunctioning autism spectrum disorder. Occup Ther Int. 2014 Mar;21(1):4-11. doi: 10.1002/oti.1357. PMID: 23893373. INCLUDE

2411. Iwanami A, Okajima Y, Ota H, et al. P300 component of event-related potentials in persons with asperger disorder. J Clin Neurophysiol. 2014 Oct;31(5):493-9. doi: 10.1097/wnp.0000000000000080. PMID: 25271690.X-1

2412. Iwata K, Matsuzaki H, Tachibana T, et al. N-ethylmaleimide-sensitive factor interacts with the serotonin transporter and modulates its trafficking: implications for pathophysiology in autism. Mol Autism. 2014;5:33. doi: 10.1186/2040-2392-5-33. PMID: 24834316.X1

2413. Jahan A, Rezina-Parvin SZ, Bugum D. Familial, Social and Environmental Risk Factors in Autism: A Case-Control Study. Bangladesh Med Res Counc Bull. 2014 Dec;40(3):113-7. PMID: 26402976.X-1

2414. Jain A, Spencer D, Yang W, et al. Injuries among children with autism spectrum disorder. Acad Pediatr. 2014 Jul-

Aug;14(4):390-7. doi:

10.1016/j.acap.2014.03.012. PMID:

24976351.X-1

2415. Jain T, Cautam V. Experience with Homoeopathic Remedies in the Treatment of ADHD and Autism. Homoeopathic Heritage. 2014;40(4):33-9 7p. PMID: 107817965. Language: English. Entry Date: 20140915. Revision Date: 20150712. Publication Type: Journal Article.X-5

2416. Jalili M, Jahangiri N, Yazdi AA, et al. The effects of imitative vs. Cognitive methods on the speech development of children with autism. Iran J Child Neurol. 2014 Winter;8(1):37-46. PMID: 24665326.X-1
2417. James LW, Pizur-Barnekow KA, Schefkind S. Online survey examining practitioners' perceived preparedness in the early identification of autism. Am J Occup Ther. 2014 Jan-Feb;68(1):e13-20. doi: 10.5014/ajot.2014.009027. PMID: 24367965.X-1

2418. Janeslatt G, Kottorp A, Granlund M. Evaluating intervention using time aids in children with disabilities. Scand J Occup Ther. 2014 May;21(3):181-90. doi: 10.3109/11038128.2013.870225. PMID: 24417452.X-1

2419. Janssen FM, Landry G, Cambraia Lopes P, et al. Factors influencing the accuracy of beam range estimation in proton therapy using prompt gamma emission. Phys Med Biol. 2014 Aug 7;59(15):4427-41. doi: 10.1088/0031-9155/59/15/4427. PMID: 25049223.X-1

2420. Jaschke AC. Music intervention as system: reversing hyper systemising in autism spectrum disorders to the comprehension of music as intervention. Med Hypotheses. 2014 Jan;82(1):40-8. doi: 10.1016/j.mehy.2013.11.001. PMID: 24280561.X-1

2421. Jasien JM, Daimon CM, Wang R, et al. The effects of aging on the BTBR mouse model of autism spectrum disorder. Front Aging Neurosci. 2014;6:225. doi: 10.3389/fnagi.2014.00225. PMID: 25225482.X-1

2422. Jeong JW, Tiwari VN, Behen ME, et al. In vivo detection of reduced Purkinje cell fibers with diffusion MRI tractography in children with autistic spectrum disorders. Front Hum Neurosci. 2014;8:110. doi: 10.3389/fnhum.2014.00110. PMID: 24592234.X-1

2423. Jeste SS, Wu JY, Senturk D, et al. Early developmental trajectories associated with ASD in infants with tuberous sclerosis complex. Neurology. 2014;83(2):160-8 9p. doi: 10.1212/WNL.0000000000000568. PMID: 107862115. Language: English. Entry Date: 20140912. Revision Date: 20150712. Publication Type: Journal Article.X-1 
2424. Ji B, Sun M, Yi R, et al. Multidisciplinary parent education for caregivers of children with autism spectrum disorders. Arch Psychiatr Nurs. 2014 Oct;28(5):319-26. doi: 10.1016/j.apnu.2014.06.003. PMID: 25439973.X-1

2425. Ji B, Zhao I, Turner C, et al. Predictors of health-related quality of life in Chinese caregivers of children with autism spectrum disorders: a cross-sectional study. Arch Psychiatr Nurs. 2014 Oct;28(5):327-32. doi: 10.1016/j.apnu.2014.06.001. PMID: 25439974.X-1

2426. Jiménez L, Lorda M, Méndez C. Emulation and Mimicry in School Students with Typical Development and with High Functioning Autism. Journal of Autism \& Developmental Disorders. 2014;44(7):1597-608 12p. doi: 10.1007/s10803-013-2027-0. PMID: 103963185. Language: English. Entry Date: 20140620. Revision Date: 20150710. Publication Type: Journal Article.X-1

2427. Johnson N, Bree O, Lalley EE, et al. Effect of a social script iPad application for children with autism going to imaging. J Pediatr Nurs. 2014 Nov-Dec;29(6):651-9. doi: 10.1016/j.pedn.2014.04.007. PMID: 24836052.X-1

2428. Johnson NL, Bekhet A, Robinson K, et al. Attributed meanings and strategies to prevent challenging behaviors of hospitalized children with autism: two perspectives. J Pediatr Health Care. 2014 Sep-Oct;28(5):38693. doi: 10.1016/j.pedhc.2013.10.001. PMID: 24239062.X-1

2429. Johnson RA, Danis M, Hafner-Eaton C. US state variation in autism insurance mandates: balancing access and fairness. Autism. 2014 Oct;18(7):803-14. doi: 10.1177/1362361314529191. PMID: 24789870.X-1

2430. Jones I, McDonald L. Living with uncertainty: antidepressants and pregnancy. $\mathrm{Br}$ J Psychiatry. 2014 Aug;205(2):103-4. doi: 10.1192/bjp.bp.113.141713. PMID: 25252318.X-1

2431. Jones J, Lerman DC, Lechago S. Assessing stimulus control and promoting generalization via video modeling when teaching social responses to children with autism. J Appl Behav Anal. 2014 Spring;47(1):37-50. doi: 10.1002/jaba.81. PMID: 24114657.X-1, X-3

2432. Jones L, Hastings RP, Totsika V, et al. Child behavior problems and parental wellbeing in families of children with autism: the mediating role of mindfulness and acceptance. Am J Intellect Dev Disabil. 2014 Mar;119(2):171-85. doi: 10.1352/1944-7558119.2.171. PMID: 24679352.X-1

2433. Joosten AV, Safe AP. Management strategies of mothers of school-age children with autism: implications for practice. Aust Occup Ther J. 2014 Aug;61(4):249-58. doi: 10.1111/1440-1630.12116. PMID: 24499184.X-1

2434. Joshi G, Faraone SV, Wozniak J, et al. Symptom Profile of ADHD in Youth With High-Functioning Autism Spectrum Disorder: A Comparative Study in Psychiatrically Referred Populations. J Atten Disord. 2014 Aug 1doi: 10.1177/1087054714543368. PMID: 25085653.X-1

2435. Jung M, Kosaka H, Saito DN, et al. Default mode network in young male adults with autism spectrum disorder: relationship with autism spectrum traits. Mol Autism. 2014;5:35. doi: 10.1186/2040-2392-5-35. PMID: 24955232.X-1

2436. Kaale A, Fagerland MW, Martinsen EW, et al. Preschool-based social communication treatment for children with autism: 12-month follow-up of a randomized trial. J Am Acad Child Adolesc Psychiatry. 2014 Feb;53(2):188-98. doi: 10.1016/j.jaac.2013.09.019. PMID: 24472253.X-1

2437. Kaboski JR, Diehl JJ, Beriont J, et al. Brief Report: A Pilot Summer Robotics Camp to Reduce Social Anxiety and Improve Social/Vocational Skills in Adolescents with ASD. J Autism Dev Disord. 2014 Jun 5doi: 10.1007/s10803-014-2153-3. PMID: 24898910.X-1, X-4

2438. Kanne SM, Mazurek MO, Sikora D, et al. The Autism Impact Measure (AIM): initial development of a new tool for treatment 
outcome measurement. J Autism Dev Disord. 2014 Jan;44(1):168-79. doi: 10.1007/s10803013-1862-3. PMID: 23748386.X-1

2439. Kara B, Mukaddes NM, Altinkaya I, et al. Using the modified checklist for autism in toddlers in a well-child clinic in Turkey: adapting the screening method based on culture and setting. Autism. 2014 Apr;18(3):331-8. doi: 10.1177/1362361312467864. PMID: 23175752.X-1

2440. Karvat G, Kimchi T. Acetylcholine elevation relieves cognitive rigidity and social deficiency in a mouse model of autism. Neuropsychopharmacology. 2014 Mar;39(4):831-40. doi: 10.1038/npp.2013.274. PMID: 24096295.X-1

2441. Kasari C, Kaiser A, Goods K, et al. Communication interventions for minimally verbal children with autism: a sequential multiple assignment randomized trial. J Am Acad Child Adolesc Psychiatry. 2014 Jun;53(6):635-46. doi: 10.1016/j.jaac.2014.01.019. PMID: 24839882.X-1

2442. Kasari C, Lawton K, Shih W, et al. Caregiver-mediated intervention for lowresourced preschoolers with autism: an RCT. Pediatrics. 2014 Jul;134(1):e72-9. doi: 10.1542/peds.2013-3229. PMID: 24958585.X-1

2443. Kasari C, Siller M, Huynh LN, et al. Randomized controlled trial of parental responsiveness intervention for toddlers at high risk for autism. Infant Behav Dev. 2014 Nov;37(4):711-21. doi: 10.1016/j.infbeh.2014.08.007. PMID: 25260191.X-1

2444. Kasarpalkar NJ, Kothari ST, Dave UP. Brain-Derived Neurotrophic Factor in children with Autism Spectrum Disorder. Ann Neurosci. 2014 Oct;21(4):129-33. doi: 10.5214/ans.0972.7531.210403. PMID: 25452672.X-1

2445. Kasirer A, Mashal N. Verbal creativity in autism: comprehension and generation of metaphoric language in high-functioning autism spectrum disorder and typical development. Front Hum Neurosci. 2014;8:615. doi: 10.3389/fnhum.2014.00615. PMID: 25157225.X-1
2446. Kato F, Iwanaga R, Chono M, et al. Relationship between Sympathetic Skin Responses and Auditory Hypersensitivity to Different Auditory Stimuli. J Phys Ther Sci. 2014 Jul;26(7):1087-91. doi: 10.1589/jpts.26.1087. PMID: 25140103.X-1

2447. Kaye L, Kurtz M, Tierney C, et al. Gaze maintenance and autism spectrum disorder. J Dev Behav Pediatr. 2014 NovDec;35(9):610-2. doi: 10.1097/dbp.0000000000000106. PMID: 25325757.X-1

2448. Kenworthy L, Anthony LG, Naiman DQ, et al. Randomized controlled effectiveness trial of executive function intervention for children on the autism spectrum. J Child Psychol Psychiatry. 2014 Apr;55(4):374-83. doi: 10.1111/jcpp.12161. PMID: 24256459.X-1

2449. Kerekes N, Lundstrom S, Chang Z, et al. Oppositional defiant- and conduct disorderlike problems: neurodevelopmental predictors and genetic background in boys and girls, in a nationwide twin study. PeerJ. 2014;2:e359. doi: 10.7717/peerj.359. PMID: 24795851.X-1

2450. Key AP, Ibanez LV, Henderson HA, et al. Positive Affect Processing and Joint Attention in Infants at High Risk for Autism: An Exploratory Study. J Autism Dev Disord. 2014 Jul 24doi: 10.1007/s10803-014-2191-x. PMID: 25056131.X-1

2451. Kim JW, Seung H, Kwon KJ, et al. Subchronic treatment of donepezil rescues impaired social, hyperactive, and stereotypic behavior in valproic acid-induced animal model of autism. PLoS One. 2014;9(8):e104927. doi: 10.1371/journal.pone.0104927. PMID: 25133713.X-1

2452. Kimhi Y. Theory of Mind Abilities and Deficits in Autism Spectrum Disorders. Topics in Language Disorders. 2014;34(4):32943 15p. doi: 10.1097/TLD. 0000000000000033. PMID: 107838773. Language: English. Entry Date: 20141124 . Revision Date: 20150712. Publication Type: Journal Article.X-1

2453. Kimple KS, Bartelt EA, Wysocki KL, et al. Performance of the Modified Checklist for Autism in Toddlers in Spanish-speaking patients. Clin Pediatr (Phila). 2014 
Jun;53(7):632-8. doi:

10.1177/0009922814522346. PMID:

24550559.X-1

2454. Klusek J, Martin GE, Losh M. A comparison of pragmatic language in boys with autism and fragile $X$ syndrome. J Speech Lang Hear Res. 2014 Oct;57(5):1692-707. doi: 10.1044/2014_jslhr-l-13-0064. PMID: 24686468.X-1

2455. Knopf A. Kids TLC helps families and children, focusing on trauma and partnerships. Behav Healthc. 2014 May-Jun;34(3):55-6. PMID: 25065158.X-1

2456. Kobari-Wright VV, Miguel CF. The effects of listener training on the emergence of categorization and speaker behavior in children with autism. J Appl Behav Anal. 2014 Summer;47(2):431-6. doi: 10.1002/jaba.115. PMID: 24740431.X-3, X-4

2457. Koegel L, Singh A, Koegel R, et al. Assessing and Improving Early Social Engagement in Infants. J Posit Behav Interv. 2014 Apr;16(2):69-80. doi: 10.1177/1098300713482977. PMID: 25313271.X-1

2458. Koegel RL, Bradshaw JL, Ashbaugh $\mathrm{K}$, et al. Improving question-asking initiations in young children with autism using pivotal response treatment. J Autism Dev Disord. 2014 Apr;44(4):816-27. doi: 10.1007/s10803-0131932-6. PMID: 24014174.X-3, X-4

2459. Koehler AD, Fagnano M, Montes G, et al. Elevated burden for caregivers of children with persistent asthma and a developmental disability. Matern Child Health J. 2014 Nov;18(9):2080-8. doi: 10.1007/s10995-0141455-6. PMID: 24619226.X-1

2460. Koelkebeck K, Riedel A, Ohrmann P, et al. [High-functioning autism spectrum disorders in adulthood]. Nervenarzt. 2014 Jul;85(7):891-900; quiz 1-2. doi: 10.1007/s00115-014-4050-6. PMID: 24969950.X-1

2461. Koenig KP, Feldman JM, Siegel D, et al. Issues in implementing a comprehensive intervention for public school children with autism spectrum disorders. J Prev Interv Community. 2014;42(4):248-63. doi:
10.1080/10852352.2014.943638. PMID: 25321640.X-1

2462. Kohl S, Wolters C, Gruendler TO, et al. Prepulse inhibition of the acoustic startle reflex in high functioning autism. PLoS One. 2014;9(3):e92372. doi: 10.1371/journal.pone.0092372. PMID: 24643088.X-1

2463. Kolevzon A, Bush L, Wang AT, et al. A pilot controlled trial of insulin-like growth factor-1 in children with Phelan-McDermid syndrome. Mol Autism. 2014;5(1):54. doi: 10.1186/2040-2392-5-54. PMID: 25685306.X$1, \mathrm{X}-4$

2464. Kontorinis G, Lloyd SK, Henderson L, et al. Cochlear implantation in children with auditory neuropathy spectrum disorders. Cochlear Implants Int. 2014 May;15 Suppl 1:S51-4. doi: 10.1179/1467010014z.000000000157. PMID: 24869444.X-1

2465. Koolen S, Vissers C, Egger J, et al. How Stimulus and Task Complexity Affect Monitoring in High-Functioning Adults with Autism Spectrum Disorder. Journal of Autism \& Developmental Disorders. 2014;44(10):2499513 15p. doi: 10.1007/s10803-014-2119-5. PMID: 103891499. Language: English. Entry Date: 20140922. Revision Date: 20151001. Publication Type: Journal Article.X-1

2466. Koren EV, Kupriyanova TA, Dubinskaya AO, et al. [An influence of mental disorder in the child on the parents in the context of differentiated approaches to psychosocial interventions on childhood psychiatry]. Zh Nevrol Psikhiatr Im S S Korsakova. 2014;114(10):14-9. PMID: 25591510.X-1

2467. Korotchenko S, Cingolani LA, Kuznetsova T, et al. Modulation of network activity and induction of homeostatic synaptic plasticity by enzymatic removal of heparan sulfates. Philos Trans R Soc Lond B Biol Sci. 2014 Oct 19;369(1654):20140134. doi: 10.1098/rstb.2014.0134. PMID: 25225107.X-1

2468. Koterba EA, Leezenbaum NB, Iverson $\mathrm{JM}$. Object exploration at 6 and 9 months in infants with and without risk for autism. Autism: The International Journal of Research 
\& Practice. 2014;18(2):97-105 9p. doi: 10.1177/1362361312464826. PMID: 104005351. Language: English. Entry Date: 20140128. Revision Date: 20150710. Publication Type: Journal Article.X-1

2469. Kover ST, Davidson MM, Sindberg $\mathrm{HA}$, et al. Use of the ADOS for assessing spontaneous expressive language in young children with ASD: a comparison of sampling contexts. J Speech Lang Hear Res. 2014 Dec;57(6):2221-33. doi: 10.1044/2014_jslhr-l13-0330. PMID: 25093577.X-1

2470. Kozhushko N, Kropotov Iu D, Matveev Iu K, et al. [Brain structures and functional pecularities in children with mental disorders and transcranial direct current stimulation]. Fiziol Cheloveka. 2014 JulAug;40(4):36-43. PMID: 25707217.X-1

2471. Kramer J, Rubin A, Coster W, et al. Strategies to address participant misrepresentation for eligibility in Web-based research. Int J Methods Psychiatr Res. 2014 Mar;23(1):120-9. doi: 10.1002/mpr.1415. PMID: 24431134.X-1

2472. Krawczyk DC, Kandalaft MR, Didehbani N, et al. An investigation of reasoning by analogy in schizophrenia and autism spectrum disorder. Front Hum Neurosci. 2014;8:517. doi: 10.3389/fnhum.2014.00517. PMID: 25191240.X-1

2473. Kronenberg LM, Slager-Visscher K, Goossens PJ, et al. Everyday life consequences of substance use in adult patients with a substance use disorder (SUD) and co-occurring attention deficit/hyperactivity disorder (ADHD) or autism spectrum disorder (ASD): a patient's perspective. BMC Psychiatry. 2014;14:264. doi: 10.1186/s12888-014-0264-1. PMID: 25234344.X-1

2474. Kuhn M, Bransfield R. Divergent opinions of proper Lyme disease diagnosis and implications for children co-morbid with autism spectrum disorder. Med Hypotheses. 2014

Sep;83(3):321-5. doi: 10.1016/j.mehy.2014.06.005. PMID: 24986703.X-1

2475. Kumar M, Duda JT, Hwang WT, et al. High resolution magnetic resonance imaging for characterization of the neuroligin-3 knock- in mouse model associated with autism spectrum disorder. PLoS One.

2014;9(10):e109872. doi: 10.1371/journal.pone.0109872. PMID: 25299583.X-1

2476. Kumazaki H, Watanabe K, Imasaka Y, et al. Risperidone-associated urinary incontinence in patients with autistic disorder with mental retardation. J Clin Psychopharmacol. 2014 Oct;34(5):624-6. doi: 10.1097/jcp.0000000000000197. PMID: 25118082.X-1, X-3

2477. Kushki A, Brian J, Dupuis A, et al. Functional autonomic nervous system profile in children with autism spectrum disorder. Mol Autism. 2014;5:39. doi: 10.1186/2040-2392-539. PMID: 25031832.X-1

2478. Kwok SY, Leung CL, Wong DF. Marital satisfaction of Chinese mothers of children with autism and intellectual disabilities in Hong Kong. J Intellect Disabil Res. 2014 Dec;58(12):1156-71. doi: 10.1111/jir.12116. PMID: 24450394.X-1

2479. LaGasse AB. Effects of a music therapy group intervention on enhancing social skills in children with autism. J Music Ther. 2014 Fall;51(3):250-75. doi: 10.1093/jmt/thu012. PMID: 25053766.X-4

2480. Lagunju IA, Bella-Awusah TT, Omigbodun OO. Autistic disorder in Nigeria: profile and challenges to management. Epilepsy Behav. 2014 Oct;39:126-9. doi: 10.1016/j.yebeh.2014.08.020. PMID: 25240124.X-1

2481. Lajiness-O'Neill R, Richard AE, Moran JE, et al. Neural synchrony examined with magnetoencephalography (MEG) during eye gaze processing in autism spectrum disorders: preliminary findings. J Neurodev Disord. 2014;6(1):15. doi: 10.1186/1866-19556-15. PMID: 24976870.X-1

2482. Lake JK, Weiss JA, Dergal J, et al. Child, parent, and service predictors of psychotropic polypharmacy among adolescents and young adults with an autism spectrum disorder. J Child Adolesc Psychopharmacol. 2014 Nov;24(9):486-93. doi: 10.1089/cap.2014.0011. PMID: 25329798.X-1 
2483. Lanning BA, Baier ME, Ivey-Hatz J, et al. Effects of equine assisted activities on autism spectrum disorder. J Autism Dev Disord. 2014 Aug;44(8):1897-907. doi: 10.1007/s10803-014-2062-5. PMID: 24526337.X-4

2484. LaRusso L. Competencies for Providing Evidence-Based Care. Nursing for Women's Health. 2014;18(2):105-10 6p. doi: 10.1111/1751-486X.12106. PMID: 103933868. Language: English. Entry Date: 20140422. Revision Date: 20150710. Publication Type: Journal Article.X-1

2485. Latham SO, Stockman IJ. Effect of augmented sensorimotor input on learning verbal and nonverbal tasks among children with autism spectrum disorders. J Autism Dev Disord. 2014 Jun;44(6):1288-302. doi: 10.1007/s10803-013-1990-9. PMID: 24234676. INCLUDE

2486. Laub WU, Crilly R. Clinical radiation therapy measurements with a new commercial synthetic single crystal diamond detector. J Appl Clin Med Phys. 2014;15(6):4890. doi: 10.1120/jacmp.v15i6.4890. PMID: 25493512.X-1

2487. Lavelle TA, Weinstein MC, Newhouse JP, et al. Economic burden of childhood autism spectrum disorders. Pediatrics. 2014 Mar;133(3):e520-9. doi: 10.1542/peds.20130763. PMID: 24515505.X-1

2488. Lazar M, Miles LM, Babb JS, et al. Axonal deficits in young adults with High Functioning Autism and their impact on processing speed. Neuroimage Clin. 2014;4:417-25. doi: 10.1016/j.nicl.2014.01.014. PMID: 24624327.X-1

2489. Lee K, Yoon K, Park KS, et al. Treatment of extensive comminuted mandibular fracture between both mandibular angles with bilateral condylar fractures using a reconstruction plate: a case report. J Korean Assoc Oral Maxillofac Surg. 2014 Jun;40(3):135-9. doi: 10.5125/jkaoms.2014.40.3.135. PMID: 25045641.X-1

2490. Leezenbaum NB, Campbell SB, Butler $\mathrm{D}$, et al. Maternal verbal responses to communication of infants at low and heightened risk of autism. Autism. 2014 Aug;18(6):694703. doi: 10.1177/1362361313491327. PMID: 24113343.X-1

2491. Legradi J, El Abdellaoui N, van Pomeren M, et al. Comparability of behavioural assays using zebrafish larvae to assess neurotoxicity. Environ Sci Pollut Res Int. 2014 Nov 18doi: 10.1007/s11356-014-3805-8. PMID: 25399529.X-1

2492. Leonard HC, Bedford R, Charman T, et al. Motor development in children at risk of autism: A follow-up study of infant siblings. Autism: The International Journal of Research \& Practice. 2014;18(3):281-91 11p. doi: 10.1177/1362361312470037. PMID: 104044086. Language: English. Entry Date: 20140321. Revision Date: 20150710. Publication Type: Journal Article.X-1

2493. Lepage JF, Lortie M, Deal CL, et al. Empathy, autistic traits, and motor resonance in adults with Turner syndrome. Soc Neurosci. 2014;9(6):601-9. doi: 10.1080/17470919.2014.944317. PMID: 25079009.X-1

2494. Lerna A, Esposito D, Conson M, et al. Long-term effects of PECS on socialcommunicative skills of children with autism spectrum disorders: a follow-up study. Int J Lang Commun Disord. 2014 JulAug;49(4):478-85. doi: 10.1111/14606984.12079. PMID: 24655345.X-1

2495. Lester JN, Karim K, O'Reilly M. Autism itself actually isn't a disability': Negotiating a 'normal' versus 'abnormal' autistic identity. Commun Med. 2014;11(2):139-52. PMID: 26596122.X-1

2496. Levey EJ. Effective Treatment Strategies for Autism During the First Five Years of Development. J Am Psychoanal Assoc. 2014 Jun 9;62(3):475-84. doi: 10.1177/0003065114539839. PMID: 24913552.X-1, X-2, X-3

2497. Levin DS, Volkert VM, Piazza CC. A multi-component treatment to reduce packing in children with feeding and autism spectrum disorders. Behav Modif. 2014 Nov;38(6):94063. doi: 10.1177/0145445514550683. PMID: 25271069.X-3, X-4 
2498. Li LY, Jiang N, Zhao Y. Could acupuncture have a role in the treatment of autism spectrum disorder via modulation of BDNF expression and activation? Acupunct Med. 2014 Dec;32(6):503-5. doi: 10.1136/acupmed-2014-010602. PMID: 25257393.X-1, X-2, X-3

2499. Li SO, Wang JL, Bjorklund G, et al. Serum copper and zinc levels in individuals with autism spectrum disorders. Neuroreport. 2014 Oct 22;25(15):1216-20. doi: 10.1097/wnr.0000000000000251. PMID: 25162784.X-1

2500. Li YW, Ma L, Sui B, et al. Etomidate with or without flumazenil anesthesia for stem cell transplantation in autistic children. Drug Metabol Drug Interact. 2014;29(1):47-51. doi: 10.1515/dmdi-2013-0043. PMID: 24225126.X1

2501. Li Z, Tang J, Li H, et al. Shorter telomere length in peripheral blood leukocytes is associated with childhood autism. Sci Rep. 2014;4:7073. doi: 10.1038/srep07073. PMID: 25399515.X-1

2502. Liao ST, Hwang YS, Chen YJ, et al. Home-based DIR/Floortime intervention program for preschool children with autism spectrum disorders: preliminary findings. Phys Occup Ther Pediatr. 2014 Nov;34(4):356-67. doi: 10.3109/01942638.2014.918074. PMID: 24865120.X-3, X-4

2503. Libero LE, Maximo JO, Deshpande $\mathrm{HD}$, et al. The role of mirroring and mentalizing networks in mediating action intentions in autism. Mol Autism. 2014;5(1):50. doi: 10.1186/2040-2392-5-50. PMID: 25352976.X1

2504. Libertus K, Landa RJ. Scaffolded reaching experiences encourage grasping activity in infants at high risk for autism. Front Psychol. 2014;5:1071. doi: 10.3389/fpsyg.2014.01071. PMID: 25295021.X-1

2505. Libertus K, Sheperd KA, Ross SW, et al. Limited fine motor and grasping skills in 6month-old infants at high risk for autism. Child Development. 2014;85(6):2218-31 14p. doi: 10.1111/cdev.12262. PMID: 109764429. Language: English. Entry Date: 20150821.
Revision Date: 20151104. Publication Type: journal article.X-1

2506. Lieberman-Betz RG, Yoder P, Stone WL, et al. An illustration of using multiple imputation versus listwise deletion analyses: the effect of Hanen's "More than words" on parenting stress. Am J Intellect Dev Disabil. 2014 Sep;119(5):472-86. doi: 10.1352/19447558-119.5.472. PMID: 25148059.X-1

2507. Li-Grining CP, Durlak JA. The design and implementation of early childhood intervention programs: informing efforts to address risk and promote resilience. J Prev Interv Community. 2014;42(4):243-7. doi: 10.1080/10852352.2014.943640. PMID: 25321639.X-1

2508. Lin IF, Kashino M, Ohta H, et al. The effect of intranasal oxytocin versus placebo treatment on the autonomic responses to human sounds in autism: a single-blind, randomized, placebo-controlled, crossover design study. Mol Autism. 2014;5(1):20. doi: 10.1186/2040-23925-20. PMID: 24576333.X-1

2509. Lin SC, Margolis B, Yu SM, et al. The role of medical home in emergency department use for children with developmental disabilities in the United States. Pediatr Emerg Care. 2014 Aug;30(8):534-9. doi: 10.1097/pec.0000000000000184. PMID: 25062298.X-1

2510. Lind SE, Bowler DM, Raber J. Spatial navigation, episodic memory, episodic future thinking, and theory of mind in children with autism spectrum disorder: evidence for impairments in mental simulation? Front Psychol. 2014;5:1411. doi:

10.3389/fpsyg.2014.01411. PMID: 25538661.X-1

2511. Lionello-Denolf KM, Farber R, Jones BM, et al. Thematic Matching as Remedial Teaching for Symbolic Matching for Individuals with Autism Spectrum Disorder. Res Autism Spectr Disord. 2014 May 1;8(5):455-62. doi: 10.1016/j.rasd.2014.01.004. PMID: 24634695.X-1, X-3, X-4

2512. Little LM, Sideris J, Ausderau K, et al. Activity participation among children with autism spectrum disorder. Am J Occup Ther. 2014 Mar-Apr;68(2):177-85. doi: 
10.5014/ajot.2014.009894. PMID:

24581404.X-1

2513. Liu L, Lei J, Sanders SJ, et al. DAWN: a framework to identify autism genes and subnetworks using gene expression and genetics. Mol Autism. 2014;5(1):22. doi: 10.1186/2040-2392-5-22. PMID: 24602502.X1

2514. Locke J, Rotheram-Fuller E, Xie M, et al. Correlation of cognitive and social outcomes among children with autism spectrum disorder in a randomized trial of behavioral intervention. Autism. 2014 May;18(4):370-5. doi: 10.1177/1362361313479181. PMID: 24104511.X-1, X-3

2515. Lofkvist U, Almkvist O, Lyxell B, et al. Lexical and semantic ability in groups of children with cochlear implants, language impairment and autism spectrum disorder. Int J Pediatr Otorhinolaryngol. 2014 Feb;78(2):25363. doi: 10.1016/j.ijporl.2013.11.017. PMID: 24332667.X-1

2516. Logan SL, Carpenter L, Leslie RS, et al. Rates and predictors of adherence to psychotropic medications in children with autism spectrum disorders. J Autism Dev Disord. 2014 Nov;44(11):2931-48. doi: 10.1007/s10803-014-2156-0. PMID: 24929833.X-1

2517. Lopez-Pison J, Garcia-Jimenez MC, Monge-Galindo L, et al. Our experience with the aetiological diagnosis of global developmental delay and intellectual disability: 2006-2010. Neurologia. 2014 Sep;29(7):402-7. doi: 10.1016/j.nrl.2013.10.006. PMID: 24332781.X-1

2518. Lotan A, Fenckova M, Bralten J, et al. Neuroinformatic analyses of common and distinct genetic components associated with major neuropsychiatric disorders. Front Neurosci. 2014;8:331. doi: 10.3389/fnins.2014.00331. PMID: 25414627.X-1

2519. Loth E, Spooren W, Murphy DG. New treatment targets for autism spectrum disorders: EU-AIMS. Lancet Psychiatry. 2014

Nov;1(6):413-5. doi: 10.1016/s22150366(14)00004-2. PMID: 26361185.X-1
2520. Loukusa S, Makinen L, KuusikkoGauffin S, et al. Theory of mind and emotion recognition skills in children with specific language impairment, autism spectrum disorder and typical development: group differences and connection to knowledge of grammatical morphology, word-finding abilities and verbal working memory. Int J Lang Commun Disord. 2014 Jul-Aug;49(4):498-507. doi: 10.1111/1460-6984.12091. PMID: 24888967.X-1

2521. Lowth M. Detecting developmental delay. Practice Nurse. 2014;44(5):24-8 5p. PMID: 107854713. Language: English. Entry Date: 20140602. Revision Date: 20150712. Publication Type: Journal Article.X-1

2522. Lozano R, Hagerman RJ, Duyzend M, et al. Genomic studies in fragile X premutation carriers. J Neurodev Disord. 2014;6(1):27. doi: 10.1186/1866-1955-6-27. PMID: 25170347.X1

2523. Lucas R, Norbury CF. Orthography facilitates vocabulary learning for children with autism spectrum disorders (ASD). Q J Exp Psychol (Hove). 2014;67(7):1317-34. doi: 10.1080/17470218.2013.859714. PMID: 24313313.X-1, X-3

2524. Ludlow A, Mohr B, Whitmore A, et al. Auditory processing and sensory behaviours in children with autism spectrum disorders as revealed by mismatch negativity. Brain Cogn. 2014 Apr;86:55-63. doi: 10.1016/j.bandc.2014.01.016. PMID: 24565813.X-1

2525. Lugo JN, Smith GD, Arbuckle EP, et al. Deletion of PTEN produces autism-like behavioral deficits and alterations in synaptic proteins. Front Mol Neurosci. 2014;7:27. doi: 10.3389/fnmol.2014.00027. PMID: 24795561.X-1

2526. Lumeng JC. Commentary on 'successful use of intravenous dexmedetomidine for magnetic resonance imaging sedation in autistic children'. Southern Medical Journal. 2014;107(9):565-6 2p. doi: 10.14423/SMJ.0000000000000161. PMID: 107828261. Language: English. Entry Date: 20141114. Revision Date: 20150712. Publication Type: Journal Article.X-1 
2527. Ma M-Y, Lee Y-H. Children with autism and composite tactile-visual toys during parent-child interaction. Interaction Studies: Social Behaviour and Communication in Biological and Artificial Systems. 2014 201502-24;15(2):260-91. doi: http://dx.doi.org/10.1075/is.15.2.13ma. PMID: 1655757528; 2014-41524-013.X-1

2528. MacDonald M, Lord C, Ulrich DA. Motor skills and calibrated autism severity in young children with autism spectrum disorder. Adapt Phys Activ Q. 2014 Apr;31(2):95-105. doi: 10.1123/apaq.2013-0068. PMID: 24762385.X-1

2529. MacDonald R, Parry-Cruwys D, Dupere $\mathrm{S}$, et al. Assessing progress and outcome of early intensive behavioral intervention for toddlers with autism. Res Dev Disabil. 2014 Dec;35(12):3632-44. doi: 10.1016/j.ridd.2014.08.036. PMID: 25241118.X-3

2530. Macpherson K, Charlop MH, Miltenberger CA. Using Portable Video Modeling Technology to Increase the Compliment Behaviors of Children with Autism During Athletic Group Play. J Autism Dev Disord. 2014 Feb 27doi: 10.1007/s10803014-2072-3. PMID: 24573335.X-3, X-4

2531. Madsen GF, Bilenberg N, Cantio C, et al. Increased prepulse inhibition and sensitization of the startle reflex in autistic children. Autism Res. 2014 Feb;7(1):94-103. doi: 10.1002/aur.1337. PMID: 24124111.X-1

2532. Majdalany LM, Wilder DA, Greif A, et al. Comparing massed-trial instruction, distributed-trial instruction, and task interspersal to teach tacts to children with autism spectrum disorders. J Appl Behav Anal. 2014 Fall;47(3):657-62. doi: 10.1002/jaba.149. PMID: 24988891.X-3, X-4

2533. Malandraki GA, Roth M, Sheppard JJ. Telepractice for pediatric Dysphagia: a case study. Int J Telerehabil. 2014 Spring;6(1):3-16. doi: 10.5195/ijt.2014.6135. PMID: 25945217.X-1

2534. Maloney A, Mick EO, Frazier J. Aripiprazole decreases irritability in 12 out of 14 youth with autism spectrum disorders. J Child Adolesc Psychopharmacol. 2014
Aug;24(6):357-9. doi: 10.1089/cap.2013.0143. PMID: 24828130.X-3, X-4

2535. Malow BA, Adkins KW, Reynolds A, et al. Parent-based sleep education for children with autism spectrum disorders. J Autism Dev Disord. 2014 Jan;44(1):216-28. doi: 10.1007/s10803-013-1866-z. PMID: 23754339.X-1

2536. Mandelberg J, Frankel F, Cunningham $\mathrm{T}$, et al. Long-term outcomes of parent-assisted social skills intervention for high-functioning children with autism spectrum disorders. Autism. 2014 Apr;18(3):255-63. doi: 10.1177/1362361312472403. PMID: 23996903.X-1, X-3

2537. Mangerud WL, Bjerkeset O, Lydersen $\mathrm{S}$, et al. Physical activity in adolescents with psychiatric disorders and in the general population. Child Adolesc Psychiatry Ment Health. 2014;8(1):2. doi: 10.1186/1753-2000-82. PMID: 24450542.X-1

2538. Manouilenko I, Eriksson JM, Humble $\mathrm{MB}$, et al. Minor physical anomalies in adults with autism spectrum disorder and healthy controls. Autism Res Treat. 2014;2014:743482. doi: 10.1155/2014/743482. PMID: 24782925.X-1

2539. Markwick L, Smith C, Mick D. Functional behavioral analysis and social scripting for the older patient with schizophrenia: a staff development program. Issues Ment Health Nurs. 2014 Nov;35(11):883-90. doi: 10.3109/01612840.2014.897777. PMID: 25353301.X-1

2540. Marroquin M, Alvero A, Sturmey P. Evaluation of the observer effect on compliance training in adolescents with autism. Res Dev Disabil. 2014 Feb;35(2):537-40. doi: 10.1016/j.ridd.2013.11.008. PMID: 24378631.X-1

2541. Marrus N, Underwood-Riordan H, Randall F, et al. Lack of effect of risperidone on core autistic symptoms: data from a longitudinal study. J Child Adolesc Psychopharmacol. 2014 Nov;24(9):513-8. doi: 10.1089/cap.2014.0055. PMID: 25361070.X-3 
2542. Marrus N, Veenstra-Vanderweele J, Hellings JA, et al. Training of child and adolescent psychiatry fellows in autism and intellectual disability. Autism. 2014

May;18(4):471-5. doi:

10.1177/1362361313477247. PMID:

24113341.X-1

2543. Marshall ES. Increasing prevalence of autism: implications for school nursing. NASN Sch Nurse. 2014 Sep;29(5):241-3. PMID: 25272410.X-1

2544. Martin HG, Manzoni OJ. Late onset deficits in synaptic plasticity in the valproic acid rat model of autism. Front Cell Neurosci. 2014;8:23. doi: 10.3389/fncel.2014.00023. PMID: 24550781.X-1

2545. Martínez-Sanchis S. Neurobiological foundations of multisensory integration in people with autism spectrum disorders: The role of the medial prefrontal cortex. Frontiers in Human Neuroscience. 2014 2015-12-09;8 PMID: 1696231239; 2015-25642-001.X-1

2546. Maskey M, Lowry J, Rodgers J, et al. Reducing specific phobia/fear in young people with autism spectrum disorders (ASDs) through a virtual reality environment intervention. PLoS One. 2014;9(7):e100374. doi: 10.1371/journal.pone.0100374. PMID: 24987957.X-3, X-4

2547. Mason R, Kamps D, Turcotte A, et al. Peer Mediation to Increase Communication and Interaction at Recess for Students with Autism Spectrum Disorders. Res Autism Spectr Disord. 2014 Mar;8(3):334-44. doi:

10.1016/j.rasd.2013.12.014. PMID:

26180543.X-1, X-3

2548. Masterson TL, Dimitriou F, Turko K, et al. Developing undergraduate coursework in autism spectrum disorders. J Autism Dev Disord. 2014 Oct;44(10):2646-9. doi: 10.1007/s10803-012-1673-y. PMID: 23054203.X-1

2549. Matsuzaki J, Kagitani-Shimono K, Sugata H, et al. Progressively increased M50 responses to repeated sounds in autism spectrum disorder with auditory hypersensitivity: a magnetoencephalographic study. PLoS One. 2014;9(7):e102599. doi: 10.1371/journal.pone.0102599. PMID: 25054201.X-1, X-3

2550. Mavranezouli I, Megnin-Viggars O, Cheema N, et al. The cost-effectiveness of supported employment for adults with autism in the United Kingdom. Autism. 2014

Nov;18(8):975-84. doi: 10.1177/1362361313505720. PMID: 24126866.X-1

2551. Maxwell BG, Lobato RL, Cason MB, et al. Perioperative morbidity and mortality of cardiothoracic surgery in patients with a do-notresuscitate order. PeerJ. 2014;2:e245. doi: 10.7717/peerj.245. PMID: 24498575.X-1

2552. Mayer JL, Heaton PF. Age and sensory processing abnormalities predict declines in encoding and recall of temporally manipulated speech in high-functioning adults with ASD. Autism Res. 2014 Feb;7(1):40-9. doi: 10.1002/aur.1333. PMID: 24106132.X-1

2553. Mazefsky CA, Schreiber DR, Olino $\mathrm{TM}$, et al. The association between emotional and behavioral problems and gastrointestinal symptoms among children with highfunctioning autism. Autism. 2014 Jul;18(5):493-501. doi: 10.1177/1362361313485164. PMID: 24104507.X-1

2554. Mazurek MO, Handen BL, Wodka EL, et al. Age at first autism spectrum disorder diagnosis: the role of birth cohort, demographic factors, and clinical features. J Dev Behav Pediatr. 2014 Nov-Dec;35(9):561-9. doi: 10.1097/dbp.0000000000000097. PMID: 25211371.X-1

2555. Mazza M, Pino MC, Mariano M, et al. Affective and cognitive empathy in adolescents with autism spectrum disorder. Front Hum Neurosci. 2014;8:791. doi:

10.3389/fnhum.2014.00791. PMID: 25339889.X-1

2556. McConachie H, McLaughlin E, Grahame V, et al. Group therapy for anxiety in children with autism spectrum disorder. Autism. 2014 Aug;18(6):723-32. doi: 10.1177/1362361313488839. PMID: 24101715.X-1 
2557. McCormick C, Hessl D, Macari SL, et al. Electrodermal and behavioral responses of children with autism spectrum disorders to sensory and repetitive stimuli. Autism Research. 2014 2015-12-09;7(4):468-80. doi: http://dx.doi.org/10.1002/aur.1382. PMID: 1560635711; 2014-18958-001.X-1

2558. McFadden B, Kamps D, HeitzmanPowell L. Social Communication Effects of Peer-Mediated Recess Intervention for Children with Autism. Res Autism Spectr Disord. 2014 Dec;8(12):1699-712. doi:

10.1016/j.rasd.2014.08.015. PMID: 26312064.X-3, X-4

2559. McGillivray JA, Evert HT. Group cognitive behavioural therapy program shows potential in reducing symptoms of depression and stress among young people with ASD. J Autism Dev Disord. 2014 Aug;44(8):2041-51. doi: 10.1007/s10803-014-2087-9. PMID: 24634065.X-1

2560. McGonigle JJ, Migyanka JM, GlorScheib SJ, et al. Development and evaluation of educational materials for pre-hospital and emergency department personnel on the care of patients with autism spectrum disorder. J Autism Dev Disord. 2014 May;44(5):1252-9. doi: 10.1007/s10803-013-1962-0. PMID: 24091472.X-1

2561. McLean RL, Johnson Harrison A, Zimak E, et al. Executive function in probands with autism with average IQ and their unaffected first-degree relatives. J Am Acad Child Adolesc Psychiatry. 2014

Sep;53(9):1001-9. doi:

10.1016/j.jaac.2014.05.019. PMID: 25151423.X-1

2562. McMahon J, Cullinan V. Education programmes for young children with Autism Spectrum Disorder: an Evaluation Framework. Res Dev Disabil. 2014 Dec;35(12):3689-97. doi: 10.1016/j.ridd.2014.09.004. PMID: 25262014.X-1

2563. Meilleur AA, Berthiaume C, Bertone A, et al. Autism-specific covariation in perceptual performances: "g" or "p" factor? PLoS One. 2014;9(8):e103781. doi: 10.1371/journal.pone.0103781. PMID: 25117450.X-1
2564. Meyenburg B. [Gender dysphoria in adolescents: difficulties in treatment]. Prax Kinderpsychol Kinderpsychiatr. 2014;63(6):510-22. PMID: 25296511.X-1

2565. Meyer MA. [Consultations with a nurse specialized in cognitive behavioural therapies]. Soins Psychiatr. 2014 NovDec(295):42-4. PMID: 25562920.X-1, X-3

2566. Miller M, Chukoskie L, Zinni M, et al. Dyspraxia, motor function and visual-motor integration in autism. Behavioural Brain Research. 2014 2015-07-22;269:95-102. doi: $10.2522 / \mathrm{ptj} .20100294$ http://dx.doi.org/10.1016/j.bbr.2014.04.011. PMID: 1697763095; 2014-23423-015.X-1

2567. Milner LC, Cho MK. Focusing on Cause or Cure?: Priorities and Stakeholder Presence in Childhood Psychiatry Research. AJOB Prim Res. 2014 Jan 1;5(1):44-55. doi: 10.1080/21507716.2013.811315. PMID: 24729931.X-1

2568. Miltenberger CA, Charlop MH. Increasing the athletic group play of children with autism. J Autism Dev Disord. 2014 Jan;44(1):41-54. doi: 10.1007/s10803-0131850-7. PMID: 23700189.X-3, X-4

2569. Mire SS, Nowell KP, Kubiszyn T, et al. Psychotropic medication use among children with autism spectrum disorders within the Simons Simplex Collection: are core features of autism spectrum disorder related? Autism. 2014 Nov;18(8):933-42. doi:

10.1177/1362361313498518. PMID: 24031086.X-1, X-3

2570. Misquiatti AR, Brito MC, Olivati AG, et al. Sociocognitive performance in autism spectrum disorders and interference of the therapeutic environment. Codas. 2014 SepOct;26(5):402-6. PMID: 25388074.X-3, X-4

2571. Missault S, Van den Eynde K, Vanden Berghe W, et al. The risk for behavioural deficits is determined by the maternal immune response to prenatal immune challenge in a neurodevelopmental model. Brain Behav Immun. 2014 Nov;42:138-46. doi: 10.1016/j.bbi.2014.06.013. PMID: 24973728.X-1 
2572. Mitchell C, Holdt N. The search for a timely diagnosis: parents' experiences of their child being diagnosed with an Autistic Spectrum Disorder. J Child Adolesc Ment Health. 2014;26(1):49-62. doi: 10.2989/17280583.2013.849606. PMID: 25391570.X-1

2573. Mito H, Matsuura N, Mukai K, et al. The impacts of elevated autism spectrum disorder traits on clinical and psychosocial features and long-term treatment outcome in adult patients with obsessive-compulsive disorder. Compr Psychiatry. 2014 Oct;55(7):1526-33. doi: 10.1016/j.comppsych.2014.05.005. PMID: 24957957.X-1

2574. Mizen CS. Narcissistic disorder and the failure of symbolisation: a Relational Affective Hypothesis. Med Hypotheses. 2014 Sep;83(3):254-62. doi: 10.1016/j.mehy.2014.05.012. PMID: 24986704.X-1

2575. Mohammadzaheri F, Koegel LK, Rezaee M, et al. A randomized clinical trial comparison between pivotal response treatment (PRT) and structured applied behavior analysis (ABA) intervention for children with autism. J Autism Dev Disord. 2014 Nov;44(11):2769-77. doi: 10.1007/s10803-014-2137-3. PMID: 24840596.X-1

2576. Mood D, Shield A. Clinical use of the autism diagnostic observation schedule-second edition with children who are deaf. Semin Speech Lang. 2014 Nov;35(4):288-300. doi: 10.1055/s-0034-1389101. PMID: 25321853.X1

2577. Mostafavi Abdolmaleky H. Horizons of psychiatric genetics and epigenetics: where are we and where are we heading? Iran J Psychiatry Behav Sci. 2014 Fall;8(3):1-10. PMID: 25780369.X-1

2578. Mouti A, Reddihough D, Marraffa C, et al. Fluoxetine for Autistic Behaviors (FAB trial): study protocol for a randomized controlled trial in children and adolescents with autism. Trials. 2014;15:230. doi: 10.1186/17456215-15-230. PMID: 24934401.X-1, X-2, X-4

2579. Mukaddes NM, Tutkunkardas MD, Sari O, et al. Characteristics of children who lost the diagnosis of autism: a sample from istanbul, Turkey. Autism Res Treat. 2014;2014:472120. doi: 10.1155/2014/472120. PMID: 24876961.X-1

2580. Mukherjee S, Rupani K, Dave M, et al. Evaluation of effectiveness of integrated intervention in autistic children. Indian J Pediatr. 2014 Apr;81(4):339-45. doi: 10.1007/s12098-013-1169-6. PMID: 24057967.X-3, X-4

2581. Mulligan RC, Reiersen AM, Todorov AA. Attention-Deficit/Hyperactivity Disorder, Autistic Traits, and Substance Use Among Missouri Adolescents. Scand J Child Adolesc Psychiatr Psychol. 2014;2(2):86-92. PMID: 26146608.X-1

2582. Mundalil Vasu M, Anitha A, Thanseem I, et al. Serum microRNA profiles in children with autism. Mol Autism. 2014;5:40. doi: 10.1186/2040-2392-5-40. PMID: 25126405.X-1

2583. Muratori F, Narzisi A. Exploratory study describing 6 month outcomes for young children with autism who receive treatment as usual in Italy. Neuropsychiatr Dis Treat. 2014;10:577-86. doi: 10.2147/ndt.s58308. PMID: 24748794.X-1, X-3

2584. Murphy CM, Christakou A, Daly EM, et al. Abnormal functional activation and maturation of fronto-striato-temporal and cerebellar regions during sustained attention in autism spectrum disorder. American Journal of Psychiatry. 2014;171(10):1107-16 10p. doi: 10.1176/appi.ajp.2014.12030352. PMID: 107802812. Corporate Author: MRC AIMS Consortium. Language: English. Entry Date: 20150313. Revision Date: 20150712. Publication Type: Journal Article.X-1

2585. Murphy JW, Foxe JJ, Peters JB, et al. Susceptibility to distraction in autism spectrum disorder: probing the integrity of oscillatory alpha-band suppression mechanisms. Autism Res. 2014 Aug;7(4):442-58. doi: 10.1002/aur.1374. PMID: 24678054.X-1

2586. Murray ML, Hsia Y, Glaser K, et al. Pharmacological treatments prescribed to people with autism spectrum disorder (ASD) in primary health care. Psychopharmacology (Berl). 2014 Mar;231(6):1011-21. doi: 
10.1007/s00213-013-3140-7. PMID: 23681164.X-1

2587. Murshid EZ. Diet, oral hygiene practices and dental health in autistic children in Riyadh, Saudi Arabia. Oral Health Dent Manag. 2014 Mar;13(1):91-6. PMID: 24603923.X-1

2588. Murza KA, Nye C, Schwartz JB, et al. A randomized controlled trial of an inference generation strategy intervention for adults with high-functioning autism spectrum disorder. Am J Speech Lang Pathol. 2014 Aug;23(3):461-73. doi: 10.1044/2014_ajslp-13-0012. PMID: 24687182.X-1

2589. Must A, Curtin C, Hubbard K, et al. Obesity Prevention for Children with Developmental Disabilities. Curr Obes Rep. 2014 Jun;3(2):156-70. doi: 10.1007/s13679014-0098-7. PMID: 25530916.X-1

2590. Muthusamy K, Thomas MM, George $\mathrm{RE}$, et al. Siblings with fucosidosis. J Pediatr Neurosci. 2014 May;9(2):156-8. doi: 10.4103/1817-1745.139331. PMID: 25250075.X-1

2591. Nagashima M, Monden Y, Dan I, et al. Neuropharmacological effect of atomoxetine on attention network in children with attention deficit hyperactivity disorder during oddball paradigms as assessed using functional nearinfrared spectroscopy. Neurophotonics. 2014 Oct;1(2):025007. doi: 10.1117/1.NPh.1.2.025007. PMID: 26157979.X-1

2592. Nair MK, Lakshmi MA, Latha S, et al. CDC Kerala 15: Developmental Evaluation Clinic (2-10 y)--developmental diagnosis and use of home intervention package. Indian J Pediatr. 2014 Dec;81 Suppl 2:S142-50. doi: 10.1007/s12098-014-1587-0. PMID: 25326156.X-1, X-3

2593. Nair MK, Mini AO, Leena ML, et al. CDC Kerala 7: Effect of early language intervention among children 0-3 y with speech and language delay. Indian J Pediatr. 2014 Dec;81 Suppl 2:S102-9. doi: 10.1007/s12098014-1555-8. PMID: 25179239.X-1

2594. Nair MK, Russell PS, George B, et al. CDC Kerala 9: Effectiveness of low intensity home based early intervention for autism spectrum disorder in India. Indian J Pediatr. 2014 Dec;81 Suppl 2:S115-9. doi: 10.1007/s12098-014-1474-8. PMID: 25141828.X-3

2595. Nair MK, Russell PS, George B, et al. CDC Kerala 10: Diagnostic accuracy of the severity scores for childhood autism rating scale in India. Indian J Pediatr. 2014 Dec;81 Suppl 2:S120-4. doi: 10.1007/s12098-014-1623-0. PMID: 25408269.X-1

2596. Nair MK, Russell PS, George B, et al. CDC Kerala 8: Effectiveness of a clinic based, low intensity, early intervention for children with autism spectrum disorder in India: a naturalistic observational study. Indian J Pediatr. 2014 Dec;81 Suppl 2:S110-4. doi: 10.1007/s12098-014-1601-6. PMID: 25366289.X-3

2597. Nakako T, Murai T, Ikejiri M, et al. Effects of lurasidone on ketamine-induced joint visual attention dysfunction as a possible disease model of autism spectrum disorders in common marmosets. Behav Brain Res. 2014 Nov 1;274:349-54. doi: 10.1016/j.bbr.2014.08.032. PMID: 25169254.X-1

2598. Nakamura T, Oinuma T. Usefulness of Photodynamic Diagnosis and Therapy using Talaporfin Sodium for an Advanced-aged Patient with Inoperable Gastric Cancer (a secondary publication). Laser Ther. 2014 Sep 30;23(3):201-10. doi: 10.5978/islsm.14-OR-16. PMID: 25368446.X-1

2599. Naviaux JC, Schuchbauer MA, Li K, et al. Reversal of autism-like behaviors and metabolism in adult mice with single-dose antipurinergic therapy. Transl Psychiatry. 2014;4:e400. doi: 10.1038/tp.2014.33. PMID: 24937094.X-1

2600. Nayfack AM, Huffman LC, Feldman HM, et al. Hospitalizations of children with autism increased from 1999 to 2009. J Autism Dev Disord. 2014 May;44(5):1087-94. doi: 10.1007/s10803-013-1965-x. PMID: 24122446.X-1

2601. Nicely TA, Lane-Loney S, Masciulli E, et al. Prevalence and characteristics of avoidant/restrictive food intake disorder in a 
cohort of young patients in day treatment for eating disorders. J Eat Disord. 2014;2(1):21. doi: 10.1186/s40337-014-0021-3. PMID: 25165558.X-1

2602. Nicolaidis C, Kripke CC, Raymaker D. Primary care for adults on the autism spectrum. Medical Clinics of North America.

2014;98(5):1169-91 23p. doi: 10.1016/j.mcna.2014.06.011. PMID: 107830100. Language: English. Entry Date: 20141024. Revision Date: 20150712. Publication Type: Journal Article. Journal Subset: Biomedical.X-1

2603. Nomura K, Okada K, Noujima Y, et al. A clinical study of attention-

deficit/hyperactivity disorder in preschool children--prevalence and differential diagnoses. Brain Dev. 2014 Oct;36(9):778-85. doi: 10.1016/j.braindev.2013.11.004. PMID: 24295540.X-1

2604. Nyffeler J, Walitza S, Bobrowski E, et al. Association study in siblings and casecontrols of serotonin- and oxytocin-related genes with high functioning autism. J Mol Psychiatry. 2014;2(1):1. doi: 10.1186/20499256-2-1. PMID: 25408912.X-1

2605. Nyp SS, Nadler CB, Call CR, et al. Driven by evidence: diagnosis and treatment for children with autism spectrum disorders. Mo Med. 2014 May-Jun;111(3):195-8. PMID: 25011339.X-1

2606. Oberman LM, Enticott PG, Casanova MF, et al. Transcranial magnetic stimulation (TMS) therapy for autism: an international consensus conference held in conjunction with the international meeting for autism research on May 13th and 14th, 2014. Front Hum Neurosci. 2014;8:1034. doi: 10.3389/fnhum.2014.01034. PMID: 25642178.X-1, X-2, X-3

2607. Oberman LM, Pascual-Leone A. Hyperplasticity in Autism Spectrum Disorder confers protection from Alzheimer's disease. Med Hypotheses. 2014 Sep;83(3):337-42. doi: 10.1016/j.mehy.2014.06.008. PMID: 25047996.X-1

2608. Oberman LM, Pascual-Leone A, Rotenberg A. Modulation of corticospinal excitability by transcranial magnetic stimulation in children and adolescents with autism spectrum disorder. Front Hum Neurosci. 2014;8:627. doi: 10.3389/fnhum.2014.00627. PMID: 25165441.X-1, X-3

2609. Odom SL, Thompson JL, Hedges S, et al. Technology-Aided Interventions and Instruction for Adolescents with Autism Spectrum Disorder. J Autism Dev Disord. 2014 Dec 3doi: 10.1007/s10803-014-2320-6. PMID: 25468409.X-1, X-2

2610. Ogawa LM, Vallender EJ. Evolutionary conservation in genes underlying human psychiatric disorders. Front Hum Neurosci. 2014;8:283. doi: 10.3389/fnhum.2014.00283. PMID: 24834046.X-1

2611. O'Haire ME, McKenzie SJ, McCune S, et al. Effects of classroom animal-assisted activities on social functioning in children with autism spectrum disorder. J Altern Complement Med. 2014 Mar;20(3):162-8. doi:

10.1089/acm.2013.0165. PMID: 24156772.X-1

2612. Olu-Lafe O, Liederman J, Tager-

Flusberg H. Is the Ability to Integrate Parts into Wholes Affected in Autism Spectrum Disorder? Journal of Autism \& Developmental Disorders. 2014;44(10):2652-60 9p. doi: 10.1007/s10803014-2120-z. PMID: 103891498. Language: English. Entry Date: 20140922. Revision Date: 20151001. Publication Type: Journal Article.X1

2613. Oner P, Oner O, Munir K. Three-item Direct Observation Screen (TIDOS) for autism spectrum disorder. Autism. 2014 Aug;18(6):733-42. doi: 10.1177/1362361313487028. PMID: 24126869.X-1

2614. Ong D, Chua NH, Vissers K. Percutaneous Disc Decompression for Lumbar Radicular Pain: A Review Article. Pain Pract. 2014 Oct 29doi: 10.1111/papr.12250. PMID: 25354274.X-1

2615. O'Nions E, Viding E, Greven CU, et al. Pathological demand avoidance: exploring the behavioural profile. Autism. 2014 Jul;18(5):538-44. doi: 10.1177/1362361313481861. PMID: 24104509.X-1 
2616. Orekhova EV, Elsabbagh M, Jones EJ, et al. EEG hyper-connectivity in high-risk infants is associated with later autism. J Neurodev Disord. 2014;6(1):40. doi: 10.1186/1866-1955-6-40. PMID: 25400705.X1

2617. Orellana LM, Martinez-Sanchis S, Silvestre FJ. Training adults and children with an autism spectrum disorder to be compliant with a clinical dental assessment using a TEACCH-based approach. J Autism Dev Disord. 2014 Apr;44(4):776-85. doi: 10.1007/s10803-013-1930-8. PMID: 24002415.X-1, X-3

2618. Orinstein AJ, Helt M, Troyb E, et al. Intervention for optimal outcome in children and adolescents with a history of autism. J Dev Behav Pediatr. 2014 May;35(4):247-56. doi: 10.1097/dbp.0000000000000037. PMID: 24799263.X-1

2619. Ouyang L, Grosse SD, Riley C, et al. A comparison of family financial and employment impacts of fragile X syndrome, autism spectrum disorders, and intellectual disability. Res Dev Disabil. 2014

Jul;35(7):1518-27. doi: 10.1016/j.ridd.2014.04.009. PMID: 24755230.X-1

2620. Pahnke J, Lundgren T, Hursti T, et al. Outcomes of an acceptance and commitment therapy-based skills training group for students with high-functioning autism spectrum disorder: a quasi-experimental pilot study. Autism. 2014 Nov;18(8):953-64. doi: 10.1177/1362361313501091. PMID: 24142796.X-1

2621. Paloyelis Y, Doyle OM, Zelaya FO, et al. A Spatiotemporal Profile of In Vivo Cerebral Blood Flow Changes Following Intranasal Oxytocin in Humans. Biol Psychiatry. 2014 Oct 18doi: 10.1016/j.biopsych.2014.10.005. PMID: 25499958.X-1

2622. Pan C-Y. Motor proficiency and physical fitness in adolescent males with and without autism spectrum disorders. Autism: The International Journal of Research \& Practice. 2014;18(2):156-65 10p. doi: 10.1177/1362361312458597. PMID: 104005345. Language: English. Entry Date:
20140128. Revision Date: 20150710. Publication Type: Journal Article.X-1

2623. Panerai S, Tasca D, Lanuzza B, et al. Effects of repetitive transcranial magnetic stimulation in performing eye-hand integration tasks: four preliminary studies with children showing low-functioning autism. Autism. 2014 Aug;18(6):638-50. doi: 10.1177/1362361313495717. PMID: 24113340.X-1, X-3

2624. Panerai S, Tasca D, Lanuzza B, et al. Effects of repetitive transcranial magnetic stimulation in performing eye-hand integration tasks: Four preliminary studies with children showing low-functioning autism. Autism: The International Journal of Research \& Practice.

2014;18(6):638-50 13p. doi: 10.1177/1362361313495717. PMID: 103977740. Language: English. Entry Date: 20140723. Revision Date: 20150710. Publication Type: Journal Article.X-1

2625. Park MJ, Aja S, Li Q, et al. Anaplerotic triheptanoin diet enhances mitochondrial substrate use to remodel the metabolome and improve lifespan, motor function, and sociability in MeCP2-null mice. PLoS One. 2014;9(10):e109527. doi: 10.1371/journal.pone.0109527. PMID: 25299635.X-1

2626. Parkinson J. Gender dysphoria in Asperger's syndrome: a caution. Australas Psychiatry. 2014 Feb;22(1):84-5. doi: 10.1177/1039856213497814. PMID: 23897727.X-1

2627. Pasalich DS, Dadds MR, Hawes DJ. Cognitive and affective empathy in children with conduct problems: additive and interactive effects of callous-unemotional traits and autism spectrum disorders symptoms. Psychiatry Res. 2014 Nov 30;219(3):625-30. doi: 10.1016/j.psychres.2014.06.025. PMID: 25015711.X-1

2628. Pasco G, Clark B, Dragan I, et al. A training and development project to improve services and opportunities for social inclusion for children and young people with autism in Romania. Autism. 2014 Oct;18(7):827-31. doi: 10.1177/1362361314524642. PMID: 24590469.X-1 
2629. Pasiali V, LaGasse AB, Penn SL. The effect of musical attention control training (MACT) on attention skills of adolescents with neurodevelopmental delays: a pilot study. J Music Ther. 2014 Winter;51(4):333-54. doi: 10.1093/jmt/thu030. PMID: 25504177.X-1

2630. Payakachat N, Tilford JM, Kuhlthau KA, et al. Predicting health utilities for children with autism spectrum disorders. Autism Res. 2014 Dec;7(6):649-63. doi: 10.1002/aur.1409. PMID: 25255789.X-1

2631. Pennington ML, Cullinan D, Southern LB. Defining autism: variability in state education agency definitions of and evaluations for autism spectrum disorders. Autism Res Treat. 2014;2014:327271. doi: 10.1155/2014/327271. PMID: 24987527.X-1

2632. Perron L. [Art therapy to support autistic people]. Soins Pediatr Pueric. 2014 JanFeb(276):36-8. PMID: 24617093.X-1, X-2, X3

2633. Petersen DB, Brown CL, Ukrainetz TA, et al. Systematic individualized narrative language intervention on the personal narratives of children with autism. Lang Speech Hear Serv Sch. 2014 Jan;45(1):67-86. doi: 10.1044/2013_lshss-12-0099. PMID: 24687768.X-3, X-4

2634. Petersen I, Evans S, Nazareth I. Prenatal exposure to selective serotonin reuptake inhibitors and autistic symptoms in young children: another red herring? $\mathrm{Br} \mathrm{J}$ Psychiatry. 2014 Aug;205(2):105-6. doi: 10.1192/bjp.bp.113.141721. PMID: 25252319.X-1

2635. Phillips KL, Schieve LA, Visser S, et al. Prevalence and impact of unhealthy weight in a national sample of US adolescents with autism and other learning and behavioral disabilities. Matern Child Health J. 2014 Oct;18(8):1964-75. doi: 10.1007/s10995-0141442-y. PMID: 24553796.X-1

2636. Pickles A, Anderson DK, Lord C. Heterogeneity and plasticity in the development of language: a 17-year follow-up of children referred early for possible autism. J Child Psychol Psychiatry. 2014 Dec;55(12):1354-62. doi: 10.1111/jcpp.12269. PMID: 24889883.X-1
2637. Pifer R, Sperandio V. The Interplay between the Microbiota and Enterohemorrhagic Escherichia coli. Microbiol Spectr. 2014 Oct;2(5)doi: 10.1128/microbiolspec.EHEC0015-2013. PMID: 26104352.X-1

2638. Pillai D, Sheppard E, Ropar D, et al. Using Other Minds as a Window Onto the World: Guessing What Happened from Clues in Behaviour. Journal of Autism \& Developmental Disorders. 2014;44(10):2430-9 10p. doi: 10.1007/s10803-014-2106-x. PMID: 103891507. Language: English. Entry Date: 20140922. Revision Date: 20151001. Publication Type: Journal Article.X-1

2639. Pineda JA, Carrasco K, Datko M, et al. Neurofeedback training produces normalization in behavioural and electrophysiological measures of high-functioning autism. Philos Trans R Soc Lond B Biol Sci. 2014;369(1644):20130183. doi: 10.1098/rstb.2013.0183. PMID: 24778378.X-3, $\mathrm{X}-4$

2640. Pineda JA, Friedrich EV, LaMarca K. Neurorehabilitation of social dysfunctions: a model-based neurofeedback approach for low and high-functioning autism. Front Neuroeng. 2014;7:29. doi: 10.3389/fneng.2014.00029. PMID: 25147521.X-1, X-2

2641. Pini G, Scusa MF, Benincasa A, et al. Repeated insulin-like growth factor 1 treatment in a patient with rett syndrome: a single case study. Front Pediatr. 2014;2:52. doi: 10.3389/fped.2014.00052. PMID: 24918098.X1

2642. Poot M. A candidate gene association study further corroborates involvement of contactin genes in autism. Mol Syndromol. 2014 Aug;5(5):229-35. doi: 10.1159/000362891. PMID: 25337070.X-1

2643. Pop-Jordanova N, PlasevskaKaranfilska D. Autism - genetics, electrophysiology and clinical syndromes. Prilozi. 2014;35(1):133-46. PMID: 24802198.X-1

2644. Porges SW, Bazhenova OV, Bal E, et al. Reducing auditory hypersensitivities in autistic spectrum disorder: preliminary findings evaluating the listening project protocol. Front Pediatr. 2014;2:80. doi: 
10.3389/fped.2014.00080. PMID: 25136545. INCLUDE

2645. Poslawsky IE, Naber FB, BakermansKranenburg MJ, et al. Development of a Videofeedback Intervention to promote Positive Parenting for Children with Autism (VIPPAUTI). Attach Hum Dev. 2014;16(4):343-55. doi: 10.1080/14616734.2014.912487. PMID: 24972103.X-1

2646. Poslawsky IE, Naber FB, Van Daalen $\mathrm{E}$, et al. Parental reaction to early diagnosis of their children's autism spectrum disorder: an exploratory study. Child Psychiatry Hum Dev. 2014 Jun;45(3):294-305. doi: 10.1007/s10578013-0400-z. PMID: 23959534.X-1

2647. Post M, Haymes L, Storey K, et al. Understanding stalking behaviors by individuals with Autism Spectrum Disorders and recommended prevention strategies for school settings. J Autism Dev Disord. 2014 Nov;44(11):2698-706. doi: 10.1007/s10803012-1712-8. PMID: 23207743.X-1, X-2, X-3

2648. Pozo P, Sarria E. Prediction of stress in mothers of children with autism spectrum disorders. Span J Psychol. 2014;17:E6. doi: 10.1017/sjp.2014.6. PMID: 25012691.X-1

2649. Pragnya B, Kameshwari JS, Veeresh B. Ameliorating effect of piperine on behavioral abnormalities and oxidative markers in sodium valproate induced autism in BALB/C mice. Behav Brain Res. 2014 Aug 15;270:8694. doi: 10.1016/j.bbr.2014.04.045. PMID: 24803211.X-1

2650. Prast JM, Schardl A, Schwarzer C, et al. Reacquisition of cocaine conditioned place preference and its inhibition by previous social interaction preferentially affect D1-medium spiny neurons in the accumbens corridor. Front Behav Neurosci. 2014;8:317. doi: 10.3389/fnbeh.2014.00317. PMID: 25309368.X-1

2651. Preis J, McKenna M. The effects of sensory integration therapy on verbal expression and engagement in children with autism. International Journal of Therapy \& Rehabilitation. 2014;21(10):476-86 11p. PMID: 103917339. Language: English. Entry Date: 20141119. Revision Date: 20150820. Publication Type: Journal Article.X-1, X-3, X-4
2652. Pretto D, Yrigollen CM, Tang HT, et al. Clinical and molecular implications of mosaicism in FMR1 full mutations. Front Genet. 2014;5:318. doi: 10.3389/fgene.2014.00318. PMID: 25278957.X-1

2653. Price AM, Coates C, Symeonides C, et al. Chocolate frogs do not increase completion of parent survey: randomised study. J Paediatr Child Health. 2014 Nov;50(11):866-8. doi: 10.1111/jpc.12606. PMID: 24925605.X-1

2654. Provenzano G, Clementi E, Genovesi S, et al. GH Dysfunction in Engrailed-2 Knockout Mice, a Model for Autism Spectrum Disorders. Front Pediatr. 2014;2:92. doi: 10.3389/fped.2014.00092. PMID: 25225635.X1

2655. Puckering C, Allely CS, Doolin O, et al. Association between parent-infant interactions in infancy and disruptive behaviour disorders at age seven: a nested, case-control ALSPAC study. BMC Pediatr. 2014;14:223. doi: 10.1186/1471-2431-14-223. PMID: 25193601.X-1

2656. Puertas-Martin V, Carreras-Saez I, Marana A, et al. [Therapeutic possibilities in refractory epilepsy in tuberous sclerosis complex]. Rev Neurol. 2014 Jun 16;58(12):529-35. PMID: 24915028.X-1

2657. Pugliese CE, White SW. Brief report: problem solving therapy in college students with autism spectrum disorders: feasibility and preliminary efficacy. J Autism Dev Disord. 2014 Mar;44(3):719-29. doi: 10.1007/s10803013-1914-8. PMID: 23963592.X-1

2658. Puscian A, Leski S, Gorkiewicz T, et al. A novel automated behavioral test battery assessing cognitive rigidity in two genetic mouse models of autism. Front Behav Neurosci. 2014;8:140. doi: 10.3389/fnbeh.2014.00140. PMID: 24808839.X-1

2659. Radell ML, Mercado E, 3rd. Modeling possible effects of atypical cerebellar processing on eyeblink conditioning in autism. Cogn Affect Behav Neurosci. 2014 Sep;14(3):1142-64. doi: 10.3758/s13415-0140263-1. PMID: 24590391.X-1 
2660. Raduege TJ. Benefits and services. Issue Brief Health Policy Track Serv. 2014 Dec 29:1-51. PMID: 25771547.X-5

2661. Raggi A, Meucci P, Leonardi M, et al. The development of a structured schedule for collecting ICF-CY-based information on disability in school and preschool children: an action research from Italy. Int J Rehabil Res. 2014 Mar;37(1):86-96. doi: 10.1097/mrr.0000000000000042. PMID: 24323140.X-1

2662. Rahbar MH, Samms-Vaughan M, Dickerson AS, et al. Role of fruits, grains, and seafood consumption in blood cadmium concentrations of Jamaican children with and without Autism Spectrum Disorder. Res Autism Spectr Disord. 2014 Sep 1;8(9):1134-45. doi: 10.1016/j.rasd.2014.06.002. PMID: 25089152.X-1

2663. Rahman FR, Maharaj V, Yates R, et al. Addressing the inverse care law: the role of community paediatric services. Perspect Public Health. 2014 Mar;134(2):85-92. doi: 10.1177/1757913913516089. PMID: 24406636.X-1

2664. Raja S, Mohapatra S, Kumar JS, et al. Prescription patterns of hypolipidaemic drugs in a tertiary care teaching hospital of southern India. J Clin Diagn Res. 2014 Apr;8(4):Hc01-3. doi: 10.7860/jcdr/2014/8010.4206. PMID: 24959461.X-1

2665. Rance G, Saunders K, Carew P, et al. The use of listening devices to ameliorate auditory deficit in children with autism. J Pediatr. 2014 Feb;164(2):352-7. doi: 10.1016/j.jpeds.2013.09.041. PMID: 24183205.X-1, X-3

2666. Rattaz C, Ledesert B, Masson O, et al. Special education and care services for children, adolescents, and adults with autism spectrum disorders in France: Families' opinion and satisfaction. Autism. 2014 Feb;18(2):18593. doi: 10.1177/1362361312460952. PMID: 23045217.X-1

2667. Rattazzi A. [The importance of early detection and early intervention for children with autism spectrum conditions]. Vertex. 2014 Jul-Aug;25(116):290-4. PMID: 25546644.X-1
2668. Rauf NK, Anis ul H, Aslam N, et al. Characteristic symptoms and adaptive behaviors of children with autism. J Coll Physicians Surg Pak. 2014 Sep;24(9):658-62. doi: 09.2014/jcpsp.658662. PMID:

25233971.X-1

2669. Reading R. Psychotropic medication use and polypharmacy in children with autism spectrum disorders. Child: Care, Health \& Development. 2014;40(1):147-8 2p. doi: 10.1111/cch.12123_6. PMID: 104168019. Language: English. Entry Date: 20131203. Revision Date: 20150711. Publication Type: Journal Article.X-1, X-2, X-3

2670. Regent L. [Bibliography. Autism in childhood and adolescence]. Soins Pediatr Pueric. 2014 Jan-Feb(276):39. PMID: 24617094.X-1

2671. Retico A, Tosetti M, Muratori F, et al. Neuroimaging-based methods for autism identification: a possible translational application? Funct Neurol. 2014 OctDec;29(4):231-9. PMID: 25764253.X-1

2672. Rhind C, Bonfioli E, Hibbs R, et al. An examination of autism spectrum traits in adolescents with anorexia nervosa and their parents. Mol Autism. 2014;5(1):56. doi: 10.1186/2040-2392-5-56. PMID: 25553237.X1

2673. Richdale AL, Baker E, Short M, et al. The role of insomnia, pre-sleep arousal and psychopathology symptoms in daytime impairment in adolescents with highfunctioning autism spectrum disorder. Sleep Med. 2014 Sep;15(9):1082-8. doi: 10.1016/j.sleep.2014.05.005. PMID: 24974199.X-1

2674. Rideau Batista Novais A, Crouzin N, Cavalier M, et al. Tiagabine improves hippocampal long-term depression in rat pups subjected to prenatal inflammation. PLoS One. 2014;9(9):e106302. doi: 10.1371/journal.pone.0106302. PMID: 25184226.X-1

2675. Rieffe C, De Bruine M, De Rooij M, et al. Approach and avoidant emotion regulation prevent depressive symptoms in children with an Autism Spectrum Disorder. Int J Dev Neurosci. 2014 Dec;39:37-43. doi: 
10.1016/j.ijdevneu.2014.06.003. PMID: 24951836.X-1, X-3

2676. Righi G, Tierney AL, Tager-Flusberg $\mathrm{H}$, et al. Functional connectivity in the first year of life in infants at risk for autism spectrum disorder: an EEG study. PLoS One.

2014;9(8):e105176. doi: 10.1371/journal.pone.0105176. PMID: 25140874.X-1

2677. Rispoli M, Camargo S, Machalicek W, et al. Functional communication training in the treatment of problem behavior maintained by access to rituals. J Appl Behav Anal. 2014 Fall;47(3):580-93. doi: 10.1002/jaba.130. PMID: 24817482.X-3, X-4

2678. Rispoli M, Camargo SH, Neely L, et al. Pre-Session Satiation as a Treatment for Stereotypy During Group Activities. Behav Modif. 2014 May;38(3):392-411. doi: 10.1177/0145445513511631. PMID: 24270401.X-1

2679. Roane HS, DeRosa NM. Reduction of emergent dropping behavior during treatment of elopement. J Appl Behav Anal. 2014

Fall;47(3):633-8. doi: 10.1002/jaba.136. PMID: 24825372.X-1, X-3, X-4

2680. Robertson CE, Thomas C, Kravitz DJ, et al. Global motion perception deficits in autism are reflected as early as primary visual cortex. Brain: A Journal of Neurology. 2014;137(9):2588-99 12p. doi: brain/awu189. PMID: 103985787. Language: English. Entry Date: 20141121. Revision Date: 20150901. Publication Type: Journal Article. Journal Subset: Allied Health.X-1

2681. Rogers SJ, Vismara L, Wagner AL, et al. Autism treatment in the first year of life: a pilot study of infant start, a parent-implemented intervention for symptomatic infants. J Autism Dev Disord. 2014 Dec;44(12):2981-95. doi: 10.1007/s10803-014-2202-y. PMID: 25212413.X-1, X-4

2682. Rosales R, Maderitz C, Garcia YA. Comparison of simple and complex auditoryvisual conditional discrimination training. J Appl Behav Anal. 2014 Summer;47(2):437-42. doi: 10.1002/jaba.121. PMID: 24764261.X-1, $\mathrm{X}-3, \mathrm{X}-4$
2683. Rossman IT, Lin L, Morgan KM, et al. Engrailed2 modulates cerebellar granule neuron precursor proliferation, differentiation and insulin-like growth factor 1 signaling during postnatal development. Mol Autism. 2014;5(1):9. doi: 10.1186/2040-2392-5-9. PMID: 24507165.X-1

2684. Ruiz-Robledillo N, Gonzalez-Bono E, Moya-Albiol L. Lack of institutional support entails disruption in cortisol awakening response in caregivers of people with highfunctioning autism. J Health Psychol. 2014 Dec;19(12):1586-96. doi: 10.1177/1359105313496444. PMID: 23933951.X-1

2685. Russo A. Decreased Mitogen Inducible Gene 6 (MIG-6) Associated with Symptom Severity in Children with Autism. Biomark Insights. 2014;9:85-9. doi: 10.4137/bmi.s15218. PMID: 25342879.X-1

2686. Russo AJ. Increased Epidermal Growth Factor Receptor (EGFR) Associated with Hepatocyte Growth Factor (HGF) and Symptom Severity in Children with Autism Spectrum Disorders (ASDs). J Cent Nerv Syst Dis. 2014;6:79-83. doi: 10.4137/jcnsd.s13767. PMID: 25249767.X-1

2687. Sadoughi HR, Nasseri S, Momennezhad M, et al. A Comparison Between GATE and MCNPX Monte Carlo Codes in Simulation of Medical Linear Accelerator. J Med Signals Sens. 2014 Jan;4(1):10-7. PMID: 24696804.X-1

2688. Saloviita T, Leppanen M, Ojalammi U. Authorship in facilitated communication: an analysis of 11 cases. Augment Altern Commun. 2014 Sep;30(3):213-25. doi: 10.3109/07434618.2014.927529. PMID: 24946681.X-1

2689. Samadi SA, McConkey R. The utility of the Gilliam autism rating scale for identifying Iranian children with autism. Disabil Rehabil. 2014;36(6):452-6. doi: 10.3109/09638288.2013.797514. PMID: 23738615.X-1

2690. Samaras K, Correll CU, Mitchell AJ, et al. Diabetes risk potentially underestimated in youth and children receiving antipsychotics. JAMA Psychiatry. 2014 Feb;71(2):209-10. doi: 
10.1001/jamapsychiatry.2013.4030. PMID: 24500630.X-1

2691. San Mauro Martin I, Garicano Vilar E, Collado Yurrutia L, et al. [Is gluten the great etiopathogenic agent of disease in the XXI century?]. Nutr Hosp. 2014;30(6):1203-10. doi: 10.3305/nh.2014.30.6.7866. PMID: 25433099.X-1

2692. Sangha S, Greba Q, Robinson PD, et al. Heightened fear in response to a safety cue and extinguished fear cue in a rat model of maternal immune activation. Front Behav Neurosci. 2014;8:168. doi: 10.3389/fnbeh.2014.00168. PMID: 24847231.X-1

2693. Sato W, Kubota Y, Kochiyama T, et al. Increased putamen volume in adults with autism spectrum disorder. Front Hum Neurosci. 2014;8:957. doi: 10.3389/fnhum.2014.00957. PMID: 25505401.X-1

2694. Savoy M. Autism: 5 misconceptions that can complicate care. J Fam Pract. 2014 Jun;63(6):310-4. PMID: 25061620.X-1

2695. Scaler Scott K, Tetnowski JA, Flaitz JR, et al. Preliminary study of disfluency in school-aged children with autism. Int J Lang Commun Disord. 2014 Jan-Feb;49(1):75-89. doi: 10.1111/1460-6984.12048. PMID: 24372887.X-1

2696. Schaaf RC, Benevides T, Mailloux Z, et al. An intervention for sensory difficulties in children with autism: a randomized trial. J Autism Dev Disord. 2014 Jul;44(7):1493-506. doi: 10.1007/s10803-013-1983-8. PMID: 24214165. INCLUDE

2697. Schaaf RC, Leiby B, Benevides T, et al. Response from authors to comments on "An intervention for sensory difficulties in children with autism: A randomized trial”. Journal of Autism and Developmental Disorders. 2014 2015-02-24;44(6):1489-91. doi:

10.1016/j.rasd.2006.08.006

10.1016/j.rasd.2008.11.001 . http://dx.doi.org/10.1007/s10803-014-2111-0. PMID: 1531964316; 2014-20681-001.X-1, X-2

2698. Schafer EC, Bryant D, Sanders K, et al. Fitting and verification of frequency modulation systems on children with normal hearing. J Am Acad Audiol. 2014

Jun;25(6):529-40. doi: 10.3766/jaaa.25.6.3. PMID: 25313543.X-1

2699. Scheele D, Kendrick KM, Khouri C, et al. An oxytocin-induced facilitation of neural and emotional responses to social touch correlates inversely with autism traits. Neuropsychopharmacology. 2014 Aug;39(9):2078-85. doi: 10.1038/npp.2014.78. PMID: 24694924.X-1

2700. Schelinski S, Riedel P, von Kriegstein K. Visual abilities are important for auditoryonly speech recognition: evidence from autism spectrum disorder. Neuropsychologia. 2014 Dec;65:1-11. doi: 10.1016/j.neuropsychologia.2014.09.031. PMID: 25283605.X-1

2701. Schieve LA, Tian LH, Baio J, et al. Population attributable fractions for three perinatal risk factors for autism spectrum disorders, 2002 and 2008 autism and developmental disabilities monitoring network. Ann Epidemiol. 2014 Apr;24(4):260-6. doi: 10.1016/j.annepidem.2013.12.014. PMID: 24529515.X-1

2702. Schindel RH, Chahine A, Anderson N, et al. Behavior modification of children with autism spectrum disorder in an orthodontic setting. J Clin Orthod. 2014 May;48(5):285-91. PMID: 25083672.X-5

2703. Schlosser RW, Koul R, Shane H, et al. Effects of animation on naming and identification across two graphic symbol sets representing verbs and prepositions. J Speech Lang Hear Res. 2014 Oct;57(5):1779-91. doi: 10.1044/2014_jslhr-l-13-0193. PMID: 24811580.X-1, X-3

2704. Schmidt RJ, Tancredi DJ, Krakowiak $\mathrm{P}$, et al. Maternal intake of supplemental iron and risk of autism spectrum disorder. Am J Epidemiol. 2014 Nov 1;180(9):890-900. doi: 10.1093/aje/kwu208. PMID: 25249546.X-1

2705. Schmitt LM, Cook EH, Sweeney JA, et al. Saccadic eye movement abnormalities in autism spectrum disorder indicate dysfunctions in cerebellum and brainstem. Mol Autism. 2014;5(1):47. doi: 10.1186/2040-2392-5-47. PMID: 25400899.X-1 
2706. Schoen Simmons E, Paul R, Volkmar F. Assessing pragmatic language in autism spectrum disorder: the Yale in vivo Pragmatic Protocol. J Speech Lang Hear Res. 2014 Dec;57(6):2162-73. doi: 10.1044/2014_jslhr-l14-0040. PMID: 25029348.X-1

2707. Schohl KA, Van Hecke AV, Carson $\mathrm{AM}$, et al. A replication and extension of the PEERS intervention: examining effects on social skills and social anxiety in adolescents with autism spectrum disorders. J Autism Dev Disord. 2014 Mar;44(3):532-45. doi: 10.1007/s10803-013-1900-1. PMID: 23893101.X-1

2708. Schore AN. Early interpersonal neurobiological assessment of attachment and autistic spectrum disorders. Front Psychol. 2014;5:1049. doi: 10.3389/fpsyg.2014.01049. PMID: 25339916.X-1

2709. Schreibman L, Stahmer AC. A randomized trial comparison of the effects of verbal and pictorial naturalistic communication strategies on spoken language for young children with autism. J Autism Dev Disord. 2014 May;44(5):1244-51. doi: 10.1007/s10803013-1972-y. PMID: 24272416.X-1

2710. Schubart JR, Camacho F, Leslie D. Psychotropic medication trends among children and adolescents with autism spectrum disorder in the Medicaid program. Autism. 2014

Aug;18(6):631-7. doi: 10.1177/1362361313497537. PMID: 24165274.X-1

2711. Schuwerk T, Langguth B, Sommer M. Modulating functional and dysfunctional mentalizing by transcranial magnetic stimulation. Front Psychol. 2014;5:1309. doi: 10.3389/fpsyg.2014.01309. PMID: 25477838.X-1

2712. Segal O, Kaplan D, Patael S, et al. Judging emotions in lexical-prosodic congruent and incongruent speech stimuli by adolescents in the autism spectrum. Folia Phoniatr Logop. 2014;66(1-2):25-36. doi: 10.1159/000363739. PMID: 25472790.X-1

2713. Segovia F, Holt R, Spencer M, et al. Identifying endophenotypes of autism: a multivariate approach. Front Comput Neurosci.
2014;8:60. doi: 10.3389/fncom.2014.00060. PMID: 24936183.X-1

2714. Sener EF, Oztop DB, Ozkul Y. MTHFR Gene C677T Polymorphism in Autism Spectrum Disorders. Genet Res Int. 2014;2014:698574. doi: 10.1155/2014/698574. PMID: 25431675.X-1

2715. Serret S, Hun S, Iakimova G, et al. Facing the challenge of teaching emotions to individuals with low- and high-functioning autism using a new Serious game: a pilot study. Mol Autism. 2014;5:37. doi: 10.1186/20402392-5-37. PMID: 25018866.X-3

2716. Shaddel F, Ghazirad M, Bryant M. What is the best available evidence for using homeopathy in patients with intellectual disabilities? Iran J Pediatr. 2014

Aug;24(4):339-44. PMID: 25755852.X-1, X-2, $\mathrm{X}-3$

2717. Shafai T, Mustafa M, Hild T, et al. The association of early weaning and formula feeding with autism spectrum disorders. Breastfeed Med. 2014 Jun;9(5):275-6. doi: 10.1089/bfm.2013.0104. PMID: 24506526.X-1

2718. Sharda M, Khundrakpam BS, Evans AC, et al. Disruption of structural covariance networks for language in autism is modulated by verbal ability. Brain Struct Funct. 2014 Dec 2doi: 10.1007/s00429-014-0953-z. PMID: 25445842.X-1

2719. Sharp WG, Burrell TL, Jaquess DL. The Autism MEAL Plan: a parent-training curriculum to manage eating aversions and low intake among children with autism. Autism. 2014 Aug;18(6):712-22. doi: 10.1177/1362361313489190. PMID: 24101716.X-4

2720. Shattuck PT, Steinberg J, Yu J, et al. Disability Identification and Self-Efficacy among College Students on the Autism Spectrum. Autism Res Treat. 2014;2014:924182. doi: 10.1155/2014/924182. PMID: 24707401.X-1

2721. Sherif T, Rioux P, Rousseau ME, et al. CBRAIN: a web-based, distributed computing platform for collaborative neuroimaging research. Front Neuroinform. 2014;8:54. doi: 
10.3389/fninf.2014.00054. PMID: 24904400.X-

1

2722. Shield A. Preliminary findings of similarities and differences in the signed and spoken language of children with autism. Semin Speech Lang. 2014 Nov;35(4):309-20. doi: 10.1055/s-0034-1389103. PMID: 25321855.X1

2723. Shih W, Patterson SY, Kasari C. Developing an Adaptive Treatment Strategy for Peer-Related Social Skills for Children With Autism Spectrum Disorders. J Clin Child Adolesc Psychol. 2014 Jun 13:1-11. doi: 10.1080/15374416.2014.915549. PMID: 24926658.X-1

2724. Shrestha M, Shrestha R. Symptom recognition to diagnosis of autism in Nepal. J Autism Dev Disord. 2014 Jun;44(6):1483-5. doi: 10.1007/s10803-013-2005-6. PMID: 24293038.X-1

2725. Siegel M, Beresford CA, Bunker M, et al. Preliminary investigation of lithium for mood disorder symptoms in children and adolescents with autism spectrum disorder. J Child Adolesc Psychopharmacol. 2014 Sep;24(7):399-402. doi: 10.1089/cap.2014.0019. PMID: 25093602.X-1

2726. Siegel M, Milligan B, Chemelski B, et al. Specialized inpatient psychiatry for serious behavioral disturbance in autism and intellectual disability. J Autism Dev Disord. 2014 Dec;44(12):3026-32. doi: 10.1007/s10803-014-2157-z. PMID: 24925543.X-3

2727. Siller M, Reyes N, Hotez E, et al. Longitudinal change in the use of services in autism spectrum disorder: understanding the role of child characteristics, family demographics, and parent cognitions. Autism. 2014 May;18(4):433-46. doi: 10.1177/1362361313476766. PMID: 24108191.X-1

2728. Siller M, Swanson M, Gerber A, et al. A parent-mediated intervention that targets responsive parental behaviors increases attachment behaviors in children with ASD: results from a randomized clinical trial. $\mathrm{J}$ Autism Dev Disord. 2014 Jul;44(7):1720-32. doi: 10.1007/s10803-014-2049-2. PMID: 24488157.X-1

2729. Silverman L, Hollway JA, Smith T, et al. A Multisite Trial of Atomoxetine and Parent Training in Children with Autism Spectrum Disorders: Rationale and Design Challenges. Res Autism Spectr Disord. 2014 Jul 1;8(7):899907. doi: 10.1016/j.rasd.2014.03.013. PMID: 25197320.X-1

2730. Singh K, Connors SL, Macklin EA, et al. Sulforaphane treatment of autism spectrum disorder (ASD). Proc Natl Acad Sci U S A. 2014 Oct 28;111(43):15550-5. doi: 10.1073/pnas.1416940111. PMID: 25313065.X-1

2731. Singleton G, Warren S, Piersel W. Clinical overview of the need for technologies for around-the-clock monitoring of the health status of severely disabled autistic children. Conf Proc IEEE Eng Med Biol Soc. 2014 Aug;2014:789-91. doi: 10.1109/embc.2014.6943709. PMID: 25570077.X-1

2732. Smith EG. Multisensory integration and temporal synchrony in autism; 2014.

2733. Smith J, Hand L, Dowrick PW. Video feedforward for rapid learning of a picturebased communication system. J Autism Dev Disord. 2014 Apr;44(4):926-36. doi: 10.1007/s10803-013-1946-0. PMID: 24068486.X-1, X-3, X-4

2734. Smith MJ, Ginger EJ, Wright K, et al. Virtual reality job interview training in adults with autism spectrum disorder. J Autism Dev Disord. 2014 Oct;44(10):2450-63. doi: 10.1007/s10803-014-2113-y. PMID: 24803366.X-1

2735. Smith T. Behavior analysts can be interdisciplinary too: a review of durand's autism spectrum disorder. J Appl Behav Anal. 2014 Oct 17doi: 10.1002/jaba.157. PMID: 25323996.X-1

2736. Sokhadze EM, El-Baz AS, Sears LL, et al. rTMS neuromodulation improves electrocortical functional measures of information processing and behavioral responses in autism. Front Syst Neurosci. 
2014;8:134. doi: 10.3389/fnsys.2014.00134. PMID: 25147508.X-1, X-3

2737. Sokhadze EM, El-Baz AS, Tasman A, et al. Neuromodulation integrating rTMS and neurofeedback for the treatment of autism spectrum disorder: an exploratory study. Appl Psychophysiol Biofeedback. 2014 Dec;39(34):237-57. doi: 10.1007/s10484-014-9264-7. PMID: 25267414.X-1

2738. Solomon R, Van Egeren LA, Mahoney G, et al. PLAY Project Home Consultation intervention program for young children with autism spectrum disorders: a randomized controlled trial. J Dev Behav Pediatr. 2014 Oct;35(8):475-85. doi: 10.1097/dbp.0000000000000096. PMID: 25264862.X-1

2739. Spriggs AD, Knight V, Sherrow L. Talking Picture Schedules: Embedding Video Models into Visual Activity Schedules to Increase Independence for Students with ASD.

J Autism Dev Disord. 2014 Nov 21doi: 10.1007/s10803-014-2315-3. PMID: 25413145.X-1

2740. Srinivasan SM, Pescatello LS, Bhat AN. Current Perspectives on Physical Activity and Exercise Recommendations for Children and Adolescents With Autism Spectrum Disorders. Physical Therapy. 2014;94(6):87589 15p. doi: 10.2522/ptj.20130157. PMID: 107854570. Language: English. Entry Date: 20140610. Revision Date: 20150820. Publication Type: Journal Article.X-1

2741. Srivastava S, Landy-Schmitt C, Clark $\mathrm{B}$, et al. Autism traits in children and adolescents with Cornelia de Lange syndrome. Am J Med Genet A. 2014 Jun;164a(6):1400-10. doi: 10.1002/ajmg.a.36573. PMID: 24718998.X-1

2742. Stagg SD, Slavny R, Hand C, et al. Does facial expressivity count? How typically developing children respond initially to children with autism. Autism. 2014 Aug;18(6):704-11. doi: 10.1177/1362361313492392. PMID: 24121180.X-1

2743. Starovoitova TE, Dolgikh VV, Mikhnovich VI, et al. [Electric status epilepticus in sleep (case report).]. Zh Nevrol Psikhiatr Im S S Korsakova. 2014;114(4
Vypusk 2 Epilepsy):8-10. PMID: 24874330.X1

2744. Steeb H, Ramsey JM, Guest PC, et al. Serum proteomic analysis identifies sexspecific differences in lipid metabolism and inflammation profiles in adults diagnosed with Asperger syndrome. Mol Autism. 2014;5(1):4. doi: 10.1186/2040-2392-5-4. PMID: 24467795.X-1

2745. Stein K. The politics and process of revising the DSM-V and the impact of changes on dietetics. J Acad Nutr Diet. 2014 Mar;114(3):350-65. doi: 10.1016/j.jand.2013.12.011. PMID: 24534369.X-1

2746. Steiner NJ, Frenette E, Hynes C, et al. A pilot feasibility study of neurofeedback for children with autism. Appl Psychophysiol Biofeedback. 2014 Jun;39(2):99-107. doi: 10.1007/s10484-014-9241-1. PMID: 24737038.X-3, X-4

2747. Stevenson RA, Segers M, Ferber S, et al. The impact of mutisensory integration deficits on speech perception in children with autism spectrum disorders. Frontiers in Psychology. 2014 2015-12-09;5doi: 10.1093/cercor/bht213. CrossRef Medline. 10.1007/s 10803-010-1000-4. PMID: 1627947213; 2014-40502-001.X-1, X-2

2748. Stevenson RA, Siemann JK, Schneider $\mathrm{BC}$, et al. Multisensory temporal integration in autism spectrum disorders. J Neurosci. 2014 Jan 15;34(3):691-7. doi: 10.1523/jneurosci.361513.2014. PMID: 24431427.X-1

2749. Stevenson RA, Siemann JK, Woynaroski TG, et al. Evidence for diminished multisensory integration in autism spectrum disorders. J Autism Dev Disord. 2014 Dec;44(12):3161-7. doi: 10.1007/s10803-0142179-6. PMID: 25022248.X-1

2750. Stevenson RA, Siemann JK, Woynaroski TG, et al. Brief report: Arrested development of audiovisual speech perception in autism spectrum disorders. J Autism Dev Disord. 2014 Jun;44(6):1470-7. doi: 10.1007/s10803-013-1992-7. PMID: 24218241.X-1 
2751. Stichter JP, Laffey J, Galyen K, et al. iSocial: delivering the Social Competence Intervention for Adolescents (SCI-A) in a 3D virtual learning environment for youth with high functioning autism. J Autism Dev Disord. 2014 Feb;44(2):417-30. doi: 10.1007/s10803013-1881-0. PMID: 23812663.X-1

2752. Stoodley CJ. Distinct regions of the cerebellum show gray matter decreases in autism, ADHD, and developmental dyslexia. Front Syst Neurosci. 2014;8:92. doi: 10.3389/fnsys.2014.00092. PMID: 24904314.X-1

2753. Strickland AD. Prevention of cerebral palsy, autism spectrum disorder, and attention deficit-hyperactivity disorder. Med Hypotheses. 2014 May;82(5):522-8. doi: 10.1016/j.mehy.2014.02.003. PMID: 24581674.X-1

2754. Strifert K. The link between oral contraceptive use and prevalence in autism spectrum disorder. Med Hypotheses. 2014 Dec;83(6):718-25. doi: 10.1016/j.mehy.2014.09.026. PMID: 25459142.X-1

2755. Strunz S, Westphal L, Ritter K, et al. Personality Pathology of Adults With Autism Spectrum Disorder Without Accompanying Intellectual Impairment in Comparison to Adults With Personality Disorders. J Autism Dev Disord. 2014 Jul 15doi: 10.1007/s10803014-2183-x. PMID: 25022250.X-1

2756. Stubendorff K, Larsson V, Ballard C, et al. Treatment effect of memantine on survival in dementia with Lewy bodies and Parkinson's disease with dementia: a prospective study. BMJ Open. 2014;4(7):e005158. doi: 10.1136/bmjopen-2014-005158. PMID: 24993765.X-1

2757. Stuttard L, Beresford B, Clarke S, et al. Riding the rapids: living with autism or disability--an evaluation of a parenting support intervention for parents of disabled children. Res Dev Disabil. 2014 Oct;35(10):2371-83. doi: 10.1016/j.ridd.2014.05.021. PMID: 24973545.X-1

2758. Sugama M, Ishizaki A. [Drug therapy for $\mathrm{AD} / \mathrm{HD}$ investigation of usefulness of extended-release methylphenidate and atomoxetine from the viewpoint of persistency rate]. No To Hattatsu. 2014 Jan;46(1):22-5. PMID: 24620426.X-1, X-3

2759. Suing M, Gollan T, Schmidt MH, et al. [In Process Citation]. Prax Kinderpsychol Kinderpsychiatr. 2014 Oct;63(8):666-84. doi: 10.13109/prkk.2014.63.8.666. PMID: 25524037.X-1

2760. Sullivan JC, Miller LJ, Nielsen DM, et al. The presence of migraines and its association with sensory hyperreactivity and anxiety symptomatology in children with autism spectrum disorder. Autism. 2014 Aug;18(6):743-7. doi: 10.1177/1362361313489377. PMID: 24072661.X-1

2761. Sun J, Chang EB. Exploring gut microbes in human health and disease: Pushing the envelope. Genes Dis. 2014 Dec;1(2):132-9. doi: 10.1016/j.gendis.2014.08.001. PMID: 25642449.X-1

2762. Sun JG, Sun XR. [Encephalopathy therapeutic tongue acupoint apparatus (ETTAA) for 42 cases of autism]. Zhongguo Zhen Jiu. 2014 Jan;34(1):96-8. PMID: 24673076.X-1

2763. Sun X, Allison C, Auyeung B, et al. Comparison between a Mandarin Chinese version of the Childhood Autism Spectrum Test and the Clancy Autism Behaviour Scale in mainland China. Res Dev Disabil. 2014 Jul;35(7):1599-608. doi: 10.1016/j.ridd.2014.02.005. PMID: 24769432.X-1

2764. Suren P, Gunnes N, Roth C, et al. Parental obesity and risk of autism spectrum disorder. Pediatrics. 2014 May;133(5):e112838. doi: 10.1542/peds.2013-3664. PMID: 24709932.X-1

2765. Suresh AP, Benjamin TE, Crasta JE, et al. Comparison of burden among primary caregivers of children with autism and intellectual disability against children with intellectual disability only in a hospital population in India. Indian J Pediatr. 2014 Dec;81 Suppl 2:S179-82. doi: 10.1007/s12098-014-1626-x. PMID: 25387661.X-1 
2766. Suzuki M, Yamada A, Watanabe N, et al. A failure to confirm the effectiveness of a brief group psychoeducational program for mothers of children with high-functioning pervasive developmental disorders: a randomized controlled pilot trial. Neuropsychiatr Dis Treat. 2014;10:1141-53. doi: 10.2147/ndt.s60058. PMID: 25061301.X-1

2767. Swanson AR, Warren ZE, Stone WL, et al. The diagnosis of autism in community pediatric settings: does advanced training facilitate practice change? Autism. 2014 Jul;18(5):555-61. doi: 10.1177/1362361313481507. PMID: 23847130.X-1

2768. Takahashi H, Nakahachi T, Komatsu S, et al. Hyperreactivity to weak acoustic stimuli and prolonged acoustic startle latency in children with autism spectrum disorders. Mol Autism. 2014;5(1):23. doi: 10.1186/2040-23925-23. PMID: 24618368.X-1

2769. Takara K, Kondo T. Comorbid atypical autistic traits as a potential risk factor for suicide attempts among adult depressed patients: a case-control study. Ann Gen Psychiatry. 2014;13(1):33. doi: 10.1186/s12991-014-0033-z. PMID: 25328535.X-1

2770. Takuma K, Hara Y, Kataoka S, et al. Chronic treatment with valproic acid or sodium butyrate attenuates novel object recognition deficits and hippocampal dendritic spine loss in a mouse model of autism. Pharmacol Biochem Behav. 2014 Nov;126:43-9. doi: 10.1016/j.pbb.2014.08.013. PMID: 25240644.X-1

2771. Tan XY, Trembath D, Bloomberg K, et al. Acquisition and generalization of key word signing by three children with autism. Dev Neurorehabil. 2014 Apr;17(2):125-36. doi: 10.3109/17518423.2013.863236. PMID: 24393060.X-3, X-4

2772. Tanidir C, Mukaddes NM. Referral pattern and special interests in children and adolescents with Asperger syndrome: a Turkish referred sample. Autism. 2014 Feb;18(2):17884. doi: 10.1177/1362361312457716. PMID: 22987894.X-1
2773. Tao VQ, Chan KY, Chu YW, et al. The clinical impact of chromosomal microarray on paediatric care in Hong Kong. PLoS One. 2014;9(10):e109629. doi: 10.1371/journal.pone.0109629. PMID: 25333781.X-1

2774. Tavassoli T, Hoekstra RA, BaronCohen S. The Sensory Perception Quotient (SPQ): development and validation of a new sensory questionnaire for adults with and without autism. Mol Autism. 2014;5:29. doi: 10.1186/2040-2392-5-29. PMID: 24791196.X1

2775. Taylor JL, Mailick MR. A longitudinal examination of 10-year change in vocational and educational activities for adults with autism spectrum disorders. Dev Psychol. 2014 Mar;50(3):699-708. doi: 10.1037/a0034297. PMID: 24001150.X-1

2776. Taylor JL, Smith LE, Mailick MR. Engagement in vocational activities promotes behavioral development for adults with autism spectrum disorders. J Autism Dev Disord. 2014 Jun;44(6):1447-60. doi: 10.1007/s10803-0132010-9. PMID: 24287880.X-1

2777. Tellegen CL, Sanders MR. A randomized controlled trial evaluating a brief parenting program with children with autism spectrum disorders. J Consult Clin Psychol. 2014 Dec;82(6):1193-200. doi: 10.1037/a0037246. PMID: 24979315.X-1

2778. Tetef S. Effectiveness of Transmucosal Sedation for Special Needs Populations in the Ambulatory Care Setting. AORN Journal. 2014;100(6):651-69 19p. doi: 10.1016/j.aorn.2014.04.019. PMID: 103923061. Language: English. Entry Date: 20141206. Revision Date: 20150818. Publication Type: Journal Article.X-1, X-2

2779. Thomas KC, Parish SL, Williams CS. Healthcare expenditures for autism during times of school transition: some vulnerable families fall behind. Matern Child Health J. 2014 Oct;18(8):1936-44. doi: 10.1007/s10995-0141439-6. PMID: 24553795.X-1

2780. Thompson DG, Tielsch-Goddard A. Improving management of patients with autism spectrum disorder having scheduled surgery: optimizing practice. J Pediatr Health Care. 2014 
Sep-Oct;28(5):394-403. doi:

10.1016/j.pedhc.2013.09.007. PMID:

24287372.X-1

2781. Thompson GA, McFerran KS, Gold C. Family-centred music therapy to promote social engagement in young children with severe autism spectrum disorder: a randomized controlled study. Child Care Health Dev. 2014 Nov;40(6):840-52. doi: 10.1111/cch.12121. PMID: 24261547. INCLUDE

2782. Thompson N, Yoshinaga-Itano C. Enhancing the development of infants and toddlers with dual diagnosis of autism spectrum disorder and deafness. Semin Speech Lang. 2014 Nov;35(4):321-30. doi: 10.1055/s-00341389104. PMID: 25321856.X-1

2783. Thrasher A. Video modeling for children with dual diagnosis of deafness or hard of hearing and autism spectrum disorder to promote peer interaction. Semin Speech Lang. 2014 Nov;35(4):331-42. doi: 10.1055/s-00341389105. PMID: 25321857.X-1, X-2, X-3, X-4

2784. Timimi S. Children's behaviour problems: a NICE mess. Int J Clin Pract. 2014 Sep;68(9):1053-5. doi: 10.1111/ijcp.12442. PMID: 25196181.X-1

2785. Titeca D, Roeyers H, Josephy H, et al. Preschool predictors of mathematics in first grade children with autism spectrum disorder. Res Dev Disabil. 2014 Nov;35(11):2714-27. doi: 10.1016/j.ridd.2014.07.012. PMID: 25068926.X-1

2786. Tomlinson M, Yasamy MT, Emerson $\mathrm{E}$, et al. Setting global research priorities for developmental disabilities, including intellectual disabilities and autism. J Intellect Disabil Res. 2014 Dec;58(12):1121-30. doi: 10.1111/jir.12106. PMID: 24397279.X-1

2787. Tonge B, Brereton A, Kiomall M, et al. A randomised group comparison controlled trial of 'preschoolers with autism': a parent education and skills training intervention for young children with autistic disorder. Autism. 2014 Feb;18(2):166-77. doi:

10.1177/1362361312458186. PMID: 22987897.X-1

2788. Tonge BJ, Bull K, Brereton A, et al. A review of evidence-based early intervention for behavioural problems in children with autism spectrum disorder: the core components of effective programs, child-focused interventions and comprehensive treatment models. Current Opinion in Psychiatry. 2014;27(2):158-65 8p. doi: 10.1097/YCO.0000000000000043. PMID: 107884078. Language: English. Entry Date: 20141010. Revision Date: 20150819.

Publication Type: Journal Article.X-2

2789. Towle PO, Vacanti-Shova K, Shah S, et al. School-aged functioning of children diagnosed with autism spectrum disorder before age three: parent-reported diagnostic, adaptive, medication, and school placement outcomes. J Autism Dev Disord. 2014 Jun;44(6):1357-72. doi: 10.1007/s10803-013-1997-2. PMID: 24346492.X-1

2790. Trembath D, Iacono T, Lyon K, et al. Augmentative and alternative communication supports for adults with autism spectrum disorders. Autism. 2014 Nov;18(8):891-902. doi: 10.1177/1362361313486204. PMID: 24104514.X-1

2791. Trent S, Fry JP, Ojarikre OA, et al. Altered brain gene expression but not steroid biochemistry in a genetic mouse model of neurodevelopmental disorder. Mol Autism. 2014;5(1):21. doi: 10.1186/2040-2392-5-21. PMID: 24602487.X-1

2792. Trezise KL, Gray KM, Taffe J, et al. Working memory in adolescent males with Down syndrome and males with autism and intellectual disability: Implications for the classroom. Journal of Intellectual \& Developmental Disability. 2014;39(1):24-34 11p. doi: 10.3109/13668250.2013.874550. PMID: 107891580. Language: English. Entry Date: 20140311. Revision Date: 20150824. Publication Type: Journal Article.X-1

2793. Troyb E, Orinstein A, Tyson K, et al. Restricted and repetitive behaviors in individuals with a history of ASDs who have achieved optimal outcomes. J Autism Dev Disord. 2014 Dec;44(12):3168-84. doi: 10.1007/s10803-014-2182-y. PMID: 25030967.X-1

2794. Troyb E, Orinstein A, Tyson K, et al. Academic abilities in children and adolescents with a history of autism spectrum disorders who have achieved optimal outcomes. Autism. 2014 
Apr;18(3):233-43. doi:

10.1177/1362361312473519. PMID:

24096312.X-1

2795. Troyb E, Rosenthal M, Eigsti IM, et al. Executive functioning in individuals with a history of ASDs who have achieved optimal outcomes. Child Neuropsychol.

2014;20(4):378-97. doi:

10.1080/09297049.2013.799644. PMID: 23731181.X-1

2796. Tsai WH, Hwang YS, Hung TY, et al. Association between mechanical ventilation and neurodevelopmental disorders in a nationwide cohort of extremely low birth weight infants.

Res Dev Disabil. 2014 Jul;35(7):1544-50. doi: 10.1016/j.ridd.2014.03.048. PMID:

24769371.X-1

2797. Tseng A, Bansal R, Liu J, et al. Using the Circumplex Model of Affect to Study Valence and Arousal Ratings of Emotional Faces by Children and Adults with Autism Spectrum Disorders. Journal of Autism \& Developmental Disorders. 2014;44(6):1332-46 15p. doi: 10.1007/s10803-013-1993-6. PMID: 103945251. Language: English. Entry Date: 20140520. Revision Date: 20150710. Publication Type: Journal Article.X-1

2798. Tsilioni I, Dodman N, Petra AI, et al. Elevated serum neurotensin and CRH levels in children with autistic spectrum disorders and tail-chasing Bull Terriers with a phenotype similar to autism. Transl Psychiatry. 2014;4:e466. doi: 10.1038/tp.2014.106. PMID: 25313509.X-1

2799. Tsui GH, Rutherford MD. Video selfmodeling is an effective intervention for an adult with autism. Case Rep Neurol Med. 2014;2014:425897. doi: 10.1155/2014/425897. PMID: 25276447.X-1

2800. Tsunemi K, Tamura A, Ogawa S, et al. Intensive exposure to narrative in story books as a possibly effective treatment of social perspective-taking in schoolchildren with autism. Front Psychol. 2014;5:2. doi: 10.3389/fpsyg.2014.00002. PMID: 24474946.X-1, X-3

2801. Turygin N, Matson JL, Adams H. Prevalence of co-occurring disorders in a sample of adults with mild and moderate intellectual disabilities who reside in a residential treatment setting. Res Dev Disabil. 2014 Jul;35(7):1802-8. doi: 10.1016/j.ridd.2014.01.027. PMID: 24656808.X-1

2802. Tye C, Battaglia M, Bertoletti E, et al. Altered neurophysiological responses to emotional faces discriminate children with ASD, ADHD and ASD+ADHD. Biol Psychol. 2014 Dec;103:125-34. doi: 10.1016/j.biopsycho.2014.08.013. PMID: 25179537.X-1

2803. Tyler K, MacDonald M, Menear K. Physical activity and physical fitness of schoolaged children and youth with autism spectrum disorders. Autism Res Treat.

2014;2014:312163. doi: 10.1155/2014/312163. PMID: 25309753.X-1

2804. Udensi UK, Tchounwou PB. Dual effect of oxidative stress on leukemia cancer induction and treatment. J Exp Clin Cancer Res. 2014;33:106. doi: 10.1186/s13046-014-0106-5. PMID: 25519934.X-1

2805. Ugur C, Saday Duman N, Bektas O, et al. Antiepileptic treatment in a child with Landau Kleffner syndrome: a case report. Turk Psikiyatri Derg. 2014 Winter;25(4):282-6. PMID: 25487625.X-1

2806. Uljarevic M, Prior MR, Leekam SR. First evidence of sensory atypicality in mothers of children with Autism Spectrum Disorder (ASD). Mol Autism. 2014;5(1):26. doi: 10.1186/2040-2392-5-26. PMID: 24694290.X1

2807. Ung D, Arnold EB, De Nadai AS, et al. Inter-rater Reliability of the Anxiety Disorders Interview Schedule for DSM-IV in High-Functioning Youth with Autism Spectrum Disorder. J Dev Phys Disabil. 2014 Feb 1;26(1):53-65. doi: 10.1007/s10882-013-93433. PMID: 24683302.X-1

2808. Urbano M, Okwara L, Manser P, et al. A trial of D-cycloserine to treat stereotypies in older adolescents and young adults with autism spectrum disorder. Clin Neuropharmacol. 2014 May-Jun;37(3):69-72. doi: 10.1097/wnf.0000000000000033. PMID: 24824660.X-1 
2809. Uutela M, Lindholm J, Rantamaki T, et al. Distinctive behavioral and cellular responses to fluoxetine in the mouse model for Fragile X syndrome. Front Cell Neurosci. 2014;8:150. doi: 10.3389/fncel.2014.00150. PMID: 24904293.X-1

2810. Valenti M, La Malfa G, Tomassini A, et al. Burnout among therapists working with persons with autism after the 2009 earthquake in L'Aquila, Italy: a longitudinal comparative study. J Psychiatr Ment Health Nurs. 2014 Apr;21(3):234-40. doi: 10.1111/jpm.12054. PMID: 23552108.X-1

2811. Valicenti-McDermott M, Burrows B, Bernstein L, et al. Use of complementary and alternative medicine in children with autism and other developmental disabilities: associations with ethnicity, child comorbid symptoms, and parental stress. J Child Neurol. 2014 Mar;29(3):360-7. doi: 10.1177/0883073812474489. PMID: 23372032.X-1, X-3

2812. Vallortigara J, Rangarajan S, Whitfield $\mathrm{D}$, et al. Dynamin1 concentration in the prefrontal cortex is associated with cognitive impairment in Lewy body dementia. F1000Res. 2014;3:108. doi: 10.12688/f1000research.3786.1. PMID: 25671083.X-1

2813. van de Lagemaat LN, Nijhof B, Bosch DG, et al. Age-related decreased inhibitory vs. excitatory gene expression in the adult autistic brain. Front Neurosci. 2014;8:394. doi: 10.3389/fnins.2014.00394. PMID: 25538548.X-1

2814. van den Bergh SF, Scheeren AM, Begeer S, et al. Age related differences of executive functioning problems in everyday life of children and adolescents in the autism spectrum. J Autism Dev Disord. 2014 Aug;44(8):1959-71. doi: 10.1007/s10803-0142071-4. PMID: 24562693.X-1

2815. van Rijn S, Stockmann L, van Buggenhout G, et al. Social cognition and underlying cognitive mechanisms in children with an extra X chromosome: a comparison with autism spectrum disorder. Genes Brain Behav. 2014 Jun;13(5):459-67. doi: 10.1111/gbb.12134. PMID: 24655419.X-1
2816. Vanderwerker L, Akincigil A, Olfson $\mathrm{M}$, et al. Foster care, externalizing disorders, and antipsychotic use among Medicaid-enrolled youths. Psychiatr Serv. 2014 Oct;65(10):12814. doi: 10.1176/appi.ps.201300455. PMID: 25124057.X-1

2817. Vara AS, Pang EW, Doyle-Thomas $\mathrm{KA}$, et al. Is inhibitory control a 'no-go' in adolescents with autism spectrum disorder? Mol Autism. 2014;5(1):6. doi: 10.1186/20402392-5-6. PMID: 24485230.X-1

2818. Varella AA, de Souza DG. Emergence of auditory-visual relations from a visual-visual baseline with auditory-specific consequences in individuals with autism. J Exp Anal Behav. 2014 Jul;102(1):139-49. doi: 10.1002/jeab.93. PMID: 24965883.X-1, X-3, X-4

2819. Ventola P, Friedman HE, Anderson LC, et al. Improvements in social and adaptive functioning following short-duration PRT program: a clinical replication. J Autism Dev Disord. 2014 Nov;44(11):2862-70. doi: 10.1007/s10803-014-2145-3. PMID: 24915928.X-3, X-4

2820. Ventola P, Saulnier C, Steinberg E, et al. Early-Emerging Social Adaptive Skills in Toddlers with Autism Spectrum Disorders: An Item Analysis. Journal of Autism \& Developmental Disorders. 2014;44(2):283-93 11p. doi: 10.1007/s10803-011-1278-X. PMID: 104006225. Language: English. Entry Date: 20140129. Revision Date: 20150710. Publication Type: Journal Article.X-1

2821. Verly M, Verhoeven J, Zink I, et al. Altered functional connectivity of the language network in ASD: Role of classical language areas and cerebellum. Neuroimage Clin. 2014;4:374-82. doi: 10.1016/j.nicl.2014.01.008. PMID: 24567909.X-1

2822. Vernon TW. Fostering a social child with autism: a moment-by-moment sequential analysis of an early social engagement intervention. J Autism Dev Disord. 2014 Dec;44(12):3072-82. doi: 10.1007/s10803-0142173-z. PMID: 24974256.X-3, X-4

2823. Vivanti G, Paynter J, Duncan E, et al. Effectiveness and feasibility of the early start denver model implemented in a group-based community childcare setting. J Autism Dev 
Disord. 2014 Dec;44(12):3140-53. doi: 10.1007/s10803-014-2168-9. PMID: 24974255.X-1

2824. Vogan VM, Morgan BR, Lee W, et al. The neural correlates of visuo-spatial working memory in children with autism spectrum disorder: effects of cognitive load. J Neurodev Disord. 2014;6(1):19. doi: 10.1186/1866-19556-19. PMID: 25057329.X-1

2825. Vogindroukas I, Chelas EN, Petridis NE. Reading the Mind in the Eyes Test (Children's Version): a comparison study between children with typical development, children with high-functioning autism and typically developed adults. Folia Phoniatr Logop. 2014;66(1-2):18-24. doi: 10.1159/000363697. PMID: 25472789.X-1

2826. Vohra R, Madhavan S, Khanna R, et al. Child's autism severity: effect on West Virginia caregiver satisfaction with school services. W V Med J. 2014 Sep-Oct;110(5):329. PMID: 25643472.X-1

2827. Vohra R, Madhavan S, Sambamoorthi $\mathrm{U}$, et al. Access to services, quality of care, and family impact for children with autism, other developmental disabilities, and other mental health conditions. Autism. 2014 Oct;18(7):81526. doi: 10.1177/1362361313512902. PMID: 24353274.X-1

2828. Wachtel LE, Reti IM, Ying H. Stability of intraocular pressure after retinal reattachment surgery during electroconvulsive therapy for intractable self-injury in a 12-yearold autistic boy. J ect. 2014 Mar;30(1):73-6. doi: 10.1097/YCT.0b013e31829b2d61. PMID: 23812023.X-1, X-3, X-4

2829. Wagner DV, Borduin CM, Kanne SM, et al. Multisystemic therapy for disruptive behavior problems in youths with autism spectrum disorders: a progress report. J Marital Fam Ther. 2014 Jul;40(3):319-31. doi: 10.1111/jmft.12012. PMID: 24749815.X-2, X-3

2830. Waite A. On an Even Keel. OT Practice. 2014;19(18):7-10 4p. PMID: 107832109. Language: English. Entry Date: 20141017. Revision Date: 20150820. Publication Type: Journal Article.X-1, X-2
2831. Wallace MT, Stevenson RA. The construct of the multisensory temporal binding window and its dysregulation in developmental disabilities. Neuropsychologia. 2014 2015-0715;64:105-23. doi: http://dx.doi.org/10.1016/j.neuropsychologia.20 14.08.005. PMID: 1696233447; 2014-48956013.X-1

2832. Wang HY, Berg C. Participation of young adults with high-functioning autism in Taiwan: a pilot study. OTJR (Thorofare N J). 2014 Winter;34(1):41-51. PMID: 24669398.X1

2833. Ward CS. Autism's cancer connection: the anti-proliferation hypothesis and why it may matter. Med Hypotheses. 2014 Jan;82(1):26-35. doi: 10.1016/j.mehy.2013.10.029. PMID: 24275522.X-1

2834. Warfield ME, Chiri G, Leutz WN, et al. Family well-being in a participant-directed autism waiver program: the role of relational coordination. J Intellect Disabil Res. 2014 Dec;58(12):1091-104. doi: 10.1111/jir.12102. PMID: 25330124.X-1

2835. Waris P, Lindberg N, Kettunen K, et al. Catatonic features in adolescents with schizophrenia with and without a comorbid pervasive developmental disorder. Child Adolesc Psychiatry Ment Health. 2014;8:16. doi: 10.1186/1753-2000-8-16. PMID: 24914405.X-1

2836. Warlaumont AS, Richards JA, Gilkerson J, et al. A social feedback loop for speech development and its reduction in autism. Psychol Sci. 2014 Jul;25(7):1314-24. doi: 10.1177/0956797614531023. PMID: 24840717.X-1

2837. Warren Z, Zheng Z, Das S, et al. Brief Report: Development of a Robotic Intervention Platform for Young Children with ASD. J Autism Dev Disord. 2014 Dec 14doi: 10.1007/s10803-014-2334-0. PMID: 25503680.X-3, X-4

2838. Warreyn P, Roeyers H. See what I see, do as I do: promoting joint attention and imitation in preschoolers with autism spectrum disorder. Autism. 2014 Aug;18(6):658-71. doi: 10.1177/1362361313493834. PMID: 24104513.X-1 
2839. Watanabe T, Abe O, Kuwabara H, et al. Mitigation of sociocommunicational deficits of autism through oxytocin-induced recovery of medial prefrontal activity: a randomized trial. JAMA Psychiatry. 2014 Feb;71(2):166-75. doi: 10.1001/jamapsychiatry.2013.3181. PMID: 24352377.X-1

2840. Watkins N, Rapp JT. Environmental enrichment and response cost: immediate and subsequent effects on stereotypy. J Appl Behav Anal. 2014 Spring;47(1):186-91. doi: 10.1002/jaba.97. PMID: 24424774.X-3, X-4

2841. Watkins N, Sparling E. The Effectiveness of the Snug Vest on Stereotypic Behaviors in Children Diagnosed With an Autism Spectrum Disorder. Behav Modif. 2014 May;38(3):412-27. doi:

10.1177/0145445514532128. PMID: 24778434.X-3, X-4

2842. Weeks S, Grimmer K, Boshoff K, et al. Conducting robust intervention trials to address the sensory needs of children with autism spectrum disorder: design challenges in an Australian context. British Journal of Occupational Therapy (College of Occupational Therapists Limited). 2014;77(10):533-5 3p. doi: 10.4276/030802214X14122630932557. PMID: 103903785. Language: English. Entry Date: 20141022. Revision Date: 20151015.

Publication Type: Journal Article. Journal Subset: Allied Health.X-1

2843. Wehman PH, Schall CM, McDonough J, et al. Competitive employment for youth with autism spectrum disorders: early results from a randomized clinical trial. J Autism Dev Disord. 2014 Mar;44(3):487-500. doi: 10.1007/s10803013-1892-X. PMID: 23893098.X-1

2844. Wei X, Wagner M, Christiano ER, et al. Special Education Services Received by Students with Autism Spectrum Disorders from Preschool through High School. J Spec Educ. 2014 Nov;48(3):167-79. doi: 10.1177/0022466913483576. PMID: 25419002.X-1

2845. Weiner RH, Greene RL. Intentionbased therapy for autism spectrum disorder: promising results of a wait-list control study in children. Explore (NY). 2014 Jan-Feb;10(1):13-
23. doi: 10.1016/j.explore.2013.10.005. PMID: 24439092.X-4

2846. Weiss AL, Rohland P. "Measuring up" to ethical standards in service delivery to college students on the Autism Spectrum: A practical application of Powell's model for ethical practices in clinical phonetics and linguistics. Clin Linguist Phon. 2014 JulAug;28(7-8):627-38. doi: 10.3109/02699206.2014.927002. PMID: 25000383.X-1

2847. Weiss JA. Transdiagnostic Case Conceptualization of Emotional Problems in Youth with ASD: An Emotion Regulation Approach. Clin Psychol (New York). 2014 Dec;21(4):331-50. doi: 10.1111/cpsp.12084. PMID: 25673923.X-1

2848. Wesseling H, Guest PC, Lee CM, et al. Integrative proteomic analysis of the NMDA NR1 knockdown mouse model reveals effects on central and peripheral pathways associated with schizophrenia and autism spectrum disorders. Mol Autism. 2014;5:38. doi: 10.1186/2040-2392-5-38. PMID: 25061506.X1

2849. West PR, Amaral DG, Bais P, et al. Metabolomics as a tool for discovery of biomarkers of autism spectrum disorder in the blood plasma of children. PLoS One. 2014;9(11):e112445. doi: 10.1371/journal.pone.0112445. PMID: 25380056.X-1

2850. Westby CE. Social neuroscience and theory of mind. Folia Phoniatr Logop. 2014;66(1-2):7-17. doi: 10.1159/000362877. PMID: 25472788.X-1

2851. Wetherby AM, Guthrie W, Woods J, et al. Parent-implemented social intervention for toddlers with autism: an RCT. Pediatrics. 2014 Dec;134(6):1084-93. doi: 10.1542/peds.20140757. PMID: 25367544.X-1

2852. White E. Science, pseudoscience, and the frontline practitioner: the vaccination/autism debate. J Evid Based Soc Work. 2014;11(3):269-74. doi: 10.1080/15433714.2012.759470. PMID: 24857555.X-1 
2853. White SW, Smith LA, Schry AR. Assessment of global functioning in adolescents with autism spectrum disorders: utility of the Developmental Disability-Child Global Assessment Scale. Autism. 2014 May;18(4):362-9. doi: 10.1177/1362361313481287. PMID: 23965288.X-1

2854. Whitfield DR, Vallortigara J, Alghamdi A, et al. Assessment of ZnT3 and PSD95 protein levels in Lewy body dementias and Alzheimer's disease: association with cognitive impairment. Neurobiol Aging. 2014 Dec;35(12):2836-44. doi:

10.1016/j.neurobiolaging.2014.06.015. PMID: 25104558.X-1

2855. Winburn E, Charlton J, McConachie $\mathrm{H}$, et al. Parents' and child health professionals' attitudes towards dietary interventions for children with autism spectrum disorders. J Autism Dev Disord. 2014 Apr;44(4):747-57. doi: 10.1007/s10803-013-1922-8. PMID: 23996225.X-1

2856. Wolff ER, Madlon-Kay DJ. Childhood vaccine beliefs reported by Somali and nonSomali parents. J Am Board Fam Med. 2014 Jul-Aug;27(4):458-64. doi:

10.3122/jabfm.2014.04.130275. PMID: 25002000.X-1

2857. Wong AY, Hsia Y, Chan EW, et al. The variation of psychopharmacological prescription rates for people with autism spectrum disorder (ASD) in 30 countries. Autism Res. 2014 Oct;7(5):543-54. doi: 10.1002/aur.1391. PMID: 24895332.X-1

2858. Wong JD, Mailick MR, Greenberg JS, et al. Daily Work Stress and Awakening Cortisol in Mothers of Individuals with Autism Spectrum Disorders or Fragile X Syndrome. Fam Relat. 2014 Feb;63(1):135-47. doi: 10.1111/fare.12055. PMID: 25313265.X-1

2859. Wood A. Prenatal exposure to sodium valproate is associated with increased risk of childhood autism and autistic spectrum disorder. Evidence Based Nursing. 2014;17(3):84- 1p. doi: 10.1136/eb-2013101422. PMID: 103967026. Language: English. Entry Date: 20140627. Revision Date: 20150820. Publication Type: Journal Article.X1
2860. Wood JJ, Fujii C, Renno P, et al. Impact of cognitive behavioral therapy on observed autism symptom severity during school recess: a preliminary randomized, controlled trial. J Autism Dev Disord. 2014 Sep;44(9):2264-76. doi: 10.1007/s10803-0142097-7. PMID: 24671750.X-4

2861. Worley MJ, Tate SR, Granholm E, et al. Mediated and moderated effects of neurocognitive impairment on outcomes of treatment for substance dependence and major depression. J Consult Clin Psychol. 2014 Jun;82(3):418-28. doi: 10.1037/a0036033. PMID: 24588403.X-1

2862. Worley MJ, Trim RS, Tate SR, et al. Self-efficacy and social networks after treatment for alcohol or drug dependence and major depression: disentangling person and time-level effects. Psychol Addict Behav. 2014 Dec;28(4):1220-9. doi: 10.1037/a0037901. PMID: 25347018.X-1

2863. Wright B, Marshall D, Collingridge Moore D, et al. Autism Spectrum Social Stories In Schools Trial (ASSSIST): study protocol for a feasibility randomised controlled trial analysing clinical and cost-effectiveness of Social Stories in mainstream schools. BMJ Open. 2014;4(7):e005952. doi: 10.1136/bmjopen-2014-005952. PMID: 25009139.X-1

2864. Wu CM, Kung PT, Li CI, et al. The difference in medical utilization and associated factors between children and adolescents with and without autism spectrum disorders. Res Dev Disabil. 2014 Oct 18;36c:78-86. doi: 10.1016/j.ridd.2014.09.019. PMID: 25462468.X-1

2865. Wu X, Bai Y, Tan T, et al. Lithium ameliorates autistic-like behaviors induced by neonatal isolation in rats. Front Behav Neurosci. 2014;8:234. doi: 10.3389/fnbeh.2014.00234. PMID: 25018711.X-1

2866. Xin JF, Leonard DA. Using iPads to Teach Communication Skills of Students with Autism. J Autism Dev Disord. 2014 Oct 8doi: 10.1007/s10803-014-2266-8. PMID: 25294648.X-3, X-4 
2867. Xiong XX, He M, Chen XQ. [Progress in autism: de novo mutation and CHD8 functions]. Sheng Li Ke Xue Jin Zhan. 2014 Jun;45(3):185-9. PMID: 25219269.X-1

2868. Xu D, Richards JA, Gilkerson J. Automated analysis of child phonetic production using naturalistic recordings. J Speech Lang Hear Res. 2014 Oct;57(5):163850. doi: 10.1044/2014_jslhr-s-13-0037. PMID: 24824489.X-1

2869. Yamkovenko S, Waite A. Industry News. OT Practice. 2014;19(1):2- 1p. PMID: 107880501. Language: English. Entry Date: 20140122. Revision Date: 20150712. Publication Type: Journal Article. Journal Subset: Allied Health.X-1

2870. Yang HC, Lee IC, Lee IC. Visual feedback and target size effects on reach-tograsp tasks in children with autism. J Autism Dev Disord. 2014 Dec;44(12):3129-39. doi: 10.1007/s10803-014-2165-z. PMID: 24974254.X-1, X-3

2871. Yang P, Chang CL. Glutamatemediated signaling and autism spectrum disorders: emerging treatment targets. Curr Pharm Des. 2014;20(32):5186-93. PMID: 24410563.X-1

2872. Yashoda R, Puranik MP. Oral health status and parental perception of child oral health related quality-of-life of children with autism in Bangalore, India. J Indian Soc Pedod Prev Dent. 2014 Apr-Jun;32(2):135-9. doi: 10.4103/0970-4388.130967. PMID: 24739913.X-1

2873. Yassa HA. Autism: a form of lead and mercury toxicity. Environ Toxicol Pharmacol. 2014 Nov;38(3):1016-24. doi: 10.1016/j.etap.2014.10.005. PMID: 25461563.X-1, X-3

2874. Yasuda Y, Hashimoto R, Ohi K, et al. Cognitive inflexibility in Japanese adolescents and adults with autism spectrum disorders. World J Psychiatry. 2014 Jun 22;4(2):42-8. doi: 10.5498/wjp.v4.i2.42. PMID: 25019056.X-1

2875. Yoo HJ, Bahn G, Cho IH, et al. A randomized controlled trial of the Korean version of the PEERS((R)) parent-assisted social skills training program for teens with
ASD. Autism Res. 2014 Feb;7(1):145-61. doi: 10.1002/aur.1354. PMID: 24408892.X-1

2876. Youngster I, Zachor DA, Gabis LV, et al. CYP2D6 genotyping in paediatric patients with autism treated with risperidone: a preliminary cohort study. Dev Med Child Neurol. 2014 Oct;56(10):990-4. doi: 10.1111/dmcn.12470. PMID: 24828442.X-1, $\mathrm{X}-3$

2877. Yu Q, Peng Y, Mishra V, et al. Microstructure, length, and connection of limbic tracts in normal human brain development. Front Aging Neurosci. 2014;6:228. doi: 10.3389/fnagi.2014.00228. PMID: 25221509.X-1

2878. Yucel GH, Belger A, Bizzell J, et al. Abnormal Neural Activation to Faces in the Parents of Children with Autism. Cereb Cortex. 2014 Jul 23doi: 10.1093/cercor/bhu147. PMID: 25056573.X-1

2879. Zablotsky B, Kalb LG, Freedman B, et al. Health care experiences and perceived financial impact among families of children with an autism spectrum disorder. Psychiatr Serv. 2014 Mar 1;65(3):395-8. doi: 10.1176/appi.ps.201200552. PMID: 24584528.X-1

2880. Zachor DA, Ben-Itzchak E. The relationship between clinical presentation and unusual sensory interests in autism spectrum disorders: A preliminary investigation. Journal of Autism and Developmental Disorders. 2014 2015-02-24;44(1):229-35. doi: http://dx.doi.org/10.1007/s10803-013-1867-y. PMID: 1373444989; 2013-23011-001.X-1

2881. Zamora I, Harley EK, Green SA, et al. How sex of children with autism spectrum disorders and access to treatment services relates to parental stress. Autism Res Treat. 2014;2014:721418. doi: 10.1155/2014/721418. PMID: 25580295.X-1

2882. Zamzow RM, Christ SE, Saklayen SS, et al. Effect of propranolol on facial scanning in autism spectrum disorder: a preliminary investigation. J Clin Exp Neuropsychol. 2014;36(4):431-45. doi: 10.1080/13803395.2014.904844. PMID: 24730708.X-3, X-4 
2883. Zeidan-Chulia F, de Oliveira BH, Salmina AB, et al. Altered expression of Alzheimer's disease-related genes in the cerebellum of autistic patients: a model for disrupted brain connectome and therapy. Cell Death Dis. 2014;5:e1250. doi: 10.1038/cddis.2014.227. PMID: 24853428.X-1

2884. Zhang W, Yan TT, Du YS, et al. Brief report: effects of solution-focused brief therapy group-work on promoting post-traumatic growth of mothers who have a child with ASD. J Autism Dev Disord. 2014 Aug;44(8):2052-6. doi: 10.1007/s10803-014-2051-8. PMID: 24488119.X-1

2885. Zhao ZQ, Jia SW, Hu S, et al. Evaluating the effectiveness of electroacupuncture as a treatment for childhood autism using single photon emission computed tomography. Chin J Integr Med. 2014 Jan;20(1):19-23. doi: 10.1007/s11655-0141680-2. PMID: 24374754.X-3

2886. Zhou WZ, Ye AY, Sun ZK, et al. Statistical analysis of twenty years (1993 to 2012) of data from mainland China's first intervention center for children with autism spectrum disorder. Mol Autism. 2014;5:52. doi: 10.1186/2040-2392-5-52. PMID: 25694804.X1

2887. Zhou Y-y, Huang C-j, Liu J, et al. Efficacy of medication combined with sensory integration therapy for children with comorbid high-functioning autism spectrum disorder and attention deficit and hyperactivity disorder. Chinese Journal of Clinical Psychology. 2014 2015-12-09;22(6):1137-40. PMID: 1697761359; 2015-13180-041.X-5

2888. Zhu H, Fan Y, Guo H, et al. Reduced interhemispheric functional connectivity of children with autism spectrum disorder: evidence from functional near infrared spectroscopy studies. Biomed Opt Express. 2014 Apr 1;5(4):1262-74. doi: 10.1364/boe.5.001262. PMID: 24761305.X-1

2889. Ziv Y, Hadad B, Khateeb Y. Social Information Processing in Preschool Children Diagnosed with Autism Spectrum Disorder. Journal of Autism \& Developmental Disorders. 2014;44(4):846-59 14p. doi: 10.1007/s10803013-1935-3. PMID: 104039484. Language: English. Entry Date: 20140311. Revision Date:
20150710. Publication Type: Journal Article.X1

2890. Zuckerman KE, Lindly OJ, Bethell $\mathrm{CD}$, et al. Family impacts among children with autism spectrum disorder: the role of health care quality. Acad Pediatr. 2014 JulAug;14(4):398-407. doi: 10.1016/j.acap.2014.03.011. PMID: 24976352.X-1

2891. Zuckerman KE, Sinche B, Mejia A, et al. Latino parents' perspectives on barriers to autism diagnosis. Acad Pediatr. 2014 MayJun;14(3):301-8. doi: 10.1016/j.acap.2013.12.004. PMID: 24767783.X-1

2892. . Circumcision and autism. Arch Dis Child. 2015 Apr;100(4):369. doi: 10.1136/archdischild-2015-308402. PMID: 25715006.X-1

2893. Identification of neuromotor deficits common to autism spectrum disorder and attention deficit/hyperactivity disorder, and imitation deficits specific to autism spectrum disorder. European Child \& Adolescent Psychiatry. 2015;24(12):1497-507 11p. doi: 10.1007/s00787-015-0753-X. PMID: 111003902. Language: English. Entry Date: 20151124. Revision Date: 20151124. Publication Type: Article. Journal Subset: Biomedical.X-1, X-2

2894. . Development of the Classroom Sensory Environment Assessment (CSEA). American Journal of Occupational Therapy. 2015;69(6):1-9 p. doi: 10.5014/ajot.2015.019430. PMID: 110629136. Language: English. Entry Date: 20151109. Revision Date: 20151112. Publication Type: Article. Journal Subset: Allied Health.X-1, X-2, $\mathrm{X}-3$

2895. . State of the Science: A Roadmap for Research in Sensory Integration. American Journal of Occupational Therapy. 2015;69(6):17 p. doi: 10.5014/ajot.2015.019539. PMID: 110629144. Language: English. Entry Date: 20151109. Revision Date: 20151112. Publication Type: Article. Journal Subset: Allied Health.X-1, X-2

2896. . Early Identification of Autism Spectrum Disorder: Recommendations for 
Practice and Research. Pediatrics.

2015;136:S10-S40 31p. doi:

10.1542/peds.2014-3667C. PMID: 110209534.

Language: English. Entry Date: 20151009.

Revision Date: 20151015. Publication Type:

Article. Supplement Title: 2015 Supplement.

Journal Subset: Biomedical.X-1, X-2

2897. . Failure in Pantomime Action

Execution Correlates with the Severity of Social

Behavior Deficits in Children with Autism: A

Praxis Study. Journal of Autism \&

Developmental Disorders. 2015;45(10):3085-97

13p. doi: 10.1007/s10803-015-2461-2. PMID:

109441299. Language: English. Entry Date:

20150925. Revision Date: 20151021.

Publication Type: Article. Journal Subset:

Biomedical.X-1, X-2

2898. . Stimulus Over-Selectivity and

Extinction-Induced Recovery of Performance as a Product of Intellectual Impairment and Autism Severity. Journal of Autism \& Developmental Disorders. 2015;45(10):3098106 9p. doi: 10.1007/s10803-015-2466-x. PMID: 109441330. Language: English. Entry

Date: 20150925. Revision Date: 20151021.

Publication Type: Article. Journal Subset:

Biomedical.X-1, X-2

2899. Patterns of Sensory Integration Dysfunction in Specific Populations: EvidenceBased Identification. Bethesda, Maryland: American Occupational Therapy Association; 2015. p. CE-1-CE-8 p.

2900. . Effectiveness of Ayres Sensory Integration ${ }^{\circledR}$ and Sensory-Based Interventions for People With Autism Spectrum Disorder: A Systematic Review. American Journal of Occupational Therapy. 2015;69(5):p1-p12 20p. doi: 10.5014/ajot.2015.018051. PMID: 109352679. Language: English. Entry Date: 20151012. Revision Date: 20151012. Publication Type: Article.X-1, X-2

2901. . Retrospective Analysis of Motor Development in Infants at High and Low Risk for Autism Spectrum Disorder. American Journal of Occupational Therapy.

2015;69(5):p1-p9 p. doi:

10.5014/ajot.2015.017525. PMID: 109352687. Language: English. Entry Date: 20151012.

Revision Date: 20151012. Publication Type: Article.X-1
2902. . Brainstem White Matter Predicts Individual Differences in Manual Motor Difficulties and Symptom Severity in Autism. Journal of Autism \& Developmental Disorders. 2015;45(9):3030-40 11p. doi: 10.1007/s10803015-2467-9. PMID: 109172089. Language: English. Entry Date: 20151016. Revision Date: 20151016. Publication Type: Article.X-1, X-2

2903. . Untitled. Adapted Physical Activity Quarterly. 2015;32(3):279-82 4p. doi: 10.1123/APAQ.2015-0044. PMID: 109828829. Language: English. Entry Date: 20150716. Revision Date: 20150923. Publication Type: Journal Article.X-5

2904. . Mom Creates Weighted Vest From Husband's Military Uniform. OT Practice. 2015;20(8):3- 1p. PMID: 109801607. Language: English. Entry Date: 20150720. Revision Date: 20150923. Publication Type: Journal Article.X-1, X-2

2905. . Keep your eye on. Brown University Child \& Adolescent Behavior Letter. 2015;31(12):2- /3p. doi: 10.1002/cbl. PMID: 110958844. Language: English. Entry Date: 20151119. Revision Date: 20151123. Publication Type: Article. Journal Subset: Biomedical.X-1, X-2

2906. . Clinical Trial Investigates Drug for Autism. ASHA Leader. 2015;20(9):15- 1p. PMID: 109838417. Language: English. Entry Date: 20150902 . Revision Date: 20150923. Publication Type: Journal Article.X-1, X-2

2907. Reduction of Aggressive Episodes After Repeated Transdermal Nicotine Administration in a Hospitalized Adolescent with Autism Spectrum Disorder. , <Blank>: Springer Science \& Business Media B.V.; 2015. p. 3061-6 6p.

2908. . LURASIDONE IMPROVES FUNCTION IN PATIENTS WITH AUTISM SPECTRUM DISORDER. Exceptional Parent. 2015;45(6):14- 1p. PMID: 109834014. Language: English. Entry Date: 20150818. Revision Date: 20150923. Publication Type: Journal Article.X-1, X-2

2909. . LEGAL NEWS ROUND-UP. Occupational Health. 2015;67(6):8- 1p. PMID: 109810214. Language: English. Entry Date: 
20150623. Revision Date: 20150923.

Publication Type: Journal Article.X-1, X-2

2910. . FDA approves first generic Abilify to treat mental illnesses. Mental Health Weekly. 2015;25(18):6-7 2p. doi: 10.1002/mhw.20472. PMID: 103798390. Language: English. Entry Date: 20150518. Revision Date: 20151102. Publication Type: Journal Article.X-1, X-2

2911. . Pharmacotherapy options in autism: Serious adverse effects, little efficacy. Brown University Child \& Adolescent Psychopharmacology Update. 2015;17(3):1-3 p. doi: 10.1002/cpu.20228. PMID: 103761955. Language: English. Entry Date: 20150302. Revision Date: 20150828. Publication Type: Journal Article. Journal Subset: Biomedical.X$1, \mathrm{X}-2$

2912. . PEDIATRICS SUMMARY. Pediatrics. 2015;135(2):D1-6 1p. PMID: 103794718. Language: English. Entry Date: 20150501. Revision Date: 20150710.

Publication Type: Journal Article.X-1, X-2

2913. Abbott AE, Nair A, Keown CL, et al. Patterns of Atypical Functional Connectivity and Behavioral Links in Autism Differ Between Default, Salience, and Executive Networks. Cereb Cortex. 2015 Sep 7doi: 10.1093/cercor/bhv191. PMID: 26351318.X-1

2914. Abdeltawwab MM, Baz H. Automatic Pre-Attentive Auditory Responses: MMN to Tone Burst Frequency Changes in Autistic School-Age Children. J Int Adv Otol. 2015 Apr;11(1):36-41. doi: 10.5152/iao.2014.438. PMID: 26223716.X-1

2915. Acab A, Muotri AR. The Use of Induced Pluripotent Stem Cell Technology to Advance Autism Research and Treatment. Neurotherapeutics. 2015 Jul;12(3):534-45. doi: 10.1007/s13311-015-0354-x. PMID: 25851569.X-1

2916. Accardo PJ, Barrow W. Toe walking in autism: further observations. J Child Neurol. 2015 Apr;30(5):606-9. doi: 10.1177/0883073814521298. PMID: 24563477.X-1

2917. Addis L, Ahn JW, Dobson R, et al. Microdeletions of ELP4 Are Associated with Language Impairment, Autism Spectrum
Disorder, and Mental Retardation. Hum Mutat. 2015 Sep;36(9):842-50. doi: 10.1002/humu.22816. PMID: 26010655.X-1

2918. Adelsky SJ. Review of Parenting on the autism spectrum: A survival guide. Journal of the American Academy of Child \& Adolescent Psychiatry. 2015 2015-0318;54(3):236-7. doi: http://dx.doi.org/10.1016/j.jaac.2015.01.004 PMID: 1664225803; 2015-08819-018.X-1, X-2

2919. Adibsereshki N, Nesayan A, Asadi Gandomani R, et al. The Effectiveness of Theory of Mind Training On the Social Skills of Children with High Functioning Autism Spectrum Disorders. Iran J Child Neurol. 2015 Summer;9(3):40-9. PMID: 26401152.X-1

2920. Adler BA, Wink LK, Early M, et al. Drug-refractory aggression, self-injurious behavior, and severe tantrums in autism spectrum disorders: a chart review study. Autism. 2015 Jan;19(1):102-6. doi: 10.1177/1362361314524641. PMID: 24571823.X-3

2921. Adler N, Dvash J, Shamay-Tsoory SG. Empathic Embarrassment Accuracy in Autism Spectrum Disorder. Autism Res. 2015 Jun;8(3):241-9. doi: 10.1002/aur.1439. PMID: 25732043.X-1

2922. Adolfsson M, Simmeborn Fleischer A. Applying the ICF to identify requirements for students with Asperger syndrome in higher education. Dev Neurorehabil. 2015 Jun;18(3):190-202. doi: 10.3109/17518423.2013.819947. PMID: 23957214.X-1

2923. Agarwal AK, Rai S, Upreti MC, et al. Day care as an innovative approach in psychiatry: Analysis of Lucknow experience. Indian J Psychiatry. 2015 Apr-Jun;57(2):162-4. doi: 10.4103/0019-5545.158157. PMID: 26124522.X-1

2924. Aggarwal S, Angus B. Misdiagnosis versus missed diagnosis: diagnosing autism spectrum disorder in adolescents. Australas Psychiatry. 2015 Apr;23(2):120-3. doi: 10.1177/1039856214568214. PMID: 25653302.X-1 
2925. Ago Y, Condro MC, Tan YV, et al. Reductions in synaptic proteins and selective alteration of prepulse inhibition in male C57BL/6 mice after postnatal administration of a VIP receptor (VIPR2) agonist. Psychopharmacology (Berl). 2015 Jun;232(12):2181-9. doi: 10.1007/s00213-0143848-z. PMID: 25575489.X-1

2926. Aguilar-Valles A, Matta-Camacho E, Khoutorsky A, et al. Inhibition of Group I Metabotropic Glutamate Receptors Reverses Autistic-Like Phenotypes Caused by Deficiency of the Translation Repressor eIF4E Binding Protein 2. J Neurosci. 2015 Aug 5;35(31):11125-32. doi: 10.1523/jneurosci.4615-14.2015. PMID: 26245973.X-1

2927. Ahouee SM, Shooshtari MH, Bidaki R. A girl with increased writing and painting activities associated with Turner's syndrome and autistic spectrum disorder. Adv Biomed Res. 2015;4:91. doi: 10.4103/22779175.156655. PMID: 26015917.X-1

2928. Aida T, Yoshida J, Nomura M, et al. Astroglial glutamate transporter deficiency increases synaptic excitability and leads to pathological repetitive behaviors in mice. Neuropsychopharmacology. 2015 Jun;40(7):1569-79. doi: 10.1038/npp.2015.26. PMID: 25662838.X-1

2929. Akintunde ME, Rose M, Krakowiak P, et al. Increased production of IL-17 in children with autism spectrum disorders and co-morbid asthma. J Neuroimmunol. 2015 Sep 15;286:3341. doi: 10.1016/j.jneuroim.2015.07.003. PMID: 26298322.X-1

2930. Al Shirian S, Al Dera H. Descriptive characteristics of children with autism at Autism Treatment Center, KSA. Physiol Behav. 2015 Nov 1;151:604-8. doi: 10.1016/j.physbeh.2015.09.001. PMID: 26343774.X-1

2931. Alaerts K, Geerlings F, Herremans L, et al. Functional Organization of the Action Observation Network in Autism: A Graph Theory Approach. PLoS One. 2015;10(8):e0137020. doi: 10.1371/journal.pone.0137020. PMID: 26317222.X-1
2932. Al-Amin MM, Rahman MM, Khan FR, et al. Astaxanthin improves behavioral disorder and oxidative stress in prenatal valproic acid-induced mice model of autism. Behav Brain Res. 2015 Jun 1;286:112-21. doi: 10.1016/j.bbr.2015.02.041. PMID: 25732953.X-1

2933. Alaniz ML, Galit E, Necesito CI, et al. Hand Strength, Handwriting, and Functional Skills in Children With Autism. Am J Occup Ther. 2015 Jul-Aug;69(4):6904220030p1-9. doi: 10.5014/ajot.2015.016022. PMID: 26114459.X-1

2934. Aldaqre I, Paulus M, Sodian B. Referential gaze and word learning in adults with autism. Autism. 2015 Nov;19(8):944-55. doi: 10.1177/1362361314556784. PMID: 25488004.X-1

2935. Aldinger KA, Lane CJ, VeenstraVanderWeele J, et al. Patterns of Risk for Multiple Co-Occurring Medical Conditions Replicate Across Distinct Cohorts of Children with Autism Spectrum Disorder. Autism Res. 2015 May 24doi: 10.1002/aur.1492. PMID: 26011086.X-1

2936. Alexander M, Petri H, Ding Y, et al. Morbidity and medication in a large population of individuals with Down syndrome compared to the general population. Dev Med Child Neurol. 2015 Aug 18doi: 10.1111/dmcn.12868. PMID: 26282180.X-1

2937. Al-Heizan MO, AlAbdulwahab SS, Kachanathu SJ, et al. Sensory processing dysfunction among Saudi children with and without autism. J Phys Ther Sci. 2015 May;27(5):1313-6. doi: 10.1589/jpts.27.1313. PMID: 26157208.X-1

2938. Allen KD, Vatland C, Bowen SL, et al. An evaluation of parent-produced video selfmodeling to improve independence in an adolescent with intellectual developmental disorder and an autism spectrum disorder: a controlled case study. Behav Modif. 2015 Jul;39(4):542-56. doi: 10.1177/0145445515583247. PMID: 25903581.X-1

2939. Allen ML, Hartley C, Cain K. Do iPads promote symbolic understanding and word learning in children with autism? Front 
Psychol. 2015;6:138. doi:

10.3389/fpsyg.2015.00138. PMID:

25729376.X-1, X-3

2940. Alokam R, Singhal S, Srivathsav GS, et al. Design of dual inhibitors of ROCK-I and NOX2 as potential leads for the treatment of neuroinflammation associated with various neurological diseases including autism spectrum disorder. Mol Biosyst. 2015 Feb;11(2):607-17. doi: 10.1039/c4mb00570h. PMID: 25465055.X-1

2941. Alquraini T, Mahoney G. An Exploratory Investigation of the Role of Parenting Stress in Relationship Focused Intervention. J Appl Res Intellect Disabil. 2015 Nov;28(6):536-47. doi: 10.1111/jar.12148. PMID: 25727239.X-1

2942. Althaus M, Groen Y, Wijers AA, et al. Oxytocin enhances orienting to social information in a selective group of highfunctioning male adults with autism spectrum disorder. Neuropsychologia. 2015 Oct 21doi: 10.1016/j.neuropsychologia.2015.10.025. PMID: 26498227.X-1

2943. Al-Zaid FS, Alhader AA, Al-Ayadhi LY. The second to fourth digit ratio (2D:4D) in Saudi boys with autism: A potential screening tool. Early Hum Dev. 2015 Jul;91(7):413-5. doi: 10.1016/j.earlhumdev.2015.04.007. PMID: 25981494.X-1

2944. Amaral CP, Simoes MA, CasteloBranco MS. Neural signals evoked by stimuli of increasing social scene complexity are detectable at the single-trial level and right lateralized. PLoS One. 2015;10(3):e0121970. doi: 10.1371/journal.pone.0121970. PMID: 25807525.X-1

2945. Ament K, Mejia A, Buhlman R, et al. Evidence for Specificity of Motor Impairments in Catching and Balance in Children with Autism. Journal of Autism \& Developmental Disorders. 2015;45(3):742-51 10p. doi: 10.1007/s10803-014-2229-0. PMID: 103763379. Language: English. Entry Date: 20150226. Revision Date: 20150710. Publication Type: Journal Article.X-1

2946. Anagnostou E, Jones N, Huerta M, et al. Measuring social communication behaviors as a treatment endpoint in individuals with autism spectrum disorder. Autism. 2015

Jul;19(5):622-36. doi:

10.1177/1362361314542955. PMID: 25096930.X-1

2947. Andell E, Tomson T, Carlsson S, et al. The incidence of unprovoked seizures and occurrence of neurodevelopmental comorbidities in children at the time of their first epileptic seizure and during the subsequent six months. Epilepsy Res. 2015 Jul;113:140-50. doi: 10.1016/j.eplepsyres.2015.04.002. PMID: 25986201.X-1

2948. Ander BP, Barger N, Stamova B, et al. Atypical miRNA expression in temporal cortex associated with dysregulation of immune, cell cycle, and other pathways in autism spectrum disorders. Mol Autism. 2015;6:37. doi: 10.1186/s13229-015-0029-9. PMID: 26146533.X-1

2949. Andersen PN, Skogli EW, Hovik KT, et al. Working memory arrest in children with high-functioning autism compared to children with attention-deficit/hyperactivity disorder: results from a 2-year longitudinal study. Autism. 2015 May;19(4):443-50. doi: 10.1177/1362361314524844. PMID: 24604922.X-1

2950. Anderson D, Byrne-Barta D. Keeping the Child with Autism Spectrum Disorder Safe. AAACN Viewpoint. 2015;37(1):11-3 3p. PMID: 103756286. Language: English. Entry Date: 20150213. Revision Date: 20150820. Publication Type: Journal Article.X-1, X-2

2951. Anderson GM, Montazeri F, de Bildt A. Network Approach to Autistic Traits: Group and Subgroup Analyses of ADOS Item Scores. J Autism Dev Disord. 2015 Oct;45(10):311532. doi: 10.1007/s10803-015-2537-z. PMID: 26206232.X-1, X-2

2952. Andoh-Noda T, Akamatsu W, Miyake $\mathrm{K}$, et al. Differentiation of multipotent neural stem cells derived from Rett syndrome patients is biased toward the astrocytic lineage. Mol Brain. 2015;8:31. doi: 10.1186/s13041-0150121-2. PMID: 26012557.X-1

2953. Angliker N, Burri M, Zaichuk M, et al. mTORC1 and mTORC2 have largely distinct functions in Purkinje cells. Eur J Neurosci. 
2015 Oct;42(8):2595-612. doi: 10.1111/ejn.13051. PMID: 26296489.X-1

2954. Angriman M, Caravale B, Novelli L, et al. Sleep in children with neurodevelopmental disabilities. Neuropediatrics. 2015 Jun;46(3):199-210. doi: 10.1055/s-00351550151. PMID: 25918987.X-1

2955. Anketell PM, Saunders KJ, Gallagher SM, et al. Brief Report: Vision in Children with Autism Spectrum Disorder: What Should Clinicians Expect? J Autism Dev Disord. 2015 Sep;45(9):3041-7. doi: 10.1007/s10803-0152431-8. PMID: 25847754.X-1

2956. Antonucci N, Cirillo A, Siniscalco D. Beneficial Effects of Palmitoylethanolamide on Expressive Language, Cognition, and Behaviors in Autism: A Report of Two Cases. Case Rep Psychiatry. 2015;2015:325061. doi: 10.1155/2015/325061. PMID: 26491593.X-3, $\mathrm{X}-4$

2957. Aoki Y, Watanabe T, Abe O, et al. Oxytocin's neurochemical effects in the medial prefrontal cortex underlie recovery of taskspecific brain activity in autism: a randomized controlled trial. Mol Psychiatry. 2015

Apr;20(4):447-53. doi: 10.1038/mp.2014.74. PMID: 25070538.X-1

2958. Arabameri E, Sotoodeh MS. Early developmental delay in children with autism: A study from a developing country. Infant Behav Dev. 2015 May;39:118-23. doi: 10.1016/j.infbeh.2015.02.017. PMID: 25827390.X-1

2959. Aramaki E, Shikata S, Miyabe M, et al. Understanding the Relationship between Social Cognition and Word Difficulty. A Language Based Analysis of Individuals with Autism Spectrum Disorder. Methods Inf Med. 2015 Sep 22;54(5)doi: 10.3414/me15-01-0038. PMID: 26391807.X-1

2960. Ardhanareeswaran K, Volkmar F. Introduction. Focus: autism spectrum disorders. Yale J Biol Med. 2015 Mar;88(1):3-4. PMID: 25902571.X-1, X-2

2961. Aresti-Bartolome N, Garcia-Zapirain B. Cognitive rehabilitation system for children with autism spectrum disorder using serious games: A pilot study. Biomed Mater Eng. 2015
Aug 17;26 Suppl 1:S811-24. doi: 10.3233/bme151373. PMID: 26406079.X-1, X-3

2962. Arnold B, Elliott A, Laohamroonvorapongse D, et al. Autistic children and anesthesia: is their perioperative experience different? Paediatr Anaesth. 2015 Nov;25(11):1103-10. doi: 10.1111/pan.12739. PMID: 26338278.X-1

2963. Arteaga G, Buritica E, Escobar MI, et al. Human prefrontal layer II interneurons in areas 46, 10 and 24. Colomb Med (Cali). 2015 Jan-Mar;46(1):19-25. PMID: 26019381.X-1

2964. Ashwin C, Hietanen JK, Baron-Cohen S. Atypical integration of social cues for orienting to gaze direction in adults with autism. Mol Autism. 2015;6(1):5. doi: 10.1186/2040-2392-6-5. PMID: 25685307.X-1

2965. Ashwood KL, Buitelaar J, Murphy D, et al. European clinical network: autism spectrum disorder assessments and patient characterisation. Eur Child Adolesc Psychiatry. 2015 Aug;24(8):985-95. doi: 10.1007/s00787014-0648-2. PMID: 25471824.X-1

2966. Ashwood KL, Tye C, Azadi B, et al. Brief Report: Adaptive Functioning in Children with ASD, ADHD and ASD + ADHD. J Autism Dev Disord. 2015 Jul;45(7):2235-42. doi: 10.1007/s10803-014-2352-y. PMID: 25614019.X-1

2967. Atladottir HO, Schendel DE, Henriksen TB, et al. Gestational Age and Autism Spectrum Disorder: Trends in Risk Over Time. Autism Res. 2015 Sep 12doi: 10.1002/aur.1525. PMID: 26363410.X-1

2968. Atladottir HO, Schendel DE, Parner ET, et al. A Descriptive Study on the Neonatal Morbidity Profile of Autism Spectrum Disorders, Including a Comparison with Other Neurodevelopmental Disorders. J Autism Dev Disord. 2015 Aug;45(8):2429-42. doi: 10.1007/s10803-015-2408-7. PMID: 25758820.X-1

2969. Auyeung B, Lombardo MV, Heinrichs $\mathrm{M}$, et al. Oxytocin increases eye contact during a real-time, naturalistic social interaction in males with and without autism. Transl Psychiatry. 2015;5:e507. doi: 10.1038/tp.2014.146. PMID: 25668435.X-1 
2970. Au-Yeung SK, Kaakinen JK, Liversedge SP, et al. Processing of Written Irony in Autism Spectrum Disorder: An EyeMovement Study. Autism Res. 2015 May 11doi: 10.1002/aur.1490. PMID: 25962666.X-1

2971. Auzou N, Dujardin K, Biundo R, et al. Diagnosing dementia in multiple system atrophy by applying Movement Disorder Society diagnostic criteria for Parkinson's disease dementia. Parkinsonism Relat Disord. 2015 Oct;21(10):1273-7. doi: 10.1016/j.parkreldis.2015.08.013. PMID: 26305998.X-1

2972. Avcil S, Baykara B, Baydur H, et al. [The validity and reliability of the Social Communication Questionnaire- Turkish form in autistics aged 4-18 years]. Turk Psikiyatri Derg. 2015 Spring;26(1):56-64. PMID: 25742038.X1

2973. Backer van Ommeren T, Koot HM, Scheeren AM, et al. Reliability and Validity of the Interactive Drawing Test: A Measure of Reciprocity for Children and Adolescents with Autism Spectrum Disorder. J Autism Dev Disord. 2015 Jul;45(7):1967-77. doi: 10.1007/s10803-014-2353-x. PMID: 25630897.X-1

2974. Backstrom DC, Eriksson Domellof M, Linder J, et al. Cerebrospinal Fluid Patterns and the Risk of Future Dementia in Early, Incident Parkinson Disease. JAMA Neurol. 2015 Oct 1;72(10):1175-82. doi: 10.1001/jamaneurol.2015.1449. PMID: 26258692.X-1

2975. Bacon C, Schneider M, Le Magueresse C, et al. Brain-specific Foxp1 deletion impairs neuronal development and causes autistic-like behaviour. Mol Psychiatry. 2015 May;20(5):632-9. doi: 10.1038/mp.2014.116. PMID: 25266127.X-1

2976. Badgett N, Falcomata TS. A comparison of methodologies of brief functional analysis. Dev Neurorehabil. 2015;18(4):224-33. doi: 10.3109/17518423.2013.792298. PMID: 23869515.X-1

2977. Baek JH, Whitfield D, Howlett D, et al. Unfolded protein response is activated in
Lewy body dementias. Neuropathol Appl Neurobiol. 2015 Jul 23doi: 10.1111/nan.12260. PMID: 26202523.X-1

2978. Baggio G, van Lambalgen M, Hagoort P. Logic as Marr's Computational Level: Four Case Studies. Top Cogn Sci. 2015

Apr;7(2):287-98. doi: 10.1111/tops.12125. PMID: 25417838.X-1

2979. Baghani HR, Aghamiri SM, Mahdavi $\mathrm{SR}$, et al. Comparing the dosimetric characteristics of the electron beam from dedicated intraoperative and conventional radiotherapy accelerators. J Appl Clin Med Phys. 2015;16(2):5017. doi: 10.1120/jacmp.v16i2.5017. PMID: 26103175.X-1

2980. Bahta L, Ashkir A. Addressing MMR Vaccine Resistance in Minnesota's Somali Community. Minn Med. 2015 Oct;98(10):33-6. PMID: 26596077.X-1

2981. Bailey KM, Blair KC. Feasibility and potential efficacy of the family-centered Prevent-Teach-Reinforce model with families of children with developmental disorders. Res Dev Disabil. 2015 Oct 6;47:218-33. doi: 10.1016/j.ridd.2015.09.019. PMID: 26451882.X-1, X-3, X-4

2982. Baker EK, Richdale AL. Sleep Patterns in Adults with a Diagnosis of HighFunctioning Autism Spectrum Disorder. Sleep. 2015 Jul 24 PMID: 26237770.X-1

2983. Baker JK, Fenning RM, Howland MA, et al. Brief Report: A Pilot Study of ParentChild Biobehavioral Synchrony in Autism Spectrum Disorder. J Autism Dev Disord. 2015 Jul 17doi: 10.1007/s10803-015-2528-0. PMID: 26183724.X-1

2984. Baker K, Scerif G, Astle DE, et al. Psychopathology and cognitive performance in individuals with membrane-associated guanylate kinase mutations: a functional network phenotyping study. J Neurodev Disord. 2015;7(1):8. doi: 10.1186/s11689-015-9105-x. PMID: 25802558.X-1

2985. Bakri MH, Ismail EA, Ali MS, et al. Behavioral and emotional effects of repeated general anesthesia in young children. Saudi J Anaesth. 2015 Apr-Jun;9(2):161-6. doi: 
10.4103/1658-354x.152843. PMID: 25829904.X-1

2986. Bal VH, Kim SH, Cheong D, et al. Daily living skills in individuals with autism spectrum disorder from 2 to 21 years of age. Autism. 2015 Oct;19(7):774-84. doi: 10.1177/1362361315575840. PMID: 25922445.X-1

2987. Bal VH, Lord C. Replication of Standardized ADOS Domain Scores in the Simons Simplex Collection. Autism Res. 2015 Oct;8(5):583-92. doi: 10.1002/aur.1474. PMID: 25712123.X-1

2988. Balardin JB, Comfort WE, Daly E, et al. Decreased centrality of cortical volume covariance networks in autism spectrum disorders. J Psychiatr Res. 2015 Oct;69:142-9. doi: 10.1016/j.jpsychires.2015.08.003. PMID: 26343606.X-1

2989. Baldwin PR, Curtis KN, Patriquin $\mathrm{MA}$, et al. Identifying diagnostically-relevant resting state brain functional connectivity in the ventral posterior complex via genetic data mining in autism spectrum disorder. Autism Res. 2015 Oct 9doi: 10.1002/aur.1559. PMID: 26451751.X-1

2990. Banire B, Jomhari N, Ahmad R. Visual Hybrid Development Learning System (VHDLS) Framework for Children with Autism. J Autism Dev Disord. 2015 Oct;45(10):3069-84. doi: 10.1007/s10803-0152469-7. PMID: 25997598.X-1, X-3

2991. Baranek GT, Watson LR, TurnerBrown L, et al. Preliminary efficacy of adapted responsive teaching for infants at risk of autism spectrum disorder in a community sample. Autism Res Treat. 2015;2015:386951. doi: 10.1155/2015/386951. PMID: 25648749.X-1

2992. Barbeau EB, Meilleur AA, Zeffiro TA, et al. Comparing Motor Skills in Autism Spectrum Individuals With and Without Speech Delay. Autism Res. 2015 Mar 29doi: 10.1002/aur.1483. PMID: 25820662.X-1

2993. Barber C. Old age and people on the autism spectrum: a focus group perspective. $\mathrm{Br}$ J Nurs. 2015 Nov 26-Dec 9;24(21):1054-7. doi: 10.12968/bjon.2015.24.21.1054. PMID: 26618675.X-1
2994. Baribeau DA, Doyle-Thomas KA, Dupuis A, et al. Examining and comparing social perception abilities across childhoodonset neurodevelopmental disorders. J Am Acad Child Adolesc Psychiatry. 2015 Jun;54(6):479-86.e1. doi: 10.1016/j.jaac.2015.03.016. PMID: 26004663.X-1

2995. Barnard-Brak L, Davis TN, Schmidt $\mathrm{M}$, et al. Effects associated with on- and offlabel stimulant treatment of core autism and ADHD symptoms exhibited by children with autism spectrum disorder. Dev Neurorehabil. 2015 Aug 24:1-8. doi:

10.3109/17518423.2014.904949. PMID: 24739141.X-3

2996. Baron-Cohen S, Auyeung B, Norgaard-Pedersen B, et al. Elevated fetal steroidogenic activity in autism. Mol Psychiatry. 2015 Mar;20(3):369-76. doi: 10.1038/mp.2014.48. PMID: 24888361.X-1

2997. Baronio D, Castro K, Gonchoroski T, et al. Effects of an H3R antagonist on the animal model of autism induced by prenatal exposure to valproic acid. PLoS One. 2015;10(1):e0116363. doi: 10.1371/journal.pone.0116363. PMID: 25560049.X-1

2998. Barrett B, Mosweu I, Jones CR, et al. Comparing service use and costs among adolescents with autism spectrum disorders, special needs and typical development. Autism. 2015 Jul;19(5):562-9. doi:

10.1177/1362361314536626. PMID: 24913778.X-1

2999. Barrett SL, Uljarevic M, Baker EK, et al. The Adult Repetitive Behaviours Questionnaire-2 (RBQ-2A): A Self-Report Measure of Restricted and Repetitive Behaviours. J Autism Dev Disord. 2015 Nov;45(11):3680-92. doi: 10.1007/s10803-0152514-6. PMID: 26155763.X-1

3000. Barua S, Kuizon S, Chadman KK, et al. Microarray analysis reveals higher gestational folic Acid alters expression of genes in the cerebellum of mice offspring-a pilot study. Brain Sci. 2015;5(1):14-31. doi: 10.3390/brainsci5010014. PMID: 25629700.X1 
3001. Bath E, Reba-Harrelson L, Peace R, et al. Correlates of Competency to Stand Trial Among Youths Admitted to a Juvenile Mental Health Court. J Am Acad Psychiatry Law. 2015 Sep;43(3):329-39. PMID: 26438811.X-1

3002. Batool SS, Khurshid S. Factors Associated with Stress Among Parents of Children with Autism. J Coll Physicians Surg Pak. 2015 Oct;25(10):752-6. doi: 10.2015/jcpsp.752756. PMID: 26454393.X-1

3003. Baucum AJ, 2nd, Shonesy BC, Rose $\mathrm{KL}$, et al. Quantitative proteomics analysis of CaMKII phosphorylation and the CaMKII interactome in the mouse forebrain. ACS Chem Neurosci. 2015 Apr 15;6(4):615-31. doi: 10.1021/cn500337u. PMID: 25650780.X-1

3004. Bauer NS, Carroll AE, Saha C, et al. Computer Decision Support Changes Physician Practice But Not Knowledge Regarding Autism Spectrum Disorders. Appl Clin Inform. 2015;6(3):454-65. doi: 10.4338/aci-2014-09-ra0084. PMID: 26448791.X-1

3005. Baum KT, Shear PK, Howe SR, et al. A comparison of WISC-IV and SB-5 intelligence scores in adolescents with autism spectrum disorder. Autism. 2015

Aug;19(6):736-45. doi:

10.1177/1362361314554920. PMID: 25398894.X-1

3006. Bawaskar HS, Bawaskar PH, Bawaskar PH. RBC acetyl cholinesterase: A poor man's early diagnostic biomarker for familial alzheimer's and Parkinson's disease dementia. J Neurosci Rural Pract. 2015 Jan;6(1):33-8. doi: 10.4103/0976-3147.143187. PMID: 25552849.X-1

3007. Bazalova-Carter M, Liu M, Palma B, et al. Comparison of film measurements and Monte Carlo simulations of dose delivered with very high-energy electron beams in a polystyrene phantom. Med Phys. 2015 Apr;42(4):1606-13. doi: 10.1118/1.4914371. PMID: 25832051.X-1

3008. Beales DL. Biome depletion in conjunction with evolutionary mismatches could play a role in the etiology of neurofibromatosis 1. Med Hypotheses. 2015 Apr;84(4):305-14. doi: 10.1016/j.mehy.2015.01.014. PMID: 25665856.X-1

3009. Bean Ellawadi A, McGregor KK. Children with ASD can use gaze to map new words. Int J Lang Commun Disord. 2015 Sep 6doi: 10.1111/1460-6984.12192. PMID: 26344421.X-1

3010. Bearss K, Burrell TL, Stewart L, et al. Parent Training in Autism Spectrum Disorder: What's in a Name? Clin Child Fam Psychol Rev. 2015 Jun;18(2):170-82. doi: 10.1007/s10567-015-0179-5. PMID: 25722072.X-1

3011. Bearss K, Johnson C, Smith T, et al. Effect of parent training vs parent education on behavioral problems in children with autism spectrum disorder: a randomized clinical trial. Jama. 2015 Apr 21;313(15):1524-33. doi: 10.1001/jama.2015.3150. PMID: 25898050.X-1

3012. Bearss K, Taylor CA, Aman MG, et al. Using qualitative methods to guide scale development for anxiety in youth with autism spectrum disorder. Autism. 2015 Sep 22doi: 10.1177/1362361315601012. PMID: 26395234.X-1

3013. Bebko JM, Rhee T, McMorris CA, et al. Spontaneous strategy use in children with autism spectrum disorder: the roles of metamemory and language skills. Front Psychol. 2015;6:182. doi: 10.3389/fpsyg.2015.00182. PMID: 25788890.X-1, X-3

3014. Begeer S, Fink E, van der Meijden S, et al. Bullying-related behaviour in a mainstream high school versus a high school for autism: Self-report and peer-report. Autism. 2015 Aug 3doi: 10.1177/1362361315597525. PMID: 26239138.X-1

3015. Begeer S, Howlin P, Hoddenbach E, et al. Effects and Moderators of a Short Theory of Mind Intervention for Children with Autism Spectrum Disorder: A Randomized Controlled Trial. Autism Res. 2015 Apr 6doi: 10.1002/aur.1489. PMID: 25847054.X-1

3016. Bellando J, Fussell JJ, Lopez M. Autism Speaks Toolkits: Resources for Busy Physicians. Clin Pediatr (Phila). 2015 Jul 6doi: 
10.1177/0009922815594587. PMID: 26149848.X-1

3017. Benjamin DP, McDuffie AS, Thurman AJ, et al. Effect of speaker gaze on word learning in fragile $\mathrm{X}$ syndrome: a comparison with nonsyndromic autism spectrum disorder. J Speech Lang Hear Res. 2015 Apr;58(2):383-95. doi: 10.1044/2015_jslhr-l-14-0136. PMID: 25629603.X-1

3018. Bennabi M, Delorme R, Oliveira J, et al. Dectin-1 Polymorphism: A Genetic Disease Specifier in Autism Spectrum Disorders? PLoS One. 2015;10(9):e0137339. doi: 10.1371/journal.pone.0137339. PMID: 26352598.X-1

3019. Bennett JA, Germani T, Haqq AM, et al. Autism spectrum disorder in Prader-Willi syndrome: A systematic review. Am J Med Genet A. 2015 Aug 29doi: 10.1002/ajmg.a.37286. PMID: 26331980.X-1

3020. Bent CA, Dissanayake C, Barbaro J. Mapping the diagnosis of autism spectrum disorders in children aged under 7 years in Australia, 2010-2012. Med J Aust. 2015 Apr 6;202(6):317-20. PMID: 25832158.X-1

3021. Benvenuto A, Battan B, Benassi F, et al. Effectiveness of community-based treatment on clinical outcome in children with autism spectrum disorders: An Italian prospective study. Dev Neurorehabil. 2015 Aug 24:1-9. doi: 10.3109/17518423.2013.864716. PMID: 24393078.X-3

3022. Berens JC, Peacock C. Implementation of an academic adult primary care clinic for adolescents and young adults with complex, chronic childhood conditions. J Pediatr Rehabil Med. 2015;8(1):3-12. doi: 10.3233/prm150313. PMID: 25737343.X-1

3023. Bergmann T, Sappok T, Diefenbacher A, et al. Music-based Autism Diagnostics (MUSAD) - A newly developed diagnostic measure for adults with intellectual developmental disabilities suspected of autism. Res Dev Disabil. 2015 Aug-Sep;43-44:123-35. doi: 10.1016/j.ridd.2015.05.011. PMID: 26183337.X-1

3024. Berry RC, Novak P, Withrow N, et al. Nutrition Management of Gastrointestinal
Symptoms in Children with Autism Spectrum Disorder: Guideline from an Expert Panel. J Acad Nutr Diet. 2015 Dec;115(12):1919-27. doi: 10.1016/j.jand.2015.05.016. PMID: 26164551.X-1

3025. Besag FM. Current controversies in the relationships between autism and epilepsy. Epilepsy Behav. 2015 Jun;47:143-6. doi: 10.1016/j.yebeh.2015.05.032. PMID: 26091860.X-1, X-2

3026. Bhandari R, Kuhad A.

Neuropsychopharmacotherapeutic efficacy of curcumin in experimental paradigm of autism spectrum disorders. Life Sci. 2015 Sep 23doi: 10.1016/j.lfs.2015.09.012. PMID: 26407474.X1

3027. Bijl N, Thys C, Wittevrongel C, et al. Platelet studies in autism spectrum disorder patients and first-degree relatives. Mol Autism. 2015;6:57. doi: 10.1186/s13229-015-0051-y. PMID: 26500752.X-1

3028. Bilaver LA, Cushing LS, Cutler AT. Prevalence and Correlates of Educational Intervention Utilization Among Children with Autism Spectrum Disorder. J Autism Dev Disord. 2015 Sep 21doi: 10.1007/s10803-0152598-z. PMID: 26391885.X-1

3029. Billeci L, Tonacci A, Tartarisco G, et al. Association Between Atopic Dermatitis and Autism Spectrum Disorders: A Systematic Review. Am J Clin Dermatol. 2015 Oct;16(5):371-88. doi: 10.1007/s40257-0150145-5. PMID: 26254000.X-1

3030. Bink M, Popma A, Bongers IL, et al. Cardiac reactivity and stimulant use in adolescents with autism spectrum disorders with comorbid ADHD versus ADHD. J Autism Dev Disord. 2015 Feb;45(2):481-94. doi: 10.1007/s10803-013-1929-1. PMID: 24005985.X-1

3031. Bink M, van Boxtel GJ, Popma A, et al. EEG theta and beta power spectra in adolescents with ADHD versus adolescents with ASD + ADHD. Eur Child Adolesc Psychiatry. 2015 Aug;24(8):873-86. doi: 10.1007/s00787-014-0632-x. PMID: 25374034.X-1 
3032. Bisgaard AM, Schonewolf-Greulich B, Ravn K, et al. Is it possible to diagnose Rett syndrome before classical symptoms become obvious? Review of 24 Danish cases born between 2003 and 2012. Eur J Paediatr Neurol. 2015 Nov;19(6):679-87. doi: 10.1016/j.ejpn.2015.07.004. PMID: 26228846.X-1

3033. Bishop JR, Najjar F, Rubin LH, et al. Escitalopram pharmacogenetics: CYP2C19 relationships with dosing and clinical outcomes in autism spectrum disorder. Pharmacogenet Genomics. 2015 Nov;25(11):548-54. doi: 10.1097/fpc.0000000000000173. PMID: 26313485.X-3

3034. Bishop-Fitzpatrick L, Mazefsky CA, Minshew NJ, et al. The relationship between stress and social functioning in adults with autism spectrum disorder and without intellectual disability. Autism Res. 2015 Apr;8(2):164-73. doi: 10.1002/aur.1433. PMID: 25524571.X-1

3035. Blackmon K, Bluvstein J, MacAllister WS, et al. Treatment Resistant Epilepsy in Autism Spectrum Disorder: Increased Risk for Females. Autism Res. 2015 Jun 26doi: 10.1002/aur.1514. PMID: 26112160.X-1

3036. Blanche EI, Diaz J, Barretto T, et al. Caregiving Experiences of Latino Families With Children With Autism Spectrum Disorder. Am J Occup Ther. 2015 SepOct;69(5):6905185010p1-11. doi: 10.5014/ajot.2015.017848. PMID: 26356658.X-1

3037. Blasi A, Lloyd-Fox S, Sethna V, et al. Atypical processing of voice sounds in infants at risk for autism spectrum disorder. Cortex. 2015 Oct;71:122-33. doi: 10.1016/j.cortex.2015.06.015. PMID: 26200892.X-1

3038. Blomqvist M, Bejerot S, Dahllof G. A cross-sectional study on oral health and dental care in intellectually able adults with autism spectrum disorder. BMC Oral Health. 2015;15:81. doi: 10.1186/s12903-015-0065-z. PMID: 26174171.X-1

3039. Blumberg SJ, Zablotsky B, Avila RM, et al. Diagnosis lost: Differences between children who had and who currently have an autism spectrum disorder diagnosis. Autism. 2015 Oct 20doi: 10.1177/1362361315607724. PMID: 26489772.X-1

3040. Bodner KE, Engelhardt CR, Minshew NJ, et al. Making Inferences: Comprehension of Physical Causality, Intentionality, and Emotions in Discourse by High-Functioning Older Children, Adolescents, and Adults with Autism. J Autism Dev Disord. 2015 Sep;45(9):2721-33. doi: 10.1007/s10803-015-2436-3. PMID: 25821925.X-1

3041. Boets B, Verhoeven J, Wouters J, et al. Fragile spectral and temporal auditory processing in adolescents with autism spectrum disorder and early language delay. J Autism Dev Disord. 2015 Jun;45(6):1845-57. doi: 10.1007/s10803-014-2341-1. PMID: 25503681.X-1

3042. Bogliacino F, Parra Forero IA. Behaviourally designed treatments that increase willingness to treatment from families with children suffering from autism spectrum disorder. J Epidemiol Community Health. 2015 Oct;69(10):958-62. doi: 10.1136/jech-2015205505. PMID: 25903754.X-1

3043. Bolling DZ, Pelphrey KA, Vander Wyk BC. Trait-level temporal lobe hypoactivation to social exclusion in unaffected siblings of children and adolescents with autism spectrum disorders. Dev Cogn Neurosci. 2015 Jun;13:75-83. doi: 10.1016/j.dcn.2015.04.007. PMID: 26011751.X-1

3044. Bolte S, Ciaramidaro A, Schlitt S, et al. Training-induced plasticity of the social brain in autism spectrum disorder. $\mathrm{Br} \mathrm{J}$ Psychiatry. 2015 Aug;207(2):149-57. doi: 10.1192/bjp.bp.113.143784. PMID: 25792694.X-1

3045. Bone D, Goodwin MS, Black MP, et al. Applying machine learning to facilitate autism diagnostics: pitfalls and promises. J Autism Dev Disord. 2015 May;45(5):1121-36. doi: 10.1007/s10803-014-2268-6. PMID: 25294649.X-1

3046. Bonete S, Calero MD, FernandezParra A. Group training in interpersonal problem-solving skills for workplace adaptation of adolescents and adults with Asperger syndrome: a preliminary study. Autism. 2015 
May;19(4):409-20. doi:

10.1177/1362361314522354. PMID:

24569569.X-1

3047. Bora E, Bartholomeusz C, Pantelis C. Meta-analysis of Theory of Mind (ToM)

impairment in bipolar disorder. Psychol Med.

2015 Oct 12:1-12. doi:

10.1017/s0033291715001993. PMID:

26456502.X-1

3048. Borders CM, Bock SJ, Szymanski C. Teacher ratings of evidence-based practices from the field of autism. J Deaf Stud Deaf Educ. 2015 Jan;20(1):91-100. doi: 10.1093/deafed/enu033. PMID: 25316908.X-1

3049. Borgi M, Loliva D, Cerino S, et al. Effectiveness of a Standardized EquineAssisted Therapy Program for Children with Autism Spectrum Disorder. J Autism Dev Disord. 2015 Jul 26doi: 10.1007/s10803-0152530-6. PMID: 26210515.X-1

3050. Borroni B, Premi E, Formenti A, et al. Structural and functional imaging study in dementia with Lewy bodies and Parkinson's disease dementia. Parkinsonism Relat Disord. 2015 Sep;21(9):1049-55. doi:

10.1016/j.parkreldis.2015.06.013. PMID: 26109553.X-1

3051. Bos DJ, Merchan-Naranjo J, Martinez $\mathrm{K}$, et al. Reduced Gyrification Is Related to Reduced Interhemispheric Connectivity in Autism Spectrum Disorders. J Am Acad Child Adolesc Psychiatry. 2015 Aug;54(8):668-76. doi: 10.1016/j.jaac.2015.05.011. PMID: 26210336.X-1

3052. Bottema-Beutel K, Li Z. Adolescent judgments and reasoning about the failure to include peers with social disabilities. J Autism Dev Disord. 2015 Jun;45(6):1873-86. doi: 10.1007/s10803-014-2348-7. PMID: 25575622.X-1

3053. Bottema-Beutel K, Mullins TS, Harvey MN, et al. Avoiding the "brick wall of awkward": Perspectives of youth with autism spectrum disorder on social-focused intervention practices. Autism. 2015 Apr 16doi: 10.1177/1362361315574888. PMID: 25882390.X-1
3054. Bottema-Beutel K, White R. By the Book: An Analysis of Adolescents with Autism Spectrum Condition Co-constructing Fictional Narratives with Peers. J Autism Dev Disord. 2015 Jul 15doi: 10.1007/s10803-015-2524-4. PMID: 26174049.X-1

3055. Boudreau BA, Vladescu JC, Kodak $\mathrm{TM}$, et al. A comparison of differential reinforcement procedures with children with autism. J Appl Behav Anal. 2015 Jul 14doi: 10.1002/jaba.232. PMID: 26174019.X-3, X-4

3056. Bowers K, Wink LK, Pottenger A, et al. Phenotypic differences in individuals with autism spectrum disorder born preterm and at term gestation. Autism. 2015 Aug;19(6):75863. doi: 10.1177/1362361314547366. PMID: 25192860.X-1

3057. Bowler DM, Gaigg SB, Gardiner JM. Brief Report: The Role of Task Support in the Spatial and Temporal Source Memory of Adults with Autism Spectrum Disorder. J Autism Dev Disord. 2015 Aug;45(8):2613-7. doi: 10.1007/s10803-015-2378-9. PMID: 25694363.X-1

3058. Braat S, D'Hulst C, Heulens I, et al. The GABAA receptor is an FMRP target with therapeutic potential in fragile $\mathrm{X}$ syndrome. Cell Cycle. 2015 Sep 17;14(18):2985-95. doi: 10.4161/15384101.2014.989114. PMID: 25790165.X-1

3059. Braddock BA, Twyman KA, Garrity MR, et al. A Few Close Friends: The Pediatrician's Role in the Management of Social Skills Deficits in Adolescent Children. Clin Pediatr (Phila). 2015 Oct;54(12):1192-9. doi: 10.1177/0009922815570619. PMID: 25676832.X-1

3060. Bradshaw J, Steiner AM, Gengoux G, et al. Feasibility and effectiveness of very early intervention for infants at-risk for autism spectrum disorder: a systematic review. J Autism Dev Disord. 2015 Mar;45(3):778-94. doi: 10.1007/s10803-014-2235-2. PMID: 25218848.X-1, X-2

3061. Brady NC, Storkel HL, Bushnell P, et al. Investigating a Multimodal Intervention for Children with Limited Expressive Vocabularies Associated with Autism. Am J Speech Lang 
Pathol. 2015 Apr 24doi: 10.1044/2015_ajslp14-0093. PMID: 25910710.X-3

3062. Braida D, Ponzoni L, Matteoli M, et al. Different attentional abilities among inbred mice strains using virtual object recognition task (VORT): SNAP25 mice as a model of attentional deficit. Behav Brain Res. 2015 Aug 20doi: 10.1016/j.bbr.2015.08.016. PMID: 26300453.X-1

3063. Brand S, Jossen S, Holsboer-Trachsler $\mathrm{E}$, et al. Impact of aerobic exercise on sleep and motor skills in children with autism spectrum disorders - a pilot study. Neuropsychiatr Dis Treat. 2015;11:1911-20. doi: 10.2147/ndt.s85650. PMID: 26346856.X-3

3064. Brandwein AB, Foxe JJ, Butler JS, et al. Neurophysiological indices of atypical auditory processing and multisensory integration are associated with symptom severity in autism. Journal of Autism and Developmental Disorders. 2015 2015-1209;45(1):230-44. doi: 10.1002/aur.56 . http://dx.doi.org/10.1007/s10803-014-2212-9. PMID: 1606044959; 2014-40415-001.X-1

3065. Breau LM, Clark B, Scott O, et al. Social communication features in children following moderate to severe acquired brain injury: a cross-sectional pilot study. J Child Neurol. 2015 Apr;30(5):588-94. doi: 10.1177/0883073814528282. PMID: 24659736.X-1

3066. Bremer E, Balogh R, Lloyd M. Effectiveness of a fundamental motor skill intervention for 4-year-old children with autism spectrum disorder: A pilot study. Autism. 2015 Nov;19(8):980-91. doi: 10.1177/1362361314557548. PMID: 25432505.X-4

3067. Brenes JC, Lackinger M, Hoglinger GU, et al. Differential effects of social and physical environmental enrichment on brain plasticity, cognition, and ultrasonic communication in rats. J Comp Neurol. 2015 Jul 1doi: 10.1002/cne.23842. PMID: 26132842.X-1

3068. Brezis RS, Weisner TS, Daley TC, et al. Parenting a child with autism in India: narratives before and after a parent-child intervention program. Cult Med Psychiatry.
2015 Jun;39(2):277-98. doi: 10.1007/s11013015-9434-y. PMID: 25739529.X-1

3069. Brian JA, Bryson SE, Zwaigenbaum L. Autism spectrum disorder in infancy: developmental considerations in treatment targets. Curr Opin Neurol. 2015 Apr;28(2):11723. doi: $10.1097 /$ wco.0000000000000182. PMID: 25695137.X-1, $x-2$

3070. Brigandi SA, Shao H, Qian SY, et al. Autistic children exhibit decreased levels of essential Fatty acids in red blood cells. Int J Mol Sci. 2015;16(5):10061-76. doi: 10.3390/ijms160510061. PMID: 25946342.X-1

3071. Brix MK, Ersland L, Hugdahl K, et al. "Brain MR spectroscopy in autism spectrum disorder-the GABA excitatory/inhibitory imbalance theory revisited". Front Hum Neurosci. 2015;9:365. doi: 10.3389/fnhum.2015.00365. PMID: 26157380.X-1

3072. Broekhof E, Ketelaar L, Stockmann L, et al. The Understanding of Intentions, Desires and Beliefs in Young Children with Autism Spectrum Disorder. J Autism Dev Disord. 2015 Jul;45(7):2035-45. doi: 10.1007/s10803-0152363-3. PMID: 25636676.X-1

3073. Brondino N, Fusar-Poli L, Panisi C, et al. Pharmacological Modulation of GABA Function in Autism Spectrum Disorders: A Systematic Review of Human Studies. J Autism Dev Disord. 2015 Oct 6doi: 10.1007/s10803015-2619-y. PMID: 26443675.X-2

3074. Brosnan M, Johnson H, Grawmeyer B, et al. Emotion recognition in animated compared to human stimuli in adolescents with autism spectrum disorder. J Autism Dev Disord. 2015 Jun;45(6):1785-96. doi: 10.1007/s10803014-2338-9. PMID: 25567528.X-1

3075. Brosnan M, Mills E. The effect of diagnostic labels on the affective responses of college students towards peers with 'Asperger's Syndrome' and 'Autism Spectrum Disorder'. Autism. 2015 Jun 4doi: 10.1177/1362361315586721. PMID: 26045542.X-1

3076. Brown A, Crowe L, Beauchamp MH, et al. Neurodevelopmental profiles of children with glutaric aciduria type I diagnosed by 
newborn screening: a follow-up case series. JIMD Rep. 2015;18:125-34. doi: 10.1007/8904_2014_360. PMID: 25503300.X1

3077. Bruce SM, Borders C. Communication and Language in Learners Who Are Deaf and Hard of Hearing With Disabilities: Theories, Research, and Practice. Am Ann Deaf. 2015;160(4):368-84. doi: 10.1353/aad.2015.0035. PMID: 26497075.X-1

3078. Brugha TS, Doos L, Tempier A, et al. Outcome measures in intervention trials for adults with autism spectrum disorders; a systematic review of assessments of core autism features and associated emotional and behavioural problems. Int J Methods Psychiatr Res. 2015 Jun;24(2):99-115. doi: 10.1002/mpr.1466. PMID: 26077193.X-1

3079. Bruining H, Matsui A, Oguro-Ando A, et al. Genetic Mapping in Mice Reveals the Involvement of Pcdh9 in Long-Term Social and Object Recognition and Sensorimotor Development. Biol Psychiatry. 2015 Oct 1;78(7):485-95. doi:

10.1016/j.biopsych.2015.01.017. PMID: 25802080.X-1

3080. Bruining H, Passtoors L, Goriounova N, et al. Paradoxical Benzodiazepine Response: A Rationale for Bumetanide in Neurodevelopmental Disorders? Pediatrics. 2015 Aug;136(2):e539-43. doi: 10.1542/peds.2014-4133. PMID: 26216321.X$1, \mathrm{X}-3, \mathrm{X}-4$

3081. Brunner D, Kabitzke P, He D, et al. Comprehensive Analysis of the 16p11.2 Deletion and Null Cntnap2 Mouse Models of Autism Spectrum Disorder. PLoS One. 2015;10(8):e0134572. doi: 10.1371/journal.pone.0134572. PMID: 26273832.X-1

3082. Brunsdon VE, Colvert E, Ames C, et al. Exploring the cognitive features in children with autism spectrum disorder, their co-twins, and typically developing children within a population-based sample. J Child Psychol Psychiatry. 2015 Aug;56(8):893-902. doi: 10.1111/jcpp.12362. PMID: 25418509.X-1

3083. Bryn V, Halvorsen B, Ueland T, et al. Brain derived neurotrophic factor (BDNF) and autism spectrum disorders (ASD) in childhood. Eur J Paediatr Neurol. 2015 Jul;19(4):411-4. doi: 10.1016/j.ejpn.2015.03.005. PMID: 25847750.X-1

3084. Bukala M, Hu MY, Lee R, et al. The effects of work-reinforcer schedules on performance and preference in students with autism. J Appl Behav Anal. 2015 Spring;48(1):215-20. doi: 10.1002/jaba.188. PMID: 25688839.X-1

3085. Bunai Y, Ishii A, Akaza K, et al. A case of sudden death after Japanese encephalitis vaccination. Leg Med (Tokyo). 2015 Jul;17(4):279-82. doi: 10.1016/j.legalmed.2015.03.003. PMID: 25819538.X-1

3086. Burckley E, Tincani M, Guld Fisher A. An iPad-based picture and video activity schedule increases community shopping skills of a young adult with autism spectrum disorder and intellectual disability. Dev Neurorehabil. 2015 Apr;18(2):131-6. doi: 10.3109/17518423.2014.945045. PMID: 25084013.X-1

3087. Burke MM, Goldman SE. Identifying the associated factors of mediation and due process in families of students with autism spectrum disorder. J Autism Dev Disord. 2015 May;45(5):1345-53. doi: 10.1007/s10803-0142294-4. PMID: 25374132.X-1

3088. Burkett K, Morris E, ManningCourtney P, et al. African American Families on Autism Diagnosis and Treatment: The Influence of Culture. J Autism Dev Disord. 2015 Oct;45(10):3244-54. doi: 10.1007/s10803015-2482-x. PMID: 26055985.X-1

3089. Busch de Ahumada LC, Ahumada JL. Contacting a 19 month-old mute autistic girl: a clinical narrative. Int J Psychoanal. 2015 Feb;96(1):11-38. doi: 10.1111/17458315.12248. PMID: 25294631.X-1

3090. Bush RA, Stahmer AC, Connelly CD. Exploring perceptions and use of the electronic health record by parents of children with autism spectrum disorder: A qualitative study. Health Informatics J. 2015 May 18doi: 10.1177/1460458215581911. PMID: 25989804.X-1 
3091. Butler EE, Ward R, Ramsey R. Investigating the Relationship between Stable Personality Characteristics and Automatic Imitation. PLoS One. 2015;10(6):e0129651. doi: 10.1371/journal.pone.0129651. PMID: 26079137.X-1

3092. Butler MG, Hossain W, Sulsona C, et al. Increased plasma chemokine levels in children with Prader-Willi syndrome. Am J Med Genet A. 2015 Mar;167a(3):563-71. doi: 10.1002/ajmg.a.36908. PMID: 25691409.X-1

3093. Butler MG, McGuire AB, Masoud H, et al. Currently recognized genes for schizophrenia: High-resolution chromosome ideogram representation. Am J Med Genet B Neuropsychiatr Genet. 2015 Oct 13doi: 10.1002/ajmg.b.32391. PMID: 26462458.X-1

3094. Buzhardt J, Rusinko L, HeitzmanPowell L, et al. Exploratory Evaluation and Initial Adaptation of a Parent Training Program for Hispanic Families of Children with Autism. Fam Process. 2015 Mar 16doi: 10.1111/famp.12146. PMID: 25776105.X-1, X2

3095. Byford S, Cary M, Barrett B, et al. Cost-effectiveness analysis of a communication-focused therapy for pre-school children with autism: results from a randomised controlled trial. BMC Psychiatry. 2015;15:316. doi: 10.1186/s12888-015-0700-x. PMID: 26691535.X-1

3096. Byrge L, Dubois J, Tyszka JM, et al. Idiosyncratic brain activation patterns are associated with poor social comprehension in autism. J Neurosci. 2015 Apr 8;35(14):5837-50. doi: 10.1523/jneurosci.5182-14.2015. PMID: 25855192.X-1, X-4

3097. Cagetti MG, Mastroberardino S, Campus G, et al. Dental care protocol based on visual supports for children with autism spectrum disorders. Med Oral Patol Oral Cir Bucal. 2015;20(5):e598-604. PMID: 26241453.X-1

3098. Calarge CA, Ziegler EE, Del Castillo $\mathrm{N}$, et al. Iron homeostasis during risperidone treatment in children and adolescents. J Clin Psychiatry. 2015 Aug 4doi: 10.4088/JCP.14m09258. PMID: 26301448.X-1, $\mathrm{X}-3$
3099. Calderon J, Bellinger DC. Executive function deficits in congenital heart disease: why is intervention important? Cardiol Young. 2015 Oct;25(7):1238-46. doi: 10.1017/s1047951115001134. PMID: 26082199.X-1, X-2

3100. Calderoni S, Fantozzi P, Balboni G, et al. The impact of internalizing symptoms on autistic traits in adolescents with restrictive anorexia nervosa. Neuropsychiatr Dis Treat. 2015;11:75-85. doi: 10.2147/ndt.s73235. PMID: 25609969.X-1

3101. Campbell JE, Morgan M, Barnett V, et al. Handheld Devices and Video Modeling to Enhance the Learning of Self-Help Skills in Adolescents With Autism Spectrum Disorder. OTJR (Thorofare N J). 2015 Apr;35(2):95-100. PMID: 26460472.X-1, X-3, X-4

3102. Campbell SB, Leezenbaum NB, Schmidt EN, et al. Concern for Another's Distress in Toddlers at High and Low Genetic Risk for Autism Spectrum Disorder. J Autism Dev Disord. 2015 Nov;45(11):3594-605. doi: 10.1007/s10803-015-2505-7. PMID: 26093390.X-1

3103. Canal CE, Felsing DE, Liu Y, et al. An Orally Active Phenylaminotetralin-Chemotype Serotonin 5-HT7 and 5-HT1A Receptor Partial Agonist that Corrects Motor Stereotypy in Mouse Models. ACS Chem Neurosci. 2015 Jul 15;6(7):1259-70. doi:

10.1021/acschemneuro.5b00099. PMID: 26011730.X-1

3104. Canal-Bedia R, Garcia-Primo P, Hernandez-Fabian A, et al. [From early detection to early care: intervention strategies based on prospective screening]. Rev Neurol. 2015 Feb 25;60 Suppl 1:S25-9. PMID: 25726819.X-1

3105. Canitano R. Mood Stabilizers in Children and Adolescents With Autism Spectrum Disorders. Clin Neuropharmacol. 2015 Sep-Oct;38(5):177-82. doi: 10.1097/wnf.0000000000000096. PMID: 26366961.X-1

3106. Canitano R, Bozzi Y. New treatment perspectives in autism spectrum disorders. Front Pediatr. 2015;3:22. doi: 
10.3389/fped.2015.00022. PMID: 25853112.X$1, \mathrm{X}-2, \mathrm{X}-3$

3107. Caplette L, Desroches P, Wicker B, et al. Autistic and neurotypical subjects extract spatial frequencies differently. J Vis. 2015 Sep 1;15(12):478. doi: 10.1167/15.12.478. PMID: 26326166.X-1

3108. Carbon M, Kapoor S, Sheridan E, et al. Neuromotor Adverse Effects in 342 Youth During 12 Weeks of Naturalistic Treatment With 5 Second-Generation Antipsychotics. J Am Acad Child Adolesc Psychiatry. 2015 Sep;54(9):718-27.e3. doi: 10.1016/j.jaac.2015.06.015. PMID: 26299293.X-1

3109. Carmichael JA, Kubas HA, Carlson HL, et al. Reconsidering "inattention" in attention-deficit hyperactivity disorder: implications for neuropsychological assessment and intervention. Appl Neuropsychol Child. 2015;4(2):97-105. doi: 10.1080/21622965.2015.1005481. PMID: 25748971.X-1

3110. Carmo JC, Duarte E, Pinho S, et al. Preserved Proactive Interference in Autism Spectrum Disorder. J Autism Dev Disord. 2015 Jul 26doi: 10.1007/s10803-015-2540-4. PMID: 26210518.X-1

3111. Carmo JC, Duarte E, Pinho S, et al. Verbal fluency as a function of time in autism spectrum disorder: An impairment of initiation processes? J Clin Exp Neuropsychol. 2015 Sep;37(7):710-21. doi: 10.1080/13803395.2015.1062082. PMID: 26207691.X-1

3112. Carper RA, Solders S, Treiber JM, et al. Corticospinal Tract Anatomy and Functional Connectivity of Primary Motor Cortex in Autism. J Am Acad Child Adolesc Psychiatry. 2015 Oct;54(10):859-67. doi: 10.1016/j.jaac.2015.07.007. PMID: 26407496.X-1

3113. Carr T, Shih W, Lawton K, et al. The relationship between treatment attendance, adherence, and outcome in a caregivermediated intervention for low-resourced families of young children with autism spectrum disorder. Autism. 2015 Aug 19doi:
10.1177/1362361315598634. PMID: 26290524.X-1, X-3

3114. Carre A, Chevallier C, Robel L, et al. Tracking Social Motivation Systems Deficits: The Affective Neuroscience View of Autism. J Autism Dev Disord. 2015 Oct;45(10):3351-63. doi: 10.1007/s10803-015-2498-2. PMID: 26123007.X-1

3115. Carroll RA, Joachim BT, St Peter CC, et al. A comparison of error-correction procedures on skill acquisition during discretetrial instruction. J Appl Behav Anal. 2015 Summer;48(2):257-73. doi: 10.1002/jaba.205. PMID: 25913105.X-1

3116. Carroll RA, Kodak T. Using Instructive Feedback to Increase Response Variability During Intraverbal Training for Children with Autism Spectrum Disorder. Anal Verbal Behav. 2015 Oct;31(2):183-99. doi: 10.1007/s40616-015-0039-x. PMID: 27606211.X-3, X-4

3117. Casanova MF, Sokhadze E, Opris I, et al. Autism spectrum disorders: linking neuropathological findings to treatment with transcranial magnetic stimulation. Acta Paediatr. 2015 Apr;104(4):346-55. doi: 10.1111/apa.12943. PMID: 25626149.X-1

3118. Cascio CJ, Gu C, Schauder KB, et al. Somatosensory Event-Related Potentials and Association with Tactile Behavioral Responsiveness Patterns in Children with ASD. Brain Topogr. 2015 Nov;28(6):895-903. doi: 10.1007/s10548-015-0439-1. PMID: 26016951.X-1

3119. Cascio MA. Rigid therapies, rigid minds: italian professionals' perspectives on autism interventions. Cult Med Psychiatry. 2015 Jun;39(2):235-53. doi: 10.1007/s11013015-9439-6. PMID: 25743186.X-1

3120. Casenhiser DM, Binns A, McGill F, et al. Measuring and supporting language function for children with autism: evidence from a randomized control trial of a social-interactionbased therapy. J Autism Dev Disord. 2015 Mar;45(3):846-57. doi: 10.1007/s10803-0142242-3. PMID: 25234481.X-1, X-2

3121. Case-Smith J, Weaver LL, Fristad MA. A systematic review of sensory processing 
interventions for children with autism spectrum disorders. Autism: The International Journal of Research \& Practice. 2015;19(2):133-48 16p. doi: 10.1177/1362361313517762. PMID: 103750467. Language: English. Entry Date: 20150130. Revision Date: 20150710. Publication Type: Journal Article.X-1, X-2

3122. Casey AF, Quenneville-Himbeault G, Normore A, et al. A therapeutic skating intervention for children with autism spectrum disorder. Pediatr Phys Ther. 2015

Summer;27(2):170-7. doi: 10.1097/pep.0000000000000139. PMID: 25822357.X-3, X-4

3123. Cash-Padgett T, Sawa A, Jaaro-Peled $\mathrm{H}$. Increased stereotypy in conditional Cxcr4 knockout mice. Neurosci Res. 2015 Oct 13doi: 10.1016/j.neures.2015.10.001. PMID: 26458529.X-1

3124. Cassidy S, Mitchell P, Chapman P, et al. Processing of Spontaneous Emotional Responses in Adolescents and Adults with Autism Spectrum Disorders: Effect of Stimulus Type. Autism Res. 2015 Oct;8(5):534-44. doi: 10.1002/aur.1468. PMID: 25735657.X-1

3125. Castro S, Pinto A. Matrix for assessment of activities and participation: Measuring functioning beyond diagnosis in young children with disabilities. Dev Neurorehabil. 2015 Jun;18(3):177-89. doi: 10.3109/17518423.2013.806963. PMID: 23869963.X-1

3126. Cermak SA, Stein Duker LI, Williams ME, et al. Sensory Adapted Dental Environments to Enhance Oral Care for Children with Autism Spectrum Disorders: A Randomized Controlled Pilot Study. J Autism Dev Disord. 2015 Sep;45(9):2876-88. doi: 10.1007/s10803-015-2450-5. PMID: 25931290.X-1

3127. Cermak SA, Stein Duker LI, Williams ME, et al. Feasibility of a sensory-adapted dental environment for children with autism. Am J Occup Ther. 2015 MayJun;69(3):6903220020p1-p10. doi: 10.5014/ajot.2015.013714. PMID: 25871593.X-1

3128. Cermakova P, Johnell K, Fastbom J, et al. Cardiovascular Diseases in approximately
30,000 Patients in the Swedish Dementia Registry. J Alzheimers Dis. 2015 Sep 16doi: 10.3233/jad-150499. PMID: 26402118.X-1

3129. Cervantes PE, Matson JL. Comorbid Symptomology in Adults with Autism Spectrum Disorder and Intellectual Disability. J Autism Dev Disord. 2015 Aug 9doi: 10.1007/s10803-015-2553-z. PMID: 26254894.X-1

3130. Chakrabarti B, Persico A, Battista N, et al. Endocannabinoid Signaling in Autism. Neurotherapeutics. 2015 Oct;12(4):837-47. doi: 10.1007/s13311-015-0371-9. PMID: 26216231.X-1

3131. Chakraborty A, Chakrabarti B. Is it me? Self-recognition bias across sensory modalities and its relationship to autistic traits. Mol Autism. 2015;6:20. doi: 10.1186/s13229015-0016-1. PMID: 25908952.X-1

3132. Chakraborty S, Thomas P, Bhatia T, et al. Assessment of severity of autism using the Indian scale for assessment of autism. Indian $\mathrm{J}$ Psychol Med. 2015 Apr-Jun;37(2):169-74. doi: 10.4103/0253-7176.155616. PMID: 25969602.X-1

3133. Chaminade T, Rosset D, Da Fonseca $\mathrm{D}$, et al. Anthropomorphic bias found in typically developing children is not found in children with autistic spectrum disorder. Autism: The International Journal of Research \& Practice. 2015;19(2):248-51 4p. doi: 10.1177/1362361313512425. PMID: 103750461. Language: English. Entry Date: 20150130. Revision Date: 20150710. Publication Type: Journal Article.X-1

3134. Chandler S, Howlin P, Simonoff E, et al. Emotional and behavioural problems in young children with autism spectrum disorder. Dev Med Child Neurol. 2015 Jun 16doi: 10.1111/dmcn.12830. PMID: 26077499.X-1

3135. Chandley MJ, Crawford JD, Szebeni A, et al. NTRK2 expression levels are reduced in laser captured pyramidal neurons from the anterior cingulate cortex in males with autism spectrum disorder. Mol Autism. 2015;6:28. doi: 10.1186/s13229-015-0023-2. PMID: 26000162.X-1 
3136. Chandrasekhar T, Sikich L. Challenges in the diagnosis and treatment of depression in autism spectrum disorders across the lifespan. Dialogues Clin Neurosci. 2015 Jun;17(2):21927. PMID: 26246795.X-1, X-2

3137. Chang L, Ho SY, Lee TF, et al. Calibration of EBT2 film using a red-channel PDD method in combination with a modified three-channel technique. Med Phys. 2015 Oct;42(10):5838. doi: 10.1118/1.4930253. PMID: 26429258.X-1

3138. Chantiluke K, Barrett N, Giampietro $\mathrm{V}$, et al. Inverse Effect of Fluoxetine on Medial Prefrontal Cortex Activation During Reward Reversal in ADHD and Autism. Cereb Cortex. 2015 Jul;25(7):1757-70. doi:

10.1093/cercor/bht365. PMID: 24451919.X-1

3139. Chantiluke K, Barrett N, Giampietro $\mathrm{V}$, et al. Disorder-dissociated effects of fluoxetine on brain function of working memory in attention deficit hyperactivity disorder and autism spectrum disorder. Psychol Med. 2015 Apr;45(6):1195-205. doi: 10.1017/s0033291714002232. PMID: 25292351.X-1, X-3

3140. Chantiluke K, Barrett N, Giampietro $\mathrm{V}$, et al. Inverse fluoxetine effects on inhibitory brain activation in non-comorbid boys with ADHD and with ASD. Psychopharmacology (Berl). 2015 Jun;232(12):2071-82. doi: 10.1007/s00213-014-3837-2. PMID: 25533997.X-1

3141. Chapman NH, Nato AQ, Jr., Bernier $\mathrm{R}$, et al. Whole exome sequencing in extended families with autism spectrum disorder implicates four candidate genes. Hum Genet. 2015 Oct;134(10):1055-68. doi: 10.1007/s00439-015-1585-y. PMID: 26204995.X-1

3142. Charman T, Baird G, Simonoff E, et al. Testing two screening instruments for autism spectrum disorder in UK community child health services. Dev Med Child Neurol. 2015 Aug 25doi: 10.1111/dmcn.12874. PMID: 26303216.X-1

3143. Charman T, Ricketts J, Dockrell JE, et al. Emotional and behavioural problems in children with language impairments and children with autism spectrum disorders. Int J
Lang Commun Disord. 2015 Jan;50(1):84-93. doi: 10.1111/1460-6984.12116. PMID: 25039810.X-1

3144. Chatterji P, Decker SL, Markowitz S. The effects of mandated health insurance benefits for autism on out-of-pocket costs and access to treatment. J Policy Anal Manage. 2015 Spring;34(2):328-53. PMID: 25893237.X-1

3145. Chen B, Fan GG, Liu H, et al. Changes in anatomical and functional connectivity of Parkinson's disease patients according to cognitive status. Eur J Radiol. 2015 Jul;84(7):1318-24. doi: 10.1016/j.ejrad.2015.04.014. PMID: 25963506.X-1

3146. Chen CK, Shyur SD, Chu SH, et al. Pachydermodactyly: Three new cases in Taiwan. J Microbiol Immunol Infect. 2015 Jun;48(3):340-4. doi: 10.1016/j.jmii.2012.09.002. PMID: 23218407.X-1

3147. Chen FC, Tsai CL. A light fingertip touch reduces postural sway in children with autism spectrum disorders. Gait Posture. 2015 Sep 25doi: 10.1016/j.gaitpost.2015.09.012. PMID: 26422366.X-3

3148. Chen JL, Sung C, Pi S. Vocational Rehabilitation Service Patterns and Outcomes for Individuals with Autism of Different Ages. J Autism Dev Disord. 2015 Sep;45(9):3015-29. doi: 10.1007/s10803-015-2465-y. PMID: 25982310.X-1

3149. Chen L, Tai WC, Brar MS, et al. Tumor grafting induces changes of gut microbiota in athymic nude mice in the presence and absence of medicinal Gynostemma saponins. PLoS One. 2015;10(5):e0126807. doi: 10.1371/journal.pone.0126807. PMID: 25992551.X-1

3150. Chen LS, Xu L, Dhar SU, et al. Autism spectrum disorders: a qualitative study of attitudes toward prenatal genetic testing and termination decisions of affected pregnancies. Clin Genet. 2015 Aug;88(2):122-8. doi: 10.1111/cge.12504. PMID: 25251361.X-1 
3151. Chhabra R, Ruozi B, Vilella A, et al. Application of Polymeric Nanoparticles for CNS Targeted Zinc Delivery In Vivo. CNS Neurol Disord Drug Targets. 2015;14(8):104153. PMID: 26295815.X-1

3152. Chi OZ, Wu CC, Liu X, et al. Restoration of Normal Cerebral Oxygen Consumption with Rapamycin Treatment in a Rat Model of Autism-Tuberous Sclerosis. Neuromolecular Med. 2015 Sep;17(3):305-13. doi: 10.1007/s12017-015-8359-5. PMID: 26048361.X-1

3153. Chiam JT, Dobson RJ, Kiddle SJ, et al. Are blood-based protein biomarkers for Alzheimer's disease also involved in other brain disorders? A systematic review. J Alzheimers Dis. 2015;43(1):303-14. doi: 10.3233/jad140816. PMID: 25096613.X-1

3154. Chiang CH, Chu CL, Lee TC. Efficacy of caregiver-mediated joint engagement intervention for young children with autism spectrum disorders. Autism. 2015 Apr 20doi: 10.1177/1362361315575725. PMID: 25896268.X-1

3155. Chiang HL, Gau SS. Comorbid psychiatric conditions as mediators to predict later social adjustment in youths with autism spectrum disorder. J Child Psychol Psychiatry. 2015 Aug 6doi: 10.1111/jcpp.12450. PMID: 26251168.X-1

3156. Chikako F, Okada A, Akagi T, et al. Analysis of the synthetic-HTP drawing test about developmental disorders. Pediatr Int. 2015 Aug 13doi: 10.1111/ped.12790. PMID: 26270796.X-1

3157. Chita-Tegmark M, Arunachalam S, Nelson CA, et al. Eye-Tracking Measurements of Language Processing: Developmental Differences in Children at High Risk for ASD. J Autism Dev Disord. 2015 Oct;45(10):3327-38. doi: 10.1007/s10803-015-2495-5. PMID: 26109246.X-1

3158. Chiu TA, Anagnostou E, Brian J, et al. Specificity of autonomic arousal to anxiety in children with autism spectrum disorder. Autism Res. 2015 Sep 21doi: 10.1002/aur.1528. PMID: 26389543.X-1
3159. Ch'ng C, Kwok W, Rogic S, et al. Meta-Analysis of Gene Expression in Autism Spectrum Disorder. Autism Res. 2015 Oct;8(5):593-608. doi: 10.1002/aur.1475. PMID: 25720351.X-1

3160. Chowdhury M, Aman MG, Lecavalier L, et al. Factor structure and psychometric properties of the revised Home Situations Questionnaire for autism spectrum disorder: The Home Situations Questionnaire-Autism Spectrum Disorder. Autism. 2015 Jul 17doi: 10.1177/1362361315593941. PMID: 26187059.X-1

3161. Christon LM, Arnold CC, Myers BJ. Professionals' reported provision and recommendation of psychosocial interventions for youth with autism spectrum disorder. Behav Ther. 2015 Jan;46(1):68-82. doi: 10.1016/j.beth.2014.02.002. PMID: 25526836.X-1

3162. Clarke AR, Barry RJ, Indraratna A, et al. EEG activity in children with Asperger's Syndrome. Clin Neurophysiol. 2015 Jun 15doi: 10.1016/j.clinph.2015.05.015. PMID: 26187351.X-1

3163. Clarke C. Autism Spectrum Disorder and Amplified Pain. Case Rep Psychiatry. 2015;2015:930874. doi: 10.1155/2015/930874. PMID: 26064754.X-1

3164. Clarke TK, Lupton MK, FernandezPujals AM, et al. Common polygenic risk for autism spectrum disorder (ASD) is associated with cognitive ability in the general population. Mol Psychiatry. 2015 Mar 10doi:

10.1038/mp.2015.12. PMID: 25754080.X-1

3165. Clausi S, Coricelli G, Pisotta I, et al. Cerebellar damage impairs the self-rating of regret feeling in a gambling task. Front Behav Neurosci. 2015;9:113. doi:

10.3389/fnbeh.2015.00113. PMID: 25999829.X-1

3166. Cleary L, Brady N, Fitzgerald M, et al. Holistic processing of faces as measured by the Thatcher illusion is intact in autism spectrum disorders. Autism. 2015 May;19(4):451-8. doi: 10.1177/1362361314526005. PMID: 24637429.X-1 
3167. Cleland N, Lieblich S, Schalling M, et al. A 16-year-old girl with anti-NMDA-receptor encephalitis and family history of psychotic disorders. Acta Neuropsychiatr. 2015 Jun 1:1-5. doi: 10.1017/neu.2015.32. PMID: 26028254.X1

3168. Clowry GJ. An enhanced role and expanded developmental origins for gammaaminobutyric acidergic interneurons in the human cerebral cortex. J Anat. 2015 Oct;227(4):384-93. doi: 10.1111/joa.12198. PMID: 24839870.X-1

3169. Cochran DM, Sikoglu EM, Hodge SM, et al. Relationship among Glutamine, gammaAminobutyric Acid, and Social Cognition in Autism Spectrum Disorders. J Child Adolesc Psychopharmacol. 2015 May;25(4):314-22. doi: 10.1089/cap.2014.0112. PMID: 25919578.X-1

3170. Cochran L, Moss J, Nelson L, et al. Contrasting age related changes in autism spectrum disorder phenomenology in Cornelia de Lange, Fragile X, and Cri du Chat syndromes: Results from a 2.5 year follow-up. Am J Med Genet C Semin Med Genet. 2015 Jun;169(2):188-97. doi: 10.1002/ajmg.c.31438. PMID: 25989416.X-1

3171. Coeckelbergh M, Pop C, Simut R, et al. A Survey of Expectations About the Role of Robots in Robot-Assisted Therapy for Children with ASD: Ethical Acceptability, Trust, Sociability, Appearance, and Attachment. Sci Eng Ethics. 2015 Apr 17doi: 10.1007/s11948015-9649-x. PMID: 25894654.X-1

3172. Coiro P, Padmashri R, Suresh A, et al. Impaired synaptic development in a maternal immune activation mouse model of neurodevelopmental disorders. Brain Behav Immun. 2015 Jul 26doi:

10.1016/j.bbi.2015.07.022. PMID: 26218293.X-1

3173. Coleman MB, Cherry RA, Moore TC, et al. Teaching Sight Words to Elementary Students With Intellectual Disability and Autism: A Comparison of Teacher-Directed Versus Computer-Assisted Simultaneous Prompting. Intellect Dev Disabil. 2015 Jun;53(3):196-210. doi: 10.1352/1934-955653.3.196. PMID: 26107853.X-1, X-3, X-4
3174. Collyer K, Harrington I. Sensory regulation for inclusive classrooms. Asperger syndrome: Risk factors, cognitive-behavioral characteristics and management strategies.: Nova Biomedical Books, Hauppauge, NY; 2015:9-21.

3175. Conner KO, McKinnon SA, Ward CJ, et al. Peer education as a strategy for reducing internalized stigma among depressed older adults. Psychiatr Rehabil J. 2015 Jun;38(2):18693. doi: 10.1037/prj0000109. PMID: 25915057.X-1

3176. Constantino JN, Zhang Y, Holzhauer $\mathrm{K}$, et al. Distribution and Within-Family Specificity of Quantitative Autistic Traits in Patients with Neurofibromatosis Type I. J Pediatr. 2015 Sep;167(3):621-6.e1. doi: 10.1016/j.jpeds.2015.04.075. PMID: 26051969.X-1

3177. Conti E, Pannek K, Calderoni S, et al. High angular resolution diffusion imaging in a child with autism spectrum disorder and comparison with his unaffected identical twin. Funct Neurol. 2015 Oct 5:1-6. PMID: 26446271.X-1

3178. Conti R. Compassionate Parenting as a Key to Satisfaction, Efficacy and Meaning Among Mothers of Children with Autism. J Autism Dev Disord. 2015 Jul;45(7):2008-18. doi: 10.1007/s10803-015-2360-6. PMID: 25601216.X-1

3179. Cook R, Shah P, Gaule A, et al. Autism and Developmental Prosopagnosia: A Cross-Disorder Study. J Vis. 2015 Sep 1;15(12):1211. doi: 10.1167/15.12.1211. PMID: 26326899.X-1

3180. Cooper M, Thapar A, Jones DK. ADHD severity is associated with white matter microstructure in the subgenual cingulum. Neuroimage Clin. 2015;7:653-60. doi: 10.1016/j.nicl.2015.02.012. PMID: 25844319.X-1

3181. Cooper RA, Plaisted-Grant KC, Hannula DE, et al. Impaired recollection of visual scene details in adults with autism spectrum conditions. J Abnorm Psychol. 2015 Aug;124(3):565-75. doi: 10.1037/abn0000070. PMID: 26120966.X-1 
3182. Coorg R, Weisenberg JL. Successful Treatment of Electrographic Status Epilepticus Of Sleep With Felbamate in a Patient With SLC9A6 Mutation. Pediatr Neurol. 2015 Jul 22doi: 10.1016/j.pediatrneurol.2015.07.007. PMID: 26421989.X-1

3183. Corbett BA, Key AP, Qualls L, et al. Improvement in Social Competence Using a Randomized Trial of a Theatre Intervention for Children with Autism Spectrum Disorder. J

Autism Dev Disord. 2015 Sep 29doi: 10.1007/s10803-015-2600-9. PMID: 26419766.X-1

3184. Corbett J, Melcher D. Evidence for global perceptual averaging in individuals with Autism Spectrum Disorder. J Vis. 2015 Sep 1;15(12):847. doi: 10.1167/15.12.847. PMID: 26326535.X-1

3185. Corcoran J, Berry A, Hill S. The lived experience of US parents of children with autism spectrum disorders: A systematic review and meta-synthesis. J Intellect Disabil. 2015 Mar 27doi: 10.1177/1744629515577876. PMID: 25819433.X-1, X-2

3186. Cordier R, Brown N, Chen YW, et al. Piloting the use of experience sampling method to investigate the everyday social experiences of children with Asperger syndrome/high functioning autism. Dev Neurorehabil. 2015 Aug 24:1-8. doi: 10.3109/17518423.2014.915244. PMID: 24840290.X-1

3187. Costales JL, Kolevzon A. PhelanMcDermid Syndrome and SHANK3: Implications for Treatment. Neurotherapeutics. 2015 Jul;12(3):620-30. doi: 10.1007/s13311015-0352-z. PMID: 25894671.X-1

3188. Costescu CA, Vanderborght B, David DO. Reversal Learning Task in Children with Autism Spectrum Disorder: A Robot-Based Approach. J Autism Dev Disord. 2015 Nov;45(11):3715-25. doi: 10.1007/s10803-0142319-z. PMID: 25479815.X-1, X-3

3189. Courchesne V, Meilleur AA, PoulinLord MP, et al. Autistic children at risk of being underestimated: school-based pilot study of a strength-informed assessment. Mol Autism. 2015;6:12. doi: 10.1186/s13229-015-0006-3. PMID: 25774281.X-1
3190. Cox CR, Eaton S, Ekas NV, et al. Death concerns and psychological well-being in mothers of children with autism spectrum disorder. Res Dev Disabil. 2015 Oct-Nov;4546:229-38. doi: 10.1016/j.ridd.2015.07.029. PMID: 26256841.X-1

3191. Craig F, Lamanna AL, Margari F, et al. Overlap Between Autism Spectrum Disorders and Attention Deficit Hyperactivity Disorder: Searching for Distinctive/Common Clinical Features. Autism Res. 2015 Jun;8(3):328-37. doi: 10.1002/aur.1449. PMID: 25604000.X-1

3192. Cranwell M, Riby D, Le Couteur A, et al. Dissociation of chromatic discrimination ability in developmental disorders: Autism Spectrum Disorder and Williams Syndrome. J Vis. 2015 Sep 1;15(12):645. doi: 10.1167/15.12.645. PMID: 26326333.X-1

3193. Crawford JD, Chandley MJ, Szebeni $\mathrm{K}$, et al. Elevated GFAP Protein in Anterior Cingulate Cortical White Matter in Males With Autism Spectrum Disorder. Autism Res. 2015 Apr 6doi: 10.1002/aur.1480. PMID: 25846779.X-1

3194. Croen LA, Zerbo O, Qian Y, et al. The health status of adults on the autism spectrum. Autism. 2015 Oct;19(7):814-23. doi: 10.1177/1362361315577517. PMID: 25911091.X-1

3195. Cromarty RA, Elder GJ, Graziadio S, et al. Neurophysiological biomarkers for Lewy body dementias. Clin Neurophysiol. 2015 Jun 27doi: 10.1016/j.clinph.2015.06.020. PMID: 26183755.X-1

3196. Crook JM, Wallace G, TomaskovicCrook E. The potential of induced pluripotent stem cells in models of neurological disorders: implications on future therapy. Expert Rev Neurother. 2015 Mar;15(3):295-304. doi: 10.1586/14737175.2015.1013096. PMID: 25664599.X-1

3197. Crutchfield SA, Mason RA, Chambers A, et al. Use of a Self-monitoring Application to Reduce Stereotypic Behavior in Adolescents with Autism: A Preliminary Investigation of IConnect. J Autism Dev Disord. 2015 
May;45(5):1146-55. doi: 10.1007/s10803-0142272-x. PMID: 25326255.X-1, X-3, X-4

3198. Curran EA, Dalman C, Kearney PM, et al. Association Between Obstetric Mode of Delivery and Autism Spectrum Disorder: A Population-Based Sibling Design Study. JAMA Psychiatry. 2015 Sep 1;72(9):935-42. doi: 10.1001/jamapsychiatry.2015.0846. PMID: 26107922.X-1

3199. Curran EA, O'Neill SM, Cryan JF, et al. Research review: Birth by caesarean section and development of autism spectrum disorder and attention-deficit/hyperactivity disorder: a systematic review and meta-analysis. J Child Psychol Psychiatry. 2015 May;56(5):500-8. doi: 10.1111/jcpp.12351. PMID: 25348074.X-1

3200. Curtin C, Hubbard K, Anderson SE, et al. Food Selectivity, Mealtime Behavior Problems, Spousal Stress, and Family Food Choices in Children with and without Autism Spectrum Disorder. J Autism Dev Disord. 2015 Oct;45(10):3308-15. doi: 10.1007/s10803-0152490-x. PMID: 26070276.X-1

3201. Curtin C, Humphrey K, Vronsky K, et al. Expanding Horizons: A Pilot Mentoring Program Linking College/Graduate Students and Teens With ASD. Clin Pediatr (Phila). 2015 May 27doi: 10.1177/0009922815588821. PMID: 26016838.X-1

3202. Dababnah S, Bulson K. "On the Sidelines": Access to Autism-Related Services in the West Bank. J Autism Dev Disord. 2015 Jul 29doi: 10.1007/s10803-015-2538-y. PMID: 26219419.X-1

3203. Dababnah S, Parish SL. A Comprehensive Literature Review of Randomized Controlled Trials for Parents of Young Children with Autism Spectrum Disorder. J Evid Inf Soc Work. 2015 Jul 15:116. doi: 10.1080/23761407.2015.1052909. PMID: 26177069.X-2

3204. Dababnah S, Parish SL. Feasibility of an empirically based program for parents of preschoolers with autism spectrum disorder. Autism. 2015 Feb 25doi:

10.1177/1362361314568900. PMID:

25717131.X-1, X-3
3205. D'Agostino G, Cristiano C, Lyons DJ, et al. Peroxisome proliferator-activated receptor alpha plays a crucial role in behavioral repetition and cognitive flexibility in mice. Mol Metab. 2015 Jul;4(7):528-36. doi: 10.1016/j.molmet.2015.04.005. PMID: 26137440.X-1

3206. Daimon CM, Jasien JM, Wood WH, 3rd, et al. Hippocampal Transcriptomic and Proteomic Alterations in the BTBR Mouse Model of Autism Spectrum Disorder. Front Physiol. 2015;6:324. doi:

10.3389/fphys.2015.00324. PMID: 26635614.X-1

3207. Daluwatte C, Miles JH, Sun J, et al. Association between pupillary light reflex and sensory behaviors in children with autism spectrum disorders. Research in Developmental Disabilities. 2015 2015-12-09;37:209-15. doi: http://dx.doi.org/10.1016/j.ridd.2014.11.019. PMID: 1660457442; 2015-05377-025.X-1

3208. Danzer E, Gerdes M, D'Agostino JA, et al. Patient characteristics are important determinants of neurodevelopmental outcome during infancy in giant omphalocele. Early Hum Dev. 2015 Mar;91(3):187-93. doi: 10.1016/j.earlhumdev.2014.12.009. PMID: 25676186.X-1

3209. Das DK, Tapias V, D'Aiuto L, et al. Genetic and morphological features of human iPSC-derived neurons with chromosome 15q11.2 (BP1-BP2) deletions. Mol Neuropsychiatry. 2015 Jul;1(2):116-23. doi: 10.1159/000430916. PMID: 26528485.X-1

3210. David M, Billette de Villemeur A, Devillard F, et al. [Schooling and care of mild intellectual disability children]. Arch Pediatr. 2015 Mar;22(3):223-34. doi: 10.1016/j.arcped.2014.12.006. PMID: 25656456.X-1

3211. Davidovitch M, Levit-Binnun N, Golan D, et al. Late diagnosis of autism spectrum disorder after initial negative assessment by a multidisciplinary team. J Dev Behav Pediatr. 2015 May;36(4):227-34. doi: 10.1097/dbp.0000000000000133. PMID: 25651066.X-1

3212. Davidson C, O'Hare A, Mactaggart F, et al. Social relationship difficulties in autism 
and reactive attachment disorder: Improving diagnostic validity through structured assessment. Res Dev Disabil. 2015 May;40:6372. doi: 10.1016/j.ridd.2015.01.007. PMID: 25754456.X-1

3213. Davis PE, Peters JM, Krueger DA, et al. Tuberous Sclerosis: A New Frontier in Targeted Treatment of Autism. Neurotherapeutics. 2015 Jul;12(3):572-83. doi: 10.1007/s13311-015-0359-5. PMID: 25986747.X-1

3214. Davis T, Clifton D, Papadopoulos C. Identifying autism early: The Toddlers at Risk of Autism Clinic model. J Paediatr Child Health. 2015 Jul;51(7):699-703. doi: 10.1111/jpc.12832. PMID: 25623060.X-1

3215. De Angelis M, Francavilla R, Piccolo $\mathrm{M}$, et al. Autism spectrum disorders and intestinal microbiota. Gut Microbes. 2015;6(3):207-13. doi: 10.1080/19490976.2015.1035855. PMID: 25835343.X-1

3216. de Araujo MF, Matsumoto J, Ono T, et al. An animal model of disengagement: Temporary inactivation of the superior colliculus impairs attention disengagement in rats. Behav Brain Res. 2015 Oct 15;293:34-40. doi: 10.1016/j.bbr.2015.07.031. PMID: 26196954.X-1

3217. de Bruin EI, Blom R, Smit FM, et al. MYmind: Mindfulness training for Youngsters with autism spectrum disorders and their parents. Autism. 2015 Nov;19(8):906-14. doi: 10.1177/1362361314553279. PMID: 25348866.X-1, X-3

3218. de Esch CE, van den Berg WE, Buijsen RA, et al. Fragile X mice have robust mGluR5-dependent alterations of social behaviour in the Automated Tube Test. Neurobiol Dis. 2015 Mar;75:31-9. doi: 10.1016/j.nbd.2014.12.021. PMID: 25562659.X-1

3219. De Filippis B, Valenti D, de Bari L, et al. Mitochondrial free radical overproduction due to respiratory chain impairment in the brain of a mouse model of Rett syndrome: protective effect of CNF1. Free Radic Biol Med. 2015 Jun;83:167-77. doi: 10.1016/j.freeradbiomed.2015.02.014. PMID: 25708779.X-1

3220. De Giacomo A, Craig F, Cristella A, et al. Can PEP-3 Provide a Cognitive Profile in Children with ASD? A Comparison Between the Developmental Ages of PEP-3 and IQ of Leiter-R. J Appl Res Intellect Disabil. 2015 Oct 6doi: 10.1111/jar.12216. PMID: 26440549.X-1

3221. De Giacomo A, De Giambattista C, Balducci R, et al. [SCQ as a tool for screening ASD comorbidities with ADHD]. Riv Psichiatr. 2015 Jan-Feb;50(1):34-7. doi:

10.1708/1794.19534. PMID: 25805353.X-1

3222. de la Hoz AB, Maortua H, GarciaRives A, et al. 3p14 De Novo Interstitial Microdeletion in a Patient with Intellectual Disability and Autistic Features with Language Impairment: A Comparison with Similar Cases. Case Rep Genet. 2015;2015:876348. doi: 10.1155/2015/876348. PMID: 26075115.X-1

3223. de Lissa P, Sorensen S, Badcock N, et al. Measuring the face-sensitive N170 with a gaming EEG system: A validation study. J Neurosci Methods. 2015 Sep 30;253:47-54. doi: 10.1016/j.jneumeth.2015.05.025. PMID: 26057115.X-1

3224. de Marchena AB, Eigsti IM, Yerys BE. Brief Report: Generalization Weaknesses in Verbally Fluent Children and Adolescents with Autism Spectrum Disorder. J Autism Dev Disord. 2015 Oct;45(10):3370-6. doi: 10.1007/s10803-015-2478-6. PMID: 26031922.X-1

3225. de Schipper E, Lundequist A, Coghill D, et al. Ability and Disability in Autism Spectrum Disorder: A Systematic Literature Review Employing the International Classification of Functioning, Disability and Health-Children and Youth Version. Autism Res. 2015 Mar 28doi: 10.1002/aur.1485. PMID: 25820780.X-1

3226. de Vaan G, Vervloed M, PetersScheffer NC, et al. Behavioural assessment of autism spectrum disorders in people with multiple disabilities. J Intellect Disabil Res. 2015 Jun 8doi: 10.1111/jir.12206. PMID: 26059997.X-1 
3227. de Vinck-Baroody O, Shui A, Macklin EA, et al. Overweight and Obesity in a Sample of Children With Autism Spectrum Disorder. Acad Pediatr. 2015 Jul-Aug;15(4):396-404. doi: 10.1016/j.acap.2015.03.008. PMID: 25937610.X-1

3228. de Vries M, Geurts H. Influence of Autism Traits and Executive Functioning on Quality of Life in Children with an Autism Spectrum Disorder. J Autism Dev Disord. 2015 Sep;45(9):2734-43. doi: 10.1007/s10803-0152438-1. PMID: 25835211.X-1

3229. de Vries M, Prins PJ, Schmand BA, et al. Working memory and cognitive flexibilitytraining for children with an autism spectrum disorder: a randomized controlled trial. J Child Psychol Psychiatry. 2015 May;56(5):566-76. doi: 10.1111/jcpp.12324. PMID: 25256627.X-1

3230. Dekker LP, van der Vegt EJ, Visser K, et al. Improving psychosexual knowledge in adolescents with autism spectrum disorder: pilot of the tackling teenage training program. J Autism Dev Disord. 2015 Jun;45(6):1532-40. doi: 10.1007/s10803-014-2301-9. PMID: 25399394.X-1

3231. Dekkers LM, Groot NA, Diaz Mosquera EN, et al. Prevalence of Autism Spectrum Disorders in Ecuador: A Pilot Study in Quito. J Autism Dev Disord. 2015 Aug 30doi: 10.1007/s10803-015-2559-6. PMID: 26319251.X-1

3232. Dell'Osso L, Dalle Luche R, Cerliani C, et al. Unexpected subthreshold autism spectrum in a 25-year-old male stalker hospitalized for delusional disorder: a case report. Compr Psychiatry. 2015 Aug;61:10-4. doi: 10.1016/j.comppsych.2015.04.003. PMID: 26031384.X-1

3233. Delude CM. Deep phenotyping: The details of disease. Nature. 2015 Nov 5;527(7576):S14-5. doi: 10.1038/527S14a. PMID: 26536218.X-1

3234. Demopoulos C, Arroyo MS, Dunn W, et al. Individuals with agenesis of the corpus callosum show sensory processing differences as measured by the sensory profile. Neuropsychology. 2015 Sep;29(5):751-8. doi: 10.1037/neu0000165. PMID: 25528608.X-1
3235. Demopoulos C, Brandes-Aitken AN, Desai SS, et al. Shared and Divergent Auditory and Tactile Processing in Children with Autism and Children with Sensory Processing Dysfunction Relative to Typically Developing Peers. J Int Neuropsychol Soc. 2015 Jul;21(6):444-54. doi: 10.1017/s1355617715000387. PMID: 26145730.X-1

3236. Demopoulos C, Hopkins J, Kopald $\mathrm{BE}$, et al. Deficits in Auditory Processing Contribute to Impairments in Vocal Affect Recognition in Autism Spectrum Disorders: A MEG Study. Neuropsychology. 2015 May 25doi: 10.1037/neu0000209. PMID: 26011112.X-1

3237. Demopoulos C, Lewine JD. Audiometric Profiles in Autism Spectrum Disorders: Does Subclinical Hearing Loss Impact Communication? Autism Res. 2015 May 11doi: 10.1002/aur.1495. PMID: 25962745.X-1

3238. Deoni SC, Zinkstok JR, Daly E, et al. White-matter relaxation time and myelin water fraction differences in young adults with autism. Psychol Med. 2015 Mar;45(4):795-805. doi: 10.1017/s0033291714001858. PMID: 25111948.X-1

3239. DeQuinzio JA, Taylor BA. Teaching children with autism to discriminate the reinforced and nonreinforced responses of others: implications for observational learning. J Appl Behav Anal. 2015 Spring;48(1):38-51. doi: 10.1002/jaba.192. PMID: 25689037.X-3, $\mathrm{X}-4$

3240. Derakhshan N. Vagal Nerve Stimulation for the Treatment of Autism. Ment Illn. 2015 Feb 24;7(1):5788. doi:

10.4081/mi.2015.5788. PMID: 26266025.X-1, $\mathrm{X}-2, \mathrm{X}-3$

3241. Derosa NM, Fisher WW, Steege MW. An evaluation of time in establishing operation on the effectiveness of functional communication training. J Appl Behav Anal. 2015 Spring;48(1):115-30. doi: 10.1002/jaba.180. PMID: 25420612.X-1

3242. Desachy G, Croen LA, Torres AR, et al. Increased female autosomal burden of rare copy number variants in human populations and 
in autism families. Mol Psychiatry. 2015 Feb;20(2):170-5. doi: 10.1038/mp.2014.179. PMID: 25582617.X-1

3243. DeThorne L, Aparicio Betancourt M, Karahalios K, et al. Visualizing Syllables: RealTime Computerized Feedback Within a SpeechLanguage Intervention. J Autism Dev Disord. 2015 Nov;45(11):3756-63. doi: 10.1007/s10803-014-2274-8. PMID: 25344794.X-1

3244. Devriese J, Dhar M, Walleghem D, et al. [Hallucinations and obsessive behaviour in an infant with autism spectrum disorder: diagnostic problems]. Tijdschr Psychiatr. 2015;57(8):608-12. PMID: 26402898.X-1

3245. Dewinter J, Vermeiren R, Vanwesenbeeck I, et al. Parental Awareness of Sexual Experience in Adolescent Boys With Autism Spectrum Disorder. J Autism Dev Disord. 2015 Oct 19doi: 10.1007/s10803-0152622-3. PMID: 26481386.X-1

3246. Di Marino D, D'Annessa I, Tancredi $\mathrm{H}$, et al. A unique binding mode of the eukaryotic translation initiation factor $4 \mathrm{E}$ for guiding the design of novel peptide inhibitors. Protein Sci. 2015 Sep;24(9):1370-82. doi: 10.1002/pro.2708. PMID: 26013047.X-1

3247. Di Renzo M, Bianchi Di Castelbianco F, Petrillo M, et al. ASSESSMENT OF A LONG-TERM DEVELOPMENTAL RELATIONSHIP-BASED APPROACH IN CHILDREN WITH AUTISM SPECTRUM DISORDER. Psychol Rep. 2015

Aug;117(1):26-49. doi: 10.2466/15.10.PR0.117c15z8. PMID: 26270989.X-3

3248. Di Venanzio C, Marinelli M, Tonnetti A, et al. Comparison between small radiation therapy electron beams collimated by Cerrobend and tubular applicators. J Appl Clin Med Phys. 2015;16(1):5186. doi: 10.1120/jacmp.v16i1.5186. PMID: 25679175.X-1

3249. Di Venanzio C, Marinelli M, Tonnetti A, et al. Characterization of a microDiamond detector in high-dose-per-pulse electron beams for intra operative radiation therapy. Phys Med. 2015 Jul 6doi: 10.1016/j.ejmp.2015.06.008. PMID: 26160702.X-1
3250. Dickerson AS, Rahbar MH, Han I, et al. Autism spectrum disorder prevalence and proximity to industrial facilities releasing arsenic, lead or mercury. Sci Total Environ. 2015 Dec 1;536:245-51. doi: 10.1016/j.scitotenv.2015.07.024. PMID: 26218563.X-1

3251. Dickerson Mayes S, Calhoun SL, Baweja R, et al. Suicide ideation and attempts in children with psychiatric disorders and typical development. Crisis. 2015;36(1):55-60. doi: 10.1027/0227-5910/a000284. PMID: 25410255.X-1

3252. Diehl JJ, Friedberg C, Paul R, et al. The use of prosody during syntactic processing in children and adolescents with autism spectrum disorders. Dev Psychopathol. 2015 Aug;27(3):867-84. doi: 10.1017/s0954579414000741. PMID: 25156911.X-1

3253. Dillen C, Steyaert J, Op de Beeck HP, et al. Visual processing in adolescents with autism spectrum disorder: evidence from embedded figures and configural superiority tests. J Autism Dev Disord. 2015 May;45(5):1281-90. doi: 10.1007/s10803-0142288-2. PMID: 25342435.X-1

3254. Dillenburger K, McKerr L, Jordan JA, et al. Creating an Inclusive Society... How Close are We in Relation to Autism Spectrum Disorder? A General Population Survey. J Appl Res Intellect Disabil. 2015 Jul;28(4):330-40. doi: 10.1111/jar.12144. PMID: 25808648.X-1

3255. Dimitriou D, Leonard HC, KarmiloffSmith A, et al. Atypical development of configural face recognition in children with autism, Down syndrome and Williams syndrome. J Intellect Disabil Res. 2015 May;59(5):422-38. doi: 10.1111/jir.12141. PMID: 25059077.X-1

3256. Dinecola CM, Lemieux CM. Practice with persons with autism spectrum disorders: predictors of self-efficacy among social work students. J Soc Work Disabil Rehabil. 2015;14(1):23-40. doi: 10.1080/1536710x.2015.989559. PMID: 25584441.X-1 
3257. Ditzian K, Wilder DA, King A, et al. An evaluation of the Performance Diagnostic Checklist-Human Services to assess an employee performance problem in a centerbased autism treatment facility. J Appl Behav Anal. 2015 Spring;48(1):199-203. doi: 10.1002/jaba.171. PMID: 25311712.X-1

3258. Divan G, Hamdani SU, Vajartkar V, et al. Adapting an evidence-based intervention for autism spectrum disorder for scaling up in resource-constrained settings: the development of the PASS intervention in South Asia. Glob Health Action. 2015;8:27278. doi: 10.3402/gha.v8.27278. PMID: 26243710.X-1, $\mathrm{X}-2$

3259. Doll CA, Broadie K. Activitydependent FMRP requirements in development of the neural circuitry of learning and memory. Development. 2015 Apr 1;142(7):1346-56. doi: 10.1242/dev.117127. PMID: 25804740.X-1

3260. Dominick K, Wink LK, McDougle CJ, et al. A Retrospective Naturalistic Study of Ziprasidone for Irritability in Youth with Autism Spectrum Disorder. J Child Adolesc Psychopharmacol. 2015 Jun;25(5):397-401. doi: 10.1089/cap.2014.0111. PMID: 26091194.X-3

3261. Dong L, Yan H, Huang X, et al. A2BP1 gene polymorphisms association with olanzapine-induced weight gain. Pharmacol Res. 2015 Sep;99:155-61. doi: 10.1016/j.phrs.2015.06.003. PMID: 26092620.X-1

3262. Donkers FC, Schipul SE, Baranek GT, et al. Attenuated auditory event-related potentials and associations with atypical sensory response patterns in children with autism. J Autism Dev Disord. 2015 Feb;45(2):506-23. doi: 10.1007/s10803-0131948-y. PMID: 24072639.X-1

3263. Donovan MD, O'Brien FE, Boylan GB, et al. The effect of organic anion transporter 3 inhibitor probenecid on bumetanide levels in the brain: an integrated in vivo microdialysis study in the rat. J Pharm Pharmacol. 2015 Apr;67(4):501-10. doi: 10.1111/jphp.12341. PMID: 25490899.X-1

3264. Doshi-Velez F, Avillach P, Palmer N, et al. Prevalence of Inflammatory Bowel
Disease Among Patients with Autism Spectrum Disorders. Inflamm Bowel Dis. 2015

Oct;21(10):2281-8. doi:

10.1097/mib.0000000000000502. PMID: 26218138.X-1

3265. Dougherty CC, Evans DW, Myers SM, et al. A Comparison of Structural Brain Imaging Findings in Autism Spectrum Disorder and Attention-Deficit Hyperactivity Disorder. Neuropsychol Rev. 2015 Oct 19doi: 10.1007/s11065-015-9300-2. PMID: 26482248.X-1

3266. Douglas NF, Campbell WN, Hinckley JJ. Implementation Science: Buzzword or Game Changer? J Speech Lang Hear Res. 2015 Oct 16doi: 10.1044/2015_jslhr-l-15-0302. PMID: 26502033.X-1

3267. Downs J, Hotopf M, Ford T, et al. Clinical predictors of antipsychotic use in children and adolescents with autism spectrum disorders: a historical open cohort study using electronic health records. Eur Child Adolesc Psychiatry. 2015 Oct 15doi: 10.1007/s00787015-0780-7. PMID: 26472118.X-1

3268. Doyen C, Contejean Y, Risler V, et al. [Cognitive remediation therapy for children: literature data and clinical application in a child and adolescent psychiatry department]. Arch Pediatr. 2015 Apr;22(4):418-26. doi: 10.1016/j.arcped.2015.01.012. PMID: 25736104.X-1, X-3, X-4

3269. Dreyer Gillette ML, Borner KB, Nadler CB, et al. Prevalence and Health Correlates of Overweight and Obesity in Children with Autism Spectrum Disorder. J Dev Behav Pediatr. 2015 Sep;36(7):489-96. doi: 10.1097/dbp.0000000000000198. PMID: 26166285.X-1

3270. D'Souza D, Booth R, Connolly M, et al. Rethinking the concepts of 'local or global processors': evidence from Williams syndrome, Down syndrome, and Autism Spectrum Disorders. Dev Sci. 2015 May 25doi: 10.1111/desc.12312. PMID: 26010432.X-1

3271. Du RY, Yiu CK, King NM, et al. Oral health among preschool children with autism spectrum disorders: A case-control study. Autism. 2015 Aug;19(6):746-51. doi: 
10.1177/1362361314553439. PMID: 25432504.X-1

3272. Duan G, Chen J, Zhang W, et al. Physical maltreatment of children with autism in Henan province in China: A cross-sectional study. Child Abuse Negl. 2015 Oct;48:140-7. doi: 10.1016/j.chiabu.2015.03.018. PMID: 25957752.X-1

3273. Dubin AH, Lieberman-Betz R, Michele Lease A. Investigation of Individual Factors Associated with Anxiety in Youth with Autism Spectrum Disorders. J Autism Dev Disord. 2015 Sep;45(9):2947-60. doi: 10.1007/s10803-015-2458-x. PMID: 25917383.X-1

3274. Ducharme S, Price BH, Larvie M, et al. Clinical Approach to the Differential Diagnosis Between Behavioral Variant Frontotemporal Dementia and Primary Psychiatric Disorders. Am J Psychiatry. 2015 Sep 1;172(9):827-37. doi: 10.1176/appi.ajp.2015.14101248. PMID: 26324301.X-1

3275. Dudova I, Kocourkova J, Koutek J. Early-onset anorexia nervosa in girls with Asperger syndrome. Neuropsychiatr Dis Treat. 2015;11:1639-43. doi: 10.2147/ndt.s83831. PMID: 26170676.X-1

3276. Duerden EG, Taylor MJ, Lee M, et al. Decreased sensitivity to thermal stimuli in adolescents with autism spectrum disorder: relation to symptomatology and cognitive ability. J Pain. 2015 May;16(5):463-71. doi: 10.1016/j.jpain.2015.02.001. PMID: 25704841.X-1

3277. Duffney LJ, Zhong P, Wei J, et al. Autism-like Deficits in Shank3-Deficient Mice Are Rescued by Targeting Actin Regulators. Cell Rep. 2015 Jun 9;11(9):1400-13. doi: 10.1016/j.celrep.2015.04.064. PMID: 26027926.X-1

3278. Duncan AW, Bishop SL. Understanding the gap between cognitive abilities and daily living skills in adolescents with autism spectrum disorders with average intelligence. Autism. 2015 Jan;19(1):64-72. doi: 10.1177/1362361313510068. PMID:

24275020.X-1
3279. Dunleavy L. Evaluation of a continuing education course for occupational therapy practitioners on the use of applied behavior analysis. Occup Ther Health Care. 2015 Jan;29(1):39-53. doi: 10.3109/07380577.2014.950784. PMID: 25180710.X-1

3280. Dupuis DL, Lerman DC, Tsami L, et al. Reduction of aggression evoked by sounds using noncontingent reinforcement and timeout. J Appl Behav Anal. 2015 Sep;48(3):66974. doi: 10.1002/jaba.220. PMID: 26118940.X3, X-4

3281. Durrleman S, Hippolyte L, Zufferey S, et al. Complex syntax in autism spectrum disorders: a study of relative clauses. Int J Lang Commun Disord. 2015 Mar-Apr;50(2):260-7. doi: 10.1111/1460-6984.12130. PMID: 25244532.X-1

3282. D'Urso G, Bruzzese D, Ferrucci R, et al. Transcranial direct current stimulation for hyperactivity and noncompliance in autistic disorder. World J Biol Psychiatry. 2015;16(5):361-6. doi: 10.3109/15622975.2015.1014411. PMID: 25800799.X-3, X-4

3283. Dutheil F, Chambres P, Hufnagel C, et al. 'Do Well B.': Design Of WELL Being monitoring systems. A study protocol for the application in autism. BMJ Open. 2015;5(2):e007716. doi: 10.1136/bmjopen2015-007716. PMID: 25710916.X-1

3284. Duvekot J, van der Ende J, Verhulst FC, et al. The Screening Accuracy of the Parent and Teacher-Reported Social Responsiveness Scale (SRS): Comparison with the 3Di and ADOS. J Autism Dev Disord. 2015 Jun;45(6):1658-72. doi: 10.1007/s10803-0142323-3. PMID: 25428292.X-1

3285. Ebrahimi-Fakhari D, Sahin M. Autism and the synapse: emerging mechanisms and mechanism-based therapies. Curr Opin Neurol. 2015 Apr;28(2):91-102. doi: 10.1097/wco.0000000000000186. PMID: 25695134.X-1

3286. Ebrahimzadeh MA, Safdari Y, Khalili M. Antioxidant Activity of Different Fractions of Methanolic Extract of the Golden Chanterelle Mushroom Cantharellus cibarius 
(Higher Basidiomycetes) from Iran. Int J Med Mushrooms. 2015;17(6):557-65. PMID: 26349513.X-1

3287. Edgar JC, Khan SY, Blaskey L, et al. Neuromagnetic oscillations predict evokedresponse latency delays and core language deficits in autism spectrum disorders. J Autism Dev Disord. 2015 Feb;45(2):395-405. doi: 10.1007/s10803-013-1904-X. PMID: 23963591.X-1

3288. Edgin JO, Tooley U, Demara B, et al. Sleep Disturbance and Expressive Language Development in Preschool-Age Children With Down Syndrome. Child Dev. 2015 Oct 5doi: 10.1111/cdev.12443. PMID: 26435268.X-1

3289. Egawa J, Watanabe Y, Wang C, et al. Novel rare missense variations and risk of autism spectrum disorder: whole-exome sequencing in two families with affected siblings and a two-stage follow-up study in a Japanese population. PLoS One.

2015;10(3):e0119413. doi:

10.1371/journal.pone.0119413. PMID: 25806950.X-1

3290. Eisenberg IW, Wallace GL, Kenworthy L, et al. Insistence on sameness relates to increased covariance of gray matter structure in autism spectrum disorder. Mol Autism. 2015;6:54. doi: 10.1186/s13229-0150047-7. PMID: 26435832.X-1

3291. El Zein F, Solis M, Lang R, et al. Embedding perseverative interest of a child with autism in text may result in improved reading comprehension: A pilot study. Dev Neurorehabil. 2015 Aug 24:1-5. doi: 10.3109/17518423.2014.915893. PMID: 24840483.X-1, X-3, X-4

3292. El-Ansary A, Bhat RS, Al-Daihan S, et al. The neurotoxic effects of ampicillinassociated gut bacterial imbalances compared to those of orally administered propionic acid in the etiology of persistent autistic features in rat pups: effects of various dietary regimens. Gut Pathog. 2015;7:7. doi: 10.1186/s13099-0150054-4. PMID: 25852770.X-1

3293. Ellis Weismer S, Kover ST. Preschool language variation, growth, and predictors in children on the autism spectrum. J Child
Psychol Psychiatry. 2015 Mar 10doi: 10.1111/jcpp.12406. PMID: 25753577.X-1

3294. Elrod MG, Hood BS. Sleep differences among children with autism spectrum disorders and typically developing peers: a meta-analysis. J Dev Behav Pediatr. 2015 Apr;36(3):166-77. doi: 10.1097/dbp.0000000000000140. PMID: 25741949.X-1

3295. Emily G, Grace I. Family quality of life and ASD: the role of child adaptive functioning and behavior problems. Autism Res. 2015 Apr;8(2):199-213. doi: 10.1002/aur.1442. PMID: 25641930.X-1

3296. Endreffy I, Bjorklund G, Dicso F, et al. Acid glycosaminoglycan (aGAG) excretion is increased in children with autism spectrum disorder, and it can be controlled by diet. Metab Brain Dis. 2015 Oct 14doi: 10.1007/s11011015-9745-2. PMID: 26464064.X-3

3297. Engelhardt CR, Mazurek MO, Hilgard $\mathrm{J}$, et al. Effects of Violent-Video-Game Exposure on Aggressive Behavior, AggressiveThought Accessibility, and Aggressive Affect Among Adults With and Without Autism Spectrum Disorder. Psychol Sci. 2015 Aug;26(8):1187-200. doi: 10.1177/0956797615583038. PMID: 26113064.X-1

3298. English MC, Maybery MT, Visser TA. Individuals with Autistic-Like Traits Show Reduced Lateralization on a Greyscales Task. J Autism Dev Disord. 2015 Oct;45(10):3390-5. doi: 10.1007/s10803-015-2493-7. PMID: 26060048.X-1

3299. Epitropakis C, DiPietro EA. Medication compliance protocol for pediatric patients with severe intellectual and behavioral disabilities. J Pediatr Nurs. 2015 MarApr;30(2):329-32. doi: 10.1016/j.pedn.2014.08.006. PMID: 25193686.X-1

3300. Erbetta A, Bulgheroni S, Contarino VE, et al. Low-Functioning Autism and Nonsyndromic Intellectual Disability: Magnetic Resonance Imaging (MRI) Findings. J Child Neurol. 2015 Oct;30(12):1658-63. doi: 10.1177/0883073815578523. PMID: 25895913.X-1 
3301. Erviti M, Semal C, Wright BA, et al. A late-emerging auditory deficit in autism.

Neuropsychology. 2015 May;29(3):454-62. doi: 10.1037/neu0000162. PMID: 25495831.X-1

3302. Eryigit-Madzwamuse S, Strauss V, Baumann N, et al. Personality of adults who were born very preterm. Arch Dis Child Fetal Neonatal Ed. 2015 Nov;100(6):F524-9. doi: 10.1136/archdischild-2014-308007. PMID: 26215406.X-1

3303. Esan F, Chester V, Gunaratna IJ, et al. The clinical, forensic and treatment outcome factors of patients with autism spectrum disorder treated in a forensic intellectual disability service. J Appl Res Intellect Disabil. 2015 May;28(3):193-200. doi: 10.1111/jar.12121. PMID: 25379816.X-1

3304. Eshraghi AA, Nazarian R, Telischi FF, et al. Cochlear Implantation in Children With Autism Spectrum Disorder. Otol Neurotol. 2015 Sep;36(8):e121-8. doi: 10.1097/mao.0000000000000757. PMID: 25899551.X-1, X-3

3305. Esler AN, Bal VH, Guthrie W, et al. The Autism Diagnostic Observation Schedule, Toddler Module: Standardized Severity Scores. J Autism Dev Disord. 2015 Sep;45(9):2704-20. doi: 10.1007/s10803-015-2432-7. PMID: 25832801.X-1

3306. Esposito G, Setoh P, Yoshida S, et al. The calming effect of maternal carrying in different mammalian species. Front Psychol. 2015;6:445. doi: 10.3389/fpsyg.2015.00445. PMID: 25932017.X-1

3307. Esposito G, Valenzi S, Islam T, et al. Three physiological responses in fathers and non-fathers' to vocalizations of typically developing infants and infants with Autism Spectrum Disorder. Res Dev Disabil. 2015 Aug-Sep;43-44:43-50. doi: 10.1016/j.ridd.2015.06.007. PMID: 26151442.X-1

3308. Estes A, Munson J, Rogers SJ, et al. Long-Term Outcomes of Early Intervention in 6-Year-Old Children With Autism Spectrum Disorder. J Am Acad Child Adolesc Psychiatry. 2015 Jul;54(7):580-7. doi: 10.1016/j.jaac.2015.04.005. PMID: 26088663.X-1, X-3
3309. Estes A, Zwaigenbaum L, Gu H, et al. Behavioral, cognitive, and adaptive development in infants with autism spectrum disorder in the first 2 years of life. J Neurodev Disord. 2015;7(1):24. doi: 10.1186/s11689-0159117-6. PMID: 26203305.X-1

3310. Evers C, Mitter D, Strobl-Wildemann G, et al. Duplication Xp11.22-p14 in females: does $\mathrm{X}$-inactivation help in assessing their significance? Am J Med Genet A. 2015 Mar;167a(3):553-62. doi: 10.1002/ajmg.a.36897. PMID: 25691408.X-1

3311. Eversole M, Collins DM, Karmarkar A, et al. Leisure Activity Enjoyment of Children with Autism Spectrum Disorders. J Autism Dev Disord. 2015 Jul 26doi: 10.1007/s10803-015-2529-z. PMID: 26210514.X-1

3312. Ewbank M, Pell P, Powell T, et al. Reduced repetition suppression to faces in the fusiform face area of adults with autism spectrum conditions. J Vis. 2015 Sep 1;15(12):1210. doi: 10.1167/15.12.1210. PMID: 26326898.X-1

3313. Faber S, Zinn GM, Boggess A, et al. A cleanroom sleeping environment's impact on markers of oxidative stress, immune dysregulation, and behavior in children with autism spectrum disorders. BMC Complement Altern Med. 2015;15:71. doi: 10.1186/s12906015-0564-0. PMID: 25887094.X-1

3314. Fadini CC, Lamonica DA, Fett-Conte $\mathrm{AC}$, et al. Influence of sleep disorders on the behavior of individuals with autism spectrum disorder. Front Hum Neurosci. 2015;9:347. doi: 10.3389/fnhum.2015.00347. PMID: 26150777.X-1

3315. Faedda GL, Wachtel LE, Higgins AM, et al. Catatonia in an adolescent with velocardio-facial syndrome. Am J Med Genet A. 2015 Sep;167(9):2150-3. doi:

10.1002/ajmg.a.37087. PMID: 25832449.X-1

3316. Fahmie TA, Iwata BA, Jann KE. Comparison of edible and leisure reinforcers. J Appl Behav Anal. 2015 Summer;48(2):331-43. doi: 10.1002/jaba.200. PMID: 25891170.X-1, $\mathrm{X}-3$ 
3317. Fairthorne J, de Klerk N, Leonard H. Brief Report: Burden of Care in Mothers of Children with Autism Spectrum Disorder or Intellectual Disability. J Autism Dev Disord. 2015 Oct 19doi: 10.1007/s10803-015-2629-9. PMID: 26481387.X-1

3318. Fairthorne J, Jacoby P, Bourke J, et al. Onset of maternal psychiatric disorders after the birth of a child with autism spectrum disorder: A retrospective cohort study. Autism. 2015 Feb 4doi: 10.1177/1362361314566048. PMID: 25653305.X-1

3319. Fakroon S, Arheiam A, Omar S. Dental caries experience and periodontal treatment needs of children with autistic spectrum disorder. Eur Arch Paediatr Dent. 2015 Apr;16(2):205-9. doi: 10.1007/s40368014-0156-6. PMID: 25385711.X-1

3320. Falck-Ytter T, Thorup E, Bolte S. Brief report: Lack of processing bias for the objects other people attend to in 3-year-olds with autism. J Autism Dev Disord. 2015 Jun;45(6):1897-904. doi: 10.1007/s10803-0142278-4. PMID: 25331324.X-1

3321. Falco MD, Masala S, Stefanini M, et al. Patient skin dose measurements using a cable free system MOSFETs based in fluoroscopically guided percutaneous vertebroplasty, percutaneous disc decompression, radiofrequency medial branch neurolysis, and endovascular critical limb ischemia. J Appl Clin Med Phys.

2015;16(1):5020. doi:

10.1120/jacmp.v16i1.5020. PMID: 25679159.X-1

3322. Falcomata TS, Muething CS, Roberts GJ, et al. Further evaluation of latency-based brief functional analysis methods: An evaluation of treatment utility. Dev Neurorehabil. 2015 Aug 24:1-7. doi: 10.3109/17518423.2014.910281. PMID: 25029101.X-1, X-3, X-4

3323. Falkmer M, Black M, Tang J, et al. Local visual perception bias in children with high-functioning autism spectrum disorders; do we have the whole picture? Dev Neurorehabil. 2015 Aug 24:1-6. doi: 10.3109/17518423.2014.928387. PMID: 24960245.X-1, X-2
3324. Falkmer M, Oehlers K, Granlund M, et al. Can you see it too? Observed and self-rated participation in mainstream schools in students with and without autism spectrum disorders. Dev Neurorehabil. 2015 Aug 24:1-10. doi: 10.3109/17518423.2013.850751. PMID: 24304126.X-1

3325. Farajollahi AR, Bouzarjomehri F, Kiani M. Comparison between Clinically Used Irregular Fields Shielded by Cerrobend and Standard Lead Blocks. J Biomed Phys Eng. 2015 Jun;5(2):77-82. PMID: 26157733.X-1

3326. Farmer C, Butter E, Mazurek MO, et al. Aggression in children with autism spectrum disorders and a clinic-referred comparison group. Autism. 2015 Apr;19(3):281-91. doi: 10.1177/1362361313518995. PMID: 24497627.X-1

3327. Fein E. Making meaningful worlds: role-playing subcultures and the autism spectrum. Cult Med Psychiatry. 2015 Jun;39(2):299-321. doi: 10.1007/s11013-0159443-x. PMID: 25812848.X-1

3328. Feinberg JI, Bakulski KM, Jaffe AE, et al. Paternal sperm DNA methylation associated with early signs of autism risk in an autismenriched cohort. Int J Epidemiol. 2015 Aug;44(4):1199-210. doi: 10.1093/ije/dyv028. PMID: 25878217.X-1

3329. Fejzo MS, Magtira A, Schoenberg FP, et al. Neurodevelopmental delay in children exposed in utero to hyperemesis gravidarum. Eur J Obstet Gynecol Reprod Biol. 2015 Jun;189:79-84. doi: 10.1016/j.ejogrb.2015.03.028. PMID: 25898368.X-1

3330. Feldman MA, Hendry AM, Ward RA, et al. Behavioral development and sociodemographics of infants and young children at higher and lower risk for autism spectrum disorders. J Autism Dev Disord. 2015 May;45(5):1167-75. doi: 10.1007/s10803-0142277-5. PMID: 25326257.X-1

3331. Fenikile TS, Ellerbeck K, Filippi MK, et al. Barriers to autism screening in family medicine practice: a qualitative study. Prim Health Care Res Dev. 2015 Jul;16(4):356-66. doi: 10.1017/s1463423614000449. PMID: 25367194.X-1 
3332. Fenlon LR, Liu S, Gobius I, et al. Formation of functional areas in the cerebral cortex is disrupted in a mouse model of autism spectrum disorder. Neural Dev. 2015;10:10. doi: 10.1186/s13064-015-0033-y. PMID: 25879444.X-1

3333. Fernandez-Andres MI, PastorCerezuela G, Sanz-Cervera P, et al. A comparative study of sensory processing in children with and without Autism Spectrum Disorder in the home and classroom environments. Res Dev Disabil. 2015 Mar;38:202-12. doi: 10.1016/j.ridd.2014.12.034. PMID: 25575284.X-1

3334. Fernell E, Bejerot S, Westerlund J, et al. Autism spectrum disorder and low vitamin D at birth: a sibling control study. Mol Autism. 2015;6:3. doi: 10.1186/2040-2392-6-3. PMID: 25874075.X-1

3335. Feron F, Gepner B, Lacassagne E, et al. Olfactory stem cells reveal MOCOS as a new player in autism spectrum disorders. Mol Psychiatry. 2015 Aug 4doi: 10.1038/mp.2015.106. PMID: 26239292.X-1

3336. Ferreira-Vasques AT, Lamonica DA. Motor, linguistic, personal and social aspects of children with Down syndrome. J Appl Oral Sci. 2015 Aug;23(4):424-30. doi: 10.1590/1678775720150102. PMID: 26398516.X-1

3337. Fiene L, Brownlow C. Investigating interoception and body awareness in adults with and without autism spectrum disorder. Autism Res. 2015 Mar 25doi: 10.1002/aur.1486. PMID: 25808391.X-1

3338. Fieremans N, Van Esch H, de Ravel T, et al. Microdeletion of the escape genes KDM5C and IQSEC2 in a girl with severe intellectual disability and autistic features. Eur J Med Genet. 2015 May;58(5):324-7. doi: 10.1016/j.ejmg.2015.03.003. PMID: 25858702.X-1

3339. Fiks AG, Mayne SL, Song L, et al. Changing patterns of alpha agonist medication use in children and adolescents 2009-2011. J Child Adolesc Psychopharmacol. 2015 May;25(4):362-7. doi: 10.1089/cap.2014.0122. PMID: 25919708.X-1, X-3
3340. Filip C, Feragen KB, Lemvik JS, et al. Multidisciplinary Aspects of 104 Patients With Pierre Robin Sequence. Cleft Palate Craniofac J. 2015 Jan 2doi: 10.1597/14-161. PMID: 25554857.X-1

3341. Filliter JH, Longard J, Lawrence MA, et al. Positive affect in infant siblings of children diagnosed with autism spectrum disorder. J Abnorm Child Psychol. 2015 Apr;43(3):567-75. doi: 10.1007/s10802-0149921-6. PMID: 25117578.X-1

3342. Finn L, Ramasamy R, Dukes C, et al. Using WatchMinder to Increase the On-Task Behavior of Students with Autism Spectrum Disorder. J Autism Dev Disord. 2015 May;45(5):1408-18. doi: 10.1007/s10803-0142300-x. PMID: 25377769.X-1, X-3

3343. Fiske KE, Isenhower RW, Bamond MJ, et al. Assessing the value of token reinforcement for individuals with autism. $\mathrm{J}$ Appl Behav Anal. 2015 Summer;48(2):448-53. doi: 10.1002/jaba.207. PMID: 25930718.X-1, $\mathrm{X}-3, \mathrm{X}-4$

3344. Fitzgerald J, Johnson K, Kehoe E, et al. Disrupted functional connectivity in dorsal and ventral attention networks during attention orienting in autism spectrum disorders. Autism Res. 2015 Apr;8(2):136-52. doi: 10.1002/aur.1430. PMID: 25428212.X-1

3345. Flanagan T, Brodeur DA, Burack JA. A Point of Departure in the Comparison of Social and Nonsocial Visual Orienting Among Persons With Autism Spectrum Disorders. Autism Res. 2015 Oct;8(5):575-82. doi: 10.1002/aur.1472. PMID: 25755149.X-1

3346. Fleury VP, Thompson JL, Wong C. Learning how to be a student: an overview of instructional practices targeting school readiness skills for preschoolers with autism spectrum disorder. Behav Modif. 2015 Jan;39(1):69-97. doi: 10.1177/0145445514551384. PMID: 25261082.X-1, X-2

3347. Fleva E, Khan A. An examination of the leftward cradling bias among typically developing adults high on autistic traits. Laterality. 2015 Nov;20(6):711-22. doi: 
10.1080/1357650x.2015.1046881. PMID: 26120878.X-1

3348. Flippin M, Watson LR. Fathers' and Mothers' Verbal Responsiveness and the Language Skills of Young Children With Autism Spectrum Disorder. Am J Speech Lang Pathol. 2015 Aug 1;24(3):400-10. doi: 10.1044/2015_ajslp-13-0138. PMID: 25836377.X-1

3349. Floris DL, Lai MC, Auer T, et al. Atypically rightward cerebral asymmetry in male adults with autism stratifies individuals with and without language delay. Hum Brain Mapp. 2015 Oct 23doi: 10.1002/hbm.23023. PMID: 26493275.X-1

3350. Foley D, Reid N, Neels A, et al. Profound hypomagnesaemia secondary to alternative therapy in a child with autism spectrum disorder. J Paediatr Child Health. 2015 Jul;51(7):744-5. doi: 10.1111/jpc.12946. PMID: 26135158.X-1, X-3, X-4

3351. Fortea-Sevilla MS, EscandellBermudez MO, Castro-Sanchez JJ, et al. [Early development of language in small children with autism spectrum disorder using alternative systems]. Rev Neurol. 2015 Feb 25;60 Suppl

1:S31-5. PMID: 25726821.X-3

3352. Fortuna RJ, Robinson L, Smith TH, et al. Health Conditions and Functional Status in Adults with Autism: A Cross-Sectional Evaluation. J Gen Intern Med. 2015 Sep 11doi: 10.1007/s11606-015-3509-x. PMID: 26361965.X-1

3353. Foss-Feig JH, McPartland JC, Anticevic A, et al. Re-conceptualizing ASD Within a Dimensional Framework: Positive, Negative, and Cognitive Feature Clusters. J Autism Dev Disord. 2015 Aug 13doi: 10.1007/s10803-015-2539-x. PMID: 26267330.X-1

3354. Foti F, De Crescenzo F, Vivanti G, et al. Implicit learning in individuals with autism spectrum disorders: a meta-analysis. Psychol Med. 2015 Apr;45(5):897-910. doi: 10.1017/s0033291714001950. PMID: 25126858.X-1, X-2

3355. Foulkes L, Bird G, Gokcen E, et al. Common and distinct impacts of autistic traits and alexithymia on social reward. PLoS One. 2015;10(4):e0121018. doi: 10.1371/journal.pone.0121018. PMID: 25853670.X-1

3356. Fountain C, Zhang Y, Kissin DM, et al. Association between assisted reproductive technology conception and autism in California, 1997-2007. Am J Public Health. 2015

May;105(5):963-71. doi:

10.2105/ajph.2014.302383. PMID:

25790396.X-1

3357. Foxe JJ, Molholm S, Del Bene VA, et al. Severe multisensory speech integration deficits in high-functioning school-aged children with Autism Spectrum Disorder (ASD) and their resolution during early adolescence. Cereb Cortex. 2015 Feb;25(2):298-312. doi: 10.1093/cercor/bht213. PMID: 23985136.X-1

3358. Fraley H. See Me, See My Child: Glimpses into Autism Spectrum Disorder. J Christ Nurs. 2015 Oct-Dec;32(4):212-8. PMID: 26548173.X-1

3359. Frazier TW, Embacher R, Tilot AK, et al. Molecular and phenotypic abnormalities in individuals with germline heterozygous PTEN mutations and autism. Mol Psychiatry. 2015 Sep;20(9):1132-8. doi: 10.1038/mp.2014.125. PMID: 25288137.X-1

3360. Freyberg J, Robertson C, Baron-Cohen S. Atypical Binocular Rivalry Dynamics of Simple and Complex Stimuli in Autism. J Vis. 2015 Sep 1;15(12):643. doi: 10.1167/15.12.643. PMID: 26326331.X-1

3361. Friedrich EV, Sivanathan A, Lim T, et al. An Effective Neurofeedback Intervention to Improve Social Interactions in Children with Autism Spectrum Disorder. J Autism Dev Disord. 2015 Jul 26doi: 10.1007/s10803-0152523-5. PMID: 26210513.X-3

3362. Frisch M, Simonsen J. Ritual circumcision and risk of autism spectrum disorder in 0- to 9-year-old boys: national cohort study in Denmark. J R Soc Med. 2015 Jul;108(7):266-79. doi: 10.1177/0141076814565942. PMID: 25573114.X-1

3363. Frolli A, Piscopo S, Conson M. Developmental changes in cognitive and 
behavioural functioning of adolescents with fragile-X syndrome. J Intellect Disabil Res. 2015 Jul;59(7):613-21. doi: 10.1111/jir.12165. PMID: 25160119.X-1

3364. Frye RE, Slattery J, MacFabe DF, et al. Approaches to studying and manipulating the enteric microbiome to improve autism symptoms. Microb Ecol Health Dis.

2015;26:26878. doi: 10.3402/mehd.v26.26878. PMID: 25956237.X-1

3365. Fu SB, Penrod B, Fernand JK, et al. The Effects of Modeling Contingencies in the Treatment of Food Selectivity in Children With Autism. Behav Modif. 2015 Jul 1doi: 10.1177/0145445515592639. PMID: 26134303.X-1, X-3, X-4

3366. Fuentes-Albero M, Puig-Alcaraz C, Cauli O. Lead excretion in spanish children with autism spectrum disorder. Brain Sci. 2015;5(1):58-68. doi: 10.3390/brainsci5010058. PMID: 25692508.X-1

3367. Fueyo M, Caldwell T, Mattern SB, et al. The Health Home: A Service Delivery Model for Autism and Intellectual Disability. Psychiatr Serv. 2015 Jul 1:appips201400443. doi: 10.1176/appi.ps.201400443. PMID: 26129999.X-1

3368. Fukuhara H, Inoue K, Kurabayashi A, et al. Performance of 5-aminolevulinic-acidbased photodynamic diagnosis for radical prostatectomy. BMC Urol. 2015;15:78. doi: 10.1186/s12894-015-0073-y. PMID: 26232024.X-1

3369. Fukuhara H, Kureishi M, Khoda T, et al. The Utility of a Flexible FluorescenceCystoscope with a Twin Mode Monitor for the 5-Aminolevulinic Acid-Mediated Photodynamic Diagnosis of Bladder Cancer. PLoS One. 2015;10(9):e0136416. doi: 10.1371/journal.pone.0136416. PMID: 26332041.X-1

3370. Fung SC. Increasing the Social Communication of a Boy With Autism Using Animal-assisted Play Therapy: A Case Report. Adv Mind Body Med. 2015 Summer;29(3):2731. PMID: 26026154.X-3, X-4

3371. Furst A, Thron J, Scheele D, et al. The neuropeptide oxytocin modulates consumer brand relationships. Sci Rep. 2015;5:14960. doi: 10.1038/srep14960. PMID: 26449882.X-1

3372. Gabriels RL, Pan Z, Dechant B, et al. Randomized Controlled Trial of Therapeutic Horseback Riding in Children and Adolescents With Autism Spectrum Disorder. J Am Acad Child Adolesc Psychiatry. 2015 Jul;54(7):5419. doi: 10.1016/j.jaac.2015.04.007. PMID: 26088658.X-1

3373. Gadke DL, McKinney C, Oliveros A. Autism Spectrum Disorder Symptoms and Comorbidity in Emerging Adults. Child Psychiatry Hum Dev. 2015 May 21doi: 10.1007/s10578-015-0556-9. PMID: 25995020.X-1

3374. Gal E, Lamash L, Bauminger-Zviely $\mathrm{N}$, et al. Using Multitouch Collaboration Technology to Enhance Social Interaction of Children with High-Functioning Autism. Phys Occup Ther Pediatr. 2015 Sep 30:1-12. doi: 10.3109/01942638.2015.1040572. PMID: 26422262.X-3

3375. Gal E, Selanikyo E, Erez AB, et al. Integration in the Vocational World: How Does It Affect Quality of Life and Subjective WellBeing of Young Adults with ASD. Int J Environ Res Public Health. 2015 Sep;12(9):10820-32. doi: 10.3390/ijerph120910820. PMID: 26404341.X1

3376. Gamsiz ED, Sciarra LN, Maguire AM, et al. Discovery of Rare Mutations in Autism: Elucidating Neurodevelopmental Mechanisms. Neurotherapeutics. 2015 Jul;12(3):553-71. doi: 10.1007/s13311-015-0363-9. PMID: 26105128.X-1

3377. Ganai SA, Ramadoss M, Mahadevan V. Histone Deacetylase (HDAC) Inhibitors emerging roles in neuronal memory, learning, synaptic plasticity and neural regeneration. Curr Neuropharmacol. 2015 Oct 21 PMID: 26487502.X-1

3378. Gandin I, Faletra F, Faletra F, et al. Excess of runs of homozygosity is associated with severe cognitive impairment in intellectual disability. Genet Med. 2015 May;17(5):396-9. doi: 10.1038/gim.2014.118. PMID: 25232855.X-1 
3379. Garbacz SA, McIntyre LL. Conjoint Behavioral Consultation for Children With Autism Spectrum Disorder. Sch Psychol Q. 2015 Feb 16doi: 10.1037/spq0000114. PMID: 25688808.X-3, X-4

3380. Gardner SJ, Gulam M, Song K, et al. Generation and verification of QFix kVue Calypso-compatible couch top model for a dedicated stereotactic linear accelerator with FFF beams. J Appl Clin Med Phys. 2015;16(4):5441. PMID: 26219010.X-1

3381. Garg S, Plasschaert E, Descheemaeker MJ, et al. Autism spectrum disorder profile in neurofibromatosis type I. J Autism Dev Disord. 2015 Jun;45(6):1649-57. doi: 10.1007/s10803014-2321-5. PMID: 25475362.X-1

3382. Gargaro BA, May T, Tonge BJ, et al. Attentional Mechanisms in Autism, ADHD, and Autism-ADHD Using a Local-Global Paradigm. J Atten Disord. 2015 Sep 15doi: 10.1177/1087054715603197. PMID: 26377113.X-1

3383. Gazina EV, Leaw BT, Richards KL, et al. 'Neonatal' Nav1.2 reduces neuronal excitability and affects seizure susceptibility and behaviour. Hum Mol Genet. 2015 Mar 1;24(5):1457-68. doi: 10.1093/hmg/ddu562. PMID: 25378553.X-1

3384. Gee B, Thompson K, Pierce A, et al. The effectiveness of auditory stimulation in children with autism spectrum disorders: A case-control study. International Journal of Therapy \& Rehabilitation. 2015;22(1):36-46 11p. PMID: 103872758. Language: English. Entry Date: 20150116. Revision Date: 20150820. Publication Type: Journal Article.X$1, \mathrm{X}-3, \mathrm{X}-4$

3385. Gengoux GW, Berquist KL, Salzman E, et al. Pivotal Response Treatment Parent Training for Autism: Findings from a 3-Month Follow-Up Evaluation. J Autism Dev Disord. 2015 Sep;45(9):2889-98. doi: 10.1007/s10803015-2452-3. PMID: 25911977.X-1, X-3

3386. Gentry T, Kriner R, Sima A, et al. Reducing the need for personal supports among workers with autism using an iPod Touch as an assistive technology: delayed randomized control trial. J Autism Dev Disord. 2015
Mar;45(3):669-84. doi: 10.1007/s10803-0142221-8. PMID: 25212414.X-1

3387. Geoffroy PA, Etain B, Franchi JA, et al. Melatonin and Melatonin Agonists as Adjunctive Treatments in Bipolar Disorders. Curr Pharm Des. 2015;21(23):3352-8. PMID: 26088111.X-1

3388. Geretsegger M, Holck U, Carpente JA, et al. Common Characteristics of Improvisational Approaches in Music Therapy for Children with Autism Spectrum Disorder: Developing Treatment Guidelines. J Music Ther. 2015 Summer;52(2):258-81. doi: 10.1093/jmt/thv005. PMID: 26019303.X-1, X-3

3389. Germain B, Eppinger MA, Mostofsky $\mathrm{SH}$, et al. Recent Advances in Understanding and Managing Autism Spectrum Disorders. J Child Neurol. 2015 Sep 2doi: 10.1177/0883073815601499. PMID: 26336201.X-1, X-2

3390. Gerow S, Rispoli M, Boles MB, et al. An analysis of contingency statements in a DRO procedure: A case report. Dev Neurorehabil. 2015 Jun;18(3):203-8. doi: 10.3109/17518423.2013.809812. PMID: 23870002.X-1

3391. Gershon ES, Grennan KS. Genetic and genomic analyses as a basis for new diagnostic nosologies. Dialogues Clin Neurosci. 2015 Mar;17(1):69-78. PMID: 25987865.X-1

3392. Gesundheit B, Ashwood P, Keating A, et al. Therapeutic properties of mesenchymal stem cells for autism spectrum disorders. Med Hypotheses. 2015 Mar;84(3):169-77. doi: 10.1016/j.mehy.2014.12.016. PMID: 25592283.X-1, X-2, X-3

3393. Gettings S, Franco F, Santosh PJ. Facilitating support groups for siblings of children with neurodevelopmental disorders using audio-conferencing: a longitudinal feasibility study. Child Adolesc Psychiatry Ment Health. 2015;9:8. doi: 10.1186/s13034015-0041-z. PMID: 25866558.X-1

3394. Ghanizadeh A, Berk M. Beta-lactam antibiotics as a possible novel therapy for managing epilepsy and autism, a case report and review of literature. Iran J Child Neurol. 
2015 Winter;9(1):99-102. PMID: 25767546.X1

3395. Ghanizadeh A, Tordjman S, Jaafari N. Aripiprazole for treating irritability in children \& adolescents with autism: A systematic review. Indian J Med Res. 2015

Sep;142(3):269-75. doi: 10.4103/09715916.166584. PMID: 26458342.X-2, X-3

3396. Ghasemtabar SN, Hosseini M, Fayyaz I, et al. Music therapy: An effective approach in improving social skills of children with autism. Adv Biomed Res. 2015;4:157. doi:

10.4103/2277-9175.161584. PMID: 26380242. INCLUDE

3397. Gilani SZ, Tan DW, Russell-Smith $\mathrm{SN}$, et al. Sexually dimorphic facial features vary according to level of autistic-like traits in the general population. J Neurodev Disord. 2015;7(1):14. doi: 10.1186/s11689-015-9109-6. PMID: 25901187.X-1

3398. Gillam SL, Hartzheim D, Studenka B, et al. Narrative Intervention for Children With Autism Spectrum Disorder (ASD). J Speech Lang Hear Res. 2015 Jun;58(3):920-33. doi: 10.1044/2015_jslhr-l-14-0295. PMID: 25837265.X-3, X-4

3399. Gillberg IC, Helles A, Billstedt E, et al. Boys with Asperger Syndrome Grow Up: Psychiatric and Neurodevelopmental Disorders 20 Years After Initial Diagnosis. J Autism Dev Disord. 2015 Jul 26doi: 10.1007/s10803-0152544-0. PMID: 26210519.X-1

3400. Ginn NC, Clionsky LN, Eyberg SM, et al. Child-Directed Interaction Training for Young Children With Autism Spectrum Disorders: Parent and Child Outcomes. J Clin Child Adolesc Psychol. 2015 Mar 18:1-9. doi: 10.1080/15374416.2015.1015135. PMID: 25785646.X-1

3401. Giovagnoli G, Postorino V, Fatta LM, et al. Behavioral and emotional profile and parental stress in preschool children with autism spectrum disorder. Res Dev Disabil. 2015 OctNov;45-46:411-21. doi: 10.1016/j.ridd.2015.08.006. PMID: 26318505.X-1

3402. Giovannetti T, Seligman SC, Britnell $\mathrm{P}$, et al. Differential effects of goal cues on everyday action errors in Alzheimer's disease versus Parkinson's disease dementia.

Neuropsychology. 2015 Jul;29(4):592-602. doi: 10.1037/neu0000167. PMID: 25495833.X-1

3403. Gipson TT, Poretti A, Thomas EA, et al. Autism Phenotypes in Tuberous Sclerosis Complex: Diagnostic and Treatment Considerations. J Child Neurol. 2015 Aug 24doi: 10.1177/0883073815600871. PMID: 26303410.X-1

3404. Globerson E, Amir N, Kishon-Rabin $\mathrm{L}$, et al. Prosody recognition in adults with high-functioning autism spectrum disorders: from psychoacoustics to cognition. Autism Res. 2015 Apr;8(2):153-63. doi: 10.1002/aur.1432. PMID: 25428545.X-1

3405. Goddard MN, Swaab H, Rombouts SA, et al. Neural systems for social cognition: gray matter volume abnormalities in boys at high genetic risk of autism symptoms, and a comparison with idiopathic autism spectrum disorder. Eur Arch Psychiatry Clin Neurosci. 2015 Aug 2doi: 10.1007/s00406-015-0623-z. PMID: 26233431.X-1

3406. Goin-Kochel RP, Mire SS, Dempsey AG. Emergence of autism spectrum disorder in children from simplex families: relations to parental perceptions of etiology. J Autism Dev Disord. 2015 May;45(5):1451-63. doi: 10.1007/s10803-014-2310-8. PMID: 25398603.X-1

3407. Goitia V, Oquendo M, Stratton R. Case of 7p22.1 Microduplication Detected by Whole Genome Microarray (REVEAL) in Workup of Child Diagnosed with Autism. Case Rep Genet. 2015;2015:212436. doi: 10.1155/2015/212436. PMID: 25893121.X-1

3408. Goldingay S, Stagnitti K, Sheppard L, et al. An intervention to improve social participation for adolescents with autism spectrum disorder: pilot study. Dev Neurorehabil. 2015 Apr;18(2):122-30. doi: 10.3109/17518423.2013.855275. PMID: 24206240.X-1

3409. Goldman S, DeNigris D. Parents' strategies to elicit autobiographical memories in autism spectrum disorders, developmental language disorders and typically developing children. J Autism Dev Disord. 2015 
May;45(5):1464-73. doi: 10.1007/s10803-0142271-y. PMID: 25312278.X-1

3410. Gona JK, Newton CR, Rimba K, et al. Parents' and Professionals' Perceptions on Causes and Treatment Options for Autism Spectrum Disorders (ASD) in a Multicultural Context on the Kenyan Coast. PLoS One. 2015;10(8):e0132729. doi: 10.1371/journal.pone.0132729. PMID: 26267668.X-1

3411. Gong Y, Du Y, Li H, et al. Parenting stress and affective symptoms in parents of autistic children. Sci China Life Sci. 2015 Oct;58(10):1036-43. doi: 10.1007/s11427-0124293-z. PMID: 26335730.X-1

3412. Gonsiorowski A, Williamson RA, Robins DL. Brief Report: Imitation of ObjectDirected Acts in Young Children with Autism Spectrum Disorders. J Autism Dev Disord. 2015 Sep 19doi: 10.1007/s10803-015-2596-1. PMID: 26386710.X-1

3413. Gonzales HK, O'Reilly M, Lang R, et al. Research involving anxiety in non-human primates has potential implications for the assessment and treatment of anxiety in autism spectrum disorder: A translational literature review. Dev Neurorehabil. 2015 Aug 24:1-18. doi: 10.3109/17518423.2014.941117. PMID: 25057887.X-1

3414. Gonzalez EC, Summers C, Mueller V, et al. Developmental Surveillance and Referral in a Traditionally Medically Underserved Border Community. Matern Child Health J. 2015 Nov;19(11):2323-8. doi: 10.1007/s10995015-1741-y. PMID: 26386709.X-1

3415. Goodkind MS, Sturm VE, Ascher EA, et al. Emotion recognition in frontotemporal dementia and Alzheimer's disease: A new filmbased assessment. Emotion. 2015 Aug;15(4):416-27. doi: 10.1037/a0039261. PMID: 26010574.X-1

3416. Goodwin A, Fein D, Naigles L. The role of maternal input in the development of wh-question comprehension in autism and typical development. Journal of Child Language. 2015;42(1):32-63 32p. doi: 10.1017/S0305000913000524. PMID: 103922637. Language: English. Entry Date:
20141208. Revision Date: 20150820. Publication Type: Journal Article.X-1

3417. Gopinath N, Muneer AK, Unnikrishnan S, et al. Children (10-12 years age) of women with epilepsy have lower intelligence, attention and memory: Observations from a prospective cohort case control study. Epilepsy Res. 2015 Sep 9;117:58-62. doi:

10.1016/j.eplepsyres.2015.09.003. PMID: 26418668.X-1

3418. Gordon EA, Corbitt C. Investigation of pre-pubertal sex differences in wheel running and social behavior in three mouse strains. J Ethol. 2015 Aug;33(3):177-87. doi: 10.1007/s10164-015-0429-5. PMID: 26316671.X-1

3419. Gordon K, Murin M, Baykaner O, et al. A randomised controlled trial of PEGASUS, a psychoeducational programme for young people with high-functioning autism spectrum disorder. J Child Psychol Psychiatry. 2015 Apr;56(4):468-76. doi: 10.1111/jcpp.12304. PMID: 25132516.X-1

3420. Gori I, Giuliano A, Muratori F, et al. Gray Matter Alterations in Young Children with Autism Spectrum Disorders: Comparing Morphometry at the Voxel and Regional Level. J Neuroimaging. 2015 Nov;25(6):866-74. doi: 10.1111/jon.12280. PMID: 26214066.X-1

3421. Gotham K, Marvin AR, Taylor JL, et al. Characterizing the daily life, needs, and priorities of adults with autism spectrum disorder from Interactive Autism Network data. Autism. 2015 Oct;19(7):794-804. doi: 10.1177/1362361315583818. PMID: 25964655.X-1

3422. Gotham K, Unruh K, Lord C. Depression and its measurement in verbal adolescents and adults with autism spectrum disorder. Autism. 2015 May;19(4):491-504. doi: 10.1177/1362361314536625. PMID: 24916450.X-1

3423. Gozes I, Yeheskel A, Pasmanik-Chor M. Activity-dependent neuroprotective protein (ADNP): a case study for highly conserved chordata-specific genes shaping the brain and mutated in cancer. J Alzheimers Dis. 
2015;45(1):57-73. doi: 10.3233/jad-142490. PMID: 25428252.X-1

3424. Graham S, Abbott A, Nair A, et al. The Influence of Task Difficulty and Participant Age on Balance Control in ASD. Journal of Autism \& Developmental Disorders. 2015;45(5):1419-27 9p. doi: 10.1007/s10803014-2303-7. PMID: 103791717. Language: English. Entry Date: 20150424. Revision Date: 20150710. Publication Type: Journal Article.X1

3425. Grahame V, Brett D, Dixon L, et al. Managing Repetitive Behaviours in Young Children with Autism Spectrum Disorder (ASD): Pilot Randomised Controlled Trial of a New Parent Group Intervention. J Autism Dev Disord. 2015 Oct;45(10):3168-82. doi: 10.1007/s10803-015-2474-x. PMID: 26036646.X-1

3426. Grandgeorge M, Bourreau Y, Alavi Z, et al. Interest towards human, animal and object in children with autism spectrum disorders: an ethological approach at home. European Child \& Adolescent Psychiatry. 2015;24(1):83-93 11p. doi: 10.1007/s00787-014-0528-9. PMID: 103873284. Language: English. Entry Date: 20150115. Revision Date: 20150710. Publication Type: Journal Article.X-1, X-3

3427. Grange P, Menashe I, Hawrylycz M. Cell-type-specific neuroanatomy of cliques of autism-related genes in the mouse brain. Front Comput Neurosci. 2015;9:55. doi: 10.3389/fncom.2015.00055. PMID: 26074809.X-1

3428. Grant N, Rodger S, Hoffmann T. Intervention decision-making processes and information preferences of parents of children with autism spectrum disorders. Child Care Health Dev. 2015 Oct 22doi: 10.1111/cch.12296. PMID: 26489390.X-1

3429. Grant N, Rodger S, Hoffmann T. Evaluation of Autism-Related Health Information on the Web. J Appl Res Intellect Disabil. 2015 Jul;28(4):276-82. doi: 10.1111/jar.12127. PMID: 25224690.X-1

3430. Grapel JN, Cicchetti DV, Volkmar FR. Sensory features as diagnostic criteria for autism: sensory features in autism. Yale J Biol
Med. 2015 Mar;88(1):69-71. PMID: 25745375.X-1

3431. Gray PH, Edwards DM, O'Callaghan MJ, et al. Screening for autism spectrum disorder in very preterm infants during early childhood. Early Hum Dev. 2015 Apr;91(4):271-6. doi: 10.1016/j.earlhumdev.2015.02.007. PMID: 25766314.X-1

3432. Grayson B, Leger M, Piercy C, et al. Assessment of disease-related cognitive impairments using the novel object recognition (NOR) task in rodents. Behav Brain Res. 2015 May 15;285:176-93. doi: 10.1016/j.bbr.2014.10.025. PMID: 25447293.X-1

3433. Green J, Charman T, Pickles A, et al. Parent-mediated intervention versus no intervention for infants at high risk of autism: a parallel, single-blind, randomised trial. Lancet Psychiatry. 2015 Feb;2(2):133-40. doi: 10.1016/s2215-0366(14)00091-1. PMID: 26359749.X-1

3434. Green JL, Rinehart N, Anderson V, et al. Autism spectrum disorder symptoms in children with ADHD: A community-based study. Res Dev Disabil. 2015 Oct 1;47:175-84. doi: 10.1016/j.ridd.2015.09.016. PMID: 26433184.X-1

3435. Green RR, Bigler ED, Froehlich A, et al. Beery VMI performance in autism spectrum disorder. Child Neuropsychol. 2015 Aug 21:123. doi: 10.1080/09297049.2015.1056131. PMID: 26292997.X-1

3436. Green SA, Hernandez L, Tottenham N, et al. Neurobiology of Sensory

Overresponsivity in Youth With Autism Spectrum Disorders. JAMA Psychiatry. 2015 Aug;72(8):778-86. doi: 10.1001/jamapsychiatry.2015.0737. PMID: 26061819.X-1

3437. Greenberg DM, Baron-Cohen S, Stillwell DJ, et al. Musical Preferences are Linked to Cognitive Styles. PLoS One. 2015;10(7):e0131151. doi: 10.1371/journal.pone.0131151. PMID: 26200656.X-1 
3438. Greener M. Research roundup: June 2015. Nurse Prescribing. 2015;13(6):274-6 3p. PMID: 109804915. Language: English. Entry Date: 20150616. Revision Date: 20151008. Publication Type: Journal Article. Journal Subset: Double Blind Peer Reviewed.X-1, X-2

3439. Greger HK, Myhre AK, Lydersen S, et al. Previous maltreatment and present mental health in a high-risk adolescent population. Child Abuse Negl. 2015 Jul;45:122-34. doi: 10.1016/j.chiabu.2015.05.003. PMID: 26003821.X-1

3440. Grice SJ, Liu JL, Webber C. Synergistic interactions between Drosophila orthologues of genes spanned by de novo human CNVs support multiple-hit models of autism. PLoS Genet. 2015 Mar;11(3):e1004998. doi: 10.1371/journal.pgen.1004998. PMID: 25816101.X-1

3441. Griesi-Oliveira K, Acab A, Gupta AR, et al. Modeling non-syndromic autism and the impact of TRPC6 disruption in human neurons. Mol Psychiatry. 2015 Nov;20(11):1350-65. doi: 10.1038/mp.2014.141. PMID: 25385366.X-1

3442. Griffin C, Lombardo MV, Auyeung B. Alexithymia in children with and without autism spectrum disorders. Autism Res. 2015 Oct 1doi: 10.1002/aur.1569. PMID: 26426084.X-1

3443. Grimm D, Assouline B, Piero A. [Schooling of patients exhibiting Autism Spectrum Disorders without mental retardation]. Encephale. 2015 Sep 7doi: 10.1016/j.encep.2015.07.005. PMID: 26358485.X-1

3444. Grinker RR, Kang-Yi CD, Ahmann C, et al. Cultural Adaptation and Translation of Outreach Materials on Autism Spectrum Disorder. J Autism Dev Disord. 2015 Aug;45(8):2329-36. doi: 10.1007/s10803-0152397-6. PMID: 25758819.X-1

3445. Groskreutz MP, Peters A, Groskreutz NC, et al. Increasing play-based commenting in children with autism spectrum disorder using a novel script-frame procedure. J Appl Behav Anal. 2015 Summer;48(2):442-7. doi: 10.1002/jaba.194. PMID: 25891090.X-1
3446. Gross C, Hoffmann A, Bassell GJ, et al. Therapeutic Strategies in Fragile $X$ Syndrome: From Bench to Bedside and Back. Neurotherapeutics. 2015 Jul;12(3):584-608. doi: 10.1007/s13311-015-0355-9. PMID: 25986746.X-1

3447. Gross JA, Bureau A, Croteau J, et al. A genome-wide copy number variant study of suicidal behavior. PLoS One.

2015;10(5):e0128369. doi: 10.1371/journal.pone.0128369. PMID: 26010658.X-1

3448. Grossman RB, Steinhart E, Mitchell T, et al. "Look who's talking!" gaze patterns for implicit and explicit audio-visual speech synchrony detection in children with highfunctioning autism. Autism Research. 2015 2015-08-26;8(3):307-16. doi: http://dx.doi.org/10.1002/aur.1447. PMID: 1707076864; 2015-27677-001.X-1, X-2

3449. Grossman RB, Steinhart E, Mitchell T, et al. "Look who's talking!" Gaze Patterns for Implicit and Explicit Audio-Visual Speech Synchrony Detection in Children With HighFunctioning Autism. Autism Res. 2015 Jun;8(3):307-16. doi: 10.1002/aur.1447. PMID: 25620208.X-1

3450. Grove R, Roth I, Hoekstra RA. The motivation for special interests in individuals with autism and controls: Development and validation of the special interest motivation scale. Autism Res. 2015 Oct 24doi: 10.1002/aur.1560. PMID: 26496939.X-1

3451. Gu C, Shen T, An H, et al. Combined therapy of Di-Huang-Yi-Zhi with Donepezil in patients with Parkinson's disease dementia. Neurosci Lett. 2015 Oct 8;606:13-7. doi: 10.1016/j.neulet.2015.08.019. PMID: 26297124.X-1

3452. Gu X, Eilam-Stock T, Zhou T, et al. Autonomic and brain responses associated with empathy deficits in autism spectrum disorder. Hum Brain Mapp. 2015 Sep;36(9):3323-38. doi: 10.1002/hbm.22840. PMID: 25995134.X-1

3453. Guastella AJ, Gray KM, Rinehart NJ, et al. The effects of a course of intranasal oxytocin on social behaviors in youth diagnosed with autism spectrum disorders: a randomized controlled trial. J Child Psychol Psychiatry. 
2015 Apr;56(4):444-52. doi:

10.1111/jcpp.12305. PMID: 25087908.X-1

3454. Guevara-Campos J, Gonzalez-Guevara L, Cauli O. Autism and intellectual disability associated with mitochondrial disease and hyperlactacidemia. Int J Mol Sci.

2015;16(2):3870-84. doi:

10.3390/ijms16023870. PMID: 25679448.X-1

3455. Guinchat V, Cravero C, Diaz L, et al. Acute behavioral crises in psychiatric inpatients with autism spectrum disorder (ASD): recognition of concomitant medical or nonASD psychiatric conditions predicts enhanced improvement. Res Dev Disabil. 2015

Mar;38:242-55. doi:

10.1016/j.ridd.2014.12.020. PMID:

25575287.X-1

3456. Guy J, Perreault A, Mottron L, et al. A systematic examination of early perceptual influences on low-, mid and high-level visual abilities in Autism Spectrum Disorder. J Vis. 2015 Sep 1;15(12):644. doi: 10.1167/15.12.644. PMID: 26326332.X-1

3457. Guyatt AL, Heron J, Knight Ble C, et al. Digit ratio and autism spectrum disorders in the Avon Longitudinal Study of Parents and Children: a birth cohort study. BMJ Open. 2015;5(8):e007433. doi: 10.1136/bmjopen2014-007433. PMID: 26307613.X-1

3458. Habibe RC, Ortega AO, Guare RO, et al. Risk factors for anterior traumatic dental injury in children and adolescents with autism spectrum disorders: a case-control study. Eur Arch Paediatr Dent. 2015 Oct 8doi: 10.1007/s40368-015-0207-7. PMID: 26449618.X-1

3459. Hadjikhani N, Zürcher NR, Rogier O, et al. Improving emotional face perception in autism with diuretic bumetanide: A proof-ofconcept behavioral and functional brain imaging pilot study. Autism: The International Journal of Research \& Practice.

2015;19(2):149-57 9p. doi:

10.1177/1362361313514141. PMID:

103750459. Language: English. Entry Date:

20150130. Revision Date: 20150710.

Publication Type: Journal Article.X-1, X-3

3460. Hagberg B, Billstedt E, Nyden A, et al. Asperger syndrome and nonverbal learning difficulties in adult males: self- and parentreported autism, attention and executive problems. Eur Child Adolesc Psychiatry. 2015 Aug;24(8):969-77. doi: 10.1007/s00787-0140646-4. PMID: 25399237.X-1

3461. Hagen E, Shprung D, Minakova E, et al. Autism-Like Behavior in BTBR Mice Is Improved by Electroconvulsive Therapy. Neurotherapeutics. 2015 Jul;12(3):657-66. doi: 10.1007/s13311-015-0357-7. PMID: 25916397.X-1

3462. Haigh SM, Heeger DJ, Dinstein I, et al. Cortical variability in the sensory-evoked response in autism. J Autism Dev Disord. 2015 May;45(5):1176-90. doi: 10.1007/s10803-0142276-6. PMID: 25326820.X-1

3463. Hall SS, Frank MC, Pusiol GT, et al. Quantifying naturalistic social gaze in fragile $\mathrm{X}$ syndrome using a novel eye tracking paradigm. Am J Med Genet B Neuropsychiatr Genet. 2015 Oct;168(7):564-72. doi: 10.1002/ajmg.b.32331. PMID: 26079280.X-1

3464. Hall T, Kriz D, Duvall S, et al. Healthcare transition challenges faced by young adults with autism spectrum disorder. Clin Pharmacol Ther. 2015 Dec;98(6):573-5. doi: 10.1002/cpt.254. PMID: 26300522.X-1

3465. Halladay AK, Bishop S, Constantino $\mathrm{JN}$, et al. Sex and gender differences in autism spectrum disorder: summarizing evidence gaps and identifying emerging areas of priority. Mol Autism. 2015;6:36. doi: 10.1186/s13229-0150019-y. PMID: 26075049.X-1

3466. Hallbook T, Sjolander A, Amark P, et al. Effectiveness of the ketogenic diet used to treat resistant childhood epilepsy in Scandinavia. Eur J Paediatr Neurol. 2015 Jan;19(1):29-36. doi: 10.1016/j.ejpn.2014.09.005. PMID: 25457511.X-1

3467. Han B, Tijus C, Le Barillier F, et al. Morphing technique reveals intact perception of object motion and disturbed perception of emotional expressions by low-functioning adolescents with Autism Spectrum Disorder. Res Dev Disabil. 2015 Dec;47:393-404. doi: 10.1016/j.ridd.2015.09.025. PMID: 26513740.X-1 
3468. Han Y, Xi QQ, Dai W, et al. Abnormal transsulfuration metabolism and reduced antioxidant capacity in Chinese children with autism spectrum disorders. Int J Dev Neurosci. 2015 Nov;46:27-32. doi: 10.1016/j.ijdevneu.2015.06.006. PMID: 26150135.X-1

3469. Hanne M. Diagnosis and Metaphor. Perspect Biol Med. 2015 Winter;58(1):35-52. doi: 10.1353/pbm.2015.0010. PMID: 26657680.X-1

3470. Hanson E, Bernier R, Porche K, et al. The cognitive and behavioral phenotype of the $16 p 11.2$ deletion in a clinically ascertained population. Biol Psychiatry. 2015 May 1;77(9):785-93. doi: 10.1016/j.biopsych.2014.04.021. PMID: 25064419.X-1

3471. Haq SS, Kodak T, Kurtz-Nelson E, et al. Comparing the effects of massed and distributed practice on skill acquisition for children with autism. J Appl Behav Anal. 2015 Summer;48(2):454-9. doi: 10.1002/jaba.213. PMID: 25962478.X-1

3472. Hardan AY, Gengoux GW, Berquist KL, et al. A randomized controlled trial of Pivotal Response Treatment Group for parents of children with autism. J Child Psychol Psychiatry. 2015 Aug;56(8):884-92. doi: 10.1111/jcpp.12354. PMID: 25346345.X-1

3473. Hare DJ, Wood C, Wastell S, et al. Anxiety in Asperger's syndrome: Assessment in real time. Autism. 2015 Jul;19(5):542-52. doi: 10.1177/1362361314531340. PMID: 24811968.X-1

3474. Harfterkamp M, van der Meer D, van der Loo-Neus G, et al. No evidence for predictors of response to atomoxetine treatment of attention-deficit/hyperactivity disorder symptoms in children and adolescents with autism spectrum disorder. J Child Adolesc Psychopharmacol. 2015 May;25(4):372-5. doi: 10.1089/cap.2014.0142. PMID: 25919900.X-2

3475. Harstad EB, Fogler J, Sideridis G, et al. Comparing Diagnostic Outcomes of Autism Spectrum Disorder Using DSM-IV-TR and DSM-5 Criteria. J Autism Dev Disord. 2015 May;45(5):1437-50. doi: 10.1007/s10803-0142306-4. PMID: 25384720.X-1
3476. Hartley SL, Schultz HM. Support needs of fathers and mothers of children and adolescents with autism spectrum disorder. $\mathrm{J}$ Autism Dev Disord. 2015 Jun;45(6):1636-48. doi: 10.1007/s10803-014-2318-0. PMID: 25433405.X-1

3477. Hasenstab K, Sugar CA, Telesca D, et al. Identifying longitudinal trends within EEG experiments. Biometrics. 2015 Jul 20doi: 10.1111/biom.12347. PMID: 26195327.X-1

3478. Hashemi B, Bassett A, Chitayat D, et al. Deletion of 15q11.2(BP1-BP2) region: Further evidence for lack of phenotypic specificity in a pediatric population. Am J Med Genet A. 2015 Sep;167(9):2098-102. doi: 10.1002/ajmg.a.37134. PMID: 25946043.X-1

3479. Heavner K, Burstyn I. A Simulation Study of Categorizing Continuous Exposure Variables Measured with Error in Autism Research: Small Changes with Large Effects. Int J Environ Res Public Health. 2015 Aug;12(8):10198-234. doi: 10.3390/ijerph120810198. PMID: 26305250.X1

3480. Heberling C, Dhurjati P. Novel systems modeling methodology in comparative microbial metabolomics: identifying key enzymes and metabolites implicated in autism spectrum disorders. Int J Mol Sci.

2015;16(4):8949-67. doi:

10.3390/ijms16048949. PMID: 25913376.X-1

3481. Hedley D, Nevill RE, Monroy-Moreno $\mathrm{Y}$, et al. Efficacy of the ADEC in Identifying Autism Spectrum Disorder in Clinically Referred Toddlers in the US. J Autism Dev Disord. 2015 Aug;45(8):2337-48. doi: 10.1007/s10803-015-2398-5. PMID: 25737020.X-1

3482. Hedvall A, Westerlund J, Fernell E, et al. Preschoolers with Autism Spectrum Disorder Followed for 2 Years: Those Who Gained and Those Who Lost the Most in Terms of Adaptive Functioning Outcome. J Autism Dev Disord. 2015 Nov;45(11):3624-33. doi: 10.1007/s10803-015-2509-3. PMID: 26123008.X-1

3483. Heffler KF, Oestreicher LM. Causation model of autism: Audiovisual brain 
specialization in infancy competes with social brain networks. Med Hypotheses. 2015 Jun 27doi: 10.1016/j.mehy.2015.06.019. PMID: 26146132.X-1

3484. Heidary A, Hosseini Shahidi L, Mohammadpuor A. Spiritual Journey in Mothers' Lived Experiences of Caring for Children With Autism Spectrum Disorders. Glob J Health Sci. 2015 Nov;7(6):79-87. doi: 10.5539/gjhs.v7n6p79. PMID: 26153169.X-1

3485. Heisler JM, Morales J, Donegan JJ, et al. The attentional set shifting task: a measure of cognitive flexibility in mice. J Vis Exp. 2015(96)doi: 10.3791/51944. PMID: 25741905.X-1

3486. Helles A, Gillberg CI, Gillberg C, et al. Asperger syndrome in males over two decades: stability and predictors of diagnosis. J Child Psychol Psychiatry. 2015 Jun;56(6):7118. doi: 10.1111/jcpp.12334. PMID: 25283685.X-1

3487. Hellings JA, Jadhav M, Jain S, et al. Low Dose Loxapine: Neuromotor Side Effects and Tolerability in Autism Spectrum Disorders. J Child Adolesc Psychopharmacol. 2015 Oct;25(8):618-24. doi: 10.1089/cap.2014.0145. PMID: 26485086.X-1, X-3

3488. Hellings JA, Reed G, Cain SE, et al. Loxapine add-on for adolescents and adults with autism spectrum disorders and irritability. J Child Adolesc Psychopharmacol. 2015 Mar;25(2):150-9. doi: 10.1089/cap.2014.0003. PMID: 25782098.X-1

3489. Hellwig S, Frings L, Amtage F, et al. 18F-FDG PET Is an Early Predictor of Overall Survival in Suspected Atypical Parkinsonism. J Nucl Med. 2015 Oct;56(10):1541-6. doi: 10.2967/jnumed.115.159822. PMID: 26229141.X-1

3490. Hepburn SL, Blakeley-Smith A, Wolff $\mathrm{B}$, et al. Telehealth delivery of cognitivebehavioral intervention to youth with autism spectrum disorder and anxiety: A pilot study. Autism. 2015 Apr 20doi: 10.1177/1362361315575164. PMID: 25896267.X-1, X-3

3491. Herbrecht E, Kievit E, Spiegel R, et al. Become Related: FIAS, an Intensive Early
Intervention for Young Children with Autism Spectrum Disorders. Psychopathology. 2015;48(3):162-72. doi: 10.1159/000375504. PMID: 25832916.X-3, X-4

3492. Herndon JM. Evidence of Coal-FlyAsh Toxic Chemical Geoengineering in the Troposphere: Consequences for Public Health. Int J Environ Res Public Health. 2015

Aug;12(8):9375-90. doi: 10.3390/ijerph120809375. PMID: 26270671.X1

3493. Herpers PC, Klip H, Rommelse NN, et al. Associations between high callousunemotional traits and quality of life across youths with non-conduct disorder diagnoses. Eur Child Adolesc Psychiatry. 2015 Sep 11doi: 10.1007/s00787-015-0766-5. PMID: 26362863.X-1

3494. Herrmann S. Counting Sheep: Sleep Disorders in Children With Autism Spectrum Disorders. J Pediatr Health Care. 2015 Aug 22doi: 10.1016/j.pedhc.2015.07.003. PMID: 26307198.X-1

3495. Hidalgo NJ, Mc IL, Mc WE. Sociodemographic differences in parental satisfaction with an autism spectrum disorder diagnosis. J Intellect Dev Disabil. 2015;40(2):147-55. doi: 10.3109/13668250.2014.994171. PMID: 26213482.X-1

3496. Hidding E, Swaab H, de Sonneville $\mathrm{LM}$, et al. Intellectual functioning in relation to autism and ADHD symptomatology in children and adolescents with 22q11.2 deletion syndrome. J Intellect Disabil Res. 2015 Sep;59(9):803-15. doi: 10.1111/jir.12187. PMID: 25683771.X-1

3497. Higdon R, Earl RK, Stanberry L, et al. The promise of multi-omics and clinical data integration to identify and target personalized healthcare approaches in autism spectrum disorders. Omics. 2015 Apr;19(4):197-208. doi: 10.1089/omi.2015.0020. PMID: 25831060.X-1

3498. Higuchi Y, Uchitomi Y, Fujimori M, et al. Exploring autistic-like traits relating to empathic attitude and psychological distress in hospital pharmacists. Int J Clin Pharm. 2015 Dec;37(6):1258-66. doi: 10.1007/s11096-0150204-2. PMID: 26441314.X-1 
3499. Hiller RM, Young RL, Weber N. Sex differences in pre-diagnosis concerns for children later diagnosed with autism spectrum disorder. Autism. 2015 Feb 25doi: 10.1177/1362361314568899. PMID: 25717130.X-1

3500. Hiraishi H, Kikuchi M, Yoshimura Y, et al. Unusual developmental pattern of brain lateralization in young boys with autism spectrum disorder: Power analysis with childsized magnetoencephalography. Psychiatry Clin Neurosci. 2015 Mar;69(3):153-60. doi: 10.1111/pcn.12261. PMID: 25439739.X-1

3501. Hirvikoski T, Blomqvist M. High selfperceived stress and poor coping in intellectually able adults with autism spectrum disorder. Autism. 2015 Aug;19(6):752-7. doi: 10.1177/1362361314543530. PMID: 25073750.X-1

3502. Hobson JA, Tarver L, Beurkens N, et al. The Relation between Severity of Autism and Caregiver-Child Interaction: a Study in the Context of Relationship Development Intervention. J Abnorm Child Psychol. 2015 Aug 23doi: 10.1007/s10802-015-0067-y. PMID: 26298470.X-1, X-3

3503. Hobson P, Meara J. Mild cognitive impairment in Parkinson's disease and its progression onto dementia: a 16-year outcome evaluation of the Denbighshire cohort. Int J Geriatr Psychiatry. 2015 Oct;30(10):1048-55. doi: 10.1002/gps.4261. PMID: 25676160.X-1

3504. Hock R, Kinsman A, Ortaglia A. Examining treatment adherence among parents of children with autism spectrum disorder. Disabil Health J. 2015 Jul;8(3):407-13. doi: 10.1016/j.dhjo.2014.10.005. PMID: 25595296.X-1

3505. Hodgetts S, Zwaigenbaum L, Nicholas D. Profile and predictors of service needs for families of children with autism spectrum disorders. Autism. 2015 Aug;19(6):673-83. doi: 10.1177/1362361314543531. PMID: 25073749.X-1

3506. Hofmann SG, Fang A, Brager DN. Effect of intranasal oxytocin administration on psychiatric symptoms: A meta-analysis of placebo-controlled studies. Psychiatry Res.
2015 Aug 30;228(3):708-14. doi: 10.1016/j.psychres.2015.05.039. PMID: 26094200.X-2, X-3

3507. Holmes GL, Tian C, Hernan AE, et al. Alterations in sociability and functional brain connectivity caused by early-life seizures are prevented by bumetanide. Neurobiol Dis. 2015 May;77:204-19. doi: 10.1016/j.nbd.2015.02.015. PMID: 25766676.X-1

3508. Hong ER, Neely L, Rispoli MJ, et al. A comparison of general and explicit delay cues to reinforcement for tangible-maintained challenging behaviour. Dev Neurorehabil. 2015 Aug 24:1-7. doi: 10.3109/17518423.2013.874378. PMID: 24392663.X-1, X-4

3509. Hong LJ, Jiang Q, Long S, et al. Valproic Acid Influences MTNR1A Intracellular Trafficking and Signaling in a beta-Arrestin 2-Dependent Manner. Mol Neurobiol. 2015 Jan 24doi: 10.1007/s12035014-9085-y. PMID: 25613019.X-1

3510. Hongkaew Y, Ngamsamut N, Puangpetch A, et al. Hyperprolactinemia in Thai children and adolescents with autism spectrum disorder treated with risperidone. Neuropsychiatr Dis Treat. 2015;11:191-6. doi: 10.2147/ndt.s76276. PMID: 25653528.X-1, X-3

3511. Horlin C, Black M, Falkmer M, et al. Proficiency of individuals with autism spectrum disorder at disembedding figures: A systematic review. Dev Neurorehabil. 2015 Aug 24:1-10. doi: 10.3109/17518423.2014.888102. PMID: 24649841.X-1, X-2

3512. Howe YJ, O'Rourke JA, Yatchmink Y, et al. Female Autism Phenotypes Investigated at Different Levels of Language and Developmental Abilities. J Autism Dev Disord. 2015 Nov;45(11):3537-49. doi: 10.1007/s10803-015-2501-y. PMID: 26100851.X-1

3513. Hranilovic D, Blazevic S, Stefulj J, et al. DNA Methylation Analysis of HTR2A Regulatory Region in Leukocytes of Autistic Subjects. Autism Res. 2015 Jul 7doi: 10.1002/aur.1519. PMID: 26149086.X-1 
3514. Hu VW, Sarachana T, Sherrard RM, et al. Investigation of sex differences in the expression of RORA and its transcriptional targets in the brain as a potential contributor to the sex bias in autism. Mol Autism. 2015;6:7. doi: 10.1186/2040-2392-6-7. PMID: 26056561.X-1

3515. Huang SF, Oi M, Taguchi A. Comprehension of figurative language in Taiwanese children with autism: The role of theory of mind and receptive vocabulary. Clin Linguist Phon. 2015 Aug;29(8-10):764-75. doi: 10.3109/02699206.2015.1027833. PMID: 25909823.X-1

3516. Hudac CM, Kresse A, Aaronson B, et al. Modulation of mu attenuation to social stimuli in children and adults with 16p11.2 deletions and duplications. J Neurodev Disord. 2015;7(1):25. doi: 10.1186/s11689-015-9118-5. PMID: 26213586.X-1

3517. Huguet G, Contejean Y, Doyen C. [Autism spectrum disorder and suicidality]. Encephale. 2015 Sep;41(4):362-9. doi: 10.1016/j.encep.2014.08.010. PMID: 25200592.X-1

3518. Hui Z, Yongchao Z, Yongqing Z. Recent progresses in molecular genetics of autism spectrum disorders. Yi Chuan. 2015 Sep;37(9):845-54. doi: 10.16288/j.yczz.15-281. PMID: 26399524.X-1

3519. Huijbregts SC, Loitfelder M, Rombouts SA, et al. Cerebral volumetric abnormalities in Neurofibromatosis type 1: associations with parent ratings of social and attention problems, executive dysfunction, and autistic mannerisms. J Neurodev Disord. 2015;7:32. doi: 10.1186/s11689-015-9128-3. PMID: 26473019.X-1

3520. Hull MM, Madhavan D, Zaroff CM. Autistic spectrum disorder, epilepsy, and vagus nerve stimulation. Childs Nerv Syst. 2015 Aug;31(8):1377-85. doi: 10.1007/s00381-0152720-8. PMID: 25922052.X-1

3521. Hurwitz S, Watson LR. Joint attention revisited: Finding strengths among children with autism. Autism. 2015 Jul 6doi: 10.1177/1362361315593536. PMID: 26148983.X-1
3522. Huskens B, Palmen A, Van der Werff $\mathrm{M}$, et al. Improving Collaborative Play Between Children with Autism Spectrum Disorders and Their Siblings: The Effectiveness of a RobotMediated Intervention Based on Lego((R)) Therapy. J Autism Dev Disord. 2015 Nov;45(11):3746-55. doi: 10.1007/s10803-0142326-0. PMID: 25428293.X-1, X-3

3523. Hustyi KM, Hall SS, Quintin EM, et al. The relationship between autistic symptomatology and independent living skills in adolescents and young adults with fragile $X$ syndrome. J Autism Dev Disord. 2015 Jun;45(6):1836-44. doi: 10.1007/s10803-0142342-0. PMID: 25518824.X-1

3524. Iadarola S, Hetherington S, Clinton C, et al. Services for children with autism spectrum disorder in three, large urban school districts: Perspectives of parents and educators. Autism. 2015 Aug;19(6):694-703. doi: 10.1177/1362361314548078. PMID: 25192859.X-1

3525. Iannuzzi DA, Cheng ER, BroderFingert S, et al. Brief report: Emergency department utilization by individuals with autism. J Autism Dev Disord. 2015 Apr;45(4):1096-102. doi: 10.1007/s10803-0142251-2. PMID: 25261249.X-1

3526. Igartua DE, Calienni MN, Feas DA, et al. Development of Nutraceutical Emulsions as Risperidone Delivery Systems: Characterization and Toxicological Studies. J Pharm Sci. 2015 Sep 11doi: 10.1002/jps.24636. PMID: 26359783.X-1

3527. Ingersoll B, Berger NI. Parent Engagement With a Telehealth-Based ParentMediated Intervention Program for Children With Autism Spectrum Disorders: Predictors of Program Use and Parent Outcomes. J Med Internet Res. 2015;17(10):e227. doi: 10.2196/jmir.4913. PMID: 26443557.X-1

3528. Inoue K, Anai S, Fujimoto K, et al. Oral 5-aminolevulinic acid mediated photodynamic diagnosis using fluorescence cystoscopy for non-muscle-invasive bladder cancer: A randomized, double-blind, multicentre phase II/III study. Photodiagnosis Photodyn Ther. 2015 Jun;12(2):193-200. doi: 10.1016/j.pdpdt.2015.03.008. PMID: 25843912.X-1 
3529. Iourov IY, Vorsanova SG, Korostelev SA, et al. Long contiguous stretches of homozygosity spanning shortly the imprinted loci are associated with intellectual disability, autism and/or epilepsy. Mol Cytogenet. 2015;8:77. doi: 10.1186/s13039-015-0182-z. PMID: 26478745.X-1

3530. Irwin J, Preston J, Brancazio L, et al. Development of an audiovisual speech perception app for children with autism spectrum disorders. Clin Linguist Phon. 2015 Jan;29(1):76-83. doi:

10.3109/02699206.2014.966395. PMID: 25313714.X-3, X-4

3531. Ishii K, Kubo K, Endo T, et al. Neuronal Heterotopias Affect the Activities of Distant Brain Areas and Lead to Behavioral Deficits. J Neurosci. 2015 Sep 9;35(36):1243245. doi: 10.1523/jneurosci.3648-14.2015. PMID: 26354912.X-1

3532. Isomura T, Ogawa S, Shibasaki M, et al. Delayed disengagement of attention from snakes in children with autism. Front Psychol. 2015;6:241. doi: 10.3389/fpsyg.2015.00241. PMID: 25784895.X-1

3533. Itahashi T, Yamada T, Watanabe H, et al. Alterations of local spontaneous brain activity and connectivity in adults with highfunctioning autism spectrum disorder. Mol Autism. 2015;6:30. doi: 10.1186/s13229-0150026-z. PMID: 26023326.X-1

3534. Izadi-Najafabadi S, Mirzakhani-Araghi N, Miri-Lavasani N, et al. Implicit and explicit motor learning: Application to children with Autism Spectrum Disorder (ASD). Res Dev Disabil. 2015 Oct 9;47:284-96. doi: 10.1016/j.ridd.2015.09.020. PMID: 26458260.X-1

3535. Izuwah DN, Okoh BA, Alikor EA. Clinical Pattern of Autism in Nigeria. Autism Res. 2015 Aug 25doi: 10.1002/aur.1531. PMID: 26304095.X-1

3536. Jaarsma P, Welin S. Autism, Accommodation and Treatment: A Rejoinder to Chong-Ming Lim's Critique. Bioethics. 2015 Nov;29(9):684-5. doi: 10.1111/bioe.12183. PMID: 26307242.X-1, X-2
3537. Jac Fredo AR, Kavitha G, Ramakrishnan S. Subcortical Region Segmentation using Fuzzy Based Augmented Lagrangian Multiphase Level Sets Method in Autistic MR Brain Images. Biomed Sci Instrum. 2015;51:323-31. PMID: 25996735.X1

3538. Jachim S, Warren PA, McLoughlin N, et al. Collinear facilitation and contour integration in autism: evidence for atypical visual integration. Front Hum Neurosci. 2015;9:115. doi: 10.3389/fnhum.2015.00115. PMID: 25805985.X-1

3539. Jacob A, Scott M, Falkmer M, et al. The Costs and Benefits of Employing an Adult with Autism Spectrum Disorder: A Systematic Review. PLoS One. 2015;10(10):e0139896. doi: 10.1371/journal.pone.0139896. PMID: 26445345.X-1

3540. Jameel L, Vyas K, Bellesi G, et al. Great Expectations: The Role of Rules in Guiding Pro-social Behaviour in Groups with High Versus Low Autistic Traits. J Autism Dev Disord. 2015 Aug;45(8):2311-22. doi: 10.1007/s10803-015-2393-x. PMID: 25697738.X-1

3541. James DM, Hall A, Lombardo C, et al. A Video Feedback Intervention for Workforce Development: Exploring Staff Perspective Using Longitudinal Qualitative Methodology. J Appl Res Intellect Disabil. 2015 Mar 16doi: 10.1111/jar.12161. PMID: 25772003.X-1

3542. James S, Stevenson SW, Silove N, et al. Chelation for autism spectrum disorder (ASD). Cochrane Database Syst Rev. 2015 May 11;5:Cd010766. doi: 10.1002/14651858.CD010766.pub2. PMID: 26114777.X-1, X-2

3543. Jamison TR, Schuttler JO. Examining social competence, self-perception, quality of life, and internalizing and externalizing symptoms in adolescent females with and without autism spectrum disorder: a quantitative design including between-groups and correlational analyses. Mol Autism. 2015;6:53. doi: 10.1186/s13229-015-0044-X. PMID: 26388982.X-1

3544. Janvier YM, Harris JF, Coffield CN, et al. Screening for autism spectrum disorder in 
underserved communities: Early childcare providers as reporters. Autism. 2015 May 19doi: 10.1177/1362361315585055. PMID: 25991845.X-1

3545. Janzen LA, David D, Walker D, et al. Pre-Morbid Developmental Vulnerabilities in Children With Newly Diagnosed Acute Lymphoblastic Leukemia (ALL). Pediatr Blood Cancer. 2015 Dec;62(12):2183-8. doi: 10.1002/pbc.25692. PMID: 26305495.X-1

3546. Jarvinen A, Ng R, Crivelli D, et al. Social functioning and autonomic nervous system sensitivity across vocal and musical emotion in Williams syndrome and autism spectrum disorder. Dev Psychobiol. 2015 Aug 6doi: 10.1002/dev.21335. PMID: 26248474.X1

3547. Jeffries T, Crosland K, Miltenberger R. Evaluating a tablet application and differential reinforcement to increase eye contact in children with autism. J Appl Behav Anal. 2015 Oct 19doi: 10.1002/jaba.262. PMID: 26477444.X-3, X-4

3548. Jeha D, Usta I, Ghulmiyyah L, et al. A review of the risks and consequences of adolescent pregnancy. J Neonatal Perinatal Med. 2015 Mar 12doi: 10.3233/npm-15814038. PMID: 25766198.X-1

3549. Jenkins AK, Paterson C, Wang Y, et al. Neurexin 1 (NRXN1) splice isoform expression during human neocortical development and aging. Mol Psychiatry. 2015 Jul 28doi: 10.1038/mp.2015.107. PMID: 26216298.X-1

3550. Jenkins J, 3rd, Chow V, Blaskey L, et al. Auditory Evoked M100 Response Latency is Delayed in Children with 16p11.2 Deletion but not 16p11.2 Duplication. Cereb Cortex. 2015 Feb 11doi: 10.1093/cercor/bhv008. PMID: 25678630.X-1

3551. Jermoumi M, Korideck H, Bhagwat $\mathrm{M}$, et al. Comprehensive quality assurance phantom for the small animal radiation research platform (SARRP). Phys Med. 2015 Jul;31(5):529-35. doi: 10.1016/j.ejmp.2015.04.010. PMID: 25964129.X-1
3552. Jeste SS. Neurodevelopmental behavioral and cognitive disorders. Continuum (Minneap Minn). 2015 Jun;21(3 Behavioral Neurology and Neuropsychiatry):690-714. doi: 10.1212/01.CON.0000466661.89908.3c. PMID: 26039849.X-1

3553. Jeste SS, Frohlich J, Loo SK. Electrophysiological biomarkers of diagnosis and outcome in neurodevelopmental disorders. Curr Opin Neurol. 2015 Apr;28(2):110-6. doi: 10.1097/wco.0000000000000181. PMID: 25710286.X-1

3554. Jeste SS, Tuchman R. Autism Spectrum Disorder and Epilepsy: Two Sides of the Same Coin? J Child Neurol. 2015 Sep 14doi: 10.1177/0883073815601501. PMID: 26374786.X-1

3555. Jiang R. Walking on multiple diseasegene networks to prioritize candidate genes. J Mol Cell Biol. 2015 Jun;7(3):214-30. doi: 10.1093/jmcb/mjv008. PMID: 25681405.X-1, $\mathrm{X}-2$

3556. Jiang YV, Palm BE, DeBolt MC, et al. High-precision visual long-term memory in children with high-functioning autism. J Abnorm Psychol. 2015 May;124(2):447-56. doi: 10.1037/abn0000022. PMID: 25436998.X1

3557. Jin Y, Wee CY, Shi F, et al. Identification of infants at high-risk for autism spectrum disorder using multiparameter multiscale white matter connectivity networks. Hum Brain Mapp. 2015 Sep 14doi: 10.1002/hbm.22957. PMID: 26368659.X-1

3558. Jo H, Schieve LA, Rice CE, et al. Age at Autism Spectrum Disorder (ASD) Diagnosis by Race, Ethnicity, and Primary Household Language Among Children with Special Health Care Needs, United States, 2009-2010. Matern Child Health J. 2015 Aug;19(8):1687-97. doi: 10.1007/s10995-015-1683-4. PMID: 25701197.X-1

3559. Johnco CJ, De Nadai AS, Lewin AB, et al. Defining Treatment Response and Symptom Remission for Anxiety Disorders in Pediatric Autism Spectrum Disorders Using the Pediatric Anxiety Rating Scale. J Autism Dev Disord. 2015 Oct;45(10):3232-42. doi: 
10.1007/s10803-015-2483-9. PMID:

26031924.X-1, X-3

3560. Johnco CJ, De Nadai AS, Lewin AB, et al. Erratum to: Defining Treatment Response and Symptom Remission for Anxiety Disorders in Pediatric Autism Spectrum Disorders Using the Pediatric Anxiety Rating Scale. J Autism Dev Disord. 2015 Oct;45(10):3243. doi: 10.1007/s10803-015-2554-y. PMID: 26272676.X-1

3561. Johnson N, Van Hecke A. Increasing Autism Awareness in Inner-City Churches: A Brief Report. J Pediatr Nurs. 2015 May 15doi: 10.1016/j.pedn.2015.04.008. PMID: 25981127.X-1

3562. Johnstone CD, LaFontaine R, Poirier $\mathrm{Y}$, et al. Modeling a superficial radiotherapy Xray source for relative dose calculations. J Appl Clin Med Phys. 2015;16(3):5162. doi: 10.1120/jacmp.v16i3.5162. PMID: 26103479.X-1

3563. Jones KB, Cottle K, Bakian A, et al. A description of medical conditions in adults with autism spectrum disorder: A follow-up of the 1980s Utah/UCLA Autism Epidemiologic Study. Autism. 2015 Jul 10doi: 10.1177/1362361315594798. PMID: 26162628.X-1

3564. Jones RM, Risi S, Wexler D, et al. How interview questions are placed in time influences caregiver description of social communication symptoms on the ADI-R. J Child Psychol Psychiatry. 2015 May;56(5):57785. doi: 10.1111/jcpp.12325. PMID: 25243378.X-1

3565. Jure R, Pogonza R, Rapin I. Autism Spectrum Disorders (ASD) in Blind Children: Very High Prevalence, Potentially Better Outlook. J Autism Dev Disord. 2015 Sep 25doi: 10.1007/s10803-015-2612-5. PMID: 26408327.X-1

3566. Jurecka A, Zikanova M, Kmoch S, et al. Adenylosuccinate lyase deficiency. J Inherit Metab Dis. 2015 Mar;38(2):231-42. doi: 10.1007/s10545-014-9755-y. PMID: 25112391.X-1

3567. Jurnak F. The Pivotal Role of Aldehyde Toxicity in Autism Spectrum
Disorder: The Therapeutic Potential of Micronutrient Supplementation. Nutr Metab Insights. 2015;8(Suppl 1):57-77. doi: 10.4137/nmi.s29531. PMID: 27330305.X-1

3568. Kaat AJ, Lecavalier L. Reliability and Validity of Parent- and Child-Rated Anxiety Measures in Autism Spectrum Disorder. J Autism Dev Disord. 2015 Oct;45(10):3219-31. doi: 10.1007/s10803-015-2481-y. PMID: 26036649.X-1

3569. Kabitzke PA, Simpson EH, Kandel ER, et al. Social behavior in a genetic model of dopamine dysfunction at different neurodevelopmental time points. Genes Brain Behav. 2015 Sep;14(7):503-15. doi: 10.1111/gbb.12233. PMID: 26176662.X-1

3570. Kadak MT, Cetin I, Tarakcioglu MC, et al. Low Serum Level alpha-Synuclein and Tau Protein in Autism Spectrum Disorder Compared to Controls. Neuropediatrics. 2015 Oct 19doi: 10.1055/s-0035-1565273. PMID: 26479762.X-1

3571. Kaiser C. SUFFERING IN SILENCE. JEMS: Journal of Emergency Medical Services. 2015;40(2):30-1 2p. PMID: 107772905. Language: English. Entry Date: 20150213. Revision Date: 20150820. Publication Type: Journal Article.X-1

3572. Kalsched DE. Revisioning Fordham's 'Defences of the self' in light of modern relational theory and contemporary neuroscience. J Anal Psychol. 2015 Sep;60(4):477-96. doi: 10.1111/14685922.12165. PMID: 26274848.X-1

3573. Kaltiala-Heino R, Sumia M, Tyolajarvi $\mathrm{M}$, et al. Two years of gender identity service for minors: overrepresentation of natal girls with severe problems in adolescent development. Child Adolesc Psychiatry Ment Health. 2015;9:9. doi: 10.1186/s13034-0150042-y. PMID: 25873995.X-1

3574. Kamio Y, Haraguchi H, Miyake A, et al. Brief report: large individual variation in outcomes of autistic children receiving lowintensity behavioral interventions in community settings. Child Adolesc Psychiatry Ment Health. 2015;9:6. doi: 10.1186/s13034-015-0039-6. PMID: 25960766.X-4 
3575. Kamps D, Thiemann-Bourque K, Heitzman-Powell L, et al. A comprehensive peer network intervention to improve social communication of children with autism spectrum disorders: a randomized trial in kindergarten and first grade. J Autism Dev Disord. 2015 Jun;45(6):1809-24. doi: 10.1007/s10803-014-2340-2. PMID: 25510450.X-1

3576. Kanduri C, Kantojarvi K, Salo PM, et al. The landscape of copy number variations in Finnish families with autism spectrum disorders. Autism Res. 2015 Jun 6doi: 10.1002/aur.1502. PMID: 26052927.X-1

3577. Kang-Yi CD, Locke J, Marcus SC, et al. School-Based Behavioral Health Service Use and Expenditures for Children With Autism and Children With Other Disorders. Psychiatr Serv. 2015 Aug 17:appips201400505. doi: 10.1176/appi.ps.201400505. PMID: 26278232.X-1

3578. Kantarcioglu AS, Kiraz N, Aydin A. Microbiota-Gut-Brain Axis: Yeast Species Isolated from Stool Samples of Children with Suspected or Diagnosed Autism Spectrum Disorders and In Vitro Susceptibility Against Nystatin and Fluconazole. Mycopathologia. 2015 Oct 6doi: 10.1007/s11046-015-9949-3. PMID: 26442855.X-1

3579. Kao YC, Kramer JM, Liljenquist K, et al. Association between impairment, function, and daily life task management in children and adolescents with autism. Dev Med Child Neurol. 2015 Jan;57(1):68-74. doi: 10.1111/dmcn.12562. PMID: 25312547.X-1

3580. Karakurt MN, Suren S. Desmopressin Use in the Treatment of Aripiprazole-Induced Nocturnal Enuresis in a Child Diagnosed with Autistic Disorder. J Child Adolesc Psychopharmacol. 2015 Aug;25(6):518-9. doi: 10.1089/cap.2015.0003. PMID: 26222623.X-3, $\mathrm{X}-4$

3581. Karaminis T, Neil L, Manning C, et al. Ensemble perception of emotions in children with autism. J Vis. 2015 Sep 1;15(12):916. doi: 10.1167/15.12.916. PMID: 26326604.X-1

3582. Karaminis T, Turi M, Neil L, et al. Atypicalities in perceptual adaptation in autism do not extend to perceptual causality. PLoS
One. 2015;10(3):e0120439. doi: 10.1371/journal.pone.0120439. PMID: 25774507.X-1

3583. Kardas F, Bayram AK, Demirci E, et al. Increased Serum Phthalates (MEHP, DEHP) and Bisphenol A Concentrations in Children With Autism Spectrum Disorder: The Role of Endocrine Disruptors in Autism Etiopathogenesis. J Child Neurol. 2015 Oct 8doi: 10.1177/0883073815609150. PMID: 26450281.X-1

3584. Kargas N, Lopez B, Reddy V, et al. The relationship between auditory processing and restricted, repetitive behaviors in adults with autism spectrum disorders. J Autism Dev Disord. 2015 Mar;45(3):658-68. doi: 10.1007/s10803-014-2219-2. PMID: 25178987.X-1

3585. Karlsson SA, Haziri K, Hansson E, et al. Effects of sex and gonadectomy on social investigation and social recognition in mice. BMC Neurosci. 2015;16:83. doi: 10.1186/s12868-015-0221-z. PMID: 26608570.X-1

3586. Karst JS, Van Hecke AV, Carson AM, et al. Parent and family outcomes of PEERS: a social skills intervention for adolescents with autism spectrum disorder. J Autism Dev Disord. 2015 Mar;45(3):752-65. doi: 10.1007/s10803014-2231-6. PMID: 25193142.X-1

3587. Karten A, Hirsch J. Brief report: Anomalous neural deactivations and functional connectivity during receptive language in autism spectrum disorder: a functional MRI study. J Autism Dev Disord. 2015 Jun;45(6):1905-14. doi: 10.1007/s10803-0142344-y. PMID: 25526952.X-1

3588. Kasari C. Update on behavioral interventions for autism and developmental disabilities. Curr Opin Neurol. 2015 Apr;28(2):124-9. doi: 10.1097/wco.0000000000000185. PMID: 25695136.X-1, X-2

3589. Kasari C, Dean M, Kretzmann M, et al. Children with autism spectrum disorder and social skills groups at school: a randomized trial comparing intervention approach and peer composition. J Child Psychol Psychiatry. 2015 
Sep 22doi: 10.1111/jcpp.12460. PMID: 26391889.X-1

3590. Kasari C, Gulsrud A, Paparella T, et al. Randomized comparative efficacy study of parent-mediated interventions for toddlers with autism. J Consult Clin Psychol. 2015 Jun;83(3):554-63. doi: 10.1037/a0039080. PMID: 25822242.X-1

3591. Kaslovsky R, Gottsegen D. Hypnosis for Asthma and Vocal Cord Dysfunction in a Patient With Autism. Am J Clin Hypn. 2015;58(2):195-203. doi: 10.1080/00029157.2014.989566. PMID: 26264542.X-1, X-3, X-4

3592. Kata SG, Aboumarzouk OM, Zreik A, et al. Photodynamic diagnostic ureterorenoscopy: A valuable tool in the detection of upper urinary tract tumour. Photodiagnosis Photodyn Ther. 2015 Aug 6doi: 10.1016/j.pdpdt.2015.08.002. PMID: 26256824.X-1

3593. Katoh M. Mutation spectra of histone methyltransferases with canonical SET domains and EZH2-targeted therapy. Epigenomics. 2015 Sep 28doi: 10.2217/epi.15.89. PMID: 26411517.X-1

3594. Katz E, Girolametto L. Peer-mediated intervention for pre-schoolers with ASD: Effects on responses and initiations. Int $\mathrm{J}$ Speech Lang Pathol. 2015 Apr 17:1-12. doi: 10.3109/17549507.2015.1024166. PMID: 25882445.X-3, X-4

3595. Kaur M, Srinivasan SM, Bhat AN. Atypical object exploration in infants at-risk for autism during the first year of lifer. Front Psychol. 2015;6:798. doi: 10.3389/fpsyg.2015.00798. PMID: 26136702.X-1

3596. Kaushik G, Thomas MA, Aho KA. Psychoactive pharmaceuticals as environmental contaminants may disrupt highly interconnected nodes in an Autism-associated protein-protein interaction network. BMC Bioinformatics. 2015;16 Suppl 7:S3. doi: 10.1186/1471-2105-16-s7-s3. PMID: 25952302.X-1

3597. Kazim SF, Cardenas-Aguayo Mdel C, Arif M, et al. Sera from children with autism induce autistic features which can be rescued with a CNTF small peptide mimetic in rats. PLoS One. 2015;10(3):e0118627. doi: 10.1371/journal.pone.0118627. PMID: 25769033.X-1

3598. Keehn B, Vogel-Farley V, TagerFlusberg $\mathrm{H}$, et al. Atypical hemispheric specialization for faces in infants at risk for autism spectrum disorder. Autism Res. 2015 Apr;8(2):187-98. doi: 10.1002/aur.1438. PMID: 25808162.X-1

3599. Keidan I, Ben-Menachem E, Tzadok $\mathrm{M}$, et al. Electroencephalography for children with autistic spectrum disorder: a sedation protocol. Paediatr Anaesth. 2015 Feb;25(2):200-5. doi: 10.1111/pan.12510. PMID: 25145661.X-1

3600. Kelley ME, Liddon CJ, Ribeiro A, et al. Basic and translational evaluation of renewal of operant responding. J Appl Behav Anal. 2015 Summer;48(2):390-401. doi: 10.1002/jaba.209. PMID: 25891414.X-1

3601. Kelly AM. Caring for Patients with ASD and Their Caregivers: Federal and State Autism-Specific Insurance Reform. AMA J Ethics. 2015 Apr;17(4):328-41. doi: 10.1001/journalofethics.2015.17.4.hlaw1-1504. PMID: 25901701.X-1

3602. Kendall W, Kingstone A, Todd R. Detecting emotions is easier in less realistic faces. J Vis. 2015 Sep 1;15(12):1379. doi: 10.1167/15.12.1379. PMID: 26327067.X-1

3603. Kent-Walsh J, Murza KA, Malani MD, et al. Effects of Communication Partner Instruction on the Communication of Individuals using AAC: A Meta-Analysis. Augment Altern Commun. 2015 Jun 10:1-14. doi: 10.3109/07434618.2015.1052153. PMID: 26059542.X-2

3604. Kerekes N, Tajnia A, Lichtenstein P, et al. Neurodevelopmental problems and extremes in BMI. PeerJ. 2015;3:e1024. doi: 10.7717/peerj.1024. PMID: 26207189.X-1

3605. Kern JK, Geier DA, Sykes LK, et al. Are ASD and ADHD a Continuum? A Comparison of Pathophysiological Similarities Between the Disorders. J Atten Disord. 2015 Sep;19(9):805-27. doi: 
10.1177/1087054712459886. PMID: 23074304.X-1

3606. Kerns CM, Maddox BB, Kendall PC, et al. Brief measures of anxiety in nontreatment-seeking youth with autism spectrum disorder. Autism. 2015 Nov;19(8):969-79. doi: 10.1177/1362361314558465. PMID: 25633222.X-1

3607. Kerr EN, Bhan A, Heon E. Exploration of the cognitive, adaptive and behavioral functioning of patients affected with BardetBiedl syndrome. Clin Genet. 2015 May 19doi: 10.1111/cge.12614. PMID: 25988237.X-1

3608. Kestemont J, Ma N, Baetens K, et al. Neural correlates of attributing causes to the self, another person and the situation. Soc Cogn Affect Neurosci. 2015 Jan;10(1):114-21. doi: 10.1093/scan/nsu030. PMID: 24633532.X-1

3609. Kestemont J, Vandekerckhove M, Bulnes LC, et al. Causal attribution in individuals with subclinical and clinical autism spectrum disorder: An fMRI study. Soc Neurosci. 2015 Aug 11:1-13. doi: 10.1080/17470919.2015.1074104. PMID: 26212902.X-1

3610. Khadem A, Hossein-Zadeh GA, Khorrami A. Long-Range Reduced Predictive Information Transfers of Autistic Youths in EEG Sensor-Space During Face Processing. Brain Topogr. 2015 Oct 3doi: 10.1007/s10548015-0452-4. PMID: 26433373.X-1

3611. Khaledi N, Arbabi A, Sardari D, et al. Simultaneous production of mixed electron-photon beam in a medical LINAC: A feasibility study. Phys Med. 2015 Jun;31(4):391-7. doi: 10.1016/j.ejmp.2015.02.014. PMID: 25773884.X-1

3612. Khamooshi M, Mirmahdi SR. The effectiveness of sensory integration procedure on reduction of stereotypical behaviors in autistic children. Journal of Iranian Psychologists. 2015 2015-11-25;11(44):417-23. PMID: 1735925639; 2015-43162-007.X-5

3613. Khan AJ, Nair A, Keown CL, et al. Cerebro-cerebellar Resting-State Functional Connectivity in Children and Adolescents with Autism Spectrum Disorder. Biol Psychiatry. 2015 Nov 1;78(9):625-34. doi: 10.1016/j.biopsych.2015.03.024. PMID: 25959247.X-1

3614. Khanna R, Jariwala K, West-Strum D. Validity and reliability of the Medical Outcomes Study Short-Form Health Survey version 2 (SF-12v2) among adults with autism. Res Dev Disabil. 2015 Aug-Sep;43-44:51-60. doi: 10.1016/j.ridd.2015.06.006. PMID: 26151443.X-1

3615. Khongrum J, Wattanathorn J. Laser Acupuncture Improves Behavioral Disorders and Brain Oxidative Stress Status in the Valproic Acid Rat Model of Autism. J Acupunct Meridian Stud. 2015 Aug;8(4):18391. doi: 10.1016/j.jams.2015.06.008. PMID: 26276454.X-1

3616. Khowaja K, Salim SS. Heuristics to Evaluate Interactive Systems for Children with Autism Spectrum Disorder (ASD). PLoS One. 2015;10(7):e0132187. doi:

10.1371/journal.pone.0132187. PMID: 26196385.X-1, X-2

3617. Kienle X, Freiberger V, Greulich H, et al. [Autism Spectrum Disorder and DSM-5: Spectrum or Cluster?]. Prax Kinderpsychol Kinderpsychiatr. 2015;64(6):412-28. doi: 10.13109/prkk.2015.64.6.412. PMID: 26289149.X-1

3618. Kihara H, Nakamura T. Early standard development assessment characteristics in very low birth weight infants later classified with autism spectrum disorder. Early Hum Dev. 2015 Jun;91(6):357-9. doi: 10.1016/j.earlhumdev.2015.03.012. PMID: 25912387.X-1

3619. Kikuchi M, Yoshimura Y, Hiraishi H, et al. Reduced long-range functional connectivity in young children with autism spectrum disorder. Soc Cogn Affect Neurosci. 2015 Feb;10(2):248-54. doi: 10.1093/scan/nsu049. PMID: 24652855.X-1

3620. Kim DJ, Sim MK, Lee SW, et al. Successful Removal of a Screw Nail in the Jejunum Using Double-Balloon Enteroscopy. Clin Endosc. 2015 Sep;48(5):444-6. doi: 10.5946/ce.2015.48.5.444. PMID: 26473131.X1 
3621. Kim SH, Macari S, Koller J, et al. Examining the phenotypic heterogeneity of early Autism Spectrum Disorder: subtypes and short-term outcomes. J Child Psychol Psychiatry. 2015 Aug 12doi: 10.1111/jcpp.12448. PMID: 26264996.X-1

3622. Kim SN, Jo GH, Kim HA, et al. Aberrant IgG isotype generation in mice with abnormal behaviors. J Immunotoxicol. 2015 Feb 18:1-5. doi: 10.3109/1547691x.2015.1014581. PMID: 25691089.X-1

3623. Kim SY, Choi US, Park SY, et al. Abnormal activation of the social brain network in children with autism spectrum disorder: an FMRI study. Psychiatry Investig. 2015 Jan;12(1):37-45. doi: 10.4306/pi.2015.12.1.37. PMID: 25670944.X-1

3624. Kim YJ, Park JK, Kang WS, et al. LAMB1 polymorphism is associated with autism symptom severity in Korean autism spectrum disorder patients. Nord J Psychiatry. 2015 Nov;69(8):594-8. doi: 10.3109/08039488.2015.1022597. PMID: 25774865.X-1

3625. Kirby AV, Little LM, Schultz B, et al. Observational Characterization of Sensory Interests, Repetitions, and Seeking Behaviors. American Journal of Occupational Therapy. 2015;69(3):p1-9 1p. doi: 10.5014/ajot.2015.015081. PMID: 103795722. Language: English. Entry Date: 20150505. Revision Date: 20150819. Publication Type: Journal Article.X-1

3626. Kirby AV, White TJ, Baranek GT. Caregiver strain and sensory features in children with autism spectrum disorder and other developmental disabilities. Am J Intellect Dev Disabil. 2015 Jan;120(1):32-45. doi: 10.1352/1944-7558-120.1.32. PMID: 25551265.X-1

3627. Kirsten TB, Chaves-Kirsten GP, Bernardes S, et al. Lipopolysaccharide Exposure Induces Maternal Hypozincemia, and Prenatal Zinc Treatment Prevents Autistic-Like Behaviors and Disturbances in the Striatal Dopaminergic and mTOR Systems of Offspring. PLoS One. 2015;10(7):e0134565. doi: 10.1371/journal.pone.0134565. PMID: 26218250.X-1
3628. Kirsten TB, Queiroz-Hazarbassanov $\mathrm{N}$, Bernardi MM, et al. Prenatal zinc prevents communication impairments and BDNF disturbance in a rat model of autism induced by prenatal lipopolysaccharide exposure. Life Sci. 2015 Jun 1;130:12-7. doi:

10.1016/j.lfs.2015.02.027. PMID: 25817235.X1

3629. Kissin DM, Zhang Y, Boulet SL, et al. Association of assisted reproductive technology (ART) treatment and parental infertility diagnosis with autism in ART-conceived children. Hum Reprod. 2015 Feb;30(2):454-65. doi: 10.1093/humrep/deu338. PMID: 25518976.X-1

3630. Kleberg JL, Selbing I, Lundqvist D, et al. Spontaneous eye movements and trait empathy predict vicarious learning of fear. Int $\mathrm{J}$ Psychophysiol. 2015 Apr 11doi: 10.1016/j.ijpsycho.2015.04.001. PMID: 25872010.X-1

3631. Kleiman GH, Barnea A, Gozes I. ADNP: A major autism mutated gene is differentially distributed (age and gender) in the songbird brain. Peptides. 2015 Apr 17doi: 10.1016/j.peptides.2015.04.008. PMID: 25895853.X-1

3632. Kleinhans NM, Reiter MA, Neuhaus $\mathrm{E}$, et al. Subregional differences in intrinsic amygdala hyperconnectivity and hypoconnectivity in autism spectrum disorder. Autism Res. 2015 Dec 15doi: 10.1002/aur.1589. PMID: 26666502.X-1

3633. Klin A, Klaiman C, Jones W. Reducing age of autism diagnosis: developmental social neuroscience meets public health challenge. Rev Neurol. 2015 Feb 25;60 Suppl 1:S3-11. PMID: 25726820.X-1

3634. Klintwall L, Eldevik S, Eikeseth S. Narrowing the gap: effects of intervention on developmental trajectories in autism. Autism. 2015 Jan;19(1):53-63. doi: 10.1177/1362361313510067. PMID: 24212258.X-1, X-2

3635. Klintwall L, Macari S, Eikeseth S, et al. Interest level in 2-year-olds with autism spectrum disorder predicts rate of verbal, nonverbal, and adaptive skill acquisition. 
Autism. 2015 Nov;19(8):925-33. doi: 10.1177/1362361314555376. PMID: 25398893.X-1

3636. Klukowski M, Wasilewska J, Lebensztejn D. Smith-Magenis syndrome and its circadian influence on development, behavior, and obesity - own experience. Dev Period Med. 2015 Apr-Jun;19(2):157-61. PMID: 26384115.X-1

3637. Klusek J, Roberts JE, Losh M. Cardiac autonomic regulation in autism and Fragile $\mathrm{X}$ syndrome: a review. Psychol Bull. 2015 Jan;141(1):141-75. doi: 10.1037/a0038237. PMID: 25420222.X-1, X-2

3638. Knutsen J, Mandell DS, Frye D. Children with autism are impaired in the understanding of teaching. Dev Sci. 2015 Dec 8doi: 10.1111/desc.12368. PMID: 26646799.X1

3639. Ko WR, Huang JY, Chiang YC, et al. Risk of autistic disorder after exposure to general anaesthesia and surgery: a nationwide, retrospective matched cohort study. Eur J Anaesthesiol. 2015 May;32(5):303-10. doi: 10.1097/eja.0000000000000130. PMID: 25101714.X-1

3640. Kobayashi T, Hirano Y, Nemoto K, et al. Correlation between Morphologic Changes and Autism Spectrum Tendency in ObsessiveCompulsive Disorder. Magn Reson Med Sci. 2015 Jun 23doi: 10.2463/mrms.2014-0146. PMID: 26104070.X-1

3641. Kobeissy F, Moshourab RA. Frontiers in Neuroengineering

Autoantibodies in CNS Trauma and

Neuropsychiatric Disorders: A New Generation of Biomarkers. In: Kobeissy FH, ed Brain Neurotrauma: Molecular, Neuropsychological, and Rehabilitation Aspects. Boca Raton (FL): CRC Press

(c) 2015 by Taylor \& Francis Group, LLC.; 2015.

3642. Koch SC, Mehl L, Sobanski E, et al. Fixing the mirrors: a feasibility study of the effects of dance movement therapy on young adults with autism spectrum disorder. Autism. 2015 Apr;19(3):338-50. doi: 10.1177/1362361314522353. PMID: 24566716.X-1
3643. Kodak T, Clements A, Paden AR, et al. Examination of the relation between an assessment of skills and performance on auditory-visual conditional discriminations for children with autism spectrum disorder. J Appl Behav Anal. 2015 Spring;48(1):52-70. doi: 10.1002/jaba.160. PMID: 25293383.X-1

3644. Kosmicki JA, Sochat V, Duda M, et al. Searching for a minimal set of behaviors for autism detection through feature selectionbased machine learning. Transl Psychiatry. 2015;5:e514. doi: 10.1038/tp.2015.7. PMID: 25710120.X-1

3645. Kovelman I, Wagley N, Hay JS, et al. Multimodal imaging of temporal processing in typical and atypical language development. Ann N Y Acad Sci. 2015 Mar;1337:7-15. doi: 10.1111/nyas.12688. PMID: 25773611.X-1

3646. Kovtun O, Sakrikar D, Tomlinson ID, et al. Single-quantum-dot tracking reveals altered membrane dynamics of an attentiondeficit/hyperactivity-disorder-derived dopamine transporter coding variant. ACS Chem Neurosci. 2015 Apr 15;6(4):526-34. doi: 10.1021/cn500202c. PMID: 25747272.X-1

3647. Krakowiak P, Goines PE, Tancredi DJ, et al. Neonatal Cytokine Profiles Associated with Autism Spectrum Disorder. Biol Psychiatry. 2015 Aug 14doi: 10.1016/j.biopsych.2015.08.007. PMID: 26392128.X-1

3648. Krakowiak P, Walker CK, Tancredi DJ, et al. Maternal Recall Versus Medical Records of Metabolic Conditions from the Prenatal Period: A Validation Study. Matern Child Health J. 2015 Sep;19(9):1925-35. doi: 10.1007/s10995-015-1723-0. PMID: 25656730.X-1

3649. Kral TV, Souders MC, Tompkins VH, et al. Child Eating Behaviors and Caregiver Feeding Practices in Children with Autism Spectrum Disorders. Public Health Nurs. 2015 Sep;32(5):488-97. doi: 10.1111/phn.12146. PMID: 25112438.X-1

3650. Kramer JM, Liljenquist K, Ni P, et al. Examining differential responses of youth with and without autism on a measure of everyday activity performance. Qual Life Res. 2015 
Dec;24(12):2993-3000. doi: 10.1007/s11136015-1035-2. PMID: 26063170.X-1

3651. Kreibich SR, Chen M, Reichle J. Teaching a Child With Autism to Request Breaks While Concurrently Increasing Task Engagement. Lang Speech Hear Serv Sch. 2015 Jul;46(3):256-65. doi: 10.1044/2015_lshss-140081. PMID: 25908538.X-3, X-4

3652. Kreslins A, Robertson AE, Melville C. The effectiveness of psychosocial interventions for anxiety in children and adolescents with autism spectrum disorder: a systematic review and meta-analysis. Child Adolesc Psychiatry Ment Health. 2015;9:22. doi: 10.1186/s13034015-0054-7. PMID: 26120361.X-2

3653. Krigbaum G, Wigton NL. A Methodology of Analysis for Monitoring Treatment Progression with 19-Channel ZScore Neurofeedback (19ZNF) in a SingleSubject Design. Appl Psychophysiol Biofeedback. 2015 Sep;40(3):139-49. doi: 10.1007/s10484-015-9274-0. PMID: 25777656.X-1

3654. Kronenberg LM, Goossens PJ, van Busschbach J, et al. Coping styles in substance use disorder (SUD) patients with and without co-occurring attention deficit/hyperactivity disorder (ADHD) or autism spectrum disorder (ASD). BMC Psychiatry. 2015;15:159. doi: 10.1186/s12888-015-0530-x. PMID: 26169586.X-1

3655. Kryzak LA, Cengher M, Feeley KM, et al. A community support program for children with autism and their typically developing siblings: Initial investigation. J Intellect Disabil. 2015 Jun;19(2):159-77. doi: 10.1177/1744629514564450. PMID: 25542700.X-1

3656. Kubota T, Miyake K, Hariya N, et al. Epigenomic-basis of Preemptive Medicine for Neurodevelopmental Disorders. Curr Genomics. 2015 Jun;16(3):175-82. doi: 10.2174/1389202916666150216221312. PMID: 26069457.X-1

3657. Kuhaneck HM, Madonna S, Novak A, et al. Effectiveness of Interventions for Children With Autism Spectrum Disorder and Their Parents: A Systematic Review of Family Outcomes. Am J Occup Ther. 2015 Sep-
Oct;69(5):6905180040p1-p14. doi: 10.5014/ajot.2015.017855. PMID: 26356656.X-2, X-3

3658. Kuhaneck HM, Watling R. Occupational Therapy: Meeting the Needs of Families of People With Autism Spectrum Disorder. Am J Occup Ther. 2015 SepOct;69(5):6905170010p1-5. doi: 10.5014/ajot.2015.019562. PMID: 26356652.X-1, X-2

3659. Kuhlthau KA, Warfield ME, Hurson J, et al. Pediatric provider's perspectives on the transition to adult health care for youth with autism spectrum disorder: current strategies and promising new directions. Autism. 2015 Apr;19(3):262-71. doi: 10.1177/1362361313518125. PMID: 24497626.X-1

3660. Kumar H, Sharma BM, Sharma B. Benefits of agomelatine in behavioral, neurochemical and blood brain barrier alterations in prenatal valproic acid induced autism spectrum disorder. Neurochem Int. 2015 Oct 20doi: 10.1016/j.neuint.2015.10.007. PMID: 26498253.X-1

3661. Kumar R, Pallagatti S, Sheikh S, et al. Correlation Between Clinical Findings of Temporomandibular Disorders and MRI Characteristics of Disc Displacement. Open Dent J. 2015;9:273-81. doi: 10.2174/1874210601509010273. PMID: 26464595.X-1

3662. Kuo N-C, Plavnick JB. Using an Antecedent Art Intervention to Improve the Behavior of a Child With Autism. Art Therapy: Journal of the American Art Therapy Association. 2015;32(2):54-9 6p. doi: 10.1080/07421656.2015.1028312. PMID: 109807439. Language: English. Entry Date: 20150730. Revision Date: 20150923. Publication Type: Journal Article.X-1, X-3, X-4

3663. Kuriakose S, Lahiri U. Understanding the Psycho-Physiological Implications of Interaction With a Virtual Reality-Based System in Adolescents With Autism: A Feasibility Study. IEEE Trans Neural Syst Rehabil Eng. 2015 Jul;23(4):665-75. doi: 10.1109/tnsre.2015.2393891. PMID: 25643409.X-1 
3664. Kurtz PF, Chin MD, Robinson AN, et al. Functional analysis and treatment of problem behavior exhibited by children with fragile X syndrome. Res Dev Disabil. 2015

Aug-Sep;43-44:150-66. doi: 10.1016/j.ridd.2015.06.010. PMID: 26183339.X-1

3665. Kuschner ES, Eisenberg IW, Orionzi B, et al. A Preliminary Study of Self-Reported Food Selectivity in Adolescents and Young Adults with Autism Spectrum Disorder. Res Autism Spectr Disord. 2015 Jul 1;15-16:53-9. doi: 10.1016/j.rasd.2015.04.005. PMID: 26309446.X-1

3666. Kushki A, Khan A, Brian J, et al. A Kalman filtering framework for physiological detection of anxiety-related arousal in children with autism spectrum disorder. IEEE Trans Biomed Eng. 2015 Mar;62(3):990-1000. doi: 10.1109/tbme.2014.2377555. PMID: 25494497.X-1

3667. Kwon KJ, Lee EJ, Kim MK, et al. The potential role of melatonin on sleep deprivationinduced cognitive impairments: implication of FMRP on cognitive function. Neuroscience. 2015 Aug 20;301:403-14. doi:

10.1016/j.neuroscience.2015.05.079. PMID: 26047724.X-1

3668. Kyzar EJ, Stewart AM, Kalueff AV. Effects of LSD on grooming behavior in serotonin transporter heterozygous (Sert) mice. Behav Brain Res. 2015 Sep 1;296:47-52. doi: 10.1016/j.bbr.2015.08.018. PMID: 26340513.X-1

3669. LaFrance DL, Miguel CF, Donahue JN, et al. A case study on the use of auditory integration training as a treatment for stereotypy. Behavioral Interventions. 2015 2015-12-09;30(3):286-93. doi: http://dx.doi.org/10.1002/bin.1415. PMID: 1690656789; 2015-27676-001.X-1

3670. Laghi F, Federico F, Lonigro A, et al. Peer and Teacher-Selected Peer Buddies for Adolescents With Autism Spectrum Disorders: The Role of Social, Emotional, and Mentalizing Abilities. J Psychol. 2015 Sep 23:1-22. doi: 10.1080/00223980.2015.1087375. PMID: 26398319.X-1
3671. Lahiri U, Bekele E, Dohrmann E, et al. A physiologically informed virtual reality based social communication system for individuals with autism. J Autism Dev Disord. 2015 Apr;45(4):919-31. doi: 10.1007/s10803-0142240-5. PMID: 25261247.X-1

3672. Lai WW, Goh TJ, Oei TP, et al. Coping and Well-Being in Parents of Children with Autism Spectrum Disorders (ASD). J Autism Dev Disord. 2015 Aug;45(8):2582-93. doi: 10.1007/s10803-015-2430-9. PMID: 25800867.X-1

3673. Lake JK, Vogan V, Sawyer A, et al. Psychotropic medication use among adolescents and young adults with an autism spectrum disorder: parent views about medication use and healthcare services. J Child Adolesc Psychopharmacol. 2015 Apr;25(3):260-8. doi: 10.1089/cap.2014.0106. PMID: 25803636.X-1

3674. Lal D, Pernhorst K, Klein KM, et al. Extending the phenotypic spectrum of RBFOX1 deletions: Sporadic focal epilepsy. Epilepsia. 2015 Sep;56(9):e129-e33. doi: 10.1111/epi.13076. PMID: 26174448.X-1

3675. Lal D, Ruppert AK, Trucks H, et al. Burden analysis of rare microdeletions suggests a strong impact of neurodevelopmental genes in genetic generalised epilepsies. PLoS Genet. 2015 May;11(5):e1005226. doi: 10.1371/journal.pgen.1005226. PMID: 25950944.X-1

3676. Lalanne L, Weiner L, Trojak B, et al. Substance-use disorder in high-functioning autism: clinical and neurocognitive insights from two case reports. BMC Psychiatry. 2015;15:149. doi: 10.1186/s12888-015-0541-7. PMID: 26149411.X-1

3677. Landy KM, Salmon DP, Filoteo JV, et al. Visual search in Dementia with Lewy Bodies and Alzheimer's disease. Cortex. 2015 Sep 21;73:228-39. doi: 10.1016/j.cortex.2015.08.020. PMID: 26476402.X-1

3678. Lane R, Kessler R, Buckley AW, et al. Evaluation of Periodic Limb Movements in Sleep and Iron Status in Children With Autism. Pediatr Neurol. 2015 Oct;53(4):343-9. doi: 10.1016/j.pediatrneurol.2015.06.014. PMID: 26231264.X-1 
3679. Lange KW, Hauser J, Reissmann A. Gluten-free and casein-free diets in the therapy of autism. Curr Opin Clin Nutr Metab Care. 2015 Nov;18(6):572-5. doi: 10.1097/mco.0000000000000228. PMID: 26418822.X-1, X-3

3680. Lange N, Travers BG, Bigler ED, et al. Longitudinal volumetric brain changes in autism spectrum disorder ages 6-35 years. Autism Res. 2015 Feb;8(1):82-93. doi: 10.1002/aur.1427. PMID: 25381736.X-1

3681. Langley EA, Krykbaeva M, Blusztajn JK, et al. High maternal choline consumption during pregnancy and nursing alleviates deficits in social interaction and improves anxiety-like behaviors in the BTBR T+Itpr3tf/J mouse model of autism. Behav Brain Res. 2015 Feb 1;278:210-20. doi: 10.1016/j.bbr.2014.09.043. PMID: 25300468.X-1

3682. Lanovaz MJ, Dufour MM, Shah S. Newspaper Coverage of Autism Treatment in Canada: 10-Year Trends (2004-2013). Can J Psychiatry. 2015 Jul;60(7):329-33. PMID: 26175392.X-1, X-2

3683. Lanovaz MJ, Rapp JT, Maciw I, et al. Preliminary effects of parent-implemented behavioural interventions for stereotypy: Brief report. Dev Neurorehabil. 2015 Aug 24:1-4. doi: 10.3109/17518423.2014.986821. PMID: 25549178.X-3, X-4

3684. Larson FV, Lai MC, Wagner AP, et al. Testing the 'Extreme Female Brain' Theory of Psychosis in Adults with Autism Spectrum Disorder with or without Co-Morbid Psychosis. PLoS One. 2015;10(6):e0128102. doi: 10.1371/journal.pone.0128102. PMID: 26069955.X-1

3685. Laue CL. CONSIDERING THE EFFECTS OF GENDER IN CHILD-ROBOT INTERACTION STUDIES: COMMENT ON SRINIVASAN, ET AL. ( 2013. Perceptual \& Motor Skills. 2015;120(1):336-42 7p. doi: 10.2466/15.10.PMS.120v16x4. PMID: 103763859. Language: English. Entry Date: 20150227. Revision Date: 20150710. Publication Type: Journal Article.X-1, X-2

3686. Laugeson EA, Gantman A, Kapp SK, et al. A Randomized Controlled Trial to
Improve Social Skills in Young Adults with Autism Spectrum Disorder: The UCLA PEERS Program. J Autism Dev Disord. 2015 Jun 25doi: 10.1007/s10803-015-2504-8. PMID: 26109247.X-1

3687. Laugeson EA, Gantman A, Kapp SK, et al. A Randomized Controlled Trial to Improve Social Skills in Young Adults with Autism Spectrum Disorder: The UCLA PEERS((R)) Program. J Autism Dev Disord. 2015 Dec;45(12):3978-89. doi: 10.1007/s10803-015-2504-8. PMID: 26109247.X-1

3688. Lawson RA, Papadakis AA, Higginson $\mathrm{CI}$, et al. Everyday executive function impairments predict comorbid psychopathology in autism spectrum and attention deficit hyperactivity disorders. Neuropsychology. 2015 May;29(3):445-53. doi: 10.1037/neu0000145. PMID: 25313979.X-1

3689. Laxman DJ, McBride BA, Jeans LM, et al. Father involvement and maternal depressive symptoms in families of children with disabilities or delays. Matern Child Health J. 2015 May;19(5):1078-86. doi: 10.1007/s10995-014-1608-7. PMID: 25326111.X-1

3690. Leaf JB, Leaf R, McEachin J, et al. Applied Behavior Analysis is a Science and, Therefore, Progressive. J Autism Dev Disord. 2015 Sep 15doi: 10.1007/s10803-015-2591-6. PMID: 26373767.X-1

3691. LeBarton ES, Iverson JM. Gesture development in toddlers with an older sibling with autism. Int J Lang Commun Disord. 2015 Sep 6doi: 10.1111/1460-6984.12180. PMID: 26343932.X-1

3692. Lechpammer M, Wintermark P, Merry KM, et al. Dysregulation of FMRP/mTOR Signaling Cascade in Hypoxic-Ischemic Injury of Premature Human Brain. J Child Neurol. 2015 Aug 3doi: 10.1177/0883073815596617. PMID: 26239490.X-1

3693. LeClerc S, Easley D. Pharmacological therapies for autism spectrum disorder: a review. P t. 2015 Jun;40(6):389-97. PMID: 26045648.X-2 
3694. Ledbetter-Cho K, Lang R, Davenport $\mathrm{K}$, et al. Effects of script training on the peer-topeer communication of children with autism spectrum disorder. J Appl Behav Anal. 2015 Aug 24doi: 10.1002/jaba.240. PMID: 26303985.X-3, X-4

3695. Ledford JR, Wehby JH. Teaching Children with Autism in Small Groups with Students Who are At-Risk for Academic Problems: Effects on Academic and Social Behaviors. J Autism Dev Disord. 2015 Jun;45(6):1624-35. doi: 10.1007/s10803-0142317-1. PMID: 25409705.X-1, X-3

3696. Lee A, Lang R, Davenport K, et al. Comparison of therapist implemented and iPadassisted interventions for children with autism. Dev Neurorehabil. 2015 Apr;18(2):97-103. doi: 10.3109/17518423.2013.830231. PMID: 24088050.X-3, X-4

3697. Lee BK, Magnusson C, Gardner RM, et al. Maternal hospitalization with infection during pregnancy and risk of autism spectrum disorders. Brain Behav Immun. 2015 Feb;44:100-5. doi: 10.1016/j.bbi.2014.09.001. PMID: 25218900.X-1

3698. Lee IS, Carvalho CM, Douvaras P, et al. Characterization of molecular and cellular phenotypes associated with a heterozygous CNTNAP2 deletion using patient-derived hiPSC neural cells. NPJ Schizophr. 2015 Jun 24;1 PMID: 26985448.X-1

3699. Lee JY, Cho KS, Kang DH, et al. A network meta-analysis of therapeutic outcomes after new image technology-assisted transurethral resection for non-muscle invasive bladder cancer: 5-aminolaevulinic acid fluorescence vs hexylaminolevulinate fluorescence vs narrow band imaging. BMC Cancer. 2015;15:566. doi: 10.1186/s12885-0151571-8. PMID: 26232037.X-1

3700. Lee KM, Chiu KB, Didier PJ, et al. Naltrexone treatment reverses astrocyte atrophy and immune dysfunction in self-harming macaques. Brain Behav Immun. 2015 Jul 17doi: 10.1016/j.bbi.2015.07.017. PMID: 26191654.X-1

3701. Lee SB, Song SH, Ham JH, et al. Idiom Comprehension Deficits in HighFunctioning Autism Spectrum Disorder Using a
Korean Autism Social Language Task. Yonsei Med J. 2015 Nov;56(6):1613-8. doi: 10.3349/ymj.2015.56.6.1613. PMID: 26446644.X-1

3702. Lefebvre A, Beggiato A, Bourgeron T, et al. Neuroanatomical Diversity of Corpus Callosum and Brain Volume in Autism: Metaanalysis, Analysis of the Autism Brain Imaging Data Exchange Project, and Simulation. Biol Psychiatry. 2015 Jul 15;78(2):126-34. doi: 10.1016/j.biopsych.2015.02.010. PMID: 25850620.X-1

3703. Lefort R. Reversing synapse loss in Alzheimer's disease: Rho-guanosine triphosphatases and insights from other brain disorders. Neurotherapeutics. 2015 Jan;12(1):19-28. doi: 10.1007/s13311-0140328-4. PMID: 25588580.X-1

3704. Lerman DC, Hawkins L, Hillman C, et al. Adults with autism spectrum disorder as behavior technicians for young children with autism: Outcomes of a behavioral skills training program. J Appl Behav Anal. 2015 Summer;48(2):233-56. doi: 10.1002/jaba.196. PMID: 25869079.X-1, X-3, X-4

3705. Lerthattasilp T, Charernboon T, Chunsuwan I, et al. Depression and burden among caregivers of children with autistic spectrum disorder. J Med Assoc Thai. 2015 Mar;98 Suppl 2:S45-52. PMID: 26211103.X-1

3706. Leung RC, Vogan VM, Powell TL, et al. The role of executive functions in social impairment in Autism Spectrum Disorder. Child Neuropsychol. 2015 Mar 3:1-9. doi: 10.1080/09297049.2015.1005066. PMID: 25731979.X-1

3707. Levin AR, Fox NA, Zeanah CH, Jr., et al. Social communication difficulties and autism in previously institutionalized children. J Am Acad Child Adolesc Psychiatry. 2015 Feb;54(2):108-15.e1. doi: 10.1016/j.jaac.2014.11.011. PMID: 25617251.X-1

3708. Levin IP, Gaeth GJ, Foley-Nicpon M, et al. Extending decision making competence to special populations: a pilot study of persons on the autism spectrum. Front Psychol. 2015;6:539. doi: 10.3389/fpsyg.2015.00539. PMID: 25972831.X-1 
3709. Li HH, Shan L, Du L, et al. [Research advances in the management of autism spectrum disorders in children]. Zhongguo Dang Dai Er Ke Za Zhi. 2015 Aug;17(8):88692. PMID: 26287360.X-1, X-2

3710. Li J, Cai T, Jiang Y, et al. Genes with de novo mutations are shared by four neuropsychiatric disorders discovered from NPdenovo database. Mol Psychiatry. 2015 Apr 7doi: 10.1038/mp.2015.40. PMID: 25849321.X-1

3711. Li Q, Chen CF, Wang DY, et al. Transplantation of umbilical cord blood mononuclear cells increases levels of nerve growth factor in the cerebrospinal fluid of patients with autism. Genet Mol Res. 2015;14(3):8725-32. doi: 10.4238/2015.July.31.21. PMID: 26345804.X-1

3712. Li YM, Ou JJ, Liu L, et al. Association Between Maternal Obesity and Autism Spectrum Disorder in Offspring: A Metaanalysis. J Autism Dev Disord. 2015 Aug 9doi: 10.1007/s10803-015-2549-8. PMID: 26254893.X-1

3713. Li Z, Yu Z, Zhang J, et al. Impact of Rivastigmine on Cognitive Dysfunction and Falling in Parkinson's Disease Patients. Eur Neurol. 2015;74(1-2):86-91. doi: 10.1159/000438824. PMID: 26288230.X-1

3714. Lichtman JS, Sonnenburg JL, Elias JE. Monitoring host responses to the gut microbiota. Isme j. 2015 Sep;9(9):1908-15. doi: 10.1038/ismej.2015.93. PMID: 26057846.X-1

3715. Light J, McNaughton D. Designing AAC Research and Intervention to Improve Outcomes for Individuals with Complex Communication Needs. Augment Altern Commun. 2015 Jun;31(2):85-96. doi: 10.3109/07434618.2015.1036458. PMID: 25904008.X-1

3716. Lim CK, Essa MM, de Paula Martins $\mathrm{R}$, et al. Altered kynurenine pathway metabolism in autism: Implication for immuneinduced glutamatergic activity. Autism Res. 2015 Oct 24doi: 10.1002/aur.1565. PMID: 26497015.X-1
3717. Lim CM. Accommodating Autistics and Treating Autism: Can We Have Both? Bioethics. 2015 Oct;29(8):564-72. doi: 10.1111/bioe.12154. PMID: 25689416.X-1, X2

3718. Lim L, Chantiluke K, Cubillo AI, et al. Disorder-specific grey matter deficits in attention deficit hyperactivity disorder relative to autism spectrum disorder. Psychol Med. 2015 Apr;45(5):965-76. doi: 10.1017/s0033291714001974. PMID: 25229248.X-1

3719. Lin IF, Mochida T, Asada K, et al. Atypical delayed auditory feedback effect and Lombard effect on speech production in highfunctioning adults with autism spectrum disorder. Front Hum Neurosci. 2015;9:510. doi: 10.3389/fnhum.2015.00510. PMID: 26441607.X-1

3720. Lin IF, Yamada T, Komine Y, et al. Enhanced segregation of concurrent sounds with similar spectral uncertainties in individuals with autism spectrum disorder. Sci Rep. 2015;5:10524. doi: 10.1038/srep10524. PMID: 26001110.X-1

3721. Lin IF, Yamada T, Komine Y, et al. Vocal Identity Recognition in Autism Spectrum Disorder. PLoS One. 2015;10(6):e0129451. doi: 10.1371/journal.pone.0129451. PMID: 26070199.X-1

3722. Lindberg D, Shan D, Ayers-Ringler J, et al. Purinergic signaling and energy homeostasis in psychiatric disorders. Curr Mol Med. 2015;15(3):275-95. PMID: 25950756.X1

3723. Lindly OJ, Sinche BK, Zuckerman KE. Variation in Educational Services Receipt Among US Children With Developmental Conditions. Acad Pediatr. 2015 SepOct;15(5):534-43. doi: 10.1016/j.acap.2015.04.001. PMID: 26344719.X-1

3724. Lisiecka DM, Holt R, Tait R, et al. Developmental white matter microstructure in autism phenotype and corresponding endophenotype during adolescence. Transl Psychiatry. 2015;5:e529. doi: 10.1038/tp.2015.23. PMID: 25781228.X-1 
3725. Little LM, Ausderau K, Sideris J, et al. Activity Participation and Sensory Features Among Children with Autism Spectrum Disorders. J Autism Dev Disord. 2015 Sep;45(9):2981-90. doi: 10.1007/s10803-0152460-3. PMID: 25975628.X-1

3726. Littlewood RA, Claus ED, Arenella P, et al. Dose specific effects of olanzapine in the treatment of alcohol dependence.

Psychopharmacology (Berl). 2015

Apr;232(7):1261-8. doi: 10.1007/s00213-014-

3757-1. PMID: 25304864.X-1

3727. Liu X, Kawashima M, Miyagawa T, et al. Novel rare variations of the oxytocin receptor (OXTR) gene in autism spectrum disorder individuals. Hum Genome Var. 2015;2:15024. doi: 10.1038/hgv.2015.24. PMID: 27081536.X-1

3728. Liu Z, Qian Y, Zhang Y, et al. Effects of several temporomandibular disorders on the stress distributions of temporomandibular joint: a finite element analysis. Comput Methods Biomech Biomed Engin. 2015 Jan 14:1-7. doi: 10.1080/10255842.2014.996876. PMID: 25587737.X-1

3729. Llorens F, Schmitz M, Gloeckner SF, et al. Increased albumin CSF/serum ratio in dementia with Lewy bodies. J Neurol Sci. 2015 Oct 14doi: 10.1016/j.jns.2015.10.011. PMID: 26476775.X-1

3730. Lloyd BP, Finley CI, Weaver ES. Experimental analysis of stereotypy with applications of nonparametric statistical tests for alternating treatments designs. Dev Neurorehabil. 2015 Nov 17:1-11. doi: 10.3109/17518423.2015.1091043. PMID: 26577886.X-1

3731. Loades ME. Evidence-Based Practice in the Face of Complexity and Comorbidity: A Case Study of an Adolescent With Asperger's Syndrome, Anxiety, Depression, and Chronic Pain. J Child Adolesc Psychiatr Nurs. 2015 May;28(2):73-83. doi: 10.1111/jcap.12108. PMID: 25865961.X-1, X-3, X-4

3732. Locke J, Olsen A, Wideman R, et al. A tangled web: the challenges of implementing an evidence-based social engagement intervention for children with autism in urban public school settings. Behav Ther. 2015 Jan;46(1):54-67. doi: 10.1016/j.beth.2014.05.001. PMID: 25526835.X-1, X-3, X-4

3733. Locke J, Shih W, Kretzmann M, et al. Examining playground engagement between elementary school children with and without autism spectrum disorder. Autism. 2015 Sep 4doi: 10.1177/1362361315599468. PMID: 26341991.X-1

3734. Logan SL, Carpenter L, Leslie RS, et al. Aberrant Behaviors and Co-occurring Conditions as Predictors of Psychotropic Polypharmacy among Children with Autism Spectrum Disorders. J Child Adolesc Psychopharmacol. 2015 May;25(4):323-36. doi: 10.1089/cap.2013.0119. PMID: 25919445.X-1

3735. Lotan Y. Promises and challenges of fluorescence cystoscopy. Urol Oncol. 2015 Jun;33(6):261-4. doi: 10.1016/j.urolonc.2015.03.019. PMID: 25937623.X-1

3736. Lough E, Hanley M, Rodgers J, et al. Violations of Personal Space in Young People with Autism Spectrum Disorders and Williams Syndrome: Insights from the Social Responsiveness Scale. J Autism Dev Disord. 2015 Jul 24doi: 10.1007/s10803-015-2536-0. PMID: 26206231.X-1

3737. Loukas KM, Raymond L, Perron AR, et al. Occupational transformation: Parental influence and social cognition of young adults with autism. Work. 2015;50(3):457-63. doi: 10.3233/wor-141956. PMID: 25248535.X-1

3738. Louw JJ, Corveleyn A, Jia Y, et al. MEIS2 involvement in cardiac development, cleft palate, and intellectual disability. Am J Med Genet A. 2015 May;167a(5):1142-6. doi: 10.1002/ajmg.a.36989. PMID: 25712757.X-1

3739. Lozano R, Martinez-Cerdeno V, Hagerman RJ. Advances In The Understanding Of The Gabaergic Neurobiology Of Fmr1 Expanded Alleles Leading To Targeted Treatments For Fragile X Spectrum Disorder. Curr Pharm Des. 2015 Sep 14 PMID: 26365141.X-1

3740. Lugnegard T, Hallerback MU, Gillberg C. Asperger syndrome and schizophrenia: Overlap of self-reported autistic traits using the Autism-spectrum Quotient 
(AQ). Nord J Psychiatry. 2015 May;69(4):26874. doi: 10.3109/08039488.2014.972452. PMID: 25389915.X-1

3741. Lukas M, Wohr M. Endogenous vasopressin, innate anxiety, and the emission of pro-social $50-\mathrm{kHz}$ ultrasonic vocalizations during social play behavior in juvenile rats. Psychoneuroendocrinology. 2015 Jun;56:35-44. doi: 10.1016/j.psyneuen.2015.03.005. PMID: 25800147.X-1

3742. Lukic A, Uphill J, Brown CA, et al. Rare structural genetic variation in human prion diseases. Neurobiol Aging. 2015

May;36(5):2004.e1-8. doi:

10.1016/j.neurobiolaging.2015.01.011. PMID: 25726360.X-1

3743. Lumu L, Albertyn L, Szabo CP. Psychiatric services for preschoolers: an emerging need. J Child Adolesc Ment Health. 2015 Jul;27(2):113-24. doi: 10.2989/17280583.2015.1081207. PMID: 26357917.X-1

3744. Lundstrom S, Reichenberg A, Melke J, et al. Autism spectrum disorders and coexisting disorders in a nationwide Swedish twin study. J Child Psychol Psychiatry. 2015 Jun;56(6):70210. doi: 10.1111/jcpp.12329. PMID: 25279993.X-1

3745. Lyall K, Van de Water J, Ashwood P, et al. Asthma and Allergies in Children With Autism Spectrum Disorders: Results From the CHARGE Study. Autism Res. 2015 Oct;8(5):567-74. doi: 10.1002/aur.1471. PMID: 25722050.X-1

3746. Lydon S, Healy O, Reed P, et al. A systematic review of physiological reactivity to stimuli in autism. Dev Neurorehabil. 2015 Aug 24:1-21. doi: 10.3109/17518423.2014.971975. PMID: 25356589.X-1, X-2

3747. Lynch BA, Weaver AL, Starr SR, et al. Developmental Screening and Follow-up by

Nurses. MCN Am J Matern Child Nurs. 2015

Nov-Dec;40(6):388-93. doi:

10.1097/nmc.0000000000000179. PMID: 26488856.X-1

3748. Ma L, Fu C, Liu H, et al.

[Retrospective study of the efficacy and safety of treatment with PDD vs PAD in de novo patients with multiple myiloma]. Zhonghua Xue Ye Xue Za Zhi. 2015 Apr;36(4):340-3. doi: 10.3760/cma.j.issn.0253-2727.2015.04.018. PMID: 25916300.X-1

3749. Ma YH, Li YW, Ma L, et al. Anesthesia for stem cell transplantation in autistic children: A prospective, randomized, double-blind comparison of propofol and etomidate following sevoflurane inhalation. Exp Ther Med. 2015 Mar;9(3):1035-9. doi: 10.3892/etm.2015.2176. PMID: 25667673.X-1

3750. MacFabe DF. Enteric short-chain fatty acids: microbial messengers of metabolism, mitochondria, and mind: implications in autism spectrum disorders. Microb Ecol Health Dis. 2015;26:28177. doi: 10.3402/mehd.v26.28177. PMID: 26031685.X-1

3751. Machado C, Estevez M, Leisman G, et al. QEEG spectral and coherence assessment of autistic children in three different experimental conditions. J Autism Dev Disord. 2015 Feb;45(2):406-24. doi: 10.1007/s10803-0131909-5. PMID: 24048514.X-1, X-3

3752. Machado CJ, Whitaker AM, Smith SE, et al. Maternal immune activation in nonhuman primates alters social attention in juvenile offspring. Biol Psychiatry. 2015 May 1;77(9):823-32. doi: 10.1016/j.biopsych.2014.07.035. PMID: 25442006.X-1

3753. MacMullin JA, Lunsky Y, Weiss JA. Plugged in: Electronics use in youth and young adults with autism spectrum disorder. Autism. 2015 Feb 18doi: 10.1177/1362361314566047. PMID: 25694586.X-1

3754. Madsen GF, Bilenberg N, Jepsen JR, et al. Normal P50 Gating in Children with Autism, Yet Attenuated P50 Amplitude in the Asperger Subcategory. Autism Res. 2015 Aug;8(4):371-8. doi: 10.1002/aur.1452. PMID: 25599888.X-1

3755. Maekawa M, Iwayama Y, Ohnishi T, et al. Investigation of the fatty acid transporterencoding genes SLC27A3 and SLC27A4 in autism. Sci Rep. 2015;5:16239. doi: 10.1038/srep16239. PMID: 26548558.X-1

3756. Magana S, Lopez K, Machalicek W. Parents Taking Action: A Psycho-Educational 
Intervention for Latino Parents of Children With Autism Spectrum Disorder. Fam Process. 2015 Aug 13doi: 10.1111/famp.12169. PMID: 26269291.X-3, X-4

3757. Magana S, Parish SL, Son E. Have Racial and Ethnic Disparities in the Quality of Health Care Relationships Changed for Children With Developmental Disabilities and ASD? Am J Intellect Dev Disabil. 2015 Nov;120(6):504-13. doi: 10.1352/1944-7558120.6.504. PMID: 26505871.X-1

3758. Magiati I, Ong C, Lim XY, et al. Anxiety symptoms in young people with autism spectrum disorder attending special schools: Associations with gender, adaptive functioning and autism symptomatology. Autism. $2015 \mathrm{Apr}$ 27doi: 10.1177/1362361315577519. PMID: 25916865.X-1

3759. Magid-Bernstein J, Mahajan K, Lincoln J, et al. Case report: cytokine and CD4+ T-cell profiles of monozygotic twins with autism and divergent comorbidities and drug treatment. J Child Neurol. 2015 Mar;30(3):386-90. doi: 10.1177/0883073814529821. PMID: 24736120.X-1

3760. Mahajan R, Mostofsky SH. Neuroimaging endophenotypes in autism spectrum disorder. CNS Spectr. 2015 Aug;20(4):412-26. doi: 10.1017/s1092852915000371. PMID: 26234701.X-1

3761. Mahajnah M, Sharkia R, Shalabe H, et al. Clinical characteristics of autism spectrum disorder in Israel: impact of ethnic and social diversities. Biomed Res Int. 2015;2015:962093. doi: 10.1155/2015/962093. PMID: 25984535.X-1

3762. Main PA, Thomas P, Angley MT, et al. Lack of evidence for genomic instability in autistic children as measured by the cytokinesis-block micronucleus cytome assay. Autism Res. 2015 Feb;8(1):94-104. doi: 10.1002/aur.1428. PMID: 25371234.X-1

3763. Mak E, Su L, Williams GB, et al. Neuroimaging correlates of cognitive impairment and dementia in Parkinson's disease. Parkinsonism Relat Disord. 2015 Aug;21(8):862-70. doi: 10.1016/j.parkreldis.2015.05.013. PMID: 26004683.X-1

3764. Malgaz M, van Kesteren CF, Sommer IE. [Auditory verbal hallucinations in an adult with autism spectrum disorder]. Tijdschr Psychiatr. 2015;57(9):684-7. PMID: 26401611.X-1

3765. Malhi P, Singhi P. Adaptive Behavior Functioning in Children with Autism. Indian J Pediatr. 2015 Aug;82(8):677-81. doi: 10.1007/s12098-015-1692-8. PMID: 25673293.X-1

3766. Mandic-Maravic V, PejovicMilovancevic M, Mitkovic-Voncina M, et al. Sex differences in autism spectrum disorders: does sex moderate the pathway from clinical symptoms to adaptive behavior? Sci Rep. 2015;5:10418. doi: 10.1038/srep10418. PMID: 25988942.X-1

3767. Mandy W, Murin M, Baykaner O, et al. Easing the transition to secondary education for children with autism spectrum disorder: An evaluation of the Systemic Transition in Education Programme for Autism Spectrum Disorder (STEP-ASD). Autism. 2015 Aug 24doi: 10.1177/1362361315598892. PMID: 26304678.X-1

3768. Mandy W, Tchanturia K. Do women with eating disorders who have social and flexibility difficulties really have autism? A case series. Mol Autism. 2015;6:6. doi: 10.1186/2040-2392-6-6. PMID: 26056560.X-1

3769. Manea S, Dalla Pozza LV, Mazzucato $\mathrm{M}$, et al. Diagnosis of pervasive developmental disorders: when and how? An area-based study about health care providers. World J Pediatr. 2015 Feb;11(1):48-53. doi: 10.1007/s12519014-0533-6. PMID: 25447633.X-1

3770. Manning C, Morgan M, Allen C, et al. Do children with autism show reduced susceptibility to the Ebbinghaus illusion? J Vis. 2015 Sep 1;15(12):646. doi: 10.1167/15.12.646. PMID: 26326334.X-1

3771. Manning C, Neil L, Karaminis T, et al. The effects of grouping on speed discrimination thresholds in adults, typically developing children, and children with autism. J Vis. 2015 
Aug 1;15(11):17. doi: 10.1167/15.11.17. PMID: 26317324.X-1

3772. Manschot GM, Hondius AJ, van Dijk MM, et al. [Resolution of a treatment impasse by combining two Dutch laws regarding a patient with paranoid thoughts and a testicular cancer]. Tijdschr Psychiatr. 2015;57(9):680-3. PMID: 26401610.X-1

3773. Margolis SS, Sell GL, Zbinden MA, et al. Angelman Syndrome. Neurotherapeutics. 2015 Jul;12(3):641-50. doi: 10.1007/s13311015-0361-y. PMID: 26040994.X-1

3774. Mariappan P, Rai B, El-Mokadem I, et al. Real-life Experience: Early Recurrence With Hexvix Photodynamic Diagnosis-assisted Transurethral Resection of Bladder Tumour vs Good-quality White Light TURBT in New Non-muscle-invasive Bladder Cancer. Urology. 2015 Aug;86(2):327-31. doi:

10.1016/j.urology.2015.04.015. PMID: 26142924.X-1

3775. Mari-Bauset S, Llopis-Gonzalez A, Zazpe I, et al. Nutritional Impact of a GlutenFree Casein-Free Diet in Children with Autism Spectrum Disorder. J Autism Dev Disord. 2015 Oct 1doi: 10.1007/s10803-015-2582-7. PMID: 26428353.X-1

3776. Mari-Bauset S, Llopis-Gonzalez A, Zazpe I, et al. Fat intake in children with autism spectrum disorder in the Mediterranean region (Valencia, Spain). Nutr Neurosci. 2015 May 28doi: 10.1179/1476830515y.0000000029. PMID: 26020227.X-1

3777. Mark DH, Civic D. Special report: early intensive behavioral intervention and other behavioral interventions for autism spectrum disorder. Technol Eval Cent Assess Program Exec Summ. 2015 Mar;30(1):1-3. PMID: 25962192.X-2

3778. Marko MK, Crocetti D, Hulst T, et al. Behavioural and neural basis of anomalous motor learning in children with autism. Brain: A Journal of Neurology. 2015;138(3):784-97 14p. doi: brain/awu394. PMID: 103761287. Language: English. Entry Date: 20150508. Revision Date: 20150710. Publication Type: Journal Article.X-1
3779. Marrus N, Glowinski AL, Jacob T, et al. Rapid video-referenced ratings of reciprocal social behavior in toddlers: a twin study. J Child Psychol Psychiatry. 2015 Feb 11doi: 10.1111/jcpp.12391. PMID: 25677414.X-1

3780. Marshall D, Goodall C. The Right to Appropriate and Meaningful Education for Children with ASD. J Autism Dev Disord. 2015 Oct;45(10):3159-67. doi: 10.1007/s10803-0152475-9. PMID: 26036647.X-1

3781. Marshall J, Hill RJ, Ware RS, et al. Multidisciplinary intervention for childhood feeding difficulties. J Pediatr Gastroenterol Nutr. 2015 May;60(5):680-7. doi: 10.1097/mpg.0000000000000669. PMID: 25534777.X-1

3782. Marshall J, Ware R, Ziviani J, et al. Efficacy of interventions to improve feeding difficulties in children with autism spectrum disorders: a systematic review and metaanalysis. Child Care Health Dev. 2015 Mar;41(2):278-302. doi: 10.1111/cch.12157. PMID: 24962184.X-2

3783. Martin L, Iceberg E. Quantifying Social Motivation in Mice Using Operant Conditioning. J Vis Exp. 2015(102):e53009. doi: 10.3791/53009. PMID: 26327305.X-1

3784. Maruani A, Huguet G, Beggiato A, et al. 11q24.2-25 micro-rearrangements in autism spectrum disorders: Relation to brain structures. Am J Med Genet A. 2015 Sep 3doi: 10.1002/ajmg.a.37345. PMID: 26334118.X-1

3785. Marzinzik F, Herrmann A, Gogarten JH, et al. Dysfunctional action control as a specific feature of Parkinson's disease. J Neural Transm (Vienna). 2015 Aug;122(8):1125-33. doi: 10.1007/s00702-014-1354-4. PMID: 25547860.X-1

3786. Masi A, Lampit A, Glozier N, et al. Predictors of placebo response in pharmacological and dietary supplement treatment trials in pediatric autism spectrum disorder: a meta-analysis. Transl Psychiatry. 2015;5:e640. doi: 10.1038/tp.2015.143. PMID: 26393486.X-2

3787. Masi A, Quintana DS, Glozier N, et al. Cytokine aberrations in autism spectrum disorder: a systematic review and meta- 
analysis. Mol Psychiatry. 2015 Apr;20(4):4406. doi: 10.1038/mp.2014.59. PMID: 24934179.X-1

3788. Maski K, Holbrook H, Manoach D, et al. Sleep Dependent Memory Consolidation in Children with Autism Spectrum Disorder. Sleep. 2015 Jul 13 PMID: 26194566.X-1

3789. Masuya Y, Okamoto Y, Inohara K, et al. Sex-different abnormalities in the right second to fourth digit ratio in Japanese individuals with autism spectrum disorders. Mol Autism. 2015;6:34. doi: 10.1186/s13229015-0028-x. PMID: 26060570.X-1

3790. Matlis S, Boric K, Chu CJ, et al. Robust disruptions in electroencephalogram cortical oscillations and large-scale functional networks in autism. BMC Neurol. 2015;15:97. doi: 10.1186/s12883-015-0355-8. PMID: 26111798.X-1

3791. Matsuda S, Minagawa Y, Yamamoto J. Gaze Behavior of Children with ASD toward Pictures of Facial Expressions. Autism Res Treat. 2015;2015:617190. doi: 10.1155/2015/617190. PMID: 26090223.X-1

3792. Matsumoto MM, Matthews KR. A Need for Renewed and Cohesive US Policy on Cord Blood Banking. Stem Cell Rev. 2015 Aug 4doi: 10.1007/s12015-015-9613-9. PMID: 26239848.X-1

3793. Matsuo J, Kamio Y, Takahashi H, et al. Autistic-like traits in adult patients with mood disorders and schizophrenia. PLoS One. 2015;10(4):e0122711. doi: 10.1371/journal.pone.0122711. PMID: 25838109.X-1

3794. Matsuo M, Fuji A, Matsuzaka T, et al. [Effectiveness and safety of long-term levetiracetam treatment in patients with refractory epilepsy]. No To Hattatsu. 2015 Jul;47(4):272-8. PMID: 26353448.X-1

3795. Matthews NL, Smith CJ, Pollard E, et al. Adaptive Functioning in Autism Spectrum Disorder During the Transition to Adulthood. J Autism Dev Disord. 2015 Aug;45(8):2349-60. doi: 10.1007/s10803-015-2400-2. PMID: 25733158.X-1
3796. Mattison ML, Dando CJ, Ormerod TC. Sketching to remember: episodic free recall task support for child witnesses and victims with autism spectrum disorder. J Autism Dev Disord. 2015 Jun;45(6):1751-65. doi: 10.1007/s10803014-2335-z. PMID: 25503484.X-1

3797. May T, Cornish K, Rinehart NJ. Parent-Child Agreement Using the Spence Children's Anxiety Scale and a Thermometer in Children with Autism Spectrum Disorder. Autism Res Treat. 2015;2015:315495. doi: 10.1155/2015/315495. PMID: 25922765.X-1

3798. May T, Cornish K, Rinehart NJ. Mechanisms of Anxiety Related Attentional Biases in Children with Autism Spectrum Disorder. J Autism Dev Disord. 2015 Oct;45(10):3339-50. doi: 10.1007/s10803-0152500-z. PMID: 26070278.X-1

3799. Mazefsky CA. Emotion Regulation and Emotional Distress in Autism Spectrum Disorder: Foundations and Considerations for Future Research. J Autism Dev Disord. 2015 Nov;45(11):3405-8. doi: 10.1007/s10803-0152602-7. PMID: 26391886.X-1

3800. Mazurek MO, Petroski GF. Sleep problems in children with autism spectrum disorder: examining the contributions of sensory over-responsivity and anxiety. Sleep Med. 2015 Feb;16(2):270-9. doi: 10.1016/j.sleep.2014.11.006. PMID: 25600781.X-1

3801. McArthur S, Pienaar IS, Siddiqi SM, et al. Sex-specific disruption of murine midbrain astrocytic and dopaminergic developmental trajectories following antenatal GC treatment. Brain Struct Funct. 2015 May 6doi: 10.1007/s00429-015-1049-0. PMID: 25944572.X-1

3802. McConachie H. Appropriate outcome measurement for children with autism spectrum disorder. Dev Med Child Neurol. 2015 Jul 30doi: 10.1111/dmcn.12862. PMID: 26235606.X-1, X-2

3803. McConachie H, Fletcher-Watson S. Building capacity for rigorous controlled trials in autism: the importance of measuring treatment adherence. Child Care Health Dev. 2015 Mar;41(2):169-77. doi: 10.1111/cch.12185. PMID: 25163398.X-1, X-2 
3804. McConachie H, Parr JR, Glod M, et al. Systematic review of tools to measure outcomes for young children with autism spectrum disorder. Health Technol Assess. 2015 Jun;19(41):1-506. doi: 10.3310/hta19410. PMID: 26065374.X-2

3805. McCrimmon AW, Matchullis RL, Altomare AA. Resilience and emotional intelligence in children with high-functioning autism spectrum disorder. Dev Neurorehabil. 2015 Aug 24:1-8. doi: 10.3109/17518423.2014.927017. PMID: 24960312.X-1

3806. McDermott S, Hardin JW, Royer JA, et al. Emergency department and inpatient hospitalizations for young people with fragile $\mathrm{X}$ syndrome. Am J Intellect Dev Disabil. 2015 May;120(3):230-43. doi: 10.1352/1944-7558120.3.230. PMID: 25928435.X-1

3807. McDonnell A, McCreadie M, Mills R, et al. The role of physiological arousal in the management of challenging behaviours in individuals with autistic spectrum disorders. Research in Developmental Disabilities. 2015 2015-12-09;36:311-22. doi: http://dx.doi.org/10.1016/j.ridd.2014.09.012. PMID: 1640012096; 2014-54687-033.X-1

3808. McDuffie A, Thurman AJ, Hagerman RJ, et al. Symptoms of Autism in Males with Fragile X Syndrome: A Comparison to Nonsyndromic ASD Using Current ADI-R Scores. J Autism Dev Disord. 2015 Jul;45(7):1925-37. doi: 10.1007/s10803-0132013-6. PMID: 24414079.X-1

3809. McEvilly M, Wicks S, Dalman C. Sick Leave and Work Participation Among Parents of Children with Autism Spectrum Disorder in the Stockholm Youth Cohort: A Register Linkage Study in Stockholm, Sweden. J Autism Dev Disord. 2015 Jul;45(7):2157-67. doi: 10.1007/s10803-015-2381-1. PMID: 25697737.X-1

3810. McGrane IR, Loveland JG, Zaluski HJ, et al. Serum Quetiapine Concentration Changes with Concomitant Oxcarbazepine Therapy in a Boy with Autism Spectrum Disorder. J Child Adolesc Psychopharmacol. 2015 Oct 15doi: 10.1089/cap.2015.0117. PMID: 26469302.X-1, X-3, X-4
3811. McGrath LM, Braaten EB, Doty ND, et al. Extending the 'cross-disorder' relevance of executive functions to dimensional neuropsychiatric traits in youth. J Child Psychol Psychiatry. 2015 Sep 28doi: 10.1111/jcpp.12463. PMID: 26411927.X-1

3812. McHale SM, Updegraff KA, Feinberg ME. Siblings of Youth with Autism Spectrum Disorders: Theoretical Perspectives on Sibling Relationships and Individual Adjustment. J Autism Dev Disord. 2015 Oct 17doi: 10.1007/s10803-015-2611-6. PMID: 26476737.X-1

3813. McKean SJ, Bartell SM, Hansen RL, et al. Prenatal mercury exposure, autism, and developmental delay, using pharmacokinetic combination of newborn blood concentrations and questionnaire data: a case control study. Environ Health. 2015;14:62. doi: 10.1186/s12940-015-0045-4. PMID: 26198445.X-1

3814. McKenna PE, Glass A, Rajendran G, et al. Strange Words: Autistic Traits and the Processing of Non-Literal Language. J Autism Dev Disord. 2015 Nov;45(11):3606-12. doi: 10.1007/s10803-015-2508-4. PMID: 26093391.X-1

3815. McKenney EL, Bristol RM. Supporting intensive interventions for students with autism spectrum disorder: performance feedback and discrete trial teaching. Sch Psychol Q. 2015 Mar;30(1):8-22. doi: 10.1037/spq0000060. PMID: 24884446.X-1

3816. McLay LK, France K. Empirical research evaluating non-traditional approaches to managing sleep problems in children with autism. Dev Neurorehabil. 2015 Aug 24:1-12. doi: 10.3109/17518423.2014.904452. PMID: 24724691.X-1, X-2

3817. McLeod BD, Wood JJ, Klebanoff S. Advances in evidence-based intervention and assessment practices for youth with an autism spectrum disorder. Behav Ther. 2015 Jan;46(1):1-6. doi: 10.1016/j.beth.2014.07.004. PMID: 25526830.X-1

3818. McMahon CM, Henderson HA. Errormonitoring in response to social stimuli in individuals with higher-functioning Autism 
Spectrum Disorder. Dev Sci. 2015

May;18(3):389-403. doi: 10.1111/desc.12220.

PMID: 25066088.X-1

3819. McMahon CM, Solomon M. Brief

Report: Parent-Adolescent Informant

Discrepancies of Social Skill Importance and

Social Skill Engagement for Higher-

Functioning Adolescents with Autism Spectrum

Disorder. J Autism Dev Disord. 2015

Oct;45(10):3396-403. doi: 10.1007/s10803-

015-2494-6. PMID: 26077952.X-1

3820. McMahon JJ, Yu W, Yang J, et al.

Seizure-dependent mTOR activation in 5-HT

neurons promotes autism-like behaviors in

mice. Neurobiol Dis. 2015 Jan;73:296-306. doi:

10.1016/j.nbd.2014.10.004. PMID:

25315683.X-1

3821. Meguid NA, Gebril OH, Khalil RO. A

study of blood serotonin and serotonin

transporter promoter variant (5-HTTLPR)

polymorphism in Egyptian autistic children.

Adv Biomed Res. 2015;4:94. doi:

10.4103/2277-9175.156658. PMID:

26015920.X-1

3822. Mehling MH, Tasse MJ. Impact of Choice on Social Outcomes of Adults with

ASD. J Autism Dev Disord. 2015

Jun;45(6):1588-602. doi: 10.1007/s10803-014-

2312-6. PMID: 25413143.X-1

3823. Mehta B, Chawla VK, Parakh M, et al. ABER Assessment in Pre-school Children with Developmental Speech and Language

Impairment. J Clin Diagn Res. 2015

May;9(5):Cc01-3. doi:

10.7860/jcdr/2015/13723.5873. PMID:

26155471.X-1

3824. Mehta UM, Waghmare AV, Thirthalli $\mathrm{J}$, et al. Is the human mirror neuron system plastic? Evidence from a transcranial magnetic stimulation study. Asian J Psychiatr. 2015 Jul 8doi: 10.1016/j.ajp.2015.06.014. PMID: 26194133.X-1

3825. Meilleur A-A, Jelenic P, Mottron L. Prevalence of Clinically and Empirically Defined Talents and Strengths in Autism. Journal of Autism \& Developmental Disorders. 2015;45(5):1354-67 14p. doi: 10.1007/s10803014-2296-2. PMID: 103791723. Language:

English. Entry Date: 20150424. Revision Date:
20150710. Publication Type: Journal Article.X1

3826. Merrick H, McConachie H, Le Couteur A, et al. Characteristics of young people with long term conditions close to transfer to adult health services. BMC Health Serv Res. 2015;15(1):435. doi: 10.1186/s12913-015-1095-6. PMID: 26424085.X-1

3827. Messinger DS, Young GS, Webb SJ, et al. Early sex differences are not autism-specific: A Baby Siblings Research Consortium (BSRC) study. Mol Autism. 2015;6:32. doi: 10.1186/s13229-015-0027-y. PMID: 26045943.X-1

3828. Meziane H, Schaller F, Bauer S, et al. An Early Postnatal Oxytocin Treatment Prevents Social and Learning Deficits in Adult Mice Deficient for Magel2, a Gene Involved in Prader-Willi Syndrome and Autism. Biol Psychiatry. 2015 Jul 15;78(2):85-94. doi: 10.1016/j.biopsych.2014.11.010. PMID: 25599930.X-1

3829. Mikita N, Hollocks MJ, Papadopoulos AS, et al. Irritability in boys with autism spectrum disorders: an investigation of physiological reactivity. J Child Psychol Psychiatry. 2015 Oct;56(10):1118-26. doi: 10.1111/jcpp.12382. PMID: 25626926.X-1

3830. Miller SA, Rodriguez NM, Rourke AJ. Do mirrors facilitate acquisition of motor imitation in children diagnosed with autism? J Appl Behav Anal. 2015 Spring;48(1):194-8. doi: 10.1002/jaba.187. PMID: 25684255.X-1, $\mathrm{X}-3, \mathrm{X}-4$

3831. Mimouni-Bloch A, Yeshaya J, Kahana $\mathrm{S}$, et al. A de-novo interstitial microduplication involving 2p16.1-p15 and mirroring 2p16.1-p15 microdeletion syndrome: Clinical and molecular analysis. Eur J Paediatr Neurol. 2015 Nov;19(6):711-5. doi: 10.1016/j.ejpn.2015.07.013. PMID: 26278498.X-1

3832. Minhas A, Vajaratkar V, Divan G, et al. Parents' perspectives on care of children with autistic spectrum disorder in South Asia Views from Pakistan and India. Int Rev Psychiatry. 2015;27(3):247-56. doi: 
10.3109/09540261.2015.1049128. PMID: 26107996.X-1

3833. Minnes P, Perry A, Weiss JA. Predictors of distress and well-being in parents of young children with developmental delays and disabilities: the importance of parent perceptions. J Intellect Disabil Res. 2015 Jun;59(6):551-60. doi: 10.1111/jir.12160. PMID: 25169777.X-1

3834. Minshawi NF, Hurwitz S, Morriss D, et al. Multidisciplinary assessment and treatment of self-injurious behavior in autism spectrum disorder and intellectual disability: integration of psychological and biological theory and approach. J Autism Dev Disord. 2015 Jun;45(6):1541-68. doi: 10.1007/s10803014-2307-3. PMID: 25395094.X-1, X-2

3835. Mirabella AC, Foster BM, Bartke T. Chromatin deregulation in disease.

Chromosoma. 2015 Jul 19doi: 10.1007/s00412015-0530-0. PMID: 26188466.X-1

3836. Mirabella G. Is art therapy a reliable tool for rehabilitating people suffering from brain/mental diseases? J Altern Complement Med. 2015 Apr;21(4):196-9. doi: 10.1089/acm.2014.0374. PMID: 25848886.X-1

3837. Miron O, Ari-Even Roth D, Gabis LV, et al. Prolonged auditory brainstem responses in infants with autism. Autism Res. 2015 Oct 19doi: 10.1002/aur.1561. PMID: 26477791.X-1

3838. Misic B, Doesburg SM, Fatima Z, et al. Coordinated Information Generation and Mental Flexibility: Large-Scale Network Disruption in Children with Autism. Cereb Cortex. 2015 Sep;25(9):2815-27. doi: 10.1093/cercor/bhu082. PMID: 24770713.X-1, $\mathrm{X}-2$

3839. Mitchell ES, Mrug S, Patterson CS, et al. Summer Treatment Program Improves Behavior of Children with High-Functioning Autism Spectrum Disorder. J Autism Dev Disord. 2015 Aug;45(8):2295-310. doi: 10.1007/s10803-014-2241-4. PMID: 25503679.X-1, X-3

3840. Mitchell GE, Locke KD. Lay beliefs about autism spectrum disorder among the general public and childcare providers. Autism. 2015 Jul;19(5):553-61. doi:
10.1177/1362361314533839. PMID: 24852751.X-1

3841. Mitteer DR, Romani PW, Greer BD, et al. Assessment and treatment of pica and destruction of holiday decorations. J Appl Behav Anal. 2015 Sep 18doi: 10.1002/jaba.255. PMID: 26380947.X-1, X-3, X-4

3842. Mohammadian M, Zarafshan H, Mohammadi MR, et al. Evaluating Reliability and Predictive Validity of the Persian Translation of Quantitative Checklist for Autism in Toddlers (Q-CHAT). Iran J Psychiatry. 2015;10(1):64-70. PMID: 26005483.X-1

3843. Mohammadzaheri F, Koegel LK, Rezaei M, et al. A Randomized Clinical Trial Comparison Between Pivotal Response Treatment (PRT) and Adult-Driven Applied Behavior Analysis (ABA) Intervention on Disruptive Behaviors in Public School Children with Autism. J Autism Dev Disord. 2015 Sep;45(9):2899-907. doi: 10.1007/s10803-0152451-4. PMID: 25953148.X-1

3844. Moieni M, Irwin MR, Jevtic I, et al. Inflammation impairs social cognitive processing: A randomized controlled trial of endotoxin. Brain Behav Immun. 2015 Aug;48:132-8. doi: 10.1016/j.bbi.2015.03.002. PMID: 25770082.X-1

3845. Molesworth C, Chevallier C, Happe F, et al. Children with autism do not show sequence effects with auditory stimuli. J Exp Psychol Gen. 2015 Feb;144(1):48-57. doi: 10.1037/a0038204. PMID: 25365532.X-1

3846. Moore DJ, Reidy J, Heavey L. Attentional allocation of autism spectrum disorder individuals: Searching for a Face-inthe-Crowd. Autism. 2015 Mar 13doi: 10.1177/1362361315573637. PMID: 25769313.X-1

3847. Moore DW, Venkatesh S, Anderson A, et al. TOBY play-pad application to teach children with ASD - A pilot trial. Dev Neurorehabil. 2015;18(4):213-7. doi: 10.3109/17518423.2013.784817. PMID: 23869435.X-1, X-3

3848. Moore KM, Cividini-Motta C, Clark $\mathrm{KM}$, et al. Sensory integration as a treatment for 
automatically maintained stereotypy.

Behavioral Interventions. 2015 2015-06-

04;30(2):95-111. doi: doi:

10.1901/jaba.1994.27-197. (Reprinted from

Analysis and Intervention in Developmental

Disabilities, 1982, Vol. 2, pp. 3-20)

http://dx.doi.org/10.1002/bin.1405. PMID:

1685828099; 2015-19248-001.X-1, X-3, X-4

3849. Mori K, Toda Y, Ito H, et al.

Neuroimaging in autism spectrum disorders:

1H-MRS and NIRS study. J Med Invest.

2015;62(1-2):29-36. doi: 10.2152/jmi.62.29.

PMID: 25817280.X-1

3850. Morin K, Guy J, Habak C, et al. Atypical Face Perception in Autism: A Point of View? Autism Res. 2015 Oct;8(5):497-506. doi: 10.1002/aur.1464. PMID: 25683613.X-1

3851. Morris SL, Foster CJ, Parsons R, et al. Differences in the use of vision and proprioception for postural control in autism spectrum disorder. Neuroscience. 2015 Oct 29;307:273-80. doi:

10.1016/j.neuroscience.2015.08.040. PMID: 26314635.X-1

3852. Moseley RL, Shtyrov Y, Mohr B, et al. Lost for emotion words: what motor and limbic brain activity reveals about autism and semantic theory. Neuroimage. 2015 Jan 1;104:413-22.

doi: 10.1016/j.neuroimage.2014.09.046. PMID: 25278250.X-1

3853. Moses L, Katz N, Weizman A. Impact of epilepsy and antiepileptic medications on the metabolic profile in adults with autism spectrum disorder and intellectual disabilities. Int Clin Psychopharmacol. 2015

Nov;30(6):351-5. doi:

10.1097/yic.0000000000000089. PMID: 26186174.X-1

3854. Mosienko V, Beis D, Alenina N, et al. Reduced isolation-induced pup ultrasonic communication in mouse pups lacking brain serotonin. Mol Autism. 2015;6:13. doi: 10.1186/s13229-015-0003-6. PMID: 25901271.X-1

3855. Mouga S, Almeida J, Cafe C, et al. Adaptive profiles in autism and other neurodevelopmental disorders. J Autism Dev Disord. 2015 Apr;45(4):1001-12. doi: 10.1007/s10803-014-2256-x. PMID: 25241010.X-1

3856. Muehlmann AM, Bliznyuk N, Duerr I, et al. Repetitive motor behavior: further characterization of development and temporal dynamics. Dev Psychobiol. 2015 Mar;57(2):201-11. doi: 10.1002/dev.21279. PMID: 25631623.X-1

3857. Mukherjee SB, Malhotra MK, Aneja $\mathrm{S}$, et al. Diagnostic accuracy of Indian Scale for Assessment of Autism (ISAA) in chidren aged 2-9 years. Indian Pediatr. 2015 Mar 8;52(3):212-6. PMID: 25848996.X-1

3858. Mullegama SV, Alaimo JT, Chen L, et al. Phenotypic and molecular convergence of 2q23.1 deletion syndrome with other neurodevelopmental syndromes associated with autism spectrum disorder. Int J Mol Sci. 2015;16(4):7627-43. doi:

10.3390/ijms16047627. PMID: 25853262.X-1

3859. Mullegama SV, Pugliesi L, Burns B, et al. MBD5 haploinsufficiency is associated with sleep disturbance and disrupts circadian pathways common to Smith-Magenis and fragile X syndromes. Eur J Hum Genet. 2015 Jun;23(6):781-9. doi: 10.1038/ejhg.2014.200. PMID: 25271084.X-1

3860. Munde V, Vlaskamp C, Ter Haar A. Social-emotional instability in individuals with Rett syndrome: parents' experiences with second stage behaviour. J Intellect Disabil Res. 2015 Oct 14doi: 10.1111/jir.12233. PMID: 26497300.X-1

3861. Muneoka K, Kuwagata M, Ogawa T, et al. Mother/offspring co-administration of the traditional herbal remedy yokukansan during the nursing period influences grooming and cerebellar serotonin levels in a rat model of neurodevelopmental disorders. Cerebellum. 2015 Apr;14(2):86-96. doi: 10.1007/s12311014-0611-2. PMID: 25315739.X-1

3862. Munoz Lasa S, Maximo Bocanegra N, Valero Alcaide R, et al. Animal assisted interventions in neurorehabilitation: a review of the most recent literature. Neurologia. 2015 Jan-Feb;30(1):1-7. doi: 10.1016/j.nrl.2013.01.012. PMID: 23642347.X$1, \mathrm{X}-2$ 
3863. Murdaugh DL, Deshpande HD, Kana RK. The Impact of Reading Intervention on Brain Responses Underlying Language in Children With Autism. Autism Res. 2015 May 25doi: 10.1002/aur.1503. PMID: 26016818.X-1

3864. Murdaugh DL, Maximo JO, Kana RK. Changes in intrinsic connectivity of the brain's reading network following intervention in children with autism. Hum Brain Mapp. 2015 Aug;36(8):2965-79. doi: 10.1002/hbm.22821. PMID: 26058572.X-1

3865. Murray K, Jassi A, Mataix-Cols D, et al. Outcomes of cognitive behaviour therapy for obsessive-compulsive disorder in young people with and without autism spectrum disorders: A case controlled study. Psychiatry Res. 2015 Jul 30;228(1):8-13. doi:

10.1016/j.psychres.2015.03.012. PMID: 25935374.X-1, X-3

3866. Muskat B, Burnham Riosa P, Nicholas DB, et al. Autism comes to the hospital: The experiences of patients with autism spectrum disorder, their parents and health-care providers at two Canadian paediatric hospitals. Autism: The International Journal of Research \& Practice. 2015;19(4):482-90 9p. doi: 10.1177/1362361314531341. PMID: 103788864. Language: English. Entry Date: 20150416. Revision Date: 20150710. Publication Type: Journal Article.X-1

3867. Mussey JL, Travers BG, Klinger LG, et al. Decision-making skills in ASD: performance on the Iowa Gambling Task. Autism Res. 2015 Feb;8(1):105-14. doi: 10.1002/aur.1429. PMID: 25371315.X-1

3868. Must A, Phillips S, Curtin C, et al. Barriers to Physical Activity in Children With Autism Spectrum Disorders: Relationship to Physical Activity and Screen Time. J Phys Act Health. 2015 Apr;12(4):529-34. doi: 10.1123/jpah.2013-0271. PMID: 25920014.X-1

3869. Mutluer T, Karakoc Demirkaya S, Abali O. Assessment of sleep problems and related risk factors observed in Turkish children with Autism spectrum disorders. Autism Res. 2015 Oct 13doi: 10.1002/aur.1542. PMID: 26459217.X-1

3870. Myers E, Davis BE, Stobbe G, et al. Community and Social Participation Among
Individuals with Autism Spectrum Disorder Transitioning to Adulthood. J Autism Dev Disord. 2015 Aug;45(8):2373-81. doi: 10.1007/s10803-015-2403-z. PMID: 25725812.X-1

3871. Nadeau JM, Arnold EB, Keene AC, et al. Frequency and Clinical Correlates of SleepRelated Problems Among Anxious Youth with Autism Spectrum Disorders. Child Psychiatry Hum Dev. 2015 Aug;46(4):558-66. doi: 10.1007/s10578-014-0496-9. PMID: 25239284.X-1, X-3

3872. Najjar F, Owley T, Mosconi MW, et al. Pharmacogenetic Study of Serotonin Transporter and 5HT2A Genotypes in Autism. J Child Adolesc Psychopharmacol. 2015 Aug;25(6):467-74. doi: 10.1089/cap.2014.0158. PMID: 26262902.X-3

3873. Nakamura T, Matsumoto J, Takamura Y, et al. Relationships among parvalbuminimmunoreactive neuron density, phase-locked gamma oscillations, and autistic/schizophrenic symptoms in PDGFR-beta knock-out and control mice. PLoS One. 2015;10(3):e0119258. doi: 10.1371/journal.pone.0119258. PMID: 25803852.X-1

3874. Nakamura T, Oinuma T, Yamagishi H, et al. Evaluation of a novel high-resolution magnifying videoendoscope that is capable of photodynamic diagnosis and therapy for gastric cancer. Photodiagnosis Photodyn Ther. 2015 Mar;12(1):115-22. doi: 10.1016/j.pdpdt.2014.10.010. PMID: 25462577.X-1

3875. Nance E, Porambo M, Zhang F, et al. Systemic dendrimer-drug treatment of ischemia-induced neonatal white matter injury. J Control Release. 2015 Sep 28;214:112-20. doi: 10.1016/j.jconrel.2015.07.009. PMID: 26184052.X-1

3876. Narzisi A, Muratori F, Buscema M, et al. Outcome predictors in autism spectrum disorders preschoolers undergoing treatment as usual: insights from an observational study using artificial neural networks. Neuropsychiatr Dis Treat. 2015;11:1587-99. doi: 10.2147/ndt.s81233. PMID: 26170671.X-1

3877. Navarro D, Alvarado M, Navarrete F, et al. Gestational and early postnatal 
hypothyroidism alters VGluT1 and VGAT bouton distribution in the neocortex and hippocampus, and behavior in rats. Front Neuroanat. 2015;9:9. doi: 10.3389/fnana.2015.00009. PMID: 25741243.X-1

3878. Navarro E, Araya M. [Non-celiac gluten sensitivity: Another condition that responds to gluten]. Rev Med Chil. 2015 May;143(5):619-26. doi: 10.4067/s003498872015000500010. PMID: 26203574.X-1, $\mathrm{X}-2$

3879. Naviaux JC, Wang L, Li K, et al. Antipurinergic therapy corrects the autism-like features in the Fragile X (Fmr1 knockout) mouse model. Mol Autism. 2015;6:1. doi: 10.1186/2040-2392-6-1. PMID: 25705365.X-1

3880. Nazneen N, Rozga A, Smith CJ, et al. A Novel System for Supporting Autism Diagnosis Using Home Videos: Iterative Development and Evaluation of System Design. JMIR Mhealth Uhealth. 2015;3(2):e68. doi: 10.2196/mhealth.4393. PMID: 26085230.X-1

3881. Neely L, Rispoli M, Gerow S, et al. Effects of antecedent exercise on academic engagement and stereotypy during instruction. Behav Modif. 2015 Jan;39(1):98-116. doi: 10.1177/0145445514552891. PMID: 25271070.X-1, X-3, X-4

3882. Nelson CA, Varcin KJ, Coman NK, et al. Shortened Telomeres in Families With a Propensity to Autism. J Am Acad Child Adolesc Psychiatry. 2015 Jul;54(7):588-94. doi: 10.1016/j.jaac.2015.04.006. PMID: 26088664.X-1

3883. Nelson TM, Sheller B, Friedman CS, et al. Educational and therapeutic behavioral approaches to providing dental care for patients with Autism Spectrum Disorder. Spec Care Dentist. 2015 May-Jun;35(3):105-13. doi: 10.1111/scd.12101. PMID: 25470557.X-1, X-2

3884. Neufeld J, Ioannou C, Korb S, et al. Spontaneous Facial Mimicry is Modulated by Joint Attention and Autistic Traits. Autism Res. 2015 Oct 7doi: 10.1002/aur.1573. PMID: 26442665.X-1

3885. Neuhaus E, Bernier RA, Beauchaine TP. Children with Autism Show Altered
Autonomic Adaptation to Novel and Familiar Social Partners. Autism Res. 2015 Aug 25doi: 10.1002/aur.1543. PMID: 26305051.X-1

3886. Neuhaus E, Bernier RA, Beauchaine TP. Electrodermal Response to Reward and Non-Reward Among Children With Autism. Autism Res. 2015 Aug;8(4):357-70. doi: 10.1002/aur.1451. PMID: 25599655.X-1

3887. Ng R, Brown TT, Erhart M, et al. Morphological differences in the mirror neuron system in Williams syndrome. Soc Neurosci. 2015 Aug 18:1-12. doi:

10.1080/17470919.2015.1070746. PMID: 26230578.X-1

3888. Ngounou Wetie AG, Wormwood KL, Charette L, et al. Comparative two-dimensional polyacrylamide gel electrophoresis of the salivary proteome of children with autism spectrum disorder. J Cell Mol Med. 2015 Aug 20doi: 10.1111/jcmm.12658. PMID: 26290361.X-1

3889. Ngounou Wetie AG, Wormwood KL, Russell S, et al. A Pilot Proteomic Analysis of Salivary Biomarkers in Autism Spectrum Disorder. Autism Res. 2015 Jun;8(3):338-50. doi: 10.1002/aur.1450. PMID: 25626423.X-1

3890. Nguyen CT, Fairclough DL, Noll RB. Problem-solving skills training for mothers of children recently diagnosed with autism spectrum disorder: A pilot feasibility study. Autism. 2015 Apr 20doi: 10.1177/1362361314567134. PMID: 25896269.X-1

3891. Nickel RE, Huang-Storms L. Early Identification of Young Children with Autism Spectrum Disorder. Indian J Pediatr. 2015 Sep 28doi: 10.1007/s12098-015-1894-0. PMID: 26411730.X-1

3892. Nielsen S, Anckarsater H, Gillberg C, et al. Effects of autism spectrum disorders on outcome in teenage-onset anorexia nervosa evaluated by the Morgan-Russell outcome assessment schedule: a controlled communitybased study. Mol Autism. 2015;6:14. doi: 10.1186/s13229-015-0013-4. PMID: 25774282.X-1

3893. Nikiforuk A. Targeting the Serotonin 5-HT7 Receptor in the Search for Treatments 
for CNS Disorders: Rationale and Progress to Date. CNS Drugs. 2015 Apr;29(4):265-75. doi: 10.1007/s40263-015-0236-0. PMID: 25721336.X-1

3894. Nishijo M, Tai PT, Anh NT, et al. Urinary amino acid alterations in 3-year-old children with neurodevelopmental effects due to perinatal dioxin exposure in Vietnam: a nested case-control study for neurobiomarker discovery. PLoS One. 2015;10(1):e0116778. doi: 10.1371/journal.pone.0116778. PMID: 25584822.X-1

3895. Nomi JS, Uddin LQ. Developmental changes in large-scale network connectivity in autism. Neuroimage Clin. 2015;7:732-41. doi: 10.1016/j.nicl.2015.02.024. PMID: 25844325.X-1

3896. Noor A, Dupuis L, Mittal K, et al. 15q11.2 Duplication Encompassing Only the UBE3A Gene Is Associated with Developmental Delay and Neuropsychiatric Phenotypes. Hum Mutat. 2015 Jul;36(7):68993. doi: 10.1002/humu.22800. PMID: 25884337.X-1

3897. Nordahl CW, Iosif AM, Young GS, et al. Sex differences in the corpus callosum in preschool-aged children with autism spectrum disorder. Mol Autism. 2015;6:26. doi: 10.1186/s13229-015-0005-4. PMID: 25973163.X-1

3898. Noriega G. A neural model to study sensory abnormalities and multisensory effects in autism. IEEE Trans Neural Syst Rehabil Eng. 2015 Mar;23(2):199-209. doi: 10.1109/tnsre.2014.2363775. PMID: 25343764.X-1

3899. Norrelgen F, Fernell E, Eriksson M, et al. Children with autism spectrum disorders who do not develop phrase speech in the preschool years. Autism. 2015 Nov;19(8):93443. doi: 10.1177/1362361314556782. PMID: 25488002.X-1

3900. Northrup JB, Iverson JM. Vocal Coordination During Early Parent-Infant Interactions Predicts Language Outcome in Infant Siblings of Children with Autism Spectrum Disorder. Infancy. 2015 SepOct;20(5):523-47. PMID: 26345517.X-1
3901. Nottingham CL, Vladescu JC, Kodak TM. Incorporating additional targets into learning trials for individuals with autism spectrum disorder. J Appl Behav Anal. 2015 Spring;48(1):227-32. doi: 10.1002/jaba.179. PMID: 25376814.X-1, X-2

3902. Nowack N, Wittsiepe J, KasperSonnenberg M, et al. Influence of Low-Level Prenatal Exposure to PCDD/Fs and PCBs on Empathizing, Systemizing and Autistic Traits: Results from the Duisburg Birth Cohort Study. PLoS One. 2015;10(6):e0129906. doi: 10.1371/journal.pone.0129906. PMID: 26066795.X-1

3903. Nowell KP, Schanding GT, Jr., Kanne SM, et al. Cognitive Profiles in Youth with Autism Spectrum Disorder: An Investigation of Base Rate Discrepancies using the Differential Ability Scales--Second Edition. J Autism Dev Disord. 2015 Jul;45(7):1978-88. doi: 10.1007/s10803-014-2356-7. PMID: 25614020.X-1

3904. Nunes ML, Bruni O. Insomnia in childhood and adolescence: clinical aspects, diagnosis, and therapeutic approach. J Pediatr (Rio J). 2015 Sep 18doi: 10.1016/j.jped.2015.08.006. PMID: 26392218.X-1

3905. Nuske HJ, Vivanti G, Dissanayake C. No Evidence of Emotional Dysregulation or Aversion to Mutual Gaze in Preschoolers with Autism Spectrum Disorder: An Eye-Tracking Pupillometry Study. J Autism Dev Disord. 2015 Nov;45(11):3433-45. doi: 10.1007/s10803-0152479-5. PMID: 26031923.X-1

3906. Nuzzi LC, Pike CM, Lewine EB, et al. Preoperative electrocardiograms for nonsyndromic children with hand syndactyly. J Hand Surg Am. 2015 Mar;40(3):452-5. doi: 10.1016/j.jhsa.2014.10.053. PMID: 25542431.X-1

3907. Nystrom P, Gredeback G, Bolte S, et al. Hypersensitive pupillary light reflex in infants at risk for autism. Mol Autism. 2015;6:10. doi: 10.1186/s13229-015-0011-6. PMID: 25750705.X-1

3908. Oakley C, Mahone EM, Morris-Berry $\mathrm{C}$, et al. Primary complex motor stereotypies in older children and adolescents: clinical features 
and longitudinal follow-up. Pediatr Neurol. 2015 Apr;52(4):398-403.e1. doi: 10.1016/j.pediatrneurol.2014.11.002. PMID: 25661287.X-1

3909. Obeid R, Daou N, DeNigris D, et al. A Cross-Cultural Comparison of Knowledge and Stigma Associated with Autism Spectrum Disorder Among College Students in Lebanon and the United States. J Autism Dev Disord. 2015 Nov;45(11):3520-36. doi: 10.1007/s10803-015-2499-1. PMID: 26084712.X-1

3910. Oberg E, Givant C, Fisk B, et al. Epigenetics in Clinical Practice: Characterizing Patient and Provider Experiences with MTHFR Polymorphisms and Methylfolate. J Nutrigenet Nutrigenomics. 2015;8(3):137-50. doi: 10.1159/000440700. PMID: 26484755.X-1

3911. Ochiai M, Ichiyama M, Iwayama M, et al. Longitudinal study of very low birth weight infants until 9years of age; attention deficit hyperactivity and autistic features are correlated with their cognitive functions. Early Hum Dev. 2015 Oct 3doi: 10.1016/j.earlhumdev.2015.09.005. PMID: 26443619.X-1

3912. Ockeloen CW, Willemsen $\mathrm{MH}$, de Munnik S, et al. Further delineation of the KBG syndrome phenotype caused by ANKRD11 aberrations. Eur J Hum Genet. 2015 Sep;23(9):1176-85. doi: 10.1038/ejhg.2014.253. PMID: 25424714.X-1

3913. Oddi D, Subashi E, Middei S, et al. Early social enrichment rescues adult behavioral and brain abnormalities in a mouse model of fragile $\mathrm{X}$ syndrome.

Neuropsychopharmacology. 2015

Apr;40(5):1113-22. doi: 10.1038/npp.2014.291. PMID: 25348604.X-1

3914. Odell AP. Treatment Challenges in Special Populations: High-functioning Autistic Patients with Pain. Journal for Nurse Practitioners. 2015;11(4):466-7 2p. doi: 10.1016/j.nurpra.2014.11.020. PMID: 109793308. Language: English. Entry Date: 20150618. Revision Date: 20151008. Publication Type: Journal Article.X-1

3915. Odriozola P, Uddin LQ, Lynch CJ, et al. Insula response and connectivity during social and non-social attention in children with autism. Soc Cogn Affect Neurosci. 2015 Oct 9doi: 10.1093/scan/nsv126. PMID: 26454817.X-1

3916. Oguro-Ando A, Rosensweig C, Herman E, et al. Increased CYFIP1 dosage alters cellular and dendritic morphology and dysregulates mTOR. Mol Psychiatry. 2015 Sep;20(9):1069-78. doi: 10.1038/mp.2014.124. PMID: 25311365.X-1

3917. Oh S, Ji H, Barzman D, et al. Pediatric asthma and autism-genomic perspectives. Clin Transl Med. 2015 Dec;4(1):37. doi: 10.1186/s40169-015-0078-X. PMID: 26668064.X-1

3918. Oh YS, Kim JS, Lee PH. Effect of Rivastigmine on Behavioral and Psychiatric Symptoms of Parkinson's Disease Dementia. J Mov Disord. 2015 May;8(2):98-102. doi: 10.14802/jmd.15041. PMID: 26090082.X-1

3919. O'Haire ME, McKenzie SJ, Beck AM, et al. Animals may act as social buffers: Skin conductance arousal in children with autism spectrum disorder in a social context. Dev Psychobiol. 2015 Jul;57(5):584-95. doi: 10.1002/dev.21310. PMID: 25913902.X-1, X-3

3920. Ohan JL, Ellefson SE, Corrigan PW. Brief Report: The Impact of Changing from DSM-IV 'Asperger's' to DSM-5 'Autistic Spectrum Disorder' Diagnostic Labels on Stigma and Treatment Attitudes. J Autism Dev Disord. 2015 Oct;45(10):3384-9. doi: 10.1007/s10803-015-2485-7. PMID: 26043847.X-1

3921. O'Hearn K, Velanova K, Lynn A, et al. Abnormalities in brain systems supporting individuation and enumeration in autism. Autism Res. 2015 May 26doi: 10.1002/aur.1498. PMID: 26011184.X-1

3922. Ohta H, Nordahl CW, Iosif AM, et al. Increased Surface Area, but not Cortical Thickness, in a Subset of Young Boys With Autism Spectrum Disorder. Autism Res. 2015 Jul 16doi: 10.1002/aur.1520. PMID: 26184828.X-1

3923. Oien R, Eisemann MR. Brief Report: Parent-Reported Problems Related to Communication, Behavior and Interests in 
Children with Autistic Disorder and Their Impact on Quality of Life. J Autism Dev Disord. 2015 Oct 5doi: 10.1007/s10803-0152577-4. PMID: 26438642.X-1

3924. Okazaki R, Takahashi T, Ueno K, et al. Changes in EEG complexity with electroconvulsive therapy in a patient with autism spectrum disorders: a multiscale entropy approach. Front Hum Neurosci. 2015;9:106. doi: 10.3389/fnhum.2015.00106. PMID: 25767444.X-1

3925. Olde Loohuis NF, Kole K, Glennon JC, et al. Elevated microRNA-181c and microRNA-30d levels in the enlarged amygdala of the valproic acid rat model of autism. Neurobiol Dis. 2015 Aug;80:42-53. doi: 10.1016/j.nbd.2015.05.006. PMID: 25986729.X-1

3926. Olfson M, King M, Schoenbaum M. Treatment of Young People With Antipsychotic Medications in the United States. JAMA Psychiatry. 2015 Sep;72(9):867-74. doi: 10.1001/jamapsychiatry.2015.0500. PMID: 26132724.X-1

3927. Ollington N. Comparison of a direct and an indirect approach for the functional assessment of insistence on sameness in a child with autism spectrum disorder and a typically developing child. Dev Neurorehabil. 2015 Aug 24:1-8. doi: 10.3109/17518423.2014.898161. PMID: 24724559.X-1

3928. Olsson MB, Westerlund J, Lundstrom $\mathrm{S}$, et al. "Recovery" from the diagnosis of autism - and then? Neuropsychiatr Dis Treat. 2015;11:999-1005. doi: 10.2147/ndt.s78707. PMID: 25897237.X-1, X-3

3929. Olzenak DL. The Association between Motor Proficiency and Participation in Schoolaged Children with Autism Spectrum Disorder: University of Rochester; 2015.

3930. Omura Y, Lu D, Jones MK, et al. Early Detection of Autism (ASD) by a Noninvasive Quick Measurement of Markedly Reduced Acetylcholine \& DHEA and Increased beta-Amyloid (1-42), Asbestos (Chrysotile), Titanium Dioxide, Al, Hg \& often Coexisting Virus Infections (CMV, HPV 16 and 18), Bacterial Infections etc. in the Brain and Corresponding Safe Individualized Effective
Treatment. Acupunct Electrother Res. 2015;40(3):157-87. PMID: 26829843.X-1

3931. Ong WY, Farooqui T, Kokotos G, et al. Synthetic and natural inhibitors of phospholipases A2: their importance for understanding and treatment of neurological disorders. ACS Chem Neurosci. 2015 Jun 17;6(6):814-31. doi: 10.1021/acschemneuro.5b00073. PMID: 25891385.X-1

3932. Ooi YP, Weng SJ, Jang LY, et al. Omega-3 fatty acids in the management of autism spectrum disorders: findings from an open-label pilot study in Singapore. Eur J Clin Nutr. 2015 Aug;69(8):969-71. doi: 10.1038/ejcn.2015.28. PMID: 25804268.X-3

3933. Orinstein A, Tyson KE, Suh J, et al. Psychiatric Symptoms in Youth with a History of Autism and Optimal Outcome. J Autism Dev Disord. 2015 Nov;45(11):3703-14. doi: 10.1007/s10803-015-2520-8. PMID: 26155764.X-1

3934. Orinstein AJ, Suh J, Porter K, et al. Social Function and Communication in Optimal Outcome Children and Adolescents with an Autism History on Structured Test Measures. J Autism Dev Disord. 2015 Aug;45(8):2443-63. doi: 10.1007/s10803-015-2409-6. PMID: 25758821.X-1

3935. Orsmond GI, Cohn ES. The Distinctive Features of a Feasibility Study: Objectives and Guiding Questions. OTJR (Thorofare N J). 2015 Jul;35(3):169-77. PMID: 26594739.X-1

3936. Osiecka BJ, Jurczyszyn K, Nockowski $\mathrm{P}$, et al. Using photodynamic therapy to estimate effectiveness of innovative combined diclofenac and tazaroten therapy of disseminated actinic keratosis. Acta Dermatovenerol Croat. 2015;23(1):52-8. PMID: 25969914.X-1

3937. Osipowicz K, Bosenbark DD, Patrick KE. Cortical Changes Across the Autism Lifespan. Autism Res. 2015 Aug;8(4):379-85. doi: 10.1002/aur.1453. PMID: 25630444.X-1

3938. Ostfeld-Etzion S, Golan O, HirschlerGuttenberg Y, et al. Neuroendocrine and behavioral response to social rupture and repair 
in preschoolers with autism spectrum disorders interacting with mother and father. Mol Autism. 2015;6:11. doi: 10.1186/s13229-015-0007-2. PMID: 25774280.X-1

3939. Oswald TM, Beck JS, Iosif AM, et al. Clinical and Cognitive Characteristics Associated with Mathematics Problem Solving in Adolescents with Autism Spectrum Disorder. Autism Res. 2015 Sep 29doi: 10.1002/aur.1524. PMID: 26418313.X-1

3940. Ouchi K, Sugiyama K. Required propofol dose for anesthesia and time to emerge are affected by the use of antiepileptics: prospective cohort study. BMC Anesthesiol. 2015;15:34. doi: 10.1186/s12871-015-0006-z. PMID: 25788855.X-1

3941. Ouhtit A, Al-Farsi Y, Al-Sharbati M, et al. Underlying Factors Behind the Low Prevalence of Autism Spectrum Disorders in Oman: Sociocultural perspective. Sultan Qaboos Univ Med J. 2015 May;15(2):e213-7. PMID: 26052454.X-1

3942. Ouwenga RL, Dougherty J. Fmrp targets or not: long, highly brain-expressed genes tend to be implicated in autism and brain disorders. Mol Autism. 2015;6:16. doi: 10.1186/s13229-015-0008-1. PMID: 25789151.X-1

3943. Overby CL, Devine EB, Abernethy N, et al. Making pharmacogenomic-based prescribing alerts more effective: A scenariobased pilot study with physicians. J Biomed Inform. 2015 Jun;55:249-59. doi: 10.1016/j.jbi.2015.04.011. PMID: 25957826.X1

3944. Owen-Smith AA, Bent S, Lynch FL, et al. Prevalence and Predictors of Complementary and Alternative Medicine Use in a Large Insured Sample of Children with Autism Spectrum Disorders. Res Autism Spectr Disord. 2015 Sep 1;17:40-51. doi: 10.1016/j.rasd.2015.05.002. PMID: 26366192.X-1

3945. Pace M, Bricout VA. Low heart rate response of children with autism spectrum disorders in comparison to controls during physical exercise. Physiol Behav. 2015 Mar 15;141:63-8. doi: 10.1016/j.physbeh.2015.01.011. PMID: 25582513.X-1

3946. Pack A, Colozzo P, Bernhardt BM, et al. A case study on vocal loudness with a young adult with autism spectrum disorder and developmental delay. Am J Speech Lang Pathol. 2015 Aug 7doi: 10.1044/2015_ajslp-140209. PMID: 26254450.X-1

3947. Paden AR, Kodak T. The effects of reinforcement magnitude on skill acquisition for children with autism. J Appl Behav Anal. 2015 Aug 17doi: 10.1002/jaba.239. PMID: 26281795.X-1

3948. Padmanabhan A, Garver K, O'Hearn $\mathrm{K}$, et al. Developmental changes in brain function underlying inhibitory control in autism spectrum disorders. Autism Res. 2015 Apr;8(2):123-35. doi: 10.1002/aur.1398. PMID: 25382787.X-1

3949. Palmer CJ, Paton B, Kirkovski M, et al. Context sensitivity in action decreases along the autism spectrum: a predictive processing perspective. Proc Biol Sci. 2015 Mar 7;282(1802)doi: 10.1098/rspb.2014.1557. PMID: 25631989.X-1

3950. Palmer EE, Leffler M, Rogers C, et al. New insights into Brunner syndrome and potential for targeted therapy. Clin Genet. 2015 Mar 23doi: 10.1111/cge.12589. PMID: 25807999.X-1

3951. Pan CY, Tsai CL, Chu CH, et al. Objectively Measured Physical Activity and Health-Related Physical Fitness in Secondary School-Aged Male Students With Autism Spectrum Disorders. Phys Ther. 2015 Sep 24doi: 10.2522/ptj.20140353. PMID: 26405090.X-1

3952. Pan X, Chang X, Leung C, et al. PAK1 regulates cortical development via promoting neuronal migration and progenitor cell proliferation. Mol Brain. 2015;8:36. doi: 10.1186/s13041-015-0124-z. PMID: 26043730.X-1

3953. Pane HM, Sidener TM, Vladescu JC, et al. Evaluating Function-Based Social Stories With Children With Autism. Behav Modif. 2015 Sep 3doi: 10.1177/0145445515603708. PMID: 26342012.X-1, X-4 
3954. Panigrahi B, Martin KA, Li Y, et al. Dopamine Is Required for the Neural Representation and Control of Movement Vigor. Cell. 2015 Sep 10;162(6):1418-30. doi: 10.1016/j.cell.2015.08.014. PMID: 26359992.X-1

3955. Pantaleon FG, Juvier RT. [Molecular basis of Rett syndrome: A current look]. Rev Chil Pediatr. 2015 May-Jun;86(3):142-51. doi: 10.1016/j.rchipe.2015.07.001. PMID: 26239053.X-1

3956. Paolicchi JM, Ross G, Lee D, et al. Clobazam and Aggression-Related Adverse Events in Pediatric Patients With LennoxGastaut Syndrome. Pediatr Neurol. 2015 Oct;53(4):338-42. doi: 10.1016/j.pediatrneurol.2015.06.021. PMID: 26245776.X-1

3957. Papadopoulos N, Sciberras E, Hiscock $\mathrm{H}$, et al. The Efficacy of a Brief Behavioral Sleep Intervention in School-Aged Children With ADHD and Comorbid Autism Spectrum Disorder. J Atten Disord. 2015 Feb 2doi: 10.1177/1087054714568565. PMID: 25646022.X-1

3958. Paquet A, Olliac B, Golse B, et al. Current knowledge on motor disorders in children with autism spectrum disorder (ASD). Child Neuropsychol. 2015 Sep 29:1-32. doi: 10.1080/09297049.2015.1085501. PMID: 26416608.X-1

3959. Parish C. Are we treating or drugging? Learning Disability Practice. 2015;18(5):5- 1p. PMID: 103807071. Language: English. Entry Date: 20150604 . Revision Date: 20150710. Publication Type: Journal Article.X-1, X-2

3960. Parisi L, Di Filippo T, Roccella M. Autism spectrum disorder in Kabuki syndrome: clinical, diagnostic and rehabilitative aspects assessed through the presentation of three cases. Minerva Pediatr. 2015 Aug;67(4):369-75. PMID: 26129805.X-1

3961. Park EY, Kim J. Factor structure of the childhood autism rating scale as per DSM-5. Pediatr Int. 2015 Aug 8doi: 10.1111/ped.12770. PMID: 26256774.X-1
3962. Park HE, Park IS, Oh YS, et al Subcortical whiter matter hyperintensities within the cholinergic pathways of patients with dementia and parkinsonism. J Neurol Sci. 2015 Jun 15;353(1-2):44-8. doi: 10.1016/j.jns.2015.03.046. PMID: 25891829.X1

3963. Parr JR, De Jonge MV, Wallace S, et al. New Interview and Observation Measures of the Broader Autism Phenotype: Description of Strategy and Reliability Findings for the Interview Measures. Autism Res. 2015 Oct;8(5):522-33. doi: 10.1002/aur.1466. PMID: 25959701.X-1

3964. Parra MA, Cubelli R, Bellamy KJ, et al. Gist-based illusions within and across stimulus modalities in autism spectrum disorder. Memory. 2015 Feb 4:1-11. doi: 10.1080/09658211.2015.1004349. PMID: 25651475.X-1

3965. Parsi K, Elster N. A Life of One's Own: Challenges in the Transition from Childhood to Adulthood with Autism Spectrum Disorder. AMA J Ethics. 2015 Apr;17(4):342-7. doi: 10.1001/journalofethics.2015.17.4.pfor11504. PMID: 25901702.X-1

3966. Partty A, Kalliomaki M, Wacklin P, et al. A possible link between early probiotic intervention and the risk of neuropsychiatric disorders later in childhood: a randomized trial. Pediatr Res. 2015 Jun;77(6):823-8. doi: 10.1038/pr.2015.51. PMID: 25760553.X-1

3967. Pastor PN, Reuben CA, Kobau R, et al. Functional difficulties and school limitations of children with epilepsy: findings from the 20092010 National Survey of Children with Special Health Care Needs. Disabil Health J. 2015 Apr;8(2):231-9. doi: 10.1016/j.dhjo.2014.09.002. PMID: 25312691.X-1

3968. Patel N, Crider A, Pandya CD, et al. Altered mRNA Levels of Glucocorticoid Receptor, Mineralocorticoid Receptor, and CoChaperones (FKBP5 and PTGES3) in the Middle Frontal Gyrus of Autism Spectrum Disorder Subjects. Mol Neurobiol. 2015 Apr 26doi: 10.1007/s12035-015-9178-2. PMID: 25912394.X-1 
3969. Paul AR, McKechanie AG, Johnstone EC, et al. Brief Report: The Association of Autistic Traits and Behavioural Patterns in Adolescents Receiving Special Educational Assistance. J Autism Dev Disord. 2015 Sep;45(9):3055-60. doi: 10.1007/s10803-0152445-2. PMID: 25894524.X-1

3970. Paynter JM, Keen D. Knowledge and use of intervention practices by communitybased early intervention service providers. J Autism Dev Disord. 2015 Jun;45(6):1614-23. doi: 10.1007/s10803-014-2316-2. PMID: 25398604.X-1

3971. Pearson A, Marsh L, Ropar D, et al. Cognitive Mechanisms underlying visual perspective taking in typical and ASC children. Autism Res. 2015 Jun 6doi: 10.1002/aur.1501. PMID: 26052836.X-1

3972. Pearson BL, Defensor EB, Blanchard DC, et al. Applying the ethoexperimental approach to neurodevelopmental syndrome research reveals exaggerated defensive behavior in Mecp2 mutant mice. Physiol Behav. 2015 Jul 1;146:98-104. doi:

10.1016/j.physbeh.2015.03.035. PMID: 26066729.X-1

3973. Pecorelli A, Cervellati F, Belmonte G, et al. Cytokines profile and peripheral blood mononuclear cells morphology in Rett and autistic patients. Cytokine. 2015 Oct 12doi: 10.1016/j.cyto.2015.10.002. PMID: 26471937.X-1

3974. Peiker I, Schneider TR, Milne E, et al. Stronger Neural Modulation by Visual Motion Intensity in Autism Spectrum Disorders. PLoS One. 2015;10(7):e0132531. doi: 10.1371/journal.pone.0132531. PMID: 26147342.X-1

3975. Pellecchia M, Connell JE, Beidas RS, et al. Dismantling the Active Ingredients of an Intervention for Children with Autism. J Autism Dev Disord. 2015 Sep;45(9):2917-27. doi: 10.1007/s10803-015-2455-0. PMID: 25911305.X-1, X-3

3976. Pellecchia M, Connell JE, Kerns CM, et al. Child characteristics associated with outcome for children with autism in a schoolbased behavioral intervention. Autism. 2015
Apr 24doi: 10.1177/1362361315577518. PMID: 25911092.X-1, X-3

3977. Pelly L, Vardy C, Fernandez B, et al. Incidence and cohort prevalence for autism spectrum disorders in the Avalon Peninsula, Newfoundland and Labrador. CMAJ Open. 2015 Jul-Sep;3(3):E276-80. doi: 10.9778/cmajo.20140056. PMID: 26442225.X1

3978. Pelz-Sherman D, Olmsted S. Sandplay therapy with individuals on the autism spectrum. Journal of Sandplay Therapy. 2015 2015-07-08;24(1):69-88. PMID: 1694709913; 2015-24420-005.X-2, X-3, X-4

3979. Penagarikano O, Lazaro MT, Lu XH, et al. Exogenous and evoked oxytocin restores social behavior in the Cntnap2 mouse model of autism. Sci Transl Med. 2015 Jan

21;7(271):271ra8. doi:

10.1126/scitranslmed.3010257. PMID: 25609168.X-1

3980. Pence ST, St Peter CC. Evaluation of treatment integrity errors on mand acquisition. J Appl Behav Anal. 2015 Sep;48(3):575-89. doi: 10.1002/jaba.238. PMID: 26235379.X-1, X-3, $\mathrm{X}-4$

3981. Penn AH, Carver LJ, Herbert CA, et al. Breast Milk Protects Against Gastrointestinal Symptoms in Infants at High Risk for Autism During Early Development. J Pediatr Gastroenterol Nutr. 2015 Jul 29doi: 10.1097/mpg.0000000000000907. PMID: 26230900.X-1

3982. Penner M, Rayar M, Bashir N, et al. Cost-Effectiveness Analysis Comparing Prediagnosis Autism Spectrum Disorder (ASD)Targeted Intervention with Ontario's Autism Intervention Program. J Autism Dev Disord. 2015 Sep;45(9):2833-47. doi: 10.1007/s10803015-2447-0. PMID: 25936527.X-1, X-2

3983. Peraza LR, Colloby SJ, Firbank MJ, et al. Resting state in Parkinson's disease dementia and dementia with Lewy bodies: commonalities and differences. Int J Geriatr Psychiatry. 2015 Nov;30(11):1135-46. doi: 10.1002/gps.4342. PMID: 26270627.X-1

3984. Percinel I, Yazici KU. The Use of Aripiprazole in Young Children with Autism 
Spectrum Disorders: The Treatment and 16 Week Follow-Up of a 23-Month-old Male Patient. J Child Adolesc Psychopharmacol. 2015 Sep 23doi: 10.1089/cap.2015.0106. PMID: 26397364.X-3, X-4

3985. Perez-Gonzalez LA, Diaz E, Fernandez-Garcia S, et al. Stimuli with identical contextual functions taught independently become functionally equivalent. Learn Behav. 2015 Jun;43(2):113-28. doi: 10.3758/s13420014-0166-6. PMID: 25673100.X-1

3986. Perkins TJ, Bittar RG, McGillivray JA, et al. Increased premotor cortex activation in high functioning autism during action observation. J Clin Neurosci. 2015 Apr;22(4):664-9. doi: 10.1016/j.jocn.2014.10.007. PMID: 25726458.X-1

3987. Perreault A, Habak C, Lepore F, et al. Behavioral evidence for a functional link between low- and mid-level visual perception in the autism spectrum. Neuropsychologia. 2015 Oct;77:380-6. doi:

10.1016/j.neuropsychologia.2015.09.022. PMID: 26384775.X-1

3988. Perry A, Isaacs B. Validity of the Family Quality of Life Survey-2006. J Appl Res Intellect Disabil. 2015 Nov;28(6):584-8. doi: 10.1111/jar.12141. PMID: 25914221.X-1

3989. Perry A, Levy-Gigi E, Richter-Levin $\mathrm{G}$, et al. Interpersonal distance and social anxiety in autistic spectrum disorders: A behavioral and ERP study. Soc Neurosci. 2015 Aug;10(4):354-65. doi:

10.1080/17470919.2015.1010740. PMID: 25666260.X-1

3990. Petasecca M, Alhujaili S, Aldosari $\mathrm{AH}$, et al. Angular independent silicon detector for dosimetry in external beam radiotherapy. Med Phys. 2015 Aug;42(8):4708-18. doi: 10.1118/1.4926778. PMID: 26233198.X-1

3991. Peters SM, Pothuizen HH, Spruijt BM. Ethological concepts enhance the translational value of animal models. Eur J Pharmacol. 2015 Jul 15;759:42-50. doi:

10.1016/j.ejphar.2015.03.043. PMID: 25823814.X-1
3992. Peterson D, Mahajan R, Crocetti D, et al. Left-hemispheric microstructural abnormalities in children with high-functioning autism spectrum disorder. Autism Res. 2015 Feb;8(1):61-72. doi: 10.1002/aur.1413. PMID: 25256103.X-1

3993. Peterson J, Reed S, Kenny R, et al. A Two-Factor Structure within the Systemizing Trait of Autism Differentially Predicts Susceptibility to Lateral and Collinear Flanker Effects. J Vis. 2015 Sep 1;15(12):647. doi: 10.1167/15.12.647. PMID: 26326335.X-1

3994. Petit LM, Girard D, GanousseMazeron S, et al. Weaning Off Prognosis Factors of Home Parenteral Nutrition for Children with Primary Digestive Disease. J Pediatr Gastroenterol Nutr. 2015 Sep 17doi: 10.1097/mpg.0000000000000980. PMID: 26398153.X-1

3995. Petrongolo M, DuBard M, Luiselli JK. Effects of an idiosyncratic stimulus on functional analysis of vocal stereotypy in two settings. Dev Neurorehabil. 2015

Jun;18(3):209-12. doi:

10.3109/17518423.2013.869271. PMID: 24354848.X-1

3996. Petrova M, Mehrabian-Spasova S, Aarsland D, et al. Clinical and Neuropsychological Differences between Mild Parkinson's Disease Dementia and Dementia with Lewy Bodies. Dement Geriatr Cogn Dis Extra. 2015 May-Aug;5(2):212-20. doi: 10.1159/000375363. PMID: 26195977.X-1

3997. Peykov S, Berkel S, Schoen M, et al. Identification and functional characterization of rare SHANK2 variants in schizophrenia. Mol Psychiatry. 2015 Jan 6doi: 10.1038/mp.2014.172. PMID: 25560758.X-1

3998. Piccoli GB, Postorino V, Cabiddu G, et al. Children of a lesser god or miracles? An emotional and behavioural profile of children born to mothers on dialysis in Italy: a multicentre nationwide study 2000-12. Nephrol Dial Transplant. 2015 Jul;30(7):1193-202. doi: 10.1093/ndt/gfv127. PMID: 26047633.X-1

3999. Pickles A, Harris V, Green J, et al. Treatment mechanism in the MRC preschool autism communication trial: implications for study design and parent-focussed therapy for 
children. J Child Psychol Psychiatry. 2015

Feb;56(2):162-70. doi: 10.1111/jcpp.12291.

PMID: 25039961.X-1, X-2, X-3

4000. Pieron M, Seassau M, Leboyer M, et al. Accelerated time course of saccadic inhibition of return in individuals with autism spectrum disorders. Exp Brain Res. 2015 Mar;233(3):767-75. doi: 10.1007/s00221-0144152-1. PMID: 25432625.X-1

4001. Pileggi LA, Malcolm-Smith S, Solms $\mathrm{M}$. Investigating the role of social-affective attachment processes in cradling bias: the absence of cradling bias in children with Autism Spectrum Disorders. Laterality. 2015;20(2):154-70. doi: 10.1080/1357650x.2014.948449. PMID: 25131653.X-1

4002. Pineda R, Melchior K, Oberle S, et al. Assessment of Autism Symptoms During the Neonatal Period: Is There Early Evidence of Autism Risk? Am J Occup Ther. 2015 JulAug;69(4):6904220010p1-p11. doi: 10.5014/ajot.2015.015925. PMID: 26114457.X-1

4003. Pisula E, Kawa R, Danielewicz D, et al. The relationship between temperament and autistic traits in a non-clinical students sample. PLoS One. 2015;10(4):e0124364. doi: 10.1371/journal.pone.0124364. PMID: 25860508.X-1

4004. Pivovarciova A, Hnilicova S, Ostatnikova D, et al. Bio-behavioral model of aggression in autism spectrum disorders-pilot study. Bratisl Lek Listy. 2015;116(12):702-6. PMID: 26924148.X-1

4005. Plavnick JB, Kaid T, MacFarland MC. Effects of a School-Based Social Skills Training Program for Adolescents with Autism Spectrum Disorder and Intellectual Disability. J Autism Dev Disord. 2015 Sep;45(9):2674-90. doi: 10.1007/s10803-015-2434-5. PMID: 25820638.X-1, X-3, X-4

4006. Poirier N, Vallee-Ouimet J. [The life path of parents and of their children presenting an autism spectrum disorder (ASD)]. Sante Ment Que. 2015 Spring;40(1):203-26. PMID: 26355488.X-1
4007. Pokorny JJ, Hatt NV, Colombi C, et al. The Action Observation System when

Observing Hand Actions in Autism and Typical Development. Autism Res. 2015 Jun;8(3):28496. doi: 10.1002/aur.1445. PMID: 25631917.X1

4008. Polyak A, Kubina RM, Girirajan S. Comorbidity of intellectual disability confounds ascertainment of autism: implications for genetic diagnosis. Am J Med Genet B Neuropsychiatr Genet. 2015 Oct;168(7):600-8. doi: 10.1002/ajmg.b.32338. PMID: 26198689.X-1

4009. Polyak A, Rosenfeld JA, Girirajan S. An assessment of sex bias in neurodevelopmental disorders. Genome Med. 2015;7(1):94. doi: 10.1186/s13073-015-0216-5. PMID: 26307204.X-1

4010. Poole D, Gowen E, Warren PA, et al. Investigating visual-tactile interactions over time and space in adults with autism. Journal of Autism and Developmental Disorders. 2015 2015-10-07;45(10):3316-26. doi: http://dx.doi.org/10.1007/s10803-015-2492-8. PMID: 1689320033; 2015-26686-001.X-1

4011. Posar A, Resca F, Visconti P. Autism according to diagnostic and statistical manual of mental disorders 5(th) edition: The need for further improvements. J Pediatr Neurosci. 2015 Apr-Jun;10(2):146-8. doi: 10.4103/18171745.159195. PMID: 26167220.X-1

4012. Poslawsky IE, Naber FB, BakermansKranenburg MJ, et al. Video-feedback Intervention to promote Positive Parenting adapted to Autism (VIPP-AUTI): A randomized controlled trial. Autism. 2015 Jul;19(5):588-603. doi: 10.1177/1362361314537124. PMID: 24919961.X-1

4013. Postorino V, Fatta LM, De Peppo L, et al. Longitudinal comparison between male and female preschool children with autism spectrum disorder. J Autism Dev Disord. 2015 Jul;45(7):2046-55. doi: 10.1007/s10803-0152366-0. PMID: 25633919.X-1

4014. Postorino V, Sanges V, Giovagnoli G, et al. Clinical differences in children with autism spectrum disorder with and without food selectivity. Appetite. 2015 Sep;92:126-32. doi: 
10.1016/j.appet.2015.05.016. PMID: 25998237.X-1

4015. Pozo P, Sarria E. Still stressed but feeling better: Well-being in autism spectrum disorder families as children become adults. Autism. 2015 Oct;19(7):805-13. doi: 10.1177/1362361315583191. PMID: 25957298.X-1

4016. Prasad H, Rao R. Applying knowledge of autism to brain cancer management: what do we know? Future Oncol. 2015;11(13):1847-50. doi: 10.2217/fon.15.93. PMID: 26161920.X-1

4017. Prather AA, Epel ES, Arenander J, et al. Longevity factor klotho and chronic psychological stress. Transl Psychiatry. 2015;5:e585. doi: 10.1038/tp.2015.81. PMID: 26080320.X-1

4018. Prayson RA, Gales JM. Coexistent ganglioglioma, focal cortical dysplasia, and hippocampal sclerosis (triple pathology) in chronic epilepsy. Ann Diagn Pathol. 2015 Oct;19(5):310-3. doi:

10.1016/j.anndiagpath.2015.07.003. PMID: 26235882.X-1

4019. Prilutsky D, Kho AT, Palmer NP, et al. Gene expression analysis in Fmr1KO mice identifies an immunological signature in brain tissue and mGluR5-related signaling in primary neuronal cultures. Mol Autism. 2015;6:66. doi: 10.1186/s13229-015-0061-9. PMID: 26697163.X-1

4020. Pritchard AE, Kalback S, McCurdy M, et al. Executive functions among youth with Down Syndrome and co-existing neurobehavioural disorders. J Intellect Disabil Res. 2015 Sep 15doi: 10.1111/jir.12217. PMID: 26369785.X-1

4021. Provenzano G, Pangrazzi L, Poli A, et al. Immunohistochemical visualization of hippocampal neuron activity after spatial learning in a mouse model of neurodevelopmental disorders. J Vis Exp. 2015(99):e52919. doi: 10.3791/52919. PMID: 25992917.X-1

4022. Puangpetch A, Suwannarat P, Chamnanphol M, et al. Significant Association of HLA-B Alleles and Genotypes in Thai Children with Autism Spectrum Disorders: A
Case-Control Study. Dis Markers. 2015;2015:724935. doi: 10.1155/2015/724935. PMID: 26819491.X-1

4023. Pugliese CE, Anthony L, Strang JF, et al. Increasing adaptive behavior skill deficits from childhood to adolescence in autism spectrum disorder: role of executive function. $\mathrm{J}$ Autism Dev Disord. 2015 Jun;45(6):1579-87. doi: 10.1007/s10803-014-2309-1. PMID: 25398602.X-1

4024. Pugliese CE, Anthony LG, Strang JF, et al. Longitudinal Examination of Adaptive Behavior in Autism Spectrum Disorders: Influence of Executive Function. J Autism Dev Disord. 2015 Sep 9doi: 10.1007/s10803-0152584-5. PMID: 26349921.X-1

4025. Pugliese CE, Fritz MS, White SW. The role of anger rumination and autism spectrum disorder-linked perseveration in the experience of aggression in the general population. Autism. 2015 Aug;19(6):704-12. doi: 10.1177/1362361314548731. PMID: 25212211.X-1

4026. Pugliese CE, Kenworthy L, Bal VH, et al. Replication and Comparison of the Newly Proposed ADOS-2, Module 4 Algorithm in ASD Without ID: A Multi-site Study. J Autism Dev Disord. 2015 Sep 18doi: 10.1007/s10803015-2586-3. PMID: 26385796.X-1

4027. Puig-Alcaraz C, Fuentes-Albero M, Calderon J, et al. Increased homocysteine levels correlate with the communication deficit in children with autism spectrum disorder. Psychiatry Res. 2015 Oct 30;229(3):1031-7. doi: 10.1016/j.psychres.2015.05.021. PMID: 26070768.X-1

4028. Pulcini CD, Perrin JM, Houtrow AJ, et al. Examining Trends and Coexisting Conditions Among Children Qualifying for SSI Under ADHD, ASD, and ID. Acad Pediatr. 2015 Jul-Aug;15(4):439-43. doi: 10.1016/j.acap.2015.05.002. PMID: 26142070.X-1

4029. Purkayastha P, Malapati A, Yogeeswari P, et al. A Review on GABA/Glutamate Pathway for Therapeutic Intervention of ASD and ADHD. Curr Med Chem. 2015 Feb 9 PMID: 25666800.X-1, X-2 
4030. Qin L, Dai X. [Effect of sulindac on improving autistic behaviors in rats]. Nan Fang Yi Ke Da Xue Xue Bao. 2015 Aug;35(8):11625. PMID: 26277514.X-1

4031. Qin M, Huang T, Kader M, et al. RBaclofen Reverses a Social Behavior Deficit and Elevated Protein Synthesis in a Mouse Model of Fragile X Syndrome. Int J Neuropsychopharmacol. 2015 Jul;18(9)doi: 10.1093/ijnp/pyv034. PMID: 25820841.X-1

4032. Qin M, Zeidler Z, Moulton K, et al. Endocannabinoid-mediated improvement on a test of aversive memory in a mouse model of fragile X syndrome. Behav Brain Res. 2015 Sep 15;291:164-71. doi: 10.1016/j.bbr.2015.05.003. PMID: 25979787.X-1

4033. Quinlan CA, McVeigh KH, Driver CR, et al. Parental Age and Autism Spectrum Disorders Among New York City Children 036 Months of Age. Matern Child Health J. 2015 Aug;19(8):1783-90. doi: 10.1007/s10995-0151692-3. PMID: 25776271.X-1

4034. Radley KC, Ford WB, McHugh MB, et al. Brief Report: Use of Superheroes Social Skills to Promote Accurate Social Skill Use in Children with Autism Spectrum Disorder. J Autism Dev Disord. 2015 Sep;45(9):3048-54. doi: 10.1007/s10803-015-2442-5. PMID: 25841884.X-3, X-4

4035. Rahko JS, Vuontela VA, Carlson S, et al. Attention and Working Memory in Adolescents with Autism Spectrum Disorder: A Functional MRI Study. Child Psychiatry Hum Dev. 2015 Sep 1doi: 10.1007/s10578-0150583-6. PMID: 26323584.X-1

4036. Rahkonen P, Lano A, Pesonen AK, et al. Atypical sensory processing is common in extremely low gestational age children. Acta Paediatr. 2015 May;104(5):522-8. doi: 10.1111/apa.12911. PMID: 25620288.X-1

4037. Rana P, Mishra D. Quality of life of unaffected siblings of children with chronic neurological disorders. Indian J Pediatr. 2015 Jun;82(6):545-8. doi: 10.1007/s12098-0141672-4. PMID: 25640066.X-1

4038. Randall M, Sciberras E, Brignell A, et al. Autism spectrum disorder: Presentation and prevalence in a nationally representative
Australian sample. Aust N Z J Psychiatry. 2015 Aug 17doi: 10.1177/0004867415595287. PMID: 26282446.X-1

4039. Randel A, Adlof S, Klusek J, et al. Teaching reading to youth with fragile $\mathrm{X}$ syndrome: Should phonemic awareness and phonics instruction be used? EBP Briefs. 2015 Mar;9(6):47-61. PMID: 26500715.X-1

4040. Rane P, Cochran D, Hodge SM, et al. Connectivity in Autism: A Review of MRI Connectivity Studies. Harv Rev Psychiatry. 2015 Jul-Aug;23(4):223-44. doi: 10.1097/hrp.0000000000000072. PMID: 26146755.X-1

4041. Rao PA, Raman V, Thomas T, et al. IQ in Autism: Is there an Alternative Global Cognitive Index? Indian J Psychol Med. 2015 Jan-Mar;37(1):48-52. doi: 10.4103/02537176.150819. PMID: 25722512.X-1, X-2

4042. Rao VS, Raman V, Mysore AV. Issues related to obtaining intelligence quotientmatched controls in autism research. Indian $\mathrm{J}$ Psychol Med. 2015 Apr-Jun;37(2):149-53. doi: 10.4103/0253-7176.155612. PMID: 25969598.X-1

4043. Ratcliffe B, Wong M, Dossetor D, et al. The Association Between Social Skills and Mental Health in School-Aged Children with Autism Spectrum Disorder, With and Without Intellectual Disability. J Autism Dev Disord. 2015 Aug;45(8):2487-96. doi: 10.1007/s10803015-2411-z. PMID: 25758822.X-1

4044. Ratni H, Rogers-Evans M, Bissantz C, et al. Discovery of highly selective brainpenetrant vasopressin 1a antagonists for the potential treatment of autism via a chemogenomic and scaffold hopping approach. J Med Chem. 2015 Mar 12;58(5):2275-89. doi: 10.1021/jm501745f. PMID: 25654260.X-1

4045. Rattaz C, Michelon C, Baghdadli A. Symptom severity as a risk factor for selfinjurious behaviours in adolescents with autism spectrum disorders. J Intellect Disabil Res. 2015 Aug;59(8):730-40. doi: 10.1111/jir.12177. PMID: 25583371.X-1

4046. Ratto AB, Anthony BJ, Kenworthy L, et al. Are Non-intellectually Disabled Black Youth with ASD Less Impaired on Parent 
Report than Their White Peers? J Autism Dev Disord. 2015 Oct 6doi: 10.1007/s10803-0152614-3. PMID: 26439481.X-1

4047. Ratto AB, Mesibov GB. Autism spectrum disorders in adolescence and adulthood: Long-term outcomes and relevant issues for treatment and research. Sci China Life Sci. 2015 Oct;58(10):1010-5. doi: 10.1007/s11427-012-4295-X. PMID: 26335732.X-1

4048. Rausa VC, Moore DW, Anderson A. Use of video modelling to teach complex and meaningful job skills to an adult with autism spectrum disorder. Dev Neurorehabil. 2015 Aug 24:1-8. doi: 10.3109/17518423.2015.1008150. PMID: 25825994.X-1

4049. Rayner C. Video-based intervention for children with autism: towards improved assessment of pre-requisite imitation skills. Dev Neurorehabil. 2015 Apr;18(2):113-21. doi: 10.3109/17518423.2014.890959. PMID: 24649844.X-1, X-4

4050. Raza S, Harker A, Richards S, et al. Tactile stimulation improves neuroanatomical pathology but not behavior in rats prenatally exposed to valproic acid. Behav Brain Res. 2015 Apr 1;282:25-36. doi: 10.1016/j.bbr.2014.12.055. PMID: 25557797.X-1

4051. Reaven J, Blakeley-Smith A, Beattie TL, et al. Improving transportability of a cognitive-behavioral treatment intervention for anxiety in youth with autism spectrum disorders: results from a US-Canada collaboration. Autism. 2015 Feb;19(2):211-22. doi: 10.1177/1362361313518124. PMID: 24463434.X-3, X-4

4052. Reaven J, Washington L, Moody EJ, et al. Examining the Relationship Between Parental Anxiety and Treatment Response in Children and Adolescents with Autism Spectrum Disorder and Anxiety. J Autism Dev Disord. 2015 Aug;45(8):2464-73. doi: 10.1007/s10803-015-2410-0. PMID: 25778837.X-1, X-3

4053. Reese RM, Braun MJ, Hoffmeier S, et al. Preliminary Evidence for the Integrated Systems Using Telemedicine. Telemed J E
Health. 2015 Jul;21(7):581-7. doi: 10.1089/tmj.2014.0124. PMID: 25898157.X-1

4054. Reese RM, Jamison TR, Braun M, et al. Brief report: use of interactive television in identifying autism in young children: methodology and preliminary data. J Autism Dev Disord. 2015 May;45(5):1474-82. doi: 10.1007/s10803-014-2269-5. PMID: 25331323.X-1

4055. Reetzke R, Zou X, Sheng L, et al. Communicative Development in Bilingually Exposed Chinese Children With Autism Spectrum Disorders. J Speech Lang Hear Res. 2015 Jun;58(3):813-25. doi:

10.1044/2015_jslhr-l-13-0258. PMID: 25679338.X-1

4056. Reilly C, Senior J, Murtagh L. ASD, ADHD, mental health conditions and psychopharmacology in neurogenetic syndromes: parent survey. J Intellect Disabil Res. 2015 Apr;59(4):307-18. doi: 10.1111/jir.12147. PMID: 24965264.X-1

4057. Rhodes G, Neumann MF, Ewing L, et al. Reduced set averaging of face identity in children and adolescents with autism. Q J Exp Psychol (Hove). 2015;68(7):1391-403. doi: 10.1080/17470218.2014.981554. PMID: 25510166.X-1

4058. Ribeiro DM, Miguel CF, Goyos C. The effects of listener training on discriminative control by elements of compound stimuli in children with disabilities. J Exp Anal Behav. 2015 Jul;104(1):48-62. doi: 10.1002/jeab.161. PMID: 26150348.X-1

4059. Rice LM, Wall CA, Fogel A, et al. Computer-Assisted Face Processing Instruction Improves Emotion Recognition, Mentalizing, and Social Skills in Students with ASD. J Autism Dev Disord. 2015 Jul;45(7):2176-86. doi: 10.1007/s10803-015-2380-2. PMID: 25694364.X-1

4060. Rice ML. Specific Language Impairment, Nonverbal IQ, ADHD, ASD, Cochlear Implants, Bilingualism and Dialectal Variants: Defining the boundaries, clarifying clinical conditions and sorting out causes. J Speech Lang Hear Res. 2015 Oct 20doi: 10.1044/2015_jslhr-l-15-0255. PMID: 26502218.X-1 
4061. Rice TR. Postnatal testosterone may be an important mediator of the association between prematurity and male neurodevelopmental disorders: a hypothesis. Int J Adolesc Med Health. 2015 Sep 10doi: 10.1515/ijamh-2015-0047. PMID: 26356360.X1

4062. Richard AE, Lajiness-O'Neill R. Visual attention shifting in autism spectrum disorders. J Clin Exp Neuropsychol. 2015 Sep;37(7):671-87. doi: 10.1080/13803395.2015.1042838. PMID: 26156236.X-1

4063. Richards C, Jones C, Groves L, et al. Prevalence of autism spectrum disorder phenomenology in genetic disorders: a systematic review and meta-analysis. Lancet Psychiatry. 2015 Oct;2(10):909-16. doi: 10.1016/s2215-0366(15)00376-4. PMID: 26341300.X-1, X-2, X-3

4064. Richdale AL, Baglin CL. Self-report and caregiver-report of sleep and psychopathology in children with highfunctioning autism spectrum disorder: A pilot study. Dev Neurorehabil. 2015;18(4):272-9. doi: 10.3109/17518423.2013.829534. PMID: 24087935.X-1

4065. Richter J, Henze R, Vomstein K, et al. Reduced cortical thickness and its association with social reactivity in children with autism spectrum disorder. Psychiatry Res. 2015 Aug 22doi: 10.1016/j.pscychresns.2015.06.011. PMID: 26329119.X-1

4066. Ricketts J, Dockrell JE, Patel N, et al. Do children with specific language impairment and autism spectrum disorders benefit from the presence of orthography when learning new spoken words? J Exp Child Psychol. 2015 Jun;134:43-61. doi: 10.1016/j.jecp.2015.01.015. PMID: 25795987.X-1, X-3

4067. Ricotti V, Mandy WP, Scoto M, et al. Neurodevelopmental, emotional, and behavioural problems in Duchenne muscular dystrophy in relation to underlying dystrophin gene mutations. Dev Med Child Neurol. 2015 Sep 14doi: 10.1111/dmcn.12922. PMID: 26365034.X-1
4068. Rieske RD, Matson JL, Beighley JS, et al. Comorbid psychopathology rates in children diagnosed with autism spectrum disorders according to the DSM-IV-TR and the proposed DSM-5. Dev Neurorehabil. 2015;18(4):218-23. doi: 10.3109/17518423.2013.790519. PMID: 23869479.X-1

4069. Rieth SR, Stahmer AC, Suhrheinrich J, et al. Examination of the prevalence of stimulus overselectivity in children with ASD. J Appl Behav Anal. 2015 Spring;48(1):71-84. doi: 10.1002/jaba.165. PMID: 25418837.X-1

4070. Ring M, Gaigg SB, Bowler DM. Relational Memory Processes in Adults with Autism Spectrum Disorder. Autism Res. 2015 May 7doi: 10.1002/aur.1493. PMID: 25952759.X-1

4071. Rios C, Costa Andrada B. The changing face of autism in Brazil. Cult Med Psychiatry. 2015 Jun;39(2):213-34. doi: 10.1007/s11013-015-9448-5. PMID: 25842350.X-1

4072. Roberts AL, Koenen KC, Lyall K, et al. Association of autistic traits in adulthood with childhood abuse, interpersonal victimization, and posttraumatic stress. Child Abuse Negl. 2015 Jul;45:135-42. doi: 10.1016/j.chiabu.2015.04.010. PMID: 25957197.X-1

4073. Roberts RM, Ejova A, Giallo R, et al. A controlled trial of the SibworkS group program for siblings of children with special needs. Res Dev Disabil. 2015 Aug-Sep;4344:21-31. doi: 10.1016/j.ridd.2015.06.002. PMID: 26151440.X-1

4074. Robertson AE, David RSR. The sensory experiences of adults with autism spectrum disorder: A qualitative analysis. Perception. 2015;44(5):569-86. PMID: 26422904.x-1

4075. Robinson EB, Neale BM, Hyman SE. Genetic research in autism spectrum disorders. Curr Opin Pediatr. 2015 Sep 18doi: 10.1097/mop.0000000000000278. PMID: 26371945.X-1

4076. Rodriguez-Lopez J, Carrera N, Arrojo $\mathrm{M}$, et al. An efficient screening method for simultaneous detection of recurrent copy 
number variants associated with psychiatric disorders. Clin Chim Acta. 2015 May 20;445:34-40. doi: 10.1016/j.cca.2015.03.013. PMID: 25797897.X-1

4077. Roine U, Salmi J, Roine T, et al. Constrained spherical deconvolution-based tractography and tract-based spatial statistics show abnormal microstructural organization in Asperger syndrome. Mol Autism. 2015;6:4. doi: 10.1186/2040-2392-6-4. PMID: 25874076.X-1

4078. Rollins PR, Campbell M, Hoffman RT, et al. A community-based early intervention program for toddlers with autism spectrum disorders. Autism. 2015 Apr 23doi: 10.1177/1362361315577217. PMID: 25907978.X-3, X-4

4079. Rommelse N, Langerak I, van der Meer J, et al. Intelligence May Moderate the Cognitive Profile of Patients with ASD. PLoS One. 2015;10(10):e0138698. doi: 10.1371/journal.pone.0138698. PMID: 26444877.X-1

4080. Romski M, Sevcik RA, Barton-Hulsey A, et al. Early Intervention and AAC: What a Difference 30 Years Makes. Augment Altern Commun. 2015;31(3):181-202. doi: 10.3109/07434618.2015.1064163. PMID: 26153901.X-1, X-2, X-3, X-4

4081. Rosales R, Gongola L, Homlitas C. An evaluation of video modeling with embedded instructions to teach implementation of stimulus preference assessments. J Appl Behav Anal. 2015 Spring;48(1):209-14. PMID: 25927112.X-1

4082. Rosander C, Hallbook T. Dravet syndrome in Sweden: a population-based study. Dev Med Child Neurol. 2015 Mar 13doi: 10.1111/dmcn.12709. PMID: 25772213.X-1

4083. Roscoe EM, Schlichenmeyer KJ, Dube WV. Functional analysis of problem behavior: A systematic approach for identifying idiosyncratic variables. J Appl Behav Anal. 2015 Summer;48(2):289-314. doi: 10.1002/jaba.201. PMID: 25930176.X-1

4084. Rose S, Wynne R, Frye RE, et al. Increased susceptibility to ethylmercuryinduced mitochondrial dysfunction in a subset of autism lymphoblastoid cell lines. J Toxicol.
2015;2015:573701. doi: 10.1155/2015/573701. PMID: 25688267.X-1

4085. Rosenberg A, Patterson JS, Angelaki DE. A computational perspective on autism.

Proc Natl Acad Sci U S A. 2015 Jul 28;112(30):9158-65. doi:

10.1073/pnas.1510583112. PMID: 26170299.X-1

4086. Rosenbloom R, Mason RA, Wills HP, et al. Technology Delivered Self-Monitoring Application to Promote Successful Inclusion of an Elementary Student with Autism. Assist Technol. 2015 Jul 7doi: 10.1080/10400435.2015.1059384. PMID: 26151808.X-1, X-3, X-4

4087. Ross LA, Del Bene VA, Molholm S, et al. Sex differences in multisensory speech processing in both typically developing children and those on the autism spectrum. Front Neurosci. 2015;9:185. doi: 10.3389/fnins.2015.00185. PMID: 26074757.X-1

4088. Rousseau P. Reflections: Neurology And The Humanities. Silvia's blessing. Neurology. 2015 Jun 9;84(23):2379-80. PMID: 26251863.X-2

4089. Rowe MP, Gillespie BM, Harris KR, et al. Redesigning a General Education Science Course to Promote Critical Thinking. CBE Life Sci Educ. 2015 Fall;14(3)doi: 10.1187/cbe.1502-0032. PMID: 26231561.X-1

4090. Roy A, Roy M, Deb S, et al. Are opioid antagonists effective in attenuating the core symptoms of autism spectrum conditions in children: a systematic review. J Intellect Disabil Res. 2015 Apr;59(4):293-306. doi: 10.1111/jir.12122. PMID: 24589346.X-2

4091. Roy M, Prox-Vagedes V, Ohlmeier $\mathrm{MD}$, et al. Beyond childhood: psychiatric comorbidities and social background of adults with Asperger syndrome. Psychiatr Danub. 2015 Mar;27(1):50-9. PMID: 25751431.X-1

4092. Rubenstein E, Wiggins LD, Lee LC. A Review of the Differences in Developmental, Psychiatric, and Medical Endophenotypes Between Males and Females with Autism Spectrum Disorder. J Dev Phys Disabil. 2015 
Feb;27(1):119-39. doi: 10.1007/s10882-0149397-x. PMID: 26146472.X-1

4093. Rubino T, Zamberletti E, Parolaro D. Endocannabinoids and Mental Disorders.

Handb Exp Pharmacol. 2015;231:261-83. doi: 10.1007/978-3-319-20825-1_9. PMID: 26408164.X-1

4094. Ruby K, Falvey K, Kulesza RJ. Abnormal neuronal morphology and neurochemistry in the auditory brainstem of Fmr1 knockout rats. Neuroscience. 2015 Sep 10;303:285-98. doi:

10.1016/j.neuroscience.2015.06.061. PMID: 26166728.X-1

4095. Rueda JR, Guillen V, Ballesteros J, et al. L-acetylcarnitine for treating fragile $\mathrm{X}$ syndrome. Cochrane Database Syst Rev. 2015;5:Cd010012. doi: 10.1002/14651858.CD010012.pub2. PMID: 25985235.X-1

4096. Ruggieri VL, Arberas CL. [Therapeutic approaches in autism spectrum disorders]. Rev Neurol. 2015 Feb 25;60 Suppl 1:S45-9. PMID: 25726823.X-1, X-2

4097. Ruiz-Robledillo N, Bellosta-Batalla M, Moya-Albiol L. Lower cardiovascular reactivity to acute stress in informal caregivers of people with autism spectrum disorder than in noncaregivers: Implications for health outcomes. Int J Psychophysiol. 2015 Oct;98(1):143-50. doi: 10.1016/j.ijpsycho.2015.07.011. PMID: 26226113.X-1

4098. Ruiz-Robledillo N, Moya-Albiol L. Lower electrodermal activity to acute stress in caregivers of people with autism spectrum disorder: an adaptive habituation to stress. J Autism Dev Disord. 2015 Feb;45(2):576-88. doi: 10.1007/s10803-013-1996-3. PMID: 24221817.X-1

4099. Russo AJ. Decreased plasma myeloperoxidase associated with probiotic therapy in autistic children. Clin Med Insights Pediatr. 2015;9:13-7. doi: 10.4137/CMPed.S17901. PMID: 25674031.X-1

4100. Russo-Ponsaran NM, McKown C, Johnson JK, et al. Social-Emotional Correlates of Early Stage Social Information Processing Skills in Children With and Without Autism
Spectrum Disorder. Autism Res. 2015

Oct;8(5):486-96. doi: 10.1002/aur.1463. PMID: 25683488.X-1

4101. Rutherford MD, Walsh J, Creighton S. Emotion perception or social cognitive complexity: What drives face processing deficits in autism spectrum disorder? J Vis. 2015 Sep 1;15(12):1223. doi: 10.1167/15.12.1223. PMID: 26326911.X-1

4102. Rutherford MD, Walsh JA, Lee V. Brief Report: Infants Developing with ASD Show a Unique Developmental Pattern of Facial Feature Scanning. J Autism Dev Disord. 2015 Aug;45(8):2618-23. doi: 10.1007/s10803015-2396-7. PMID: 25703032.X-1

4103. Ruzich E, Allison C, Smith P, et al. Subgrouping siblings of people with autism: Identifying the broader autism phenotype. Autism Res. 2015 Sep 2doi: 10.1002/aur.1544. PMID: 26332889.X-1

4104. Saad K, Abdel-Rahman AA, Elserogy YM, et al. Vitamin D status in autism spectrum disorders and the efficacy of vitamin $\mathrm{D}$ supplementation in autistic children. Nutr Neurosci. 2015 Apr 15doi: 10.1179/1476830515y.0000000019. PMID: 25876214.X-3

4105. Saban-Bezalel R, Mashal N. The effects of intervention on the comprehension of irony and on hemispheric processing of irony in adults with ASD. Neuropsychologia. 2015 Oct;77:233-41. doi: 10.1016/j.neuropsychologia.2015.09.004. PMID: 26359714.X-1

4106. Sacco R, Gabriele S, Persico AM. Head circumference and brain size in autism spectrum disorder: A systematic review and meta-analysis. Psychiatry Res. 2015 Sep 28doi: 10.1016/j.pscychresns.2015.08.016. PMID: 26456415.X-1

4107. Sacks GC, Altarescu G, Guedalia J, et al. Developmental neuropsychological assessment of 4- to 5-year-old children born following Preimplantation Genetic Diagnosis (PGD): A pilot study. Child Neuropsychol. 2015 Mar 16:1-14. doi: 10.1080/09297049.2015.1014900. PMID: 25774437.X-1 
4108. Sacrey LA, Zwaigenbaum L, Bryson $\mathrm{S}$, et al. Can parents' concerns predict autism spectrum disorder? A prospective study of highrisk siblings from 6 to 36 months of age. J Am Acad Child Adolesc Psychiatry. 2015

Jun;54(6):470-8. doi:

10.1016/j.jaac.2015.03.014. PMID:

26004662.X-1

4109. Sacrey LR, Bennett JA, Zwaigenbaum L. Early Infant Development and Intervention for Autism Spectrum Disorder. J Child Neurol. 2015 Aug 31doi: 10.1177/0883073815601500. PMID: 26323499.X-1, X-2

4110. Sagiv SK, Kalkbrenner AE, Bellinger DC. Of decrements and disorders: assessing impairments in neurodevelopment in prospective studies of environmental toxicant exposures. Environ Health. 2015;14:8. doi: 10.1186/1476-069x-14-8. PMID: 25609433.X$1, \mathrm{X}-2$

4111. Saiano M, Pellegrino L, Casadio M, et al. Natural interfaces and virtual environments for the acquisition of street crossing and path following skills in adults with Autism Spectrum Disorders: a feasibility study. J Neuroeng Rehabil. 2015;12:17. doi: 10.1186/s12984-0150010-z. PMID: 25885279.X-1

4112. Saini V, Gregory MK, Uran KJ, et al. Parametric analysis of response interruption and redirection as treatment for stereotypy. J Appl Behav Anal. 2015 Spring;48(1):96-106. doi: 10.1002/jaba.186. PMID: 25640901.X-1

4113. Salcuni S. New frontiers and applications of attachment theory. Front Psychol. 2015;6:273. doi: 10.3389/fpsyg.2015.00273. PMID: 25814972.X-1

4114. Saleh M, Nashaat NH, Fahim C, et al. MRI Surface-Based Brain Morphometry in Egyptian Autistic and Typically Developing Children. Folia Phoniatr Logop. 2015;67(1):2935. doi: 10.1159/000368962. PMID: 25967922.X-1

4115. Salomone E, Beranova S, BonnetBrilhault F, et al. Use of early intervention for young children with autism spectrum disorder across Europe. Autism. 2015 Apr 27doi: 10.1177/1362361315577218. PMID: 25916866.X-1
4116. Salomone E, Charman T, McConachie $\mathrm{H}$, et al. Prevalence and correlates of use of complementary and alternative medicine in children with autism spectrum disorder in Europe. Eur J Pediatr. 2015 Oct;174(10):127785. doi: 10.1007/s00431-015-2531-7. PMID: 25855095.X-1

4117. Salomone E, Charman T, McConachie $\mathrm{H}$, et al. Child's verbal ability and gender are associated with age at diagnosis in a sample of young children with ASD in Europe. Child Care Health Dev. 2015 May 28doi: 10.1111/cch.12261. PMID: 26016520.X-1

4118. Salowitz NMG. Multimodal sensory integration for perception and action in high functioning children with autism spectrum disorder; 2015.

4119. Samango-Sprouse CA, Stapleton EJ, Aliabadi F, et al. Identification of infants at risk for autism spectrum disorder and developmental language delay prior to 12 months. Autism. 2015 Apr;19(3):327-37. doi: 10.1177/1362361314521329. PMID: 24550549.X-1

4120. Samson AC, Hardan AY, Lee IA, et al. Maladaptive Behavior in Autism Spectrum Disorder: The Role of Emotion Experience and Emotion Regulation. J Autism Dev Disord. 2015 Nov;45(11):3424-32. doi: 10.1007/s10803-015-2388-7. PMID: 25711546.X-1

4121. Samson AC, Hardan AY, Podell RW, et al. Emotion regulation in children and adolescents with autism spectrum disorder. Autism Res. 2015 Feb;8(1):9-18. doi: 10.1002/aur.1387. PMID: 24863869.X-1

4122. Samson AC, Wells WM, Phillips JM, et al. Emotion regulation in autism spectrum disorder: evidence from parent interviews and children's daily diaries. J Child Psychol Psychiatry. 2015 Aug;56(8):903-13. doi: 10.1111/jcpp.12370. PMID: 25442191.X-1, X3

4123. Samson F, Zeffiro TA, Doyon J, et al. Speech acquisition predicts regions of enhanced cortical response to auditory stimulation in autism spectrum individuals. J Psychiatr Res. 2015 Sep;68:285-92. doi: 
10.1016/j.jpsychires.2015.05.011. PMID: 26037888.X-1, X-2

4124. Santangelo G, Vitale C, Picillo M, et al. Mild Cognitive Impairment in newly diagnosed Parkinson's disease: A longitudinal prospective study. Parkinsonism Relat Disord. 2015 Oct;21(10):1219-26. doi: 10.1016/j.parkreldis.2015.08.024. PMID: 26321021.X-1

4125. Santiago JL, Hanley GP, Moore K, et al. The Generality of Interview-Informed Functional Analyses: Systematic Replications in School and Home. J Autism Dev Disord. 2015 Oct 3doi: 10.1007/s10803-015-2617-0. PMID: 26433877.X-1, X-3, X-4

4126. Santomauro D, Sheffield J, Sofronoff K. Depression in Adolescents with ASD: A Pilot RCT of a Group Intervention. J Autism Dev Disord. 2015 Sep 24doi: 10.1007/s10803015-2605-4. PMID: 26404701.X-1

4127. Santoso TB, Ito Y, Ohshima N, et al. Resilience in Daily Occupations of Indonesian Mothers of Children With Autism Spectrum Disorder. Am J Occup Ther. 2015 SepOct;69(5):6905185020p1-8. doi: 10.5014/ajot.2015.017566. PMID: 26356659.X-1

4128. Sarmento S, Costa F, Pereira A, et al. Attenuation measurements show that the presence of a TachoSil surgical patch will not compromise target irradiation in intra-operative electron radiation therapy or high-dose-rate brachytherapy. Radiat Oncol. 2015;10(1):7. doi: 10.1186/s13014-014-0316-1. PMID: 25572977.X-1

4129. Sarrett JC. Custodial Homes, Therapeutic Homes, and Parental Acceptance: Parental Experiences of Autism in Kerala, India and Atlanta, GA USA. Cult Med Psychiatry. 2015 Jun;39(2):254-76. doi: 10.1007/s11013015-9441-z. PMID: 25772598.X-1

4130. Sasaki T, Hashimoto K, Oda Y, et al. Decreased levels of serum oxytocin in pediatric patients with Attention Deficit/Hyperactivity Disorder. Psychiatry Res. 2015 Aug 30;228(3):746-51. doi: 10.1016/j.psychres.2015.05.029. PMID: 26168929.X-1
4131. Sasaki Y, Usami M, Sasayama D, et al. Concerns expressed by parents of children with pervasive developmental disorders for different time periods of the day: a case-control study. PLoS One. 2015;10(4):e0124692. doi: 10.1371/journal.pone.0124692. PMID: 25898260.X-1

4132. Sauce B, Wass C, Netrakanti M, et al. Heterozygous L1-deficient mice express an autism-like phenotype. Behav Brain Res. 2015 Oct 1;292:432-42. doi: 10.1016/j.bbr.2015.05.040. PMID: 26079769.X-1

4133. Saunders BS, Tilford JM, Fussell JJ, et al. Financial and employment impact of intellectual disability on families of children with autism. Fam Syst Health. 2015 Mar;33(1):36-45. doi: 10.1037/fsh0000102. PMID: 25581557.X-1

4134. Say GN, Karabekiroglu K, Babadagi $Z$, et al. Maternal stress and perinatal features in autism \& attention deficit/ hyperactivity disorder. Pediatr Int. 2015 Sep 4doi: 10.1111/ped.12822. PMID: 26338105.X-1

4135. Sceniak MP, Lang M, Enomoto AC, et al. Mechanisms of Functional Hypoconnectivity in the Medial Prefrontal Cortex of Mecp2 Null Mice. Cereb Cortex. 2015 Feb 7doi: 10.1093/cercor/bhv002. PMID: 25662825.X-1

4136. Schaaf RC, Cohn ES, Burke J, et al. Linking Sensory Factors to Participation: Establishing Intervention Goals With Parents for Children With Autism Spectrum Disorder. Am J Occup Ther. 2015 SepOct;69(5):6905185005p1-8. doi: 10.5014/ajot.2015.018036. PMID: 26356657.X-1

4137. Schaer M, Kochalka J, Padmanabhan A, et al. Sex differences in cortical volume and gyrification in autism. Mol Autism. 2015;6:42. doi: 10.1186/s13229-015-0035-y. PMID: 26146534.X-1

4138. Schalkwyk G, Peluso F, Qayyum Z, et al. Varieties of Misdiagnosis in ASD: An Illustrative Case Series. Journal of Autism \& Developmental Disorders. 2015;45(4):911-8 8p. doi: 10.1007/s10803-014-2239-y. PMID: 103775657. Language: English. Entry Date: 
20150324. Revision Date: 20150710. Publication Type: Journal Article.X-1

4139. Scharoun SM, Bryden PJ. Is strength of handedness reliable over repeated testing? An examination of typical development and autism spectrum disorder. Front Psychol. 2015;6:17. doi: 10.3389/fpsyg.2015.00017. PMID: 25691875.X-1

4140. Scheeren AM, Banerjee R, Koot HM, et al. Self-Presentation and the Role of Perspective Taking and Social Motivation in Autism Spectrum Disorder. J Autism Dev Disord. 2015 Sep 25doi: 10.1007/s10803-0152610-7. PMID: 26408326.X-1

4141. Schenkelberg MA, Rosenkranz RR, Milliken GA, et al. Social Environmental Influences on Physical Activity of Children With Autism Spectrum Disorders. J Phys Act Health. 2015 May;12(5):636-41. doi: 10.1123/jpah.2013-0312. PMID: 25109324.X-1

4142. Schieve LA, Clayton HB, Durkin MS, et al. Comparison of Perinatal Risk Factors Associated with Autism Spectrum Disorder (ASD), Intellectual Disability (ID), and Cooccurring ASD and ID. J Autism Dev Disord. 2015 Aug;45(8):2361-72. doi: 10.1007/s10803015-2402-0. PMID: 25739693.X-1

4143. Schieve LA, Fountain C, Boulet SL, et al. Does Autism Diagnosis Age or Symptom Severity Differ Among Children According to Whether Assisted Reproductive Technology was Used to Achieve Pregnancy? J Autism Dev Disord. 2015 Sep;45(9):2991-3003. doi: 10.1007/s10803-015-2462-1. PMID: 25997596.X-1

4144. Schlenz AM, Carpenter LA, Bradley $\mathrm{C}$, et al. Age Differences in Emergency Department Visits and Inpatient Hospitalizations in Preadolescent and Adolescent Youth with Autism Spectrum Disorders. J Autism Dev Disord. 2015 Aug;45(8):2382-91. doi: 10.1007/s10803-0152405-x. PMID: 25739694.X-1

4145. Schlichenmeyer KJ, Dube WV, Vargas-Irwin M. Stimulus Fading and Response Elaboration in Differential Reinforcement for Alternative Behavior. Behav Interv. 2015 Feb;30(1):51-64. doi: 10.1002/bin.1402. PMID: 25844032.X-1, X-3, X-4
4146. Schlosser RW, Koul RK. Speech Output Technologies in Interventions for Individuals with Autism Spectrum Disorders: A Scoping Review. Augment Altern Commun. 2015 Jul 14:1-25. doi: 10.3109/07434618.2015.1063689. PMID: 26170252.X-1, X-2, X-3

4147. Schmeck K. [Pros and cons of antipsychotics in children and adolescents]. Praxis (Bern 1994). 2015 Aug 5;104(16):85964. doi: 10.1024/1661-8157/a002089. PMID: 26242421.X-1

4148. Schmidt C. Mental health: thinking from the gut. Nature. 2015 Feb 26;518(7540):S12-5. doi: 10.1038/518S13a. PMID: 25715275.X-1

4149. Schmidt L, Kirchner J, Strunz S, et al. Psychosocial Functioning and Life Satisfaction in Adults With Autism Spectrum Disorder Without Intellectual Impairment. J Clin Psychol. 2015 Sep 25doi: 10.1002/jclp.22225. PMID: 26406481.X-1

4150. Schmitz EA, Banerjee R, Pouw LBC, et al. Better to be equal? Challenges to equality for cognitively able children with autism spectrum disorders in a social decision game. Autism: The International Journal of Research \& Practice. 2015;19(2):178-86 9p. doi: 10.1177/1362361313516547. PMID: 103750466. Language: English. Entry Date: 20150130. Revision Date: 20150710. Publication Type: Journal Article.X-1

4151. Schmock H, Vangkilde A, Larsen KM, et al. The Danish 22q11 research initiative. BMC Psychiatry. 2015;15(1):220. doi: 10.1186/s12888-015-0594-7. PMID: 26384214.X-1

4152. Schneider I, Regenbogen C, Kohn N, et al. Reduced Responsiveness to Social Provocation in Autism Spectrum Disorder. Autism Res. 2015 Jun;8(3):297-306. doi: 10.1002/aur.1446. PMID: 25603913.X-1

4153. Schreibman L, Dawson G, Stahmer AC, et al. Naturalistic Developmental Behavioral Interventions: Empirically Validated Treatments for Autism Spectrum Disorder. J Autism Dev Disord. 2015 Aug;45(8):2411-28. 
doi: 10.1007/s10803-015-2407-8. PMID:

25737021.X-1, X-2

4154. Schunke O, Schottle D, Vettorazzi E, et al. Mirror me: Imitative responses in adults with autism. Autism. 2015 Mar 13doi: 10.1177/1362361315571757. PMID: 25769312.X-1

4155. Schuwerk T, Vuori M, Sodian B. Implicit and explicit Theory of Mind reasoning in autism spectrum disorders: the impact of experience. Autism. 2015 May;19(4):459-68. doi: 10.1177/1362361314526004. PMID: 24627427.X-1

4156. Searles Quick VB, Davis JM, Olincy A, et al. DUF1220 copy number is associated with schizophrenia risk and severity: implications for understanding autism and schizophrenia as related diseases. Transl Psychiatry. 2015;5:e697. doi: 10.1038/tp.2015.192. PMID: 26670282.X-1

4157. Segal-Gavish H, Karvat G, Barak N, et al. Mesenchymal Stem Cell Transplantation Promotes Neurogenesis and Ameliorates Autism Related Behaviors in BTBR Mice. Autism Res. 2015 Aug 10doi: 10.1002/aur.1530. PMID: 26257137.X-1

4158. Segar DJ, Chodakiewitz YG, Torabi R, et al. Deep brain stimulation for the obsessivecompulsive and Tourette-like symptoms of Kleefstra syndrome. Neurosurg Focus. 2015 Jun;38(6):E12. doi: 10.3171/2015.3.focus1528. PMID: 26030700.X-1

4159. Segura M, Pedreno C, Obiols J, et al. Neurotrophin blood-based gene expression and social cognition analysis in patients with autism spectrum disorder. Neurogenetics. 2015 Apr;16(2):123-31. doi: 10.1007/s10048-0140434-9. PMID: 25535174.X-1

4160. Sekine K, Matsune S, Shiiba K, et al. Treatment of nostril and nasal stenosis due to facial burn using a self-expandable metallic esophageal stent. Auris Nasus Larynx. 2015 Aug;42(4):348-52. doi: 10.1016/j.anl.2015.02.013. PMID: 25748514.X-1

4161. Selim ME, Abd-Elhakim YM, AlAyadhi LY. Pancreatic response to gold nanoparticles includes decrease of oxidative stress and inflammation in autistic diabetic model. Cell Physiol Biochem. 2015;35(2):586600. doi: 10.1159/000369721. PMID: 25612738.X-1

4162. Selles RR, Arnold EB, Phares V, et al. Cognitive-behavioral therapy for anxiety in youth with an autism spectrum disorder: A follow-up study. Autism. 2015 Jul;19(5):61321. doi: $10.1177 / 1362361314537912$. PMID: 24923895.X-1, X-3

4163. Senju A, Vernetti A, Ganea N, et al. Early Social Experience Affects the Development of Eye Gaze Processing. Curr Biol. 2015 Dec 7;25(23):3086-91. doi: 10.1016/j.cub.2015.10.019. PMID: 26752077.X-1

4164. Serret S, Thummler S, Dor E, et al. Lithium as a rescue therapy for regression and catatonia features in two SHANK3 patients with autism spectrum disorder: case reports. BMC Psychiatry. 2015;15:107. doi: 10.1186/s12888-015-0490-1. PMID: 25947967.X-1

4165. Shaaya EA, Pollack SF, Boronat S, et al. Gastrointestinal problems in $15 q$ duplication syndrome. Eur J Med Genet. 2015

Mar;58(3):191-3. doi:

10.1016/j.ejmg.2014.12.012. PMID: 25573720.X-1

4166. Shafritz KM, Bregman JD, Ikuta T, et al. Neural systems mediating decision-making and response inhibition for social and nonsocial stimuli in autism. Prog Neuropsychopharmacol Biol Psychiatry. 2015 Jul 3;60:112-20. doi: 10.1016/j.pnpbp.2015.03.001. PMID: 25765593.X-1

4167. Shao EH, Liolios V, Lindfield D, et al. Topical Adrenaline (1: 1000) for the Management of Severe Tarsal Conjunctival Chemosis. Ophthal Plast Reconstr Surg. 2015 Jul-Aug;31(4):e115-6. doi: 10.1097/iop.0000000000000143. PMID: 26168209.X-1

4168. Shapira I, Sequeira JM, Quadros EV. Folate receptor autoantibodies in pregnancy related complications. Birth Defects Res A Clin Mol Teratol. 2015 Sep 21doi: 10.1002/bdra.23436. PMID: 26390016.X-1, X3 
4169. Shapiro EG, Nestrasil I, Ahmed A, et al. Quantifying behaviors of children with Sanfilippo syndrome: the Sanfilippo Behavior Rating Scale. Mol Genet Metab. 2015 Apr;114(4):594-8. doi: 10.1016/j.ymgme.2015.02.008. PMID: 25770355.X-1

4170. Sharda M, Midha R, Malik S, et al. Fronto-temporal connectivity is preserved during sung but not spoken word listening, across the autism spectrum. Autism Res. 2015 Apr;8(2):174-86. doi: 10.1002/aur.1437. PMID: 25377165.X-1, X-3

4171. Sharer EA, Mostofsky SH, PascualLeone A, et al. Isolating Visual and Proprioceptive Components of Motor Sequence Learning in ASD. Autism Res. 2015 Oct 7doi: 10.1002/aur.1537. PMID: 26442448.X-1

4172. Sharma R, Agarwal A, Rohra VK, et al. Effects of increased paternal age on sperm quality, reproductive outcome and associated epigenetic risks to offspring. Reprod Biol Endocrinol. 2015;13:35. doi: 10.1186/s12958015-0028-X. PMID: 25928123.X-1

4173. Sharp RA, Mudford OC, Elliffe D. Representativeness of direct observations selected using a work-sampling equation. J Appl Behav Anal. 2015 Spring;48(1):153-66. doi: 10.1002/jaba.193. PMID: 25754895.X-1

4174. Shattuck PT, Roux AM. Commentary on employment supports research. Autism.

2015 Feb;19(2):246-7. doi:

10.1177/1362361313518996. PMID:

24449605.X-1

4175. Sheehan R, Hassiotis A, Walters K, et al. Mental illness, challenging behaviour, and psychotropic drug prescribing in people with intellectual disability: UK population based cohort study. Bmj. 2015;351:h4326. doi: 10.1136/bmj.h4326. PMID: 26330451.X-1

4176. Shen Y, Xun G, Guo H, et al. Association and gene-gene interactions study of reelin signaling pathway related genes with autism in the Han Chinese population. Autism Res. 2015 Aug 19doi: 10.1002/aur.1540. PMID: 26285919.X-1
4177. Shi L, Zhou Y, Ou J, et al. Different visual preference patterns in response to simple and complex dynamic social stimuli in preschool-aged children with autism spectrum disorders. PLoS One. 2015;10(3):e0122280. doi: 10.1371/journal.pone.0122280. PMID: 25781170.X-1

4178. Shic F, Smith D, Horsburgh B, et al. Catalysts for Change: The Role of Small Business Funders in the Creation and Dissemination of Innovation. J Autism Dev Disord. 2015 Dec;45(12):3900-4. doi: 10.1007/s10803-015-2636-x. PMID: 26481385.X-1

4179. Shield A, Meier RP, Tager-Flusberg H. The Use of Sign Language Pronouns by Native-Signing Children with Autism. J Autism Dev Disord. 2015 Jul;45(7):2128-45. doi: 10.1007/s10803-015-2377-x. PMID: 25643865.X-1

4180. Shigemori T, Sakai A, Takumi T, et al. Altered Microglia in the Amygdala Are Involved in Anxiety-related Behaviors of a Copy Number Variation Mouse Model of Autism. J Nippon Med Sch. 2015;82(2):92-9. doi: 10.1272/jnms.82.92. PMID: 25959200.X-1

4181. Shiozawa BJ. It's About Time for Autism Reform Legislation in Utah. J Autism Dev Disord. 2015 May;45(5):1495-6. doi: 10.1007/s10803-014-2302-8. PMID: 25395093.X-1

4182. Shire SY, Goods K, Shih W, et al. Parents' Adoption of Social Communication Intervention Strategies: Families Including Children with Autism Spectrum Disorder Who are Minimally Verbal. J Autism Dev Disord. 2015 Jun;45(6):1712-24. doi: 10.1007/s10803014-2329-x. PMID: 25475363.X-3

4183. Shmaya Y, Eilat-Adar S, Leitner Y, et al. Nutritional deficiencies and overweight prevalence among children with autism spectrum disorder. Res Dev Disabil. 2015 Mar;38:1-6. doi: 10.1016/j.ridd.2014.11.020. PMID: 25532026.X-1

4184. Shubrata KS, Sinha S, Seshadri SP, et al. Childhood autism spectrum disorders with and without epilepsy: clinical implications. J Child Neurol. 2015 Mar;30(4):476-82. doi: 
10.1177/0883073814540521. PMID: 25038126.X-1

4185. Shultz SR, Aziz NA, Yang L, et al. Intracerebroventricular injection of propionic acid, an enteric metabolite implicated in autism, induces social abnormalities that do not differ between seizure-prone (FAST) and seizureresistant (SLOW) rats. Behav Brain Res. 2015 Feb 1;278:542-8. doi: 10.1016/j.bbr.2014.10.050. PMID: 25446754.X-1

4186. Siegel M, Smith KA, Mazefsky C, et al. The autism inpatient collection: methods and preliminary sample description. Mol Autism. 2015;6:61. doi: 10.1186/s13229-015-0054-8. PMID: 26557975.X-1

4187. Silva D, Houghton S, Hagemann E, et al. Child attention deficit hyperactive disorder co morbidities on family stress: effect of medication. Community Ment Health J. 2015 Apr;51(3):347-53. doi: 10.1007/s10597-0149770-3. PMID: 25154408.X-1

4188. Silva LM, Schalock M, Gabrielsen $\mathrm{KR}$, et al. Early Intervention with a ParentDelivered Massage Protocol Directed at Tactile Abnormalities Decreases Severity of Autism and Improves Child-to-Parent Interactions: A Replication Study. Autism Res Treat. 2015;2015:904585. doi: 10.1155/2015/904585. PMID: 25878901. INCLUDE

4189. Silverman JL, Gastrell PT, Karras MN, et al. Cognitive abilities on transitive inference using a novel touchscreen technology for mice. Cereb Cortex. 2015 May;25(5):1133-42. doi: 10.1093/cercor/bht293. PMID: 24293564.X-1

4190. Silverman JL, Pride MC, Hayes JE, et al. GABAB Receptor Agonist R-Baclofen Reverses Social Deficits and Reduces Repetitive Behavior in Two Mouse Models of Autism. Neuropsychopharmacology. 2015 Aug;40(9):2228-39. doi: 10.1038/npp.2015.66. PMID: 25754761.X-1

4191. Simberlund J, Ferretti CJ, Hollander E. Mesenchymal stem cells in autism spectrum and neurodevelopmental disorders: pitfalls and potential promises. World J Biol Psychiatry. 2015 Jul 31:1-8. PMID: 26230216.X-1, X-2
4192. Simmons ES, Paul R, Shic F. Brief Report: A Mobile Application to Treat Prosodic Deficits in Autism Spectrum Disorder and Other Communication Impairments: A Pilot Study. J Autism Dev Disord. 2015 Sep 2doi: 10.1007/s10803-015-2573-8. PMID: 26329637.X-1, X-3

4193. Simut RE, Vanderfaeillie J, Peca A, et al. Children with Autism Spectrum Disorders Make a Fruit Salad with Probo, the Social Robot: An Interaction Study. J Autism Dev Disord. 2015 Aug 25doi: 10.1007/s10803-0152556-9. PMID: 26304031.X-1

4194. Singer A, Ravi R. Complementary and alternative treatments for autism part 2: identifying and avoiding non-evidence-based treatments. AMA J Ethics. 2015 Apr;17(4):37580. doi:

10.1001/journalofethics.2015.17.4.sect2-1504. PMID: 25901708.X-1

4195. Singer L. Thoughts about sex and gender differences from the next generation of autism scientists. Mol Autism. 2015;6:52. doi: 10.1186/s13229-015-0046-8. PMID: 26388981.X-1

4196. Singh HN, Rajeswari MR. Role of long purine stretches in controlling the expression of genes associated with neurological disorders. Gene. 2015 Nov 10;572(2):175-83. doi: 10.1016/j.gene.2015.07.007. PMID: 26149656.X-1

4197. Sinha S, McGovern RA, Sheth SA. Deep brain stimulation for severe autism: from pathophysiology to procedure. Neurosurg Focus. 2015 Jun;38(6):E3. doi: 10.3171/2015.3.focus1548. PMID: 26030703.X-1, X-3

4198. Sizoo BB, van der Gaag RJ, van den Brink W. Temperament and character as endophenotype in adults with autism spectrum disorders or attention deficit/hyperactivity disorder. Autism. 2015 May;19(4):400-8. doi: 10.1177/1362361314522352. PMID: 24535690.X-1

4199. Skewes JC, Jegindo EM, Gebauer L. Perceptual inference and autistic traits. Autism. 2015 Apr;19(3):301-7. doi: 
10.1177/1362361313519872. PMID: 24523412.X-1

4200. Skinner HJ, Walther RB, Dolwick MF, et al. Management of obstructive sleep apnea in a developmentally delayed pediatric patient with aggressive behavior and Pierre Robin sequence. J Clin Sleep Med. 2015;11(2):181-3. doi: 10.5664/jcsm.4470. PMID: 25580604.X-1

4201. Skokauskas N, Frodl T. Overlap between Autism Spectrum Disorder and Bipolar Affective Disorder. Psychopathology. 2015;48(4):209-16. doi: 10.1159/000435787. PMID: 26278909.X-1

4202. Skorich DP, May AR, Talipski LA, et al. Is Social Categorization the Missing Link Between Weak Central Coherence and Mental State Inference Abilities in Autism? Preliminary Evidence from a General Population Sample. J Autism Dev Disord. 2015 Oct 5doi: 10.1007/s10803-015-2623-2. PMID: 26438641.X-1

4203. Slocum SK, Vollmer TR. A comparison of positive and negative reinforcement for compliance to treat problem behavior maintained by escape. J Appl Behav Anal. 2015 Sep;48(3):563-74. doi: 10.1002/jaba.216. PMID: 25969379.X-1, X-3, $\mathrm{X}-4$

4204. Small BL. Discovery and Development of Pyridine-bis(imine) and Related Catalysts for Olefin Polymerization and Oligomerization. Acc Chem Res. 2015 Sep 15;48(9):2599-611. doi: 10.1021/acs.accounts.5b00252. PMID: 26267011.X-1

4205. Small DM, Pelphrey KA. Autism spectrum disorder: sniffing out a new biomarker. Curr Biol. 2015 Aug 3;25(15):R674-6. doi: 10.1016/j.cub.2015.06.050. PMID: 26241147.X-1, X-2

4206. Smith D, Ropar D, Allen HA. Visual integration in autism. Frontiers in Human Neuroscience. 2015 2015-09-09;9 PMID: 1710262987; 2015-35634-001.X-1

4207. Smith IM, Flanagan HE, Garon N, et al. Effectiveness of community-based early intervention based on pivotal response treatment. J Autism Dev Disord. 2015 Jun;45(6):1858-72. doi: 10.1007/s10803-0142345-x. PMID: 25563454.X-1, X-3

4208. Smith MJ, Fleming MF, Wright MA, et al. Brief Report: Vocational Outcomes for Young Adults with Autism Spectrum Disorders at Six Months After Virtual Reality Job Interview Training. J Autism Dev Disord. 2015 Oct;45(10):3364-9. doi: 10.1007/s10803-0152470-1. PMID: 25986176.X-1

4209. Smith Roley S, Mailloux Z, Parham LD, et al. Sensory Integration and Praxis Patterns in Children With Autism. American Journal of Occupational Therapy. 2015;69(1):18 p. doi: 10.5014/ajot.2015.012476. PMID: 103870119. Language: English. Entry Date: 20150110. Revision Date: 20150819. Publication Type: Journal Article.X-1

4210. Smith T, Iadarola S. Evidence Base Update for Autism Spectrum Disorder. J Clin Child Adolesc Psychol. 2015 NovDec;44(6):897-922. doi: 10.1080/15374416.2015.1077448. PMID: 26430947.X-2

4211. Smith T, Klorman R, Mruzek DW. Predicting Outcome of Community-Based Early Intensive Behavioral Intervention for Children with Autism. J Abnorm Child Psychol. 2015 Oct;43(7):1271-82. doi: 10.1007/s10802-0150002-2. PMID: 25778537.X-1, X-3

4212. Smith T, Sharp S, Manzardo AM, et al. Pharmacogenetics informed decision making in adolescent psychiatric treatment: a clinical case report. Int J Mol Sci. 2015;16(3):4416-28. doi: 10.3390/ijms16034416. PMID: 25710722.X-1, $\mathrm{X}-3, \mathrm{X}-4$

4213. Smoot Reinert S, Jackson K, Bigelow K. Using Posturography to Examine the Immediate Effects of Vestibular Therapy for Children with Autism Spectrum Disorders: A Feasibility Study. Phys Occup Ther Pediatr. 2015 Aug 19:1-16. doi: 10.3109/01942638.2014.975313. PMID: 25374155.X-4

4214. Sniezyk CJ, Zane TL. Investigating the effects of sensory integration therapy in decreasing stereotypy. Focus on Autism and Other Developmental Disabilities. 2015 201512-09;30(1):13-22. doi: 
http://dx.doi.org/10.1177/1088357614525663. PMID: 1666304692; 2015-06965-002.X-1, X-3, $\mathrm{X}-4$

4215. So WC, Lui M, Wong TK, et al. The use of hand gestures to communicate about nonpresent objects in mind among children with autism spectrum disorder. J Speech Lang Hear Res. 2015 Apr;58(2):373-82. doi: 10.1044/2015_jslhr-l-14-0213. PMID: 25785685.X-1

4216. So WC, Wong MK, Lui M, et al. The development of co-speech gesture and its semantic integration with speech in 6- to 12 year-old children with autism spectrum disorders. Autism. 2015 Nov;19(8):956-68. doi: 10.1177/1362361314556783. PMID: 25488001.X-1

4217. Sobotka SA, Francis A, Vander Ploeg Booth K. Associations of family characteristics with perceptions of care among parents of children with autism. Child Care Health Dev. 2015 Oct 16doi: 10.1111/cch.12290. PMID: 26470756.X-1

4218. Solomon M, Frank MJ, Ragland JD, et al. Feedback-driven trial-by-trial learning in autism spectrum disorders. Am J Psychiatry. 2015 Feb 1;172(2):173-81. doi:

10.1176/appi.ajp.2014.14010036. PMID: 25158242.X-1

4219. Solomon O. "But-He'll Fall!": Children with Autism, Interspecies Intersubjectivity, and the Problem of 'Being Social'. Cult Med Psychiatry. 2015 Jun;39(2):323-44. doi: 10.1007/s11013-015-9446-7. PMID: 25926308.X-1

4220. Soltanifar A, Akbarzadeh F, Moharreri $\mathrm{F}$, et al. Comparison of parental stress among mothers and fathers of children with autistic spectrum disorder in Iran. Iran J Nurs Midwifery Res. 2015 Jan-Feb;20(1):93-8. PMID: 25709697.X-1

4221. Son JS, Zheng LJ, Rowehl LM, et al. Comparison of Fecal Microbiota in Children with Autism Spectrum Disorders and Neurotypical Siblings in the Simons Simplex Collection. PLoS One. 2015;10(10):e0137725. doi: 10.1371/journal.pone.0137725. PMID: 26427004.X-1
4222. Song J, Bergen SE, Kuja-Halkola R, et al. Bipolar disorder and its relation to major psychiatric disorders: a family-based study in the Swedish population. Bipolar Disord. 2015 Mar;17(2):184-93. doi: 10.1111/bdi.12242. PMID: 25118125.X-1

4223. Song Y, Hakoda Y, Sanefuji W, et al. Can They See It? The Functional Field of View Is Narrower in Individuals with Autism Spectrum Disorder. PLoS One. 2015;10(7):e0133237. doi: 10.1371/journal.pone.0133237. PMID: 26204121.X-1

4224. Soorya LV, Siper PM, Beck T, et al. Randomized comparative trial of a social cognitive skills group for children with autism spectrum disorder. J Am Acad Child Adolesc Psychiatry. 2015 Mar;54(3):208-16.e1. doi: 10.1016/j.jaac.2014.12.005. PMID: 25721186.X-1

4225. Sorensen EM, Bertelsen F, Weikop P, et al. Hyperactivity and lack of social discrimination in the adolescent Fmr1 knockout mouse. Behav Pharmacol. 2015 Jun 23doi: 10.1097/fbp.0000000000000152. PMID: 26110222.X-1

4226. Sowden S, Koehne S, Catmur C, et al. Intact Automatic Imitation and Typical Spatial Compatibility in Autism Spectrum Disorder: Challenging the Broken Mirror Theory. Autism Res. 2015 Jun 26doi: 10.1002/aur.1511. PMID: 26112060.X-1

4227. Spain D, Blainey SH. Group social skills interventions for adults with highfunctioning autism spectrum disorders: A systematic review. Autism. 2015

Oct;19(7):874-86. doi:

10.1177/1362361315587659. PMID: 26045543.X-1

4228. Spek AA. [Eating problems in individuals with autism spectrum disorder (ASD) but no intellectual impairment]. Tijdschr Psychiatr. 2015;57(10):749-56. PMID: 26479255.X-1

4229. Srinivasan SM, Park IK, Neelly LB, et al. A comparison of the effects of rhythm and robotic interventions on repetitive behaviors and affective states of children with Autism Spectrum Disorder (ASD). Res Autism Spectr 
Disord. 2015 Oct 1;18:51-63. doi: 10.1016/j.rasd.2015.07.004. PMID: 26251668.X-1

4230. Srinivasjois R, Rao S, Patole S. Probiotic supplementation in children with autism spectrum disorder. Arch Dis Child. 2015 May;100(5):505-6. doi: 10.1136/archdischild2014-308002. PMID: 25809345.X-2

4231. Staal WG. Autism, DRD3 and repetitive and stereotyped behavior, an overview of the current knowledge. Eur Neuropsychopharmacol. 2015 Sep;25(9):14216. doi: 10.1016/j.euroneuro.2014.08.011. PMID: 25224105.X-1

4232. Stadnick N, Brookman-Frazee L, Williams KN, et al. A Pilot Study Examining the Use of the Autism Diagnostic Observation Schedule in Community-Based Mental Health Clinics. Res Autism Spectr Disord. 2015 Dec 1;20:39-46. doi: 10.1016/j.rasd.2015.08.007. PMID: 26379765.X-1

4233. Stadnick NA, Stahmer A, BrookmanFrazee L. Preliminary Effectiveness of Project ImPACT: A Parent-Mediated Intervention for Children with Autism Spectrum Disorder Delivered in a Community Program. J Autism Dev Disord. 2015 Jul;45(7):2092-104. doi: 10.1007/s10803-015-2376-y. PMID: 25633920.X-1

4234. Stagi P, Galeotti S, Mimmi S, et al. Continuity of care from child and adolescent to adult mental health services: evidence from a regional survey in Northern Italy. Eur Child Adolesc Psychiatry. 2015 Jul 4doi: 10.1007/s00787-015-0735-z. PMID: 26141538.X-1

4235. Stahmer AC, Reed S, Lee E, et al. Training Teachers to use Evidence-Based Practices for Autism: Examining Procedural Implementation fidelity. Psychol Sch. 2015 Feb 1;52(2):181-95. doi: 10.1002/pits.21815. PMID: 25593374.X-1

4236. Stanish H, Curtin C, Must A, et al. Enjoyment, Barriers, and Beliefs About Physical Activity in Adolescents With and Without Autism Spectrum Disorder. Adapt Phys Activ Q. 2015 Oct;32(4):302-17. doi: 10.1123/apaq.2015-0038. PMID: 26485735.X-1
4237. Stark KH, Barnes JC, Young ND, et al. Brief Report: Understanding Crisis Behaviors in Hospitalized Psychiatric Patients with Autism Spectrum Disorder--Iceberg Assessment Interview. J Autism Dev Disord. 2015 Nov;45(11):3468-74. doi: 10.1007/s10803-015-2552-0. PMID: 26324248.X-1

4238. Starkstein S, Gellar S, Parlier M, et al. High rates of parkinsonism in adults with autism. J Neurodev Disord. 2015;7(1):29. doi: 10.1186/s11689-015-9125-6. PMID: 26322138.X-1

4239. Steenweg-de Graaff J, Ghassabian A, Jaddoe VW, et al. Folate concentrations during pregnancy and autistic traits in the offspring. The Generation R Study. Eur J Public Health. 2015 Jun;25(3):431-3. doi: 10.1093/eurpub/cku126. PMID: 25085472.X-1

4240. Stefanik P, Olexova L, Krskova L. Increased sociability and gene expression of oxytocin and its receptor in the brains of rats affected prenatally by valproic acid. Pharmacol Biochem Behav. 2015 Apr;131:42-50. doi: 10.1016/j.pbb.2015.01.021. PMID: 25662821.X-1

4241. Steiner H, Kertesz Z. Effects of therapeutic horse riding on gait cycle parameters and some aspects of behavior of children with autism. Acta Physiol Hung. 2015 Sep;102(3):324-35. doi: 10.1556/036.102.2015.3.10. PMID: 26551748.X-1, X-3

4242. Stendal K, Balandin S. Virtual worlds for people with autism spectrum disorder: a case study in Second Life. Disabil Rehabil. 2015;37(17):1591-8. doi: 10.3109/09638288.2015.1052577. PMID: 26023707.X-1

4243. Stephanie D, Julie F. Exploring links between language and cognition in autism spectrum disorders: Complement sentences, false belief, and executive functioning. J Commun Disord. 2015 Mar-Apr;54:15-31. doi: 10.1016/j.jcomdis.2014.12.001. PMID: 25637130.X-1

4244. Stewart CR, Sanchez SS, Grenesko EL, et al. Sensory Symptoms and Processing of Nonverbal Auditory and Visual Stimuli in 
Children with Autism Spectrum Disorder. J

Autism Dev Disord. 2015 Feb 5doi:

10.1007/s10803-015-2367-z. PMID: 25652601.X-1

4245. Stewart LT. Cell adhesion proteins and the pathogenesis of autism spectrum disorders. J Neurophysiol. 2015 Mar 1;113(5):1283-6. doi: 10.1152/jn.00780.2013. PMID: 24990562.X-1

4246. Stewart PA, Hyman SL, Schmidt BL, et al. Dietary Supplementation in Children with Autism Spectrum Disorders: Common, Insufficient, and Excessive. J Acad Nutr Diet. 2015 Aug;115(8):1237-48. doi: 10.1016/j.jand.2015.03.026. PMID: 26052041.X-1

4247. Stiegler LN. Examining the Echolalia Literature: Where Do Speech-Language Pathologists Stand? Am J Speech Lang Pathol. 2015 Jul 10doi: 10.1044/2015_ajslp-14-0166. PMID: 26161804.X-1, X-2

4248. Stilling RM, Ryan FJ, Hoban AE, et al. Microbes \& neurodevelopment - Absence of microbiota during early life increases activityrelated transcriptional pathways in the amygdala. Brain Behav Immun. 2015 Jul 14doi: 10.1016/j.bbi.2015.07.009. PMID: 26184083.X-1

4249. Stins JF, Emck C, de Vries EM, et al. Attentional and sensory contributions to postural sway in children with autism spectrum disorder. Gait Posture. 2015 Jul;42(2):199-203. doi: 10.1016/j.gaitpost.2015.05.010. PMID: 26028526.X-1

4250. Storch EA, Lewin AB, Collier AB, et al. A randomized controlled trial of cognitivebehavioral therapy versus treatment as usual for adolescents with autism spectrum disorders and comorbid anxiety. Depress Anxiety. 2015 Mar;32(3):174-81. doi: 10.1002/da.22332. PMID: 25424398.X-1

4251. Storch EA, Nadeau JM, Rudy B, et al. A Case Series of Cognitive-Behavioral Therapy Augmentation of Antidepressant Medication for Anxiety in Children With Autism Spectrum Disorders. Children's Health Care.

2015;44(2):183-98 16p. doi:

10.1080/02739615.2014.906310. PMID:

103790337. Language: English. Entry Date:
20150424. Revision Date: 20150710.

Publication Type: Journal Article.X-3, X-4

4252. Storch EA, Zavrou S, Collier AB, et al. Preliminary study of family accommodation in youth with autism spectrum disorders and anxiety: Incidence, clinical correlates, and behavioral treatment response. J Anxiety Disord. 2015 Aug;34:94-9. doi: 10.1016/j.janxdis.2015.06.007. PMID: 26188615.X-1, X-3

4253. Strauss K, Benvenuto A, Battan B, et al. Promoting Shared Decision Making to strengthen outcome of young children with Autism Spectrum Disorders: the role of staff competence. Res Dev Disabil. 2015 Mar;38:4863. doi: 10.1016/j.ridd.2014.11.016. PMID: 25544428.X-1

4254. Stuttard L, Beresford B, Clarke S, et al. A preliminary investigation into the effectiveness of a group-delivered sleep management intervention for parents of children with intellectual disabilities. J Intellect Disabil. 2015 Mar 19doi:

10.1177/1744629515576610. PMID: 25792540.X-3

4255. Stuttard L, Clarke S, Thomas M, et al. Replacing home visits with telephone calls to support parents implementing a sleep management intervention: findings from a pilot study and implications for future research. Child Care Health Dev. 2015 Apr 10doi: 10.1111/cch.12250. PMID: 25865216.X-1, X-4

4256. Suades-Gonzalez E, Gascon M, Guxens M, et al. Air Pollution and Neuropsychological Development: A Review of the Latest Evidence. Endocrinology. 2015 Oct;156(10):3473-82. doi: 10.1210/en.20151403. PMID: 26241071.X-1

4257. Suganya V, Geetha A, Sujatha S. Urine proteome analysis to evaluate protein biomarkers in children with autism. Clin Chim Acta. 2015 Oct 23;450:210-9. doi: 10.1016/j.cca.2015.08.015. PMID: 26296899.X-1

4258. Suhrheinrich J. A sustainable model for training teachers to use pivotal response training. Autism. 2015 Aug;19(6):713-23. doi: 10.1177/1362361314552200. PMID: 25319208.X-1 
4259. Sullivan AL, Sadeh S.

Psychopharmacological treatment among adolescents with disabilities: Prevalence and predictors in a nationally representative sample. Sch Psychol Q. 2015 Sep;30(3):443-55. doi: 10.1037/spq0000105. PMID: 25528591.X-1

4260. Sun F, Oristaglio J, Levy SE, et al. AHRQ Comparative Effectiveness Technical Briefs. Genetic Testing for Developmental Disabilities, Intellectual Disability, and Autism Spectrum Disorder. Rockville (MD): Agency for Healthcare Research and Quality (US); 2015.

4261. Sun Y. Supercritical Fluid Particle Design of DPI Formulations (Review). Curr Pharm Des. 2015;21(19):2516-42. PMID: 25876911.X-1

4262. Sung C, Sanchez J, Kuo HJ, et al. Gender differences in vocational rehabilitation service predictors of successful competitive employment for transition-aged individuals with autism. J Autism Dev Disord. 2015 Oct;45(10):3204-18. doi: 10.1007/s10803-0152480-z. PMID: 26060047.X-1

4263. Supekar K, Menon V. Sex differences in structural organization of motor systems and their dissociable links with repetitive/restricted behaviors in children with autism. Mol Autism. 2015;6:50. doi: 10.1186/s13229-015-0042-z. PMID: 26347127.X-1

4264. Suryadevara M, Handel A, Bonville CA, et al. Pediatric provider vaccine hesitancy: An under-recognized obstacle to immunizing children. Vaccine. 2015 Nov 27;33(48):662934. doi: 10.1016/j.vaccine.2015.10.096. PMID: 26529071.X-1

4265. Sussman D, Leung RC, Vogan VM, et al. The autism puzzle: Diffuse but not pervasive neuroanatomical abnormalities in children with ASD. Neuroimage Clin. 2015;8:170-9. doi: 10.1016/j.nicl.2015.04.008. PMID: 26106541.X-1

4266. Sutherland B, Middlebrook N, Kairn $\mathrm{T}$, et al. A comparison between direct TMR measurements and TMRs calculated from PDDs using BJR Supplement 25 data for flattened and unflattened photon beams. Australas Phys Eng Sci Med. 2015
Sep;38(3):503-7. doi: 10.1007/s13246-0150359-0. PMID: 26123946.X-1

4267. Suzuki K, Kobayashi T, Moriyama K, et al. [Parenting resilience for rearing a child with autism spectrum disorder: a qualitative study]. No To Hattatsu. 2015 Jul;47(4):283-8. PMID: 26353450.X-1

4268. Suzumura S. Quality of life in mothers of preschoolers with high-functioning pervasive developmental disorders. Pediatr Int. 2015 Feb;57(1):149-54. doi: 10.1111/ped.12560. PMID: 25495867.X-1

4269. Swain NR, Eadie PA, Prior MR, et al. Assessing early communication skills at 12 months: a retrospective study of Autism Spectrum Disorder. Int J Lang Commun Disord. 2015 Jul;50(4):488-98. doi: 10.1111/14606984.12150. PMID: 25630989.X-1

4270. Swanger SA, Mattheyses AL, Gentry EG, et al. ROCK1 and ROCK2 inhibition alters dendritic spine morphology in hippocampal neurons. Cell Logist. 2015 Oct-

Dec;5(4):e1133266. doi: 10.1080/21592799.2015.1133266. PMID: 27054047.X-1

4271. Swineford LB, Guthrie W, Thurm A. Convergent and Divergent Validity of the Mullen Scales of Early Learning in Young Children With and Without Autism Spectrum Disorder. Psychol Assess. 2015 Apr 20doi: 10.1037/pas0000116. PMID: 25894712.X-1

4272. Swinth Y, Tomlin G, Luthman M. Content Analysis of Qualitative Research on Children and Youth With Autism, 1993-2011: Considerations for Occupational Therapy Services. Am J Occup Ther. 2015 SepOct;69(5):6905185030p1-9. doi: 10.5014/ajot.2015.017970. PMID: 26356660.X-1, X-2

4273. Szakacs A, Hallbook T, Tideman P, et al. Psychiatric comorbidity and cognitive profile in children with narcolepsy with or without association to the H1N1 influenza vaccination. Sleep. 2015 Apr;38(4):615-21. doi: 10.5665/sleep.4582. PMID: 25325473.X-1

4274. Tager-Flusberg H. Risk Factors Associated with Language in Autism Spectrum Disorder: Clues to Underlying Mechanisms. J 
Speech Lang Hear Res. 2015 Oct 20doi: 10.1044/2015_jslhr-l-15-0146. PMID: 26502110.X-1

4275. Takahashi H, Komatsu S, Nakahachi $\mathrm{T}$, et al. Relationship of the Acoustic Startle Response and Its Modulation to Emotional and Behavioral Problems in Typical Development Children and Those with Autism Spectrum Disorders. J Autism Dev Disord. 2015 Sep 12doi: 10.1007/s10803-015-2593-4. PMID: 26362152.X-1

4276. Takai T, Inamoto T, Komura K, et al. Feasibility of photodynamic diagnosis for challenging TUR- Bt cases including muscle invasive bladder cancer, BCG failure or 2ndTUR. Asian Pac J Cancer Prev. 2015;16(6):2297-301. PMID: 25824753.X-1

4277. Takano T. Role of Microglia in Autism: Recent Advances. Dev Neurosci. 2015;37(3):195-202. doi: 10.1159/000398791. PMID: 25998072.X-1

4278. Takeuchi H, Taki Y, Sekiguchi A, et al. Brain structures in the sciences and humanities. Brain Struct Funct. 2015 Nov;220(6):3295-305. doi: 10.1007/s00429014-0857-y. PMID: 25079346.X-1

4279. Talbott EO, Marshall LP, Rager JR, et al. Air toxics and the risk of autism spectrum disorder: the results of a population based casecontrol study in southwestern Pennsylvania.

Environ Health. 2015;14:80. doi: 10.1186/s12940-015-0064-1. PMID: 26444407.X-1

4280. Talbott MR, Nelson CA, TagerFlusberg H. Maternal Vocal Feedback to 9Month-Old Infant Siblings of Children with ASD. Autism Res. 2015 Jul 14doi: 10.1002/aur.1521. PMID: 26174704.X-1

4281. Talpos JC, Riordan J, Olley J, et al. Opposing effects of glutamatergic and GABAergic pharmacological manipulations on a visual perception task with relevance to schizophrenia. Psychopharmacology (Berl). 2015 Nov;232(21-22):3967-76. doi: 10.1007/s00213-015-3964-4. PMID: 26014109.X-1

4282. Tamasaki A, Saito Y, Ueda R, et al. Effects of donepezil and serotonin reuptake inhibitor on acute regression during adolescence in Down syndrome. Brain Dev. 2015 Jul 2doi: 10.1016/j.braindev.2015.06.006. PMID: 26143664.X-1

4283. Tang B, Wang T, Wan H, et al. Fmr1 deficiency promotes age-dependent alterations in the cortical synaptic proteome. Proc Natl Acad Sci U S A. 2015 Aug 25;112(34):E4697706. doi: 10.1073/pnas.1502258112. PMID: 26307763.X-1

4284. Tang KL, Antshel KM, Fremont WP, et al. Behavioral and Psychiatric Phenotypes in 22q11.2 Deletion Syndrome. J Dev Behav

Pediatr. 2015 Oct;36(8):639-50. doi: 10.1097/dbp.0000000000000210. PMID: 26372046.X-1

4285. Tanner K, Hand BN, O'Toole G, et al. Effectiveness of Interventions to Improve Social Participation, Play, Leisure, and Restricted and Repetitive Behaviors in People With Autism Spectrum Disorder: A Systematic Review. Am J Occup Ther. 2015 SepOct;69(5):6905180010p1-p12. doi: 10.5014/ajot.2015.017806. PMID: 26356653.X-2

4286. Tarazi FI, Sahli ZT, Pleskow J, et al. Asperger's syndrome: diagnosis, comorbidity and therapy. Expert Rev Neurother. 2015 Mar;15(3):281-93. doi: 10.1586/14737175.2015.1009898. PMID: 25655905.X-1

4287. Tell D, Davidson D. Emotion recognition from congruent and incongruent emotional expressions and situational cues in children with autism spectrum disorder. Autism. 2015 Apr;19(3):375-9. doi: 10.1177/1362361314535676. PMID: 24852752.X-1

4288. Ten Eycke K, Müller U. Brief Report: New Evidence for a Social-Specific Imagination Deficit in Children with Autism Spectrum Disorder. Journal of Autism \& Developmental Disorders. 2015;45(1):213-20 8p. doi: 10.1007/s10803-014-2206-7. PMID: 103871007. Language: English. Entry Date: 20150109. Revision Date: 20150710. Publication Type: Journal Article.X-1

4289. Tessier S, Lambert A, Chicoine M, et al. Intelligence measures and stage 2 sleep in 
typically-developing and autistic children. Int J Psychophysiol. 2015 Jul;97(1):58-65. doi: 10.1016/j.ijpsycho.2015.05.003. PMID: 25958790.X-1

4290. Tessier S, Lambert A, Scherzer P, et al. REM sleep and emotional face memory in typically-developing children and children with autism. Biol Psychol. 2015 Sep;110:107-14. doi: 10.1016/j.biopsycho.2015.07.012. PMID: 26219603.X-1

4291. Thanos PK, Restif C, O'Rourke JR, et al. Mouse Social Interaction Test (MoST): a quantitative computer automated analysis of behavior. J Neural Transm (Vienna). 2015 Dec 24doi: 10.1007/s00702-015-1487-0. PMID: 26704381.X-1, X-2

4292. Theoharides TC, Stewart JM, Panagiotidou S, et al. Mast cells, brain inflammation and autism. Eur J Pharmacol. 2015 May 1doi: 10.1016/j.ejphar.2015.03.086. PMID: 25941080.X-1

4293. Thiebaut FI, White SJ, Walsh A, et al. Does Faux Pas Detection in Adult Autism Reflect Differences in Social Cognition or Decision-Making Abilities? J Autism Dev Disord. 2015 Aug 15doi: 10.1007/s10803-0152551-1. PMID: 26276266.X-1

4294. Thienpont L, Verhofstadt M, Van Loon $\mathrm{T}$, et al. Euthanasia requests, procedures and outcomes for 100 Belgian patients suffering from psychiatric disorders: a retrospective, descriptive study. BMJ Open.

2015;5(7):e007454. doi: 10.1136/bmjopen2014-007454. PMID: 26216150.X-1

4295. Thoelking J, Sekar Y, Fleckenstein J, et al. Characterization of a new transmission detector for patient individualized online plan verification and its influence on 6MV x-ray beam characteristics. Z Med Phys. 2015 Aug 21doi: 10.1016/j.zemedi.2015.08.001. PMID: 26303190.X-1

4296. Thomas MS, Davis R, KarmiloffSmith A, et al. The over-pruning hypothesis of autism. Dev Sci. 2015 Apr 6doi: 10.1111/desc.12303. PMID: 25845529.X-1

4297. Thomas S, Sciberras E, Lycett K, et al. Physical Functioning, Emotional, and Behavioral Problems in Children With ADHD and Comorbid ASD: A Cross-Sectional Study. J Atten Disord. 2015 May 25doi: 10.1177/1087054715587096. PMID: 26006166.X-1

4298. Thomeer ML, Smith RA, Lopata C, et al. Randomized Controlled Trial of Mind Reading and In Vivo Rehearsal for HighFunctioning Children with ASD. J Autism Dev Disord. 2015 Jul;45(7):2115-27. doi: 10.1007/s10803-015-2374-0. PMID: 25643864.X-1

4299. Thompson JI, Peck CE, Karvelas G, et al. Temporal processing as a source of altered visual perception in high autistic tendency. Neuropsychologia. 2015 Mar;69:148-53. doi: 10.1016/j.neuropsychologia.2015.01.046. PMID: 25645512.X-1

4300. Thomson K, Burnham Riosa P, Weiss JA. Brief Report of Preliminary Outcomes of an Emotion Regulation Intervention for Children with Autism Spectrum Disorder. J Autism Dev Disord. 2015 Nov;45(11):3487-95. doi: 10.1007/s10803-015-2446-1. PMID: 25877014.X-1, X-3

4301. Thurman AJ, McDuffie A, Kover ST, et al. Autism Symptomatology in Boys with Fragile X Syndrome: A Cross Sectional Developmental Trajectories Comparison with Nonsyndromic Autism Spectrum Disorder. J Autism Dev Disord. 2015 Sep;45(9):2816-32. doi: 10.1007/s10803-015-2443-4. PMID: 25904201.X-1

4302. Tian D, Stoppel LJ, Heynen AJ, et al. Contribution of mGluR5 to pathophysiology in a mouse model of human chromosome 16p11.2 microdeletion. Nat Neurosci. 2015

Feb;18(2):182-4. doi: 10.1038/nn.3911. PMID: 25581360.X-1

4303. Tilford JM, Payakachat N, Kuhlthau KA, et al. Treatment for Sleep Problems in Children with Autism and Caregiver Spillover Effects. J Autism Dev Disord. 2015 Nov;45(11):3613-23. doi: 10.1007/s10803-0152507-5. PMID: 26126749.X-1, X-3

4304. Tillmann J, Olguin A, Tuomainen J, et al. The Effect of Visual Perceptual Load on Auditory Awareness in Autism Spectrum Disorder. J Autism Dev Disord. 2015 
Oct;45(10):3297-307. doi: 10.1007/s10803015-2491-9. PMID: 26043848.X-1

4305. Tilot AK, Bebek G, Niazi F, et al. Neural transcriptome of constitutional Pten dysfunction in mice and its relevance to human idiopathic autism spectrum disorder. Mol Psychiatry. 2015 Mar 10doi:

10.1038/mp.2015.17. PMID: 25754085.X-1

4306. Timonen-Soivio L, Sourander A, Malm H, et al. The Association Between Autism Spectrum Disorders and Congenital Anomalies by Organ Systems in a Finnish National Birth Cohort. J Autism Dev Disord. 2015 Oct;45(10):3195-203. doi: 10.1007/s10803-015-2477-7. PMID: 26036648.X-1

4307. Tomchek SD, Little LM, Dunn W. Sensory Pattern Contributions to Developmental Performance in Children With Autism Spectrum Disorder. Am J Occup Ther. 2015 Sep-Oct;69(5):6905185040p1-p10. doi: 10.5014/ajot.2015.018044. PMID: 26356661.X-1

4308. Tomlin GS, Swinth Y. Contribution of Qualitative Research to Evidence in Practice for People With Autism Spectrum Disorder. Am J Occup Ther. 2015 SepOct;69(5):6905360010p1-4. doi: 10.5014/ajot.2015.017988. PMID: 26356667.X-1, X-2, X-3

4309. Tomova A, Husarova V, Lakatosova $\mathrm{S}$, et al. Gastrointestinal microbiota in children with autism in Slovakia. Physiol Behav. 2015 Jan;138:179-87. doi:

10.1016/j.physbeh.2014.10.033. PMID: 25446201.X-1

4310. Tomson SN, Schreiner MJ, Narayan $\mathrm{M}$, et al. Resting state functional MRI reveals abnormal network connectivity in neurofibromatosis 1. Hum Brain Mapp. 2015 Nov;36(11):4566-81. doi: 10.1002/hbm.22937. PMID: 26304096.X-1

4311. Tonhajzerova I, Ondrejka I, Mestanik $\mathrm{M}$, et al. Inflammatory Activity in Autism Spectrum Disorder. Adv Exp Med Biol. 2015;861:93-8. doi: 10.1007/5584_2015_145. PMID: 26022900.X-1
4312. Tourian L, LeBoeuf A, Breton JJ, et al. Treatment Options for the Cardinal Symptoms of Disruptive Mood Dysregulation Disorder. J Can Acad Child Adolesc Psychiatry. 2015 Winter;24(1):41-54. PMID: 26336379.X-1

4313. Travers BG, Kana RK, Klinger LG, et al. Motor learning in individuals with autism spectrum disorder: activation in superior parietal lobule related to learning and repetitive behaviors. Autism Res. 2015 Feb;8(1):38-51. doi: 10.1002/aur.1403. PMID: 25258047.X-1

4314. Trembath D, Vivanti G, Iacono T, et al. Accurate or assumed: visual learning in children with ASD. J Autism Dev Disord. 2015 Oct;45(10):3276-87. doi: 10.1007/s10803-0152488-4. PMID: 26070275.X-1

4315. Trivedi MS, Hodgson NW, Walker SJ, et al. Epigenetic effects of casein-derived opioid peptides in SH-SY5Y human neuroblastoma cells. Nutr Metab (Lond). 2015;12:54. doi: 10.1186/s12986-015-0050-1. PMID: 26664459.X-1

4316. Trivisano M, Specchio N, Vigevano F. Extending the use of stiripentol to other epileptic syndromes: a case of PCDH19-related epilepsy. Eur J Paediatr Neurol. 2015 Mar;19(2):248-50. doi: 10.1016/j.ejpn.2014.11.008. PMID: 25510386.X-1

4317. Trontel HG, Duffield TC, Bigler ED, et al. Mesial temporal lobe and memory function in autism spectrum disorder: an exploration of volumetric findings. J Clin Exp Neuropsychol. 2015;37(2):178-92. doi: 10.1080/13803395.2014.997677. PMID: 25749302.X-1

4318. Truong DT, Rendall AR, Castelluccio BC, et al. Auditory Processing and Morphological Anomalies in Medial Geniculate Nucleus of Cntnap2 Mutant Mice. Behav Neurosci. 2015 Oct 26doi: 10.1037/bne0000096. PMID: 26501174.X-1

4319. Tsai SJ. Is riluzole a potential therapy for Rett syndrome? Med Hypotheses. 2015 Jul;85(1):76-8. doi:

10.1016/j.mehy.2015.03.025. PMID: 25858436.X-1 
4320. Tsilioni I, Taliou A, Francis K, et al. Children with autism spectrum disorders, who improved with a luteolin-containing dietary formulation, show reduced serum levels of TNF and IL-6. Transl Psychiatry. 2015;5:e647. doi: 10.1038/tp.2015.142. PMID: 26418275.X-3

4321. Tsuji S, Yuhi T, Furuhara K, et al. Salivary oxytocin concentrations in seven boys with autism spectrum disorder received massage from their mothers: a pilot study. Front Psychiatry. 2015;6:58. doi: 10.3389/fpsyt.2015.00058. PMID: 25954210.X-3, X-4

4322. Tsutiya A, Nishihara M, Goshima Y, et al. Mouse pups lacking collapsin response mediator protein 4 manifest impaired olfactory function and hyperactivity in the olfactory bulb. Eur J Neurosci. 2015 Sep;42(6):2335-45. doi: 10.1111/ejn.12999. PMID: 26118640.X-1

4323. Tudor ME, Walsh CE, Mulder EC, et al. Pain as a predictor of sleep problems in youth with autism spectrum disorders. Autism. 2015 Apr;19(3):292-300. doi: 10.1177/1362361313518994. PMID: 24497628.X-1

4324. Turygin N, Matson JL, Tureck K. The relationship of attention-deficit hyperactivity disorder and autism spectrum disorder to adaptive skills in young children. Dev Neurorehabil. 2015 Oct;18(5):317-21. doi: 10.3109/17518423.2013.846947. PMID: 24303981.X-1

4325. Tye C, Farroni T, Volein A, et al. Autism diagnosis differentiates neurophysiological responses to faces in adults with tuberous sclerosis complex. J Neurodev Disord. 2015;7:33. doi: 10.1186/s11689-0159129-2. PMID: 26451165.X-1

4326. Uchida M, Spencer TJ, Faraone SV, et al. Adult Outcome of ADHD: An Overview of Results From the MGH Longitudinal Family Studies of Pediatrically and Psychiatrically Referred Youth With and Without ADHD of Both Sexes. J Atten Disord. 2015 Sep 22doi: 10.1177/1087054715604360. PMID: 26396145.X-1

4327. Ueyama Y. A Bayesian Model of the Uncanny Valley Effect for Explaining the Effects of Therapeutic Robots in Autism
Spectrum Disorder. PLoS One. 2015;10(9):e0138642. doi: 10.1371/journal.pone.0138642. PMID: 26389805.X-1, X-2, X-3

4328. Ujiie Y, Asai T, Wakabayashi A. The relationship between level of autistic traits and local bias in the context of the McGurk effect. Frontiers in Psychology. 2015 2016-01-06;6doi: 10.1017/CBO9780511526770 10.1007/s 10803-010-1000-4. PMID: 1753449054; 2015-55530-001.X-1

4329. Ung D, Selles R, Small BJ, et al. A Systematic Review and Meta-Analysis of Cognitive-Behavioral Therapy for Anxiety in Youth with High-Functioning Autism Spectrum Disorders. Child Psychiatry Hum Dev. 2015 Aug;46(4):533-47. doi: 10.1007/s10578-0140494-y. PMID: 25246292.X-2

4330. Ungar WJ. Next Generation Sequencing and Health Technology Assessment in Autism Spectrum Disorder. J Can Acad Child Adolesc Psychiatry. 2015 Fall;24(2):1237. PMID: 26379724.X-1

4331. Unwin LM, Maybery MT, Murphy A, et al. A Prospective Ultrasound Study of Prenatal Growth in Infant Siblings of Children With Autism. Autism Res. 2015 Jul 7doi: 10.1002/aur.1518. PMID: 26148908.X-1

4332. Uppal N, Puri R, Yuk F, et al. Ultrastructural analyses in the hippocampus CA1 field in Shank3-deficient mice. Mol Autism. 2015;6:41. doi: 10.1186/s13229-0150036-x. PMID: 26137200.X-1

4333. Urbain CM, Pang EW, Taylor MJ. Atypical spatiotemporal signatures of working memory brain processes in autism. Transl Psychiatry. 2015;5:e617. doi: 10.1038/tp.2015.107. PMID: 26261885.X-1

4334. Urbano M, Okwara L, Manser P, et al. A trial of d-cycloserine to treat the social deficit in older adolescents and young adults with autism spectrum disorders. J Neuropsychiatry Clin Neurosci. 2015 Spring;27(2):133-8. doi: 10.1176/appi.neuropsych.13070155. PMID: 25923852.X-1

4335. Ure AM, Treyvaud K, Thompson DK, et al. Neonatal brain abnormalities associated with autism spectrum disorder in children born 
very preterm. Autism Res. 2015 Oct 7doi: 10.1002/aur.1558. PMID: 26442616.X-1

4336. Usami M, Iwadare Y, Watanabe K, et al. A case-control study of the difficulties in daily functioning experienced by children with depressive disorder. J Affect Disord. 2015 Jul 1;179:167-74. doi: 10.1016/j.jad.2015.03.023. PMID: 25863914.X-1

4337. Usher LV, Burrows CA, Schwartz CB, et al. Social Competence with an Unfamiliar Peer in Children and Adolescents with High Functioning Autism: Measurement and Individual Differences. Res Autism Spectr Disord. 2015 Sep 1;17:25-39. doi: 10.1016/j.rasd.2015.05.005. PMID: 26161136.X-1

4338. Uzunova G, Pallanti S, Hollander E. Excitatory/inhibitory imbalance in autism spectrum disorders: Implications for interventions and therapeutics. World J Biol Psychiatry. 2015 Oct 15:1-13. doi: 10.3109/15622975.2015.1085597. PMID: 26469219.X-1

4339. Vaiouli P, Grimmet K, Ruich LJ. "Bill is now singing": joint engagement and the emergence of social communication of three young children with autism. Autism. 2015 Jan;19(1):73-83. doi: 10.1177/1362361313511709. PMID: 24254638.X-3, X-4

4340. Valdez C, Scroggs R, Chassen R, et al. Variation in Dube3a expression affects neurotransmission at the Drosophila neuromuscular junction. Biol Open. 2015;4(7):776-82. doi: 10.1242/bio.20148045. PMID: 25948754.X-1

4341. van Asselt-Goverts AE, Embregts PJ, Hendriks AH, et al. Do social networks differ? Comparison of the social networks of people with intellectual disabilities, people with autism spectrum disorders and other people living in the community. J Autism Dev Disord. 2015 May;45(5):1191-203. doi: 10.1007/s10803-0142279-3. PMID: 25326258.X-1

4342. van Bennekom MJ, Blom RM, Vulink $\mathrm{N}$, et al. A case of digital hoarding. BMJ Case Rep. 2015;2015doi: 10.1136/bcr-2015-210814. PMID: 26452411.X-1
4343. van Bon BW, Coe BP, Bernier R, et al. Disruptive de novo mutations of DYRK1A lead to a syndromic form of autism and ID. Mol Psychiatry. 2015 Feb 24doi: 10.1038/mp.2015.5. PMID: 25707398.X-1

4344. Van der Hallen R, Chamberlain R, deWit L, et al. The Leuven Embedded Figures Test (L-EFT): Re-embedding the EFT into vision sciences. J Vis. 2015 Sep 1;15(12):336. doi: 10.1167/15.12.336. PMID: 26326024.X-1

4345. van der Meer JM, Hartman CA, Thissen AJ, et al. How 'core' are motor timing difficulties in ADHD? A latent class comparison of pure and comorbid ADHD classes. Eur Child Adolesc Psychiatry. 2015 Jul 8doi: 10.1007/s00787-015-0734-0. PMID: 26154019.X-1

4346. Van der Paelt S, Warreyn P, Roeyers H. Effect of community interventions on socialcommunicative abilities of preschoolers with autism spectrum disorder. Dev Neurorehabil. 2015 Aug 24:1-13. doi:

10.3109/17518423.2014.933983. PMID: 25029102.X-1

4347. Van Eylen L, Boets B, Steyaert J, et al. Executive functioning in autism spectrum disorders: influence of task and sample characteristics and relation to symptom severity. Eur Child Adolesc Psychiatry. 2015 Feb 20doi: 10.1007/s00787-015-0689-1. PMID: 25697266.X-1

4348. Van Hecke AV, Stevens S, Carson AM, et al. Measuring the plasticity of social approach: a randomized controlled trial of the effects of the PEERS intervention on EEG asymmetry in adolescents with autism spectrum disorders. J Autism Dev Disord. 2015 Feb;45(2):316-35. doi: 10.1007/s10803-0131883-y. PMID: 23812665.X-1

4349. van Iterson L, de Jong PF, Zijlstra BJ. Pediatric epilepsy and comorbid reading disorders, math disorders, or autism spectrum disorders: Impact of epilepsy on cognitive patterns. Epilepsy Behav. 2015 Mar;44:159-68. doi: 10.1016/j.yebeh.2015.02.007. PMID: 25723912.X-1

4350. Van Naarden Braun K, Christensen D, Doernberg $\mathrm{N}$, et al. Trends in the prevalence of autism spectrum disorder, cerebral palsy, 
hearing loss, intellectual disability, and vision impairment, metropolitan atlanta, 1991-2010. PLoS One. 2015;10(4):e0124120. doi: 10.1371/journal.pone.0124120. PMID: 25923140.X-1

4351. van Rijn S. Social Attention in 47,XXY (Klinefelter Syndrome): Visual Scanning of Facial Expressions Using Eyetracking. J Int Neuropsychol Soc. 2015 May;21(5):364-72. doi: 10.1017/s1355617715000302. PMID: 26018944.X-1

4352. van Steensel FJ, Bogels SM. CBT for anxiety disorders in children with and without autism spectrum disorders. J Consult Clin Psychol. 2015 Jun;83(3):512-23. doi: 10.1037/a0039108. PMID: 25894668.X-1

4353. van Tongerloo MA, van Wijngaarden PJ, van der Gaag RJ, et al. Raising a child with an Autism Spectrum Disorder: 'If this were a partner relationship, I would have quit ages ago'. Fam Pract. 2015 Feb;32(1):88-93. doi: 10.1093/fampra/cmu076. PMID: 25425636.X-1

4354. VanDam M, Oller DK, Ambrose SE, et al. Automated Vocal Analysis of Children With Hearing Loss and Their Typical and Atypical Peers. Ear Hear. 2015 JulAug;36(4):e146-52. doi: 10.1097/aud.0000000000000138. PMID: 25587667.X-1

4355. VanderLaan DP, Postema L, Wood H, et al. Do children with gender dysphoria have intense/obsessional interests? J Sex Res. 2015;52(2):213-9. doi: 10.1080/00224499.2013.860073. PMID: 24558954.X-1

4356. Vandermeer J, Beamish W, Milford T, et al. iPad-presented social stories for young children with autism. Dev Neurorehabil. 2015 Apr;18(2):75-81. doi: 10.3109/17518423.2013.809811. PMID: 23815083.X-3, X-4

4357. Vanmarcke S, Van Der Hallen R, Evers K, et al. Ultra-Rapid Categorization of Meaningful Real-Life Scenes in Adults With and Without ASD. J Autism Dev Disord. 2015 Sep 9doi: 10.1007/s10803-015-2583-6. PMID: 26349920.X-1
4358. Vasconcellos LF, Pereira JS. Parkinson's disease dementia: Diagnostic criteria and risk factor review. J Clin Exp Neuropsychol. 2015 Nov;37(9):988-93. doi: 10.1080/13803395.2015.1073227. PMID: 26332178.X-1

4359. Veale D, Miles S, Read J, et al. Penile Dysmorphic Disorder: Development of a Screening Scale. Arch Sex Behav. 2015 Mar 3doi: 10.1007/s10508-015-0484-6. PMID: 25731908.X-1

4360. Veatch OJ, Goldman SE, Adkins KW, et al. Melatonin in Children with Autism Spectrum Disorders: How Does the Evidence Fit Together? J Nat Sci. 2015;1(7):e125. PMID: 26120597.X-1

4361. Veatch OJ, Maxwell-Horn AC, Malow BA. Sleep in Autism Spectrum Disorders. Curr Sleep Med Rep. 2015 Jun;1(2):131-40. doi: 10.1007/s40675-015-0012-1. PMID: 26046012.X-1

4362. Vedora J, Grandelski K. A comparison of methods for teaching receptive language to toddlers with autism. J Appl Behav Anal. 2015 Spring;48(1):188-93. doi: 10.1002/jaba.167. PMID: 25303362.X-1, X-3

4363. Ventola P, Yang DY, Friedman HE, et al. Heterogeneity of neural mechanisms of response to pivotal response treatment. Brain Imaging Behav. 2015 Mar;9(1):74-88. doi: 10.1007/s11682-014-9331-y. PMID: 25370452.X-1

4364. Verhoeff B. Two kinds of autism: a comparison of distinct understandings of psychiatric disease. Med Health Care Philos. 2015 Jun 28doi: 10.1007/s11019-015-9655-4. PMID: 26122535.X-1

4365. Ververi A, Vargiami E, Papadopoulou V, et al. Brainstem Auditory Evoked Potentials in Boys with Autism: Still Searching for the Hidden Truth. Iran J Child Neurol. 2015 Spring;9(2):21-8. PMID: 26221159.X-1

4366. Vesperini S, Askenazy F, Renaudo N, et al. [Prosocial intervention in children and adolescents with autism spectrum disorder without intellectual disability: benefits of videotaping therapy sessions]. Encephale. 2015 Feb;41(1):47-55. doi: 
10.1016/j.encep.2013.04.015. PMID: 25439857.X-3, X-4

4367. Viaggi C, Gerace C, Pardini C, et al. Serotonin abnormalities in Engrailed-2 knockout mice: New insight relevant for a model of Autism Spectrum Disorder. Neurochem Int. 2015 Aug;87:34-42. doi: 10.1016/j.neuint.2015.05.004. PMID: 26002543.X-1

4368. Viggiano D, Ianiro G, Vanella G, et al. Gut barrier in health and disease: focus on childhood. Eur Rev Med Pharmacol Sci. 2015;19(6):1077-85. PMID: 25855935.X-1

4369. Vinet E, Pineau CA, Clarke AE, et al. Increased Risk of Autism Spectrum Disorders in Children Born to Women with Systemic Lupus Erythematosus: Results from the OSLER Cohort. Arthritis Rheumatol. 2015 Aug 28doi: 10.1002/art.39320. PMID: 26315754.X-1

4370. Virk J, Liew Z, Olsen J, et al. Preconceptional and prenatal supplementary folic acid and multivitamin intake and autism spectrum disorders. Autism. 2015 Sep 25doi: 10.1177/1362361315604076. PMID: 26408631.X-1

4371. Visser K, Greaves-Lord K, Tick NT, et al. Study protocol: a randomized controlled trial investigating the effects of a psychosexual training program for adolescents with autism spectrum disorder. BMC Psychiatry. 2015;15(1):207. doi: 10.1186/s12888-0150586-7. PMID: 26310577.X-1

4372. Vitiello B, Lazzaretto D, Yershova K, et al. Pharmacotherapy of the Preschool ADHD Treatment Study (PATS) Children Growing Up. J Am Acad Child Adolesc Psychiatry. 2015 Jul;54(7):550-6. doi: 10.1016/j.jaac.2015.04.004. PMID: 26088659.X-1

4373. Vogt D, Cho KK, Lee AT, et al. The parvalbumin/somatostatin ratio is increased in Pten mutant mice and by human PTEN ASD alleles. Cell Rep. 2015 May 12;11(6):944-56. doi: 10.1016/j.celrep.2015.04.019. PMID: 25937288.X-1

4374. von Gontard A, Pirrung M, Niemczyk $\mathrm{J}$, et al. Incontinence in children with autism spectrum disorder. J Pediatr Urol. 2015
Oct;11(5):264.e1-.e7. doi:

10.1016/j.jpurol.2015.04.015. PMID: 26052001.X-1

4375. von Stulpnagel C, Funke C, Haberl C, et al. SYNGAP1 Mutation in Focal and Generalized Epilepsy: A Literature Overview and A Case Report with Special Aspects of the EEG. Neuropediatrics. 2015 Aug;46(4):287-91. doi: 10.1055/s-0035-1554098. PMID:

26110312.X-1

4376. Vonck S, Swinnen SP, Wenderoth N, et al. Effects of Transcranial Direct Current Stimulation on the Recognition of Bodily Emotions from Point-Light Displays. Front Hum Neurosci. 2015;9:438. doi: 10.3389/fnhum.2015.00438. PMID: 26283952.X-1

4377. Wachtel L, Commins E, Park M, et al. Neuroleptic malignant syndrome and delirious mania as malignant catatonia in autism: prompt relief with electroconvulsive therapy. Acta Psychiatr Scand. 2015 Oct;132(4):319-20. doi: 10.1111/acps.12441. PMID: 26018851.X-1, X2

4378. Wainer AL, Ingersoll BR. Increasing Access to an ASD Imitation Intervention Via a Telehealth Parent Training Program. J Autism Dev Disord. 2015 Dec;45(12):3877-90. doi: 10.1007/s10803-014-2186-7. PMID: 25035089.X-2, X-3

4379. Wakeford S, Hinvest N, Ring H, et al. Autistic characteristics in adults with epilepsy and perceived seizure activity. Epilepsy Behav. 2015 Oct 13;52(Pt A):244-50. doi: 10.1016/j.yebeh.2015.08.031. PMID: 26474368.X-1

4380. Wallace GL, Eisenberg IW, Robustelli $\mathrm{B}$, et al. Longitudinal cortical development during adolescence and young adulthood in autism spectrum disorder: increased cortical thinning but comparable surface area changes. J Am Acad Child Adolesc Psychiatry. 2015 Jun;54(6):464-9. doi: 10.1016/j.jaac.2015.03.007. PMID: 26004661.X-1

4381. Walsh JA, Vida MD, Morrisey MN, et al. Adults with autism spectrum disorder show evidence of figural aftereffects with male and female faces. Vision Res. 2015 Oct;115(Pt 
A):104-12. doi: 10.1016/j.visres.2015.08.010. PMID: 26322382.X-1

4382. Walton KM, Ingersoll BR. The influence of maternal language responsiveness on the expressive speech production of children with autism spectrum disorders: a microanalysis of mother-child play interactions. Autism. 2015 May;19(4):421-32. doi: 10.1177/1362361314523144. PMID: 24566717.X-1, X-3

4383. Walton KM, Ingersoll BR. Psychosocial Adjustment and Sibling Relationships in Siblings of Children with Autism Spectrum Disorder: Risk and Protective Factors. J Autism Dev Disord. 2015 Sep;45(9):2764-78. doi: 10.1007/s10803-0152440-7. PMID: 25847756.X-1

4384. Waltz MM. Mothers and autism: the evolution of a discourse of blame. AMA J Ethics. 2015 Apr;17(4):353-8. doi: 10.1001/journalofethics.2015.17.4.mhst1-1504. PMID: 25901704.X-1

4385. Wang C, Shimojo E, Shimojo S. Don't look at the eyes: Live interaction reveals strong eye avoidance behavior in autism. J Vis. 2015

Sep 1;15(12):648. doi: 10.1167/15.12.648. PMID: 26326336.X-1

4386. Wang G, Liu Z, Xu G, et al. Sleep Disturbances and Associated Factors in Chinese Children with Autism Spectrum Disorder: A Retrospective and Cross-Sectional Study. Child Psychiatry Hum Dev. 2015 Jun 14doi: 10.1007/s10578-015-0561-z. PMID: 26072264.X-1

4387. Wang H, Doering LC. Autism spectrum disorders: emerging mechanisms and mechanism-based treatment. Front Cell Neurosci. 2015;9:183. doi: 10.3389/fncel.2015.00183. PMID: 26029053.X-1, X-2, X-3

4388. Wang KS, Tonarelli S, Luo X, et al. Polymorphisms within ASTN2 gene are associated with age at onset of Alzheimer's disease. J Neural Transm (Vienna). 2015 May;122(5):701-8. doi: 10.1007/s00702-0141306-z. PMID: 25410587.X-1

4389. Wang L, Almeida LE, Spornick NA, et al. Modulation of social deficits and repetitive behaviors in a mouse model of autism: the role of the nicotinic cholinergic system. Psychopharmacology (Berl). 2015 Dec;232(23):4303-16. doi: 10.1007/s00213015-4058-z. PMID: 26337613.X-1

4390. Wang M, Chen H, Yu T, et al. Increased serum levels of brain-derived neurotrophic factor in autism spectrum disorder. Neuroreport. 2015 Aug 5;26(11):638-41. doi: 10.1097/wnr.0000000000000404. PMID: 26103118.X-1

4391. Wang T, de Kok L, Willemsen R, et al. In vivo synaptic transmission and morphology in mouse models of Tuberous sclerosis, Fragile $\mathrm{X}$ syndrome, Neurofibromatosis type 1 , and Costello syndrome. Front Cell Neurosci. 2015;9:234. doi: 10.3389/fncel.2015.00234. PMID: 26190969.X-1

4392. Wang Z, Magnon GC, White SP, et al. Individuals with autism spectrum disorder show abnormalities during initial and subsequent phases of precision gripping. J Neurophysiol. 2015 Apr 1;113(7):1989-2001. doi: 10.1152/jn.00661.2014. PMID: 25552638.X-1

4393. Warfield ME, Crossman MK, Delahaye J, et al. Physician Perspectives on Providing Primary Medical Care to Adults with Autism Spectrum Disorders (ASD). J Autism Dev Disord. 2015 Jul;45(7):2209-17. doi: 10.1007/s10803-015-2386-9. PMID: 25724445.X-1

4394. Warnell F, George B, McConachie H, et al. Designing and recruiting to UK autism spectrum disorder research databases: do they include representative children with valid ASD diagnoses? BMJ Open. 2015;5(9):e008625. doi: 10.1136/bmjopen-2015-008625. PMID: 26341584.X-1

4395. Warren ZE, Zheng Z, Swanson AR, et al. Can Robotic Interaction Improve Joint Attention Skills? J Autism Dev Disord. 2015 Nov;45(11):3726-34. doi: 10.1007/s10803-0131918-4. PMID: 24014194.X-3, X-4

4396. Watanabe T, Kuroda M, Kuwabara H, et al. Clinical and neural effects of six-week administration of oxytocin on core symptoms of autism. Brain. 2015 Sep 3doi:

10.1093/brain/awv249. PMID: 26336909.X-1 
4397. Watling R, Hauer S. Effectiveness of Ayres Sensory Integration((R)) and SensoryBased Interventions for People With Autism Spectrum Disorder: A Systematic Review. Am J Occup Ther. 2015 SepOct;69(5):6905180030p1-p12. doi: 10.5014/ajot.2015.018051. PMID: 26356655.X-2

4398. Weaver LL. Effectiveness of Work, Activities of Daily Living, Education, and Sleep Interventions for People With Autism Spectrum Disorder: A Systematic Review. Am J Occup Ther. 2015 Sep-Oct;69(5):6905180020p1-p11. doi: 10.5014/ajot.2015.017962. PMID: 26356654.X-1, X-2

4399. Webb S, Bernier R, Henderson H, et al. Guidelines and Best Practices for Electrophysiological Data Collection, Analysis and Reporting in Autism. Journal of Autism \& Developmental Disorders. 2015;45(2):425-43 19p. doi: 10.1007/s10803-013-1916-6. PMID: 103751306. Language: English. Entry Date: 20150203. Revision Date: 20150710. Publication Type: Journal Article.X-1

4400. Weber J, Gutierrez A, Jr. A Treatment Package without Escape Extinction to Address Food Selectivity. J Vis Exp. 2015(102):e52898. doi: 10.3791/52898. PMID: 26325108.X-5

4401. Weeks S, Atlas A. Clinical audit for occupational therapy intervention for children with autism spectrum disorder: sampling steps and sample size calculation. BMC Res Notes. 2015;8:282. doi: 10.1186/s13104-015-1247-0. PMID: 26122044.X-1

4402. Wei D, Lee D, Cox CD, et al. Endocannabinoid signaling mediates oxytocindriven social reward. Proc Natl Acad Sci U S A. 2015 Nov 10;112(45):14084-9. doi: 10.1073/pnas.1509795112. PMID: 26504214.X-1

4403. Weimer K, Colloca L, Enck P. Placebo effects in psychiatry: mediators and moderators. Lancet Psychiatry. 2015 Mar 1;2(3):246-57. doi: 10.1016/s2215-0366(14)00092-3. PMID: 25815249.X-1, X-2

4404. Weir RK, Forghany R, Smith SE, et al. Preliminary evidence of neuropathology in nonhuman primates prenatally exposed to maternal immune activation. Brain Behav
Immun. 2015 Aug;48:139-46. doi: 10.1016/j.bbi.2015.03.009. PMID: 25816799.X-1

4405. Weismuller B, Thienel R, Youlden AM, et al. Psychophysiological Correlates of Developmental Changes in Healthy and Autistic Boys. J Autism Dev Disord. 2015 Jul;45(7):2168-75. doi: 10.1007/s10803-0152385-x. PMID: 25663626.X-1

4406. Weiss JA, Burnham Riosa P. Thriving in Youth with Autism Spectrum Disorder and Intellectual Disability. J Autism Dev Disord. 2015 Aug;45(8):2474-86. doi: 10.1007/s10803015-2412-y. PMID: 25772536.X-1

4407. Weiss JA, Tint A, Paquette-Smith M, et al. Perceived self-efficacy in parents of adolescents and adults with autism spectrum disorder. Autism. 2015 May 27doi: 10.1177/1362361315586292. PMID: 26019305.X-1

4408. Welch MG, Firestein MR, Austin J, et al. Family Nurture Intervention in the Neonatal Intensive Care Unit improves socialrelatedness, attention, and neurodevelopment of preterm infants at 18 months in a randomized controlled trial. J Child Psychol Psychiatry. 2015 Nov;56(11):1202-11. doi: 10.1111/jcpp.12405. PMID: 25763525.X-1

4409. Werling DM, Geschwind DH. Recurrence rates provide evidence for sexdifferential, familial genetic liability for autism spectrum disorders in multiplex families and twins. Mol Autism. 2015;6:27. doi: 10.1186/s13229-015-0004-5. PMID: 25973164.X-1

4410. West JD, Cheetham TD, Dane C, et al. Should radioiodine be the first-line treatment for paediatric Graves' disease? J Pediatr Endocrinol Metab. 2015 Jul;28(7-8):797-804. doi: 10.1515/jpem-2014-0176. PMID: 25719296.X-1

4411. West M, Targett P, Wehman P, et al. Separation from supported employment: a retrospective chart review study. Disabil Rehabil. 2015;37(12):1055-9. doi: 10.3109/09638288.2014.955133. PMID: 25162839.X-1 
4412. Weston B, Fogal B, Cook D, et al. An agent-based modeling framework for evaluating hypotheses on risks for developing autism: effects of the gut microbial environment. Med Hypotheses. 2015 Apr;84(4):395-401. doi: 10.1016/j.mehy.2015.01.027. PMID: 25670416.X-1

4413. Whipple CM, Gfeller K, Driscoll V, et al. Do communication disorders extend to musical messages? An answer from children with hearing loss or autism spectrum disorders. J Music Ther. 2015 Spring;52(1):78-116. doi: 10.1093/jmt/thu039. PMID: 25691513.X-1

4414. Whissell PD, Cajanding JD, Fogel N, et al. Comparative density of CCK- and PVGABA cells within the cortex and hippocampus. Front Neuroanat. 2015;9:124. doi: 10.3389/fnana.2015.00124. PMID: 26441554.X-1

4415. White SW, Maddox BB, Panneton RK. Fear of Negative Evaluation Influences Eye Gaze in Adolescents with Autism Spectrum Disorder: A Pilot Study. J Autism Dev Disord. 2015 Nov;45(11):3446-57. doi: 10.1007/s10803-014-2349-6. PMID: 25578337.X-1

4416. White SW, Schry AR, Miyazaki Y, et al. Effects of Verbal Ability and Severity of Autism on Anxiety in Adolescents With ASD: One-Year Follow-Up After Cognitive Behavioral Therapy. J Clin Child Adolesc Psychol. 2015;44(5):839-45. doi: 10.1080/15374416.2014.893515. PMID: 24731184.X-1

4417. Whiteley P. Nutritional management of (some) autism: a case for gluten- and caseinfree diets? Proc Nutr Soc. 2015 Aug;74(3):2027. doi: 10.1017/s0029665114001475. PMID: 25311313.X-2

4418. Whitney RV, Smith G. Emotional Disclosure Through Journal Writing: Telehealth Intervention for Maternal Stress and MotherChild Relationships. J Autism Dev Disord. 2015 Nov;45(11):3735-45. doi: 10.1007/s10803-014-2332-2. PMID: 25503483.X-1

4419. Whyte E, Elbich D, Behrmann M, et al. Altered functional connectivity in the core and extended face-processing network in adolescents with autism. J Vis. 2015 Sep 1;15(12):1209. doi: 10.1167/15.12.1209. PMID: 26326897.X-1

4420. Whyte EM, Nelson KE. Trajectories of pragmatic and nonliteral language development in children with autism spectrum disorders. J Commun Disord. 2015 Mar-Apr;54:2-14. doi: 10.1016/j.jcomdis.2015.01.001. PMID: 25638464.X-1

4421. Wiggins LD, Levy SE, Daniels J, et al. Autism Spectrum Disorder Symptoms Among Children Enrolled in the Study to Explore Early Development (SEED). J Autism Dev Disord. 2015 Oct;45(10):3183-94. doi: 10.1007/s10803015-2476-8. PMID: 26048040.X-1

4422. Wiggins LD, Reynolds A, Rice CE, et al. Using standardized diagnostic instruments to classify children with autism in the study to explore early development. J Autism Dev Disord. 2015 May;45(5):1271-80. doi: 10.1007/s10803-014-2287-3. PMID: 25348175.X-1

4423. Wijma RA, van der Nagel BC, Dierckx B, et al. Identification and quantification of the antipsychotics risperidone, aripiprazole, pipamperone and their major metabolites in plasma using ultra-high performance liquid chromatography-mass spectrometry. Biomed Chromatogr. 2015 Sep 7doi: 10.1002/bmc.3610. PMID: 26447610.X-1

4424. Wijnhoven LA, Creemers DH, Engels $\mathrm{RC}$, et al. The effect of the video game Mindlight on anxiety symptoms in children with an Autism Spectrum Disorder. BMC Psychiatry. 2015;15:138. doi: 10.1186/s12888015-0522-X. PMID: 26129831.X-1, X-2

4425. Wilkes BJ, T BC, Patel KP, et al. Oculomotor performance in children with highfunctioning Autism Spectrum Disorders. Res Dev Disabil. 2015 Mar;38:338-44. doi: 10.1016/j.ridd.2014.12.022. PMID: 25590171.X-1

4426. Williamson KE, Jakobson LS, Saunders DR, et al. Local and global aspects of biological motion perception in children born at very low birth weight. Child Neuropsychol.

2015 Sep;21(5):603-28. doi: 10.1080/09297049.2014.945407. PMID: 25103588.X-1 
4427. Willing J, Wagner CK. Progesterone Receptor Expression in the Developing Mesocortical Dopamine Pathway: Importance for Complex Cognitive Behavior in Adulthood. Neuroendocrinology. 2015 Jun 10doi: 10.1159/000434725. PMID: 26065828.X-1

4428. Winarni TI, Schneider A, Ghaziuddin $\mathrm{N}$, et al. Psychosis and catatonia in fragile $\mathrm{X}$ : Case report and literature review. Intractable Rare Dis Res. 2015 Aug;4(3):139-46. doi: 10.5582/irdr.2015.01028. PMID: 26361565.X-1

4429. Windham GC, Lyall K, Anderson M, et al. Autism Spectrum Disorder Risk in Relation to Maternal Mid-Pregnancy Serum Hormone and Protein Markers from Prenatal Screening in California. J Autism Dev Disord. 2015 Sep 14doi: 10.1007/s10803-015-2587-2. PMID: 26370672.X-1

4430. Wink LK, Fitzpatrick S, Shaffer R, et al. The neurobehavioral and molecular phenotype of Angelman Syndrome. Am J Med Genet A. 2015 Nov;167(11):2623-8. doi: 10.1002/ajmg.a.37254. PMID: 26219744.X-1

4431. Wink LK, Pedapati EV, Horn PS, et al. Multiple Antipsychotic Medication Use in Autism Spectrum Disorder. J Child Adolesc Psychopharmacol. 2015 Oct 14doi: 10.1089/cap.2015.0123. PMID: 26465194.X-1

4432. Wohr M. Effect of social odor context on the emission of isolation-induced ultrasonic vocalizations in the BTBR $\mathrm{T}+\mathrm{tf} / \mathrm{J}$ mouse model for autism. Front Neurosci. 2015;9:73. doi: 10.3389/fnins.2015.00073. PMID: 25852455.X-1

4433. Wolfberg P, DeWitt M, Young GS, et al. Integrated play groups: promoting symbolic play and social engagement with typical peers in children with ASD across settings. J Autism Dev Disord. 2015 Mar;45(3):830-45. doi: 10.1007/s10803-014-2245-0. PMID: 25231289.X-1, X-3

4434. Wolfe FH, Auzias G, Deruelle C, et al. Focal atrophy of the hypothalamus associated with third ventricle enlargement in autism spectrum disorder. Neuroreport. 2015 Oct 6doi: 10.1097/wnr.0000000000000461. PMID: 26445284.X-1
4435. Wolff AR, Bilkey DK. Prenatal immune activation alters hippocampal place cell firing characteristics in adult animals. Brain Behav Immun. 2015 Aug;48:232-43. doi: 10.1016/j.bbi.2015.03.012. PMID: 25843370.X-1

4436. Wong C, Odom SL, Hume KA, et al. Evidence-Based Practices for Children, Youth, and Young Adults with Autism Spectrum Disorder: A Comprehensive Review. J Autism Dev Disord. 2015 Jul;45(7):1951-66. doi: 10.1007/s10803-014-2351-z. PMID: 25578338.X-2

4437. Wong PH, White KM. Impact of Immunoglobulin Therapy in Pediatric Disease: a Review of Immune Mechanisms. Clin Rev Allergy Immunol. 2015 Jul 4doi: 10.1007/s12016-015-8499-2. PMID: 26142065.X-1, X-2

4438. Wong VC, Fung CW, Kwong AK. SCN2A mutation in a Chinese boy with infantile spasm - response to Modified Atkins Diet. Brain Dev. 2015 Aug;37(7):729-32. doi: 10.1016/j.braindev.2014.10.008. PMID: 25459969.X-1

4439. Wong VC, Fung CW, Lee SL, et al. Review of evolution of clinical, training and educational services and research program for autism spectrum disorders in Hong Kong. Sci China Life Sci. 2015 Oct;58(10):991-1009. doi: 10.1007/s11427-012-4294-y. PMID: 26335731.X-1, X-2

4440. Wong VC, Kwong AK. CDKL5 variant in a boy with infantile epileptic encephalopathy: case report. Brain Dev. 2015 Apr;37(4):446-8. doi: 10.1016/j.braindev.2014.07.003. PMID: 25085838.X-1

4441. Woo CC, Donnelly JH, SteinbergEpstein R, et al. Environmental enrichment as a therapy for autism: A clinical trial replication and extension. Behav Neurosci. 2015 Aug;129(4):412-22. doi: 10.1037/bne0000068. PMID: 26052790. INCLUDE

4442. Wood AG, Nadebaum C, Anderson V, et al. Prospective assessment of autism traits in children exposed to antiepileptic drugs during pregnancy. Epilepsia. 2015 Jul;56(7):1047-55. doi: 10.1111/epi.13007. PMID: 25963613.X-1 
4443. Wood JJ, Ehrenreich-May J, Alessandri M, et al. Cognitive behavioral therapy for early adolescents with autism spectrum disorders and clinical anxiety: a randomized, controlled trial. Behav Ther. 2015 Jan;46(1):7-19. doi:

10.1016/j.beth.2014.01.002. PMID: 25526831.X-1

4444. Worley G, Crissman BG, Cadogan E, et al. Down Syndrome Disintegrative Disorder: New-Onset Autistic Regression, Dementia, and Insomnia in Older Children and Adolescents With Down Syndrome. J Child Neurol. 2015 Aug;30(9):1147-52. doi: 10.1177/0883073814554654. PMID: 25367918.X-1

4445. Wright HF, Hall S, Hames A, et al. Acquiring a Pet Dog Significantly Reduces Stress of Primary Carers for Children with Autism Spectrum Disorder: A Prospective Case Control Study. J Autism Dev Disord. 2015 Aug;45(8):2531-40. doi: 10.1007/s10803-0152418-5. PMID: 25832799.X-1

4446. Ws A, Zwaigenbaum L, Nicholas D, et al. Factors influencing autism spectrum disorder screening by community paediatricians.

Paediatr Child Health. 2015 Jun-Jul;20(5):e20-

4. PMID: 26175565.X-1

4447. Wu J, de Theije CG, da Silva SL, et al. mTOR plays an important role in cow's milk allergy-associated behavioral and immunological deficits. Neuropharmacology. 2015 Oct;97:220-32. doi: 10.1016/j.neuropharm.2015.04.035. PMID: 26027949.X-1

4448. Wunderlich KL, Vollmer TR. Data analysis of response interruption and redirection as a treatment for vocal stereotypy. J Appl Behav Anal. 2015 Jul 14doi: 10.1002/jaba.227. PMID: 26173813.X-3, X-4

4449. Wynter K, Hammarberg K, Sartore GM, et al. Brief online surveys to monitor and evaluate facilitated peer support groups for caregivers of children with special needs. Eval Program Plann. 2015 Apr;49:70-5. doi: 10.1016/j.evalprogplan.2014.12.019. PMID: 25559709.X-1
4450. Xiang J, Korostenskaja M, Molloy C, et al. Multi-frequency localization of aberrant brain activity in autism spectrum disorder. Brain Dev. 2015 Apr 27doi: 10.1016/j.braindev.2015.04.007. PMID: 25937458.X-1

4451. Xie B, Agam G, Balasubramanian S, et al. Disease gene prioritization using network and feature. J Comput Biol. 2015

Apr;22(4):313-23. doi: 10.1089/cmb.2015.0001. PMID: 25844670.X-1

4452. Xu H, Wang Y, He Z, et al. Direct conversion of mouse fibroblasts to GABAergic neurons with combined medium without the introduction of transcription factors or miRNAs. Cell Cycle. 2015 Aug 3;14(15):245160. doi: 10.1080/15384101.2015.1060382. PMID: 26114472.X-1

4453. Xu L, Ma X, Zhao W, et al. Oxytocin enhances attentional bias for neutral and positive expression faces in individuals with higher autistic traits.

Psychoneuroendocrinology. 2015 Sep 5;62:3528. doi: 10.1016/j.psyneuen.2015.09.002. PMID: 26372768.X-1

4454. Yakubova G, Hughes EM, Hornberger E. Video-Based Intervention in Teaching Fraction Problem-Solving to Students with Autism Spectrum Disorder. J Autism Dev Disord. 2015 Sep;45(9):2865-75. doi: 10.1007/s10803-015-2449-y. PMID: 25911304.X-1, X-3, X-4

4455. Yan CL, Zhang J, Hou Y. Decreased plasma levels of lipoxin A4 in children with autism spectrum disorders. Neuroreport. 2015 Apr 15;26(6):341-5. doi: 10.1097/wnr.0000000000000350. PMID: 25714424.X-1

4456. Yang C, Hao Z, Zhang LL, et al. Efficacy and safety of acupuncture in children: an overview of systematic reviews. Pediatr Res. 2015 Aug;78(2):112-9. doi: 10.1038/pr.2015.91. PMID: 25950453.X-2, X-3

4457. Yang CJ, Liu CL, Sang B, et al. The combined role of serotonin and interleukin- 6 as biomarker for autism. Neuroscience. 2015 Jan 22;284:290-6. doi:

10.1016/j.neuroscience.2014.10.011. PMID: 25453766.X-1 
4458. Yang H, Lu X, He H, et al. Longer plate-to-disc distance prevents adjacent-level ossification development but does not influence adjacent-segment degeneration. Spine (Phila $\mathrm{Pa}$ 1976). 2015 Apr 1;40(7):E388-93. doi: 10.1097/brs.0000000000000800. PMID: 25627288.X-1

4459. Yang J, Hofmann J. Action observation and imitation in autism spectrum disorders: an ALE meta-analysis of fMRI studies. Brain Imaging Behav. 2015 Sep 24doi: 10.1007/s11682-015-9456-7. PMID: 26404019.X-1

4460. Yang M, Lewis F, Foley G, et al. In tribute to Bob Blanchard: Divergent behavioral phenotypes of 16p11.2 deletion mice reared in same-genotype versus mixed-genotype cages. Physiol Behav. 2015 Jul 1;146:16-27. doi: 10.1016/j.physbeh.2015.04.023. PMID: 26066718.X-1

4461. Yang M, Mahrt EJ, Lewis F, et al. 16p11.2 Deletion Syndrome Mice Display Sensory and Ultrasonic Vocalization Deficits During Social Interactions. Autism Res. 2015 Oct;8(5):507-21. doi: 10.1002/aur.1465. PMID: 25663600.X-1

4462. Yang Z, Matsumoto A, Nakayama K, et al. Circadian-relevant genes are highly polymorphic in autism spectrum disorder patients. Brain Dev. 2015 May 6doi: 10.1016/j.braindev.2015.04.006. PMID: 25957987.X-1

4463. Yerys BE, Antezana L, Weinblatt R, et al. Neural Correlates of Set-Shifting in Children With Autism. Autism Res. 2015 Aug;8(4):38697. doi: 10.1002/aur.1454. PMID: 25599972.X1

4464. Yeung MK, Han YM, Sze SL, et al. Abnormal Frontal Theta Oscillations Underlie the Cognitive Flexibility Deficits in Children With High-Functioning Autism Spectrum Disorders. Neuropsychology. 2015 Aug 10doi: 10.1037/neu0000231. PMID: 26375380.X-1

4465. Yirmiya N, Seidman I, Koren-Karie N, et al. Stability and change in resolution of diagnosis among parents of children with autism spectrum disorder: Child and parental contributions. Dev Psychopathol. 2015
Nov;27(4 Pt 1):1045-57. doi: 10.1017/s095457941500067x. PMID: 26439061.X-1

4466. Yoder P, Watson LR, Lambert W. Value-added predictors of expressive and receptive language growth in initially nonverbal preschoolers with autism spectrum disorders. J Autism Dev Disord. 2015 May;45(5):1254-70. doi: 10.1007/s10803-014-2286-4. PMID: 25344152.X-1

4467. Yokoyama S, Al Mahmuda N, Munesue T, et al. Association Study between the CD157/BST1 Gene and Autism Spectrum Disorders in a Japanese Population. Brain Sci. 2015;5(2):188-200. doi: 10.3390/brainsci5020188. PMID: 26010484.X1

4468. Young AS, Cooke MR, Taiclet LM. Management of patient with acrometageria for routine dental treatment: a case report. Spec Care Dentist. 2015 Aug 11doi: 10.1111/scd.12127. PMID: 26260731.X-1

4469. Young HE, Falco RA, Hanita M. Randomized, Controlled Trial of a Comprehensive Program for Young Students with Autism Spectrum Disorder. J Autism Dev Disord. 2015 Oct 5doi: 10.1007/s10803-0152597-0. PMID: 26438637.X-1

4470. Young LJ, Barrett CE. Neuroscience. Can oxytocin treat autism? Science. $2015 \mathrm{Feb}$ 20;347(6224):825-6. doi: 10.1126/science.aaa8120. PMID: 25700501.X1

4471. Young NJ, Findling RL. An update on pharmacotherapy for autism spectrum disorder in children and adolescents. Current Opinion in Psychiatry. 2015;28(2):91-101 11p. doi: 10.1097/YCO.0000000000000132. PMID: 109778580. Language: English. Entry Date: 20150821. Revision Date: 20150923. Publication Type: Journal Article.X-2

4472. Yu L, Fan Y, Deng Z, et al. Pitch Processing in Tonal-Language-Speaking Children with Autism: An Event-Related Potential Study. J Autism Dev Disord. 2015 Nov;45(11):3656-67. doi: 10.1007/s10803-0152510-x. PMID: 26111738.X-1 
4473. Yui K, Sato A, Imataka G.

Mitochondrial Dysfunction and Its Relationship with mTOR Signaling and Oxidative Damage in Autism Spectrum Disorders. Mini Rev Med Chem. 2015;15(5):373-89. PMID: 25910652.X-1

4474. Zablotsky B, Pringle BA, Colpe LJ, et al. Service and treatment use among children diagnosed with autism spectrum disorders. J Dev Behav Pediatr. 2015 Feb-Mar;36(2):98105. doi: 10.1097/dbp.0000000000000127. PMID: 25650952.X-1

4475. Zaidel A, Goin-Kochel RP, Angelaki DE. Self-motion perception in autism is compromised by visual noise but integrated optimally across multiple senses. PNAS Proceedings of the National Academy of Sciences of the United States of America. 2015 2016-06-23;112(20):6461-6. doi: 10.1007/s 10803-010-1000-4 http://dx.doi.org/10.1073/pnas.1506582112. PMID: 1799003583; 2016-20383-002.X-1

4476. Zalaquett DF, Schonstedt MG, Angeli $\mathrm{M}$, et al. [Basics of early intervention in children with autism spectrum disorders]. Rev Chil Pediatr. 2015 Mar-Apr;86(2):126-31. doi: 10.1016/j.rchipe.2015.04.025. PMID: 26235694.X-1, X-2

4477. Zamora I, Williams ME, Higareda M, et al. Brief Report: Recruitment and Retention of Minority Children for Autism Research. J Autism Dev Disord. 2015 Sep 24doi: 10.1007/s10803-015-2603-6. PMID: 26404703.X-1

4478. Zapf AC, Glindemann LA, Vogeley K, et al. Sex differences in mental rotation and how they add to the understanding of autism. PLoS One. 2015;10(4):e0124628. doi: 10.1371/journal.pone.0124628. PMID: 25884501.X-1

4479. Zeidler S, Hukema RK, Willemsen R. The quest for targeted therapy in fragile $\mathrm{X}$ syndrome. Expert Opin Ther Targets. 2015 Oct;19(10):1277-81. doi: 10.1517/14728222.2015.1079176. PMID: 26294013.X-1

4480. Zerbo O, Yoshida C, Gunderson EP, et al. Interpregnancy Interval and Risk of Autism Spectrum Disorders. Pediatrics. 2015
Oct;136(4):651-7. doi: 10.1542/peds.20151099. PMID: 26371204.X-1

4481. Zhan Y. Theta frequency prefrontalhippocampal driving relationship during free exploration in mice. Neuroscience. 2015 Aug 6;300:554-65. doi:

10.1016/j.neuroscience.2015.05.063. PMID: 26037805.X-1

4482. Zhang L, Tang J, Dong Y, et al. Similarities and Differences in DecisionMaking Impairments between Autism Spectrum Disorder and Schizophrenia. Front Behav Neurosci. 2015;9:259. doi: 10.3389/fnbeh.2015.00259. PMID: 26441583.X-1

4483. Zhang M, Reyhan M, Kim LH. Depth dose perturbation by a hydrogel fiducial marker in a proton beam. J Appl Clin Med Phys.

2015;16(1):5090. doi:

10.1120/jacmp.v16i1.5090. PMID: 25679167.X-1

4484. Zhang Y, Yang C, Yuan G, et al. Sulindac attenuates valproic acid-induced oxidative stress levels in primary cultured cortical neurons and ameliorates repetitive/stereotypic-like movement disorders in Wistar rats prenatally exposed to valproic acid. Int J Mol Med. 2015 Jan;35(1):263-70. doi: 10.3892/ijmm.2014.1996. PMID: 25384498.X-1

4485. Zhao G, Gao J, Liang S, et al. Study of the serum levels of polyunsaturated fatty acids and the expression of related liver metabolic enzymes in a rat valproate-induced autism model. Int J Dev Neurosci. 2015 Aug;44:14-21. doi: 10.1016/j.ijdevneu.2015.04.350. PMID: 25916973.X-1

4486. Zhao HX, Yin SS, Fan JG. High plasma neopterin levels in Chinese children with autism spectrum disorders. Int J Dev Neurosci. 2015 Apr;41:92-7. doi: 10.1016/j.ijdevneu.2015.02.002. PMID: 25660944.X-1

4487. Zhen B, Blackwell AF, Coulouris G. Using Augmented Reality to Elicit Pretend Play for Children with Autism. IEEE Trans Vis Comput Graph. 2015 May;21(5):598-610. doi: 10.1109/tvcg.2014.2385092. PMID: 26357207.X-1, X-3 
4488. Zheng Z, Young E, Swanson A, et al. Robot-mediated Imitation Skill Training for Children with Autism. IEEE Trans Neural Syst Rehabil Eng. 2015 Sep 3doi:

10.1109/tnsre.2015.2475724. PMID:

26353376.X-1, X-3

4489. Zhu H, Li J, Fan Y, et al. Atypical prefrontal cortical responses to joint/non-joint attention in children with autism spectrum disorder (ASD): A functional near-infrared spectroscopy study. Biomed Opt Express. 2015 Mar 1;6(3):690-701. doi:

10.1364/boe.6.000690. PMID: 25798296.X-1

4490. Zivoder I, Martic-Biocina S, Kosic AV, et al. Neurofeedback application in the treatment of autistic spectrum disorders (ASD). Psychiatr Danub. 2015 Sep;27 Suppl 1:391-4. PMID: 26417802.X-3, X-4

4491. Zobel-Lachiusa J, Andrianopoulos MV, Mailloux Z, et al. Sensory Differences and Mealtime Behavior in Children With Autism.

Am J Occup Ther. 2015 Sep-

Oct;69(5):6905185050p1-8. doi:

10.5014/ajot.2015.016790. PMID:

26379266.X-1

4492. Zuckerman KE, Lindly OJ, Sinche BK, et al. Parent health beliefs, social determinants of health, and child health services utilization among U.S. school-age children with autism. J Dev Behav Pediatr. 2015

Apr;36(3):146-57. doi: 10.1097/dbp.0000000000000136. PMID: 25741947.X-1

4493. Zwaigenbaum L, Bauman ML, Choueiri R, et al. Early Identification and Interventions for Autism Spectrum Disorder: Executive Summary. Pediatrics. 2015 Oct;136 Suppl 1:S1-9. doi: 10.1542/peds.2014-3667B. PMID: 26430167.X-1, X-2

4494. Zwaigenbaum L, Bauman ML, Choueiri R, et al. Early Intervention for Children With Autism Spectrum Disorder Under 3 Years of Age: Recommendations for Practice and Research. Pediatrics. 2015 Oct;136 Suppl 1:S60-81. doi: 10.1542/peds.20143667E. PMID: 26430170.X-1

4495. . Antidepressants in pregnancy and autism. Arch Dis Child. 2016 Mar;101(3):240. doi: 10.1136/archdischild-2016-310462. PMID: 26787608.X-1

4496. . Neurodevelopmental disorders: Epilepsy and autism spectrum disorders may have a shared aetiology. Nat Rev Neurol. 2016 Jul 1doi: 10.1038/nrneurol.2016.100. PMID: 27364744.X-1

4497. . A Prospective Open-Label Trial of Memantine Hydrochloride for the Treatment of Social Deficits in Intellectually Capable Adults With Autism Spectrum Disorder: Erratum. J Clin Psychopharmacol. 2016 Aug;36(4):339. doi: $10.1097 /$ jcp.0000000000000534. PMID: 27355795.X-1

4498. Aabbassi B, Benali A, Asri F. Risperidone-induced priapism in an autistic child: a case report. J Med Case Rep. 2016;10:164. doi: 10.1186/s13256-016-0956-X. PMID: 27266696.X-1, X-3, X-4

4499. Abdelkarim A, Levi DS, Tran B, et al. Fenestrated Transcatheter ASD Closure in Adults with Diastolic Dysfunction and/or Pulmonary Hypertension: Case Series and Review of the Literature. Congenit Heart Dis. 2016 Apr 29doi: 10.1111/chd.12367. PMID: 27125263.X-1

4500. Aberle B, Jemmali R, Dirks JH. Effect of sample treatment on biomechanical properties of insect cuticle. Arthropod Struct Dev. 2016 Aug 2doi: 10.1016/j.asd.2016.08.001. PMID: 27495946.X-1

4501. Abrams DA, Chen T, Odriozola P, et al. Neural circuits underlying mother's voice perception predict social communication abilities in children. Proc Natl Acad Sci U S A. 2016 May 31;113(22):6295-300. doi: 10.1073/pnas.1602948113. PMID: 27185915.X-1

4502. Abu-Akel A, Apperly IA, Wood SJ, et al. Autism Tendencies and Psychosis Proneness Interactively Modulate Saliency Cost. Schizophr Bull. 2016 May 23doi: 10.1093/schbul/sbw066. PMID: 27217269.X-1

4503. Abu-Akel AM, Apperly IA, Wood SJ, et al. Autism and psychosis expressions diametrically modulate the right temporoparietal junction. Soc Neurosci. 2016 
Jun 3:1-13. doi:

10.1080/17470919.2016.1190786. PMID:

27187170.X-1

4504. Acaroglu E, Yavuz AC, Guler UO, et al. A decision analysis to identify the ideal treatment for adult spinal deformity: is surgery better than non-surgical treatment in improving health-related quality of life and decreasing the disease burden? Eur Spine J. 2016 Jan 28doi: 10.1007/s00586-016-4413-8. PMID: 26821143.X-1

4505. Achuta VS, Grym H, Putkonen N, et al. Metabotropic glutamate receptor 5 responses dictate differentiation of neural progenitors to NMDA-responsive cells in fragile $\mathrm{X}$ syndrome. Dev Neurobiol. 2016 Jul 13doi: 10.1002/dneu.22419. PMID: 27411166.X-1

4506. Ackerlund Brandt JA, Weinkauf S, Zeug N, et al. An evaluation of constant time delay and simultaneous prompting procedures in skill acquisition for young children with autism. Education and Training in Autism and Developmental Disabilities. 2016;51(1):5566.X-1, X-3, X-4

4507. Adams DJ, Susi A, Erdie-Lalena CR, et al. Otitis media and related complications among children with autism spectrum disorders. Journal of Autism and Developmental Disorders. 2016;46(5):1636-42. doi: http://dx.doi.org/10.1007/s10803-015-2689-x.x1

4508. Adams ILJ, Ferguson GD, Lust JM, et al. Action planning and position sense in children with Developmental Coordination Disorder. Human Movement Science.

2016;46:196-208. doi:

http://dx.doi.org/10.1016/j.humov.2016.01.006. $\mathrm{X}-1$

4509. Adams ILJ, Steenbergen B, Lust JM, et al. Motor imagery training for children with developmental coordination disorder-Study protocol for a randomized controlled trial. BMC Neurology. 2016;16doi: http://dx.doi.org/10.1186/s12883-016-05306.X-1

4510. Aggarwala V, Voight BF. An expanded sequence context model broadly explains variability in polymorphism levels across the human genome. Nat Genet. 2016
Apr;48(4):349-55. doi: 10.1038/ng.3511. PMID: 26878723.X-1

4511. Agius MM, Vance M. A Comparison of PECS and iPad to Teach Requesting to Preschoolers with Autistic Spectrum Disorders. Augment Altern Commun. 2016;32(1):58-68. doi: 10.3109/07434618.2015.1108363. PMID: 26586580.X-1, X-3, X-4

4512. Aguilar JM, White PJ, Fragale C, et al. Preference for language of instruction of an English language learner with autism. Developmental Neurorehabilitation. 2016;19(3):207-10.X-1, X-3, X-4

4513. Ahlgrim-Delzell L, Browder DM, Wood L, et al. Systematic instruction of phonics skills using an iPad for students with developmental disabilities who are AAC users. The Journal of Special Education.

2016;50(2):86-97. doi: http://dx.doi.org/10.1177/0022466915622140.X $-1, \mathrm{X}-4$

4514. Ahmad SF, Zoheir KM, Ansari MA, et al. Dysregulation of Th1, Th2, Th17, and T regulatory cell-related transcription factor signaling in children with autism. Mol Neurobiol. 2016 Jun 25doi: 10.1007/s12035016-9977-0. PMID: 27344332.X-1

4515. Ahn S, Kim TG, Kim KS, et al. Differentiation of human pluripotent stem cells into Medial Ganglionic Eminence vs. Caudal Ganglionic Eminence cells. Methods. 2016 May 15;101:103-12. doi: 10.1016/j.ymeth.2015.09.009. PMID: 26364591.X-1

4516. Ainsworth MK, Evmenova AS, Behrmann M, et al. Teaching phonics to groups of middle school students with autism, intellectual disabilities and complex communication needs. Res Dev Disabil. 2016 Sep;56:165-76. doi: 10.1016/j.ridd.2016.06.001. PMID: 27318612.X-1

4517. Akcakaya NH, Tekturk P, Cagatay A, et al. Atypical enterovirus encephalitis causing behavioral changes and autism-like clinical manifestations: case report. Acta Neurol Belg. 2016 Feb 19doi: 10.1007/s13760-016-0614-5. PMID: 26895551.X-1 
4518. Akers JS, Higbee TS, Pollard JS, et al. An evaluation of photographic activity schedules to increase independent playground skills in young children with autism. J Appl Behav Anal. 2016 Jun 10doi: 10.1002/jaba.327. PMID: 27283759.X-1

4519. Akiyama T, Osaka H, Shimbo H, et al. SSADH deficiency possibly associated with enzyme activity-reducing SNPs. Brain Dev. 2016 Apr 4doi: 10.1016/j.braindev.2016.03.008. PMID: 27056292.X-1

4520. Akula VS, Durgaprasad R, Velam V, et al. Right Ventricle before and after Atrial Septal Defect Device Closure.

Echocardiography. 2016 Apr 24doi: 10.1111/echo.13250. PMID: 27109837.X-1

4521. Alba G, Pereda E, Mañas S, et al. The variability of EEG functional connectivity of young ADHD subjects in different resting states. Clinical Neurophysiology.

2016;127(2):1321-30. doi: http://dx.doi.org/10.1016/j.clinph.2015.09.134. $\mathrm{X}-1$

4522. Aldaqre I, Schuwerk T, Daum MM, et al. Sensitivity to communicative and noncommunicative gestures in adolescents and adults with autism spectrum disorder: saccadic and pupillary responses. Exp Brain Res. 2016 Apr 27doi: 10.1007/s00221-016-4656-y. PMID: 27119361.X-1

4523. Alentado VJ, Lubelski D, Healy AT, et al. Predisposing Characteristics of Adjacent Segment Disease Following Lumbar Fusion. Spine (Phila Pa 1976). 2016 Feb 6doi: 10.1097/brs.0000000000001493. PMID: 26863261.X-1

4524. Ali MA, Tazmin T, Latif T, et al. Incomplete Pentalogy of Cantrell--A Case Report. Mymensingh Med J. 2016 Jan;25(1):153-7. PMID: 26931266.X-1

4525. Aliaga SM, Slater HR, Francis D, et al. Identification of Males with Cryptic Fragile X Alleles by Methylation-Specific Quantitative Melt Analysis. Clin Chem. 2016 Feb;62(2):34352. doi: 10.1373/clinchem.2015.244681. PMID: 26715660.X-1
4526. Allard MJ, Bergeron JD, Baharnoori $\mathrm{M}$, et al. A sexually dichotomous, autistic-like phenotype is induced by Group B Streptococcus maternofetal immune activation. Autism Res. 2016 May 25doi: 10.1002/aur.1647. PMID: 27220806.X-1

4527. Allely CS, Wilson P, Minnis H, et al. Violence is Rare in Autism: When It Does Occur, Is It Sometimes Extreme? J Psychol. 2016 May 16:1-20. doi: 10.1080/00223980.2016.1175998. PMID: 27185105.X-1

4528. Allen JL, Morris A, Chhoa CY. Callous-unemotional (CU) traits in adolescent boys and response to teacher reward and discipline strategies. Emotional \& Behavioural Difficulties. 2016;21(3):329-42. doi: http://dx.doi.org/10.1080/13632752.2016.11659 68.X-1

4529. Allen ML, Craig E. Brief report: Imaginative drawing in children with autism spectrum disorder and learning disabilities. Journal of Autism and Developmental Disorders. 2016;46(2):704-12. doi: http://dx.doi.org/10.1007/s10803-015-2599y.X-1

4530. Allin MP. Novel insights from quantitative imaging of the developing cerebellum. Semin Fetal Neonatal Med. 2016 Jun 26doi: 10.1016/j.siny.2016.06.003. PMID: 27357079.X-1

4531. Allman MJ, Mareschal D. Possible evolutionary and developmental mechanisms of mental time travel (and implications for autism). Curr Opin Behav Sci. 2016 Apr;8:2205. doi: 10.1016/j.cobeha.2016.02.018. PMID: 27019863.X-1

4532. Al-Mendalawi MD. Prevalence of autism spectrum disorders among children (110 years of age): Findings of a midterm report from Northwest India. J Postgrad Med. 2016 Jan-Mar;62(1):52-3. doi: 10.4103/00223859.173219. PMID: 26732201.X-1

4533. Almirall D, DiStefano C, Chang YC, et al. Longitudinal Effects of Adaptive Interventions With a Speech-Generating Device in Minimally Verbal Children With ASD. J Clin Child Adolesc Psychol. 2016 JulAug;45(4):442-56. doi: 
10.1080/15374416.2016.1138407. PMID: 26954267.X-1

4534. Alrayes N, Mohamoud HSA, Ahmed $\mathrm{S}$, et al. The alkylglycerol monooxygenase (AGMO) gene previously involved in autism also causes a novel syndromic form of primary microcephaly in a consanguineous Saudi family. Journal of the Neurological Sciences. 2016;363:240-4. doi: http://dx.doi.org/10.1016/j.jns.2016.02.063.X-1

4535. Alsop B, Furukawa E, Sowerby P, et al. Behavioral sensitivity to changing reinforcement contingencies in attention-deficit hyperactivity disorder. Journal of Child Psychology and Psychiatry. 2016;57(8):947-56. doi: http://dx.doi.org/10.1111/jcpp.12561.X-1

4536. Althaus M, Groen Y, A AW, et al. Do blood plasma levels of oxytocin moderate the effect of nasally administered oxytocin on social orienting in high-functioning male adults with autism spectrum disorder?

Psychopharmacology (Berl). 2016 Jul;233(14):2737-51. doi: 10.1007/s00213-0164339-1. PMID: 27256356.X-1

4537. Altieri N, Yang CT. Parallel linear dynamic models can mimic the McGurk effect in clinical populations. J Comput Neurosci. 2016 Jun 7doi: 10.1007/s10827-016-0610-z. PMID: 27272510.X-1

4538. Alvares GA, Balleine BW, Whittle L, et al. Reduced goal-directed action control in autism spectrum disorder. Autism Res. 2016 Mar 21doi: 10.1002/aur.1613. PMID: 26999793.X-1

4539. Alvarez-Mora MI, Calvo Escalona R, Puig Navarro O, et al. Comprehensive molecular testing in patients with high functioning autism spectrum disorder. Mutat Res. 2016 Feb-Mar;784-785:46-52. doi: 10.1016/j.mrfmmm.2015.12.006. PMID: 26845707.X-1

4540. Alwan S, Friedman JM, Chambers C. Safety of Selective Serotonin Reuptake Inhibitors in Pregnancy: A Review of Current Evidence. CNS Drugs. 2016 Jun;30(6):499-515. doi: 10.1007/s40263-016-0338-3. PMID: 27138915.X-1
4541. Amaral F, Balthazar Jacob MF, Manso $\mathrm{PH}$, et al. Desmoid Tumor After a Minimally Invasive Atrial Septal Defect Closure in an Adolescent. World J Pediatr Congenit Heart Surg. 2016 Feb 16doi: 10.1177/2150135115607190. PMID: 26884451.X-1

4542. Ameis SH, Lerch JP, Taylor MJ, et al. A Diffusion Tensor Imaging Study in Children With ADHD, Autism Spectrum Disorder, OCD, and Matched Controls: Distinct and Non-

Distinct White Matter Disruption and

Dimensional Brain-Behavior Relationships. Am J Psychiatry. 2016 Jul 1:appiajp201615111435. doi: 10.1176/appi.ajp.2016.15111435. PMID: 27363509.X-1

4543. Amodeo DA, Rivera E, Dunn JT, et al. M100907 attenuates elevated grooming behavior in the BTBR mouse. Behav Brain Res. 2016 Jul 1;313:67-70. doi: 10.1016/j.bbr.2016.06.064. PMID: 27378338.X-1

4544. Amos LE, Carpenter SL, Hoeltzel MF. Lost at Sea in Search of a Diagnosis: A Case of Unexplained Bleeding. Pediatr Blood Cancer. 2016 Jul;63(7):1305-6. doi: 10.1002/pbc.25980. PMID: 27062477.X-1

4545. Amram N, Hacohen-Kleiman G, Sragovich S, et al. Sexual divergence in microtubule function: the novel intranasal microtubule targeting SKIP normalizes axonal transport and enhances memory. Mol Psychiatry. 2016 Jan 19doi: 10.1038/mp.2015.208. PMID: 26782054.X-1

4546. An JY, Claudianos C. Genetic heterogeneity in autism: From single gene to a pathway perspective. Neurosci Biobehav Rev. 2016 Jun 16;68:442-53. doi: 10.1016/j.neubiorev.2016.06.013. PMID: 27317861.X-1

4547. Anagnostou E, Aman MG, Handen BL, et al. Metformin for Treatment of Overweight Induced by Atypical Antipsychotic Medication in Young People With Autism Spectrum Disorder: A Randomized Clinical Trial. JAMA Psychiatry. 2016 Aug 24doi: 10.1001/jamapsychiatry.2016.1232. PMID: 27556593.X-1 
4548. Andakyan L, Fryling MJ, Benjamin K. Further evaluation of the displacement of leisure items by food during stimulus preference assessments with children with autism. Journal of Developmental and Physical Disabilities. 2016;28(3):461-7. doi: http://dx.doi.org/10.1007/s10882-016-94833.X-1, X-3, X-4

4549. Anderson A, Locke J, Kretzmann M, et al. Social network analysis of children with autism spectrum disorder: Predictors of fragmentation and connectivity in elementary school classrooms. Autism. 2016

Aug;20(6):700-9. doi: 10.1177/1362361315603568. PMID: 26567264.X-1

4550. Anderson S, Bucholz JL, Hazelkorn $\mathrm{M}$, et al. Using narrated literacy-based behavioural interventions to decrease episodes of physical aggression in elementary students with disabilities. Support for Learning. 2016;31(2):90-103. doi: http://dx.doi.org/10.1111/1467-9604.12118.X1, X-3, X-4

4551. Anderson S, Meints K. Brief Report: The Effects of Equine-Assisted Activities on the Social Functioning in Children and Adolescents with Autism Spectrum Disorder. J Autism Dev Disord. 2016 Jul 25doi: 10.1007/s10803-016-2869-3. PMID: 27457363.X-3

4552. Anderst W. Narrative review of the in vivo mechanics of the cervical spine after anterior arthrodesis as revealed by dynamic biplane radiography. J Orthop Res. 2016 Jan;34(1):22-30. doi: 10.1002/jor.23042. PMID: 26331480.X-1

4553. Andrade NS, Dutra TT, Fernandes RF, et al. Retrospective study of dental trauma in children with autism spectrum disorders: a paired study. Spec Care Dentist. 2016 Apr 26doi: 10.1111/scd.12180. PMID: 27114111.X1

4554. Angioni L, Cocco C, Ferri GL, et al. Involvement of nigral oxytocin in locomotor activity: A behavioral, immunohistochemical and lesion study in male rats. Horm Behav. 2016 Jul;83:23-38. doi: 10.1016/j.yhbeh.2016.05.012. PMID: 27189764.X-1
4555. Annavarapu RN, Kathi S. Cognitive disorders in children associated with urban vehicular emissions. Environ Pollut. 2016 Jan;208(Pt A):74-8. doi: 10.1016/j.envpol.2015.09.036. PMID: 26476694.X-1

4556. Antezana L, Mosner MG, Troiani V, et al. Social-emotional inhibition of return in children with autism spectrum disorder versus typical development. Journal of Autism and Developmental Disorders. 2016;46(4):1236-46. doi: http://dx.doi.org/10.1007/s10803-0152661-9.X-1

4557. Anzures G, Goyet L, Ganea N, et al. Enhanced ERPs to visual stimuli in unaffected male siblings of ASD children. Child Neuropsychol. 2016;22(2):220-37. doi: 10.1080/09297049.2014.988609. PMID: 25506753.X-1

4558. Aponte CA, Romanczyk RG. Assessment of feeding problems in children with autism spectrum disorder. Research in Autism Spectrum Disorders. 2016;21:61-72. doi: http://dx.doi.org/10.1016/j.rasd.2015.09.007.X1

4559. Arbabshirani MR, Plis S, Sui J, et al. Single subject prediction of brain disorders in neuroimaging: Promises and pitfalls. Neuroimage. 2016 Mar 21doi: 10.1016/j.neuroimage.2016.02.079. PMID: 27012503.X-1

4560. Arbogast T, Ouagazzal AM, Chevalier C, et al. Reciprocal Effects on Neurocognitive and Metabolic Phenotypes in Mouse Models of 16p11.2 Deletion and Duplication Syndromes. PLoS Genet. 2016 Feb;12(2):e1005709. doi: 10.1371/journal.pgen.1005709. PMID: 26872257.X-1

4561. Archer T, Kostrzewa RM. Neuroteratology and Animal Modeling of Brain Disorders. Curr Top Behav Neurosci. 2016 Feb 9doi: 10.1007/7854_2015_434. PMID: 26857462.X-1

4562. Arevalo C, DelRosso LM, Khaytin I, et al. A 7-Year-Old Boy with Intractable Seizures and Snoring. J Clin Sleep Med. 2016 Feb 29 PMID: 26951404.X-1 
4563. Arias VB, Ponce FP, Martínez-Molina A, et al. General and specific attentiondeficit/hyperactivity disorder factors of children 4 to 6 years of age: An exploratory structural equation modeling approach to assessing symptom multidimensionality. Journal of Abnormal Psychology. 2016;125(1):125-37. doi: http://dx.doi.org/10.1037/abn0000115.X-1

4564. Arildskov TW, Hojgaard DR, Skarphedinsson G, et al. Subclinical autism spectrum symptoms in pediatric obsessivecompulsive disorder. Eur Child Adolesc Psychiatry. 2016 Jul;25(7):711-23. doi: 10.1007/s00787-015-0782-5. PMID: 26518580.X-1

4565. Arildskov TW, Højgaard DRMA, Skarphedinsson G, et al. Subclinical autism spectrum symptoms in pediatric obsessivecompulsive disorder. European Child \& Adolescent Psychiatry. 2016;25(7):711-23. doi: http://dx.doi.org/10.1007/s00787-015-07825.X-1

4566. Arima H, Yamato Y, Hasegawa T, et al. Discrepancy between Standing Posture and Sagittal Balance during Walking in Adult Spinal Deformity Patients. Spine (Phila Pa 1976). 2016 May 23doi: 10.1097/brs.0000000000001709. PMID: 27220034.X-1

4567. Arkilo D, Devinsky O, Mudigoudar B, et al. Electroencephalographic patterns during sleep in children with chromosome 15q11.213.1 duplications (Dup15q). Epilepsy \& Behavior. 2016;57(Part A):133-6. doi: http://dx.doi.org/10.1016/j.yebeh.2016.02.010. $\mathrm{X}-1$

4568. Arnevik EA, Helverschou SB. Autism Spectrum Disorder and Co-occurring Substance Use Disorder - A Systematic Review. Subst Abuse. 2016;10:69-75. doi: 10.4137/sart.s39921. PMID: 27559296.X-1, X2

4569. Ashburner J, Vickerstaff S, Beetge J, et al. Remote versus face-to-face delivery of early intervention programs for children with autism spectrum disorders: Perceptions of rural families and service providers. Research in Autism Spectrum Disorders. 2016;23:1-14. doi:
http://dx.doi.org/10.1016/j.rasd.2015.11.011.X1

4570. Ashwood KL, Gillan N, Horder J, et al. Predicting the diagnosis of autism in adults using the Autism-Spectrum Quotient (AQ) questionnaire. Psychol Med. 2016 Jun 29:1-10. doi: 10.1017/s0033291716001082. PMID: 27353452.X-1

4571. Ausderau KK, Sideris J, Little LM, et al. Sensory subtypes and associated outcomes in children with autism spectrum disorders. Autism Res. 2016 May 2doi: 10.1002/aur.1626. PMID: 27135214.X-1

4572. Austin JE, Zinke VL, Davies WH. Influencing Perception About Children with Autism and their Parents Using Disclosure Cards. J Autism Dev Disord. 2016 May 30doi: 10.1007/s10803-016-2821-6. PMID: 27241346.X-1

4573. Avella-Garcia CB, Julvez J, Fortuny J, et al. Acetaminophen use in pregnancy and neurodevelopment: attention function and autism spectrum symptoms. Int J Epidemiol. 2016 Jun 28doi: 10.1093/ije/dyw115. PMID: 27353198.X-1

4574. Avrech Bar M, Shelef L, Bart O. Do participation and self-efficacy of mothers to children with ASD predict their children's participation? Research in Autism Spectrum Disorders. 2016;24:1-10. doi: http://dx.doi.org/10.1016/j.rasd.2016.01.002.X1

4575. Azad GF, Locke J, Kasari C, et al. Race, disability, and grade: Social relationships in children with autism spectrum disorders. Autism. 2016 Mar 10doi: 10.1177/1362361315627792. PMID: 26966285.X-1

4576. Azmitia EC, Saccomano ZT, Alzoobaee MF, et al. Persistent angiogenesis in the autism brain: An immunocytochemical study of postmortem cortex, brainstem and cerebellum. Journal of Autism and Developmental Disorders. 2016;46(4):1307-18. doi: http://dx.doi.org/10.1007/s10803-0152672-6.X-1

4577. Baarsma ME, Boonmann C, t HartKerkhoffs LA, et al. Sexuality and Autistic- 
Like Symptoms in Juvenile Sex Offenders: A Follow-Up After 8 Years. J Autism Dev Disord. 2016 May 18doi: 10.1007/s10803-016-2805-6. PMID: 27193182.X-1

4578. Badone E, Nicholas D, Roberts W, et al. Asperger's Syndrome, Subjectivity and the Senses. Cult Med Psychiatry. 2016 Feb 2doi: 10.1007/s11013-016-9484-9. PMID: 26838589.X-1, X-2, X-3

4579. Baez S, Garcia AM, Ibanez A. The Social Context Network Model in Psychiatric and Neurological Diseases. Curr Top Behav Neurosci. 2016 Apr 30doi: 10.1007/7854_2016_443. PMID: 27130326.X1

4580. Baghel S, Cathcart H, O'Reilly NJ. Theoretical and experimental investigation of drug-polymer interaction and miscibility and its impact on drug supersaturation in aqueous medium. Eur J Pharm Biopharm. 2016 Jul 1doi: 10.1016/j.ejpb.2016.06.024. PMID: 27378287.X-1

4581. Bahi A. Sustained lentiviral-mediated overexpression of microRNA124a in the dentate gyrus exacerbates anxiety- and autismlike behaviors associated with neonatal isolation in rats. Behav Brain Res. 2016 Sep 15;311:298-308. doi: 10.1016/j.bbr.2016.05.033. PMID: 27211062.X-1

4582. Bahrami F, Movahedi A, Marandi SM, et al. The effect of Karate techniques training on communication deficit of children with autism spectrum disorders. Journal of Autism and Developmental Disorders. 2016;46(3):97886. doi: http://dx.doi.org/10.1007/s10803-0152643-y.X-1

4583. Bai JY, Cowie S, Podlesnik CA. Quantitative analysis of local-level resurgence. Learn Behav. 2016 Aug 5doi: 10.3758/s13420016-0242-1. PMID: 27495929.X-1

4584. Baijot S, Slama H, Söderlund G, et al. Neuropsychological and neurophysiological benefits from white noise in children with and without ADHD. Behavioral and Brain Functions. 2016;12doi: http://dx.doi.org/10.1186/s12993-016-0095y.X-1
4585. Bakroon A, Lakshminarayanan V. Visual function in autism spectrum disorders: a critical review. Clin Exp Optom. 2016 May 10doi: 10.1111/cxo.12383. PMID: 27161596.X-1

4586. Bala KA, Dogan M, Mutluer T, et al. Plasma amino acid profile in autism spectrum disorder (ASD). Eur Rev Med Pharmacol Sci. 2016 Mar;20(5):923-9. PMID: 27010152.X-1

4587. Baldwin PR, Curtis KN, Patriquin MA, et al. Identifying diagnostically-relevant resting state brain functional connectivity in the ventral posterior complex via genetic data mining in autism spectrum disorder. Autism Research. 2016;9(5):553-62. doi: http://dx.doi.org/10.1002/aur.1559.X-1

4588. Ballantyne CJ, Núñez M. Developmental trajectories of hierarchical visuo-spatial processing in fragile $\mathrm{X}$ syndrome and ASD: Within- and cross-syndrome variability. Research in Developmental Disabilities. 2016;51-52:103-15. doi: http://dx.doi.org/10.1016/j.ridd.2015.12.016.X1

4589. Ballard J, Crane DR, Harper JM, et al. Mental health service utilization in autism spectrum disorders. Research in Autism Spectrum Disorders. 2016;23:84-93. doi: http://dx.doi.org/10.1016/j.rasd.2015.12.002.X1

4590. Balz J, Keil J, Roa Romero Y, et al. GABA concentration in superior temporal sulcus predicts gamma power and perception in the sound-induced flash illusion. Neuroimage. 2016 Jan 15;125:724-30. doi: 10.1016/j.neuroimage.2015.10.087. PMID: 26546865.X-1

4591. Bancroft SL, Thompson RH, Peters LC, et al. Behavioral variability in the play of children with autism and their typically developing peers. Behavioral Interventions. 2016;31(2):107-19. doi: http://dx.doi.org/10.1002/bin.1438.X-1

4592. Banerjee A, Luong JA, Ho A, et al. Overexpression of Homer1a in the basal and lateral amygdala impairs fear conditioning and induces an autism-like social impairment. Mol Autism. 2016;7:16. doi: 10.1186/s13229-0160077-9. PMID: 26929812.X-1 
4593. Barbaresi WJ. The meaning of "regression" in children with autism spectrum disorder: Why does it matter? Journal of Developmental and Behavioral Pediatrics. 2016;37(6):506-7. doi: http://dx.doi.org/10.1097/DBP.0000000000000 325.X-1, X-2

4594. Barbaro J, Dissanayake C. Diagnostic stability of autism spectrum disorder in toddlers prospectively identified in a community-based setting: Behavioural characteristics and predictors of change over time. Autism. 2016 Jul 28doi: 10.1177/1362361316654084. PMID: 27474118.X-1

4595. Barber AB, Saffo RW, Gilpin AT, et al. Peers as clinicians: Examining the impact of Stay Play Talk on social communication in young preschoolers with autism. Journal of Communication Disorders. 2016;59:1-15. doi: http://dx.doi.org/10.1016/j.jcomdis.2015.06.009 .X-3, X-4

4596. Bariselli S, Tzanoulinou S, Glangetas C, et al. SHANK3 controls maturation of social reward circuits in the VTA. Nat Neurosci. 2016 Jul;19(7):926-34. doi: 10.1038/nn.4319. PMID: 27273769.X-1

4597. Barker-Haliski ML, Vanegas F, Mau $\mathrm{MJ}$, et al. Acute cognitive impact of antiseizure drugs in naive rodents and corneal-kindled mice. Epilepsia. 2016 Jul 28doi: 10.1111/epi.13476. PMID: 27466022.X-1

4598. Barnard-Brak L, Stevens T, Xiao F, et al. Approaches to learning and medicated ADHD: The potential impact on learning and assessment. Learning and Individual Differences. 2016;47:298-303. doi: http://dx.doi.org/10.1016/j.lindif.2015.12.029.X $-1$

4599. Barnett R. Attention deficit hyperactivity disorder. The Lancet. 2016;387(10020):737. doi: http://dx.doi.org/10.1016/S01406736(16)00332-9.X-1

4600. Barnevik Olsson M, Lundstrom S, Westerlund J, et al. Preschool to School in Autism: Neuropsychiatric Problems 8 Years After Diagnosis at 3 Years of Age. J Autism
Dev Disord. 2016 May 26doi: 10.1007/s10803016-2819-0. PMID: 27230761.X-1

4601. Baroncelli L, Molinaro A, Cacciante F, et al. A mouse model for creatine transporter deficiency reveals early onset cognitive impairment and neuropathology associated with brain aging. Hum Mol Genet. 2016 Jul 27doi: 10.1093/hmg/ddw252. PMID: 27466184.X-1

4602. Barone R, Sturiale L, Fiumara A, et al. Csf n-glycan profile reveals sialylation deficiency in a patient with gm2 gangliosidosis presenting as childhood disintegrative disorder. Autism Research. 2016;9(4):423-8. doi: http://dx.doi.org/10.1002/aur.1541.X-1

4603. Bartolo A, Ham HS. A Cognitive Overview of Limb Apraxia. Curr Neurol Neurosci Rep. 2016 Aug;16(8):75. doi: 10.1007/s11910-016-0675-0. PMID: 27349561.X-1

4604. Bartolome-Villar B, MourelleMartinez MR, Dieguez-Perez M, et al. Incidence of oral health in paediatric patients with disabilities: Sensory disorders and autism spectrum disorder. Systematic review II. J Clin Exp Dent. 2016 Jul;8(3):e344-51. doi: 10.4317/jced.52923. PMID: 27398188.X-1

4605. Barton EE, Harris B, Leech N, et al. An analysis of state autism educational assessment practices and requirements. Journal of Autism and Developmental Disorders. 2016;46(3):737-48. doi: http://dx.doi.org/10.1007/s10803-015-25890.X-1

4606. Basay BK, Buber A, Basay O, et al. White matter alterations related to attentiondeficit hyperactivity disorder and COMT val $^{158}$ met polymorphism: Children with valine homozygote attention-deficit hyperactivity disorder have altered white matter connectivity in the right cingulum (cingulate gyrus). Neuropsychiatric Disease and Treatment. 2016;12.X-1

4607. Baugh EH, Simmons-Edler R, Muller $\mathrm{CL}$, et al. Robust classification of protein variation using structural modelling and largescale data integration. Nucleic Acids Res. 2016 Apr 7;44(6):2501-13. doi: 10.1093/nar/gkw120. PMID: 26926108.X-1 
4608. Bayram AK, Kardas F, Demirci EO, et al. Lack of serum antineuronal antibodies in children with autism. Bratisl Lek Listy. 2016;117(2):77-9. PMID: 26830036.X-1

4609. Beal SJ, Riddle IK, Kichler JC, et al. The Associations of Chronic Condition Type and Individual Characteristics with Transition Readiness. Acad Pediatr. 2016 Jun 23doi: 10.1016/j.acap.2016.06.007. PMID: 27345693.X-1

4610. Becker MM, Riesgo RS, Roesler R, et al. Improvement in Symptoms of Autism Spectrum Disorder in Children With the Use of Gastrin-Releasing Peptide: An Open Trial. Clin Neuropharmacol. 2016 Jun 21doi: 10.1097/wnf.0000000000000165. PMID: 27332629.X-3

4611. Becker SP, Leopold DR, Burns GL, et al. The internal, external, and diagnostic validity of sluggish cognitive tempo: A metaanalysis and critical review. Journal of the American Academy of Child \& Adolescent Psychiatry. 2016;55(3):163-78. doi: http://dx.doi.org/10.1016/j.jaac.2015.12.006.X1

4612. Becker SP, Withrow AR, Stoppelbein $\mathrm{L}$, et al. Sluggish cognitive tempo is associated with suicide risk in psychiatrically hospitalized children. J Child Psychol Psychiatry. 2016 Jun 1doi: 10.1111/jcpp.12580. PMID: 27245482.X1

4613. Beckman L, Janson S, von Kobyletzki L. Associations between neurodevelopmental disorders and factors related to school, health, and social interaction in schoolchildren: Results from a Swedish population-based survey. Disabil Health J. 2016 May 18doi: 10.1016/j.dhjo.2016.05.002. PMID: 27343047.X-1

4614. Bedard P. Prenatal Antidepressant Use and Risk of Autism Spectrum Disorders in Children. JAMA Pediatr. 2016 May 31doi: 10.1001/jamapediatrics.2016.0730. PMID: 27244697.X-1

4615. Bedford R, Pellicano E, Mareschal D, et al. Flexible integration of visual cues in adolescents with autism spectrum disorder. Autism Res. 2016 Feb;9(2):272-81. doi: 10.1002/aur.1509. PMID: 26097109.X-1
4616. Begeer S, Bernstein DM, Aßfalg A, et al. Reprint of: Equal egocentric bias in schoolaged children with and without autism spectrum disorders. Journal of Experimental Child Psychology. 2016;149:134-45. doi: http://dx.doi.org/10.1016/j.jecp.2016.05.017.X1

4617. Begeer S, Bernstein DM, Aßfalg A, et al. Equal egocentric bias in school-aged children with and without autism spectrum disorders. Journal of Experimental Child Psychology. 2016;144:15-26. doi: http://dx.doi.org/10.1016/j.jecp.2015.10.018.X1

4618. Bekes V, Perry JC, Robertson BM. Psychological masochism: A systematic review of the literature on conflicts, defenses, and motives. Psychother Res. 2016 Jun 1:1-14. doi: 10.1080/10503307.2016.1189618. PMID: 27248648.X-1

4619. Bekhet AK, Matel-Anderson D. Risk and Protective Factors in the Lives of Caregivers of Persons With Autism: Caregivers' Perspectives. Perspect Psychiatr Care. 2016 Jun 16doi: 10.1111/ppc.12158. PMID: 27306504.X-1

4620. Belagodu AP, Zendeli L, Slater BJ, et al. Blocking elevated VEGF-A attenuates nonvasculature Fragile X Syndrome abnormalities. Dev Neurobiol. 2016 Jun 6doi: 10.1002/dneu.22404. PMID: 27265443.X-1

4621. Belinson H, Nakatani J, Babineau BA, et al. Prenatal beta-catenin/Brn2/Tbr2 transcriptional cascade regulates adult social and stereotypic behaviors. Mol Psychiatry. 2016 Feb 2doi: 10.1038/mp.2015.207. PMID: 26830142.X-1

4622. Belisle J, Dixon MR, Stanley CR, et al. Teaching foundational perspective-taking skills to children with autism using the PEAK-T curriculum: single-reversal "I-You" deictic frames. J Appl Behav Anal. 2016 Jun 9doi: 10.1002/jaba.324. PMID: 27279459.X-1, X-3

4623. Beltrao-Braga PC, Muotri AR. Modeling autism spectrum disorders with human neurons. Brain Res. 2016 Feb 5doi: 10.1016/j.brainres.2016.01.057. PMID: 26854137.X-1 
4624. Benedek-Wood E, McNaughton D, Light J. Instruction in letter-sound correspondences for children with autism and limited speech. Topics in Early Childhood Special Education. 2016;36(1):43-54. doi: http://dx.doi.org/10.1177/0271121415593497.X $-1, \mathrm{X}-3, \mathrm{X}-4$

4625. Ben-Itzchak E, Abutbul S, Bela H, et al. Understanding one's own emotions in cognitively-able preadolescents with autism spectrum disorder. Journal of Autism and Developmental Disorders. 2016;46(7):2363-71. doi: http://dx.doi.org/10.1007/s10803-0162769-6.X-1

4626. Ben-Itzchak E, Zukerman G, Zachor DA. Having Older Siblings is Associated with Less Severe Social Communication Symptoms in Young Children with Autism Spectrum Disorder. J Abnorm Child Psychol. 2016 Feb 3doi: 10.1007/s10802-016-0133-0. PMID: 26843212.X-1

4627. Bennett M, Goodall E. Towards an Agenda for Research for Lesbian, Gay, Bisexual, Transgendered and/or Intersexed People with an Autism Spectrum Diagnosis. J Autism Dev Disord. 2016 Jun 20doi: 10.1007/s10803-016-2844-z. PMID: 27324493.X-1

4628. Bennett M, Hodgson V. The missing voices of Indigenous Australians with autism in research. Autism. 2016 May 25doi: 10.1177/1362361316643696. PMID: 27231338.X-1

4629. Benning SD, Kovac M, Campbell A, et al. Late Positive Potential ERP Responses to Social and Nonsocial Stimuli in Youth with Autism Spectrum Disorder. J Autism Dev Disord. 2016 Jun 25doi: 10.1007/s10803-0162845-y. PMID: 27344337.X-1, X-3

4630. Benson PR. The longitudinal effects of network characteristics on the mental health of mothers of children with ASD: The mediating role of parent cognitions. Journal of Autism and Developmental Disorders. 2016;46(5):1699715. doi: http://dx.doi.org/10.1007/s10803-0162699-3.X-1

4631. Berard A, Boukhris T. Prenatal Antidepressant Use and Risk of Autism
Spectrum Disorders in Children-Reply. JAMA Pediatr. 2016 May 31doi: 10.1001/jamapediatrics.2016.0748. PMID: 27244224.X-1

4632. Berg KL, Shiu CS, Acharya K, et al. Disparities in adversity among children with autism spectrum disorder: a population-based study. Dev Med Child Neurol. 2016 Jun 2doi: 10.1111/dmcn.13161. PMID: 27251442.X-1

4633. Berger J, Dorninger F, Forss-Petter S, et al. Peroxisomes in brain development and function. Biochim Biophys Acta. 2016 May;1863(5):934-55. doi: 10.1016/j.bbamcr.2015.12.005. PMID: 26686055.X-1

4634. Berger NI, Manston L, Ingersoll B. Establishing a Scale for Assessing the Social Validity of Skill Building Interventions for Young Children with Autism Spectrum Disorder. J Autism Dev Disord. 2016 Jul 27doi: 10.1007/s10803-016-2863-9. PMID: 27465242.X-1

4635. Berglund IG, Huus K, Enskär K, et al. Perioperative and anesthesia guidelines for children with autism: A nationwide survey from Sweden. Journal of Developmental and Behavioral Pediatrics. 2016;37(6):457-64. doi: http://dx.doi.org/10.1097/DBP.0000000000000 289.X-1

4636. Bergstrom R, Najdowski AC, Alvarado $\mathrm{M}$, et al. Teaching children with autism to tell socially appropriate lies. J Appl Behav Anal. 2016 Jun;49(2):405-10. doi: 10.1002/jaba.295. PMID: 26831011.X-1, X-3

4637. Bergwerff CE, Luman M, Blom HJ, et al. No Tryptophan, Tyrosine and Phenylalanine Abnormalities in Children with AttentionDeficit/Hyperactivity Disorder. PLoS One. 2016;11(3):e0151100. doi: 10.1371/journal.pone.0151100. PMID: 26938936.X-1

4638. Berna F, Goritz AS, Schroder J, et al. Self-Disorders in Individuals with Autistic Traits: Contribution of Reduced Autobiographical Reasoning Capacities. J Autism Dev Disord. 2016 Apr 21doi: 10.1007/s10803-016-2797-2. PMID: 27101235.X-1 
4639. Bernhardt BC, Di Martino A, Valk SL, et al. Neuroimaging-Based Phenotyping of the Autism Spectrum. Curr Top Behav Neurosci. 2016 Mar 6doi: 10.1007/7854_2016_438. PMID: 26946501.X-1

4640. Berryessa CM. Brief Report: Judicial Attitudes Regarding the Sentencing of Offenders with High Functioning Autism. J Autism Dev Disord. 2016 Apr 22doi: 10.1007/s10803-016-2798-1. PMID: 27106568.X-1

4641. Berry-Kravis E, Des Portes V, Hagerman R, et al. Mavoglurant in fragile X syndrome: Results of two randomized, doubleblind, placebo-controlled trials. Sci Transl Med. 2016 Jan 13;8(321):321ra5. doi: 10.1126/scitranslmed.aab4109. PMID: 26764156.X-1

4642. Berto S, Usui N, Konopka G, et al. ELAVL2-regulated transcriptional and splicing networks in human neurons link neurodevelopment and autism. Hum Mol Genet. 2016 Jun 3doi: 10.1093/hmg/ddw110. PMID: 27260404.X-1

4643. Bertorelli TE. Hope and doubt in the promise of neuroimaging: The case of autism spectrum disorder. Health (London). 2016 Jul 29doi: 10.1177/1363459316660860. PMID: 27474754.X-1

4644. Bertram J, Koschutzke L, Pfannmoller JP, et al. Morphological and behavioral characterization of adult mice deficient for SrGAP3. Cell Tissue Res. 2016 May 17doi: 10.1007/s00441-016-2413-y. PMID: 27184948.X-1

4645. Berzhanskaya J, Phillips MA, Gorin A, et al. Disrupted Cortical State Regulation in a Rat Model of Fragile X Syndrome. Cereb Cortex. 2016 Jan 5doi: 10.1093/cercor/bhv331. PMID: 26733529.X-1

4646. Besag F, Aldenkamp A, Caplan R, et al. Psychiatric and Behavioural Disorders in Children with Epilepsy (ILAE Task Force Report): Epilepsy and autism. Epileptic Disord. 2016 May 16doi: 10.1684/epd.2016.0812. PMID: 27184301.X-1

4647. Besag F, Gobbi G, Aldenkamp A, et al. Psychiatric and Behavioural Disorders in
Children with Epilepsy (ILAE Task Force Report): Behavioural and psychiatric disorders associated with childhood epilepsy syndromes. Epileptic Disord. 2016 May 16doi: 10.1684/epd.2016.0815. PMID: 27184676.X-1

4648. Beunders G, van de Kamp J, Vasudevan P, et al. A detailed clinical analysis of 13 patients with AUTS2 syndrome further delineates the phenotypic spectrum and underscores the behavioural phenotype. J Med Genet. 2016 Apr 13doi: 10.1136/jmedgenet2015-103601. PMID: 27075013.X-1

4649. Bhat G, LaGrave D, Millson A, et al. Xq11.1-11.2 deletion involving ARHGEF9 in a girl with autism spectrum disorder. Eur J Med Genet. 2016 May 27doi: 10.1016/j.ejmg.2016.05.014. PMID: 27238888.X-1

4650. Bhattacharya A, Mamcarz M, Mullins C, et al. Targeting Translation Control with p70 S6 Kinase 1 Inhibitors to Reverse Phenotypes in Fragile X Syndrome Mice. Neuropsychopharmacology. 2016 Jul;41(8):1991-2000. doi: 10.1038/npp.2015.369. PMID: 26708105.X-1

4651. Bhattacharyya S. Inside story of Group I Metabotropic Glutamate Receptors (mGluRs). Int J Biochem Cell Biol. 2016 Mar 14doi: 10.1016/j.biocel.2016.03.003. PMID: 26987586.X-1, X-3

4652. Bidinosti M, Botta P, Kruttner S, et al. CLK2 inhibition ameliorates autistic features associated with SHANK3 deficiency. Science. 2016 Mar 11;351(6278):1199-203. doi: 10.1126/science.aad5487. PMID: 26847545.X1

4653. Billeci L, Tonacci A, Tartarisco G, et al. An Integrated Approach for the Monitoring of Brain and Autonomic Response of Children with Autism Spectrum Disorders during Treatment by Wearable Technologies. Front Neurosci. 2016;10:276. doi: 10.3389/fnins.2016.00276. PMID: 27445652.X-1

4654. Billen J, Hashim R, Ito F. Ultrastructure of the mandibular gland of the ant Myrmoteras iriodum. Arthropod Struct Dev. 2016 May 1doi: 10.1016/j.asd.2016.04.003. PMID: 27130260.X-1 
4655. Bin S, Xiangwang H, Sheng X, et al. Artificial Cervical Disc Replacement for the Treatment of Adjacent Segment Disease after Anterior Cervical Decompression and Fusion. Clin Spine Surg. 2016 Jun 28doi: 10.1097/bsd.0000000000000173. PMID: 27355704.X-1

4656. Biran J, Levkowitz G. Zebrafish Reel in Phenotypic Suppressors of Autism. Neuron. 2016 Feb 17;89(4):673-5. doi: 10.1016/j.neuron.2016.02.005. PMID: 26889805.X-1

4657. Birch RC, Hocking DR, Trollor JN. Prevalence and predictors of subjective memory complaints in adult male carriers of the FMR1 premutation. Clin Neuropsychol. 2016 Jun 29:1-15. doi: 10.1080/13854046.2016.1145905. PMID: 27355815.X-1

4658. Biscaldi M, Bednorz N, Weissbrodt K, et al. Cognitive endophenotypes of attention deficit/hyperactivity disorder and intra-subject variability in patients with autism spectrum disorder. Biol Psychol. 2016 Apr 30;118:25-34. doi: 10.1016/j.biopsycho.2016.04.064. PMID: 27143193.X-1

4659. Bishop SL, Havdahl KA, Huerta M, et al. Subdimensions of social-communication impairment in autism spectrum disorder. J Child Psychol Psychiatry. 2016 Jan 8doi: 10.1111/jcpp.12510. PMID: 26748412.X-1

4660. Bishop SL, Havdahl KA, Huerta M, et al. Subdimensions of social-communication impairment in autism spectrum disorder. Journal of Child Psychology and Psychiatry. 2016;57(8):909-16. doi: http://dx.doi.org/10.1111/jcpp.12510.X-1

4661. Bishop SL, Huerta M, Gotham K, et al. The autism symptom interview, school-age: A brief telephone interview to identify autism spectrum disorders in 5-to-12-year-old children. Autism Res. 2016 Jun 10doi: 10.1002/aur.1645. PMID: 27282463.X-1

4662. Bishop-Fitzpatrick L, Hong J, Smith LE, et al. Characterizing Objective Quality of Life and Normative Outcomes in Adults with Autism Spectrum Disorder: An Exploratory Latent Class Analysis. J Autism Dev Disord.
2016 May 20doi: 10.1007/s10803-016-2816-3. PMID: 27207091.X-1

4663. Biskup CS, Helmbold K, Baurmann D, et al. Resting state default mode network connectivity in children and adolescents with ADHD after acute tryptophan depletion. Acta Psychiatrica Scandinavica. 2016;134(2):161-71. doi: http://dx.doi.org/10.1111/acps.12573.X-1

4664. Bissonette GB, Roesch MR. Development and function of the midbrain dopamine system: what we know and what we need to. Genes Brain Behav. 2016 Jan;15(1):6273. doi: 10.1111/gbb.12257. PMID: 26548362.X-1

4665. Bitsika V, Sharpley CF. The association between social responsivity and depression in high-functioning boys with an Autism Spectrum Disorder. Journal of Developmental and Physical Disabilities. 2016;28(2):317-31. doi: http://dx.doi.org/10.1007/s10882-015-9470$0 . \mathrm{X}-1$

4666. Bitsika V, Sharpley CF, Christie DR, et al. Measuring Personal and Functional Changes in Prostate Cancer Survivors: Development and validation of the FADE: Data from the TROG 03.04 RADAR trial. Psychooncology. 2016 May 30doi: 10.1002/pon.4182. PMID: 27240216.X-1

4667. Bitsika V, Sharpley CF, Mills R. Are Sensory Processing Features Associated with Depressive Symptoms in Boys with an ASD? J Autism Dev Disord. 2016 Jan;46(1):242-52. doi: 10.1007/s10803-015-2569-4. PMID: 26319255.X-1

4668. Bitsika V, Sharpley CF, Mills R. How are Sensory Features associated with seven anxiety disorders in boys with Autism Spectrum Disorder? International Journal of Developmental Neuroscience. 2016;50:47-54. doi:

http://dx.doi.org/10.1016/j.ijdevneu.2016.03.00 5.X-1

4669. Bjornsdotter M, Wang N, Pelphrey K, et al. Evaluation of Quantified Social Perception Circuit Activity as a Neurobiological Marker of Autism Spectrum Disorder. JAMA Psychiatry. 2016 Jun 1;73(6):614-21. doi: 
10.1001/jamapsychiatry.2016.0219. PMID: 27096285.X-1

4670. Blanco-Suarez E, Caldwell AL, Allen NJ. Role of astrocyte-synapse interactions in CNS disorders. J Physiol. 2016 Jul 5doi: 10.1113/jp270988. PMID: 27381164.X-1

4671. Bland VJ, Bai JY, Fullerton JA, et al. Signaled alternative reinforcement and the persistence of operant behavior. J Exp Anal Behav. 2016 Jun 10doi: 10.1002/jeab.212. PMID: 27282131.X-1

4672. Blanquie O, Liebmann L, Hubner CA, et al. NKCC1-Mediated GABAergic Signaling Promotes Postnatal Cell Death in Neocortical Cajal-Retzius Cells. Cereb Cortex. 2016 Jan 26doi: 10.1093/cercor/bhw004. PMID: 26819276.X-1

4673. Bliksted V, Ubukata S, Koelkebeck K. Discriminating autism spectrum disorders from schizophrenia by investigation of mental state attribution on an on-line mentalizing task: A review and meta-analysis. Schizophr Res. 2016 Mar;171(1-3):16-26. doi:

10.1016/j.schres.2016.01.037. PMID: 26817402.X-1

4674. Bo J, Lee C-M, Colbert A, et al. Do children with autism spectrum disorders have motor learning difficulties? Research in Autism Spectrum Disorders. 2016;23:50-62. doi: http://dx.doi.org/10.1016/j.rasd.2015.12.001.X$1, \mathrm{X}-2$

4675. Bohnert A, Lieb R, Arola N. More than Leisure: Organized Activity Participation and Socio-Emotional Adjustment Among Adolescents with Autism Spectrum Disorder. J Autism Dev Disord. 2016 May 3doi:

10.1007/s10803-016-2783-8. PMID:

27141864.X-1, X-3

4676. Boilson AM, Staines A, Ramirez A, et al. Operationalisation of the European Protocol for Autism Prevalence (EPAP) for Autism

Spectrum Disorder Prevalence Measurement in Ireland. J Autism Dev Disord. 2016 Jun 30doi: 10.1007/s10803-016-2837-y. PMID:

27364514.X-1

4677. Bokshan SL, Godzik J, Dalton J, et al. Reliability of the revised Scoliosis Research Society-22 and Oswestry Disability Index
(ODI) questionnaires in adult spinal deformity when administered by telephone. Spine J. 2016 Mar 17doi: 10.1016/j.spinee.2016.03.022. PMID: 26997110.X-1

4678. Bolkan S, Gordon JA. Neuroscience: Untangling autism. Nature. 2016 Apr 7;532(7597):45-6. doi: 10.1038/nature17311. PMID: 27007842.X-1, X-2

4679. Bolkvadze T, Puhakka N, Pitkanen A. Epileptogenesis after traumatic brain injury in Plaur-deficient mice. Epilepsy Behav. 2016 Jul;60:187-96. doi:

10.1016/j.yebeh.2016.04.038. PMID: 27208924.X-1

4680. Bonatto SJ, Kerner M, Merelles S, et al. The prevalence of symptoms of attentiondeficit/hyperactivity disorder in parents of children with autism spectrum disorder. Psychiatry Research. 2016;240:1-3. doi: http://dx.doi.org/10.1016/j.psychres.2016.04.00 6.X-1

4681. Bone D, Bishop S, Black MP, et al. Use of machine learning to improve autism screening and diagnostic instruments: effectiveness, efficiency, and multi-instrument fusion. J Child Psychol Psychiatry. 2016 Apr 19doi: 10.1111/jcpp.12559. PMID: 27090613.X-1

4682. Bone D, Bishop SL, Black MP, et al. Use of machine learning to improve autism screening and diagnostic instruments: Effectiveness, efficiency, and multi-instrument fusion. Journal of Child Psychology and Psychiatry. 2016;57(8):927-37. doi: http://dx.doi.org/10.1111/jcpp.12559.X-1

4683. Bono V, Narzisi A, Jouen A-L, et al. GOLIAH: A gaming platform for home-based intervention in autism-Principles and design. Frontiers in Psychiatry. 2016;7.X-1, X-3, X-4

4684. Booms S, Hill E, Kulhanek L, et al. Iodine Deficiency and Hypothyroidism From Voluntary Diet Restrictions in the US: Case Reports. Pediatrics. 2016 Jun;137(6)doi: 10.1542/peds.2015-4003. PMID: 27244854.X-1

4685. Boonen S, Billen J. Functional morphology of the maxillary and propharyngeal glands of Monomorium pharaonis (L.). Arthropod Struct Dev. 2016 May 15doi: 
10.1016/j.asd.2016.04.005. PMID:

27179446.X-1

4686. Booth RD, Happe FG. Evidence of Reduced Global Processing in Autism Spectrum Disorder. J Autism Dev Disord. 2016 Feb 10doi: 10.1007/s10803-016-2724-6. PMID: 26864159.X-1

4687. Bos PA, Hofman D, Hermans EJ, et al. Testosterone reduces functional connectivity during the 'Reading the Mind in the Eyes' Test. Psychoneuroendocrinology. 2016 Jun;68:194201. doi: 10.1016/j.psyneuen.2016.03.006. PMID: 26994483.X-1

4688. Bostrom C, Yau SY, Majaess N, et al. Hippocampal Dysfunction and Cognitive Impairment in Fragile-X Syndrome. Neurosci Biobehav Rev. 2016 Jun 23doi:

10.1016/j.neubiorev.2016.06.033. PMID: 27345143.X-1

4689. Bottema-Beutel K. Associations between joint attention and language in autism spectrum disorder and typical development: A systematic review and meta-regression analysis. Autism Res. 2016 Apr 5doi: 10.1002/aur.1624. PMID: 27059941.X-1

4690. Bottema-Beutel K, Turiel E, DeWitt $\mathrm{MN}$, et al. To include or not to include: Evaluations and reasoning about the failure to include peers with autism spectrum disorder in elementary students. Autism. 2016 Mar 7doi: 10.1177/1362361315622412. PMID: 26951326.X-1

4691. Bouck EC, Satsangi R, Bartlett W. Comparing a number line and audio prompts in supporting price comparison by students with intellectual disability. Res Dev Disabil. 2016 Jun-Jul;53-54:342-57. doi:

10.1016/j.ridd.2016.02.011. PMID: 26977936.X-1

4692. Boujut E, Dean A, Grouselle A, et al. Comparative Study of Teachers in Regular Schools and Teachers in Specialized Schools in France, Working with Students with an Autism Spectrum Disorder: Stress, Social Support, Coping Strategies and Burnout. J Autism Dev Disord. 2016 Jun 9doi: 10.1007/s10803-0162833-2. PMID: 27282858.X-1
4693. Boukhris T, Sheehy O, Mottron L, et al. Antidepressant Use During Pregnancy and the Risk of Autism Spectrum Disorder in Children. JAMA Pediatr. 2016 Feb;170(2):11724. doi: 10.1001/jamapediatrics.2015.3356. PMID: 26660917.X-1

4694. Bourgeois JA. Neuropsychiatry of fragile X-premutation carriers with and without fragile $\mathrm{X}$-associated tremor-ataxia syndrome: implications for neuropsychology. Clin Neuropsychol. 2016 Jun 29:1-16. doi: 10.1080/13854046.2016.1192134. PMID: 27355575.X-1

4695. Bourgeron T. Current knowledge on the genetics of autism and propositions for future research. C R Biol. 2016 Jun 8doi: 10.1016/j.crvi.2016.05.004. PMID: 27289453.X-1

4696. Bourgeron T. The genetics and neurobiology of ESSENCE: The third Birgit Olsson lecture. Nord J Psychiatry.

2016;70(1):1-9. doi: 10.3109/08039488.2015.1042519. PMID: 25971862.X-1

4697. Bouvet L, Mottron L, Valdois S, et al. Auditory stream segregation in autism spectrum disorder: Benefits and downsides of superior perceptual processes. Journal of Autism and Developmental Disorders. 2016;46(5):1553-61. doi: http://dx.doi.org/10.1007/s10803-0132003-8.X-1

4698. Bowman P, Broadbridge E, Knight BA, et al. Psychiatric morbidity in children with KCNJ11 neonatal diabetes. Diabet Med. 2016 Apr 18doi: 10.1111/dme.13135. PMID: 27086753.X-1

4699. Boyer BE, Doove LL, Geurts HM, et al. Qualitative Treatment-Subgroup Interactions in a Randomized Clinical Trial of Treatments for Adolescents with ADHD: Exploring What Cognitive-Behavioral Treatment Works for Whom. PLoS One. 2016;11(3):e0150698. doi: 10.1371/journal.pone.0150698. PMID: 26977602.X-1

4700. Bozkus-Genc G, Yucesoy-Ozkan S. Meta-analysis of pivotal response training for children with autism spectrum disorder. Education and Training in Autism and 
Developmental Disabilities. 2016;51(1):1326.X-2

4701. Bozzatello P, Brignolo E, De Grandi E, et al. Supplementation with Omega-3 Fatty Acids in Psychiatric Disorders: A Review of Literature Data. J Clin Med. 2016;5(8)doi: 10.3390/jcm5080067. PMID: 27472373.X-1, $\mathrm{X}-2$

4702. Brabson LA, Brown JL, Capriotti MR, et al. Patterned changes in urge ratings with tic suppression in youth with chronic tic disorders. Journal of Behavior Therapy and Experimental Psychiatry. 2016;50:162-70. doi: http://dx.doi.org/10.1016/j.jbtep.2015.07.004.X $-1$

4703. Braddock BA, Gabany C, Shah M, et al. Patterns of Gesture Use in Adolescents With Autism Spectrum Disorder. Am J Speech Lang Pathol. 2016 Jun 3:1-8. doi: 10.1044/2015_ajslp-14-0112. PMID: 27258802.X-1

4704. Braddock BA, Hilton JC. Arm and hand movement in children suspected of having autism spectrum disorder. Communication Disorders Quarterly. 2016;37(3):148-59. doi: http://dx.doi.org/10.1177/1525740114562065.X $-1$

4705. Bradley CC, Boan AD, Cohen AP, et al. Reported History of Developmental Regression and Restricted, Repetitive Behaviors in Children with Autism Spectrum Disorders. J Dev Behav Pediatr. 2016 JulAug;37(6):451-6. doi: 10.1097/dbp.0000000000000316. PMID: 27366956.X-1

4706. Bradstreet LE, Juechter JI, Kamphaus RW, et al. Using the BASC-2 Parent Rating Scales to Screen for Autism Spectrum Disorder in Toddlers and Preschool-Aged Children. J Abnorm Child Psychol. 2016 May 14doi: 10.1007/s10802-016-0167-3. PMID: 27177744.X-1

4707. Brady LJ, Bartley AF, Li Q, et al. Transcriptional dysregulation causes altered modulation of inhibition by haloperidol. Neuropharmacology. 2016 Jul 29doi: 10.1016/j.neuropharm.2016.07.034. PMID: 27480797.X-1
4708. Branigan HP, Tosi A, Gillespie-Smith K. Spontaneous Lexical Alignment in Children With an Autistic Spectrum Disorder and Their Typically Developing Peers. J Exp Psychol Learn Mem Cogn. 2016 Apr 14doi: 10.1037/xlm0000272. PMID: 27078162.X-1

4709. Breckpot J, Vercruyssen M, Weyts E, et al. Copy number variation analysis in adults with catatonia confirms haploinsufficiency of SHANK3 as a predisposing factor. Eur J Med Genet. 2016 Aug 9;59(9):436-43. doi: 10.1016/j.ejmg.2016.08.003. PMID: 27519580.X-1

4710. Bremer E, Crozier M, Lloyd M. A systematic review of the behavioural outcomes following exercise interventions for children and youth with autism spectrum disorder. Autism. 2016 Jan 28doi: 10.1177/1362361315616002. PMID: 26823546.X-1, X-2

4711. Brett D, Warnell F, McConachie H, et al. Factors affecting age at ASD diagnosis in UK: No evidence that diagnosis age has decreased between 2004 and 2014. Journal of Autism and Developmental Disorders. 2016;46(6):1974-84. doi: http://dx.doi.org/10.1007/s10803-016-27166.X-1

4712. Breuillard D, Leunen D, Chemaly N, et al. Autism spectrum disorder phenotype and intellectual disability in females with epilepsy and PCDH-19 mutations. Epilepsy \& Behavior. 2016;60:75-80. doi: http://dx.doi.org/10.1016/j.yebeh.2016.04.009. $\mathrm{X}-1$

4713. Brewer CC, Zalewski CK, King KA, et al. Heritability of non-speech auditory processing skills. Eur J Hum Genet. 2016 Feb 17doi: 10.1038/ejhg.2015.277. PMID: 26883091.X-1

4714. Brewer N, Zoanetti J, Young RL. The influence of media suggestions about links between criminality and autism spectrum disorder. Autism. 2016 Apr 30doi: 10.1177/1362361316632097. PMID: 27132012.X-1

4715. Brignell A, Williams K, Prior M, et al. Parent-reported patterns of loss and gain in communication in 1- to 2-year-old children are 
not unique to autism spectrum disorder. Autism. 2016 May 12doi: 10.1177/1362361316644729. PMID: 27178996.X-1

4716. Brockman M, Hussain K, Sanchez B, et al. Managing child behavior problems in children with Autism Spectrum Disorders: Utilizing structural and solution focused therapy with primary caregivers. American Journal of Family Therapy. 2016;44(1):1-10. doi:

http://dx.doi.org/10.1080/01926187.2015.10994 14.X-1, X-2, X-3, X-4

4717. Brodhead MT, Al-Dubayan MN, Mates M, et al. An evaluation of a brief videobased multiple-stimulus without replacement preference assessment. Behavior Analysis in Practice. 2016;9(2):160-4. doi: http://dx.doi.org/10.1007/s40617-015-0081$0 . \mathrm{X}-1, \mathrm{X}-4$

4718. Brodhead MT, Higbee TS, Gerencser $\mathrm{KR}$, et al. The use of a discrimination-training procedure to teach mand variability to children with autism. Journal of Applied Behavior Analysis. 2016;49(1):34-48. doi: http://dx.doi.org/10.1002/jaba.280.X-1, X-3, X4

4719. Broek JA, Lin Z, de Gruiter HM, et al. Synaptic vesicle dynamic changes in a model of fragile X. Mol Autism. 2016;7:17. doi: 10.1186/s13229-016-0080-1. PMID: 26933487.X-1

4720. Bromley R. The treatment of epilepsy in pregnancy: The neurodevelopmental risks associated with exposure to antiepileptic drugs. Reprod Toxicol. 2016 Jun 14doi: 10.1016/j.reprotox.2016.06.007. PMID: 27312074.X-1

4721. Brook K. A response to 'Notes on a Puzzle Piece'. Autism. 2016 Feb;20(2):251-2. doi: 10.1177/1362361315604077. PMID: 26408633.X-1

4722. Brown JA, Woods JJ. Parentimplemented communication intervention: Sequential analysis of triadic relationships. Topics in Early Childhood Special Education. 2016;36(2):115-24. doi: http://dx.doi.org/10.1177/0271121416628200.X $-1, \mathrm{X}-3, \mathrm{X}-4$
4723. Brown RC, Nugent NR, Hawn SE, et al. Predicting the Transition From Acute Stress Disorder to Posttraumatic Stress Disorder in Children With Severe Injuries. J Pediatr Health Care. 2016 Jan 14doi:

10.1016/j.pedhc.2015.11.015. PMID: 26776839.X-1

4724. Bruckheimer E, Rotschild C, Dagan T, et al. Computer-generated real-time digital holography: first time use in clinical medical imaging. Eur Heart J Cardiovasc Imaging. 2016 Jun 9doi: 10.1093/ehjci/jew087. PMID: 27283456.X-1

4725. Brukner-Wertman Y, Laor N, Golan O. Social (Pragmatic) Communication Disorder and Its Relation to the Autism Spectrum: Dilemmas Arising From the DSM-5 Classification. J Autism Dev Disord. 2016 May 26doi: 10.1007/s10803-016-2814-5. PMID: 27230759.X-1

4726. Bruno JL, Hosseini SM, Saggar M, et al. Altered Brain Network Segregation in Fragile X Syndrome Revealed by Structural Connectomics. Cereb Cortex. 2016 Mar 22doi: 10.1093/cercor/bhw055. PMID: 27009247.X-1

4727. Bryant CD, Yazdani N. RNA-binding proteins, neural development and the addictions. Genes Brain Behav. 2016 Jan;15(1):169-86. doi: 10.1111/gbb.12273. PMID: 26643147.X-1

4728. Buckland AJ, Vira S, Oren JH, et al. When is compensation for lumbar spinal stenosis a clinical sagittal plane deformity? Spine J. 2016 Apr 5doi:

10.1016/j.spinee.2016.03.047. PMID: 27063925.X-1

4729. Bueno C, Tabares-Seisdedos R, Moraleda JM, et al. Rett Syndrome Mutant Neural Cells Lacks MeCP2 Immunoreactive Bands. PLoS One. 2016;11(4):e0153262. doi: 10.1371/journal.pone.0153262. PMID: 27064487.X-1

4730. Buffington SA, Di Prisco GV, Auchtung TA, et al. Microbial Reconstitution Reverses Maternal Diet-Induced Social and Synaptic Deficits in Offspring. Cell. 2016 Jun 16;165(7):1762-75. doi: 10.1016/j.cell.2016.06.001. PMID: 27315483.X-1 
4731. Bulkeley K, Bundy A, Roberts J, et al. Family-Centered Management of Sensory Challenges of Children With Autism: SingleCase Experimental Design. Am J Occup Ther. 2016 Sep-Oct;70(5):7005220040p1-8. doi: 10.5014/ajot.2016.017822. PMID: 27548868.X-3, X-4

4732. Bull LE, Oliver C, Woodcock KA. Signalling changes to individuals who show resistance to change can reduce challenging behaviour. J Behav Ther Exp Psychiatry. 2016 Jun 22;54:58-70. doi: 10.1016/j.jbtep.2016.06.006. PMID: 27367567.X-1

4733. Bultas MW, Johnson NL, Burkett K, et al. Translating Research to Practice for Children With Autism Spectrum Disorder: Part 2: Behavior Management in Home and Health Care Settings. J Pediatr Health Care. 2016 JanFeb;30(1):27-37. doi: 10.1016/j.pedhc.2015.09.009. PMID: 26525946.X-1

4734. Bultas MW, McMillin SE, Zand DH. Reducing Barriers to Care in the Office-Based Health Care Setting for Children With Autism. J Pediatr Health Care. 2016 Jan-Feb;30(1):5-14. doi: 10.1016/j.pedhc.2015.08.007. PMID: 26455786.X-1

4735. Bulut MO, Yucel IK, Kucuk M, et al. Initial Experience with the Nit-Occlud ASD-R: Short-Term Results. Pediatr Cardiol. 2016 Jun 9doi: 10.1007/s00246-016-1426-3. PMID: 27278631.X-1

4736. Burbach JP. AUTISM. Unraveling a pathway to autism. Science. 2016 Mar 11;351(6278):1153-4. doi: 10.1126/science.aaf5097. PMID: 26965613.X$1, \mathrm{X}-2$

4737. Burger-Caplan R, Saulnier C, Jones $\mathrm{W}$, et al. Predicting social and communicative ability in school-age children with autism spectrum disorder: A pilot study of the Social Attribution Task, Multiple Choice. Autism. 2016 Apr 27doi: 10.1177/1362361315617589. PMID: 27121244.X-1

4738. Burger-Veltmeijer AEJ, Minnaert AEMG, Van den Bosch EJ. Intellectually gifted students with possible characteristics of ASD:
A multiple case study of psycho-educational assessment practices. European Journal of Special Needs Education. 2016;31(1):76-95. doi:

http://dx.doi.org/10.1080/08856257.2015.10871 47.X-1

4739. Burke M, Heller T. Individual, parent and social-environmental correlates of caregiving experiences among parents of adults with autism spectrum disorder. J Intellect Disabil Res. 2016 May;60(5):401-11. doi: 10.1111/jir.12271. PMID: 27120984.X-1

4740. Burke MM, Magana S, Garcia M, et al. Brief Report: The Feasibility and Effectiveness of an Advocacy Program for Latino Families of Children with Autism Spectrum Disorder. J Autism Dev Disord. 2016 Jul;46(7):2532-8. doi: 10.1007/s10803-016-2765-x. PMID: 26944592.X-1

4741. Burkhardt BW, Brielmaier M, Schwerdtfeger K, et al. Smith-Robinson Procedure with an Autologus Iliac Crest for Degenerative Cervical Disc Disease A 28-year Follow-Up of 95 Patients. World Neurosurg. 2016 May 26doi: 10.1016/j.wneu.2016.05.036. PMID: 27237417.X-1

4742. Burkhardt BW, Brielmeier M, Schwerdtfeger K, et al. 311 Smith-Robinson Procedure With an Autologous Iliac Crest Bone Graft With and Without Caspar Plating as a Treatment for Soft Cervical Disc Herniation: Report of 122 Patients With an Average Follow-up of 25 Years. Neurosurgery. 2016 Aug;63 Suppl 1:189-90. doi: 10.1227/01.neu.0000489800.11738.0d. PMID: 27399509.X-1

4743. Burns CO, Matson JL, Cervantes PE, et al. Hearing impairment, autism spectrum disorder, and developmental functioning in infants and toddlers. Journal of Developmental and Physical Disabilities. 2016;28(4):495-507. doi: http://dx.doi.org/10.1007/s10882-0169486-0.X-1

4744. Burrows CA, Laird AR, Uddin LQ. Functional connectivity of brain regions for self- and other-evaluation in children, adolescents and adults with autism. Dev Sci. 2016 Jan 11doi: 10.1111/desc.12400. PMID: 26750447.X-1, X-2, X-3 
4745. Burrows CA, Laird AR, Uddin LQ. Functional connectivity of brain regions for self- and other-evaluation in children, adolescents and adults with autism. Developmental Science. 2016;19(4):564-80. doi: http://dx.doi.org/10.1111/desc.12400.X-1

4746. Burrows CA, Usher LV, Schwartz CB, et al. Supporting the spectrum hypothesis: Selfreported temperament in children and adolescents with high functioning autism. Journal of Autism and Developmental Disorders. 2016;46(4):1184-95. doi: http://dx.doi.org/10.1007/s10803-015-26539.X-1

4747. Burt SA, Klump KL, Gorman-Smith $D$, et al. Neighborhood disadvantage alters the origins of children's nonaggressive conduct problems. Clinical Psychological Science. 2016;4(3):511-26. doi: http://dx.doi.org/10.1177/2167702615618164.X $-1$

4748. Burton CL, Crosbie J, Dupuis A, et al. Clinical correlates of hoarding with and without comorbid obsessive-compulsive symptoms in a community pediatric sample. Journal of the American Academy of Child \& Adolescent Psychiatry. 2016;55(2):114-21. doi: http://dx.doi.org/10.1016/j.jaac.2015.11.014.X1

4749. Butera G, Lovin N, Basile DP. How to deal with atrial septal defect closure from right internal jugular vein: Role of venous-arterial circuit for sizing and over-the-wire device implantation. Catheter Cardiovasc Interv. 2016 Jun 17doi: 10.1002/ccd.26621. PMID: 27315359.X-1

4750. Butera G, Lovin N, Basile DP. Patent ductus arteriosus balloon sizing: A new technique to evaluate the size in complex cases. Catheter Cardiovasc Interv. 2016 May;87(6):1135-7. doi: 10.1002/ccd.26331. PMID: 26602995.X-1

4751. Cadette JN, Wilson CL, Brady MP, et al. The Effectiveness of Direct Instruction in Teaching Students with Autism Spectrum Disorder to Answer "Wh-" Questions. J Autism Dev Disord. 2016 Jun 16doi: 10.1007/s10803016-2825-2. PMID: 27312714.X-1, X-3, X-4
4752. Caeyenberghs K, Taymans T, Wilson $\mathrm{PH}$, et al. Neural signature of developmental coordination disorder in the structural connectome independent of comorbid autism. Dev Sci. 2016 May 4doi: 10.1111/desc.12424. PMID: 27147441.X-1

4753. Cage E, Bird G, Pellicano L. 'I am who I am': Reputation concerns in adolescents on the autism spectrum. Research in Autism Spectrum Disorders. 2016;25:12-23. doi: http://dx.doi.org/10.1016/j.rasd.2016.01.010.X1

4754. Cai J, Ding L, Zhang J-S, et al. Elevated plasma levels of glutamate in children with autism spectrum disorders. NeuroReport: For Rapid Communication of Neuroscience Research. 2016;27(4):272-6. doi: http://dx.doi.org/10.1097/WNR.000000000000 0532.X-1

4755. Cairns J, Swanson D, Yeung J, et al. Abnormalities in the Structure and Function of Cerebellar Neurons and Neuroglia in the Lc/+ Chimeric Mouse Model of Variable Developmental Purkinje Cell Loss. Cerebellum. 2016 Feb 2doi: 10.1007/s12311-015-0756-7. PMID: 26837618.X-1

4756. Call NA, Alvarez JP, Simmons CA, et al. Clinical outcomes of behavioral treatments for elopement in individuals with autism spectrum disorder and other developmental disabilities. Autism. 2016 May 12doi: 10.1177/1362361316644732. PMID: 27178995.X-1, X-3

4757. Campbell SB, Leezenbaum NB, Mahoney AS, et al. Pretend play and social engagement in toddlers at high and low genetic risk for autism spectrum disorder. Journal of Autism and Developmental Disorders. 2016;46(7):2305-16. doi: http://dx.doi.org/10.1007/s10803-016-2764y.X-1

4758. Campione GC, Piazza C, Villa L, et al. Three-Dimensional Kinematic Analysis of Prehension Movements in Young Children with Autism Spectrum Disorder: New Insights on Motor Impairment. J Autism Dev Disord. 2016 Jun;46(6):1985-99. doi: 10.1007/s10803-0162732-6. PMID: 26861718.X-1 
4759. Campistol J, Diez-Juan M, Callejon L, et al. Inborn error metabolic screening in individuals with nonsyndromic autism spectrum disorders. Dev Med Child Neurol. 2016 Mar 31doi: 10.1111/dmcn.13114. PMID: 27038397.X-1

4760. Campos LK, Fernandes FD. School profile and language and cognitive abilities of children and adolescents with autism spectrum disorders. Codas. 2016 Jun 16;0:0. doi: 10.1590/2317-1782/20162015023. PMID: 27332949.X-1

4761. Canals J, Morales-Hidalgo P, Jane MC, et al. ADHD Prevalence in Spanish Preschoolers: Comorbidity, Socio-Demographic Factors, and Functional Consequences. J Atten Disord. 2016 Mar 23doi:

10.1177/1087054716638511. PMID: 27009923.X-1

4762. Candini M, Giuberti V, Manattini A, et al. Personal space regulation in childhood autism: Effects of social interaction and person's perspective. Autism Res. 2016 May 9doi: 10.1002/aur.1637. PMID: 27157094.X-1

4763. Canfield AR, Eigsti IM, de Marchena A, et al. Story Goodness in Adolescents With Autism Spectrum Disorder (ASD) and in Optimal Outcomes From ASD. J Speech Lang Hear Res. 2016 Jun 1;59(3):533-45. doi: 10.1044/2015_jslhr-l-15-0022. PMID: 27280731.X-1

4764. Cantio C, Jepsen JR, Madsen GF, et al. Exploring 'The autisms' at a cognitive level. Autism Res. 2016 Apr 19doi: 10.1002/aur.1630. PMID: 27091289.X-1

4765. Cao Y, Wang J, Wei C, et al. Genetic variations of NKX2-5 in sporadic atrial septal defect and ventricular septal defect in Chinese Yunnan population. Gene. 2016 Jan 1;575(1):29-33. doi:

10.1016/j.gene.2015.08.033. PMID: 26297999.X-1

4766. Capriola NN, Maddox BB, White SW. No Offense Intended: Fear of Negative Evaluation in Adolescents and Adults with Autism Spectrum Disorder. J Autism Dev Disord. 2016 Jun 20doi: 10.1007/s10803-0162827-0. PMID: 27324492.X-1
4767. Carbone PS, Norlin C, Young PC. Improving Early Identification and Ongoing Care of Children With Autism Spectrum Disorder. Pediatrics. 2016 Jun;137(6)doi: 10.1542/peds.2015-1850. PMID: 27244841.X-1

4768. Cariveau T, Kodak T, Campbell V. The effects of intertrial interval and instructional format on skill acquisition and maintenance for children with autism spectrum disorders. J Appl Behav Anal. 2016 May 26doi: 10.1002/jaba.322. PMID: 27227899.X-1

4769. Carr ME. Self-management of challenging behaviours associated with autism spectrum disorder: A meta-analysis. Australian Psychologist. 2016;51(4):316-33. doi: http://dx.doi.org/10.1111/ap.12227.X-2

4770. Carr T, Lord C. A Pilot Study Promoting Participation of Families with Limited Resources in Early Autism Intervention. Res Autism Spectr Disord. 2016 May 1;2:87-96. doi:

10.1016/j.rasd.2016.02.003. PMID: 27019670.X-1

4771. Carrigan N, Allez K. Cognitive Behaviour Therapy for Post-Traumatic Stress Disorder in a person with an Autism Spectrum Condition and Intellectual Disability: A Case Study. J Appl Res Intellect Disabil. 2016 Feb 12doi: 10.1111/jar.12243. PMID: 26868276.X$1, \mathrm{X}-3$

4772. Carroll RA, Kodak T, Adolf KJ. Effect of delayed reinforcement on skill acquisition during discrete-trial instruction: Implications for treatment-integrity errors in academic settings. Journal of Applied Behavior Analysis. 2016;49(1):176-81. doi: http://dx.doi.org/10.1002/jaba.268.X-3, X-4

4773. Carson TB, Wilkes BJ, Patel K, et al. Vestibulo-ocular reflex function in children with high-functioning autism spectrum disorders. Autism Res. 2016 May 25doi: 10.1002/aur.1642. PMID: 27220548.X-1

4774. Carter CJ. The barrier, airway particle clearance, placental and detoxification functions of autism susceptibility genes. Neurochem Int. 2016 Jun 11;99:42-51. doi: 10.1016/j.neuint.2016.06.003. PMID: 27296113.X-1 
4775. Casanova EL, Sharp JL, Chakraborty $\mathrm{H}$, et al. Genes with high penetrance for syndromic and non-syndromic autism typically function within the nucleus and regulate gene expression. Mol Autism. 2016;7:18. doi: 10.1186/s13229-016-0082-z. PMID: 26985359.X-1

4776. Casartelli L, Chiamulera C. The motor way: Clinical implications of understanding and shaping actions with the motor system in autism and drug addiction. Cogn Affect Behav Neurosci. 2016 Apr;16(2):191-206. doi: 10.3758/s13415-015-0399-7. PMID: 26679723.X-1

4777. Cascia J, Barr JJ. Associations Among Vocabulary, Executive Function Skills and Empathy in Individuals with Autism Spectrum Disorder. J Appl Res Intellect Disabil. 2016 May 2doi: 10.1111/jar.12257. PMID: 27137895.X-1

4778. Cascio CJ, Woynaroski T, Baranek GT, et al. Toward an interdisciplinary approach to understanding sensory function in autism spectrum disorder. Autism Res. 2016 Apr 19doi: 10.1002/aur.1612. PMID: 27090878.X-1

4779. Case LK, Laubacher CM, Olausson H, et al. Encoding of Touch Intensity But Not Pleasantness in Human Primary Somatosensory Cortex. J Neurosci. 2016 May 25;36(21):585060. doi: 10.1523/jneurosci.1130-15.2016. PMID: 27225773.X-1

4780. Casella G, Bordo BM, Schalling R, et al. Neurological disorders and celiac disease. Minerva Gastroenterol Dietol. 2016 Apr 6 PMID: 27050467.X-1

4781. Cashin A. Autism Spectrum Disorder and Psychosis: A Case Study. J Child Adolesc Psychiatr Nurs. 2016 Jun 17doi: 10.1111/jcap.12145. PMID: 27311754.X-1

4782. Cassidy SA, Stenger B, Van Dongen L, et al. Expressive visual text-to-speech as an assistive technology for individuals with autism spectrum conditions. Comput Vis Image Underst. 2016 Jul;148:193-200. doi: 10.1016/j.cviu.2015.08.011. PMID: 27375348.X-1

4783. Castro K, Baronio D, Perry IS, et al. The effect of ketogenic diet in an animal model of autism induced by prenatal exposure to valproic acid. Nutr Neurosci. 2016 Feb 9doi: 10.1080/1028415x.2015.1133029. PMID: 26856821.X-1

4784. Castro VM, Kong SW, Clements CC, et al. Absence of evidence for increase in risk for autism or attention-deficit hyperactivity disorder following antidepressant exposure during pregnancy: a replication study. Transl Psychiatry. 2016;6:e708. doi: 10.1038/tp.2015.190. PMID: 26731445.X-1

4785. Catani M, Dell'Acqua F, Budisavljevic $\mathrm{S}$, et al. Frontal networks in adults with autism spectrum disorder. Brain. 2016 Feb;139(Pt 2):616-30. doi: 10.1093/brain/awv351. PMID: 26912520.X-1

4786. Çelik G, Taş D, Tahiroğlu A, et al. Vitamin D deficiency in obsessive-compulsive disorder patients with pediatric autoimmune neuropsychiatric disorders associated with streptococcal infections: A case control study. Nöropsikiyatri Arşivi / Archives of Neuropsychiatry. 2016;53(1):33-7. doi: http://dx.doi.org/10.5152/npa.2015.8763.X-1

4787. Cengher M, Shamoun K, Moss P, et al. A comparison of the effects of two promptfading strategies on skill acquisition in children with autism spectrum disorders. Behavior Analysis in Practice. 2016;9(2):115-25. doi: http://dx.doi.org/10.1007/s40617-015-00966.X-3, X-4

4788. Cesta CE, Mansson M, Palm C, et al. Polycystic ovary syndrome and psychiatric disorders: Co-morbidity and heritability in a nationwide Swedish cohort.

Psychoneuroendocrinology. 2016 Aug

3;73:196-203. doi:

10.1016/j.psyneuen.2016.08.005. PMID:

27513883.X-1

4789. Cetin I, Tezdig I, Tarakcioglu MC, et al. Serum levels of glial fibrillary acidic protein and Nogo-A in children with autism spectrum disorders. Biomarkers. 2016 Apr 21:1-5. doi: 10.3109/1354750x.2016.1171901. PMID: 27097671.X-1

4790. Chaaya M, Saab D, Maalouf FT, et al. Prevalence of autism spectrum disorder in nurseries in Lebanon: A cross sectional study. Journal of Autism and Developmental 
Disorders. 2016;46(2):514-22. doi: http://dx.doi.org/10.1007/s10803-015-25907.X-1

4791. Chambers N, Stronach ST, Wetherby AM. Performance of South African children on the Communication and Symbolic Behavior Scales_-Developmental Profile (CSBS DP). International Journal of Language \& Communication Disorders. 2016;51(3):265-75. doi: http://dx.doi.org/10.1111/14606984.12203.X-1

4792. Chambers NJ, Wetherby AM, Stronach ST, et al. Early detection of autism spectrum disorder in young isiZulu-speaking children in South Africa. Autism. 2016 Jun 22doi: 10.1177/1362361316651196. PMID: 27335105.X-1

4793. Chamie F, Maia J, Giuliano LC. Device-in-device: A transcatheter alternative to surgical explantation of a failing atrial septal defect intracardiac prosthesis. Catheter Cardiovasc Interv. 2016 Feb 19doi: 10.1002/ccd.26456. PMID: 26893203.X-1

4794. Chan JS, Langer A, Kaiser J. Temporal integration of multisensory stimuli in autism spectrum disorder: a predictive coding perspective. J Neural Transm (Vienna). 2016 Jun 20doi: 10.1007/s00702-016-1587-5. PMID: 27324803.X-1

4795. Chandler S, Howlin P, Simonoff E, et al. Comparison of parental estimate of developmental age with measured IQ in children with neurodevelopmental disorders. Child: Care, Health and Development. 2016;42(4):486-93. doi: http://dx.doi.org/10.1111/cch.12346.X-1

4796. Chang AD, Berges VA, Chung SJ, et al. High-Frequency Stimulation at the Subthalamic Nucleus Suppresses Excessive Self-Grooming in Autism-Like Mouse Models. Neuropsychopharmacology. 2016 Jun;41(7):1813-21. doi: 10.1038/npp.2015.350. PMID: 26606849.X-1

4797. Chang J, Go YY, Park MK, et al. Asian Sand Dust Enhances the Inflammatory Response and Mucin Gene Expression in the Middle Ear. Clin Exp Otorhinolaryngol. 2016 Apr 20doi: 10.21053/ceo.2015.01060. PMID: 27095518.X-1
4798. Chang YC, Shire SY, Shih W, et al. Preschool Deployment of Evidence-Based Social Communication Intervention: JASPER in the Classroom. J Autism Dev Disord. 2016 Jun;46(6):2211-23. doi: 10.1007/s10803-0162752-2. PMID: 26936161.X-1

4799. Chang Y-C, Locke J. A systematic review of peer-mediated interventions for children with autism spectrum disorder. Research in Autism Spectrum Disorders. 2016;27:1-10. doi: http://dx.doi.org/10.1016/j.rasd.2016.03.010.X2

4800. Chang Y-C, Shih W, Kasari C. Friendships in preschool children with autism spectrum disorder: What holds them back, child characteristics or teacher behavior? Autism. 2016;20(1):65-74. doi: http://dx.doi.org/10.1177/1362361314567761.X $-1$

4801. Chang YS, Owen JP, Pojman NJ, et al. Reciprocal white matter alterations due to 16p11.2 chromosomal deletions versus duplications. Hum Brain Mapp. 2016 May 24doi: 10.1002/hbm.23211. PMID: 27219475.X-1

4802. Charlson FJ, Baxter AJ, Dua T, et al. Excess Mortality from Mental, Neurological, and Substance Use Disorders in the Global Burden of Disease Study 2010. In: Patel V, Chisholm D, Dua T, Laxminarayan R, MedinaMora ME, eds. Mental, Neurological, and Substance Use Disorders: Disease Control Priorities, Third Edition (Volume 4). Washington (DC): The International Bank for Reconstruction and Development / The World Bank (c) 2016 International Bank for Reconstruction and Development / The World Bank.; 2016.

4803. Charman T, Chakrabarti B.

Commentary: Not just genes--reclaiming a role for environmental influences on aetiology and outcome in autism. A commentary on Mandy and Lai (2016). J Child Psychol Psychiatry. 2016 Mar;57(3):293-5. doi: 10.1111/jcpp.12527. PMID: 26889893.X-1

4804. Chawarska K, Macari S, Powell K, et al. Enhanced social attention in female infant siblings at risk for autism. Journal of the 
American Academy of Child \& Adolescent Psychiatry. 2016;55(3):188-95. doi: http://dx.doi.org/10.1016/j.jaac.2015.11.016.X1

4805. Chawarska K, Ye S, Shic F, et al. Multilevel differences in spontaneous social attention in toddlers with autism spectrum disorder. Child Development. 2016;87(2):54357. doi:

http://dx.doi.org/10.1111/cdev.12473.X-1

4806. Chebli SS, Lanovaz MJ. Using computer tablets to assess preference for videos in children with autism. Behavior Analysis in Practice. 2016;9(1):50-3. doi: http://dx.doi.org/10.1007/s40617-016-0109$0 . \mathrm{X}-1$

4807. Chen C, Hung AY, Fan YT, et al. Linkage between pain sensitivity and empathic response in adolescents with autism spectrum conditions and conduct disorder symptoms. Autism Res. 2016 Jun 16doi: 10.1002/aur.1653. PMID: 27305862.X-1

4808. Chen C, Van Horn JD. Developmental neurogenetics and multimodal neuroimaging of sex differences in autism. Brain Imaging Behav. 2016 Jan 19doi: 10.1007/s11682-015-9504-3. PMID: 26781567.X-1

4809. Chen CA, Bosch DG, Cho MT, et al. The expanding clinical phenotype of BoschBoonstra-Schaaf optic atrophy syndrome: 20 new cases and possible genotype-phenotype correlations. Genet Med. 2016 Mar 17doi: 10.1038/gim.2016.18. PMID: 26986877.X-1

4810. Chen C-H, Lee IJ, Lin L-Y. Augmented reality-based video-modeling storybook of nonverbal facial cues for children with autism spectrum disorder to improve their perceptions and judgments of facial expressions and emotions. Computers in Human Behavior. 2016;51(Part A):477-85. doi: http://dx.doi.org/10.1016/j.chb.2015.09.033.X$1, \mathrm{X}-3$

4811. Chen F. Risperidone-Induced Acute Respiratory Distress in an Adolescent with Autism. J Child Adolesc Psychopharmacol. 2016 May 24doi: 10.1089/cap.2016.0051. PMID: 27220113.X-1
4812. Chen H, Duan X, Liu F, et al. Multivariate classification of autism spectrum disorder using frequency-specific resting-state functional connectivity--A multi-center study. Prog Neuropsychopharmacol Biol Psychiatry. 2016 Jan 4;64:1-9. doi: 10.1016/j.pnpbp.2015.06.014. PMID: 26148789.X-1

4813. Chen H, Duan X, Liu F, et al. Multivariate classification of autism spectrum disorder using frequency-specific resting-state functional connectivity-A multi-center study. Progress in Neuro-Psychopharmacology \& Biological Psychiatry. 2016;64:1-9. doi: http://dx.doi.org/10.1016/j.pnpbp.2015.06.014. $\mathrm{X}-1$

4814. Chen H, Zhao B, Porges EC, et al. Edgewise and subgraph-level tests for brain networks. Stat Med. 2016 Jul 11doi: 10.1002/sim.7039. PMID: 27397632.X-1

4815. Chen J, Qi B, Zhao J, et al. A novel mutation of GATA4 (K300T) associated with familial atrial septal defect. Gene. 2016 Jan 10;575(2 Pt 2):473-7. doi: 10.1016/j.gene.2015.09.021. PMID: 26376067.X-1

4816. Chen PJ, Gau SS, Lee SH, et al. Differences in age-dependent neural correlates of semantic processing between youths with autism spectrum disorder and typically developing youths. Autism Res. 2016 Feb 21doi: 10.1002/aur.1616. PMID: 26899269.X-1

4817. Chen R, Nixon E, Herskovits E. Advanced Connectivity Analysis (ACA): A large scale functional connectivity data mining environment. Neuroinformatics.

2016;14(2):191-9. doi: http://dx.doi.org/10.1007/s12021-015-92905.X-1

4818. Chen SF, Chien YL, Wu CT, et al. Deficits in executive functions among youths with autism spectrum disorders: An agestratified analysis. Psychological Medicine. 2016;46(8):1625-38. doi: http://dx.doi.org/10.1017/S0033291715002238. $\mathrm{X}-1$

4819. Chen S-w, Zhong X-s, Jiang L-n, et al. Maternal autoimmune diseases and the risk of autism spectrum disorders in offspring: A 
systematic review and meta-analysis.

Behavioural Brain Research. 2016;296:61-9.

doi:

http://dx.doi.org/10.1016/j.bbr.2015.08.035.X-1

4820. Chen XL, Guan L, Liu YZ, et al. Interspinous dynamic stabilization adjacent to fusion versus double-segment fusion for treatment of lumbar degenerative disease with a minimum follow-up of three years. Int Orthop. 2016 Jun;40(6):1275-83. doi: 10.1007/s00264016-3199-y. PMID: 27118374.X-1

4821. Chen Y, Wang S, Wang S, et al. Sodium Lauryl Sulfate Competitively Interacts with HPMC-AS and Consequently Reduces Oral Bioavailability of Posaconazole/HPMCAS Amorphous Solid Dispersion. Mol Pharm. 2016 Jul 1doi:

10.1021/acs.molpharmaceut.6b00391. PMID: 27337060.X-1

4822. Chen Y, Wang S, Wang S, et al. Initial Drug Dissolution from Amorphous Solid Dispersions Controlled by Polymer Dissolution and Drug-Polymer Interaction. Pharm Res. 2016 Jun 9doi: 10.1007/s11095-016-1969-2. PMID: 27283830.X-1

4823. Chen YW, Bundy AC, Cordier R, et al. A cross-cultural exploration of the everyday social participation of individuals with autism spectrum disorders in Australia and Taiwan: An experience sampling study. Autism. 2016 Apr 27doi: 10.1177/1362361316636756. PMID: 27121243.X-1

4824. Chen Z, Trapp BD. Microglia and neuroprotection. J Neurochem. 2016 Jan;136 Suppl 1:10-7. doi: 10.1111/jnc.13062. PMID: 25693054.X-1

4825. Cheng C, Lau SK, Doering LC. Astrocyte-secreted thrombospondin-1 modulates synapse and spine defects in the fragile X mouse model. Mol Brain. 2016;9(1):74. doi: 10.1186/s13041-016-0256-9. PMID: 27485117.X-1

4826. Chenxi L, Chen Y, Li Y, et al. Complexity analysis of brain activity in attention-deficit/hyperactivity disorder: A multiscale entropy analysis. Brain Research Bulletin. 2016;124:12-20. doi: http://dx.doi.org/10.1016/j.brainresbull.2016.03 .007.X-1
4827. Cheuk S, Lashewicz B. How are they doing? Listening as fathers of children with autism spectrum disorder compare themselves to fathers of children who are typically developing. Autism. 2016;20(3):343-52. doi: http://dx.doi.org/10.1177/1362361315584464.X $-1$

4828. Chezan L, Drasgow E, Legg J, et al. Effects of conditional discrimination training and choice opportunities on manding for two young children with Autism Spectrum Disorder and language delays. Journal of Developmental and Physical Disabilities. 2016;28(4):557-79. doi: http://dx.doi.org/10.1007/s10882-0169493-1.X-3, X-4

4829. Chezan LC, Drasgow E, Martin CA, et al. Negatively-Reinforced Mands: An Examination of Resurgence to Existing Mands in Two Children With Autism and Language Delays. Behav Modif. 2016 May 9doi: 10.1177/0145445516648664. PMID: 27161587.X-1, X-3, X-4

4830. Chi DL, Momany ET, Mancl LA, et al. Dental homes for children with autism: A longitudinal analysis of Iowa Medicaid's ISmile Program. American Journal of Preventive Medicine. 2016;50(5):609-15. doi: http://dx.doi.org/10.1016/j.amepre.2015.08.022. $\mathrm{X}-1$

4831. Chien HY, Gau SS, Isaac Tseng WY. Deficient visuospatial working memory functions and neural correlates of the defaultmode network in adolescents with autism spectrum disorder. Autism Res. 2016 Feb 1doi: 10.1002/aur.1607. PMID: 26829405.X-1

4832. Chiocchetti AG, Haslinger D, Stein JL, et al. Transcriptomic signatures of neuronal differentiation and their association with risk genes for autism spectrum and related neuropsychiatric disorders. Transl Psychiatry. 2016;6(8):e864. doi: 10.1038/tp.2016.119. PMID: 27483382.X-1

4833. Chiu MM, Roberts CA. Improved analyses of single cases: Dynamic multilevel analysis. Dev Neurorehabil. 2016 Feb 10:1-13. doi: 10.3109/17518423.2015.1119904. PMID: 26864247.X-1 
4834. Choi CH, Schoenfeld BP, Bell AJ, et al. Multiple Drug Treatments That Increase cAMP Signaling Restore Long-Term Memory and Aberrant Signaling in Fragile X Syndrome Models. Front Behav Neurosci. 2016;10:136. doi: 10.3389/fnbeh.2016.00136. PMID: 27445731.X-1

4835. Choi GB, Yim YS, Wong H, et al. The maternal interleukin-17a pathway in mice promotes autism-like phenotypes in offspring. Science. 2016 Feb 26;351(6276):933-9. doi: 10.1126/science.aad0314. PMID: 26822608.X1

4836. Cholemkery H, Medda J, Lempp T, et al. Classifying autism spectrum disorders by ADI-R: Subtypes or severity gradient? Journal of Autism and Developmental Disorders. 2016;46(7):2327-39. doi: http://dx.doi.org/10.1007/s10803-016-27602.X-1

4837. Chong WH, Kua SM. Parenting SelfEfficacy Beliefs in Parents of Children With Autism: Perspectives From Singapore. Am J Orthopsychiatry. 2016 Apr 14doi: 10.1037/ort0000169. PMID: 27078047.X-1, X3, $\mathrm{X}-4$

4838. Choque Olsson N, Rautio D, Asztalos $\mathrm{J}$, et al. Social skills group training in highfunctioning autism: A qualitative responder study. Autism. 2016 Feb 24doi: 10.1177/1362361315621885. PMID: 26912485.X-1

4839. Christensen DL, Baio J, Van Naarden Braun K, et al. Prevalence and Characteristics of Autism Spectrum Disorder Among Children Aged 8 Years--Autism and Developmental Disabilities Monitoring Network, 11 Sites, United States, 2012. MMWR Surveill Summ. 2016 Apr 1;65(3):1-23. doi: 10.15585/mmwr.ss6503a1. PMID: 27031587.X-1

4840. Christensen DL, Bilder DA, Zahorodny W, et al. Prevalence and characteristics of autism spectrum disorder among 4-year-old children in the autism and developmental disabilities monitoring network. Journal of Developmental and Behavioral Pediatrics. 2016;37(1):1-8. doi: http://dx.doi.org/10.1097/DBP.0000000000000 235.X-1
4841. Christian EJ, Meltzer CL, Thede LL, et al. The Relationship Between Early Life Events, Parental Attachment, and Psychopathic Tendencies in Adolescent Detainees. Child Psychiatry Hum Dev. 2016 Mar 30doi: 10.1007/s10578-016-0638-3. PMID: 27027884.X-1, X-3

4842. Christiansz JA, Gray KM, Taffe J, et al. Autism spectrum disorder in the DSM-5: Diagnostic sensitivity and specificity in early childhood. Journal of Autism and Developmental Disorders. 2016;46(6):2054-63. doi: http://dx.doi.org/10.1007/s10803-0162734-4.X-1

4843. Christoffersen GR, Schachtman TR. Electrophysiological CNS-processes related to associative learning in humans. Behav Brain Res. 2016 Jan 1;296:211-32. doi: 10.1016/j.bbr.2015.09.011. PMID: 26367470.X-1

4844. Christou AI, Endo S, Wallis Y, et al. Variation in serotonin transporter linked polymorphic region (5-HTTLPR) short/long genotype modulates resting frontal electroencephalography asymmetries in children. Development and Psychopathology. 2016;28(1):239-50. doi: http://dx.doi.org/10.1017/S0954579415000413. $\mathrm{X}-1$

4845. Chrobak AA, Soltys Z. Bergmann Glia, Long-Term Depression, and Autism Spectrum Disorder. Mol Neurobiol. 2016 Jan 26doi: 10.1007/s12035-016-9719-3. PMID: 26809583.X-1

4846. Chung J, Tchaconas A, Meryash D, et al. Treatment of attention-deficit/hyperactivity disorder in preschool-age children: Child and adolescent psychiatrists' adherence to clinical practice guidelines. Journal of Child and Adolescent Psychopharmacology. 2016;26(4):335-43. doi: http://dx.doi.org/10.1089/cap.2015.0108.X-1

4847. Cianfaglione R, Meek A, Clarke A, et al. Direct observation of the behaviour of females with Rett syndrome. Journal of Developmental and Physical Disabilities. 2016;28(3):425-41. doi: http://dx.doi.org/10.1007/s10882-016-9478$0 . \mathrm{X}-1$ 
4848. Ciernia AV, LaSalle J. The landscape of DNA methylation amid a perfect storm of autism aetiologies. Nat Rev Neurosci. 2016 Jul;17(7):411-23. doi: 10.1038/nrn.2016.41. PMID: 27150399.X-1

4849. Cioffi CL, Guzzo PR. Inhibitors of Glycine Transporter-1: Potential Therapeutics for the Treatment of CNS Disorders. Curr Top Med Chem. 2016 Apr 5 PMID: 27048272.X-1

4850. Clarke C, Hill V, Charman T. School based cognitive behavioural therapy targeting anxiety in children with autistic spectrum disorder: a quasi-experimental randomised controlled trail incorporating a mixed methods approach. J Autism Dev Disord. 2016 Apr 30doi: 10.1007/s10803-016-2801-x. PMID: 27138893.X-1, X-3

4851. Clince M, Connolly L, Nolan C. Comparing and Exploring the Sensory Processing Patterns of Higher Education Students With Attention Deficit Hyperactivity Disorder and Autism Spectrum Disorder. Am J Occup Ther. 2016 Mar-

Apr;70(2):7002250010p1-9. doi: 10.5014/ajot.2016.016816. PMID: 26943106.X-1, X-3

4852. Clipperton-Allen AE, Chen Y, Page DT. Autism-relevant behaviors are minimally impacted by conditional deletion of Pten in oxytocinergic neurons. Autism Res. 2016 May 25doi: 10.1002/aur.1641. PMID: 27220363.X-1

4853. Cohen IL, Liu X, Hudson M, et al. Using the PDD Behavior Inventory as a Level 2 Screener: A Classification and Regression Trees Analysis. J Autism Dev Disord. 2016 Jun 18doi: 10.1007/s10803-016-2843-0. PMID: 27318809.X-1

4854. Colbert AM, Webber J, Graham R. Factors that Influence Autism Knowledge in Hispanic Cultures: a Pilot Study. J Racial Ethn Health Disparities. 2016 Mar 11doi: 10.1007/s40615-016-0213-4. PMID: 26969159.X-1

4855. Conallen K, Reed P. A teaching procedure to help children with autistic spectrum disorder to label emotions. Research in Autism Spectrum Disorders. 2016;23:63-72. doi:
http://dx.doi.org/10.1016/j.rasd.2015.11.006.X$1, \mathrm{X}-3$

4856. Congiu S, Fadda R, Doneddu G, et al. Impaired representational gaze following in children with autism spectrum disorder. Res Dev Disabil. 2016 Jun 24;57:11-7. doi: 10.1016/j.ridd.2016.06.008. PMID: 27348855.X-1

4857. Connolly A, Fielding J, Papadopoulos $\mathrm{N}$, et al. Factors Associated With Accidental Injuries in Children With ADHD-Combined Type: More Than a Motor Problem? J Atten Disord. 2016 Mar 14doi: 10.1177/1087054716633857. PMID: 26975838.X-1

4858. Connolly N, Anixt J, Manning P, et al. Maternal metabolic risk factors for autism spectrum disorder-An analysis of electronic medical records and linked birth data. Autism Res. 2016 Jan 29doi: 10.1002/aur.1586. PMID: 26824581.X-1

4859. Connolly SC, Millians M, Peterman R, et al. The clinical application of applied behavior analysis in a child with partial fetal alcohol syndrome: A case study. Clinical Case Studies. 2016;15(3):225-42. doi: http://dx.doi.org/10.1177/1534650116632298.X $-1$

4860. Conson M, Hamilton A, De Bellis F, et al. Body constraints on motor simulation in autism spectrum disorders. Journal of Autism and Developmental Disorders.

2016;46(3):1051-60. doi: http://dx.doi.org/10.1007/s10803-015-2652x.X-1

4861. Constantino JN, Charman T. Diagnosis of autism spectrum disorder: reconciling the syndrome, its diverse origins, and variation in expression. Lancet Neurol. 2016 Mar;15(3):279-91. doi: 10.1016/s14744422(15)00151-9. PMID: 26497771.X-1

4862. Contarino VE, Bulgheroni S, Annunziata S, et al. Widespread Focal Cortical Alterations in Autism Spectrum Disorder with Intellectual Disability Detected by ThresholdFree Cluster Enhancement. AJNR Am J Neuroradiol. 2016 Apr 21doi: 10.3174/ajnr.A4779. PMID: 27102310.X-1 
4863. Contreras BP, Betz AM. Using lag schedules to strengthen the intraverbal repertoires of children with autism. Journal of Applied Behavior Analysis. 2016;49(1):3-16. doi: http://dx.doi.org/10.1002/jaba.271.X-1, X3, $\mathrm{X}-4$

4864. Coogle CG, Hanline MF. An exploratory study of family-centred help-giving practices in early intervention: Families of young children with autism spectrum disorder. Child \& Family Social Work. 2016;21(2):24960. doi: http://dx.doi.org/10.1111/cfs.12148.X-1

4865. Cope ZA, Powell SB, Young JW. Modeling neurodevelopmental cognitive deficits in tasks with cross-species translational validity. Genes Brain Behav. 2016 Jan;15(1):27-44. doi: 10.1111/gbb.12268. PMID: 26667374.X-1

4866. Copf T. Impairments in dendrite morphogenesis as etiology for neurodevelopmental disorders and implications for therapeutic treatments. Neurosci Biobehav Rev. 2016 Apr 30doi: 10.1016/j.neubiorev.2016.04.008. PMID: 27143622.X-1

4867. Copping NA, Berg EL, Foley GM, et al. Touchscreen learning deficits and normal social approach behavior in the Shank3B model of Phelan-McDermid Syndrome and autism. Neuroscience. 2016 May 14doi: 10.1016/j.neuroscience.2016.05.016. PMID: 27189882.X-1

4868. Corbett BA, Blain SD, Ioannou S, et al. Changes in anxiety following a randomized control trial of a theatre-based intervention for youth with autism spectrum disorder. Autism. 2016 May 5doi: 10.1177/1362361316643623. PMID: 27154909.X-1

4869. Corbett BA, Muscatello RA, Blain SD. Impact of Sensory Sensitivity on Physiological Stress Response and Novel Peer Interaction in Children with and without Autism Spectrum Disorder. Front Neurosci. 2016;10:278. doi: 10.3389/fnins.2016.00278. PMID: 27445653.X-1

4870. Corona LL, Fox SA, Christodulu KV, et al. Providing education on sexuality and relationships to adolescents with autism spectrum disorder and their parents. Sexuality and Disability. 2016;34(2):199-214. doi: http://dx.doi.org/10.1007/s11195-015-94246.X-1

4871. Cortelazzo A, De Felice C, Guerranti $\mathrm{R}$, et al. Expression and oxidative modifications of plasma proteins in autism spectrum disorders: Interplay between inflammatory response and lipid peroxidation. Proteomics Clin Appl. 2016 Jun 1doi: 10.1002/prca.201500076. PMID: 27246309.X-1

4872. Coshway L, Broussard J, Acharya K, et al. Medical Therapy for Inappropriate Sexual Behaviors in a Teen With Autism Spectrum Disorder. Pediatrics. 2016 Apr;137(4)doi: 10.1542/peds.2015-4366. PMID: 26936858.X1, X-3, X-4

4873. Coskun S, Simsek S, Camkurt MA, et al. Association of polymorphisms in the vitamin D receptor gene and serum 25-hydroxyvitamin D levels in children with autism spectrum disorder. Gene. 2016 Aug 22;588(2):109-14. doi: 10.1016/j.gene.2016.05.004. PMID: 27155524.X-1

4874. Costa V, Aigner S, Vukcevic M, et al. mTORC1 Inhibition Corrects Neurodevelopmental and Synaptic Alterations in a Human Stem Cell Model of Tuberous Sclerosis. Cell Rep. 2016 Apr 5;15(1):86-95. doi: 10.1016/j.celrep.2016.02.090. PMID: 27052171.X-1

4875. Coster WJ, Kramer JM, Tian F, et al. Evaluating the appropriateness of a new computer-administered measure of adaptive function for children and youth with autism spectrum disorders. Autism. 2016;20(1):14-25. doi:

http://dx.doi.org/10.1177/1362361314564473.X $-1$

4876. Courtemanche AB, Black WR. Everyday expressions of pain in children with and without autism spectrum disorder. Research in Autism Spectrum Disorders. 2016;26:65-70. doi: http://dx.doi.org/10.1016/j.rasd.2016.02.010.X1

4877. Courtemanche AB, Black WR, Reese RM. The Relationship Between Pain, SelfInjury, and Other Problem Behaviors in Young Children With Autism and Other 
Developmental Disabilities. Am J Intellect Dev Disabil. 2016 May;121(3):194-203. doi: 10.1352/1944-7558-121.3.194. PMID: 27119211.X-1

4878. Cowley B, Kirjanen S, Partanen J, et al. Epileptic Electroencephalography Profile Associates with Attention Problems in Children with Fragile X Syndrome: Review and Case Series. Front Hum Neurosci. 2016;10:353. doi: 10.3389/fnhum.2016.00353. PMID:

27462212.X-1

4879. Craciun EC, Bjorklund G, Tinkov AA, et al. Evaluation of whole blood zinc and copper levels in children with autism spectrum disorder. Metab Brain Dis. 2016 Apr 8doi: 10.1007/s11011-016-9823-0. PMID: 27059237.X-1

4880. Craig F, Operto FF, De Giacomo A, et al. Parenting stress among parents of children with Neurodevelopmental Disorders. Psychiatry Res. 2016 Jun 1;242:121-9. doi: 10.1016/j.psychres.2016.05.016. PMID: 27280521.X-1

4881. Crawford E, Aplin T, Rodger S. Human rights in occupational therapy education: A step towards a more occupationally just global society. Aust Occup Ther J. 2016 Aug 4doi: 10.1111/14401630.12321. PMID: 27488809.X-1

4882. Creer S, Enderby P, Judge S, et al. Prevalence of people who could benefit from augmentative and alternative communication (AAC) in the UK: determining the need. Int $\mathrm{J}$ Lang Commun Disord. 2016 Apr 26doi: 10.1111/1460-6984.12235. PMID: 27113569.X-1

4883. Crespi BJ. Autism As a Disorder of High Intelligence. Front Neurosci. 2016;10:300. doi: 10.3389/fnins.2016.00300. PMID: 27445671.X-1

4884. Cribb SJ, Olaithe M, Di Lorenzo R, et al. Embedded Figures Test Performance in the Broader Autism Phenotype: A Meta-analysis. J Autism Dev Disord. 2016 Jun 16doi: 10.1007/s10803-016-2832-3. PMID: 27312717.X-1, X-2

4885. Crone RM, Mehta SS. Parent training on generalized use of behavior analytic strategies for decreasing the problem behavior of children with autism spectrum disorder: A data-based case study. Education \& Treatment of Children. 2016;39(1):64-94.X-1, X-3

4886. Crossman MK, Kazdin AE. Additional Evidence is Needed to Recommend Acquiring a Dog to Families of Children with Autism Spectrum Disorder: A Response to Wright and Colleagues. J Autism Dev Disord. 2016 Jan;46(1):332-5. doi: 10.1007/s10803-0152542-2. PMID: 26231204.X-1

4887. Cruchet S, Lucero Y, Cornejo V. Truths, Myths and Needs of Special Diets: Attention-Deficit/Hyperactivity Disorder, Autism, Non-Celiac Gluten Sensitivity, and Vegetarianism. Ann Nutr Metab. 2016;68 Suppl 1:43-50. doi: 10.1159/000445393. PMID: 27356007.X-1

4888. Crutcher E, Ali M, Harrison J, et al. Assessment of Cognitive Outcome Measures in Teenagers with 15q13.3 Microdeletion Syndrome. J Autism Dev Disord. 2016 Apr;46(4):1455-63. doi: 10.1007/s10803-0152694-0. PMID: 26754479.X-1, X-3, X-4

4889. Cubells JF, Schroeder JP, Barrie ES, et al. Human Bacterial Artificial Chromosome (BAC) Transgenesis Fully Rescues Noradrenergic Function in Dopamine betaHydroxylase Knockout Mice. PLoS One. 2016;11(5):e0154864. doi: 10.1371/journal.pone.0154864. PMID: 27148966.X-1

4890. Cui T, Wang PP, Liu S, et al. P300 amplitude and latency in autism spectrum disorder: a meta-analysis. Eur Child Adolesc Psychiatry. 2016 Jun 14doi: 10.1007/s00787016-0880-z. PMID: 27299750.X-1

4891. Cummings JR, Lynch FL, Rust KC, et al. Health services utilization among children with and without autism spectrum disorders. Journal of Autism and Developmental Disorders. 2016;46(3):910-20. doi: http://dx.doi.org/10.1007/s10803-015-2634z.X-1

4892. Cupertino RB, Kappel DB, Bandeira $\mathrm{CE}$, et al. SNARE complex in developmental psychiatry: neurotransmitter exocytosis and beyond. J Neural Transm (Vienna). 2016 Feb 
8doi: 10.1007/s00702-016-1514-9. PMID: 26856328.X-1

4893. Curiel ESL, Sainato DM, Goldstein H. Matrix training of receptive language skills with a toddler with autism spectrum disorder: A case study. Education \& Treatment of Children. 2016;39(1):95-109.X-1, X-3, X-4

4894. Curran EA, Cryan JF, Kenny LC, et al. Obstetrical mode of delivery and childhood behavior and psychological development in a British cohort. Journal of Autism and Developmental Disorders. 2016;46(2):603-14. doi: http://dx.doi.org/10.1007/s10803-0152616-1.X-1

4895. Curtin C, Must A, Phillips S, et al. The healthy weight research network: a research agenda to promote healthy weight among youth with autism spectrum disorder and other developmental disabilities. Pediatr Obes. 2016 Feb 24doi: 10.1111/ijpo.12109. PMID: 26916513.X-1

4896. Cuzzocrea F, Murdaca AM, Costa S, et al. Parental stress, coping strategies and social support in families of children with a disability. Child Care in Practice. 2016;22(1):3-19. doi: http://dx.doi.org/10.1080/13575279.2015.10643 57.X-1

4897. Cwik JC, Sartory G, Nuyken M, et al. Posterior and prefrontal contributions to the development posttraumatic stress disorder symptom severity: an fMRI study of symptom provocation in acute stress disorder. Eur Arch Psychiatry Clin Neurosci. 2016 Jul 25doi: 10.1007/s00406-016-0713-6. PMID: 27455992.X-1

4898. Cyranoski D. Monkeys genetically modified to show autism symptoms. Nature. 2016 Jan 28;529(7587):449. doi: 10.1038/529449a. PMID: 26819024.X-1

4899. Dababnah S, Parish SL. Incredible Years program tailored to parents of preschoolers with autism: Pilot results. Research on Social Work Practice. 2016;26(4):372-85. doi: http://dx.doi.org/10.1177/1049731514558004.X $-3$

4900. D'Agati D, Chang AD, Wachtel LE, et al. Treatment of Severe Self-Injurious Behavior in Autism Spectrum Disorder by Neuromodulation. J ect. 2016 Jul 16doi: 10.1097/yct.0000000000000346. PMID: 27428475.X-1, X-2

4901. Dajani DR, Uddin LQ. Local brain connectivity across development in autism spectrum disorder: A cross-sectional investigation. Autism Res. 2016 Jan;9(1):43-54. doi: 10.1002/aur.1494. PMID: 26058882.X-1

4902. Dajani DR, Uddin LQ. Local brain connectivity across development in autism spectrum disorder: A cross-sectional investigation. Autism Research. 2016;9(1):4354. doi: http://dx.doi.org/10.1002/aur.1494.X-1

4903. Dallai R, Mercati D, Mashimo Y, et al. The fine structure of the rectal pads of Zorotypus caudelli Karny (Zoraptera, Insecta). Arthropod Struct Dev. 2016 Jun 28doi: 10.1016/j.asd.2016.06.004. PMID: 27368527.X-1

4904. Dallerac G, Rouach N. Astrocytes as new targets to improve cognitive functions. Prog Neurobiol. 2016 Mar 8doi: 10.1016/j.pneurobio.2016.01.003. PMID: 26969413.X-1

4905. Dalton C. Interaction Design in the Built Environment: Designing for the 'Universal User'. Stud Health Technol Inform. 2016;229:314-23. PMID: 27534322.X-1

4906. Danforth AL, Struble CM, YazarKlosinski B, et al. MDMA-assisted therapy: A new treatment model for social anxiety in autistic adults. Prog Neuropsychopharmacol Biol Psychiatry. 2016 Jan 4;64:237-49. doi: 10.1016/j.pnpbp.2015.03.011. PMID: 25818246.X-1

4907. D'Angelo D, Lebon S, Chen Q, et al. Defining the Effect of the 16p11.2 Duplication on Cognition, Behavior, and Medical Comorbidities. JAMA Psychiatry. 2016 Jan;73(1):20-30. doi: 10.1001/jamapsychiatry.2015.2123. PMID: 26629640.X-1

4908. Danielsson H, Henry L, Messer D, et al. Developmental delays in phonological recoding among children and adolescents with Down syndrome and Williams syndrome. Research in Developmental Disabilities. 
2016;55:64-76. doi: http://dx.doi.org/10.1016/j.ridd.2016.03.012.X1

4909. Darbro BW, Singh R, Zimmerman $\mathrm{MB}$, et al. Autism Linked to Increased Oncogene Mutations but Decreased Cancer Rate. PLoS One. 2016;11(3):e0149041. doi: 10.1371/journal.pone.0149041. PMID: 26934580.X-1

4910. Darville H, Poulet A, Rodet-Amsellem F, et al. Human Pluripotent Stem Cell-derived Cortical Neurons for High Throughput Medication Screening in Autism: A Proof of Concept Study in SHANK3 Haploinsufficiency Syndrome. EBioMedicine. 2016 May 27doi: 10.1016/j.ebiom.2016.05.032. PMID: 27333044.X-1

4911. Das S, Kumar P, Bhardwaj V, et al. Anesthetic management of Amplatzer atrial septal defect closure device embolization to right ventricular outflow tract. Saudi J Anaesth. 2016 Jul-Sep;10(3):335-8. doi: 10.4103/1658354x.174911. PMID: 27375392.X-1

4912. Daurio NA, Tuttle SW, Worth AJ, et al. AMPK Activation and Metabolic Reprogramming by Tamoxifen through Estrogen Receptor-Independent Mechanisms Suggests New Uses for This Therapeutic Modality in Cancer Treatment. Cancer Res. 2016 Jun 1;76(11):3295-306. doi: 10.1158/0008-5472.can-15-2197. PMID: 27020861.X-1

4913. Davids RC, Groen Y, Berg IJ, et al. Executive Functions in Older Adults With Autism Spectrum Disorder: Objective Performance and Subjective Complaints. J Autism Dev Disord. 2016 Jun 8doi: 10.1007/s10803-016-2831-4. PMID: 27278313.X-1

4914. Davidson JM, Wong CT, Rai-Bhogal $\mathrm{R}$, et al. Prostaglandin E2 elevates calcium in differentiated neuroectodermal stem cells. Mol Cell Neurosci. 2016 Apr 10;74:71-7. doi: 10.1016/j.mcn.2016.03.010. PMID: 27074429.X-1

4915. Davis J, McKone E, Zirnsak M, et al. Social and attention-to-detail subclusters of autistic traits differentially predict looking at eyes and face identity recognition ability. $\mathrm{Br} \mathrm{J}$
Psychol. 2016 Mar 14doi: 10.1111/bjop.12188. PMID: 26988108.X-1

4916. Daviss WB, Barnett E, Neubacher K, et al. Use of antipsychotic medications for nonpsychotic children: Risks and implications for mental health services. Psychiatric Services. 2016;67(3):339-41. doi: http://dx.doi.org/10.1176/appi.ps.201500272.X1

4917. De Felice A, Greco A, Calamandrei G, et al. Prenatal exposure to the organophosphate insecticide chlorpyrifos enhances brain oxidative stress and prostaglandin E2 synthesis in a mouse model of idiopathic autism. J Neuroinflammation. 2016;13(1):149. doi: 10.1186/s12974-016-0617-4. PMID: 27301868.X-1

4918. de Jong S, Newhouse SJ, Patel H, et al. Immune signatures and disorder-specific patterns in a cross-disorder gene expression analysis. Br J Psychiatry. 2016 May 5doi: 10.1192/bjp.bp.115.175471. PMID: 27151072.X-1

4919. De la Garza Ramos R, Passias PG, Schwab F, et al. Incidence, Risk Factors, and Mortality of Reintubation in Adult Spinal Deformity Surgery. Clin Spine Surg. 2016 Jun 27doi: 10.1097/bsd.0000000000000404. PMID: 27352366.X-1

4920. De la Garza-Ramos R, Jain A, Kebaish $\mathrm{KM}$, et al. Inpatient morbidity and mortality after adult spinal deformity surgery in teaching versus nonteaching hospitals. J Neurosurg Spine. 2016 Jul;25(1):15-20. doi: 10.3171/2015.11.spine151021. PMID: 26943252.X-1

4921. De La Torre L, Cogley K, Calisto J, et al. Primary sigmoidectomy and appendicostomy for chronic idiopathic constipation. Pediatr Surg Int. 2016 Jul 2doi: 10.1007/s00383-016-3913-2. PMID: 27372298.X-1

4922. de la Torre-Ubieta L, Won H, Stein JL, et al. Advancing the understanding of autism disease mechanisms through genetics. Nat Med. 2016 Apr;22(4):345-61. doi: 10.1038/nm.4071. PMID: 27050589.X-1 
4923. de Schipper E, Mahdi S, de Vries P, et al. Functioning and disability in autism spectrum disorder: A worldwide survey of experts. Autism Res. 2016 Jan 8doi: 10.1002/aur.1592. PMID: 26749373.X-1, X-2

4924. Decety J, Bartal IB, Uzefovsky F, et al. Empathy as a driver of prosocial behaviour: highly conserved neurobehavioural mechanisms across species. Philos Trans R Soc Lond B Biol Sci. 2016 Jan 19;371(1686):20150077. doi: 10.1098/rstb.2015.0077. PMID: 26644596.X-1

4925. Deckers A, Muris P, Roelofs J, et al. A Group-Administered social Skills Training for 8- to 12- Year-Old, high-Functioning Children With Autism Spectrum Disorders: An Evaluation of its Effectiveness in a Naturalistic Outpatient Treatment Setting. J Autism Dev Disord. 2016 Aug 13doi: 10.1007/s10803-0162887-1. PMID: 27522220.X-1

4926. Degenhardt F, Niklowitz P, Szymczak $\mathrm{S}$, et al. Genome-wide association study of serum coenzyme Q10 levels identifies susceptibility loci linked to neuronal diseases. Hum Mol Genet. 2016 May 5doi: 10.1093/hmg/ddw134. PMID: 27149984.X-1

4927. deJong NA, Williams CS, Thomas KC. Parent-reported health consequences and relationship to expenditures in children with ADHD. Maternal and Child Health Journal. 2016;20(4):915-24. doi: http://dx.doi.org/10.1007/s10995-015-18801.X-1

4928. Dekker AP, Salar O, Karuppiah SV, et al. Anxiety and depression predict poor outcomes in arthroscopic subacromial decompression. J Shoulder Elbow Surg. 2016 Jun;25(6):873-80. doi: 10.1016/j.jse.2016.01.031. PMID: 27068379.X1

4929. Dekker V, Nauta MH, Mulder EJ, et al. A Fresh Pair of Eyes: A Blind Observation Method for Evaluating Social Skills of Children with ASD in a Naturalistic Peer Situation in School. J Autism Dev Disord. 2016 Jun 11doi: 10.1007/s10803-016-2829-y. PMID: 27289230.X-1

4930. Del Casale A, Kotzalidis GD, Sacco $\mathrm{M}$, et al. Effectiveness of switching from oral ziprasidone to risperidone in a patient with comorbid autistic disorder, profound intellectual disability, Gilbert syndrome, and exacerbation of psychosis. Psychiatr Danub. 2016 Mar;28(1):91-4. PMID: 26938829.X-1, $\mathrm{X}-3$

4931. Dell'Osso L, Abelli M, Carpita B, et al. Historical evolution of the concept of anorexia nervosa and relationships with orthorexia nervosa, autism, and obsessive-compulsive spectrum. Neuropsychiatr Dis Treat. 2016;12:1651-60. doi: 10.2147/ndt.s108912. PMID: 27462158.X-1

4932. Demirci A, Kartal M. The prevalence of developmental delay among children aged 360 months in Izmir, Turkey. Child: Care, Health and Development. 2016;42(2):213-9. doi: http://dx.doi.org/10.1111/cch.12289.X-1

4933. Demopoulos C, Hopkins J, Lewine JD. Relations Between Nonverbal and Verbal Social Cognitive Skills and Complex Social Behavior in Children and Adolescents with Autism. J Abnorm Child Psychol. 2016 Jul;44(5):913-21. doi: 10.1007/s10802-0150082-z. PMID: 26386582.X-1

4934. Dempsey J, Dempsey AG, Guffey D, et al. Brief report: Further examination of selfinjurious behaviors in children and adolescents with autism spectrum disorders. Journal of Autism and Developmental Disorders. 2016;46(5):1872-9. doi: http://dx.doi.org/10.1007/s10803-016-2704$\mathrm{x} . \mathrm{X}-1$

4935. Demurie E, Roeyers H, Wiersema JR, et al. No evidence for inhibitory deficits or altered reward processing in ADHD: Data from a new integrated monetary incentive delay go/no-go task. Journal of Attention Disorders. 2016;20(4):353-67. doi: http://dx.doi.org/10.1177/1087054712473179.X $-1$

4936. Derguy C, M’Bailara K, Michel G, et al. The need for an ecological approach to parental stress in autism spectrum disorders: The combined role of individual and environmental factors. Journal of Autism and Developmental Disorders. 2016;46(6):1895905. doi: http://dx.doi.org/10.1007/s10803-0162719-3.X-1 
4937. DeRosa NM, Roane HS, Bishop JR, et al. The combined effects of noncontingent reinforcement and punishment on the reduction of rumination. J Appl Behav Anal. 2016 Mar

21doi: 10.1002/jaba.304. PMID: 26996890.X-1

4938. Desai A, Sequeira JM, Quadros EV. The metabolic basis for developmental disorders due to defective folate transport. Biochimie. 2016 Jul;126:31-42. doi: 10.1016/j.biochi.2016.02.012. PMID: 26924398.X-1

4939. Deutsch SI, Burket JA, Benson AD, et al. The 15q13.3 deletion syndrome: Deficient alpha7-containing nicotinic acetylcholine receptor-mediated neurotransmission in the pathogenesis of neurodevelopmental disorders. Prog Neuropsychopharmacol Biol Psychiatry. 2016 Jan 4;64:109-17. doi:

10.1016/j.pnpbp.2015.08.001. PMID: 26257138.X-1

4940. Devescovi R, Monasta L, Mancini A, et al. Early diagnosis and Early Start Denver Model intervention in autism spectrum disorders delivered in an Italian Public Health System service. Neuropsychiatr Dis Treat. 2016;12:1379-84. doi: 10.2147/ndt.s106850. PMID: 27366069.X-3

4941. Dewinter J, Van Parys H, Vermeiren $\mathrm{R}$, et al. Adolescent boys with an autism spectrum disorder and their experience of sexuality: An interpretative phenomenological analysis. Autism. 2016 Mar 7doi: 10.1177/1362361315627134. PMID: 26951327.X-1

4942. Dewinter J, Vermeiren R, Vanwesenbeeck I, et al. Adolescent boys with autism spectrum disorder growing up: followup of self-reported sexual experience. Eur Child Adolesc Psychiatry. 2016 Jan 20doi: 10.1007/s00787-016-0816-7. PMID: 26791194.X-1

4943. Di Biasi S, Trimarco B, D’Ardia C, et al. Psychological adjustment, social responsiveness and parental distress in an Italian sample of siblings of children with highfunctioning autism spectrum disorder. Journal of Child and Family Studies. 2016;25(3):88390. doi: http://dx.doi.org/10.1007/s10826-0150256-x.X-1
4944. Di Palma S, Tonacci A, Narzisi A, et al. Monitoring of autonomic response to sociocognitive tasks during treatment in children with Autism Spectrum Disorders by wearable technologies: A feasibility study. Comput Biol Med. 2016 Apr 5doi: 10.1016/j.compbiomed.2016.04.001. PMID: 27080078.X-1

4945. Di Rezze B, Rosenbaum P, Zwaigenbaum L, et al. Developing a classification system of social communication functioning of preschool children with autism spectrum disorder. Dev Med Child Neurol. 2016 May 17doi: 10.1111/dmcn.13152. PMID: 27189758.X-1

4946. Dias APBH, de Vasconcelos MM, de Souza Maia Filho H, et al. Assessment of pragmatic language in verbal and nonverbal autistic children. Revista de Logopedia, Foniatría y Audiología. 2016;36(1):15-22. doi: http://dx.doi.org/10.1016/j.rlfa.2015.02.001.X-1

4947. Diaz Heijtz R. Fetal, neonatal, and infant microbiome: Perturbations and subsequent effects on brain development and behavior. Semin Fetal Neonatal Med. 2016 May 30doi: 10.1016/j.siny.2016.04.012. PMID: 27255860.X-1

4948. Dick IE, Joshi-Mukherjee R, Yang W, et al. Arrhythmogenesis in Timothy Syndrome is associated with defects in $\mathrm{Ca}(2+)$-dependent inactivation. Nat Commun. 2016;7:10370. doi: 10.1038/ncomms10370. PMID: 26822303.X-1

4949. Dickerson AS, Rahbar MH, Bakian AV, et al. Autism spectrum disorder prevalence and associations with air concentrations of lead, mercury, and arsenic. Environ Monit Assess. 2016 Jul;188(7):407. doi: 10.1007/s10661-0165405-1. PMID: 27301968.X-1

4950. Dickinson A, Jones M, Milne E. Measuring neural excitation and inhibition in autism: different approaches, different findings and different interpretations. Brain Res. 2016 Jul 12doi: 10.1016/j.brainres.2016.07.011. PMID: 27421181.X-1

4951. Dickinson K, Place M. The Impact of a Computer-Based Activity Program on the Social Functioning of Children with Autistic Spectrum Disorder. Games Health J. 2016 
Jun;5(3):209-15. doi: 10.1089/g4h.2015.0063. PMID: 27104364.X-1

4952. Dickson PE, Cairns J, Goldowitz D, et al. Cerebellar contribution to higher and lower order rule learning and cognitive flexibility in mice. Neuroscience. 2016 Mar 21doi: 10.1016/j.neuroscience.2016.03.040. PMID: 27012612.X-1

4953. Dickstein DP, Axelson D, Weissman $\mathrm{AB}$, et al. Cognitive flexibility and performance in children and adolescents with threshold and sub-threshold bipolar disorder. Eur Child Adolesc Psychiatry. 2016 Jun;25(6):625-38. doi: 10.1007/s00787-015-0769-2. PMID: 26438382.X-1

4954. DiCriscio AS, Miller SJ, Hanna EK, et al. Brief Report: Cognitive Control of Social and Nonsocial Visual Attention in Autism. J Autism Dev Disord. 2016 May 13doi: 10.1007/s10803-016-2804-7. PMID: 27177893.X-1

4955. Didehbani N, Allen T, Kandalaft M, et al. Virtual Reality Social Cognition Training for children with high functioning autism. Computers in Human Behavior. 2016;62:70311. doi: http://dx.doi.org/10.1016/j.chb.2016.04.033.X-3

4956. Didriksen M, Fejgin K, Nilsson SR, et al. Persistent gating deficit and increased sensitivity to NMDA receptor antagonism after puberty in a new mouse model of the human 22q11.2 microdeletion syndrome: a study in male mice. J Psychiatry Neurosci. 2016 Jul 7;41(5):150381. doi: 10.1503/jpn.150381. PMID: 27391101.X-1

4957. Didry P, Didry E. Psychological care. Rev Infirm. 2016 Jan(217):47-8. doi: 10.1016/j.revinf.2015.10.027. PMID: 26743375.X-1

4958. Diebo BG, Gammal I, Ha Y, et al. Role of Ethnicity in Alignment Compensation: Propensity Matched Analysis of Differential Compensatory Mechanism Recruitment Patterns for Sagittal Malalignment in 288 ASD Patients from Japan, Korea and United States. Spine (Phila Pa 1976). 2016 Jun 17doi: 10.1097/brs.0000000000001744. PMID: 27323222.X-1
4959. Diener ML, Wright CA, Dunn L, et al. A creative 3D design programme: Building on interests and social engagement for students with autism spectrum disorder (ASD). International Journal of Disability, Development and Education. 2016;63(2):181200. doi: http://dx.doi.org/10.1080/1034912X.2015.1053 436.X-1, X-3

4960. DiGuiseppi CG, Daniels JL, Fallin DM, et al. Demographic profile of families and children in the Study to Explore Early Development (SEED): Case-control study of autism spectrum disorder. Disability and Health Journal. 2016;9(3):544-51. doi:

http://dx.doi.org/10.1016/j.dhjo.2016.01.005.x1

4961. Dima I, Deshpande A. Right heart dilatation: a rare vascular cause. Heart. 2016 Mar 21doi: 10.1136/heartjnl-2015-309172. PMID: 27000054.X-1

4962. Dimitrova N, Özçalışkan Ş, Adamson LB. Parents' translations of child gesture facilitate word learning in children with autism, Down syndrome and typical development. Journal of Autism and Developmental Disorders. 2016;46(1):221-31. doi: http://dx.doi.org/10.1007/s10803-015-25667.X-1

4963. Ding Y, Ruan DK, He Q, et al. Imaging Evaluation and Relative Significance in Cases of Cervical Disc Allografting: Radiographic Character Following Total Disc Transplantation. Clin Spine Surg. 2016 May 11doi: 10.1097/BSD.0b013e318290fc41. PMID: 27171660.X-1

4964. DiStefano C, Gulsrud A, Huberty S, et al. Identification of a distinct developmental and behavioral profile in children with Dup15q syndrome. J Neurodev Disord. 2016;8:19. doi: 10.1186/s11689-016-9152-y. PMID: 27158270.X-1

4965. DiStefano C, Shih W, Kaiser A, et al. Communication growth in minimally verbal children with ASD: The importance of interaction. Autism Res. 2016 Jan 29doi: 10.1002/aur.1594. PMID: 26824676.X-1, X-3

4966. Dittrich S, Sigler M, Priessmann H. Late complete atrioventricular block after 
closure of an atrial septal defect with a gore septal occluder (GSO). Catheter Cardiovasc Interv. 2016 Apr;87(5):945-50. doi: 10.1002/ccd.26154. PMID: 26354228.X-1

4967. Dixon MR, Stanley CR, Belisle J, et al. The test-retest and interrater reliability of the Promoting the Emergence of Advanced Knowledge-Direct Training assessment for use with individuals with autism and related disabilities. Behavior Analysis: Research and Practice. 2016;16(1):34-40. doi: http://dx.doi.org/10.1037/bar0000027.X-1

4968. Doenyas C. The Social Living Complex: A New, All Day, Yearlong Intervention Model for Individuals with Autism Spectrum Disorder and Their Parents. J Autism Dev Disord. 2016 Jun 22doi: 10.1007/s10803016-2846-x. PMID: 27334874.X-1, X-2, X-3

4969. Doernberg E, Hollander E. Neurodevelopmental Disorders (ASD and ADHD): DSM-5, ICD-10, and ICD-11. CNS Spectr. 2016 Jul 1:1-5. doi: 10.1017/s1092852916000262. PMID: 27364515.X-1

4970. Dolan BK, Van Hecke AV, Carson AM, et al. Brief Report: Assessment of Intervention Effects on In Vivo Peer Interactions in Adolescents with Autism Spectrum Disorder (ASD). J Autism Dev Disord. 2016 Jun;46(6):2251-9. doi: 10.1007/s10803-016-2738-0. PMID: 26886470.X-1

4971. Dolev S, Sher-Censor E, Baransi N, et al. Resolution of the child's ASD diagnosis among Arab-Israeli mothers: Associations with maternal sensitivity and wellbeing. Research in Autism Spectrum Disorders. 2016;21:73-83. doi: http://dx.doi.org/10.1016/j.rasd.2015.09.004.X1

4972. Doll CA, Broadie K. Neuron classspecific requirements for Fragile X Mental Retardation Protein in critical period development of calcium signaling in learning and memory circuitry. Neurobiol Dis. 2016 May;89:76-87. doi: 10.1016/j.nbd.2016.02.006. PMID: 26851502.X-1

4973. Donaldson CK, Stauder JE, Donkers FC. Increased Sensory Processing Atypicalities in Parents of Multiplex ASD Families Versus Typically Developing and Simplex ASD Families. J Autism Dev Disord. 2016 Aug 18doi: 10.1007/s10803-016-2888-0. PMID: 27538965.X-1

4974. Dong F, Jiang J, McSweeney C, et al. Deletion of CTNNB1 in inhibitory circuitry contributes to autism-associated behavioral defects. Hum Mol Genet. 2016 Apr 30doi: 10.1093/hmg/ddw131. PMID: 27131348.X-1

4975. Dong T, He J, Wang S, et al. Inability to activate Rac1-dependent forgetting contributes to behavioral inflexibility in mutants of multiple autism-risk genes. Proc Natl Acad Sci U S A. 2016 Jun 22doi: 10.1073/pnas.1602152113. PMID: 27335463.X-1

4976. Doumas M, McKenna R, Murphy B. Postural control deficits in autism spectrum disorder: The role of sensory integration. Journal of Autism and Developmental Disorders. 2016 2016-02-25;46(3):853-61. doi: 10.1176/appi.books.9780890423349 . 10.1016/j.rasd.2013.09.016 . http://dx.doi.org/10.1007/s10803-015-2621-4. PMID: 1721931437; 2015-46759-001.X-1

4977. Dow D, Guthrie W, Stronach ST, et al. Psychometric analysis of the Systematic Observation of Red Flags for autism spectrum disorder in toddlers. Autism. 2016 Apr 30doi: 10.1177/1362361316636760. PMID: 27132013.X-1

4978. Downs J, Torode I, Ellaway C, et al. Family satisfaction following spinal fusion in Rett syndrome. Developmental Neurorehabilitation. 2016;19(1):31-7. doi: http://dx.doi.org/10.3109/17518423.2014.89810 7.X-1

4979. Drasgow E, Martin CA, Chezan LC, et al. Mand training: An examination of responseclass structure in three children with autism and severe language delays. Behavior Modification. 2016;40(3):347-76. doi: http://dx.doi.org/10.1177/0145445515613582.X $-1, \mathrm{X}-3, \mathrm{X}-4$

4980. Du F, Nguyen MV, Karten A, et al. Acute and crucial requirement for MeCP2 function upon transition from early to late adult stages of brain maturation. Hum Mol Genet. 
2016 Feb 16doi: 10.1093/hmg/ddw038. PMID: 26908602.X-1

4981. Dua K, McAvoy WC, Klaus SA, et al. Hospitalist Co-management of Pediatric Orthopaedic Surgical Patients at a Community Hospital. Md Med. 2016;17(1):34-6. PMID: 27443131.X-1

4982. Dube WV, Farber RS, Mueller MR, et al. Stimulus Overselectivity in Autism, Down Syndrome, and Typical Development. Am J Intellect Dev Disabil. 2016 May;121(3):219-35. doi: 10.1352/1944-7558-121.3.219. PMID: 27119213.X-1

4983. Dubiel A, Kulesza RJ, Jr. Prenatal valproic acid exposure disrupts tonotopic c-Fos expression in the rat brainstem. Neuroscience. 2016 Jun 2;324:511-23. doi:

10.1016/j.neuroscience.2016.01.030. PMID: 27094734.X-1

4984. DuBois D, Ameis SH, Lai MC, et al. Interoception in Autism Spectrum Disorder: A review. Int J Dev Neurosci. 2016 Jun 3;52:10411. doi: 10.1016/j.ijdevneu.2016.05.001. PMID: 27269967.X-1

4985. Duda M, Daniels J, Wall DP. Clinical evaluation of a Novel and Mobile Autism Risk Assessment. Journal of Autism and Developmental Disorders. 2016;46(6):1953-61. doi: http://dx.doi.org/10.1007/s10803-0162718-4.X-1

4986. Dungan JA, Stepanovic M, Young L. Theory of mind for processing unexpected events across contexts. Soc Cogn Affect Neurosci. 2016 Mar 12doi: 10.1093/scan/nsw032. PMID: 26969865.X-1, $\mathrm{X}-2$

4987. Dunkel-Jackson SM, Dixon MR, Szekely S. Self-control as generalized operant behavior by adults with autism spectrum disorder. J Appl Behav Anal. 2016 May 14doi: 10.1002/jaba.315. PMID: 27198865.X-1

4988. Dunlop JA, Lamsdell JC. Segmentation and tagmosis in Chelicerata. Arthropod Struct Dev. 2016 Jun 21doi: 10.1016/j.asd.2016.05.002. PMID: 27240897.X-1
4989. Durante AS, Wieselberg MB, Roque $\mathrm{N}$, et al. Assessment of hearing threshold in adults with hearing loss using an automated system of cortical auditory evoked potential detection. Braz J Otorhinolaryngol. 2016 Apr 29doi: 10.1016/j.bjorl.2016.02.016. PMID: 27229658.X-1

4990. Duringer J, Fombonne E, Craig M. No Association between Mycotoxin Exposure and Autism: A Pilot Case-Control Study in SchoolAged Children. Toxins (Basel). 2016;8(7)doi: 10.3390/toxins8070224. PMID: 27447670.X-1

4991. Durkin MS, Benedict RE, Christensen D, et al. Prevalence of Cerebral Palsy among 8Year-Old Children in 2010 and Preliminary Evidence of Trends in Its Relationship to Low Birthweight. Paediatr Perinat Epidemiol. 2016 May 23doi: 10.1111/ppe.12299. PMID: 27215680.X-1

4992. Durrleman S, Burnel M, Thommen E, et al. The language cognition interface in ASD: Complement sentences and false belief reasoning. Research in Autism Spectrum Disorders. 2016;21:109-20. doi: http://dx.doi.org/10.1016/j.rasd.2015.10.003.X1

4993. Dutta MM, Sanyal N. A comparative study of emotional characteristics of children with and without ADHD by "Draw a Man test". Journal of Projective Psychology \& Mental Health. 2016;23(1):27-33.X-1

4994. Duvekot J, Ende J, Constantino JN, et al. Symptoms of autism spectrum disorder and anxiety: Shared familial transmission and crossassortative mating. Journal of Child Psychology and Psychiatry. 2016;57(6):759-69. doi: http://dx.doi.org/10.1111/jcpp.12508.X-1

4995. Duyzend MH, Nuttle X, Coe BP, et al. Maternal Modifiers and Parent-of-Origin Bias of the Autism-Associated 16p11.2 CNV. Am J Hum Genet. 2016 Jan 7;98(1):45-57. doi: 10.1016/j.ajhg.2015.11.017. PMID: 26749307.X-1

4996. Dynia JM, Brock ME, Logan JAR, et al. Comparing children with ASD and their peers' growth in print knowledge. Journal of Autism and Developmental Disorders. 2016;46(7):2490-500. doi: 
http://dx.doi.org/10.1007/s10803-016-27909.X-1

4997. Eapen V. Early identification of autism spectrum disorder: Do we need a paradigm shift? Aust N Z J Psychiatry. 2016 Jun 9doi: 10.1177/0004867416652733. PMID: 27282164.X-1

4998. Earle JF. An Introduction to the Psychopharmacology of Children and Adolescents With Autism Spectrum Disorder. J Child Adolesc Psychiatr Nurs. 2016 Jun 9doi: 10.1111/jcap.12144. PMID: 27279529.X-1

4999. Eberhardt M, Nadig A. Reduced sensitivity to context in language comprehension: A characteristic of Autism Spectrum Disorders or of poor structural language ability? Res Dev Disabil. 2016 Feb 18doi: 10.1016/j.ridd.2016.01.017. PMID: 26898317.X-1

5000. Ecker C. The neuroanatomy of autism spectrum disorder: An overview of structural neuroimaging findings and their translatability to the clinical setting. Autism. 2016 Mar 14doi: 10.1177/1362361315627136. PMID: 26975670.X-1, X-2

5001. Eddy CM. The junction between self and other? Temporo-parietal dysfunction in neuropsychiatry. Neuropsychologia. 2016 Jul 25;89:465-77. doi:

10.1016/j.neuropsychologia.2016.07.030. PMID: 27457686.X-1

5002. Edgar JC, Fisk CLIV, Liu S, et al. Translating adult electrophysiology findings to younger patient populations: Difficulty measuring $40-\mathrm{Hz}$ auditory steady-state responses in typically developing children and children with autism spectrum disorder. Developmental Neuroscience. 2016;38(1):1-14. doi: http://dx.doi.org/10.1159/000441943.X-1

5003. Edmiston EK, Blain SD, Corbett BA. Salivary cortisol and behavioral response to social evaluative threat in adolescents with autism spectrum disorder. Autism Res. $2016 \mathrm{Jul}$ 15doi: 10.1002/aur.1660. PMID: 27417507.X-1

5004. Edmiston EK, Jones RM, Corbett BA. Physiological Response to Social Evaluative Threat in Adolescents with Autism Spectrum Disorder. J Autism Dev Disord. 2016 Jun 18doi: 10.1007/s10803-016-2842-1. PMID: 27318810.X-1

5005. Edmunds SR, Ibanez LV, Warren Z, et al. Longitudinal prediction of language emergence in infants at high and low risk for autism spectrum disorder. Dev Psychopathol. 2016 Apr 6:1-11. doi: 10.1017/s0954579416000146. PMID: 27048735.X-1

5006. Edwards A, Brebner C, McCormack PF, et al. "More than blowing bubbles": What parents want from therapists working with children with autism spectrum disorder. Int J Speech Lang Pathol. 2016 Apr 4:1-13. doi: 10.3109/17549507.2015.1112835. PMID: 27063689.X-1

5007. Egger JI, Zwanenburg RJ, van Ravenswaaij-Arts CM, et al.

Neuropsychological phenotype and psychopathology in seven adult patients with Phelan-McDermid syndrome: implications for treatment strategy. Genes Brain Behav. 2016 Apr;15(4):395-404. doi: 10.1111/gbb.12285. PMID: 26824576.X-1

5008. Egilson ST, Jakobsdottir G, Olafsson $\mathrm{K}$, et al. Community participation and environment of children with and without autism spectrum disorder: parent perspectives. Scand J Occup Ther. 2016 Jun 22:1-10. doi: 10.1080/11038128.2016.1198419. PMID: 27329683.X-1

5009. Egilson ST, Olafsdottir LB, Leosdottir $\mathrm{T}$, et al. Quality of life of high-functioning children and youth with autism spectrum disorder and typically developing peers: Selfand proxy-reports. Autism. 2016 Apr 5doi: 10.1177/1362361316630881. PMID: 27048354.X-1

5010. Einarsson ID, Jóhannsson E, Daly D, et al. Physical activity during school and after school among youth with and without intellectual disability. Research in Developmental Disabilities. 2016;56:60-70. doi: http://dx.doi.org/10.1016/j.ridd.2016.05.016.X1

5011. Ekas NV, Ghilain C, Pruitt M, et al. The role of family cohesion in the psychological adjustment of non-Hispanic 
White and Hispanic mothers of children with autism spectrum disorder. Research in Autism Spectrum Disorders. 2016;21:10-24. doi: http://dx.doi.org/10.1016/j.rasd.2015.09.002.X1

5012. Ekberg TL, Falck-Ytter T, Bölte S, et al. Reduced prospective motor control in 10month-olds at risk for autism spectrum disorder. Clinical Psychological Science. 2016;4(1):12935. doi: http://dx.doi.org/10.1177/2167702615576697.X $-1$

5013. Elagoz Yuksel M, Yuceturk B, Karatas OF, et al. The Altered Promoter Methylation Of Oxytocin Receptor Gene In Autism. J

Neurogenet. 2016 Jun 16:1-15. doi: 10.1080/01677063.2016.1202951. PMID: 27309964.X-1

5014. El-Ansary A. Data of multiple regressions analysis between selected biomarkers related to glutamate excitotoxicity and oxidative stress in Saudi autistic patients. Data Brief. 2016 Jun;7:111-6. doi: 10.1016/j.dib.2016.02.025. PMID: 26933667.X-1

5015. Elberling H, Linneberg A, Rask CU, et al. Psychiatric disorders in Danish children aged 5-7 years: A general population study of prevalence and risk factors from the Copenhagen Child Cohort (CCC 2000). Nordic Journal of Psychiatry. 2016;70(2):146-55. doi: http://dx.doi.org/10.3109/08039488.2015.10701 99.X-1

5016. Elder JH, Brasher S, Alexander B. Identifying the Barriers to Early Diagnosis and Treatment in Underserved Individuals with Autism Spectrum Disorders (ASD) and Their Families: A Qualitative Study. Issues Ment Health Nurs. 2016 Jun;37(6):412-20. doi: 10.3109/01612840.2016.1153174. PMID: 27070190.X-1

5017. Ella B, Ghorayeb I, Burbaud P, et al. Bruxism in Movement Disorders: A Comprehensive Review. J Prosthodont. 2016 Apr 14doi: 10.1111/jopr.12479. PMID: 27077925.X-1

5018. Elliott C, Dillenburger K. The effect of choice on motivation for young children on the autism spectrum during discrete trial teaching.
Journal of Research in Special Educational Needs. 2016;16(3):187-98. doi: http://dx.doi.org/10.1111/1471-3802.12073.X3, X-4

5019. Elrod MG, Nylund CM, Susi AL, et al. Prevalence of Diagnosed Sleep Disorders and Related Diagnostic and Surgical Procedures in Children with Autism Spectrum Disorders. J Dev Behav Pediatr. 2016 Jun;37(5):377-84. doi: 10.1097/dbp.0000000000000248. PMID: 26982246.X-1

5020. Elton A, Di Martino A, Hazlett HC, et al. Neural connectivity evidence for a categorical-dimensional hybrid model of autism spectrum disorder. Biological Psychiatry. 2016;80(2):120-8. doi: http://dx.doi.org/10.1016/j.biopsych.2015.10.02 $0 . \mathrm{X}-1$

5021. Eluri Z, Andrade I, Trevino N, et al. Assessment and treatment of problem behavior maintained by mand compliance. J Appl Behav Anal. 2016 Jun;49(2):383-7. doi: 10.1002/jaba.296. PMID: 26831251.X-1, X-4

5022. Elwin M, Schroder A, Ek L, et al. Development and pilot validation of a sensory reactivity scale for adults with high functioning autism spectrum conditions: Sensory Reactivity in Autism Spectrum (SR-AS). Nord J Psychiatry. 2016;70(2):103-10. doi: 10.3109/08039488.2015.1053984. PMID: 26158770.X-1

5023. Ely E, Chen-Lim ML, Carpenter KM, II, et al. Pain assessment of children with autism spectrum disorders. Journal of Developmental and Behavioral Pediatrics. 2016;37(1):53-61. doi: http://dx.doi.org/10.1097/DBP.0000000000000 240.X-1

5024. Emamalizadeh B, Jamshidi J, Movafagh A, et al. RIT2 Polymorphisms: Is There a Differential Association? Mol Neurobiol. 2016 Mar 3doi: 10.1007/s12035016-9815-4. PMID: 26941103.X-1

5025. Emberti Gialloreti L, Benvenuto A, Battan B, et al. Can biological components predict short-term evolution in Autism Spectrum Disorders? A proof-of-concept study. Ital J Pediatr. 2016;42(1):70. doi: 
10.1186/s13052-016-0281-4. PMID:

27448796.X-1

5026. Emerson ND, Morrell HER, Neece C. Predictors of age of diagnosis for children with autism spectrum disorder: The role of a consistent source of medical care, race, and condition severity. Journal of Autism and Developmental Disorders. 2016;46(1):127-38. doi: http://dx.doi.org/10.1007/s10803-0152555-x.X-1

5027. Engevik LI, Næss K-AB, Hagtvet BE. Cognitive stimulation of pupils with Down syndrome: A study of inferential talk during book-sharing. Research in Developmental Disabilities. 2016;55:287-300. doi: http://dx.doi.org/10.1016/j.ridd.2016.05.004.X1

5028. Engle K, Rada R. Knowledge-guided mutation in classification rules for autism treatment efficacy. Health Informatics J. 2016 Feb 11doi: 10.1177/1460458215627187. PMID: 26868770.X-1

5029. Ercan ES, Suren S, Bacanlı A, et al. Decreasing ADHD phenotypic heterogeneity: Searching for neurobiological underpinnings of the restrictive inattentive phenotype. European Child \& Adolescent Psychiatry.

2016;25(3):273-82. doi: http://dx.doi.org/10.1007/s00787-015-07313.X-1

5030. Ergaz Z, Weinstein-Fudim L, Ornoy A. Genetic and non-genetic animal models for autism spectrum disorders (ASD). Reprod Toxicol. 2016 Apr 30doi: 10.1016/j.reprotox.2016.04.024. PMID: 27142188.X-1

5031. Erickson CA, Wink LK, Baindu B, et al. Analysis of peripheral amyloid precursor protein in Angelman Syndrome. Am J Med Genet A. 2016 Jun 21doi:

10.1002/ajmg.a.37811. PMID: 27327493.X-1

5032. Eriksson JM, Lundstrom S, Lichtenstein P, et al. Effect of co-twin gender on neurodevelopmental symptoms: a twin register study. Mol Autism. 2016;7:8. doi: 10.1186/s13229-016-0074-z. PMID: 26793297.X-1
5033. Erskine HE, Baxter AJ, Patton G, et al. The global coverage of prevalence data for mental disorders in children and adolescents. Epidemiol Psychiatr Sci. 2016 Jan 20:1-8. doi: 10.1017/s2045796015001158. PMID: 26786507.X-1

5034. Erturk O, Korkmaz B, Alev G, et al. Startle and blink reflex in high functioning autism. Neurophysiol Clin. 2016 Mar 17doi: 10.1016/j.neucli.2016.02.001. PMID: 26997128.X-1

5035. Estabillo JA, Matson JL, Jiang X. The association between familial ASD diagnosis, autism symptomatology and developmental functioning in young children. Eur Child Adolesc Psychiatry. 2016 Mar 16doi: 10.1007/s00787-016-0838-1. PMID: 26983421.X-1

5036. Estes ML, McAllister AK. IMMUNOLOGY. Maternal TH17 cells take a toll on baby's brain. Science. 2016 Feb 26;351(6276):919-20. doi: 10.1126/science.aaf2850. PMID: 26917755.X-1

5037. Estes ML, McAllister AK. Maternal immune activation: Implications for neuropsychiatric disorders. Science. 2016 Aug 19;353(6301):772-7. doi: 10.1126/science.aag3194. PMID: 27540164.X1

5038. Evrensel A, Ceylan ME. Fecal Microbiota Transplantation and Its Usage in Neuropsychiatric Disorders. Clin Psychopharmacol Neurosci. 2016 Aug 31;14(3):231-7. doi: 10.9758/cpn.2016.14.3.231. PMID: 27489376.X-1

5039. Ewen JB, Lakshmanan BM, Pillai AS, et al. Decreased Modulation of EEG Oscillations in High-Functioning Autism during a Motor Control Task. Front Hum Neurosci. 2016;10:198. doi: 10.3389/fnhum.2016.00198. PMID: 27199719.X-1

5040. Factor RS, Condy EE, Farley JP, et al. Brief Report: Insistence on Sameness, Anxiety, and Social Motivation in Children with Autism Spectrum Disorder. J Autism Dev Disord. 2016 Jul;46(7):2548-54. doi: 10.1007/s10803-0162781-x. PMID: 27040556.X-1 
5041. Fairthorne J, Hammond G, Bourke J, et al. Maternal psychiatric disorder and the risk of autism spectrum disorder or intellectual disability in subsequent offspring. Journal of Autism and Developmental Disorders. 2016;46(2):523-33. doi: http://dx.doi.org/10.1007/s10803-015-25943.X-1, X-3, X-4

5042. Fairthorne JC, de Klerk NH, Leonard HM, et al. Mothers of Children with Autism have Different Rates of Cancer According to the Presence of Intellectual Disability in Their Child. J Autism Dev Disord. 2016 Sep;46(9):3106-14. doi: 10.1007/s10803-0162847-9. PMID: 27384537.X-1

5043. Faja S, Dawson G, Aylward E, et al. Early event-related potentials to emotional faces differ for adults with autism spectrum disorder and by serotonin transporter genotype. Clin Neurophysiol. 2016 Jun;127(6):2436-47. doi: 10.1016/j.clinph.2016.02.022. PMID: 27178863.X-1

5044. Faja S, Dawson G, Sullivan K, et al. Executive function predicts the development of play skills for verbal preschoolers with autism spectrum disorders. Autism Res. 2016 Feb 18doi: 10.1002/aur.1608. PMID: 26890821.X-1

5045. Falahpour M, Thompson WK, Abbott $\mathrm{AE}$, et al. Underconnected, but not broken? Dynamic functional connectivity MRI shows underconnectivity in autism is linked to increased intra-individual variability across time. Brain Connectivity. 2016;6(5):403-14 . doi:

http://dx.doi.org/10.1089/brain.2015.0389.X-1

5046. Falk A, Heine VM, Harwood AJ, et al. Modeling psychiatric disorders: from genomic findings to cellular phenotypes. Mol Psychiatry. 2016 May 31doi: 10.1038/mp.2016.89. PMID: 27240529.X-1

5047. Faller P, Hunt J, van Hooydonk E, et al. Application of Data-Driven Decision Making Using Ayres Sensory Integration((R)) With a Child With Autism. Am J Occup Ther. 2016 Jan-Feb;70(1):7001220020p1-9. doi: 10.5014/ajot.2016.016881. PMID: 26709422.X-1

5048. Fallon LM, Collier-Meek MA, Sanetti LMH, et al. Implementation planning to promote parents' treatment integrity of behavioral interventions for children with autism. Journal of Educational \& Psychological Consultation. 2016;26(1):87-109. doi: http://dx.doi.org/10.1080/10474412.2015.10391 24.X-1, X-3, X-4

5049. Farahmand D, Saehle T, Eide PK, et al. A double-blind randomized trial on the clinical effect of different shunt valve settings in idiopathic normal pressure hydrocephalus. J Neurosurg. 2016 Feb;124(2):359-67. doi: 10.3171/2015.1.jns141301. PMID: 26315004.X-1

5050. Farber RS, Dube WV, Dickson CA. A sorting-to-matching method to teach compound matching to sample. Journal of Applied Behavior Analysis. 2016;49(2):294-307. doi: http://dx.doi.org/10.1002/jaba.290.X-1, X-3, X4

5051. Farhat F, Hsairi I, Baati H, et al. The effect of a motor skills training program in the improvement of practiced and non-practiced tasks performance in children with developmental coordination disorder (DCD). Human Movement Science. 2016;46:10-22. doi: http://dx.doi.org/10.1016/j.humov.2015.12.001. $\mathrm{X}-1$

5052. Farmer C, Golden C, Thurm A. Concurrent validity of the differential ability scales, second edition with the Mullen Scales of Early Learning in young children with and without neurodevelopmental disorders. Child Neuropsychology. 2016;22(5):556-69. doi: http://dx.doi.org/10.1080/09297049.2015.10207 75.X-1

5053. Farmer M, Echenne B, Bentourkia Mh. Study of clinical characteristics in young subjects with developmental coordination disorder. Brain \& Development.

2016;38(6):538-47. doi: http://dx.doi.org/10.1016/j.braindev.2015.12.01 $0 . \mathrm{X}-1$

5054. Farrant K, Uddin LQ. Atypical developmental of dorsal and ventral attention networks in autism. Developmental Science. 2016;19(4):550-63. doi: http://dx.doi.org/10.1111/desc.12359.X-1

5055. Faught GG, Conners FA, Barber AB, et al. Addressing phonological memory in 
language therapy with clients who have Down syndrome: Perspectives of speech-language pathologists. Int J Lang Commun Disord. 2016 May 5doi: 10.1111/1460-6984.12241. PMID: 27150499.X-1

5056. Fausey CM, Jayaraman S, Smith LB. From faces to hands: Changing visual input in the first two years. Cognition. 2016;152:101-7. doi:

http://dx.doi.org/10.1016/j.cognition.2016.03.0 05.X-1

5057. Feinberg E, Abufhele M, Sandler J, et al. Reducing Disparities in Timely Autism Diagnosis Through Family Navigation: Results From a Randomized Pilot Trial. Psychiatr Serv. 2016 May 2:appips201500162. doi: 10.1176/appi.ps.201500162. PMID: 27133722.X-1

5058. Feng J, Shan L, Du L, et al. Clinical improvement following vitamin D3 supplementation in Autism Spectrum Disorder. Nutr Neurosci. 2016 Jan 18doi: 10.1080/1028415x.2015.1123847. PMID: 26783092.X-3

5059. Ferguson BJ, Marler S, Altstein LL, et al. Psychophysiological Associations with Gastrointestinal Symptomatology in Autism Spectrum Disorder. Autism Res. 2016 Jun 20doi: 10.1002/aur.1646. PMID: 27321113.X-1

5060. Ferguson BJ, Marler S, Altstein LL, et al. Associations between cytokines, endocrine stress response, and gastrointestinal symptoms in autism spectrum disorder. Brain Behav Immun. 2016 May 12doi:

10.1016/j.bbi.2016.05.009. PMID:

27181180.X-1

5061. Fernandez-Alcantara M, Garcia-Caro MP, Perez-Marfil MN, et al. Feelings of loss and grief in parents of children diagnosed with autism spectrum disorder (ASD). Res Dev Disabil. 2016 May 24;55:312-21. doi: 10.1016/j.ridd.2016.05.007. PMID: 27235768.X-1

5062. Ferrero E, Liabaud B, Challier V, et al. Role of pelvic translation and lower-extremity compensation to maintain gravity line position in spinal deformity. J Neurosurg Spine. 2016 Mar;24(3):436-46. doi: 10.3171/2015.5.spine14989. PMID: 26565764.X-1

5063. Ferron L. Fragile X mental retardation protein controls ion channel expression and activity. J Physiol. 2016 Feb 10doi: 10.1113/jp270675. PMID: 26864773.X-1

5064. Field SS. Nutrition and Autism: Intervention Compared with Identification. Adv Nutr. 2016 Mar;7(2):420-1. doi: 10.3945/an.115.011700. PMID: 26980829.X-2

5065. Field T. Massage therapy research review. Complement Ther Clin Pract. 2016 Aug;24:19-31. doi: 10.1016/j.ctcp.2016.04.005. PMID: 27502797.X-1

5066. Filice F, Vorckel KJ, Sungur AO, et al. Reduction in parvalbumin expression not loss of the parvalbumin-expressing GABA interneuron subpopulation in genetic parvalbumin and shank mouse models of autism. Mol Brain. 2016;9:10. doi: 10.1186/s13041-016-0192-8. PMID: 26819149.X-1

5067. Findon J, Cadman T, Stewart CS, et al. Screening for co-occurring conditions in adults with autism spectrum disorder using the strengths and difficulties questionnaire: A pilot study. Autism Res. 2016 Mar 28doi: 10.1002/aur.1625. PMID: 27120552.X-1

5068. Finnegan E, Mazin AL. Strategies for increasing reading comprehension skills in students with Autism Spectrum Disorder: A review of the literature. Education \& Treatment of Children. 2016;39(2):187-220. doi: http://dx.doi.org/10.1353/etc.2016.0007.X-1, X2

5069. Fisch GS, Falk RE, Carey JC, et al. Deletion 2q37 syndrome: Cognitive-behavioral trajectories and autistic features related to breakpoint and deletion size. Am J Med Genet A. 2016 Jun 9doi: 10.1002/ajmg.a.37782. PMID: 27282419.X-1

5070. Fisher MH, Lense MD, Dykens EM. Longitudinal trajectories of intellectual and adaptive functioning in adolescents and adults with Williams syndrome. J Intellect Disabil Res. 2016 Jun 8doi: 10.1111/jir.12303. PMID: 27273269.X-1 
5071. Fitzgerald J, Gallagher L, McGrath J. Widespread Disrupted White Matter Microstructure in Autism Spectrum Disorders. J Autism Dev Disord. 2016 May 20doi: 10.1007/s10803-016-2803-8. PMID: 27207090.X-1

5072. Fitzpatrick SE, Srivorakiat L, Wink LK, et al. Aggression in autism spectrum disorder: presentation and treatment options. Neuropsychiatr Dis Treat. 2016;12:1525-38. doi: 10.2147/ndt.s84585. PMID: 27382295.X-1, $\mathrm{X}-2, \mathrm{X}-3$

5073. Fletcher FE, Foster-Owens MD, Conduit R, et al. The development trajectory of parent-report and objective sleep profiles in autism spectrum disorder: Associations with anxiety and bedtime routines. Autism. 2016 Jun 26doi: 10.1177/1362361316653365. PMID: 27354432.X-1

5074. Fletcher-Watson S, Apicella F, Auyeung B, et al. Attitudes of the autism community to early autism research. Autism. 2016 Mar 14doi: 10.1177/1362361315626577. PMID: 26975669.X-1

5075. Flore G, Cioffi S, Bilio M, et al. Cortical Development Requires Mesodermal Expression of Tbx1, a Gene Haploinsufficient in 22q11.2 Deletion Syndrome. Cereb Cortex. 2016 Mar 21doi: 10.1093/cercor/bhw076. PMID: 27005988.X-1

5076. Fluegge K. Does MeCP2 deficiency in autism confer protection against later development of Alzheimer's disease? A reply to Oberman and Pascual-Leone (2014). Med Hypotheses. 2016 Jul;92:18-20. doi: 10.1016/j.mehy.2016.04.020. PMID: 27241247.X-1

5077. Fluegge K. Maternal infection during pregnancy, risk of offspring autism, and the role of bacterial denitrification. Brain Behav Immun. 2016 Jun 24doi: 10.1016/j.bbi.2016.06.013. PMID: 27350210.X-1

5078. Fluegge K. The role of air pollution exposures in mediating nutritional biochemical profiles in autism: A reply to Jory (2015). Nutrition. 2016 Mar 6doi: 10.1016/j.nut.2016.02.017. PMID: 27158054.X-1
5079. Fluegge K. Methyl B12 and Autism Spectrum Disorders: Any Clues to Etiology? J Child Adolesc Psychopharmacol. 2016 Apr 22doi: 10.1089/cap.2016.0038. PMID: 27105341.X-1

5080. Fluegge K. Do Toxic Synergies of Underlying Etiologies Predispose the Positive Association Between Traumatic Brain Injury and ADHD? J Atten Disord. 2016 Mar 8doi: 10.1177/1087054716633858. PMID: 26957549.X-1, X-2

5081. Fluegge K. Environmental factors in the development of autism spectrum disorders: A reply to Sealey et al. (2016). Environ Int. 2016 Feb 26doi: 10.1016/j.envint.2016.02.024. PMID: 26926711.X-1, X-2, X-3

5082. Fluegge K. Does the Clinical Benefit of Ketamine Treatment Offer Any Clues to Autism Spectrum Disorder Etiology? J Clin Psychiatry. 2016 Jul;77(7):e903. doi: 10.4088/JCP.16lr10663. PMID: 27464326.X-1

5083. Fluegge K. A reply to 'Metabolic effects of sapropterin treatment in autism spectrum disorder: a preliminary study'. Transl Psychiatry. 2016;6:e793. doi: 10.1038/tp.2016.24. PMID: 27115124.X-1, X-2

5084. Flynn SD, Lo Y-y. Teacher implementation of trial-based functional analysis and differential reinforcement of alternative behavior for students with challenging behavior. Journal of Behavioral Education. 2016;25(1):1-31. doi: http://dx.doi.org/10.1007/s10864-015-92312.X-1, X-3, X-4

5085. Fogarty MJ, Kanjhan R, Bellingham MC, et al. Glycinergic Neurotransmission: A Potent Regulator of Embryonic Motor Neuron Dendritic Morphology and Synaptic Plasticity. J Neurosci. 2016 Jan 6;36(1):80-7. doi: 10.1523/jneurosci.1576-15.2016. PMID: 26740651.X-1

5086. Fombonne E. Prenatal Antidepressant Use and Risk of Autism Spectrum Disorders in the Children. JAMA Pediatr. 2016 May 31doi: 10.1001/jamapediatrics.2016.0745. PMID: 27244054.X-1 
5087. Fombonne E, Marcin C, Manero AC, et al. Prevalence of autism spectrum disorders in Guanajuato, Mexico: The Leon survey. Journal of Autism and Developmental Disorders. 2016;46(5):1669-85. doi: http://dx.doi.org/10.1007/s10803-016-26966.X-1

5088. Ford TC, Crewther DP. A Comprehensive Review of the (1)H-MRS Metabolite Spectrum in Autism Spectrum Disorder. Front Mol Neurosci. 2016;9:14. doi: 10.3389/fnmol.2016.00014. PMID: 27013964.X-1

5089. Forgeot d'Arc B, Ramus F, Lefebvre A, et al. Atypical social judgment and sensitivity to perceptual cues in autism spectrum disorders. Journal of Autism and Developmental Disorders. 2016;46(5):1574-81. doi: http://dx.doi.org/10.1007/s10803-0142208-5.X-1

5090. Forgeot d'Arc B, Delorme R, Zalla T, et al. Gaze direction detection in autism spectrum disorder. Autism. 2016 Apr 30doi: 10.1177/1362361316630880. PMID: 27132008.X-1

5091. Forsingdal A, Fejgin K, Nielsen V, et al. 15q13.3 homozygous knockout mouse model display epilepsy-, autism- and schizophrenia-related phenotypes. Transl Psychiatry. 2016;6(7):e860. doi: 10.1038/tp.2016.125. PMID: 27459725.X-1

5092. Forslund T, Brocki KC, Bohlin G, et al. The heterogeneity of attentiondeficit/hyperactivity disorder symptoms and conduct problems: Cognitive inhibition, emotion regulation, emotionality, and disorganized attachment. British Journal of Developmental Psychology. 2016;34(3):371-87. doi: http://dx.doi.org/10.1111/bjdp.12136.X-1

5093. Foss-Feig JH, McGugin RW, Gauthier I, et al. A functional neuroimaging study of fusiform response to restricted interests in children and adolescents with autism spectrum disorder. Journal of Neurodevelopmental Disorders. 2016;8doi: http://dx.doi.org/10.1186/s11689-016-91496.X-1

5094. Foster NEV, Ouimet T, Tryfon A, et al. Effects of age and attention on auditory global-local processing in children with autism spectrum disorder. Journal of Autism and Developmental Disorders. 2016;46(4):1415-28. doi: http://dx.doi.org/10.1007/s10803-0152684-2.X-1

5095. Fountain MD, Aten E, Cho MT, et al. The phenotypic spectrum of Schaaf-Yang syndrome: 18 new affected individuals from 14 families. Genet Med. 2016 May 19doi: 10.1038/gim.2016.53. PMID: 27195816.X-1

5096. Franzetti E, Casartelli M, D'Antona P, et al. Midgut epithelium in molting silkworm: A fine balance among cell growth, differentiation, and survival. Arthropod Struct Dev. 2016 Jul;45(4):368-79. doi: 10.1016/j.asd.2016.06.002. PMID: 27349418.X-1

5097. Frazier TW, Klingemier EW, Beukemann M, et al. Development of an objective autism risk index using remote eye tracking. Journal of the American Academy of Child \& Adolescent Psychiatry.

2016;55(4):301-9. doi: http://dx.doi.org/10.1016/j.jaac.2016.01.011.X1

5098. Freeman NC, Gray KM, Taffe JR, et al. A cross-syndrome evaluation of a new attention rating scale: The Scale of Attention in intellectual Disability. Res Dev Disabil. 2016 Jun 24;57:18-28. doi: 10.1016/j.ridd.2016.06.005. PMID: 27348856.X-1

5099. Freeman SM, Samineni S, Allen PC, et al. Plasma and CSF oxytocin levels after intranasal and intravenous oxytocin in awake macaques. Psychoneuroendocrinology. 2016 Apr;66:185-94. doi: 10.1016/j.psyneuen.2016.01.014. PMID: 26826355.X-1

5100. Freitag CM, Jensen K, Elsuni L, et al. Group-based cognitive behavioural psychotherapy for children and adolescents with ASD: The randomized, multicentre, controlled SOSTA - net trial. Journal of Child Psychology and Psychiatry. 2016;57(5):596605. doi: http://dx.doi.org/10.1111/jcpp.12509.X-1, X-4

5101. Frey AJ, Feldman DR, Trefely S, et al. LC-quadrupole/Orbitrap high-resolution mass 
spectrometry enables stable isotope-resolved simultaneous quantification and (1)(3)Cisotopic labeling of acyl-coenzyme A thioesters. Anal Bioanal Chem. 2016 May;408(13):3651-8. doi: 10.1007/s00216-016-9448-5. PMID: 26968563.X-1

5102. Fried R, Joshi G, Bhide P, et al. A study of the neuropsychological correlates in adults with high functioning autism spectrum disorders. Acta Neuropsychiatr. 2016 Apr 4:110. doi: 10.1017/neu.2016.12. PMID:

27040318.X-1

5103. Frye RE, Rossignol DA. Identification and Treatment of Pathophysiological Comorbidities of Autism Spectrum Disorder to Achieve Optimal Outcomes. Clin Med Insights Pediatr. 2016;10:43-56. doi: 10.4137/CMPed.S38337. PMID: 27330338.X-1

5104. Fujioka T, Inohara K, Okamoto Y, et al. Gazefinder as a clinical supplementary tool for discriminating between autism spectrum disorder and typical development in male adolescents and adults. Mol Autism. 2016;7:19. doi: 10.1186/s13229-016-0083-y. PMID: 27011784.X-1

5105. Fujiwara T, Sanada M, Kofuji T, et al. Unusual social behavior in HPC-1/syntaxin1A knockout mice is caused by disruption of the oxytocinergic neural system. J Neurochem. 2016 Jul;138(1):117-23. doi:

10.1111/jnc.13634. PMID: 27059771.X-1

5106. Fukumura K. Development of audience design in children with and without ASD. Developmental Psychology. 2016;52(1):71-87. doi: http://dx.doi.org/10.1037/dev0000064.X-1

5107. Furey H. Aristotle and Autism: Reconsidering a Radical Shift to Virtue Ethics in Engineering. Sci Eng Ethics. 2016 Jun 29doi: 10.1007/s11948-016-9787-9. PMID: 27357575.X-1

5108. Gabis LV, Gruber N, Berkenstadt M, et al. Fragile X Premutation Carrier Epidemiology and Symptomatology in IsraelResults from a Tertiary Child Developmental Center. Cerebellum. 2016 Jun 17doi: 10.1007/s12311-016-0804-y. PMID: 27312842.X-1
5109. Gabriele S, Sacco R, Altieri L, et al. Slow intestinal transit contributes to elevate urinary p-cresol level in Italian autistic children. Autism Research. 2016;9(7):752-9. doi: http://dx.doi.org/10.1002/aur.1571.X-1

5110. Gadow KD, Perlman G, Ramdhany L, et al. Clinical correlates of co-occurring psychiatric and autism spectrum disorder (ASD) symptom-induced impairment in children with ASD. Journal of Abnormal Child Psychology. 2016;44(1):129-39. doi: http://dx.doi.org/10.1007/s10802-015-99799.X-1

5111. Galling B, Roldan A, Nielsen RE, et al. Type 2 Diabetes Mellitus in Youth Exposed to Antipsychotics: A Systematic Review and Meta-analysis. JAMA Psychiatry. 2016 Mar;73(3):247-59. doi: 10.1001/jamapsychiatry.2015.2923. PMID: 26792761.X-1

5112. Gandal MJ, Geschwind DH. The Genetics-Driven Revival in Neuropsychiatric Drug Development. Biol Psychiatry. 2016 Apr 15;79(8):628-30. doi:

10.1016/j.biopsych.2016.02.024. PMID: 26997117.X-1

5113. Gangi DN, Messinger DS, Martin ER, et al. Dopaminergic variants in siblings at high risk for autism: Associations with initiating joint attention. Autism Res. 2016 Mar 15doi: 10.1002/aur.1623. PMID: 26990357.X-1

5114. Gao Y, Wang F, Eisinger BE, et al. Integrative Single-Cell Transcriptomics Reveals Molecular Networks Defining Neuronal Maturation During Postnatal Neurogenesis. Cereb Cortex. 2016 Mar 17doi: 10.1093/cercor/bhw040. PMID: 26989163.X-1

5115. Garcia D, Dukes C, Brady MP, et al. Using modeling and rehearsal to teach fire safety to children with autism. J Appl Behav Anal. 2016 Jun 30doi: 10.1002/jaba.331. PMID: 27357240.X-1, X-3

5116. Garcia-Lopez C, Sarria E, Pozo P. Parental Self-Efficacy and Positive Contributions Regarding Autism Spectrum Condition: An Actor-Partner Interdependence Model. J Autism Dev Disord. 2016 Jul;46(7):2385-98. doi: 10.1007/s10803-0162771-z. PMID: 27007725.X-1 
5117. García-López C, Sarriá E, Pozo P. Parental self-efficacy and positive contributions regarding autism spectrum condition: An actorpartner interdependence model. Journal of Autism and Developmental Disorders. 2016;46(7):2385-98. doi: http://dx.doi.org/10.1007/s10803-016-2771z.X-1

5118. Garcia-Villamisar D, Dattilo J, Muela C. Effects of therapeutic recreation on adults with ASD and ID: a preliminary randomized control trial. J Intellect Disabil Res. 2016 Jul 28doi: 10.1111/jir.12320. PMID: 27465318.X-1

5119. Garg P, Lillystone D, Dossetor D, et al. A Pilot Study for Understanding the Perceptions of Australian General Practitioners Regarding Psychopharmacology for Children With Autism Spectrum Disorders. J Prim Care Community Health. 2016 Apr 20doi: 10.1177/2150131916644030. PMID: 27103117.X-1

5120. Garman HD, Spaulding CJ, Webb SJ, et al. Wanting it Too Much: An Inverse Relation Between Social Motivation and Facial Emotion Recognition in Autism Spectrum Disorder. Child Psychiatry Hum Dev. 2016 Jan 7doi: 10.1007/s10578-015-0620-5. PMID: 26743637.X-1

5121. Garon N, Zwaigenbaum L, Bryson S, et al. Temperament and its association with autism symptoms in a high-risk population. Journal of Abnormal Child Psychology. 2016;44(4):757-69. doi: http://dx.doi.org/10.1007/s10802-015-00641.X-1

5122. Gart N, Zamora I, Williams ME. Parallel models of assessment: Infant mental health and therapeutic assessment models intersect through early childhood case studies. Infant Mental Health Journal. 2016;37(4):45265. doi: http://dx.doi.org/10.1002/imhj.21573.X-1

5123. Gassowska M, Baranowska-Bosiacka I, Moczydlowska J, et al. Perinatal exposure to lead $(\mathrm{Pb})$ promotes Tau phosphorylation in the rat brain in a GSK-3beta and CDK5 dependent manner: Relevance to neurological disorders. Toxicology. 2016 Mar 10;347-349:17-28. doi: 10.1016/j.tox.2016.03.002. PMID: 27012722.X-1

5124. Gaudi S, Guffanti G, Fallon J, et al. Epigenetic mechanisms and associated brain circuits in the regulation of positive emotions: A role for Transposable Elements. J Comp Neurol. 2016 May 25doi: 10.1002/cne.24046. PMID: 27224878.X-1

5125. Geary N, Bachler T, Whiting L, et al. RYGB progressively increases avidity for a low-energy, artificially sweetened diet in female rats. Appetite. 2016 Mar 1;98:133-41. doi: 10.1016/j.appet.2015.11.029. PMID: 26707654.X-1

5126. Gee BM, Peterson TW. Changes in Caregiver Knowledge and Perceived Competency Following Group Education about Sensory Processing Disturbances: An Exploratory Study. Occup Ther Int. 2016 Jul 4doi: 10.1002/oti.1435. PMID: 27374620.X-1

5127. Gellen B, Volgyi K, Gyorffy BA, et al. Proteomic investigation of the prefrontal cortex in the rat clomipramine model of depression. $\mathrm{J}$ Proteomics. 2016 Jun 28doi: 10.1016/j.jprot.2016.06.027. PMID: 27371348.X-1

5128. Geretsegger M, Holck U, Bieleninik L, et al. Feasibility of a Trial on Improvisational Music Therapy for Children with Autism Spectrum Disorder. J Music Ther. 2016 Summer;53(2):93-120. doi: 10.1093/jmt/thv038. PMID: 27084832.X-1

5129. Getz KD, Anderka MT, Werler MM, et al. Maternal Pre-pregnancy Body Mass Index and Autism Spectrum Disorder among Offspring: A Population-Based Case-Control Study. Paediatr Perinat Epidemiol. 2016 May 30doi: 10.1111/ppe.12306. PMID: 27239935.X-1

5130. Getz SA, DeSpenza T, Jr., Li M, et al. Rapamycin prevents, but does not reverse, aberrant migration in Pten knockout neurons. Neurobiol Dis. 2016 Sep;93:12-20. doi: 10.1016/j.nbd.2016.03.010. PMID: 26992888.X-1

5131. Gevarter C, O'Reilly MF, Kuhn M, et al. Increasing the vocalizations of individuals with autism during intervention with a speech- 
generating device. Journal of Applied Behavior Analysis. 2016;49(1):17-33. doi:

http://dx.doi.org/10.1002/jaba.270.X-3, X-4

5132. Ghilain CS, Parlade MV, McBee MT, et al. Validation of the Pictorial Infant Communication Scale for preschool-aged children with autism spectrum disorder. Autism. 2016 Apr 30doi: 10.1177/1362361316636757. PMID: 27132009.X-1

5133. Gialluisi A, Visconti A, Willcutt EG, et al. Investigating the effects of copy number variants on reading and language performance. Journal of Neurodevelopmental Disorders. 2016;8doi: http://dx.doi.org/10.1186/s11689016-9147-8.X-1

5134. Giannakopoulos A, Fryssira H, Tzetis $\mathrm{M}$, et al. Central precocious puberty in a boy with 22q13 deletion syndrome and NOTCH-1 gene duplication. J Pediatr Endocrinol Metab. 2016 May 28doi: 10.1515/jpem-2015-0484. PMID: 27235670.X-1

5135. Giannopulu I, Montreynaud V, Watanabe T. Minimalistic toy robot to analyze a scenery of speaker-listener condition in autism. Cognitive Processing. 2016;17(2):195203. doi: http://dx.doi.org/10.1007/s10339-0160752-y.X-1, X-3

5136. Gidaya NB, Lee BK, Burstyn I, et al. In utero Exposure to beta-2-Adrenergic

Receptor Agonist Drugs and Risk for Autism Spectrum Disorders. Pediatrics. 2016

Feb;137(2):e20151316. doi: 10.1542/peds.2015-1316. PMID: 26738885.X-1

5137. Giles LL, Martini DR. Challenges and Promises of Pediatric Psychopharmacology.

Acad Pediatr. 2016 Apr 5doi:

10.1016/j.acap.2016.03.011. PMID:

27064142.X-1

5138. Ginevra MC, Nota L, Stokes MA. The differential effects of autism and down's syndrome on sexual behavior. Autism Research. 2016;9(1):131-40. doi: http://dx.doi.org/10.1002/aur.1504.X-1

5139. Gnanasekaran S, Choueiri R, Neumeyer A, et al. Impact of employee benefits on families with children with autism spectrum disorders. Autism. 2016;20(5):616-22. doi:
http://dx.doi.org/10.1177/1362361315598891.X $-1$

5140. Gogliotti RG, Senter RK, Rook JM, et al. mGlu5 positive allosteric modulation normalizes synaptic plasticity defects and motor phenotypes in a mouse model of Rett syndrome. Hum Mol Genet. 2016 Mar 2doi:

10.1093/hmg/ddw074. PMID: 26936821.X-1

5141. Gogou M, Spilioti M, Tramma D, et al. Succinic Semialdehyde Dehydrogenase Deficiency Presenting as Autism Spectrum Disorder. Indian J Pediatr. 2016 Jan 25doi: 10.1007/s12098-015-2003-0. PMID: 26806207.X-1

5142. Goin-Kochel RP. Musings on the puzzle piece. Autism. 2016 Feb;20(2):250. doi: 10.1177/1362361315602135. PMID: 26395235.X-1

5143. Goldberg MC, Allman MJ, Hagopian LP, et al. Examining the reinforcing value of stimuli within social and non-social contexts in children with and without high-functioning autism. Autism. 2016 Jul 1doi: 10.1177/1362361316655035. PMID: 27368350.X-1

5144. Goldfarb C, Genore L, Hunt C, et al. Hyperbaric oxygen therapy for the treatment of children and youth with Autism Spectrum disorders: An evidence-based systematic review. Research in Autism Spectrum Disorders. 2016;29-30:1-7. doi: http://dx.doi.org/10.1016/j.rasd.2016.05.004.X2

5145. Goldin RL, Matson JL. Premature birth as a risk factor for autism spectrum disorder. Developmental Neurorehabilitation. 2016;19(3):203-6.X-1

5146. Goldschmidt J. What Happened to Paul? Manifestation of Abnormal Pain Response for Individuals With Autism Spectrum Disorder. Qual Health Res. 2016 Apr 26doi: 10.1177/1049732316644415. PMID: 27117957.X-1

5147. Golub MS, Hogrefe CE, Bulleri AM. Peer social interaction is facilitated in juvenile rhesus monkeys treated with fluoxetine. Neuropharmacology. 2016 Jun;105:553-60. doi: 
10.1016/j.neuropharm.2016.02.025. PMID: 26905291.X-1

5148. Gomez-Perez MM, Calero MD, Mata $\mathrm{S}$, et al. Discrepancies between direct and indirect measures of interpersonal and neurocognitive skills in autism spectrum disorder children. J Clin Exp Neuropsychol. 2016 Oct;38(8):875-86. doi: 10.1080/13803395.2016.1170106. PMID: 27192042.X-1

5149. Gonthier C, Longuepee L, Bouvard M. Sensory Processing in Low-Functioning Adults with Autism Spectrum Disorder: Distinct Sensory Profiles and Their Relationships with Behavioral Dysfunction. J Autism Dev Disord. 2016 Jun 30doi: 10.1007/s10803-016-2850-1. PMID: 27364513.X-1

5150. Gordon-Lipkin E, Foster J, Peacock G. Whittling Down the Wait Time: Exploring Models to Minimize the Delay from Initial Concern to Diagnosis and Treatment of Autism Spectrum Disorder. Pediatr Clin North Am. 2016 Oct;63(5):851-9. doi: 10.1016/j.pcl.2016.06.007. PMID: 27565363.X-1

5151. Gorman K, Olson L, Hill AP, et al. Uh and um in children with autism spectrum disorders or language impairment. Autism Res. 2016 Jan 22doi: 10.1002/aur.1578. PMID: 26800246.X-1

5152. Goulardins JB, Rigoli D, Piek JP, et al. The relationship between motor skills, ADHD symptoms, and childhood body weight. Research in Developmental Disabilities. 2016;55:279-86. doi: http://dx.doi.org/10.1016/j.ridd.2016.05.005.X1

5153. Grabb MC, Gobburu JV. Challenges in developing drugs for pediatric CNS disorders: A focus on psychopharmacology. Prog Neurobiol. 2016 May 20doi: 10.1016/j.pneurobio.2016.05.003. PMID: 27216638.X-1

5154. Grainger C, Williams DM, Lind SE. Recognition memory and source memory in autism spectrum disorder: A study of the intention superiority and enactment effects. Autism. 2016 Jun 22doi:
10.1177/1362361316653364. PMID: 27335106.X-1

5155. Grainger C, Williams DM, Lind SE. Metacognitive monitoring and control processes in children with autism spectrum disorder: Diminished judgement of confidence accuracy. Consciousness and Cognition: An International Journal. 2016;42:65-74. doi: http://dx.doi.org/10.1016/j.concog.2016.03.003. $\mathrm{X}-1, \mathrm{X}-3$

5156. Granich J, Lin A, Hunt A, et al. Obesity and associated factors in youth with an autism spectrum disorder. Autism. 2016 Feb 17doi: 10.1177/1362361315616345. PMID: 26893400.X-1

5157. Grant N, Rodger S, Hoffmann T. Intervention decision-making processes and information preferences of parents of children with autism spectrum disorders. Child: Care, Health and Development. 2016;42(1):125-34. doi: http://dx.doi.org/10.1111/cch.12296.X-1

5158. Gray C, Climie EA. Children with attention deficit/hyperactivity disorder and reading disability: A review of the efficacy of medication treatments. Frontiers in Psychology. 2016;7.X-1, X-2

5159. Graziano PA, Garcia A. Attentiondeficit hyperactivity disorder and children's emotion dysregulation: A meta-analysis. Clinical Psychology Review. 2016;46:106-23. doi:

http://dx.doi.org/10.1016/j.cpr.2016.04.011.X1, $\mathrm{X}-2$

5160. Green AE, Kenworthy L, Gallagher $\mathrm{NM}$, et al. Social analogical reasoning in school-aged children with autism spectrum disorder and typically developing peers.

Autism. 2016 May 12doi:

10.1177/1362361316644728. PMID: 27178998.X-1

5161. Green D, Chandler S, Charman T, et al. Brief Report: DSM-5 Sensory Behaviours in Children With and Without an Autism Spectrum Disorder. J Autism Dev Disord. 2016 Jul 30doi: 10.1007/s10803-016-2881-7. PMID: 27475418.X-1

5162. Green J, Leadbitter K, Kay C, et al. Autism spectrum disorder in children adopted 
after early care breakdown. Journal of Autism and Developmental Disorders.

2016;46(4):1392-402. doi:

http://dx.doi.org/10.1007/s10803-015-2680-

6.X-1

5163. Green JL, Rinehart N, Anderson V, et al. Association between autism symptoms and family functioning in children with attentiondeficit/hyperactivity disorder: a communitybased study. Eur Child Adolesc Psychiatry. 2016 May 6doi: 10.1007/s00787-016-0861-2. PMID: 27154048.X-1

5164. Green JL, Sciberras E, Anderson V, et al. Association between autism symptoms and functioning in children with ADHD. Arch Dis Child. 2016 Apr 26doi: 10.1136/archdischild2015-310257. PMID: 27117836.X-1

5165. Green SA, Hernandez L, Bookheimer SY, et al. Salience Network Connectivity in Autism Is Related to Brain and Behavioral Markers of Sensory Overresponsivity. J Am Acad Child Adolesc Psychiatry. 2016 Jul;55(7):618-26.e1. doi: 10.1016/j.jaac.2016.04.013. PMID: 27343889.X-1

5166. Green Snyder L, D'Angelo D, Chen Q, et al. Autism Spectrum Disorder, Developmental and Psychiatric Features in 16p11.2 Duplication. J Autism Dev Disord. 2016 May 21doi: 10.1007/s10803-016-2807-4. PMID: 27207092.X-1

5167. Greenslade KJ, Coggins TE. Brief Report: An Independent Replication and Extension of Psychometric Evidence Supporting the Theory of Mind Inventory. J Autism Dev Disord. 2016 Apr 22doi: 10.1007/s10803-016-2784-7. PMID: 27106567.X-1

5168. Greer BD, Neidert PL, Dozier CL. A component analysis of toilet-training procedures recommended for young children. Journal of Applied Behavior Analysis. 2016;49(1):69-84. doi: http://dx.doi.org/10.1002/jaba.275.X-1

5169. Griguoli M, Sgritta M, Cherubini E. Presynaptic BK channels control transmitter release: physiological relevance and potential therapeutic implications. J Physiol. 2016 Jul
1;594(13):3489-500. doi: 10.1113/jp271841. PMID: 26969302.X-1

5170. Grodberg D, Siper P, Jamison J, et al. A simplified diagnostic observational assessment of autism spectrum disorder in early childhood. Autism Research. 2016;9(4):443-9. doi: http://dx.doi.org/10.1002/aur.1539.X-1

5171. Gross C. Defective phosphoinositide metabolism in autism. J Neurosci Res. 2016 Jul 4doi: 10.1002/jnr.23797. PMID: 27376697.X-1

5172. Grossi E, Melli S, Dunca D, et al. Unexpected improvement in core autism spectrum disorder symptoms after long-term treatment with probiotics. SAGE Open Med Case Rep. 2016;4:2050313x16666231. doi: 10.1177/2050313x16666231. PMID: 27621806.X-3, X-4

5173. Grossman ES, Hoffman YS, Shrira A. Trauma-Related Context Increases Sleep Disturbances in People with Acute Stress Disorder Symptoms. Stress Health. 2016 Apr 6doi: 10.1002/smi.2679. PMID: 27062710.X-1

5174. Grzadzinski R, Carr T, Colombi C, et al. Measuring Changes in Social Communication Behaviors: Preliminary Development of the Brief Observation of Social Communication Change (BOSCC). J Autism Dev Disord. 2016 Jul;46(7):2464-79. doi: 10.1007/s10803-016-2782-9. PMID: 27062034.X-1

5175. Grzadzinski R, Dick C, Lord C, et al. Parent-reported and clinician-observed autism spectrum disorder (ASD) symptoms in children with attention deficit/hyperactivity disorder (ADHD): implications for practice under DSM5. Mol Autism. 2016;7:7. doi: 10.1186/s13229016-0072-1. PMID: 26788284.X-1

5176. Guardino C, Cannon JE. Deafness and Diversity: Reflections and Directions. Am Ann Deaf. 2016 Spring;161(1):104-12. doi: 10.1353/aad.2016.0016. PMID: 27156921.X-1

5177. Guffanti G, Gaudi S, Klengel T, et al. LINE1 insertions as a genomic risk factor for schizophrenia: Preliminary evidence from an affected family. Am J Med Genet B Neuropsychiatr Genet. 2016 Jun;171(4):534-45. doi: 10.1002/ajmg.b.32437. PMID: 26990047.X-1 
5178. Guler UO, Yuksel S, Yakici S, et al. Analysis of the reliability of surgeons' ability to differentiate between idiopathic and degenerative spinal deformity in adults radiologically. What descriptive parameters help them decide? Eur Spine J. 2016 Jan 14doi: 10.1007/s00586-015-4366-3. PMID: 26769036.X-1

5179. Gulsrud AC, Hellemann G, Shire S, et al. Isolating active ingredients in a parentmediated social communication intervention for toddlers with autism spectrum disorder. J Child Psychol Psychiatry. 2016 May;57(5):606-13. doi: 10.1111/jcpp.12481. PMID: 26525461.X-1

5180. Gulsrud AC, Hellemann G, Shire S, et al. Isolating active ingredients in a parentmediated social communication intervention for toddlers with autism spectrum disorder. Journal of Child Psychology and Psychiatry. 2016;57(5):606-13. doi: http://dx.doi.org/10.1111/jcpp.12481.X-1

5181. Gunn KCM, Delafield-Butt JT. Teaching children with autism spectrum disorder with restricted interests: A review of evidence for best practice. Review of Educational Research. 2016;86(2):408-30. doi: http://dx.doi.org/10.3102/0034654315604027.X $-1, \mathrm{X}-2$

5182. Guo L, Worth AJ, Mesaros C, et al. Diisopropylethylamine/hexafluoroisopropanolmediated ion-pairing ultra-high-performance liquid chromatography/mass spectrometry for phosphate and carboxylate metabolite analysis: utility for studying cellular metabolism. Rapid Commun Mass Spectrom. 2016 Aug 30;30(16):1835-45. doi: 10.1002/rcm.7667. PMID: 27476658.X-1

5183. Guo W, Molinaro G, Collins KA, et al. Selective Disruption of Metabotropic Glutamate Receptor 5-Homer Interactions Mimics Phenotypes of Fragile X Syndrome in Mice. J Neurosci. 2016 Feb 17;36(7):2131-47. doi: 10.1523/jneurosci.2921-15.2016. PMID: 26888925.X-1

5184. Guy J, Mottron L, Berthiaume C, et al. A Developmental Perspective of Global and Local Visual Perception in Autism Spectrum Disorder. J Autism Dev Disord. 2016 Jul 1doi: 10.1007/s10803-016-2834-1. PMID:

27371139.X-1

5185. Haack LM, Gerdes AC, Lawton KE, et al. Understanding and measuring functional impairment in diverse children with ADHD: Development of the ADHD-FX scale with an at-risk, community sample. Journal of Attention Disorders. 2016;20(6):487-500. doi: http://dx.doi.org/10.1177/1087054714527791.X $-1$

5186. Haas NA, Soetemann DB, Ates I, et al. Closure of Secundum Atrial Septal Defects by Using the Occlutech Occluder Devices in More Than 1300 Patients: The IRFACODE Project: A Retrospective Case Series. Catheter Cardiovasc Interv. 2016 Mar 31doi: 10.1002/ccd.26497. PMID: 27029396.X-1

5187. Habela CW, Song H, Ming GL. Modeling synaptogenesis in schizophrenia and autism using human iPSC derived neurons. Mol Cell Neurosci. 2016 Jun;73:52-62. doi: 10.1016/j.mcn.2015.12.002. PMID: 26655799.X-1

5188. Hage A, Banaschewski T, Buitelaar $\mathrm{JK}$, et al. Glutamatergic medication in the treatment of obsessive compulsive disorder (OCD) and autism spectrum disorder (ASD) study protocol for a randomised controlled trial. Trials. 2016;17(1):141. doi: 10.1186/s13063016-1266-8. PMID: 26983548.X-1

5189. Hagenaars SP, Harris SE, Davies G, et al. Shared genetic aetiology between cognitive functions and physical and mental health in UK Biobank (N=112 151) and 24 GWAS consortia. Mol Psychiatry. 2016 Jan 26doi: 10.1038/mp.2015.225. PMID: 26809841.X-1

5190. Haggarty SJ, Silva MC, Cross A, et al. Advancing drug discovery for neuropsychiatric disorders using patient-specific stem cell models. Mol Cell Neurosci. 2016 Jun;73:10415. doi: 10.1016/j.mcn.2016.01.011. PMID: 26826498.X-1

5191. Hagmann CE, Wyble B, Shea N, et al. Children with autism detect targets at very rapid presentation rates with similar accuracy as adults. Journal of Autism and Developmental Disorders. 2016;46(5):1762-72. doi: http://dx.doi.org/10.1007/s10803-016-27059.X-1, X-3 
5192. Haigh SM, Gupta A, Barb SM, et al. Differential sensory fMRI signatures in autism and schizophrenia: Analysis of amplitude and trial-to-trial variability. Schizophr Res. 2016 Apr 12doi: 10.1016/j.schres.2016.03.036. PMID: 27083780.X-1

5193. Haker H, Schneebeli M, Stephan KE. Can Bayesian Theories of Autism Spectrum Disorder Help Improve Clinical Practice? Front Psychiatry. 2016;7:107. doi: 10.3389/fpsyt.2016.00107. PMID: 27378955.X-1

5194. Hall CL, Valentine AZ, Groom MJ, et al. The clinical utility of the continuous performance test and objective measures of activity for diagnosing and monitoring ADHD in children: A systematic review. European Child \& Adolescent Psychiatry.

2016;25(7):677-99. doi:

http://dx.doi.org/10.1007/s00787-015-0798x.X-1, X-2

5195. Hall DA, Robertson E, Shelton AL, et al. Update on the Clinical, Radiographic, and Neurobehavioral Manifestations in FXTAS and FMR1 Premutation Carriers. Cerebellum. 2016 Jun 10doi: 10.1007/s12311-016-0799-4. PMID: 27287737.X-1

5196. Hall SS, Wright HF, Mills DS. What Factors Are Associated with Positive Effects of Dog Ownership in Families with Children with Autism Spectrum Disorder? The Development of the Lincoln Autism Pet Dog Impact Scale. PLoS One. 2016;11(2):e0149736. doi: 10.1371/journal.pone.0149736. PMID: 26894820.X-1

5197. Halpin J. What do nurses think they are doing in pre-school autism assessment? $\mathrm{Br} \mathrm{J}$ Nurs. 2016 Mar 24-Apr 13;25(6):319-23. doi: 10.12968/bjon.2016.25.6.319. PMID: 27019169.X-1

5198. Hames EC, Murphy B, Rajmohan R, et al. Visual, Auditory, and Cross Modal Sensory Processing in Adults with Autism: An EEG Power and BOLD fMRI Investigation. Front Hum Neurosci. 2016;10:167. doi: 10.3389/fnhum.2016.00167. PMID: 27148020.X-1
5199. Hamilton DK, Kanter AS, Bolinger $\mathrm{BD}$, et al. Reoperation rates in minimally invasive, hybrid and open surgical treatment for adult spinal deformity with minimum 2-year follow-up. Eur Spine J. 2016 Feb 24doi: 10.1007/s00586-016-4443-2. PMID: 26909764.X-1

5200. Hamilton J, Stevens G, Girdler S. Becoming a Mentor: The Impact of Training and the Experience of Mentoring University Students on the Autism Spectrum. PLoS One. 2016;11(4):e0153204. doi:

10.1371/journal.pone.0153204. PMID: 27070418.X-1

5201. Hampton LH, Kaiser AP. Intervention effects on spoken-language outcomes for children with autism: A systematic review and meta-analysis. Journal of Intellectual Disability Research. 2016;60(5):444-63. doi: http://dx.doi.org/10.1111/jir.12283.X-2

5202. Han Y, Sun J, Luo C, et al. Comparison of pedicle screw-based dynamic stabilization and fusion surgery in the treatment of radiographic adjacent-segment degeneration: a retrospective analysis of single L5-S1 degenerative spondylosis covering 4 years. J Neurosurg Spine. 2016 Jun 24:1-7. doi: 10.3171/2016.4.spine1679. PMID: 27341057.X-1

5203. Hanaie R, Mohri I, Kagitani-Shimono $\mathrm{K}$, et al. White matter volume in the brainstem and inferior parietal lobule is related to motor performance in children with autism spectrum disorder: A voxel-based morphometry study. Autism Res. 2016 Jan 25doi: 10.1002/aur.1605. PMID: 26808675.X-1

5204. Hanamsagar R, Bilbo SD. Sex differences in neurodevelopmental and neurodegenerative disorders: Focus on microglial function and neuroinflammation during development. J Steroid Biochem Mol Biol. 2016 Jun;160:127-33. doi: 10.1016/j.jsbmb.2015.09.039. PMID: 26435451.X-1, X-2, X-3, X-4

5205. Haraya S, Karasawa K, Sano Y, et al. Development of a highly specific enzyme immunoassay for oxytocin and its use in plasma samples. Ann Clin Biochem. 2016 May 10doi: 10.1177/0004563216645122. PMID: 27166312.X-1 
5206. Hardan AY, Fung LK, Frazier T, et al. A proton spectroscopy study of white matter in children with autism. Progress in NeuroPsychopharmacology \& Biological Psychiatry. 2016;66:48-53. doi: http://dx.doi.org/10.1016/j.pnpbp.2015.11.005. $\mathrm{X}-1$

5207. Harden SW, Frazier CJ. Oxytocin Depolarizes Fast-Spiking Hilar Interneurons and Induces GABA Release onto Mossy Cells of the Rat Dentate Gyrus. Hippocampus. 2016 Apr 12doi: 10.1002/hipo.22595. PMID: 27068005.X-1

5208. Hare DJ, Gracey C, Wood C. Anxiety in high-functioning autism: A pilot study of experience sampling using a mobile platform. Autism. 2016 Aug;20(6):730-43. doi: 10.1177/1362361315604817. PMID: 26514793.X-1

5209. Harker CM, Ibañez LV, Nguyen TP, et al. The effect of parenting style on social smiling in infants at high and low risk for ASD. Journal of Autism and Developmental Disorders. 2016;46(7):2399-407. doi: http://dx.doi.org/10.1007/s10803-016-2772y.X-1

5210. Harkin LF, Gerrelli D, Gold Diaz DC, et al. Distinct expression patterns for type II topoisomerases IIA and IIB in the early foetal human telencephalon. J Anat. 2016 Mar;228(3):452-63. doi: 10.1111/joa.12416. PMID: 26612825.X-1

5211. Harmon P, Galipeau K, Xu W, et al. Mechanism of Dissolution-Induced Nanoparticle Formation from a CopovidoneBased Amorphous Solid Dispersion. Mol Pharm. 2016 May 2;13(5):1467-81. doi: 10.1021/acs.molpharmaceut.5b00863. PMID: 27019407.X-1

5212. Harriage B, Blair KS, Miltenberger R. An Evaluation of a Parent Implemented In Situ Pedestrian Safety Skills Intervention for Individuals with Autism. J Autism Dev Disord. 2016 Jun;46(6):2017-27. doi: 10.1007/s10803016-2730-8. PMID: 26864158.X-1, X-3

5213. Harrison AJ, Long KA, Manji KP, et al. Development of a Brief Intervention to Improve Knowledge of Autism and Behavioral
Strategies Among Parents in Tanzania. Intellect Dev Disabil. 2016 Jun;54(3):187-201. doi: 10.1352/1934-9556-54.3.187. PMID: 27268474.X-1

5214. Harrison AJ, Lu Z, McLean RL, et al. Cognitive and adaptive correlates of an ADOSderived joint attention composite. Research in Autism Spectrum Disorders. 2016;29-30:66-78. doi: http://dx.doi.org/10.1016/j.rasd.2016.07.001.X1

5215. Harrison AJ, Slane MM, Hoang L, et al. An international review of autism knowledge assessment measures. Autism. 2016 May 5doi: 10.1177/1362361316638786. PMID: 27154908.X-1

5216. Harrop C, Green J, Hudry K. Play complexity and toy engagement in preschoolers with autism spectrum disorder: Do girls and boys differ? Autism. 2016 Mar 2doi: 10.1177/1362361315622410. PMID: 26936930.X-1

5217. Harrop C, Gulsrud A, Shih W, et al. Characterizing caregiver responses to restricted and repetitive behaviors in toddlers with autism spectrum disorder. Autism. 2016;20(3):330-42. doi:

http://dx.doi.org/10.1177/1362361315580443.X $-1$

5218. Harrop C, McBee M, Boyd BA. How are child restricted and repetitive behaviors associated with caregiver stress over time? A parallel process multilevel growth model. Journal of Autism and Developmental Disorders. 2016;46(5):1773-83. doi: http://dx.doi.org/10.1007/s10803-016-27077.X-1

5219. Harstad E, Blum N, Gahman A, et al. Management of Attention-Deficit/Hyperactivity Disorder by Developmental-Behavioral Pediatricians: A DBPNet Study. J Dev Behav Pediatr. 2016 Jul 13doi: 10.1097/dbp.0000000000000329. PMID: 27429355.X-1

5220. Hartley SL, Papp LM, Blumenstock SM, et al. The Effect of Daily Challenges in Children With Autism on Parents' Couple Problem-Solving Interactions. J Fam Psychol. 
2016 Jun 23doi: 10.1037/fam0000219. PMID: 27336179.X-1

5221. Hartley SL, Papp LM, Bolt D. Spillover of Marital Interactions and Parenting Stress in Families of Children With Autism Spectrum Disorder. J Clin Child Adolesc Psychol. 2016 May 24:1-12. doi: 10.1080/15374416.2016.1152552. PMID: 27218268.X-1

5222. Hashemi E, Ariza J, Rogers H, et al. The Number of Parvalbumin-Expressing Interneurons Is Decreased in the Medial Prefrontal Cortex in Autism. Cereb Cortex. 2016 Feb 27doi: 10.1093/cercor/bhw021. PMID: 26922658.X-1

5223. Hashimoto R, Nakazawa T, Tsurusaki $\mathrm{Y}$, et al. Whole-exome sequencing and neurite outgrowth analysis in autism spectrum disorder. J Hum Genet. 2016 Mar;61(3):199-206. doi: 10.1038/jhg.2015.141. PMID: 26582266.X-1

5224. Hatakenaka Y, Fernell E, Sakaguchi M, et al. ESSENCE-Q - a first clinical validation study of a new screening questionnaire for young children with suspected neurodevelopmental problems in south Japan. Neuropsychiatr Dis Treat. 2016;12:1739-46. doi: 10.2147/ndt.s108411. PMID: 27478377.X1

5225. Hatakenaka Y, Fernell E, Sakaguchi $\mathrm{M}$, et al. ESSENCE-Q-A first clinical validation study of a new screening questionnaire for young children with suspected neurodevelopmental problems in south Japan. Neuropsychiatric Disease and Treatment. 2016;12.X-1

5226. Havdahl KA, von Tetzchner S, Huerta $\mathrm{M}$, et al. Utility of the child behavior checklist as a screener for autism spectrum disorder. Autism Research. 2016;9(1):33-42. doi: http://dx.doi.org/10.1002/aur.1515.X-1

5227. Heaton TJ, Freeth M. Reduced visual exploration when viewing photographic scenes in individuals with autism spectrum disorder. Journal of Abnormal Psychology. 2016;125(3):399-411. doi: http://dx.doi.org/10.1037/abn0000145.X-1

5228. Hebron J, Oldfield J, Humphrey N. Cumulative risk effects in the bullying of children and young people with autism spectrum conditions. Autism. 2016 Apr 19doi: 10.1177/1362361316636761. PMID: 27095833.X-1

5229. Hecht PM, Hudson M, Connors SL, et al. Maternal serotonin transporter genotype affects risk for ASD with exposure to prenatal stress. Autism Res. 2016 Apr 19doi: 10.1002/aur.1629. PMID: 27091118.X-1

5230. Hedley D. New rapid autism screening test. J Pediatr. 2016 Jan;168:255-6. doi: 10.1016/j.jpeds.2015.10.075. PMID: 26719185.X-1

5231. Hedley D, Brewer N, Nevill R, et al. The relationship between clinicians' confidence and accuracy, and the influence of child characteristics, in the screening of autism spectrum disorder. Journal of Autism and Developmental Disorders. 2016;46(7):2340-8. doi: http://dx.doi.org/10.1007/s10803-0162766-9.X-1

5232. Heifert TA, Susi A, Hisle-Gorman E, et al. Feeding Disorders in Children with Autism Spectrum Disorders Are Associated with Eosinophilic Esophagitis. J Pediatr Gastroenterol Nutr. 2016 Jun 8doi: 10.1097/mpg.0000000000001282. PMID: 27276430.X-1

5233. Heimann M, Nordqvist E, Strid K, et al. Children with autism respond differently to spontaneous, elicited and deferred imitation. Journal of Intellectual Disability Research. 2016;60(5):491-501. doi: http://dx.doi.org/10.1111/jir.12272.X-1, X-3

5234. Heinen CA, Jongejan A, Watson PJ, et al. A specific mutation in TBL1XR1 causes Pierpont syndrome. J Med Genet. 2016 May;53(5):330-7. doi: 10.1136/jmedgenet2015-103233. PMID: 26769062.X-1

5235. Helland WA, Posserud M-B, Helland $\mathrm{T}$, et al. Language impairments in children with ADHD and in children with reading disorder. Journal of Attention Disorders. 2016;20(7):5819. doi: http://dx.doi.org/10.1177/1087054712461530.X $-1$

5236. Helles A, Gillberg IC, Gillberg C, et al. Asperger syndrome in males over two 
decades: Quality of life in relation to diagnostic stability and psychiatric comorbidity. Autism. 2016 May 26doi: 10.1177/1362361316650090. PMID: 27233289.X-1

5237. Hellmuth SG, Pedersen LH, Miltoft $\mathrm{CB}$, et al. Increased nuchal translucency thickness and the risk of neurodevelopmental disorders. Ultrasound Obstet Gynecol. 2016 May 17doi: 10.1002/uog.15961. PMID: 27183961.X-1

5238. Helt MS, Fein DA. Facial feedback and social input: Effects on laughter and enjoyment in children with autism spectrum disorders. Journal of Autism and Developmental Disorders. 2016;46(1):83-94. doi: http://dx.doi.org/10.1007/s10803-0152545-z.X-1

5239. Hemmingsson H, Olafsdottir LB, Egilson ST. Agreements and disagreements between children and their parents in healthrelated assessments. Disabil Rehabil. 2016 Jun 13:1-14. doi: 10.1080/09638288.2016.1189603. PMID: 27291406.X-1, X-2

5240. Hendriksen JG, Vles JS. Muscle and brain: a dyad with important diagnostic and therapeutic implications. Dev Med Child Neurol. 2016 Jan;58(1):13. doi: 10.1111/dmcn.12937. PMID: 26456166.X-1

5241. Hendy H, Harclerode W, Williams KE. The Parent Mealtime Action Scale revised (PMAS-R): Psychometric characteristics and associations with variables of clinical interest. Appetite. 2016 May 21;105:283-90. doi: 10.1016/j.appet.2016.05.025. PMID: 27221356.X-1

5242. Henning B, Cordier R, Wilkes-Gillan $\mathrm{S}$, et al. A pilot play-based intervention to improve the social play interactions of children with autism spectrum disorder and their typically developing playmates. Aust Occup Ther J. 2016 Apr 26doi: 10.1111/14401630.12285. PMID: 27118688.X-3, X-4

5243. Hens K, Peeters H, Dierickx K. Genetic testing and counseling in the case of an autism diagnosis: A caregivers perspective. Eur J Med Genet. 2016 Aug 17;59(9):452-8. doi: 10.1016/j.ejmg.2016.08.007. PMID: 27544064.X-1
5244. Heon E, Kim G, Qin S, et al. Mutations in C8ORF37 cause Bardet Biedl syndrome (BBS21). Hum Mol Genet. 2016 Mar 22doi: 10.1093/hmg/ddw096. PMID: 27008867.X-1

5245. Herpers PCM, Klip H, Rommelse NNJ, et al. Associations between high callousunemotional traits and quality of life across youths with non-conduct disorder diagnoses. European Child \& Adolescent Psychiatry. 2016;25(5):547-55. doi: http://dx.doi.org/10.1007/s00787-015-07665.X-1

5246. Herrick RT, Tavarez MA, Harsh BN, et al. Effect of immunological castration management strategy on lipid oxidation and sensory characteristics of bacon stored under simulated food service conditions. J Anim Sci. 2016 Jul;94(7):3084-92. doi: 10.2527/jas.20160366. PMID: 27482695.X-1

5247. Herringshaw AJ, Ammons CJ, DeRamus TP, et al. Hemispheric differences in language processing in autism spectrum disorders: A meta-analysis of neuroimaging studies. Autism Res. 2016 Jan 11doi: 10.1002/aur.1599. PMID: 26751141.X-1, X-2

5248. Hessl D, Grigsby J. Fragile Xassociated tremor/ataxia syndrome: another phenotype of the fragile $X$ gene. Clin Neuropsychol. 2016 Jun 29:1-5. doi: 10.1080/13854046.2016.1186661. PMID: 27355274.X-1

5249. Heunis TM, Aldrich C, de Vries PJ. Recent Advances in Resting-State Electroencephalography Biomarkers for Autism Spectrum Disorder-A Review of Methodological and Clinical Challenges. Pediatr Neurol. 2016 Apr 1doi: 10.1016/j.pediatrneurol.2016.03.010. PMID: 27255413.X-1

5250. Hewavitharana SS, Mazzola M. Carbon source-dependent effects of anaerobic soil disinfestation on soil microbiome and suppression of Rhizoctonia solani AG-5 and Pratylenchus penetrans. Phytopathology. 2016 May 4doi: 10.1094/phyto-12-15-0329-r. PMID: 27143411.X-1

5251. Hewitt A, Hall-Lande J, Hamre K, et al. Autism Spectrum Disorder (ASD) 
Prevalence in Somali and Non-Somali Children. J Autism Dev Disord. 2016 Apr 22doi: 10.1007/s10803-016-2793-6. PMID: 27106569.X-1

5252. Heys M, Alexander A, Medeiros E, et al. Understanding parents' and professionals' knowledge and awareness of autism in Nepal. Autism. 2016 May 19doi: 10.1177/1362361316646558. PMID: 27197696.X-1

5253. Hidding E, Swaab H, de Sonneville LM, et al. The role of COMT and plasma proline in the variable penetrance of autistic spectrum symptoms in 22q11.2 Deletion Syndrome. Clin Genet. 2016 Feb 26doi: 10.1111/cge.12766. PMID: 26919535.X-1

5254. Higbee TS, Aporta AP, Resende A, et al. Interactive computer training to teach discrete-trial instruction to undergraduates and special educators in Brazil: A replication and extension. J Appl Behav Anal. 2016 Jun 17doi: 10.1002/jaba.329. PMID: 27312360.X-1

5255. Higuchi Y, Inagaki M, Koyama T, et al. A cross-sectional study of psychological distress, burnout, and the associated risk factors in hospital pharmacists in Japan. BMC Public Health. 2016;16:534. doi: 10.1186/s12889-0163208-5. PMID: 27392676.X-1

5256. Hilvert E, Davidson D, Gámez PB. Examination of script and non-script based narrative retellings in children with autism spectrum disorders. Research in Autism Spectrum Disorders. 2016;29-30:79-92. doi: http://dx.doi.org/10.1016/j.rasd.2016.06.002.X$1, \mathrm{X}-3$

5257. Hiniker A, Rosenberg-Lee M, Menon $\mathrm{V}$. Distinctive role of symbolic number sense in mediating the mathematical abilities of children with autism. Journal of Autism and Developmental Disorders. 2016;46(4):1268-81. doi: http://dx.doi.org/10.1007/s10803-0152666-4.X-1

5258. Hippolyte L, Maillard AM, RodriguezHerreros B, et al. The number of genomic copies at the 16p11.2 locus modulates language, verbal memory, and inhibition. Biological Psychiatry. 2016;80(2):129-39. doi: http://dx.doi.org/10.1016/j.biopsych.2015.10.02 1.X-1
5259. Hoch J, Spofford L, Dimian A, et al. A direct comparison of self-injurious and stereotyped motor behavior between preschoolaged children with and without developmental delays. Journal of Pediatric Psychology. 2016;41(5):566-72. doi: http://dx.doi.org/10.1093/jpepsy/jsv102.X-1

5260. Hochstenbach R, Nowakowska B, Volleth M, et al. Multiple Small Supernumerary Marker Chromosomes Resulting from Maternal Meiosis I or II Errors. Mol Syndromol. 2016 Feb;6(5):210-21. doi: 10.1159/000441408. PMID: 26997941.X-1

5261. Hodgetts S, Park E. Preparing for the future: a review of tools and strategies to support autonomous goal setting for children and youth with autism spectrum disorders. Disabil Rehabil. 2016 Mar 30:1-9. doi: 10.3109/09638288.2016.1161084. PMID: 27027477.X-1, X-2

5262. Hodgson AR, Freeston MH, Honey E, et al. Facing the Unknown: Intolerance of Uncertainty in Children with Autism Spectrum Disorder. J Appl Res Intellect Disabil. 2016 Feb 12doi: 10.1111/jar.12245. PMID: 26868412.X1

5263. Hofer J, Hoffmann F, Bachmann C. Use of complementary and alternative medicine in children and adolescents with autism spectrum disorder: A systematic review. Autism. 2016 May 25doi: 10.1177/1362361316646559. PMID: 27231337.X-1, X-2

5264. Hoffman EJ, Turner KJ, Fernandez JM, et al. Estrogens Suppress a Behavioral Phenotype in Zebrafish Mutants of the Autism Risk Gene, CNTNAP2. Neuron. 2016 Feb 17;89(4):725-33. doi: 10.1016/j.neuron.2015.12.039. PMID: 26833134.X-1

5265. Hoffmann E, Bruck C, Kreifelts B, et al. Reduced functional connectivity to the frontal cortex during processing of social cues in autism spectrum disorder. J Neural Transm (Vienna). 2016 Apr 19doi: 10.1007/s00702016-1544-3. PMID: 27094176.X-1

5266. Hoffmann W, Weber L, König U, et al. The role of the CBCL in the assessment of 
autism spectrum disorders: An evaluation of symptom profiles and screening characteristics. Research in Autism Spectrum Disorders. 2016;27:44-53. doi: http://dx.doi.org/10.1016/j.rasd.2016.04.002.X1

5267. Hoglund Carlsson L, Saltvedt S, Anderlid BM, et al. Ultrasound in the first and second trimester and autism; a prospective randomized study. Ultrasound Obstet Gynecol. 2016 May 17doi: 10.1002/uog.15962. PMID: 27184020.X-1

5268. Höher Camargo SP, Rispoli M, Ganz $\mathrm{J}$, et al. Behaviorally based interventions for teaching social interaction skills to children with ASD in inclusive settings: A metaanalysis. Journal of Behavioral Education. 2016;25(2):223-48. doi: http://dx.doi.org/10.1007/s10864-015-92401.X-2

5269. Holbrook JR, Cuffe SP, Cai B, et al. Persistence of parent-reported ADHD symptoms from childhood through adolescence in a community sample. Journal of Attention Disorders. 2016;20(1):11-20. doi: http://dx.doi.org/10.1177/1087054714539997.X $-1$

5270. Hollway JA, Aman MG, MendozaBurcham MI, et al. Caregiver Satisfaction with a Multisite Trial of Atomoxetine and Parent Training for Attention-Deficit/Hyperactivity Disorder and Behavioral Noncompliance in Children with Autism Spectrum Disorder. J Child Adolesc Psychopharmacol. 2016 Jan 21doi: 10.1089/cap.2015.0130. PMID: 26797318.X-1

5271. Holmes LG, Himle MB, Strassberg DS. Parental romantic expectations and parentchild sexuality communication in autism spectrum disorders. Autism. 2016

Aug;20(6):687-99. doi: 10.1177/1362361315602371. PMID: 26408632.X-1

5272. Holmes LG, Himle MB, Strassberg DS. Parental romantic expectations and parentchild sexuality communication in autism spectrum disorders. Autism. 2016;20(6):687-99. doi:

http://dx.doi.org/10.1177/1362361315602371.X $-1$
5273. Holmes LG, Himle MB, Strassberg DS. Parental sexuality-related concerns for adolescents with autism spectrum disorders and average or above IQ. Research in Autism Spectrum Disorders. 2016;21:84-93. doi: http://dx.doi.org/10.1016/j.rasd.2015.10.001.X1

5274. Holt V, Skagerberg E, Dunsford M. Young people with features of gender dysphoria: Demographics and associated difficulties. Clinical Child Psychology and Psychiatry. 2016;21(1):108-18. doi: http://dx.doi.org/10.1177/1359104514558431.X $-1$

5275. Homberg JR, Kyzar EJ, Scattoni ML, et al. Genetic and environmental modulation of neurodevelopmental disorders: Translational insights from labs to beds. Brain Res Bull. 2016 Apr 23;125:79-91. doi: 10.1016/j.brainresbull.2016.04.015. PMID: 27113433.X-1

5276. Honeycutt JA, Keary Iii KM, Kania VM, et al. Developmental Age Differentially Mediates the Calcium-Binding Protein Parvalbumin in the Rat: Evidence for a Selective Decrease in Hippocampal Parvalbumin Cell Counts. Dev Neurosci. 2016;38(2):105-14. doi: 10.1159/000444447. PMID: 27002731.X-1

5277. Hong ER, Ganz JB, Mason R, et al. The effects of video modeling in teaching functional living skills to persons with ASD: A meta-analysis of single-case studies. Res Dev Disabil. 2016 Jul 18;57:158-69. doi: 10.1016/j.ridd.2016.07.001. PMID: 27442687.X-1, X-2

5278. Hong ER, Ganz JB, Neely L, et al. A review of the quality of primary caregiverimplemented communication intervention research for children with ASD. Research in Autism Spectrum Disorders. 2016;25:122-36. doi:

http://dx.doi.org/10.1016/j.rasd.2016.02.005.X$1, \mathrm{X}-2, \mathrm{X}-3$

5279. Hong H, Kim BS, Im HI.

Pathophysiological Role of Neuroinflammation in Neurodegenerative Diseases and Psychiatric Disorders. Int Neurourol J. 2016 May;20(Suppl 
1):S2-7. doi: 10.5213/inj.1632604.302. PMID: 27230456.X-1

5280. Honkasilta J, Vehmas S, Vehkakoski T. Self-pathologizing, self-condemning, selfliberating: Youths' accounts of their ADHDrelated behavior. Social Science \& Medicine. 2016;150:248-55. doi: http://dx.doi.org/10.1016/j.socscimed.2015.12.0 30.X-1

5281. Hopf KP, Madren E, Santianni KA. Use and Perceived Effectiveness of Complementary and Alternative Medicine to Treat and Manage the Symptoms of Autism in Children: A Survey of Parents in a Community Population. J Altern Complement Med. 2016 Jan;22(1):25-32. doi: 10.1089/acm.2015.0163. PMID: 26654976.X-1, X-3

5282. Hopkins Z, Yuill N, Keller B. Children with autism align syntax in natural conversation. Applied Psycholinguistics. 2016;37(2):347-70. doi: http://dx.doi.org/10.1017/S0142716414000599. $\mathrm{X}-1$

5283. Horvath G, Petrovszki Z, Kekesi G, et al. Electrophysiological alterations in a complex rat model of schizophrenia. Behav Brain Res.

2016 Jul 1;307:65-72. doi:

10.1016/j.bbr.2016.03.051. PMID:

27036646.X-1

5284. House SA, Goodman DC, Weinstein SJ, et al. Prescription Use among Children with Autism Spectrum Disorders in Northern New England: Intensity and Small Area Variation. J Pediatr. 2016 Feb;169:277-83.e2. doi: 10.1016/j.jpeds.2015.10.027. PMID: 26561379.X-1

5285. Howe FE, Stagg SD. Erratum to: How Sensory Experiences Affect Adolescents with an Autistic Spectrum Condition within the Classroom. J Autism Dev Disord. 2016 Jun;46(6):2273. doi: 10.1007/s10803-016-27918. PMID: 27101236.X-1

5286. Howe FEJ, Stagg SD. How sensory experiences affect adolescents with an autistic spectrum condition within the classroom. Journal of Autism and Developmental Disorders. 2016;46(5):1656-68. doi: http://dx.doi.org/10.1007/s10803-015-26931.X-1
5287. Howie EK, Campbell AC, Straker LM. An active video game intervention does not improve physical activity and sedentary time of children at-risk for developmental coordination disorder: A crossover randomized trial. Child: Care, Health and Development.

2016;42(2):253-60. doi: http://dx.doi.org/10.1111/cch.12305.X-1

5288. Hrdlicka M, Vacova M, Oslejskova H, et al. Age at diagnosis of autism spectrum disorders: is there an association with socioeconomic status and family self-education about autism? Neuropsychiatr Dis Treat. 2016;12:1639-44. doi: 10.2147/ndt.s107239. PMID: 27462156.X-1

5289. Hsiao K, Harony-Nicolas H, Buxbaum JD, et al. Cyfip1 Regulates Presynaptic Activity during Development. J Neurosci. 2016 Feb 3;36(5):1564-76. doi: 10.1523/jneurosci.051115.2016. PMID: 26843638.X-1

5290. Huang CT, Chiang CH, Hung CY. Young children with autism spectrum disorders imitate in the context of others' prior intention. Autism. 2016 Apr 5doi: 10.1177/1362361315627135. PMID: 27048355.X-1

5291. Huang JY, Tian Y, Wang HJ, et al. Functional Genomic Analyses Identify Pathways Dysregulated in Animal Model of Autism. CNS Neurosci Ther. 2016 Jun 20doi: 10.1111/cns.12582. PMID: 27321591.X-1

5292. Hubner LM, Feldman HM, Huffman LC. Parent-reported shared decision making: Autism spectrum disorder and other neurodevelopmental disorders. Journal of Developmental and Behavioral Pediatrics. 2016;37(1):20-32.X-1

5293. Huijnen CA, Lexis MA, Jansens R, et al. Mapping Robots to Therapy and Educational Objectives for Children with Autism Spectrum Disorder. J Autism Dev Disord. 2016 Jun;46(6):2100-14. doi: 10.1007/s10803-0162740-6. PMID: 26909762.X-1, X-3

5294. Humphreys KL, Galán CA, Tottenham $\mathrm{N}$, et al. Impaired social decision-making mediates the association between ADHD and social problems. Journal of Abnormal Child Psychology. 2016;44(5):1023-32. doi: 
http://dx.doi.org/10.1007/s10802-015-00957.X-1

5295. Hundley RJ, Shui A, Malow BA. Relationship Between Subtypes of Restricted and Repetitive Behaviors and Sleep Disturbance in Autism Spectrum Disorder. J Autism Dev Disord. 2016 Aug 10doi: 10.1007/s10803-016-2884-4. PMID: 27511195.X-1

5296. Hunter JE, Irving SA, Biesecker LG, et al. A standardized, evidence-based protocol to assess clinical actionability of genetic disorders associated with genomic variation. Genet Med. 2016 Apr 28doi: 10.1038/gim.2016.40. PMID: 27124788.X-1

5297. Husk JS, Keim SA. Breastfeeding and dietary variety among preterm children aged 13 years. Appetite. 2016;99:130-7. doi: http://dx.doi.org/10.1016/j.appet.2016.01.016.X $-1$

5298. Hutchins TL, Brien A. Conversational topic moderates social attention in autism spectrum disorder: Talking about emotions is like driving in a snowstorm. Research in Autism Spectrum Disorders. 2016;26:99-110. doi: http://dx.doi.org/10.1016/j.rasd.2016.03.006.X1

5299. Hutchins TL, Prelock PA, Morris H, et al. Explicit vs. applied theory of mind competence: A comparison of typically developing males, males with ASD, and males with ADHD. Research in Autism Spectrum Disorders. 2016;21:94-108. doi: http://dx.doi.org/10.1016/j.rasd.2015.10.004.X1

5300. Hutsler JJ, Casanova MF. Review: Cortical construction in autism spectrum disorder: columns, connectivity and the subplate. Neuropathol Appl Neurobiol. 2016 Feb;42(2):115-34. doi: 10.1111/nan.12227. PMID: 25630827.X-1

5301. Huynh K. Antiplatelet therapy: Clopidogrel plus aspirin reduces migrane attacks after ASD closure. Nat Rev Cardiol. 2016 Jan;13(1):2. doi: 10.1038/nrcardio.2015.186. PMID: 26606954.X-1
5302. Hwang S-K, Lee J-H, Yang J-e, et al. Everolimus improves neuropsychiatric symptoms in a patient with tuberous sclerosis carrying a novel TSC2 mutation. Molecular Brain. 2016;9.X-1

5303. Iacobucci G. Government slow to improve services for people with learning disabilities, report finds. Bmj. 2016;352:i1118. doi: 10.1136/bmj.i1118. PMID: 26905485.X-1

5304. Igelstrom KM, Webb TW, Graziano MS. Functional Connectivity Between the Temporoparietal Cortex and Cerebellum in Autism Spectrum Disorder. Cereb Cortex. 2016 Apr 12doi: 10.1093/cercor/bhw079. PMID: 27073219.X-1

5305. Iijima Y, Behr K, Iijima T, et al. Distinct Defects in Synaptic Differentiation of Neocortical Neurons in Response to Prenatal Valproate Exposure. Sci Rep. 2016;6:27400. doi: 10.1038/srep27400. PMID: 27264355.X-1

5306. Ikejiri K, Hosozawa M, Mitomo S, et al. Reduced growth during early infancy in very low birth weight children with autism spectrum disorder. Early Hum Dev. 2016 Jun 28;98:23-7. doi: 10.1016/j.earlhumdev.2016.05.001. PMID: 27367970.X-1

5307. Imagama S, Kawakami N, Kanemura T, et al. Radiographic Adjacent Segment Degeneration at Five Years After L4/5 Posterior Lumbar Interbody Fusion With Pedicle Screw Instrumentation: Evaluation by Computed Tomography and Annual Screening With Magnetic Resonance Imaging. Clin Spine Surg. 2016 May 2doi:

10.1097/BSD.0b013e31828aec78. PMID: 27137156.X-1

5308. Imms C, Adair B, Keen D, et al. 'Participation': a systematic review of language, definitions, and constructs used in intervention research with children with disabilities. Dev Med Child Neurol. 2016 Jan;58(1):29-38. doi: 10.1111/dmcn.12932. PMID: 26411643.X-1, X-2

5309. Inami S, Moridaira H, Takeuchi D, et al. Optimum pelvic incidence minus lumbar lordosis value can be determined by individual pelvic incidence. Eur Spine J. 2016 Apr 12doi: 10.1007/s00586-016-4563-8. PMID: 27072550.X-1 
5310. Inan M, Zhao M, Manuszak M, et al. Energy deficit in parvalbumin neurons leads to circuit dysfunction, impaired sensory gating and social disability. Neurobiol Dis. 2016 Sep;93:35-46. doi: 10.1016/j.nbd.2016.04.004. PMID: 27105708.X-1

5311. Ingersoll B, Wainer AL, Berger NI, et al. Comparison of a Self-Directed and Therapist-Assisted Telehealth Parent-Mediated Intervention for Children with ASD: A Pilot RCT. J Autism Dev Disord. 2016 Jul;46(7):2275-84. doi: 10.1007/s10803-0162755-z. PMID: 26922192.X-1

5312. Inskip M, Mavros Y, Sachdev PS, et al. Exercise for Individuals with Lewy Body Dementia: A Systematic Review. PLoS One. 2016;11(6):e0156520. doi: 10.1371/journal.pone.0156520. PMID: 27258533.X-1

5313. Ionita-Laza I, McCallum K, Xu B, et al. A spectral approach integrating functional genomic annotations for coding and noncoding variants. Nat Genet. 2016 Feb;48(2):214-20. doi: 10.1038/ng.3477. PMID: 26727659.X-1

5314. Iorio JA, Reid P, Kim HJ. Neurological complications in adult spinal deformity surgery. Curr Rev Musculoskelet Med. 2016 Jun 1doi: 10.1007/s12178-0169350-y. PMID: 27250041.X-1

5315. Irvine CA, Eigsti I-M, Fein DA. Uh, um, and autism: Filler disfluencies as pragmatic markers in adolescents with optimal outcomes from autism spectrum disorder. Journal of Autism and Developmental Disorders. 2016;46(3):1061-70. doi: http://dx.doi.org/10.1007/s10803-015-2651y.X-1

5316. Ishizuka Y, Yamamoto JI. Contingent imitation increases verbal interaction in children with autism spectrum disorders. Autism. 2016 Apr 19doi: 10.1177/1362361315622856. PMID: 27095832.X-1

5317. Isles AR, Ingason A, Lowther C, et al. Parental Origin of Interstitial Duplications at 15q11.2-q13.3 in Schizophrenia and Neurodevelopmental Disorders. PLoS Genet. 2016 May;12(5):e1005993. doi: 10.1371/journal.pgen.1005993. PMID: 27153221.X-1

5318. Ito S. GABA and glycine in the developing brain. J Physiol Sci. 2016 Mar 7doi: 10.1007/s12576-016-0442-7. PMID: 26951057.X-1

5319. Jacobs M, Woolfson LM, Hunter SC. Attributions of stability, control and responsibility: How parents of children with intellectual disabilities view their child's problematic behaviour and its causes. Journal of Applied Research in Intellectual Disabilities. 2016;29(1):58-70. doi: http://dx.doi.org/10.1111/jar.12158.X-1

5320. Jain A, Lafage V, Kelly MP, et al. Validity, Reliability, and Responsiveness of SRS-7 as an Outcomes Assessment Instrument for Operatively Treated Patients with Adult Spinal Deformity. Spine (Phila Pa 1976). 2016 Feb 29doi: 10.1097/brs.0000000000001540. PMID: 26937607.X-1

5321. Jain A, Marshall J, Buikema A, et al. Correction of Description of MMR Vaccine Receipt Coding and Minor Errors in MMR Vaccine and Autism Study. Jama. 2016 Jan 12;315(2):202-4. doi: 10.1001/jama.2015.17065. PMID: 26757474.X1

5322. Jakobsen KD, Bruhn CH, Pagsberg AK, et al. Neurological, Metabolic, and Psychiatric Adverse Events in Children and Adolescents Treated With Aripiprazole. J Clin Psychopharmacol. 2016 Aug 6doi: 10.1097/jcp.0000000000000548. PMID: 27504593.X-1, X-3

5323. Jalai CM, Passias PG, Lafage V, et al. A comparative analysis of the prevalence and characteristics of cervical malalignment in adults presenting with thoracolumbar spine deformity based on variations in treatment approach over 2 years. Eur Spine J. 2016 Apr 13doi: 10.1007/s00586-016-4564-7. PMID: 27076049.X-1

5324. Jalal Z, Hascoet S, Baruteau AE, et al. Long-term Complications After Transcatheter Atrial Septal Defect Closure: A Review of the Medical Literature. Can J Cardiol. 2016 Mar 3doi: 10.1016/j.cjca.2016.02.068. PMID: 27179546.X-1 
5325. Jao Keehn RJ, Sanchez SS, Stewart $\mathrm{CR}$, et al. Impaired downregulation of visual cortex during auditory processing is associated with autism symptomatology in children and adolescents with autism spectrum disorder. Autism Res. 2016 May 20doi: 10.1002/aur.1636. PMID: 27205875.X-1

5326. Jarczok TA, Fritsch M, Kroger A, et al. Maturation of interhemispheric signal propagation in autism spectrum disorder and typically developing controls: a TMS-EEG study. J Neural Transm (Vienna). 2016 May 13doi: 10.1007/s00702-016-1550-5. PMID: 27177879.X-1

5327. Jarrett MA, Gilpin AT, Pierucci JM, et al. Cognitive and reactive control processes: Associations with ADHD symptoms in preschoolers. International Journal of Behavioral Development. 2016;40(1):53-7. doi: http://dx.doi.org/10.1177/0165025415575625.X $-1$

5328. Jeevanantham D, Bartlett D. Perspectives on classification of selected childhood neurodisabilities based on a review of literature. Dev Neurorehabil. 2016 Apr 8:113. doi: 10.3109/17518423.2016.1139009. PMID: 27057781.X-1, X-2

5329. Jenkins DD, Wiest DB, Mulvihill DM, et al. Fetal and Neonatal Effects of N-

Acetylcysteine When Used for Neuroprotection in Maternal Chorioamnionitis. J Pediatr. 2016 Jan;168:67-76.e6. doi: 10.1016/j.jpeds.2015.09.076. PMID: 26545726.X-1

5330. Jeon JP, Cho YD, Han MH. Intraarterial thrombolysis of central retinal artery occlusion following percutaneous atrial septal defect closure. Catheter Cardiovasc Interv. 2016 Jul;88(1):157-60. doi: 10.1002/ccd.26100. PMID: 26153142.X-1

5331. Jessel J, Hanley GP, Ghaemmaghami M. A translational evaluation of transitions. J Appl Behav Anal. 2016 Jun;49(2):359-76. doi: 10.1002/jaba.283. PMID: 26916573.X-1

5332. Jeste SS, Geschwind DH. Clinical trials for neurodevelopmental disorders: At a therapeutic frontier. Sci Transl Med. 2016 Jan 13;8(321):321fs1. doi: 10.1126/scitranslmed.aad9874. PMID: 26764154.X-1

5333. Jeste SS, Varcin KJ, Hellemann GS, et al. Symptom profiles of autism spectrum disorder in tuberous sclerosis complex. Neurology. 2016 Jul 20doi: 10.1212/wnl.0000000000003002. PMID: 27440144.X-1

5334. Jeyabalan N, Clement JP. SYNGAP1: Mind the Gap. Front Cell Neurosci. 2016;10:32. doi: 10.3389/fncel.2016.00032. PMID: 26912996.X-1

5335. Jia F, Cui M, Than MT, et al. Developmental Defects of Caenorhabditis elegans Lacking Branched-chain alphaKetoacid Dehydrogenase Are Mainly Caused by Monomethyl Branched-chain Fatty Acid Deficiency. J Biol Chem. 2016 Feb 5;291(6):2967-73. doi: 10.1074/jbc.M115.676650. PMID: 26683372.X-1

5336. Jiang HY, Xu LL, Shao L, et al. Maternal infection during pregnancy and risk of autism spectrum disorders: A systematic review and meta-analysis. Brain Behav Immun. 2016 Jun 7doi: 10.1016/j.bbi.2016.06.005. PMID: 27287966.X-1

5337. Jiang HY, Zhou K, Ruan B. Re: Maternal infection during pregnancy, risk of offspring autism, and the role of bacterial denitrification. Brain Behav Immun. 2016 Jun 24doi: 10.1016/j.bbi.2016.06.012. PMID: 27350209.X-1

5338. Jiang M, Polepalli J, Chen LY, et al. Conditional ablation of neuroligin-1 in CA1 pyramidal neurons blocks LTP by a cellautonomous NMDA receptor-independent mechanism. Mol Psychiatry. 2016 May 24doi: 10.1038/mp.2016.80. PMID: 27217145.X-1

5339. Jin J. JAMA PATIENT PAGE.

Screening for Autism Spectrum Disorder. Jama. 2016 Feb 16;315(7):718. doi: 10.1001/jama.2016.0930. PMID: 26881385.X-1

5340. Johannessen J, Nærland T, Bloss C, et al. Parents' attitudes toward genetic research in autism spectrum disorder. Psychiatric Genetics. 2016;26(2):74-80. doi: 
http://dx.doi.org/10.1097/YPG.0000000000000 121.X-1

5341. John TS, Estes AM, Dager SR, et al. Emerging executive functioning and motor development in infants at high and low risk for autism spectrum disorder. Frontiers in Psychology. 2016;7.X-1

5342. Johnson HL, DeLeon PH. Accessing care for children with special health care needs. Practice Innovations. 2016;1(2):105-16. doi: http://dx.doi.org/10.1037/pri0000019.X-1

5343. Johnson NL, Burkett K, Reinhold J, et al. Translating Research to Practice for Children With Autism Spectrum Disorder: Part I: Definition, Associated Behaviors, Prevalence, Diagnostic Process, and Interventions. J Pediatr Health Care. 2016 Jan-Feb;30(1):15-26. doi: 10.1016/j.pedhc.2015.09.008. PMID: 26530271.X-1

5344. Johnson S, Kochhar P, Hennessy E, et al. Antecedents of attention-

deficit/hyperactivity disorder symptoms in children born extremely preterm. Journal of Developmental and Behavioral Pediatrics. 2016;37(4):285-97. doi:

http://dx.doi.org/10.1097/DBP.0000000000000 298.X-1

5345. Johnson SA, Javurek AB, Painter MS, et al. Effects of developmental exposure to bisphenol A on spatial navigational learning and memory in rats: A CLARITY-BPA study. Horm Behav. 2016 Apr;80:139-48. doi: 10.1016/j.yhbeh.2015.09.005. PMID: 26436835.X-1

5346. Johnston SB, Raines RT. PTENpred: A Designer Protein Impact Predictor for PTENrelated Disorders. J Comput Biol. 2016 Jun 16doi: 10.1089/cmb.2016.0058. PMID: 27310656.X-1

5347. Jone PN, Ross MM, Bracken JA, et al. Feasibility and Safety of Using a Fused Echocardiography/Fluoroscopy Imaging System in Patients with Congenital Heart Disease. J Am Soc Echocardiogr. 2016 Jun;29(6):513-21. doi: 10.1016/j.echo.2016.03.014. PMID: 27143284.X-1
5348. Jones EJ, Venema K, Earl R, et al. Reduced engagement with social stimuli in 6month-old infants with later autism spectrum disorder: a longitudinal prospective study of infants at high familial risk. J Neurodev Disord. 2016;8:7. doi: 10.1186/s11689-016-9139-8. PMID: 26981158.X-1

5349. Jones KL, Croen LA, Yoshida CK, et al. Autism with intellectual disability is associated with increased levels of maternal cytokines and chemokines during gestation. Mol Psychiatry. 2016 May 24doi: 10.1038/mp.2016.77. PMID: 27217154.X-1

5350. Jones S, Bremer E, Lloyd M. Autism spectrum disorder: family quality of life while waiting for intervention services. Qual Life Res. 2016 Aug 2doi: 10.1007/s11136-016-1382-7. PMID: 27485916.X-1

5351. Joosten A, Girdler S, Albrecht MA, et al. Gaze and visual search strategies of children with Asperger syndrome/high functioning autism viewing a magic trick. Developmental Neurorehabilitation. 2016;19(2):95-102.X-1

5352. Jorge Garcia A, Polidori C, NievesAldrey JL. Pheomelanin in the secondary sexual characters of male parasitoid wasps (Hymenoptera: Pteromalidae). Arthropod Struct Dev. 2016 May 21doi:

10.1016/j.asd.2016.05.001. PMID: 27224206.X-1

5353. Joseph RM, O'Shea TM, Allred EN, et al. Prevalence and associated features of autism spectrum disorder in extremely low gestational age newborns at age 10 years. Autism Res. 2016 May 25doi: 10.1002/aur.1644. PMID: 27220677.X-1

5354. Joshi G, Wozniak J, Faraone SV, et al. A Prospective Open-Label Trial of Memantine Hydrochloride for the Treatment of Social Deficits in Intellectually Capable Adults With Autism Spectrum Disorder. J Clin Psychopharmacol. 2016 Jun;36(3):262-71. doi: 10.1097/jcp.0000000000000499. PMID: 27043118.X-1

5355. Jowett Hirst ES, Dozier CL, Payne SW. Efficacy of and preference for reinforcement and response cost in token economies. Journal of Applied Behavior 
Analysis. 2016;49(2):329-45. doi:

http://dx.doi.org/10.1002/jaba.294.X-1

5356. Julien HM, Reichle J. A comparison of high and low dosages of a component of Milieu teaching strategies for two preschool-age learners with autism spectrum disorder. Language, Speech, and Hearing Services in Schools. 2016;47(1):87-98. doi: http://dx.doi.org/10.1044/2015_LSHSS-150035.X-3, X-4

5357. Jull S, Mirenda P. Effects of a staff training program on community instructors' ability to teach swimming skills to children with autism. Journal of Positive Behavior Interventions. 2016;18(1):29-40. doi: http://dx.doi.org/10.1177/1098300715576797.X $-1, \mathrm{X}-3, \mathrm{X}-4$

5358. Julvez J, Mendez M, Fernandez-Barres $\mathrm{S}$, et al. Maternal Consumption of Seafood in Pregnancy and Child Neuropsychological Development: A Longitudinal Study Based on a Population With High Consumption Levels. Am J Epidemiol. 2016 Feb 1;183(3):169-82. doi: 10.1093/aje/kwv195. PMID: 26740026.X-1

5359. Kabir ZD, Lee AS, Rajadhyaksha AM. L-type Ca2+ channels in mood, cognition and addiction: Integrating human and rodent studies with a focus on behavioural endophenotypes. J Physiol. 2016 Feb 23doi: 10.1113/jp270673. PMID: 26913808.X-1

5360. Kaden E, Kelm ND, Carson RP, et al. Multi-compartment microscopic diffusion imaging. Neuroimage. 2016 Jun 6doi: 10.1016/j.neuroimage.2016.06.002. PMID: 27282476.X-1

5361. Kaganovich N. Development of sensitivity to audiovisual temporal asynchrony during midchildhood. Developmental Psychology. 2016;52(2):232-41. doi: http://dx.doi.org/10.1037/dev0000073.X-1

5362. Kahle KT, Khanna AR, Duan J, et al. The KCC2 Cotransporter and Human Epilepsy: Getting Excited About Inhibition. Neuroscientist. 2016 Apr 29doi: 10.1177/1073858416645087. PMID: 27130838.X-1

5363. Kaiser MD, Yang DY, Voos AC, et al. Brain Mechanisms for Processing Affective (and Nonaffective) Touch Are Atypical in Autism. Cereb Cortex. 2016 Jun;26(6):2705-14. doi: 10.1093/cercor/bhv125. PMID: 26048952.X-1

5364. Kalb LG, Vasa RA, Ballard ED, et al. Epidemiology of Injury-Related Emergency Department Visits in the US Among Youth with Autism Spectrum Disorder. J Autism Dev Disord. 2016 May 30doi: 10.1007/s10803-0162820-7. PMID: 27241347.X-1

5365. Kaldy Z, Giserman I, Carter AS, et al. The mechanisms underlying the ASD advantage in visual search. Journal of Autism and Developmental Disorders.

2016;46(5):1513-27. doi: http://dx.doi.org/10.1007/s10803-013-1957$\mathrm{x} . \mathrm{X}-1$

5366. Kalkman HO, Feuerbach D. Modulatory effects of alpha7 nAChRs on the immune system and its relevance for CNS disorders. Cell Mol Life Sci. 2016 Jul;73(13):2511-30. doi: 10.1007/s00018-0162175-4. PMID: 26979166.X-1

5367. Kalman S, Garbett KA, Janka Z, et al. Human dermal fibroblasts in psychiatry research. Neuroscience. 2016 Apr 21;320:10521. doi: 10.1016/j.neuroscience.2016.01.067. PMID: 26855193.X-1

5368. Kalueff AV, Stewart AM, Song C, et al. Neurobiology of rodent self-grooming and its value for translational neuroscience. Nat Rev Neurosci. 2016 Jan;17(1):45-59. doi: 10.1038/nrn.2015.8. PMID: 26675822.X-1

5369. Kang CN, Kim CW, Moon JK. The outcomes of instrumented posterolateral lumbar fusion in patients with rheumatoid arthritis. Bone Joint J. 2016 Jan;98-b(1):102-8. doi: 10.1302/0301-620x.98b1.36247. PMID: 26733522.X-1

5370. Kang J, Park H, Kim E. IRSp53/BAIAP2 in dendritic spine development, NMDA receptor regulation, and psychiatric disorders. Neuropharmacology. 2016 Jan;100:27-39. doi: 10.1016/j.neuropharm.2015.06.019. PMID: 26275848.X-1

5371. Karaminis T, Cicchini GM, Neil L, et al. Central tendency effects in time interval 
reproduction in autism. Sci Rep. 2016;6:28570. doi: 10.1038/srep28570. PMID: 27349722.X-1

5372. Karhson DS, Golob EJ. Atypical sensory reactivity influences auditory attentional control in adults with autism spectrum disorders. Autism Res. 2016 Jan 18doi: 10.1002/aur.1593. PMID: 26778164.X-1

5373. Kashima R, Roy S, Ascano M, et al. Augmented noncanonical BMP type II receptor signaling mediates the synaptic abnormality of fragile X syndrome. Sci Signal.

2016;9(431):ra58. doi: 10.1126/scisignal.aaf6060. PMID: 27273096.X1

5374. Kaskowitz AP, Dendrinos M, Murray PJ, et al. The Effect of Menstrual Issues on Young Women with Angelman Syndrome. J Pediatr Adolesc Gynecol. 2016 Aug;29(4):34852. doi: 10.1016/j.jpag.2015.12.004. PMID: 26718530.X-1

5375. Katayama K, Yamashita Y, Yatsuga S, et al. ADHD-like behavior in a patient with hypothalamic hamartoma. Brain \& Development. 2016;38(1):145-8. doi: http://dx.doi.org/10.1016/j.braindev.2015.05.01 1.X-1

5376. Kato T, Mandai T, Iwatani S, et al. Extremely preterm infants small for gestational age are at risk for motor impairment at 3 years corrected age. Brain \& Development. 2016;38(2):188-95. doi: http://dx.doi.org/10.1016/j.braindev.2015.07.00 8.X-1

5377. Katz J, d'Albis MA, Boisgontier J, et al. Similar white matter but opposite grey matter changes in schizophrenia and highfunctioning autism. Acta Psychiatr Scand. 2016 Jul;134(1):31-9. doi: 10.1111/acps.12579. PMID: 27105136.X-1

5378. Kaufmann FN, Gazal M, Bastos CR, et al. Curcumin in depressive disorders: An overview of potential mechanisms, preclinical and clinical findings. Eur J Pharmacol. 2016 May 24;784:192-8. doi: 10.1016/j.ejphar.2016.05.026. PMID: 27235294.X-1

5379. Kauschke C, van der Beek B, KampBecker I. Narratives of girls and boys with autism spectrum disorders: Gender differences in narrative competence and internal state language. Journal of Autism and Developmental Disorders. 2016;46(3):840-52. doi: http://dx.doi.org/10.1007/s10803-0152620-5.X-1

5380. Kaushik G, Huber DP, Aho K, et al. Maternal exposure to carbamazepine at environmental concentrations can cross intestinal and placental barriers. Biochem Biophys Res Commun. 2016 May 27;474(2):291-5. doi: 10.1016/j.bbrc.2016.04.088. PMID: 27105911.X-1

5381. Kaushik G, Xia Y, Yang L, et al. Psychoactive pharmaceuticals at environmental concentrations induce in vitro gene expression associated with neurological disorders. BMC Genomics. 2016;17 Suppl 3:435. doi: 10.1186/s12864-016-2784-1. PMID: 27356971.X-1

5382. Kawabe K, Kondo S, Matsumoto M, et al. Developmental quotient to estimate intelligence in autism spectrum disorder. Pediatr Int. 2016 Mar 2doi: 10.1111/ped.12969. PMID: 26933939.X-1

5383. Kawalekar OU, O'Connor RS, Fraietta JA, et al. Distinct Signaling of Coreceptors Regulates Specific Metabolism Pathways and Impacts Memory Development in CAR T Cells. Immunity. 2016 Feb 16;44(2):380-90. doi: 10.1016/j.immuni.2016.01.021. PMID: 26885860.X-1

5384. Kawamura A, Mylopoulos M, Orsino A, et al. Promoting the Development of Adaptive Expertise: Exploring a Simulation Model for Sharing a Diagnosis of Autism With Parents. Acad Med. 2016 May 31doi: 10.1097/acm.0000000000001246. PMID: 27254013.X-1

5385. Kawanai T, Ago Y, Watanabe R, et al. Prenatal Exposure to Histone Deacetylase Inhibitors Affects Gene Expression of AutismRelated Molecules and Delays Neuronal Maturation. Neurochem Res. 2016 Jun 14doi: 10.1007/s11064-016-1969-y. PMID: 27300699.X-1

5386. Kay-Raining Bird E, Genesee F, Verhoeven L. Bilingualism in children with 
developmental disorders: A narrative review. J Commun Disord. 2016 Jul 18doi:

10.1016/j.jcomdis.2016.07.003. PMID:

27461977.X-1

5387. Kazdoba TM, Leach PT, Yang M, et al. Translational Mouse Models of Autism: Advancing Toward Pharmacological Therapeutics. Curr Top Behav Neurosci. 2016 Jun 16doi: 10.1007/7854_2015_5003. PMID: 27305922.X-1

5388. Kazlauskas N, Campolongo M, Lucchina L, et al. Postnatal behavioral and inflammatory alterations in female pups prenatally exposed to valproic acid. Psychoneuroendocrinology. 2016 Jun 7;72:1121. doi: 10.1016/j.psyneuen.2016.06.001. PMID: 27337090.X-1

5389. Keefer A, Kreiser NL, Singh V, et al. Intolerance of Uncertainty Predicts Anxiety Outcomes Following CBT in Youth with ASD. J Autism Dev Disord. 2016 Jul 12doi: 10.1007/s10803-016-2852-z. PMID: 27405445.X-1, X-3

5390. Keehn B, Joseph RM. Exploring what's missing: What do target absent trials reveal about autism search superiority? Journal of Autism and Developmental Disorders. 2016;46(5):1686-98. doi: http://dx.doi.org/10.1007/s10803-016-27001.X-1

5391. Keehn B, Nair A, Lincoln AJ, et al. Under-reactive but easily distracted: An fMRI investigation of attentional capture in autism spectrum disorder. Developmental Cognitive Neuroscience. 2016;17:46-56. doi: http://dx.doi.org/10.1016/j.dcn.2015.12.002.X-1

5392. Keen D, Webster A, Ridley G. How well are children with autism spectrum disorder doing academically at school? An overview of the literature. Autism. 2016;20(3):276-94. doi: http://dx.doi.org/10.1177/1362361315580962.X $-1$

5393. Keenan BM, Newman LK, Gray KM, et al. Parents of Children with ASD Experience More Psychological Distress, Parenting Stress, and Attachment-Related Anxiety. J Autism Dev Disord. 2016 Jun 16doi: 10.1007/s10803-0162836-z. PMID: 27312716.X-1
5394. Keil KP, Lein PJ. DNA methylation: a mechanism linking environmental chemical exposures to risk of autism spectrum disorders? Environ Epigenet. 2016 Mar;2(1)doi: 10.1093/eep/dvv012. PMID: 27158529.X-1

5395. Kelley ME, Shillingsburg MA, Bowen CN. Stability of daily preference across multiple individuals. J Appl Behav Anal. 2016 Jun;49(2):394-8. doi: 10.1002/jaba.288. PMID: 26816192.X-1

5396. Kelly MP, Zebala LP, Kim HJ, et al. Effectiveness of preoperative autologous blood donation for protection against allogeneic blood exposure in adult spinal deformity surgeries: a propensity-matched cohort analysis. J

Neurosurg Spine. 2016 Jan;24(1):124-30. doi: 10.3171/2015.4.spine141329. PMID: 26407086.X-1

5397. Kennedy AJ, Rahn EJ, Paulukaitis BS, et al. Tcf4 Regulates Synaptic Plasticity, DNA Methylation, and Memory Function. Cell Rep. 2016 Aug 24doi: 10.1016/j.celrep.2016.08.004. PMID: 27568567.X-1

5398. Kennedy M, Kreppner J, Knights N, et al. Early severe institutional deprivation is associated with a persistent variant of adult attention-deficit/hyperactivity disorder: clinical presentation, developmental continuities and life circumstances in the English and Romanian Adoptees study. J Child Psychol Psychiatry. 2016 Jun 6doi: 10.1111/jcpp.12576. PMID: 27264475.X-1

5399. Kentner AC, Khoury A, Lima Queiroz $\mathrm{E}$, et al. Environmental enrichment rescues the effects of early life inflammation on markers of synaptic transmission and plasticity. Brain Behav Immun. 2016 Mar 18doi: 10.1016/j.bbi.2016.03.013. PMID: 27002704.X-1

5400. Kerns KA, Macoun S, MacSween J, et al. Attention and Working Memory Training: A Feasibility Study in Children with Neurodevelopmental Disorders. Appl Neuropsychol Child. 2016 Apr 6:1-18. doi: 10.1080/21622965.2015.1109513. PMID: 27049769.X-1

5401. Kerr DJ, Marsillo A, Guariglia SR, et al. Aberrant hippocampal Atp8a1 levels are associated with altered synaptic strength, 
electrical activity, and autistic-like behavior.

Biochim Biophys Acta. 2016 Jun

7;1862(9):1755-65. doi:

10.1016/j.bbadis.2016.06.005. PMID:

27287255.X-1

5402. Kessler D, Angstadt M, Sripada C. Growth charting of brain connectivity networks and the identification of attention impairment in youth. JAMA Psychiatry. 2016;73(5):481-9.

doi:

http://dx.doi.org/10.1001/jamapsychiatry.2016. 0088.X-1

5403. Ketcheson L, Hauck J, Ulrich D. The effects of an early motor skill intervention on motor skills, levels of physical activity, and socialization in young children with autism spectrum disorder: A pilot study. Autism. 2016 Jun 26doi: 10.1177/1362361316650611. PMID: 27354429.X-1

5404. Key AP, Yoder PJ, Stone WL. Consonant differentiation mediates the discrepancy between non-verbal and verbal abilities in children with ASD. Journal of Intellectual Disability Research. 2016;60(5):478-90. doi: http://dx.doi.org/10.1111/jir.12286.X-1

5405. Khadmaoui A, Gómez C, Poza J, et al. MEG analysis of neural interactions in attention-deficit/hyperactivity disorder. Computational Intelligence and Neuroscience. 2016;2016doi:

http://dx.doi.org/10.1155/2016/8450241.X-1

5406. Khemir S, Halayem S, Azzouz H, et al. Autism in phenylketonuria patients: From clinical presentation to molecular defects. Journal of Child Neurology. 2016;31(7):843-9. doi:

http://dx.doi.org/10.1177/0883073815623636.X $-1$

5407. Khemka I, Hickson L, Mallory SB. Evaluation of a Decision-Making Curriculum for Teaching Adolescents with Disabilities to Resist Negative Peer Pressure. J Autism Dev Disord. 2016 Jul;46(7):2372-84. doi: 10.1007/s10803-016-2770-0. PMID: 26993636.X-1

5408. Kiely B, Migdal TR, Vettam S, et al. Prevalence and Correlates of Elopement in a Nationally Representative Sample of Children with Developmental Disabilities in the United States. PLoS One. 2016;11(2):e0148337. doi: 10.1371/journal.pone.0148337. PMID: 26845701.X-1

5409. Kijima Y, Akagi T, Takaya Y, et al. Deficient Surrounding Rims in Patients Undergoing Transcatheter Atrial Septal Defect Closure. J Am Soc Echocardiogr. 2016 May 25doi: 10.1016/j.echo.2016.04.010. PMID: 27236281.X-1

5410. Kilincaslan A, Mutluer TD, Pasabeyoglu B, et al. Effects of Atomoxetine in Individuals with Attention-

Deficit/Hyperactivity Disorder and LowFunctioning Autism Spectrum Disorder. J Child Adolesc Psychopharmacol. 2016 May 26doi: 10.1089/cap.2015.0179. PMID: 27228116.X-2, $\mathrm{X}-3$

5411. Kim H, Carlson AG, Curby TW, et al. Relations among motor, social, and cognitive skills in pre-kindergarten children with developmental disabilities. Research in Developmental Disabilities. 2016;53-54:43-60. doi: http://dx.doi.org/10.1016/j.ridd.2016.01.016.X1

5412. Kim H, Lim CS, Kaang BK. Neuronal mechanisms and circuits underlying repetitive behaviors in mouse models of autism spectrum disorder. Behav Brain Funct. 2016;12(1):3. doi: 10.1186/s12993-016-0087-y. PMID: 26790724.X-1

5413. Kim HJ, Cho MH, Shim WH, et al. Deficient autophagy in microglia impairs synaptic pruning and causes social behavioral defects. Mol Psychiatry. 2016 Jul 12doi: 10.1038/mp.2016.103. PMID: 27400854.X-1

5414. Kim HJ, Iyer S, Zebala LP, et al. Perioperative Neurologic Complications in Adult Spinal Deformity Surgery: Incidence and Risk factors in 564 Patients. Spine (Phila $\mathrm{Pa}$ 1976). 2016 Jul 7doi: 10.1097/brs.0000000000001774. PMID: 27398890.X-1

5415. Kim HY, Korade Z, Tallman KA, et al. Inhibitors of 7-Dehydrocholesterol Reductase: Screening of a Collection of Pharmacologically Active Compounds in Neuro2a Cells. Chem Res Toxicol. 2016 May 16;29(5):892-900. doi: 
10.1021/acs.chemrestox.6b00054. PMID: 27097157.X-1

5416. Kim I, Ekas NV, Hock R. Associations between child behavior problems, family management, and depressive symptoms for mothers of children with autism spectrum disorder. Research in Autism Spectrum Disorders. 2016;26:80-90. doi: http://dx.doi.org/10.1016/j.rasd.2016.03.009.X1

5417. Kim JM, Eom TH. The Pseudosubarachnoid Sign: Clinical Implications of Subarachnoid Hemorrhage Misdiagnosis. Pediatr Emerg Care. 2016 May 12doi: 10.1097/pec.0000000000000746. PMID: 27176908.X-1

5418. Kim JY, Ryu DS, Paik HK, et al. Paraspinal muscle, facet joint, and disc problems: risk factors for adjacent segment degeneration after lumbar fusion. Spine J. 2016 Mar 9doi: 10.1016/j.spinee.2016.03.010. PMID: 26970600.X-1

5419. Kim S, Boylan K. Effectiveness of Antidepressant Medications for Symptoms of Irritability and Disruptive Behaviors in Children and Adolescents. J Child Adolesc Psychopharmacol. 2016 Aug 2doi: 10.1089/cap.2015.0127. PMID: 27482998.X-1

5420. Kim SH, Kim YS, Koh YJ, et al. Often Asked but Rarely Answered: Can Asians Meet DSM-5/ICD-10 Autism Spectrum Disorder Criteria? J Child Adolesc Psychopharmacol. 2016 Jun 17doi: 10.1089/cap.2016.0021. PMID: 27315155.X-1

5421. Kim SH, Lyu I, Fonov VS, et al. Development of cortical shape in the human brain from 6 to 24 months of age via a novel measure of shape complexity. NeuroImage. 2016;135:163-76. doi: http://dx.doi.org/10.1016/j.neuroimage.2016.04. 053.X-1

5422. Kim SH, Macari S, Koller J, et al. Examining the phenotypic heterogeneity of early autism spectrum disorder: Subtypes and short-term outcomes. Journal of Child Psychology and Psychiatry. 2016;57(1):93-102. doi: http://dx.doi.org/10.1111/jcpp.12448.X-1
5423. Kim SY, De Vries RG, Peteet JR. Euthanasia and Assisted Suicide of Patients With Psychiatric Disorders in the Netherlands 2011 to 2014. JAMA Psychiatry. 2016 Apr;73(4):362-8. doi: 10.1001/jamapsychiatry.2015.2887. PMID: 26864709.X-1

5424. Kimura M, Yamazaki Y. Physical punishment, mental health and sense of coherence among parents of children with intellectual disability in Japan. Journal of Applied Research in Intellectual Disabilities. 2016;29(5):455-67. doi: http://dx.doi.org/10.1111/jar.12198.X-1

5425. King BH. Assessing Risk of Autism Spectrum Disorder in Children After Antidepressant Use During Pregnancy. JAMA Pediatr. 2016 Feb;170(2):111-2. doi: 10.1001/jamapediatrics.2015.3493. PMID: 26660503.X-1

5426. King LB, Koch M, Murphy KR, et al. Neurofibromin Loss of Function Drives Excessive Grooming in Drosophila. G3 (Bethesda). 2016;6(4):1083-93. doi: 10.1534/g3.115.026484. PMID: 26896440.X-1

5427. King LB, Walum H, Inoue K, et al. Variation in the Oxytocin Receptor Gene Predicts Brain Region-Specific Expression and Social Attachment. Biol Psychiatry. 2016 Jul 15;80(2):160-9. doi: 10.1016/j.biopsych.2015.12.008. PMID: 26893121.X-1

5428. Kinnear SH, Link BG, Ballan MS, et al. Understanding the experience of stigma for parents of children with autism spectrum disorder and the role stigma plays in families' lives. Journal of Autism and Developmental Disorders. 2016;46(3):942-53. doi: http://dx.doi.org/10.1007/s10803-015-26379.X-1

5429. Kirby AV, Boyd BA, Williams KL, et al. Sensory and repetitive behaviors among children with autism spectrum disorder at home. Autism. 2016 Apr 18doi: 10.1177/1362361316632710. PMID: 27091950.X-1

5430. Kirby AV, Little LM, Schultz B, et al. Development and Pilot of the Caregiver Strategies Inventory. Am J Occup Ther. 2016 
Jul-Aug;70(4):7004360010p1-6. doi: 10.5014/ajot.2016.019901. PMID: 27294999.X-1

5431. Kirkovski M, Rogasch NC, Saeki T, et al. Single Pulse Transcranial Magnetic Stimulation-Electroencephalogram Reveals No Electrophysiological Abnormality in Adults with High-Functioning Autism Spectrum Disorder. J Child Adolesc Psychopharmacol. 2016 Jun 10doi: 10.1089/cap.2015.0181. PMID: 27284688.X-1

5432. Kister T, Dahnert I, Lurz P. Fatal Erosion Atrial Septal Defect Device. Catheter Cardiovasc Interv. 2016 Apr;87(5):951-4. doi: 10.1002/ccd.26219. PMID: 26354498.X-1

5433. Kitazoe N, Fujita N, Izumoto Y, et al. Whether the Autism Spectrum Quotient consists of two different subgroups? Cluster analysis of the Autism Spectrum Quotient in general population. Autism. 2016 Apr 30doi: 10.1177/1362361316638787. PMID: 27132011.X-1

5434. Klein N, Kemper KJ. Integrative Approaches to Caring for Children with Autism. Curr Probl Pediatr Adolesc Health Care. 2016 Jun;46(6):195-201. doi: 10.1016/j.cppeds.2015.12.004. PMID: 26776326.X-1, X-2

5435. Klionsky DJ, Abdelmohsen K, Abe A, et al. Guidelines for the use and interpretation of assays for monitoring autophagy (3rd edition). Autophagy. 2016;12(1):1-222. doi: 10.1080/15548627.2015.1100356. PMID: 26799652.X-1

5436. Kloo D, Kain W. The direct way may not be the best way: Children with ADHD and their understanding of self-presentation in social interactions. European Journal of Developmental Psychology. 2016;13(1):40-51. doi:

http://dx.doi.org/10.1080/17405629.2015.10519 60.X-1

5437. Knight AM, Xie M, Mandell DS. Disparities in Psychiatric Diagnosis and Treatment for Youth with Systemic Lupus Erythematosus: Analysis of a National US Medicaid Sample. J Rheumatol. 2016 Jul;43(7):1427-33. doi: 10.3899/jrheum.150967. PMID: 27134262.X-1
5438. Ko C, Kim N, Kim E, et al. The effect of epilepsy on autistic symptom severity assessed by the Social Responsiveness Scale in children with autism spectrum disorder. Behavioral and Brain Functions. 2016;12doi: http://dx.doi.org/10.1186/s12993-016-0105$0 . \mathrm{X}-1$

5439. Kodak T, Campbell V, Bergmann S, et al. Examination of efficacious, efficient, and socially valid error-correction procedures to teach sight words and prepositions to children with autism spectrum disorder. J Appl Behav Anal. 2016 May 6doi: 10.1002/jaba.310. PMID: 27150389.X-1

5440. Koehne S, Behrends A, Fairhurst MT, et al. Fostering Social Cognition through an Imitation- and Synchronization-Based Dance/Movement Intervention in Adults with Autism Spectrum Disorder: A Controlled Proof-of-Concept Study. Psychother Psychosom. 2016;85(1):27-35. doi: 10.1159/000441111. PMID: 26609704.X-1

5441. Koks N, Ghassabian A, Greaves-Lord $\mathrm{K}$, et al. Maternal C-Reactive Protein Concentration in Early Pregnancy and Child Autistic Traits in the General Population. Paediatr Perinat Epidemiol. 2016 Mar;30(2):181-9. doi: 10.1111/ppe.12261. PMID: 26860445.X-1

5442. Kondolot M, Özmert EN, Öztop DB, et al. The modified checklist for autism in Turkish toddlers: A different cultural adaptation sample. Research in Autism Spectrum Disorders. 2016;21:121-7. doi: http://dx.doi.org/10.1016/j.rasd.2015.10.006.X1

5443. Konopka G, Roberts TF. Animal Models of Speech and Vocal Communication Deficits Associated With Psychiatric Disorders. Biol Psychiatry. 2016 Jan 1;79(1):53-61. doi: 10.1016/j.biopsych.2015.07.001. PMID: 26232298.X-1

5444. Konrad M, Drosselmeyer J, Kostev K. Referral for occupational therapy after diagnosis of developmental disorder by German child psychiatrists. Early Child Development and Care. 2016;186(6):959-70. doi: http://dx.doi.org/10.1080/03004430.2015.10702 62.X-1 
5445. Koolschijn PC, Geurts HM. Gray Matter Characteristics in Mid and Old Aged Adults with ASD. J Autism Dev Disord. 2016 May 13doi: 10.1007/s10803-016-2810-9. PMID: 27177894.X-1

5446. Koren' EV, Kupriyanova TA, Drobinskaya AO, et al. Effects of mental disorders in children on parents in the context of differentiated approaches to psychosocial interventions in pediatric psychiatry. Neuroscience and Behavioral Physiology. 2016;46(4):394-9. doi: http://dx.doi.org/10.1007/s11055-016-02483.X-1

5447. Korhonen V, Werner S. Autistic traits and attention to speech: Evidence from typically developing individuals. Logoped Phoniatr Vocol. 2016 May 23:1-7. doi: 10.1080/14015439.2016.1186731. PMID: 27216974.X-1

5448. Korkiakangas T, Dindar K, Laitila A, et al. The Sally-Anne test: an interactional analysis of a dyadic assessment. Int J Lang Commun Disord. 2016 May 17doi: 10.1111/1460-6984.12240. PMID: 27184176.X-1

5449. Kos A, Olde Loohuis N, Meinhardt J, et al. MicroRNA-181 promotes synaptogenesis and attenuates axonal outgrowth in cortical neurons. Cell Mol Life Sci. 2016 Mar 26doi: 10.1007/s00018-016-2179-0. PMID: 27017280.X-1

5450. Kosaka H, Okamoto Y, Munesue T, et al. Oxytocin efficacy is modulated by dosage and oxytocin receptor genotype in young adults with high-functioning autism: a 24-week randomized clinical trial. Transl Psychiatry. 2016;6(8):e872. doi: 10.1038/tp.2016.152. PMID: 27552585.X-1

5451. Koshiba M, Karino G, Mimura K, et al. Psycho-Cognitive Intervention for ASD from Cross-Species Behavioral Analyses of Infants, Chicks and Common Marmosets. CNS Neurol Disord Drug Targets. 2016;15(5):57886. PMID: 27071788.X-1

5452. Koski S, Gabriels RL, Beresford C. Interventions for paediatric surgery patients with comorbid autism spectrum disorder: a systematic literature review. Arch Dis Child. 2016 May 25doi: 10.1136/archdischild-2016310814. PMID: 27226525.X-1

5453. Kossyvaki L, Jones G, Guldberg K. Training teaching staff to facilitate spontaneous communication in children with autism: Adult Interactive Style Intervention (AISI). Journal of Research in Special Educational Needs. 2016;16(3):156-68. doi: http://dx.doi.org/10.1111/1471-3802.12068.X3, X-4

5454. Kossyvaki L, Papoudi D. A review of play interventions for children with autism at school. International Journal of Disability, Development and Education. 2016;63(1):45-63. doi: http://dx.doi.org/10.1080/1034912X.2015.1111 303.X-1, X-2

5455. Kot M, Buning J, Jankowska W, et al. Development of ovary structures in the last larval and adult stages of psyllids (Insecta, Hemiptera, Sternorrhyncha: Psylloidea). Arthropod Struct Dev. 2016 May 6doi: 10.1016/j.asd.2016.04.004. PMID: 27140505.X-1

5456. Kovac M, Mosner M, Miller S, et al. Experience sampling of positive affect in adolescents with autism: Feasibility and preliminary findings. Research in Autism Spectrum Disorders. 2016;29-30:57-65. doi: http://dx.doi.org/10.1016/j.rasd.2016.06.003.X1

5457. Kovarski K, Thillay A, Houy-Durand E, et al. Brief Report: Early VEPs to PatternReversal in Adolescents and Adults with Autism. J Autism Dev Disord. 2016 Jul 30doi: 10.1007/s10803-016-2880-8. PMID: 27475419.X-1

5458. Kover ST, Edmunds SR, Ellis Weismer S. Brief Report: Ages of Language Milestones as Predictors of Developmental Trajectories in Young Children with Autism Spectrum Disorder. J Autism Dev Disord. 2016 Jul;46(7):2501-7. doi: 10.1007/s10803-0162756-y. PMID: 26936159.X-1

5459. Kozol RA, Abrams AJ, James DM, et al. Function Over Form: Modeling Groups of Inherited Neurological Conditions in Zebrafish. Front Mol Neurosci. 2016;9:55. doi: 
10.3389/fnmol.2016.00055. PMID:

27458342.X-1

5460. Krahe TE, Filgueiras CC, Medina AE. Effects of developmental alcohol and valproic acid exposure on play behavior of ferrets. Int $\mathrm{J}$ Dev Neurosci. 2016 May 18;52:75-81. doi: 10.1016/j.ijdevneu.2016.03.007. PMID: 27208641.X-1

5461. Krakovich TM, McGrew JH, Yu Y, et al. Stress in Parents of Children with Autism Spectrum Disorder: An Exploration of Demands and Resources. J Autism Dev Disord. 2016 Jun;46(6):2042-53. doi: 10.1007/s10803016-2728-2. PMID: 26883644.X-1

5462. Krakowiak P, Walker CK, Tancredi D, et al. Autism-specific maternal anti-fetal brain autoantibodies are associated with metabolic conditions. Autism Res. 2016 Jun 17doi: 10.1002/aur.1657. PMID: 27312731.X-1

5463. Kramer JM, Liljenquist K, Coster WJ. Validity, reliability, and usability of the Pediatric Evaluation of Disability InventoryComputer Adaptive Test for autism spectrum disorders. Dev Med Child Neurol. 2016 Mar;58(3):255-61. doi: 10.1111/dmcn.12837. PMID: 26104112.X-1

5464. Kranz TM, Kopp M, Waltes R, et al. Meta-analysis and association of two common polymorphisms of the human oxytocin receptor gene in autism spectrum disorder. Autism Res. 2016 Jan 20doi: 10.1002/aur.1597. PMID: 26788924.X-1

5465. Kreider CM, Bendixen RM, Young ME, et al. Social networks and participation with others for youth with learning, attention, and autism spectrum disorders. Can J Occup Ther. 2016 Feb;83(1):14-26. doi: 10.1177/0008417415583107. PMID: 26755040.X-1

5466. Kronenberg LM, Goossens PJ, van Busschbach JT, et al. Burden and Expressed Emotion of Caregivers in Cases of Adult Substance Use Disorder with and Without Attention Deficit/Hyperactivity Disorder or Autism Spectrum Disorder. Int J Ment Health Addict. 2016;14:49-63. doi: 10.1007/s11469015-9567-9. PMID: 26798327.X-1
5467. Krstovska-Guerrero I, Jones EA. Social-communication intervention for toddlers with Autism Spectrum Disorder: Eye gaze in the context of requesting and joint attention. Journal of Developmental and Physical Disabilities. 2016;28(2):289-316. doi: http://dx.doi.org/10.1007/s10882-015-94669.X-3, X-4

5468. Krystal JH, Abi-Dargham A, Akbarian $\mathrm{S}$, et al. Constance E. Lieber, Theodore R. Stanley, and the Enduring Impact of Philanthropy on Psychiatry Research. Biol Psychiatry. 2016 Jul 15;80(2):84-6. doi: 10.1016/j.biopsych.2016.05.004. PMID: 27346079.X-1

5469. Krystal JH, Abi-Dargham A, Barch DM, et al. Biological Psychiatry and Biological Psychiatry: Cognitive Neuroscience and Neuroimaging Adopt Neuroscience-Based Nomenclature. Biol Psychiatry. 2016 Jul 1;80(1):2-3. doi: 10.1016/j.biopsych.2016.03.2103. PMID: 27312230.X-1

5470. Kuiper MW, Verhoeven EW, Geurts HM. The role of interstimulus interval and "Stimulus-type" in prepotent response inhibition abilities in people with ASD: A quantitative and qualitative review. Autism Res. 2016 Apr 19doi: 10.1002/aur.1631. PMID: 27091394.X-1

5471. Kulakova E, Nieuwland MS. Pragmatic skills predict online counterfactual comprehension: Evidence from the N400. Cogn Affect Behav Neurosci. 2016 May 9doi: 10.3758/s13415-016-0433-4. PMID: 27160367.X-1

5472. Kumar A, Juneja M, Mishra D. Prevalence of autism spectrum disorders in siblings of Indian children with autism spectrum disorders. Journal of Child Neurology. 2016;31(7):873-8. doi: http://dx.doi.org/10.1177/0883073815624764.X $-1$

5473. Kumar H, Sharma B. Memantine ameliorates autistic behavior, biochemistry \& blood brain barrier impairments in rats. Brain Res Bull. 2016 Mar 23;124:27-39. doi: 10.1016/j.brainresbull.2016.03.013. PMID: 27034117.X-1 
5474. Kumazaki H, Muramatsu T, Fujisawa TX, et al. Assessment of olfactory detection thresholds in children with autism spectrum disorders using a pulse ejection system. Mol Autism. 2016;7:6. doi: 10.1186/s13229-0160071-2. PMID: 26788283.X-1

5475. Kung KT, Constantinescu M, Browne $\mathrm{WV}$, et al. No relationship between early postnatal testosterone concentrations and autistic traits in 18 to 30-month-old children. Mol Autism. 2016;7:15. doi: 10.1186/s13229016-0078-8. PMID: 26893820.X-1

5476. Kurita T, Kikuchi M, Yoshimura Y, et al. Atypical Bilateral Brain Synchronization in the Early Stage of Human Voice Auditory Processing in Young Children with Autism. PLoS One. 2016;11(4):e0153077. doi: 10.1371/journal.pone.0153077. PMID: 27074011.X-1

5477. Kuroda T, Cancado CR, Podlesnik CA. Resistance to change and resurgence in humans engaging in a computer task. Behav Processes. 2016 Apr;125:1-5. doi: 10.1016/j.beproc.2016.01.010. PMID: 26836390.X-1

5478. Kurth JA, Mastergeorge AM, Paschall $\mathrm{K}$. Economic and demographic factors impacting placement of students with autism. Education and Training in Autism and Developmental Disabilities. 2016;51(1):3-12.X1

5479. Kwon M-K, Setoodehnia M, Baek J, et al. The development of visual search in infancy: Attention to faces versus salience.

Developmental Psychology. 2016;52(4):537-55. doi: http://dx.doi.org/10.1037/dev0000080.X-1

5480. Kyzar EJ, Stewart AM, Kalueff AV. Effects of LSD on grooming behavior in serotonin transporter heterozygous (Sert(+)/(-)) mice. Behav Brain Res. 2016 Jan 1;296:47-52. doi: 10.1016/j.bbr.2015.08.018. PMID: 26340513.X-1

5481. Ladd-Acosta C, Shu C, Lee BK, et al. Presence of an epigenetic signature of prenatal cigarette smoke exposure in childhood. Environ Res. 2016 Jan;144(Pt A):139-48. doi: 10.1016/j.envres.2015.11.014. PMID: 26610292.X-1
5482. Lai JK, Lerch JP, Doering LC, et al. Regional brain volumes changes in adult male FMR1-KO mouse on the FVB strain. Neuroscience. 2016 Mar 24;318:12-21. doi: 10.1016/j.neuroscience.2016.01.021. PMID: 26794591.X-1

5483. Lambert A, Tessier S, Rochette A-C, et al. Poor sleep affects daytime functioning in typically developing and autistic children not complaining of sleep problems: A questionnaire-based and polysomnographic study. Research in Autism Spectrum Disorders. 2016;23:94-106. doi: http://dx.doi.org/10.1016/j.rasd.2015.11.010.X1

5484. Landa R, Hanley GP. An evaluation of multiple-schedule variations to reduce high-rate requests in the picture exchange communication system. J Appl Behav Anal. 2016 Jun;49(2):388-93. doi: 10.1002/jaba.285. PMID: 26814152.X-1, X-4

5485. Lane JD, Ledford JR. A review of interventions designed to increase sharing behaviors in children with social delays or deficits. Journal of Behavioral Education. 2016;25(1):69-94. doi: http://dx.doi.org/10.1007/s10864-015-9235y.X-1, X-2

5486. Lane JD, Lieberman-Betz R, Gast DL. An analysis of naturalistic interventions for increasing spontaneous expressive language in children with autism spectrum disorder. The Journal of Special Education. 2016;50(1):49-61. doi:

http://dx.doi.org/10.1177/0022466915614837.X $-2$

5487. Lane JD, Shepley C, Lieberman-Betz R. Promoting Expressive Language in Young Children with or At-Risk for Autism Spectrum Disorder in a Preschool Classroom. J Autism Dev Disord. 2016 Jul 15doi: 10.1007/s10803016-2856-8. PMID: 27422402.X-3, X-4

5488. Lange H, Buse J, Bender S, et al. Accident proneness in children and adolescents affected by ADHD and the impact of medication. Journal of Attention Disorders. 2016;20(6):501-9. doi: http://dx.doi.org/10.1177/1087054713518237.X $-1$ 
5489. Langh U, Hammar M, Klintwall L, et al. Allegiance and knowledge levels of professionals working with early intensive behavioural intervention in autism. Early Interv Psychiatry. 2016 Apr 6doi: 10.1111/eip.12335. PMID: 27060473.X-1

5490. Lanovaz MJ, Rapp JT, Maciw I, et al. Preliminary effects of parent-implemented behavioural interventions for stereotypy. Dev Neurorehabil. 2016 Jun;19(3):193-6. doi: 10.3109/17518423.2014.986821. PMID: 25549178.X-3, X-4

5491. Laskowski RA, Tyagi N, Johnson D, et al. Integrating population variation and protein structural analysis to improve clinical interpretation of missense variation: application to the WD40 domain. Hum Mol Genet. 2016 Mar 1;25(5):927-35. doi: 10.1093/hmg/ddv625. PMID: 26740553.X-1

5492. Latifi R, Gunn JK, Bakiu E, et al. Access to Specialized Care Through Telemedicine in Limited-Resource Country: Initial 1,065 Teleconsultations in Albania. Telemed J E Health. 2016 May 24doi: 10.1089/tmj.2016.0050. PMID: 27219617.X-1

5493. Lau WYP, Peterson CC, Attwood T, et al. Parents on the autism continuum: Links with parenting efficacy. Research in Autism Spectrum Disorders. 2016;26:57-64. doi: http://dx.doi.org/10.1016/j.rasd.2016.02.007.X1

5494. Lawson LM, Foster L. Sensory Patterns, Obesity, and Physical Activity Participation of Children With Autism Spectrum Disorder. Am J Occup Ther. 2016 Sep-Oct;70(5):7005180070p1-8. doi: 10.5014/ajot.2016.021535. PMID: 27548863.X-1

5495. Lawson SK, Gray AC, Woehrle NS. Effects of oxytocin on serotonin 1B agonistinduced autism-like behavior in mice. Behav Brain Res. 2016 Jul 18;314:52-64. doi: 10.1016/j.bbr.2016.07.027. PMID: 27439030.X-1

5496. Lazaro CP, Ponde MP, Rodrigues LE. Opioid peptides and gastrointestinal symptoms in autism spectrum disorders. Rev Bras Psiquiatr. 2016 Jun 14:0. doi: 10.1590/15164446-2015-1777. PMID: 27304256.X-1
5497. Lazenby DC, Sideridis GD, Huntington N, et al. Language differences at 12 months in infants who develop autism spectrum disorder. Journal of Autism and Developmental Disorders. 2016;46(3):899-909. doi: http://dx.doi.org/10.1007/s10803-015-26321.X-1

5498. Leach EL, van Karnebeek CD, Townsend KN, et al. Episodic ataxia associated with a de novo SCN2A mutation. Eur J Paediatr Neurol. 2016 Jun 14doi: 10.1016/j.ejpn.2016.05.020. PMID: 27328862.X-1

5499. Leaf JA, Leaf JB, Milne C, et al. The effects of the cool versus not cool procedure to teach social game play to individuals diagnosed with autism spectrum disorder. Behavior Analysis in Practice. 2016;9(1):34-49. doi: http://dx.doi.org/10.1007/s40617-016-01125.X-3, X-4

5500. Leaf JB, Leaf JA, Alcalay A, et al. Comparison of most-to-least prompting to flexible prompt fading for children with autism spectrum disorder. Exceptionality.

2016;24(2):109-22. doi:

http://dx.doi.org/10.1080/09362835.2015.10644 19.X-1, X-4

5501. Leaf JB, Mitchell E, Townley-Cochran D, et al. Comparing Social Stories ${ }^{\mathrm{TM}}$ to cool versus not cool. Education \& Treatment of Children. 2016;39(2):173-86. doi: http://dx.doi.org/10.1353/etc.2016.0006.X-1, X$3, \mathrm{X}-4$

5502. Leaf JB, Oppenheim-Leaf ML, Townley-Cochran $\mathrm{D}$, et al. Changing preference from tangible to social activities through an observation procedure. Journal of Applied Behavior Analysis. 2016;49(1):49-57. doi: http://dx.doi.org/10.1002/jaba.276.X-1

5503. Leaf JB, Taubman M, Milne C, et al. Teaching social communication skills using a cool versus not cool procedure plus role-playing and a social skills taxonomy. Education \& Treatment of Children. 2016;39(1):44-63.X-3, $\mathrm{X}-4$

5504. Leaf JB, Townley-Cochran D, Mitchell E, et al. Evaluation of multiplealternative prompts during tact training. J Appl 
Behav Anal. 2016 Jun;49(2):399-404. doi: 10.1002/jaba.289. PMID: 26792578.X-4

5505. Leaf JB, Townley-Cochran D, Mitchell E, et al. Evaluation of multiplealternative prompts during tact training. Journal of Applied Behavior Analysis. 2016;49(2):399404. doi: http://dx.doi.org/10.1002/jaba.289.X-4

5506. LeBarton ES, Iverson JM.

Associations between gross motor and communicative development in at-risk infants. Infant Behav Dev. 2016 Jun 14;44:59-67. doi: 10.1016/j.infbeh.2016.05.003. PMID: 27314943.X-1

5507. LeBlanc JJ, Nelson CA. Deletion and duplication of $16 \mathrm{p} 11.2$ are associated with opposing effects on visual evoked potential amplitude. Mol Autism. 2016;7:30. doi: 10.1186/s13229-016-0095-7. PMID: 27354901.X-1

5508. Lechpammer M, Tran YP, Wintermark $\mathrm{P}$, et al. Upregulation of cystathione betasynthase and p70S6K/S6 in neonatal hypoxic ischemic brain injury. Brain Pathol. 2016 Jul 28doi: 10.1111/bpa.12421. PMID: 27465493.X-1

5509. Lee B. What are the benefits of phenotype analyses to special education? Am J Med Genet B Neuropsychiatr Genet. 2016 Mar;171b(2):301. doi: 10.1002/ajmg.b.32405. PMID: 26614532.X-1

5510. Lee C-Y, Su C-T, Chiang F-M, et al. Developing a short form of the Psychoeducational Profile-Third Edition for children with autism spectrum disorder. Research in Autism Spectrum Disorders. 2016;21:37-50. doi: http://dx.doi.org/10.1016/j.rasd.2015.09.008.X1

5511. Lee HJ, Dreyfus C, DiCicco-Bloom E. Valproic acid stimulates proliferation of glial precursors during cortical gliogenesis in developing rat. Dev Neurobiol. 2016 Jul;76(7):780-98. doi: 10.1002/dneu.22359. PMID: 26505176.X-1

5512. Lee JA, Damianov A, Lin CH, et al. Cytoplasmic Rbfox1 Regulates the Expression of Synaptic and Autism-Related Genes.

Neuron. 2016 Jan 6;89(1):113-28. doi: 10.1016/j.neuron.2015.11.025. PMID: 26687839.X-1

5513. Lee JH, Kim KT, Lee SH, et al. Overcorrection of lumbar lordosis for adult spinal deformity with sagittal imbalance: comparison of radiographic outcomes between overcorrection and undercorrection. Eur Spine J. 2016 Feb 16doi: 10.1007/s00586-016-44414. PMID: 26883266.X-1

5514. Lee K, Goodman L, Fourie C, et al. AMPA Receptors as Therapeutic Targets for Neurological Disorders. Adv Protein Chem Struct Biol. 2016;103:203-61. doi: 10.1016/bs.apcsb.2015.10.004. PMID: 26920691.X-1

5515. Lee K, Lambert H, Wittich W, et al. The use of movement-based interventions with children diagnosed with autism for psychosocial outcomes-a scoping review. Research in Autism Spectrum Disorders. 2016 2016-06-30;24:52-67. doi: 10.1016/j.newideapsych.2010.03.004. http://dx.doi.org/10.1016/j.rasd.2015.12.011. PMID: 1800417137; 2016-08981-007.X-1, X-2

5516. Lee KYS, Crooke PJ, Lui ALY, et al. The outcome of a social cognitive training for mainstream adolescents with social communication deficits in a Chinese community. International Journal of Disability, Development and Education. 2016;63(2):201-

23. doi: http://dx.doi.org/10.1080/1034912X.2015.1065 960.X-1, X-3

5517. Lee SM, Kim BK, Kim TW, et al. Music application alleviates short-term memory impairments through increasing cell proliferation in the hippocampus of valproic acid-induced autistic rat pups. J Exerc Rehabil. 2016 Jun;12(3):148-55. doi: 10.12965/jer.1632638.319. PMID: 27419108.X-1

5518. Lee Y-C, Chan P-C, Lin S-K, et al. Correlation patterns between pretend play and playfulness in children with autism spectrum disorder, developmental delay, and typical development. Research in Autism Spectrum Disorders. 2016;24:29-38. doi: http://dx.doi.org/10.1016/j.rasd.2016.01.006.X1 
5519. Lee Y-c, Yang H-J, Chen VC-h, et al. Meta-analysis of quality of life in children and adolescents with ADHD: By both parent proxyreport and child self-report using PedsQLTM.

Research in Developmental Disabilities.

2016;51-52:160-72. doi:

http://dx.doi.org/10.1016/j.ridd.2015.11.009.X1

5520. Lee YH, Lenhart PD, Lambert SR. Cataract secondary to self-inflicted blunt trauma in children with autism spectrum disorder. J aapos. 2016 May 17doi: 10.1016/j.jaapos.2016.02.013. PMID: 27223635.X-1

5521. Leffa DD, Daumann F, Damiani AP, et al. DNA damage after chronic oxytocin administration in rats: a safety yellow light? Metab Brain Dis. 2016 Aug 3doi: 10.1007/s11011-016-9885-z. PMID: 27488109.X-1

5522. Lehnhardt FG, Falter CM, Gawronski A, et al. Sex-Related Cognitive Profile in Autism Spectrum Disorders Diagnosed Late in Life: Implications for the Female Autistic Phenotype. J Autism Dev Disord. 2016 Jan;46(1):139-54. doi: 10.1007/s10803-0152558-7. PMID: 26319250.X-1

5523. Lehti V, Chudal R, Suominen A, et al. Association between immigrant background and ADHD: A nationwide population-based case-control study. Journal of Child Psychology and Psychiatry. 2016;57(8):967-75. doi: http://dx.doi.org/10.1111/jcpp.12570.X-1

5524. Leigh JP, Grosse SD, Cassady D, et al. Spending by California's Department of Developmental Services for Persons with Autism across Demographic and Expenditure Categories. PLoS One. 2016;11(3):e0151970. doi: 10.1371/journal.pone.0151970. PMID: 27015098.X-1

5525. Lense MD, Dykens EM. Beat perception and sociability: Evidence from Williams syndrome. Frontiers in Psychology. 2016;7.X-1

5526. Leon Guerrero CR, Pathak S, Grange DK, et al. Neurologic and neuroimaging manifestations of Cantu syndrome: A case series. Neurology. 2016 Jun 17doi: 10.1212/wnl.0000000000002861. PMID: 27316244.X-1

5527. Leon Guerrero CR, Pathak S, Grange DK, et al. Neurologic and neuroimaging manifestations of Cantú syndrome: A case series. Neurology. 2016;87(3):270-6. doi: http://dx.doi.org/10.1212/WNL.0000000000002 861.X-1

5528. Leone E, Dorstyn D, Ward L. Defining resilience in families living with neurodevelopmental disorder: A preliminary examination of Walsh's framework. Journal of Developmental and Physical Disabilities. 2016;28(4):595-608. doi: http://dx.doi.org/10.1007/s10882-016-9497$\mathrm{x} . \mathrm{X}-1$

5529. Lepeta K, Lourenco MV, Schweitzer BC, et al. Synaptopathies: synaptic dysfunction in neurological disorders. J Neurochem. 2016 Jun 22doi: 10.1111/jnc.13713. PMID: 27333343.X-1

5530. Leppa VM, Kravitz SN, Martin CL, et al. Rare Inherited and De Novo CNVs Reveal Complex Contributions to ASD Risk in Multiplex Families. Am J Hum Genet. 2016 Aug 25doi: 10.1016/j.ajhg.2016.06.036. PMID: 27569545.X-1

5531. Lepper TL, Devine B, Petursdottir AI. Application of a lag contingency to reduce perseveration on circumscribed interests. Dev Neurorehabil. 2016 Apr 8:1-4. doi: 10.3109/17518423.2016.1152612. PMID: 27058176.X-1, X-3

5532. Lera-Miguel S, Rosa M, Puig O, et al. Assessing advanced theory of mind in children and adolescents with high-functioning autism: The Spanish version of the Stories of Everyday Life. Journal of Autism and Developmental Disorders. 2016;46(1):294-304. doi: http://dx.doi.org/10.1007/s10803-015-25854.X-1

5533. Lesch KP. Maturing insights into the genetic architecture of neurodevelopmental disorders - from common and rare variant interplay to precision psychiatry. J Child Psychol Psychiatry. 2016 Jun;57(6):659-61. doi: 10.1111/jcpp.12574. PMID: 27192951.X-1 
5534. Leslie NR, Longy M. Inherited PTEN mutations and the prediction of phenotype. Semin Cell Dev Biol. 2016 Apr;52:30-8. doi: 10.1016/j.semcdb.2016.01.030. PMID: 26827793.X-1

5535. Leung C, Chan S, Lam T, et al. The effect of parent education program for preschool children with developmental disabilities: A randomized controlled trial. Research in Developmental Disabilities. 2016;56:18-28. doi: http://dx.doi.org/10.1016/j.ridd.2016.05.015.X1

5536. Leung C, Jia Z. Mouse Genetic Models of Human Brain Disorders. Front Genet. 2016;7:40. doi: 10.3389/fgene.2016.00040. PMID: 27047540.X-1

5537. Levato LE, Aponte CA, Wilkins J, et al. Use of urine alarms in toilet training children with intellectual and developmental disabilities: A review. Res Dev Disabil. 2016 Jun-Jul;5354:232-41. doi: 10.1016/j.ridd.2016.02.007. PMID: 26942703.X-1

5538. Leventakou V, Micali N, Georgiou V, et al. Is there an association between eating behaviour and attention-deficit/hyperactivity disorder symptoms in preschool children? Journal of Child Psychology and Psychiatry. 2016;57(6):676-84. doi:

http://dx.doi.org/10.1111/jcpp.12504.X-1

5539. Levin K. Aesthetics of hyperactivity: A study of the role of expressive movement in ADHD and capoeira. American Journal of Dance Therapy. 2016;38(1):41-62. doi: http://dx.doi.org/10.1007/s10465-016-92117.X-1

5540. Leviton A, Allred EN, Kuban KCK, et al. Brain disorders associated with corticotropin-releasing hormone expression in the placenta among children born before the 28th week of gestation. Acta Paediatrica. 2016;105(1):e7-e11. doi: http://dx.doi.org/10.1111/apa.13174.X-1

5541. Levy SE, Frasso R, Colantonio S, et al. Shared Decision Making and Treatment Decisions for Young Children With Autism Spectrum Disorder. Acad Pediatr. 2016 Apr 27doi: 10.1016/j.acap.2016.04.007. PMID: 27132050.X-1

5542. Lewis LF. Realizing a diagnosis of autism spectrum disorder as an adult. Int J Ment Health Nurs. 2016 Mar 4doi: 10.1111/inm.12200. PMID: 26940281.X-1

5543. Li B, Tadross MR, Tsien RW. Sequential ionic and conformational signaling by calcium channels drives neuronal gene expression. Science. $2016 \mathrm{Feb}$ 19;351(6275):863-7. doi: 10.1126/science.aad3647. PMID: 26912895.X1

5544. Li H, Zhao P, Xu Q, et al. The autismrelated gene SNRPN regulates cortical and spine development via controlling nuclear receptor Nr4a1. Sci Rep. 2016;6:29878. doi: 10.1038/srep29878. PMID: 27430727.X-1

5545. Li J, You Y, Yue W, et al. Chromatin remodeling gene EZH2 involved in the genetic etiology of autism in Chinese Han population. Neurosci Lett. 2016 Jan 1;610:182-6. doi: 10.1016/j.neulet.2015.10.074. PMID: 26552012.X-1

5546. Li M, Amuta A, Xu L, et al. Autism genetic testing information needs among parents of affected children: A qualitative study. Patient Education and Counseling. 2016;99(6):1011-6. doi: http://dx.doi.org/10.1016/j.pec.2015.12.023.X-1

5547. Li M, Liu Y. Topoisomerase I in Human Disease Pathogenesis and Treatments. Genomics Proteomics Bioinformatics. 2016 May 12doi: 10.1016/j.gpb.2016.02.004. PMID: 27181710.X-1

5548. Li M, Zhang ND, Wang Y, et al. Coordinate regulatory osteogenesis effects of icariin, timosaponin B II and ferulic acid from traditional Chinese medicine formulas on UMR-106 osteoblastic cells and osteoblasts in neonatal rat calvaria cultures. J

Ethnopharmacol. 2016 Jun 5;185:120-31. doi: 10.1016/j.jep.2016.03.023. PMID: 26983755.X-1

5549. Li M, Zhao H, Ananiev GE, et al. Establishment of Reporter Lines for Detecting Fragile X Mental Retardation (FMR1) Gene Reactivation in Human Neural Cells. Stem 
Cells. 2016 Jul 16doi: 10.1002/stem.2463. PMID: 27422057.X-1

5550. Li P, Zhang C, Yi L. Brief report: Sensitivity of children with autism spectrum disorders to face appearance in selective trust. Journal of Autism and Developmental Disorders. 2016;46(7):2520-5. doi: http://dx.doi.org/10.1007/s10803-016-27611.X-1

5551. Li Q, Chen CF, Wang DY, et al. Changes in growth factor levels in the cerebrospinal fluid of autism patients after transplantation of human umbilical cord blood mononuclear cells and umbilical cord-derived mesenchymal stem cells. Genet Mol Res. 2016;15(2)doi: 10.4238/gmr.15027526. PMID: 27323064.X-1

5552. Li Q, Zhou JM. The microbiota-gutbrain axis and its potential therapeutic role in autism spectrum disorder. Neuroscience. 2016 Jun 2;324:131-9. doi:

10.1016/j.neuroscience.2016.03.013. PMID: 26964681.X-1

5553. Li YC, Yang SC, Hsu CT, et al. Capsulated Metallic Debris Tumor Mass Mimicking Adjacent Segment Disease: A Case Report. Clin Spine Surg. 2016 May 11doi: 10.1097/BSD.0b013e318292e685. PMID: 27171666.X-1

5554. Liao TC, Lien YT, Wang S, et al. Comorbidity of Atopic Disorders with Autism Spectrum Disorder and Attention Deficit/Hyperactivity Disorder. J Pediatr. 2016 Apr;171:248-55. doi: 10.1016/j.jpeds.2015.12.063. PMID: 26846570.X-1

5555. Libero LE, Nordahl CW, Li DD, et al. Persistence of megalencephaly in a subgroup of young boys with autism spectrum disorder. Autism Res. 2016 Jun 8doi: 10.1002/aur.1643. PMID: 27273931.X-1

5556. Lim BO, O'Sullivan D, Choi BG, et al. Comparative gait analysis between children with autism and age-matched controls: analysis with temporal-spatial and foot pressure variables. J Phys Ther Sci. 2016 Jan;28(1):28692. doi: 10.1589/jpts.28.286. PMID:

26957776.X-1
5557. Lim SS. Through the tablet glass: Transcendent parenting in an era of mobile media and cloud computing. Journal of Children and Media. 2016;10(1):21-9. doi: http://dx.doi.org/10.1080/17482798.2015.11218 96.X-1

5558. Limberg K, Gruber K, Noterdaeme M. The German version of the Child Behavior Checklist $1.5-5$ to identify children with a risk of autism spectrum disorder. Autism. 2016 May 22doi: 10.1177/1362361316645932. PMID: 27217430.X-1

5559. Lin IF, Agus TR, Suied C, et al. Fast response to human voices in autism. Sci Rep. 2016;6:26336. doi: 10.1038/srep26336. PMID: 27193919.X-1

5560. Lindsay G, Ricketts J, Peacey LV, et al. Meeting the educational and social needs of children with language impairment or autism spectrum disorder: the parents' perspectives. Int J Lang Commun Disord. 2016 Mar 8doi: 10.1111/1460-6984.12226. PMID: 26952185.X-1

5561. Lindsay S. Systematic review of factors affecting driving and motor vehicle transportation among people with autism spectrum disorder. Disabil Rehabil. 2016 Apr 4:1-10. doi: 10.3109/09638288.2016.1161849. PMID: 27045872.X-1, X-2

5562. Lindstrom R, Lepisto-Paisley T, Vanhala R, et al. Impaired neural discrimination of emotional speech prosody in children with autism spectrum disorder and language impairment. Neurosci Lett. 2016 Jun 9;628:4751. doi: 10.1016/j.neulet.2016.06.016. PMID: 27291458.X-1

5563. Lingren T, Chen P, Bochenek J, et al. Electronic Health Record Based Algorithm to Identify Patients with Autism Spectrum Disorder. PLoS One. 2016;11(7):e0159621. doi: 10.1371/journal.pone.0159621. PMID: 27472449.X-1

5564. Linkovski O, Katzin N, Salti M. Mirror Neurons and Mirror-Touch Synesthesia. Neuroscientist. 2016 May 30doi: 10.1177/1073858416652079. PMID: 27242280.X-1 
5565. Linsell L, Malouf R, Johnson S, et al. Prognostic factors for behavioral problems and psychiatric disorders in children born very preterm or very low birth weight: A systematic review. Journal of Developmental and Behavioral Pediatrics. 2016;37(1):88-102.X-1

5566. Lipstein EA, Lindly OJ, Anixt JS, et al. Shared Decision Making in the Care of Children with Developmental and Behavioral Disorders. Matern Child Health J. 2016 Mar;20(3):665-73. doi: 10.1007/s10995-0151866-z. PMID: 26518006.X-1

5567. Little EE, Carver LJ, Legare CH. Cultural variation in triadic infant-caregiver object exploration. Child Development. 2016;87(4):1130-45. doi: http://dx.doi.org/10.1111/cdev.12513.X-1

5568. Liu CX, Peng XL, Hu CC, et al. Developmental profiling of ASD-related shank3 transcripts and their differential regulation by valproic acid in zebrafish. Dev Genes Evol. 2016 Aug 26doi: 10.1007/s00427-016-0561-4. PMID: 27562614.X-1

5569. Liu J, Morey RA, Wilson JK, et al. Practices and outcomes of self-treatment with helminths based on physicians' observations. J Helminthol. 2016 May 31:1-11. doi: 10.1017/s0022149x16000316. PMID: 27240605.X-1

5570. Liu W, Li M, Yi L. Identifying children with autism spectrum disorder based on their face processing abnormality: A machine learning framework. Autism Res. 2016 Apr 1doi: 10.1002/aur.1615. PMID: 27037971.X-1

5571. Liu X, Campanac E, Cheung HH, et al. Idiopathic Autism: Cellular and Molecular Phenotypes in Pluripotent Stem Cell-Derived Neurons. Mol Neurobiol. 2016 Jun 29doi: 10.1007/s12035-016-9961-8. PMID: 27356918.X-1

5572. Liu Y, Ao NI, Zhao H. Gallbladder agenesis and atrial septal defect: A case report. Exp Ther Med. 2016 May;11(5):1795-8. doi: 10.3892/etm.2016.3115. PMID: 27168805.X-1

5573. Liu Z, Li X, Zhang JT, et al. Autismlike behaviours and germline transmission in transgenic monkeys overexpressing MeCP2.
Nature. 2016 Feb 4;530(7588):98-102. doi: 10.1038/nature16533. PMID: 26808898.X-1

5574. Lo FS, Erzurumlu RS, Powell EM. Insulin-Independent GABAA ReceptorMediated Response in the Barrel Cortex of Mice with Impaired Met Activity. J Neurosci. 2016 Mar 30;36(13):3691-7. doi: 10.1523/jneurosci.0006-16.2016. PMID: 27030755.X-1

5575. Lockwood PL. The anatomy of empathy: Vicarious experience and disorders of social cognition. Behav Brain Res. 2016 Sep 15;311:255-66. doi: 10.1016/j.bbr.2016.05.048. PMID: 27235714.X-1

5576. Loebel A, Brams M, Goldman RS, et al. Lurasidone for the treatment of irritability associated with autistic disorder. Journal of Autism and Developmental Disorders. 2016;46(4):1153-63. doi: http://dx.doi.org/10.1007/s10803-015-2628x.X-1, X-4

5577. Longo N, Frigeni M, Pasquali M. Carnitine transport and fatty acid oxidation. Biochim Biophys Acta. 2016 Jan 29doi: 10.1016/j.bbamcr.2016.01.023. PMID: 26828774.X-1

5578. Lopata C, Lipinski AM, Thomeer ML, et al. Open-trial pilot study of a comprehensive outpatient psychosocial treatment for children with high-functioning autism spectrum disorder. Autism. 2016 Apr 7doi: 10.1177/1362361316630201. PMID: 27056846.X-3

5579. Lopata C, Thomeer ML, Rodgers JD, et al. RCT of mind reading as a component of a psychosocial treatment for high-functioning children with ASD. Research in Autism Spectrum Disorders. 2016;21:25-36. doi: http://dx.doi.org/10.1016/j.rasd.2015.09.003.X1

5580. Lopes F, Barbosa M, Ameur A, et al. Identification of novel genetic causes of Rett syndrome-like phenotypes. J Med Genet. 2016 Mar;53(3):190-9. doi: 10.1136/jmedgenet2015-103568. PMID: 26740508.X-1

5581. Lopez-Cacho JM, Gallardo S, Posada $\mathrm{M}$, et al. Characterization of immune cell phenotypes in adults with autism spectrum 
disorders. J Investig Med. 2016 Jun 13doi: 10.1136/jim-2016-000070. PMID: 27296457.X-1

5582. Lopez-Mourelo O, Mur E, Madrigal I, et al. Social anxiety and autism spectrum traits among adult FMR1 premutation carriers. Clin Genet. 2016 Apr 22doi: 10.1111/cge.12791. PMID: 27102723.X-1

5583. Lord C, Veenstra-VanderWeele J. Following the Trail From Genotype to Phenotypes. JAMA Psychiatry. 2016 Jan;73(1):7-8. doi: 10.1001/jamapsychiatry.2015.2344. PMID: 26630619.X-1

5584. Lorenz T, Frischling C, Cuadros R, et al. Autism and Overcoming Job Barriers: Comparing Job-Related Barriers and Possible Solutions in and outside of Autism-Specific Employment. PLoS One. 2016;11(1):e0147040. doi: 10.1371/journal.pone.0147040. PMID: 26766183.X-1

5585. Lorenzo G, Lledó A, Pomares J, et al. Design and application of an immersive virtual reality system to enhance emotional skills for children with autism spectrum disorders.

Computers \& Education. 2016;98:192-205. doi: http://dx.doi.org/10.1016/j.compedu.2016.03.01 8.X-1

5586. Loring WA, Johnston R, Gray L, et al. A brief behavioral intervention for insomnia in adolescents with autism spectrum disorders. Clinical Practice in Pediatric Psychology. 2016;4(2):112-24. doi: http://dx.doi.org/10.1037/cpp0000141.X-1, X-3

5587. Loth E, Spooren W, Ham LM, et al. Identification and validation of biomarkers for autism spectrum disorders. Nat Rev Drug Discov. 2016 Jan;15(1):70-3. doi: 10.1038/nrd.2015.7. PMID: 26718285.X-1

5588. Lough E, Fisher MH. Parent and SelfReport Ratings on the Perceived Levels of Social Vulnerability of Adults with Williams Syndrome. J Autism Dev Disord. 2016 Aug 9doi: 10.1007/s10803-016-2885-3. PMID: 27506644.X-1

5589. Loukisas TD, Papoudi D. Mothers' experiences of children in the autistic spectrum in Greece: Narratives of development, education and disability across their blogs. International Journal of Disability, Development and Education. 2016;63(1):64-78. doi:

http://dx.doi.org/10.1080/1034912X.2015.1111 304.X-1

5590. Louros SR, Osterweil EK. Perturbed proteostasis in autism spectrum disorders. J Neurochem. 2016 Jul 1doi: 10.1111/jnc.13723. PMID: 27365114.X-1

5591. Loveall SJ, Channell MM, Phillips BA, et al. Receptive vocabulary analysis in Down syndrome. Research in Developmental Disabilities. 2016;55:161-72. doi: http://dx.doi.org/10.1016/j.ridd.2016.03.018.X1

5592. Lovelace JW, Wen TH, Reinhard S, et al. Matrix metalloproteinase-9 deletion rescues auditory evoked potential habituation deficit in a mouse model of Fragile X Syndrome.

Neurobiol Dis. 2016 May;89:126-35. doi: 10.1016/j.nbd.2016.02.002. PMID: 26850918.X-1

5593. Lovell B, Wetherell MA. Behaviour problems of children with ASD and perceived stress in their caregivers: The moderating role of trait emotional intelligence? Research in Autism Spectrum Disorders. 2016;28:1-6. doi: http://dx.doi.org/10.1016/j.rasd.2016.05.002.X1

5594. Loviglio MN, Leleu M, Mannik K, et al. Chromosomal contacts connect loci associated with autism, BMI and head circumference phenotypes. Mol Psychiatry. 2016 May 31doi: 10.1038/mp.2016.84. PMID: 27240531.X-1

5595. Lozano R, Saito N, Reed D, et al. Aging in Fragile X Premutation Carriers. Cerebellum. 2016 Jun 22doi: 10.1007/s12311016-0805-X. PMID: 27334385.X-1

5596. Lu C, Qi Z, Harris A, et al. Shared neuroanatomical substrates of impaired phonological working memory across reading disability and autism. Biol Psychiatry Cogn Neurosci Neuroimaging. 2016 Mar 1;1(2):16977. doi: 10.1016/j.bpsc.2015.11.001. PMID: 26949750.X-1 
5597. Luchsinger K, Lau H, Hedlund JL, et al. Parental-reported pain insensitivity in Dup15q. Epilepsy \& Behavior. 2016;55:124-7. doi:

http://dx.doi.org/10.1016/j.yebeh.2015.10.007. $\mathrm{X}-1$

5598. Ludlow AK, Wilkins AJ. Atypical

Sensory behaviours in children with Tourette's Syndrome and in children with Autism

Spectrum Disorders. Res Dev Disabil. 2016

Sep;56:108-16. doi:

10.1016/j.ridd.2016.05.019. PMID:

27286465.X-1

5599. Lushchekina EA, Khaerdinova OY, Novototskii-Vlasov VY, et al. Synchronization of EEG rhythms in baseline conditions and during counting in children with autism spectrum disorders. Neuroscience and Behavioral Physiology. 2016;46(4):382-9. doi: http://dx.doi.org/10.1007/s11055-016-0246-

5.X-1

5600. Luxford S, Hadwin JA, Kovshoff H. Evaluating the Effectiveness of a School-Based Cognitive Behavioural Therapy Intervention for Anxiety in Adolescents Diagnosed with Autism Spectrum Disorder. J Autism Dev Disord. 2016 Jul 20doi: 10.1007/s10803-016-2857-7. PMID: 27440250.X-1

5601. Lyons GL, Huber HB, Carter EW, et al. Assessing the Social Skills and Problem Behaviors of Adolescents With Severe Disabilities Enrolled in General Education Classes. Am J Intellect Dev Disabil. 2016 Jul;121(4):327-45. doi: 10.1352/1944-7558121.4.327. PMID: 27351700.X-1

5602. Ma NS, Thompson C, Weston S. Brief report: Scurvy as a manifestation of food selectivity in children with autism. Journal of Autism and Developmental Disorders. 2016;46(4):1464-70. doi: http://dx.doi.org/10.1007/s10803-015-2660$\mathrm{x} . \mathrm{X}-1$

5603. Mache MA, Todd TA. Gross motor skills are related to postural stability and age in children with autism spectrum disorder.

Research in Autism Spectrum Disorders. 2016;23:179-87. doi: http://dx.doi.org/10.1016/j.rasd.2016.01.001.X1
5604. Macizo P, Soriano MF, Paredes N. Phonological and Visuospatial Working Memory in Autism Spectrum Disorders. J Autism Dev Disord. 2016 Jun 17doi: 10.1007/s10803-016-2835-0. PMID: 27314268.X-1

5605. Macoun SJ, Kerns KA. Evidence of motor-control difficulties in children with attention deficit hyperactivity disorder, explored through a hierarchical motor-systems perspective. Journal of Clinical and Experimental Neuropsychology. 2016;38(2):183-96. doi: http://dx.doi.org/10.1080/13803395.2015.10940 28.X-1

5606. Maddox BB, Miyazaki Y, White SW. Long-Term Effects of CBT on Social Impairment in Adolescents with ASD. J Autism Dev Disord. 2016 Apr 4doi: 10.1007/s10803016-2779-4. PMID: 27040557.X-1, X-4

5607. Maddox BB, Trubanova A, White SW. Untended wounds: Non-suicidal self-injury in adults with autism spectrum disorder. Autism. 2016 May 12doi: 10.1177/1362361316644731. PMID: 27178994.X-1

5608. Magana S, Parish SL, Son E. Functional severity and Latino ethnicity in specialty services for children with autism spectrum disorder. J Intellect Disabil Res. 2016 May;60(5):424-34. doi: 10.1111/jir.12293. PMID: 27120986.X-1

5609. Magliaro C, Cocito C, Bagatella S, et al. The number of Purkinje neurons and their topology in the cerebellar vermis of normal and reln haplodeficient mouse. Ann Anat. 2016 Mar 17doi: 10.1016/j.aanat.2016.02.009. PMID: 26996540.X-1

5610. Magnúsdóttir K, Saemundsen E, Einarsson BL, et al. The impact of attention deficit/hyperactivity disorder on adaptive functioning in children diagnosed late with autism spectrum disorder-A comparative analysis. Research in Autism Spectrum Disorders. 2016;23:28-35. doi: http://dx.doi.org/10.1016/j.rasd.2015.11.012.X1

5611. Mahaj M, Sharkia R, Shorbaji N, et al. Clinical Profile of Attention Deficit Hyperactivity Disorder: Impact of Ethnic and 
Social Diversities in Israel. Isr Med Assoc J. 2016 Jun;18(6):322-5. PMID: 27468523.X-1

5612. Mahoney G, Solomon R. Mechanism of developmental change in the PLAY Project Home Consultation Intervention Program: Evidence from a randomized control trial. Journal of Autism and Developmental Disorders. 2016;46(5):1860-71. doi: http://dx.doi.org/10.1007/s10803-016-2720x.X-1

5613. Maïano C, Normand CL, Salvas MC, et al. Prevalence of school bullying among youth with autism spectrum disorders: A systematic review and meta-analysis. Autism Research. 2016;9(6):601-15. doi: http://dx.doi.org/10.1002/aur.1568.X-1

5614. Mailend ML, Plante E, Anderson MA, et al. Reliability of the Test of Integrated Language and Literacy Skills (TILLS). International Journal of Language \& Communication Disorders. 2016;51(4):447-59. doi: http://dx.doi.org/10.1111/14606984.12222.X-1

5615. Maillard AM, Hippolyte L, RodriguezHerreros B, et al. 16p11.2 Locus modulates response to satiety before the onset of obesity. Int J Obes (Lond). 2016 May;40(5):870-6. doi: 10.1038/ijo.2015.247. PMID: 26620891.X-1

5616. Maisel ME, Stephenson KG, South M, et al. Modeling the cognitive mechanisms linking autism symptoms and anxiety in adults. J Abnorm Psychol. 2016 Jul;125(5):692-703. doi: 10.1037/abn0000168. PMID: 27196436.X1

5617. Majdalany L, Wilder DA, Smeltz L, et al. The effect of brief delays to reinforcement on the acquisition of tacts in children with autism. J Appl Behav Anal. 2016 Jun;49(2):411-5. doi: 10.1002/jaba.282. PMID: 26792114.X-1, X-4

5618. Majoko T. Inclusion of children with autism spectrum disorders: Listening and hearing to voices from the grassroots. Journal of Autism and Developmental Disorders. 2016;46(4):1429-40. doi: http://dx.doi.org/10.1007/s10803-015-26851.X-1
5619. Malinverni L, Mora-Guiard J, Pares N. Towards methods for evaluating and communicating participatory design: A multimodal approach. International Journal of Human-Computer Studies. 2016;94:53-63. doi: http://dx.doi.org/10.1016/j.ijhcs.2016.03.004.X1

5620. Malow BA, Connolly HV, Weiss SK, et al. The Pediatric Sleep Clinical Global Impressions Scale-A new tool to measure pediatric insomnia in autism spectrum disorders. Journal of Developmental and Behavioral Pediatrics. 2016;37(5):370-6. doi: http://dx.doi.org/10.1097/DBP.0000000000000 307.X-1

5621. Malow BA, MacDonald LL, Fawkes $\mathrm{DB}$, et al. Teaching children with autism spectrum disorder how to sleep better: A pilot educational program for parents. Clinical Practice in Pediatric Psychology. 2016;4(2):125-36. doi: http://dx.doi.org/10.1037/cpp0000138.X-3

5622. Maltete D. Adult-onset stereotypical motor behaviors. Rev Neurol (Paris). 2016 Aug 3doi: 10.1016/j.neurol.2016.07.002. PMID: 27498241.X-1

5623. Mammas IN, Greenough A, Theodoridou M, et al. Current views and advances on Paediatric Virology: An update for paediatric trainees. Exp Ther Med. 2016 Jan;11(1):6-14. doi: 10.3892/etm.2015.2890. PMID: 26889211.X-1

5624. Mancil GR, Haydon T, Boman M. Differentiated effects of sensory activities as abolishing operations via non-contingent reinforcement on academic and aberrant behavior. Education and Training in Autism and Developmental Disabilities. 2016;51(1):93104.X-3, X-4

5625. Mandell DS, Barry CL, Marcus SC, et al. Effects of Autism Spectrum Disorder Insurance Mandates on the Treated Prevalence of Autism Spectrum Disorder. JAMA Pediatr. 2016 Jul 11doi:

10.1001/jamapediatrics.2016.1049. PMID: 27399053.X-1

5626. Manduca A, Servadio M, Damsteegt $\mathrm{R}$, et al. Dopaminergic Neurotransmission in the Nucleus Accumbens Modulates Social Play 
Behavior in Rats. Neuropsychopharmacology. 2016 Feb 10doi: 10.1038/npp.2016.22. PMID: 26860202.X-1

5627. Marchese M, Valvo G, Moro F, et al. Targeted gene resequencing (astrochip) to explore the tripartite synapse in autismepilepsy phenotype with macrocephaly. NeuroMolecular Medicine. 2016;18(1):69-80. doi: http://dx.doi.org/10.1007/s12017-0158378-2.X-1

5628. Marchetto MC, Belinson H, Tian Y, et al. Altered proliferation and networks in neural cells derived from idiopathic autistic individuals. Mol Psychiatry. 2016 Jul 5doi: 10.1038/mp.2016.95. PMID: 27378147.X-1

5629. Mariano M, Pino MC, Peretti S, et al. Understanding criminal behavior: Empathic impairment in criminal offenders. Soc Neurosci. 2016 May 9:1-7. doi: 10.1080/17470919.2016.1179670. PMID: 27108546.X-1

5630. Mari-Bauset S, Llopis-Gonzalez A, Zazpe I, et al. Comparison of nutritional status between children with autism spectrum disorder and typically developing children in the Mediterranean Region (Valencia, Spain). Autism. 2016 Apr 30doi: 10.1177/1362361316636976. PMID: 27132010.X-1

5631. Marko D, Boege F. A possible link between nutritional uptake of ubiquitous topoisomerase inhibitors and autism? Int J Dev Neurosci. 2016 Jun 11;53:8-9. doi: 10.1016/j.ijdevneu.2016.06.003. PMID: 27296517.X-1

5632. Marshall D, Wright B, Allgar V, et al. Social Stories in mainstream schools for children with autism spectrum disorder: a feasibility randomised controlled trial. BMJ Open. 2016;6(8):e011748. doi: 10.1136/bmjopen-2016-011748. PMID: 27515756.X-1

5633. Martin BS, Martinez-Botella G, Loya $\mathrm{CM}$, et al. Rescue of deficient amygdala tonic gamma-aminobutyric acidergic currents in the Fmr(-/y) mouse model of fragile X syndrome by a novel gamma-aminobutyric acid type $\mathrm{A}$ receptor-positive allosteric modulator. J
Neurosci Res. 2016 Jun;94(6):568-78. doi: 10.1002/jnr.23632. PMID: 26308557.X-1

5634. Martin S, Lazzarini M, Dullin C, et al. SK3 Channel Overexpression in Mice Causes Hippocampal Shrinkage Associated with Cognitive Impairments. Mol Neurobiol. 2016 Jan 23doi: 10.1007/s12035-015-9680-6. PMID: 26803493.X-1

5635. Martinez CK, Betz AM, Liddon CJ, et al. A progression to transfer RIRD to the natural environment. Behavioral Interventions. 2016;31(2):144-62. doi: http://dx.doi.org/10.1002/bin.1444.X-1

5636. Martin-Trias P, Bragulat V, PenaGomez C, et al. Translational challenge models in support of efficacy studies: Neurobehavioral and cognitive changes induced by Transcranial Magnetic Stimulation in healthy volunteers. CNS Neurol Disord Drug Targets. 2016 May 18 PMID: 27189466.X-1

5637. Mashayekhi F, Mizban N, Bidabadi E, et al. The association of SHANK3 gene polymorphism and autism. Minerva Pediatr. 2016 Jun 8 PMID: 27271042.X-1

5638. Mason LL, Davis D, Andrews A. Mentalistic explanations for autistic behavior: A behavioral phenomenological analysis. Behavior and Philosophy. 2016;43:62-84.X-1

5639. Masse JJ, McNeil CB, Wagner S, et al. Examining the efficacy of parent-child interaction therapy with children on the autism spectrum. Journal of Child and Family Studies. 2016;25(8):2508-25. doi: http://dx.doi.org/10.1007/s10826-016-04247.X-3, X-4

5640. Matson JL, Cervantes PE, Peters WJ. Autism spectrum disorders: management over the lifespan. Expert Rev Neurother. 2016 Jun 30:1-10. doi: 10.1080/14737175.2016.1203255. PMID: 27322023.X-1

5641. Matsushima K, Matsubayashi J, Toichi $\mathrm{M}$, et al. Unusual sensory features are related to resting-state cardiac vagus nerve activity in autism spectrum disorders. Research in Autism Spectrum Disorders. 2016;25:37-46. doi: http://dx.doi.org/10.1016/j.rasd.2015.12.006.X1 
5642. Mattila I, Patila T, Rautiainen P, et al. The effect of continuous wound infusion of ropivacaine on postoperative pain after median sternotomy and mediastinal drain in children. Paediatr Anaesth. 2016 Jul;26(7):727-33. doi: 10.1111/pan.12919. PMID: 27184591.X-1

5643. Maule J, Stanworth K, Pellicano E, et al. Color Afterimages in Autistic Adults. J Autism Dev Disord. 2016 Apr 28doi: 10.1007/s10803-016-2786-5. PMID: 27121352.X-1

5644. May T, Cornish K, Rinehart NJ. Exploring factors related to the anger superiority effect in children with Autism Spectrum Disorder. Brain and Cognition. 2016;106:65-71. doi: http://dx.doi.org/10.1016/j.bandc.2016.05.004. $\mathrm{X}-1, \mathrm{X}-3$

5645. May T, McGinley J, Murphy A, et al. A multidisciplinary perspective on motor impairment as an early behavioural marker in children with autism spectrum disorder. Australian Psychologist. 2016;51(4):296-303. doi: http://dx.doi.org/10.1111/ap.12225.X-1, X2

5646. Mayer JL, Hannent I, Heaton PF. Mapping the developmental trajectory and correlates of enhanced pitch perception on speech processing in adults with ASD. Journal of Autism and Developmental Disorders. 2016;46(5):1562-73. doi: http://dx.doi.org/10.1007/s10803-014-22076.X-1

5647. Maynard DW, McDonald TA, Stickle T. Parents as a team: Mother, father, a child with autism spectrum disorder, and a spinning toy. Journal of Autism and Developmental Disorders. 2016;46(2):406-23. doi: http://dx.doi.org/10.1007/s10803-015-25685.X-1, X-3, X-4

5648. Mazahery H, Conlon C, Beck KL, et al. Vitamin D and omega-3 fatty acid supplements in children with autism spectrum disorder: a study protocol for a factorial randomised, double-blind, placebo-controlled trial. Trials. 2016;17(1):295. doi: 10.1186/s13063-016-1428-8. PMID: 27334138.X-1
5649. Mazurek MO, Brown R, Curran A, et al. ECHO Autism: A New Model for Training Primary Care Providers in Best-Practice Care for Children With Autism. Clin Pediatr (Phila). 2016 May 11doi: 10.1177/0009922816648288. PMID: 27169714.X-1

5650. Mazurek MO, Engelhardt CR, Hilgard $\mathrm{J}$, et al. Bedtime Electronic Media Use and Sleep in Children with Autism Spectrum Disorder. J Dev Behav Pediatr. 2016 Jun 28doi: 10.1097/dbp.0000000000000314. PMID: 27355885.X-1, X-3

5651. Mazurek MO, Sohl K. Sleep and behavioral problems in children with autism spectrum disorder. Journal of Autism and Developmental Disorders. 2016;46(6):1906-15. doi: http://dx.doi.org/10.1007/s10803-0162723-7.X-1

5652. McCarthy A, Asghar S, Wilens T, et al. Using a brief parent-report measure to track outcomes for children and teens with ADHD. Child Psychiatry and Human Development. 2016;47(3):407-16. doi: http://dx.doi.org/10.1007/s10578-015-05756.X-1

5653. McClendon J, Jr., Graham RB, Sugrue PA, et al. Cranial Center of Mass Compared to C7 Plumb Line Alignment in Adult Spinal Deformity. World Neurosurg. 2016 Mar 30doi: 10.1016/j.wneu.2016.03.078. PMID: 27046014.X-1

5654. McConkey R, Kelly C, Craig S, et al. A decade of change in mainstream education for children with intellectual disabilities in the Republic of Ireland. European Journal of Special Needs Education. 2016;31(1):96-110. doi: http://dx.doi.org/10.1080/08856257.2015.10871 51.X-1

5655. McCormick C, Hepburn S, Young GS, et al. Sensory symptoms in children with autism spectrum disorder, other developmental disorders and typical development: A longitudinal study. Autism. $2016 \mathrm{Jul} ; 20$ (5):5729. doi: 10.1177/1362361315599755. PMID: 26395236.X-1

5656. McCoy SM, Jakicic JM, Gibbs BB. Comparison of obesity, physical activity, and sedentary behaviors between adolescents with 
autism spectrum disorders and without. Journal of Autism and Developmental Disorders. 2016;46(7):2317-26. doi: http://dx.doi.org/10.1007/s10803-016-2762$0 . \mathrm{X}-1$

5657. McDaniels J. Family Advocates for Parents With Children With an Autism Spectrum Disorder. A Letter to the Editor on the Article "Parent Perceptions of Care Received by Children With an Autism Spectrum Disorder". J Pediatr Nurs. 2016 May 9doi: 10.1016/j.pedn.2016.04.005. PMID: 27174784.X-1

5658. McDonald NM, Baker JK, Messinger DS. Oxytocin and parent-child interaction in the development of empathy among children at risk for autism. Developmental Psychology. 2016;52(5):735-45. doi: http://dx.doi.org/10.1037/dev0000104.X-1

5659. McDougall J, Baldwin P, Evans J, et al. Quality of life and self-determination: Youth with chronic health conditions make the connection. Applied Research in Quality of Life. 2016;11(2):571-99. doi: http://dx.doi.org/10.1007/s11482-014-93827.X-1

5660. McEwen FS, Stewart CS, Colvert E, et al. Diagnosing autism spectrum disorder in community settings using the Development and Well-Being Assessment: Validation in a UK population-based twin sample. Journal of Child Psychology and Psychiatry. 2016;57(2):161-70. doi: http://dx.doi.org/10.1111/jcpp.12447.X-1

5661. McGrane IR, Loveland JG, Zaluski HJ. Adjunctive Amantadine Treatment for Aggressive Behavior in Children: A Series of Eight Cases. J Child Adolesc Psychopharmacol. 2016 Aug 2doi: 10.1089/cap.2016.0042. PMID: 27483360.X-1

5662. McGrath LM, Braaten EB, Doty ND, et al. Extending the 'cross-disorder' relevance of executive functions to dimensional neuropsychiatric traits in youth. Journal of Child Psychology and Psychiatry.

2016;57(4):462-71. doi:

http://dx.doi.org/10.1111/jcpp.12463.X-1

5663. McGrath LM, Oates JM, Dai YG, et al. Attention Bias to Emotional Faces Varies by IQ and Anxiety in Williams Syndrome. J Autism
Dev Disord. 2016 Jun;46(6):2174-85. doi: 10.1007/s10803-016-2748-y. PMID: 26886469.X-1

5664. McHale P, Keenan A, Ghebrehewet S. Reasons for measles cases not being vaccinated with MMR: investigation into parents' and carers' views following a large measles outbreak. Epidemiol Infect. 2016

Mar;144(4):870-5. doi: 10.1017/s0950268815001909. PMID: 26265115.X-1

5665. McKenna C. Review of Autism spectrum disorder in children and adolescents: Evidence-based assessment and intervention in schools. Child and Adolescent Mental Health. 2016;21(2):136. doi: http://dx.doi.org/10.1111/camh.12135.X-1, X-2

5666. McKenzie K, Forsyth K, O’Hare A, et al. The relationship between waiting times and 'adherence' to the Scottish Intercollegiate Guidelines Network 98 guideline in autism spectrum disorder diagnostic services in Scotland. Autism. 2016;20(4):395-401. doi: http://dx.doi.org/10.1177/1362361315586136.X $-1$

5667. McKenzie K, Ouellette-Kuntz H, Blinkhorn A, et al. Out of School and Into Distress: Families of Young Adults with Intellectual and Developmental Disabilities in Transition. J Appl Res Intellect Disabil. 2016 Jun 9doi: 10.1111/jar.12264. PMID: 27276996.X-1

5668. McKinnon A, Brewer N, MeiserStedman R, et al. Trauma memory characteristics and the development of acute stress disorder and post-traumatic stress disorder in youth. J Behav Ther Exp Psychiatry. 2016 Jul 22;54:112-9. doi: 10.1016/j.jbtep.2016.07.009. PMID: 27467024.X-1

5669. McKnight LM, O'Malley-Keighran MP, Carroll C. 'Just wait then and see what he does': a speech act analysis of healthcare professionals' interaction coaching with parents of children with autism spectrum disorders. Int J Lang Commun Disord. 2016 Jun 9doi: 10.1111/1460-6984.12246. PMID: 27277838.X-1 
5670. McLay L, Church J, Sutherland D. Variables affecting the emergence of untaught equivalence relations in children with and without autism. Developmental Neurorehabilitation. 2016;19(2):75-87.X-1, X-3

5671. McMahon CM, Henderson HA, Newell L, et al. Metacognitive awareness of facial affect in higher-functioning children and adolescents with autism spectrum disorder. Journal of Autism and Developmental Disorders. 2016;46(3):882-98. doi: http://dx.doi.org/10.1007/s10803-015-26303.X-1

5672. McManus BM, Robinson CC, Rosenberg SA. Identifying infants and toddlers at high risk for persistent delays. Maternal and Child Health Journal. 2016;20(3):639-45. doi: http://dx.doi.org/10.1007/s10995-015-18632.X-1

5673. Meador KJ, Loring DW. Developmental effects of antiepileptic drugs and the need for improved regulations. Neurology. 2016 Jan 19;86(3):297-306. doi: 10.1212/wnl.0000000000002119. PMID: 26519545.X-1

5674. Medhasi S, Pasomsub E, Vanwong N, et al. Clinically relevant genetic variants of drug-metabolizing enzyme and transporter genes detected in Thai children and adolescents with autism spectrum disorder.

Neuropsychiatric Disease and Treatment. 2016;12.X-1

5675. Mei Y, Monteiro P, Zhou Y, et al. Adult restoration of Shank3 expression rescues selective autistic-like phenotypes. Nature. 2016 Feb 25;530(7591):481-4. doi:

10.1038/nature16971. PMID: 26886798.X-1

5676. Meisel HJ, Jurak L, Antinheimo J, et al. Four-year results of a prospective single-arm study on 200 semi-constrained total cervical disc prostheses: clinical and radiographic outcome. J Neurosurg Spine. 2016 Jun 3:1-10. doi: 10.3171/2016.3.spine15810. PMID: 27258476.X-1

5677. Menassa DA, Sloan C, Chance SA. Primary olfactory cortex in autism and epilepsy: Increased glial cells in autism. Brain Pathol. 2016 Jul 13doi: 10.1111/bpa.12415. PMID: 27409070.X-1
5678. Mendelson JL, Gates JA, Lerner MD. Friendship in school-age boys with autism spectrum disorders: A meta-analytic summary and developmental, process-based model. Psychological Bulletin. 2016;142(6):601-22. doi: http://dx.doi.org/10.1037/bul0000041.X-1, $\mathrm{X}-2$

5679. Meng WD, Sun SJ, Yang J, et al. Elevated Serum Brain-Derived Neurotrophic Factor (BDNF) but not BDNF Gene Val66Met Polymorphism Is Associated with Autism Spectrum Disorders. Mol Neurobiol. 2016 Jan 28doi: 10.1007/s12035-016-9721-9. PMID: 26820673.X-1

5680. Meng X, Wang W, Lu H, et al. Manipulations of MeCP2 in glutamatergic neurons highlight their contributions to Rett and other neurological disorders. Elife. 2016 Jun 21;5doi: 10.7554/eLife.14199. PMID: 27328325.X-1

5681. Menga EN, Spiegel MA, Vira S, et al. Variability Over Time of Preoperative Sagittal Alignment Parameters: Radiographic and Clinical Considerations. Spine (Phila Pa 1976). 2016 Apr 23doi: 10.1097/brs.0000000000001648. PMID: 27120056.X-1

5682. Meppelink R, de Bruin EI, Bögels SM. Meditation or Medication? Mindfulness training versus medication in the treatment of childhood ADHD: A randomized controlled trial. BMC Psychiatry. 2016;16.X-1

5683. Mercado E, 3rd, Church BA. Brief Report: Simulations Suggest Heterogeneous Category Learning and Generalization in Children with Autism is a Result of Idiosyncratic Perceptual Transformations. J Autism Dev Disord. 2016 May 18doi: 10.1007/s10803-016-2815-4. PMID: 27193184.X-1

5684. Mercati O, Huguet G, Danckaert A, et al. CNTN6 mutations are risk factors for abnormal auditory sensory perception in autism spectrum disorders. Mol Psychiatry. 2016 May 10doi: 10.1038/mp.2016.61. PMID: 27166760.X-1

5685. Merner N, Forgeot d'Arc B, Bell SC, et al. A de novo frameshift mutation in 
chromodomain helicase DNA-binding domain 8 (CHD8): A case report and literature review.

Am J Med Genet A. 2016 May;170(5):1225-35. doi: 10.1002/ajmg.a.37566. PMID:

26789910.X-1

5686. Merner ND, Mercado A, Khanna AR, et al. Gain-of-function missense variant in SLC12A2, encoding the bumetanide-sensitive NKCC1 cotransporter, identified in human schizophrenia. J Psychiatr Res. 2016 Jun;77:226. doi: 10.1016/j.jpsychires.2016.02.016. PMID: 26955005.X-1

5687. Merrick AD, Grieve A, Cogan N. Psychological impacts of challenging behaviour and motivational orientation in staff supporting individuals with autistic spectrum conditions. Autism. 2016 Jun 26doi: 10.1177/1362361316654857. PMID: 27354430.X-1

5688. Mevel K, Fransson P. The functional brain connectome of the child and autism spectrum disorders. Acta Paediatr. 2016 May 26doi: 10.1111/apa.13484. PMID: 27228241.X1

5689. Meziane H, Khelfaoui M, Morello N, et al. Fasudil treatment in adult reverses behavioural changes and brain ventricular enlargement in Oligophrenin-1 mouse model of intellectual disability. Hum Mol Genet. 2016 May 4doi: 10.1093/hmg/ddw102. PMID: 27146843.X-1

5690. Mhatre D, Bapat D, Udani V. Longterm outcomes in children diagnosed with autism spectrum disorders in India. Journal of Autism and Developmental Disorders. 2016;46(3):760-72. doi: http://dx.doi.org/10.1007/s10803-015-26134.X-1

5691. Micalizzi L, Ronald A, Saudino KJ. A genetically informed cross-lagged analysis of autistic-like traits and affective problems in early childhood. Journal of Abnormal Child Psychology. 2016;44(5):937-47. doi: http://dx.doi.org/10.1007/s10802-015-00886.X-1

5692. Michna I, Trestman R. Correctional Management and Treatment of Autism Spectrum Disorder. J Am Acad Psychiatry Law. 2016 Jun;44(2):253-8. PMID: 27236182.X-1
5693. Mieses AM, Tavassoli T, Li E, et al. Brief Report: Sensory Reactivity in Children with Phelan-McDermid Syndrome. J Autism Dev Disord. 2016 Jul;46(7):2508-13. doi: 10.1007/s10803-016-2754-0. PMID: 26914612.X-1

5694. Mieses AM, Tavassoli T, Li E, et al. Brief report: Sensory reactivity in children with Phelan-McDermid syndrome. Journal of Autism and Developmental Disorders. 2016;46(7):2508-13. doi: http://dx.doi.org/10.1007/s10803-016-2754$0 . \mathrm{X}-1$

5695. Mihic T, Rainkie D, Wilby KJ, et al. The Therapeutic Effects of Camel Milk: A Systematic Review of Animal and Human Trials. J Evid Based Complementary Altern Med. 2016 Jul 18doi: 10.1177/2156587216658846. PMID: 27432772.X-1, X-2

5696. Miller M, Iosif AM, Young GS, et al. School-age outcomes of infants at risk for autism spectrum disorder. Autism Research. 2016;9(6):632-42. doi: http://dx.doi.org/10.1002/aur.1572.X-1

5697. Miller NA, Merryman MB, Eskow $\mathrm{KG}$, et al. State Design and Use of Medicaid 1915(c) Waivers and Related Benefits to Provide Services to Children and Youth With Autism Spectrum Disorder. Am J Intellect Dev Disabil. 2016 Jul;121(4):295-311. doi: 10.1352/1944-7558-121.4.295. PMID: 27351698.X-1

5698. Mills BD, Pearce HL, Khan O, et al. Prenatal domoic acid exposure disrupts mouse pro-social behavior and functional connectivity MRI. Behav Brain Res. 2016 Jul 15;308:14-23. doi: 10.1016/j.bbr.2016.03.039. PMID: 27050322.X-1

5699. Milosavljevic B, Carter Leno V, Simonoff E, et al. Alexithymia in adolescents with autism spectrum disorder: Its relationship to internalising difficulties, sensory modulation and social cognition. Journal of Autism and Developmental Disorders. 2016;46(4):1354-67. doi: http://dx.doi.org/10.1007/s10803-0152670-8.X-1 
5700. Ming X, Patel R, Kang V, et al. Respiratory and autonomic dysfunction in children with autism spectrum disorders. Brain \& Development. 2016;38(2):225-32. doi: http://dx.doi.org/10.1016/j.braindev.2015.07.00 3.X-1

5701. Minshawi NF, Wink LK, Shaffer R, et al. A randomized, placebo-controlled trial of Dcycloserine for the enhancement of social skills training in autism spectrum disorders. Mol Autism. 2016;7:2. doi: 10.1186/s13229-0150062-8. PMID: 26770664.X-1

5702. Mintz M. Evolution in the Understanding of Autism Spectrum Disorder: Historical Perspective. Indian J Pediatr. 2016 Apr 7doi: 10.1007/s12098-016-2080-8. PMID: 27053182.X-1, X-2, X-3

5703. Mitra A, Zhu W, Kesisoglou F. Physiologically Based Absorption Modeling for Amorphous Solid Dispersion Formulations. Mol Pharm. 2016 Jul 29doi: 10.1021/acs.molpharmaceut.6b00424. PMID: 27442959.X-1

5704. Miyajima M, Omiya H, Yamashita K, et al. The effects of cognitive remediation therapy using the frontal/executive program for autism spectrum disorder. Int J Psychiatry Med. 2016 Apr;51(3):223-35. doi: 10.1177/0091217416651254. PMID: 27284115.X-1, X-4

5705. Miyawaki D, Iwakura Y, Seto T, et al. Psychogenic nonepileptic seizures as a manifestation of psychological distress associated with undiagnosed autism spectrum disorder. Neuropsychiatric Disease and Treatment. 2016;12.X-1

5706. Mizukami T, Kohno T, Hattori M. CUB and Sushi multiple domains 3 regulates dendrite development. Neurosci Res. 2016 Mar 28doi: 10.1016/j.neures.2016.03.003. PMID: 27033969.X-1

5707. Moavero R, Napolitano A, Cusmai R, et al. White matter disruption is associated with persistent seizures in tuberous sclerosis complex. Epilepsy \& Behavior. 2016;60:63-7. doi: http://dx.doi.org/10.1016/j.yebeh.2016.04.026. $\mathrm{X}-1$
5708. Modabbernia A, Mollon J, Boffetta P, et al. Impaired gas exchange at birth and risk of intellectual disability and autism: A metaanalysis. Journal of Autism and Developmental Disorders. 2016;46(5):1847-59. doi: http://dx.doi.org/10.1007/s10803-016-27175.X-1

5709. Modabbernia A, Velthorst E, Gennings $\mathrm{C}$, et al. Early-life metal exposure and schizophrenia: A proof-of-concept study using novel tooth-matrix biomarkers. Eur Psychiatry. 2016 Jun 13;36:1-6. doi: 10.1016/j.eurpsy.2016.03.006. PMID: 27311101.X-1

5710. Modi ME, Majchrzak MJ, Fonseca KR, et al. Peripheral Administration of a LongActing Peptide Oxytocin Receptor Agonist Inhibits Fear-Induced Freezing. J Pharmacol Exp Ther. 2016 Aug;358(2):164-72. doi: 10.1124/jpet.116.232702. PMID: 27217590.X1

5711. Mohamed FE, Zaky EA, Youssef A, et al. Screening of Egyptian toddlers for autism spectrum disorder using an Arabic validated version of M-CHAT; report of a communitybased study (Stage I). European Psychiatry. 2016;34:43-8. doi: http://dx.doi.org/10.1016/j.eurpsy.2016.01.2421 .X-1

5712. Mohammad-Rezazadeh I, Frohlich J, Loo SK, et al. Brain connectivity in autism spectrum disorder. Curr Opin Neurol. 2016 Apr;29(2):137-47. doi: 10.1097/wco.0000000000000301. PMID: 26910484.X-1

5713. Mohr-Jensen C, Koch SV, Lauritsen MB, et al. The validity and reliability of the diagnosis of hyperkinetic disorders in the Danish Psychiatric Central Research Registry. European Psychiatry. 2016;35:16-24. doi: http://dx.doi.org/10.1016/j.eurpsy.2016.01.2427 .X-1

5714. Momen S. Special care dentistry: Treating autistic children. Br Dent J. 2016 Feb 12;220(3):89. doi: 10.1038/sj.bdj.2016.68. PMID: 26868774.X-1

5715. Montagna MT, Cristina ML, Giglio OD, et al. Serological and molecular identification of Legionella spp. isolated from 
water and surrounding air samples in Italian healthcare facilities. Environ Res. 2016

Apr;146:47-50. doi:

10.1016/j.envres.2015.12.015. PMID: 26717079.X-1

5716. Monteiro SA, Dempsey J, Broton S, et al. Early intervention before autism diagnosis in children referred to a regional autism clinic. Journal of Developmental and Behavioral Pediatrics. 2016;37(1):15-9. doi: http://dx.doi.org/10.1097/DBP.0000000000000 241.X-1

5717. Monyak RE, Emerson D, Schoenfeld $\mathrm{BP}$, et al. Insulin signaling misregulation underlies circadian and cognitive deficits in a Drosophila fragile X model. Mol Psychiatry. 2016 Apr 19doi: 10.1038/mp.2016.51. PMID: 27090306.X-1

5718. Moore RD, Pindus DM, Raine LB, et al. The persistent influence of concussion on attention, executive control and neuroelectric function in preadolescent children. International Journal of Psychophysiology. 2016;99:85-95. doi:

http://dx.doi.org/10.1016/j.ijpsycho.2015.11.01 $0 . \mathrm{X}-1$

5719. Moos WH, Faller DV, Harpp DN, et al. Microbiota and Neurological Disorders: A Gut Feeling. Biores Open Access. 2016;5(1):137-45. doi: 10.1089/biores.2016.0010. PMID: 27274912.X1

5720. Moraru C-A, Gomez J-C, McGuigan $\mathrm{N}$. Developmental changes in the influence of conventional and instrumental cues on overimitation in 3- to 6-year-old children. Journal of Experimental Child Psychology. 2016;145:3447. doi: http://dx.doi.org/10.1016/j.jecp.2015.11.017.X1

5721. Morava E, Tiemes V, Tiel C, et al. ALG6-CDG: a recognizable phenotype with epilepsy, proximal muscle weakness, ataxia and behavioral and limb anomalies. J Inherit Metab Dis. 2016 Jun 10doi: 10.1007/s10545-0169945-x. PMID: 27287710.X-1

5722. Moreau PE, Ferrero E, Riouallon G, et al. Radiologic adjacent segment degeneration 2 years after lumbar fusion for degenerative spondylolisthesis. Orthop Traumatol Surg Res. 2016 Jun 1doi: 10.1016/j.otsr.2016.03.012.

PMID: 27262830.X-1

5723. Moreira ES, Silva IMW, Lourenço N, et al. Detection of small copy number variations (CNVs) in autism spectrum disorder (ASD) by custom array comparative genomic hybridization (aCGH). Research in Autism Spectrum Disorders. 2016;23:145-51. doi: http://dx.doi.org/10.1016/j.rasd.2015.12.012.X1

5724. Morett LM, O’Hearn K, Luna B, et al. Altered gesture and speech production in ASD detract from in-person communicative quality. Journal of Autism and Developmental Disorders. 2016;46(3):998-1012. doi: http://dx.doi.org/10.1007/s10803-015-26459.X-1

5725. Morris G, Berk M, Carvalho A, et al. The Role of the Microbial Metabolites Including Tryptophan Catabolites and Short Chain Fatty Acids in the Pathophysiology of Immune-Inflammatory and Neuroimmune Disease. Mol Neurobiol. 2016 Jun 27doi: 10.1007/s12035-016-0004-2. PMID: 27349436.X-1

5726. Mostafa GA, Bjorklund G, Urbina MA, et al. The positive association between elevated blood lead levels and brain-specific autoantibodies in autistic children from low lead-polluted areas. Metab Brain Dis. 2016 Jun 1doi: 10.1007/s11011-016-9836-8. PMID: 27250967.X-1

5727. Mostafa GA, Bjorklund G, Urbina MA, et al. The levels of blood mercury and inflammatory-related neuropeptides in the serum are correlated in children with autism spectrum disorder. Metab Brain Dis. 2016 Jun;31(3):593-9. doi: 10.1007/s11011-0159784-8. PMID: 26738726.X-1

5728. Mostafavi M, Hardy P, Arnold LE. Varenicline in Autism: Theory and Case Report of Clinical and Biochemical Changes. J Child Adolesc Psychopharmacol. 2016 Apr 28doi: 10.1089/cap.2015.0230. PMID: 27123827.X-1

5729. Mouga S, Cafe C, Almeida J, et al. Intellectual Profiles in the Autism Spectrum and Other Neurodevelopmental Disorders. J Autism 
Dev Disord. 2016 Jun 16doi: 10.1007/s10803016-2838-x. PMID: 27312715.X-1

5730. Moulton E, Barton M, Robins DL, et al. Early characteristics of children with ASD who demonstrate optimal progress between age two and four. Journal of Autism and Developmental Disorders. 2016;46(6):2160-73. doi: http://dx.doi.org/10.1007/s10803-0162745-1.X-1

5731. Mouridsen SE, Rich B, Isager T. Risk of cancer in adult people diagnosed with infantile autism in childhood: A longitudinal case control study based on hospital discharge diagnoses. Research in Autism Spectrum Disorders. 2016;23:203-9. doi: http://dx.doi.org/10.1016/j.rasd.2015.12.010.X1

5732. Mucke HA. From Psychiatry to Flower Power and Back Again: The Amazing Story of Lysergic Acid Diethylamide. Assay Drug Dev Technol. 2016 Jul 8doi: 10.1089/adt.2016.747. PMID: 27392130.X-1

5733. Mulhern T, Lydon S, Healy O, et al. A systematic review and evaluation of procedures for the induction of speech among persons with developmental disabilities. Dev Neurorehabil. 2016 Apr 8:1-21. doi:

10.3109/17518423.2016.1150360. PMID:

27058303.X-1, X-2

5734. Muller E, Cannon LR, Kornblum C, et al. Description and Preliminary Evaluation of a Curriculum for Teaching Conversational Skills to Children With High-Functioning Autism and Other Social Cognition Challenges. Lang Speech Hear Serv Sch. 2016 Jun 8:1-18. doi: 10.1044/2016_lshss-15-0042. PMID: 27276535.X-1

5735. Muller N, Baumeister S, Dziobek I, et al. Validation of the Movie for the Assessment of Social Cognition in Adolescents with ASD: Fixation Duration and Pupil Dilation as Predictors of Performance. J Autism Dev Disord. 2016 Jun 6doi: 10.1007/s10803-0162828-z. PMID: 27271932.X-1

5736. Muller-Pinzler L, Krach S, Kramer UM, et al. The Social Neuroscience of Interpersonal Emotions. Curr Top Behav Neurosci. 2016 Mar 6doi:
10.1007/7854_2016_437. PMID: 26946503.X$1, \mathrm{X}-2$

5737. Munde V, Vlaskamp C, ter Haar A. Social-emotional instability in individuals with Rett syndrome: Parents' experiences with second stage behaviour. Journal of Intellectual Disability Research. 2016;60(1):43-53. doi: http://dx.doi.org/10.1111/jir.12233.X-1

5738. Mundy P, Kim K, McIntyre N, et al. Brief report: Joint attention and information processing in children with higher functioning autism spectrum disorders. Journal of Autism and Developmental Disorders.

2016;46(7):2555-60. doi: http://dx.doi.org/10.1007/s10803-016-27856.X-1, X-3

5739. Muotri AR. The Human Model: Changing Focus on Autism Research. Biol Psychiatry. 2016 Apr 15;79(8):642-9. doi: 10.1016/j.biopsych.2015.03.012. PMID: 25861701.X-1

5740. Murakami T, Nakazawa G, Horinouchi $\mathrm{H}$, et al. Transcatheter closure of atrial septal defect protects from pulmonary edema: septal occluder device gradually reduces LR shunt. Heart Vessels. 2016 Jun 17doi: 10.1007/s00380-016-0863-5. PMID: 27314266.X-1

5741. Murala J, Sassalos P, Si MS. 'Near death' thromboembolic episode following device closure of atrial septal defect. Interact Cardiovasc Thorac Surg. 2016 May 14doi: 10.1093/icvts/ivw145. PMID: 27179351.X-1

5742. Murphy CM, Wilson CE, Robertson $\mathrm{DM}$, et al. Autism spectrum disorder in adults: diagnosis, management, and health services development. Neuropsychiatr Dis Treat. 2016;12:1669-86. doi: 10.2147/ndt.s65455. PMID: 27462160.X-1

5743. Murphy J, Zlomke KR. A behavioral parent-training intervention for a child with avoidant/restrictive food intake disorder. Clinical Practice in Pediatric Psychology. 2016;4(1):23-34. doi: http://dx.doi.org/10.1037/cpp0000128.X-1

5744. Murza KA, Schwartz JB, HahsVaughn DL, et al. Joint attention interventions for children with autism spectrum disorder: A 
systematic review and meta-analysis. International Journal of Language \& Communication Disorders. 2016;51(3):236-51. doi: http://dx.doi.org/10.1111/14606984.12212.X-2

5745. Muscogiuri G, Altieri B, Annweiler C, et al. Vitamin D and chronic diseases: the current state of the art. Arch Toxicol. 2016 Jul 18doi: 10.1007/s00204-016-1804-X. PMID: 27425218.X-1

5746. Muskat B, Greenblatt A, Nicholas DB, et al. Parent and health care provider perspectives related to disclosure of autism spectrum disorder in pediatric emergency departments. Autism. 2016 Feb 5doi: 10.1177/1362361315621520. PMID: 26851228.X-1

5747. Mussap M, Noto A, Fanos V. Metabolomics of autism spectrum disorders: early insights regarding mammalian-microbial cometabolites. Expert Rev Mol Diagn. 2016 Jun 30:1-13. doi: 10.1080/14737159.2016.1202765. PMID: 27310602.X-1

5748. Muszkat D, Polanczyk GV, Dias TG, et al. Transcranial Direct Current Stimulation in Child and Adolescent Psychiatry. J Child Adolesc Psychopharmacol. 2016 Mar 30doi: 10.1089/cap.2015.0172. PMID: 27027666.X-2, $\mathrm{X}-3$

5749. Muzio L, Brambilla V, Calcaterra L, et al. Increased neuroplasticity and hippocampal microglia activation in a mice model of rapid antidepressant treatment. Behav Brain Res. 2016 Sep 15;311:392-402. doi: 10.1016/j.bbr.2016.05.063. PMID: 27265783.X-1

5750. Mychasiuk R, Rho JM. Genetic modifications associated with ketogenic diet treatment in the BTBRT+Tf/J mouse model of autism spectrum disorder. Autism Res. 2016 Aug 16doi: 10.1002/aur.1682. PMID: 27529337.X-1

5751. Nader A-M, Courchesne V, Dawson $\mathrm{M}$, et al. Does WISC-IV underestimate the intelligence of autistic children? Journal of Autism and Developmental Disorders. 2016;46(5):1582-9. doi: http://dx.doi.org/10.1007/s10803-014-2270z.X-1
5752. Nadim WD, Simion V, Benedetti H, et al. MicroRNAs in Neurocognitive Dysfunctions: New Molecular Targets for Pharmacological Treatments? Curr Neuropharmacol. 2016 Jul 8 PMID: 27396304.X-1

5753. Nagra MK, White R, Appiah A, et al. Intensive Interaction Training for Paid Carers: 'Looking, Looking and Find Out When They Want to Relate to You'. J Appl Res Intellect Disabil. 2016 Jun 8doi: 10.1111/jar.12259. PMID: 27279387.X-1

5754. Nahum Sacks K, Friger M, ShohamVardi I, et al. Prenatal exposure to gestational diabetes mellitus as an independent risk factor for long-term neuropsychiatric morbidity of the offspring. Am J Obstet Gynecol. 2016 Mar 24doi: 10.1016/j.ajog.2016.03.030. PMID: 27018463.X-1

5755. Naigles LR, Cheng M, Rattansone NX, et al. "You're telling me!" The Prevalence and Predictors of Pronoun Reversals in Children with Autism Spectrum Disorders and Typical Development. Res Autism Spectr Disord. 2016 Jul;27:11-20. doi: 10.1016/j.rasd.2016.03.008. PMID: 27103941.X-1

5756. Nakagawa Y, Chiba K. Involvement of neuroinflammation during brain development in social cognitive deficits in autism spectrum disorder and schizophrenia. J Pharmacol Exp Ther. 2016 Jul 6doi: 10.1124/jpet.116.234476. PMID: 27384073.X-1

5757. Napoli E, Song G, Wong S, et al. Altered Bioenergetics in Primary Dermal Fibroblasts from Adult Carriers of the FMR1 Premutation Before the Onset of the Neurodegenerative Disease Fragile XAssociated Tremor/Ataxia Syndrome. Cerebellum. 2016 Apr 18doi: 10.1007/s12311016-0779-8. PMID: 27089882.X-1

5758. Narita S, Nagahori K, Nishizawa D, et al. Association between AUTS2 haplotypes and alcohol dependence in a Japanese population. Acta Neuropsychiatr. 2016 Aug;28(4):214-20. doi: 10.1017/neu.2015.70. PMID: 26763194.X1

5759. Nassif M, Abdelghani M, Bouma BJ, et al. Historical developments of atrial septal 
defect closure devices: what we learn from the past. Expert Rev Med Devices. 2016

Jun;13(6):555-68. doi:

10.1080/17434440.2016.1182860. PMID:

27112301.X-1

5760. Navon D, Eyal G. Looping Genomes: Diagnostic Change and the Genetic Makeup of the Autism Population. Ajs. 2016

Mar;121(5):1416-71. PMID: 27092389.X-1

5761. Navot N, Jorgenson AG, Vander Stoep A, et al. Family planning and family vision in mothers after diagnosis of a child with autism spectrum disorder. Autism. 2016 Jul;20(5):60515. doi: 10.1177/1362361315602134. PMID: 26395237.X-1

5762. Nebel MB, Eloyan A, Nettles CA, et al. Intrinsic visual-motor synchrony correlates with social deficits in autism. Biological Psychiatry. 2016;79(8):633-41. doi: http://dx.doi.org/10.1016/j.biopsych.2015.08.02 9.X-1

5763. Neely KA, Mohanty S, Schmitt LM, et al. Motor Memory Deficits Contribute to Motor Impairments in Autism Spectrum Disorder. J Autism Dev Disord. 2016 May 7doi: 10.1007/s10803-016-2806-5. PMID: 27155985.X-1

5764. Neil L, Cappagli G, Karaminis T, et al. Recognizing the same face in different contexts: Testing within-person face recognition in typical development and in autism. Journal of Experimental Child Psychology. 2016;143:13953. doi: http://dx.doi.org/10.1016/j.jecp.2015.09.029.X1

5765. Neil L, Olsson NC, Pellicano E. The Relationship Between Intolerance of Uncertainty, Sensory Sensitivities, and Anxiety in Autistic and Typically Developing Children. J Autism Dev Disord. 2016 Jun;46(6):1962-73. doi: 10.1007/s10803-016-2721-9. PMID: 26864157.X-1

5766. Nelson AT, Lopata C, Volker MA, et al. Exploratory Factor Analysis of SRS-2 Teacher Ratings for Youth with ASD. J Autism Dev Disord. 2016 Jun 22doi: 10.1007/s10803016-2822-5. PMID: 27334872.X-1
5767. Nestor MW, Phillips AW, Artimovich E, et al. Human Inducible Pluripotent Stem Cells and Autism Spectrum Disorder: Emerging Technologies. Autism Res. 2016 May;9(5):51335. doi: 10.1002/aur.1570. PMID: 26426199.X1

5768. Nesvag R, Hartz I, Bramness JG, et al. Mental disorder diagnoses among children and adolescents who use antipsychotic drugs. Eur Neuropsychopharmacol. 2016 Jul 21doi: 10.1016/j.euroneuro.2016.07.001. PMID: 27452144.X-1

5769. Neuhaus E, Jones EJH, Barnes K, et al. The relationship between early neural responses to emotional faces at age 3 and later autism and anxiety symptoms in adolescents with autism. Journal of Autism and Developmental Disorders. 2016;46(7):2450-63. doi: http://dx.doi.org/10.1007/s10803-016-2780y.X-1

5770. Neuhaus E, Kresse A, Faja S, et al. Face processing among twins with and without autism: Social correlates and twin concordance. Social Cognitive and Affective Neuroscience. 2016;11(1):44-54. doi: http://dx.doi.org/10.1093/scan/nsv085.X-1

5771. Neumann ID, Slattery DA. Oxytocin in General Anxiety and Social Fear: A

Translational Approach. Biol Psychiatry. 2016

Feb 1;79(3):213-21. doi: 10.1016/j.biopsych.2015.06.004. PMID: 26208744.X-1

5772. Newbigin A, Uljarevic M, Vivanti G, et al. Brief Report: Empathic Responsiveness of High Functioning Children with Autism to Expressed and Anticipated Distress. J Autism Dev Disord. 2016 Jul 19doi: 10.1007/s10803016-2862-x. PMID: 27436192.X-1

5773. Newbutt N, Sung C, Kuo HJ, et al. Brief Report: A Pilot Study of the Use of a Virtual Reality Headset in Autism Populations. J Autism Dev Disord. 2016 Jun 7doi: 10.1007/s10803-016-2830-5. PMID: 27272115.X-1

5774. Newhouse-Oisten MK, Kestner KM, Frieder JE. An evaluation of modified exposure therapy for a child diagnosed with obsessive compulsive disorder and pervasive developmental disorder-not otherwise 
specified. Behavior Analysis: Research and Practice. 2016;16(3):147-55. doi: http://dx.doi.org/10.1037/bar0000048.X-1, X-3, $\mathrm{X}-4$

5775. Newschaffer CJ, Schriver E, Berrigan $\mathrm{L}$, et al. Development and validation of a streamlined autism case confirmation approach for use in epidemiologic risk factor research in prospective cohorts. Autism Res. 2016 Aug 3doi: 10.1002/aur.1659. PMID: 27484054.X-1

5776. Neymotin F, Nemzer LR. Linking autism and epilepsy. Children's Health Care. 2016;45(1):84-108. doi: http://dx.doi.org/10.1080/02739615.2014.97992 1.X-1

5777. Ngamsamut N, Hongkaew Y, Vanwong N, et al. 9-Hydroxyrisperidoneinduced Hyperprolactinaemia in Thai Children and Adolescents with Autism Spectrum Disorder. Basic Clin Pharmacol Toxicol. 2016 Feb 16doi: 10.1111/bcpt.12570. PMID: 26879343.X-1

5778. Nguyen LS, Lepleux M, Makhlouf M, et al. Profiling olfactory stem cells from living patients identifies miRNAs relevant for autism pathophysiology. Mol Autism. 2016;7:1. doi: 10.1186/s13229-015-0064-6. PMID: 26753090.X-1

5779. Nicholas DB, Zwaigenbaum L, Ing S, et al. "Live it to understand it": The experiences of mothers of children with autism spectrum disorder. Qualitative Health Research. 2016;26(7):921-34. doi: http://dx.doi.org/10.1177/1049732315616622.X $-1$

5780. Nicholas DB, Zwaigenbaum L, Muskat B, et al. Experiences of emergency department care from the perspective of families in which a child has autism spectrum disorder. Soc Work Health Care. 2016 Jul;55(6):409-26. doi: 10.1080/00981389.2016.1178679. PMID: 27315287.X-1

5781. Nicolaidis C, Raymaker D, McDonald $\mathrm{K}$, et al. The Development and Evaluation of an Online Healthcare Toolkit for Autistic Adults and their Primary Care Providers. J Gen Intern Med. 2016 Jun 6doi: 10.1007/s11606-0163763-6. PMID: 27271730.X-1
5782. Niinomi K, Asano M, Kadoma A, et al. Developing the "Skippu-Mama" program for mothers of children with autism spectrum disorder. Nurs Health Sci. 2016 Mar 4doi: 10.1111/nhs.12264. PMID: 26940071.X-1, X-3

5783. Nikolas MA, Elmore AL, Franzen L, et al. Risky bicycling behavior among youth with and without attention-deficit hyperactivity disorder. Journal of Child Psychology and Psychiatry. 2016;57(2):141-8. doi: http://dx.doi.org/10.1111/jcpp.12491.X-1

5784. Noel JP, De Niear MA, Stevenson R, et al. Atypical rapid audio-visual temporal recalibration in autism spectrum disorders. Autism Res. 2016 May 9doi: 10.1002/aur.1633. PMID: 27156926.X-1

5785. Nordahl CW, Mello M, Shen AM, et al. Methods for acquiring MRI data in children with autism spectrum disorder and intellectual impairment without the use of sedation. Journal of Neurodevelopmental Disorders. 2016;8doi: http://dx.doi.org/10.1186/s11689-016-91549.X-1

5786. Nordahl-Hansen A, Fletcher-Watson $\mathrm{S}$, McConachie $\mathrm{H}$, et al. Relations between specific and global outcome measures in a social-communication intervention for children with autism spectrum disorder. Research in Autism Spectrum Disorders. 2016;29-30:19-29. doi: http://dx.doi.org/10.1016/j.rasd.2016.05.005.X1

5787. Nordgren PM. F0, F0 range and duration of utterances-Longitudinal singlesubject studies of prosody in two Swedish children with ASC. Clinical Linguistics \& Phonetics. 2016;30(1):29-48. doi: http://dx.doi.org/10.3109/02699206.2015.10987 28.X-1, X-3, X-4

5788. Nordt M, Hoehl S, Weigelt S. The use of repetition suppression paradigms in developmental cognitive neuroscience. Cortex: A Journal Devoted to the Study of the Nervous System and Behavior. 2016;80:61-75. doi: http://dx.doi.org/10.1016/j.cortex.2016.04.002. $\mathrm{X}-1$

5789. Norkett R, Modi S, Birsa N, et al. DISC1-dependent Regulation of Mitochondrial 
Dynamics Controls the Morphogenesis of Complex Neuronal Dendrites. J Biol Chem. 2016 Jan 8;291(2):613-29. doi: 10.1074/jbc.M115.699447. PMID: 26553875.X-1

5790. Noroozi R, Taheri M, Movafagh A, et al. Glutamate receptor, metabotropic 7 (GRM7) gene variations and susceptibility to autism: A case-control study. Autism Res. 2016 Jun 17doi: 10.1002/aur.1640. PMID: 27312574.X-1

5791. Notwell JH, Heavner WE, Fazel Darbandi S, et al. TBR1 regulates autism risk genes in the developing neocortex. Genome Res. 2016 Jun 20doi: 10.1101/gr.203612.115. PMID: 27325115.X-1

5792. Nunes DRP, Araújo ER, Walter E, et al. Augmenting caregiver responsiveness: An intervention proposal for youngsters with autism in Brazil. Early Childhood Education Journal. 2016;44(1):39-49. doi: http://dx.doi.org/10.1007/s10643-014-0677y.X-3, X-4

5793. Nwaobi SE, Cuddapah VA, Patterson KC, et al. The role of glial-specific Kir4.1 in normal and pathological states of the CNS. Acta Neuropathol. 2016 Jul;132(1):1-21. doi: 10.1007/s00401-016-1553-1. PMID: 26961251.X-1

5794. O’Neill S, Thornton V, Marks DJ, et al. Early language mediates the relations between preschool inattention and school-age reading achievement. Neuropsychology. 2016;30(4):398-404. doi: http://dx.doi.org/10.1037/neu0000247.X-1

5795. O’Nions E, Gould J, Christie P, et al. Identifying features of 'pathological demand avoidance' using the Diagnostic Interview for Social and Communication Disorders (DISCO). European Child \& Adolescent Psychiatry. 2016;25(4):407-19. doi: http://dx.doi.org/10.1007/s00787-015-07402.X-1

5796. Oaks AW, Zamarbide M, Tambunan DE, et al. Cc2d1a Loss of Function Disrupts Functional and Morphological Development in Forebrain Neurons Leading to Cognitive and Social Deficits. Cereb Cortex. 2016 Jan 29doi: 10.1093/cercor/bhw009. PMID: 26826102.X-1
5797. Obeid I, Boissiere L, Yilgor C, et al. Global tilt: a single parameter incorporating spinal and pelvic sagittal parameters and least affected by patient positioning. Eur Spine J. 2016 Jun 20doi: 10.1007/s00586-016-4649-3. PMID: 27323962.X-1

5798. Obel C, Zhu JL, Olsen J, et al. The risk of attention deficit hyperactivity disorder in children exposed to maternal smoking during pregnancy-A re-examination using a sibling design. Journal of Child Psychology and Psychiatry. 2016;57(4):532-7. doi: http://dx.doi.org/10.1111/jcpp.12478.X-1

5799. Oberg AS, D'Onofrio BM, Rickert ME, et al. Association of Labor Induction With Offspring Risk of Autism Spectrum Disorders. JAMA Pediatr. 2016 Jul 25:e160965. doi: 10.1001/jamapediatrics.2016.0965. PMID: 27454803.X-1

5800. Oberman LM, Enticott PG, Casanova MF, et al. Transcranial magnetic stimulation in autism spectrum disorder: Challenges, promise, and roadmap for future research. Autism Res. 2016 Feb;9(2):184-203. doi: 10.1002/aur.1567. PMID: 26536383.X-1, X-2

5801. Oberman LM, Ifert-Miller F, Najib U, et al. Abnormal Mechanisms of Plasticity and Metaplasticity in Autism Spectrum Disorders and Fragile X Syndrome. J Child Adolesc Psychopharmacol. 2016 May 24doi: 10.1089/cap.2015.0166. PMID: 27218148.X-1

5802. O'Byrne ML, Gillespie MJ, Kennedy $\mathrm{KF}$, et al. The influence of deficient retro-aortic rim on technical success and early adverse events following device closure of secundum atrial septal defects: An Analysis of the IMPACT Registry(R). Catheter Cardiovasc Interv. 2016 May 18doi: 10.1002/ccd.26585. PMID: 27189502.X-1

5803. Ochi M, Kimura K, Kanda A, et al. Physicochemical and Pharmacokinetic Characterization of Amorphous Solid Dispersion of Meloxicam with Enhanced Dissolution Property and Storage Stability. AAPS PharmSciTech. 2016 Aug;17(4):932-9. doi: 10.1208/s12249-015-0422-x. PMID: 27435198.X-1

5804. O'Connor E. The use of 'Circle of Friends' strategy to improve social interactions 
and social acceptance: A case study of a child with Asperger's Syndrome and other associated needs. Support for Learning. 2016;31(2):13847. doi: http://dx.doi.org/10.1111/14679604.12122.X-3, X-4

5805. Odent MR. The future of neonatal BCG. Med Hypotheses. 2016 Jun;91:34-6. doi: 10.1016/j.mehy.2016.04.010. PMID: 27142139.X-1

5806. Odom SL. Review of Neurotribes: The legacy of autism and the future of neurodiversity. Journal of Autism and Developmental Disorders. 2016;46(5):1885-6. doi: http://dx.doi.org/10.1007/s10803-0162726-4.X-1, X-2

5807. Oe S, Yamato Y, Togawa D, et al. Preoperative T1 Slope More Than 40 degrees as a Risk Factor of Correction Loss in Patients with Adult Spinal Deformity. Spine (Phila Pa 1976). 2016 Mar 26doi: 10.1097/brs.0000000000001578. PMID: 27031766.X-1

5808. Oerlemans AM, Burmanje MJ, Franke $\mathrm{B}$, et al. Identifying unique versus shared preand perinatal risk factors for ASD and ADHD using a simplex-multiplex stratification. Journal of Abnormal Child Psychology.

2016;44(5):923-35. doi: http://dx.doi.org/10.1007/s10802-015-0081$0 . \mathrm{X}-1$

5809. Oerlemans AM, Hartman CA, Franke $\mathrm{B}$, et al. Does the cognitive architecture of simplex and multiplex ASD families differ? Journal of Autism and Developmental Disorders. 2016;46(2):489-501. doi: http://dx.doi.org/10.1007/s10803-015-25729.X-1

5810. Ogden KK, Ozkan ED, Rumbaugh G. Prioritizing the development of mouse models for childhood brain disorders.

Neuropharmacology. 2016 Jan;100:2-16. doi: 10.1016/j.neuropharm.2015.07.029. PMID: 26231830.X-1

5811. Ogg JA, Bateman L, Dedrick RF, et al. The relationship between life satisfaction and ADHD symptoms in middle school students: Using a bifactor model. Journal of Attention Disorders. 2016;20(5):390-9. doi:
http://dx.doi.org/10.1177/1087054714521292.X $-1$

5812. O'Hare A, Bremner L. Management of developmental speech and language disorders: Part 1. Arch Dis Child. 2016 Mar;101(3):272-7. doi: 10.1136/archdischild-2014-307394. PMID: 26208514.X-1

5813. Oien RA, Siper P, Kolevzon A, et al. Detecting Autism Spectrum Disorder in Children With ADHD and Social Disability. J Atten Disord. 2016 Apr 13doi: 10.1177/1087054716642518. PMID: 27074940.X-1

5814. Oja L, Huotilainen M, Nikkanen E, et al. Behavioral and electrophysiological indicators of auditory distractibility in children with ADHD and comorbid ODD. Brain Research. 2016;1632:42-50. doi: http://dx.doi.org/10.1016/j.brainres.2015.12.003 .X-1

5815. Olçay-Gül S, Tekin-Iftar E. Family generated and delivered social story intervention: Acquisition, maintenance, and generalization of social skills in youths with ASD. Education and Training in Autism and Developmental Disabilities. 2016;51(1):6778.X-1, X-3

5816. Olexova L, Stefanik P, Krskova L. Increased anxiety-like behaviour and altered GABAergic system in the amygdala and cerebellum of VPA rats-An animal model of autism. Neurosci Lett. 2016 Jun 25doi: 10.1016/j.neulet.2016.06.035. PMID: 27353514.X-1

5817. Olivito G, Clausi S, Laghi F, et al. Resting-State Functional Connectivity Changes Between Dentate Nucleus and Cortical Social Brain Regions in Autism Spectrum Disorders. Cerebellum. 2016 Jun 1doi: 10.1007/s12311016-0795-8. PMID: 27250977.X-1

5818. Olmsted D, Blaxill M. Leo Kanner's mention of 1938 in his report on autism refers to his first patient. Journal of Autism and Developmental Disorders. 2016;46(1):340-1. doi: http://dx.doi.org/10.1007/s10803-0152541-3.X-1

5819. O'Neill KR, Wilson RJ, Burns KM, et al. Anterior Cervical Discectomy and Fusion 
for Adjacent Segment Disease: Clinical Outcomes and Cost Utility of Surgical Intervention. Clin Spine Surg. 2016 Jul;29(6):234-41. doi: 10.1097/BSD.0b013e31828ffc54. PMID: 27137162.X-1

5820. Ooi KL, Ong YS, Jacob SA, et al. A meta-synthesis on parenting a child with autism. Neuropsychiatric Disease and Treatment. 2016;12.X-1, X-2, X-3

5821. Ooi YP, Weng S-J, Magiati I, et al. Factors influencing agreement between parent and child reports of anxiety symptoms among children with high-functioning Autism Spectrum Disorders. Journal of Developmental and Physical Disabilities. 2016;28(3):407-24. doi: http://dx.doi.org/10.1007/s10882-0169481-5.X-1

5822. Orefice LL, Zimmerman AL, Chirila AM, et al. Peripheral Mechanosensory Neuron Dysfunction Underlies Tactile and Behavioral Deficits in Mouse Models of ASDs. Cell. 2016 Jun 8doi: 10.1016/j.cell.2016.05.033. PMID: 27293187.X-1

5823. Ormond DR, Albert L, Jr., Das K. Polyetheretherketone (PEEK) Rods in Lumbar Spine Degenerative Disease: A Case Series. Clin Spine Surg. 2016 Aug;29(7):E371-5. doi: 10.1097/BSD.0b013e318277cb9b. PMID: 23075859.X-1

5824. O'Shea KS, McInnis MG.

Neurodevelopmental origins of bipolar disorder: iPSC models. Mol Cell Neurosci. 2016 Jun;73:63-83. doi: 10.1016/j.mcn.2015.11.006. PMID: 26608002.X-1

5825. Oswald TM, Winter-Messiers MA, Gibson B, et al. Sex differences in internalizing problems during adolescence in autism spectrum disorder. Journal of Autism and Developmental Disorders. 2016;46(2):624-36. doi: http://dx.doi.org/10.1007/s10803-0152608-1.X-1

5826. Ottley JR, Ferron JM, Hanline MF. Explaining variance and identifying predictors of children's communication via a multilevel model of single-case design research. Developmental Neurorehabilitation. 2016;19(3):197-202.X-1
5827. Ottosen C, Petersen L, Larsen JT, et al. Gender differences in associations between attention-deficit/hyperactivity disorder and substance use disorder. Journal of the American Academy of Child \& Adolescent Psychiatry. 2016;55(3):227-34. doi: http://dx.doi.org/10.1016/j.jaac.2015.12.010.X1

5828. Ouyang M, Cheng H, Mishra V, et al. Atypical age-dependent effects of autism on white matter microstructure in children of 2-7 years. Human Brain Mapping. 2016;37(2):81932. doi: http://dx.doi.org/10.1002/hbm.23073.X-1

5829. Ouyang M, Jeon T, Mishra V, et al. Global and regional cortical connectivity maturation index (CCMI) of developmental human brain with quantification of short-range association tracts. Proc SPIE Int Soc Opt Eng. 2016 Feb 27;9788doi: 10.1117/12.2218029. PMID: 27076697.X-1, X-3

5830. Overgaard KR, Aase H, Torgersen S, et al. Co-occurrence of ADHD and anxiety in preschool children. Journal of Attention Disorders. 2016;20(7):573-80. doi: http://dx.doi.org/10.1177/1087054712463063.X $-1$

5831. Oxelgren UW, Myrelid A, Anneren G, et al. Prevalence of autism and attention-deficithyperactivity disorder in Down syndrome: a population-based study. Dev Med Child Neurol. 2016 Aug 9doi: 10.1111/dmcn.13217. PMID: 27503703.X-1

5832. Özçalışkan Ş, Adamson LB, Dimitrova N. Early deictic but not other gestures predict later vocabulary in both typical development and autism. Autism. 2016;20(6):754-63. doi: http://dx.doi.org/10.1177/1362361315605921.X $-1, \mathrm{X}-3$

5833. Ozsivadjian A, Hollocks MJ, Southcott J, et al. Anxious Imagery in Children With and Without Autism Spectrum Disorder: An Investigation into Occurrence, Content, Features and Implications for Therapy. J Autism Dev Disord. 2016 Jun 21doi: 10.1007/s10803-016-2840-3. PMID: 27328680.X-1 
5834. Ozturk Y, Vivanti G, Uljarevic M, et al. Treatment-related changes in children's communication impact on maternal satisfaction and psychological distress. Res Dev Disabil. 2016 Sep;56:128-38. doi:

10.1016/j.ridd.2016.05.021. PMID: 27295207.X-1

5835. Paasch V, Leibowitz L, Accardo J, et al. Preparing children with autism spectrum disorders for overnight sleep studies: A case series. Clinical Practice in Pediatric Psychology. 2016;4(2):153-63. doi: http://dx.doi.org/10.1037/cpp0000139.X-1, X3, X-4

5836. Page TF, Pelham WE, III, Fabiano GA, et al. Comparative cost analysis of sequential, adaptive, behavioral, pharmacological, and combined treatments for childhood ADHD. Journal of Clinical Child and Adolescent Psychology. 2016;45(4):416-27. doi:

http://dx.doi.org/10.1080/15374416.2015.10558 59.X-1

5837. Palm U, Segmiller FM, Epple AN, et al. Transcranial direct current stimulation in children and adolescents: a comprehensive review. J Neural Transm (Vienna). 2016 May 12doi: 10.1007/s00702-016-1572-z. PMID: 27173384.X-1

5838. Pan CC, Davis R, Nichols D, et al. Prevalence of overweight and obesity among students with intellectual disabilities in Taiwan: A secondary analysis. Res Dev Disabil. 2016 Jun-Jul;53-54:305-13. doi: 10.1016/j.ridd.2016.02.018. PMID: 26986697.X-1

5839. Pan CY, Chu CH, Tsai CL, et al. The impacts of physical activity intervention on physical and cognitive outcomes in children with autism spectrum disorder. Autism. 2016 Apr 7doi: 10.1177/1362361316633562. PMID: 27056845.X-1

5840. Panchision DM. Concise Review: Progress and Challenges in Using Human Stem Cells for Biological and Therapeutics Discovery: Neuropsychiatric Disorders. Stem Cells. 2016 Mar;34(3):523-36. doi: 10.1002/stem.2295. PMID: 26840228.X-1
5841. Pandya NJ, Klaassen RV, van der Schors RC, et al. Group 1 metabotropic glutamate receptors 1 and 5 form a protein complex in mouse hippocampus and cortex. Proteomics. 2016 Jul 9doi: 10.1002/pmic.201500400. PMID: 27392515.X1

5842. Pantelis PC, Kennedy DP. Estimation of the prevalence of autism spectrum disorder in South Korea, revisited. Autism. 2016;20(5):517-27. doi: http://dx.doi.org/10.1177/1362361315592378.X $-1$

5843. Papatola KJ, Lustig SL. Navigating a managed care peer review: Guidance for clinicians using applied behavior analysis in the treatment of children on the autism spectrum. Behavior Analysis in Practice. 2016;9(2):13545. doi: http://dx.doi.org/10.1007/s40617-0160120-5.X-1

5844. Park HK, Shin HJ, Park YH, et al. The importance of preoperative oxygen saturation as a predictor of pulmonary arterial hypertension after surgery of atrial septal defects. Interact Cardiovasc Thorac Surg. 2016 May 24doi: 10.1093/icvts/ivw162. PMID: 27222001.X-1

5845. Park P, Okonkwo DO, Nguyen S, et al. Can a Minimal Clinically Important Difference Be Achieved in Elderly Patients with Adult Spinal Deformity Who Undergo Minimally Invasive Spinal Surgery? World Neurosurg. 2016 Feb;86:168-72. doi: 10.1016/j.wneu.2015.09.072. PMID: 26431736.X-1

5846. Park P, Wang MY, Nguyen S, et al. Comparison of Complications and Clinical and Radiographic Outcomes Between Nonobese and Obese Patients with Adult Spinal Deformity Undergoing Minimally Invasive Surgery. World Neurosurg. 2016 Mar;87:55-60. doi: 10.1016/j.wneu.2015.12.024. PMID: 26724613.X-1

5847. Park SJ, Lee CS, Chung SS, et al. Different Risk Factors of Proximal Junctional Kyphosis and Proximal Junctional Failure Following Long Instrumented Fusion to the Sacrum for Adult Spinal Deformity: Survivorship Analysis of 160 Patients. Neurosurgery. 2016 Apr 8doi: 
10.1227/neu.0000000000001240. PMID: 27065296.X-1

5848. Park SY, Cervesi C, Galling B, et al. Antipsychotic Use Trends in Youth With Autism Spectrum Disorder and/or Intellectual Disability: A Meta-Analysis. J Am Acad Child Adolesc Psychiatry. 2016 Jun;55(6):456-68.e4. doi: 10.1016/j.jaac.2016.03.012. PMID: 27238064.X-1

5849. Parker A, Corkum P. ADHD diagnosis: As simple as administering a questionnaire or a complex diagnostic process? Journal of Attention Disorders. 2016;20(6):47886. doi: http://dx.doi.org/10.1177/1087054713495736.X $-1$

5850. Parker CH. TRUST A v X AND OTHERS: THE GHOST OF NIELSEN RETURNS? Med Law Rev. 2016 Spring;24(2):268-77. doi: 10.1093/medlaw/fwv047. PMID: 27273919.X1

5851. Pasco Fearon RM. Editorial: Looking beyond the horizon - innovation in child psychology and psychiatry. Journal of Child Psychology and Psychiatry. 2016;57(3):213-5. doi: http://dx.doi.org/10.1111/jcpp.12545.X-1, $\mathrm{X}-2$

5852. Passias PG, Klineberg EO, Jalai CM, et al. Hospital Readmission within Two Years Following Adult Thoracolumbar Spinal Deformity Surgery: Prevalence, Predictors, and Effect on Patient-Derived Outcome Measures. Spine (Phila Pa 1976). 2016 Mar 8doi: 10.1097/brs.0000000000001552. PMID: 26967123.X-1

5853. Passias PG, Oh C, Jalai CM, et al. Predictive Model for Cervical Alignment and Malalignment Following Surgical Correction of Adult Spinal Deformity. Spine (Phila Pa 1976). 2016 Apr 19doi: 10.1097/brs.0000000000001640. PMID: 27105461.X-1

5854. Pastor-Cerezuela G, Fernández-Andrés M-I, Feo-Álvarez M, et al. Semantic verbal fluency in children with and without Autism Spectrum Disorder: Relationship with chronological age and IQ. Frontiers in Psychology. 2016;7.X-1
5855. Patak J, Zhang-James Y, Faraone SV. Endosomal system genetics and autism spectrum disorders: A literature review. Neurosci Biobehav Rev. 2016 Jun;65:95-112. doi: 10.1016/j.neubiorev.2016.03.022. PMID: 27048963.X-1

5856. Patel I, Erickson SR, Caldwell CH, et al. Predictors of medication adherence and persistence in Medicaid enrollees with developmental disabilities and type 2 diabetes. Res Social Adm Pharm. 2016 Jul-

Aug;12(4):592-603. doi: 10.1016/j.sapharm.2015.09.008. PMID: 26522400.X-1

5857. Patel S, Day TN, Jones N, et al. Association between anger rumination and autism symptom severity, depression symptoms, aggression, and general dysregulation in adolescents with autism spectrum disorder. Autism. 2016 Apr 19doi: 10.1177/1362361316633566. PMID: 27095831.X-1

5858. Patriquin MA, DeRamus T, Libero LE, et al. Neuroanatomical and neurofunctional markers of social cognition in autism spectrum disorder. Hum Brain Mapp. 2016 Jun 22doi: 10.1002/hbm.23288. PMID: 27329401.X-1

5859. Patros CHG, Alderson RM, Kasper LJ, et al. Choice-impulsivity in children and adolescents with attention-deficit/hyperactivity disorder (ADHD): A meta-analytic review. Clinical Psychology Review. 2016;43:162-74. doi: http://dx.doi.org/10.1016/j.cpr.2015.11.001.X-1

5860. Pawar J, Tayade A, Gangurde A, et al. Solubility and dissolution enhancement of efavirenz hot melt extruded amorphous solid dispersions using combination of polymeric blends: A QbD approach. Eur J Pharm Sci. 2016 Jun 10;88:37-49. doi: 10.1016/j.ejps.2016.04.001. PMID: 27049050.X-1

5861. Paynter JM, Ferguson S, Fordyce K, et al. Utilisation of evidence-based practices by ASD early intervention service providers. Autism. 2016 Apr 18doi: 10.1177/1362361316633032. PMID: 27091949.X-1 
5862. Pearson BL, Crawley JN, Eilam D, et al. Curiosity as an approach to ethoexperimental analysis: Behavioral neuroscience as seen by students and colleagues of Bob Blanchard. Neurosci Biobehav Rev.

2016 Mar 24doi:

10.1016/j.neubiorev.2016.03.012. PMID:

27018381.X-1

5863. Pearson BL, Simon JM, McCoy ES, et al. Identification of chemicals that mimic transcriptional changes associated with autism, brain aging and neurodegeneration. Nat Commun. 2016;7:11173. doi:

10.1038/ncomms11173. PMID: 27029645.X-1

5864. Peckett H, MacCallum F, Knibbs J. Maternal experience of Lego Therapy in families with children with autism spectrum conditions: What is the impact on family relationships? Autism. 2016 Feb 5doi: 10.1177/1362361315621054. PMID: 26851230.X-1

5865. Pedapati EV, Gilbert DL, Erickson CA, et al. Abnormal Cortical Plasticity in Youth with Autism Spectrum Disorder: A Transcranial Magnetic Stimulation CaseControl Pilot Study. J Child Adolesc Psychopharmacol. 2016 Mar 23doi: 10.1089/cap.2015.0183. PMID: 27007257.X-1

5866. Peeters B, Noens I, Kuppens S, et al. Toilet training in children with a functional defecation disorder and concomitant symptoms of autism spectrum disorder. Research in Autism Spectrum Disorders. 2016;26:91-8. doi: http://dx.doi.org/10.1016/j.rasd.2016.02.009.X1

5867. Pegado F, Vankrunkelsven H, Steyaert $\mathrm{J}$, et al. Exploring the Use of Sensorial LTP/LTD-Like Stimulation to Modulate Human Performance for Complex Visual Stimuli. PLoS One. 2016;11(6):e0158312. doi: 10.1371/journal.pone.0158312. PMID: 27341210.X-1

5868. Pelham WE, Jr., Fabiano GA, Waxmonsky JG, et al. Treatment sequencing for childhood ADHD: A multiplerandomization study of adaptive medication and behavioral interventions. Journal of Clinical Child and Adolescent Psychology.

2016;45(4):396-415. doi: http://dx.doi.org/10.1080/15374416.2015.11051 38.X-1

5869. Pennisi P, Tonacci A, Tartarisco G, et al. Autism and social robotics: A systematic review. Autism Res. 2016 Feb;9(2):165-83. doi: 10.1002/aur.1527. PMID: 26483270.X-2

5870. Peralta F, Fuentealba C, Fiedler J, et al. Prenatal valproate treatment produces autistic-like behavior and increases metabotropic glutamate receptor $1 \mathrm{~A}$ immunoreactivity in the hippocampus of juvenile rats. Mol Med Rep. 2016 Sep;14(3):2807-14. doi: 10.3892/mmr.2016.5529. PMID: 27430241.X-1

5871. Perathoner S, Cordero-Maldonado ML, Crawford AD. Potential of zebrafish as a model for exploring the role of the amygdala in emotional memory and motivational behavior. J Neurosci Res. 2016 Jun;94(6):445-62. doi: 10.1002/jnr.23712. PMID: 26833658.X-1

5872. Perera H, Jeewandara KC, Seneviratne $\mathrm{S}$, et al. Outcome of Home-Based Early Intervention for Autism in Sri Lanka: FollowUp of a Cohort and Comparison with a Nonintervention Group. Biomed Res Int. 2016;2016:3284087. doi: 10.1155/2016/3284087. PMID: 27419131.X-1

5873. Perez-Pouchoulen M, Miquel M, Saft $\mathrm{P}$, et al. Prenatal exposure to sodium valproate alters androgen receptor expression in the developing cerebellum in a region and age specific manner in male and female rats. Int $\mathrm{J}$ Dev Neurosci. 2016 Jul 14;53:46-52. doi: 10.1016/j.ijdevneu.2016.07.001. PMID: 27423376.X-1

5874. Peschansky VJ, Pastori C, Zeier Z, et al. The long non-coding RNA FMR4 promotes proliferation of human neural precursor cells and epigenetic regulation of gene expression in trans. Mol Cell Neurosci. 2016 Mar 19;74:4957. doi: 10.1016/j.mcn.2016.03.008. PMID: 27001315.X-1

5875. Peters B. A model for enhancing social communication and interaction in everyday activities for primary school children with ASD. Journal of Research in Special Educational Needs. 2016;16(2):89-101. doi: http://dx.doi.org/10.1111/1471-3802.12059.X$1, \mathrm{X}-3, \mathrm{X}-4$ 
5876. Peters S, Van Duijvenvoorde ACK, Koolschijn PCMP, et al. Longitudinal development of frontoparietal activity during feedback learning: Contributions of age, performance, working memory and cortical thickness. Developmental Cognitive Neuroscience. 2016;19:211-22. doi: http://dx.doi.org/10.1016/j.dcn.2016.04.004.X-1

5877. Peters SU, Byiers BJ, Symons FJ. Diurnal salivary cortisol and regression status in MECP2 duplication syndrome. Journal of Child Neurology. 2016;31(2):159-63. doi: http://dx.doi.org/10.1177/0883073815585577.X $-1$

5878. Peterson BT, Anderer P, Moreau A, et al. A novel actigraphy data analysis tool and its application to identifying the optimal threshold value in three subject populations. Physiol Meas. 2016 Jun 20;37(7):N49-n61. doi: 10.1088/0967-3334/37/7/n49. PMID: 27319572.X-1

5879. Peterson C, Lerman DC, Nissen MA. Reinforcer choice as an antecedent versus consequence. Journal of Applied Behavior Analysis. 2016;49(2):286-93. doi: http://dx.doi.org/10.1002/jaba.284.X-1, X-3, X4

5880. Peterson C, Slaughter V, Moore C, et al. Peer social skills and theory of mind in children with autism, deafness, or typical development. Developmental Psychology. 2016;52(1):46-57. doi: http://dx.doi.org/10.1037/a0039833.X-1

5881. Peterson KM, Piazza CC, Volkert VM. A comparison of a modified sequential oral sensory approach to an applied behavioranalytic approach in the treatment of food selectivity in children with autism spectrum disorders. J Appl Behav Anal. 2016 Jul 23doi: 10.1002/jaba.332. PMID: 27449267.X-1, X-4

5882. Petrina N, Carter M, Stephenson J, et al. Perceived friendship quality of children with autism spectrum disorder as compared to their peers in mixed and non-mixed dyads. Journal of Autism and Developmental Disorders.

2016;46(4):1334-43. doi: http://dx.doi.org/10.1007/s10803-015-26735.X-1
5883. Pham AV. Differentiating behavioral ratings of inattention, impulsivity, and hyperactivity in children: Effects on reading achievement. Journal of Attention Disorders. 2016;20(8):674-83. doi: http://dx.doi.org/10.1177/1087054712473833.X $-1$

5884. Phan P, Ploumis A, Hess K, et al. Evaluating the Extent of Clinical Variability Among Treatment Options for Patients With Adult Spinal Deformity. Clin Spine Surg. 2016 Jun 27doi: 10.1097/bsd.0000000000000407. PMID: 27352371.X-1

5885. Phillips D, Choleris E, Ervin KS, et al. Cage-induced stereotypic behaviour in laboratory mice covaries with nucleus accumbens FosB/DeltaFosB expression. Behav Brain Res. 2016 Mar 15;301:238-42. doi: 10.1016/j.bbr.2015.12.035. PMID: 26731014.X-1

5886. Philpott-Robinson K, Lane AE, Harpster K. Sensory Features of Toddlers at Risk for Autism Spectrum Disorder. Am J Occup Ther. 2016 Jul-

Aug;70(4):7004220010p1-8. doi: 10.5014/ajot.2016.019497. PMID: 27294987.X-1

5887. Pickard KE, Kilgore AN, Ingersoll BR. Using Community Partnerships to Better Understand the Barriers to Using an EvidenceBased, Parent-Mediated Intervention for Autism Spectrum Disorder in a Medicaid System. Am J Community Psychol. 2016 Jun;57(3-4):391-403. doi: 10.1002/ajcp.12050. PMID: 27216766.X-1

5888. Pickard KE, Wainer AL, Bailey KM, et al. A mixed-method evaluation of the feasibility and acceptability of a telehealthbased parent-mediated intervention for children with autism spectrum disorder. Autism. 2016 Feb 9doi: 10.1177/1362361315614496. PMID: 26862084.X-1

5889. Pierce K, Courchesne E, Bacon E. To Screen or Not to Screen Universally for Autism is not the Question: Why the Task Force Got It Wrong. J Pediatr. 2016 Jul 12doi: 10.1016/j.jpeds.2016.06.004. PMID: 27421956.X-1 
5890. Pierce K, Marinero S, Hazin R, et al. Eye tracking reveals abnormal visual preference for geometric images as an early biomarker of an autism spectrum disorder subtype associated with increased symptom severity. Biological Psychiatry. 2016;79(8):657-66. doi: http://dx.doi.org/10.1016/j.biopsych.2015.03.03 2.X-1

5891. Pierucci JM. Mothers’ scaffolding techniques used during play in toddlers with autism spectrum disorder. Journal of Developmental and Physical Disabilities. 2016;28(2):217-35. doi: http://dx.doi.org/10.1007/s10882-015-94598.X-1

5892. Pietropaolo S, Crusio WE, D'Amato F R. Treatment Approaches in Rodent Models for Autism Spectrum Disorder. Curr Top Behav Neurosci. 2016 Feb 9doi: 10.1007/7854_2015_433. PMID: 26857461.X1

5893. Piletic K, Kunej T. MicroRNA epigenetic signatures in human disease. Arch Toxicol. 2016 Aug 24doi: 10.1007/s00204-0161815-7. PMID: 27557899.X-1

5894. Pinggera A, Striessnig J. Cav 1.3 (CACNA1D) L-type Ca2+ channel dysfunction in CNS disorders. J Physiol. 2016 Feb 4doi: 10.1113/jp270672. PMID: 26842699.X-1

5895. Pini G, Congiu L, Benincasa A, et al. Illness Severity, Social and Cognitive Ability, and EEG Analysis of Ten Patients with Rett Syndrome Treated with Mecasermin (Recombinant Human IGF-1). Autism Res Treat. 2016;2016:5073078. doi: 10.1155/2016/5073078. PMID: 26925263.X-1

5896. Pinto AM, Bianciardi L, Mencarelli MA, et al. Exome sequencing analysis in a pair of monozygotic twins re-evaluates the genetics behind their intellectual disability and reveals a CHD2 mutation. Brain \& Development. 2016;38(6):590-6. doi: http://dx.doi.org/10.1016/j.braindev.2015.12.00 6.X-1

5897. Pinto R, Rijsdijk F, Ouellet-Morin I, et al. The aetiological association between the dynamics of cortisol productivity and ADHD. Journal of Neural Transmission. 2016;123(8):991-1000. doi: http://dx.doi.org/10.1007/s00702-016-15345.X-1

5898. Pinto R, Rijsdijk F, Ronald A, et al. The genetic overlap of attentiondeficit/hyperactivity disorder and autistic-like traits: An investigation of individual symptom scales and cognitive markers. Journal of Abnormal Child Psychology. 2016;44(2):33545. doi: http://dx.doi.org/10.1007/s10802-0150037-4.X-1

5899. Pivovarciova A, Durdiakova J, Babinska K, et al. Testosterone and androgen receptor sensitivity in relation to hyperactivity symptoms in boys with Autism spectrum disorders. PLoS ONE. 2016;11(2).X-1

5900. Plaisance L, Lerman DC, Laudont C, et al. Inserting mastered targets during error correction when teaching skills to children with autism. Journal of Applied Behavior Analysis. 2016;49(2):251-64. doi: http://dx.doi.org/10.1002/jaba.292.X-1, X-3, X4

5901. Plasschaert E, Van Eylen L, Descheemaeker MJ, et al. Executive functioning deficits in children with neurofibromatosis type 1 : The influence of intellectual and social functioning. American Journal of Medical Genetics Part B: Neuropsychiatric Genetics. 2016;171(3):34862. doi: http://dx.doi.org/10.1002/ajmg.b.32414.X-1

5902. Plavnick JB, Vitale FA. A comparison of vocal mand training strategies for children with autism spectrum disorders. Journal of Positive Behavior Interventions. 2016;18(1):5262. doi:

http://dx.doi.org/10.1177/1098300714548800.X $-3, \mathrm{X}-4$

5903. Plesa Skwerer D, Jordan SE, Brukilacchio BH, et al. Comparing methods for assessing receptive language skills in minimally verbal children and adolescents with autism spectrum disorders. Autism. 2016;20(5):591604. doi: http://dx.doi.org/10.1177/1362361315600146.x $-1$

5904. Pletz J, Sanchez-Bayo F, Tennekes HA. Dose-response analysis indicating timedependent neurotoxicity caused by organic and 
inorganic mercury-Implications for toxic effects in the developing brain. Toxicology. 2016 Mar 10;347-349:1-5. doi:

10.1016/j.tox.2016.02.006. PMID: 26945727.X-1

5905. Podlesnik CA, Jimenez-Gomez C. Contrafreeloading, reinforcement rate, and behavioral momentum. Behav Processes. 2016 Jul;128:24-8. doi: 10.1016/j.beproc.2016.03.022. PMID: 27045696.X-1

5906. Polfuss M, Johnson N, Bonis SA, et al. Autism Spectrum Disorder and the Child's Weight-Related Behaviors: A Parents' Perspective. J Pediatr Nurs. 2016 Jun 20doi: 10.1016/j.pedn.2016.05.006. PMID: 27339734.X-1, X-4

5907. Politi Y, Pippel E, Licuco-Massouh AC, et al. Nano-channels in the spider fang for the transport of $\mathrm{Zn}$ ions to cross-link His-rich proteins pre-deposited in the cuticle matrix. Arthropod Struct Dev. 2016 Jun 18doi: 10.1016/j.asd.2016.06.001. PMID: 27329320.X-1

5908. Pollack B, Hojnoski R, DuPaul GJ, et al. Play behavior differences among preschoolers with ADHD: Impact of comorbid ODD and anxiety. Journal of Psychopathology and Behavioral Assessment. 2016;38(1):66-75. doi: http://dx.doi.org/10.1007/s10862-0159497-7.X-1

5909. Polzin A, Dannenberg L, Sophia Popp $\mathrm{V}$, et al. Antiplatelet effects of clopidogrel and aspirin after interventional patent foramen ovale/ atrium septum defect closure. Platelets. 2016 Jun;27(4):317-21. doi: 10.3109/09537104.2015.1096335. PMID: 26556101.X-1

5910. Ponce P, Molina A, Grammatikou D. Design based on fuzzy signal detection theory for a semi-autonomous assisting robot in children autism therapy. Computers in Human Behavior. 2016;51(Part A):28-42. doi: http://dx.doi.org/10.1016/j.chb.2015.08.036.X-1

5911. Poon KK, Yang X. The student profile, service delivery model, and support practices of four early childhood intervention environments in Singapore. Asia Pacific Journal of Education. 2016;36(3):437-49. doi: http://dx.doi.org/10.1080/02188791.2014.94003 $0 . \mathrm{X}-1$

5912. Poopal AC, Schroeder LM, Horn PS, et al. Increased expression of the PI3K catalytic subunit p110delta underlies elevated S6 phosphorylation and protein synthesis in an individual with autism from a multiplex family. Mol Autism. 2016;7:3. doi: 10.1186/s13229015-0066-4. PMID: 26770665.X-1

5913. Popple B, Wall C, Flink L, et al. Brief Report: Remotely Delivered Video Modeling for Improving Oral Hygiene in Children with ASD: A Pilot Study. J Autism Dev Disord. 2016 Apr 22doi: 10.1007/s10803-016-2795-4. PMID: 27106570.X-1

5914. Portales-Casamar E, Lussier AA, Jones MJ, et al. DNA methylation signature of human fetal alcohol spectrum disorder. Epigenetics Chromatin. 2016;9:25. doi: 10.1186/s13072-016-0074-4. PMID: 27358653.X-1

5915. Posner DS. Review of Autism and understanding: The Waldon approach to child development. Journal of Autism and Developmental Disorders. 2016;46(4):1498-9. doi: http://dx.doi.org/10.1007/s10803-0152658-4.X-1, X-2

5916. Posserud M, Hysing M, Helland W, et al. Autism traits: The importance of "comorbid" problems for impairment and contact with services. Data from the Bergen Child Study. Res Dev Disabil. 2016 Jan 27doi: 10.1016/j.ridd.2016.01.002. PMID: 26826893.X-1

5917. Poustka L, Kamp-Becker I. Current Practice and Future Avenues in Autism Therapy. Curr Top Behav Neurosci. 2016 Apr 30doi: 10.1007/7854_2016_442. PMID: 27130325.X-1

5918. Powell G, Wass SV, Erichsen JT, et al. First evidence of the feasibility of gazecontingent attention training for school children with autism. Autism. 2016 Feb 9doi: 10.1177/1362361315617880. PMID: 26862085.X-1

5919. Prager EM, Bergstrom HC, Wynn GH, et al. The basolateral amygdala gammaaminobutyric acidergic system in health and 
disease. J Neurosci Res. 2016 Jun;94(6):548-67. doi: 10.1002/jnr.23690. PMID: 26586374.X-1

5920. Preckel K, Kanske P, Singer T, et al. Clinical trial of modulatory effects of oxytocin treatment on higher-order social cognition in autism spectrum disorder: a randomized, placebo-controlled, double-blind and crossover trial. BMC Psychiatry. 2016;16(1):329. doi: 10.1186/s12888-016-1036-X. PMID: 27655235.X-2, X-3

5921. Preis J, Amon R, Robinette DS, et al. Does music matter? The effects of background music on verbal expression and engagement in children with autism spectrum disorders. Music Therapy Perspectives. 2016;34(1):106-15. doi: http://dx.doi.org/10.1093/mtp/miu044.X-3, X-4

5922. Prince EB, Kim ES, Wall CA, et al. The relationship between autism symptoms and arousal level in toddlers with autism spectrum disorder, as measured by electrodermal activity. Autism. 2016 Jun 10doi:

10.1177/1362361316648816. PMID: 27289132.X-1

5923. Prins BP, Abbasi A, Wong A, et al. Investigating the Causal Relationship of CReactive Protein with 32 Complex Somatic and Psychiatric Outcomes: A Large-Scale CrossConsortium Mendelian Randomization Study. PLoS Med. 2016 Jun;13(6):e1001976. doi: 10.1371/journal.pmed.1001976. PMID: 27327646.X-1

5924. Pritchard MA, de Dassel T, Beller E, et al. Autism in Toddlers Born Very Preterm. Pediatrics. 2016 Feb;137(2):e20151949. doi: 10.1542/peds.2015-1949. PMID: 26798043.X-1

5925. Pruett JR, Jr., Povinelli DJ. Commentary - Autism Spectrum Disorder: Spectrum or Cluster? Autism Res. 2016 Jun 22doi: 10.1002/aur.1650. PMID: 27333214.X$1, \mathrm{X}-2$

5926. Pruitt MM, Willis K, Timmons L, et al. The impact of maternal, child, and family characteristics on the daily well-being and parenting experiences of mothers of children with autism spectrum disorder. Autism. 2016 Feb 5doi: 10.1177/1362361315620409. PMID: 26851229.X-1
5927. Puente AE, Heller S, Sekely A. Neuropsychological Analysis of an Idiot Savant: A Case Study. Appl Neuropsychol Adult. 2016 May 9:1-5. doi: 10.1080/23279095.2016.1159563. PMID: 27158857.X-1

5928. Puig-Alcaraz C, Fuentes-Albero M, Cauli O. Relationship between adipic acid concentration and the core symptoms of autism spectrum disorders. Psychiatry Res. 2016 May 25;242:39-45. doi:

10.1016/j.psychres.2016.05.027. PMID: 27259135.X-1

5929. Pujals E, Batlle S, Camprodon E, et al. Brief report: Translation and adaptation of the Theory of Mind Inventory to Spanish. Journal of Autism and Developmental Disorders. 2016;46(2):685-90. doi: http://dx.doi.org/10.1007/s10803-015-25765.X-1

5930. Puka K, Smith ML, Moineddin R, et al. Health resource utilization varies by comorbidities in children with epilepsy. Epilepsy \& Behavior. 2016;57(Part A):151-4. doi:

http://dx.doi.org/10.1016/j.yebeh.2016.02.011. $\mathrm{X}-1$

5931. Pusponegoro HD, Efar P, Soedjatmiko, et al. Gross Motor Profile and Its Association with Socialization Skills in Children with Autism Spectrum Disorders. Pediatr Neonatol. 2016 Apr 2doi: 10.1016/j.pedneo.2016.02.004. PMID: 27161079.X-1

5932. Putnam PT, Roman JM, Zimmerman $\mathrm{PE}$, et al. Oxytocin enhances gaze-following responses to videos of natural social behavior in adult male rhesus monkeys.

Psychoneuroendocrinology. 2016 Jun 3;72:47-

53. doi: 10.1016/j.psyneuen.2016.05.016. PMID: 27343726.X-1

5933. Puvanesarajah V, Shen FH, Cancienne JM, et al. Risk factors for revision surgery following primary adult spinal deformity surgery in patients 65 years and older. $\mathrm{J}$ Neurosurg Spine. 2016 May 6:1-8. doi: 10.3171/2016.2.spine151345. PMID: 27153147.X-1

5934. Qin L, Dai X, Yin Y. Valproic acid exposure sequentially activates Wnt and mTOR 
pathways in rats. Mol Cell Neurosci. 2016 Jun 23;75:27-35. doi: 10.1016/j.mcn.2016.06.004. PMID: 27343825.X-1

5935. Qiu T, Chang C, Li Y, et al. Two years changes in the development of caudate nucleus are involved in restricted repetitive behaviors in 2-5-year-old children with autism spectrum disorder. Developmental Cognitive Neuroscience. 2016;19:137-43. doi: http://dx.doi.org/10.1016/j.dcn.2016.02.010.X-1

5936. Quigley J, McNally S, Lawson S. Prosodic patterns in interaction of low-risk and at-risk-of-autism spectrum disorders infants and their mothers at 12 and 18 months. Language Learning and Development. 2016;12(3):295310. doi: http://dx.doi.org/10.1080/15475441.2015.10754 05.X-1

5937. Rademacher L, Schulte-Ruther M, Hanewald B, et al. Reward: From Basic Reinforcers to Anticipation of Social Cues. Curr Top Behav Neurosci. 2016 Jan 5doi: 10.1007/7854_2015_429. PMID: 26728170.X1

5938. Radley KC, Dart EH, Moore JW, et al. Promoting Accurate Variability of Social Skills in Children With Autism Spectrum Disorder. Behav Modif. 2016 Jul 5doi: 10.1177/0145445516655428. PMID: 27385411.X-3, X-4

5939. Radley KC, Hanglein J, Arak M. School-based social skills training for preschool-age children with autism spectrum disorder. Autism. 2016 Feb 5doi: 10.1177/1362361315617361. PMID: 26851232.X-1, X-3, X-4

5940. Raffard S, Bortolon C, Khoramshahi $\mathrm{M}$, et al. Humanoid robots versus humans: How is emotional valence of facial expressions recognized by individuals with schizophrenia? An exploratory study. Schizophr Res. 2016 Jun 9doi: 10.1016/j.schres.2016.06.001. PMID: 27293136.X-1

5941. Rafie F, Ghasemi A, Zamani Jam A, et al. Effect of exercise intervention on the perceptual- motor skills in adolescents with autism. J Sports Med Phys Fitness. 2016 Mar 30 PMID: 27028719.X-1, X-3
5942. Rahimi-Golkhandan S, Steenbergen B, Piek JP, et al. Revealing hot executive function in children with motor coordination problems: What's the go? Brain and Cognition.

2016;106:55-64. doi: http://dx.doi.org/10.1016/j.bandc.2016.04.010. $\mathrm{X}-1$

5943. Rahman A, Divan G, Hamdani SU, et al. Effectiveness of the parent-mediated intervention for children with autism spectrum disorder in south Asia in India and Pakistan (PASS): a randomised controlled trial. Lancet Psychiatry. 2016 Feb;3(2):128-36. doi: 10.1016/s2215-0366(15)00388-0. PMID: 26704571.X-1

5944. Rai V. Association of methylenetetrahydrofolate reductase (MTHFR) gene C677T polymorphism with autism: evidence of genetic susceptibility. Metab Brain Dis. 2016 Mar 8doi: 10.1007/s11011-016-98150. PMID: 26956130.X-1

5945. Ramaekers VT, Sequeira JM, Quadros $\mathrm{EV}$. The basis for folinic acid treatment in neuro-psychiatric disorders. Biochimie. 2016 Jul;126:79-90. doi: 10.1016/j.biochi.2016.04.005. PMID: 27068282.X-1

5946. Ramoglu MG, Ucar T, Tutar E. Early malfunction of polyvinyl alcohol membrane of septal occluder. Catheter Cardiovasc Interv. 2016 Mar;87(4):E151-e3. doi: 10.1002/ccd.26057. PMID: 26106065.X-1

5947. Ranasinghe KG, Rankin KP, Pressman PS, et al. Distinct Subtypes of Behavioral Variant Frontotemporal Dementia Based on Patterns of Network Degeneration. JAMA Neurol. 2016 Jul 18doi: 10.1001/jamaneurol.2016.2016. PMID: 27429218.X-1

5948. Rangarajan P, Karthikeyan A, Dheen ST. Role of dietary phenols in mitigating microglia-mediated neuroinflammation. Neuromolecular Med. 2016 Jul 27doi: 10.1007/s12017-016-8430-x. PMID: 27465151.X-1

5949. Ranger P, Ellenbroek BA. Perinatal Influences of Valproate on Brain and Behaviour: An Animal Model for Autism. Curr Top Behav Neurosci. 2016;29:363-86. doi: 
10.1007/7854_2015_404. PMID: 26510739.X1

5950. Rankin JA, Weber RJ, Kang E, et al. Parent- and self-reported social skills importance in autism spectrum disorder. Journal of Autism and Developmental Disorders. 2016;46(1):273-86. doi: http://dx.doi.org/10.1007/s10803-015-25747.X-1

5951. Rapp JT, Cook JL, McHugh C, et al. Decreasing Stereotypy Using NCR and DRO With Functionally Matched Stimulation: Effects on Targeted and Non-Targeted Stereotypy.

Behav Modif. 2016 Jun 7doi: 10.1177/0145445516652370. PMID: 27268457.X-1

5952. Rapp JT, Gunby K. Task interspersal for individuals with autism and other neurodevelopmental disorders. J Appl Behav Anal. 2016 May 27doi: 10.1002/jaba.319. PMID: 27228967.X-1

5953. Rappaport SR, Kanter C, Anast K. Special considerations in child custody evaluations for children with high-functioning autism spectrum disorder. Family Court Review. 2016;54(1):81-95. doi: http://dx.doi.org/10.1111/fcre.12206.X-1

5954. Ratsep MT, Paolozza A, Hickman AF, et al. Brain Structural and Vascular Anatomy Is Altered in Offspring of Pre-Eclamptic Pregnancies: A Pilot Study. AJNR Am J Neuroradiol. 2016 May;37(5):939-45. doi: 10.3174/ajnr.A4640. PMID: 26721772.X-1

5955. Ratto AB, Anthony BJ, Pugliese C, et al. Lessons learned: Engaging culturally diverse families in neurodevelopmental disorders intervention research. Autism. 2016 Jun 15doi: 10.1177/1362361316650394. PMID: 27313190.X-1, X-2

5956. Rauh VA, Margolis AE. Research review: Environmental exposures, neurodevelopment, and child mental healthNew paradigms for the study of brain and behavioral effects. Journal of Child Psychology and Psychiatry. 2016;57(7):775-93. doi: http://dx.doi.org/10.1111/jcpp.12537.X-1

5957. Rausch A, Zhang W, Haak KV, et al. Altered functional connectivity of the amygdaloid input nuclei in adolescents and young adults with autism spectrum disorder: a resting state fMRI study. Mol Autism. 2016;7:13. doi: 10.1186/s13229-015-0060-x. PMID: 26823966.X-1

5958. Rayan A, Ahmad M. Effectiveness of mindfulness-based interventions on quality of life and positive reappraisal coping among parents of children with autism spectrum disorder. Res Dev Disabil. 2016 Apr 20;55:18596. doi: 10.1016/j.ridd.2016.04.002. PMID: 27107368.X-1

5959. Raynal P, Melioli T, Chabrol H. Personality profiles in young adults with disordered eating behavior. Eat Behav. 2016 Jun 3;22:119-23. doi: 10.1016/j.eatbeh.2016.06.020. PMID: 27289047.X-1

5960. Reddish P, Tok P, Kundt R. Religious cognition and behaviour in autism: The role of mentalizing. International Journal for the Psychology of Religion. 2016;26(2):95-112. doi:

http://dx.doi.org/10.1080/10508619.2014.10035 18.X-1

5961. Reed P, Sejunaite K, Osborne LA. Relationship between self-reported health and stress in mothers of children with autism spectrum disorders. Journal of Autism and Developmental Disorders. 2016;46(3):934-41. doi: http://dx.doi.org/10.1007/s10803-0152638-8.X-1

5962. Reid DB. Hypnosis Without Empathy? Perspectives From Autistic Spectrum Disorder and Stage Hypnosis. Am J Clin Hypn. 2016 Jan;58(3):304-20. doi: 10.1080/00029157.2015.1103204. PMID: 26675158.X-1

5963. Reiner G. Inborn error metabolic screening in nonsyndromic autism spectrum disorders. Dev Med Child Neurol. 2016 May 12doi: 10.1111/dmcn.13150. PMID: 27169684.X-1

5964. Reinvall O, Moisio A-L, LahtiNuuttila P, et al. Psychiatric symptoms in children and adolescents with higher functioning autism spectrum disorders on the Development and Well-Being Assessment. Research in Autism Spectrum Disorders. 
2016;25:47-57. doi: http://dx.doi.org/10.1016/j.rasd.2016.01.009.X1

5965. Rendall AR, Truong DT, Fitch RH. Learning delays in a mouse model of Autism Spectrum Disorder. Behav Brain Res. 2016 Apr 15;303:201-7. doi: 10.1016/j.bbr.2016.02.006. PMID: 26873041.X-1

5966. Rengit AC, McKowen JW, O'Brien J, et al. Brief Report: Autism Spectrum Disorder and Substance Use Disorder: A Review and Case Study. J Autism Dev Disord. 2016 Jul;46(7):2514-9. doi: 10.1007/s10803-0162763-z. PMID: 26944591.X-1

5967. Retico A, Giuliano A, Tancredi R, et al. The effect of gender on the neuroanatomy of children with autism spectrum disorders: a support vector machine case-control study. Mol Autism. 2016;7:5. doi: 10.1186/s13229-0150067-3. PMID: 26788282.X-1

5968. Rice K, Redcay E. Interaction matters: A perceived social partner alters the neural processing of human speech. Neuroimage. 2016 Apr 1;129:480-8. doi:

10.1016/j.neuroimage.2015.11.041. PMID: 26608245.X-1

5969. Richard AC, Rovelet-Lecrux A, Delaby E, et al. The 22q11 PRODH/DGCR6 deletion is frequent in hyperprolinemic subjects but is not a strong risk factor for ASD.

American Journal of Medical Genetics Part B: Neuropsychiatric Genetics. 2016;171(3):37782. doi: http://dx.doi.org/10.1002/ajmg.b.32416.X-1

5970. Richards C, Moss J, Nelson L, et al. Persistence of self-injurious behaviour in autism spectrum disorder over 3 years: A prospective cohort study of risk markers. Journal of Neurodevelopmental Disorders. 2016;8doi: http://dx.doi.org/10.1186/s11689016-9153-X.X-1

5971. Riddle K, Cascio CJ, Woodward ND. Brain structure in autism: a voxel-based morphometry analysis of the Autism Brain Imaging Database Exchange (ABIDE). Brain Imaging Behav. 2016 Mar 3doi: 10.1007/s11682-016-9534-5. PMID: 26941174.X-1
5972. Rigatelli G, Dell'Avvocata F, Giordan $\mathrm{M}$, et al. Incomplete Cor Triatriatum Dexter and Its Clinical and Technical Implications in Interatrial Shunt Device-Based Closure: An Intracardiac Echocardiography Study. Congenit Heart Dis. 2016 Feb 17doi: 10.1111/chd.12326. PMID: 26887534.X-1

5973. Rigatelli G, Pedon L, Zecchel R, et al. Long-Term Outcomes and Complications of Intracardiac Echocardiography-Assisted Patent Foramen Ovale Closure in 1,000 Consecutive Patients. J Interv Cardiol. 2016 Aug 8doi: 10.1111/joic.12325. PMID: 27500752.X-1

5974. Rizzo R, Pavone P. Aripiprazole for the treatment of irritability and aggression in children and adolescents affected by autism spectrum disorders. Expert Rev Neurother. 2016 Aug;16(8):867-74. doi: 10.1080/14737175.2016.1211007. PMID: 27388494.X-1, X-2

5975. Roane HS, Fisher WW, Carr JE. Applied Behavior Analysis as Treatment for Autism Spectrum Disorder. J Pediatr. 2016 May 11doi: 10.1016/j.jpeds.2016.04.023. PMID: 27179552.X-1, X-2

5976. Roberts JE, McCary LM, Shinkareva $\mathrm{SV}$, et al. Infant development in fragile $\mathrm{X}$ syndrome: Cross-syndrome comparisons. Journal of Autism and Developmental Disorders. 2016;46(6):2088-99. doi: http://dx.doi.org/10.1007/s10803-016-27371.X-1

5977. Roberts RM, Ejova A, Giallo R, et al. Support group programme for siblings of children with special needs: predictors of improved emotional and behavioural functioning. Disabil Rehabil. 2016 Jan 5:1-10. doi: 10.3109/09638288.2015.1116621. PMID: 26728633.X-1

5978. Robins DL, Adamson LB, Barton M, et al. Universal autism screening for toddlers: Recommendations at odds. Journal of Autism and Developmental Disorders.

2016;46(5):1880-2. doi: http://dx.doi.org/10.1007/s10803-016-26975.X-1

5979. Robinson AN, Roberts JE, Brady NC, et al. Physiological correlates of maternal responsivity in mothers of preschoolers with 
fragile X syndrome. American Journal on Intellectual and Developmental Disabilities. 2016;121(2):111-20. doi: http://dx.doi.org/10.1352/1944-7558121.2.111.X-1

5980. Robinson KJ, Hurd PL, Read S, et al. The PCSK6 gene is associated with handedness, the autism spectrum, and magical ideation in a non-clinical population.

Neuropsychologia. 2016 Apr;84:205-12. doi: 10.1016/j.neuropsychologia.2016.02.020.

PMID: 26921480.X-1

5981. Robinson S, Howlin P, Russell A. Personality traits, autobiographical memory and knowledge of self and others: A comparative study in young people with autism spectrum disorder. Autism. 2016 May 19doi: 10.1177/1362361316645429. PMID: 27197697.X-1

5982. Robinson-Shelton A, Malow BA. Sleep Disturbances in Neurodevelopmental Disorders. Curr Psychiatry Rep. 2016 Jan;18(1):6. doi: 10.1007/s11920-015-0638-1. PMID: 26719309.X-1

5983. Rodenas-Cuadrado P, Pietrafusa N, Francavilla T, et al. Characterisation of CASPR2 deficiency disorder--a syndrome involving autism, epilepsy and language impairment. BMC Med Genet. 2016;17:8. doi: 10.1186/s12881-016-0272-8. PMID: 26843181.X-1

5984. Rodgers J, Wigham S, McConachie H, et al. Development of the anxiety scale for children with autism spectrum disorder (ASCASD). Autism Res. 2016 Feb 17doi: 10.1002/aur.1603. PMID: 26887910.X-1

5985. Rodgers JD, Warhol A, Fox JD, et al. Minimal risk of internalizing problems in typically-developing siblings of children with high-functioning autism spectrum disorder. Journal of Child and Family Studies. 2016;25(8):2554-61. doi: http://dx.doi.org/10.1007/s10826-016-04078.X-1

5986. Rodogno R, Krause-Jensen K, Ashcroft RE. 'Autism and the good life': a new approach to the study of well-being. J Med Ethics. 2016 Jun;42(6):401-8. doi: 10.1136/medethics-2016-103595. PMID: 27174806.X-1

5987. Rodríguez-Barranco M, Gil F, Hernández AF, et al. Postnatal arsenic exposure and attention impairment in school children. Cortex: A Journal Devoted to the Study of the Nervous System and Behavior. 2016;74:370-82. doi:

http://dx.doi.org/10.1016/j.cortex.2014.12.018. $\mathrm{X}-1$

5988. Romano E, Cosentino L, Laviola G, et al. Genes and sex hormones interaction in neurodevelopmental disorders. Neurosci Biobehav Rev. 2016 Mar 4doi: 10.1016/j.neubiorev.2016.02.019. PMID: 26952805.X-1

5989. Ronconi L, Casartelli L, Carna S, et al. When one is Enough: Impaired Multisensory Integration in Cerebellar Agenesis. Cereb Cortex. 2016 Mar 5doi: 10.1093/cercor/bhw049. PMID: 26946125.X-1

5990. Ronconi L, Franchin L, Valenza E, et al. The attentional 'zoom-lens' in 8-month-old infants. Developmental Science.

2016;19(1):145-54. doi:

http://dx.doi.org/10.1111/desc.12288.X-1

5991. Rooney GE, Goodwin AF, Depeille P, et al. Human iPS Cell-Derived Neurons Uncover the Impact of Increased Ras Signaling in Costello Syndrome. J Neurosci. 2016 Jan 6;36(1):142-52. doi: 10.1523/jneurosci.154715.2016. PMID: 26740656.X-1

5992. Rosa M, Puig O, Lazaro L, et al. Socioeconomic status and intelligence quotient as predictors of psychiatric disorders in children and adolescents with high-functioning autism spectrum disorder and in their siblings. Autism. 2016 Feb 5doi: 10.1177/1362361315617881. PMID: 26851231.X-1

5993. Rosch KS, Dirlikov B, Mostofsky SH. Corrigendum to "Reduced intrasubject variability with reinforcement in boys, but not girls, with ADHD: Associations with prefrontal anatomy" [Biol. Psychol. 110 (2015) 12-23].

Biol Psychol. 2016 May;117:239. doi: 10.1016/j.biopsycho.2016.01.004. PMID: 27180014.X-1 
5994. Rose V, Trembath D, Keen D, et al. The proportion of minimally verbal children with autism spectrum disorder in a communitybased early intervention programme. Journal of Intellectual Disability Research.

2016;60(5):464-77. doi:

http://dx.doi.org/10.1111/jir.12284.X-1

5995. Rosen TE, Lerner MD. Externalizing and Internalizing Symptoms Moderate Longitudinal Patterns of Facial Emotion Recognition in Autism Spectrum Disorder. J Autism Dev Disord. 2016 Apr 28doi: 10.1007/s10803-016-2800-y. PMID: 27126817.x-1

5996. Roskam I, Stievenart M, de Mol J. The transactional process between the relationships with caregivers and children's externalizing behavior. Journal of Applied Developmental Psychology. 2016;42:31-9. doi: http://dx.doi.org/10.1016/j.appdev.2015.11.003. $\mathrm{X}-1$

5997. Ruiz LM, Damron M, Jones KB, et al. Antipsychotic use and metabolic monitoring in individuals with developmental disabilities served in a Medicaid medical home. Journal of Autism and Developmental Disorders. 2016;46(6):1887-94. doi: http://dx.doi.org/10.1007/s10803-016-2712$\mathrm{x} . \mathrm{X}-1$

5998. Rumondor AC, Dhareshwar SS, Kesisoglou F. Amorphous Solid Dispersions or Prodrugs: Complementary Strategies to Increase Drug Absorption. J Pharm Sci. 2016 Jan 22doi: 10.1016/j.xphs.2015.11.004. PMID: 26886316.X-1

5999. Ruparelia K, Abubakar A, Badoe E, et al. Autism Spectrum Disorders in Africa: Current Challenges in Identification, Assessment, and Treatment: A Report on the International Child Neurology Association Meeting on ASD in Africa, Ghana, April 3-5, 2014. J Child Neurol. 2016 Jul;31(8):1018-26. doi: 10.1177/0883073816635748. PMID: 26979098.X-1

6000. Russell AE, Ford T, Williams R, et al. The association between socioeconomic disadvantage and attention deficit/hyperactivity disorder (ADHD): A systematic review. Child Psychiatry and Human Development. 2016;47(3):440-58. doi: http://dx.doi.org/10.1007/s10578-015-05783.X-1

6001. Russo-Ponsaran NM, Evans-Smith B, Johnson J, et al. Efficacy of a facial emotion training program for children and adolescents with autism spectrum disorders. Journal of Nonverbal Behavior. 2016;40(1):13-38. doi: http://dx.doi.org/10.1007/s10919-015-02175.X-1

6002. Rutherford M, McKenzie K, Johnson $\mathrm{T}$, et al. Gender ratio in a clinical population sample, age of diagnosis and duration of assessment in children and adults with autism spectrum disorder. Autism. 2016;20(5):628-34. doi: http://dx.doi.org/10.1177/1362361315617879.X $-1$

6003. Rutherford M, McKenzie K, McClure I, et al. A national study to investigate the clinical use of standardised instruments in autism spectrum disorder assessment of children and adults in Scotland. Research in Autism Spectrum Disorders. 2016;29-30:93100. doi: http://dx.doi.org/10.1016/j.rasd.2016.05.003.X1

6004. Rutherford MD, Subiaul F. Children with autism spectrum disorder have an exceptional explanatory drive. Autism. 2016;20(6):744-53. doi: http://dx.doi.org/10.1177/1362361315605973.X $-1$

6005. Ruzich E, Allison C, Smith P, et al. The Autism-Spectrum Quotient in Siblings of People With Autism. Autism Res. 2016 Jun 22doi: 10.1002/aur.1651. PMID: 27333365.X-1

6006. Ruzzo EK, Geschwind DH. Schizophrenia genetics complements its mechanistic understanding. Nat Neurosci. 2016 Apr;19(4):523-5. doi: 10.1038/nn.4277. PMID: 26998600.X-1

6007. Ryan C, Furley P, Mulhall K. Judgments of Nonverbal Behaviour by Children with High-Functioning Autism Spectrum Disorder: Can they Detect Signs of Winning and Losing from Brief Video Clips? J Autism Dev Disord. 2016 Jun 15doi: 10.1007/s10803016-2839-9. PMID: 27306454.X-1 
6008. Rynkiewicz A, Schuller B, Marchi E, et al. An investigation of the 'female camouflage effect' in autism using a computerized ADOS-2 and a test of sex/gender differences. Mol Autism. 2016;7:10. doi: 10.1186/s13229-016-0073-0. PMID: 26798446.X-1

6009. Rzhepetskyy Y, Lazniewska J, Proft J, et al. A Cav3.2/Stac1 molecular complex controls T-type channel expression at the plasma membrane. Channels (Austin). 2016 May 5:1-9. doi: 10.1080/19336950.2016.1186318. PMID: 27149520.X-1

6010. Saad AF, Alshehri W, Lei J, et al. Maternal Fructose Consumption Disrupts Brain Development of Offspring in a Murine Model of Autism Spectrum Disorder. Am J Perinatol. 2016 May 2doi: 10.1055/s-0036-1583292. PMID: 27135955.X-1

6011. Sabatos-DeVito M, Schipul SE, Bulluck JC, et al. Eye tracking reveals impaired attentional disengagement associated with sensory response patterns in children with autism. Journal of Autism and Developmental Disorders. 2016 2016-03-30;46(4):1319-33. doi: http://dx.doi.org/10.1007/s10803-0152681-5. PMID: 1762039533; 2016-05140001.X-1

6012. Saeedi Saravi SS, Dehpour AR. Potential role of organochlorine pesticides in the pathogenesis of neurodevelopmental, neurodegenerative, and neurobehavioral disorders: A review. Life Sci. 2016 Jan 15;145:255-64. doi: 10.1016/j.lfs.2015.11.006. PMID: 26549647.X-1, X-2

6013. Sah SP, Bartakian S, El-Said H, et al. Preprocedural Transthoracic Echocardiography Can Predict Amplatzer Septal Occluder Device Size for Transcatheter Atrial Septal Defect Closure. Congenit Heart Dis. 2016 Apr 14doi: 10.1111/chd.12365. PMID: 27079542.X-1

6014. Saitovitch A, Popa T, Lemaitre H, et al. Tuning Eye-Gaze Perception by Transitory STS Inhibition. Cereb Cortex. 2016 Jun;26(6):2823-31. doi: 10.1093/cercor/bhw045. PMID: 26946130.X-1

6015. Sajith SG, Liew SF, Tor PC. Response to Electroconvulsive Therapy in Patients With
Autism Spectrum Disorder and Intractable Challenging Behaviors Associated With Symptoms of Catatonia. J ect. 2016 Jul 16doi: 10.1097/yct.0000000000000338. PMID: 27428481.X-1

6016. Sakai K, Shoji H, Kohno T, et al. Mice that lack the C-terminal region of Reelin exhibit behavioral abnormalities related to neuropsychiatric disorders. Sci Rep. 2016;6:28636. doi: 10.1038/srep28636. PMID: 27346785.X-1

6017. Sakaura H, Miwa T, Yamashita T, et al. Posterior lumbar interbody fusion with cortical bone trajectory screw fixation versus posterior lumbar interbody fusion using traditional pedicle screw fixation for degenerative lumbar spondylolisthesis: a comparative study. J Neurosurg Spine. 2016 May 27:1-5. doi: 10.3171/2016.3.spine151525. PMID: 27231813.X-1

6018. Salatino-Oliveira A, AkutagavaMartins GC, Bruxel EM, et al. NOS1 and SNAP25 polymorphisms are associated with Attention-Deficit/Hyperactivity Disorder symptoms in adults but not in children. Journal of Psychiatric Research. 2016;75:75-81. doi: http://dx.doi.org/10.1016/j.jpsychires.2016.01.0 10.X-1

6019. Salatino-Oliveira A, Wagner F, Akutagava-Martins GC, et al. MAP1B and NOS1 genes are associated with working memory in youths with attentiondeficit/hyperactivity disorder. European Archives of Psychiatry and Clinical Neuroscience. 2016;266(4):359-66. doi: http://dx.doi.org/10.1007/s00406-015-06269.X-1

6020. Salles J, Strelnikov K, Carine M, et al. Deficits in voice and multisensory processing in patients with Prader-Willi syndrome.

Neuropsychologia. 2016 May;85:137-47. doi: 10.1016/j.neuropsychologia.2016.03.015. PMID: 26994593.X-1

6021. Salomone E, Maurizio Arduino G. Parental attitudes to a telehealth parent coaching for autism spectrum disorder. J Telemed Telecare. 2016 Apr 6doi: 10.1177/1357633x16642067. PMID: 27056906.X-1, X-3 
6022. Salter K, Beamish W, Davies M. The effects of child-centered play therapy (CCPT) on the social and emotional growth of young Australian children with autism. International Journal of Play Therapy. 2016;25(2):78-90. doi: http://dx.doi.org/10.1037/pla0000012.X-3, X-4

6023. Salvano-Pardieu V, Blanc R, Combalbert $\mathrm{N}$, et al. Judgment of blame in teenagers with Asperger's syndrome. Thinking \& Reasoning. 2016;22(3):251-73. doi: http://dx.doi.org/10.1080/13546783.2015.11272 88.X-1

6024. Samanta D. Severe developmental delay and complete agenesis of corpus callosum in a Noonan syndrome patient with SOS1 mutation. Acta Neurologica Belgica.

2016;116(2):223-4. doi: http://dx.doi.org/10.1007/s13760-015-05269.X-1

6025. Sanders EJ, Irvin DW, Belardi K, et al. The questions verbal children with autism spectrum disorder encounter in the inclusive preschool classroom. Autism. 2016;20(1):96105. doi: http://dx.doi.org/10.1177/1362361315569744.X $-1$

6026. Sanefuji M, Craig M, Parlatini V, et al. Double-dissociation between the mechanism leading to impulsivity and inattention in Attention Deficit Hyperactivity Disorder: A resting-state functional connectivity study. Cortex. 2016 Jun 16doi: 10.1016/j.cortex.2016.06.005. PMID: 27394716.X-1

6027. Sanmann JN, Casas KA, Bevilacqua J, et al. The first patient with tandem duplication of 6q14q16: Molecular and phenotypic characterization. Am J Med Genet A. 2016 Jun 23doi: 10.1002/ajmg.a.37797. PMID: 27338032.X-1

6028. Santa Maria L, Aliaga S, Faundes V, et al. FMR1 gene mutations in patients with fragile X syndrome and obligate carriers: 30 years of experience in Chile. Genet Res (Camb). 2016;98:e11. doi:

10.1017/s0016672316000082. PMID: 27350105.X-1

6029. Santarosa LMC, Conforto D. Educational and digital inclusion for subjects with autism spectrum disorders in 1:1 technological configuration. Computers in Human Behavior. 2016;60:293-300. doi: http://dx.doi.org/10.1016/j.chb.2016.02.021.X1, X-3, X-4

6030. Santocchi E, Guiducci L, Fulceri F, et al. Gut to brain interaction in Autism Spectrum Disorders: a randomized controlled trial on the role of probiotics on clinical, biochemical and neurophysiological parameters. BMC Psychiatry. 2016;16:183. doi: 10.1186/s12888016-0887-5. PMID: 27260271.X-2, X-3

6031. Saresella M, Piancone F, Marventano I, et al. Multiple inflammasome complexes are activated in autistic spectrum disorders. Brain Behav Immun. 2016 Mar 12doi: 10.1016/j.bbi.2016.03.009. PMID: 26979869.X-1

6032. Sarnat H, Samuel E, Ashkenazi-Alfasi $\mathrm{N}$, et al. Oral Health Characteristics of Preschool Children with Autistic Syndrome Disorder. J Clin Pediatr Dent. 2016 Winter;40(1):21-5. doi: 10.17796/1053-462840.1.21. PMID: 26696102.X-1

6033. Sarowar T, Chhabra R, Vilella A, et al. Activity and circadian rhythm influence synaptic Shank3 protein levels in mice. J Neurochem. 2016 Jun 22doi: 10.1111/jnc.13709. PMID: 27329942.X-1

6034. Satoh M, Obara T, Nishigori H, et al. Prescription trends in children with pervasive developmental disorders (PDD): A claims databased study in Japan. World J Pediatr. 2016 Jun 10doi: 10.1007/s12519-016-0036-8. PMID: 27286689.X-1

6035. Scahill L, Bearss K, Lecavalier L, et al. Effect of Parent Training on Adaptive Behavior in Children With Autism Spectrum Disorder and Disruptive Behavior: Results of a Randomized Trial. J Am Acad Child Adolesc Psychiatry. 2016 Jul;55(7):602-9.e3. doi: 10.1016/j.jaac.2016.05.001. PMID: 27343887.X-1

6036. Scahill L, Jeon S, Boorin SJ, et al. Weight gain and metabolic consequences of risperidone in young children with autism spectrum disorder. Journal of the American Academy of Child \& Adolescent Psychiatry. 2016;55(5):415-23. doi: 
http://dx.doi.org/10.1016/j.jaac.2016.02.016.M EDICAL X-1

6037. Scemama C, Magrino B, Gillet P, et al. Risk of adjacent-segment disease requiring surgery after short lumbar fusion: results of the French Spine Surgery Society Series. J Neurosurg Spine. 2016 Jul;25(1):46-51. doi: 10.3171/2015.11.spine15700. PMID: 26967992.X-1

6038. Schall U. Is it time to move mismatch negativity into the clinic? Biol Psychol. 2016 Apr;116:41-6. doi: 10.1016/j.biopsycho.2015.09.001. PMID: 26342995.X-1

6039. Schatz RB, Peterson RK, Bellini S. The use of video self-modeling to increase ontask behavior in children with high-functioning autism. Journal of Applied School Psychology. 2016;32(3):234-53. doi:

http://dx.doi.org/10.1080/15377903.2016.11835 42.X-3, X-4

6040. Schauder KB, Bennetto L. Toward an Interdisciplinary Understanding of Sensory Dysfunction in Autism Spectrum Disorder: An Integration of the Neural and Symptom Literatures. Front Neurosci. 2016;10:268. doi: 10.3389/fnins.2016.00268. PMID: 27378838.X-1

6041. Scheckel C, Drapeau E, Frias MA, et al. Regulatory consequences of neuronal ELAV-like protein binding to coding and noncoding RNAs in human brain. Elife. 2016;5doi: 10.7554/eLife.10421. PMID: 26894958.X-1

6042. Scheer JK, Hostin R, Robinson C, et al. Operative Management of Adult Spinal Deformity Results in Significant Increases in QALYs Gained Compared to Non-operative Management: Analysis of 479 patients with Minimum 2-year Follow-up. Spine (Phila Pa 1976). 2016 Apr 11doi:

10.1097/brs.0000000000001626. PMID:

27253084.X-1

6043. Scheer JK, Osorio JA, Smith JS, et al. Development of Validated Computer Based Pre-operative Predictive Model for Proximal Junction Failure (PJF) or Clinically Significant PJK with 86\% Accuracy Based on 510 ASD Patients with 2-year Follow-up. Spine (Phila Pa 1976). 2016 Apr 1doi: 10.1097/brs.0000000000001598. PMID: 27046638.X-1

6044. Scheer JK, Passias PG, Sorocean AM, et al. Association between preoperative cervical sagittal deformity and inferior outcomes at 2year follow-up in patients with adult thoracolumbar deformity: analysis of 182 patients. J Neurosurg Spine. 2016 Jan;24(1):108-15. doi: 10.3171/2015.3.spine141098. PMID: 26360147.X-1

6045. Schelinski S, Borowiak K, von Kriegstein K. Temporal voice areas exist in autism spectrum disorder but are dysfunctional for voice identity recognition. Soc Cogn Affect Neurosci. 2016 Jun 30doi: 10.1093/scan/nsw089. PMID: 27369067.X-1

6046. Scherr JF, Hahn LJ, Hooper SR, et al. HPA axis function predicts development of working memory in boys with FXS. Brain and Cognition. 2016;102:80-90. doi: http://dx.doi.org/10.1016/j.bandc.2015.12.002. $\mathrm{X}-1$

6047. Schlebusch L, Samuels AE, Dada S. South African families raising children with autism spectrum disorders: Relationship between family routines, cognitive appraisal and family quality of life. Journal of Intellectual Disability Research. 2016;60(5):412-23. doi: http://dx.doi.org/10.1111/jir.12292.X-1

6048. Schneider A, Johnston C, Tassone F, et al. Broad autism spectrum and obsessivecompulsive symptoms in adults with the fragile X premutation. Clin Neuropsychol. 2016 Jun 29:1-15. doi: 10.1080/13854046.2016.1189536. PMID: 27355445.X-1

6049. Schneider HE, Lam JC, Mahone EM. Sleep disturbance and neuropsychological function in young children with ADHD. Child Neuropsychology. 2016;22(4):493-506. doi: http://dx.doi.org/10.1080/09297049.2015.10181 53.X-1

6050. Schneider M, de Vries PJ, Schonig K, et al. mTOR inhibitor reverses autistic-like social deficit behaviours in adult rats with both Tsc2 haploinsufficiency and developmental status epilepticus. Eur Arch Psychiatry Clin Neurosci. 2016 Jun 4doi: 10.1007/s00406-0160703-8. PMID: 27263037.X-1 
6051. Schoch H, Kreibich AS, Ferri SL, et al. Sociability Deficits and Altered Amygdala Circuits in Mice Lacking Pcdh10, an Autism Associated Gene. Biol Psychiatry. 2016 Jun 16doi: 10.1016/j.biopsych.2016.06.008. PMID: 27567313.X-1

6052. Schrantee A, Tamminga HG, Bouziane $\mathrm{C}$, et al. Age-Dependent Effects of Methylphenidate on the Human Dopaminergic System in Young vs Adult Patients With Attention-Deficit/Hyperactivity Disorder: A Randomized Clinical Trial. JAMA Psychiatry. 2016 Aug 3doi:

10.1001/jamapsychiatry.2016.1572. PMID: 27487479.X-1

6053. Schrooten I, Scholte RH, Cillessen AH, et al. Participant Roles in Bullying Among Dutch Adolescents With Autism Spectrum Disorders. J Clin Child Adolesc Psychol. 2016 Mar 30:1-14. doi:

10.1080/15374416.2016.1138411. PMID: 27029898.X-1

6054. Schuch JB, Müller D, Endres RG, et al. Psychomotor agitation and mood instability in patients with autism spectrum disorders: A possible effect of SLC6A4 gene? Research in Autism Spectrum Disorders. 2016;26:48-56. doi: http://dx.doi.org/10.1016/j.rasd.2016.03.001.X1

6055. Schuetze M, Park MT, Cho IY, et al. Morphological Alterations in the Thalamus, Striatum, and Pallidum in Autism Spectrum Disorder. Neuropsychopharmacology. 2016 Jun 8doi: 10.1038/npp.2016.64. PMID:

27125303.X-1

6056. Schuh JM, Eigsti IM, Mirman D. Discourse comprehension in autism spectrum disorder: Effects of working memory load and common ground. Autism Res. 2016 Apr 19doi: 10.1002/aur.1632. PMID: 27091496.X-1

6057. Schulte-Ruther M, Otte E, Adiguzel K, et al. Intact mirror mechanisms for automatic facial emotions in children and adolescents with autism spectrum disorder. Autism Res. 2016 Jun 28doi: 10.1002/aur.1654. PMID: 27349835.X-1
6058. Schulze A, Bauman M, Tsai AC, et al. Prevalence of Creatine Deficiency Syndromes in Children With Nonsyndromic Autism. Pediatrics. 2016 Jan;137(1)doi: 10.1542/peds.2015-2672. PMID: 26684475.X-1

6059. Schur RR, Draisma LW, Wijnen JP, et al. Brain GABA levels across psychiatric disorders: A systematic literature review and meta-analysis of $1 \mathrm{H}$-MRS studies. Hum Brain Mapp. 2016 May 4doi: 10.1002/hbm.23244. PMID: 27145016.X-1

6060. Schwartzberg ET, Silverman MJ. Effects of a music-based short story on shortand long-term reading comprehension of individuals with Autism Spectrum Disorder: A cluster randomized study. The Arts in Psychotherapy. 2016;48:54-61. doi: http://dx.doi.org/10.1016/j.aip.2016.01.001.X$1, \mathrm{X}-4$

6061. Schwartzer JJ, Onore CE, Rose D, et al. C57BL/6J bone marrow transplant increases sociability in BTBR T+ Itpr3tf/J mice. Brain Behav Immun. 2016 May 26doi: 10.1016/j.bbi.2016.05.019. PMID: 27235929.X-1

6062. Schweitzer J, James C, Jenkins W, et al. Acute Agitation and Self-Injury in a 5-Year Old with Autism. J Dev Behav Pediatr. 2016 Jun 28doi: 10.1097/dbp.0000000000000324. PMID: 27355884.X-1

6063. Schwichtenberg AJ, Hensle T, Honaker S, et al. Sibling sleep-What can it tell us about parental sleep reports in the context of autism? Clinical Practice in Pediatric Psychology. 2016;4(2):137-52. doi: http://dx.doi.org/10.1037/cpp0000143.X-1

6064. Sciberras E, Bisset M, Hazell P, et al. Health-related impairments in young children with ADHD: A community-based study. Child: Care, Health and Development.

2016;42(5):709-17. doi: http://dx.doi.org/10.1111/cch.12363.X-1

6065. Sciubba DM, Scheer JK, Yurter A, et al. Patients with spinal deformity over the age of 75: a retrospective analysis of operative versus non-operative management. Eur Spine J. 2016 Aug;25(8):2433-41. doi: 10.1007/s00586015-3759-7. PMID: 25657104.X-1 
6066. Scott RC, Tuchman R. Epilepsy and autism spectrum disorders: Relatively related. Neurology. 2016 Jun 15doi:

10.1212/wnl.0000000000002852. PMID: 27306638.X-1

6067. Scroggins ML, Litchke LG, Liu T. Effects of multisensory yoga on behavior in a male child with Apert and Asperger syndrome. Int J Yoga. 2016 Jan-Jun;9(1):81-4. doi: 10.4103/0973-6131.171716. PMID: 26865777.X-1, X-3, X-4

6068. Sedgewick F, Hill V, Yates R, et al. Gender differences in the social motivation and friendship experiences of autistic and nonautistic adolescents. Journal of Autism and Developmental Disorders. 2016;46(4):1297306. doi: http://dx.doi.org/10.1007/s10803-0152669-1.X-1

6069. Sedney CL, Daffner SD, Stefanko JJ, et al. Fracture of fusion mass after hardware removal in patients with high sagittal imbalance. J Neurosurg Spine. 2016 Apr;24(4):639-43. doi: 10.3171/2015.7.spine15153. PMID: 26682604.X-1

6070. Self TL, Parham DF. Students' selfperceptions of interprofessional education following participation on a diagnostic team for autism spectrum disorder. J Interprof Care. 2016 Jun 17:1-3. doi: 10.1080/13561820.2016.1181615. PMID: 27314474.X-1

6071. Sellers TP, Kelley K, Higbee TS, et al. Effects of simultaneous script training on use of varied mand frames by preschoolers with autism. Analysis of Verbal Behavior. 2016;32(1):15-26. doi: http://dx.doi.org/10.1007/s40616-015-00498. X-1, X-3, X-4

6072. Sellinger VJ, Elder JH. Parent Training Intervention to Manage Externalizing Behaviors in Children With Autism. J Pediatr Health Care. 2016 Jan-Feb;30(1):73-7. doi: 10.1016/j.pedhc.2015.10.002. PMID: 26559136.X-3, X-4

6073. Semrud-Clikeman M, Fine JG, Bledsoe J. Social functioning using direct and indirect measures with children with High Functioning Autism, nonverbal learning disability, and typically developing children. Child Neuropsychol. 2016;22(3):318-35. doi: 10.1080/09297049.2014.994487. PMID: 25711370.X-1

6074. Sener EF, Canatan H, Ozkul Y. Recent Advances in Autism Spectrum Disorders: Applications of Whole Exome Sequencing Technology. Psychiatry Investig. 2016 May;13(3):255-64. doi: 10.4306/pi.2016.13.3.255. PMID: 27247591.X1

6075. Sener EF, Uytun MC, Bayramov KK, et al. The roles of CC2D1A and HTR1A gene expressions in autism spectrum disorders. Metabolic Brain Disease. 2016;31(3):613-9. doi: http://dx.doi.org/10.1007/s11011-0169795-0.X-1

6076. Senland AK, Higgins-D'Alessandro A. Sociomoral Reasoning, Empathy, and Meeting Developmental Tasks During the Transition to Adulthood in Autism Spectrum Disorder. J Autism Dev Disord. 2016 Jul 1doi: 10.1007/s10803-016-2849-7. PMID: 27371138.X-1

6077. Senokossoff GW. Developing reading comprehension skills in high-functioning children with autism spectrum disorder: A review of the research, 1990-2012. Reading \& Writing Quarterly: Overcoming Learning Difficulties. 2016;32(3):223-46. doi: http://dx.doi.org/10.1080/10573569.2014.93657 4.X-1, X-2

6078. Sequeira JM, Desai A, BerrocalZaragoza MI, et al. Exposure to Folate Receptor Alpha Antibodies during Gestation and Weaning Leads to Severe Behavioral Deficits in Rats: A Pilot Study. PLoS One.

2016;11(3):e0152249. doi: 10.1371/journal.pone.0152249. PMID: 27011008.X-1

6079. Shah P. Interoception: The Eighth Sensory System: Practical Solutions for Improving Self-Regulation, Self-Awareness and Social Understanding of Individuals with Autism Spectrum and Related Disorders : K. J. Mahler: Shawnee Mission KS, AAPC, 2015, 186 pp, $\$ 29.95$ (paper), ISBN 978-1-94219714-0. J Autism Dev Disord. 2016 Jun 25doi: 10.1007/s10803-016-2848-8. PMID: 27344338.X-1 
6080. Shah P, Hall R, Catmur C, et al. Alexithymia, not autism, is associated with impaired interoception. Cortex. $2016 \mathrm{Apr}$ 5;81:215-20. doi: 10.1016/j.cortex.2016.03.021. PMID: 27253723.X-1

6081. Shamblin S, Graham D, Bianco JA. Creating trauma-informed schools for rural appalachia: The partnerships program for enhancing resiliency, confidence and workforce development in early childhood education. School Mental Health. 2016;8(1):189-200. doi: http://dx.doi.org/10.1007/s12310-016-91814.X-1

6082. Shang L, Henderson LB, Cho MT, et al. De novo missense variants in PPP2R5D are associated with intellectual disability, macrocephaly, hypotonia, and autism. Neurogenetics. 2016;17(1):43-9. doi: http://dx.doi.org/10.1007/s10048-015-04669.X-1

6083. Shapiro E, King K, Ahmed A, et al. The Neurobehavioral Phenotype in Mucopolysaccharidosis Type IIIB: an Exploratory Study. Mol Genet Metab Rep. 2016 Mar 1;6:41-7. doi: 10.1016/j.ymgmr.2016.01.003. PMID: 26918231.X-1

6084. Sharda M, Foster NE, Tryfon A, et al. Language Ability Predicts Cortical Structure and Covariance in Boys with Autism Spectrum Disorder. Cereb Cortex. 2016 Feb 17doi: 10.1093/cercor/bhw024. PMID: 26891985.X-1

6085. Sharpe K, Di Pietro N, Jacob KJ, et al. A Dichotomy of Information-Seeking and Information-Trusting: Stem Cell Interventions and Children with Neurodevelopmental Disorders. Stem Cell Rev. 2016 Aug;12(4):43847. doi: 10.1007/s12015-016-9667-3. PMID: 27286955.X-1

6086. Sharpley CF, Bitsika V, Andronicos $\mathrm{NM}$, et al. Is afternoon cortisol more reliable than waking cortisol in association studies of children with an ASD? Physiology \& Behavior. 2016;155:218-23. doi:

http://dx.doi.org/10.1016/j.physbeh.2015.12.02 $0 . \mathrm{X}-1$

6087. Sharpley CF, Bitsika V, Jesulola E, et al. The association between aspects of psychological resilience and subtypes of depression: implications for focussed clinical treatment models. Int J Psychiatry Clin Pract. 2016 Sep;20(3):151-6. doi: 10.1080/13651501.2016.1199810. PMID: 27332989.X-1

6088. Sharpley CF, Christie DR, Bitsika V, et al. Trajectories of total depression and depressive symptoms in prostate cancer patients receiving six months of hormone therapy. Psychooncology. 2016 Feb 9doi: 10.1002/pon.4100. PMID: 26857160.X-1

6089. Shatrov AB, Soldatenko EV. Dermal glands in freshwater mites Limnesia undulata (O.F. Muller, 1776) and Limnesia fulgida (C.L. Koch, 1836) (Acariformes, Limnesiidae). Arthropod Struct Dev. 2016 Jun 14doi: 10.1016/j.asd.2016.05.003. PMID: 27256546.X-1

6090. Shehata AI, Hassanein FI, AbdulGhani R. Seroprevalence of Toxoplasma gondii infection among patients with non-

schizophrenic neurodevelopmental disorders in Alexandria, Egypt. Acta Trop. 2016

Feb;154:155-9. doi:

10.1016/j.actatropica.2015.11.016. PMID: 26656562.X-1

6091. Shelton AL, Cornish KM, Kraan CM, et al. Executive Dysfunction in Female FMR1 Premutation Carriers. Cerebellum. 2016 Apr 28doi: 10.1007/s12311-016-0782-0. PMID: 27126308.X-1

6092. Shen Q, Wang G, Li S, et al. ASD v3.0: unraveling allosteric regulation with structural mechanisms and biological networks. Nucleic Acids Res. 2016 Jan 4;44(D1):D52735. doi: 10.1093/nar/gkv902. PMID: 26365237.X-1

6093. Shen Y, Xun G, Guo H, et al. Association and gene-gene interactions study of reelin signaling pathway related genes with autism in the Han Chinese population. Autism Research. 2016;9(4):436-42. doi: http://dx.doi.org/10.1002/aur.1540.X-1

6094. Shermer M. The Quack of the Gaps Problem. Sci Am. 2016 Aug;315(2):75. doi: 10.1038/scientificamerican0816-75. PMID: 27459575.X-1 
6095. Shield A, Pyers J, Martin A, et al. Relations between language and cognition in native-signing children with autism spectrum disorder. Autism Res. 2016 Mar 3doi: 10.1002/aur.1621. PMID: 26938935.X-1

6096. Shillingsburg MA, Gayman CM, Walton W. Using textual prompts to teach mands for information using "who?”. Analysis of Verbal Behavior. 2016;32(1):1-14. doi: http://dx.doi.org/10.1007/s40616-016-00537.X-1, X-3, X-4

6097. Shire SY, Gulsrud A, Kasari C. Increasing responsive parent-child interactions and joint engagement: Comparing the influence of parent-mediated intervention and parent psychoeducation. Journal of Autism and Developmental Disorders. 2016;46(5):1737-47. doi: http://dx.doi.org/10.1007/s10803-0162702-z.X-1

6098. Shireman ML, Lerman DC, Hillman CB. Teaching social play skills to adults and children with autism as an approach to building rapport. J Appl Behav Anal. 2016 Mar 15doi: 10.1002/jaba.299. PMID: 26990643.X-1

6099. Shirley MD, Frelin L, López JS, et al. Copy number variants associated with 14 cases of self-injurious behavior. PLoS ONE.

2016;11(3).X-1

6100. Shochet IM, Saggers BR, Carrington SB, et al. The Cooperative Research Centre for Living with Autism (Autism CRC) Conceptual Model to Promote Mental Health for Adolescents with ASD. Clin Child Fam Psychol Rev. 2016 Jun;19(2):94-116. doi: 10.1007/s10567-016-0203-4. PMID: 27072681.X-1

6101. Shrader-Frechette K, ChoGlueck C. Pesticides, Neurodevelopmental Disagreement, and Bradford Hill's Guidelines. Account Res. 2016 Jun 27doi: 10.1080/08989621.2016.1203786. PMID: 27348618.X-1

6102. Shtayermman O. Review of CBT for children and adolescents with high functioning autism spectrum disorders. Journal of Autism and Developmental Disorders.

2016;46(4):1500-1. doi: http://dx.doi.org/10.1007/s10803-015-2686$0 . \mathrm{X}-1, \mathrm{X}-2$
6103. Silbaugh BC, Falcomata TS.

Translational evaluation of a lag schedule and variability in food consumed by a boy with autism and food selectivity. Dev Neurorehabil. 2016 Mar 28:1-4. doi:

10.3109/17518423.2016.1146364. PMID: 27019276.X-1, X-3

6104. Silva G, Ribeiro MJ, Costa GN, et al. Peripheral Attentional Targets under Covert Attention Lead to Paradoxically Enhanced Alpha Desynchronization in Neurofibromatosis Type 1. PLoS One. 2016;11(2):e0148600. doi: 10.1371/journal.pone.0148600. PMID: 26881921.X-1

6105. Silva-Martinez GA, Rodriguez-Rios D, Alvarado-Caudillo Y, et al. Arachidonic and oleic acid exert distinct effects on the DNA methylome. Epigenetics. 2016 May 3;11(5):321-34. doi: 10.1080/15592294.2016.1161873. PMID: 27088456.X-1

6106. Silverstein M, Radesky J. Embrace the Complexity: The US Preventive Services Task Force Recommendation on Screening for Autism Spectrum Disorder. Jama. 2016 Feb 16;315(7):661-2. doi: 10.1001/jama.2016.0051. PMID: 26881368.X-1

6107. Simon DM, Wallace MT. Dysfunction of sensory oscillations in Autism Spectrum Disorder. Neurosci Biobehav Rev. 2016 Jul 19;68:848-61. doi:

10.1016/j.neubiorev.2016.07.016. PMID: 27451342.X-1

6108. Simoni ZR. Do social skills mediate the relationship between ADHD and depression? Sociological Spectrum. 2016;36(2):109-22. doi: http://dx.doi.org/10.1080/02732173.2015.10956 62.X-1

6109. Simonstein F, Mashiach-Eizenberg M. Attitudes Toward Autism Spectrum Disorders Among Students of Allied Health Professions. J Genet Couns. 2016 Jun 2doi: 10.1007/s10897016-9969-2. PMID: 27250068.X-1

6110. Simsek S, Cetin I, Cim A, et al. Elevated levels of tissue plasminogen activator and E-selectin in male children with autism 
spectrum disorder. Autism Res. 2016 May 19doi: 10.1002/aur.1638. PMID: 27194368.X-1

6111. Sinclair D, Oranje B, Razak KA, et al. Sensory processing in autism spectrum disorders and Fragile $\mathrm{X}$ syndrome-From the clinic to animal models. Neurosci Biobehav Rev. 2016 May 24doi: 10.1016/j.neubiorev.2016.05.029. PMID: 27235081.X-1

6112. Singh JS. Parenting work and autism trajectories of care. Sociol Health Illn. 2016 Apr 25doi: 10.1111/1467-9566.12437. PMID: 27111881.X-1

6113. Singh NN, Lancioni GE, Karazsia BT, et al. Effects of Samatha meditation on active academic engagement and math performance of students with attention deficit/hyperactivity disorder. Mindfulness. 2016;7(1):68-75. doi: http://dx.doi.org/10.1007/s12671-015-04245.X-1

6114. Singh SK, Stogsdill JA, Pulimood NS, et al. Astrocytes Assemble Thalamocortical Synapses by Bridging NRX1alpha and NL1 via Hevin. Cell. 2016 Jan 14;164(1-2):183-96. doi: 10.1016/j.cell.2015.11.034. PMID: 26771491.X-1

6115. Singman EL, Daphalapurkar N, White $\mathrm{H}$, et al. Indirect traumatic optic neuropathy. Mil Med Res. 2016;3:2. doi: 10.1186/s40779016-0069-2. PMID: 26759722.X-1

6116. Sirin N, Tekin-Iftar E. Opinions of Turkish Parents and Teachers About Safety Skills Instruction to Children with Autism Spectrum Disorders: A Preliminary Investigation. J Autism Dev Disord. 2016 May 11doi: 10.1007/s10803-016-2809-2. PMID: 27166959.X-1

6117. Siu AL, Bibbins-Domingo K, Grossman DC, et al. Screening for Autism Spectrum Disorder in Young Children: US Preventive Services Task Force

Recommendation Statement. Jama. 2016 Feb 16;315(7):691-6. doi: 10.1001/jama.2016.0018. PMID: 26881372.X-1

6118. Siu W-K, Lam C-W, Gao W-W, et al. Unmasking a novel disease gene NEO1 associated with autism spectrum disorders by a hemizygous deletion on chromosome 15 and a functional polymorphism. Behavioural Brain Research. 2016;300:135-42. doi: http://dx.doi.org/10.1016/j.bbr.2015.10.041.X-1

6119. Sivarao DV, Chen P, Senapati A, et al. $40 \mathrm{~Hz}$ Auditory Steady-State Response Is a Pharmacodynamic Biomarker for Cortical NMDA Receptors. Neuropsychopharmacology. 2016 Feb 3doi: 10.1038/npp.2016.17. PMID: 26837462.X-1

6120. Skewes JC, Gebauer L. Brief Report: Suboptimal Auditory Localization in Autism Spectrum Disorder: Support for the Bayesian Account of Sensory Symptoms. J Autism Dev Disord. 2016 Jul;46(7):2539-47. doi: 10.1007/s10803-016-2774-9. PMID: 27011323.X-1

6121. Skold A, Janeslatt GK. Self-rating of daily time management in children: psychometric properties of the Time-S. Scand J Occup Ther. 2016 Jul 8:1-9. doi: 10.1080/11038128.2016.1185465. PMID: 27387553.X-1

6122. Skonieczna-Zydecka K, Gorzkowska I, Pierzak-Sominka J, et al. The Prevalence of Autism Spectrum Disorders in West Pomeranian and Pomeranian Regions of Poland. J Appl Res Intellect Disabil. 2016 Jan 14doi: 10.1111/jar.12238. PMID: 26771078.X1

6123. Skosnik PD, Cortes-Briones JA. Targeting the ecology within: The role of the gut-brain axis and human microbiota in drug addiction. Med Hypotheses. 2016 Aug;93:7780. doi: 10.1016/j.mehy.2016.05.021. PMID: 27372861.X-1

6124. Slappendel G, Mandy W, van der Ende $\mathrm{J}$, et al. Utility of the 3Di short version for the diagnostic assessment of autism spectrum disorder and compatibility with DSM-5. Journal of Autism and Developmental Disorders. 2016;46(5):1834-46. doi: http://dx.doi.org/10.1007/s10803-016-27139.X-1

6125. Slattery L, Crosland K, Iovannone R. An evaluation of a self-management intervention to increase on-task behavior with individuals diagnosed with attentiondeficit/hyperactivity disorder. Journal of Positive Behavior Interventions. 
2016;18(3):168-79. doi:

http://dx.doi.org/10.1177/1098300715588282.X $-1$

6126. Smallwood M, Sareen A, Baker E, et al. Increased Risk of Autism Development in Children Whose Mothers Experienced Birth Complications or Received Labor and Delivery Drugs. ASN Neuro. 2016 Aug;8(4)doi: 10.1177/1759091416659742. PMID: 27511908.X-1

6127. Smith CE, Carr EG, Moskowitz LJ. Fatigue as a biological setting event for severe problem behavior in autism spectrum disorder. Research in Autism Spectrum Disorders. 2016;23:131-44. doi: http://dx.doi.org/10.1016/j.rasd.2015.12.003.X$1, \mathrm{X}-3, \mathrm{X}-4$

6128. Smith CJ, Rozga A, Matthews N, et al. Investigating the Accuracy of a Novel Telehealth Diagnostic Approach for Autism Spectrum Disorder. Psychol Assess. 2016 May 19doi: 10.1037/pas0000317. PMID: 27196689.X-1

6129. Smith DP, Eikeseth S, Fletcher SE, et al. Emergent intraverbal forms may occur as a result of listener training for children with autism. Analysis of Verbal Behavior. 2016;32(1):27-37. doi: http://dx.doi.org/10.1007/s40616-016-00573.X-1, X-4

6130. Smith E, Meyer BJ, Koerting J, et al. Preschool hyperactivity specifically elevates long-term mental health risks more strongly in males than females: a prospective longitudinal study through to young adulthood. Eur Child Adolesc Psychiatry. 2016 Jun 13doi: 10.1007/s00787-016-0876-8. PMID: 27295115.X-1

6131. Smith E, Thurm A, Greenstein D, et al. Cortical thickness change in autism during early childhood. Human Brain Mapping.

2016;37(7):2616-29. doi:

http://dx.doi.org/10.1002/hbm.23195.X-1

6132. Smith IM. Expanding our understanding of behavioural difficulties associated with autism spectrum disorder. Dev Med Child Neurol. 2016 Feb;58(2):121-2. doi: 10.1111/dmcn.12855. PMID: 26219422.X-1
6133. Smith J, Rho JM, Teskey GC. Ketogenic diet restores aberrant cortical motor maps and excitation-to-inhibition imbalance in the BTBR mouse model of autism spectrum disorder. Behav Brain Res. 2016 May 1;304:6770. doi: 10.1016/j.bbr.2016.02.015. PMID: 26876011.X-1

6134. Smith JC. The embodied becoming of autism and childhood: A storytelling methodology. Disability \& Society. 2016;31(2):180-91.X-1

6135. Smith JS, Klineberg E, Lafage V, et al. Prospective multicenter assessment of perioperative and minimum 2-year postoperative complication rates associated with adult spinal deformity surgery. $J$ Neurosurg Spine. 2016 Jul;25(1):1-14. doi: 10.3171/2015.11.spine151036. PMID: 26918574.X-1

6136. Smith JS, Lafage V, Shaffrey CI, et al. Outcomes of Operative and Nonoperative Treatment for Adult Spinal Deformity: A Prospective, Multicenter, Propensity-Matched Cohort Assessment With Minimum 2-Year Follow-up. Neurosurgery. 2016 Jun;78(6):85161. doi: 10.1227/neu.0000000000001116. PMID: 26579966.X-1

6137. Smith L, Malcolm-Smith S, de Vries PJ. Translation and cultural appropriateness of the Autism Diagnostic Observation Schedule-2 in Afrikaans. Autism. 2016 May 25doi: 10.1177/1362361316648469. PMID: 27231336.X-1

6138. Smith LE, Hong J, Greenberg JS, et al. Change in the behavioral phenotype of adolescents and adults with FXS: Role of the family environment. Journal of Autism and Developmental Disorders. 2016;46(5):1824-33. doi: http://dx.doi.org/10.1007/s10803-0162714-8.X-1

6139. Smithers-Sheedy H, McIntyre S, Gibson C, et al. A special supplement: Findings from the australian cerebral palsy register, birth years 1993 to 2006. Developmental Medicine \& Child Neurology. 2016;58(Suppl 2):5-10. doi: http://dx.doi.org/10.1111/dmcn.13026.X-1

6140. Smolensky MH, Hermida RC,

Reinberg A, et al. Circadian disruption: New clinical perspective of disease pathology and 
basis for chronotherapeutic intervention. Chronobiol Int. 2016 Jun 16:1-19. doi: 10.1080/07420528.2016.1184678. PMID: 27308960.X-1

6141. Snow M, Donnelly J. Factors Mediating Dysphoric Moods and Help Seeking Behaviour Among Australian Parents of Children with Autism. J Autism Dev Disord. 2016 Jun;46(6):1941-52. doi: 10.1007/s10803016-2725-5. PMID: 26858033.X-1

6142. So W-C, Wong MK-Y. I use my space not yours: Use of gesture space for referential identification among children with autism spectrum disorders. Research in Autism Spectrum Disorders. 2016;26:33-47. doi: http://dx.doi.org/10.1016/j.rasd.2016.03.005.X1

6143. Söderlund GBW, Jobs EN. Differences in speech recognition between children with attention deficits and typically developed children disappear when exposed to $65 \mathrm{~dB}$ of auditory noise. Frontiers in Psychology. 2016;7.X-1

6144. Sokhadze EM, Tasman A, Sokhadze GE, et al. Behavioral, cognitive, and motor preparation deficits in a visual cued spatial attention task in autism spectrum disorder. Applied Psychophysiology and Biofeedback. 2016;41(1):81-92. doi: http://dx.doi.org/10.1007/s10484-015-9313x.X-1

6145. Sollis E, Graham SA, Vino A, et al. Identification and functional characterization of de novo FOXP1 variants provides novel insights into the etiology of neurodevelopmental disorder. Hum Mol Genet. 2016 Feb 1;25(3):546-57. doi: 10.1093/hmg/ddv495. PMID: 26647308.X-1

6146. Solomon M, McCauley JB, Iosif AM, et al. Cognitive control and episodic memory in adolescents with autism spectrum disorders. Neuropsychologia. 2016 May 13;89:31-41. doi: 10.1016/j.neuropsychologia.2016.05.013. PMID: 27184119.X-1

6147. Solomon O, Heritage J, Yin L, et al. 'What brings him here today?': Medical problem presentation involving children with autism spectrum disorders and typically developing children. Journal of Autism and
Developmental Disorders. 2016;46(2):378-93. doi: http://dx.doi.org/10.1007/s10803-0152550-2.X-1

6148. Solso S, Xu R, Proudfoot J, et al. Diffusion tensor imaging provides evidence of possible axonal overconnectivity in frontal lobes in autism spectrum disorder toddlers. Biological Psychiatry. 2016;79(8):676-84. doi: http://dx.doi.org/10.1016/j.biopsych.2015.06.02 9. $x-1$

6149. Somogyi E, Kapitány E, Kenyeres K, et al. Visual feedback increases postural stability in children with autism spectrum disorder. Research in Autism Spectrum Disorders. 2016;29-30:48-56. doi: http://dx.doi.org/10.1016/j.rasd.2016.06.001.X1

6150. Song Y, Hakoda Y, Sang B. A selective impairment in extracting fearful information from another's eyes in Autism. Autism Res. 2016 Jan 18doi: 10.1002/aur.1583. PMID: 26777988.X-1

6151. Soroceanu A, Burton DC, Oren JH, et al. Medical Complications after Adult Spinal Deformity Surgery: Incidence, Risk factors, and Clinical Impact. Spine (Phila Pa 1976). 2016 Apr 19doi: 10.1097/brs.0000000000001636. PMID: 27105460.X-1

6152. Soumiya H, Godai A, Araiso H, et al. Neonatal Whisker Trimming Impairs Fear/Anxiety-Related Emotional Systems of the Amygdala and Social Behaviors in Adult Mice. PLoS One. 2016;11(6):e0158583. doi: 10.1371/journal.pone.0158583. PMID: 27362655.X-1

6153. Sparapani N, Morgan L, Reinhardt VP, et al. Evaluation of classroom active engagement in elementary students with autism spectrum disorder. Journal of Autism and Developmental Disorders. 2016;46(3):782-96. doi: http://dx.doi.org/10.1007/s10803-0152615-2.X-1

6154. Spaulding CJ, Lerner MD, Gadow KD. Trajectories and correlates of special education supports for youth with autism spectrum disorder and psychiatric comparisons. Autism. 2016 May 12doi: 10.1177/1362361316645428. PMID: 27178997.X-1 
6155. Specht MW, Mahone EM, Kline T, et al. Efficacy of parent-delivered behavioral therapy for primary complex motor stereotypies. Dev Med Child Neurol. 2016 Jun 4doi: 10.1111/dmcn.13164. PMID: 27259464.X-1

6156. Spjut Jansson B, Miniscalco C, Westerlund J, et al. Children who screen positive for autism at 2.5 years and receive early intervention: a prospective naturalistic 2year outcome study. Neuropsychiatr Dis Treat. 2016;12:2255-63. doi: 10.2147/ndt.s108899. PMID: 27621636.X-1

6157. Sprafkin J, Steinberg EA, Gadow KD, et al. Agreement among categorical, dimensional, and impairment criteria for ADHD and common comorbidities. Journal of Attention Disorders. 2016;20(8):665-73. doi: http://dx.doi.org/10.1177/1087054712475083.X $-1$

6158. Spriggs AD, Gast DL, Knight VF. Video Modeling and Observational Learning to Teach Gaming Access to Students with ASD. J Autism Dev Disord. 2016 Jun 6doi: 10.1007/s10803-016-2824-3. PMID: 27271933.X-1

6159. Squeglia LM, Brammer WA, Ray LA, et al. Attention deficit/hyperactivity disorder (ADHD) symptoms predict alcohol expectancy development. Journal of Child \& Adolescent Substance Abuse. 2016;25(2):169-79. doi: http://dx.doi.org/10.1080/1067828X.2014.9698 56.X-1

6160. Srinath S, Jacob P. Challenges in parent-mediated training in autism spectrum disorder. Lancet Psychiatry. 2016 Feb;3(2):935. doi: 10.1016/s2215-0366(15)00453-8. PMID: 26704572.X-1, X-2, X-3, X-4

6161. Srinivasan SM, Bhat AN. Differences in object sharing between infants at risk for autism and typically developing infants from 9 to 15 months of age. Infant Behavior \& Development. 2016;42:128-41. doi: http://dx.doi.org/10.1016/j.infbeh.2015.12.003. $\mathrm{X}-1$

6162. Srinivasan SM, Eigsti IM, Neelly L, et al. The effects of embodied rhythm and robotic interventions on the spontaneous and responsive social attention patterns of children with Autism Spectrum Disorder (ASD): A pilot randomized controlled trial. Res Autism Spectr Disord. 2016 Jul;27:54-72. doi:

10.1016/j.rasd.2016.01.004. PMID: 27453721. INCLUDE

6163. Srinivasan SM, Eigsti I-M, Gifford T, et al. The effects of embodied rhythm and robotic interventions on the spontaneous and responsive verbal communication skills of children with Autism Spectrum Disorder (ASD): A further outcome of a pilot randomized controlled trial. Research in Autism Spectrum Disorders. 2016;27:73-87. doi: http://dx.doi.org/10.1016/j.rasd.2016.04.001. INCLUDE

6164. Stace LB. Welcoming max: Increasing pediatric provider knowledge of service dogs. Complement Ther Clin Pract. 2016 Aug;24:5766. doi: 10.1016/j.ctcp.2016.05.005. PMID: 27502802.X-1

6165. Stadnick N, Chlebowski C, BakerEriczen M, et al. Psychiatric comorbidity in autism spectrum disorder: Correspondence between mental health clinician report and structured parent interview. Autism. $2016 \mathrm{Jul}$ 11doi: 10.1177/1362361316654083. PMID: 27407039.X-1

6166. Stahmer AC, Brookman-Frazee L, Rieth SR, et al. Parent perceptions of an adapted evidence-based practice for toddlers with autism in a community setting. Autism. 2016 Apr 27doi: 10.1177/1362361316637580. PMID: 27121242.X-1

6167. Starck M, Grünwald J, Schlarb AA. Occurrence of ADHD in parents of ADHD children in a clinical sample. Neuropsychiatric Disease and Treatment. 2016;12.X-1

6168. Steel C. The Asperger's defence in digital child pornography investigations. Psychiatry, Psychology and Law. 2016;23(3):473-82. doi: http://dx.doi.org/10.1080/13218719.2015.10801 50.X-1

6169. Stefanelli G, Gandaglia A, Costa M, et al. Brain phosphorylation of MeCP2 at serine 164 is developmentally regulated and globally alters its chromatin association. Sci Rep. 2016;6:28295. doi: 10.1038/srep28295. PMID: 27323888.X-1 
6170. Steinbach JM, Garza ET, Ryan BC. Novel Object Exploration as a Potential Assay for Higher Order Repetitive Behaviors in Mice. J Vis Exp. 2016(114)doi: 10.3791/54324. PMID: 27583676.X-1

6171. Steinfeld H, Cho MT, Retterer K, et al. Mutations in HIVEP2 are associated with developmental delay, intellectual disability, and dysmorphic features. Neurogenetics.

2016;17(3):159-64. doi: http://dx.doi.org/10.1007/s10048-016-0479z.X-1

6172. Stephens RL, Sabatos-DeVito M, Reznick JS. The Development and Validation of Attention Constructs From the First Year Inventory. Psychol Assess. 2016 Aug 8doi: 10.1037/pas0000380. PMID: 27504901.X-1

6173. Stephenson J. Using the Choiceboard Creator $^{\mathrm{TM}}$ app on an iPad(C) to teach choice making to a student with severe disabilities. AAC: Augmentative and Alternative Communication. 2016;32(1):49-57. doi: http://dx.doi.org/10.3109/07434618.2015.11366 88.X-1, X-3, X-4

6174. Stephenson KG, Quintin EM, South M. Erratum to: Age-Related Differences in Response to Music-Evoked Emotion Among Children and Adolescents with Autism Spectrum Disorders. J Autism Dev Disord. 2016 Apr;46(4):1152. doi: 10.1007/s10803015-2676-2. PMID: 26701672.X-1

6175. Stephenson KG, Quintin EM, South M. Age-related differences in response to music-evoked emotion among children and adolescents with autism spectrum disorders. Journal of Autism and Developmental Disorders. 2016;46(4):1142-51. doi: http://dx.doi.org/10.1007/s10803-015-26241.X-1, X-3

6176. Sterponi L, de Kirby K. A Multidimensional Reappraisal of Language in Autism: Insights from a Discourse Analytic Study. J Autism Dev Disord. 2016 Feb;46(2):394-405. doi: 10.1007/s10803-0152679-z. PMID: 26701673.X-1

6177. Stessman HA, Willemsen MH, Fenckova M, et al. Disruption of POGZ Is Associated with Intellectual Disability and
Autism Spectrum Disorders. Am J Hum Genet. 2016 Mar 3;98(3):541-52. doi: 10.1016/j.ajhg.2016.02.004. PMID: 26942287.X-1

6178. Stevenson MT, Ghezzi PM, Valenton KG. FCT and delay fading for elopement with a child with autism. Behavior Analysis in Practice. 2016;9(2):169-73. doi: http://dx.doi.org/10.1007/s40617-015-00895.X-1, X-3

6179. Stevenson RA, Segers M, Ferber S, et al. Keeping time in the brain: Autism spectrum disorder and audiovisual temporal processing. Autism Res. 2016 Jul;9(7):720-38. doi: 10.1002/aur.1566. PMID: 26402725.X-1

6180. Stevenson RA, Sun SZ, Hazlett N, et al. Seeing the Forest and the Trees: Default Local Processing in Individuals with High Autistic Traits Does Not Come at the Expense of Global Attention. J Autism Dev Disord. 2016 Feb 9doi: 10.1007/s10803-016-2711-y. PMID: 26861715.X-1

6181. Stewart E, Cancilliere MK, Freeman J, et al. Elevated Autism Spectrum Disorder Traits in Young Children with OCD. Child Psychiatry Hum Dev. 2016 Feb 8doi: 10.1007/s10578-0160629-4. PMID: 26858231.X-1

6182. Stichter JP, Christ SE, Herzog MJ, et al. Exploring the role of executive functioning measures for social competence research. Assessment for Effective Intervention. 2016;41(4):243-54. doi: http://dx.doi.org/10.1177/1534508416644179.X $-1$

6183. Stichter JP, Herzog MJ, Owens SA, et al. Manualization, feasibility, and effectiveness of the school-based Social Competence Intervention for Adolescents (SCI-A). Psychology in the Schools. 2016;53(6):583600. doi:

http://dx.doi.org/10.1002/pits.21928.X-1, X-3

6184. Stokl J, Herzner G. Morphology and structure of the allomone and sex-pheromone producing mandibular gland of the parasitoid wasp Leptopilina heterotoma (Hymenoptera: Figitidae). Arthropod Struct Dev. 2016 Jun 24doi: 10.1016/j.asd.2016.06.003. PMID: 27349419.X-1 
6185. Stoodley CJ, Limperopoulos C. Structure-function relationships in the developing cerebellum: Evidence from earlylife cerebellar injury and neurodevelopmental disorders. Semin Fetal Neonatal Med. 2016 May 12doi: 10.1016/j.siny.2016.04.010. PMID: 27184461.X-1

6186. Storch EA, Nadeau JM, Johnco C, et al. Hoarding in youth with autism spectrum disorders and anxiety: Incidence, clinical correlates, and behavioral treatment response. Journal of Autism and Developmental Disorders. 2016;46(5):1602-12. doi: http://dx.doi.org/10.1007/s10803-015-2687z.X-1

6187. Strom RG, Bae J, Mizutani J, et al. Lateral interbody fusion combined with open posterior surgery for adult spinal deformity. $\mathrm{J}$ Neurosurg Spine. 2016 Jun 24:1-9. doi: 10.3171/2016.4.spine16157. PMID: 27341052.X-1

6188. Strunz S, Schermuck C, Ballerstein S, et al. Romantic Relationships and Relationship Satisfaction Among Adults With Asperger Syndrome and High-Functioning Autism. J Clin Psychol. 2016 May 16doi: 10.1002/jclp.22319. PMID: 27196958.X-1

6189. Stuttard L, Beresford B, Clarke S, et al. An evaluation of the Cygnet parenting support programme for parents of children with autism spectrum conditions. Research in Autism Spectrum Disorders. 2016;23:166-78. doi: http://dx.doi.org/10.1016/j.rasd.2015.12.004.X$1, \mathrm{X}-4$

6190. Su CC, Chi MH, Lin SH, et al. Bidirectional association between autism spectrum disorder and epilepsy in child and adolescent patients: a population-based cohort study. Eur Child Adolesc Psychiatry. 2016 Jan 20doi: 10.1007/s00787-016-0817-6. PMID: 26791195.X-1

6191. Sudarshan S, Gupta N, Kabra M. Genetic Studies in Autism. Indian J Pediatr. 2016 Mar 3doi: 10.1007/s12098-015-1989-7. PMID: 26935198.X-1, X-2

6192. Suess AN, Wacker DP, Schwartz JE, et al. Preliminary Evidence on the use of Telehealth in an Outpatient Behavior Clinic. J
Appl Behav Anal. 2016 Mar 22doi: 10.1002/jaba.305. PMID: 27001117.X-1

6193. Sugimoto D, Bowen SL, Meehan WP, III, et al. Effects of neuromuscular training on children and young adults with Down syndrome: Systematic review and metaanalysis. Research in Developmental Disabilities. 2016;55:197-206. doi: http://dx.doi.org/10.1016/j.ridd.2016.04.003.X1

6194. Sukasem C, Hongkaew Y, Ngamsamut $\mathrm{N}$, et al. Impact of pharmacogenetic markers of CYP2D6 and DRD2 on prolactin response in risperidone-treated Thai children and adolescents with autism spectrum disorders. Journal of Clinical Psychopharmacology. 2016;36(2):141-6. doi: http://dx.doi.org/10.1097/JCP.00000000000004 74.X-1

6195. Suma K, Adamson LB, Bakeman R, et al. After Early Autism Diagnosis: Changes in Intervention and Parent-Child Interaction. J Autism Dev Disord. 2016 May 18doi: 10.1007/s10803-016-2808-3. PMID: 27193183.X-1

6196. Suma K, Adamson LB, Bakeman R, et al. After early autism diagnosis: Changes in intervention and parent-child interaction. Journal of Autism and Developmental Disorders. 2016;46(8):2720-33. doi: http://dx.doi.org/10.1007/s10803-016-28083.X-1

6197. Sumner E, Leonard HC, Hill EL. Overlapping Phenotypes in Autism Spectrum Disorder and Developmental Coordination Disorder: A Cross-Syndrome Comparison of Motor and Social Skills. J Autism Dev Disord. 2016 Apr 28doi: 10.1007/s10803-016-2794-5. PMID: 27126816.X-1

6198. Sumner E, Pratt ML, Hill EL. Examining the cognitive profile of children with Developmental Coordination Disorder. Research in Developmental Disabilities. 2016;56:10-7. doi: http://dx.doi.org/10.1016/j.ridd.2016.05.012.X1

6199. Sundelin HE, Larsson H, Lichtenstein P, et al. Autism and epilepsy: A populationbased nationwide cohort study. Neurology. 
2016 Jun 15doi:

10.1212/wnl.0000000000002836. PMID:

27306624.X-1

6200. Sutherland R, Trembath D, Hodge A, et al. Telehealth language assessments using consumer grade equipment in rural and urban settings: Feasible, reliable and well tolerated. J Telemed Telecare. 2016 Jan 13doi: 10.1177/1357633x15623921. PMID: 26768598.X-1

6201. Sy JR, Green L, Gratz O, et al. An Evaluation of the Effects of a Mild Delayed Verbal Punisher on Choice of an Immediate Reinforcer by Children With Autism. Behav Modif. 2016 Jan 12doi: 10.1177/0145445515622382. PMID: 26762626.X-1

6202. Syriopoulou-Delli CK, Cassimos DC, Polychronopoulou SA. Collaboration between teachers and parents of children with ASD on issues of education. Res Dev Disabil. 2016 May 24;55:330-45. doi: 10.1016/j.ridd.2016.04.011. PMID: 27235770.X-1

6203. Szatmari P, Chawarska K, Dawson G, et al. Prospective longitudinal studies of infant siblings of children with autism: Lessons learned and future directions. Journal of the American Academy of Child \& Adolescent Psychiatry. 2016;55(3):179-87. doi: http://dx.doi.org/10.1016/j.jaac.2015.12.014.X1

6204. Tadayyon H, Sannachi L, Gangeh M, et al. Quantitative ultrasound assessment of breast tumor response to chemotherapy using a multi-parameter approach. Oncotarget. 2016 Apr 20doi: 10.18632/oncotarget.8862. PMID: 27105515.X-1

6205. Tager-Flusberg H, Plesa Skwerer D, Joseph RM, et al. Conducting research with minimally verbal participants with autism spectrum disorder. Autism. 2016 Jun 26doi: 10.1177/1362361316654605. PMID: 27354431.X-1

6206. Taheri A, Perry A, Minnes P. Examining the social participation of children and adolescents with Intellectual Disabilities and Autism Spectrum Disorder in relation to peers. Journal of Intellectual Disability
Research. 2016;60(5):435-43. doi: http://dx.doi.org/10.1111/jir.12289.X-1

6207. Tait K, Fung F, Hu A, et al. Understanding Hong Kong Chinese families’ experiences of an autism/ASD diagnosis. Journal of Autism and Developmental Disorders. 2016;46(4):1164-83. doi: http://dx.doi.org/10.1007/s10803-015-2650z.X-1

6208. Takahashi K, Miyatake N, Kurato R, et al. Prevalence of attention deficit hyperactivity disorder and/or autism spectrum disorder and its relation to lifestyle in female college students. Environ Health Prev Med. 2016 Jun 27doi: 10.1007/s12199-016-0548-9. PMID: 27350055.X-1

6209. Takahashi T, Yoshimura Y, Hiraishi $\mathrm{H}$, et al. Enhanced brain signal variability in children with autism spectrum disorder during early childhood. Human Brain Mapping. 2016;37(3):1038-50. doi: http://dx.doi.org/10.1002/hbm.23089.X-1

6210. Takarae Y, Sablich SR, White SP, et al. Neurophysiological hyperresponsivity to sensory input in autism spectrum disorders. J Neurodev Disord. 2016;8:29. doi: 10.1186/s11689-016-9162-9. PMID: 27504143.X-1

6211. Takemoto M, Boissiere L, Novoa F, et al. Sagittal malalignment has a significant association with postoperative leg pain in adult spinal deformity patients. Eur Spine J. 2016 May 25doi: 10.1007/s00586-016-4616-z. PMID: 27225901.X-1

6212. Talbott MR, Nelson CA, TagerFlusberg H. Maternal vocal feedback to 9month-old infant siblings of children with ASD. Autism Research. 2016;9(4):460-70. doi: http://dx.doi.org/10.1002/aur.1521.X-1

6213. Tamm G, Kreegipuu K, Harro J. Perception of emotion in facial stimuli: The interaction of ADRA2A and COMT genotypes, and sex. Prog Neuropsychopharmacol Biol Psychiatry. 2016 Jan 4;64:87-95. doi: 10.1016/j.pnpbp.2015.07.012. PMID: 26234518.X-1

6214. Tan BW, Pooley JA, Speelman CP. A Meta-Analytic Review of the Efficacy of 
Physical Exercise Interventions on Cognition in Individuals with Autism Spectrum Disorder and ADHD. J Autism Dev Disord. 2016 Sep;46(9):3126-43. doi: 10.1007/s10803-0162854-x. PMID: 27412579.X-1

6215. Tan W, Schauder C, Naryshkina T, et al. Zfrp8 forms a complex with fragile-X mental retardation protein and regulates its localization and function. Dev Biol. 2016 Feb 15;410(2):202-12. doi: 10.1016/j.ydbio.2015.12.008. PMID: 26772998.X-1

6216. Tanaka JW, Simonyi D. The "parts and wholes" of face recognition: A review of the literature. Q J Exp Psychol (Hove). 2016 Mar 4:1-14. doi: 10.1080/17470218.2016.1146780. PMID: 26886495.X-1, X-2

6217. Tang X, Kim J, Zhou L, et al. KCC2 rescues functional deficits in human neurons derived from patients with Rett syndrome. Proc Natl Acad Sci U S A. 2016 Jan 19;113(3):7516. doi: 10.1073/pnas.1524013113. PMID: 26733678.X-1

6218. Tao Y, Gao H, Ackerman B, et al. Evidence for contribution of common genetic variants within chromosome 8p21.2-8p21.1 to restricted and repetitive behaviors in autism spectrum disorders. BMC Genomics. 2016;17:163. doi: 10.1186/s12864-016-2475-y. PMID: 26931105.X-1

6219. Tasse MJ, Schalock RL, Thissen D, et al. Development and Standardization of the Diagnostic Adaptive Behavior Scale: Application of Item Response Theory to the Assessment of Adaptive Behavior. Am J Intellect Dev Disabil. 2016 Mar;121(2):79-94. doi: 10.1352/1944-7558-121.2.79. PMID: 26914464.X-1

6220. Tavassoli T, Bellesheim K, Siper PM, et al. Measuring Sensory Reactivity in Autism Spectrum Disorder: Application and Simplification of a Clinician-Administered Sensory Observation Scale. J Autism Dev Disord. 2016 Jan;46(1):287-93. doi: 10.1007/s10803-015-2578-3. PMID: 26340959.X-1

6221. Tavassoli T, Bellesheim K, Tommerdahl M, et al. Altered tactile processing in children with autism spectrum disorder.
Autism Res. 2016 Jun;9(6):616-20. doi: 10.1002/aur.1563. PMID: 26568449.X-1

6222. Taylor CE, Bush K. Unintended consequences of inhibiting dihydrofolate reductase through folic acid supplementation: inattentive-type attention deficit hyperactivity disorder and ASD connections. Int J Food Sci Nutr. 2016 Jun 26:1-2. doi: 10.1080/09637486.2016.1200017. PMID: 27346490.X-1

6223. Taylor LS, Zhang GG. Physical chemistry of supersaturated solutions and implications for oral absorption. Adv Drug Deliv Rev. 2016 Jun 1;101:122-42. doi: 10.1016/j.addr.2016.03.006. PMID: 27013254.X-1

6224. Taylor MJ, Lichtenstein P, Larsson H, et al. Is there a female protective effect against attention-deficit/hyperactivity disorder? Evidence from two representative twin samples. Journal of the American Academy of Child \& Adolescent Psychiatry. 2016;55(6):504-12. doi: http://dx.doi.org/10.1016/j.jaac.2016.04.004.X1

6225. Tede Z, Cohen MO, Riskin A, et al. The reliability and validity of the Greenspan Social Emotional Growth Chart (GSEGC) in Israeli children with developmental delay and autism-A pilot study. Research in Developmental Disabilities. 2016;55:226-34. doi: http://dx.doi.org/10.1016/j.ridd.2016.04.013.X1

6226. Tede Z, Ophir Cohen M, Riskin A, et al. The reliability and validity of the Greenspan Social Emotional Growth Chart (GSEGC) in Israeli children with developmental delay and autism-A pilot study. Res Dev Disabil. 2016 Apr 30;55:226-34. doi: 10.1016/j.ridd.2016.04.013. PMID: 27138232.X-1

6227. Teng BL, Nikolova VD, Riddick NV, et al. Reversal of social deficits by subchronic oxytocin in two autism mouse models. Neuropharmacology. 2016 Jun;105:61-71. doi: 10.1016/j.neuropharm.2015.12.025. PMID: 26748053.X-1

6228. Terzi A, Marinis T, Francis K. The Interface of Syntax with Pragmatics and 
Prosody in Children with Autism Spectrum Disorders. J Autism Dev Disord. 2016 May 21doi: 10.1007/s10803-016-2811-8. PMID: 27209514.X-1

6229. Than KD, Park P, Fu KM, et al. Clinical and radiographic parameters associated with best versus worst clinical outcomes in minimally invasive spinal deformity surgery. J Neurosurg Spine. 2016 Jul;25(1):21-5. doi: 10.3171/2015.12.spine15999. PMID: 26943254.X-1

6230. Thapa R. Retrospective descriptive study of cerebral palsy in Nepal. Journal of Autism and Developmental Disorders. 2016;46(7):2285-91. doi: http://dx.doi.org/10.1007/s10803-016-2757$\mathrm{x} . \mathrm{X}-1$

6231. Theoharides TC, Tsilioni I, Patel AB, et al. Atopic diseases and inflammation of the brain in the pathogenesis of autism spectrum disorders. Transl Psychiatry. 2016;6(6):e844. doi: 10.1038/tp.2016.77. PMID: 27351598.X-1

6232. Theologis AA, Ailon T, Scheer JK, et al. Impact of preoperative depression on 2-year clinical outcomes following adult spinal deformity surgery: the importance of risk stratification based on type of psychological distress. J Neurosurg Spine. 2016 May 6:1-9. doi: 10.3171/2016.2.spine15980. PMID: 27153146.X-1

6233. Theologis AA, Miller L, Callahan M, et al. The Economic Impact of Revision Surgery for Proximal Junctional Failure after Adult Spinal Deformity Surgery: A Cost Analysis of 57 Operations in A 10-Year Experience at a Major Deformity Center. Spine (Phila Pa 1976). 2016 Feb 18doi: 10.1097/brs.0000000000001523. PMID: 26909838.X-1

6234. Therrien MC, Light J, Pope L. Systematic Review of the Effects of Interventions to Promote Peer Interactions for Children who use Aided AAC. Augment Altern Commun. 2016 Jun;32(2):81-93. doi: 10.3109/07434618.2016.1146331. PMID: 26903484.X-1, X-2

6235. Thomas BL, Viljoen M. EEG brain wave activity at rest and during evoked attention in children with attention- deficit/hyperactivity disorder and effects of methylphenidate. Neuropsychobiology.

2016;73(1):16-22. doi: http://dx.doi.org/10.1159/000441523.X-1

6236. Thomas MSC, Davis R, KarmiloffSmith A, et al. The over-pruning hypothesis of autism. Developmental Science. 2016;19(2):284-305. doi: http://dx.doi.org/10.1111/desc.12303.X-1

6237. Thomas RE, Spragins W, Mazloum G, et al. Rates of detection of developmental problems at the 18-month well-baby visit by family physicians' using four evidence-based screening tools compared to usual care: A randomized controlled trial. Child: Care, Health and Development. 2016;42(3):382-93. doi: http://dx.doi.org/10.1111/cch.12333.X-1

6238. Thorup E, Nystrom P, Gredeback G, et al. Altered gaze following during live interaction in infants at risk for autism: an eye tracking study. Mol Autism. 2016;7:12. doi: 10.1186/s13229-016-0069-9. PMID: 26819699.X-1

6239. Thurm A, Himelstein D, D'Souza P, et al. Creatine transporter deficiency: Screening of males with neurodevelopmental disorders and neurocognitive characterization of a case. Journal of Developmental and Behavioral Pediatrics. 2016;37(4):322-6. doi: http://dx.doi.org/10.1097/DBP.0000000000000 299.X-1

6240. Thurm A, Tierney E, Farmer C, et al. Development, behavior, and biomarker characterization of Smith-Lemli-Opitz syndrome: an update. J Neurodev Disord. 2016;8:12. doi: 10.1186/s11689-016-9145-X. PMID: 27053961.X-1

6241. Tian W, Yan K, Han X, et al. Comparison of the Clinical and Radiographic Results between Cervical Artificial Disc Replacement and Anterior Cervical Fusion: A Six-year Prospective Non-randomized Comparative Study. Clin Spine Surg. 2016 Jun 28doi: 10.1097/bsd.0000000000000206. PMID: 27355702.X-1

6242. Tian Y, Wang L, Jia M, et al. Association of oligodendrocytes differentiation regulator gene DUSP15 with autism. World J Biol Psychiatry. 2016 May 25:1-8. doi: 
10.1080/15622975.2016.1178395. PMID: 27223645.X-1

6243. Tick B, Bolton P, Happé F, et al. Heritability of autism spectrum disorders: A meta-analysis of twin studies. Journal of Child Psychology and Psychiatry. 2016;57(5):585-95. doi: http://dx.doi.org/10.1111/jcpp.12499.X-1

6244. Tick B, Colvert E, McEwen F, et al. Autism spectrum disorders and other mental health problems: Exploring etiological overlaps and phenotypic causal associations. Journal of the American Academy of Child \& Adolescent Psychiatry. 2016;55(2):106-13. doi: http://dx.doi.org/10.1016/j.jaac.2015.11.013.X1

6245. Timmons L, Willis KD, Pruitt MM, et al. Predictors of Daily Relationship Quality in Mothers of Children with Autism Spectrum Disorder. J Autism Dev Disord. 2016 Apr 20doi: 10.1007/s10803-016-2799-0. PMID: 27097814.X-1

6246. Timonen-Soivio L, Vanhala R, Malm $\mathrm{H}$, et al. Brief Report: Syndromes in Autistic Children in a Finnish Birth Cohort. J Autism Dev Disord. 2016 Apr 21doi: 10.1007/s10803016-2789-2. PMID: 27101237.X-1

6247. Tint A, Maughan AL, Weiss JA. Community participation of youth with intellectual disability and autism spectrum disorder. J Intellect Disabil Res. 2016 Aug 4doi: 10.1111/jir.12311. PMID: 27492816.X-1

6248. Tirado MJ, Saldaña D. Readers with autism can produce inferences, but they cannot answer inferential questions. Journal of Autism and Developmental Disorders.

2016;46(3):1025-37. doi:

http://dx.doi.org/10.1007/s10803-015-2648-

6.X-1

6249. Tomeny TS. Parenting stress as an indirect pathway to mental health concerns among mothers of children with autism spectrum disorder. Autism. 2016 Jul 1doi: 10.1177/1362361316655322. PMID: 27368349.X-1

6250. Tong HJ, Lee HY, Lee YT, et al. Factors influencing the inclusion of oral health education in individualized education plans of children with autism spectrum disorders in
Singapore. Int J Paediatr Dent. 2016 Aug 1doi: 10.1111/ipd.12257. PMID: 27478971.X-1

6251. Torelli JN, Lambert JM, Da Fonte MA, et al. Assessing acquisition of and preference for mand topographies during functional communication training. Behavior Analysis in Practice. 2016;9(2):165-8. doi: http://dx.doi.org/10.1007/s40617-015-0083y.X-1, X-3, X-4

6252. Torras-Mañá M, Gómez-Morales A, González-Gimeno I, et al. Assessment of cognition and language in the early diagnosis of autism spectrum disorder: Usefulness of the Bayley Scales of infant and toddler development, third edition. Journal of Intellectual Disability Research. 2016;60(5):502-11. doi: http://dx.doi.org/10.1111/jir.12291.X-1

6253. Torres EB, Nguyen J, Mistry S, et al. Characterization of the Statistical Signatures of Micro-Movements Underlying Natural Gait Patterns in Children with Phelan McDermid Syndrome: Towards Precision-Phenotyping of Behavior in ASD. Front Integr Neurosci. 2016;10:22. doi: 10.3389/fnint.2016.00022. PMID: 27445720.X-1

6254. Torrico B, Chiocchetti AG, Bacchelli E, et al. Lack of replication of previous autism spectrum disorder GWAS hits in European populations. Autism Res. 2016 Jul 15doi: 10.1002/aur.1662. PMID: 27417655.X-1

6255. Toussaint KA, Kodak T, Vladescu JC. An evaluation of choice on instructional efficacy and individual preferences among children with autism. Journal of Applied Behavior Analysis. 2016;49(1):170-5. doi: http://dx.doi.org/10.1002/jaba.263.X-1, X-3, X4

6256. Tovo-Rodrigues L, RecamondeMendoza M, Paixao-Cortes VR, et al. The role of protein intrinsic disorder in major psychiatric disorders. Am J Med Genet B Neuropsychiatr Genet. 2016 May 17doi: 10.1002/ajmg.b.32455. PMID: 27184105.X-1

6257. Traboulsi EI, Vanderveen D, Morrison D, et al. Associated systemic and ocular disorders in patients with congenital unilateral cataracts: the Infant Aphakia Treatment Study 
experience. Eye (Lond). 2016 Jun 17doi: 10.1038/eye.2016.124. PMID: 27315350.X-1

6258. Tran NN, Pham TT, Ozawa K, et al. Impacts of Perinatal Dioxin Exposure on Motor Coordination and Higher Cognitive Development in Vietnamese Preschool Children: A Five-Year Follow-Up. PLoS One. 2016;11(1):e0147655. doi: 10.1371/journal.pone.0147655. PMID: 26824471.X-1

6259. Trasande L, Zoeller RT, Hass U, et al. Burden of disease and costs of exposure to endocrine disrupting chemicals in the European Union: an updated analysis. Andrology. 2016 Mar 22doi: 10.1111/andr.12178. PMID: 27003928.X-1

6260. Travers BG, Bigler ED, Duffield TC, et al. Longitudinal development of manual motor ability in autism spectrum disorder from childhood to mid-adulthood relates to adaptive daily living skills. Dev Sci. 2016 Apr 7doi: 10.1111/desc.12401. PMID: 27061223.X-1

6261. Trembath D, Hawtree R, Arciuli J, et al. What do speech-language pathologists think parents expect when treating their children with autism spectrum disorder? Int J Speech Lang Pathol. 2016 Jun;18(3):250-8. doi: 10.3109/17549507.2016.1139625. PMID: 27063700.X-1

6262. Trimmer E, McDonald S, Rushby JA. Not knowing what I feel: Emotional empathy in autism spectrum disorders. Autism. 2016 May 30doi: 10.1177/1362361316648520. PMID: 27246093.X-1

6263. Tropeano M, Howley D, Gazzellone MJ, et al. Microduplications at the pseudoautosomal SHOX locus in autism spectrum disorders and related neurodevelopmental conditions. J Med Genet. 2016 Apr 12doi: 10.1136/jmedgenet-2015103621. PMID: 27073233.X-1

6264. Trotter PD, McGlone F, McKie S, et al. Effects of acute tryptophan depletion on central processing of CT-targeted and discriminatory touch in humans. Eur J Neurosci. 2016 Jun 16doi: 10.1111/ejn.13298. PMID: 27307373.X-1
6265. Troyb E, Knoch K, Herlihy L, et al. Restricted and repetitive behaviors as predictors of outcome in autism spectrum disorders. Journal of Autism and Developmental Disorders. 2016;46(4):1282-96. doi: http://dx.doi.org/10.1007/s10803-015-26682.X-1

6266. Truszkowski TL, James EJ, Hasan M, et al. Fragile $\mathrm{X}$ mental retardation protein knockdown in the developing Xenopus tadpole optic tectum results in enhanced feedforward inhibition and behavioral deficits. Neural Dev. 2016;11(1):14. doi: 10.1186/s13064-016-00697. PMID: 27503008.X-1

6267. Truzzi A, Setoh P, Shinohara K, et al. Physiological responses to dyadic interactions are influenced by neurotypical adults' levels of autistic and empathy traits. Physiol Behav. 2016 Jun 28;165:7-14. doi: 10.1016/j.physbeh.2016.06.034. PMID: 27371501.X-1

6268. Tsai PT. Autism and cerebellar dysfunction: Evidence from animal models. Semin Fetal Neonatal Med. 2016 May 11doi: 10.1016/j.siny.2016.04.009. PMID: 27179922.X-1

6269. Tseng A, Wang Z, Huo Y, et al. Differences in neural activity when processing emotional arousal and valence in autism spectrum disorders. Human Brain Mapping. 2016;37(2):443-61. doi: http://dx.doi.org/10.1002/hbm.23041.X-1

6270. Tseng KC, Tseng SH, Cheng HY. Design, development, and clinical validation of therapeutic toys for autistic children. J Phys Ther Sci. 2016 Jul;28(7):1972-80. doi: 10.1589/jpts.28.1972. PMID: 27512246.X-1

6271. Tu PC, Hsu JW, Lan CC, et al. Structural and functional correlates of a quantitative autistic trait measured using the social responsive scale in neurotypical male adolescents. Autism Research. 2016;9(5):570-8. doi: http://dx.doi.org/10.1002/aur.1535.X-1

6272. Turcotte P, Mathew M, Shea LL, et al. Service needs across the lifespan for individuals with autism. Journal of Autism and Developmental Disorders. 2016;46(7):2480-9. doi: http://dx.doi.org/10.1007/s10803-0162787-4.X-1 
6273. Türkoğlu S, Türkoğlu G. Comorbid gender dysphoria in a preadolescent boy with fragile X syndrome. Archives of Sexual Behavior. 2016;45(4):791-2. doi: http://dx.doi.org/10.1007/s10508-016-0710$\mathrm{x} . \mathrm{X}-1$

6274. Turner AH, Greenspan KS, van Erp TG. Pallidum and lateral ventricle volume enlargement in autism spectrum disorder. Psychiatry Res. 2016 Jun 30;252:40-5. doi: 10.1016/j.pscychresns.2016.04.003. PMID: 27179315.X-1

6275. Turner JD, Eastlack RK, Mirzadeh Z, et al. Fluctuations in Spinal Cord Perfusion During Adult Spinal Deformity Correction Identify Neurologic Changes: Proof of Concept. World Neurosurg. 2016 Jan;85:365.e1-6. doi: 10.1016/j.wneu.2015.08.067. PMID: 26361322.X-1

6276. Turner TN, Hormozdiari F, Duyzend $\mathrm{MH}$, et al. Genome Sequencing of AutismAffected Families Reveals Disruption of Putative Noncoding Regulatory DNA. Am J Hum Genet. 2016 Jan 7;98(1):58-74. doi: 10.1016/j.ajhg.2015.11.023. PMID: 26749308.X-1

6277. Turner-Brown L, Hume K, Boyd BA, et al. Preliminary Efficacy of Family Implemented TEACCH for Toddlers: Effects on Parents and Their Toddlers with Autism Spectrum Disorder. J Autism Dev Disord. 2016 May 30doi: 10.1007/s10803-016-2812-7. PMID: 27241348.X-1

6278. Tustin F. Revisiting Frances Tustin’s 'Autistic processes'. Journal of Child Psychotherapy. 2016;42(1):54-68. doi: http://dx.doi.org/10.1080/0075417X.2016.1140 924.X-1

6279. Tyner S, Brewer A, Helman M, et al. Nice doggie! Contact desensitization plus reinforcement decreases dog phobias for children with autism. Behavior Analysis in Practice. 2016;9(1):54-7. doi: http://dx.doi.org/10.1007/s40617-016-01134.X-1, X-3, X-4

6280. Ucak A, Temizkan V, Ugur M, et al. Comparison of primary repair and patch plasty procedure on the $\mathrm{P}$ wave in adult atrial septal defect closure. Cardiovasc J Afr. 2016 May 4;27:1-4. doi: 10.5830/cvja-2016-013. PMID: 27149297.X-1

6281. Uemura H. Surgical aspects of atrial arrhythmia : Right atrial ablation and antiarrhythmic surgery in congenital heart disease. Herzschrittmacherther Elektrophysiol. 2016 Jun;27(2):137-42. doi: 10.1007/s00399-0160434-6. PMID: 27225164.X-1

6282. Ulbrich L, Favaloro FL, Trobiani L, et al. Autism-associated R451C mutation in neuroligin3 leads to activation of the unfolded protein response in a PC12 Tet-On inducible system. Biochem J. 2016 Feb 15;473(4):423-34. doi: 10.1042/bj20150274. PMID: 26621873.X1

6283. Uljarevic M, Carrington S, Leekam S. Brief Report: Effects of Sensory Sensitivity and Intolerance of Uncertainty on Anxiety in Mothers of Children with Autism Spectrum Disorder. J Autism Dev Disord. 2016 Jan;46(1):315-9. doi: 10.1007/s10803-0152557-8. PMID: 26254895.X-1

6284. Uljarevic M, Evans DW. Relationship between repetitive behaviour and fear across normative development, autism spectrum disorder, and down syndrome. Autism Res. 2016 Jul 26doi: 10.1002/aur.1674. PMID: 27459229.X-1

6285. Uljarevic M, Katsos N, Hudry K, et al. Practitioner Review: Multilingualism and neurodevelopmental disorders - an overview of recent research and discussion of clinical implications. J Child Psychol Psychiatry. 2016 Jul 22doi: 10.1111/jcpp.12596. PMID: 27443172.X-1

6286. Uljarevic M, Lane A, Kelly A, et al. Sensory subtypes and anxiety in older children and adolescents with autism spectrum disorder. Autism Res. 2016 Jan 13doi: 10.1002/aur.1602. PMID: 26765165.X-1

6287. Ung D, Johnco C, McBride NM, et al. Optimizing the screening of autism spectrum disorders in outpatient clinics: An examination of the Social Communication QuestionnaireLifetime. Research in Autism Spectrum Disorders. 2016;27:21-8. doi: http://dx.doi.org/10.1016/j.rasd.2016.03.011.X1 
6288. Unterrainer JM, Rauh R, Rahm B, et al. Development of planning in children with high-functioning autism spectrum disorders and/or attention deficit/hyperactivity disorder. Autism Research. 2016;9(7):739-51. doi: http://dx.doi.org/10.1002/aur.1574.X-1

6289. Urbain C, Vogan VM, Ye AX, et al. Desynchronization of fronto-temporal networks during working memory processing in autism. Human Brain Mapping. 2016;37(1):153-64. doi: http://dx.doi.org/10.1002/hbm.23021.X-1

6290. Urbanowicz A, Leonard H, Girdler S, et al. Parental perspectives on the communication abilities of their daughters with Rett syndrome. Developmental Neurorehabilitation. 2016;19(1):17-25. doi: http://dx.doi.org/10.3109/17518423.2013.87994 $0 . \mathrm{X}-1$

6291. Usami M. Functional consequences of attention-deficit hyperactivity disorder on children and their families. Psychiatry and Clinical Neurosciences. 2016;70(8):303-17. doi: http://dx.doi.org/10.1111/pcn.12393.X-1

6292. Uzefovsky F, Allison C, Smith P, et al. Brief Report: The Go/No-Go Task Online: Inhibitory Control Deficits in Autism in a Large Sample. J Autism Dev Disord. 2016 Apr 21doi: 10.1007/s10803-016-2788-3. PMID: 27103120.X-1

6293. Vaillancourt T, Haltigan JD, Smith I, et al. Joint trajectories of internalizing and externalizing problems in preschool children with autism spectrum disorder. Dev Psychopathol. 2016 Feb 5:1-12. doi: 10.1017/s0954579416000043. PMID: 26847324.X-1

6294. Valenzuela CF, Medina AE, Wozniak JR, et al. Proceedings of the 2015 Annual Meeting of the Fetal Alcohol Spectrum Disorders Study Group. Alcohol. 2016;50:3742. doi: http://dx.doi.org/10.1016/j.alcohol.2015.10.002. $\mathrm{X}-1$

6295. Valvo G, Baldini S, Retico A, et al. Temporal lobe connects regression and macrocephaly to autism spectrum disorders. European Child \& Adolescent Psychiatry. 2016;25(4):421-9. doi: http://dx.doi.org/10.1007/s00787-015-07469.X-1, X-3

6296. van Amen-Hellebrekers CJ, Jansen S, Pfundt R, et al. Duplications of SLC1A3:

Associated with ADHD and autism. Eur J Med Genet. 2016 Jun 11;59(8):373-6. doi: 10.1016/j.ejmg.2016.06.003. PMID: 27296938.X-1

6297. van Boxtel JJ, Dapretto M, Lu H. Intact recognition, but attenuated adaptation, for biological motion in youth with autism spectrum disorder. Autism Res. 2016 Jan 25doi: 10.1002/aur.1595. PMID: 26808343.X-1

6298. van den Heuvel L, Suliman S, MalanMuller S, et al. Brain-derived neurotrophic factor Val66met polymorphism and plasma levels in road traffic accident survivors. Anxiety Stress Coping. 2016 Mar 29:1-14. doi: 10.1080/10615806.2016.1163545. PMID: 26999419.X-1

6299. Van der Hallen R, Evers K, Boets B, et al. Visual Search in ASD: Instructed Versus Spontaneous Local and Global Processing. J Autism Dev Disord. 2016 Jun 22doi: 10.1007/s10803-016-2826-1. PMID: 27334873.X-1

6300. van der Steen SL, Riedijk SR, Verhagen-Visser J, et al. The Psychological Impact of Prenatal Diagnosis and Disclosure of Susceptibility Loci: First Impressions of Parents' Experiences. J Genet Couns. 2016 May 25doi: 10.1007/s10897-016-9960-y. PMID: 27220741.X-1

6301. van Goozen SHM, Langley K, Northover C, et al. Identifying mechanisms that underlie links between COMT genotype and aggression in male adolescents with ADHD. Journal of Child Psychology and Psychiatry. 2016;57(4):472-80. doi: http://dx.doi.org/10.1111/jcpp.12464.X-1

6302. van IMH, Bakermans-Kranenburg MJ. The Role of Oxytocin in Parenting and as Augmentative Pharmacotherapy: Critical Issues and Bold Conjectures. J Neuroendocrinol. 2016 Aug;28(8)doi: 10.1111/jne.12355. PMID: 26709101.X-1

6303. van Schalkwyk GI, Beyer C, Martin A, et al. College students with Autism Spectrum 
Disorders: A growing role for adult psychiatrists. J Am Coll Health. 2016 Jun 27:0. doi: 10.1080/07448481.2016.1205072. PMID: 27347704.X-1

6304. van Scheppingen J, Iyer AM, Prabowo AS, et al. Expression of microRNAs miR21, miR146a, and miR155 in tuberous sclerosis complex cortical tubers and their regulation in human astrocytes and SEGA-derived cell cultures. Glia. 2016 Jun;64(6):1066-82. doi: 10.1002/glia.22983. PMID: 27014996.X-1

6305. van Scheppingen J, Iyer AM, Prabowo AS, et al. Expression of microRNAs miR21, miR146a, and miR155 in tuberous sclerosis complex cortical tubers and their regulation in human astrocytes and SEGA-derived cell cultures. Glia. 2016;64(6):1066-82. doi: http://dx.doi.org/10.1002/glia.22983.X-1

6306. Vanegas SB, Magaña S, Morales M, et al. Clinical validity of the ADI-R in a US-Based Latino population. Journal of Autism and Developmental Disorders. 2016;46(5):1623-35. doi: http://dx.doi.org/10.1007/s10803-0152690-4.X-1

6307. Vanmarcke S, Mullin C, Van der Hallen R, et al. In the Eye of the Beholder: Rapid Visual Perception of Real-Life Scenes by Young Adults with and Without ASD. J Autism Dev Disord. 2016 Apr 28doi: 10.1007/s10803016-2802-9. PMID: 27126818.X-1

6308. Vanvuchelen M. A qualitative analysis of imitation performances of preschoolers with Down syndrome. American Journal on Intellectual and Developmental Disabilities. 2016;121(3):266-75. doi: http://dx.doi.org/10.1352/1944-7558121.3.266.X-1

6309. Vanvuchelen M, Van Schuerbeeck L, Braeken MA. Screening accuracy of the parentcompleted Ages and Stages Questionnaires second edition as a broadband screener for motor problems in preschoolers with autism spectrum disorders. Autism. 2016 Feb 5doi: 10.1177/1362361315621703. PMID: 26851227.X-1

6310. Vanwong N, Ngamsamut N, Medhasi S, et al. Impact of CYP2D6 Polymorphism on Steady-State Plasma Levels of Risperidone and 9-Hydroxyrisperidone in Thai Children and
Adolescents with Autism Spectrum Disorder. J Child Adolesc Psychopharmacol. 2016 Jan 18doi: 10.1089/cap.2014.0171. PMID: 26780783.X-1

6311. Vanwong N, Prommas S, Puangpetch A, et al. Development and Validation of Liquid Chromatography/Tandem Mass Spectrometry Analysis for Therapeutic Drug Monitoring of Risperidone and 9-Hydroxyrisperidone in Pediatric Patients with Autism Spectrum Disorders. J Clin Lab Anal. 2016 Jun 26doi: 10.1002/jcla.22009. PMID: 27346210.X-1

6312. Varcin KJ, Nelson CA, III, Ko J, et al. Visual evoked potentials as a readout of cortical function in infants with tuberous sclerosis complex. Journal of Child Neurology. 2016;31(2):195-202. doi: http://dx.doi.org/10.1177/0883073815587328.X $-1$

6313. Vargas S, Lucker JR. A Quantitative Summary of The Listening Program (TLP) Efficacy Studies: What Areas Were Found to Improve by TLP Intervention? Occup Ther Int. 2016 Jun;23(2):206-17. doi: 10.1002/oti.1425. PMID: 26890280.X-1

6314. Vasli N, Ahmed I, Mittal K, et al. Identification of a homozygous missense mutation in LRP2 and a hemizygous missense mutation in TSPYL2 in a family with mild intellectual disability. Psychiatric Genetics. 2016;26(2):66-73. doi: http://dx.doi.org/10.1097/YPG.0000000000000 114.X-1

6315. Veazey SE, Valentino AL, Low AI, et al. Teaching feminine hygiene skills to young females with autism spectrum disorder and intellectual disability. Behavior Analysis in Practice. 2016;9(2):184-9. doi: http://dx.doi.org/10.1007/s40617-015-0065$0 . \mathrm{X}-1, \mathrm{X}-3, \mathrm{X}-4$

6316. Velott DL, Agbese E, Mandell D, et al. Medicaid 1915(c) Home- and CommunityBased Services waivers for children with autism spectrum disorder. Autism. 2016;20(4):473-82. doi:

http://dx.doi.org/10.1177/1362361315590806.X $-1$

6317. Venkataraman A, Yang D, Pelphrey K, et al. Bayesian Community Detection in the 
Space of Group-Level Functional Differences. IEEE Trans Med Imaging. 2016 Mar 2doi: 10.1109/tmi.2016.2536559. PMID: 26955022.X-1

6318. Venker CE. Spoken word recognition in children with autism spectrum disorder: The role of visual disengagement. Autism. 2016 Jun 22doi: 10.1177/1362361316653230. PMID: 27335107.X-1

6319. Venker CE, Haebig E, Edwards J, et al. Brief report: Early lexical comprehension in young children with ASD: Comparing eye-gaze methodology and parent report. Journal of Autism and Developmental Disorders. 2016;46(6):2260-6. doi: http://dx.doi.org/10.1007/s10803-016-2747z.X-1

6320. Venker CE, Kover ST, Weismer SE. Brief report: Fast mapping predicts differences in concurrent and later language abilities among children with ASD. Journal of Autism and Developmental Disorders. 2016;46(3):1118-23. doi: http://dx.doi.org/10.1007/s10803-0152644-X.X-1

6321. Ventola PE, Yang D, Abdullahi SM, et al. Brief Report: Reduced Restricted and Repetitive Behaviors after Pivotal Response Treatment. J Autism Dev Disord. 2016 May 26doi: 10.1007/s10803-016-2813-6. PMID: 27230762.X-3

6322. Verhaeghe L, Dereu M, Warreyn P, et al. Erratum to: Extremely Preterm Born Children at Very High Risk for Developing Autism Spectrum Disorder. Child Psychiatry Hum Dev. 2016 Apr 30doi: 10.1007/s10578016-0647-2. PMID: 27130292.X-1

6323. Vernon TW, Miller AR, Ko JA, et al. Social tools and rules for teens (The START program): Program description and preliminary outcomes of an experiential socialization intervention for adolescents with autism spectrum disorder. Journal of Autism and Developmental Disorders. 2016;46(5):1806-23. doi: http://dx.doi.org/10.1007/s10803-0162715-7.X-1, X-3, X-4

6324. Verpeut JL, DiCicco-Bloom E, Bello NT. Ketogenic diet exposure during the juvenile period increases social behaviors and forebrain neural activation in adult Engrailed 2 null mice. Physiol Behav. 2016 Jul 1;161:90-8. doi: 10.1016/j.physbeh.2016.04.001. PMID: 27080080.X-1

6325. Verriden AL, Roscoe EM. A comparison of preference-assessment methods. Journal of Applied Behavior Analysis. 2016;49(2):265-85. doi: http://dx.doi.org/10.1002/jaba.302.X-1

6326. Vicidomini C, Ponzoni L, Lim D, et al. Pharmacological enhancement of mGlu5 receptors rescues behavioral deficits in SHANK3 knock-out mice. Mol Psychiatry. 2016 Mar 29doi: 10.1038/mp.2016.30. PMID: 27021819.X-1

6327. Vied C, Ray S, Badger CD, et al. Transcriptomic analysis of the hippocampus from six inbred strains of mice suggests basis for sex-specific susceptibility and severity of neurological disorders. J Comp Neurol. 2016 Feb 24doi: 10.1002/cne.23989. PMID: 26917114.X-1

6328. Virring A, Lambek R, Thomsen PH, et al. Disturbed sleep in attention-deficit hyperactivity disorder (ADHD) is not a question of psychiatric comorbidity or ADHD presentation. Journal of Sleep Research. 2016;25(3):333-40. doi: http://dx.doi.org/10.1111/jsr.12377.X-1

6329. Visser JC, Rommelse NN, Greven CU, et al. Autism spectrum disorder and attentiondeficit/hyperactivity disorder in early childhood: A review of unique and shared characteristics and developmental antecedents. Neurosci Biobehav Rev. 2016 Jun;65:229-63. doi: 10.1016/j.neubiorev.2016.03.019. PMID: 27026637.X-1

6330. Vittori M, Tusek-Znidaric M, Strus J. Exoskeletal cuticle of cavernicolous and epigean terrestrial isopods: A review and perspectives. Arthropod Struct Dev. 2016 Aug 5doi: 10.1016/j.asd.2016.08.002. PMID: 27502824.X-1

6331. Vivanti G, Dissanayake C. Outcome for Children Receiving the Early Start Denver Model Before and After 48 Months. J Autism Dev Disord. 2016 Jul;46(7):2441-9. doi: 10.1007/s10803-016-2777-6. PMID: 27020055.X-3 
6332. Vogan VM, Morgan BR, Leung RC, et al. Widespread white matter differences in children and adolescents with autism spectrum disorder. Journal of Autism and Developmental Disorders. 2016;46(6):2138-47. doi: http://dx.doi.org/10.1007/s10803-016-27442.X-1

6333. Voigt V, Neufeld F, Kaste J, et al. Clinically assessed posttraumatic stress in patients with breast cancer during the first year after diagnosis in the prospective, longitudinal, controlled COGNICARES study. Psychooncology. 2016 Feb 22doi: 10.1002/pon.4102. PMID: 26898732.X-1

6334. Volkmann RA, Fanger CM, Anderson DR, et al. MPX-004 and MPX-007: New Pharmacological Tools to Study the Physiology of NMDA Receptors Containing the GluN2A Subunit. PLoS One. 2016;11(2):e0148129. doi: 10.1371/journal.pone.0148129. PMID: 26829109.X-1

6335. Vollebregt MA, Zumer JM, ter Huurne $\mathrm{N}$, et al. Posterior alpha oscillations reflect attentional problems in boys with Attention Deficit Hyperactivity Disorder. Clinical Neurophysiology. 2016;127(5):2182-91. doi: http://dx.doi.org/10.1016/j.clinph.2016.01.021. $\mathrm{X}-1$

6336. Vomund S, de Souza Silva MA, Huston JP, et al. Behavioral Resilience and Sensitivity to Locally Restricted Cortical Migration Deficits Induced by In Utero Knockdown of Disabled-1 in the Adult Rat. Cereb Cortex. 2016 Mar 10doi: 10.1093/cercor/bhw060. PMID: 26965907.X-1

6337. Voon V, Dalley JW. Translatable and Back-Translatable Measurement of Impulsivity and Compulsivity: Convergent and Divergent Processes. Curr Top Behav Neurosci. 2016 Jul 15doi: 10.1007/7854_2015_5013. PMID: 27418067.X-1

6338. Wade MG, Tsai C-L, Stoffregen T, et al. Perception of object length via manual wielding in children with and without developmental coordination disorder. Journal of Motor Behavior. 2016;48(1):13-9. doi: http://dx.doi.org/10.1080/00222895.2015.10259 02.X-1, X-3
6339. Wainer AL, Hepburn S, McMahon Griffith E. Remembering parents in parentmediated early intervention: An approach to examining impact on parents and families. Autism. 2016 Mar 7doi: 10.1177/1362361315622411. PMID: 26951325.X-1

6340. Waite J, Beck SR, Heald M, et al. Dissociation of cross-sectional trajectories for verbal and visuo-spatial working memory development in Rubinstein-Taybi syndrome. Journal of Autism and Developmental Disorders. 2016;46(6):2064-71. doi: http://dx.doi.org/10.1007/s10803-016-27362.X-1

6341. Walerius DM, Fogleman ND, Rosen PJ. The role of ADHD and negative emotional lability in predicting changes in parenting daily hassles. Journal of Child and Family Studies. 2016;25(7):2279-91. doi: http://dx.doi.org/10.1007/s10826-016-03811.X-1

6342. Wallace C, Ievers-Landis CE, Scherer C, et al. Attention-deficit/hyperactivity disorder, behavior regulation and virtual school support. Journal of Developmental and Behavioral Pediatrics. 2016;37(3):254-6. doi: http://dx.doi.org/10.1097/DBP.0000000000000 292.X-1

6343. Wallace GL, Budgett J, Charlton RA. Aging and autism spectrum disorder: Evidence from the broad autism phenotype. Autism Res. 2016 Mar 11doi: 10.1002/aur.1620. PMID: 26970433.X-1

6344. Walton KM, Ingersoll BR. The utility of Thin Slice ratings for predicting language growth in children with autism spectrum disorder. Autism. 2016;20(3):374-80. doi: http://dx.doi.org/10.1177/1362361315584465.X $-1$

6345. Wan SH, McKie PM, Schirger JA, et al. Chronic Peptide Therapy With B-Type Natriuretic Peptide in Patients With PreClinical Diastolic Dysfunction (Stage B Heart Failure). JACC Heart Fail. 2016 Jul;4(7):53947. doi: 10.1016/j.jchf.2015.12.014. PMID: 26874387.X-1

6346. Wang AT, Lim T, Jamison J, et al. Neural selectivity for communicative auditory 
signals in Phelan-McDermid syndrome. Journal of Neurodevelopmental Disorders. 2016;8doi: http://dx.doi.org/10.1186/s11689-016-91389.X-1

6347. Wang H, Lee IS, Braun C, et al. Effect of probiotics on central nervous system functions in animals and humans - a systematic review. J Neurogastroenterol Motil. 2016 Jul 13doi: 10.5056/jnm16018. PMID: 27413138.X1

6348. Wang H, Liang S, Wang M, et al. Potential serum biomarkers from a metabolomics study of autism. Journal of Psychiatry \& Neuroscience. 2016;41(1):27-37. doi: http://dx.doi.org/10.1503/jpn.140009.X-1

6349. Wang HG, Jeffries JJ, Wang TF. Genetic and developmental perspective of language abnormality in autism and schizophrenia: One disease occurring at different ages in humans? The Neuroscientist. 2016;22(2):119-31. doi: http://dx.doi.org/10.1177/1073858415572078.X $-1$

6350. Wang KY. Updated findings on neurodevelopmental disorders in Taiwan: impact of the institutionalized national healthcare system on prevalence and health outcomes. Curr Opin Psychiatry. 2016 Mar;29(2):144-8. doi: 10.1097/yсо.0000000000000229. PMID: 26731557.X-1

6351. Wang L, Jia J, Zhang J, et al. Serum levels of sod and risk of autism spectrum disorder: A case-control study. International Journal of Developmental Neuroscience. 2016;51:12-6. doi: http://dx.doi.org/10.1016/j.ijdevneu.2016.04.00 4.X-1

6352. Wang L, Wee C-Y, Tang X, et al. Multi-task feature selection via supervised canonical graph matching for diagnosis of autism spectrum disorder. Brain Imaging and Behavior. 2016;10(1):33-40. doi: http://dx.doi.org/10.1007/s11682-015-93601.X-1

6353. Wang M, Spiegelman D, Kuchiba A, et al. Statistical methods for studying disease subtype heterogeneity. Stat Med. 2016 Feb
28;35(5):782-800. doi: 10.1002/sim.6793. PMID: 26619806.X-1

6354. Wang Q, Hong P, Gao H, et al. An interneuron progenitor maintains neurogenic potential in vivo and differentiates into GABAergic interneurons after transplantation in the postnatal rat brain. Sci Rep. 2016;6:19003. doi: 10.1038/srep19003. PMID: 26750620.X-1

6355. Wang W, Niu Z, Wang Y, et al. Comparative transcriptome analysis of atrial septal defect identifies dysregulated genes during heart septum morphogenesis. Gene. 2016 Jan 10;575(2 Pt 1):303-12. doi: 10.1016/j.gene.2015.09.016. PMID: 26375510.X-1

6356. Wang X, Laffey J, Xing W, et al. Exploring embodied social presence of youth with Autism in 3D collaborative virtual learning environment: A case study. Computers in Human Behavior. 2016;51(Part A):310-21. doi: http://dx.doi.org/10.1016/j.chb.2015.09.006.X-1

6357. Wang Y, Billon C, Walker JK, et al. Therapeutic Effect of a Synthetic RORalpha/gamma Agonist in an Animal Model of Autism. ACS Chem Neurosci. 2016 Feb 17;7(2):143-8. doi:

10.1021/acschemneuro.5b00159. PMID: 26625251.X-1

6358. Ward B, Tanner BS, Mandleco B, et al. Sibling Experiences: Living with Young Persons with Autism Spectrum Disorders. Pediatr Nurs. 2016 Mar-Apr;42(2):69-76. PMID: 27254975.X-1

6359. Watanabe T, Rees G. Anatomical imbalance between cortical networks in autism. Sci Rep. 2016;6:31114. doi: 10.1038/srep31114. PMID: 27484308.X-1

6360. Watson KJ, DiCarlo CF. Increasing completion of classroom routines through the use of picture activity schedules. Early Childhood Education Journal. 2016;44(2):8996. doi: http://dx.doi.org/10.1007/s10643-0150697-2.X-1, X-3, X-4

6361. Wauthia E, Rossignol M. Emotional processing and attention control impairments in children with anxiety: An integrative review of 
event-related potentials findings. Frontiers in Psychology. 2016;7.X-1

6362. Webb SJ, Neuhaus E, Faja S. Face perception and learning in autism spectrum disorders. Q J Exp Psychol (Hove). 2016 Mar 16:1-17. doi: 10.1080/17470218.2016.1151059. PMID: 26886246.X-1

6363. Weber-Stadlbauer U, Richetto J, Labouesse MA, et al. Transgenerational transmission and modification of pathological traits induced by prenatal immune activation. Mol Psychiatry. 2016 Mar 29doi: 10.1038/mp.2016.41. PMID: 27021823.X-1

6364. Wee CY, Yap PT, Shen D. Diagnosis of autism spectrum disorders using temporally distinct resting-state functional connectivity networks. CNS Neuroscience \& Therapeutics. 2016;22(3):212-9. doi: http://dx.doi.org/10.1111/cns.12499.X-1

6365. Wehman P, Schall CM, McDonough J, et al. Effects of an employer-based intervention on employment outcomes for youth with significant support needs due to autism. Autism. 2016 May 5doi: 10.1177/1362361316635826. PMID: 27154907.X-1

6366. Wei G, Deng X, Agarwal S, et al. Patient Mutations of the Intellectual Disability Gene KDM5C Downregulate Netrin G2 and Suppress Neurite Growth in Neuro2a Cells. J Mol Neurosci. 2016 Jul 16doi: 10.1007/s12031016-0770-3. PMID: 27421841.X-1

6367. Wei H, Ma Y, Liu J, et al. Inhibition of IL-6 trans-signaling in the brain increases sociability in the BTBR mouse model of autism. Biochim Biophys Acta. 2016 Jul 25;1862(10):1918-25. doi: 10.1016/j.bbadis.2016.07.013. PMID: 27460706.X-1

6368. Weiss JA, Ting V, Perry A. Psychosocial correlates of psychiatric diagnoses and maladaptive behaviour in youth with severe developmental disability. J Intellect Disabil Res. 2016 Jun;60(6):583-93. doi: 10.1111/jir.12278. PMID: 27073035.X-1

6369. Weitzman C, Sia JH, Baroody OD, et al. Journal article reviews. Journal of Developmental and Behavioral Pediatrics. 2016;37(3):257-61. doi:
http://dx.doi.org/10.1097/DBP.0000000000000 275.X-1

6370. Welch CD, Polatajko HJ. Applied Behavior Analysis, Autism, and Occupational Therapy: A Search for Understanding. Am J Occup Ther. 2016 Jul-

Aug;70(4):7004360020p1-5. doi: 10.5014/ajot.2016.018689. PMID: 27295000.X-1, X-2

6371. Welles BF. Computational CAM: Studying children and media in the age of big data. Journal of Children and Media. 2016;10(1):72-80. doi: http://dx.doi.org/10.1080/17482798.2015.11231 65.X-1

6372. Wells MF, Wimmer RD, Schmitt LI, et al. Thalamic reticular impairment underlies attention deficit in Ptchd1(Y/-) mice. Nature. 2016 Apr 7;532(7597):58-63. doi: 10.1038/nature17427. PMID: 27007844.X-1

6373. Wells PG, Bhatia S, Drake DM, et al. Fetal oxidative stress mechanisms of neurodevelopmental deficits and exacerbation by ethanol and methamphetamine. Birth Defects Res C Embryo Today. 2016 Jun;108(2):108-30. doi: 10.1002/bdrc.21134. PMID: 27345013.X-1

6374. Welsh JP, Oristaglio JT. Autism and Classical Eyeblink Conditioning: Performance Changes of the Conditioned Response Related to Autism Spectrum Disorder Diagnosis. Front Psychiatry. 2016;7:137. doi: 10.3389/fpsyt.2016.00137. PMID: 27563293.X-1

6375. Wenger TL, Miller JS, DePolo LM, et al. 22q11.2 duplication syndrome: elevated rate of autism spectrum disorder and need for medical screening. Mol Autism. 2016;7:27. doi: 10.1186/s13229-016-0090-z. PMID: 27158440.X-1

6376. Werling AM, Bobrowski E, Taurines $\mathrm{R}$, et al. CNTNAP2 gene in high functioning autism: no association according to family and meta-analysis approaches. J Neural Transm (Vienna). 2016 Mar;123(3):353-63. doi: 10.1007/s00702-015-1458-5. PMID: 26559825.X-1 
6377. Werling DM, Parikshak NN, Geschwind DH. Gene expression in human brain implicates sexually dimorphic pathways in autism spectrum disorders. Nat Commun. 2016;7:10717. doi: 10.1038/ncomms10717. PMID: 26892004.X-1

6378. Wernicke T, Kalbantner-Wernicke K. Fit for School: The Samurai School Program. Med Acupunct. 2016 Feb 1;28(1):33-9. doi: 10.1089/acu.2015.1147. PMID: 26909127.X-1, $\mathrm{X}-2, \mathrm{X}-3$

6379. Wertz J, Zavos HMS, Matthews T, et al. Etiology of pervasive versus situational antisocial behaviors: A multi-informant longitudinal cohort study. Child Development. 2016;87(1):312-25. doi: http://dx.doi.org/10.1111/cdev.12456.X-1

6380. Westerveld MF, Trembath D, Shellshear L, et al. A systematic review of the literature on emergent literacy skills of preschool children with autism spectrum disorder. The Journal of Special Education. 2016;50(1):37-48. doi: http://dx.doi.org/10.1177/0022466915613593.X $-1, \mathrm{X}-2$

6381. Whitaker L, Jones CRG, Wilkins AJ, et al. Judging the intensity of emotional expression in faces: The effects of colored tints on individuals with autism spectrum disorder. Autism Research. 2016;9(4):450-9. doi: http://dx.doi.org/10.1002/aur.1506.X-1

6382. White D, Hillier A, Frye A, et al. College Students' Knowledge and Attitudes Towards Students on the Autism Spectrum. J Autism Dev Disord. 2016 May 26doi: 10.1007/s10803-016-2818-1. PMID: 27230760.X-1

6383. Wichnick-Gillis AM, Vener SM, Poulson CL. The effect of a script-fading procedure on social interactions among young children with autism. Research in Autism Spectrum Disorders. 2016;26:1-9. doi: http://dx.doi.org/10.1016/j.rasd.2016.03.004.X3, X-4

6384. Wicker B, Monfardini E, Royet JP. Olfactory processing in adults with autism spectrum disorders. Mol Autism. 2016;7:4. doi: 10.1186/s13229-016-0070-3. PMID: 26788281.X-1
6385. Wiener RC, Vohra R, Sambamoorthi $\mathrm{U}$, et al. Caregiver Burdens and Preventive Dental Care for Children with Autism Spectrum Disorder, Developmental Disability and/or Mental Health Conditions: National Survey of CSHCN, 2009-2010. Matern Child Health J. 2016 Jul 27doi: 10.1007/s10995-016-2083-0. PMID: 27465058.X-1

6386. Wilde L, Mitchell A, Oliver C. Differences in social motivation in children with Smith-Magenis syndrome and Down syndrome. Journal of Autism and Developmental Disorders. 2016;46(6):2148-59. doi: http://dx.doi.org/10.1007/s10803-0162743-3.X-1

6387. Wilkes-Gillan S, Joosten A. Technology-based interventions were found to have evidence of effectiveness on a range of outcomes, including social problem solving and facial and emotional processing skills for individuals with autism spectrum disorders. Aust Occup Ther J. 2016 Apr;63(2):135-6. doi: 10.1111/1440-1630.12274. PMID: 27045530.X-1, X-2, X-3, X-4

6388. Wilkinson M, Wang R, van der Kouwe A, et al. White and gray matter fiber pathways in autism spectrum disorder revealed by ex vivo diffusion MR tractography. Brain Behav. 2016 May 5:e00483. doi: 10.1002/brb3.483. PMID: 27247853.X-1

6389. Williams KE, Sciberras E. Sleep and self-regulation from birth to 7 years: A retrospective study of children with and without attention-deficit hyperactivity disorder at 8 to 9 years. Journal of Developmental and Behavioral Pediatrics. 2016;37(5):385-94. doi: http://dx.doi.org/10.1097/DBP.0000000000000 281.X-1

6390. Williamson AA, Patrick KE, Rubens SL, et al. Pediatric sleep disorders in an outpatient sleep clinic: Clinical presentation and needs of children with neurodevelopmental conditions. Clinical Practice in Pediatric Psychology. 2016;4(2):188-99. doi: http://dx.doi.org/10.1037/cpp0000135.X-1

6391. Willis K, Timmons L, Pruitt M, et al. The Relationship Between Optimism, Coping, and Depressive Symptoms in Hispanic Mothers and Fathers of Children with Autism Spectrum 
Disorder. J Autism Dev Disord. 2016

Jul;46(7):2427-40. doi: 10.1007/s10803-016-

2776-7. PMID: 27017210.X-1

6392. Wilmot B, Fry R, Smeester L, et al. Methylomic analysis of salivary DNA in childhood ADHD identifies altered DNA methylation in VIPR2. Journal of Child Psychology and Psychiatry. 2016;57(2):152-60. doi: http://dx.doi.org/10.1111/jcpp.12457.X-1

6393. Wilson CE, Murphy CM, McAlonan $\mathrm{G}$, et al. Does sex influence the diagnostic evaluation of autism spectrum disorder in adults? Autism. 2016 Jan 22doi: 10.1177/1362361315611381. PMID: 26802113.X-1

6394. Wilson TW, Heinrichs-Graham E, Proskovec AL, et al. Neuroimaging with magnetoencephalography: A dynamic view of brain pathophysiology. Transl Res. 2016 Jan 25doi: 10.1016/j.trsl.2016.01.007. PMID: 26874219.X-1

6395. Wise T, Radua J, Via E, et al. Common and distinct patterns of grey-matter volume alteration in major depression and bipolar disorder: evidence from voxel-based meta-analysis. Mol Psychiatry. 2016 May 24doi: 10.1038/mp.2016.72. PMID: 27217146.X-1

6396. Witteveen JS, Willemsen MH, Dombroski TC, et al. Haploinsufficiency of MeCP2-interacting transcriptional co-repressor SIN3A causes mild intellectual disability by affecting the development of cortical integrity. Nat Genet. 2016 Aug;48(8):877-87. doi: 10.1038/ng.3619. PMID: 27399968.X-1

6397. Wodka EL, Puts NA, Mahone EM, et al. The Role of Attention in Somatosensory Processing: A Multi-trait, Multi-method Analysis. J Autism Dev Disord. 2016 Jul 22doi: 10.1007/s10803-016-2866-6. PMID: 27448580.X-1

6398. Wolff N, Rubia K, Knopf H, et al. Reduced pain perception in children and adolescents with ADHD is normalized by methylphenidate. Child and Adolescent Psychiatry and Mental Health. 2016;10.X-1, X4
6399. Wong CM, Koh HC. Brief Report: Investigating the Implications of Applying the New DSM-5 Criteria for Diagnosing Autism Spectrum Disorder in a Preschool Population in Singapore. J Autism Dev Disord. 2016 Jun 18doi: 10.1007/s10803-016-2841-2. PMID: 27318808.X-1

6400. Wong S, Giulivi C. Autism, Mitochondria and Polybrominated Diphenyl Ether Exposure. CNS Neurol Disord Drug Targets. 2016;15(5):614-23. PMID: 27071785.X-1

6401. Woodman AC, Smith LE, Greenberg JS, et al. Contextual Factors Predict Patterns of Change in Functioning over 10 Years Among Adolescents and Adults with Autism Spectrum Disorders. J Autism Dev Disord. 2016 Jan;46(1):176-89. doi: 10.1007/s10803-0152561-z. PMID: 26319253.X-1

6402. Worth AJ, Marchione DM, Parry RC, et al. LC-MS Analysis of Human Platelets as a Platform for Studying Mitochondrial Metabolism. J Vis Exp. 2016(110):e53941. doi: 10.3791/53941. PMID: 27077278.X-1

6403. Woynaroski T, Watson L, Gardner E, et al. Early predictors of growth in diversity of key consonants used in communication in initially preverbal children with autism spectrum disorder. Journal of Autism and Developmental Disorders. 2016;46(3):1013-24. doi: http://dx.doi.org/10.1007/s10803-0152647-7.X-1

6404. Wright HF, Hall S, Mills DS. Additional evidence is needed to recommend acquiring a dog to families of children with Autism Spectrum Disorder: A response to Crossman and Kazdin. Journal of Autism and Developmental Disorders. 2016;46(1):336-9. doi: http://dx.doi.org/10.1007/s10803-0152548-9.X-1

6405. Wright K, Kelley E, Poulin-Dubois D. Biological motion and the animate-inanimate distinction in children with high-functioning Autism Spectrum Disorder. Research in Autism Spectrum Disorders. 2016;25:1-11. doi: http://dx.doi.org/10.1016/j.rasd.2016.01.005.X1

6406. Wu YE, Parikshak NN, Belgard TG, et al. Genome-wide, integrative analysis 
implicates microRNA dysregulation in autism spectrum disorder. Nat Neurosci. 2016 Aug 29doi: 10.1038/nn.4373. PMID: 27571009.X-1

6407. Wu Y-T, Maenner MJ, Wiggins LD, et al. Retention of autism spectrum disorder diagnosis: The role of co-occurring conditions in males and females. Research in Autism Spectrum Disorders. 2016;25:76-86. doi: http://dx.doi.org/10.1016/j.rasd.2016.02.001.X1

6408. Xavier A, Pinto Gouveia J, Cunha M. Non-suicidal self-injury in adolescence: The role of shame, self-criticism and fear of selfcompassion. Child \& Youth Care Forum. 2016;45(4):571-86. doi: http://dx.doi.org/10.1007/s10566-016-93461.X-1

6409. Xie P, Zang LQ, Li XK, et al. An epigenetic view of developmental diseases: new targets, new therapies. World J Pediatr. 2016 Aug;12(3):291-7. doi: 10.1007/s12519-0160020-3. PMID: 27351564.X-1

6410. Xie Z, Li J, Baker J, et al. Receptor Tyrosine Kinase MET Interactome and Neurodevelopmental Disorder Partners at the Developing Synapse. Biol Psychiatry. 2016 Feb 26doi: 10.1016/j.biopsych.2016.02.022. PMID: 27086544.X-1

6411. Xu L, Mitchell LC, Richman AR, et al. What Do Parents Think about Chromosomal Microarray Testing? A Qualitative Report from Parents of Children with Autism Spectrum Disorders. Autism Res Treat. 2016;2016:6852539. doi: 10.1155/2016/6852539. PMID: 27413549.X-1

6412. Xu Q, Goldstein J, Wang P, et al. Chromosomal microarray analysis in clinical evaluation of neurodevelopmental disordersreporting a novel deletion of SETDB1 and illustration of counseling challenge. Pediatr Res. 2016 Jun 1doi: 10.1038/pr.2016.101. PMID: 27119313.X-1

6413. Xu Z, Jiang H, Zhong P, et al. Direct conversion of human fibroblasts to induced serotonergic neurons. Mol Psychiatry. 2016 Jan;21(1):62-70. doi: 10.1038/mp.2015.101. PMID: 26216300.X-1
6414. Yaari M, Yitzhak N, Harel A, et al. Stability of early risk assessment for autism spectrum disorder in preterm infants. Autism. 2016 Jan 22doi: 10.1177/1362361315614758. PMID: 26802114.X-1

6415. Yagi M, Ohne H, Konomi T, et al. Teriparatide improves volumetric bone mineral density and fine bone structure in the UIV+1 vertebra, and reduces bone failure type PJK after surgery for adult spinal deformity. Osteoporos Int. 2016 Jun 24doi: 10.1007/s00198-016-3676-6. PMID: 27341809.X-1

6416. Yakubova G, Hughes EM, Shinaberry M. Learning with Technology: Video Modeling with Concrete-Representational-Abstract Sequencing for Students with Autism Spectrum Disorder. J Autism Dev Disord. 2016 Jul;46(7):2349-62. doi: 10.1007/s10803-0162768-7. PMID: 26983919.X-1, X-3, X-4

6417. Yakubova G, Hughes EM, Shinaberry M. Learning with technology: Video modeling with concrete-representational-abstract sequencing for students with autism spectrum disorder. Journal of Autism and Developmental Disorders. 2016;46(7):2349-62. doi: http://dx.doi.org/10.1007/s10803-016-27687.X-3, X-4

6418. Yalcin O, Kaymak G, Erdogan A, et al. A Retrospective Investigation of Clozapine Treatment in Autistic and Nonautistic Children and Adolescents in an Inpatient Clinic in Turkey. J Child Adolesc Psychopharmacol. 2016 Jan 15doi: 10.1089/cap.2015.0020. PMID: 26771824.X-1, X-3

6419. Yamasaki K, Hoshino M, Omori K, et al. Risk Factors of Adjacent Segment Disease after Transforaminal Inter-Body Fusion for Degenerative Lumbar Disease. Spine (Phila Pa 1976). 2016 Jun 6doi: 10.1097/brs.0000000000001728. PMID: 27270640.X-1

6420. Yamasaki M, Miyagawa T, Toyoda H, et al. Evaluation of polygenic risks for narcolepsy and essential hypersomnia. J Hum Genet. 2016 Jun 16doi: 10.1038/jhg.2016.65. PMID: 27305985.X-1

6421. Yamasaki Y, Nagao M, Kawanami S, et al. One-stop shop assessment for atrial septal 
defect closure using 256-slice coronary CT angiography. Eur Radiol. 2016 May 28doi: 10.1007/s00330-016-4407-2. PMID: 27236816.X-1

6422. Yang DY, Beam D, Pelphrey KA, et al. Cortical morphological markers in children with autism: a structural magnetic resonance imaging study of thickness, area, volume, and gyrification. Mol Autism. 2016;7:11. doi: 10.1186/s13229-016-0076-X. PMID: 26816612.X-1

6423. Yang EJ, Ahn S, Lee K, et al. Correction: Early Behavioral Abnormalities and Perinatal Alterations of PTEN/AKT Pathway in Valproic Acid Autism Model Mice. PLoS One. 2016;11(6):e0157202. doi:

10.1371/journal.pone.0157202. PMID: 27258156.X-1

6424. Yang S, Paynter JM, Gilmore L. Vineland Adaptive Behavior Scales: II profile of young children with autism spectrum disorder. Journal of Autism and Developmental Disorders. 2016;46(1):64-73. doi: http://dx.doi.org/10.1007/s10803-015-25431.X-1

6425. Yang X, Meng X. Dissociation between exact and approximate addition in developmental dyslexia. Research in Developmental Disabilities. 2016;56:139-52. doi: http://dx.doi.org/10.1016/j.ridd.2016.05.018.X1

6426. Yang X, Si T, Gong Q, et al. Brain gray matter alterations and associated demographic profiles in adults with autism spectrum disorder: A meta-analysis of voxelbased morphometry studies. Aust N Z J Psychiatry. 2016 Jan 14doi: 10.1177/0004867415623858. PMID: 26769980.X-1

6427. Yang Y, Zhang W, Wu Q, et al. Transcatheter closure of atrial septal defects without fluoroscopy: a well-established procedure for alternative use in children. EuroIntervention. 2016;12(5):e652-7. doi: 10.4244/eijv12i5a106. PMID: 27497366.X-1

6428. Yang YH. Parents and Young Children with Disabilities: The Effects of a Home-Based Music Therapy Program on Parent-Child
Interactions. J Music Ther. 2016

Spring;53(1):27-54. doi: 10.1093/jmt/thv018.

PMID: 26647404.X-3

6429. Yang Z, Ren Y, Liu D, et al. Prevalence of systemic autoimmune rheumatic diseases and clinical significance of ANA profile: data from a tertiary hospital in Shanghai, China. Apmis. 2016 Jun 22doi: 10.1111/apm.12564. PMID: 27328803.X-1

6430. Yau SH, Brock J, McArthur G. The relationship between spoken language and speech and nonspeech processing in children with autism: a magnetic event-related field study. Dev Sci. 2016 May 4doi: 10.1111/desc.12328. PMID: 27146167.X-1

6431. Yeetong P, Vilboux T, Ciccone C, et al. Delayed diagnosis in a house of correction: Smith-Magenis syndrome due to a de novo nonsense RAI1 variant. Am J Med Genet A. 2016 Jun 17doi: 10.1002/ajmg.a.37602. PMID: 27311559.X-1

6432. Yen CP, Mosley YI, Uribe JS. Role of minimally invasive surgery for adult spinal deformity in preventing complications. Curr Rev Musculoskelet Med. 2016 Sep;9(3):309-15. doi: 10.1007/s12178-016-9355-6. PMID: 27411527.X-1

6433. Yeramaneni S, Robinson C, Hostin R. Impact of spine surgery complications on costs associated with management of adult spinal deformity. Curr Rev Musculoskelet Med. 2016 Jun 9doi: 10.1007/s12178-016-9352-9. PMID: 27278531.X-1

6434. Yi F, Danko T, Botelho SC, et al. Autism-associated SHANK3 haploinsufficiency causes Ih channelopathy in human neurons. Science. 2016 May 6;352(6286):aaf2669. doi: 10.1126/science.aaf2669. PMID: 26966193.X-1

6435. Yi L, Quinn PC, Fan Y, et al. Children with Autism Spectrum Disorder scan own-race faces differently from other-race faces. Journal of Experimental Child Psychology. 2016;141:177-86. doi: http://dx.doi.org/10.1016/j.jecp.2015.09.011.X1

6436. Yildiz A, Tarakci D, Hajebrahimi F, et al. Disabled children's functionality and their mother's Quality of life and psychological 
status. Pediatr Int. 2016 Apr 16doi: 10.1111/ped.13020. PMID: 27085099.X-1

6437. Yimgang DP, Albury RA, Leppert ML. Do Parental Concerns Predict Developmental and Behavioral Diagnoses in a Developmental Clinic? Clin Pediatr (Phila). 2016 May 19doi: 10.1177/0009922816648289. PMID: 27207866.X-1

6438. Yitzhak N, Harel A, Yaari M, et al. The Mullen scales of early learning: Ceiling effects among preschool children. European Journal of Developmental Psychology. 2016;13(1):138-51. doi: http://dx.doi.org/10.1080/17405629.2015.10735 84.X-1

6439. Yochum A. Autism Spectrum/Pervasive Developmental Disorder. Prim Care. 2016 Jun;43(2):285-300. doi: 10.1016/j.pop.2016.01.010. PMID: 27262008.X-1

6440. Yoo MH, Kim TY, Yoon YH, et al. Autism phenotypes in ZnT3 null mice: Involvement of zinc dyshomeostasis, MMP-9 activation and BDNF upregulation. Sci Rep. 2016;6:28548. doi: 10.1038/srep28548. PMID: 27352957.X-1

6441. Yoon Y, Wink LK, Pedapati EV, et al. Weight Gain Effects of Second-Generation Antipsychotic Treatment in Autism Spectrum Disorder. J Child Adolesc Psychopharmacol. 2016 Jul 7doi: 10.1089/cap.2016.0049. PMID: 27389348.X-1, X-3

6442. Yoshida G, Boissiere L, Larrieu D, et al. Advantages and Disadvantages of Adult Spinal Deformity Surgery and its Impact on Health-related Quality of Life. Spine (Phila Pa 1976). 2016 Jul 7doi: 10.1097/brs.0000000000001770. PMID: 27398887.X-1

6443. Yoshimura Y, Kikuchi M, Hiraishi H, et al. Atypical development of the central auditory system in young children with Autism spectrum disorder. Autism Res. 2016 Jan 25doi: 10.1002/aur.1604. PMID: 26808455.X-1

6444. Young AM, Chakrabarti B, Roberts D, et al. From molecules to neural morphology: understanding neuroinflammation in autism spectrum condition. Mol Autism. 2016;7:9. doi: 10.1186/s13229-016-0068-x. PMID: 26793298.X-1

6445. Young N, Hudry K, Trembath D, et al. Children With Autism Show Reduced Information Seeking When Learning New Tasks. Am J Intellect Dev Disabil. 2016 Jan;121(1):65-73. doi: 10.1352/1944-7558121.1.65. PMID: 26701075.X-1

6446. Young RL, Nah YH. Examining autism detection in early childhood (ADEC) in the early identification of young children with autism spectrum disorders (ASD). Australian Psychologist. 2016;51(4):261-71. doi: http://dx.doi.org/10.1111/ap.12223.X-1

6447. Yu B. Bilingualism as conceptualized and bilingualism as lived: A critical examination of the monolingual socialization of a child with autism in a bilingual family. Journal of Autism and Developmental Disorders. 2016;46(2):424-35. doi: http://dx.doi.org/10.1007/s10803-015-2625$0 . \mathrm{X}-1$

6448. Yu H, Liu J, Yang A, et al. Lack of association between polymorphisms in dopa decarboxylase and dopamine receptor- 1 genes with childhood autism in Chinese Han population. Journal of Child Neurology. 2016;31(5):560-4. doi: http://dx.doi.org/10.1177/0883073815601496.X $-1$

6449. Yuan X, Devine DP. The role of anxiety in vulnerability for self-injurious behaviour: studies in a rodent model. Behav Brain Res. 2016 Sep 15;311:201-9. doi: 10.1016/j.bbr.2016.05.041. PMID: 27217100.X-1

6450. Yui K, Imataka G, Kawasak Y, et al. Increased omega-3 polyunsaturated fatty acid/arachidonic acid ratios and upregulation of signaling mediator in individuals with autism spectrum disorders. Life Sci. 2016 Jan 15;145:205-12. doi: 10.1016/j.lfs.2015.12.039. PMID: 26724495.X-1

6451. Yui K, Imataka G, Kawasaki Y, et al. Down-regulation of a signaling mediator in association with lowered plasma arachidonic acid levels in individuals with autism spectrum disorders. Neurosci Lett. 2016 Jan 1;610:223-8. 
doi: 10.1016/j.neulet.2015.11.006. PMID:

26552013.X-1

6452. Yun S-S, Kim H, Choi J, et al. A robot-assisted behavioral intervention system for children with autism spectrum disorders. Robotics and Autonomous Systems. 2016;76:58-67. doi: http://dx.doi.org/10.1016/j.robot.2015.11.004.X $-1$

6453. Zahorakova D, Lelkova P, Gregor V, et al. MECP2 mutations in Czech patients with Rett syndrome and Rett-like phenotypes: novel mutations, genotype-phenotype correlations and validation of high-resolution melting analysis for mutation scanning. J Hum Genet. 2016 Mar 17doi: 10.1038/jhg.2016.19. PMID:

26984561.X-1

6454. Zaidman-Zait A, Curle D. Complexity: An interpretative phenomenological analysis of the experiences of mothers of deaf children with cochlear implants and autism. J Health Psychol. 2016 May 16doi:

10.1177/1359105316646171. PMID: 27185249.X-1

6455. Zaidman-Zait A, Mirenda P, Duku E, et al. Impact of personal and social resources on parenting stress in mothers of children with autism spectrum disorder. Autism. 2016 Apr 18doi: 10.1177/1362361316633033. PMID: 27091948.X-1

6456. Zainal H, Magiati I. A Comparison Between Caregiver-Reported Anxiety and Other Emotional and Behavioral Difficulties in Children and Adolescents with Autism Spectrum Disorders Attending Specialist or Mainstream Schools. J Autism Dev Disord. 2016 May 10doi: 10.1007/s10803-016-2792-7. PMID: 27160302.X-1

6457. Zamponi GW. Targeting voltage-gated calcium channels in neurological and psychiatric diseases. Nat Rev Drug Discov. 2016 Jan;15(1):19-34. doi: 10.1038/nrd.2015.5. PMID: 26542451.X-1, X-2, X-3, X-4

6458. Zanatta G, Della Flora Nunes G, Bezerra EM, et al. Two Binding Geometries for Risperidone in Dopamine D3 Receptors: Insights on the Fast-Off Mechanism through Docking, Quantum Biochemistry, and Molecular Dynamics Simulations. ACS Chem
Neurosci. 2016 Aug 5doi:

10.1021/acschemneuro.6b00074. PMID: 27434874.X-1

6459. Zander E, Willfors C, Berggren S, et al. The objectivity of the Autism Diagnostic Observation Schedule (ADOS) in naturalistic clinical settings. European Child \& Adolescent Psychiatry. 2016;25(7):769-80. doi: http://dx.doi.org/10.1007/s00787-015-07932.X-1

6460. Zarafshan H, Khaleghi A, Mohammadi MR, et al. Electroencephalogram complexity analysis in children with attentiondeficit/hyperactivity disorder during a visual cognitive task. Journal of Clinical and Experimental Neuropsychology. 2016;38(3):361-9. doi: http://dx.doi.org/10.1080/13803395.2015.11192 52.X-1

6461. Zauche LH, Thul TA, Mahoney AED, et al. Influence of language nutrition on children's language and cognitive development: An integrated review. Early Childhood Research Quarterly. 2016;36:318-33. doi: http://dx.doi.org/10.1016/j.ecresq.2016.01.015. $\mathrm{X}-1$

6462. Zavadenko NN, Suvorinova NY. Results of pharmacotherapy of attention deficit hyperactivity disorder: Assessment using neuropsychological methods. Neuroscience and Behavioral Physiology. 2016;46(3):354-9. doi: http://dx.doi.org/10.1007/s11055-016-0241x.X-1

6463. Zeedyk SM, Cohen SR, Eisenhower A, et al. Perceived social competence and loneliness among young children with ASD: Child, parent and teacher reports. Journal of Autism and Developmental Disorders. 2016;46(2):436-49. doi: http://dx.doi.org/10.1007/s10803-015-25756.X-1

6464. Zeidan-Chulia F, de Oliveira BH, Casanova MF, et al. Up-Regulation of Oligodendrocyte Lineage Markers in the Cerebellum of Autistic Patients: Evidence from Network Analysis of Gene Expression. Mol Neurobiol. 2016 Aug;53(6):4019-25. doi: 10.1007/s12035-015-9351-7. PMID: 26189831.X-1 
6465. Zerbo O, Traglia M, Yoshida C, et al. Maternal mid-pregnancy C-reactive protein and risk of autism spectrum disorders: the early markers for autism study. Transl Psychiatry. 2016;6:e783. doi: 10.1038/tp.2016.46. PMID: 27093065.X-1

6466. Zettergren A, Karlsson S, Hovey D, et al. Further investigations of the relation between polymorphisms in sex steroid related genes and autistic-like traits.

Psychoneuroendocrinology. 2016;68:1-5. doi: http://dx.doi.org/10.1016/j.psyneuen.2016.02.0 20.X-1

6467. Zhang B, Zou J, Han L, et al. Microglial activation during epileptogenesis in a mouse model of tuberous sclerosis complex. Epilepsia. 2016 Jun 6doi: 10.1111/epi.13429. PMID: 27263494.X-1

6468. Zhang D, Cui H, Zhang L, et al. Is maternal smoking during pregnancy associated with an increased risk of congenital heart defects among offspring? A systematic review and meta-analysis of observational studies. J Matern Fetal Neonatal Med. 2016 May 16:1-13. doi: 10.1080/14767058.2016.1183640. PMID: 27126055.X-1

6469. Zhang H, Harris L, Split M, et al. Anhedonia and individual differences in orbitofrontal cortex sulcogyral morphology. Hum Brain Mapp. 2016 Jun 22doi: 10.1002/hbm.23282. PMID: 27329212.X-1

6470. Zhang HF, Dai YC, Wu J, et al. Plasma Oxytocin and Arginine-Vasopressin Levels in Children with Autism Spectrum Disorder in China: Associations with Symptoms. Neurosci Bull. 2016 Jun 24doi: 10.1007/s12264-016-0046-5. PMID: 27342432.X-1

6471. Zhang J, Cheng W, Liu Z, et al. Neural, electrophysiological and anatomical basis of brain-network variability and its characteristic changes in mental disorders. Brain. 2016 Aug;139(Pt 8):2307-21. doi: 10.1093/brain/aww143. PMID: 27421791.X-1

6472. Zhang J, Zhang JX, Zhang QL. PI3K/AKT/mTOR-mediated autophagy in the development of autism spectrum disorder. Brain Res Bull. 2016 Jun 16doi: 10.1016/j.brainresbull.2016.06.007. PMID: 27320472.X-1

6473. Zhang T, Shao Z, Zhang Y. Developmental steps in theory of mind of typical Chinese children and Chinese children with autism spectrum disorder. Research in Autism Spectrum Disorders. 2016;23:210-20. doi: http://dx.doi.org/10.1016/j.rasd.2015.10.005.X1

6474. Zhang W, Baranek G. The Impact of Insurance Coverage Types on Access to and Utilization of Health Services for U.S. Children With Autism. Psychiatr Serv. 2016 Apr 15:appips201500206. doi: 10.1176/appi.ps.201500206. PMID: 27079986.X-1

6475. Zhang Y, Chen M, Qiu Z, et al. MiR130a regulates neurite outgrowth and dendritic spine density by targeting MeCP2. Protein Cell. 2016 Jul;7(7):489-500. doi: 10.1007/s13238016-0272-7. PMID: 27245166.X-1

6476. Zhang Y, Hodgson NW, Trivedi MS, et al. Decreased Brain Levels of Vitamin B12 in Aging, Autism and Schizophrenia. PLoS One. 2016;11(1):e0146797. doi: 10.1371/journal.pone.0146797. PMID: 26799654.X-1

6477. Zhang Y, Shan JL, Liu XM, et al. Comparison of the Dynesys Dynamic Stabilization System and Posterior Lumbar Interbody Fusion for Lumbar Degenerative Disease. PLoS One. 2016;11(1):e0148071. doi: 10.1371/journal.pone.0148071. PMID: 26824851.X-1

6478. Zhao F, Qiao L, Shi F, et al. Feature fusion via hierarchical supervised local CCA for diagnosis of autism spectrum disorder. Brain Imaging Behav. 2016 Aug 17doi: 10.1007/s11682-016-9587-5. PMID: 27535871.X-1

6479. Zhao Y, Castellanos FX. Annual research review: Discovery science strategies in studies of the pathophysiology of child and adolescent psychiatric disorders-Promises and limitations. Journal of Child Psychology and Psychiatry. 2016;57(3):421-39. doi: http://dx.doi.org/10.1111/jcpp.12503.X-1 
6480. Zhao Y, Chen H, Li Y, et al. Connectome-scale group-wise consistent resting-state network analysis in autism spectrum disorder. Neuroimage Clin. 2016;12:23-33. doi: 10.1016/j.nicl.2016.06.004. PMID: 27358766.X-1

6481. Zhong J, Amina S, Liang M, et al. Cyclic ADP-Ribose and Heat Regulate Oxytocin Release via CD38 and TRPM2 in the Hypothalamus during Social or Psychological Stress in Mice. Front Neurosci. 2016;10:304. doi: 10.3389/fnins.2016.00304. PMID: 27499729.X-1

6482. Zhou J, Zhang X, Ren J, et al. Validation of reference genes for quantitative real-time PCR in valproic acid rat models of autism. Mol Biol Rep. 2016 Jun 10doi: 10.1007/s11033-016-4015-X. PMID: 27287459.X-1

6483. Zhou P, Zhang Y, Wei C, et al. Acute stress disorder as a predictor of posttraumatic stress: A longitudinal study of Chinese children exposed to the Lushan earthquake. Psych J. 2016 Jun 22doi: 10.1002/pchj.136. PMID: 27329012.X-1

6484. Zhou Y, Kaiser T, Monteiro P, et al. Mice with Shank3 Mutations Associated with ASD and Schizophrenia Display Both Shared and Distinct Defects. Neuron. 2016 Jan 6;89(1):147-62. doi: 10.1016/j.neuron.2015.11.023. PMID: 26687841.X-1

6485. Zhu Y, Xing B, Dang W, et al. AUTS2 in the nucleus accumbens is essential for heroin-induced behavioral sensitization. Neuroscience. 2016 Jul 14;333:35-43. doi: 10.1016/j.neuroscience.2016.07.007. PMID: 27423627.X-1

6486. Zhu Y, Zhang B, Liu H, et al. Cervical Disc Arthroplasty Versus Anterior Cervical Discectomy and Fusion for Incidence of Symptomatic Adjacent Segment Disease: A Meta-Analysis of Prospective Randomized Controlled Trials. Spine (Phila Pa 1976). 2016 Feb 26doi: 10.1097/brs.0000000000001537. PMID: 26926472.X-1

6487. Ziats MN, Goin-Kochel RP, Berry LN, et al. The complex behavioral phenotype of 15q13.3 microdeletion syndrome. Genet Med.
2016 Mar 10doi: 10.1038/gim.2016.9. PMID: 26963284.X-1

6488. Ziegler A, Neues F, Janacek J, et al. Mineral in skeletal elements of the terrestrial crustacean Porcellio scaber: SRmuCT of function related distribution and changes during the moult cycle. Arthropod Struct Dev. 2016 Jun 30doi: 10.1016/j.asd.2016.05.004. PMID: 27288588.X-1

6489. Zilkha N, Kuperman Y, Kimchi T. High-fat diet exacerbates cognitive rigidity and social deficiency in the BTBR mouse model of autism. Neuroscience. 2016 Feb 11doi: 10.1016/j.neuroscience.2016.01.070. PMID: 26855190.X-1

6490. Zimmermann FF, Gaspary KV, Siebel AM, et al. Oxytocin reversed MK-801-induced social interaction and aggression deficits in zebrafish. Behav Brain Res. 2016 Sep 15;311:368-74. doi: 10.1016/j.bbr.2016.05.059. PMID: 27247142.X-1

6491. Zimmermann FF, Gaspary KV, Siebel AM, et al. Analysis of Extracellular Nucleotide Metabolism in Adult Zebrafish After Embryological Exposure to Valproic Acid. Mol Neurobiol. 2016 May 17doi: 10.1007/s12035016-9917-z. PMID: 27189619.X-1

6492. Zink AG, Diniz MB, Rodrigues Dos Santos MT, et al. Use of a Picture Exchange Communication System for preventive procedures in individuals with autism spectrum disorder: pilot study. Spec Care Dentist. 2016 Apr 5doi: 10.1111/scd.12183. PMID: 27059442.X-1

6493. Zohar AH, Dahan D. Young children's ritualistic compulsive-like behavior and executive function: A cross sectional study. Child Psychiatry and Human Development. 2016;47(1):13-22. doi: http://dx.doi.org/10.1007/s10578-015-0539$\mathrm{x} . \mathrm{X}-1$

6494. Zuckerman K, Lindly OJ, Chavez AE. Timeliness of Autism Spectrum Disorder Diagnosis and Use of Services Among U.S. Elementary School-Aged Children. Psychiatr Serv. 2016 Aug 1:appips201500549. doi: 10.1176/appi.ps.201500549. PMID: 27476809.X-1 
6495. Zuko A, Oguro-Ando A, van Dijk R, et al. Developmental role of the cell adhesion molecule Contactin-6 in the cerebral cortex and hippocampus. Cell Adh Migr. 2016 Mar 3:1-15. doi: 10.1080/19336918.2016.1155018. PMID: 26939565.X-1

6496. Zwaigenbaum L, Bryson SE, Brian J, et al. Stability of diagnostic assessment for autism spectrum disorder between 18 and 36 months in a high-risk cohort. Autism Research. 2016;9(7):790-800. doi:

http://dx.doi.org/10.1002/aur.1585.X-1

6497. Zwanenburg RJ, Bocca G, Ruiter SA, et al. Is there an effect of intranasal insulin on development and behaviour in Phelan-

McDermid syndrome? A randomized, doubleblind, placebo-controlled trial. Eur J Hum Genet. 2016 Aug 31doi:

10.1038/ejhg.2016.109. PMID: 27577546.X-1

6498. Zwanenburg RJ, Ruiter SAJ, van den Heuvel ER, et al. Developmental phenotype in Phelan-McDermid (22q13.3 deletion) syndrome: A systematic and prospective study in 34 children. Journal of Neurodevelopmental Disorders. 2016;8doi:

http://dx.doi.org/10.1186/s11689-016-9150-

$0 . \mathrm{X}-1$ 


\section{Appendix D. Risk of Bias Ratings}

Table D-1. Risk of bias assessments

\begin{tabular}{|c|c|c|c|c|c|c|c|c|c|c|c|c|c|c|c|c|c|c|c|}
\hline 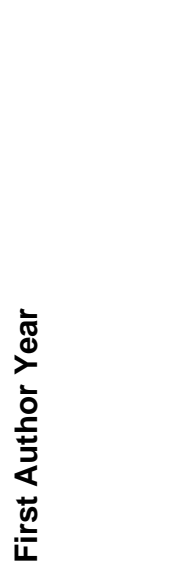 & 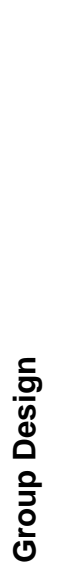 & 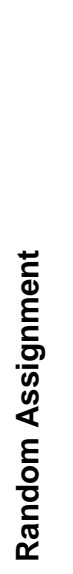 & 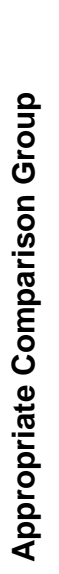 & 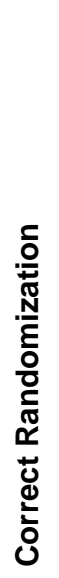 & 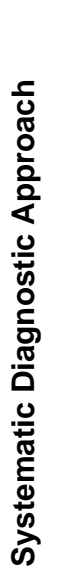 & 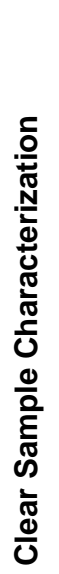 & 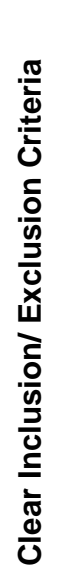 & 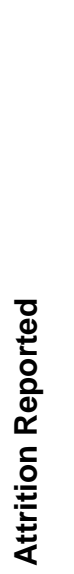 & 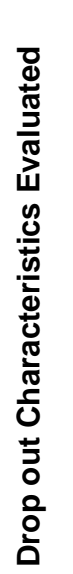 & 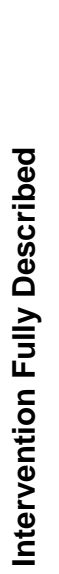 & 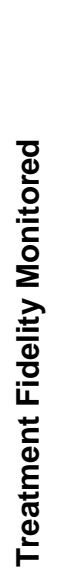 & 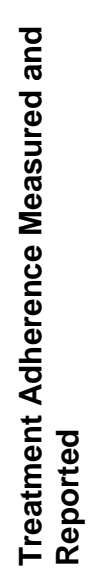 & 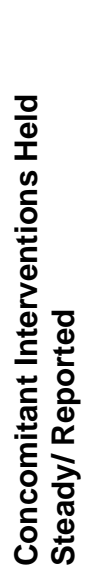 & 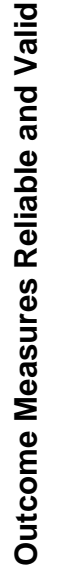 & 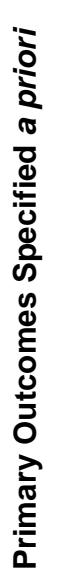 & 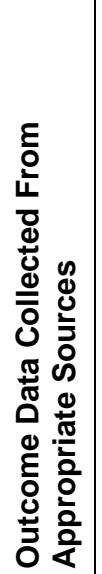 & 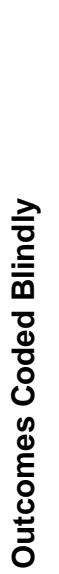 & 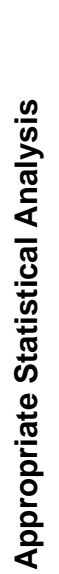 & 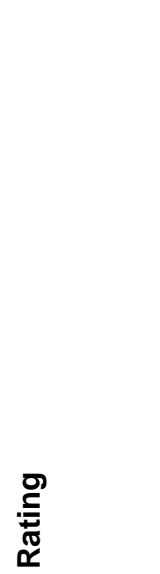 \\
\hline $\begin{array}{l}\text { Aman } \\
2016^{1}\end{array}$ & + & + & + & + & ++ & + & + & + & - & + & NA & + & + & + & + & + & + & - & Low \\
\hline $\begin{array}{l}\text { Aman } \\
2015^{2-12}\end{array}$ & + & + & + & + & ++ & + & + & + & + & + & + & + & + & + & + & + & + & + & Low \\
\hline $\begin{array}{l}\text { Amatachay } \\
\text { a } 2015^{13,14}\end{array}$ & + & + & + & + & + & - & + & - & NA & + & NA & + & + & + & + & + & + & - & Moderate \\
\hline $\begin{array}{l}\text { Arnold } \\
2^{2012^{15}}\end{array}$ & + & + & + & + & ++ & + & + & + & NA & + & NA & + & + & + & + & + & + & - & Low \\
\hline $\begin{array}{l}\text { Akhondza } \\
\text { deh } 2010^{16}\end{array}$ & + & + & + & + & - & - & + & NA & NA & + & NA & - & - & + & + & + & + & - & Moderate \\
\hline $\begin{array}{l}\text { Akhondza } \\
\text { deh } 2008^{17}\end{array}$ & + & + & + & + & - & - & + & + & NA & + & NA & - & + & + & + & + & + & + & Moderate \\
\hline $\begin{array}{l}\text { Arnold } \\
2006^{18}\end{array}$ & + & + & + & - & + & + & + & + & - & + & NA & - & + & + & + & + & + & - & moderate \\
\hline $\begin{array}{l}\text { Al Ayadhi } \\
2013^{19}\end{array}$ & + & + & + & - & + & - & - & + & NA & + & - & - & + & + & + & + & + & - & High \\
\hline $\begin{array}{l}\text { Asadabadi } \\
2013^{20}\end{array}$ & + & + & + & + & ++ & + & + & + & NA & + & NA & + & + & + & + & + & + & - & Low \\
\hline Bent & + & + & & + & - & + & + & + & NA & + & NA & + & + & + & + & + & + & - & Moderate \\
\hline
\end{tabular}




\begin{tabular}{|c|c|c|c|c|c|c|c|c|c|c|c|c|c|c|c|c|c|c|c|}
\hline 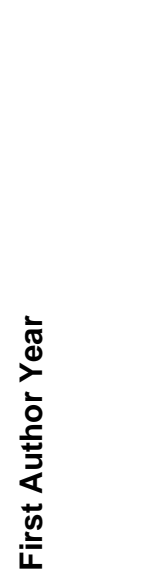 & $\begin{array}{l}\frac{5}{0} \\
\frac{0}{0} \\
0 \\
\frac{0}{3} \\
\frac{0}{0}\end{array}$ & 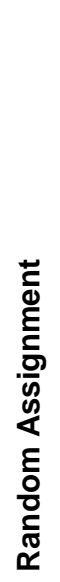 & 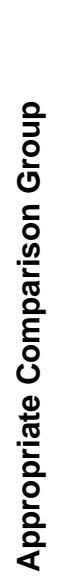 & 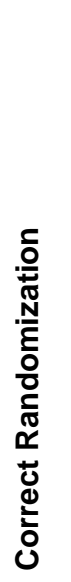 & 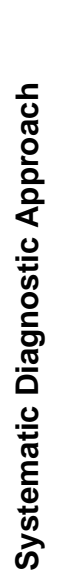 & 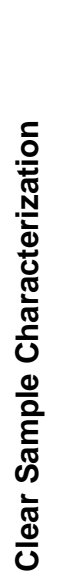 & 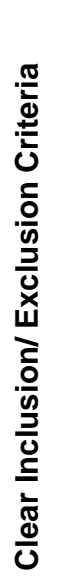 & 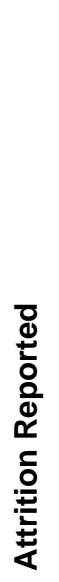 & 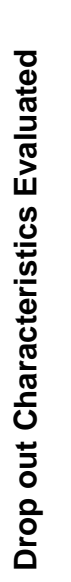 & 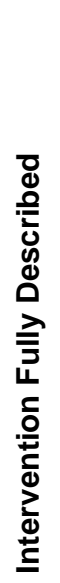 & 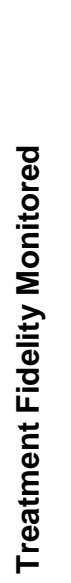 & 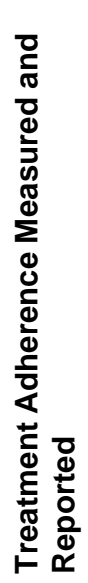 & 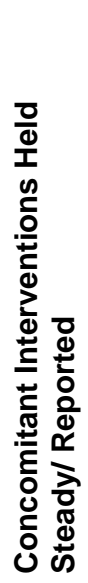 & 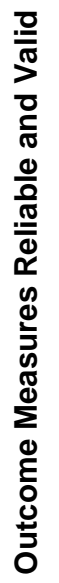 & 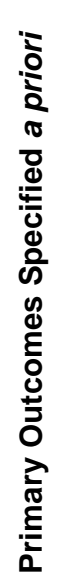 & 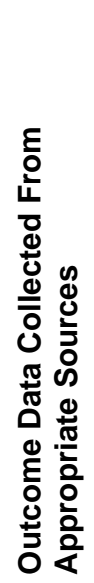 & 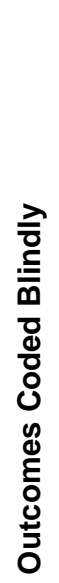 & 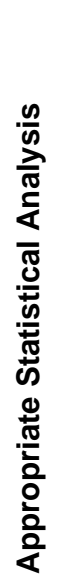 & 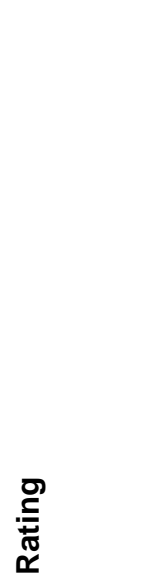 \\
\hline $2014^{21}$ & & & & & & & & & & & & & & & & & & & \\
\hline $\begin{array}{l}\text { Bent } \\
2011^{22}\end{array}$ & + & + & + & + & ++ & + & + & + & NA & + & NA & + & + & + & + & + & + & + & Low \\
\hline $\begin{array}{l}\text { Bertoglio } \\
2010^{23}\end{array}$ & + & + & + & - & ++ & + & + & + & NA & + & NA & + & + & + & + & + & + & - & Low \\
\hline $\begin{array}{l}\text { Chugani } \\
2016^{24}\end{array}$ & + & + & + & - & ++ & + & + & + & - & + & NA & + & + & + & + & + & + & - & Low \\
\hline $\begin{array}{l}\text { Cortesi } \\
2012^{25}\end{array}$ & + & + & + & + & ++ & + & + & + & + & + & NA & + & + & + & + & + & + & - & Low \\
\hline $\begin{array}{l}\text { Chez } \\
2003^{26}\end{array}$ & + & + & + & - & + & + & + & + & + & + & NA & - & + & + & + & + & + & - & Moderate \\
\hline $\begin{array}{l}\text { Dean } \\
2016^{27}\end{array}$ & + & + & + & + & + & + & + & + & - & + & NA & + & + & + & + & + & - & - & Moderate \\
\hline $\begin{array}{l}\text { Dadds } \\
2014^{28}\end{array}$ & + & + & + & - & + & + & + & + & - & + & NA & + & + & + & - & + & + & - & Low \\
\hline $\begin{array}{l}\text { Duffy } \\
2014^{29}\end{array}$ & + & - & - & NA & + & - & + & - & NA & + & NA & + & + & - & + & - & - & - & High \\
\hline $\begin{array}{l}\text { Findling } \\
2014^{30}\end{array}$ & + & + & + & - & ++ & + & + & + & - & + & NA & - & + & + & + & + & + & + & Low \\
\hline Fahmy & + & + & + & - & - & + & + & + & - & + & NA & - & - & + & - & + & + & - & Moderate \\
\hline
\end{tabular}

D-2 


\begin{tabular}{|c|c|c|c|c|c|c|c|c|c|c|c|c|c|c|c|c|c|c|c|}
\hline 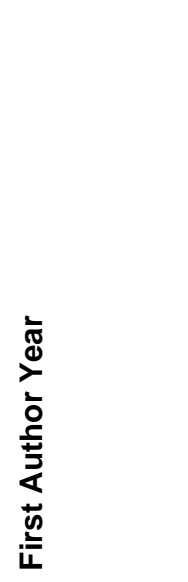 & $\begin{array}{l}\frac{c}{0} \\
\frac{0}{0} \\
0 \\
0 \\
\frac{0}{2} \\
\frac{0}{0}\end{array}$ & 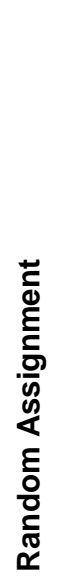 & 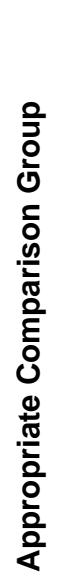 & 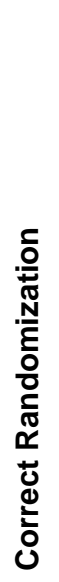 & 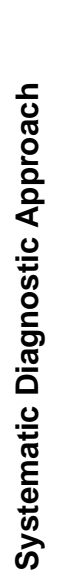 & 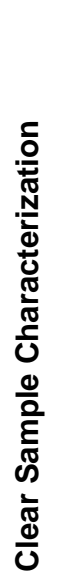 & 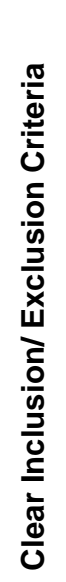 & 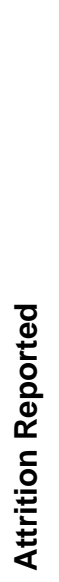 & 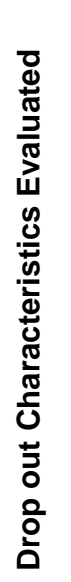 & 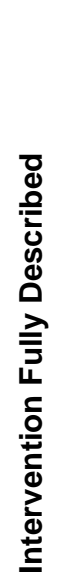 & 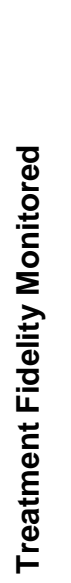 & 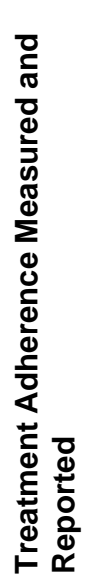 & 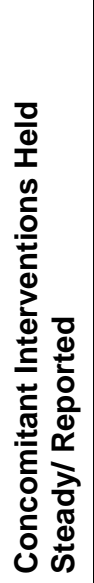 & 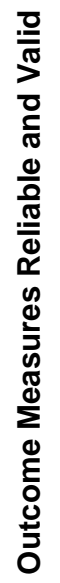 & 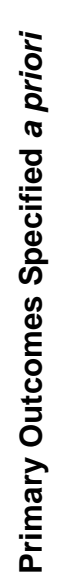 & 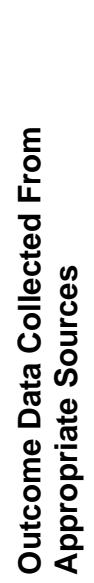 & 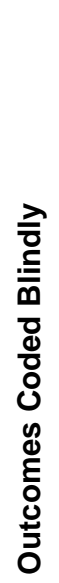 & 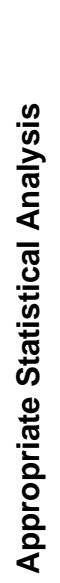 & 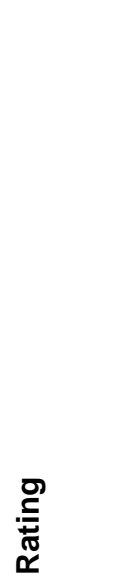 \\
\hline $2013^{31}$ & & & & & & & & & & & & & & & & & & & \\
\hline $\begin{array}{l}\text { Ghaleiha } \\
2016^{32}\end{array}$ & + & + & + & + & ++ & + & + & + & NA & + & NA & + & + & + & + & + & + & - & Low \\
\hline $\begin{array}{l}\text { Ghalichi } \\
2016^{33}\end{array}$ & + & + & + & - & - & + & + & + & $\mathrm{NA}$ & + & $\mathrm{NA}$ & + & - & + & + & + & - & - & High \\
\hline $\begin{array}{l}\text { Ghanizade } \\
\text { h } 2014^{34}\end{array}$ & + & + & + & - & ++ & + & + & + & - & + & NA & + & + & + & + & + & + & + & Low \\
\hline $\begin{array}{l}\text { Geier } \\
2011^{35}\end{array}$ & + & + & + & - & - & - & - & + & - & + & NA & + & + & + & + & + & + & - & High \\
\hline $\begin{array}{l}\text { Granpeesh } \\
\text { eh } 2010^{36}\end{array}$ & + & + & + & - & ++ & - & + & + & - & + & NA & + & + & + & + & + & + & + & Low \\
\hline $\begin{array}{l}\text { Ghaleiha } \\
2015^{37}\end{array}$ & + & + & + & + & ++ & + & + & + & NA & + & NA & + & + & + & + & + & + & - & Low \\
\hline $\begin{array}{l}\text { Ghaleiha } \\
2013^{38}\end{array}$ & + & + & + & + & ++ & + & + & + & - & + & NA & + & + & + & + & + & + & + & Low \\
\hline $\begin{array}{l}\text { Ghaleiha } \\
2013^{39}\end{array}$ & + & + & + & + & ++ & + & + & + & NA & + & NA & + & + & + & + & + & + & - & Low \\
\hline $\begin{array}{l}\text { Ghaleiha } \\
2013^{40}\end{array}$ & + & + & + & + & ++ & + & + & + & - & + & NA & + & + & + & + & + & + & - & Low \\
\hline $\begin{array}{l}\text { Ghanizade } \\
\text { h } 2015^{41}\end{array}$ & + & + & + & + & ++ & - & + & + & - & + & NA & - & + & + & + & + & + & + & Low \\
\hline
\end{tabular}

D-3 


\begin{tabular}{|c|c|c|c|c|c|c|c|c|c|c|c|c|c|c|c|c|c|c|c|}
\hline 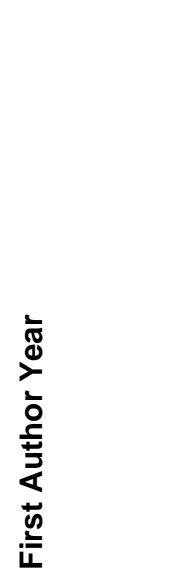 & $\begin{array}{l}\text { s. } \\
\frac{0}{0} \\
0 \\
0 \\
\frac{0}{\overline{0}} \\
\frac{0}{0}\end{array}$ & 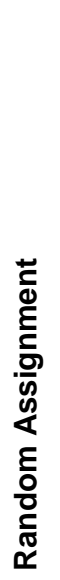 & 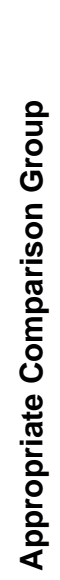 & 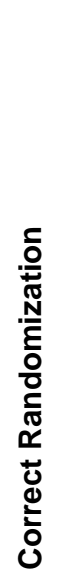 & 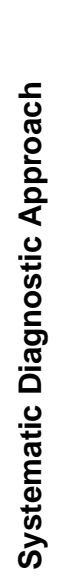 & 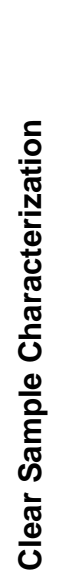 & 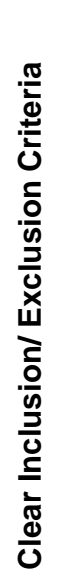 & 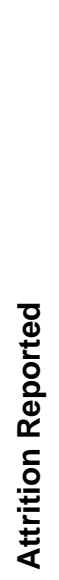 & 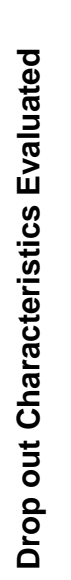 & 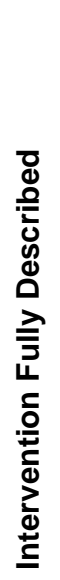 & 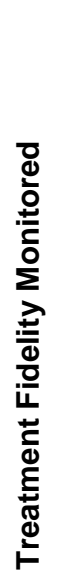 & 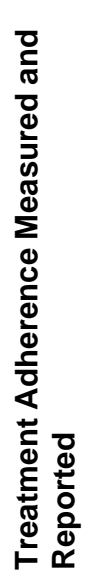 & 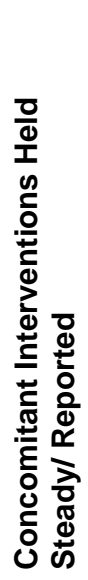 & 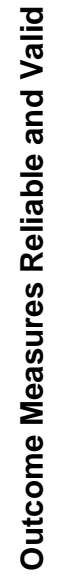 & 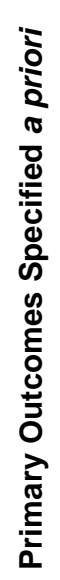 & 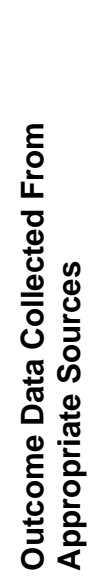 & 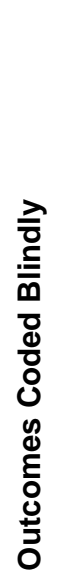 & 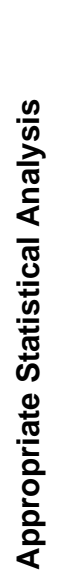 & 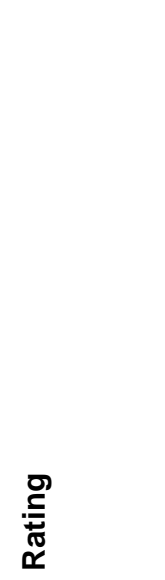 \\
\hline $\begin{array}{l}\text { Ghanizade } \\
\text { h } 2013^{42}\end{array}$ & + & + & + & + & ++ & - & + & + & - & + & NA & - & + & + & + & + & + & + & Low \\
\hline $\begin{array}{l}\text { Hendren } \\
2016^{43}\end{array}$ & + & + & + & + & + & + & + & + & - & + & NA & + & - & + & + & + & + & - & Moderate \\
\hline $\begin{array}{l}\text { Handen } \\
2015^{44,45}\end{array}$ & + & + & + & + & ++ & + & + & + & - & + & NA & + & + & + & + & + & + & - & Low \\
\hline $\begin{array}{l}\text { Hyman } \\
2015^{46}\end{array}$ & + & + & + & + & ++ & + & + & + & NA & + & NA & + & + & + & + & + & + & - & Low \\
\hline $\begin{array}{l}\text { Handen } \\
2011^{47}\end{array}$ & + & + & + & - & ++ & + & + & + & NA & + & NA & + & + & + & + & + & + & + & Low \\
\hline $\begin{array}{l}\text { Hollander } \\
2010^{48}\end{array}$ & + & + & + & - & ++ & + & + & + & - & + & NA & + & + & + & + & + & + & + & Low \\
\hline $\begin{array}{l}\text { Hardan } \\
2012^{49}\end{array}$ & + & + & + & + & ++ & + & + & + & - & + & NA & + & + & + & + & + & + & - & Low \\
\hline $\begin{array}{l}\text { Hasanzade } \\
\text { h } 2012^{50}\end{array}$ & + & + & + & + & ++ & + & + & + & $\mathrm{NA}$ & + & $\mathrm{NA}$ & - & + & + & + & + & + & - & Low \\
\hline $\begin{array}{l}\text { Harrison } \\
2006^{51,52}\end{array}$ & + & + & - & - & ++ & - & + & + & - & + & NA & - & - & + & + & + & + & - & Moderate \\
\hline $\begin{array}{l}\text { Johnson } \\
2011^{53}\end{array}$ & + & + & + & - & ++ & + & + & + & NA & + & NA & + & - & + & + & + & - & - & Moderate \\
\hline $\begin{array}{l}\text { King } \\
2001^{54}\end{array}$ & + & + & + & - & + & + & + & + & NA & + & NA & - & + & + & + & + & + & - & Moderate \\
\hline
\end{tabular}




\begin{tabular}{|c|c|c|c|c|c|c|c|c|c|c|c|c|c|c|c|c|c|c|c|}
\hline 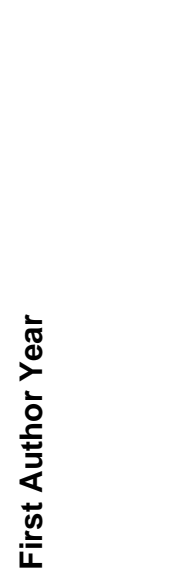 & $\begin{array}{l}\frac{5}{0} \\
\frac{0}{0} \\
0 \\
0 \\
\frac{0}{3} \\
0 \\
0\end{array}$ & 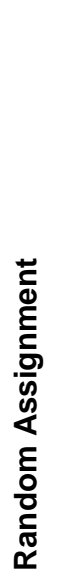 & 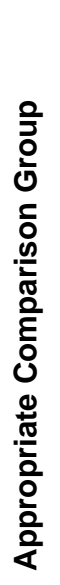 & 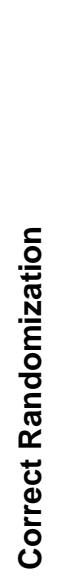 & 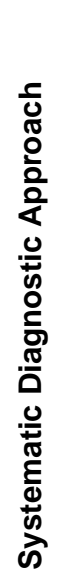 & 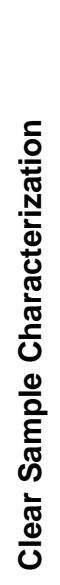 & 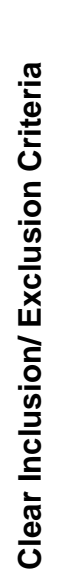 & 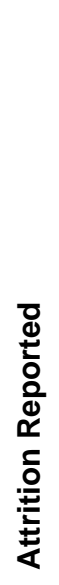 & 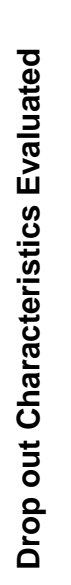 & 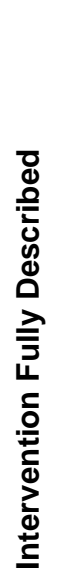 & 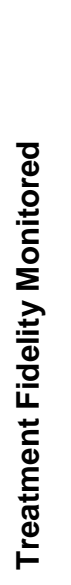 & 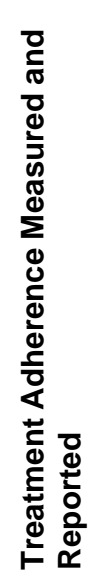 & 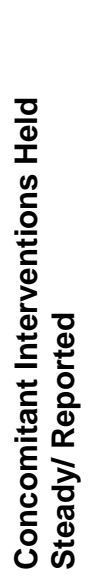 & 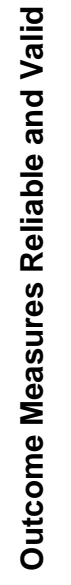 & 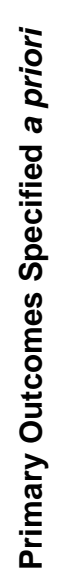 & 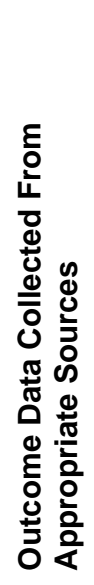 & 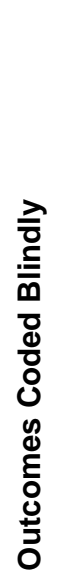 & 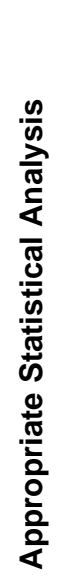 & 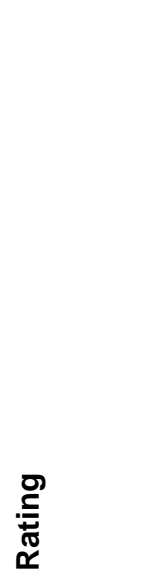 \\
\hline $\begin{array}{l}\text { Kent } \\
2013^{55,56}\end{array}$ & + & + & + & + & ++ & + & + & + & - & + & NA & - & + & + & + & + & + & + & Low \\
\hline $\begin{array}{l}\text { King } \\
2013^{57,58}\end{array}$ & + & + & + & + & ++ & + & + & + & + & + & NA & + & + & + & + & + & + & + & Low \\
\hline $\begin{array}{l}\text { Klaiman } \\
2013^{59}\end{array}$ & + & + & + & + & ++ & - & + & + & - & + & NA & + & + & + & + & + & + & - & Low \\
\hline $\begin{array}{l}\text { Lamberti } \\
2016^{60}\end{array}$ & + & + & + & - & ++ & + & + & + & - & + & NA & - & - & + & + & + & - & + & Low \\
\hline $\operatorname{Lv} 2013^{61}$ & + & - & - & NA & ++ & - & + & + & NA & + & NA & + & + & + & + & + & - & + & High \\
\hline $\begin{array}{l}\text { Lemonnier } \\
2012^{62}\end{array}$ & + & + & + & - & ++ & + & + & + & NA & + & NA & - & + & + & + & + & + & - & Moderate \\
\hline $\begin{array}{l}\text { Mankad } \\
2015^{63}\end{array}$ & + & + & + & + & ++ & + & + & + & - & + & NA & + & + & + & + & + & + & - & Low \\
\hline $\begin{array}{l}\text { McCracken } \\
2014^{64-67}\end{array}$ & + & + & + & + & ++ & + & + & + & - & + & NA & - & + & + & + & + & + & + & Moderate \\
\hline $\begin{array}{l}\text { Mohamma } \\
\text { di } 2013^{68}\end{array}$ & + & + & + & + & ++ & + & + & + & NA & + & NA & + & + & + & + & + & + & + & Low \\
\hline $\begin{array}{l}\text { Marcus } \\
2011^{69-75}\end{array}$ & + & + & + & + & ++ & + & + & + & - & + & NA & + & + & + & + & + & + & + & Low \\
\hline
\end{tabular}

D-5 


\begin{tabular}{|c|c|c|c|c|c|c|c|c|c|c|c|c|c|c|c|c|c|c|c|}
\hline 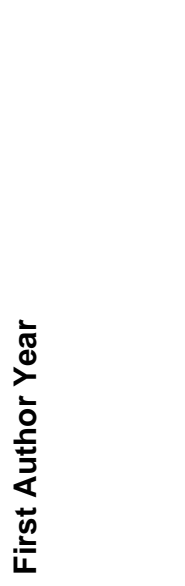 & $\begin{array}{l}\frac{c}{0} \\
\frac{0}{0} \\
0 \\
0 \\
\frac{0}{z} \\
\frac{0}{0}\end{array}$ & 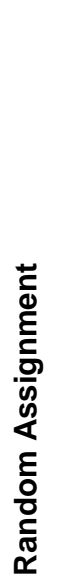 & 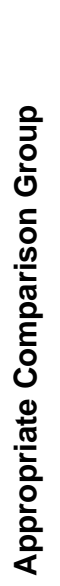 & 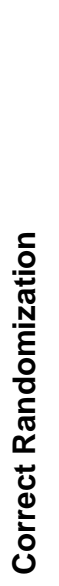 & 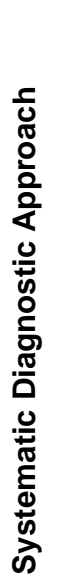 & 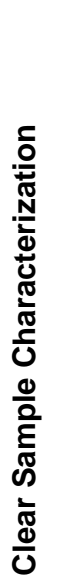 & 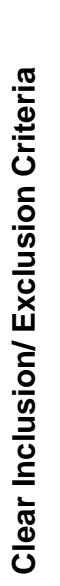 & 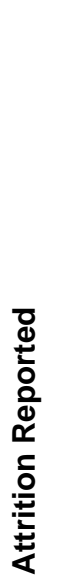 & 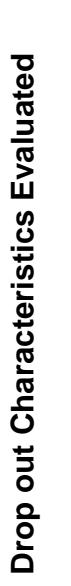 & 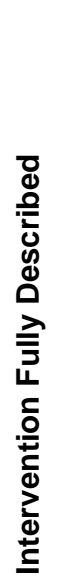 & 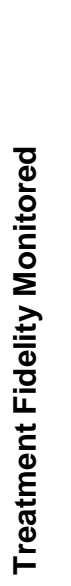 & 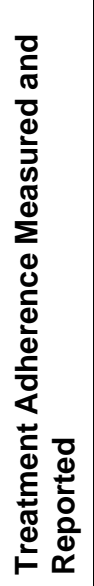 & 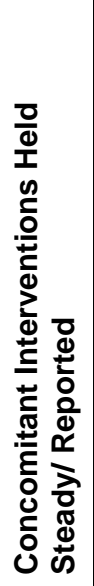 & 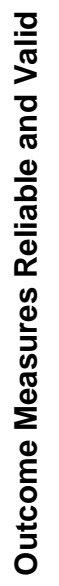 & 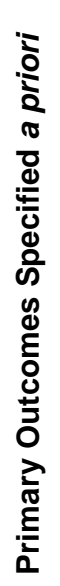 & 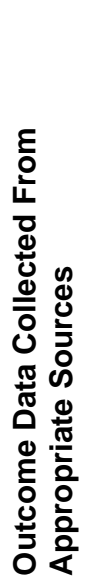 & 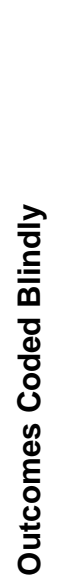 & 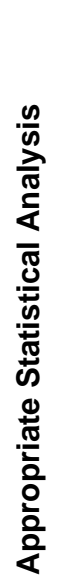 & 号 \\
\hline $\begin{array}{l}\text { Munasingh } \\
\text { e } 2010^{76}\end{array}$ & + & + & + & - & - & - & + & + & - & + & NA & + & + & + & + & + & + & - & Moderate \\
\hline $\begin{array}{l}\text { Miral } \\
2008^{77}\end{array}$ & + & + & + & + & + & + & + & + & NA & + & NA & + & + & + & + & + & + & + & Moderate \\
\hline $\begin{array}{l}\text { Nikvarz } \\
2016^{78}\end{array}$ & + & + & + & + & + & - & + & + & - & + & NA & - & + & + & + & + & - & - & Moderate \\
\hline $\begin{array}{l}\text { Navarro } \\
2015^{79}\end{array}$ & + & + & + & + & ++ & + & + & + & NA & + & NA & + & - & + & + & + & + & - & Low \\
\hline $\begin{array}{l}\text { Nikoo } \\
2014^{80}\end{array}$ & + & + & + & + & + & + & + & + & - & + & NA & - & + & + & + & + & + & + & Moderate \\
\hline $\begin{array}{l}\text { Nagaraj } \\
2006^{81}\end{array}$ & + & + & + & + & + & + & + & + & - & + & NA & - & + & + & + & + & + & - & Moderate \\
\hline $\begin{array}{l}\text { Pusponeg } \\
\text { oro } 2015^{82}\end{array}$ & + & + & + & + & - & + & + & + & - & + & + & + & - & + & + & + & + & - & Moderate \\
\hline $\begin{array}{l}\text { Pearson } \\
2013^{83}\end{array}$ & + & + & + & - & ++ & + & + & + & NA & + & NA & + & + & + & + & + & + & + & Low \\
\hline $\begin{array}{l}\text { Rezaei } \\
2010^{84}\end{array}$ & + & + & + & + & ++ & + & + & + & NA & + & NA & + & + & + & + & + & + & + & Low \\
\hline Rossignol & + & + & + & + & ++ & + & + & + & + & + & NA & - & + & + & + & + & + & - & Low \\
\hline
\end{tabular}




\begin{tabular}{|c|c|c|c|c|c|c|c|c|c|c|c|c|c|c|c|c|c|c|c|}
\hline 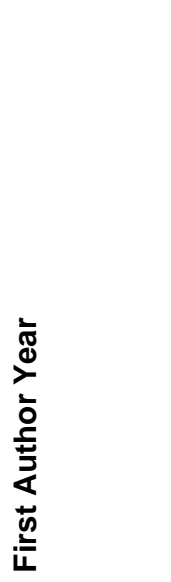 & $\begin{array}{l}\text { s } \\
\frac{0}{0} \\
0 \\
0 \\
\frac{0}{\overline{0}} \\
\frac{0}{0}\end{array}$ & 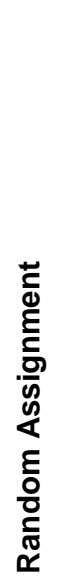 & 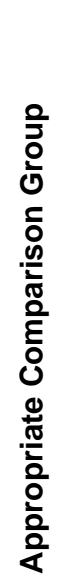 & 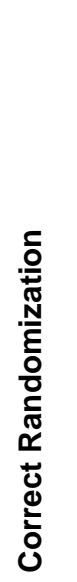 & 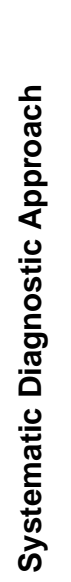 & 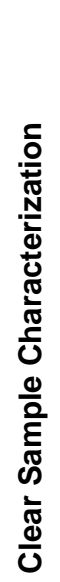 & 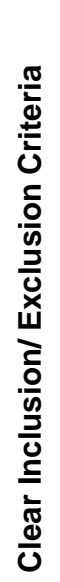 & 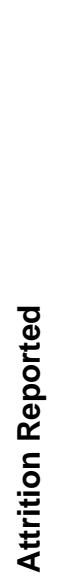 & 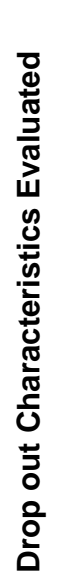 & 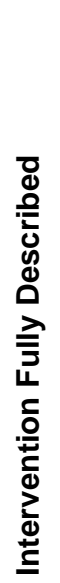 & 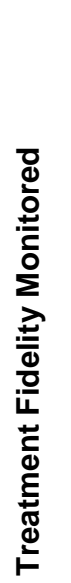 & 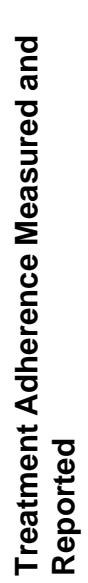 & 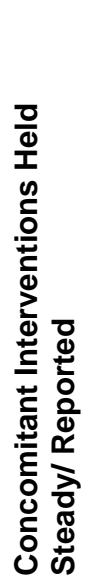 & 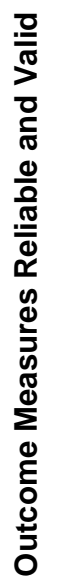 & 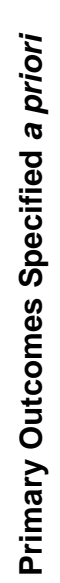 & 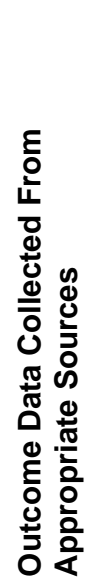 & 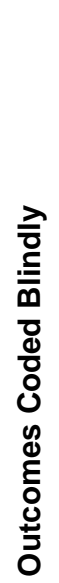 & 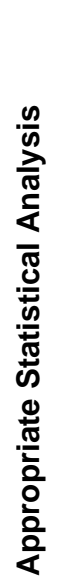 & 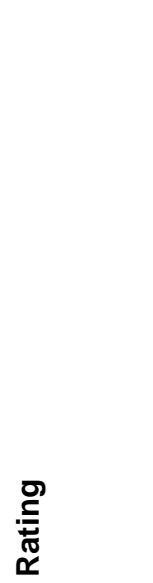 \\
\hline \multicolumn{20}{|l|}{$2009^{85}$} \\
\hline Sun $2016^{86}$ & + & - & + & NA & + & + & + & + & - & + & NA & + & + & + & + & + & - & - & Moderate \\
\hline $\begin{array}{l}\text { Sampanth } \\
\text { avivat } \\
2012^{87}\end{array}$ & + & + & + & + & + & - & + & + & NA & + & NA & + & - & + & + & + & + & - & Moderate \\
\hline $\begin{array}{l}\text { Shea } \\
2004^{88,89}\end{array}$ & + & + & + & - & + & + & + & + & - & + & $\mathrm{NA}$ & + & + & + & + & + & + & - & Moderate \\
\hline $\begin{array}{l}\text { Saad } \\
2015^{90}\end{array}$ & + & + & + & + & ++ & + & - & + & $\mathrm{NA}$ & + & NA & - & + & + & + & + & + & - & Moderate \\
\hline $\begin{array}{l}\text { Scahill } \\
2015^{91}\end{array}$ & + & + & + & + & ++ & + & + & + & + & + & NA & + & + & + & + & + & + & + & Low \\
\hline $\begin{array}{l}\text { Shan } \\
2015^{92}\end{array}$ & + & + & + & - & - & - & + & + & - & + & NA & - & - & + & - & + & - & - & High \\
\hline $\begin{array}{l}\text { Troost } \\
2005^{93}\end{array}$ & + & + & + & - & ++ & + & + & + & $\mathrm{NA}$ & + & $\mathrm{NA}$ & - & + & + & + & + & + & + & Low \\
\hline $\begin{array}{l}\text { Voigt } \\
2014^{94}\end{array}$ & + & + & + & + & ++ & + & + & + & - & + & NA & + & + & + & + & + & + & + & Low \\
\hline $\begin{array}{l}\text { Van der } \\
\text { Meer } \\
2013^{95-98}\end{array}$ & + & + & + & + & ++ & + & + & + & NA & + & $\mathrm{NA}$ & + & + & + & + & + & + & - & Low \\
\hline
\end{tabular}

D-7 


\begin{tabular}{|c|c|c|c|c|c|c|c|c|c|c|c|c|c|c|c|c|c|c|c|}
\hline 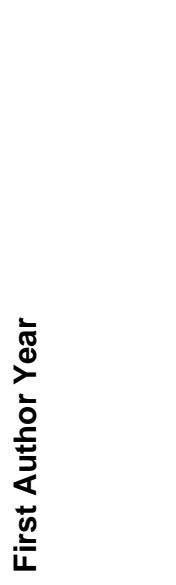 & 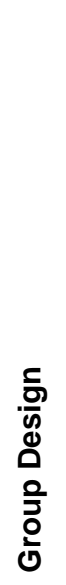 & 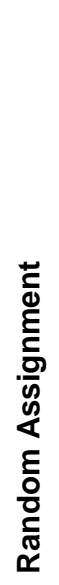 & 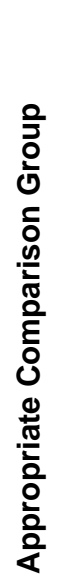 & 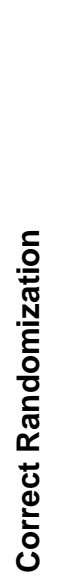 & 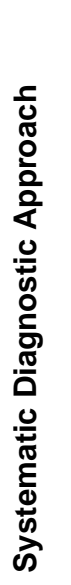 & 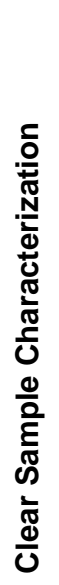 & 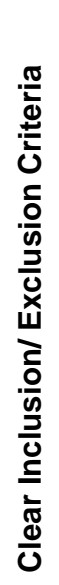 & 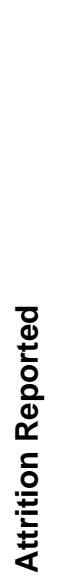 & 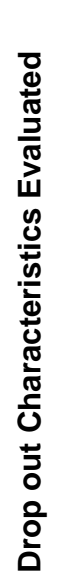 & 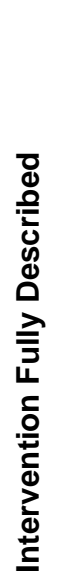 & 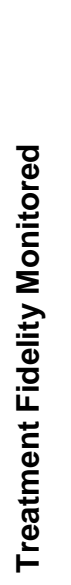 & 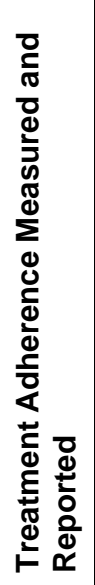 & 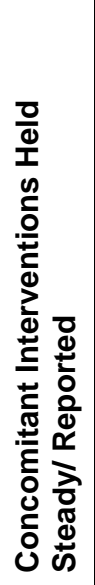 & 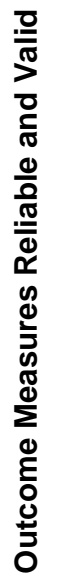 & 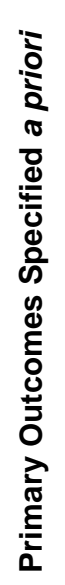 & 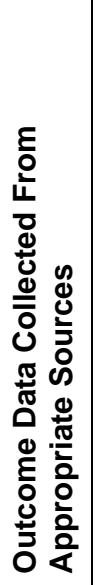 & 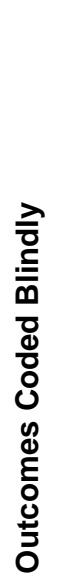 & 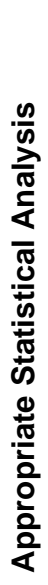 & 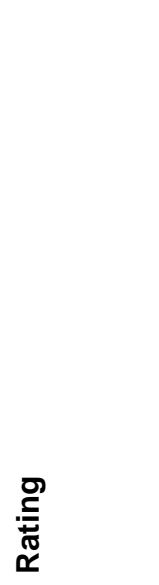 \\
\hline $\begin{array}{l}\text { Wink } \\
2014^{99} \\
\end{array}$ & + & - & + & NA & + & + & - & - & - & + & - & + & + & + & + & - & + & + & Moderate \\
\hline $\begin{array}{l}\text { Wright } \\
2011^{100}\end{array}$ & + & + & + & + & ++ & + & + & + & - & + & NA & + & + & + & + & + & + & - & Low \\
\hline $\begin{array}{l}\text { Whiteley } \\
2010^{101,102}\end{array}$ & + & + & + & - & ++ & + & + & + & - & + & NA & + & + & + & + & + & - & - & Moderate \\
\hline $\begin{array}{l}\text { Wasserma } \\
\text { n } 2006^{103}\end{array}$ & + & + & + & - & ++ & + & + & + & - & + & NA & - & + & + & + & + & + & - & Low \\
\hline $\begin{array}{l}\text { Danfors } \\
2005^{104}\end{array}$ & + & + & + & - & + & + & + & + & NA & + & NA & + & - & + & + & + & + & + & Moderate \\
\hline $\begin{array}{l}\text { Knivsberg } \\
2002^{105 \_4575}\end{array}$ & + & + & + & - & - & + & + & + & NA & - & NA & - & - & + & + & + & + & - & High \\
\hline
\end{tabular}




\section{References}

1. Aman MG, Findling RL, Hardan AY, et al. Safety and Efficacy of Memantine in Children with Autism: Randomized, PlaceboControlled Study and Open-Label Extension. J Child Adolesc Psychopharmacol 2016 Mar 15PMID: 26978327.

2. Aman M, Rettiganti M, Nagaraja HN, et al. Tolerability, Safety, and Benefits of Risperidone in Children and Adolescents with Autism: 21-Month Follow-up After 8-Week Placebo-Controlled Trial. J Child Adolesc Psychopharmacol 2015 Aug;25(6):482-93. PMID: 26262903.

3. Scahill L, Sukhodolsky DG, Anderberg E, et al. Sensitivity of the modified Children's Yale-Brown Obsessive Compulsive Scale to detect change: Results from two multi-site trials. Autism 2015 Apr 16PMID: 25882391.

4. Scahill L, Hallett V, Aman MG, et al. Brief Report: social disability in autism spectrum disorder: results from Research Units on Pediatric Psychopharmacology (RUPP) Autism Network trials. J Autism Dev Disord 2013 Mar;43(3):739-46. PMID: 23104617. 5. Arnold LE, Farmer C, Kraemer HC, et al. Moderators, mediators, and other predictors of risperidone response in children with autistic disorder and irritability. J Child Adolesc Psychopharmacol 2010 Apr;20(2):83-93. PMID: 20415603.

6. Aman MG, Hollway JA, McDougle CJ, et al. Cognitive effects of risperidone in children with autism and irritable behavior. J Child Adolesc Psychopharmacol 2008 Jun;18(3):227-36. PMID: 18582177.

7. Aman MG, Arnold LE, McDougle CJ, et al. Acute and long-term safety and tolerability of risperidone in children with autism. J Child Adolesc Psychopharmacol 2005 Dec;15(6):869-84. PMID: 16379507.

8. McDougle CJ, Scahill L, Aman MG, et al. Risperidone for the core symptom domains of autism: results from the study by the autism network of the research units on pediatric psychopharmacology. Am J Psychiatry 2005 Jun;162(6):1142-8. PMID: 15930063.

9. Martin A, Scahill L, Anderson GM, et al. Weight and leptin changes among risperidone-treated youths with autism: 6-month prospective data. Am J Psychiatry 2004 Jun;161(6):1125-7. PMID: 15169706.

10. McCracken JT, McGough J, Shah B, et al. Risperidone in children with autism and serious behavioral problems. N Engl J Med 2002 Aug 1;347(5):314-21. PMID: 12151468.

11. Arnold LE, Vitiello B, McDougle C, et al. Parent-defined target symptoms respond to risperidone in RUPP autism study: customer approach to clinical trials. J Am Acad Child Adolesc Psychiatry 2003 Dec;42(12):1443-50. PMID: 14627879.

12. . Risperidone treatment of autistic disorder: longer-term benefits and blinded discontinuation after 6 months. Am J Psychiatry 2005 Jul;162(7):1361-9. PMID: 15994720.

13. Amatachaya A, Jensen MP, Patjanasoontorn N, et al. The short-term effects of transcranial direct current stimulation on electroencephalography in children with autism: a randomized crossover controlled trial. Behav Neurol 2015;2015:928631. PMID: 25861158.

14. Amatachaya A, Auvichayapat N, Patjanasoontorn N, et al. Effect of anodal transcranial direct current stimulation on autism: a randomized double-blind crossover trial. Behav Neurol 2014;2014:173073. PMID: 25530675.

15. Arnold LE, Aman MG, Hollway J, et al. Placebo-controlled pilot trial of mecamylamine for treatment of autism spectrum disorders. J Child Adolesc Psychopharmacol 2012 Jun;22(3):198-205. PMID: 22537359. 
16. Akhondzadeh S, Fallah J, Mohammadi MR, et al. Double-blind placebo-controlled trial of pentoxifylline added to risperidone: effects on aberrant behavior in children with autism. Prog Neuropsychopharmacol Biol Psychiatry 2010 Feb 1;34(1):32-6. PMID: 19772883.

17. Akhondzadeh S, Tajdar H, Mohammadi MR, et al. A double-blind placebo controlled trial of piracetam added to risperidone in patients with autistic disorder. Child Psychiatry Hum Dev 2008 Sep;39(3):237-45. PMID: 17929164.

18. Arnold LE, Aman MG, Cook AM, et al. Atomoxetine for hyperactivity in autism spectrum disorders: placebo-controlled crossover pilot trial. J Am Acad Child Adolesc Psychiatry 2006 Oct;45(10):1196-205. PMID: 17003665.

19. Al-Ayadhi LY, Elamin NE. Camel Milk as a Potential Therapy as an Antioxidant in Autism Spectrum Disorder (ASD). Evid Based Complement Alternat Med 2013;2013:602834. PMID: 24069051.

20. Asadabadi M, Mohammadi MR, Ghanizadeh A, et al. Celecoxib as adjunctive treatment to risperidone in children with autistic disorder: a randomized, double-blind, placebo-controlled trial. Psychopharmacology (Berl) 2013 Jan;225(1):51-9. PMID: 22782459.

21. Bent S, Hendren RL, Zandi T, et al. Internet-based, randomized, controlled trial of omega-3 fatty acids for hyperactivity in autism. J Am Acad Child Adolesc Psychiatry 2014 Jun;53(6):658-66. PMID: 24839884.

22. Bent S, Bertoglio K, Ashwood P, et al. A pilot randomized controlled trial of omega-3 fatty acids for autism spectrum disorder. J Autism Dev Disord 2011 May;41(5):545-54. PMID: 20683766.

23. Bertoglio K, Jill James S, Deprey L, et al. Pilot study of the effect of methyl B12 treatment on behavioral and biomarker measures in children with autism. J Altern Complement Med 2010 May;16(5):555-60. PMID: 20804367.

24. Chugani DC, Chugani HT, Wiznitzer M, et al. Efficacy of Low-Dose Buspirone for Restricted and Repetitive Behavior in Young Children with Autism Spectrum Disorder: A Randomized Trial. J Pediatr 2016 Mar;170:45-53.e1-4. PMID: 26746121.

25. Cortesi F, Giannotti F, Sebastiani T, et al. Controlled-release melatonin, singly and combined with cognitive behavioural therapy, for persistent insomnia in children with autism spectrum disorders: a randomized placebo-controlled trial. J Sleep Res 2012 Dec;21(6):700-9. PMID: 22616853.

26. Chez MG, Buchanan TM, Becker M, et al. Donepezil hydrochloride: A double-blind study in autistic children. Journal of Pediatric Neurology 2003 Oct-Dec;1(2):83-8. PMID: 2004-12732-002.

27. Dean OM, Gray KM, Villagonzalo KA, et al. A randomised, double blind, placebo-controlled trial of a fixed dose of $\mathrm{N}$-acetyl cysteine in children with autistic disorder. Aust N Z J Psychiatry 2016 Jun 17PMID: 27316706.

28. Dadds MR, MacDonald E, Cauchi A, et al. Nasal oxytocin for social deficits in childhood autism: a randomized controlled trial. J Autism Dev Disord 2014 Mar;44(3):521-31. PMID: 23888359.

29. Duffy FH, Shankardass A, McAnulty GB, et al. Corticosteroid therapy in regressive autism: a retrospective study of effects on the Frequency Modulated Auditory Evoked Response (FMAER), language, and behavior. BMC Neurol 2014;14:70. PMID: 24885033.

30. Findling RL, Mankoski R, Timko K, et al. A randomized controlled trial investigating the safety and efficacy of aripiprazole in the long-term maintenance treatment of pediatric patients with irritability associated with autistic disorder. J Clin Psychiatry 2014 Jan;75(1):22-30. PMID: 24502859. 
31. Fahmy SF, El-hamamsy MH, Zaki OK, et al. L-Carnitine supplementation improves the behavioral symptoms in autistic children. Research in Autism Spectrum Disorders 2013;7(1):159-66. PMID: 1267033812; 2012-29769-018.

32. Ghaleiha A, Alikhani R, Kazemi MR, et al. Minocycline as Adjunctive Treatment to Risperidone in Children with Autistic

Disorder: A Randomized, Double-Blind Placebo-Controlled Trial. J Child Adolesc Psychopharmacol 2016 Apr 29 PMID: 27128958.

33. Ghalichi F, Ghaemmaghami J, Malek A, et al. Effect of gluten free diet on gastrointestinal and behavioral indices for children with autism spectrum disorders: a randomized clinical trial. World J Pediatr 2016 Jun 10PMID: 27286693.

34. Ghanizadeh A, Sahraeizadeh A, Berk M. A head-to-head comparison of aripiprazole and risperidone for safety and treating autistic disorders, a randomized double blind clinical trial. Child Psychiatry Hum Dev 2014;45(2):185-92. PMID: 23801256.

35. Geier DA, Kern JK, Davis G, et al. A prospective double-blind, randomized clinical trial of levocarnitine to treat autism spectrum disorders. Med Sci Monit 2011 Jun;17(6):Pi15-23. PMID: 21629200.

36. Granpeesheh D, Tarbox J, Dixon DR, et al. Randomized trial of hyperbaric oxygen therapy for children with autism. Research in Autism Spectrum Disorders 2010 April 2010/June;4(2):268-75. PMID: 2010025300.

37. Ghaleiha A, Rasa SM, Nikoo M, et al. A pilot double-blind placebo-controlled trial of pioglitazone as adjunctive treatment to risperidone: Effects on aberrant behavior in children with autism. Psychiatry Res 2015 Sep 30;229(1-2):181-7. PMID: 26208985. 38. Ghaleiha A, Mohammadi E, Mohammadi MR, et al. Riluzole as an adjunctive therapy to risperidone for the treatment of irritability in children with autistic disorder: a double-blind, placebo-controlled, randomized trial. Paediatr Drugs 2013 Dec;15(6):505-14. PMID: 23821414.

39. Ghaleiha A, Asadabadi M, Mohammadi MR, et al. Memantine as adjunctive treatment to risperidone in children with autistic disorder: a randomized, double-blind, placebo-controlled trial. Int J Neuropsychopharmacol 2013 May;16(4):783-9. PMID: 22999292. 40. Ghaleiha A, Ghyasvand M, Mohammadi MR, et al. Galantamine efficacy and tolerability as an augmentative therapy in autistic children: A randomized, double-blind, placebo-controlled trial. J Psychopharmacol 2013 Oct 15;28(7):677-85. PMID: 24132248. 41. Ghanizadeh A, Ayoobzadehshirazi A. A randomized double-blind placebo-controlled clinical trial of adjuvant buspirone for irritability in autism. Pediatr Neurol 2015 Jan;52(1):77-81. PMID: 25451017.

42. Ghanizadeh A, Moghimi-Sarani E. A randomized double blind placebo controlled clinical trial of N-Acetylcysteine added to risperidone for treating autistic disorders. BMC Psychiatry 2013;13:196. PMID: 23886027.

43. Hendren RL, James SJ, Widjaja F, et al. Randomized, Placebo-Controlled Trial of Methyl B12 for Children with Autism. J Child Adolesc Psychopharmacol 2016 Feb 18PMID: 26889605.

44. Handen BL, Aman MG, Arnold LE, et al. Atomoxetine, Parent Training, and Their Combination in Children With Autism Spectrum Disorder and Attention-Deficit/Hyperactivity Disorder. J Am Acad Child Adolesc Psychiatry 2015 Nov;54(11):905-15. PMID: 26506581.

45. Smith T, Aman MG, Arnold LE, et al. Atomoxetine and Parent Training for Children With Autism and AttentionDeficit/Hyperactivity Disorder: A 24-Week Extension Study. J Am Acad Child Adolesc Psychiatry 2016 Oct;55(10):868-76.e2. PMID: 27663942. 
46. Hyman SL, Stewart PA, Foley J, et al. The Gluten-Free/Casein-Free Diet: A Double-Blind Challenge Trial in Children with Autism. J Autism Dev Disord 2015 Sep 5PMID: 26343026.

47. Handen BL, Johnson CR, McAuliffe-Bellin S, et al. Safety and efficacy of donepezil in children and adolescents with autism: neuropsychological measures. J Child Adolesc Psychopharmacol 2011 Feb;21(1):43-50. PMID: 21309696.

48. Hollander E, Chaplin W, Soorya L, et al. Divalproex sodium vs placebo for the treatment of irritability in children and adolescents with autism spectrum disorders. Neuropsychopharmacology 2010 Mar;35(4):990-8. PMID: 20010551.

49. Hardan AY, Fung LK, Libove RA, et al. A randomized controlled pilot trial of oral N-acetylcysteine in children with autism. Biol Psychiatry 2012 Jun 1;71(11):956-61. PMID: 22342106.

50. Hasanzadeh E, Mohammadi MR, Ghanizadeh A, et al. A double-blind placebo controlled trial of Ginkgo biloba added to risperidone in patients with autistic disorders. Child Psychiatry Hum Dev 2012 Oct;43(5):674-82. PMID: 22392415.

51. Harrison Elder J, Shankar M, Shuster J, et al. The Gluten-Free, Casein-Free Diet In Autism: Results of A Preliminary Double Blind Clinical Trial. Journal of Autism and Developmental Disorders 2006 Apr;36(3):413-20. PMID: 2006-07517-011.

52. Seung H, Rogalski Y, Shankar M, et al. The gluten- and casein-free diet and autism: Communication outcomes from a preliminary double-blind clinical trial. Journal of Medical Speech-Language Pathology 2007 Dec;15(4):337-45.

53. Johnson CR, Handen BL, Zimmer M, et al. Effects of gluten free / casein free diet in young children with autism: A pilot study. Journal of Developmental and Physical Disabilities 2011 2015-12-09;23(3):213-25. PMID: 888750170; 2011-08445-004.

54. King BH, Wright DM, Handen BL, et al. Double-blind, placebo-controlled study of amantadine hydrochloride in the treatment of children with autistic disorder. J Am Acad Child Adolesc Psychiatry 2001 Jun;40(6):658-65. PMID: 11392343.

55. Kent JM, Kushner S, Ning X, et al. Risperidone dosing in children and adolescents with autistic disorder: a double-blind, placebocontrolled study. J Autism Dev Disord 2013 Aug;43(8):1773-83. PMID: 23212807.

56. Kent JM, Hough D, Singh J, et al. An open-label extension study of the safety and efficacy of risperidone in children and adolescents with autistic disorder. J Child Adolesc Psychopharmacol 2013 Dec;23(10):676-86. PMID: 24350813.

57. King BH, Dukes K, Donnelly CL, et al. Baseline factors predicting placebo response to treatment in children and adolescents with autism spectrum disorders: a multisite randomized clinical trial. JAMA Pediatr 2013 Nov;167(11):1045-52. PMID: 24061784.

58. King BH, Hollander E, Sikich L, et al. Lack of efficacy of citalopram in children with autism spectrum disorders and high levels of repetitive behavior: citalopram ineffective in children with autism. Arch Gen Psychiatry 2009 Jun;66(6):583-90. PMID: 19487623.

59. Klaiman C, Huffman L, Masaki L, et al. Tetrahydrobiopterin as a treatment for autism spectrum disorders: a double-blind, placebocontrolled trial. J Child Adolesc Psychopharmacol 2013 Jun;23(5):320-8. PMID: 23782126.

60. Lamberti M, Siracusano R, Italiano D, et al. Head-to-Head Comparison of Aripiprazole and Risperidone in the Treatment of ADHD Symptoms in Children with Autistic Spectrum Disorder and ADHD: A Pilot, Open-Label, Randomized Controlled Study. Paediatr Drugs 2016 Aug;18(4):319-29. PMID: 27278054.

61. Lv YT, Zhang Y, Liu M, et al. Transplantation of human cord blood mononuclear cells and umbilical cord-derived mesenchymal stem cells in autism. J Transl Med 2013;11:196. PMID: 23978163. 
62. Lemonnier E, Degrez C, Phelep M, et al. A randomised controlled trial of bumetanide in the treatment of autism in children. Transl Psychiatry 2012;2:e202. PMID: 23233021.

63. Mankad D, Dupuis A, Smile S, et al. A randomized, placebo controlled trial of omega-3 fatty acids in the treatment of young children with autism. Mol Autism 2015;6:18. PMID: 25798215.

64. McCracken JT, Badashova KK, Posey DJ, et al. Positive effects of methylphenidate on hyperactivity are moderated by monoaminergic gene variants in children with autism spectrum disorders. Pharmacogenomics J 2014 Jun;14(3):295-302. PMID: 23856854.

65. Posey DJ, Aman MG, McCracken JT, et al. Positive effects of methylphenidate on inattention and hyperactivity in pervasive developmental disorders: an analysis of secondary measures. Biol Psychiatry 2007 Feb 15;61(4):538-44. PMID: 17276750.

66. Jahromi LB, Kasari CL, McCracken JT, et al. Positive Effects of Methylphenidate on Social Communication and Self-Regulation in Children with Pervasive Developmental Disorders and Hyperactivity. Journal of Autism and Developmental Disorders 2009 Mar;39(3):395-404. PMID: EJ827753.

67. . Randomized, controlled, crossover trial of methylphenidate in pervasive developmental disorders with hyperactivity. Arch Gen Psychiatry 2005 Nov;62(11):1266-74. PMID: 16275814.

68. Mohammadi MR, Yadegari N, Hassanzadeh E, et al. Double-blind, placebo-controlled trial of risperidone plus amantadine in children with autism: a 10-week randomized study. Clin Neuropharmacol 2013 Nov-Dec;36(6):179-84. PMID: 24201232.

69. Marcus RN, Owen R, Manos G, et al. Aripiprazole in the treatment of irritability in pediatric patients (aged 6-17 years) with autistic disorder: results from a 52-week, open-label study. J Child Adolesc Psychopharmacol 2011 Jun;21(3):229-36. PMID: 21663425.

70. Aman MG, Kasper W, Manos G, et al. Line-item analysis of the Aberrant Behavior Checklist: results from two studies of aripiprazole in the treatment of irritability associated with autistic disorder. J Child Adolesc Psychopharmacol 2010 Oct;20(5):415-22. PMID: 20973712.

71. Varni JW, Handen BL, Corey-Lisle PK, et al. Effect of aripiprazole 2 to $15 \mathrm{mg} / \mathrm{d}$ on health-related quality of life in the treatment of irritability associated with autistic disorder in children: a post hoc analysis of two controlled trials. Clin Ther 2012 Apr;34(4):980-92. PMID: 22444782.

72. Amoros E, Tzvetkovitch A. [Aquatic therapy for children with autism]. Soins Pediatr Pueric 2014 Jan-Feb(276):29-30. PMID: 24617090.

73. Robb AS, Andersson C, Bellocchio EE, et al. Safety and tolerability of aripiprazole in the treatment of irritability associated with autistic disorder in pediatric subjects (6-17 years old):results from a pooled analysis of 2 studies. Prim Care Companion CNS Disord 2011;13(1)PMID: 21731831.

74. Marcus RN, Owen R, Kamen L, et al. A placebo-controlled, fixed-dose study of aripiprazole in children and adolescents with irritability associated with autistic disorder. J Am Acad Child Adolesc Psychiatry 2009 Nov;48(11):1110-9. PMID: 19797985. 
75. Owen R, Sikich L, Marcus RN, et al. Aripiprazole in the treatment of irritability in children and adolescents with autistic disorder. Pediatrics 2009 Dec;124(6):1533-40. PMID: 19948625.

76. Munasinghe SA, Oliff C, Finn J, et al. Digestive enzyme supplementation for autism spectrum disorders: a double-blind randomized controlled trial. J Autism Dev Disord 2010 Sep;40(9):1131-8. PMID: 20204691.

77. Miral S, Gencer O, Inal-Emiroglu FN, et al. Risperidone versus haloperidol in children and adolescents with AD : a randomized, controlled, double-blind trial. Eur Child Adolesc Psychiatry 2008 Feb;17(1):1-8. PMID: 18080171.

78. Nikvarz N, Alaghband-Rad J, Tehrani-Doost M, et al. Comparing Efficacy and Side Effects of Memantine vs. Risperidone in the Treatment of Autistic Disorder. Pharmacopsychiatry 2016 Jun 14PMID: 27299475.

79. Navarro F, Pearson DA, Fatheree N, et al. Are 'leaky gut' and behavior associated with gluten and dairy containing diet in children with autism spectrum disorders? Nutr Neurosci 2015 May;18(4):177-85. PMID: 24564346.

80. Nikoo M, Radnia H, Farokhnia M, et al. N-acetylcysteine as an adjunctive therapy to risperidone for treatment of irritability in autism: a randomized, double-blind, placebo-controlled clinical trial of efficacy and safety. Clin Neuropharmacol 2015 JanFeb;38(1):11-7. PMID: 25580916.

81. Nagaraj R, Singhi P, Malhi P. Risperidone in children with autism: randomized, placebo-controlled, double-blind study. J Child Neurol 2006 Jun;21(6):450-5. PMID: 16948927.

82. Pusponegoro HD, Ismael S, Firmansyah A, et al. Gluten and casein supplementation does not increase symptoms in children with autism spectrum disorder. Acta Paediatr 2015 Nov;104(11):e500-e5. PMID: 26148018.

83. Pearson DA, Santos CW, Aman MG, et al. Effects of extended release methylphenidate treatment on ratings of attentiondeficit/hyperactivity disorder (ADHD) and associated behavior in children with autism spectrum disorders and ADHD symptoms. J Child Adolesc Psychopharmacol 2013 Jun;23(5):337-51. PMID: 23782128.

84. Rezaei V, Mohammadi MR, Ghanizadeh A, et al. Double-blind, placebo-controlled trial of risperidone plus topiramate in children with autistic disorder. Prog Neuropsychopharmacol Biol Psychiatry 2010 Oct 1;34(7):1269-72. PMID: 20637249.

85. Rossignol DA, Rossignol LW, Smith S, et al. Hyperbaric treatment for children with autism: a multicenter, randomized, doubleblind, controlled trial. BMC Pediatr 2009;9:21. PMID: 19284641.

86. Sun C, Zou M, Zhao D, et al. Efficacy of Folic Acid Supplementation in Autistic Children Participating in Structured Teaching: An Open-Label Trial. Nutrients 2016;8(6)PMID: 27338456.

87. Sampanthavivat M, Singkhwa W, Chaiyakul T, et al. Hyperbaric oxygen in the treatment of childhood autism: a randomised controlled trial. Diving Hyperb Med 2012 Sep;42(3):128-33. PMID: 22987458.

88. Shea S, Turgay A, Carroll A, et al. Risperidone in the treatment of disruptive behavioral symptoms in children with autistic and other pervasive developmental disorders. Pediatrics 2004 Nov;114(5):e634-41. PMID: 15492353.

89. Pandina GJ, Bossie CA, Youssef E, et al. Risperidone improves behavioral symptoms in children with autism in a randomized, double-blind, placebo-controlled trial. J Autism Dev Disord 2007 Feb;37(2):367-73. PMID: 17019624. 
90. Saad K, Eltayeb AA, Mohamad IL, et al. A Randomized, Placebo-controlled Trial of Digestive Enzymes in Children with Autism Spectrum Disorders. Clin Psychopharmacol Neurosci 2015 Aug 31;13(2):188-93. PMID: 26243847.

91. Scahill L, McCracken JT, King BH, et al. Extended-Release Guanfacine for Hyperactivity in Children With Autism Spectrum Disorder. Am J Psychiatry 2015 Aug 28:appiajp201515010055. PMID: 26315981.

92. Du L, Shan L, Wang B, et al. A Pilot Study on the Combination of Applied Behavior Analysis and Bumetanide Treatment for Children with Autism. J Child Adolesc Psychopharmacol 2015 Sep;25(7):585-8. PMID: 26258842.

93. Troost PW, Lahuis BE, Steenhuis MP, et al. Long-term effects of risperidone in children with autism spectrum disorders: a placebo discontinuation study. J Am Acad Child Adolesc Psychiatry 2005 Nov;44(11):1137-44. PMID: 16239862.

94. Voigt RG, Mellon MW, Katusic SK, et al. Dietary docosahexaenoic acid supplementation in children with autism. J Pediatr Gastroenterol Nutr 2014 Jun;58(6):715-22. PMID: 24345834.

95. van der Meer JM, Harfterkamp M, van de Loo-Neus G, et al. A randomized, double-blind comparison of atomoxetine and placebo on response inhibition and interference control in children and adolescents with autism spectrum disorder and comorbid attentiondeficit/hyperactivity disorder symptoms. J Clin Psychopharmacol 2013 Dec;33(6):824-7. PMID: 24018545.

96. Harfterkamp M, Buitelaar JK, Minderaa RB, et al. Atomoxetine in autism spectrum disorder: no effects on social functioning; some beneficial effects on stereotyped behaviors, inappropriate speech, and fear of change. J Child Adolesc Psychopharmacol 2014 Nov;24(9):481-5. PMID: 25369243.

97. Harfterkamp M, van de Loo-Neus G, Minderaa RB, et al. A randomized double-blind study of atomoxetine versus placebo for attention-deficit/hyperactivity disorder symptoms in children with autism spectrum disorder. J Am Acad Child Adolesc Psychiatry 2012 Jul;51(7):733-41. PMID: 22721596.

98. Harfterkamp M, Buitelaar JK, Minderaa RB, et al. Long-term treatment with atomoxetine for attention-deficit/hyperactivity disorder symptoms in children and adolescents with autism spectrum disorder: an open-label extension study. J Child Adolesc Psychopharmacol 2013 Apr;23(3):194-9. PMID: 23578015.

99. Wink LK, Early M, Schaefer T, et al. Body mass index change in autism spectrum disorders: comparison of treatment with risperidone and aripiprazole. J Child Adolesc Psychopharmacol 2014 Mar;24(2):78-82. PMID: 24564519.

100. Wright B, Sims D, Smart S, et al. Melatonin versus placebo in children with autism spectrum conditions and severe sleep problems not amenable to behaviour management strategies: a randomised controlled crossover trial. J Autism Dev Disord 2011 Feb;41(2):175-84. PMID: 20535539.

101. Whiteley P, Haracopos D, Knivsberg AM, et al. The ScanBrit randomised, controlled, single-blind study of a gluten- and caseinfree dietary intervention for children with autism spectrum disorders. Nutr Neurosci 2010 Apr;13(2):87-100. PMID: 20406576.

102. Pedersen L, Parlar S, Kvist K, et al. Data mining the ScanBrit study of a gluten- and casein-free dietary intervention for children with autism spectrum disorders: behavioural and psychometric measures of dietary response. Nutr Neurosci 2014 Sep;17(5):207-13. PMID: 24075141. 
103. Wasserman S, Iyengar R, Chaplin WF, et al. Levetiracetam versus placebo in childhood and adolescent autism: a double-blind placebo-controlled study. Int Clin Psychopharmacol 2006 Nov;21(6):363-7. PMID: 17012983.

104. Danfors T, von Knorring AL, Hartvig P, et al. Tetrahydrobiopterin in the treatment of children with autistic disorder: a doubleblind placebo-controlled crossover study. J Clin Psychopharmacol 2005 Oct;25(5):485-9. PMID: 16160627.

105. Knivsberg AM, Reichelt KL, Hoien T, et al. A randomised, controlled study of dietary intervention in autistic syndromes. Nutr Neurosci 2002 Sep;5(4):251-61. PMID: 12168688. 


\title{
Appendix E. Applicability Summary Tables
}

\author{
Table E-1. Antipsychotics
}

\begin{tabular}{ll}
\hline Domain & Description of applicability of evidence compared to question \\
\hline & $\begin{array}{l}\text { Studies typically included participants recruited from non-primary care populations } \\
\text { (university/academic-affiliated clinics, research centers), which may limit applicability to the wider } \\
\text { population; participants in RCTs had an average age of roughly 9 years in the treatment and } \\
\text { comparison groups and included a mojority male subjects. In studies providing IQ data, the } \\
\text { majority of subjects had IQs in the intellectual disability range. Studies reported use of } \\
\text { concurrent medications but typically did not report on co-morbid conditions. Most of the studies } \\
\text { report including children with at least moderate level of severity of ASD. }\end{array}$ \\
\hline Population & $\begin{array}{l}\text { Antipsychotics evaluated included risperidone and aripiprazole in dose ranges used in some } \\
\text { children with ASD. }\end{array}$ \\
\hline Intervention & $\begin{array}{l}\text { RCTs compared treatment to placebo or to another medication (e.g., haloperidol, aripiprazole, } \\
\text { memantine). }\end{array}$ \\
\hline Studies primarily targeted outcomes related to problem behavior (irritability, hyperactivity) and \\
repetitive behavior, which are significant concerns in the ASD population. Outcome measures \\
used to assess change were appropriate; although the duration of studies was typically less than \\
6 months, and evaluation was typically immediately post-treatment. Some studies included open \\
label, uncontrolled extensions. Studies reported adverse effects including sedation, weight gain, \\
and extrapyramidal symptoms. Short-term harms and outcomes generally reflect longer-term \\
outcomes measured over roughly 24 months.
\end{tabular}

Table E-2. Medications to treat ADHD

\begin{tabular}{|c|c|}
\hline Domain & Description of applicability of evidence \\
\hline Population & $\begin{array}{l}\text { Studies included children } 5 \text { to } 17 \text { years old with autistic disorder; majority male. Studies typically } \\
\text { included children with cognitive impairment }(I Q \text { or } D Q \leq 70) \text { and comorbidities including ADHD, } \\
\text { ODD, OCD, typically moderate to severe. Use of concomitant medications ranged from none to } \\
\text { roughly a third of children in studies. }\end{array}$ \\
\hline Intervention & Stimulants evaluated include different doses of methylphenidate, atomoxetine, and guanfacine. \\
\hline Comparators & Placebo \\
\hline Outcomes & $\begin{array}{l}\text { Studies typically assessed outcomes in the short-term including hyperactivity/inattention, } \\
\text { oppositional behavior, and social behaviors. The dose and type of medication were similar to } \\
\text { reports in the literature; the duration of treatment across studies was } 4 \text { to } 28 \text { weeks with evaluation } \\
\text { conducted immediately after treatment. }\end{array}$ \\
\hline Setting & $\begin{array}{l}\text { Studies were conducted in the US, The MPH trial included drug-free children in } 5 \text { academic } \\
\text { outpatient clinics in the US and Netherlands, with clinic-based populations,likely representing a } \\
\text { highly selected population. }\end{array}$ \\
\hline
\end{tabular}


Table E-3. Nutritional supplements

\begin{tabular}{|c|c|}
\hline Domain & Description of applicability of evidence \\
\hline Population & $\begin{array}{l}\text { Studies included children ages 2-11 years with differing requirements for inclusion. Two omega-3 } \\
\text { fatty acid studies targeted younger children (ages 3-8) with hyperactive behavior. The degree of } \\
\text { intellectual disability was not well described in the studies. }\end{array}$ \\
\hline Intervention & $\begin{array}{l}\text { Nutritional supplements including omega-3 fatty acids, methyl B-12, peptizyde, N,N- } \\
\text { dimethylglycine, digestive enzymes, docosahex-aenoic acid and levocarnitine. }\end{array}$ \\
\hline Comparators & Comparator for all studies in this group was placebo. \\
\hline Outcomes & $\begin{array}{l}\text { Study outcomes included behavior, autism severity, communication, cognitive, and gastrointestinal } \\
\text { symptoms and sleep disturbances. Data were collected using a wide range of assessment tools } \\
\text { including CGI, ABC, CARS, SRS, BASC, GBRS, PDDBI, MCDI, PPVT, Stanford-Binet, hand } \\
\text { muscle testing, ATEC, PIA-CV, Expressive Vocabulary test, Rescorla Language Development } \\
\text { Survey, CBCL, and Childhood Psychiatric Rating Scale. Most studies were of } 3 \text { months duration } \\
\text { (range } 1 \text { to } 6 \text { months). Outcomes were collected at end of treatment with no long term follow-up. }\end{array}$ \\
\hline Setting & $\begin{array}{l}\text { Five studies were conducted in the United States, two in Egypt and one each in the Netherlands } \\
\text { and Australia }\end{array}$ \\
\hline \multicolumn{2}{|c|}{$\begin{array}{l}\text { CGI-Clinical Global Impression; ABC-Aberrant Behavior Checklist; CARS-Childhood Autism Rating Scale; SRS-Social Responsiveness Scale; } \\
\text { BASC-Behavioral Assessment System for Children; GBRS; Global Rating Scale; PDDBI-Pervasive Developmental Disorders Behavior } \\
\text { Inventory; MCDI- MacArthur Communicative Development Inventory; PPVT-Peabody Picture Vocabulary Test; ATEC-Autism Treatment } \\
\text { Evaluation Checklist; PIA-CV-Parent Interview for Autism-Clinical Version; CBCL-Child Behavior Checklist }\end{array}$} \\
\hline Domain & Description of applicability of evidence \\
\hline Population & $\begin{array}{l}\text { Study participants ranged in age from } 2 \text { to } 12 \text { years, with no information available about co- } \\
\text { morbidities, except one study that included children with severe maladaptive behavior. Severity of } \\
\text { autism was not well described and the degree of intellectual disability was not well characterized in } \\
\text { most studies. One GFCF study required participants to be concurrently enrolled in a } \\
\text { comprehensive ABA program. Use of concomitant medications was not noted in most studies. } \\
\text { One study recruited children with known allergies or food intolerances. }\end{array}$ \\
\hline Intervention & Gluten-free casein-free diet, gluten-free diet, gluten dairy-free diet, raw and boiled camel milk. \\
\hline Comparators & $\begin{array}{l}\text { Comparators included gluten and dairy diet, gluten-containig challenge foods, usual diet, placebo } \\
\text { and cow milk. }\end{array}$ \\
\hline Outcomes & $\begin{array}{l}\text { Outcomes included autism severity scores, problem behaviors including maladaptive behavior, } \\
\text { sleep, and gastrointestinal symptoms. Assessments used in the studies included ADOS, CARS, } \\
\text { Gilliam Autism Rating Scale (GARS), Ritvo-Freeman Real Life rating scales, ADHD-IV scale, } \\
\text { Conner's Behavior Rating scale, VABS, Pervasive Developmental Disorder Behavior Inventory, } \\
\text { sleep diaries, and Gastrointestinal symptom severity index. }\end{array}$ \\
\hline Setting & $\begin{array}{l}\text { Studies were conducted in academic medical centers located in the United States, Belgium, } \\
\text { Denmark, Iran, Norway, and Saudi Arabia. }\end{array}$ \\
\hline
\end{tabular}


Table E-5. Risperidone adjuncts

\begin{tabular}{|c|c|}
\hline Domain & Description of applicability of evidence compared to question \\
\hline Population & $\begin{array}{l}\text { Studies typically included participants with autism spectrum disorder (ASD) recruited from non- } \\
\text { primary care populations (university/academic-affiliated clinics, research centers), which may } \\
\text { limit applicability to the wider population; participants had an average age in a range between 3- } \\
16 \text { years and included } 17-23 \text { subjects in the treatment arm to } 14-24 \text { in the placebo arm with more } \\
\text { male subjects ( } 73 \% \text { in treatment groups and } 75 \% \text { in control). Three studies reported including } \\
\text { subjects with cognitive impairment while majority did not mention the intellectual disability status. } \\
\text { Most of the studies included subjects with severe disruptive symptoms and typically did not } \\
\text { report comorbidity status or use of concomitant medications or therapies. }\end{array}$ \\
\hline Intervention & $\begin{array}{l}\text { Risperidone adjuncts evaluated included celecoxib, Ginkgo biloba, memantine, topiramate, } \\
\text { riluzole, buspirone, N-acetyl cysteine, amantadine, pioglitazone, galantamine, pentoxifylline, and } \\
\text { piracetam in children with ASD. }\end{array}$ \\
\hline Comparators & Studies compared adjuncts to risperidone therapy to placebo added to risperidone. \\
\hline Outcomes & $\begin{array}{l}\text { Studies primarily targeted outcomes related to core and associated behavior symptoms } \\
\text { (irritability, hyperactivity, lethargy/withdrawal, stereotypy and inappropriate speech) which are } \\
\text { significant concerns in the ASD population along with global clinical improvement. Outcome } \\
\text { measures used to assess change were appropriate; although the duration of studies was } \\
\text { typically } \leq 10 \text { weeks and evaluation was typically immediately post-treatment. }\end{array}$ \\
\hline Setting & $\begin{array}{l}\text { All the studies were conducted in Iran and primarily in academic clinics or research centers } \\
\text { which may have yielded more selected populations. }\end{array}$ \\
\hline \multicolumn{2}{|c|}{ ASD-autsim spectrum disorder } \\
\hline Domain & Description of applicability of evidence \\
\hline Population & $\begin{array}{l}\text { Studies included children between } 3 \text { and } 14 \text { years old. Across studies ASD symptoms were } \\
\text { generally considered mild to moderate. In one study of atomoxetine plus parent training, children } \\
\text { had concomitant ADHD symptoms. }\end{array}$ \\
\hline Intervention & $\begin{array}{l}\text { Atomoxetine plus/minus parent training, bumetanide plus applied behavioral analysis; cord blood or } \\
\text { stem cell transplantation plus rehabilitation therapy; melatonin plus/minus cognitive behavioral } \\
\text { therapy; folic acid plus TEACCH. }\end{array}$ \\
\hline Comparators & Behavioral therapy alone, placebo. \\
\hline Outcomes & $\begin{array}{l}\text { Most studies assessed challenging behaviors (e.g., irritability, inattention, oppositional behavior) } \\
\text { and symptom severity; one study evaluated cognitive skills and one evaluated sleep outcomes. }\end{array}$ \\
\hline Setting & $\begin{array}{l}\text { Studies were conducted in China, the US, and Italy and were recruited from research centers or } \\
\text { clinics. }\end{array}$ \\
\hline
\end{tabular}


Table E-7. N-acetylcysteine

\begin{tabular}{ll}
\hline Domain & Description of applicability of evidence \\
\hline & $\begin{array}{l}\text { Studies included children between } 3 \text { and } 10 \text { years with participants recruited form the community in } \\
\text { one study and from a clinic/hospital in another. Studies included children with and without } \\
\text { intellectual disability. Treatment duration ranged from 12-24 weeks. }\end{array}$ \\
\hline Population & N-acetylcysteine in doses of 500-900 milligrams. \\
\hline Comparators & Placebo \\
\hline Outcomes & Challenging behaviors, social and communication outcomes, adaptive behavior. \\
\hline & $\begin{array}{l}\text { Studies were conducted in the US and Australia, with participants recruited form the community in } \\
\text { one study and from a clinic/hospital in another. }\end{array}$ \\
\hline
\end{tabular}

Table E-8. Tetrahydrobiopterin

\begin{tabular}{ll}
\hline Domain & Description of applicability of evidence \\
\hline Population & $\begin{array}{l}\text { Studies included children between } 3 \text { and } 7 \text { years old. One study included only males. Treatment } \\
\text { duration ranged from } 16-24 \text { weeks. }\end{array}$ \\
\hline Intervention & Tetrahydrobiopterin at doses of 3 to $20 \mathrm{mg} / \mathrm{kg}$ of body weight. \\
\hline Comparators & Placebo \\
\hline Outcomes & The primary outcome in studies was symptom severity. \\
\hline & $\begin{array}{l}\text { Studies were conducted in Sweden and the US with participants recruited form the community and } \\
\text { Setting }\end{array}$
\end{tabular}

Table E-9. Other--Hyperbaric oxygen therapy

\begin{tabular}{|c|c|}
\hline Domain & Description of applicability of evidence \\
\hline Population & $\begin{array}{l}\text { Studies included children ages } 2-14 \text { years with autism. The degree of disease severity was not } \\
\text { well categorized. Participants were overwhelmingly male in the two studies that reported gender } \\
\text { ( } 84 \% \text { and 93\%) The degree of intellectual disability was not reported in any of the studies. } \\
\text { Concomitant behavioral and medication interventions were reported in all studies. }\end{array}$ \\
\hline Intervention & Hyperbaric oxygen therapy- dosages ranged from $24 \%$ to $100 \%$ oxygen. \\
\hline Comparators & Comparators for these studies included treatments of room air, sham air and free air flow. \\
\hline Outcomes & $\begin{array}{l}\text { Study outcomes included autism severity and change in behavior. Assessment tools used in the } \\
\text { studies included CGI (used in all three trials), ATEC, ADOS, SRS, ABC. VABS, VMI-5, BRIEF, } \\
\text { PPVT-III, RBS and direct observation of toy play sessions. The number of treatment sessions } \\
\text { ranged from } 20 \text { up to } 80 \text { one hour treatments occurring over a three up to } 15 \text { week period of time. } \\
\text { Outcomes were collected at end of treatment with no long term follow-up. }\end{array}$ \\
\hline Setting & $\begin{array}{l}\text { Two studies were conducted in the United States and one was performed in Thailand. Participants } \\
\text { in one study were recruited from a large community based ABA provider. }\end{array}$ \\
\hline
\end{tabular}


Table E-10. Other--Bumetanide

\begin{tabular}{|c|c|}
\hline Domain & Description of applicability of evidence \\
\hline Population & $\begin{array}{l}\text { Study participants ranged in age from } 3 \text { to } 11 \text { years with a wide range of disease severity among } \\
\text { study participants. The Chinese study was restricted to younger children ( } 2.5 \text { to } 6.5 \text { years) who } \\
\text { were concurrently enrolled in an ABA program. The degree of intellectual disability was not } \\
\text { described. Concomitant medications were stopped prior to study entry in both studies. The } \\
\text { placebo-controlled trial did allow use of melatonin. }\end{array}$ \\
\hline Intervention & Bumetanide , $1 \mathrm{mg}$ dose \\
\hline Comparators & Placebo and no medical treatment in one study in which all participants were receiving ABA. \\
\hline Outcomes & $\begin{array}{l}\text { Outcomes included autism severity scores and symptoms. Assessments used in the studies } \\
\text { included CARS, CGI, ADOS-G and ABC scales. Both studies were of } 3 \text { months duration. The } \\
\text { placebo controlled trial also assessed subjects after a one month wash-out period post- } \\
\text { intervention. }\end{array}$ \\
\hline Setting & One placebo-controlled trial was conducted in France and the other study was done in China. \\
\hline \multicolumn{2}{|c|}{$\begin{array}{l}\text { ABA-Applied Behavioral Analysis; CGI-Clinical Global Impression; CARS-Childhood Autism Rating Scale; ADOS-G-Autism Diagnostic } \\
\text { Observation Schedule-Generic; ABC-Aberrant Behavior Checklist }\end{array}$} \\
\hline \multicolumn{2}{|c|}{ Table E-11. Other--Donepezil } \\
\hline Domain & Description of applicability of evidence \\
\hline Population & $\begin{array}{l}\text { Studies included children } 2 \text { to } 17 \text { years old with autistic disorder; with majority being male children } \\
\text { (>90\%). One study reported a total ADOS score ranging from } 10.2 \text { to } 11.2 \text {; with IQ ranging from } \\
73-146 \text { while the other did not exclude children based on intelligence or language development } \\
\text { level. Studies allowed use of concomitant medications but did not include children with co-morbid } \\
\text { conditions. }\end{array}$ \\
\hline Intervention & The acetylcholinesterase inhibitor used was donepezil hydrochloride. \\
\hline Comparators & $\begin{array}{l}\text { The trial included a placebo controlled comparison group, which was an appropriate comparison } \\
\text { group. }\end{array}$ \\
\hline Outcomes & $\begin{array}{l}\text { The trials ranged from } 10 \text { to } 12 \text { weeks in duration with the evaluation conducted immediately after } \\
\text { treatment. Studies assessed outcomes in the short-term including expressive, receptive language } \\
\text { autistic behaviors and cognitive functioning. The dose used was similar to reports in the literature; } \\
\text { treatment episodes were most commonly associated with mood swings, lability, diarrhea, } \\
\text { headache and fatigue as side effects. One trial also evaluated the effect of age, IQ and autism } \\
\text { severity on treatment response and found no significant group difference. }\end{array}$ \\
\hline Setting & Both the trials were conducted in USA. \\
\hline
\end{tabular}




\section{Appendix F. Detailed Tables of Findings}

Table F-1. Key findings in studies of antipsychotics

\begin{tabular}{|c|c|c|c|}
\hline $\begin{array}{c}\text { Author, Year } \\
\text { Study Design } \\
\text { Groups (Dose), N Enrollment / } \\
\text { N Final } \\
\\
\text { Treatment Duration/Follow- } \\
\text { Up Time Point Post- } \\
\text { Treatment } \\
\text { Risk Of Bias }\end{array}$ & $\begin{array}{l}\text { Mean Age, } \\
\text { Years } \pm \text { SD } \\
\text { Mean IQ } \pm S D\end{array}$ & $\begin{array}{c}\text { Outcome } \\
\text { Measure/Baseline } \\
\text { Scores, Mean } \pm \text { SD }\end{array}$ & $\begin{array}{c}\text { Outcome } \\
\text { Measure/Post- } \\
\text { Treatment Scores, } \\
\text { Mean } \pm \text { SD }\end{array}$ \\
\hline Atomoxetine & & & \\
\hline $\begin{array}{l}\text { Handen et al., } 2015^{1,2} \\
\text { RCT } \\
\text { G1: Atomoxetine + Parent } \\
\text { Training, up to } 1.8 \mathrm{mg} / \mathrm{kg} / \mathrm{day} \text {, } \\
\text { 32/ } \\
\text { G2: Atomoxetine, up to } 1.8 \\
\mathrm{mg} / \mathrm{kg} / \text { day, } 32 / \\
\text { G3: Placebo + Parent Training, } \\
\text { up to } 1.8 \mathrm{mg} / \mathrm{kg} / \text { day, } 32 / \\
\text { G4: Placebo, up to } 1.8 \\
\mathrm{mg} / \mathrm{kg} / \text { day, } 32 / \\
\text { 10 weeks/EOT } \\
\text { Low RoB }\end{array}$ & $\begin{array}{l}\text { Age } \\
\text { G1: } 8 \pm 1.9 \\
\text { G2: } 8.6 \pm 2.3 \\
\text { G3: } 7.7 \pm 1.5 \\
\text { G4: } 8.2 \pm 2.4 \\
\text { IQ (Stanford- } \\
\text { Binet/Mullen } \\
\text { Scales) } \\
\text { G1: } 83.3 \pm \\
21.6 \\
\text { G2: } 78.7 \pm \\
\text { 25.9 } \\
\text { G3: } 77.92 \pm \\
5.7 \\
\text { G4: } 86.7 \pm \\
23.7\end{array}$ & $\begin{array}{l}\text { SNAP-IV-Parent - } \\
\text { ADHD } \\
\text { G1: } 2.21 \pm 0.38 \\
\text { G2: } 2.18 \pm 0.44 \\
\text { G3: } 2.22 \pm 0.37 \\
\text { G4: } 2.2 \pm 0.52 \\
\text { SNAP-IV-Parent - } \\
\text { Inattention } \\
\text { G1: } 2.28 \pm 0.46 \\
\text { G2: } 2.3 \pm 0.43 \\
\text { G3: } 2.23 \pm 0.49 \\
\text { G4: } 2.27 \pm 0.51 \\
\text { SNAP-IV-Parent - } \\
\text { Hyperactivity } \\
\text { G1: } 2.14 \pm 0.49 \\
\text { G2: } 2.07 \pm 0.65 \\
\text { G3: } 2.2 \pm 0.48 \\
\text { G4: } 2.13 \pm 0.69 \\
\text { SNAP-IV-Parent }- \\
\text { Oppositional Defiant } \\
\text { Disorder } \\
\text { G1: } 1.33 \pm 0.74 \\
\text { G2: } 1.31 \pm 0.65 \\
\text { G3: } 1.37 \pm 0.64 \\
\text { G4: } 1.28 \pm 0.82 \\
\text { SNAP-IV-Teacher - } \\
\text { ADHD } \\
\text { G1: } 1.99 \pm 0.46 \\
\text { G2: } 2 \pm 0.53 \\
\text { G3: } 1.98 \pm 0.52 \\
\text { G4: } 1.96 \pm 0.59 \\
\text { SNAP-IV-Teacher - } \\
\text { Inattention } \\
\text { G1: } 2.13 \pm 0.52 \\
\text { (a) }\end{array}$ & 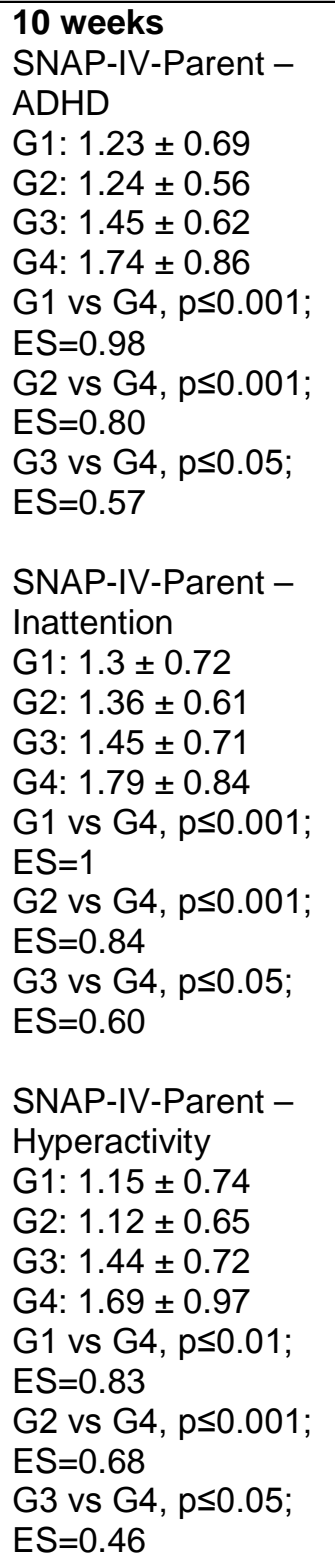 \\
\hline
\end{tabular}




\begin{tabular}{|c|c|c|c|}
\hline $\begin{array}{c}\text { Author, Year } \\
\text { Study Design } \\
\text { Groups (Dose), N Enrollment I } \\
\text { N Final } \\
\\
\text { Treatment Duration/Follow- } \\
\text { Up Time Point Post- } \\
\text { Treatment } \\
\text { Risk Of Bias }\end{array}$ & $\begin{array}{c}\text { Mean Age, } \\
\text { Years } \pm \text { SD } \\
\text { Mean IQ } \pm S D\end{array}$ & $\begin{array}{c}\text { Outcome } \\
\text { Measure/Baseline } \\
\text { Scores, Mean } \pm \text { SD }\end{array}$ & $\begin{array}{c}\text { Outcome } \\
\text { Measure/Post- } \\
\text { Treatment Scores, } \\
\text { Mean } \pm \text { SD }\end{array}$ \\
\hline & & $\begin{array}{l}\text { G2: } 2.15 \pm 0.54 \\
\text { G3: } 2.16 \pm 0.46 \\
\text { G4: } 2.28 \pm 0.49 \\
\text { SNAP-IV-Teacher - } \\
\text { Hyperactivity } \\
\text { G1: } 1.86 \pm 0.68 \\
\text { G2: } 1.85 \pm 0.72 \\
\text { G3: } 1.81 \pm 0.79 \\
\text { G4: } 1.64 \pm 0.9 \\
\text { SNAP-IV-Teacher - } \\
\text { Oppositional Defiant } \\
\text { Disorder } \\
\text { G1: } 1.07 \pm 0.73 \\
\text { G2: } 1.21 \pm 0.62 \\
\text { G3: } 1.03 \pm 0.76 \\
\text { G4: } 1.04 \pm 0.71 \\
\text { ABC-Parent }- \\
\text { Irritability } \\
\text { G1: } 17.88 \pm 9.25 \\
\text { G2: } 16 \pm 9.74 \\
\text { G3: } 18.16 \pm 9.24 \\
\text { G4: } 16.97 \pm 8.36 \\
\text { ABC-Parent }- \text { Social } \\
\text { Withdrawal } \\
\text { G1: } 12.09 \pm 7.68 \\
\text { G2: } 12.53 \pm 9.22 \\
\text { G3: } 8.56 \pm 5.69 \\
\text { G4: } 12.09 \pm 7.12 \\
\text { ABC-Parent }- \\
\text { Stereotypic Behavior } \\
\text { G1: } 5.81 \pm 4.69 \\
\text { G2: } 7.41 \pm 5.5 \\
\text { G3: } 5.34 \pm 5.03 \\
\text { G4: } 5.06 \pm 5.2 \\
\text { ABC-Parent }- \\
\text { Hyperactivity } \\
\text { G1: } 29.94 \pm 8.74 \\
\text { G2: } 29.34 \pm 9.52 \\
\text { G3: } 31.31 \pm 8.96\end{array}$ & 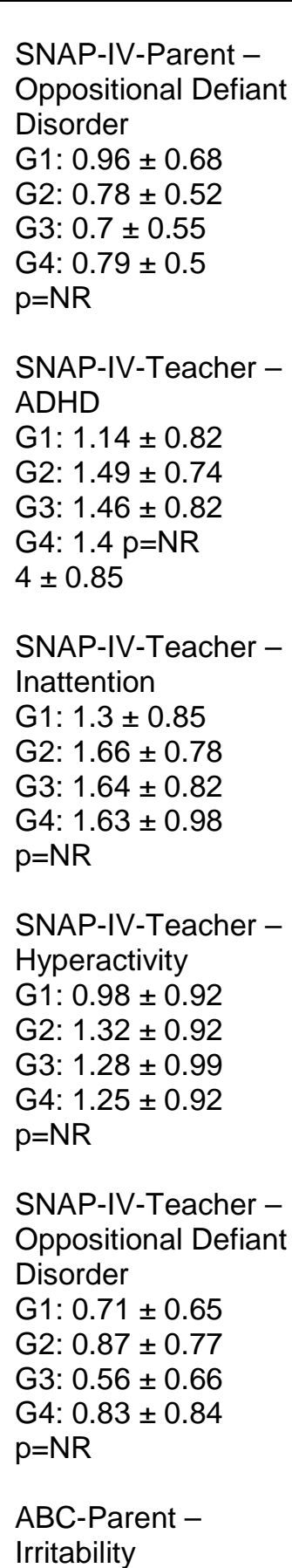 \\
\hline
\end{tabular}

F-2 


\begin{tabular}{|c|c|c|c|}
\hline $\begin{array}{c}\text { Author, Year } \\
\text { Study Design } \\
\text { Groups (Dose), N Enrollment I } \\
\text { N Final } \\
\\
\text { Treatment Duration/Follow- } \\
\text { Up Time Point Post- } \\
\text { Treatment } \\
\text { Risk Of Bias }\end{array}$ & $\begin{array}{c}\text { Mean Age, } \\
\text { Years } \pm \text { SD } \\
\text { Mean IQ } \pm S D\end{array}$ & $\begin{array}{c}\text { Outcome } \\
\text { Measure/Baseline } \\
\text { Scores, Mean } \pm \text { SD }\end{array}$ & $\begin{array}{c}\text { Outcome } \\
\text { Measure/Post- } \\
\text { Treatment Scores, } \\
\text { Mean } \pm \text { SD }\end{array}$ \\
\hline & & 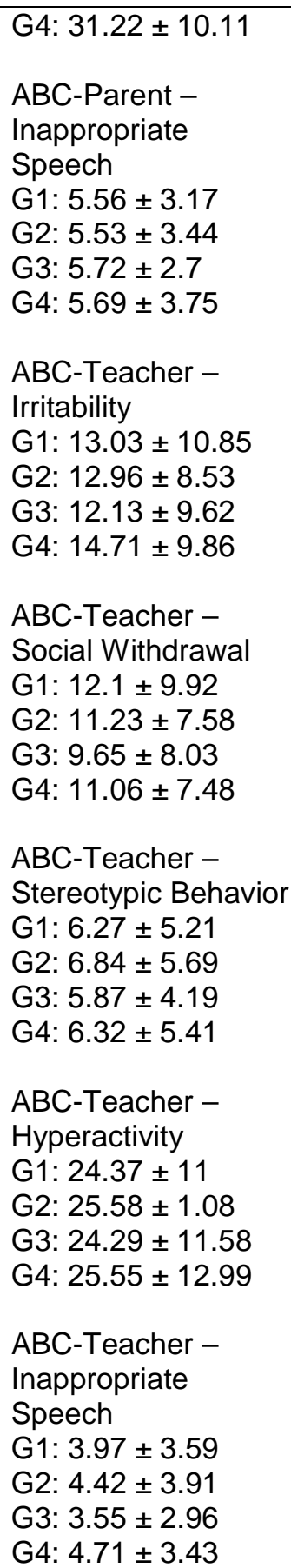 & 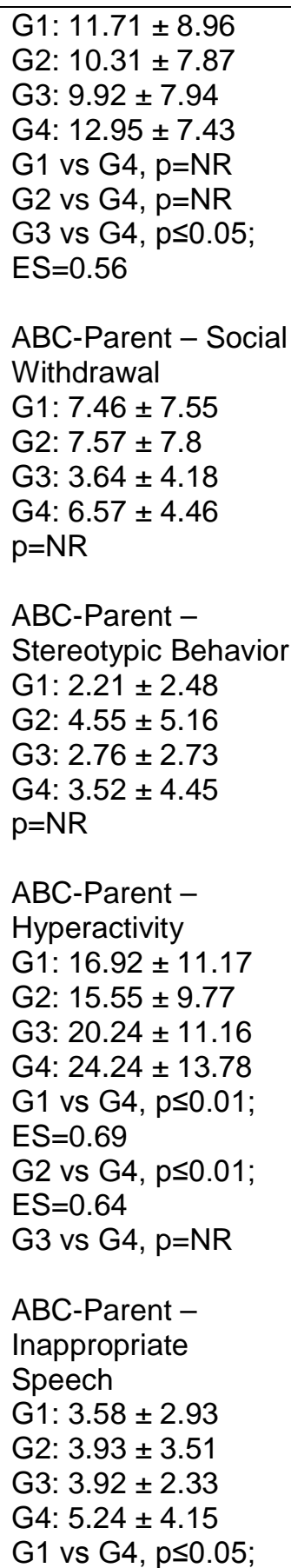 \\
\hline
\end{tabular}




\begin{tabular}{|c|c|c|c|}
\hline $\begin{array}{c}\text { Author, Year } \\
\text { Study Design } \\
\text { Groups (Dose), N Enrollment I } \\
\text { N Final } \\
\\
\text { Treatment Duration/Follow- } \\
\text { Up Time Point Post- } \\
\text { Treatment } \\
\text { Risk Of Bias }\end{array}$ & $\begin{array}{c}\text { Mean Age, } \\
\text { Years } \pm \text { SD } \\
\text { Mean IQ } \pm S D\end{array}$ & $\begin{array}{c}\text { Outcome } \\
\text { Measure/Baseline } \\
\text { Scores, Mean } \pm \text { SD }\end{array}$ & $\begin{array}{c}\text { Outcome } \\
\text { Measure/Post- } \\
\text { Treatment Scores, } \\
\text { Mean } \pm \text { SD }\end{array}$ \\
\hline & & 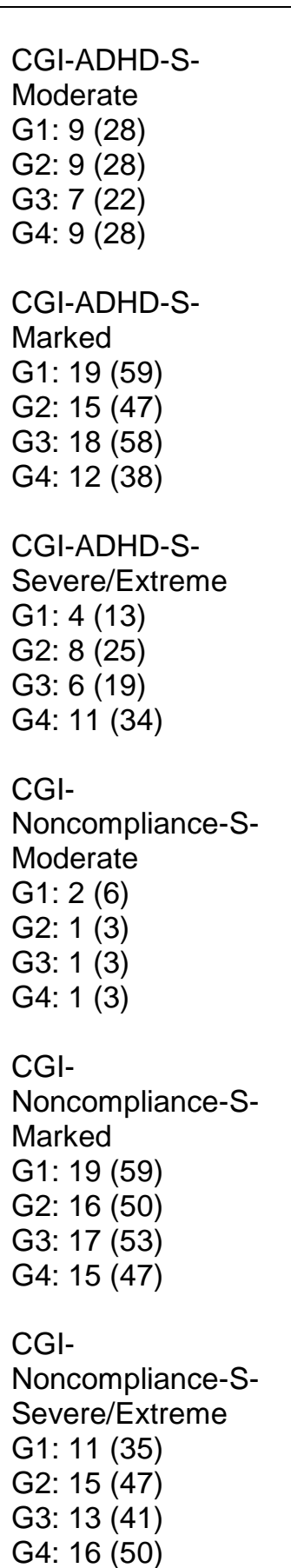 & $\begin{array}{l}\text { ES=0.76 } \\
\text { G2 vs G4, p=NR } \\
\text { G3 vs G4, p } \leq 0.05 \text {; } \\
\text { ES=0.86 } \\
\text { ABC-Teacher - } \\
\text { Irritability } \\
\text { G1: } 7.65 \pm 7.74 \\
\text { G2: } 8.97 \pm 6.92 \\
\text { G3: } 6.27 \pm 7.4 \\
\text { G4: } 12.6 \pm 13.06 \\
\text { p=NR } \\
\text { ABC-Teacher - } \\
\text { Social Withdrawal } \\
\text { G1: } 7.59 \pm 6.61 \\
\text { G2: } 10.54 \pm 7.63 \\
\text { G3: } 7.93 \pm 8.14 \\
\text { G4: } 8.67 \pm 10.4 \\
\text { p=NR } \\
\text { ABC-Teacher }- \\
\text { Stereotypic Behavior } \\
\text { G1: } 4.24 \pm 5.84 \\
\text { G2: } 5 \pm 5.3 \\
\text { G3: } 5.2 \pm 5.48 \\
\text { G4: } 5.4 \pm 6.29 \\
\text { p=NR } \\
\text { ABC-Teacher - } \\
\text { Hyperactivity } \\
\text { G1: } 13.88 \pm 11.13 \\
\text { G2: } 20.64 \pm 12.52 \\
\text { G3: } 19 \pm 12.84 \\
\text { G4: } 20.67 \pm 14.18 \\
\text { p=NR } \\
\text { ABC-Teacher - } \\
\text { Inappropriate } \\
\text { Speech } \\
\text { G1: } 2.76 \pm 2.97 \\
\text { G2: } 3.89 \pm 3.38 \\
\text { G3: } 4.13 \pm 3.38 \\
\text { G4: } 4.13 \pm 3.98 \\
\text { G1 vs G4, p=NR }\end{array}$ \\
\hline
\end{tabular}




\begin{tabular}{|c|c|c|c|}
\hline $\begin{array}{c}\text { Author, Year } \\
\text { Study Design } \\
\text { Groups (Dose), N Enrollment I } \\
\text { N Final } \\
\\
\text { Treatment Duration/Follow- } \\
\text { Up Time Point Post- } \\
\text { Treatment } \\
\text { Risk Of Bias }\end{array}$ & $\begin{array}{c}\text { Mean Age, } \\
\text { Years } \pm \text { SD } \\
\text { Mean IQ } \pm S D\end{array}$ & $\begin{array}{c}\text { Outcome } \\
\text { Measure/Baseline } \\
\text { Scores, Mean } \pm \text { SD }\end{array}$ & $\begin{array}{c}\text { Outcome } \\
\text { Measure/Post- } \\
\text { Treatment Scores, } \\
\text { Mean } \pm \text { SD }\end{array}$ \\
\hline & & $\begin{array}{l}\text { HSQ-Severity } \\
\text { G1: } 4.01 \pm 1.7 \\
\text { G2: } 3.69 \pm 1.58 \\
\text { G3: } 3.81 \pm 1.49 \\
\text { G4: } 3.99 \pm 1.82 \\
\\
\text { SSQ-Severity } \\
\text { G1: } 3.67 \pm 2.08 \\
\text { G2: } 3.4 \pm 1.93 \\
\text { G3: } 3.23 \pm 1.93 \\
\text { G4: } 3.75 \pm 2.29\end{array}$ & 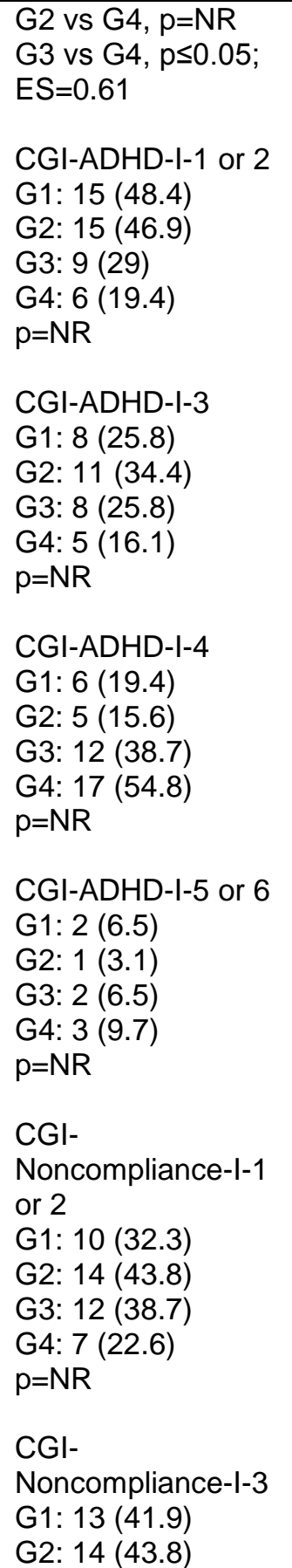 \\
\hline
\end{tabular}




\begin{tabular}{|c|c|c|c|}
\hline $\begin{array}{c}\text { Author, Year } \\
\text { Study Design } \\
\text { Groups (Dose), N Enrollment I } \\
\text { N Final } \\
\\
\text { Treatment Duration/Follow- } \\
\text { Up Time Point Post- } \\
\text { Treatment } \\
\text { Risk Of Bias }\end{array}$ & $\begin{array}{l}\text { Mean Age, } \\
\text { Years } \pm \text { SD } \\
\text { Mean IQ } \pm \text { SD }\end{array}$ & $\begin{array}{c}\text { Outcome } \\
\text { Measure/Baseline } \\
\text { Scores, Mean } \pm \text { SD }\end{array}$ & $\begin{array}{c}\text { Outcome } \\
\text { Measure/Post- } \\
\text { Treatment Scores, } \\
\text { Mean } \pm \text { SD }\end{array}$ \\
\hline & & & 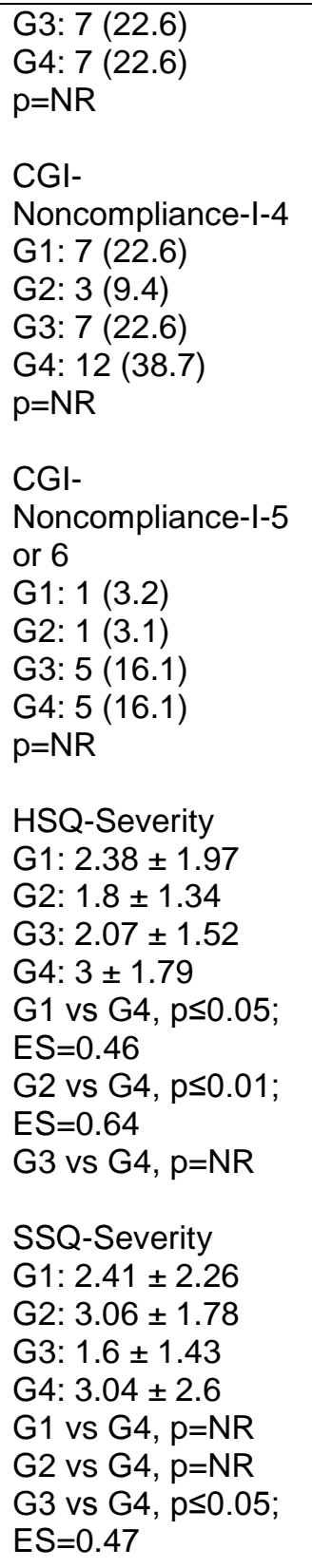 \\
\hline $\begin{array}{l}\text { Handen et al., } 2015^{1,2} \\
\text { RCT }\end{array}$ & & & $\begin{array}{l}\text { Open Label }(20 \\
\text { weeks/Last }\end{array}$ \\
\hline
\end{tabular}




\begin{tabular}{|c|c|c|c|}
\hline $\begin{array}{c}\text { Author, Year } \\
\text { Study Design } \\
\text { Groups (Dose), N Enrollment I } \\
\text { N Final } \\
\\
\text { Treatment Duration/Follow- } \\
\text { Up Time Point Post- } \\
\text { Treatment } \\
\text { Risk Of Bias }\end{array}$ & $\begin{array}{l}\text { Mean Age, } \\
\text { Years } \pm \text { SD } \\
\text { Mean IQ } \pm \text { SD }\end{array}$ & $\begin{array}{c}\text { Outcome } \\
\text { Measure/Baseline } \\
\text { Scores, Mean } \pm \text { SD }\end{array}$ & $\begin{array}{c}\text { Outcome } \\
\text { Measure/Post- } \\
\text { Treatment Scores, } \\
\text { Mean } \pm \text { SD }\end{array}$ \\
\hline $\begin{array}{l}\text { G1a: Atomoxetine + Parent } \\
\text { Training (responders) } \\
\text { G2a: Atomoxetine (responders) } \\
\text { G3a: Placebo + Parent Training } \\
\text { (responders) } \\
\text { G3b: Atomoxetine + Parent } \\
\text { Training (placebo + PT } \\
\text { nonresponders) } \\
\text { G4a: Placebo (responders) } \\
\text { G4b: Atomoxetine (placebo } \\
\text { nonresponders) } \\
24 \text { Week OLE } \\
\text { Low RoB }\end{array}$ & & & $\begin{array}{l}\text { Observation } \\
\text { Carried Forward) } \\
\text { SNAP-IV-Parent - } \\
\text { ADHD } \\
\text { G3b: } 1.78 \pm 0.65 \\
\text { G4b: } 2 . \text { p=NR } \\
\text { 18 } \pm 0.56 \\
\text { SNAP-IV-Parent - } \\
\text { Inattention } \\
\text { G3b: } 1.75 \pm 0.75 \\
\text { G4b: } 2.27 \pm 0.5 \\
\text { p=NR } \\
\text { SNAP-IV-Parent - } \\
\text { Hyperactivity } \\
\text { G3b: } 1.8 \pm 0.73 \\
\text { G4b: } 2.08 \pm 0.79 \\
\text { p=NR } \\
\text { SNAP-IV-Parent - } \\
\text { Oppositional } \\
\text { Defiance Disorder } \\
\text { G3b: } 0.97 \pm 0.72 \\
\text { G4b: } 1.02 \pm 0.76 \\
\text { p=NR } \\
\text { HSQ-Severity } \\
\text { G3b: } 2.96 \pm 1.94 \\
\text { G4b: } 3.73 \pm 1.78 \\
\text { p=NR } \\
\text { ABC-Parent- } \\
\text { Irritability } \\
\text { G3b: } 13.84 \pm 10.3 \\
\text { G4b: } 15.64 \pm 9.38 \\
\text { p=NR } \\
\text { ABC-Parent- } \\
\text { Hyperactivity } \\
\text { G3b: } 28.26 \pm 11.66 \\
\text { G4b: } 30.23 \pm 12.37 \\
\text { p=NR }\end{array}$ \\
\hline
\end{tabular}




\begin{tabular}{|c|c|c|c|}
\hline $\begin{array}{c}\text { Author, Year } \\
\text { Study Design } \\
\text { Groups (Dose), N Enrollment I } \\
\text { N Final } \\
\\
\text { Treatment Duration/Follow- } \\
\text { Up Time Point Post- } \\
\text { Treatment } \\
\text { Risk Of Bias }\end{array}$ & $\begin{array}{c}\text { Mean Age, } \\
\text { Years } \pm \text { SD } \\
\text { Mean IQ } \pm \text { SD }\end{array}$ & $\begin{array}{c}\text { Outcome } \\
\text { Measure/Baseline } \\
\text { Scores, Mean } \pm \text { SD }\end{array}$ & $\begin{array}{c}\text { Outcome } \\
\text { Measure/Post- } \\
\text { Treatment Scores, } \\
\text { Mean } \pm \text { SD }\end{array}$ \\
\hline & & & $\begin{array}{l}\text { Open Label (24 } \\
\text { weeks) } \\
\text { SNAP-IV-Parent - } \\
\text { Total Score } \\
\text { G1a: } 2.01 \pm 0.34 \\
\text { G2a: } 2.24 \pm 0.35 \\
\text { G3a: } 2.31 \pm 0.08 \\
\text { G4a: } 1.69 \pm 0.21 \\
\text { p=NR } \\
\text { HSQ-Severity } \\
\text { G1a: } 3.17 \pm 1.77 \\
\text { G2a: } 3.75 \pm 1.34 \\
\text { G3a: } 4.08 \pm 1.51 \\
\text { G4a: } 2.31 \pm 1.71 \\
\text { p=NR }\end{array}$ \\
\hline Risperidone & & & \\
\hline $\begin{array}{l}\text { Lamberti et al., } 2016^{3} \\
\text { RCT } \\
\text { G1: Aripirazole (up to } 15 \mathrm{mg} / \text { day), } \\
\text { 22/19 } \\
\text { G2: Risperidone (up tp } 3 \mathrm{mg} / \text { day), } \\
\text { 22/18 } \\
24 \text { weeks/EOT } \\
\text { Low ROB }\end{array}$ & $\begin{array}{l}\text { Age } \\
\text { G1: } 8.4 \pm 2.9 \\
\text { G2: } 7.9 \pm 2.3 \\
\text { IQ } \\
\text { NR }\end{array}$ & $\begin{array}{l}\text { CGI - Severity } \\
\text { G1: } 5.4 \pm 0.5 \\
\text { G2: } 5.5 \pm 0.6 \\
\text { CGAS - Total Score } \\
\text { G1: } 38 \pm 8.3 \\
\text { G2: } 31.42 \pm 12.4 \\
\text { ADHD Rating Scale - } \\
\text { Total Score } \\
\text { G1: } 39.4 \pm 2.8 \\
\text { G2: } 37.4 \pm 3.9 \\
\text { CPRS - Hyperactivity } \\
\text { G1: } 5.7 \pm 0.7 \\
\text { G2: } 5.4 \pm 0.7 \\
\text { CPRS - Inattention } \\
\text { G1: } 5.4 \pm 0.8 \\
\text { G2: } 4.9 \pm 0.9\end{array}$ & 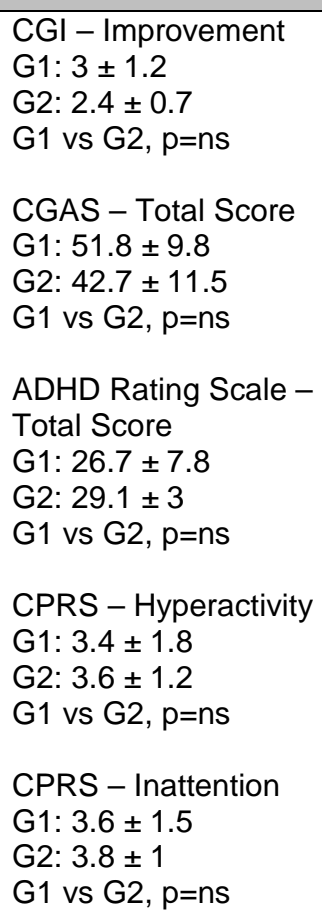 \\
\hline
\end{tabular}




\begin{tabular}{|c|c|c|c|}
\hline $\begin{array}{c}\text { Author, Year } \\
\text { Study Design } \\
\text { Groups (Dose), N Enrollment I } \\
\text { N Final } \\
\\
\text { Treatment Duration/Follow- } \\
\text { Up Time Point Post- } \\
\text { Treatment } \\
\text { Risk Of Bias }\end{array}$ & $\begin{array}{l}\text { Mean Age, } \\
\text { Years } \pm \text { SD } \\
\text { Mean IQ } \pm S D\end{array}$ & $\begin{array}{c}\text { Outcome } \\
\text { Measure/Baseline } \\
\text { Scores, Mean } \pm S D\end{array}$ & $\begin{array}{c}\text { Outcome } \\
\text { Measure/Post- } \\
\text { Treatment Scores, } \\
\text { Mean } \pm \text { SD }\end{array}$ \\
\hline $\begin{array}{l}\text { aScahill } 2015^{4} \\
\text { RCT } \\
\text { G1: Risperidone ( } 2.5 \mathrm{mg} / \text { day), } \\
\text { 57/55 } \\
\text { G2: Placebo (NA), } 27 / 26 \\
21 \text { months/2 Years post-treatment } \\
\text { Low RoB }\end{array}$ & $\begin{array}{l}\text { Age } \\
\text { G1: } 8.6 \pm 2.97 \\
\text { G2: } 9.1 \pm 2.58 \\
\text { IQ } \\
\geq 70 \\
\text { G1: } 11(23.9) \\
\text { G2: } 6(13.3) \\
<70 \\
\text { G1: } 35(76.1) \\
\text { G2: } 39(86.7)\end{array}$ & $\begin{array}{l}\text { ABC-Irritability } \\
\text { G1: } 27.22 \pm 7.28 \\
\text { G2: } 23.44 \pm 7.24 \\
\text { ABC-Social } \\
\text { Withdrawal } \\
\text { G1: } 16.05 \pm 8.55 \\
\text { G2: } 18.52 \pm 9.72 \\
\text { ABC-Stereotypic } \\
\text { Behavior } \\
\text { G1: } 10.5 \pm 4.43 \\
\text { G2: } 8.84 \pm 5.22 \\
\text { ABC- } \\
\text { Hyperactivity/Noncomp } \\
\text { liance } \\
\text { G1: } 34.3 \pm 7.95 \\
\text { G2: } 28.58 \pm 10.4 \\
\text { ABC-Inappropriate } \\
\text { Speech } \\
\text { G1: } 5.71 \pm 3.93 \\
\text { G2: } 5.59 \pm 4.03 \\
\text { CYBOCS } \\
\text { G1: } 15.29 \pm 3.15 \\
\text { G2: } 16 \pm 3.29 \\
\text { CGI-Severity } \\
\text { G1: } 5.090 .7 \\
\text { G2: } 5.230 .65 \\
\text { VABS-Communication } \\
\text { G1: } 42.81 \pm 14.75 \\
\text { G2: } 44.21 \pm 17.43 \\
\text { VABS-Daily Living } \\
\text { Skills } \\
\text { G1: } 38.56 \pm 18.44 \\
\text { G2: } 33.25 \pm 15.2 \\
\text { VABS-Social Skills } \\
\text { G1: } 47.4 \pm 11.71 \\
\text { G2: } 48.83 \pm 18.19 \\
\text { VABS-Motor Skills } \\
\text { G1: } 60.34 \pm 19.86 \\
\text { G2: } 56.88 \pm 24.63 \\
\text { (a) }\end{array}$ & 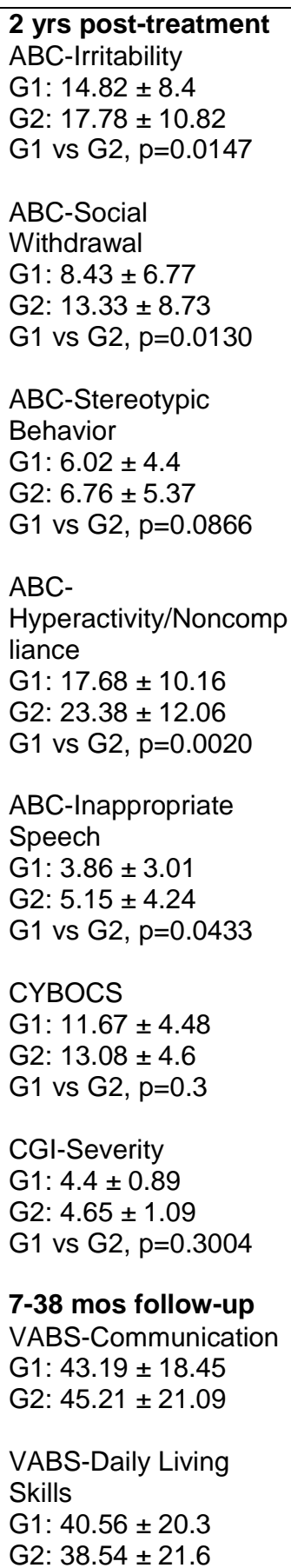 \\
\hline
\end{tabular}




\begin{tabular}{|c|c|c|c|}
\hline $\begin{array}{c}\text { Author, Year } \\
\text { Study Design } \\
\text { Groups (Dose), N Enrollment I } \\
\text { N Final } \\
\\
\text { Treatment Duration/Follow- } \\
\text { Up Time Point Post- } \\
\text { Treatment } \\
\text { Risk Of Bias }\end{array}$ & $\begin{array}{l}\text { Mean Age, } \\
\text { Years } \pm \text { SD } \\
\text { Mean IQ } \pm S D\end{array}$ & $\begin{array}{c}\text { Outcome } \\
\text { Measure/Baseline } \\
\text { Scores, Mean } \pm \text { SD }\end{array}$ & $\begin{array}{c}\text { Outcome } \\
\text { Measure/Post- } \\
\text { Treatment Scores, } \\
\text { Mean } \pm \text { SD }\end{array}$ \\
\hline & & $\begin{array}{l}\text { VABS-Adaptive } \\
\text { Behavior } \\
\text { G1: } 41.55 \pm 15.63 \\
\text { G2: } 38.58 \pm 15.38 \\
\text { IQ Tests } \\
\text { G1: } 50.52 \pm 23.07 \\
\text { G2: } 46.74 \pm 30.57 \\
\text { M-RLRS - Sensory } \\
\text { Motor } \\
\text { G1: } 6.95 \pm 4.28 \\
\text { G2: } 4.76 \pm 2.98 \\
\text { M-RLRS - Social } \\
\text { Relationships } \\
\text { G1: } 6.55 \pm 4.29 \\
\text { G2: } 6.28 \pm 4.32 \\
\text { M-RLRS - Affectual } \\
\text { Responses } \\
\text { G1: } 6.13 \pm 1.9 \\
\text { G2: } 5.2 \pm 1.94 \\
\text { M-RLRS - Sensory } \\
\text { Responses } \\
\text { G1: } 20.96 \pm 9.06 \\
\text { G2: } 19.24 \pm 8.8 \\
\text { M-RLRS - Language } \\
\text { G1: } 2.67 \pm 4.31 \\
\text { G2:4.92 } \pm 4.65\end{array}$ & $\begin{array}{l}\text { VABS-Social Skills } \\
\text { G1: } 50.63 \pm 15.1 \\
\text { G2: } 44.21 \pm 18.07 \\
\text { VABS-Motor Skills } \\
\text { G1: } 61.94 \pm 15.72 \\
\text { G2: } 63.06 \pm 27.17 \\
\text { VABS-Adaptive } \\
\text { Behavior } \\
\text { G1: } 40.25 \pm 15.51 \\
\text { G2: } 38.96 \pm 18.02 \\
\text { IQ Tests } \\
\text { G1: } 48.73 \pm 23.92 \\
\text { G2: } 45.85 \pm 32.22 \\
\text { M-RLRS - Sensory } \\
\text { Motor } \\
\text { G1: } 4.67 \pm 3.06 \\
\text { G2: } 4.2 \pm 3.42 \\
\text { M-RLRS }- \text { Social } \\
\text { Relationships } \\
\text { G1: } 1.02 \pm 4.23 \\
\text { G2: } 2.4 \pm 3.16 \\
\text { M-RLRS - Affectual } \\
\text { Responses } \\
\text { G1: } 3.49 \pm 1.88 \\
\text { G2: } 4.04 \pm 1.88 \\
\text { M-RLRS - Sensory } \\
\text { Responses } \\
\text { G1: } 11.02 \pm 7.1 \\
\text { G2: } 14.8 \pm 7.08 \\
\text { M-RLRS }- \text { Language } \\
\text { G1: }-0.15 \pm 3.99 \\
\text { G2:1.96 } \pm 4.3\end{array}$ \\
\hline $\begin{array}{l}{ }^{2} \text { Scahill et al., } 2015^{5} \\
\text { RCT } \\
\text { G1: Risperidone (up to } 2.5 \mathrm{mg} / \mathrm{kg} \text { ), } \\
49 / 43 \\
\text { G2: Placebo (NA), } 52 / 38 \\
8 \text { weeks/EOT }\end{array}$ & $\begin{array}{l}\text { Age } \\
\text { G1: } 8.6 \pm 2.97 \\
\text { G2: } 9.1 \pm 2.58 \\
\text { IQ } \\
\geq 70 \\
\text { G1: } 11(23.9) \\
\text { G2: } 6(13.3)\end{array}$ & $\begin{array}{l}\text { CYBOCS } \\
\text { G1: } 15.25 \pm 3.25 \\
\text { G2: } 14.8 \pm 4.27\end{array}$ & $\begin{array}{l}\text { EOT } \\
\text { CYBOCS } \\
\text { G1: } 11.3 \pm 3.77 \\
\text { G2: } 14.1 \pm 4.43 \\
\text { G1 vs } \mathrm{G} 2, \mathrm{p}=0.0005 \\
\text { ES=0.74 }\end{array}$ \\
\hline
\end{tabular}




\begin{tabular}{|c|c|c|c|}
\hline $\begin{array}{c}\text { Author, Year } \\
\text { Study Design } \\
\text { Groups (Dose), N Enrollment I } \\
\text { N Final } \\
\\
\text { Treatment Duration/Follow- } \\
\text { Up Time Point Post- } \\
\text { Treatment } \\
\text { Risk Of Bias }\end{array}$ & $\begin{array}{l}\text { Mean Age, } \\
\text { Years } \pm \text { SD } \\
\text { Mean IQ } \pm \text { SD }\end{array}$ & $\begin{array}{c}\text { Outcome } \\
\text { Measure/Baseline } \\
\text { Scores, Mean } \pm \text { SD }\end{array}$ & $\begin{array}{c}\text { Outcome } \\
\text { Measure/Post- } \\
\text { Treatment Scores, } \\
\text { Mean } \pm \text { SD }\end{array}$ \\
\hline Low RoB & $\begin{array}{l}<70 \\
\text { G1: } 35(76.1) \\
\text { G2: } 39(86.7)\end{array}$ & & \\
\hline $\begin{array}{l}{ }^{a} \text { Scahill et al., } 2015^{6} \\
\text { RCT } \\
\text { G1: Risperidone (up to } 2.5 \mathrm{mg} / \text { day), } \\
\text { 49/49 } \\
\text { G2: Placebo (NA), } 52 / 52 \\
8 \text { weeks/EOT } \\
\text { Low RoB }\end{array}$ & $\begin{array}{l}\text { Age } \\
\text { G1: } 7.5 \pm 2.80 \\
\text { G2: } 9.1 \pm 2.58 \\
\text { IQ } \\
\geq 70 \\
\text { G1: } 23(46.9) \\
\text { G2: } 6(13.3) \\
<70 \\
\text { NR }\end{array}$ & $\begin{array}{l}\text { ABC-Social } \\
\text { Withdrawal/Lethargy } \\
\text { G1: } 16.4 \pm 8.2 \\
\text { G2: } 16.1 \pm 8.7\end{array}$ & $\begin{array}{l}\text { EOT } \\
\text { ABC-Social } \\
\text { Withdrawal/Lethargy } \\
\text { G1 vs G2, p=0.05; } \\
\text { ES=0.42 }\end{array}$ \\
\hline $\begin{array}{l}{ }^{\mathrm{D} S c a h i l l} \text { et al., } 2015^{5} \\
\text { RCT } \\
\text { G1: Risperidone (up to } 3.5 \mathrm{mg} / \mathrm{day} \text { ), } \\
\text { 49/47 } \\
\text { G2: Risperidone + Parent Training } \\
\text { (up to } 3.5 \mathrm{mg} / \mathrm{day}+\text { mean of } 10.9 \\
\text { sessions), } 75 / 70 \\
8 \text { weeks } \\
\text { Low RoB }\end{array}$ & $\begin{array}{l}\text { Age } \\
\text { G1: } 7.5 \pm 2.80 \\
\text { G2: } 9.1 \pm 2.58 \\
\text { IQ } \\
\geq 70 \\
\text { G1: } 23(46.9) \\
\text { G2: } 6(13.3) \\
<70 \\
\text { NR }\end{array}$ & $\begin{array}{l}\text { CYBOCS } \\
\text { G1: } 16.2 \pm 2.47 \\
\text { G2: } 14.8 \pm 4.27\end{array}$ & $\begin{array}{l}\text { EOT } \\
\text { CYBOCS } \\
\text { G1: } 11 \pm 3.73 \\
\text { G2: } 14.1 \pm 4.43 \\
\text { G1 vs } \mathrm{G} 2, \mathrm{p}=0.0001 \\
\text { ES }=0.88\end{array}$ \\
\hline $\begin{array}{l}\text { Wink et al., } 2014^{7} \\
\text { Retrospective Cohort } \\
\text { G1: Risperidone ( } 2.23 \mathrm{mg} / \text { day), } \\
\text { 72/72 } \\
\text { G2: Aripiprazole (11.85 mg/day), } \\
\text { 70/70 } \\
\text { Varies/EOT } \\
\text { Moderate RoB }\end{array}$ & $\begin{array}{l}\text { Age (at } \\
\text { initiation) } \\
\text { G1: } 8.41 \pm 3.59 \\
\text { G2: } 9.74 \pm 3.46 \\
\text { IQ } \\
\text { NR }\end{array}$ & $\begin{array}{l}\text { CGI-Severity } \\
\text { NR }\end{array}$ & $\begin{array}{l}\text { EOT } \\
\text { CGI-Improvement } \\
\text { G1: } 3.2 \pm 1.2 \\
\text { G2: } 2.9 \pm 1.2 \\
\text { G1 vs } \mathrm{G} 2, \mathrm{p}=\mathrm{ns}\end{array}$ \\
\hline
\end{tabular}




\begin{tabular}{|c|c|c|c|}
\hline $\begin{array}{c}\text { Author, Year } \\
\text { Study Design } \\
\text { Groups (Dose), N Enrollment I } \\
\text { N Final } \\
\text { Treatment Duration/Follow- } \\
\text { Up Time Point Post- } \\
\text { Treatment } \\
\text { Risk Of Bias }\end{array}$ & $\begin{array}{l}\text { Mean Age, } \\
\text { Years } \pm \text { SD } \\
\text { Mean IQ } \pm S D\end{array}$ & $\begin{array}{c}\text { Outcome } \\
\text { Measure/Baseline } \\
\text { Scores, Mean } \pm S D\end{array}$ & $\begin{array}{c}\text { Outcome } \\
\text { Measure/Post- } \\
\text { Treatment Scores, } \\
\text { Mean } \pm \text { SD }\end{array}$ \\
\hline $\begin{array}{l}{ }^{\mathrm{D}} \mathrm{Kent} \text { et al., } 2013^{8} \\
\text { RCT } \\
\text { G1: Risperidone (0.125-0.175 } \\
\text { mg/day; low dose), 30/25 } \\
\text { G2: Risperidone }(1.25-1.75 \mathrm{mg} / \text { day; } \\
\text { high dose), 31/25 } \\
\text { G3: Placebo (NA), 35/27 } \\
6 \text { weeks/EOT } \\
\text { Low RoB }\end{array}$ & $\begin{array}{l}\text { Age } \\
\text { G1: } 10 \pm 3.4 \\
\text { G2: } 9 \pm 3.1 \\
\text { G3: } 9 \pm 2.6 \\
\text { IQ } \\
\text { Mental age } \\
\text { G1 + G2 + G3: } \\
5.5 \text { yrs }\end{array}$ & $\begin{array}{l}\text { ABC-Irritability } \\
\text { G1: } 27.1 \pm 6.26 \\
\text { G2: } 28.0 \pm 7.81 \\
\text { G3: } 28.9 \pm 6.10 \\
\text { CGI - Severity } \\
\text { G1: } 5.1 \pm 0.92 \\
\text { G2: } 5.0 \pm 0.78 \\
\text { G3: } 4.9 \pm 0.67\end{array}$ & $\begin{array}{l}\text { EOT } \\
\text { Mean change in: } \\
\text { ABC-Irritability } \\
\text { G1: }-7.4 \pm 8.12 \\
\text { G2: }-12.4 \pm 6.52 \\
\text { G3: }-3.5 \pm 10.67 \\
\text { G1 vs G3: } p=n s \\
\text { G2 vs G3: } p<0.001 \\
\text { CGI - Severity } \\
\text { G1: }-0.4 \pm 0.73 \\
\text { G2: }-1.0 \pm 0.78 \\
\text { G3: }-0.3 \pm 0.79 \\
\text { G1 vs } G 3: p=n s \\
\text { G2 vs } G 3: p<0.001\end{array}$ \\
\hline $\begin{array}{l}{ }^{\mathrm{C}} \text { Kent et al., } 2013^{9} \\
\text { RCT } \\
\text { G1: Risperidone (0.125-0.175 } \\
\text { mg/day; low dose)/Risperidone, } \\
\text { 30/25 } \\
\text { G2: Risperidone ( } 1.25-1.75 \mathrm{mg} / \text { day; } \\
\text { high dose)/Risperidone, } 31 / 25 \\
\text { G3: Placebo/Risperidone (NA), } \\
\text { 35/27 } \\
6 \text { weeks/End of open label trial } \\
\text { Low RoB }\end{array}$ & $\begin{array}{l}\text { Age } \\
\text { G1: } 10 \pm 3.4 \\
\text { G2: } 9 \pm 3.1 \\
\text { G3: } 9 \pm 2.6 \\
\text { IQ } \\
\text { Mental age } \\
\text { G1 + G2 + G3: } \\
5.5 \text { yrs }\end{array}$ & $\begin{array}{l}\text { ABC-Irritability } \\
\text { G1: } 13.4 \pm 3.99 \\
\text { G2: } 14.4 \pm 4.64 \\
\text { G3: } 13.7 \pm 2.66 \\
\text { ABC-Hyperactivity } \\
\text { G1: } 30.1 \pm 11.46 \\
\text { G2: } 33.8 \pm 9.75 \\
\text { G3: } 31.4 \pm 8.60 \\
\text { ABC-Stereotypic } \\
\text { Behavior } \\
\text { G1: } 9.3 \pm 5.17 \\
\text { G2: } 11.5 \pm 5.06 \\
\text { G3: } 10.5 \pm 5.26 \\
\text { ABC-Inappropriate } \\
\text { Speech } \\
\text { G1: } 6.6 \pm 3.49 \\
\text { G2: } 7.5 \pm 2.78 \\
\text { G3: } 5.9 \pm 3.42 \\
\text { ABC-Social } \\
\text { Withdrawal } \\
\text { G1: } 18.2 \pm 9.71 \\
\text { G2: } 21.4 \pm 9.09 \\
\text { G3: } 18.1 \pm 10.16 \\
\text { CYBOCS } \\
\text { G1: } 13.4 \pm 3.99 \\
\text { G2: } 14.4 \pm 4.64 \\
\text { G3: } 13.7 \pm 2.66 \\
\text { CGI-Severity } \\
\text { G1: } 5.1 \pm 0.93 \\
\text { a } 19\end{array}$ & 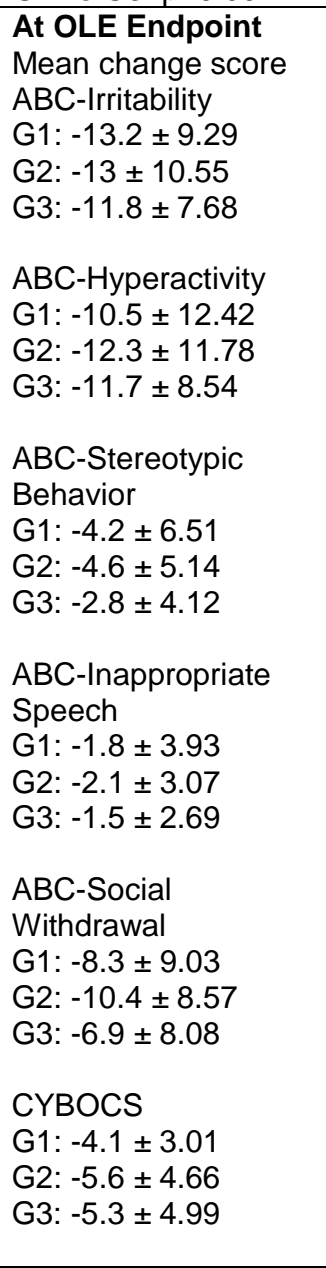 \\
\hline
\end{tabular}




\begin{tabular}{|c|c|c|c|}
\hline $\begin{array}{c}\text { Author, Year } \\
\text { Study Design } \\
\text { Groups (Dose), N Enrollment I } \\
\text { N Final } \\
\\
\text { Treatment Duration/Follow- } \\
\text { Up Time Point Post- } \\
\text { Treatment } \\
\text { Risk Of Bias }\end{array}$ & $\begin{array}{c}\text { Mean Age, } \\
\text { Years } \pm \text { SD } \\
\text { Mean IQ } \pm S D\end{array}$ & $\begin{array}{c}\text { Outcome } \\
\text { Measure/Baseline } \\
\text { Scores, Mean } \pm \text { SD }\end{array}$ & $\begin{array}{c}\text { Outcome } \\
\text { Measure/Post- } \\
\text { Treatment Scores, } \\
\text { Mean } \pm \text { SD }\end{array}$ \\
\hline & & $\begin{array}{l}\text { G2: } 5 \pm 0.75 \\
\text { G3: } 4.9 \pm 0.67\end{array}$ & $\begin{array}{l}\text { CGI-Severity } \\
\text { G1: }-1 \pm 1.02 \\
\text { G2: }-1.3 \pm 1.17 \\
\text { G3:- } 0.9 \pm 0.88 \\
\text { CGI-Much or Very } \\
\text { Much Improved } \\
\text { G1: } 14 \text { (58) } \\
\text { G2: } 15 \text { (60) } \\
\text { G3: } 20 \text { (69) } \\
\text { *\% of patients with } \\
\text { CGI-C ratings of } \\
\text { "much" or "very much" } \\
\text { improved at OLE } \\
\text { endpoint was not } \\
\text { significant among the } 3 \\
\text { groups (p=0.684) } \\
\text { *The percentage of } \\
\text { patients with } \\
\text { ABC-I response at } \\
\text { OLE endpoint (defined } \\
\text { as } \neq 25 \% \text { improvement } \\
\text { from DB baseline) was } \\
\text { not statistically } \\
\text { significantly different } \\
\text { among the three } \\
\text { groups ( } p=0.800 \text { ). } \\
\text { Mean change scores } \\
\text { from baseline } \\
\text { ABC-Irritability } \\
\text { G1: }-7.4 \pm 8.12 \\
\text { G2: }-12.4 \pm 6.52 \\
\text { G3: }-3.5 \pm 10.67 \\
\text { G1 Vs G3, p=0.164, } \\
\text { ES=0.36 } \\
\text { G2 Vs G3, } p<0.001, \\
\text { ES=0.94 } \\
\text { ABC-Hyperactivity } \\
\text { G1: }-10.5 \pm 12.42 \\
\text { G2: }-12.3 \pm 11.78 \\
\text { G3: }-11.7 \pm 8.54 \\
\text { G1 Vs G3, p=0.008 } \\
\text { G2 Vs G3, p=0.019 } \\
\text { ABC-Stereotypy } \\
\text { G1: }-4.2 \pm 6.51\end{array}$ \\
\hline
\end{tabular}




\begin{tabular}{|c|c|c|c|}
\hline $\begin{array}{c}\text { Author, Year } \\
\text { Study Design } \\
\text { Groups (Dose), N Enrollment I } \\
\text { N Final } \\
\\
\text { Treatment Duration/Follow- } \\
\text { Up Time Point Post- } \\
\text { Treatment } \\
\text { Risk Of Bias }\end{array}$ & $\begin{array}{l}\text { Mean Age, } \\
\text { Years } \pm \text { SD } \\
\text { Mean IQ } \pm \text { SD }\end{array}$ & $\begin{array}{c}\text { Outcome } \\
\text { Measure/Baseline } \\
\text { Scores, Mean } \pm S D\end{array}$ & $\begin{array}{c}\text { Outcome } \\
\text { Measure/Post- } \\
\text { Treatment Scores, } \\
\text { Mean } \pm \text { SD }\end{array}$ \\
\hline & & & $\begin{array}{l}\text { G2: }-4.6 \pm 5.14 \\
\text { G3: }-2.8 \pm 4.12 \\
\text { G1 Vs } G 3, p=n s \\
\text { G2 Vs G3, p=ns } \\
\text { ABC-Inappropriate } \\
\text { Speech } \\
\text { G1: }-1.8 \pm 3.93 \\
\text { G2: }-2.1 \pm 3.07 \\
\text { G3: }-1.5 \pm 2.69 \\
\text { G1 Vs G3, p=ns } \\
\text { G2 Vs G3, p=ns } \\
\text { ABC-Social } \\
\text { Withdrawal/Lethargy } \\
\text { G1: }-8.3 \pm 9.03 \\
\text { G2: }-10.4 \pm 8.57 \\
\text { G3: }-6.9 \pm 8.08 \\
\text { G1 Vs G3, p=0.817 } \\
\text { G2 Vs G3, p=0.004 } \\
\text { Change from } \\
\text { baseline } \\
\text { CGI-Severity } \\
\text { G1: }-0.4 \pm 0.73 \\
\text { G2: }-1 \pm 0.78 \\
\text { G3: }-0.3 \pm 0.79 \\
\text { G1 Vs G3, p=0.769, } \\
\text { ES=0.08 } \\
\text { G2 Vs G3, p<0.001, } \\
\text { ES=1.02 } \\
\text { EOT } \\
\text { CGI-Improvement }- \\
\text { much or very much } \\
\text { improved } \\
\text { G1: } 5(17) \\
\text { G2: } 18 \text { (63) } \\
\text { G3: } 5(15) \\
\text { G1 Vs G3, p=0.985 } \\
\text { G2 Vs G3, p<0.001 } \\
\text { CYBOCs } \\
\text { NR }\end{array}$ \\
\hline
\end{tabular}




\begin{tabular}{|c|c|c|c|}
\hline $\begin{array}{c}\text { Author, Year } \\
\text { Study Design } \\
\text { Groups (Dose), N Enrollment I } \\
\text { N Final } \\
\text { Treatment Duration/Follow- } \\
\text { Up Time Point Post- } \\
\text { Treatment } \\
\text { Risk Of Bias }\end{array}$ & $\begin{array}{l}\text { Mean Age, } \\
\text { Years } \pm \text { SD } \\
\text { Mean IQ } \pm S D\end{array}$ & $\begin{array}{c}\text { Outcome } \\
\text { Measure/Baseline } \\
\text { Scores, Mean } \pm \text { SD }\end{array}$ & $\begin{array}{c}\text { Outcome } \\
\text { Measure/Post- } \\
\text { Treatment Scores, } \\
\text { Mean } \pm \text { SD }\end{array}$ \\
\hline $\begin{array}{l}{ }^{5} \text { Scahill et al., }{ }^{6} 2013 \\
\text { RCT } \\
\text { G1: Risperidone (up to } 3.5 \text { mg/day), } \\
49 / 47 \\
\text { G2: Risperidone + Parent Training } \\
\text { (up to } 3.5 \text { mg/day + mean of } 10.9 \\
\text { sessions), } 75 / 70 \\
8 \text { weeks/EOT } \\
\text { Low RoB }\end{array}$ & $\begin{array}{l}\text { Age } \\
\text { G1: } 7.5 \pm 2.80 \\
\text { G2: } 9.1 \pm 2.58 \\
\text { IQ } \\
\geq 70 \\
\text { G1: } 23(46.9) \\
\text { G2: } 6(13.3) \\
<70 \\
\text { NR }\end{array}$ & $\begin{array}{l}\text { ABC-Social } \\
\text { Withdrawal/Lethargy } \\
\text { G1: } 17.1 \pm 8.5 \\
\text { G2: } 15.2 \pm 9\end{array}$ & $\begin{array}{l}\text { EOT } \\
\text { ABC-Social } \\
\text { Withdrawal/Lethargy } \\
\text { NR }\end{array}$ \\
\hline $\begin{array}{l}{ }^{d} \text { Shea et al., } 2004^{10} \\
\text { RCT } \\
\text { G1: Risperidone }(1 \mathrm{mg} / \mathrm{mL}), 24 \\
\text { G2: Placebo (NA), } 28 \\
8 \text { weeks/EOT } \\
\text { Moderate RoB }\end{array}$ & $\begin{array}{l}\text { Age } \\
\text { G1: } 7.4 \pm 2.7 \\
\text { G2: } 7.1 \pm 2.1 \\
\text { IQ - } \leq 84 \\
\text { G1: } 19(100) \\
\text { G2: } 18(75) \\
\text { IQ - >84 } \\
\text { G1: } 0(0) \\
\text { G2: } 6(25)\end{array}$ & $\begin{array}{l}\text { ABC-Irritability } \\
\text { G1: } 20.6 \pm 8.1 \\
\text { G2: } 21.6 \pm 10.2 \\
\text { ABC-Hyperactivity } \\
\text { G1: } 29.2 \pm 9.5 \\
\text { G2: } 33.6 \pm 6.8 \\
\text { ABC-Social } \\
\text { Withdrawal/Lethargy } \\
\text { G1: } 14 \pm 6.8 \\
\text { G2: } 13.6 \pm 8.6 \\
\text { ABC-Stereotypy } \\
\text { G1: } 8.4 \pm 5.8 \\
\text { G2: } 9.4 \pm 5.5 \\
\text { ABC-Inappropriate } \\
\text { Speech } \\
\text { G1: } 4.5 \pm 3.7 \\
\text { G2: } 4.5 \pm 3.7 \\
\text { Nisonger-Child } \\
\text { Behavior Rating Form } \\
\text { - Adaptive/Social } \\
\text { G1: } 3.8 \pm 2.3 \\
\text { G2: } 3.9 \pm 2 \\
\text { Nisonger-Child } \\
\text { Behavior Rating Form } \\
\text { - Compliant/Calm } \\
\text { G1: } 3.8 \pm 2.7 \\
\text { G2: } 6.2 \pm 2.4 \\
\text { Nisonger-Child } \\
\text { Behavior Rating Form } \\
\text { - Conduct Problem } \\
\text { G1: } 17.2 \pm 8 \\
\text { G2: } 21.5 \pm 10.7\end{array}$ & $\begin{array}{l}\text { During Treatment (1 } \\
\text { wk) } \\
\text { ABC-Irritability } \\
\text { G1: } 13.7 \pm 9.1 \\
\text { G2: } 16.9 \pm 10.7 \\
\text { During Treatment (2 } \\
\text { wks) } \\
\text { ABC-Irritability } \\
\text { G1: } 10.3 \pm 7.6 \\
\text { G2: } 15.8 \pm 10.8 \\
\text { During Treatment (3 } \\
\text { wks) } \\
\text { ABC-Irritability } \\
\text { G1: } 8.8 \pm 7.3 \\
\text { G2: } 14.4 \pm 11.7 \\
\text { During Treatment (5 } \\
\text { wks) } \\
\text { ABC-Irritability } \\
\text { G1: } 8.8 \pm 7.2 \\
\text { G2: } 13.7 \pm 11.1 \\
\text { During Treatment (8 } \\
\text { wks) } \\
\text { ABC-Irritability } \\
\text { G1: } 7.5 \pm 6.4 \\
\text { G2: } 13.8 \pm 12.2 \\
\text { EOT } \\
\text { ABC-Irritability } \\
\text { G1: } 7.2 \pm 5.9 \\
\text { G2: } 14.1 \pm 11.3 \\
\text { G1 vs G2, p=0.002; } \\
\text { ES = -0.7 } \\
\text { ABC-Hyperactivity }\end{array}$ \\
\hline
\end{tabular}




\begin{tabular}{|c|c|c|c|}
\hline $\begin{array}{c}\text { Author, Year } \\
\text { Study Design } \\
\text { Groups (Dose), N Enrollment I } \\
\text { N Final } \\
\\
\text { Treatment Duration/Follow- } \\
\text { Up Time Point Post- } \\
\text { Treatment } \\
\text { Risk Of Bias }\end{array}$ & $\begin{array}{l}\text { Mean Age, } \\
\text { Years } \pm \text { SD } \\
\text { Mean IQ } \pm \text { SD }\end{array}$ & $\begin{array}{c}\text { Outcome } \\
\text { Measure/Baseline } \\
\text { Scores, Mean } \pm \text { SD }\end{array}$ & $\begin{array}{c}\text { Outcome } \\
\text { Measure/Post- } \\
\text { Treatment Scores, } \\
\text { Mean } \pm \text { SD }\end{array}$ \\
\hline & & $\begin{array}{l}\text { Nisonger-Child } \\
\text { Behavior Rating Form } \\
\text { - Hyperactive } \\
\text { G1: } 17.7 \pm 5.6 \\
\text { G2: } 19.6 \pm 5.2 \\
\text { Nisonger-Child } \\
\text { Behavior Rating Form } \\
\text { - Insecure/Anxious } \\
\text { G1: } 6.3 \pm 6.7 \\
\text { G2: } 8.7 \pm 6.7 \\
\text { Nisonger-Child } \\
\text { Behavior Rating Form } \\
\text { - Overly Sensitive } \\
\text { G1: } 6.7 \pm 3.4 \\
\text { G2: } 6.6 \pm 3.4 \\
\text { Nisonger-Child } \\
\text { Behavior Rating Form } \\
\text { - Self- } \\
\text { Injury/Stereotypic } \\
\text { G1: } 4.5 \pm 4.4 \\
\text { G2: } 4.1 \pm 4.4 \\
\text { Nisonger-Child } \\
\text { Behavior Rating Form } \\
\text { - Self- } \\
\text { Isolated/Ritualistic } \\
\text { G1: } 7.3 \pm 4 \\
\text { G2: } 7.8 \pm 4.2 \\
\text { VAS-Aggression } \\
\text { G1: } 86 \pm 14.5 \\
\text { G2: } 88.3 \pm 9 \\
\text { VAS- } \\
\text { Defiance/Disobedience } \\
\text { G1: } 75 \pm 47 \\
\text { G2: } 94.8 \pm 19.6 \\
\text { VAS-Hyperactivity } \\
\text { G1: } 68.7 \pm 28.6 \\
\text { G2: } 96.3 \pm 4.2 \\
\text { VAS- } \\
\text { Obsessive/Repetitive } \\
\text { G1: } 86.3 \pm 19.4 \\
\text { G2: } 98 \pm 0\end{array}$ & 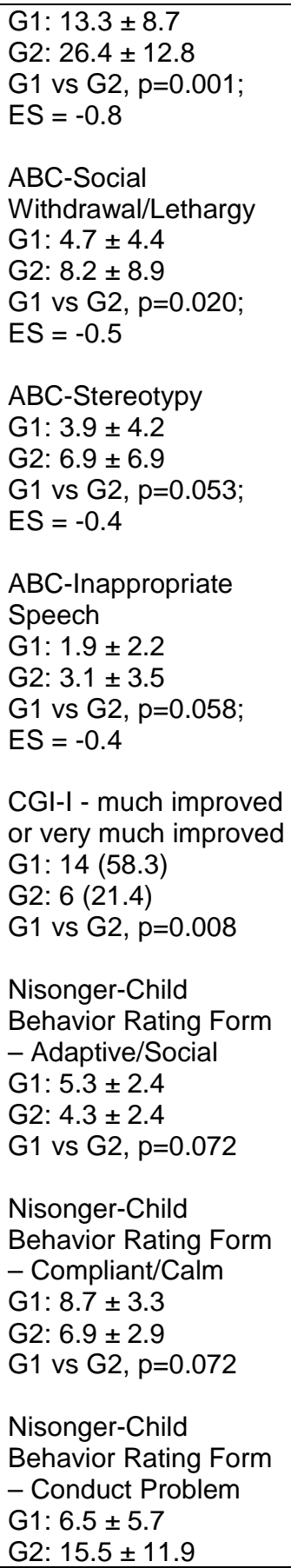 \\
\hline
\end{tabular}




\begin{tabular}{|c|c|c|c|}
\hline $\begin{array}{c}\text { Author, Year } \\
\text { Study Design } \\
\text { Groups (Dose), N Enrollment I } \\
\text { N Final } \\
\\
\text { Treatment Duration/Follow- } \\
\text { Up Time Point Post- } \\
\text { Treatment } \\
\text { Risk Of Bias }\end{array}$ & $\begin{array}{l}\text { Mean Age, } \\
\text { Years } \pm \text { SD } \\
\text { Mean IQ } \pm S D\end{array}$ & $\begin{array}{c}\text { Outcome } \\
\text { Measure/Baseline } \\
\text { Scores, Mean } \pm \text { SD }\end{array}$ & $\begin{array}{c}\text { Outcome } \\
\text { Measure/Post- } \\
\text { Treatment Scores, } \\
\text { Mean } \pm \text { SD }\end{array}$ \\
\hline & & $\begin{array}{l}\text { VAS- } \\
\text { Tantrums/Negative } \\
\text { Mood } \\
\text { G1: } 80.8 \pm 10.1 \\
\text { G2:81.2 } \pm 13.9\end{array}$ & 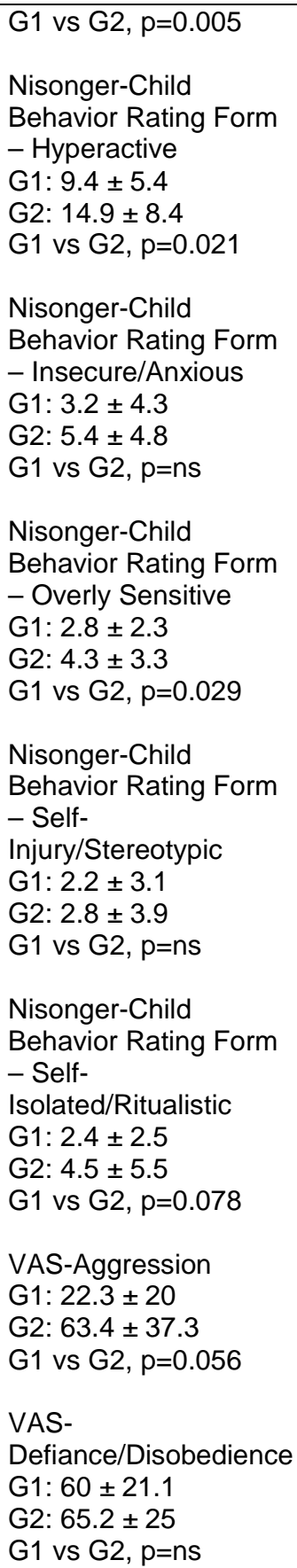 \\
\hline
\end{tabular}




\begin{tabular}{|c|c|c|c|}
\hline $\begin{array}{c}\text { Author, Year } \\
\text { Study Design } \\
\text { Groups (Dose), N Enrollment I } \\
\text { N Final } \\
\text { Treatment Duration/Follow- } \\
\text { Up Time Point Post- } \\
\text { Treatment } \\
\text { Risk Of Bias }\end{array}$ & $\begin{array}{l}\text { Mean Age, } \\
\text { Years } \pm \text { SD } \\
\text { Mean IQ } \pm S D\end{array}$ & $\begin{array}{l}\text { Outcome } \\
\text { Measure/Baseline } \\
\text { Scores, Mean } \pm \text { SD }\end{array}$ & $\begin{array}{c}\text { Outcome } \\
\text { Measure/Post- } \\
\text { Treatment Scores, } \\
\text { Mean } \pm \text { SD }\end{array}$ \\
\hline & & & $\begin{array}{l}\text { VAS-Hyperactivity } \\
\text { G1: } 39.7 \pm 19.7 \\
\text { G2: } 80.5 \pm 14.2 \\
\text { G1 vs } G 2, p=0.040 \\
\text { VAS- } \\
\text { Obsessive/Repetitive } \\
\text { G1: } 70 \pm 16.8 \\
\text { G2: } 48 \pm 63.4 \\
\text { G1 vs } \mathrm{G} 2, \mathrm{p}=\mathrm{ns} \\
\text { VAS- } \\
\text { Tantrums/Negative } \\
\text { Mood } \\
\text { G1: } 28 \pm 20.9 \\
\text { G2:43.4 } \pm 28.8 \\
\text { G1 vs } \mathrm{G} 2, \mathrm{p}=0.496\end{array}$ \\
\hline $\begin{array}{l}{ }^{a} \text { Scahill et al., } 2006^{11} \\
\text { RCT } \\
\text { G1: Risperidone }(2.5 \mathrm{mg} / \mathrm{day}) \text {, } \\
48 / 48 \\
16 \text { weeks/end of } 4 \text { months } \\
\text { Low RoB }\end{array}$ & G1: 5-16 yrs & $\begin{array}{l}\text { VABS-Communication } \\
\text { G1: } 43.5813 .48 \\
\text { VABS-Daily Living } \\
\text { Skills } \\
\text { G1: } 39.4218 .06 \\
\text { VABS-Socialization } \\
\text { G1: } 48.679 .78 \\
\text { VABS-Composite } \\
\text { G1: } 42.3515 .79\end{array}$ & $\begin{array}{l}\text { End of } 4 \text { month open } \\
\text { label extension } \\
\text { VABS-Communication } \\
\text { G1: } 45.2118 .85 \\
d=0.11 \\
\text { VABS-Daily Living } \\
\text { Skills } \\
\text { G1: } 40.3818 .11 \\
d=0.06 \\
\text { VABS-Socialization } \\
\text { G1: } 5.512 .94 \\
d=0.14 \\
\text { VABS-Composite } \\
\text { G1: } 42.0613 .53 \\
d=-0.02\end{array}$ \\
\hline $\begin{array}{l}{ }^{a} \text { Scahill et al., } 2005^{12} \\
\text { RCT } \\
\text { G1: Risperidone ( } 2.5 \mathrm{mg} / \text { day), } \\
\text { 49/49 } \\
\text { G2: Placebo (NA), 52/42 } \\
8 \text { weeks/EOT } \\
\text { Low RoB }\end{array}$ & $\begin{array}{l}\text { Age } \\
\text { G1 + G2: } 8.82 \pm \\
2.69 \\
\text { IQ } \\
\text { NR }\end{array}$ & $\begin{array}{l}\text { Ritvo-Freeman Real } \\
\text { Life Rating Scales - } \\
\text { Overall Score } \\
\text { G1: } 0.94 \pm 0.36 \\
\text { G2: } 1.03 \pm 0.37 \\
\text { VABS-Total Score } \\
\text { G1: } 33.26 \pm 8.38 \\
\text { G2: } 33.51 \pm 8.29\end{array}$ & $\begin{array}{l}\text { 4wks During } \\
\text { Treatment } \\
\text { Ritvo-Freeman Real } \\
\text { Life Rating Scales - } \\
\text { Overall Score } \\
\text { G1: } 0.54 \pm 0.36 \\
\text { G2: } 0.84 \pm 0.39 \\
\text { EOT (8 wks) } \\
\text { Ritvo-Freeman Real } \\
\text { Life Rating Scales - } \\
\text { Overall Score } \\
\text { G1: } 0.45 \pm 0.31 \\
\text { G2: } 0.88 \pm 0.4 \\
\text { G1 vs G2, ES }=1.08\end{array}$ \\
\hline
\end{tabular}




\begin{tabular}{|c|c|c|c|}
\hline $\begin{array}{c}\text { Author, Year } \\
\text { Study Design } \\
\text { Groups (Dose), N Enrollment I } \\
\text { N Final } \\
\text { Treatment Duration/Follow- } \\
\text { Up Time Point Post- } \\
\text { Treatment } \\
\text { Risk Of Bias }\end{array}$ & $\begin{array}{l}\text { Mean Age, } \\
\text { Years } \pm \text { SD } \\
\text { Mean IQ } \pm S D\end{array}$ & $\begin{array}{c}\text { Outcome } \\
\text { Measure/Baseline } \\
\text { Scores, Mean } \pm \text { SD }\end{array}$ & $\begin{array}{c}\text { Outcome } \\
\text { Measure/Post- } \\
\text { Treatment Scores, } \\
\text { Mean } \pm \text { SD }\end{array}$ \\
\hline & & & $\begin{array}{l}\text { VABS-Total Score } \\
\text { G1: } 20.34 \pm 7.93 \\
\text { G2: } 30.27 \pm 8.87 \\
\text { G1 vs G2, ES }=1.03 \\
\text { Group X time, } p<0.001\end{array}$ \\
\hline $\begin{array}{l}\text { a Scahill et al., } 2005^{13} \\
\text { RCT } \\
\text { G1: Risperidone ( } 2.5 \mathrm{mg} / \text { day), } \\
63 / 63 \\
\text { G2: Placebo-Substitution (NA), NA } \\
4 \text { weeks during open label } \\
\text { extension/EOT } \\
\text { Low RoB }\end{array}$ & $\begin{array}{l}\text { Age } \\
\text { G1 + G2: } 8.82 \pm \\
2.69 \\
\text { IQ } \\
\text { NR }\end{array}$ & 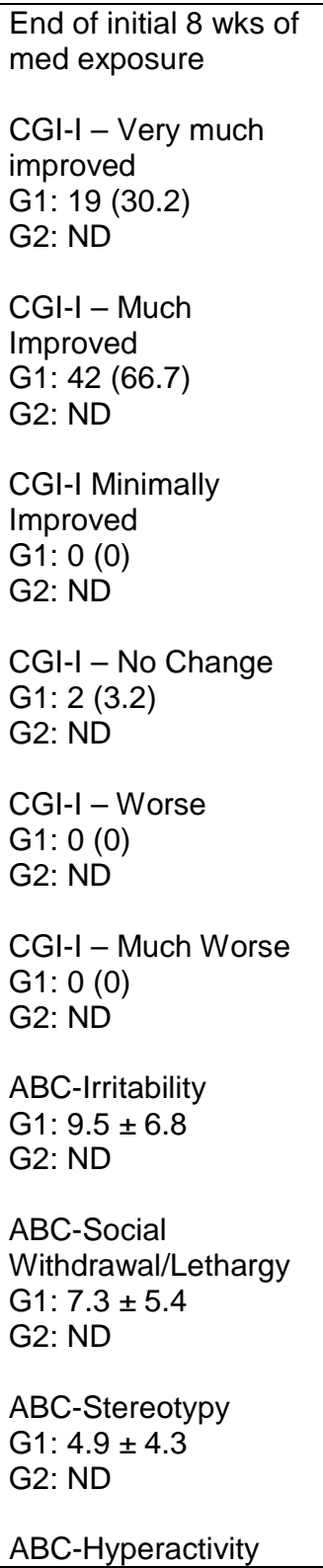 & $\begin{array}{l}4 \text { wks During } \\
\text { Treatment } \\
\text { CGI-I - Very much } \\
\text { improved } \\
\text { G1: } 13 \text { (20.6) } \\
\text { G2: ND } \\
\text { CGI-I - Much } \\
\text { Improved } \\
\text { G1: } 38 \text { (60.3) } \\
\text { G2: ND } \\
\text { CGI-I Minimally } \\
\text { Improved } \\
\text { G1: } 7 \text { (11.1) } \\
\text { G2: ND } \\
\text { CGI-I - No Change } \\
\text { G1: } 1 \text { (1.6) } \\
\text { G2: ND } \\
\text { CGI-I - Worse } \\
\text { G1: } 20 \text { (33.9) } \\
\text { G2: ND } \\
\text { CGI-I - Much Worse } \\
\text { G1: } 1 \text { (1.6) } \\
\text { G2: ND } \\
\text { 16 wks EOT } \\
\text { CGI-I - Very much } \\
\text { improved } \\
\text { G1: } 20 \text { (33.9) } \\
\text { G2: ND } \\
\text { CGI-I - Much } \\
\text { Improved } \\
\text { G1: } 31 \text { (5.5) } \\
\text { G2: ND } \\
\text { CGI-I Minimally } \\
\text { Improved } \\
\text { G1: } 5 \text { (8.5) } \\
\text { G }\end{array}$ \\
\hline
\end{tabular}




\begin{tabular}{|c|c|c|c|}
\hline $\begin{array}{c}\text { Author, Year } \\
\text { Study Design } \\
\text { Groups (Dose), N Enrollment I } \\
\text { N Final } \\
\\
\text { Treatment Duration/Follow- } \\
\text { Up Time Point Post- } \\
\text { Treatment } \\
\text { Risk Of Bias }\end{array}$ & $\begin{array}{l}\text { Mean Age, } \\
\text { Years } \pm \text { SD } \\
\text { Mean IQ } \pm \text { SD }\end{array}$ & $\begin{array}{c}\text { Outcome } \\
\text { Measure/Baseline } \\
\text { Scores, Mean } \pm \text { SD }\end{array}$ & $\begin{array}{c}\text { Outcome } \\
\text { Measure/Post- } \\
\text { Treatment Scores, } \\
\text { Mean } \pm \text { SD }\end{array}$ \\
\hline & & $\begin{array}{l}\text { G1: } 15.1 \pm 10 \\
\text { G2: ND } \\
\text { ABC-Inappropriate } \\
\text { Speech } \\
\text { G1: } 3.4 \pm 3.6 \\
\text { G2: ND }\end{array}$ & $\begin{array}{l}\text { G2: ND } \\
\text { CGI-I - No Change } \\
\text { G1: } 1 \text { (1.7) } \\
\text { G2: ND } \\
\text { CGI-I - Worse } \\
\text { G1: } 1 \text { (1.7) } \\
\text { G2: ND } \\
\text { CGI-I - Much Worse } \\
\text { G1: } 1 \text { (1.7) } \\
\text { G2: ND } \\
\text { EOT (Last } \\
\text { Observation) } \\
\text { ABC-Irritability } \\
\text { G1: 11.7 } 8 \\
\text { G2: ND } \\
\text { ABC-Social } \\
\text { Withdrawal/Lethargy } \\
\text { G1: } 6.8 \pm 5.9 \\
\text { G2: ND } \\
\text { ABC-Stereotypy } \\
\text { G1: } 5.8 \pm 4.7 \\
\text { G2: ND } \\
\text { ABC-Hyperactivity } \\
\text { G1: } 15.8 \pm 10.2 \\
\text { G2: ND } \\
\text { ABC-Inappropriate } \\
\text { Speech } \\
\text { G1: } 3.4 \pm 3.2 \\
\text { G2: ND } \\
\text { 8 wks During } \\
\text { Treatment } \\
\text { Relapse Rate } \\
\text { G1: } 10 \text { (62.5) } \\
\text { G2: } 2 \text { (12.5) } \\
\end{array}$ \\
\hline
\end{tabular}




\begin{tabular}{|c|c|c|c|}
\hline $\begin{array}{c}\text { Author, Year } \\
\text { Study Design } \\
\text { Groups (Dose), N Enrollment I } \\
\text { N Final } \\
\text { Treatment Duration/Follow- } \\
\text { Up Time Point Post- } \\
\text { Treatment } \\
\text { Risk Of Bias }\end{array}$ & $\begin{array}{l}\text { Mean Age, } \\
\text { Years } \pm \text { SD } \\
\text { Mean IQ } \pm S D\end{array}$ & $\begin{array}{c}\text { Outcome } \\
\text { Measure/Baseline } \\
\text { Scores, Mean } \pm \text { SD }\end{array}$ & $\begin{array}{c}\text { Outcome } \\
\text { Measure/Post- } \\
\text { Treatment Scores, } \\
\text { Mean } \pm \text { SD }\end{array}$ \\
\hline $\begin{array}{l}{ }^{\mathrm{c}} \text { Shea et al., } 2004^{14} \\
\text { RCT } \\
\text { G1: Risperidone (0.02 mg/kg/day), } \\
39 / 39 \\
\text { G2: Placebo (NA), } 38 / 38 \\
8 \text { weeks/EOT } \\
\text { Moderate RoB }\end{array}$ & $\begin{array}{l}\text { Age } \\
\text { G1: } 7.6 \pm 2.3 \\
\text { G2: } 7.3 \pm 2.3 \\
\text { IQ - Normal } \\
\text { G1: } 3(9.7) \\
\text { G2: } 11(31.4) \\
\text { IQ - Borderline } \\
\text { G1: } 6(19.4) \\
\text { G2: } 4(11.4) \\
\text { IQ - Mild } \\
\text { G1: } 12(38.7) \\
\text { G2: } 8 \text { (22.9) } \\
\text { IQ - Moderate } \\
\text { G1: } 10(32.3) \\
\text { G2: } 12 \text { (34.3) }\end{array}$ & $\begin{array}{l}\text { ABC-Irritability } \\
\text { G1: } 18.9 \pm 8.8 \\
\text { G2: } 21.2 \pm 9.7 \\
\text { ABC-Hyperactivity } \\
\text { G1: } 13.7 \pm 7 \\
\text { G2: } 14.3 \pm 8.2 \\
\text { ABC-Social } \\
\text { Withdrawal/Lethargy } \\
\text { G1: } 27.3 \pm 9.7 \\
\text { G2: } 30.9 \pm 8.8 \\
\text { ABC-Stereotypy } \\
\text { G1: } 4.6 \pm 3.4 \\
\text { G2: } 4.8 \pm 3.7 \\
\text { ABC-Inappropriate } \\
\text { Speech } \\
\text { G1: } 7.9 \pm 5 \\
\text { G2: } 8.1 \pm 5.6\end{array}$ & 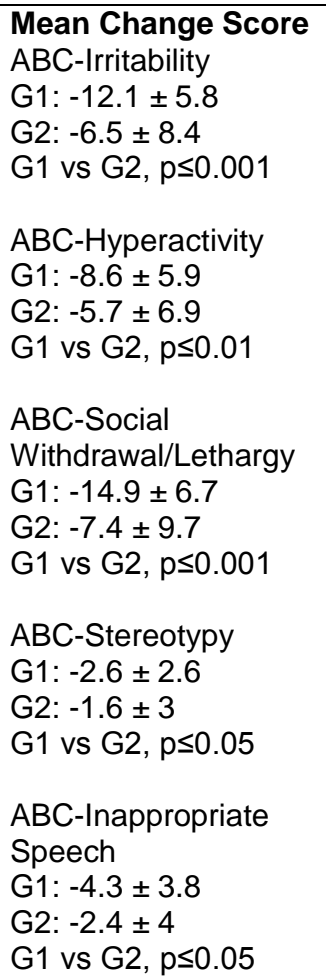 \\
\hline $\begin{array}{l}{ }^{a} \text { Scahill et al., } 2002^{15} \\
\text { RCT } \\
\text { G1: Risperidone (up to } 2.5 \mathrm{mg} / \mathrm{kg} \text { ), } \\
49 / 49 \\
\text { G2: Placebo (NA), 52/52 } \\
8 \text { weeks/EOT } \\
\text { Low RoB }\end{array}$ & $\begin{array}{l}\text { Age } \\
\text { G1 + G2: } 8.82 \pm \\
2.69 \\
\text { IQ } \\
\text { NR }\end{array}$ & $\begin{array}{l}\text { ABC-Irritability } \\
\text { G1: } 26.2 \pm 7.9 \\
\text { G2: } 25.5 \pm 6.6 \\
\text { ABC-Lethargy/Social } \\
\text { Withdrawal } \\
\text { G1: } 16.4 \pm 8.2 \\
\text { G2: } 16.1 \pm 8.7 \\
\text { ABC-Stereotypic } \\
\text { Behavior } \\
\text { G1: } 10.6 \pm 4.9 \\
\text { G2: } 9 \pm 4.4 \\
\text { ABC-Hyperactivity } \\
\text { G1: } 31.8 \pm 9.6 \\
\text { G2: } 32.3 \pm 8.5 \\
\text { ABC-Inappropriate } \\
\text { Speech } \\
\text { G1: } 4.8 \pm 4.1 \\
\text { G2: } 6.5 \pm 3.6\end{array}$ & $\begin{array}{l}\text { EOT } \\
\text { ABC-Irritability } \\
\text { G1: } 11.3 \pm 7.4 \\
\text { G2: } 21.9 \pm 9.5 \\
\text { G1 vs } \mathrm{G} 2, \mathrm{p}<0.001 \\
\text { ES }=1.2 \\
\text { ABC-Lethargy/Social } \\
\text { Withdrawal } \\
\text { G1: } 8.9 \pm 6.4 \\
\text { G2: } 12 \pm 8.3 \\
\text { G1 vs G2, p=0.03, } \\
\text { ES=0.4 } \\
\text { ABC-Stereotypic } \\
\text { Behavior } \\
\text { G1: } 5.8 \pm 4.6 \\
\text { G2: } 7.3 \pm 4.8 \\
\text { G1 vs G2, p<0.001 } \\
\text { ES=0.8 } \\
\text { ABC-Hyperactivity } \\
\text { G1: } 17 \pm 9.7\end{array}$ \\
\hline
\end{tabular}




\begin{tabular}{|c|c|c|c|}
\hline $\begin{array}{c}\text { Author, Year } \\
\text { Study Design } \\
\text { Groups (Dose), N Enrollment I } \\
\text { N Final } \\
\\
\text { Treatment Duration/Follow- } \\
\text { Up Time Point Post- } \\
\text { Treatment } \\
\text { Risk Of Bias }\end{array}$ & $\begin{array}{l}\text { Mean Age, } \\
\text { Years } \pm \text { SD } \\
\text { Mean IQ } \pm \text { SD }\end{array}$ & $\begin{array}{c}\text { Outcome } \\
\text { Measure/Baseline } \\
\text { Scores, Mean } \pm \text { SD }\end{array}$ & $\begin{array}{c}\text { Outcome } \\
\text { Measure/Post- } \\
\text { Treatment Scores, } \\
\text { Mean } \pm \text { SD }\end{array}$ \\
\hline & & $\begin{array}{l}\text { CGI-S - Moderate } \\
\text { G1: } 9 \text { (18) } \\
\text { G2: } 9 \text { (18) } \\
\text { CGI-S - Marked } \\
\text { G1: } 27 \text { (55) } \\
\text { G2: } 28 \text { (57) } \\
\text { CGI-S - Severe } \\
\text { G1: } 12(24) \\
\text { G2: } 12(24) \\
\text { CGI-S - Extreme } \\
\text { G1: } 1(2) \\
\text { G2: } 0(0)\end{array}$ & $\begin{array}{l}\text { G2: } 27.6 \pm 10.6 \\
\text { G1 vs } G 2, p<0.001 \\
\\
\text { ABC-Inappropriate } \\
\text { Speech } \\
\text { G1: } 3 \pm 3.1 \\
\text { G2: } 5.9 \pm 3.8 \\
\text { G1 vs G2, } p=0.03 \text {. } \\
\text { ES=0.3 } \\
\text { CGI-I - Much } \\
\text { Improved or very much } \\
\text { improved + } 25 \% \\
\text { reduction on ABI-I } \\
\text { G1: } 34 \text { (69) } \\
\text { G2: } 6 \text { (12) }\end{array}$ \\
\hline $\begin{array}{l}\text { Nagaraj et al., } 2006^{16} \\
\text { RCT } \\
\text { G1: Risperidone (0.5-1.0 mg/day), } \\
\text { 19/19 } \\
\text { G2: Placebo (NA), 20/20 } \\
6 \text { months/EOT } \\
\text { Moderate RoB }\end{array}$ & $\begin{array}{l}\text { Age, months } \\
\text { G1: } 57.95 \pm \\
\text { 20.84 } \\
\text { G2: } 63 \pm 20.12 \\
\text { IQ - Borderline } \\
\text { IQ } \\
\text { G1: } 9 \text { (47.7) } \\
\text { G2: } 8 \text { (40) } \\
\text { IQ - Mild } \\
\text { Retardation } \\
\text { G1: } 6 \text { (31.6) } \\
\text { G2: } 5 \text { (25) } \\
\text { IQ - Moderate } \\
\text { Retardation } \\
\text { G1: } 4 \text { (21.1) } \\
\text { G2: } 7 \text { (35) }\end{array}$ & $\begin{array}{l}\text { CARS, median } \\
\text { G1: } 39.5(32.5-46) \\
\text { G2: } 38.5(31.5-43) \\
\text { CGAS } \\
\text { G1: } 29.79 \pm 7.27 \\
\text { G2: } 32.65 \pm 7.95\end{array}$ & $\begin{array}{l}\text { EOT } \\
\text { CARS, median } \\
\text { G1: } 32(24.5-40.5) \\
\text { G2: } 37.5(30-42.5) \\
\text { G1 vs G2, } p<0.001 \\
\text { CGAS } \\
\text { G1: } 40.94 \pm 7.83 \\
\text { G2: } 35.2 \pm 9.38 \\
\text { G1 vs G2, } p=0.035 \\
\text { CARS ( } \geq 25 \% \\
\text { Improvement from } \\
\text { Baseline) } \\
\text { G1: } 12 \text { (63.2) } \\
\text { G2: } 0 \text { (0) } \\
\text { CGAS ( } \geq 25 \% \\
\text { Improvement from } \\
\text { Baseline) } \\
\text { G1: } 17 \text { (89.5) } \\
\text { G2: } 2 \text { (10) } \\
\text { Global Impression of } \\
\text { Parents - } \\
\text { Improvement to Some } \\
\text { Extent } \\
\text { G1: } 9 \text { (47.4) } \\
\text { G2: } 6 \text { (30) } \\
\text { Global Impression of } \\
\text { Parents - } \\
\text { Considerably Improved } \\
\text { G1: } 9 \text { (47.4) }\end{array}$ \\
\hline
\end{tabular}




\begin{tabular}{|c|c|c|c|}
\hline $\begin{array}{c}\text { Author, Year } \\
\text { Study Design } \\
\text { Groups (Dose), N Enrollment I } \\
\text { N Final } \\
\\
\text { Treatment Duration/Follow- } \\
\text { Up Time Point Post- } \\
\text { Treatment } \\
\text { Risk Of Bias }\end{array}$ & $\begin{array}{c}\text { Mean Age, } \\
\text { Years } \pm \text { SD } \\
\text { Mean IQ } \pm S D\end{array}$ & $\begin{array}{c}\text { Outcome } \\
\text { Measure/Baseline } \\
\text { Scores, Mean } \pm S D\end{array}$ & $\begin{array}{c}\text { Outcome } \\
\text { Measure/Post- } \\
\text { Treatment Scores, } \\
\text { Mean } \pm \text { SD }\end{array}$ \\
\hline & & & $\begin{array}{l}\text { G2: } 0(0) \\
\text { Global Impression of } \\
\text { Parents - Worsened } \\
\text { G1: } 1(5.3) \\
\text { G2: } 4(20) \\
\text { Global Impression of } \\
\text { Parents - No Change } \\
\text { G1: } 0(0) \\
\text { G2: } 9(45)\end{array}$ \\
\hline $\begin{array}{l}\text { Miral et al., } 2008^{17} \\
\text { RCT } \\
\text { G1: Risperidone (1.2-4.0 mg/day), } \\
\text { 15/13 } \\
\text { G2: Haloperidol (1.0-5.7 mg/day), } \\
\text { 15/15 } \\
12 \text { weeks/EOT } \\
\text { Moderate ROB }\end{array}$ & $\begin{array}{l}\text { Age } \\
\text { G1: } 10 \pm 2.7 \\
\text { G2: } 10.9 \pm 2.9 \\
\text { IQ } \\
\text { NR }\end{array}$ & $\begin{array}{l}\text { CGI-S-Mildly III } \\
\text { G1: } 0 \text { (0) } \\
\text { G2: } 1 \text { (6.7) } \\
\text { CGI-S-Moderately III } \\
\text { G1: } 3 \text { (20) } \\
\text { G2: } 2 \text { (13.3) } \\
\text { CGI-S-Markedly III } \\
\text { G1: } 4 \text { (26.7) } \\
\text { G2: } 7 \text { (46.7) } \\
\text { CGI-S-Severely III } \\
\text { G1: } 5 \text { (33.3) } \\
\text { G2: } 4 \text { (26.7) } \\
\text { CGI-S-Very Severely } \\
\text { III } \\
\text { G1: } 1 \text { (6.7) } \\
\text { G2: } 1 \text { (6.7) } \\
\text { ABC-Total Score } \\
\text { G1: } 85.6 \pm 27.3 \\
\text { G2: } 67.1 \pm 25.1 \\
\text { Ritvo-Freeman Real } \\
\text { Life Rating Scales- } \\
\text { Social } \\
\text { G1: 0.62 } \pm 0.5 \\
\text { G2: } 0.5 \pm 0.41 \\
\text { Ritvo-Freeman Real } \\
\text { Life Rating Scales- } \\
\text { Sensory Motor } \\
\text { G1: } 0.9 \pm 0.52 \\
\text { G2: } 0.69 \pm 0.47 \\
\text { Ritvo-Freeman Real } \\
\text { Life Rating Scales- }\end{array}$ & $\begin{array}{l}\text { CGI-I-Marked } \\
\text { Improvement } \\
\text { G1: } 2(15.4) \\
\text { G2: } 0(0) \\
\text { G1 vs } \mathrm{G} 2, \mathrm{p}=\mathrm{ns} \\
\text { CGI-I-Moderate } \\
\text { Improvement } \\
\text { G1: } 9 \text { (69.2) } \\
\text { G2: } 9 \text { (60) } \\
\text { G1 vs G2, p=ns } \\
\text { CGI-I-Slight } \\
\text { Improvement } \\
\text { G1: } 2(15.4) \\
\text { G2: } 5 \text { (33.3) } \\
\text { G1 vs G2, p=ns } \\
\text { CGI-I-No Change } \\
\text { G1: } 0 \text { (0) } \\
\text { G2: } 1(6.7) \\
\text { CGI degree of } \\
\text { improvement } \\
\text { G1 vs G2, p=ns } \\
\text { ABC-Total Score } \\
\text { G1: } 36.8 \pm 13.8 \\
\text { G2: } 45.8 \pm 20.2 \\
\text { G1 vs G2, p=0.0063 } \\
\text { Ritvo-Freeman Real } \\
\text { Life Rating Scales- } \\
\text { Social } \\
\text { G1: }-0.11 \pm 0.38 \\
\text { G2: } 0.02 \pm 0.57 \\
\text { G1 vs G2, p=ns } \\
\text { Ritvo-Freeman Real }\end{array}$ \\
\hline
\end{tabular}




\begin{tabular}{|c|c|c|c|}
\hline $\begin{array}{c}\text { Author, Year } \\
\text { Study Design } \\
\text { Groups (Dose), N Enrollment I } \\
\text { N Final } \\
\\
\text { Treatment Duration/Follow- } \\
\text { Up Time Point Post- } \\
\text { Treatment } \\
\text { Risk Of Bias }\end{array}$ & $\begin{array}{l}\text { Mean Age, } \\
\text { Years } \pm \text { SD } \\
\text { Mean IQ } \pm \text { SD }\end{array}$ & $\begin{array}{c}\text { Outcome } \\
\text { Measure/Baseline } \\
\text { Scores, Mean } \pm \text { SD }\end{array}$ & $\begin{array}{c}\text { Outcome } \\
\text { Measure/Post- } \\
\text { Treatment Scores, } \\
\text { Mean } \pm \text { SD }\end{array}$ \\
\hline & & $\begin{array}{l}\text { Affect } \\
\text { G1: } 1.09 \pm 0.41 \\
\text { G2: } 1.05 \pm 0.61 \\
\text { Ritvo-Freeman Real } \\
\text { Life Rating Scales- } \\
\text { Sensory } \\
\text { G1: } 0.98 \pm 0.46 \\
\text { G2: } 0.860 .44 \\
\text { Ritvo-Freeman Real } \\
\text { Life Rating Scales- } \\
\text { Language } \\
\text { G1: } 0.52 \pm 0.37 \\
\text { G2: } 0.15 \pm 0.44\end{array}$ & $\begin{array}{l}\text { Life Rating Scales- } \\
\text { Sensory Motor } \\
\text { G1: } 0.36 \pm 0.34 \\
\text { G2: } 0.5 \pm 0.44 \\
\text { G1 vs } G 2, p=n s \\
\text { Ritvo-Freeman Real } \\
\text { Life Rating Scales- } \\
\text { Affect } \\
\text { G1: } 0.54 \pm 0.34 \\
\text { G2: } 0.64 \pm 0.48 \\
\text { G1 vs G2, } p=n s \\
\text { Ritvo-Freeman Real } \\
\text { Life Rating Scales- } \\
\text { Sensory } \\
\text { G1: } 0.51 \pm 0.25 \\
\text { G2: } 0.58 \pm 0.49 \\
\text { G1 vs G2, p=ns } \\
\text { Ritvo-Freeman Real } \\
\text { Life Rating Scales- } \\
\text { Language } \\
\text { G1: } 0.04 \pm 0.25 \\
\text { G2: }-0.05 \pm 0.5 \\
\text { G1 vs G2, p=ns }\end{array}$ \\
\hline $\begin{array}{l}\text { Troost et al., } 2005^{18} \\
\text { RCT } \\
\text { G1: Risperidone (0.5-3.5 mg/day), } \\
\text { 12/12 } \\
\text { G2: Placebo (ND), 12/12 } \\
8 \text { weeks/EOT } \\
\text { Low ROB }\end{array}$ & $\begin{array}{l}\text { Age } \\
\text { G1: } 9.4 \pm 3.4 \\
\text { G2: } 8.7 \pm 1.2 \\
\text { Average or } \\
\text { above-average } \\
\text { IQ } \\
\text { G1: } 6 \text { (50) } \\
\text { G2: } 9 \text { (75) } \\
\text { Borderline IQ } \\
\text { G1: } 4 \text { (33) } \\
\text { G2: } 3 \text { (25) } \\
\text { Mild or } \\
\text { Moderate } \\
\text { Retardation } \\
\text { G1: } 2(17) \\
\text { G2: } 0 \text { (0) }\end{array}$ & $\begin{array}{l}\text { ABC-Irritability } \\
\text { G1: } 11.1 \pm 8.1 \\
\text { G2: } 12.7 \pm 7.7 \\
\text { ABC-Social } \\
\text { Withdrawal/Lethargy } \\
\text { G1: } 5 \pm 6 \\
\text { G2: } 6.7 \pm 6.9 \\
\text { ABC-Stereotypic } \\
\text { Behavior } \\
\text { G1: } 2.3 \pm 3.2 \\
\text { G2: } 4.7 \pm 4.3 \\
\text { ABC-Hyperactivity } \\
\text { G1: } 16.8 \pm 11.5 \\
\text { G2: } 15.8 \pm 9.4 \\
\text { ABC-Inappropriate } \\
\text { Speech } \\
\text { G1: } 3.2 \pm 3.2 \\
\text { G2: } 2.3 \pm 1.9\end{array}$ & $\begin{array}{l}\text { CGI-Minimally } \\
\text { Improved } \\
\text { G1: } 5 \text { (42) } \\
\text { G2: } 3(25) \\
\text { CGI-Much } \\
\text { Improvement } \\
\text { G1: } 3(25) \\
\text { G2: } 6 \text { (50) } \\
\text { CGI-Very Much } \\
\text { Improved } \\
\text { G1: } 4 \text { (33) } \\
\text { G2: } 3 \text { (25) } \\
\text { ABC-Irritability } \\
\text { G1: } 12.6 \pm 9.8 \\
\text { G2: } 20.3 \pm 10.2 \\
\text { G1 vs G2, p=0.043 } \\
\text { ABC-Social } \\
\text { Withdrawal/Lethargy } \\
\text { G1: } 2.8 \pm 3.1 \\
\text { G2: } 4.8 \pm 3.5\end{array}$ \\
\hline
\end{tabular}




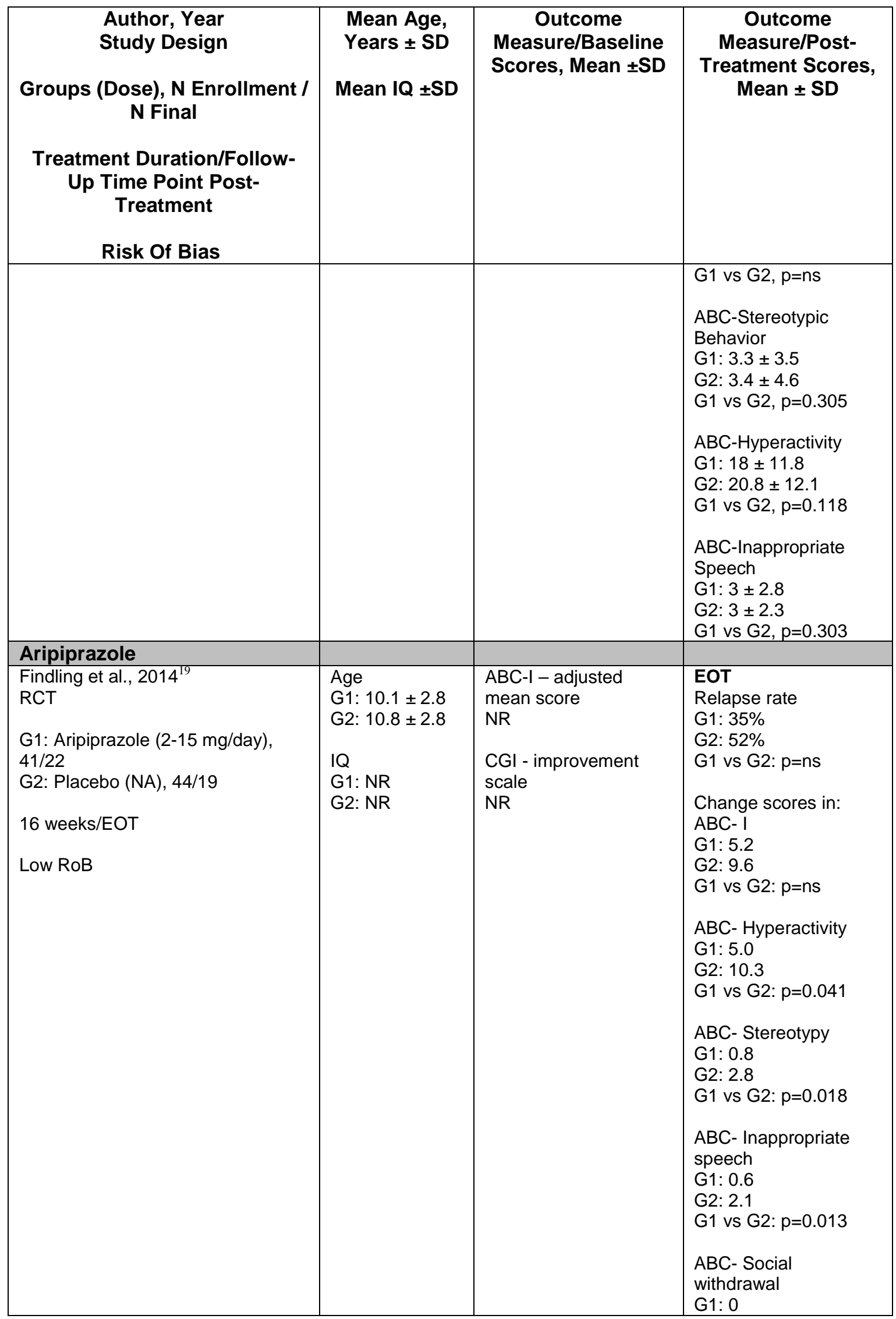




\begin{tabular}{|c|c|c|c|}
\hline $\begin{array}{c}\text { Author, Year } \\
\text { Study Design } \\
\text { Groups (Dose), N Enrollment I } \\
\text { N Final } \\
\\
\text { Treatment Duration/Follow- } \\
\text { Up Time Point Post- } \\
\text { Treatment } \\
\text { Risk Of Bias }\end{array}$ & $\begin{array}{l}\text { Mean Age, } \\
\text { Years } \pm \text { SD } \\
\text { Mean IQ } \pm S D\end{array}$ & $\begin{array}{c}\text { Outcome } \\
\text { Measure/Baseline } \\
\text { Scores, Mean } \pm S D\end{array}$ & $\begin{array}{c}\text { Outcome } \\
\text { Measure/Post- } \\
\text { Treatment Scores, } \\
\text { Mean } \pm \text { SD }\end{array}$ \\
\hline & & & $\begin{array}{l}\text { G2: } 1.5 \\
\text { G1 vs } G 2: p=n s \\
\text { CG1- I } \\
\text { G1: } 4.2 \\
\text { G2: } 4.8 \\
\text { G1 vs } G 2: p=n s\end{array}$ \\
\hline $\begin{array}{l}\text { Ghanizadeh et al., } 2014^{20} \\
\text { RCT } \\
\text { G1: Aripiprazole (1.25-10 mg/day), } \\
\text { 29/29 } \\
\text { G2: Risperidone (0.25-3 mg/day), } \\
\text { 30/30 } \\
2 \text { months/EOT } \\
\text { Low RoB }\end{array}$ & $\begin{array}{l}\text { Age } \\
\text { G1: } 9.6 \pm 3.3 \\
\text { G2: } 9.5 \pm 4.6 \\
\text { IQ } \\
\text { NR }\end{array}$ & $\begin{array}{l}\text { ABC-Irritability } \\
\text { G1: } 26.2 \pm 4.1 \\
\text { G2: } 21.5 \pm 7.4 \\
\text { ABC-Hyperactivity } \\
\text { G1: } 37.1 \pm 7 \\
\text { G2: } 36 \pm 6.2 \\
\text { ABC-Lethargy } \\
\text { G1: } 27.5 \pm 8.4 \\
\text { G2: } 25.3 \pm 8.9 \\
\text { ABC-Stereotypy } \\
\text { G1: } 13.6 \pm 5.7 \\
\text { G2: } 13.2 \pm 4.2 \\
\text { ABC-Speech } \\
\text { G1: } 8.6 \pm 3.1 \\
\text { G2: } 8.9 \pm 3.6\end{array}$ & $\begin{array}{l}\text { EOT } \\
\text { ABC-Irritability } \\
\text { G1: } 14.6 \pm 5.5 \\
\text { G2: } 12.5 \pm 5.4 \\
\text { G1 vs } \mathrm{G} 2, \mathrm{p}=\mathrm{ns} \\
\text { ABC-Hyperactivity } \\
\text { G1: } 21.1 \pm 9 \\
\text { G2: } 19.1 \pm 6.1 \\
\text { G1 vs } \mathrm{G} 2, \mathrm{p}=\mathrm{ns} \\
\text { ABC-Lethargy } \\
\text { G1: } 17.3 \pm 7.4 \\
\text { G2: } 16.1 \pm 6.9 \\
\mathrm{G} 1 \text { vs } \mathrm{G} 2, \mathrm{p}=\mathrm{ns} \\
\text { ABC-Stereotypy } \\
\text { G1: } 8.2 \pm 5 \\
\text { G2: } 7.4 \pm 3.9 \\
\text { G1 vs } \mathrm{G} 2, \mathrm{p}=\mathrm{ns} \\
\text { ABC-Speech } \\
\text { G1: } 4.9 \pm 2.3 \\
\text { G2: } 5.7 \pm 3.1 \\
\text { G1 vs } \mathrm{G} 2, \mathrm{p}=\mathrm{ns} \\
\text { CGI-Improvement, } \mathrm{n} \\
\text { Much improved } \\
\text { G1: } 9 \\
\text { G2: } 5 \\
\text { Minimally improved } \\
\text { G1: } 7 \\
\text { G2: } 12 \\
\text { No change } \\
\text { G1: } 5 \\
\text { G2: } 8 \\
\text { Minimally worse } \\
\text { G1: } 3\end{array}$ \\
\hline
\end{tabular}




\begin{tabular}{|c|c|c|c|}
\hline $\begin{array}{c}\text { Author, Year } \\
\text { Study Design } \\
\text { Groups (Dose), N Enrollment I } \\
\text { N Final } \\
\text { Treatment Duration/Follow- } \\
\text { Up Time Point Post- } \\
\text { Treatment } \\
\text { Risk Of Bias }\end{array}$ & $\begin{array}{l}\text { Mean Age, } \\
\text { Years } \pm \text { SD } \\
\text { Mean IQ } \pm S D\end{array}$ & $\begin{array}{c}\text { Outcome } \\
\text { Measure/Baseline } \\
\text { Scores, Mean } \pm \text { SD }\end{array}$ & $\begin{array}{c}\text { Outcome } \\
\text { Measure/Post- } \\
\text { Treatment Scores, } \\
\text { Mean } \pm \text { SD }\end{array}$ \\
\hline & & & $\begin{array}{l}\text { G2: } 2 \\
\text { G1 vs G2: } p=n s\end{array}$ \\
\hline 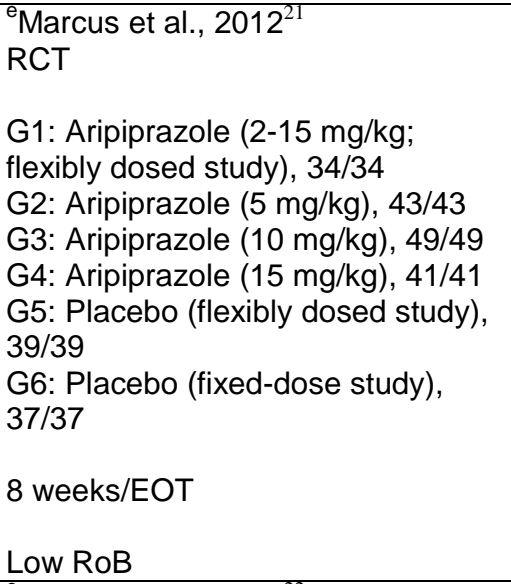 & $\begin{array}{l}\text { Age } \\
\text { G1: } 9.5 \pm 3.1 \\
\text { G2: } 9.4 \pm 3 \\
\text { IQ } \\
\text { NR }\end{array}$ & $\begin{array}{l}\text { Pediatric QoL- } \\
\text { Combined Scales } \\
\text { G1: } 45 \pm 2.9 \\
\text { G2: } 41.5 \pm 2.3 \\
\text { G3: } 43.4 \pm 2.1 \\
\text { G4: } 38.3 \pm 2.3 \\
\text { G5: } 38.1 \pm 2.7 \\
\text { G6: } 42.8 \pm 2.5\end{array}$ & $\begin{array}{l}\text { EOT } \\
\text { Pediatric QoL- } \\
\text { Combined Scales } \\
\text { G1: } 13.4 \pm 1.9 \\
\text { G2: } 14 \pm 2.4 \\
\text { G3: } 10.4 \pm 2.2 \\
\text { G4: } 18.7 \pm 2.4 \\
\text { G5: } 2 \pm 1.8 \\
\text { G6:10.6 } \pm 2.6\end{array}$ \\
\hline $\begin{array}{l}{ }^{\mathrm{e}} \text { Marcus et al., } 2011^{22} \\
\text { RCT } \\
\text { G1: De Novo Subjects }(2-15 \\
\mathrm{mg} / \mathrm{kg}), 84 / 55 \\
\text { G2: Prior Placebo }(2-15 \mathrm{mg} / \mathrm{kg}) \text {, } \\
\text { 69/37 } \\
\text { G3: Prior Aripiprazole }(2-15 \mathrm{mg} / \mathrm{kg}) \text {, } \\
\text { 169/107 } \\
52 \text { weeks } \\
\text { Low RoB }\end{array}$ & $\begin{array}{l}\text { Age } \\
9.6 \text { (mean) } \\
\text { IQ } \\
\text { NR }\end{array}$ & $\begin{array}{l}\text { CGI-Severity } \\
\text { G1: } 4.8 \pm 1 \\
\text { G2: } 4.2 \pm 1 \\
\text { G3: } 3.9 \pm 1.1 \\
\text { ABC-Irritability } \\
\text { G1: } 23.2 \pm 8.9 \\
\text { G2: } 21.5 \pm 9.8 \\
\text { G3: } 15 \pm 9.2 \\
\text { ABC-Lethargy/Social } \\
\text { Withdrawal } \\
\text { G1: } 14.6 \pm 8.6 \\
\text { G2: } 11.3 \pm 9.2 \\
\text { G3: } 10.4 \pm 8.9 \\
\text { ABC-Stereotypic } \\
\text { Behavior } \\
\text { G1: } 8.1 \pm 5.2 \\
\text { G2: } 9.1 \pm 5.6 \\
\text { G3: } 6.4 \pm 5.5 \\
\text { ABC-Hyperactivity } \\
\text { G1: } 28.4 \pm 10.9 \\
\text { G2: } 25.8 \pm 13.2\end{array}$ & $\begin{array}{l}\text { EOT } \\
\text { CGI-Severity } \\
\text { G1: }-1 \pm 0.8 \\
\text { G2: }-0.6 \pm 1.2 \\
\text { G3: }-0.1 \pm 1 \\
\text { ABC-Irritability } \\
\text { G1: }-8 \pm 10.1 \\
\text { G2: }-6.1 \pm 1.9 \\
\text { G3: } 0.7 \pm 10.2 \\
\text { ABC-Lethargy/Social } \\
\text { Withdrawal } \\
\text { G1: }-6.4 \pm 7.9 \\
\text { G2: }-4.1 \pm 7.2 \\
\text { G3: }-2.3 \pm 6.4 \\
\text { ABC-Stereotypic } \\
\text { Behavior } \\
\text { G1: }-2.7 \pm 3.1 \\
\text { G2: }-1.9 \pm 4.1 \\
\text { G3: }-0.5 \pm 4.4 \\
\text { ABC-Hyperactivity } \\
\text { G1: }-12.3 \pm 8.5\end{array}$ \\
\hline
\end{tabular}




\begin{tabular}{|c|c|c|c|}
\hline $\begin{array}{c}\text { Author, Year } \\
\text { Study Design } \\
\text { Groups (Dose), N Enrollment I } \\
\text { N Final } \\
\\
\text { Treatment Duration/Follow- } \\
\text { Up Time Point Post- } \\
\text { Treatment } \\
\text { Risk Of Bias }\end{array}$ & $\begin{array}{l}\text { Mean Age, } \\
\text { Years } \pm \text { SD } \\
\text { Mean IQ } \pm S D\end{array}$ & $\begin{array}{c}\text { Outcome } \\
\text { Measure/Baseline } \\
\text { Scores, Mean } \pm S D\end{array}$ & $\begin{array}{c}\text { Outcome } \\
\text { Measure/Post- } \\
\text { Treatment Scores, } \\
\text { Mean } \pm \text { SD }\end{array}$ \\
\hline & & $\begin{array}{l}\text { G3: } 18.4 \pm 12 \\
\text { ABC-Inappropriate } \\
\text { Speech } \\
\text { G1: } 5.8 \pm 3.2 \\
\text { G2: } 5.7 \pm 4.2 \\
\text { G3: } 4.2 \pm 3.6 \\
\text { CYBOCS } \\
\text { G1: } 12.6 \pm 4.6 \\
\text { G2: } 12.1 \pm 4 \\
\text { G3: } 10.4 \pm 3.9\end{array}$ & $\begin{array}{l}\text { G2: }-9.1 \pm 11.5 \\
\text { G3: } 0.6 \pm 10.3 \\
\text { ABC-Inappropriate } \\
\text { Speech } \\
\text { G1: }-2 \pm 2.5 \\
\text { G2: }-1.8 \pm 3 \\
\text { G3: }-0.3 \pm 2.4 \\
\text { CYBOCS } \\
\text { G1: }-2.8 \pm 3.5 \\
\text { G2: }-2.6 \pm 5.4 \\
\text { G3: } 0.2 \pm 4.1\end{array}$ \\
\hline $\begin{array}{l}{ }^{\mathrm{e}} \text { Marcus et al., } 2010^{23} \\
\text { RCT } \\
\text { G1: Aripiprazole (2-15 mg/kg; } \\
\text { flexibly dosed study), } 46 / 46 \\
\text { G2: Aripiprazole }(5 \mathrm{mg} / \mathrm{kg}), 52 / 52 \\
\text { G3: Aripiprazole }(10 \mathrm{mg} / \mathrm{kg}), 59 / 59 \\
\text { G4: Aripiprazole }(15 \mathrm{mg} / \mathrm{kg}), 53 / 53 \\
\text { G5: Placebo (flexibly dosed study) } \\
\text { 49/49 } \\
\text { G6: Placebo (fixed-dose study) } \\
\text { 49/49 } \\
\text { 8 weeks/EOT } \\
\text { Low RoB }\end{array}$ & $\begin{array}{l}\text { Age } \\
\text { Flexibly dosed } \\
\text { study: } 9.3 \pm 2.9 \\
\text { Fixed dose } \\
\text { study: } 9.7 \pm 3.1 \\
\text { IQ } \\
\text { NR }\end{array}$ & $\begin{array}{l}\text { ABC-Irritability } \\
\text { G1: } 29.6 \pm 1 \\
\text { G2: } 28.3 \pm 1 \\
\text { G3: } 27.6 \pm 0.9 \\
\text { G4: } 28.3 \pm 1 \\
\text { G5: } 30.8 \pm 1 \\
\text { G6: } 26.9 \pm 1 \\
\text { ABC-Social } \\
\text { Withdrawal/Lethargy } \\
\text { G1: } 19.9 \pm 1.6 \\
\text { G2: } 17.7 \pm 1.4 \\
\text { G3: } 16.8 \pm 1.3 \\
\text { G4: } 18.9 \pm 1.4 \\
\text { G5: } 18.1 \pm 1.6 \\
\text { G6: } 18 \pm 1.5 \\
\text { ABC-Stereotypic } \\
\text { Behavior } \\
\text { G1: } 11.9 \pm 0.9 \\
\text { G2: } 11.4 \pm 0.8 \\
\text { G3: } 11.6 \pm 0.8 \\
\text { G4: } 10.7 \pm 0.9 \\
\text { G5: } 10.7 \pm 0.8 \\
\text { G6: } 10.7 \pm 0.8 \\
\text { ABC-Hyperactivity } \\
\text { G1: } 34.1 \pm 1.4 \\
\text { G2: } 33.1 \pm 1.4 \\
\text { G3: } 33.7 \pm 1.3 \\
\text { G4: } 32.2 \pm 1.4 \\
\text { G5: } 34.7 \pm 1.4 \\
\text { G6: } 311 . \pm 4 \\
\text { ABC-Inappropriate } \\
\text { Speech } \\
\text { G1: } 7 \pm 0.6\end{array}$ & $\begin{array}{l}\text { Change score } \\
\text { EOT } \\
\text { ABC-Irritability } \\
\text { G1: }-12.9 \pm 1.4 \\
\text { G2: }-12.4 \pm 1.4 \\
\text { G3: }-13.2 \pm 1.3 \\
\text { G4: }-14.4 \pm 1 \\
\text { G5: }-5 \pm 1.4 \\
\text { G6: }-8.4 \pm 1.4 \\
\text { G1 vs G6, } p<0.05 \\
\text { G2 vs G6, } p<0.05 \\
\text { G3 vs G6, } p<0.05 \\
\text { G4 vs G6, p<0.05 } \\
\text { ABC-Social } \\
\text { Withdrawal/Lethargy } \\
\text { G1: }-7.9 \pm 1.2 \\
\text { G2: }-5.8 \pm 1.2 \\
\text { G3: }-4.9 \pm 1.1 \\
\text { G4: }-7.9 \pm 1.1 \\
\text { G5: }-62 \pm 1.1 \\
\text { G6: }-5.2 \pm 1.2 \\
\text { G5 vs G6, p=ns } \\
\text { ABC-Stereotypic } \\
\text { Behavior } \\
\text { G1: }-4.8 \pm 0.6 \\
\text { G2: }-4.5 \pm 0.7 \\
\text { G3: }-4.2 \pm 0.6 \\
\text { G4: }-4.5 \pm 0.7 \\
\text { G5: }-2 \pm 0.6 \\
\text { G6: }-1.8 \pm 0.7 \\
\text { G1 vs G6, }<<0.05 \\
\text { G2 vs G6, } p<0.05 \\
\text { G3 vs G6, } p<0.05 \\
\text { G4 vs G6, } p<0.05\end{array}$ \\
\hline
\end{tabular}




\begin{tabular}{|c|c|c|c|}
\hline $\begin{array}{c}\text { Author, Year } \\
\text { Study Design } \\
\text { Groups (Dose), N Enrollment I } \\
\text { N Final } \\
\\
\text { Treatment Duration/Follow- } \\
\text { Up Time Point Post- } \\
\text { Treatment } \\
\text { Risk Of Bias }\end{array}$ & $\begin{array}{l}\text { Mean Age, } \\
\text { Years } \pm \text { SD } \\
\text { Mean IQ } \pm \text { SD }\end{array}$ & $\begin{array}{c}\text { Outcome } \\
\text { Measure/Baseline } \\
\text { Scores, Mean } \pm \text { SD }\end{array}$ & $\begin{array}{c}\text { Outcome } \\
\text { Measure/Post- } \\
\text { Treatment Scores, } \\
\text { Mean } \pm \text { SD }\end{array}$ \\
\hline & & $\begin{array}{l}\text { G2: } 5.8 \pm 0.6 \\
\text { G3: } 6.8 \pm 0.5 \\
\text { G4: } 6.3 \pm 0.5 \\
\text { G5: } 7 \pm 0.6 \\
\text { G6: } 5.9 \pm 0.6\end{array}$ & $\begin{array}{l}\text { ABC-Hyperactivity } \\
\text { G1: } 12.7 \pm 1.5 \\
\text { G2: }-14 \pm 1.6 \\
\text { G3: }-13.3 \pm 1.5 \\
\text { G4: }-16.3 \pm 1.6 \\
\text { G5: }-2.8 \pm 1.5 \\
\text { G6: }-7.7 \pm 1.7 \\
\text { G1 vs G6, p<0.05 } \\
\text { G2 vs G6, p<0.05 } \\
\text { G3 vs G6, p<0.05 } \\
\text { G4 vs G6, p<0.05 } \\
\text { ABC-Inappropriate } \\
\text { Speech } \\
\text { G1: }-2.5 \pm 0.4 \\
\text { G2: }-2 \pm 0.5 \\
\text { G3: }-1.8 \pm 0.4 \\
\text { G4: }-2.3 \pm 0.4 \\
\text { G5: }-0.4 \pm 0.4 \\
\text { G6: }-1.1 \pm 5 \\
\text { G4 vs } \mathrm{G} 6, p<0.05\end{array}$ \\
\hline $\begin{array}{l}{ }^{\mathrm{e}} \text { Marcus et al., } 2009^{24} \\
\text { RCT } \\
\text { G1: Aripiprazole }(2-15 \mathrm{mg} / \mathrm{kg}) \text {, } \\
\text { 47/39 } \\
\text { G2: Placebo (NA), 51/36 } \\
8 \text { weeks/EOT } \\
\text { Low RoB }\end{array}$ & $\begin{array}{l}\text { Age } \\
\text { G1: } 9.7 \pm 3.2 \\
\text { G2: } 8.8 \pm 2.6 \\
\text { IQ } \\
\text { NR }\end{array}$ & $\begin{array}{l}\text { ABC-Irritability } \\
\text { G1: } 29.6 \pm 6.4 \\
\text { G2: } 30.2 \pm 6.5 \\
\text { ABC- } \\
\text { Hyperactivity/Noncomp } \\
\text { liance } \\
\text { G1: } 34.1 \\
\text { G2: } 34.7 \\
\text { ABC-Stereotypic } \\
\text { Behavior } \\
\text { G1: } 11.9 \\
\text { G2: } 10.7 \\
\text { ABC-Inappropriate } \\
\text { Speech } \\
\text { G1: } 7 \\
\text { G2: } 7 \\
\text { CYBOCS } \\
\text { G1: } 12.8 \\
\text { G2: } 13.7\end{array}$ & $\begin{array}{l}\text { Change Score } \\
\text { EOT } \\
\text { ABC-Irritability } \\
\text { G1: }-12.9 \\
\text { G2: }-5 \\
\text { G1 vs } G 2, p<0.001 \\
\text { ABC- } \\
\text { Hyperactivity/Noncomp } \\
\text { liance } \\
\text { G1: }-12.7 \\
\text { G2: }-2.8 \\
\text { G1 vs G2, p<0.001 } \\
\text { ABC-Stereotypic } \\
\text { Behavior } \\
\text { G1: }-4.8 \\
\text { G2: }-2 \\
\text { G1 vs G2, p<0.001 } \\
\text { ABC-Inappropriate } \\
\text { Speech } \\
\text { G1: }-2.5 \\
\text { G2: }-0.4 \\
\text { G1 vs } G 2, p<0.001 \\
\text { CYBOCS } \\
\text { G1: }-3.8 \\
\text { G2: }-0.8\end{array}$ \\
\hline
\end{tabular}




\begin{tabular}{|c|c|c|c|}
\hline $\begin{array}{c}\text { Author, Year } \\
\text { Study Design } \\
\text { Groups (Dose), N Enrollment I } \\
\text { N Final } \\
\\
\text { Treatment Duration/Follow- } \\
\text { Up Time Point Post- } \\
\text { Treatment } \\
\text { Risk Of Bias }\end{array}$ & $\begin{array}{l}\text { Mean Age, } \\
\text { Years } \pm \text { SD } \\
\text { Mean IQ } \pm S D\end{array}$ & $\begin{array}{c}\text { Outcome } \\
\text { Measure/Baseline } \\
\text { Scores, Mean } \pm \text { SD }\end{array}$ & $\begin{array}{c}\text { Outcome } \\
\text { Measure/Post- } \\
\text { Treatment Scores, } \\
\text { Mean } \pm \text { SD }\end{array}$ \\
\hline & & & $\begin{array}{l}\text { G1 vs G2, p<0.001 } \\
\text { CGI-Severity } \\
\text { G1: -1.2 } \\
\text { G2: -0.4 } \\
\text { CGI-I - Very much } \\
\text { improved or much } \\
\text { improved } \\
\text { G1: } 31 \text { (67) } \\
\text { G2: } 8 \text { (16) } \\
\text { CGI-I - Minimally } \\
\text { improved } \\
\text { G1: } 7 \text { (15) } \\
\text { G2: } 10 \text { (20) } \\
\text { CGI-I - No change } \\
\text { G1: } 6 \text { (13) } \\
\text { G2: } 22 \text { (45) } \\
\text { CGI-I - Minimally } \\
\text { worse } \\
\text { G1: } 2 \text { (4) } \\
\text { G2: } 5 \text { (10) } \\
\text { CGI-I - Much or very } \\
\text { much worse } \\
\text { G1: } 0 \text { (0) } \\
\text { G2: } 4 \text { (8) }\end{array}$ \\
\hline $\begin{array}{l}{ }^{\mathrm{d}} \text { Marcus et al., } 2009^{25} \\
\text { RCT } \\
\text { G1: Aripiprazole }(5 \mathrm{mg} / \mathrm{kg}), 53 / 44 \\
\text { G2: Aripiprazole }(10 \mathrm{mg} / \mathrm{kg}), 59 / 49 \\
\text { G3: Aripiprazole }(15 \mathrm{mg} / \mathrm{kg}), 54 / 47 \\
\text { G4: Placebo (NA), 52/38 } \\
8 \text { weeks/EOT } \\
\text { Low RoB }\end{array}$ & $\begin{array}{l}\text { Age } \\
\text { G1: } 9 \pm 2.8 \\
\text { G2: } 10 \pm 3.2 \\
\text { G3: } 9.5 \pm 3.1 \\
\text { G4: } 10.2 \pm 3.1 \\
\text { IQ } \\
\text { NR }\end{array}$ & $\begin{array}{l}\text { ABC-Irritability } \\
\text { G1: } 28.6 \pm 7.6 \\
\text { G2: } 28.2 \pm 7.4 \\
\text { G3: } 28.9 \pm 6.4 \\
\text { G4: } 28 \pm 6.9 \\
\text { ABC- } \\
\text { Hyperactivity/Noncomp } \\
\text { liance } \\
\text { G1: } 33.1 \pm 1.4 \\
\text { G2: } 33.7 \pm 1.3 \\
\text { G3: } 32.2 \pm 1.4 \\
\text { G4: } 31 \pm 1.4 \\
\text { ABC-Stereotypic } \\
\text { Behavior } \\
\text { G1: } 11.4 \pm 0.8 \\
\text { G2: } 11.6 \pm 0.8 \\
\text { G3: } 11.6 \pm 0.8 \\
\text { G4:10.7 } \pm 0.8\end{array}$ & 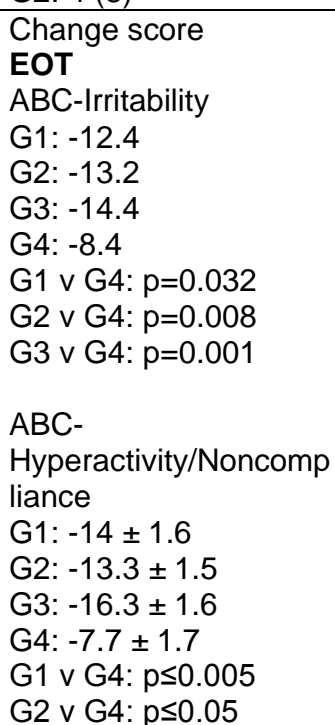 \\
\hline
\end{tabular}




\begin{tabular}{|c|c|c|c|}
\hline $\begin{array}{c}\text { Author, Year } \\
\text { Study Design } \\
\text { Groups (Dose), N Enrollment I } \\
\text { N Final } \\
\\
\text { Treatment Duration/Follow- } \\
\text { Up Time Point Post- } \\
\text { Treatment } \\
\text { Risk Of Bias }\end{array}$ & $\begin{array}{l}\text { Mean Age, } \\
\text { Years } \pm \text { SD } \\
\text { Mean IQ } \pm \text { SD }\end{array}$ & $\begin{array}{c}\text { Outcome } \\
\text { Measure/Baseline } \\
\text { Scores, Mean } \pm \text { SD }\end{array}$ & $\begin{array}{c}\text { Outcome } \\
\text { Measure/Post- } \\
\text { Treatment Scores, } \\
\text { Mean } \pm \text { SD }\end{array}$ \\
\hline & & $\begin{array}{l}\text { ABC-Social } \\
\text { Withdrawal/Lethargy } \\
\text { G1: } 17.7 \pm 1.4 \\
\text { G2: } 16.8 \pm 1.3 \\
\text { G3: } 18.9 \pm 1.4 \\
\text { G4: } 18 \pm 1.5 \\
\text { ABC-Inappropriate } \\
\text { Speech } \\
\text { G1: } 5.8 \pm 0.6 \\
\text { G2: } 6.8 \pm 0.5 \\
\text { G3: } 6.3 \pm 0.5 \\
\text { G4: } 5.9 \pm 0.6 \\
\text { CYBOCS } \\
\text { G1: } 13.9 \pm 0.6 \\
\text { G2: } 13.5 \pm 0.5 \\
\text { G3: } 14.1 \pm 05 \\
\text { G4:13.7 } \pm 0.6 \\
\text { CGI-S } \\
\text { G1: } 5 \pm 0.1 \\
\text { G2: } 4.9 \pm 0.1 \\
\text { G3: } 5.1 \pm 0.1 \\
\text { G4: } 4.7 \pm 0.1\end{array}$ & 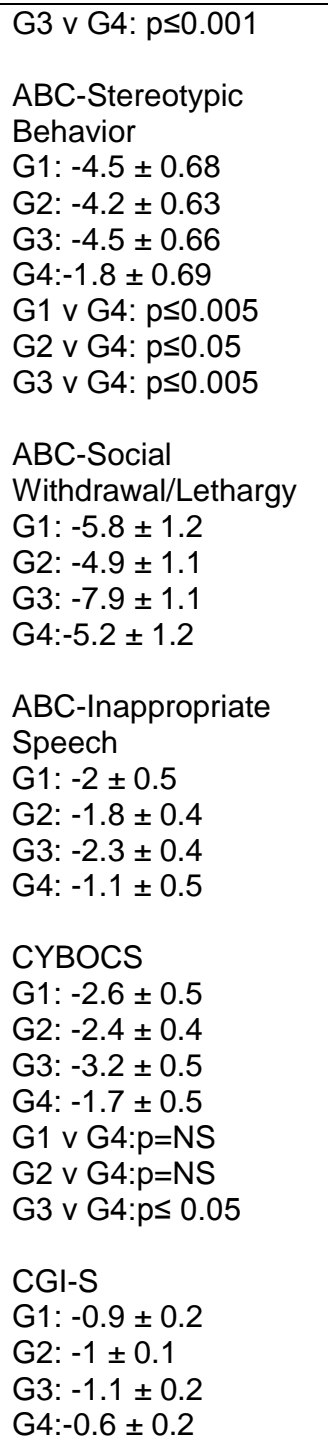 \\
\hline
\end{tabular}

${ }^{\mathrm{a}}$ Publication in a larger RCT

${ }^{\mathrm{b}}$ Publication in a larger RCT ${ }^{8,9}$

${ }^{\text {cPublication in a larger RCT }}{ }^{10,14}$

${ }^{\mathrm{d}}$ Publication in a larger RCT ${ }^{21-25,31,32}$

ABC-Aberrant Behavior Checklist; CGI-Clinical Global Impression; VABS-Vineland Adaptive Behavior Scale; CYBOCS-Children's YaleBrown Obsessive Compulsive Scale; M-RLRS-Modified Real Life Rating Scale; EOT-End of Treatment; OLE-Open-label Extension; VAS-

Visual Analog Scale; CGAS-Children’s Global Assessment Scale 
Table F-2. Key findings in studies of methylphenidate

\begin{tabular}{|c|c|c|c|}
\hline $\begin{array}{c}\text { Author, Year } \\
\text { Study Design } \\
\text { Groups (dose), N enrollment / N } \\
\text { final } \\
\text { Treatment duration/Follow-up } \\
\text { timepoint post-treatment } \\
\text { Risk of Bias }\end{array}$ & $\begin{array}{c}\text { Mean age, years } \\
\pm \text { SD } \\
\text { Mean IQ } \pm S D\end{array}$ & $\begin{array}{c}\text { Outcome } \\
\text { measure/Baseline } \\
\text { scores, mean } \pm S D\end{array}$ & $\begin{array}{c}\text { Outcome } \\
\text { measure/Post- } \\
\text { treatment } \\
\text { scores, mean } \pm \\
\text { SD }\end{array}$ \\
\hline $\begin{array}{l}\text { RUPP } 2005^{33-36} \\
\text { RCT } \\
\text { G1: Methylphenidate - low dose } \\
\text { (0.125 mg/kg), 66/45 } \\
\text { G2: Methylphenidate - medium } \\
\text { dose }(0.250 \mathrm{mg} / \mathrm{kg}), 66 / 52 \\
\text { G3: Methylphenidate - high dose } \\
\text { (0.500 } \mathrm{mg} / \mathrm{kg}), 66 / 33 \\
\text { G4: Methylphenidate - optimal dose } \\
\text { (NA), } 66 / 58 \\
\text { G5: Placebo (NA), } 66 / 46 \\
\text { 13 weeks/EOT } \\
\text { Low RoB }\end{array}$ & $\begin{array}{l}\text { Age } \\
\text { G1+G2+G3+G4+ } \\
\text { G5: } 7.5 \\
\text { IQ } \\
\text { NR }\end{array}$ & 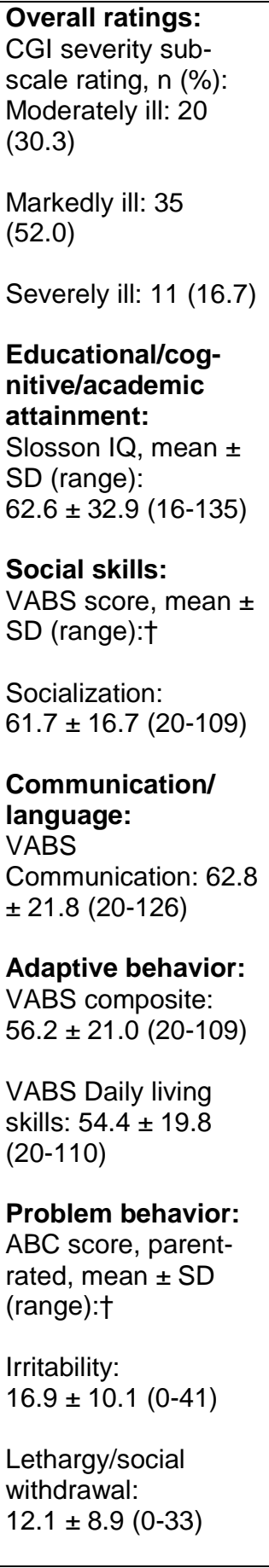 & $\begin{array}{l}\text { Overall ratings: } \\
\text { Responded to } \\
\text { treatment, sub- } \\
\text { jects completing } \\
\text { cross-over } \\
\text { phase, } \mathrm{n}(\%): \\
\text { Total: } 44 \text { (76) } \\
\text { Optimal/best } \\
\text { treatment: } \\
\text { Placebo: } 9(20) \\
\text { Low: } 11(25) \\
\text { Medium: } 14(32) \\
\text { High: } 10(23) \\
\text { Response rate, } \\
\text { by dose, } \mathrm{n}(\%): \dagger \\
\text { G1: } 20 / 61(33) \\
\text { G2: } 27 / 77(35) \\
\text { G3: } 18 / 47(38) \\
\text { G5: } 12 / 61(20) \\
\text { G1/G5: } P=0.18 \\
\text { G2/G5: } P=0.05 \\
\text { (including first } \\
\text { medium dose) } \\
\text { G2/G5: } P=0.06 \\
\text { (including first } \\
\text { and second } \\
\text { medium dose } \\
\text { when applicable) } \\
\text { G3/G5: } P=0.07 \\
\text { Social skills:^ } \\
\text { Joint attention } \\
\text { (n=33), mean } \pm \\
\text { SD } \\
\text { Initiations: } \\
\text { G1: } 23.29 \pm \\
\text { 16.62 } \\
\text { G2: } 20.85 \pm \\
\text { 13.01 } \\
\text { G3: } 19.27 \pm \\
\text { 14.23 } \\
\text { G4: } 25.09 \pm \\
\text { 15.55 } \\
\text { G5: } 18.59 \pm \\
\text { 12.03 } \\
\text { G1/G5: } P<0.05 \\
\text { G2/G5: } P=\mathrm{NS} \\
\text { G3/G5: } P=\mathrm{NS}\end{array}$ \\
\hline
\end{tabular}




\begin{tabular}{|c|c|c|c|}
\hline $\begin{array}{c}\text { Author, Year } \\
\text { Study Design } \\
\text { Groups (dose), N enrollment / N } \\
\text { final } \\
\text { Treatment duration/Follow-up } \\
\text { timepoint post-treatment } \\
\text { Risk of Bias }\end{array}$ & $\begin{array}{c}\text { Mean age, years } \\
\pm \text { SD } \\
\text { Mean IQ } \pm S D\end{array}$ & $\begin{array}{c}\text { Outcome } \\
\text { measure/Baseline } \\
\text { scores, mean } \pm S D\end{array}$ & $\begin{array}{c}\text { Outcome } \\
\text { measure/Post- } \\
\text { treatment } \\
\text { scores, mean } \pm \\
\text { SD }\end{array}$ \\
\hline & & 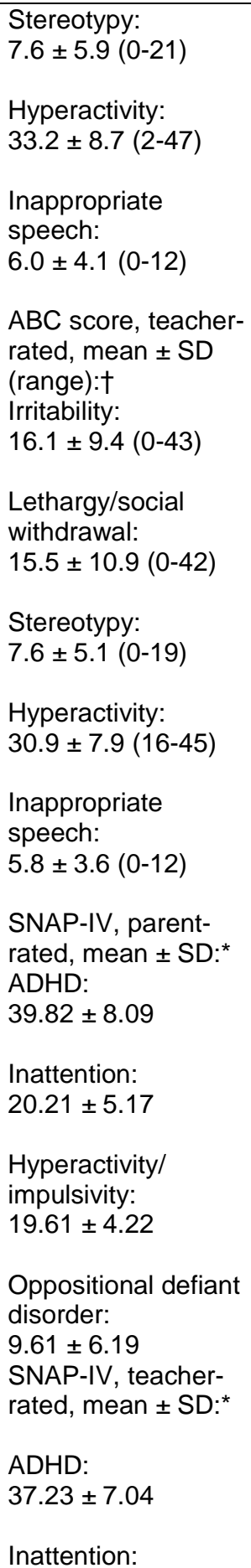 & 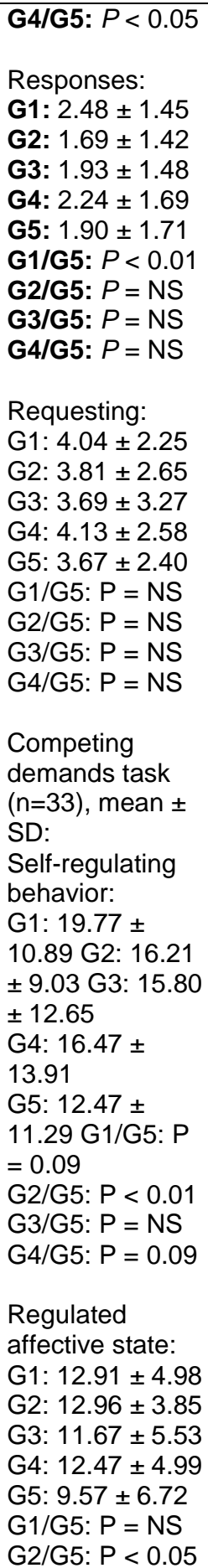 \\
\hline
\end{tabular}




\begin{tabular}{|c|c|c|c|}
\hline $\begin{array}{c}\text { Author, Year } \\
\text { Study Design } \\
\text { Groups (dose), N enrollment / N } \\
\text { final } \\
\\
\text { Treatment duration/Follow-up } \\
\text { timepoint post-treatment } \\
\text { Risk of Bias }\end{array}$ & $\begin{array}{c}\text { Mean age, years } \\
\pm \text { SD } \\
\text { Mean IQ } \pm S D\end{array}$ & $\begin{array}{c}\text { Outcome } \\
\text { measure/Baseline } \\
\text { scores, mean } \pm S D\end{array}$ & $\begin{array}{c}\text { Outcome } \\
\text { measure/Post- } \\
\text { treatment } \\
\text { scores, mean } \pm \\
\text { SD }\end{array}$ \\
\hline & & $\begin{array}{l}19.30 \pm 4.32 \\
\text { Hyperactivity/ } \\
\text { impulsivity: } \\
17.93 \pm 4.81 \\
\text { Oppositional defiant } \\
\text { disorder: } \\
8.83 \pm 5.19 \\
\text { CYBOCS-PDD, } \\
\text { clinician-rated, } \\
\text { mean } \pm \text { SD:* } \\
13.30 \pm 3.74 \\
\text { VABS Maladaptive } \\
\text { behaviors total: } \\
29.2 \pm 9.2(13-51) \\
\text { Motor skills: } \\
69.2 \pm 17.8 \text { (44-113) }\end{array}$ & 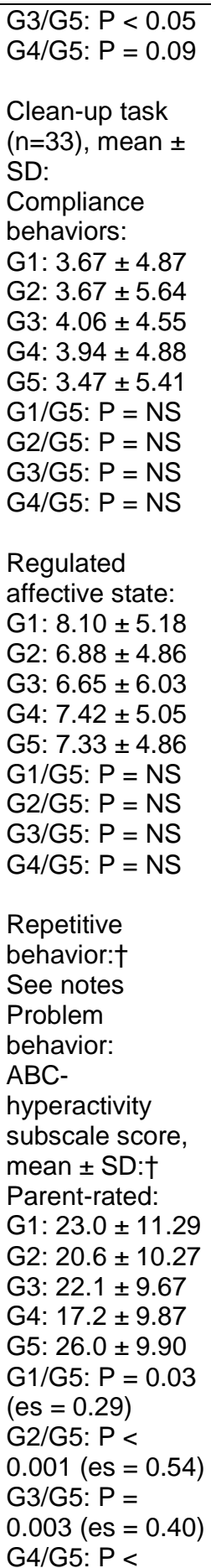 \\
\hline
\end{tabular}




\begin{tabular}{|c|c|c|c|}
\hline $\begin{array}{c}\text { Author, Year } \\
\text { Study Design } \\
\text { Groups (dose), N enrollment / N } \\
\text { final } \\
\text { Treatment duration/Follow-up } \\
\text { timepoint post-treatment } \\
\text { Risk of Bias }\end{array}$ & $\begin{array}{c}\text { Mean age, years } \\
\pm \text { SD } \\
\text { Mean IQ } \pm S D\end{array}$ & $\begin{array}{c}\text { Outcome } \\
\text { measure/Baseline } \\
\text { scores, mean } \pm S D\end{array}$ & $\begin{array}{c}\text { Outcome } \\
\text { measure/Post- } \\
\text { treatment } \\
\text { scores, mean } \pm \\
\text { SD }\end{array}$ \\
\hline & & & 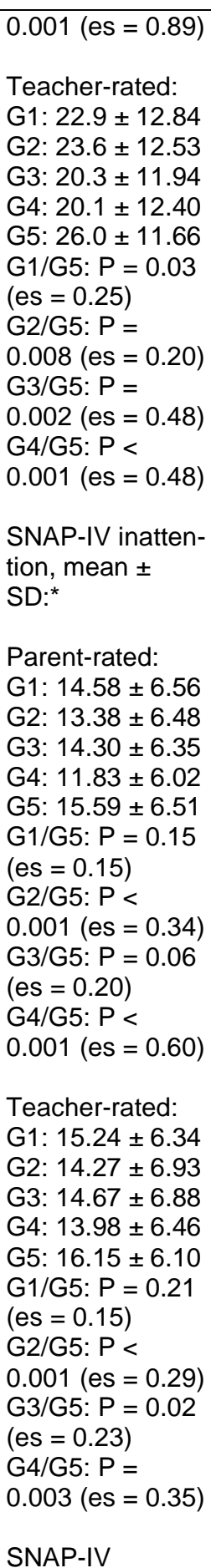 \\
\hline
\end{tabular}




\begin{tabular}{|c|c|c|c|}
\hline $\begin{array}{c}\text { Author, Year } \\
\text { Study Design } \\
\text { Groups (dose), N enrollment / N } \\
\text { final } \\
\text { Treatment duration/Follow-up } \\
\text { timepoint post-treatment } \\
\text { Risk of Bias }\end{array}$ & $\begin{array}{c}\text { Mean age, years } \\
\pm \text { SD } \\
\text { Mean IQ } \pm S D\end{array}$ & $\begin{array}{c}\text { Outcome } \\
\text { measure/Baseline } \\
\text { scores, mean } \pm S D\end{array}$ & $\begin{array}{c}\text { Outcome } \\
\text { measure/Post- } \\
\text { treatment } \\
\text { scores, mean } \pm \\
\text { SD }\end{array}$ \\
\hline & & & 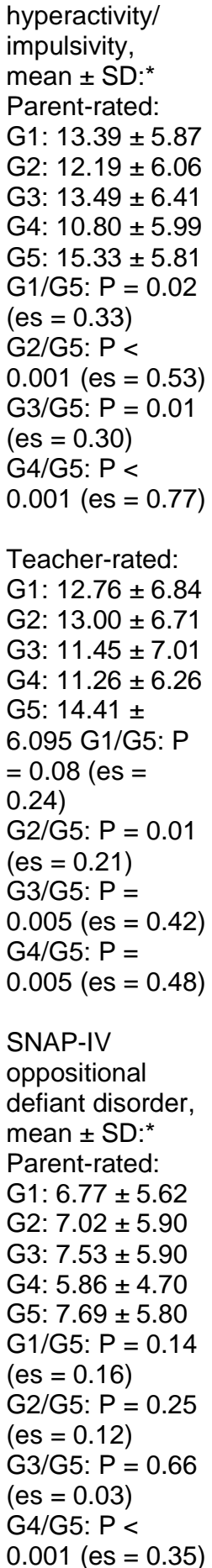 \\
\hline
\end{tabular}




\begin{tabular}{|c|c|c|c|}
\hline $\begin{array}{c}\text { Author, Year } \\
\text { Study Design } \\
\text { Groups (dose), N enrollment / N } \\
\text { final } \\
\text { Treatment duration/Follow-up } \\
\text { timepoint post-treatment } \\
\text { Risk of Bias } \\
\end{array}$ & $\begin{array}{c}\text { Mean age, years } \\
\pm \text { SD } \\
\text { Mean IQ } \pm \text { SD }\end{array}$ & $\begin{array}{c}\text { Outcome } \\
\text { measure/Baseline } \\
\text { scores, mean } \pm S D\end{array}$ & $\begin{array}{c}\text { Outcome } \\
\text { measure/Post- } \\
\text { treatment } \\
\text { scores, mean } \pm \\
\text { SD }\end{array}$ \\
\hline & & & 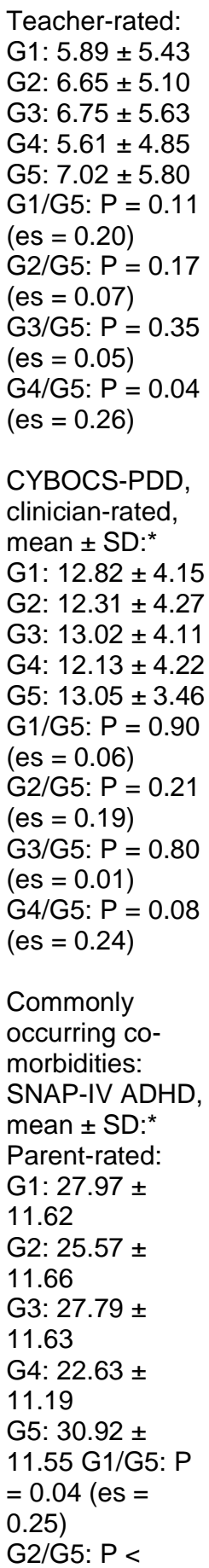 \\
\hline
\end{tabular}




\begin{tabular}{|c|c|c|c|}
\hline $\begin{array}{c}\text { Author, Year } \\
\text { Study Design } \\
\text { Groups (dose), N enrollment / N } \\
\text { final } \\
\text { Treatment duration/Follow-up } \\
\text { timepoint post-treatment } \\
\text { Risk of Bias }\end{array}$ & $\begin{array}{c}\text { Mean age, years } \\
\pm \text { SD } \\
\text { Mean IQ } \pm S D\end{array}$ & $\begin{array}{c}\text { Outcome } \\
\text { measure/Baseline } \\
\text { scores, mean } \pm S D\end{array}$ & $\begin{array}{c}\text { Outcome } \\
\text { measure/Post- } \\
\text { treatment } \\
\text { scores, mean } \pm \\
\text { SD }\end{array}$ \\
\hline & & & $\begin{array}{l}\text { 0.001 }(\mathrm{es}=0.46) \\
\text { G3/G5: } \mathrm{P}=0.02 \\
(\mathrm{es}=0.27) \\
\text { G4/G5: } \mathrm{P}< \\
0.001(\mathrm{es}=0.73) \\
\text { Teacher-rated: } \\
\text { G1: } 28.00 \pm \\
12.12 \\
\text { G2: } 27.27 \pm \\
12.21 \\
\text { G3: } 26.12 \pm \\
12.64 \\
\text { G4: } 25.24 \pm \\
\text { 11.53 } \\
\text { G5: 30.57 } \pm \\
\text { 11.84 G1/G5: P } \\
=0.10(\mathrm{es}= \\
0.21) \\
\text { G2/G5: P }= \\
0.001(\mathrm{es}=0.27) \\
\text { G3/G5: P }= \\
0.005(\mathrm{es}=0.36) \\
\text { G4/G5: P }= \\
0.003(\mathrm{es}=0.46)\end{array}$ \\
\hline $\begin{array}{l}\text { Pearson et al., } 2013^{37} \\
\text { RCT } \\
\text { G1: Methylphenidate - low dose } \\
\text { (0.14-0.21 } \mathrm{mg} / \mathrm{kg}), 24 / 24 \\
\text { G2: Methylphenidate - medium } \\
\text { dose }(0.24-0.35 \mathrm{mg} / \mathrm{kg}), 24 / 24 \\
\text { G3: Methylphenidate - high dose } \\
(0.27-0.48 \mathrm{mg} / \mathrm{kg}), 24 / 24 \\
\text { G4: Placebo (NA), 24/24 } \\
4 \text { weeks/EOT } \\
\text { Low RoB }\end{array}$ & $\begin{array}{l}\text { Age } \\
\mathrm{G} 1+\mathrm{G} 2+\mathrm{G} 3+\mathrm{G} 4: \\
8.8 \pm 1.6 \\
\mathrm{IQ} \\
\mathrm{NR}\end{array}$ & $\begin{array}{l}\text { Parent Ratings } \\
\text { CPRS } \\
\text { NR } \\
\text { CPRS-ADHD scale } \\
\text { G1+G2+G3+G4: } \\
76.4 \pm 6.7 \\
\text { SNAP-IV } \\
\text { NR } \\
\text { ACTeRS } \\
\text { NR } \\
\text { ABC } \\
\text { NR } \\
\text { SCQ } \\
\text { NR } \\
\text { Teacher Ratings } \\
\text { CTRS } \\
\text { NR } \\
\text { CTRS-ADHD scale } \\
\text { G1+G2+G3+G4: } \\
67.2 \pm 8.7 \\
\text { SNAP-IV } \\
\text { NR }\end{array}$ & $\begin{array}{l}\text { EOT } \\
\text { Parent Ratings } \\
\text { CPRS - CGI } \\
\text { Total } \\
\text { G1: } 61.9 \pm 11.7 \\
\text { G2: } 59.9 \pm 9.8 \\
\text { G3: } 55.8 \pm 9.3 \\
\text { G4: } 66.8 \pm 12.7 \\
\text { G4 vs G2, } \\
\text { p<0.05 } \\
\text { G4 vs G3, } \\
\text { p<0.05 } \\
\text { G1 vs G3, } \\
\text { p<0.05 } \\
\text { CPRS - } \\
\text { Oppositional } \\
\text { G1: } 53.1 \pm 15.3 \\
\text { G2: } 53.8 \pm 12.4 \\
\text { G3: } 49.8 \pm 11.4 \\
\text { G4: } 59.2 \pm 16 \\
\text { ANOVA p-value } \\
\text { for all groups, } \\
\text { p<0.05 } \\
\text { CPRS - } \\
\text { Cognitive }\end{array}$ \\
\hline
\end{tabular}




\begin{tabular}{|c|c|c|c|}
\hline $\begin{array}{c}\text { Author, Year } \\
\text { Study Design } \\
\text { Groups (dose), N enrollment / N } \\
\text { final } \\
\text { Treatment duration/Follow-up } \\
\text { timepoint post-treatment } \\
\text { Risk of Bias }\end{array}$ & $\begin{array}{c}\text { Mean age, years } \\
\pm \text { SD } \\
\text { Mean IQ } \pm S D\end{array}$ & $\begin{array}{c}\text { Outcome } \\
\text { measure/Baseline } \\
\text { scores, mean } \pm S D\end{array}$ & $\begin{array}{c}\text { Outcome } \\
\text { measure/Post- } \\
\text { treatment } \\
\text { scores, mean } \pm \\
\text { SD }\end{array}$ \\
\hline & & $\begin{array}{l}\text { ACTeRS } \\
\text { NR }\end{array}$ & $\begin{array}{l}\text { Problems/Inatten } \\
\text { tion } \\
\text { G1: } 67.7 \pm 11.6 \\
\text { G2: } 63.9 \pm 12.5 \\
\text { G3: } 60 \pm 11.7 \\
\text { G4: } 70.3 \pm 12.8 \\
\text { G4 vs G2, } \\
\text { p<0.05 } \\
\text { G4 vs G3, } \\
\text { p<0.05 } \\
\text { G1 vs G3, } \\
\text { p<0.05 } \\
\text { CPRS - } \\
\text { Hyperactivity } \\
\text { G1: } 62.6 \pm 12.2 \\
\text { G2: } 62.1 \pm 12 \\
\text { G3: } 58.3 \pm 10.2 \\
\text { G4: } 70.8 \pm 15.2 \\
\text { G4 vs G2, } \\
\text { p<0.05 } \\
\text { G4 vs G3, } \\
\text { p<0.05 } \\
\text { CPRS }- \text { ADHD } \\
\text { Index } \\
\text { G1: } 64.9 \pm 9.3 \\
\text { G2: } 62.3 \pm 10 \\
\text { G3: } 59.9 \pm 10.5 \\
\text { G4: } 70.1 \pm 11.9 \\
\text { G4 vs G2, } \\
\text { p<0.05 } \\
\text { G4 vs G3, } \\
\text { p<0.05 } \\
\text { SNAP-IV - } \\
\text { Inattentive/Hyper } \\
\text { active } \\
\text { G1: } 26 \pm 11.1 \\
\text { G2: } 22.8 \pm 9.6 \\
\text { G3: } 21.6 \pm 9.6 \\
\text { G4: } 31.6 \pm 11 \\
\text { G4 vs G2, } \\
\text { p<0.05 } \\
\text { G4 vs G3, } \\
\text { p<0.05 } \\
\text { ACTeRS - } \\
\text { Attention } \\
\text { G1: } 12.4 \pm 5.3 \\
\text { G2: } 13.4 \pm 5\end{array}$ \\
\hline
\end{tabular}




\begin{tabular}{|c|c|c|c|}
\hline $\begin{array}{c}\text { Author, Year } \\
\text { Study Design } \\
\text { Groups (dose), N enrollment / N } \\
\text { final } \\
\text { Treatment duration/Follow-up } \\
\text { timepoint post-treatment } \\
\text { Risk of Bias }\end{array}$ & $\begin{array}{c}\text { Mean age, years } \\
\pm \text { SD } \\
\text { Mean IQ } \pm S D\end{array}$ & $\begin{array}{c}\text { Outcome } \\
\text { measure/Baseline } \\
\text { scores, mean } \pm S D\end{array}$ & $\begin{array}{c}\text { Outcome } \\
\text { measure/Post- } \\
\text { treatment } \\
\text { scores, mean } \pm \\
\text { SD }\end{array}$ \\
\hline & & & $\begin{array}{l}\text { G3: } 14 \pm 4.2 \\
\text { G4:10.9 } 4.7 \\
\text { G4 vs G3, p=ns } \\
\text { ACTeRS - } \\
\text { Hyperactivity } \\
\text { G1: } 13.3 \pm 4.5 \\
\text { G2: } 12 \pm 4 \\
\text { G3: } 12 \pm 4.3 \\
\text { G4: } 15.7 \pm 4.5 \\
\text { G4 vs G2, } \\
\text { p<0.05 } \\
\text { G4 vs G3, } \\
\text { p<0.05 } \\
\text { ACTeRS - } \\
\text { Social Skills } \\
\text { G1: } 13.3 \pm 4.4 \\
\text { G2: } 12 \pm 3.7 \\
\text { G3: } 14 \pm 4.3 \\
\text { G4: } 12.8 \pm 4.5 \\
\text { G2 vs G3, } \\
\text { p<0.05 } \\
\text { ACTeRS }- \\
\text { Oppositional } \\
\text { G1: } 7.8 \pm 5.6 \\
\text { G2: } 6.8 \pm 4 \\
\text { G3: } 6.6 \pm 2.6 \\
\text { G4: } 8.4 \pm 5.3 \\
\text { G4 vs G2, } \\
\text { p<0.05 } \\
\text { ABC-Irritability } \\
\text { G1: } 10 \pm 9.2 \\
\text { G2: } 8.2 \pm 8.1 \\
\text { G3: } 7.2 \pm 6.9 \\
\text { G4: } 12.6 \pm 10.4 \\
\text { G4 vs G3, } \\
\text { p<0.05 } \\
\text { ABC-Social } \\
\text { Withdrawal/Leth } \\
\text { argy } \\
\text { G1: } 7.3 \pm 5.6 \\
\text { G2: } 8.1 \pm 5.9 \\
\text { G3: } 8.5 \pm 6.6 \\
\text { G4: } 9.3 \pm 8.1 \\
\text { p=ns } \\
\text { ABC-Stereotypy } \\
\text { G1: } 4.3 \pm 4.5 \\
\text { G2: } 4 \pm 3.8 \\
\text { G3: } 3.5 \pm 3.8 \\
\text { a } 5\end{array}$ \\
\hline
\end{tabular}




\begin{tabular}{|c|c|c|c|}
\hline $\begin{array}{c}\text { Author, Year } \\
\text { Study Design } \\
\text { Groups (dose), N enrollment / N } \\
\text { final } \\
\text { Treatment duration/Follow-up } \\
\text { timepoint post-treatment } \\
\text { Risk of Bias }\end{array}$ & $\begin{array}{c}\text { Mean age, years } \\
\pm \text { SD } \\
\text { Mean IQ } \pm S D\end{array}$ & $\begin{array}{c}\text { Outcome } \\
\text { measure/Baseline } \\
\text { scores, mean } \pm S D\end{array}$ & $\begin{array}{c}\text { Outcome } \\
\text { measure/Post- } \\
\text { treatment } \\
\text { scores, mean } \pm \\
\text { SD }\end{array}$ \\
\hline & & & $\begin{array}{l}\text { G4: } 4.9 \pm 5.4 \\
\text { p=ns } \\
\text { ABC- } \\
\text { Hyperactivity } \\
\text { G1: } 18.1 \pm 10.5 \\
\text { G2: } 14.5 \pm 7.7 \\
\text { G3: } 14.5 \pm 9.2 \\
\text { G4: } 24.1 \pm 13 \\
\text { G4 vs G2, } \\
\text { p<0.05 } \\
\text { G4 vs G3, } \\
\text { p<0.05 } \\
\text { ABC- } \\
\text { Inappropriate } \\
\text { Speech } \\
\text { G1: } 4.3 \pm 3.2 \\
\text { G2: } 4 \pm 3.1 \\
\text { G3: } 3.9 \pm 3.1 \\
\text { G4: } 5.2 \pm 3.1 \\
\text { G4 vs G2, } \\
\text { p<0.05 } \\
\text { G4 vs G3, } \\
\text { p<0.05 } \\
\text { sCQ - Total } \\
\text { G1: } 14.2 \pm 6.6 \\
\text { G2: } 15.2 \pm 6.2 \\
\text { G3: } 13.4 \pm 6.2 \\
\text { G4: } 15.5 \pm 6.1 \\
\text { ANOVA p-value } \\
\text { for all groups, } \\
\text { p<0.05 } \\
\text { Teacher } \\
\text { Ratings } \\
\text { CTRS }- \text { CGI- } \\
\text { Total } \\
\text { G1: } 63.4 \pm 12.8 \\
\text { G2: } 62.4 \pm 12.5 \\
\text { G3: } 59.3 \pm 12.7 \\
\text { G4: } 75.6 \pm 11.5 \\
\text { G4 vs G2, } \\
\text { p<0.05 } \\
\text { G4 vs G3, } \\
\text { p<0.05 } \\
\text { G1 vs G3, } \\
\text { p<0.05 } \\
\text { CTRS- } \\
\text { Oppositional } \\
\end{array}$ \\
\hline
\end{tabular}




\begin{tabular}{|c|c|c|c|}
\hline $\begin{array}{c}\text { Author, Year } \\
\text { Study Design } \\
\text { Groups (dose), N enrollment / N } \\
\text { final } \\
\\
\text { Treatment duration/Follow-up } \\
\text { timepoint post-treatment } \\
\text { Risk of Bias }\end{array}$ & $\begin{array}{c}\text { Mean age, years } \\
\pm \text { SD } \\
\text { Mean IQ } \pm S D\end{array}$ & $\begin{array}{c}\text { Outcome } \\
\text { measure/Baseline } \\
\text { scores, mean } \pm S D\end{array}$ & $\begin{array}{c}\text { Outcome } \\
\text { measure/Post- } \\
\text { treatment } \\
\text { scores, mean } \pm \\
\text { SD }\end{array}$ \\
\hline & & & $\begin{array}{l}\text { G1: } 56.6 \pm 13.2 \\
\text { G2: } 58.1 \pm 15.4 \\
\text { G3: } 55.1 \pm 13.5 \\
\text { G4: } 65.1 \pm 19.5 \\
\text { p=ns } \\
\text { CTRS-Cognitive } \\
\text { Problems/Inatten } \\
\text { tion } \\
\text { G1: } 58.3 \pm 10.7 \\
\text { G2: } 57.8 \pm 9.5 \\
\text { G3: } 59.3 \pm 11.1 \\
\text { G4: } 63 \pm 11.2 \\
\text { G4 vs G2, } \\
\text { p<0.05 } \\
\text { G4 vs G3, } \\
\text { p<0.05 } \\
\text { G1 vs G3, } \\
\text { p<0.05 } \\
\text { CTRS- } \\
\text { Hyperactivity } \\
\text { G1: } 59.9 \pm 13.6 \\
\text { G2: } 59.9 \pm 11.4 \\
\text { G3: } 57.7 \pm 11.3 \\
\text { G4: } 70.3 \pm 13.5 \\
\text { G4 vs G2, } \\
\text { p<0.05 } \\
\text { G4 vs G3, } \\
\text { p<0.05 } \\
\text { G1 vs G3, } \\
\text { p<0.05 } \\
\text { CTRS-ADHD } \\
\text { Index } \\
\text { G1: } 63.1 \pm 11.2 \\
\text { G2: } 63.6 \pm 10.4 \\
\text { G3: } 61.5 \pm 13 \\
\text { G4: } 72.8 \pm 12 \\
\text { G4 vs G2, } \\
\text { p<0.05 } \\
\text { G4 vs G3, } \\
\text { p<0.05 } \\
\text { G1 vs G3, } \\
\text { p<0.05 } \\
\text { SNAP-IV }- \\
\text { Inattentive/Hyper } \\
\text { active } \\
\text { G1: } 24.7 \pm 10.9\end{array}$ \\
\hline
\end{tabular}




\begin{tabular}{|c|c|c|c|}
\hline $\begin{array}{c}\text { Author, Year } \\
\text { Study Design } \\
\text { Groups (dose), N enrollment / N } \\
\text { final } \\
\text { Treatment duration/Follow-up } \\
\text { timepoint post-treatment } \\
\text { Risk of Bias }\end{array}$ & $\begin{array}{c}\text { Mean age, years } \\
\pm \text { SD } \\
\text { Mean IQ } \pm S D\end{array}$ & $\begin{array}{c}\text { Outcome } \\
\text { measure/Baseline } \\
\text { scores, mean } \pm S D\end{array}$ & $\begin{array}{c}\text { Outcome } \\
\text { measure/Post- } \\
\text { treatment } \\
\text { scores, mean } \pm \\
\text { SD }\end{array}$ \\
\hline & & & $\begin{array}{l}\text { G2: } 23.7 \pm 11.1 \\
\text { G3: } 21.8 \pm 11.6 \\
\text { G4: } 32.7 \pm 12.4 \\
\text { G4 vs G2, } \\
\text { p<0.05 } \\
\text { G4 vs G3, } \\
\text { p<0.05 } \\
\text { G1 vs G3, } \\
\text { p<0.05 } \\
\text { ACTeRS }- \\
\text { Attention } \\
\text { G1: } 14.3 \pm 6.2 \\
\text { G2: } 13.7 \pm 5.5 \\
\text { G3: } 15.9 \pm 8.5 \\
\text { G4: } 11.3 \pm 4 \\
\text { G4 vs G3, } \\
\text { p<0.05 } \\
\text { ACTeRS }- \\
\text { Hyperactivity } \\
\text { G1: } 11.8 \pm 5.6 \\
\text { G2: } 12.8 \pm 5.5 \\
\text { G3: } 11.2 \pm 5 \\
\text { G4: } 18.7 \pm 5.2 \\
\text { G4 vs G2, } \\
\text { p<0.05 } \\
\text { G4 vs G3, } \\
\text { p<0.05 } \\
\text { G1 vs G3, } \\
\text { p<0.05 } \\
\text { ACTeRS }- \\
\text { Social Skills } \\
\text { G1: } 19.2 \pm 4.2 \\
\text { G2: } 16.7 \pm 4 \\
\text { G3: } 18.5 \pm 5.8 \\
\text { G4: } 15.8 \pm 3.6 \\
\text { p=ns } \\
\text { ACTeRS }- \\
\text { Oppositional } \\
\text { G1: } 7.6 \pm 3.5 \\
\text { G2: } 9.2 \pm 5.5 \\
\text { G3: } 7.1 \pm 2.1 \\
\text { G4: } 10.6 \pm 5.8 \\
\text { G4 vs G3, } \\
\text { p<0.05 } \\
\text { CGI-Severity } \\
\text { (clinician } 1) \\
\text { G1: } 4 \pm 0.81 \\
\end{array}$ \\
\hline
\end{tabular}




\begin{tabular}{|c|c|c|c|}
\hline $\begin{array}{c}\text { Author, Year } \\
\text { Study Design } \\
\text { Groups (dose), N enrollment / N } \\
\text { final } \\
\\
\text { Treatment duration/Follow-up } \\
\text { timepoint post-treatment } \\
\text { Risk of Bias }\end{array}$ & $\begin{array}{c}\text { Mean age, years } \\
\pm \text { SD } \\
\text { Mean IQ } \pm S D\end{array}$ & $\begin{array}{c}\text { Outcome } \\
\text { measure/Baseline } \\
\text { scores, mean } \pm S D\end{array}$ & $\begin{array}{c}\text { Outcome } \\
\text { measure/Post- } \\
\text { treatment } \\
\text { scores, mean } \pm \\
\text { SD }\end{array}$ \\
\hline & & & 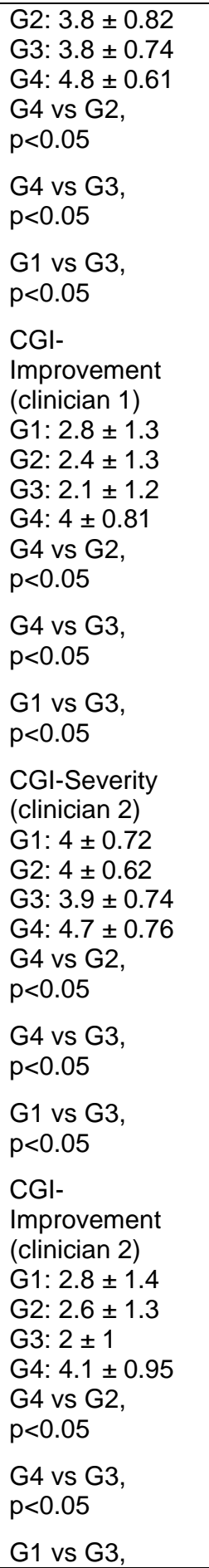 \\
\hline
\end{tabular}




\begin{tabular}{|c|c|c|c|}
\hline $\begin{array}{c}\text { Author, Year } \\
\text { Study Design }\end{array}$ & $\begin{array}{c}\text { Mean age, years } \\
\pm \text { SD }\end{array}$ & $\begin{array}{c}\text { Outcome } \\
\text { measure/Baseline } \\
\text { scores, mean } \pm \text { SD }\end{array}$ & $\begin{array}{c}\text { Outcome } \\
\text { measure/Post- } \\
\text { treatment } \\
\text { scores, mean } \pm \\
\text { SD }\end{array}$ \\
$\begin{array}{c}\text { Groups (dose), N enrollment I N } \\
\text { final } \\
\text { timepoint post-treatment }\end{array}$ & Mean IQ \pm SD & & \\
Risk of Bias & & & \\
\hline & & & $\mathrm{p}<0.05$ \\
\hline
\end{tabular}

ABC-Aberrant Behavior Checklist; CGI-Clinical Global Impression; VABS-Vineland Adaptive Behavior Scale; CYBOCS-PDD-Children's Yale-Brown Obsessive Compulsive Scale Modified for Pervasive Developmental Disorder; EOT-End of Treatment; VBS-Visual Analog Scale; CGAS-Children's Global Assessment Scale; SNAP-IV-; ADHD-Attention Deficit/Hyperactivity Disorder; CPRS-Conners Parent Rating Scale; ACTeRS-ADD-H Comprehensive Teacher Rating Scale; CTRS-Conners Teacher Rating Scale; SCQ-Social Communication Questionnaire 
Table F-3. Key findings in studies of nutritional interventions

\begin{tabular}{|c|c|c|c|}
\hline $\begin{array}{c}\text { Author, Year } \\
\text { Study Design } \\
\text { Groups (dose), N enrollment I } \\
\text { N final } \\
\text { Treatment duration/Follow-up } \\
\text { timepoint post-treatment } \\
\text { Risk of Bias }\end{array}$ & $\begin{array}{l}\text { Mean age, } \\
\text { years } \pm \text { SD } \\
\text { Mean IQ } \pm S D\end{array}$ & $\begin{array}{c}\text { Outcome } \\
\text { measure/Baseline } \\
\text { scores, mean } \pm S D\end{array}$ & $\begin{array}{c}\text { Outcome } \\
\text { measure/Post- } \\
\text { treatment scores, } \\
\text { mean } \pm \text { SD }\end{array}$ \\
\hline $\begin{array}{l}\text { Sun et al., } 2016^{38} \\
\text { RCT } \\
\text { G1: TEACCH+folic acid, } 44 / 44 \\
\text { G2: TEACCH alone, } 22 / 22 \\
3 \text { months/EOT } \\
\text { Moderate ROB } \\
\text { (Note: Discussed in section on } \\
\text { combined treatments in main } \\
\text { report) }\end{array}$ & $\begin{array}{l}\text { Age (months) } \\
\text { G1: } 57.23 \pm \\
15.06 \\
\text { G2: } 51.75 \pm \\
12.72 \\
\text { IQ } \\
\text { NR }\end{array}$ & 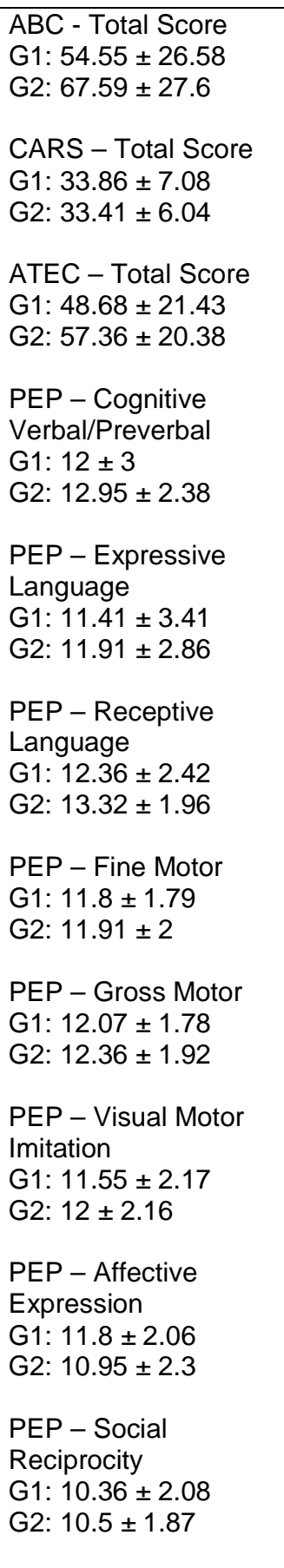 & $\begin{array}{l}\text { ABC - Total Score } \\
\text { G1: } 39.4 \pm 26.73 \\
\text { G2: } 46.18 \pm 22.71 \\
\text { G1 vs G2, p=ns } \\
\text { CARS - Total Score } \\
\text { G1: } 29.34 \pm 5.52 \\
\text { G2: } 30.82 \pm 5.06 \\
\text { G1 vs G2, p=ns } \\
\text { ATEC }- \text { Total Score } \\
\text { G1: } 36.3 \pm 17.49 \\
\text { G2: } 46.36 \pm 18.56 \\
\text { G1 vs G2, p=0.052 } \\
\text { PEP - Cognitive } \\
\text { Verbal/Preverbal } \\
\text { G1: } 14.2 \pm 1.97 \\
\text { G2: } 13.91 \pm 2.79 \\
\text { G1 vs G2, p=ns } \\
\text { PEP - Expressive } \\
\text { Language } \\
\text { G1: } 13.07 \pm 3.19 \\
\text { G2: } 13.14 \pm 2.87 \\
\text { G1 vs G2, p=ns } \\
\text { PEP - Receptive } \\
\text { Language } \\
\text { G1: } 13.75 \pm 1.4 \\
\text { G2: } 13.73 \pm 1.75 \\
\text { G1 vs G2, p=ns } \\
\text { PEP }- \text { Fine Motor } \\
\text { G1: } 12.48 \pm 1.68 \\
\text { G2: } 13 \pm 1.75 \\
\text { G1 vs G2, p=ns } \\
\text { PEP - Gross Motor } \\
\text { G1: } 12.77 \pm 0.99 \\
\text { G2: } 13.09 \pm 1.31 \\
\text { G1 vs G2, p=ns } \\
\text { PEP - Visual Motor } \\
\text { Imitation } \\
\text { G1: } 12.27 \pm 1.48 \\
\text { G2: } 13.64 \pm 1.97 \\
\text { G1 vs G2, p=ns } \\
\end{array}$ \\
\hline
\end{tabular}




\begin{tabular}{|c|c|c|c|}
\hline $\begin{array}{c}\text { Author, Year } \\
\text { Study Design } \\
\text { Groups (dose), N enrollment I } \\
\mathrm{N} \text { final } \\
\\
\text { Treatment duration/Follow-up } \\
\text { timepoint post-treatment } \\
\text { Risk of Bias }\end{array}$ & $\begin{array}{c}\text { Mean age, } \\
\text { years } \pm \text { SD } \\
\text { Mean IQ } \pm S D\end{array}$ & $\begin{array}{c}\text { Outcome } \\
\text { measure/Baseline } \\
\text { scores, mean } \pm S D\end{array}$ & $\begin{array}{c}\text { Outcome } \\
\text { measure/Post- } \\
\text { treatment scores, } \\
\text { mean } \pm \text { SD }\end{array}$ \\
\hline & & $\begin{array}{l}\text { PEP - Characteristic } \\
\text { Motor Behaviors } \\
\text { G1: } 11.98 \pm 2.39 \\
\text { G2: } 11.95 \pm 2.3 \\
\\
\text { PEP - Characteristic } \\
\text { Verbal Behaviors } \\
\text { G1: } 8 \pm 3.58 \\
\text { G2: } 9.05 \pm 2.9 \\
\text { PEP - Communication } \\
\text { G1: } 35.77 \pm 8.21 \\
\text { G2: } 38.18 \pm 6.71 \\
\\
\text { PEP - Motor } \\
\text { G1: } 35.41 \pm 5.18 \\
\text { G2: } 36.27 \pm 5.54 \\
\text { PEP - Maladaptive } \\
\text { Behavior } \\
\text { G1: } 42.14 \pm 7.84 \\
\text { G2: } 42.45 \pm 7.31 \\
\text { PEP - Problem } \\
\text { Behaviors } \\
\text { G1: } 8.25 \pm 2.36 \\
\text { G2: } 8.27 \pm 2.39 \\
\text { PEP - Personal Self- } \\
\text { Care } \\
\text { G1: } 11.7 \pm 2.41 \\
\text { G2: } 12.09 \pm 2.24 \\
\text { PEP - Adaptive } \\
\text { Behaviors } \\
\text { G1: } 11.14 \pm 2.36 \\
\text { G2: } 11.36 \pm 2.63\end{array}$ & $\begin{array}{l}\text { Expression } \\
\text { G1: } 11.98 \pm 2.25 \\
\text { G2: } 12.45 \pm 1.26 \\
\text { G1 vs } \mathrm{G} 2, \mathrm{p}=\mathrm{ns} \\
\text { PEP }- \text { Social } \\
\text { Reciprocity } \\
\text { G1: } 11.3 \pm 2.09 \\
\text { G2: } 11.64 \pm 1.65 \\
\text { G1 vs } \mathrm{G} 2, \mathrm{p}=\mathrm{ns} \\
\text { PEP }- \text { Characteristic } \\
\text { Motor Behaviors } \\
\text { G1: } 11.66 \pm 2.58 \\
\text { G2: } 12.59 \pm 2.26 \\
\text { G1 vs } \mathrm{G} 2, \mathrm{p}=\mathrm{ns} \\
\text { PEP }- \text { Characteristic } \\
\text { Verbal Behaviors } \\
\text { G1: } 9.64 \pm 3.72 \\
\text { G2: } 10.5 \pm 2.65 \\
\text { G1 vs } \mathrm{G} 2, \mathrm{p}=\mathrm{ns} \\
\text { PEP }- \text { Communication } \\
\text { G1: } 41.02 \pm 5.91 \\
\text { G2: } 40.77 \pm 7.04 \\
\text { G1 vs } \mathrm{G} 2, \mathrm{p}=\mathrm{ns} \\
\text { PEP }- \text { Motor } \\
\text { G1: } 37.52 \pm 3.79 \\
\text { G2: } 38.73 \pm 4.69 \\
\text { G1 vs } \mathrm{G} 2, \mathrm{p}=\mathrm{ns} \\
\text { PEP }- \text { Maladaptive } \\
\text { Behavior } \\
\text { G1: } 44.57 \pm 9.59 \\
\text { G2: } 47.18 \pm 6.53 \\
\text { G1 vs } \mathrm{G} 2, \mathrm{p}=\mathrm{ns} \\
\text { PEP }- \text { Problem } \\
\text { Behaviors } \\
\text { G1: } 9.05 \pm 2.9 \\
\text { G2: } 9 \pm 2.65 \\
\text { G1 vs } \mathrm{G} 2, \mathrm{p}=\mathrm{ns} \\
\text { PEP }- \text { Personal Self- } \\
\text { Care } \\
\text { G1: } 12.34 \pm 1.7 \\
\text { G2: } 12.41 \pm 2.48 \\
\text { G1 vs } \mathrm{G} 2, \mathrm{p}=\mathrm{ns} \\
\end{array}$ \\
\hline
\end{tabular}




\begin{tabular}{|c|c|c|c|}
\hline $\begin{array}{c}\text { Author, Year } \\
\text { Study Design } \\
\text { Groups (dose), N enrollment I } \\
\mathrm{N} \text { final } \\
\\
\text { Treatment duration/Follow-up } \\
\text { timepoint post-treatment } \\
\text { Risk of Bias }\end{array}$ & $\begin{array}{c}\text { Mean age, } \\
\text { years } \pm \text { SD } \\
\text { Mean IQ } \pm S D\end{array}$ & $\begin{array}{c}\text { Outcome } \\
\text { measure/Baseline } \\
\text { scores, mean } \pm S D\end{array}$ & $\begin{array}{c}\text { Outcome } \\
\text { measure/Post- } \\
\text { treatment scores, } \\
\text { mean } \pm \text { SD }\end{array}$ \\
\hline & & & $\begin{array}{l}\text { PEP - Adaptive } \\
\text { Behaviors } \\
\text { G1: } 12.32 \pm 2.59 \\
\text { G2: } 11.77 \pm 2.51 \\
\text { G1 vs } \mathrm{G} 2, \mathrm{p}=\mathrm{ns}\end{array}$ \\
\hline $\begin{array}{l}\text { Mankad et al., } 2015^{39} \\
\text { RCT } \\
\text { G1: Omega-3 fatty acid ( } 3.5 \\
\text { mL/day), } 19 / 18 \\
\text { G2: Placebo, } 19 / 19 \\
6 \text { months/EOT } \\
\text { Moderate RoB }\end{array}$ & $\begin{array}{l}\text { Age } \\
\text { G1: } 3.5 \pm 1.1 \\
\text { G2: } 3.8 \pm 1.0 \\
\text { IQ } \\
\text { NR }\end{array}$ & $\begin{array}{l}\text { Baseline scores } \\
\text { NR }\end{array}$ & $\begin{array}{l}\text { Change from } \\
\text { Baseline } \\
\text { PDDBI - Autism } \\
\text { Composite } \\
\text { G1: }-4.5 \\
\text { G2: }-6.4 \\
\text { G1 vs } G 2: p=n s \\
\text { BASC - Externalizing } \\
\text { G1: } 3.2 \\
\text { G2: }-3.0 \\
\text { G1 vs } G 2: p=0.02 \\
\text { CGI } \\
\text { G1: NR } \\
\text { G2: NR } \\
\text { G1 vs } G 2: p=n s \\
\text { VABS } \\
\text { G1: } 2.8 \\
\text { G2: }-0.2 \\
\text { G1 vs } G 2: p=n s \\
\text { Preschool Language } \\
\text { Scale (PLS4) } \\
\text { G1: } 0.7 \\
\text { G2: }-0.6 \\
\text { G1 vs G2: } p=n s\end{array}$ \\
\hline $\begin{array}{l}\text { Saad et al., } 2015^{40} \\
\text { RCT } \\
\text { G1: Digestive enzymes (15 } \\
\text { mL/day), 51/47 } \\
\text { G2: Placebo (NA), 50/45 } \\
3 \text { months/EOT } \\
\text { Moderate RoB }\end{array}$ & $\begin{array}{l}\text { Age } \\
\text { G1: } 5.94 \pm 2.01 \\
\text { G2: } 5.87 \pm 2.12 \\
\text { IQ } \\
\text { NR }\end{array}$ & $\begin{array}{l}\text { CARS } \\
\text { G1: } 36.1 \pm 3.7 \\
\text { G2: } 35.3 \pm 4.0 \\
\text { GBRS } \\
\text { Child's General } \\
\text { Behavior } \\
\text { G1: } 3.01 \pm 1.3 \\
\text { G2: } 3.50 \pm 0.7 \\
\text { Nighttime sleeping } \\
\text { G1: } 3.89 \pm 1.3 \\
\text { G2: } 3.84 \pm 1.0\end{array}$ & $\begin{array}{l}\text { EOT } \\
\text { CARS } \\
\text { G1: } 31.2 \pm 1.2 \\
\text { G2: } 35.5 \pm 2.8 \\
\text { G1 vs G2: } p=0.034 \\
\text { GBRS } \\
\text { Child's General } \\
\text { Behavior } \\
\text { G1: } 5.5 \pm 0.75 \\
\text { G2: } 3.39 \pm 1.01 \\
\text { G1 vs G2:, } p<0.001 \\
\text { Nighttime sleeping }\end{array}$ \\
\hline
\end{tabular}




\begin{tabular}{|c|c|c|c|}
\hline $\begin{array}{c}\text { Author, Year } \\
\text { Study Design } \\
\text { Groups (dose), N enrollment I } \\
\mathrm{N} \text { final } \\
\\
\text { Treatment duration/Follow-up } \\
\text { timepoint post-treatment } \\
\text { Risk of Bias }\end{array}$ & $\begin{array}{c}\text { Mean age, } \\
\text { years } \pm \text { SD } \\
\text { Mean IQ } \pm S D\end{array}$ & $\begin{array}{c}\text { Outcome } \\
\text { measure/Baseline } \\
\text { scores, mean } \pm S D\end{array}$ & $\begin{array}{c}\text { Outcome } \\
\text { measure/Post- } \\
\text { treatment scores, } \\
\text { mean } \pm \text { SD }\end{array}$ \\
\hline & & $\begin{array}{l}\text { Gastrointestinal } \\
\text { symptoms } \\
\text { G1: } 3.79 \pm 0.61 \\
\text { G2: } 4.02 \pm 0.28 \\
\text { Daytime wetting } \\
\text { G1: } 3.86 \pm 0.67 \\
\text { G2: } 3.92 \pm 0.83 \\
\\
\text { Nighttime wetting } \\
\text { G1: } 4.60 \pm 0.97 \\
\text { G2: } 3.92 \pm 0.6\end{array}$ & $\begin{array}{l}\text { G1: } 4.02 \pm 1.20 \\
\text { G2: } 3.98 \pm 0.89 \\
\text { G1 vs } G 2: p=n s \\
\text { Gastrointestinal } \\
\text { symptoms } \\
\text { G1: } 6.03 \pm 0.56 \\
\text { G2: } 4.02 \pm 0.23 \\
\text { G1 vs G2: } p<0.001 \\
\text { Daytime wetting } \\
\text { G1: } 3.97 \pm 0.88 \\
\text { G2: } 4.03 \pm 1.04 \\
\text { G1 vs } \mathrm{G} 2: \mathrm{p}=\mathrm{ns} \\
\text { Nighttime wetting } \\
\text { G1: } 4.23 \pm 1.25 \\
\text { G2: } 4.09 \pm 0.64 \\
\text { G1 vs } \mathrm{G} 2: \mathrm{p}=\mathrm{ns}\end{array}$ \\
\hline $\begin{array}{l}\text { Bent et al., } 2014^{41} \\
\text { RCT } \\
\text { G1: Omega-3 fatty acid (1.3g/day), } \\
\text { 29/29 } \\
\text { G2: Placebo, } 28 / 28 \\
6 \text { weeks/EOT } \\
\text { Moderate RoB }\end{array}$ & $\begin{array}{l}\text { Age, months } \\
\text { G1: } 88.2 \pm 12.3 \\
\text { G2: } 85 \pm 13.2 \\
\text { mos } \\
\text { IQ } \\
\text { NR }\end{array}$ & $\begin{array}{l}\text { ABC- Hyperactivity } \\
\text { Parent Ratings } \\
\text { G1: } 28.4 \pm 8.4 \\
\text { G2: } 28.1 \pm 7.6 \\
\text { ABC-Hyperactivity } \\
\text { Teacher Ratings } \\
\text { G1: } 18.1 \pm 12.0 \\
\text { G2: } 14.6 \pm .4 \\
\text { ABC-Irritability Parent } \\
\text { G1: } 20.0 \pm 8.9 \\
\text { G2: } 16.8 \pm 8.3 \\
\text { ABC-Irritability } \\
\text { Teacher } \\
\text { G1: } 14.3 \pm 9.8 \\
\text { G2: } 9.4 \pm 9.3 \\
\text { ABC-Stereotypy } \\
\text { Parent } \\
\text { G1: } 8.0 \pm 6.0 \\
\text { G2: } 5.4 \pm 4.7 \\
\text { ABC-Stereotypy } \\
\text { Teacher } \\
\text { G1: } 5.1 \pm 4.8 \\
\text { G2: } 3.7 \pm 4.1 \\
\text { ABC-Lethargy Parent } \\
\text { G1: } 12.2 \pm 8.8 \\
\text { G2: } 8.8 \pm 4.2\end{array}$ & $\begin{array}{l}\text { Mean change score } \\
\text { ABC - Hyperactivity } \\
\text { Parent } \\
\text { G1: }-5.3 \pm 7.2 \\
\text { G2: }-3.4 \pm 7.5 \\
\text { G1 vs G2: } p=n s \\
\text { ABC- Hyperactivity } \\
\text { Teacher } \\
\text { G1: }-2.6 \pm 8.0 \\
\text { G2: }-1.0 \pm 10.0 \\
\text { G1 vs G2: } p=n s \\
\text { ABC-Irritability Parent } \\
\text { G1: }-2.0 \pm 6.9 \\
\text { G2: }-2.1 \pm 4.4 \\
\text { G1 vs G2: } p=n s \\
\text { ABC-Irritability } \\
\text { Teacher } \\
\text { G1: }-1.3 \pm 8.6 \\
\text { G2: }-0.2 \pm 6.8 \\
\text { G1 vs G2: } p=n s \\
\text { ABC-Stereotypy } \\
\text { Parent } \\
\text { G1: }-2.0 \pm 3.7 \\
\text { G2: }-0.5 \pm 2.6 \\
\text { G1 vs G2: } p=0.05 \\
\text { ABC-Stereotypy } \\
\text { Teacher }\end{array}$ \\
\hline
\end{tabular}




\begin{tabular}{|c|c|c|c|}
\hline $\begin{array}{c}\text { Author, Year } \\
\text { Study Design } \\
\text { Groups (dose), N enrollment I } \\
\text { N final } \\
\\
\text { Treatment duration/Follow-up } \\
\text { timepoint post-treatment } \\
\text { Risk of Bias }\end{array}$ & $\begin{array}{c}\text { Mean age, } \\
\text { years } \pm \text { SD } \\
\text { Mean IQ } \pm S D\end{array}$ & $\begin{array}{l}\text { Outcome } \\
\text { measure/Baseline } \\
\text { scores, mean } \pm S D\end{array}$ & $\begin{array}{c}\text { Outcome } \\
\text { measure/Post- } \\
\text { treatment scores, } \\
\text { mean } \pm \text { SD }\end{array}$ \\
\hline & & $\begin{array}{l}\text { ABC-Lethargy Teacher } \\
\text { G1: } 9.9 \pm 8.9 \\
\text { G2: } 8.3 \pm 7.1 \\
\text { ABC-Inappropriate } \\
\text { speech Parent } \\
\text { G1: } 7.0 \pm 3.4 \\
\text { G2: } 5.8 \pm 2.8 \\
\text { ABC-Inappropriate } \\
\text { speech Teacher } \\
\text { G1: } 4.2 \pm 3.9 \\
\text { G2: } 3.1 \pm 3.3 \\
\text { SRS-total } \\
\text { G1: } 89.7 \pm 12.7 \\
\text { G2: } 88.3 \pm .7 \\
\text { CGI-S } \\
\text { G1: } 3.8 \pm 1.0 \\
\text { G2: } 4.0 \pm 0.79\end{array}$ & $\begin{array}{l}\text { G1: }-1.0 \pm 4.2 \\
\text { G2: }-0.7 \pm 3.2 \\
\text { G1 vs } G 2: p=n s \\
\text { ABC-Lethargy Parent } \\
\text { G1: }-2.1 \pm 4.2 \\
\text { G2: } 0.1 \pm 2.6 \\
\text { G1 vs } G 2: p=0.01 \\
\text { ABC-Lethargy Teacher } \\
\text { G1: }-2.5 \pm 7.9 \\
\text { G2: }-1.6 \pm 5.5 \\
\text { G1 vs } \mathrm{G} 2: p=n s \\
\text { ABC-Inappropriate } \\
\text { speech Parent } \\
\text { G1: }-0.6 \pm 2.7 \\
\text { G2: }-0.9 \pm 2.2 \\
\text { G1 vs } G 2: p=n s \\
\text { ABC-Inappropriate } \\
\text { speech Teacher } \\
\text { G1: }-1.0 \pm 2.6 \\
\text { G2: }-0.1 \pm 5.5 \\
\text { G1 vs } \mathrm{G} 2: p=n s \\
\text { SRS-total } \\
\text { G1: }-2.6 \pm 8.3 \\
\text { G2: }-6.1 \pm 7.8 \\
\text { G1 vs G2:p=ns } \\
\text { CG1-S } \\
\text { G1: NR } \\
\text { G2: NR }\end{array}$ \\
\hline $\begin{array}{l}\text { Voigt et al., } 2014^{42} \\
\text { RCT } \\
\text { G1: Docosahexaenoic acid (DHA) } \\
\text { (500 mg/day), } 24 / 19 \\
\text { G2: Placebo, } 24 / 15 \\
6 \text { months/EOT } \\
\text { Moderate RoB }\end{array}$ & $\begin{array}{l}\text { Age } \\
\text { G1: } 5.8 \pm 1.8 \\
\text { G2: } 6.5 \pm 2.2 \\
\text { IQ } \\
\text { NR }\end{array}$ & $\begin{array}{l}\text { CGI-I Parent } \\
\text { NR } \\
\text { BASC - Parent } \\
\text { G1: } 26.5 \pm 7.1 \\
\text { G2: } 30.3 \pm 9.1 \\
\text { BASC - Teacher } \\
\text { G1: } 32.2 \pm 7.6 \\
\text { G2: } 38.5 \pm 4.3\end{array}$ & $\begin{array}{l}\text { CGI-I Parent } \\
3 \text { months } \\
\text { G1: } 4 / 21(19) \\
\text { G2: } 5 / 16(31) \\
\text { G1 vs G2: } p=n s \\
6 \text { months } \\
\text { G1: } 5 / 18(28) \\
\text { G2: } 2 / 13(15) \text { G1 vs } \\
\text { G2: } p=n s \\
\text { CGI-I Investigator } \\
3 \text { months } \\
\text { G1: } 1 / 17(6)) \\
\text { G2: } 0 / 13(0) \\
\text { G1 vs G2: } p=n s\end{array}$ \\
\hline
\end{tabular}




\begin{tabular}{|c|c|c|c|}
\hline $\begin{array}{c}\text { Author, Year } \\
\text { Study Design } \\
\text { Groups (dose), N enrollment } / \\
\mathrm{N} \text { final }\end{array}$ & $\begin{array}{l}\text { Mean age, } \\
\text { years } \pm \text { SD } \\
\text { Mean IQ } \pm S D\end{array}$ & $\begin{array}{l}\text { Outcome } \\
\text { measure/Baseline } \\
\text { scores, mean } \pm S D\end{array}$ & $\begin{array}{c}\text { Outcome } \\
\text { measure/Post- } \\
\text { treatment scores, } \\
\text { mean } \pm \text { SD }\end{array}$ \\
\hline & & & $\begin{array}{l}6 \text { months } \\
\text { G1: } 0 / 18(0) \\
\text { G2: } 1 / 14(7) \\
\text { G1 vs } G 2: p=n s \\
\text { EOT } \\
\text { BASC - Parent } \\
\text { G1: } 26.3 \pm 6.8 \\
\text { G2: } 33.3 \pm 9.7 \\
\text { G1 vs } G 2: p=0.04 \\
\text { BASC }- \text { Teacher } \\
\text { G1: } 33.6 \pm 9.3 \\
\text { G2: } 34.0 \pm 5.6 \\
\text { G1 vs } G 2: p=0.02\end{array}$ \\
\hline $\begin{array}{l}\text { Al-Ayadhi et al., } 2013^{43} \\
\text { RCT } \\
\text { G1: Raw camel milk ( } 500 \mathrm{~mL} / \text { day), } \\
\text { 24/24 } \\
\text { G2: Boiled camel milk ( } 500 \\
\mathrm{~mL} / \text { day), } 25 / 25 \\
\text { G3: Cow milk ( } 500 \mathrm{~mL} / \text { day), 11/11 } \\
2 \text { weeks/EOT } \\
\text { High RoB }\end{array}$ & $\begin{array}{l}\text { Age } \\
2-12 \\
\text { IQ } \\
\text { NR }\end{array}$ & $\begin{array}{l}\text { CARS - total score } \\
\text { G1: } 37.63 \pm 6.31 \\
\text { G2: } 36.82 \pm 3.27 \\
\text { G3: } 34.18 \pm 3.25\end{array}$ & $\begin{array}{l}\text { EOT } \\
\text { CARS - total score } \\
\text { G1: } 34.54 \pm 5.19 \\
\text { G2: } 33.8 \pm 4.91 \\
\text { G3: } 34.41 \pm 3.25 \\
p=N R\end{array}$ \\
\hline $\begin{array}{l}\text { Fahmy et al. } 2013^{44} \\
\text { RCT } \\
\text { G1: Levocarnitine, 16/16 } \\
\text { G2: Placebo,14/14 } \\
6 \text { months/EOT } \\
\text { Moderate RoB }\end{array}$ & $\begin{array}{l}\text { Age (months) } \\
\text { G1: } 69 \text { (29-103) } \\
\text { G2: } 68.5 \text { (32- } \\
98) \\
\text { IQ } \\
\text { NR }\end{array}$ & $\begin{array}{l}\text { CARS - Mild/Moderate } \\
\text { Severity } \\
\text { G1: } 1 \text { (6.3) } \\
\text { G2: } 9 \text { (64.3) } \\
\text { CARS - Severe } \\
\text { G1: } 15 \text { (93.80) } \\
\text { G2: } 5 \text { (35.7) } \\
\text { CARS-Total Score } \\
\text { G1: } 45.25 \pm 6.191 \\
\text { G2: } 36.71 \pm 5.594\end{array}$ & $\begin{array}{l}\text { EOT } \\
\text { Mild/Moderate Severity } \\
\text { G1: } 9(56.3) \\
\text { G2: } 9(64.3) \\
\text { G1 vs } \mathrm{G} 2, \mathrm{p}=\mathrm{ns} \\
\text { CARS - Severe } \\
\text { G1: } 7(43.8) \\
\text { G2: } 5(35.7) \\
\text { G1 vs G2, p=ns } \\
\text { CARS-Total Score } \\
\text { G1: } 37.06 \pm 5.882 \\
\text { G2: } 34.71 \pm 4.631 \\
\text { G1 vs } \mathrm{G} 2, p<0.001\end{array}$ \\
\hline $\begin{array}{l}\text { Bent et al., } 2011^{45} \\
\text { RCT } \\
\text { G1: Omega-3 fatty acid ( } 1.3 \mathrm{~g} / \text { day), } \\
\text { 14/13 } \\
\text { G2: Placebo, } 13 / 12 \\
12 \text { weeks/EOT }\end{array}$ & $\begin{array}{l}\text { Age, months } \\
\text { G1: } 70.2 \pm 22 \\
\text { G2: } 69.8 \pm 17 \\
\text { IQ } \\
\text { G1: } 77.5 \pm 27 \\
\text { G2: } 77.5 \pm 17\end{array}$ & $\begin{array}{l}\text { ABC-Hyperactivity } \\
\text { G1: } 16.8 \pm 13 \\
\text { G2: } 20.3 \pm 8 \\
\text { PPVT } \\
\text { G1: } 72.2 \pm 28 \\
\text { G2: } 85.8 \pm 12 \\
\text { EVT }\end{array}$ & $\begin{array}{l}\text { Mean change score } \\
\text { ABC }- \text { Hyperactivity } \\
\text { G1: } 2.7 \pm 4.8 \\
\text { G2: } 0.3 \pm 7.2 \\
\text { G1 vs } G 2: p=n s \\
\text { PPVT } \\
\text { G1: } 2.7 \pm 11.6 \\
\text { G2: } 1.9 \pm 12.4\end{array}$ \\
\hline
\end{tabular}




\begin{tabular}{|c|c|c|c|}
\hline $\begin{array}{c}\text { Author, Year } \\
\text { Study Design } \\
\text { Groups (dose), N enrollment I } \\
\mathrm{N} \text { final } \\
\\
\text { Treatment duration/Follow-up } \\
\text { timepoint post-treatment } \\
\text { Risk of Bias }\end{array}$ & $\begin{array}{c}\text { Mean age, } \\
\text { years } \pm \text { SD } \\
\text { Mean IQ } \pm S D\end{array}$ & $\begin{array}{c}\text { Outcome } \\
\text { measure/Baseline } \\
\text { scores, mean } \pm S D\end{array}$ & $\begin{array}{c}\text { Outcome } \\
\text { measure/Post- } \\
\text { treatment scores, } \\
\text { mean } \pm \text { SD }\end{array}$ \\
\hline Low RoB & & $\begin{array}{l}\text { G1: } 70.8 \pm 33 \\
\text { G2: } 86.4 \pm 14 \\
\text { SRS } \\
\text { G1: } 76.9 \pm 11 \\
\text { G2: } 79.0 \pm \\
\text { BASC-Externalizing } \\
\text { G1: } 53.8 \pm 13 \\
\text { G2: } 66.3 \pm 25 \\
\text { BASC- Internalizing } \\
\text { G1: } 43.3 \pm 10 \\
\text { G2: } 50.1 \pm 9 \\
\text { BASC-Behavioral } \\
\text { G1: } 60.9 \pm 14 \\
\text { G2: } 65.4 \pm 3 \\
\text { BASC- Adaptive skill } \\
\text { G1: } 29.8 \pm 9 \\
\text { G2: } 31.9 \pm 9 \\
\text { BASC-Hyperactivity } \\
\text { G1: } 61.8 \pm 17 \\
\text { G2: } 64.6 \pm 7\end{array}$ & 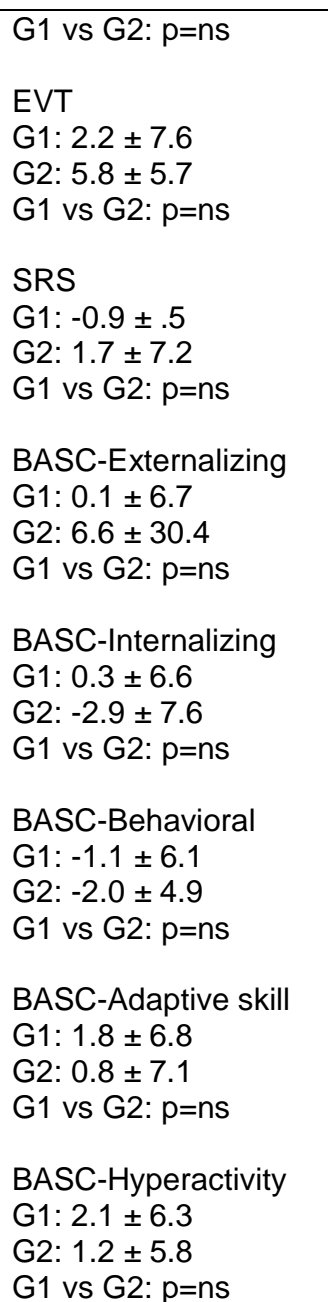 \\
\hline $\begin{array}{l}\text { Geier, et al., } 2011^{46} \\
\text { RCT } \\
\text { G1: Levocarnitine (50 mg/kg/day), } \\
\text { 19/16 } \\
\text { G2: Placebo, } 11 / 11 \\
3 \text { months/EOT } \\
\text { High RoB }\end{array}$ & $\begin{array}{l}\text { Age: } \\
\text { G1: } 6.3 \pm 2.4 \\
\text { G2: } 6.7 \pm 1.6 \\
\text { IQ } \\
\text { NR }\end{array}$ & $\begin{array}{l}\text { CARS } \\
\text { G1: } 35.7 \pm 5.3 \\
\text { G2: } 38.2 \pm 6.0 \\
\text { CGI } \\
\text { G1: } 2.0 \\
\text { G2: } 2.0 \\
\text { Hand Muscle Testing } \\
\text { G1: } 32.7 \pm 13.9 \\
\text { G2: } 35.3 \pm 13.2 \\
\text { ATEC Total } \\
\text { G1: } 55.1 \pm 23.3 \\
\text { G2: } 62.8 \pm 31.7\end{array}$ & $\begin{array}{l}\text { EOT } \\
\text { CARS } \\
\text { G1: } 33.8 \pm 5.8 \\
\text { G2: } 38.4 \pm 6.3 \\
p=.02 \\
\text { CGI } \\
\text { G1: } 1.5 \pm 0.63 \\
\text { G2: } 2.09 \pm 0.7 \\
p=.03 \\
\text { Hand Muscle Testing } \\
\text { G1: } 34.3 \pm 16.7 \\
\text { G2: } 35.1 \pm 7.5 \\
p=\text { ns }\end{array}$ \\
\hline
\end{tabular}




\begin{tabular}{|c|c|c|c|}
\hline $\begin{array}{c}\text { Author, Year } \\
\text { Study Design } \\
\text { Groups (dose), N enrollment I } \\
\mathrm{N} \text { final } \\
\\
\text { Treatment duration/Follow-up } \\
\text { timepoint post-treatment } \\
\text { Risk of Bias }\end{array}$ & $\begin{array}{c}\text { Mean age, } \\
\text { years } \pm \text { SD } \\
\text { Mean IQ } \pm S D\end{array}$ & $\begin{array}{c}\text { Outcome } \\
\text { measure/Baseline } \\
\text { scores, mean } \pm S D\end{array}$ & $\begin{array}{c}\text { Outcome } \\
\text { measure/Post- } \\
\text { treatment scores, } \\
\text { mean } \pm \text { SD }\end{array}$ \\
\hline & & $\begin{array}{l}\text { ATEC Speech } \\
\text { G1: } 9.9 \pm 6.3 \\
\text { G2: } 10.5 \pm 6.4 \\
\text { ATEC Sociability } \\
\text { G1: } 12 \pm 7.1 \\
\text { G2: } 11.9 \pm 7.7 \\
\text { ATEC Cognitive } \\
\text { G1: } 12.7 \pm 6.1 \\
\text { G2: } 14.4 \pm 7.6 \\
\text { ATEC Health/Behavior } \\
\text { G1: } 20.5 \pm 8.1 \\
\text { G2: } 26 \pm 14.8\end{array}$ & $\begin{array}{l}\text { ATEC Total } \\
\text { G1: } 40.1 \pm 22.8 \\
\text { G2: } 56 \pm 27.6 \\
p=n s \\
\text { ATEC Speech } \\
\text { G1: } 7.8 \pm 5.9 \\
\text { G2: } 10.9 \pm 7.2 \\
p=\text { ns } \\
\text { ATEC Sociability } \\
\text { G1: } 8.3 \pm 5.9 \\
\text { G2: } 10.8 \pm 8.4 \\
p=n s \\
\text { ATEC Cognitive } \\
\text { G1: } 9.2 \pm 5.5 \\
\text { G2: } 14.9 \pm 7.3 \\
p=.009 \\
\text { ATEC Health/Behavior } \\
\text { G1: } 14.8 \pm 7.9 \\
\text { G2: } 19.5 \pm 10.9 \\
p=n s\end{array}$ \\
\hline $\begin{array}{l}\text { Bertoglio et al., } 2010^{47} \\
\text { RCT } \\
\text { G1: Methyl B12 }(64.5 \mu \mathrm{g} / \mathrm{kg}) \\
\text { G2: Placebo } \\
\text { Crossover design } \mathrm{N}=30 \text { total } \\
12 \text { weeks/EOT } \\
\text { Low RoB }\end{array}$ & $\begin{array}{l}\text { Age } \\
\text { G1 + G2: 3-8 } \\
\text { IQ } \\
\text { NR }\end{array}$ & $\begin{array}{l}\text { CGIS } \\
\text { NR }\end{array}$ & $\begin{array}{l}\text { No significant group } \\
\text { difference in behavior } \\
\text { measures } \\
\text { EOT } \\
\text { CGIS Improvement ( } \geq 1 \\
\text { point) } \\
\mathrm{n}(\%) \\
\text { G1+G2: } 9 \text { (30) } \\
3 \text { received placebo first } \\
\text { and } 6 \text { received active } \\
\text { treatment } \\
\text { CBCL improvement } \\
\text { ( } \geq 5 \text { points): } \\
\text { G1+G2: } 3 \text { (10) } \\
\text { ABC improvement }(\geq 5 \\
\text { points): } \\
\text { G1+G2: } 5 \text { (16.7) }\end{array}$ \\
\hline
\end{tabular}




\begin{tabular}{|c|c|c|c|}
\hline $\begin{array}{c}\text { Author, Year } \\
\text { Study Design } \\
\text { Groups (dose), N enrollment I } \\
\text { N final } \\
\\
\text { Treatment duration/Follow-up } \\
\text { timepoint post-treatment } \\
\text { Risk of Bias }\end{array}$ & $\begin{array}{c}\text { Mean age, } \\
\text { years } \pm \text { SD } \\
\text { Mean IQ } \pm S D\end{array}$ & $\begin{array}{c}\text { Outcome } \\
\text { measure/Baseline } \\
\text { scores, mean } \pm S D\end{array}$ & $\begin{array}{c}\text { Outcome } \\
\text { measure/Post- } \\
\text { treatment scores, } \\
\text { mean } \pm \text { SD }\end{array}$ \\
\hline $\begin{array}{l}\text { Munasinghe et al., } 2010^{48} \\
\text { RCT } \\
\text { G1: Digestive enzyme supplement- } \\
\text { Peptizyde (up to } 2 \text { capsules/day) } \\
\text { G2: Placebo ( } 360 \mathrm{mg} / \mathrm{day} \text { ) } \\
\text { Crossover design } \mathrm{N}=43 \text { total } \\
3 \text { months/EOT } \\
\text { Moderate RoB }\end{array}$ & $\begin{array}{l}\text { Age, months } \\
\text { G1 + G2: } 5.78 \pm \\
1.77 \\
\text { IQ } \\
\text { NR }\end{array}$ & $\begin{array}{l}\text { GBRS } \\
\text { G1+G2: } 4 \\
\text { ARS } \\
\text { G1+G2: } 4 \\
\text { LDS } \\
\text { NR }\end{array}$ & $\begin{array}{l}\text { EOT } \\
\text { Food variety score } \\
\text { G1: } 4.42 \pm 0.62 \\
\text { G2: } 4.06 \pm 0.45 \\
p=0.02 \\
\text { Parent Behavior score } \\
\text { G1: } 4.29 \pm 0.79 \\
\text { G2: } 4.11 \pm 0.73 \\
p=n s \\
\text { Gl symptoms score } \\
\text { G1: } 4.01 \pm 0.68 \\
\text { G2: } 3.87 \pm 0.36 \\
p=n s \\
\text { Sleep Quality score } \\
\text { G1: } 3.95 \pm 0.75 \\
\text { G2: } 3.87 \pm 0.56 \\
p=n s \\
\text { Therapist engagement } \\
\text { score } \\
\text { G1: } 4.59 \pm 0.96 \\
\text { G2: } 4.43 \pm 0.55 \\
p=n s \\
\text { LDS Vocabulary } \\
\text { percentile score } \\
\text { G1: } 56.95 \pm 28.6 \\
\text { G2: } 55.59 \pm 28.6 \\
p=n s \\
\text { LDS Sentence length } \\
\text { percentile score } \\
\text { G1: } 62.38 \pm 26.2 \\
\text { G2: } 63.91 \pm 24.9 \\
p=n s\end{array}$ \\
\hline $\begin{array}{l}\text { Hendren et al., } 2016^{49} \\
\text { RCT } \\
\text { G1: Methyl B-12 }(75 \mu \mathrm{g} / \mathrm{kg} \text { every } 3 \\
\text { days), } 28 / 27 \\
\text { G2: Placebo ( } 75 \mu \mathrm{g} / \mathrm{kg} \text { every } 3 \\
\text { days), } 29 / 23 \\
8 \text { weeks/EOT } \\
\text { Moderate ROB }\end{array}$ & $\begin{array}{l}\text { Age (months) } \\
\text { G1: } 67 \pm 16 \\
\text { G2: } 58 \pm 14 \\
\text { IQ-Stanford } \\
\text { Binet } \\
\text { G1: } 64 \pm 18 \\
\text { G2: } 67 \pm 20\end{array}$ & $\begin{array}{l}\text { CGI-Severity } \\
\text { G1: } 5.2 \pm 0.7 \\
\text { G2: } 5.1 \pm 0.6 \\
\text { ABC-Hyperactivity } \\
\text { G1: } 25 \pm 10.1 \\
\text { G2: } 22.4 \pm 11.5 \\
\text { ABC-Inappropriate } \\
\text { Speech } \\
\text { G1: } 4.2 \pm 3.2 \\
\text { G2: } 2.9 \pm 3.2\end{array}$ & $\begin{array}{l}\text { Change Score } \\
\text { CGI-Improvement } \\
\text { G1: } 2.4 \pm 0.8 \\
\text { G2: } 3.1 \pm 0.8 \\
\text { G1 vs } G 2, p=0.005 \\
\\
\text { ABC-Hyperactivity } \\
\text { G1: }-0.9 \pm 4.8 \\
\text { G2: }-3.9 \pm 7.1 \\
\text { G1 vs } G 2, p=n s ; \text { ES=- } \\
0.48 \\
\text { ABC-Inappropriate }\end{array}$ \\
\hline
\end{tabular}




\begin{tabular}{|c|c|c|c|}
\hline $\begin{array}{c}\text { Author, Year } \\
\text { Study Design } \\
\text { Groups (dose), N enrollment I } \\
\mathrm{N} \text { final } \\
\\
\text { Treatment duration/Follow-up } \\
\text { timepoint post-treatment } \\
\text { Risk of Bias }\end{array}$ & $\begin{array}{c}\text { Mean age, } \\
\text { years } \pm \text { SD } \\
\text { Mean IQ } \pm S D\end{array}$ & $\begin{array}{c}\text { Outcome } \\
\text { measure/Baseline } \\
\text { scores, mean } \pm S D\end{array}$ & $\begin{array}{c}\text { Outcome } \\
\text { measure/Post- } \\
\text { treatment scores, } \\
\text { mean } \pm \text { SD }\end{array}$ \\
\hline & & $\begin{array}{l}\text { ABC-Irritability } \\
\text { G1: } 15.2 \pm 10.4 \\
\text { G2: } 11.1 \pm 8.4 \\
\text { ABC-Social } \\
\text { Withdrawal/Lethargy } \\
\text { G1: } 15 \pm 9.1 \\
\text { G2: } 14.2 \pm 8.5 \\
\text { ABC-Stereotypic } \\
\text { Behavior } \\
\text { G1: } 7.1 \pm 4.6 \\
\text { G2: } 7.4 \pm 5.1 \\
\text { SRS-Total Score } \\
\text { G1: } 90 \pm 13.7 \\
\text { G2: } 83.5 \pm 10.6\end{array}$ & $\begin{array}{l}\text { Speech } \\
\text { G1: } 0.3 \pm 1.4 \\
\text { G2: }-0.3 \pm 1.6 \\
\text { G1 vs } G 2, p=n s ; E S=- \\
0.43 \\
\text { ABC-Irritability } \\
\text { G1: }-0.1 \pm 3.7 \\
\text { G2: }-2.6 \pm 4.3 \\
\text { G1 vs } G 2, p=0.08 \text {; } \\
\text { ES=-0.61 } \\
\text { ABC-Social } \\
\text { Withdrawal/Lethargy } \\
\text { G1: }-1.9 \pm 5.8 \\
\text { G2: }-1.2 \pm 7.1 \\
\text { G1 vs } G 2, p=n s ; \\
\text { ES=0.12 } \\
\text { ABC-Stereotypic } \\
\text { Behavior } \\
\text { G1: }-0.3 \pm 2.2 \\
\text { G2: } 0.3 \pm 3.2 \\
\text { G1 vs G2, } p=n s ; \\
\text { ES=0.23 } \\
\text { SRS-Total Score } \\
\text { G1: }-1.6 \pm 7.7 \\
\text { G2: }-4.1 \pm 7.7 \\
\text { G1 vs } G 2, p=n s ; E S=- \\
0.32\end{array}$ \\
\hline
\end{tabular}

ABC-Aberrant Behavior Checklist; CGI-Clinical Global Impression; VABS-Vineland Adaptive Behavior Scale; EOT-End of Treatment; PDDBIPervasive Developmental Disorders Behavior Inventory; BASC-Behavior Assessment System for Children; CARS-Childhood Autism Rating Scale; GBRS-Global Rating Scale; SRS-Social Responsiveness Scale; PPVT-Peabody Picture Vocabulary Test; EVT-Expressive Vocabulary Test; ATEC-Autism Treatment Evaluation Checklist; CGIS-Clinical Global Impressions Scale; CBCL-Child Behavior Checklist; GBRS-Global Rating Scale; ARS-Additional Rating Scale; LDS-Language Development Survey

Table F-4. Key findings in studies of specialized diets

\begin{tabular}{|c|c|c|c|}
\hline $\begin{array}{c}\text { Author, Year } \\
\text { Study Design } \\
\text { Groups (dose), N enrollment I } \\
\mathrm{N} \text { final } \\
\\
\text { Treatment duration/Follow-up } \\
\text { timepoint post-treatment } \\
\text { Risk of Bias }\end{array}$ & $\begin{array}{l}\text { Mean age, } \\
\text { years } \pm \text { SD } \\
\text { Mean IQ } \pm S D\end{array}$ & $\begin{array}{l}\text { Outcome } \\
\text { measure/Baseline } \\
\text { scores, mean } \pm S D\end{array}$ & $\begin{array}{c}\text { Outcome } \\
\text { measure/Post- } \\
\text { treatment scores, } \\
\text { mean } \pm \text { SD }\end{array}$ \\
\hline
\end{tabular}




\begin{tabular}{|c|c|c|c|}
\hline $\begin{array}{c}\text { Author, Year } \\
\text { Study Design } \\
\text { Groups (dose), N enrollment } / \\
\mathrm{N} \text { final } \\
\\
\text { Treatment duration/Follow-up } \\
\text { timepoint post-treatment } \\
\text { Risk of Bias }\end{array}$ & $\begin{array}{c}\text { Mean age, } \\
\text { years } \pm \text { SD } \\
\text { Mean IQ } \pm S D\end{array}$ & $\begin{array}{c}\text { Outcome } \\
\text { measure/Baseline } \\
\text { scores, mean } \pm S D\end{array}$ & $\begin{array}{c}\text { Outcome } \\
\text { measure/Post- } \\
\text { treatment scores, } \\
\text { mean } \pm \text { SD }\end{array}$ \\
\hline $\begin{array}{l}\text { Hyman et al., } 2016^{50} \\
\text { RCT } \\
\text { Gluten-free/casein-free diet } \\
\text { followed by weekly challenges of } \\
\text { gluten, casein, gluten and casein } \\
\text { and placebo } \\
\text { G1: gluten } \\
\text { G2: casein } \\
\text { G3: gluten and casein } \\
\text { G4: placebo } \\
\text { Crossover study n=14 } \\
12 \text { weeks/EOT } \\
\text { Low RoB }\end{array}$ & $\begin{array}{l}\text { Age } \\
3.78 \pm 0.60 \\
\text { IQ } \\
\text { NR }\end{array}$ & $\begin{array}{l}\text { LS means } \\
\text { Day before challenge } \\
\text { Conners- research } \\
\text { asst rater } \\
\text { G1: } 6,65 \pm 2.38 \\
\text { G2: } 5.71 \pm 2.18 \\
\text { G3: } 5.32 \pm 2.13 \\
\text { G4: } 6.17 \pm 3.01 \\
\text { Conners- teacher } \\
\text { G1: } 8.50 \pm 3.72 \\
\text { G2: } 8.29 \pm 3.73 \\
\text { G3: } 6.89 \pm 2.40 \\
\text { G4: } 8.84 \pm 5.92 \\
\text { Conners- parent } \\
\text { G1: } 6.10 \pm 3.53 \\
\text { G2: } 6.48 \pm 3.99 \\
\text { G3: } 5.79 \pm 4.17 \\
\text { G4: } 5.71 \pm 3.50 \\
\text { Day before challenge: } \\
\text { NR } \\
\text { Day of challenge } \\
\text { Sensory motor } \\
\text { G1: } 0.34 \pm 0.27 \\
\text { G2: } 0.32 \pm 0.28 \\
\text { G3: } 0.31 \pm 0.37 \\
\text { G4: } 0.33 \pm 0.30 \\
\text { Social relationships } \\
\text { G1: }-0.30 \pm 0.24 \\
\text { G2: }-0.30 \pm 0.21 \\
\text { G3: }-0.27 \pm 0.17 \\
\text { G4: }-0.20 \pm 0.16 \\
\\
\text { Affectual relationships } \\
\text { G1: } 0.08 \pm 0.12 \\
\text { G2: } 0.09 \pm 0.13 \\
\text { G3: } 0.05 \pm 0.11 \\
\text { G4: } 0.09 \pm 0.11 \\
\text { Sensory responses } \\
\text { G1: } 0.25 \pm 0.14 \\
\text { G2: } 0.26 \pm 0.19 \\
\text { G3: } 0.21 \pm 0.14 \\
\text { G4: } 0.30 \pm 0.19 \\
\\
\text { Language } \\
\text { G1: }-0.07 \pm 0.31 \\
\text { G2: }-0.04 \pm 0.27 \\
\text { G3: }-0.03 \pm 0.30\end{array}$ & $\begin{array}{l}\text { EOT } \\
\text { LS means } \\
\text { Day after challenge } \\
\text { Conners- research } \\
\text { asst rater } \\
\text { G1: } 5.57 \pm 1.61 \\
\text { G2: } 6.19 \pm 2.47 \\
\text { G3: } 6.73 \pm 3.24 \\
\text { G4: } 6.55 \pm 2.83 \\
\text { p=ns } \\
\text { Conners- teacher } \\
\text { G1: } 7.76 \pm 3.08 \\
\text { G2: } 7.42 \pm 2.79 \\
\text { G3: } 7.19 \pm 2.81 \\
\text { G4: } 7.43 \pm 3.88 \\
\text { p=ns } \\
\text { Conners- parent } \\
\text { G1: } 6.75 \pm 3.69 \\
\text { G2: } 6.31 \pm 3.75 \\
\text { G3: } 7.15 \pm 4.42 \\
\text { G4: } 7.15 \pm 4.25 \\
\text { p=ns } \\
\text { Day after challenge } \\
\text { Sensory motor } \\
\text { G1: } 0.28 \pm 0.29 \\
\text { G2: } 0.29 \pm 0.22 \\
\text { G3: } 0.43 \pm 0.42 \\
\text { G4: } 0.32 \pm 0.21 \\
\text { p=ns } \\
\text { Social relationships } \\
\text { G1: }-0.27 \pm 0.21 \\
\text { G2: }-0.28 \pm 0.18 \\
\text { G3: }-0.30 \pm 0.19 \\
\text { G4: }-0.26 \pm 0.16 \\
\text { p=ns } \\
\text { Affectual relationships } \\
\text { G1: } 0.04 \pm 0.06 \\
\text { G2: } 0.11 \pm 0.13 \\
\text { G3: } 0.09 \pm 0.15 \\
\text { G4: } 0.07 \pm 0.09 \\
\text { p=ns } \\
\text { Sensory responses } \\
\text { G1: } 0.29 \pm 0.17 \\
\text { G2: } 0.27 \pm 0.16 \\
\text { G3: } 0.26 \pm 0.17 \\
\text { (a) }\end{array}$ \\
\hline
\end{tabular}




\begin{tabular}{|c|c|c|c|}
\hline $\begin{array}{c}\text { Author, Year } \\
\text { Study Design } \\
\text { Groups (dose), N enrollment I } \\
\mathrm{N} \text { final } \\
\\
\text { Treatment duration/Follow-up } \\
\text { timepoint post-treatment } \\
\text { Risk of Bias }\end{array}$ & $\begin{array}{c}\text { Mean age, } \\
\text { years } \pm \text { SD } \\
\text { Mean IQ } \pm S D\end{array}$ & $\begin{array}{c}\text { Outcome } \\
\text { measure/Baseline } \\
\text { scores, mean } \pm S D\end{array}$ & $\begin{array}{c}\text { Outcome } \\
\text { measure/Post- } \\
\text { treatment scores, } \\
\text { mean } \pm \text { SD }\end{array}$ \\
\hline & & $\begin{array}{l}\text { G4: } 0.00 \pm 0.24 \\
\text { Day before challenge } \\
\text { Minutes of sleep } \\
\text { G1: } 631 \pm 67.3 \\
\text { G2: } 627 \pm 76.6 \\
\text { G3: } 616.7 \pm 48.3 \\
\text { G4: } 693 \pm 56.4 \\
\text { Number of wakings } \\
\text { G1: } 0.34 \pm 1.53 \\
\text { G2: } 0.10 \pm 0.30 \\
\text { G3: } 0.11 \pm 0.40 \\
\text { G4: } 0.08 \pm 0.27\end{array}$ & $\begin{array}{l}\text { G4: } 0.32 \pm 0.18 \\
p=n s \\
\text { Language } \\
\text { G1: }-0.03 \pm 0.25 \\
\text { G2: }-0.03 \pm 0.25 \\
\text { G3: }-0.07 \pm 0.32 \\
\text { G4: } 0.00 \pm 0.25 \\
\text { p=ns } \\
\text { Day after challenge } \\
\text { Minutes of sleep } \\
\text { G1: } 646 \pm 69.1 \\
\text { G2: } 641 \pm 68.2 \\
\text { G3: } 633 \pm 70.7 \\
\text { G4: } 641 \pm 61.3 \\
\text { p=ns } \\
\text { Number of wakings } \\
\text { G1: } 0.20 \pm 0.58 \\
\text { G2: } 0.03 \pm 0.16 \\
\text { G3: } 0.17 \pm 0.45 \\
\text { G4: } 0.05 \pm 0.22 \\
\text { p=ns }\end{array}$ \\
\hline $\begin{array}{l}\text { Navarro et al., } 2015^{51} \\
\text { RCT } \\
\text { G1: "gluten-dairy containing } \\
\text { (GD(+)) diet", 6/6 } \\
\text { G2: gluten-dairy free }(\mathrm{GD}(-)) \text { diet, } \\
\text { 6/6 } \\
4 \text { weeks/EOT } \\
\text { Low RoB }\end{array}$ & $\begin{array}{l}\text { Age } \\
\text { G1: } 6(6-7) \\
\text { G2: } 5.5(4-7) \\
\text { IQ } \\
\text { G1: } 55(52-88) \\
\text { G2: } 79(76-97)\end{array}$ & $\begin{array}{l}\text { ABC-Irritability } \\
\text { G1: } 19.77 \pm 15.15 \\
\text { G2: } 10.50 \pm 8.55 \\
\text { ABC- } \\
\text { Hyperactivity } \\
\text { G1: } 23.20 \pm 11.28 \\
\text { G2: } 22.17 \pm 7.31 \\
\text { Connors Parent Rating } \\
\text { Scale-Revised- } \\
\text { Cognitive } \\
\text { Problems/Inattention } \\
\text { G1: } 12.17 \pm 7.11 \\
\text { G2: } 15.83 \pm 3.82\end{array}$ & $\begin{array}{l}\text { EOT } \\
\text { ABC-Irritability } \\
\text { G1: } 16.0 \\
\text { G2: } 14.50 \\
\text { G1 vs G2, p= NR } \\
\text { ABC-Hyperactivity } \\
\text { G1: } 26.5 \\
\text { G2: NR } \\
\text { Connors Parent Rating } \\
\text { Scale-Revised } \\
\text { G1: } 21 \\
\text { G2: NR }\end{array}$ \\
\hline $\begin{array}{l}\text { Pusponegoro et al., } 2015^{52} \\
\text { RCT } \\
\text { G1: Gluten-casein, 38/24 } \\
\text { G2: Placebo, 36/26 } \\
7 \text { days/EOT } \\
\text { Moderate RoB }\end{array}$ & $\begin{array}{l}\text { Age, median } \\
\text { (range) } \\
\text { G1: } 5.4(4.8- \\
6.7) \\
\text { G2: } 5.1(4.3- \\
6.1) \\
\text { IQ } \\
\text { NR }\end{array}$ & $\begin{array}{l}\text { Pervasive } \\
\text { Developmental } \\
\text { Disorder Behavior } \\
\text { Inventory - Approach } \\
\text { Withdrawal problems } \\
\text { composite } \\
\text { AWPC } \\
\text { G1: } 56.96 \pm 11.72 \\
\text { G2: } 57.08 \pm 11.46 \\
\text { Gastrointestinal }\end{array}$ & $\begin{array}{l}\text { EOT } \\
\text { Pervasive } \\
\text { Developmental } \\
\text { Disorder Behavior } \\
\text { Inventory - Approach } \\
\text { Withdrawal problems } \\
\text { composite } \\
\text { AWPC } \\
\text { G1: } 39.38 \pm 2.89 \\
\text { G2: } 39.63 \pm 3.05 \\
\text { G1 vs G2: } p=n s\end{array}$ \\
\hline
\end{tabular}




\begin{tabular}{|c|c|c|c|}
\hline $\begin{array}{c}\text { Author, Year } \\
\text { Study Design } \\
\text { Groups (dose), N enrollment I } \\
\mathrm{N} \text { final } \\
\\
\text { Treatment duration/Follow-up } \\
\text { timepoint post-treatment } \\
\text { Risk of Bias }\end{array}$ & $\begin{array}{c}\text { Mean age, } \\
\text { years } \pm \text { SD } \\
\text { Mean IQ } \pm \text { SD }\end{array}$ & $\begin{array}{c}\text { Outcome } \\
\text { measure/Baseline } \\
\text { scores, mean } \pm S D\end{array}$ & $\begin{array}{c}\text { Outcome } \\
\text { measure/Post- } \\
\text { treatment scores, } \\
\text { mean } \pm \text { SD }\end{array}$ \\
\hline & & $\begin{array}{l}\text { Symptom Severity } \\
\text { Index, median } \\
\text { G1: } 0 \\
\text { G2: } 0\end{array}$ & $\begin{array}{l}\text { Gastrointestinal } \\
\text { Symptom Severity } \\
\text { Index, median (range) } \\
\text { G1: } 3(0-9) \\
\text { G2: } 0(0-7) \\
\text { G1 vs G2: } \mathrm{p}=\mathrm{ns}\end{array}$ \\
\hline $\begin{array}{l}\text { Whiteley et al., } 2010^{53}{ }^{746} \\
\text { RCT } \\
\text { G1: Gluten and casein-free diet, } \\
\text { 38/26 } \\
\text { G2: No diet, } 34 / 29 \\
24 \text { months/EOT } \\
\text { Moderate RoB }\end{array}$ & $\begin{array}{l}\text { Age, months } \\
\text { G1: } 94.2 \text { [IQR } \\
\text { 76.6-118.0] } \\
\text { G2: 96.4 [IQR } \\
76.3-120.3] \\
\text { IQ } \\
\text { NR }\end{array}$ & $\begin{array}{l}\text { ADOS-Communication } \\
\text { G1: } 1.13 \\
\text { G2: } 1.00 \\
\text { ADOS-Social } \\
\text { G1: } 1.18 \\
\text { G2: } 1.22 \\
\text { ADOS-Repetitive } \\
\text { G1: } 0.46 \\
\text { G2: } 0.36 \\
\text { GARS-Social } \\
\text { G1: } 6.96 \\
\text { G2: } 6.41 \\
\text { GARS-Communication } \\
\text { G1: } 7.23 \\
\text { G2: } 7.28 \\
\text { GARS-Stereotyped } \\
\text { G1: } 6.81 \\
\text { G2: } 6.76 \\
\text { VABS-Communication } \\
\text { G1: } 66.46 \\
\text { G2: } 62.45 \\
\text { VABS-Social } \\
\text { G1: } 64.58 \\
\text { G2: } 64.03 \\
\text { VABS-Daily Living } \\
\text { G1: } 59.88 \\
\text { G2: } 55.55 \\
\text { ADHD-IV scale- } \\
\text { Inattention } \\
\text { G1: } 11.96 \\
\text { G2: } 11.21\end{array}$ & $\begin{array}{l}\text { 8 months } \\
\text { ADOS-Communication } \\
\text { G1: } 0.87 \\
\text { G2: } 1.07 \\
\text { G1 vs G2: } p=n s \\
\text { ADOS-Social } \\
\text { G1: } 1.09 \\
\text { G2: } 1.30 \\
\text { G1 vs G2: p=ns } \\
\text { ADOS-Repetitive } \\
\text { G1: } 0.35 \\
\text { G2: } 0.34 \\
\text { G1 vs G2: p=ns } \\
\text { GARS-Social (8 mos) } \\
\text { G1: } 5.88 \\
\text { G2: } 6.34 \\
\text { GARS- Social (12 } \\
\text { mos) } \\
\text { G1: } 5.38 \\
\text { G2: } 6.00 \\
\text { G1 vs G2: p=ns } \\
\text { GARS-Communication } \\
\text { (8 mos) } \\
\text { G1: } 6.00 \\
\text { G2: } 6.76 \\
\text { GARS-Communication } \\
\text { (12 mos) } \\
\text { G1: } 5.74 \\
\text { G2: } 5.81 \\
\text { G1 vs G2: p=ns } \\
\text { GARS-Stereotyped (8 } \\
\text { mos) } \\
\text { G1: } 5.85 \\
\text { G2: } 5.97 \\
\end{array}$ \\
\hline
\end{tabular}




\begin{tabular}{|c|c|c|c|}
\hline $\begin{array}{c}\text { Author, Year } \\
\text { Study Design } \\
\text { Groups (dose), N enrollment I } \\
\mathrm{N} \text { final } \\
\\
\text { Treatment duration/Follow-up } \\
\text { timepoint post-treatment } \\
\text { Risk of Bias }\end{array}$ & $\begin{array}{c}\text { Mean age, } \\
\text { years } \pm \text { SD } \\
\text { Mean IQ } \pm S D\end{array}$ & $\begin{array}{c}\text { Outcome } \\
\text { measure/Baseline } \\
\text { scores, mean } \pm S D\end{array}$ & $\begin{array}{c}\text { Outcome } \\
\text { measure/Post- } \\
\text { treatment scores, } \\
\text { mean } \pm \text { SD }\end{array}$ \\
\hline & & $\begin{array}{l}\text { ADHD-IV scale- } \\
\text { Hyperactivity } \\
\text { G1: } 10.31 \\
\text { G2: } 10.79\end{array}$ & $\begin{array}{l}\text { GARS-Stereotyped (12 } \\
\text { mos) } \\
\text { G1: } 5.35 \\
\text { G2: } 5.37 \\
\text { G1 vs G2: p=ns } \\
\text { VABS-Communication } \\
\text { (8 mos) } \\
\text { G1: } 66.69 \\
\text { G2: } 63.46 \\
\text { VABS-Communication } \\
\text { (12 mos) } \\
\text { G1: } 67.38 \\
\text { G2: } 64.07 \\
\text { G1 vs G2: p=ns } \\
\text { VABS-Social (8 mos) } \\
\text { G1: 66.12 } \\
\text { G2: } 65.39 \\
\text { VABS-Social (12 mos) } \\
\text { G1: } 60.42 \\
\text { G2: } 61.21 \\
\text { G1 vs G2: p=ns } \\
\text { VABS-Daily Living (8 } \\
\text { mos) } \\
\text { G1: 62.92 } \\
\text { G2: } 55.18 \\
\text { VABS-Daily Living (12 } \\
\text { mos) } \\
\text { G1: } 63.35 \\
\text { G2: } 53.96 \\
\text { G1 vs G2: p=ns } \\
\text { ADHD-IV scale- } \\
\text { Inattention (8 mos) } \\
\text { G1: } 9.81 \\
\text { G2: } 11.52 \\
\text { ADHD-IV scale- } \\
\text { Inattention (12 mos) } \\
\text { G1: } 9.77 \\
\text { G2: } 11.11 \\
\text { G1 vs G2: p=ns } \\
\text { ADHD-IV scale- } \\
\text { Hyperactivity (8 mos) } \\
\text { G1: } 8.27\end{array}$ \\
\hline
\end{tabular}




\begin{tabular}{|c|c|c|c|}
\hline $\begin{array}{c}\text { Author, Year } \\
\text { Study Design } \\
\text { Groups (dose), N enrollment I } \\
\mathrm{N} \text { final } \\
\\
\text { Treatment duration/Follow-up } \\
\text { timepoint post-treatment } \\
\text { Risk of Bias }\end{array}$ & $\begin{array}{c}\text { Mean age, } \\
\text { years } \pm \text { SD } \\
\text { Mean IQ } \pm S D\end{array}$ & $\begin{array}{l}\text { Outcome } \\
\text { measure/Baseline } \\
\text { scores, mean } \pm S D\end{array}$ & $\begin{array}{c}\text { Outcome } \\
\text { measure/Post- } \\
\text { treatment scores, } \\
\text { mean } \pm \text { SD }\end{array}$ \\
\hline & & & $\begin{array}{l}\text { G2:10.62 } \\
\text { ADHD-IV scale- } \\
\text { Hyperactivity(12 mos) } \\
\text { G1: } 8.62 \\
\text { G2:10.00 } \\
\text { G1 vs } \mathrm{G} 2: \mathrm{p}=\mathrm{ns}\end{array}$ \\
\hline $\begin{array}{l}\text { Knivsberg et al., } 2002^{54,55} \\
\text { RCT } \\
\text { G1: Gluten and Casein free diet, } \\
\text { 10/10 } \\
\text { G2: Normal Diet, } 10 / 10 \\
12 \text { months/EOT } \\
\text { Moderate ROB }\end{array}$ & $\begin{array}{l}\text { Age (months) } \\
\text { G1: } 91 \text { (62-120) } \\
\text { G2: } 86 \text { (59-127) } \\
\text { IQ } \\
\text { NR }\end{array}$ & $\begin{array}{l}\text { Leiter R-Nonverbal } \\
\text { Cognitive Level } \\
\text { G1: } 81 \pm 35.9 \\
\text { G2: } 84.6 \pm 36.6 \\
\text { Parent Questionnaire } \\
\text { ND } \\
\text { DPBC-Communication } \\
\text { and Interaction } \\
\text { G1: } 3.9 \pm 0.9 \\
\text { G2: } 4.3 \pm 1.3 \\
\text { DPBC-Resistance to } \\
\text { Communication and } \\
\text { Interaction } \\
\text { G1: } 2.51 \\
\text { G2: } 2.31 .7 \\
\text { DPBC-Social } \\
\text { Interaction and } \\
\text { Isolation } \\
\text { G1: } 7.6 \pm 1.7 \\
\text { G2: } 7.1 \pm 2.8 \\
\text { DPBC-Unusual and } \\
\text { Bizarre Behavior } \\
\text { G1: } 4.9 \pm 1.5 \\
\text { G2: } 4.5 \pm 1.6 \\
\text { DPBC-Autistic } \\
\text { Behavior } \\
\text { G1: } 12.5 \pm 2.2 \\
\text { G2: } 11.5 \pm 3.9\end{array}$ & $\begin{array}{l}\text { Leiter R-Nonverbal } \\
\text { Cognitive Level } \\
\text { G1: } 86.7 \pm 38.5 \\
\text { G2: } 74.3 \pm 31.4 \\
\text { G1 vs } \mathrm{G} 2, \mathrm{p}=0.004 \\
\text { Parent Questionnaire- } \\
\text { Attention } \\
\text { G1 vs } \mathrm{G} 2, \mathrm{p}<0.028 \\
\text { Parent Questionnaire- } \\
\text { Social and Emotional } \\
\text { Factors } \\
\text { G1 vs G2, p<0.004 } \\
\text { Parent Questionnaire- } \\
\text { Communicative } \\
\text { Factors } \\
\text { G1 vs } \mathrm{G} 2, \mathrm{p}<0.007 \\
\text { Parent Questionnaire- } \\
\text { Cognitive Factors } \\
\text { G1 vs } \mathrm{G} 2, \mathrm{p}<0.019 \\
\\
\text { Parent Questionnaire- } \\
\text { Sensory/Motor Factors } \\
\text { G1 vs G2, } \mathrm{p}=\text { ns } \\
\text { DPBC-Communication } \\
\text { and Interaction } \\
\text { G1: } 6.2 \pm 1.1 \\
\text { G2: } 4.5 \pm 1.6 \\
\text { G1 vs } \mathrm{G} 2, \mathrm{p}<0.004 \\
\text { DPBC-Resistance to } \\
\text { Communication and } \\
\text { Interaction } \\
\text { G1: } 0.2 \pm 0.4 \\
\text { G2: } 1.9 \pm 1.4 \\
\text { G1 vs } \mathrm{G} 2, \mathrm{p}<0.004\end{array}$ \\
\hline
\end{tabular}




\begin{tabular}{|c|c|c|c|}
\hline $\begin{array}{c}\text { Author, Year } \\
\text { Study Design } \\
\text { Groups (dose), N enrollment I } \\
\mathrm{N} \text { final } \\
\\
\text { Treatment duration/Follow-up } \\
\text { timepoint post-treatment } \\
\text { Risk of Bias }\end{array}$ & $\begin{array}{c}\text { Mean age, } \\
\text { years } \pm \text { SD } \\
\text { Mean IQ } \pm S D\end{array}$ & $\begin{array}{c}\text { Outcome } \\
\text { measure/Baseline } \\
\text { scores, mean } \pm S D\end{array}$ & $\begin{array}{c}\text { Outcome } \\
\text { measure/Post- } \\
\text { treatment scores, } \\
\text { mean } \pm \text { SD }\end{array}$ \\
\hline & & & $\begin{array}{l}\text { DPBC-Social } \\
\text { Interaction and } \\
\text { Isolation } \\
\text { G1: } 3 \pm 1.4 \\
\text { G2: } 6.2 \pm 2.9 \\
\text { G1 vs } \mathrm{G} 2, \mathrm{p}<0.003 \\
\\
\text { DPBC-Unusual and } \\
\text { Bizarre Behavior } \\
\text { G1: } 2.6 \pm 1.7 \\
\text { G2: } 4.8 \pm 2.6 \\
\text { G1 vs G2, p<0.007 } \\
\text { DPBC-Autistic } \\
\text { Behavior } \\
\text { G1: } 5.6 \pm 2.4 \\
\text { G2: } 11.2 \pm 5 \\
\text { G1 vs } \mathrm{G} 2, \mathrm{p}<0.001\end{array}$ \\
\hline $\begin{array}{l}\text { Elder et al., } 2006^{56,57} \\
\text { RCT } \\
\text { G1: Gluten and Casein free } \\
\text { diet/Regular Diet, } 15 \\
\text { G2: Regular Diet/Gluten and } \\
\text { Casein free diet, } 15 \\
12 \text { weeks/EOT } \\
\text { Moderate ROB }\end{array}$ & $\begin{array}{l}\text { Age } \\
7.32 \pm 4.1(2- \\
16) \\
\text { IQ } \\
\text { NR }\end{array}$ & $\begin{array}{l}\text { CARS-Total Score } \\
\text { ND } \\
\text { Ecological } \\
\text { Communication } \\
\text { Orientation Language } \\
\text { Sampling Summary } \\
\text { ND } \\
\text { Behavioral Response } \\
\text { Frequencies-Child } \\
\text { Initiating } \\
\text { ND } \\
\text { Behavioral Response } \\
\text { Frequencies-Child } \\
\text { Responding } \\
\text { ND } \\
\text { Behavioral Response } \\
\text { Frequencies-Intelligible } \\
\text { Words } \\
\text { ND } \\
\text { Verbal Social } \\
\text { Exchanges-Verbal } \\
\text { Responses } \\
\text { G1: } 16 \pm 27 \\
\text { G2: } 16 \pm 27 \\
\text { Verbal Social } \\
\text { Exchanges-Verbal }\end{array}$ & $\begin{array}{l}6 \text { Weeks } \\
\text { CARS-Total Score } \\
\text { G1: } 33.6 \pm 8.6 \\
\text { G2: } 31.2 \pm 8.7 \\
\text { G1 vs } G 2, p=n s \\
\text { Ecological } \\
\text { Communication } \\
\text { Orientation Language } \\
\text { Sampling Summary } \\
\text { G1: } 175.8 \pm 86.4 \\
\text { G2: } 174.4 \pm 86 \\
\text { G1 vs } \mathrm{G} 2, \mathrm{p}=\mathrm{ns} \\
\text { Behavioral Response } \\
\text { Frequencies-Child } \\
\text { Initiating } \\
\text { G1: } 9.5 \pm 9.6 \\
\text { G2: } 7.5 \pm 6.1 \\
\text { G1 vs } \mathrm{G} 2, \mathrm{p}=\mathrm{ns} \\
\text { Behavioral Response } \\
\text { Frequencies-Child } \\
\text { Responding } \\
\text { G1: } 27.7 \pm 21.8 \\
\text { G2: } 14.3 \pm 6.5 \\
\text { G1 vs } \mathrm{G} 2, \mathrm{p}=\mathrm{ns} \\
\\
\text { Behavioral Response } \\
\text { Frequencies-Intelligible } \\
\text { Words } \\
\text { G1: } 26.8 \pm 35.1\end{array}$ \\
\hline
\end{tabular}




\begin{tabular}{|c|c|c|c|}
\hline $\begin{array}{c}\text { Author, Year } \\
\text { Study Design } \\
\text { Groups (dose), N enrollment I } \\
\mathrm{N} \text { final } \\
\\
\text { Treatment duration/Follow-up } \\
\text { timepoint post-treatment } \\
\text { Risk of Bias }\end{array}$ & $\begin{array}{c}\text { Mean age, } \\
\text { years } \pm \text { SD } \\
\text { Mean IQ } \pm S D\end{array}$ & $\begin{array}{c}\text { Outcome } \\
\text { measure/Baseline } \\
\text { scores, mean } \pm S D\end{array}$ & $\begin{array}{c}\text { Outcome } \\
\text { measure/Post- } \\
\text { treatment scores, } \\
\text { mean } \pm \text { SD }\end{array}$ \\
\hline & & $\begin{array}{l}\text { Imitation } \\
\text { G1: } 3.85 \pm 6.9 \\
\text { G2: } 3.85 \pm 6.9 \\
\text { Lexical Productions } \\
\text { G1: } 35 \pm 37 \\
\text { G2: } 35 \pm 37\end{array}$ & $\begin{array}{l}\text { G2: } 24 \pm 43.5 \\
\text { G1 vs } \mathrm{G} 2, \mathrm{p}=\mathrm{ns} \\
\text { Verbal Social } \\
\text { Exchanges-Verbal } \\
\text { Responses } \\
\text { G1: } 11 \pm 12 \\
\text { G2: } 11 \pm 12 \\
\text { G1 vs } \mathrm{G} 2, \mathrm{p}=\mathrm{ns} \\
\text { Verbal Social } \\
\text { Exchanges-Verbal } \\
\text { Imitation } \\
\text { G1: } 1.85 \pm 2.23 \\
\text { G2: } 1.85 \pm 2.23 \\
\text { G1 vs } \mathrm{G} 2, \mathrm{p}=\mathrm{ns} \\
\\
\text { Lexical Productions } \\
\text { G1: } 32 \pm 33 \\
\text { G2: } 32 \pm 33 \\
\text { G1 vs } \mathrm{G} 2, \mathrm{p}=\mathrm{ns} \\
\\
\text { 12 Weeks } \\
\text { CARS-Total Score } \\
\text { G1: } 37.5 \pm 6.6 \\
\text { G2: } 33.5 \pm 8.4 \\
\text { G1 vs G2, } \mathrm{p}=\mathrm{ns} \\
\text { Change score } \\
\text { G1: } 1.2 \pm 6 \\
\text { G2: } 2 \pm 7.7 \\
\text { Ecological } \\
\text { Communication } \\
\text { Orientation Language } \\
\text { Sampling Summary } \\
\text { G1: } 111.6 \pm 46.6 \\
\text { G2: } 162.9 \pm 108.8 \\
\text { G1 vs } \mathrm{G} 2, \mathrm{p}=\mathrm{ns} \\
\text { Change score } \\
\text { G1: }-42.2 \pm 38.1 \\
\text { G2: }-4.1 \pm 68 \\
\text { Behavioral Response } \\
\text { Frequencies-Child } \\
\text { Initiating } \\
\text { G1: } 5.2 \pm 3.2 \\
\text { G2: } 10.8 \pm 8.4 \\
\text { G1 vs G2, } \mathrm{p}=\mathrm{ns} \\
\text { Change score } \\
\text { G1: }-0.6 \pm 4.5 \\
\text { G2 } 33+844\end{array}$ \\
\hline
\end{tabular}




\begin{tabular}{|c|c|c|c|}
\hline $\begin{array}{c}\text { Author, Year } \\
\text { Study Design } \\
\text { Groups (dose), N enrollment I } \\
\text { N final } \\
\\
\text { Treatment duration/Follow-up } \\
\text { timepoint post-treatment } \\
\text { Risk of Bias }\end{array}$ & $\begin{array}{c}\text { Mean age, } \\
\text { years } \pm \text { SD } \\
\text { Mean IQ } \pm S D\end{array}$ & $\begin{array}{l}\text { Outcome } \\
\text { measure/Baseline } \\
\text { scores, mean } \pm S D\end{array}$ & $\begin{array}{c}\text { Outcome } \\
\text { measure/Post- } \\
\text { treatment scores, } \\
\text { mean } \pm \text { SD }\end{array}$ \\
\hline & & & $\begin{array}{l}\text { Behavioral Response } \\
\text { Frequencies-Child } \\
\text { Responding } \\
\text { G1: } 15 \pm 15 \\
\text { G2: } 11.9 \pm 4.5 \\
\text { G1 vs } \mathrm{G} 2, \mathrm{p}=\mathrm{ns} \\
\text { Change score } \\
\text { G1: }-8.4 \pm 7 \\
\text { G2: }-2.4 \pm 7.7 \\
\text { Behavioral Response } \\
\text { Frequencies-Intelligible } \\
\text { Words } \\
\text { G1: } 12.4 \pm 14.2 \\
\text { G2: } 30.9 \pm 36 \\
\text { G1 vs } \mathrm{G} 2, \mathrm{p}=\mathrm{ns} \\
\text { Change score } \\
\text { G1: }-2.6 \pm 12.4 \\
\mathrm{G} 2: 6.9 \pm 17 \\
\text { Verbal Social } \\
\text { Exchanges-Verbal } \\
\text { Responses } \\
\text { G1: } 15 \pm 18 \\
\text { G2: } 15 \pm 18 \\
\text { G1 vs } \mathrm{G} 2, \mathrm{p}=\mathrm{ns} \\
\text { Verbal Social } \\
\text { Exchanges-Verbal } \\
\text { Imitation } \\
\text { G1: } 3.54 \pm 7.84 \\
\text { G2: } 3.54 \pm 7.84 \\
\text { G1 vs } \mathrm{G} 2, \mathrm{p}=\mathrm{ns} \\
\text { Lexical Productions } \\
\text { G1: } 37 \pm 34 \\
\text { G2: } 37 \pm 34 \\
\text { G1 vs } \mathrm{G} 2, \mathrm{p}=\mathrm{ns}\end{array}$ \\
\hline $\begin{array}{l}\text { Ghalichi et al., } 2016^{58} \\
\text { RCT } \\
\text { G1: Gluten free diet, 38/38 } \\
\text { G2: Regular diet, 38/38 } \\
6 \text { weeks/EOT } \\
\text { High ROB }\end{array}$ & $\begin{array}{l}\text { Age } \\
\text { G1: } 7.84 \pm 3.55 \\
\text { G2: } 8 \pm 3.22 \\
\text { IQ } \\
\text { NR }\end{array}$ & $\begin{array}{l}\text { GARS-Steretyped } \\
\text { Behaviors } \\
\text { G1: } 17.61 \pm 6.74 \\
\text { G2: } 18.92 \pm 5.89 \\
\\
\text { GARS-Communication } \\
\text { G1: } 33.87 \pm 8.31 \\
\text { G2: } 32.53 \pm 9.48 \\
\text { GARS-Social } \\
\text { Interaction }\end{array}$ & $\begin{array}{l}\text { GARS-Stereotyped } \\
\text { Behaviors } \\
\text { G1: } 14.5 \pm 7.92 \\
\text { G2: } 19.32 \pm 6.27 \\
\text { G1 vs } G 2, p<0.001 \\
\\
\text { GARS-Communication } \\
\text { G1: } 32.97 \pm 9.28 \\
\text { G2: } 32.58 \pm 9.4 \\
\text { G1 vs } G 2, p=0.005\end{array}$ \\
\hline
\end{tabular}




\begin{tabular}{|c|c|c|c|}
\hline $\begin{array}{c}\text { Author, Year } \\
\text { Study Design } \\
\text { Groups (dose), N enrollment I } \\
\mathrm{N} \text { final }\end{array}$ & $\begin{array}{c}\text { Mean age, } \\
\text { years } \pm \text { SD } \\
\text { Mean IQ } \pm S D\end{array}$ & $\begin{array}{l}\text { Outcome } \\
\text { measure/Baseline } \\
\text { scores, mean } \pm S D\end{array}$ & $\begin{array}{c}\text { Outcome } \\
\text { measure/Post- } \\
\text { treatment scores, } \\
\text { mean } \pm \text { SD }\end{array}$ \\
\hline & & $\begin{array}{l}\text { G1: } 19 \pm 50 \\
\text { G2: } 14 \pm 36.8 \\
\\
\text { GARS-Total Score } \\
\text { G1: } 18.14 \pm 19.36 \\
\text { G2: } 18.99 \pm 21.5 \\
\text { Rome-III-Stomachache } \\
\text { G1: } 11(28.9) \\
\text { G2: } 10 \text { (26.3) } \\
\text { Rome-III-Bloating } \\
\text { G1: } 21 \text { (55.3) } \\
\text { G2: } 18 \text { (47.4) } \\
\text { Rome-III-Constipation } \\
\text { G1: } 19 \text { (50) } \\
\text { G2: } 14 \text { (36.8) } \\
\text { Rome-III-Diarrhea } \\
\text { G1: } 1 \text { (2.6) } \\
\text { G2: } 3(7.9)\end{array}$ & $\begin{array}{l}\text { GARS-Social } \\
\text { Interaction } \\
\text { G1: } 2 \pm 5.3 \\
\text { G2: } 18 \pm 47.4 \\
\text { G1 vs } G 2, p=0.66 \\
\text { GARS-Total Score } \\
\text { G1: } 14.91 \pm 16.94 \\
\text { G2: } 20.72 \pm 22.72 \\
\text { G1 vs G2, p=NR } \\
\text { Rome-III-Stomachache } \\
\text { G1: } 7(18.4) \\
\text { G2: } 12(31.6) \\
\text { G1 vs G2, p=NR } \\
\text { Rome-III-Bloating } \\
\text { G1: } 13 \text { (34.2) } \\
\text { G2: } 19 \text { (50) } \\
\text { G1 vs G2, p=NR } \\
\text { Rome-III-Constipation } \\
\text { G1: } 2(5.3) \\
\text { G2: } 18 \text { (47.4) } \\
\text { G1 vs G2, p=NR } \\
\text { Rome-III-Diarrhea } \\
\text { G1: } 1 \text { (2.6) } \\
\text { G2: } 5 \text { (13.2) } \\
\text { G1 vs G2, p=NR }\end{array}$ \\
\hline $\begin{array}{l}\text { Johnson et al., } 2011^{59} \\
\text { RCT } \\
\text { G1: Gluten free and Casein free } \\
\text { diet, 8/8 } \\
\text { G2: Healthy, low sugar diet, 14/14 } \\
3 \text { months/EOT } \\
\text { Moderate ROB }\end{array}$ & $\begin{array}{l}\text { Age (months) } \\
\text { G1: } 40.13 \pm \\
9.26 \\
\text { G2: } 39.5 \pm 8.72 \\
\text { IQ } \\
\text { NR }\end{array}$ & $\begin{array}{l}\text { Mullen Scales of Early } \\
\text { Learning-Visual } \\
\text { Reception } \\
\text { G1: } 30.6 \pm 11.5 \\
\text { G2: } 26.1 \pm 10.9 \\
\text { Mullen Scales of Early } \\
\text { Learning-Fine Motor } \\
\text { G1: } 28.312 .8 \\
\text { G2: } 27.910 .8 \\
\text { Mullen Scales of Early } \\
\text { Learning-Receptive } \\
\text { Language } \\
\text { G1: } 26.1 \pm 13.6 \\
\text { G2: } 23.5 \pm 13.6 \\
\text { Mullen Scales of Early } \\
\text { Learning-Expressive } \\
\text { Language } \\
\text { G1: } 21 \pm 10.2\end{array}$ & $\begin{array}{l}\text { Mullen Scales of Early } \\
\text { Learning-Visual } \\
\text { Reception } \\
\text { G1: } 28.9 \pm 11.9 \\
\text { G2: } 30.1 \pm 10.6 \\
\text { G1 vs } G 2, p=0.005 \\
\text { Mullen Scales of Early } \\
\text { Learning-Fine Motor } \\
\text { G1: } 28 \pm 12.1 \\
\text { G2: } 30.1 \pm 10.3 \\
\text { G1 vs } G 2, p=n s \\
\text { Mullen Scales of Early } \\
\text { Learning-Receptive } \\
\text { Language } \\
\text { G1: } 26.9 \pm 11.6 \\
\text { G2: } 25 \pm 13.5 \\
\text { G1 vs G2, p=NS } \\
\text { Mullen Scales of Early }\end{array}$ \\
\hline
\end{tabular}




\begin{tabular}{|c|c|c|c|}
\hline $\begin{array}{c}\text { Author, Year } \\
\text { Study Design } \\
\text { Groups (dose), N enrollment I } \\
\text { N final } \\
\text { Treatment duration/Follow-up } \\
\text { timepoint post-treatment } \\
\text { Risk of Bias }\end{array}$ & $\begin{array}{l}\text { Mean age, } \\
\text { years } \pm \text { SD } \\
\text { Mean IQ } \pm S D\end{array}$ & $\begin{array}{c}\text { Outcome } \\
\text { measure/Baseline } \\
\text { scores, mean } \pm S D\end{array}$ & $\begin{array}{c}\text { Outcome } \\
\text { measure/Post- } \\
\text { treatment scores, } \\
\text { mean } \pm \text { SD }\end{array}$ \\
\hline & & $\begin{array}{l}\text { G2: } 21.5 \pm 11.6 \\
\text { CBCL-Total Score } \\
\text { G1: } 64.3 \pm 8.35 \\
\text { G2: } 60.2 \pm 11 \\
\text { Direct Behavior } \\
\text { Observation Measure- } \\
\text { Verbal } \\
\text { G1: } 19 \pm 6.37 \\
\text { G2: } 16 \pm 9.79 \\
\text { Direct Behavior } \\
\text { Observation Measure- } \\
\text { Attend } \\
\text { G1: } 28.1 \pm 5.46 \\
\text { G2: } 24.2 \pm 6.68 \\
\text { Direct Behavior } \\
\text { Observation Measure- } \\
\text { Initiate } \\
\text { G1: } 2.38 \pm 1.92 \\
\text { G2: } 3.46 \pm 3.28\end{array}$ & $\begin{array}{l}\text { Learning-Expressive } \\
\text { Language } \\
\text { G1: } 25 \pm 10.5 \\
\text { G2: } 22.9 \pm 12.9 \\
\text { G1 vs } \mathrm{G} 2, \mathrm{p}=\mathrm{ns} \\
\text { CBCL-Total Score } \\
\text { G1: } 62.1 \pm 4.8 \\
\text { G2: } 60.3 \pm 9.38 \\
\text { G1 vs } \mathrm{G} 2, \mathrm{p}=\mathrm{ns} \\
\text { Direct Behavior } \\
\text { Observation Measure- } \\
\text { Verbal } \\
\text { G1: } 15.5 \pm 8.93 \\
\text { G2: } 14.9 \pm 9.77 \\
\text { G1 vs } \mathrm{G} 2, \mathrm{p}=\mathrm{ns} \\
\text { Direct Behavior } \\
\text { Observation Measure- } \\
\text { Attend } \\
\text { G1: } 23.4 \pm 10.3 \\
\text { G2: } 23.3 \pm 7.84 \\
\text { G1 vs G2, p=ns } \\
\text { Direct Behavior } \\
\text { Observation Measure- } \\
\text { Initiate } \\
\text { G1: } 3.5 \pm 3.67 \\
\text { G2: } 3.58 \pm 4.52 \\
\text { G1 vs } \mathrm{G} 2 . \mathrm{p}=\mathrm{ns}\end{array}$ \\
\hline
\end{tabular}

ABC-Aberrant Behavior Checklist; VABS-Vineland Adaptive Behavior Scale; EOT-End of Treatment; AWPC-Approach Withdrawal Problems Composite; ADOS-Autism Diagnostic Observation Schedule; GARS-Gilliam Autism Rating Scale 
Table F-5. Key findings in studies of risperidone adjuncts

\begin{tabular}{|c|c|c|c|}
\hline $\begin{array}{c}\text { Author, Year } \\
\text { Study Design } \\
\text { Groups (dose), N enrollment I } \\
\text { N final } \\
\text { Treatment duration/Follow-up } \\
\text { timepoint post-treatment } \\
\text { Risk of Bias }\end{array}$ & $\begin{array}{c}\text { Mean age, } \\
\text { years } \pm \text { SD } \\
\text { Mean IQ } \pm S D\end{array}$ & $\begin{array}{c}\text { Outcome } \\
\text { measure/Baseline } \\
\text { scores, mean } \pm S D\end{array}$ & $\begin{array}{c}\text { Outcome } \\
\text { measure/Post- } \\
\text { treatment scores, } \\
\text { mean } \pm \text { SD }\end{array}$ \\
\hline $\begin{array}{l}\text { Ghaleiha et al., } 2015^{60} \\
\text { RCT } \\
\text { G1: Risperidone + pioglitazone (up } \\
\text { to } 2 \text { mg/day + } 30 \text { mg/day), } 22 / 20 \\
\text { G2: Risperidone + placebo (NA), } \\
22 / 20 \\
10 \text { weeks/EOT } \\
\text { Low RoB }\end{array}$ & $\begin{array}{l}\text { Age } \\
\text { G1: } 6.95 \pm 2.40 \\
\text { G2: } 6.20 \pm 1.32 \\
\text { IQ } \\
\text { NR }\end{array}$ & $\begin{array}{l}\text { ABC-Irritability } \\
\text { G1: } 18.25 \pm 4.38 \\
\text { G2: } 19.00 \pm 5.70 \\
\text { ABC-Lethargy } \\
\text { G1: } 15.05 \pm 7.56 \\
\text { G2: } 13.66 \pm 7.26 \\
\text { ABC-Stereotypic } \\
\text { behavior } \\
\text { G1: } 7.70 \pm 4.61 \\
\text { G2: } 9.40 \pm 5.28 \\
\text { ABC-Hyperactivity } \\
\text { G1: } 25.00 \pm 10.05 \\
\text { G2: } 27.86 \pm 9.85 \\
\text { ABC-Inappropriate } \\
\text { speech } \\
\text { G1: } 5.70 \pm 3.60 \\
\text { G2: } 4.70 \pm 3.78\end{array}$ & 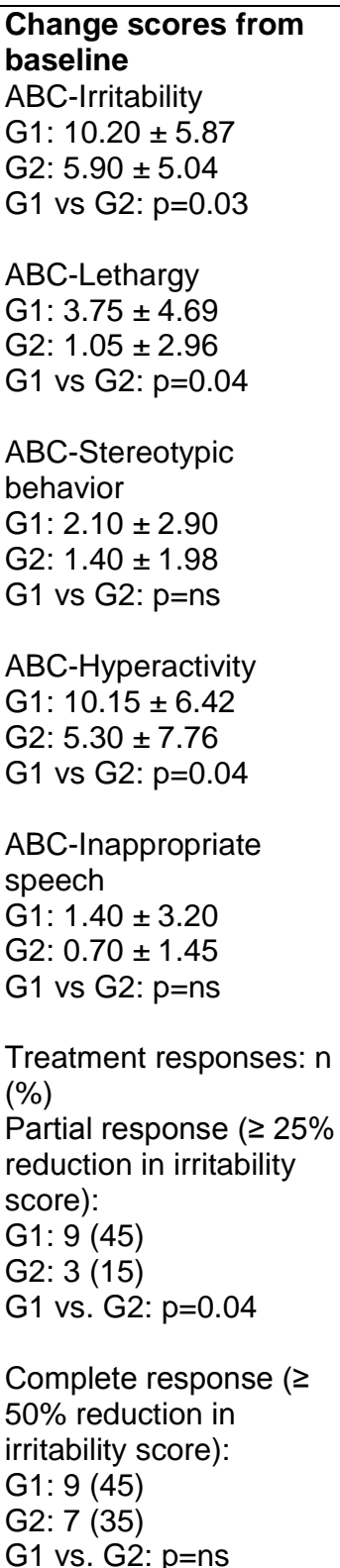 \\
\hline
\end{tabular}




\begin{tabular}{|c|c|c|c|}
\hline $\begin{array}{c}\text { Author, Year } \\
\text { Study Design } \\
\text { Groups (dose), N enrollment I } \\
\text { N final } \\
\text { Treatment duration/Follow-up } \\
\text { timepoint post-treatment } \\
\text { Risk of Bias }\end{array}$ & $\begin{array}{l}\text { Mean age, } \\
\text { years } \pm S D \\
\text { Mean IQ } \pm S D\end{array}$ & $\begin{array}{c}\text { Outcome } \\
\text { measure/Baseline } \\
\text { scores, mean } \pm S D\end{array}$ & $\begin{array}{c}\text { Outcome } \\
\text { measure/Post- } \\
\text { treatment scores, } \\
\text { mean } \pm \text { SD }\end{array}$ \\
\hline $\begin{array}{l}\text { Ghanizadeh et al., } 2015^{61} \\
\text { RCT } \\
\text { G1: Risperidone + buspirone (up to } \\
3 \text { mg/day + up to } 20 \mathrm{mg} / \mathrm{kg} \text { ), } 20 / 16 \\
\text { G2: Risperidone + placebo (NA), } \\
\text { 20/18 } \\
8 \text { weeks/EOT } \\
\text { Low RoB }\end{array}$ & $\begin{array}{l}\text { Age } \\
\text { G1: } 7.05 \pm 2.3 \\
\text { G2: } 7.5 \pm 2.6 \\
\text { IQ } \\
\text { NR }\end{array}$ & $\begin{array}{l}\text { ABC-Irritability } \\
\text { G1: } 25.7 \pm 5.7 \\
\text { G2: } 24.7 \pm 7.6\end{array}$ & $\begin{array}{l}\text { EOT } \\
\text { ABC-Irritability } \\
\text { G1: } 16.6 \pm 7.6 \\
\text { G2: } 18.2 \pm 7.7 \\
\text { Treatment response, } n \\
\text { (\%): } \\
\text { >25\% reduction in } \\
\text { irritability score: } n(\%) \text { : } \\
\text { G1: } 13 \text { (81.2) } \\
\text { G2: } 8(44.4) \\
\text { G1 vs. G2: } p<0.02, \\
\text { RR=1.82 } \\
\geq 30 \% \text { reduction in } \\
\text { irritability score: } \\
\text { G1: } 13(81.2) \\
\text { G2: } 7(38.9) \\
\text { G1 vs. G2: } p<0.01, \\
\text { RR=2.1 }\end{array}$ \\
\hline $\begin{array}{l}\text { Nikoo et al., } 2015^{62} \\
\text { RCT } \\
\text { G1: Risperidone + N- } \\
\text { acetylcysteine }(1-2.0 \mathrm{mg} / \text { day + 600- } \\
900 \mathrm{mg} / \text { day), } 25 / 20 \\
\text { G2: Risperidone + placebo (NA), } \\
25 / 20 \\
10 \text { weeks/EOT } \\
\text { Low RoB }\end{array}$ & $\begin{array}{l}\text { Age } \\
\text { G1: } 7.50 \pm 2.63 \\
\text { G2: } 7.60 \pm 2.60 \\
\text { IQ } \\
\text { G1: NR } \\
\text { G2: NR }\end{array}$ & $\begin{array}{l}\text { ABC-Irritability } \\
\text { G1: } 21.20 \pm 5.16 \\
\text { G2: } 19.70 \pm 7.61 \\
\text { ABC-Lethargy } \\
\text { G1: } 21.10 \pm 6.39 \\
\text { G2: } 20.65 \pm 9.62 \\
\text { ABC-Stereotypic } \\
\text { behavior } \\
\text { G1: } 10.55 \pm 4.22 \\
\text { G2: } 10.05 \pm 5.35 \\
\text { ABC-Hyperactivity } \\
\text { G1: } 27.65 \pm 6.16 \\
\text { G2: } 25.10 \pm 9.44 \\
\text { ABC-Inappropriate } \\
\text { speech } \\
\text { G1: } 5.70 \pm 2.92 \\
\text { G2: } 4.75 \pm 3.72\end{array}$ & $\begin{array}{l}\text { EOT } \\
\text { ABC-Irritability } \\
\text { G1: } 11.95 \pm 4.87 \\
\text { G2: } 14.35 \pm 6.27 \\
\text { G1 vs G2: } p<0.05 \\
\text { ABC-Lethargy } \\
\text { G1: } 17.15 \pm 5.46 \\
\text { G2: } 17.95 \pm 8.40 \\
\text { G1 vs G2: } p=n s \\
\text { ABC-Stereotypic } \\
\text { behavior } \\
\text { G1: } 7.75 \pm 4.90 \\
\text { G2: } 8.70 \pm 5.44 \\
\text { G1 vs G2: p=ns } \\
\text { ABC-Hyperactivity } \\
\text { G1: } 21.45 \pm 8.47 \\
\text { G2: } 23.05 \pm 9.03 \\
\text { G1 vs G2: } p<0.05 \\
\text { ABC-Inappropriate } \\
\text { speech } \\
\text { G1: } 4.95 \pm 2.85 \\
\text { G2: } 4.80 \pm 3.47 \\
\text { G1 vs G2: p=ns }\end{array}$ \\
\hline
\end{tabular}




\begin{tabular}{|c|c|c|c|}
\hline $\begin{array}{c}\text { Author, Year } \\
\text { Study Design } \\
\text { Groups (dose), N enrollment / } \\
\mathrm{N} \text { final }\end{array}$ & $\begin{array}{c}\text { Mean age, } \\
\text { years } \pm \text { SD } \\
\text { Mean IQ } \pm S D\end{array}$ & $\begin{array}{c}\text { Outcome } \\
\text { measure/Baseline } \\
\text { scores, mean } \pm S D\end{array}$ & $\begin{array}{c}\text { Outcome } \\
\text { measure/Post- } \\
\text { treatment scores, } \\
\text { mean } \pm \text { SD }\end{array}$ \\
\hline $\begin{array}{l}\text { Ghanizadeh, et al., } 2013^{63} \\
\text { RCT } \\
\text { G1: Risperidone + N-acetylcysteine } \\
\text { (up to } 3 \mathrm{mg} / \text { day + } 1200 \mathrm{mg} / \text { day), } \\
\text { 20/17 } \\
\text { G2: Risperidone + placebo (NA), } \\
\text { 20/14 } \\
8 \text { weeks/EOT } \\
\text { Low RoB }\end{array}$ & $\begin{array}{l}\text { Age: } \\
\text { G1: } 8.8 \pm 3.1 \\
\text { G2: } 7.9 \pm 2.4 \\
\text { IQ } \\
\text { NR }\end{array}$ & $\begin{array}{l}\text { ABC-Irritability } \\
\text { G1: } 13.2 \pm 5.3 \\
\text { G2: } 16.7 \pm 7.8 \\
\text { ABC-Hyperactivity } \\
\text { G1: } 29.3 \pm 6.4 \\
\text { G2: } 31.9 \pm 8.9 \\
\text { ABC-Lethargy } \\
\text { G1:11.9 } \pm 6.5 \\
\text { G2: } 12.2 \pm 8.3 \\
\text { ABC-Stereotypic } \\
\text { behavior } \\
\text { G1: } 6.6 \pm 4.5 \\
\text { G2: } 8.5 \pm 6.3 \\
\text { ABC-Inappropriate } \\
\text { speech } \\
\text { G1: } 3.9 \pm 3.7 \\
\text { G2: } 5.7 \pm 3.8\end{array}$ & $\begin{array}{l}\text { EOT } \\
\text { ABC-Irritability } \\
\text { G1: } 9.7 \pm 4.1 \\
\text { G2: } 15.1 \pm 7.8 \\
\text { G1 vs G2: } p<0.035 \\
\text { ABC-Hyperactivity } \\
\text { G1: } 18.3 \pm 6.9 \\
\text { G2: } 24.3 \pm 12.1 \\
\text { G1 vs G2: } p=n s \\
\text { ABC-Lethargy } \\
\text { G1: } 8.5 \pm 6.5 \\
\text { G2: } 10.9 \pm 7.6 \\
\text { G1 vs } G 2: p=n s \\
\text { ABC-Stereotypic } \\
\text { behavior } \\
\text { G1: } 3.9 \pm 2.7 \\
\text { G2: } 7.8 \pm 6.6 \\
\text { G1 vs G2: } p=n s \\
\text { ABC-Inappropriate } \\
\text { speech } \\
\text { G1: } 3.2 \pm 3.4 \\
\text { G2: } 5.2 \pm 4.0 \\
\text { G1 vs } \text { G2: } p=n s\end{array}$ \\
\hline $\begin{array}{l}\text { Asadabadi, et al., } 2013^{64} \\
\text { RCT } \\
\text { G1: Risperidone + celecoxib (up to } \\
3 \mathrm{mg} / \text { day + up to } 300 \mathrm{mg} / \text { day), } \\
\text { 20/20 } \\
\text { G2: Risperidone + placebo (NA), } \\
20 / 20 \\
10 \text { weeks/EOT } \\
\text { Low RoB }\end{array}$ & $\begin{array}{l}\text { Age } \\
\text { G1: } 7.6 \pm 1.7 \\
\text { G2: } 7.5 \pm 1.5 \\
\text { IQ } \\
\text { NR }\end{array}$ & $\begin{array}{l}\text { ABC-Irritability } \\
\text { G1: } 17.3 \pm 1.6 \\
\text { G2: } 17.6 \pm 2.4 \\
\text { ABC-Lethargy } \\
\text { G1: } 17.0 \pm 3.0 \\
\text { G2: } 17.1 \pm 3.2 \\
\text { ABC-Stereotypic } \\
\text { behavior } \\
\text { G1: } 9.1 \pm 2.2 \\
\text { G2: } 9.2 \pm 2.3 \\
\text { ABC-Hyperactivity } \\
\text { G1: } 22.0 \pm 2.9 \\
\text { G2: } 22.6 \pm 3.0 \\
\text { ABC-Inappropriate } \\
\text { speech } \\
\text { G1: } 5.4 \pm 1.4 \\
\text { G2: } 5.5 \pm 0.9\end{array}$ & $\begin{array}{l}\text { EOT } \\
\text { ABC-Irritability } \\
\text { G1: } 8.7 \pm 1.6 \\
\text { G2: } 12.4 \pm 3.0 \\
\text { G1 vs } \mathrm{G} 2: p<0.001 \\
\\
\text { ABC-Lethargy } \\
\text { G1: } 9.9 \pm 3.1 \\
\text { G2: } 14.3 \pm 2.1 \\
\text { G1 vs G2: } p<0.001 \\
\text { ABC-Stereotypic } \\
\text { behavior } \\
\text { G1: } 4.0 \pm 1.2 \\
\text { G2: } 6.9 \pm 2.3 \\
\text { G1 vs } \mathrm{G} 2: p<0.001 \\
\text { ABC-Hyperactivity } \\
\text { G1: } 11.2 \pm 2.1 \\
\text { G2: } 12.9 \pm 2.6 \\
\text { G1 vs } \text { G2: } p=n s \\
\text { ABC-Inappropriate } \\
\text { speech }\end{array}$ \\
\hline
\end{tabular}




\begin{tabular}{|c|c|c|c|}
\hline $\begin{array}{c}\text { Author, Year } \\
\text { Study Design } \\
\text { Groups (dose), N enrollment } / \\
\text { N final } \\
\\
\text { Treatment duration/Follow-up } \\
\text { timepoint post-treatment } \\
\text { Risk of Bias }\end{array}$ & $\begin{array}{c}\text { Mean age, } \\
\text { years } \pm \text { SD } \\
\text { Mean IQ } \pm S D\end{array}$ & $\begin{array}{c}\text { Outcome } \\
\text { measure/Baseline } \\
\text { scores, mean } \pm S D\end{array}$ & $\begin{array}{c}\text { Outcome } \\
\text { measure/Post- } \\
\text { treatment scores, } \\
\text { mean } \pm \text { SD }\end{array}$ \\
\hline & & & $\begin{array}{l}\text { G1: } 4.1 \pm 2.0 \\
\text { G2: } 4.3 \pm 1.2 \\
\text { G1 vs } G 2: p=n s\end{array}$ \\
\hline $\begin{array}{l}\text { Ghaleiha, et al., } 2013^{65} \\
\text { RCT } \\
\text { G1: Risperidone + memantine (up } \\
\text { to } 3 \mathrm{mg} / \mathrm{day}+\text { up to } 20 \mathrm{mg} / \mathrm{day} \text { ), } \\
20 / 20 \\
\text { G2: Risperidone + placebo (NA), } \\
20 / 20 \\
10 \text { weeks/EOT } \\
\text { Low RoB }\end{array}$ & $\begin{array}{l}\text { Age } \\
\text { G1: } 7.42 \pm 1.48 \\
\text { G2: } 7.97 \pm 1.68 \\
\text { IQ } \\
\text { NR }\end{array}$ & $\begin{array}{l}\text { ABC-Irritability } \\
\text { G1: } 18.25 \pm 1.55 \\
\text { G2: } 17.65 \pm 3.74 \\
\text { ABC-Lethargy } \\
\text { G1: } 16.55 \pm 4.26 \\
\text { G2: } 16.85 \pm 3.48 \\
\text { ABC-Stereotypic } \\
\text { behavior } \\
\text { G1: } 8.83 \pm 3.08 \\
\text { G2: } 8.26 \pm 2.67 \\
\text { ABC-Hyperactivity } \\
\text { G1: } 23.00 \pm 4.69 \\
\text { G2: } 22.45 \pm 7.91 \\
\text { ABC-Inappropriate } \\
\text { speech } \\
\text { G1: } 6.00 \pm 1.36 \\
\text { G2: } 5.85 \pm 1.46\end{array}$ & $\begin{array}{l}\text { EOT } \\
\text { ABC-Irritability } \\
\text { G1: } 8.90 \pm 1.55 \\
\text { G2: } 12.75 \pm 3.05 \\
\text { G1 vs } \mathrm{G} 2: \mathrm{p}<0.01 \\
\text { ABC-Lethargy } \\
\text { G1: } 11.65 \pm 3.39 \\
\text { G2: } 13.85 \pm 2.10 \\
\text { G1 vs G2: } \mathrm{p}=\mathrm{ns} \\
\text { ABC-Stereotypic } \\
\text { behavior } \\
\text { G1: } 3.30 \pm 1.30 \\
\text { G2: } 6.99 \pm 1.97 \\
\text { G1 vs G2: } p<0.01 \\
\text { ABC-Hyperactivity } \\
\text { G1: } 8.25 \pm 2.19 \\
\text { G2: } 13.85 \pm 3.28 \\
\text { G1 vs } \mathrm{G} 2: \mathrm{p}=<0.01 \\
\text { ABC-Inappropriate } \\
\text { speech } \\
\text { G1: } 4.50 \pm 1.75 \\
\text { G2: } 4.69 \pm 1.60 \\
\text { G1 vs G2: } \mathrm{p}=\text { ns }\end{array}$ \\
\hline $\begin{array}{l}\text { Ghaleiha, et al., } 2013^{66} \\
\text { RCT } \\
\text { G1: Risperidone + riluzole (up to } 3 \\
\text { mg/day + up to } 100 \text { mg/day), } 25 / 20 \\
\text { G2: Risperidone + placebo (NA), } \\
24 / 20 \\
10 \text { weeks/EOT } \\
\text { Low RoB }\end{array}$ & $\begin{array}{l}\text { Age } \\
\text { G1: } 8.4 \pm 2.3 \\
\text { G2: } 7.6 \pm 1.7 \\
\text { IQ } \\
\text { NR }\end{array}$ & $\begin{array}{l}\text { ABC-Irritability } \\
\text { G1: } 21.40 \pm 4.18 \\
\text { G2: } 22.10 \pm 9.98 \\
\text { ABC-Lethargy } \\
\text { G1: } 23.95 \pm 8.04 \\
\text { G2: } 24.30 \pm 10.98 \\
\text { ABC-Stereotypic } \\
\text { behavior } \\
\text { G1: } 7.80 \pm 3.43 \\
\text { G2: } 8.25 \pm 4.93\end{array}$ & $\begin{array}{l}\text { EOT } \\
\text { ABC-Irritability } \\
\text { G1: } 11.85 \pm 5.57 \\
\text { G2: } 16.25 \pm 7.86 \\
\text { G1 vs } \mathrm{G} 2: \mathrm{p}=0.03 \\
\text { Treatment responses: } n \\
\text { (\%) } \\
\text { Partial response }(\geq 25 \% \\
\text { reduction in irritability } \\
\text { score): } \\
\text { G1: } 17 \text { (85) }\end{array}$ \\
\hline
\end{tabular}




\begin{tabular}{|c|c|c|c|}
\hline $\begin{array}{c}\text { Author, Year } \\
\text { Study Design } \\
\text { Groups (dose), N enrollment I } \\
\mathrm{N} \text { final } \\
\\
\text { Treatment duration/Follow-up } \\
\text { timepoint post-treatment } \\
\text { Risk of Bias }\end{array}$ & $\begin{array}{c}\text { Mean age, } \\
\text { years } \pm \text { SD } \\
\text { Mean IQ } \pm S D\end{array}$ & $\begin{array}{c}\text { Outcome } \\
\text { measure/Baseline } \\
\text { scores, mean } \pm S D\end{array}$ & $\begin{array}{c}\text { Outcome } \\
\text { measure/Post- } \\
\text { treatment scores, } \\
\text { mean } \pm \text { SD }\end{array}$ \\
\hline & & $\begin{array}{l}\text { ABC-Hyperactivity } \\
\text { G1: } 26.35 \pm 7.67 \\
\text { G2: } 27.95 \pm 10.68 \\
\text { ABC-Inappropriate } \\
\text { speech } \\
\text { G1: } 5.85 \pm 3.38 \\
\text { G2: } 5.80 \pm 3.10\end{array}$ & 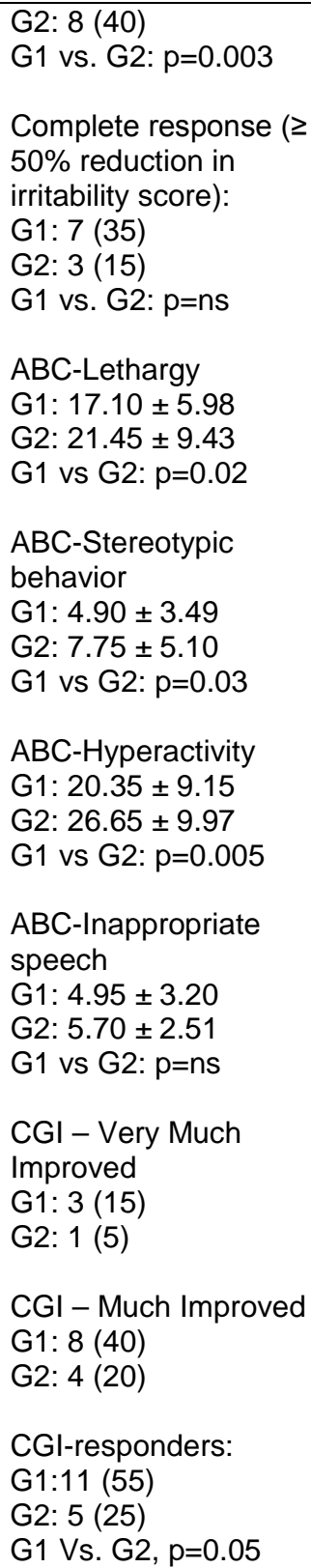 \\
\hline
\end{tabular}




\begin{tabular}{|c|c|c|c|}
\hline $\begin{array}{c}\text { Author, Year } \\
\text { Study Design } \\
\text { Groups (dose), N enrollment I } \\
\text { N final } \\
\text { Treatment duration/Follow-up } \\
\text { timepoint post-treatment } \\
\text { Risk of Bias }\end{array}$ & $\begin{array}{l}\text { Mean age, } \\
\text { years } \pm \text { SD } \\
\text { Mean IQ } \pm S D\end{array}$ & $\begin{array}{c}\text { Outcome } \\
\text { measure/Baseline } \\
\text { scores, mean } \pm S D\end{array}$ & $\begin{array}{c}\text { Outcome } \\
\text { measure/Post- } \\
\text { treatment scores, } \\
\text { mean } \pm \text { SD }\end{array}$ \\
\hline $\begin{array}{l}\text { Ghaleiha } 2013^{67} \\
\text { RCT } \\
\text { G1: Galantamine + Risperidone (2- } \\
24 \text { mg/day + 0.5-2 mg/day), 23/20 } \\
\text { G2: Risperidone + Placebo (0.5- } \\
\text { 2mg/day), 23/20 } \\
10 \text { weeks/EOT } \\
\text { Low RoB }\end{array}$ & $\begin{array}{l}\text { Age } \\
\text { G1: } 6.85 \pm 1.98 \\
\text { G2: } 5.90 \pm 1.38 \\
\text { IQ } \\
\text { NR }\end{array}$ & $\begin{array}{l}\text { ABC-C-Irritability } \\
\text { G1: } 15.59 \pm 5.83 \\
\text { G2: } 14.95 \pm 4.38 \\
\text { ABC-C- } \\
\text { Lethargy/Social } \\
\text { withdrawal } \\
\text { G1: } 11.10 \pm 5.34 \\
\text { G2: } 10.40 \pm 5.27 \\
\text { ABC-C-Stereotypical } \\
\text { behavior } \\
\text { G1: } 6.10 \pm 5.93 \\
\text { G2: } 5.80 \pm 5.08 \\
\text { ABC-C- } \\
\text { Hyperactivity/noncom } \\
\text { pliance } \\
\text { G1: } 20.85 \pm 11.69 \\
\text { G2: } 22.30 \pm 9.83 \\
\text { ABC-C-Inappropriate } \\
\text { Speech } \\
\text { G1: } 3.50 \pm 3.95 \\
\text { G2: } 3.25 \pm 4.43\end{array}$ & $\begin{array}{l}\text { EOT } \\
\text { ABC-C-Irritability } \\
\text { G1: } 5.30 \pm 4.25 \\
\text { G2: } 8.80 \pm 5.03 \\
\text { G1 vs G2: } p=0.017 \\
\text { ABC-C-Lethargy/Social } \\
\text { Withdrawal } \\
\text { G1: } 5.60 \pm 4.43 \\
\text { G2: } 8.35 \pm 5.59 \\
\text { G1 vs G2: } p=0.003 \\
\text { ABC-C-Stereotypical } \\
\text { Behavior } \\
\text { G1: } 3.95 \pm 5.06 \\
\text { G2: } 4.50 \pm 4.34 \\
\text { G1 vs G2: } p=n s \\
\text { ABC-C- } \\
\text { Hyperactivity/noncompli } \\
\text { ance } \\
\text { G1:10.85 } \pm 9.49 \\
\text { G2:16.05 } \pm 7.52 \\
\text { G1 vs G2: } p=n s \\
\text { ABC-C-Inappropriate } \\
\text { Speech } \\
\text { G1: } 2.15 \pm 2.68 \\
\text { G2: } 2.80 \pm 3.63 \\
\text { G1 vs G2: } p=n s\end{array}$ \\
\hline $\begin{array}{l}\text { Mohammadi, et al., } 2013^{68} \\
\text { RCT } \\
\text { G1: Risperidone + amantadine (up } \\
\text { to } 2 \text { mg/day + up to } 150 \mathrm{mg} / \text { day), } \\
20 / 20 \\
\text { G2: Risperidone + placebo (NA), } \\
\text { 20/19 } \\
10 \text { weeks/EOT } \\
\text { Low RoB }\end{array}$ & $\begin{array}{l}\text { Age } \\
\text { G1: } 6.4 \pm 2.3 \\
\text { G2: } 7.1 \pm 2.4 \\
\text { IQ } \\
\text { NR }\end{array}$ & $\begin{array}{l}\text { ABC-Irritability } \\
\text { G1: } 20.00 \pm 5.30 \\
\text { G2: } 20.90 \pm 6.61 \\
\text { ABC-Lethargy } \\
\text { G1: } 18.55 \pm 7.13 \\
\text { G2: } 18.15 \pm 4.80 \\
\text { ABC-Stereotypic } \\
\text { behavior } \\
\text { G1: } 10.90 \pm 4.03 \\
\text { G2: } 11.30 \pm 5.42 \\
\text { ABC-Hyperactivity } \\
\text { G1: } 28.15 \pm 6.88 \\
\text { G2: } 28.55 \pm 8.67 \\
\text { ABC-Inappropriate } \\
\text { speech } \\
\text { G1: } 5.70 \pm 3.11 \\
\text { G2: } 4.55 \pm 3.48\end{array}$ & $\begin{array}{l}\text { Change (reduction } \\
\text { from baseline) } \\
\text { ABC-Irritability } \\
\text { G1: } 8.60 \pm 4.65 \\
\text { G2: } 5.35 \pm 3.95 \\
\text { p=0.02 } \\
\text { ABC-Lethargy } \\
\text { G1: } 1.35 \pm 3.18 \\
\text { G2: } 1.30 \pm 3.33 \\
\text { p=ns } \\
\text { ABC-Stereotypic } \\
\text { behavior } \\
\text { G1: } 1.20 \pm 2.33 \\
\text { G2: } 1.20 \pm 2.09 \\
\text { p=ns } \\
\text { ABC-Hyperactivity } \\
\text { G1: } 6.15 \pm 5.11 \\
\text { G2: } 2.50 \pm 5.00\end{array}$ \\
\hline
\end{tabular}




\begin{tabular}{|c|c|c|c|}
\hline $\begin{array}{c}\text { Author, Year } \\
\text { Study Design } \\
\text { Groups (dose), N enrollment I } \\
\mathrm{N} \text { final } \\
\\
\text { Treatment duration/Follow-up } \\
\text { timepoint post-treatment } \\
\text { Risk of Bias }\end{array}$ & $\begin{array}{c}\text { Mean age, } \\
\text { years } \pm \text { SD } \\
\text { Mean IQ } \pm \text { SD }\end{array}$ & $\begin{array}{c}\text { Outcome } \\
\text { measure/Baseline } \\
\text { scores, mean } \pm S D\end{array}$ & $\begin{array}{c}\text { Outcome } \\
\text { measure/Post- } \\
\text { treatment scores, } \\
\text { mean } \pm \text { SD }\end{array}$ \\
\hline & & & $\begin{array}{l}\mathrm{P}=0.03 \\
\text { ABC-Inappropriate } \\
\text { speech } \\
\text { G1: } 0.40 \pm 0.99 \\
\mathrm{G} 2: 0.05 \pm 0.22 \\
\mathrm{p}=\mathrm{ns} \\
\mathrm{CGI} \\
\text { Very much improved, } \mathrm{n} \text { : } \\
\text { G1: } 2 \\
\text { G2: } 1 \\
\text { Much improved, } \mathrm{n}: \\
\text { G1: } 8 \\
\text { G2: } 3 \\
\text { Improvement, } \mathrm{n}(\%): \\
\text { G1: } 10 \text { (50) } \\
\text { G2: } 4 \text { (20) } \\
\text { G1 vs. G2: } \mathrm{p}=0.047\end{array}$ \\
\hline $\begin{array}{l}\text { Hasanzadeh, et al., } 2012^{69} \\
\text { RCT } \\
\text { G1: Risperidone + Ginko T.D (up to } \\
3 \text { mg/day + up to } 120 \text { mg/day), } \\
23 / 23 \\
\text { G2: Risperidone + placebo (NA), } \\
\text { 24/ } 24 \\
10 \text { weeks/EOT } \\
\text { Low RoB }\end{array}$ & $\begin{array}{l}\text { Age } \\
\text { G1: } 6.04 \pm 1.61 \\
\text { G2: } 6.76 \pm 2.60 \\
\text { IQ } \\
\text { NR }\end{array}$ & $\begin{array}{l}\text { ABC-Irritability } \\
\text { G1: } 14.95 \pm 7.86 \\
\text { G2: } 14.08 \pm 7.55 \\
\text { ABC-Lethargy } \\
\text { G1: } 11.63 \pm 7.90 \\
\text { G2: } 12.32 \pm 9.38 \\
\text { ABC-Stereotypic } \\
\text { behavior } \\
\text { G1: } 6.77 \pm 4.42 \\
\text { G2: } 6.32 \pm 4.11 \\
\text { ABC-Hyperactivity } \\
\text { G1: } 22.68 \pm 9.88 \\
\text { G2: } 21.36 \pm 6.96 \\
\text { ABC-Inappropriate } \\
\text { speech } \\
\text { G1: } 3.36 \pm 1.96 \\
\text { G2: } 3.92 \pm 2.11\end{array}$ & $\begin{array}{l}\text { EOT } \\
\text { ABC-Irritability } \\
\text { G1: } 10.54 \pm 5.75 \\
\text { G2: } 9.88 \pm 7.35 \\
\text { G1 vs } G 2: p=n s \\
\text { ABC-Lethargy } \\
\text { G1: } 6.86 \pm 6.08 \\
\text { G2: } 7.36 \pm 6.14 \\
\text { G1 vs G2: } p=n s \\
\text { ABC-Stereotypic } \\
\text { behavior } \\
\text { G1: } 4.7 \pm 23.28 \\
\text { G2: } 5.00 \pm 3.80 \\
\text { G1 vs G2: } p=n s \\
\text { ABC-Hyperactivity } \\
\text { G1: } 16.54 \pm 8.51 \\
\text { G2: } 14.84 \pm 6.25 \\
\text { G1 vs } G 2: p=n s \\
\text { ABC-Inappropriate } \\
\text { speech } \\
\text { G1: } 2.09 \pm 1.63 \\
\text { G2: } 2.44 \pm 1.58 \\
\text { G1 vs } G 2: p=n s\end{array}$ \\
\hline
\end{tabular}




\begin{tabular}{|c|c|c|c|}
\hline $\begin{array}{c}\text { Author, Year } \\
\text { Study Design } \\
\text { Groups (dose), N enrollment I } \\
\text { N final } \\
\text { Treatment duration/Follow-up } \\
\text { timepoint post-treatment } \\
\text { Risk of Bias }\end{array}$ & $\begin{array}{l}\text { Mean age, } \\
\text { years } \pm \text { SD } \\
\text { Mean IQ } \pm S D\end{array}$ & $\begin{array}{c}\text { Outcome } \\
\text { measure/Baseline } \\
\text { scores, mean } \pm S D\end{array}$ & $\begin{array}{c}\text { Outcome } \\
\text { measure/Post- } \\
\text { treatment scores, } \\
\text { mean } \pm \text { SD }\end{array}$ \\
\hline $\begin{array}{l}\text { Akhondzadeh, et al., } 2010^{70} \\
\text { RCT } \\
\text { G1: Risperidone + pentoxifylline (up } \\
\text { to } 3 \mathrm{mg} / \text { day + up to } 600 \mathrm{mg} / \text { day), } \\
20 / 20 \\
\text { G2: Risperidone + placebo (NA), } \\
20 / 20 \\
10 \text { weeks/EOT } \\
\text { Moderate RoB }\end{array}$ & $\begin{array}{l}\text { Age: } \\
\text { G1: } 8.05 \pm 2.01 \\
\text { G2: } 7.37 \pm 2.41 \\
\text { IQ } \\
\text { NR }\end{array}$ & $\begin{array}{l}\text { ABC-Irritability } \\
\text { G1: } 16.67 \pm 2.71 \\
\text { G2: } 16.06 \pm 3.00 \\
\text { ABC-Lethargy } \\
\text { G1: } 18.27 \pm 2.97 \\
\text { G2: } 17.29 \pm 3.23 \\
\text { ABC-Stereotypic } \\
\text { behavior } \\
\text { G1: } 8.01 \pm 1.30 \\
\text { G2: } 7.72 \pm 1.44 \\
\text { ABC-Hyperactivity } \\
\text { G1: } 16.03 \pm 2.60 \\
\text { G2: } 15.44 \pm 2.88 \\
\text { ABC-Inappropriate } \\
\text { speech } \\
\text { G1: } 5.13 \pm 0.83 \\
\text { G2: } 4.94 \pm 0.92\end{array}$ & 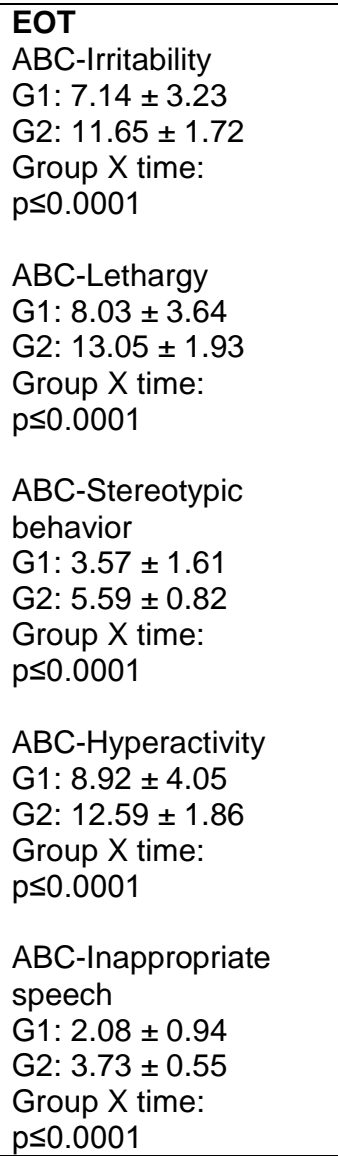 \\
\hline $\begin{array}{l}\text { Ghaleiha et al., } 2016^{71} \\
\text { RCT } \\
\text { G1: Risperidone + Minocycline ( up } \\
\text { to } 2 \mathrm{mg} / \text { day + } 50 \mathrm{mg} \text { 2/day), 25/23 } \\
\text { G2: Risperidone + Placebo ( up to } \\
2 \text { mg/day + } 1 \text { capsule 2/day), 25/23 } \\
10 \text { weeks/EOT } \\
\text { Low ROB }\end{array}$ & $\begin{array}{l}\text { Age } \\
\text { G1: } 7.39 \pm 2.48 \\
\text { G2: } 7.78 \pm 2.59 \\
\text { IQ } \\
\text { NR }\end{array}$ & $\begin{array}{l}\text { ABC-Irritability } \\
\text { G1: } 21.26 \pm 4.82 \\
\text { G2: } 19.91 \pm 7.2 \\
\text { ABC-Social } \\
\text { Withdrawal/Lethargy } \\
\text { G1: } 21.39 \pm 6.05 \\
\text { G2: } 20.3 \pm 9.12 \\
\text { ABC-Stereotypic } \\
\text { Behavior } \\
\text { G1: } 10.96 \pm 4.09 \\
\text { G2: } 11.09 \pm 5.77 \\
\text { ABC-Hyperactivity } \\
\text { G1: } 28.22 \pm 5.97 \\
\text { G2: } 25.04 \pm 9.04 \\
\text { ABC-Inappropriate }\end{array}$ & $\begin{array}{l}\text { ABC-Irritability } \\
\text { G1: } 12.09 \pm 4.55 \\
\text { G2: } 14.04 \pm 5.98 \\
\text { G1 vs G2, p=0.003; } \\
\text { d=0.91; MD }(95 \% \mathrm{Cl})= \\
3.30(1.15-5.46) \\
\text { ABC-Social } \\
\text { Withdrawal/Lethargy } \\
\text { G1: } 17.87 \pm 5.69 \\
\text { G2: } 17.7 \pm 8.18 \\
\text { G1 vs G2, p=ns; } \\
\text { d=0.22; MD (95\% } \\
\text { Cl) }=0.91(-1.57 \text { to } \\
\text { 4.00) } \\
\text { ABC-Stereotypic } \\
\text { Behavior } \\
\text { G1: } 8.22 \pm 4.81\end{array}$ \\
\hline
\end{tabular}




\begin{tabular}{|c|c|c|c|}
\hline $\begin{array}{c}\text { Author, Year } \\
\text { Study Design } \\
\text { Groups (dose), N enrollment } / \\
\mathrm{N} \text { final } \\
\\
\text { Treatment duration/Follow-up } \\
\text { timepoint post-treatment } \\
\text { Risk of Bias }\end{array}$ & $\begin{array}{c}\text { Mean age, } \\
\text { years } \pm \text { SD } \\
\text { Mean IQ } \pm S D\end{array}$ & $\begin{array}{c}\text { Outcome } \\
\text { measure/Baseline } \\
\text { scores, mean } \pm S D\end{array}$ & $\begin{array}{c}\text { Outcome } \\
\text { measure/Post- } \\
\text { treatment scores, } \\
\text { mean } \pm \text { SD }\end{array}$ \\
\hline & & $\begin{array}{l}\text { Speech } \\
\text { G1: } 6.17 \pm 3.01 \\
\text { G2: } 5.04 \pm 3.56\end{array}$ & $\begin{array}{l}\text { G2: } 9.43 \pm 5.69 \\
\text { G1 vs } \mathrm{G} 2, \mathrm{p}=\mathrm{ns} ; \\
\mathrm{d}=0.36 ; \mathrm{MD}(95 \% \mathrm{Cl})= \\
1.08(-0.68 \text { to } 2.86) \\
\\
\text { ABC-Hyperactivity } \\
\text { G1: } 21.3 \pm 7.9 \\
\mathrm{G} 2: 22.91 \pm 8.98 \\
\mathrm{G} 1 \text { vs } \mathrm{G} 2, \mathrm{p}=0.002 ; \\
\mathrm{d}=0.95 ; \mathrm{MD} \\
(95 \% \mathrm{Cl})=4.78(1.80- \\
7.70) \\
\text { ABC-Inappropriate } \\
\text { Speech } \\
\text { G1: } 5.35 \pm 2.9 \\
\text { G2: } 4.78 \pm 3.45 \\
\text { G1 vs } \mathrm{G} 2, \mathrm{p}=\mathrm{ns} ; \\
\mathrm{d}=0.34 ; \mathrm{MD}(95 \% \mathrm{Cl})= \\
0.56(-0.40 \text { to } 1.53)\end{array}$ \\
\hline $\begin{array}{l}\text { Rezaei, et al., } 2010^{72} \\
\text { RCT } \\
\text { G1: Risperidone + topiramate (up } \\
\text { to } 3 \mathrm{mg} / \text { day + up to } 200 \mathrm{mg} / \mathrm{day} \text { ), } \\
20 / 20 \\
\text { G2: Risperidone + placebo (NA), } \\
\text { 20/20 } \\
8 \text { weeks/EOT } \\
\text { Low RoB }\end{array}$ & $\begin{array}{l}\text { Age } \\
\text { G1: } 8.17 \pm 1.85 \\
\text { G2: } 7.85 \pm 1.82 \\
\text { IQ } \\
\text { NR }\end{array}$ & $\begin{array}{l}\text { ABC-Irritability } \\
\text { G1: } 17.25 \pm 3.12 \\
\text { G2: } 16.80 \pm 4.22 \\
\text { ABC-Lethargy } \\
\text { G1: } 17.65 \pm 6.02 \\
\text { G2: } 17.55 \pm 4.28 \\
\text { ABC-Stereotypic } \\
\text { behavior } \\
\text { G1: } 8.83 \pm 3.89 \\
\text { G2: } 8.71 \pm 3.21 \\
\text { ABC-Hyperactivity } \\
\text { G1: } 22.75 \pm 4.85 \\
\text { G2: } 22.00 \pm 9.17 \\
\text { ABC-Inappropriate } \\
\text { speech } \\
\text { G1: } 5.25 \pm 2.01 \\
\text { G2: } 5.10 \pm 1.80\end{array}$ & $\begin{array}{l}\text { EOT } \\
\text { ABC-Irritability } \\
\text { G1: } 8.20 \pm 2.44 \\
\text { G2: } 15.30 \pm 4.64 \\
\text { G1 vs } \mathrm{G} 2: \mathrm{p}=0.04 \\
\text { ABC-Lethargy } \\
\text { G1: } 14.15 \pm 6.67 \\
\text { G2: } 15.60 \pm 4.28 \\
\text { G1 vs } \mathrm{G} 2: \mathrm{p}=\mathrm{ns} \\
\text { ABC-Stereotypic } \\
\text { behavior } \\
\text { G1: } 3.40 \pm 1.04 \\
\text { G2: } 8.09 \pm 3.04 \\
\mathrm{G} 1 \text { vs } \mathrm{G} 2: \mathrm{p}=0.04 \\
\text { ABC-Hyperactivity } \\
\text { G1: } 7.60 \pm 2.37 \\
\text { G2: } 19.25 \pm 8.30 \\
\text { G1 vs } \mathrm{G} 2: \mathrm{p}=0.04 \\
\text { ABC-Inappropriate } \\
\text { speech } \\
\text { G1: } 3.93 \pm 2.17 \\
\text { G2: } 4.24 \pm 1.59 \\
\text { G1 vs } \mathrm{G} 2: \mathrm{p}=\mathrm{ns}\end{array}$ \\
\hline
\end{tabular}




\begin{tabular}{|c|c|c|c|}
\hline $\begin{array}{c}\text { Author, Year } \\
\text { Study Design } \\
\text { Groups (dose), N enrollment I } \\
\text { N final } \\
\\
\text { Treatment duration/Follow-up } \\
\text { timepoint post-treatment } \\
\text { Risk of Bias }\end{array}$ & $\begin{array}{c}\text { Mean age, } \\
\text { years } \pm \text { SD } \\
\text { Mean IQ } \pm S D\end{array}$ & $\begin{array}{c}\text { Outcome } \\
\text { measure/Baseline } \\
\text { scores, mean } \pm S D\end{array}$ & $\begin{array}{c}\text { Outcome } \\
\text { measure/Post- } \\
\text { treatment scores, } \\
\text { mean } \pm \text { SD }\end{array}$ \\
\hline $\begin{array}{l}\text { Akhondzadeh et al., } 2008^{73} \\
\text { RCT } \\
\text { G1: Risperidone + Piracetam (+ } 3 \\
\text { mg/day + } 800 \mathrm{mg} / \text { day), } 20 / 20 \\
\text { G2: Risperidone + Placebo (NA), } \\
\text { 20/20 } \\
10 \text { weeks/EOT } \\
\text { Moderate RoB }\end{array}$ & $\begin{array}{l}\text { Age } \\
\text { G1: } 6.90 \pm 1.86 \\
\text { G2: } 6.75 \pm 1.80 \\
\text { IQ } \\
\text { NR }\end{array}$ & $\begin{array}{l}\text { ABC-Community } \\
\text { Total Score } \\
\text { G1: } 23.15 \pm 5.8 \\
\text { G2: } 24 \pm 8.25\end{array}$ & $\begin{array}{l}\text { Mean change score } \\
\text { from baseline } \\
\text { ABC-Community Total } \\
\text { Score } \\
\text { G1: }-11.9 \pm 3.79 \\
\text { G2: }-5.15 \pm 3.04 \\
\text { G1 vs } G 2, p<0.0001\end{array}$ \\
\hline
\end{tabular}

EOT-End of Treatment; ABC-Aberrant Behavior Checklist; CGI-Clinical Global Impression 
Table F-6. Key findings in studies of other medical interventions

\begin{tabular}{|c|c|c|c|}
\hline $\begin{array}{c}\text { Author, Year } \\
\text { Study Design } \\
\text { Groups (dose), N enrollment I } \\
\text { N final } \\
\text { Treatment duration/Follow-up } \\
\text { timepoint post-treatment } \\
\text { Risk of Bias }\end{array}$ & $\begin{array}{l}\text { Mean age, } \\
\text { years } \pm \text { SD } \\
\text { Mean IQ } \pm S D\end{array}$ & $\begin{array}{c}\text { Outcome } \\
\text { measure/Baseline } \\
\text { scores, mean } \pm S D\end{array}$ & $\begin{array}{c}\text { Outcome } \\
\text { measure/Post- } \\
\text { treatment scores, } \\
\text { mean } \pm \text { SD }\end{array}$ \\
\hline \multicolumn{4}{|l|}{ Donepezil } \\
\hline $\begin{array}{l}\text { Handen et al., } 2005^{74} \\
\text { RCT } \\
\text { G1: Donepezil (2.5-10 mg/day), } \\
\text { 18/15 } \\
\text { G2: Placebo (NA), 16/16 } \\
10 \text { weeks/EOT } \\
\text { Moderate RoB }\end{array}$ & 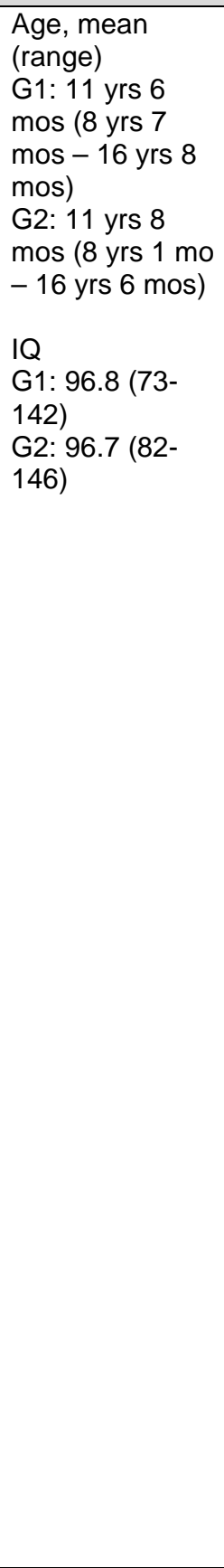 & $\begin{array}{l}\text { Trail Making Test } \\
\text { G1: } 123.656 .5 \\
\text { G2: } 154.977 .8 \\
\text { Verbal Fluency-total } \\
\text { number of verbal } \\
\text { fluency switches } \\
\text { G1: } 6.833 .17 \\
\text { G2: } 8.312 .73 \\
\text { Design Fluency- } \\
\text { number of design } \\
\text { fluency test switches } \\
\text { G1: } 3.442 .5 \\
\text { G2: } 4.752 .79 \\
\text { Color-Word } \\
\text { Interference- } \\
\text { inhibition/switch time } \\
\text { G1: } 93.338 .3 \\
\text { G2: } 90.525 .4 \\
\text { Sorting Test (STCC } \\
\text { and STFS)-number of } \\
\text { confirmed correct sorts } \\
\text { G1: } 6.722 .97 \\
\text { G2: } 7.191 .87 \\
\text { Sorting Test (STCC } \\
\text { and STFS)-total } \\
\text { number of free sorts } \\
\text { G1: } 25.211 .1 \\
\text { G2: } 26.67 .6 \\
\text { Twenty Questions } \\
\text { Test-total number of } \\
\text { questions asked } \\
\text { G1: } 24.713 .7 \\
\text { G2: } 18.98 .9 \\
\text { Tower of California } \\
\text { test-total number of } \\
\text { moves } \\
\text { G1: } 12.14 .1 \\
\text { G2: } 12.34 .9 \\
\text { Word Context Test- } \\
\text { tolmber of points }\end{array}$ & $\begin{array}{l}\text { EOT } \\
\text { Trail Making Test } \\
\text { G1: } 118.379 .1 \\
\text { G2: } 104.753 .4 \\
\text { G1 Vs G2, } p=0.72 \\
\text { Verbal Fluency-total } \\
\text { number of verbal } \\
\text { fluency switches } \\
\text { G1: } 6.175 .12 \\
\text { G2: } 6.944 .97 \\
\text { G1 Vs G2, p=0.372 } \\
\text { Design Fluency- } \\
\text { number of design } \\
\text { fluency test switches } \\
\text { G1: } 5.222 .69 \\
\text { G2: } 4.314 .25 \\
\text { G1 Vs G2, p=0.57 } \\
\text { Color-Word } \\
\text { Interference- } \\
\text { inhibition/switch time } \\
\text { G1: } 81.328 .9 \\
\text { G2: } 79.734 .2 \\
\text { G1 Vs G2, p=0.74 } \\
\text { Sorting Test (STCC } \\
\text { and STFS)-number of } \\
\text { confirmed correct sorts } \\
\text { G1: } 5.893 .1 \\
\text { G2: } 5.694 .36 \\
\text { G1 Vs G2, p=0.89 } \\
\text { Sorting Test (STCC } \\
\text { and STFS)-total } \\
\text { nuestions asked } \\
\text { G2: } 15.1 \text { } 10.8 \\
\text { G1 Vs G2, p=0.06 } \\
\text { G2: } 19.411 .6 \\
\text { G1 Vs G2, p=0.57 } \\
\end{array}$ \\
\hline
\end{tabular}




\begin{tabular}{|c|c|c|c|}
\hline $\begin{array}{c}\text { Author, Year } \\
\text { Study Design } \\
\text { Groups (dose), N enrollment I } \\
\text { N final } \\
\\
\text { Treatment duration/Follow-up } \\
\text { timepoint post-treatment } \\
\text { Risk of Bias }\end{array}$ & $\begin{array}{c}\text { Mean age, } \\
\text { years } \pm \text { SD } \\
\text { Mean IQ } \pm S D\end{array}$ & $\begin{array}{l}\text { Outcome } \\
\text { measure/Baseline } \\
\text { scores, mean } \pm S D\end{array}$ & $\begin{array}{c}\text { Outcome } \\
\text { measure/Post- } \\
\text { treatment scores, } \\
\text { mean } \pm \text { SD }\end{array}$ \\
\hline & & $\begin{array}{l}\text { earned } \\
\text { G1: } 4.94 .9 \\
\text { G2: } 6.13 .3 \\
\text { EOWPVT-Expressive } \\
\text { Language Scaled } \\
\text { Score } \\
\text { G1: } 104.622 .4 \\
\text { G2: } 108.717 \\
\text { Memory Test-Percent } \\
\text { Correct } \\
\text { G1: } 40.714 .1 \\
\text { G2: } 47.110 .3\end{array}$ & $\begin{array}{l}\text { Tower of California } \\
\text { test-total number of } \\
\text { moves } \\
\text { G1: } 15.24 .7 \\
\text { G2: } 14.97 .7 \\
\text { G1 Vs G2, } p=0.94 \\
\text { Word Context Test- } \\
\text { total number of points } \\
\text { earned } \\
\text { G1: } 33.2 \\
\text { G2: } 3.84 .9 \\
\text { G1 Vs G2, } p=0.43 \\
\text { EOWPVT-Expressive } \\
\text { Language Scaled } \\
\text { Score } \\
\text { G1: } 3.84 .9 \\
\text { G2: } 109.721 \\
\text { G1 Vs G2, } p=0.5 \\
\text { Memory Test-Percent } \\
\text { Correct } \\
\text { G1: } 114.516 .1 \\
\text { G2: } 50.211 \\
\text { G1 Vs G2, } p=0.28\end{array}$ \\
\hline \multicolumn{4}{|l|}{ Anti-epileptics } \\
\hline $\begin{array}{l}\text { Hollander et al., } 2010^{75} \\
\text { RCT } \\
\text { G1: Divalproex sodium (125mg qhs } \\
\text { - 500mg bid), 16/16 } \\
\text { G2: Placebo (NA), 11/11 } \\
12 \text { weeks/EOT } \\
\text { Low RoB }\end{array}$ & $\begin{array}{l}\text { Age } \\
\text { G1: } 9.66 \pm 2.64 \\
\text { G2: } 8.97 \pm 2.8 \\
\text { IQ } \\
\text { G1: } 52.92 \pm \\
18.5 \\
\text { G2: } 76.1 \pm \\
26.45\end{array}$ & $\begin{array}{l}\text { ABC - Irritability } \\
\text { G1: } 22 \pm 7.81 \\
\text { G2: } 20.30 \pm 7.36 \\
\text { CGI - Irritability } \\
\text { G1: } 5.13 \pm 0.72 \\
\text { G2: } 4.73 \pm 0.47 \\
\text { The Overt Aggression } \\
\text { Scale-Modified (OAS- } \\
\text { M) } \\
\text { G1: } 6.43 \pm 1.41 \\
\text { G2: } 5.36 \pm 2.2 \\
\text { CYBOCS } \\
\text { G1: NR } \\
\text { G2: NR } \\
\text { VABS } \\
\text { G1: } 37.13 \pm 15.9 \\
\text { G2: } 42.4 \pm 17.21\end{array}$ & $\begin{array}{l}\text { EOT } \\
\text { ABC - Irritability } \\
\text { G1: } 14.5 \pm 6.67 \\
\text { G2: } 17.70 \pm 7.94 \\
\text { G1 vs G2: p= NR } \\
\text { CGI - Irritability } \\
\text { Response, } n \text { (\%) } \\
\text { G1: } 10(62,5) \\
\text { G2: } 1(9.1) \\
\text { G1 vs G2, p=0.008 } \\
\text { The Overt Aggression } \\
\text { Scale-Modified (OAS- } \\
\text { M) } \\
\text { G1: } 5.42 \pm 2.17 \\
\text { G2: } 6.25 \pm 1.28 \\
\text { G1 vs G2: p=ns } \\
\text { CYBOCS } \\
\text { G1: NR } \\
\text { G2: NR } \\
\text { G1 vs G2:p=ns }\end{array}$ \\
\hline
\end{tabular}




\begin{tabular}{|c|c|c|c|}
\hline $\begin{array}{c}\text { Author, Year } \\
\text { Study Design } \\
\text { Groups (dose), N enrollment I } \\
\mathrm{N} \text { final } \\
\\
\text { Treatment duration/Follow-up } \\
\text { timepoint post-treatment } \\
\text { Risk of Bias } \\
\end{array}$ & $\begin{array}{c}\text { Mean age, } \\
\text { years } \pm \text { SD } \\
\text { Mean IQ } \pm S D\end{array}$ & $\begin{array}{c}\text { Outcome } \\
\text { measure/Baseline } \\
\text { scores, mean } \pm S D\end{array}$ & $\begin{array}{c}\text { Outcome } \\
\text { measure/Post- } \\
\text { treatment Scores, } \\
\text { mean } \pm \text { SD }\end{array}$ \\
\hline & & & $\begin{array}{l}\text { VABS } \\
\text { G1: NR } \\
\text { G2: NR } \\
\text { G1 vs G2: } p=n s\end{array}$ \\
\hline Bumetanide & & & \\
\hline $\begin{array}{l}\text { Du et al., } 2012^{76} \\
\text { RCT } \\
\text { G1: Bumetanide + ABA } \\
\text { (0.5mg/bid), 32/29 } \\
\text { G2: ABA only ( } 30-40 \text { mins/day), } \\
\text { 28/26 } \\
3 \text { months/EOT } \\
\text { High RoB } \\
\text { (Note: Discussed in section on } \\
\text { combined treatments in main } \\
\text { report) }\end{array}$ & $\begin{array}{l}\text { Age } \\
\text { G1: } 4.60 \pm 1.90 \\
\text { G2: } 4.50 \pm 1.67 \\
\text { IQ } \\
\text { NR }\end{array}$ & $\begin{array}{l}\text { Autism Behavior } \\
\text { Checklist } \\
\text { G2: } 74.68 \pm 4.47 \\
\text { G1: } 75.61 \pm 11.38 \\
\text { G1 vs } G 2, p=0.700 \\
\text { CARS } \\
\text { G2: } 37.28 \pm 3.53 \\
\text { G1: } 36.39 \pm 3.85 \\
\text { G1 vs } G 2, p=0.362 \\
\text { Clinical Global } \\
\text { Impressions (CGI) - } \\
\text { Improvement and } \\
\text { severity } \\
\text { G2: } 4.48 \pm 0.82 \\
\text { G1: } 4.47 \pm 1.05 \\
\text { G1 vs } G 2, p=0.975\end{array}$ & $\begin{array}{l}\text { EOT } \\
\text { Autism Behavior } \\
\text { Checklist } \\
\text { G2: } 62.88 \pm 4.96 \\
\text { G1: } 57.47 \pm 7.19 \\
\text { G1 vs G2, } p<0.01 \\
\text { CARS } \\
\text { G2: } 31.64 \pm 1.72 \\
\text { G1: } 30.79 \pm 2.45 \\
\text { G1 vs G2, p=ns } \\
\text { Clinical Global } \\
\text { Impressions (CGI) - } \\
\text { Improvement and } \\
\text { severity } \\
\text { G1: } \\
\text { G1: } 3.17 \pm 0.38 \\
\text { G2: } 3.40 \pm 0.50 \\
\text { G1 vs } \mathrm{G} 2, \mathrm{p}<0.05\end{array}$ \\
\hline $\begin{array}{l}\text { Lemonnier et al., } 2012^{77} \\
\text { RCT } \\
\text { G1: Bumetanide }(0.5 \mathrm{mg} / \mathrm{bid}), 30 / 27 \\
\text { G2: Placebo (NA), 30/27 } \\
3 \text { months/EOT } \\
\text { Moderate RoB }\end{array}$ & $\begin{array}{l}\text { Age, months } \\
\text { G1: } 82.5 \pm 23.2 \\
\text { G2: } 85.3 \pm 21.3 \\
\text { mos } \\
\text { IQ } \\
\text { NR }\end{array}$ & $\begin{array}{l}\text { CARS } \\
\text { G1: } 41.6 \pm 3.6 \\
\text { G2: } 41.1 \pm 4.1 \\
\text { CGI } \\
\text { NR } \\
\text { ADOS-G } \\
\text { NR }\end{array}$ & $\begin{array}{l}\text { CARS (day 90) } \\
\text { G1: } 36 \pm 5.7 \\
\text { G2: } 39.3 \pm 4.9 G 1 \text { vs } \\
\text { G2: } p=0.004 \\
\text { CARS (day 120) } \\
\text { G1: } 38.8 \pm 4.7 \\
\text { G2: } 40.5 \pm 3.8 \\
\text { EOT } \\
\text { CGI } \\
\text { G1: } 2.04 \pm 0.87 \\
\text { G2: } 1.56 \pm 0.85 \\
\text { G1 vs G2: } p=0.017 \\
\text { Significant } \\
\text { amelioration } \\
\text { G1: } 14 \text { (51.8\% } \\
\text { G2: } 6 \text { (22.2) } \\
\text { Small amelioration } \\
\text { G1: } 7 \text { (25.9) } \\
\text { G2: } 3 \text { (11.1) }\end{array}$ \\
\hline
\end{tabular}




\begin{tabular}{|c|c|c|c|}
\hline $\begin{array}{c}\text { Author, Year } \\
\text { Study Design } \\
\text { Groups (dose), N enrollment I } \\
\text { N final } \\
\\
\text { Treatment duration/Follow-up } \\
\text { timepoint post-treatment } \\
\text { Risk of Bias }\end{array}$ & $\begin{array}{c}\text { Mean age, } \\
\text { years } \pm \text { SD } \\
\text { Mean IQ } \pm S D\end{array}$ & $\begin{array}{c}\text { Outcome } \\
\text { measure/Baseline } \\
\text { scores, mean } \pm S D\end{array}$ & $\begin{array}{c}\text { Outcome } \\
\text { measure/Post- } \\
\text { treatment scores, } \\
\text { mean } \pm \text { SD }\end{array}$ \\
\hline & & & $\begin{array}{l}\text { No amelioration } \\
\text { G1: } 6(22.2) \\
\text { G2: } 18(66.6) \\
\text { ADOS-G, mean } \\
\text { change scores } \\
\text { G1: }+7.8 \pm 7.4 \\
\text { G2: }+5.3 \pm 6.6 \\
\text { G1 vs } G 2: p=n s\end{array}$ \\
\hline HBOT & & & \\
\hline $\begin{array}{l}\text { Sampanthavivat et al., } 2012^{78} \\
\text { RCT } \\
\text { G1: HBOT ( } 20,1 \mathrm{hr} \text { sessions/day at } \\
\text { 153kPa), } 29 / 29 \\
\text { G2: Sham air }(20,1 \mathrm{hr} \\
\text { sessions/day at } 116 \mathrm{kPa}), 29 / 29 \\
20 \text { days/EOT } \\
\text { Moderate RoB }\end{array}$ & $\begin{array}{l}\text { Age } \\
\text { G1: } 6.10 \pm 1.17 \\
\text { G2: } 5.67 \pm 1.01 \\
\text { IQ } \\
\text { NR }\end{array}$ & $\begin{array}{l}\text { ATEC-Parent } \\
\text { G1: } 68.07 \pm 25.43 \\
\text { G2: } 64.86 \pm 22.80 \\
\text { ATEC-Clinician } \\
\text { G1: } 60.21 \pm 19.92 \\
\text { G2: } 60.55 \pm 21.36 \\
\text { CGIS-Parent } \\
\text { G1: } 4.03 \pm 1.05 \\
\text { G2: } 3.79 \pm 0.98 \\
\text { CGIS-Clinician } \\
\text { G1: } 3.62 \pm 0.78 \\
\text { G2: } 3.83 \pm 0.93\end{array}$ & $\begin{array}{l}\text { EOT } \\
\text { ATEC-Parent } \\
\text { G1: } 58.31 \pm 21.94 \\
\text { G2: } 55.86 \pm 24.93 \\
\text { G1 vs G2: } \mathrm{p}=\mathrm{ns} \\
\text { ATEC-Clinician } \\
\text { G1: } 52.38 \pm 19.11 \\
\text { G2: } 52.93 \pm 18.93 \\
\text { G1 vs G2: } \mathrm{p}=\mathrm{ns} \\
\text { CGIS-Parent } \\
\text { G1: } 3.69 \pm 0.93 \\
\text { G2: } 3.66 \pm 0.86 \\
\text { G1, p=0.005 } \\
\text { G2, p=NS } \\
\text { CGIS-Clinician } \\
\text { G1: } 3.48 \pm 0.78 \\
\text { G2: } 3.76 \pm 0.83 \\
\text { G1 vs G2: } \mathrm{p}=\mathrm{ns} \\
\text { Change scores } \\
\text { CGIC-Parent } \\
\text { G1: } 2.34 \pm 0.61 \\
\text { G2: } 2.55 \pm 0.83 \\
\text { G1 vs. G2:p=ns } \\
\text { CGIC-Clinician } \\
\text { G1: } 2.31 \pm 0.6 \\
\text { G2: } 2.72 \pm 0.8 \\
\text { G1 vs G2: } p=0.03\end{array}$ \\
\hline $\begin{array}{l}\text { Granpeesheh et al., } 2010^{79} \\
\text { RCT } \\
\text { G1: HBOT ( } 24 \% \text { oxygen at } 1.3 \text { atm } \\
\text { pressure), } 18 / 17 \\
\text { G2: Placebo (NA), } 16 / 16 \\
15 \text { weeks/EOT }\end{array}$ & $\begin{array}{l}\text { Age } \\
\text { G1: } 6.11 \\
\text { G2: } 6.25 \\
\text { IQ } \\
\text { NR }\end{array}$ & $\begin{array}{l}\text { SRS } \\
\text { NR } \\
\text { ADOS } \\
\text { NR }\end{array}$ & $\begin{array}{l}\text { Mean change score: } \\
\text { SRS- Social } \\
\text { Awareness } \\
\text { G1: }-3.14 \pm 14.21 \\
\text { G2: }-1.33 \pm 16.62 \\
\text { G1 vs G2: } p=n s \\
\text { SRS-Social Cognition }\end{array}$ \\
\hline
\end{tabular}




\begin{tabular}{|c|c|c|c|}
\hline $\begin{array}{c}\text { Author, Year } \\
\text { Study Design } \\
\text { Groups (dose), N enrollment I } \\
\mathrm{N} \text { final } \\
\\
\text { Treatment duration/Follow-up } \\
\text { timepoint post-treatment } \\
\text { Risk of Bias }\end{array}$ & $\begin{array}{c}\text { Mean age, } \\
\text { years } \pm \text { SD } \\
\text { Mean IQ } \pm S D\end{array}$ & $\begin{array}{c}\text { Outcome } \\
\text { measure/Baseline } \\
\text { scores, mean } \pm S D\end{array}$ & $\begin{array}{c}\text { Outcome } \\
\text { measure/Post- } \\
\text { treatment scores, } \\
\text { mean } \pm \text { SD }\end{array}$ \\
\hline Low RoB & & & $\begin{array}{l}\text { G1: } 1.79 \pm 8.82 \\
\text { G2: }-5.33 \pm 16.01 \\
\text { G1 vs } G 2: p=n s \\
\text { SRS-Social } \\
\text { Communication } \\
\text { G1: }-1.00 \pm 13.06 \\
\text { G2: } 3.13 \pm 12.02 \\
\text { G1 vs G2:p=ns } \\
\text { SRS-Social Motivation } \\
\text { G1: }-5.50 \pm 12.45 \\
\text { G2: }-6.33 \pm 13.12 \\
\text { G1 vs } G 2: p=n s \\
\text { SRS-Autistic } \\
\text { Mannerisms } \\
\text { G1: } 1.64 \pm 14.58 \\
\text { G2: }-3.33 \pm 12.49 \\
\text { G1 vs } G 2, p=0.33 \\
\text { Number (\%) improving } \\
\text { ADOS-Total } \\
\text { G1: } 5 / 18(27.8) \\
\text { G2: } 4 / 16(25) \\
\text { G1 vs G2: } p=n s \\
\text { ADOS-Communication } \\
\text { G1: } 3 / 18(16.7) \\
\text { G2: } 2 / 16(12.5) \\
\text { G1 vs G2: } p=n s \\
\text { ADOS-Socialization } \\
\text { G1: } 3 / 18(16.7) \\
\text { G2: } 2 / 16(12.5) \\
\text { G1 vs G2: } p=n s\end{array}$ \\
\hline $\begin{array}{l}\text { Rossignol } 2009^{80} \\
\text { RCT } \\
\text { G1: HBOT ( } 1.3 \mathrm{~atm} \text { and } 24 \% \\
\text { oxygen), } 33 / 30 \\
\text { G2: Room air (1.03 atm and } \\
21 \% \text { oxygen), } 29 / 26 \\
4 \text { weeks/EOT } \\
\text { Low RoB }\end{array}$ & $\begin{array}{l}\text { Age } \\
\text { G1: } 4.97 \pm 1.29 \\
\text { G2: } 4.86 \pm 1.13 \\
\text { IQ } \\
\text { NR }\end{array}$ & $\begin{array}{l}\text { ABC - Irritability } \\
\text { G1: } 13.2 \pm 9.5 \\
\text { G2: } 12.2 \pm 7.9 \\
\text { ABC - Social } \\
\text { Withdrawal/Lethargy } \\
\text { G1: } 10.5 \pm 6.9 \\
\text { G2: } 11.2 \pm 6.9 \\
\text { ABC - Stereotypic } \\
\text { Behavior } \\
\text { G1: } 7.5 \pm 4.9 \\
\text { G2: } 6.2 \pm 4.7\end{array}$ & $\begin{array}{l}\text { ABC - Irritability } \\
\text { G1: } 10.5 \pm 7.4 \\
\text { G2: } 11.3 \pm 6.4 \\
\text { G1 vs G2: } p=0.0976 \\
\text { ABC - Social } \\
\text { Withdrawal/Lethargy } \\
\text { G1: } 9.3 \pm 6.7 \\
\text { G2: } 8.9 \pm 5.6 \\
\text { G1 vs G2: } p=n s \\
\text { ABC - Stereotypic } \\
\text { Behavior }\end{array}$ \\
\hline
\end{tabular}




\begin{tabular}{|c|c|c|c|}
\hline $\begin{array}{c}\text { Author, Year } \\
\text { Study Design } \\
\text { Groups (dose), N enrollment } / \\
\mathrm{N} \text { final }\end{array}$ & $\begin{array}{c}\text { Mean age, } \\
\text { years } \pm \text { SD } \\
\text { Mean IQ } \pm S D\end{array}$ & $\begin{array}{l}\text { Outcome } \\
\text { measure/Baseline } \\
\text { scores, mean } \pm S D\end{array}$ & $\begin{array}{c}\text { Outcome } \\
\text { measure/Post- } \\
\text { treatment scores, } \\
\text { mean } \pm \text { SD }\end{array}$ \\
\hline & & $\begin{array}{l}\text { ABC - Hyperactivity } \\
\text { G1: } 20.7 \pm 9.9 \\
\text { G2: } 20.1 \pm 8.2 \\
\text { ABC - Inappropriate } \\
\text { Speech } \\
\text { G1: } 3.4 \pm 3.1 \\
\text { G2: } 3.6 \pm 3.6 \\
\text { ATEC - Total Score } \\
\text { G1: } 75.3 \pm 19.5 \\
\text { G2: } 75.6 \pm 21\end{array}$ & $\begin{array}{l}\text { G1: } 6.2 \pm 5.1 \\
\text { G2: } 5.4 \pm 4 \\
\text { G1 vs } G 2: p=n s \\
\text { ABC }- \text { Hyperactivity } \\
\text { G1: } 17.8 \pm 9.2 \\
\text { G2: } 16.8 \pm 7.7 \\
\text { G1 vs } G 2: p=n s \\
\text { ABC - Inappropriate } \\
\text { Speech } \\
\text { G1: } 2.6 \pm 2.5 \\
\text { G2: } 3.3 \pm 3.2 \\
\text { ATEC }- \text { Total Score } \\
\text { G1: } 65.9 \pm 16.4 \\
\text { G2: } 70.1 \pm 21.9 \\
\text { G1 vs G2: p=ns } \\
\text { CGI }- \text { Improvement } \\
\text { (Much or very much } \\
\text { improved) } \\
\text { G1: } 9 \text { (30) } \\
\text { G2: } 2 \text { (7.7) } \\
\text { G1 vs G2: } p=0.0471\end{array}$ \\
\hline \multicolumn{4}{|l|}{ Melatonin } \\
\hline $\begin{array}{l}\text { Cortesi et al., 2012 } \\
\text { RCT } \\
\text { G1: Controlled-release melatonin + } \\
\text { CBT (3mg/day), 40/35 } \\
\text { G2: Controlled-release melatonin } \\
\text { (3mg/day), 40/34 } \\
\text { G3: CBT (4 sessions/week), 40/33 } \\
\text { G4: Placebo (NA), 40/32 } \\
12 \text { weeks/EOT } \\
\text { Low } \\
\text { (Note: Discussed in section on } \\
\text { combined treatments in main } \\
\text { report) }\end{array}$ & $\begin{array}{l}\text { Age } \\
\text { G1: } 6.4 \pm 1.1 \\
\text { G2: } 6.8 \pm 0.9 \\
\text { G3: } 7.1 \pm 0.7 \\
\text { G4: } 6.3 \pm 1.2 \\
\text { IQ } \\
\text { NR }\end{array}$ & $\begin{array}{l}\text { CSHQ } \\
\text { CSHQ-Total score } \\
\text { G1: } 66.11 \pm 5.47 \\
\text { G2: } 66.67 \pm 8.55 \\
\text { G3: } 64.48 \pm 5.48 \\
\text { G4: } 64.20 \pm 4.85 \\
\text { Bed resistance } \\
\text { G1: } 14.53 \pm 1.82 \\
\text { G2: } 13.85 \pm 2.23 \\
\text { G3: } 13.44 \pm 2.08 \\
\text { G4: } 13.63 \pm 1.82 \\
\text { SOD } \\
\text { G1: } 2.88 \pm 0.32 \\
\text { G2: } 2.85 \pm 0.35 \\
\text { G3: } 2.89 \pm 0.30 \\
\text { G4: } 2.90 \pm 0.31 \\
\text { Sleep anxiety } \\
\text { G1: } 7.95 \pm 1.83 \\
\text { G2: } 8.35 \pm 2.19\end{array}$ & $\begin{array}{l}\text { EOT } \\
\text { CSHQ } \\
\text { CSHQ-Total score } \\
\text { G1: } 47.84 \pm 2.94 \\
\text { G2: } 54.78 \pm 6.22 \\
\text { G3: } 60.06 \pm 4.71 \\
\text { G4: } 64.80 \pm 4.52 \\
\text { Time } \times \text { group } p<0.001 \\
\text { Bed resistance } \\
\text { G1: } 8.46 \pm 1.39 \\
\text { G2: } 10.50 \pm 2.20 \\
\text { G3: } 11.62 \pm 2.22 \\
\text { G4: } 14.10 \pm 1.93 \\
\text { Time } \times \text { group } p<0.001 \\
\text { SOD } \\
\text { G1: } 1.69 \pm 0.73 \\
\text { G2: } 2.10 \pm 0.68 \\
\text { G3: } 2.51 \pm 0.57 \\
\text { G4: } 2.93 \pm 0.25 \\
\text { Time } x \text { group } p<0.001\end{array}$ \\
\hline
\end{tabular}




\begin{tabular}{|c|c|c|c|}
\hline $\begin{array}{c}\text { Author, Year } \\
\text { Study Design } \\
\text { Groups (dose), N enrollment I } \\
\mathrm{N} \text { final } \\
\\
\text { Treatment duration/Follow-up } \\
\text { timepoint post-treatment } \\
\text { Risk of Bias }\end{array}$ & $\begin{array}{c}\text { Mean age, } \\
\text { years } \pm \text { SD } \\
\text { Mean IQ } \pm S D\end{array}$ & $\begin{array}{l}\text { Outcome } \\
\text { measure/Baseline } \\
\text { scores, mean } \pm S D\end{array}$ & $\begin{array}{c}\text { Outcome } \\
\text { measure/Post- } \\
\text { treatment scores, } \\
\text { mean } \pm \text { SD }\end{array}$ \\
\hline & & $\begin{array}{l}\text { G3: } 8.62 \pm 1.98 \\
\text { G4: } 7.66 \pm 1.73 \\
\text { Night-wakings } \\
\text { G1: } 7.61 \pm 0.89 \\
\text { G2: } 7.67 \pm 0.94 \\
\text { G3: } 7.62 \pm 0.94 \\
\text { G4: } 7.76 \pm 0.93 \\
\text { Sleep duration } \\
\text { G1: } 7.34 \pm 1.35 \\
\text { G2: } 7.17 \pm 1.51 \\
\text { G3: } 7.01 \pm 1.48 \\
\text { G4: } 6.46 \pm 1.25 \\
\text { Parasomnias } \\
\text { G1: } 9.15 \pm 1.68 \\
\text { G2: } 9.10 \pm 2.42 \\
\text { G3: } 9.75 \pm 2.11 \\
\text { G4: } 8.96 \pm 1.80 \\
\text { SDB } \\
\text { G1: } 3.18 \pm 0.40 \\
\text { G2: } 3.20 \pm 0.44 \\
\text { G3: } 3.10 \pm 0.30 \\
\text { G4: } 3.15 \pm 0.40 \\
\text { DS } \\
\text { G1: } 13.92 \pm 2.86 \\
\text { G2: } 13.35 \pm 3.84 \\
\text { G3: } 13.31 \pm 2.67 \\
\text { G4: } 13.13 \pm 3.11 \\
\text { Sleep Diary } \\
\text { TST } \\
\text { G1: } 414.03 \pm 45.34 \\
\text { G2: } 410.28 \pm 45.07 \\
\text { G3: } 408.08 \pm 49.03 \\
\text { G4: } 413.00 \pm 45.13 \\
\text { SOL } \\
\text { G1: } 85.84 \pm 20.02 \\
\text { G2: } 81.21 \pm 32.35 \\
\text { G3: } 76.34 \pm 31.70 \\
\text { G4: } 78.20 \pm 33.83 \\
\text { WASO } \\
\text { G1: } 69.50 \pm 23.35 \\
\text { G2: } 73.71 \pm 45.00 \\
\text { G3: } 68.72 \pm 31.77 \\
\text { G4: } 69.75 \pm 45.21\end{array}$ & $\begin{array}{l}\text { Sleep anxiety } \\
\text { G1: } 5.23 \pm 0.95 \\
\text { G2: } 7.21 \pm 1.87 \\
\text { G3: } 7.17 \pm 1.48 \\
\text { G4: } 7.93 \pm 1.99 \\
\text { Time } \times \text { group } p<0.001 \\
\text { Night-wakings } \\
\text { G1: } 4.42 \pm 0.90 \\
\text { G2: } 5.03 \pm 1.10 \\
\text { G3: } 7.06 \pm 1.06 \\
\text { G4: } 7.86 \pm 0.81 \\
\text { Time } \times \text { group } p<0.001 \\
\text { Sleep duration } \\
\text { G1: } 4.38 \pm 1.02 \\
\text { G2: } 4.82 \pm 0.94 \\
\text { G3: } 6.68 \pm 1.16 \\
\text { G4: } 6.40 \pm 1.29 \\
\text { Time } \times \text { group } p<0.001 \\
\text { Parasomnias } \\
\text { G1: } 8.92 \pm 1.38 \\
\text { G2: } 9.35 \pm 1.78 \\
\text { G3: } 9.82 \pm 2.25 \\
\text { G4: } 9.16 \pm 1.53 \\
\text { Time } \times \text { group } p=n s \\
\text { SDB } \\
\text { G1: } 3.22 \pm 0.35 \\
\text { G2: } 3.15 \pm 0.48 \\
\text { G3: } 3.20 \pm 0.41 \\
\text { G4: } 3.20 \pm 0.44 \\
\text { Time } \times \text { group } p=n s \\
\text { DS } \\
\text { G1: } 10.84 \pm 1.68 \\
\text { G2: } 11.39 \pm 2.34 \\
\text { G3: } 11.96 \pm 1.97 \\
\text { G4: } 12.96 \pm 1.97 \\
\text { Time } \times \text { group } p<0.001 \\
\text { Sleep Diary } \\
\text { TST } \\
\text { G1: } 505.01 \pm 31.18 \\
\text { G2: } 481.10 \pm 33.15 \\
\text { G3: } 445.13 \pm 48.37 \\
\text { G4: } 416.23 \pm 43.60 \\
\text { Time } \times \text { group } p<0.001\end{array}$ \\
\hline
\end{tabular}




\begin{tabular}{|c|c|c|c|}
\hline $\begin{array}{c}\text { Author, Year } \\
\text { Study Design } \\
\text { Groups (dose), N enrollment } / \\
\mathrm{N} \text { final } \\
\\
\text { Treatment duration/Follow-up } \\
\text { timepoint post-treatment } \\
\text { Risk of Bias }\end{array}$ & $\begin{array}{c}\text { Mean age, } \\
\text { years } \pm \text { SD } \\
\text { Mean IQ } \pm \text { SD }\end{array}$ & $\begin{array}{c}\text { Outcome } \\
\text { measure/Baseline } \\
\text { scores, mean } \pm S D\end{array}$ & $\begin{array}{c}\text { Outcome } \\
\text { measure/Post- } \\
\text { treatment scores, } \\
\text { mean } \pm \text { SD }\end{array}$ \\
\hline & & $\begin{array}{l}\text { NAPTIME } \\
\text { G1: } 28.26 \pm 49.13 \\
\text { G2: } 33.57 \pm 56.63 \\
\text { G3: } 35.31 \pm 60.17 \\
\text { G4: } 37.33 \pm 56.19 \\
\text { SE } \\
\text { G1: } 70.26 \pm 4.83 \\
\text { G2: } 71.10 \pm 4.91 \\
\text { G3: } 71.37 \pm 4.77 \\
\text { G4: } 71.13 \pm 4.99 \\
\text { BEDTIME } \\
\text { G1: } 23.33 \pm 1.35 \\
\text { G2: } 23.45 \pm 1.15 \\
\text { G3: } 23.39 \pm 1.03 \\
\text { G4: } 23.41 \pm 1.19\end{array}$ & $\begin{array}{l}\text { SOL } \\
\text { G1: } 33.69 \pm 14.40 \\
\text { G2: } 45.21 \pm 23.21 \\
\text { G3: } 59.13 \pm 27.60 \\
\text { G4: } 79.60 \pm 31.85 \\
\text { Time } \times \text { group } p<0.001 \\
\text { WASO } \\
\text { G1: } 29.69 \pm 12.97 \\
\text { G2: } 42.21 \pm 22.35 \\
\text { G3: } 61.17 \pm 28.93 \\
\text { G4: } 70.15 \pm 42.76 \\
\text { Time } \times \text { group p }<0.001 \\
\text { NAPTIME } \\
\text { G1: } 9.20 \pm 22.48 \\
\text { G2: } 17.00 \pm 33.11 \\
\text { G3: } 12.29 \pm 24.24 \\
\text { G4: } 36.10 \pm 33.28 \\
\text { Time } \times \text { group p }=n s \\
\text { SE } \\
\text { G1: } 84.46 \pm 4.23 \\
\text { G2: } 82.71 \pm 4.00 \\
\text { G3: } 79.58 \pm 2.82 \\
\text { G4: } 71.93 \pm 4.62 \\
\text { Time } \times \text { group } p<0.001 \\
\text { BEDTIME } \\
\text { G1: } 22.06 \pm 1.05 \\
\text { G2: } 22.30 \pm 1.10 \\
\text { G3: } 22.55 \pm 1.01 \\
\text { G4: } 23.51 \pm 1.12 \\
\text { Time } \times \text { group p }<0.001\end{array}$ \\
\hline $\begin{array}{l}\text { Wright et al., } 2011^{82} \\
\text { RCT } \\
\text { G1: Melatonin (2-10mg/day), 9/6 } \\
\text { G2: Placebo (NA), 11/10 } \\
\text { Crossover } \\
9 \text { months/EOT } \\
\text { Moderate RoB }\end{array}$ & $\begin{array}{l}\text { Age } \\
\text { G1: } 8.9 \pm 3.0 \\
\text { G2: } 8.5 \pm 2.3 \\
\text { IQ } \\
\text { NR }\end{array}$ & $\begin{array}{l}\text { Dysomnias, } \mathrm{n}=12 \\
\mathrm{G} 1+\mathrm{G} 2: 34.0 \pm 9.4 \\
\text { Parasomnias, } \mathrm{n}=14 \\
\mathrm{G} 1+\mathrm{G} 2: 18.7 \pm 7.7 \\
\text { Sleep apneas, } \mathrm{n}=13 \\
\mathrm{G} 1+\mathrm{G} 2: 13.0 \pm 9.4 \\
\text { Other sleep disorders, } \\
\mathrm{n}=10 \\
\mathrm{G} 1+\mathrm{G} 2: 25.1 \pm 5.6 \\
\\
\text { DBC-Total, } \mathrm{n}=17 \\
\mathrm{G} 1+\mathrm{G} 2: 90.1 \pm 18.7\end{array}$ & $\begin{array}{l}\text { EOT } \\
\text { Dysomnias, } n=12 \\
\text { G1: } 22.2 \pm 6.6 \\
\text { G2: } 27.8 \pm 6.3 \\
p=0.04 \\
\text { Parasomnias, } n=14 \\
\text { G1: } 17.3 \pm 4.5 \\
\text { G2: } 20.1 \pm 8.1 \\
p=n s \\
\text { Sleep apneas, } n=13 \\
\text { G1: } 11.4 \pm 4.2 \\
\text { G2: } 12.7 \pm 3.4 \\
p=n s\end{array}$ \\
\hline
\end{tabular}




\begin{tabular}{|c|c|c|c|}
\hline $\begin{array}{c}\text { Author, Year } \\
\text { Study Design } \\
\text { Groups (dose), N enrollment I } \\
\mathrm{N} \text { final } \\
\\
\text { Treatment duration/Follow-up } \\
\text { timepoint post-treatment } \\
\text { Risk of Bias }\end{array}$ & $\begin{array}{c}\text { Mean age, } \\
\text { years } \pm \text { SD } \\
\text { Mean IQ } \pm S D\end{array}$ & $\begin{array}{c}\text { Outcome } \\
\text { measure/Baseline } \\
\text { scores, mean } \pm S D\end{array}$ & $\begin{array}{c}\text { Outcome } \\
\text { measure/Post- } \\
\text { treatment scores, } \\
\text { mean } \pm \text { SD }\end{array}$ \\
\hline & & $\begin{array}{l}\text { DBC-Disruptive, } \mathrm{n}=17 \\
\mathrm{G} 1+\mathrm{G} 2: 26.2 \pm 13.0 \\
\text { DBC- Self-absorption, } \\
\mathrm{n}=17 \\
\mathrm{G} 1+\mathrm{G} 2: 33.0 \pm 11.0 \\
\text { DBC-Communication, } \\
\mathrm{n}=17 \\
\mathrm{G} 1+\mathrm{G} 2: 11.4 \pm 3.6 \\
\text { DBC-Anxiety, } \mathrm{n}=17 \\
\mathrm{G} 1+\mathrm{G} 2: 8.8 \pm 2.8 \\
\mathrm{DBC}-\mathrm{Social} \text { relating, } \\
\mathrm{n}=17 \\
\mathrm{G} 1+\mathrm{G} 2: \mathrm{:} .4 \pm 3.1 \\
\mathrm{GHQ} \text { total } \mathrm{n}=17 \\
\mathrm{G} 1+\mathrm{G} 2: 3.7 \pm 7.1 \\
\text { No. of wakenings } \mathrm{n}=17 \\
\mathrm{G} 1+\mathrm{G} 2: 0.5 \pm 0.5 \\
\text { Total sleep minutes, } \\
\mathrm{n}=17 \\
\mathrm{G} 1+\mathrm{G} 2: 499.9 \pm 66.4 \\
\text { Sleep latency, } \mathrm{n}=17 \\
\mathrm{G} 1+\mathrm{G} 2: 135.0 \pm 63.0\end{array}$ & $\begin{array}{l}\text { Other sleep disorders, } \\
n=10 \\
\text { G1: } 23.8 \pm 3.4 \\
\text { G2: } 25.9 \pm 6.2 \\
p=n s \\
\text { DBC-Total } \\
\text { G1: } 75.12 \pm 23.29 \\
\text { G2: } 82.25 \pm 25.79 \\
p=0.05 \\
\text { DBC-Disruptive } \\
\text { G1: } 21.82 \pm 11.97 \\
\text { G2: } 23.88 \pm 11.12 \\
p=n s \\
\text { DBC- Self-absorption } \\
\text { G1: } 28.94 \pm 13.41 \\
\text { G2: } 30.06 \pm 14.05 \\
\text { p=ns } \\
\text { DBC-Communication } \\
\text { G1: } 8.24 \pm 3.29 \\
\text { G2: } 9.75 \pm 5.04 \\
p=0.045 \\
\text { DBC-Anxiety } \\
\text { G1: } 7.53 \pm 3.39 \\
\text { G2: } 7.56 \pm 3.86 \\
\text { p=ns } \\
\text { DBC-Social relating } \\
\text { G1: } 7.82 \pm 3.11 \\
\text { G2: } 9.06 \pm 4.27 \\
p=n s \\
\text { GHQ total } \\
\text { G1: } 1.4 \pm 2.7 \\
\text { G2: } 3.2 \pm 4.6 \\
p=n s \\
\text { No. of wakenings } \\
\text { G1: } 0.43 \pm 0.64 \\
\text { G2: } 0.58 \pm 0.74 \\
p=n s \\
\text { Total sleep minutes } \\
\text { G1: } 556.11 \pm 53.59 \\
\text { G2: } 507.66 \pm 70.67 \\
p=0.002\end{array}$ \\
\hline
\end{tabular}




\begin{tabular}{|c|c|c|c|}
\hline $\begin{array}{c}\text { Author, Year } \\
\text { Study Design } \\
\text { Groups (dose), N enrollment I } \\
\mathrm{N} \text { final } \\
\\
\text { Treatment duration/Follow-up } \\
\text { timepoint post-treatment } \\
\text { Risk of Bias }\end{array}$ & $\begin{array}{c}\text { Mean age, } \\
\text { years } \pm \text { SD } \\
\text { Mean IQ } \pm S D\end{array}$ & $\begin{array}{c}\text { Outcome } \\
\text { measure/Baseline } \\
\text { scores, mean } \pm S D\end{array}$ & $\begin{array}{c}\text { Outcome } \\
\text { measure/Post- } \\
\text { treatment scores, } \\
\text { mean } \pm \text { SD }\end{array}$ \\
\hline & & & $\begin{array}{l}\text { Sleep latency } \\
\text { G1: } 82.84 \pm 50.61 \\
\text { G2: } 124.79 \pm N R \\
p=0.004\end{array}$ \\
\hline Other & & & \\
\hline $\begin{array}{l}\text { Amatachaya et al., } 2014^{83,84} \\
\text { RCT } \\
\text { G1: Transcranial direct current } \\
\text { stimulation (1mA), 10/10 } \\
\text { G2: Sham (NA), 10/10 } \\
8 \text { weeks/EOT } \\
\text { Moderate RoB }\end{array}$ & $\begin{array}{l}\text { Age } \\
\text { G1 + G2: 5-8 } \\
\text { IQ } \\
\text { NR }\end{array}$ & $\begin{array}{l}\text { CARS } \\
\text { G1: } 34.95 \pm 4.73 \\
\text { G2: } 34.6 \pm 4.41 \\
\text { ATEC-Total } \\
\text { G1: } 67.25 \pm 9.88 \\
\text { G2: } 69.15 \pm 8.98 \\
\text { ATEC-Language } \\
\text { G1: } 10.6 \pm 5.59 \\
\text { G2: } 10.75 \pm 4.72 \\
\text { ATEC- Social } \\
\text { G1: } 16.4 \pm 4.5 \\
\text { G2: } 17.45 \pm 2.67 \\
\text { ATEC-Sensory and } \\
\text { Cognitive awareness } \\
\text { G1: } 20.1 \pm 3.91 \\
\text { G2: } 20.5 \pm 3.4 \\
\text { ATEC-Health and } \\
\text { behavioral problem } \\
\text { G1: } 20.15 \pm 8.34 \\
\text { G2: } 20.45 \pm 7.21 \\
\text { CGAS } \\
\text { G1: } 54.35 \pm 11.07 \\
\text { G2: } 53.35 \pm 10.31 \\
\text { CGI-Severity } \\
\text { G1: } 4.05 \pm 0.94 \\
\text { G2: } 4.15 \pm 0.99\end{array}$ & $\begin{array}{l}\text { EOT } \\
\text { CARS } \\
\text { G1: } 32.2 \pm 3.98 \\
\text { G2: } 35 \pm 4.3 \\
\text { p < } 0.05 \\
\text { ATEC-Total } \\
\text { G1: } 58 \pm 5.28 \\
\text { G2: } 69.65 \pm 9.13 \\
\text { p } \leq 0.001 \\
\text { ATEC-Language } \\
\text { G1: } 10.5 \pm 5.39 \\
\text { G2: } 10.55 \pm 5.2 \\
\text { ATEC- Social } \\
\text { G1: } 14.45 \pm 4.85 \\
\text { G2: } 17.7 \pm 2.98 \\
\text { p }<0.05 \\
\text { ATEC-Sensory and } \\
\text { cognitive awareness } \\
\text { G1: } 18.35 \pm 5.35 \\
\text { G2: } 22.3 \pm 4.47 \\
\text { p }<0.05 \\
\text { ATEC-Health and } \\
\text { behavioral problem } \\
\text { G1: } 14.7 \pm 6.21 \\
\text { G2: } 19.1 \pm 6.47 \\
\text { p }<0.05 \\
\text { CGAS } \\
\text { G1: } 60 \pm 10.57 \\
\text { G2: } 53.1 \pm 10.14 \\
\text { p }<0.05 \\
\text { CGI-Improvement, } n \\
(\%) \\
\text { Very much improved } \\
\text { G1: } 0 \\
\text { G2: } 0 \\
\text { p=ns }\end{array}$ \\
\hline
\end{tabular}




\begin{tabular}{|c|c|c|c|}
\hline $\begin{array}{c}\text { Author, Year } \\
\text { Study Design } \\
\text { Groups (dose), N enrollment I } \\
\mathrm{N} \text { final } \\
\\
\text { Treatment duration/Follow-up } \\
\text { timepoint post-treatment } \\
\text { Risk of Bias }\end{array}$ & $\begin{array}{c}\text { Mean age, } \\
\text { years } \pm \text { SD } \\
\text { Mean IQ } \pm S D\end{array}$ & $\begin{array}{c}\text { Outcome } \\
\text { measure/Baseline } \\
\text { scores, mean } \pm S D\end{array}$ & $\begin{array}{c}\text { Outcome } \\
\text { measure/Post- } \\
\text { treatment scores, } \\
\text { mean } \pm \text { SD }\end{array}$ \\
\hline & & & $\begin{array}{l}\text { Much improved } \\
\text { G1: } 9 \text { (45) } \\
\text { G2: } 3(15) \\
\text { p } 0.05 \\
\text { Minimally improved } \\
\text { G1: } 8 \text { (40) } \\
\text { p=ns } \\
\text { No change } \\
\text { G1: } 1(5) \\
\text { G2: } 6 \text { (30) } \\
\text { p=ns } \\
\text { Minimally worse } \\
\text { G1: } 2(10) \\
\text { G2: } 2 \text { (10) } \\
\text { p=ns } \\
\text { Much worse } \\
\text { G1: } 0 \\
\text { G2: } 5 \text { (25) } \\
\text { p }<0.05 \\
\text { Very much worse } \\
\text { G1: } 0 \\
\text { G2: } 0 \\
p=n s\end{array}$ \\
\hline $\begin{array}{l}\text { Dadds et al., } 2014^{85} \\
\text { RCT } \\
\text { G1: Intranasal oxytocin (12 or } 24 \\
\text { IU), } 19 / 19 \\
\text { G2: Placebo (NA), 19/189 } \\
4 \text { days/EOT } \\
\text { Moderate RoB }\end{array}$ & $\begin{array}{l}\text { Age } \\
\text { G1: } 11.79 \pm \\
2.82 \\
\text { G2: } 10.74 \pm \\
2.38 \\
\text { IQ } \\
\text { G1: } 90.47 \pm \\
11.70 \\
\text { G2: } 88.64 \pm \\
7.98\end{array}$ & $\begin{array}{l}\text { SRS } \\
\text { G1: } 17.13 \pm 5.57 \\
\text { G2: } 20.56 \pm 6.70 \\
\text { Video Observation } \\
\text { Repetitive Behaviors } \\
\text { G1: } 0.24 \pm 0.26 \\
\text { G2: } 0.20 \pm 0.25 \\
\text { Video Observation } \\
\text { Social Interaction Skills } \\
\text { G1: } 1.63 \pm 0.50 \\
\text { G2: } 1.69 \pm 0.38 \\
\text { Facial Emotion } \\
\text { Recognition } \\
\text { G1: } 0.79 \pm 0.11 \\
\text { G2: } 0.77 \pm 0.14 \\
\text { OSU } \\
\text { G1: } 3.76 \pm 0.70 \\
\text { G2: } 4.17 \pm 0.79\end{array}$ & $\begin{array}{l}\text { 3 mos follow-up } \\
\text { SRS } \\
\text { G1:16.47 } \pm 5.94 \\
\text { G2: } 15.78 \pm 7.85 \\
\text { G1 vs G2: } p=n s \\
\text { Video Observation } \\
\text { Repetitive Behaviors } \\
\text { G1: } 0.27 \pm 0.24 \\
\text { G2: } 0.25 \pm 0.24 \\
\text { G1 vs G2: } p=n s \\
\text { Video Observation } \\
\text { Social Interaction Skills } \\
\text { G1: } 1.92 \pm 0.51 \\
\text { G2: } 1.96 \pm 0.49 \\
\text { G1 vs G2: } p=n s \\
\text { Facial } E \text { motion } \\
\text { Recognition } \\
\text { G1: } 0.87 \pm 0.08 \\
\text { G2: } 0.86 \pm 0.07 \\
\text { G1 vs G2: } p=n s\end{array}$ \\
\hline
\end{tabular}




\begin{tabular}{|c|c|c|c|}
\hline $\begin{array}{c}\text { Author, Year } \\
\text { Study Design } \\
\text { Groups (dose), N enrollment I } \\
\mathrm{N} \text { final } \\
\\
\text { Treatment duration/Follow-up } \\
\text { timepoint post-treatment } \\
\text { Risk of Bias }\end{array}$ & $\begin{array}{c}\text { Mean age, } \\
\text { years } \pm \text { SD } \\
\text { Mean IQ } \pm S D\end{array}$ & $\begin{array}{c}\text { Outcome } \\
\text { measure/Baseline } \\
\text { scores, mean } \pm S D\end{array}$ & $\begin{array}{c}\text { Outcome } \\
\text { measure/Post- } \\
\text { treatment scores, } \\
\text { mean } \pm \text { SD }\end{array}$ \\
\hline & & $\begin{array}{l}\text { CARS } \\
\text { G1: } 32.26 \pm 3.25 \\
\text { G2: } 33.84 \pm 3.70\end{array}$ & $\begin{array}{l}\text { OSU } \\
\text { G1: } 3.60 \pm 0.63 \\
\text { G2: } 3.78 \pm 0.94 \\
\text { G1 vs } G 2: p=n s \\
\text { CARS } \\
\text { G1: } 30.07 \pm 2.78 \\
\text { G2: } 31.06 \pm 5.20 \\
\text { G1 vs } G 2: p=n s\end{array}$ \\
\hline $\begin{array}{l}\text { Klaiman et al., } 2013^{86} \\
\text { RCT } \\
\text { G1: Tetrahydrobiopterin } \\
\text { (20mg/kg/day), 23/23 } \\
\text { G2: Placebo (NA), 23/23 } \\
16 \text { weeks/EOT } \\
\text { Low RoB }\end{array}$ & $\begin{array}{l}\text { Age, months } \\
\text { G1: } 60.1 \pm 12.0 \\
\text { G2: } 60.2 \pm 12.6 \\
\text { IQ } \\
\text { NR }\end{array}$ & $\begin{array}{l}\text { CGI-Severity [N (\%)] } \\
\text { Frequency of } \\
\text { markedly, severely, or } \\
\text { extremely ill } \\
\text { G1: } 10 \text { (48) } \\
\text { G2: } 15 \text { (68) } \\
\text { ABC-Irritability } \\
\text { G1: } 11.1 \pm 7.7 \\
\text { G2: } 11.9 \pm 7.8 \\
\text { ABC-Social } \\
\text { withdrawal/lethargy } \\
\text { G1: } 9.5 \pm 7.5 \\
\text { G2: } 16.2 \pm 10.0 \\
\text { ABC-Stereotypy } \\
\text { G1: } 6.1 \pm 3.9 \\
\text { G2: } 6.1 \pm 3.6 \\
\text { ABC-Hyperactivity } \\
\text { G1: } 21.5 \pm 10.3 \\
\text { G2: } 22.9 \pm 11.6 \\
\text { ABC-Inappropriate } \\
\text { speech } \\
\text { G1: } 3.7 \pm 2.4 \\
\text { G2: } 3.4 \pm 4.1 \\
\text { SRS } \\
\text { G1: } 81.4 \pm 10.3 \\
\text { G2: } 83.6 \pm 9.2 \\
\text { PLS } \\
\text { G1: } 77.8 \pm 29.2 \\
\text { G2: } 57.1 \pm 25.7 \\
\text { Vineland } \\
\text { G1: } 320.5 \pm 47.9 \\
\text { G2: } 274.4 \pm 51.4\end{array}$ & 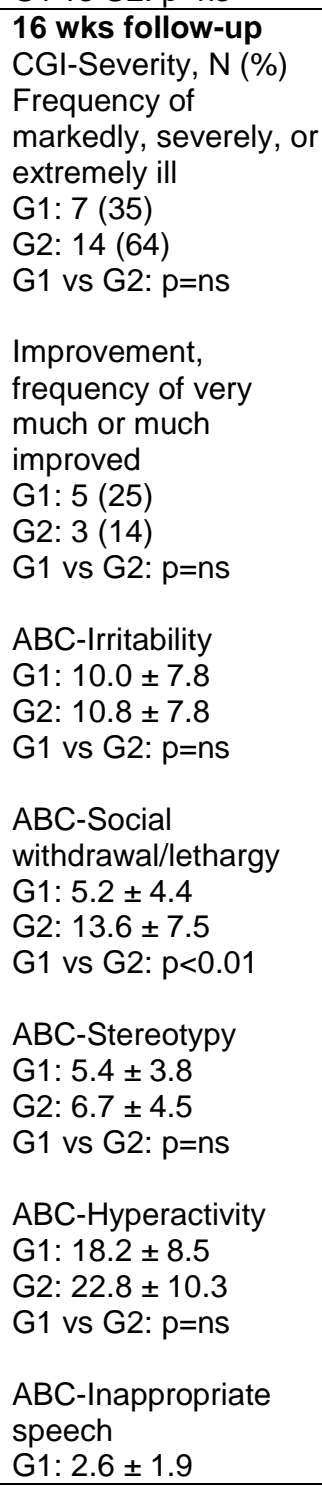 \\
\hline
\end{tabular}




\begin{tabular}{|c|c|c|c|}
\hline $\begin{array}{c}\text { Author, Year } \\
\text { Study Design } \\
\text { Groups (dose), N enrollment I } \\
\mathrm{N} \text { final } \\
\\
\text { Treatment duration/Follow-up } \\
\text { timepoint post-treatment } \\
\text { Risk of Bias }\end{array}$ & $\begin{array}{c}\text { Mean age, } \\
\text { years } \pm \text { SD } \\
\text { Mean IQ } \pm S D\end{array}$ & $\begin{array}{c}\text { Outcome } \\
\text { measure/Baseline } \\
\text { scores, mean } \pm S D\end{array}$ & $\begin{array}{c}\text { Outcome } \\
\text { measure/Post- } \\
\text { treatment scores, } \\
\text { mean } \pm \text { SD }\end{array}$ \\
\hline & & & $\begin{array}{l}\text { G2: } 3.9 \pm 3.6 \\
\text { G1 vs } G 2: p=n s \\
\text { SRS } \\
\text { G1: } 76.7 \pm 10.9 \\
\text { G2: } 83.2 \pm 10.4 \\
\text { G1 vs } G 2: p=n s \\
\text { PLS } \\
\text { G1: } 84.0 \pm 28.8 \\
\text { G2: } 60.4 \pm 25.4 \\
\text { G1 vs } G 2: p=0.01 \\
\text { Vineland } \\
\text { G1: } 344.76 \pm 50.0 \\
\text { G2: } 294.9 \pm 70.1 \\
\text { G1 vs } G 2: p=0.02\end{array}$ \\
\hline $\begin{array}{l}\text { LV et al., } 2013^{87} \\
\text { NRCT } \\
\text { G1: Human cord blood } \\
\text { mononuclear cells (CBMNCs) }(2 \times \\
\left.10^{6} / \mathrm{kg}\right)+ \text { rehabilitation therapy, } \\
14 / 14 \\
\text { G2: CBMNCs + umbilical cord- } \\
\text { derived mesenchymal stem cells } \\
(\mathrm{UCMSCs})\left(2 \times 10^{6} / \mathrm{kg} \text { and } 1 \times\right. \\
\left.10^{6} / \mathrm{kg}\right)+ \text { rehabilitation, } 9 / 9 \\
\text { G3: Rehabilitation therapy, } 14 / 13 \\
5-7 \text { days ( } 4 \text { transplants)/EOT } \\
\text { High RoB }\end{array}$ & $\begin{array}{l}\text { Age } \\
\text { G1: } 7.41 \pm 2.63 \\
\text { G2: } 6.20 \pm 2.12 \\
\text { G3: } 5.60 \pm 2.01 \\
\text { IQ } \\
\text { G1: NR } \\
\text { G2: NR } \\
\text { G3: NR }\end{array}$ & $\begin{array}{l}\text { CARS } \\
\text { G1: } 46.43 \pm 8.65 \\
\text { G2: } 45.11 \pm 4.31 \\
\text { G3: } 43.15 \pm 4.38 \\
\text { CGI } \\
\text { NR } \\
\text { ABC-Total } \\
\text { G1: } 94.07 \pm 21.98 \\
\text { G2: } 91.78 \pm 25.92 \\
\text { G3: } 75.46 \pm 12.05 \\
\text { ABC-Irritability } \\
\text { G1: } 16.36 \pm 9.61 \\
\text { G2: } 15.00 \pm 7.81 \\
\text { G3: } 9.15 \pm 5.58 \\
\text { ABC-Lethargy/Social } \\
\text { withdrawal } \\
\text { G1: } 30.71 \pm 6.08 \\
\text { G2: } 31.00 \pm 6.98 \\
\text { G3: } 35.08 \pm 4.96 \\
\text { ABC-Stereotypic } \\
\text { behavior } \\
\text { G1: } 29.43 \pm 9.77 \\
\text { G2: } 28.33 \pm 8.47 \\
\text { G3: } 22.77 \pm 6.86 \\
\text { ABC-Hyperactivity } \\
\text { G1: } 11.86 \pm 4.55 \\
\text { G2: } 11.89 \pm 6.88 \\
\text { G3: } 6.08 \pm 3.15\end{array}$ & $\begin{array}{l}\text { EOT } \\
\text { CARS } \\
\text { G1: } 37.14 \pm 10.15 \\
\text { G2: } 28.00 \pm 6.18 \\
\text { G3: } 37.23 \pm 3.42 \\
\text { CGI-GI [N (\%)] } \\
\text { Very much improved } \\
\text { G1: } 1(7.14) \\
\text { G2: } 3 \text { (33.33) } \\
\text { G3: } 0 \text { (0) } \\
\text { Much improved } \\
\text { G1: } 6 \text { (42.86) } \\
\text { G2: } 5 \text { (55.56) } \\
\text { G3: } 1 \text { (7.69) } \\
\text { Minimally improved } \\
\text { G1: } 2 \text { (14.29) } \\
\text { G2: } 1 \text { (11.11) } \\
\text { G3: } 11 \text { (84.62) } \\
\text { No change } \\
\text { G1: } 5 \text { (35.71) } \\
\text { G2: } 0 \text { (0.00) } \\
\text { G3: } 1 \text { (7.69) } \\
\text { Minimally worse } \\
\text { G1: } 0 \text { (0.00) } \\
\text { G2: } 0 \text { (0.00) } \\
\text { G3: } 0 \text { (0.00) } \\
\text { Much worse } \\
\text { G1: } 0 \text { (0.00) }\end{array}$ \\
\hline
\end{tabular}




\begin{tabular}{|c|c|c|c|}
\hline $\begin{array}{c}\text { Author, Year } \\
\text { Study Design } \\
\text { Groups (dose), N enrollment I } \\
\mathrm{N} \text { final } \\
\\
\text { Treatment duration/Follow-up } \\
\text { timepoint post-treatment } \\
\text { Risk of Bias }\end{array}$ & $\begin{array}{c}\text { Mean age, } \\
\text { years } \pm \text { SD } \\
\text { Mean IQ } \pm S D\end{array}$ & $\begin{array}{c}\text { Outcome } \\
\text { measure/Baseline } \\
\text { scores, mean } \pm S D\end{array}$ & $\begin{array}{c}\text { Outcome } \\
\text { measure/Post- } \\
\text { treatment scores, } \\
\text { mean } \pm \text { SD }\end{array}$ \\
\hline & & $\begin{array}{l}\text { ABC-Inappropriate } \\
\text { speech } \\
\text { G1: } 5.71 \pm 4.30 \\
\text { G2: } 5.56 \pm 2.83 \\
\text { G3: } 2.38 \pm 2.10\end{array}$ & $\begin{array}{l}\text { G2: } 0(0.00) \\
\text { G3: } 0(0.00) \\
\text { Very much worse } \\
\text { G1: } 0(0.00) \\
\text { G2: } 0(0.00) \\
\text { G3: } 0 \text { (0.00) } \\
\text { CGI-El [N (\%)] } \\
\text { Unchanged or worse } \\
\text { G1: } 5 \text { (35.71) } \\
\text { G2: } 0 \text { (0.00) } \\
\text { G3: } 1 \text { (7.69) } \\
\text { Minimal } \\
\text { G1: } 2(14.29) \\
\text { G2: } 1(11.11) \\
\text { G3: } 11(84.62) \\
\text { Moderate } \\
\text { G1: } 7(50.00) \\
\text { G2: } 6(66.67) \\
\text { G3: } 1(7.69) \\
\text { Marked } \\
\text { G1: } 0(0.00) \\
\text { G2: } 2(22.22) \\
\text { G3: } 0(0.00) \\
\text { ABC-Total } \\
\text { G1: } 58.36 \pm 31.73 \\
\text { G2: } 36.78 \pm 16.95 \\
\text { G3: } 62.31 \pm 11.3 \\
\text { G1 vs G3+ }<<0.05 \\
\text { G2 vs G3: p<0.05 } \\
\text { ABC-Irritability } \\
\text { G1: } 8.14 \pm 8.37 \\
\text { G2: } 4.22 \pm 3.19 \\
\text { G3: } 6.92 \pm 4.96 \\
\text { p=ns } \\
\text { ABC-Lethargy/Social } \\
\text { withdrawal } \\
\text { G1: } 24.14 \pm 9.65 \\
\text { G2: } 16.00 \pm 7.92 \\
\text { G3: } 30.54 \pm 5.03 \\
\text { G1 vs G3: }<<0.05 \\
\text { G2 vs G3: } p<0.05 \\
\text { ABC-Stereotvnic } \\
\text { AB }\end{array}$ \\
\hline
\end{tabular}




\begin{tabular}{|c|c|c|c|}
\hline $\begin{array}{c}\text { Author, Year } \\
\text { Study Design } \\
\text { Groups (dose), N enrollment I } \\
\mathrm{N} \text { final } \\
\\
\text { Treatment duration/Follow-up } \\
\text { timepoint post-treatment } \\
\text { Risk of Bias }\end{array}$ & $\begin{array}{c}\text { Mean age, } \\
\text { years } \pm \text { SD } \\
\text { Mean IQ } \pm S D\end{array}$ & $\begin{array}{c}\text { Outcome } \\
\text { measure/Baseline } \\
\text { scores, mean } \pm S D\end{array}$ & $\begin{array}{c}\text { Outcome } \\
\text { measure/Post- } \\
\text { treatment scores, } \\
\text { mean } \pm \text { SD }\end{array}$ \\
\hline & & & $\begin{array}{l}\text { behavior } \\
\text { G1: } 17.07 \pm 9.93 \\
\text { G2: } 9.33 \pm 5.81 \\
\text { G3: } 17.31 \pm 4.05 \\
\text { G2 vs G3: p,0.05 } \\
\text { ABC-Hyperactivity } \\
\text { G1: } 6.86 \pm 5.26 \\
\text { G2: } 4.67 \pm 3.74 \\
\text { G3: } 5.08 \pm 2.40 \\
\text { p=ns } \\
\text { ABC-Inappropriate } \\
\text { speech } \\
\text { G1: } 2.14 \pm 2.32 \\
\text { G2: } 2.56 \pm 2.19 \\
\text { G3: } 2.46 \pm 2.63 \\
\text { p=ns }\end{array}$ \\
\hline $\begin{array}{l}\text { Arnold et al., } 2012^{88} \\
\text { RCT } \\
\text { G1: Mecamylamine (0.5-5 } \\
\text { mg/kg/day), 12/10 } \\
\text { G2: Placebo (NA), 8/8 } \\
14 \text { weeks/EOT } \\
\text { Low RoB }\end{array}$ & $\begin{array}{l}\text { Age } \\
\text { G1: } 6.76 \pm 2.24 \\
\text { G2: } 8.36 \pm 2.83 \\
\text { IQ } \\
\text { G1: } 77.58 \pm \\
21.12 \\
\text { G2: } 62.62 \pm \\
32.53\end{array}$ & $\begin{array}{l}\text { OACIS-S } \\
\text { G1: } 5.25 \pm 0.75 \\
\text { G2: } 5.63 \pm 0.74 \\
\text { OARS } \\
\text { G1: } 1.83 \pm 0.42 \\
\text { G2: } 2.06 \pm 0.45 \\
\text { ABC-Irritability } \\
\text { G1: } 12.75 \pm 9.42 \\
\text { G2: } 12.88 \pm 9.60 \\
\text { ABC-Lethargy } \\
\text { G1: } 10.42 \pm 6.61 \\
\text { G2: } 17.00 \pm 9.37 \\
\text { ABC-Stereotype } \\
\text { G1: } 4.17 \pm 3.54 \\
\text { G2: } 9.75 \pm 6.25 \\
\text { ABC-Hyperactivity } \\
\text { G1: } 21.08 \pm 11.74 \\
\text { G2: } 19.13 \pm 13.02 \\
\text { ABC-Inappropriate } \\
\text { Speech } \\
\text { G1: } 4.50 \pm 3.73 \\
\text { G2: } 4.38 \pm 3.16 \\
\text { RBS-Stereotypy } \\
\text { G1: } 4.58 \pm 2.87 \\
\text { G2: } 7.38 \pm 3.16\end{array}$ & $\begin{array}{l}\text { Mean change score } \\
\text { OACIS-S } \\
\text { G1: }-0.58 \pm 0.79 \\
\text { G2: }-0.63 \pm 0.74 \\
\text { OARS } \\
\text { G1: }-0.30 \pm 0.33 \\
\text { G2: }-0.32 \pm 0.34 \\
\text { ABC-Irritability } \\
\text { G1: }-3.17 \pm 8.76 \\
\text { G2: }-5.00 \pm 10.78 \\
\text { ABC-Lethargy } \\
\text { G1: }-4.25 \pm 6.97 \\
\text { G2: }-7.50 \pm 9.56 \\
\text { ABC-Stereotype } \\
\text { G1: }-1.42 \pm 2.64 \\
\text { G2: }-1.63 \pm 7.11 \\
\text { ABC-Hyperactivity } \\
\text { G1: }-6.92 \pm 11.25 \\
\text { G2: }-5.50 \pm 12.06 \\
\text { ABC-Inappropriate } \\
\text { speech } \\
\text { G1: }-1.50 \pm 3.90 \\
\text { G2: }-1.75 \pm 2.38 \\
\text { RBS-Stereotypy } \\
\text { G1: }-1.92 \pm 2.94 \\
\text { G2: }-0.63 \pm 6.21\end{array}$ \\
\hline
\end{tabular}




\begin{tabular}{|c|c|c|c|}
\hline $\begin{array}{c}\text { Author, Year } \\
\text { Study Design } \\
\text { Groups (dose), N enrollment I } \\
\mathrm{N} \text { final } \\
\\
\text { Treatment duration/Follow-up } \\
\text { timepoint post-treatment } \\
\text { Risk of Bias }\end{array}$ & $\begin{array}{c}\text { Mean age, } \\
\text { years } \pm \text { SD } \\
\text { Mean IQ } \pm S D\end{array}$ & $\begin{array}{c}\text { Outcome } \\
\text { measure/Baseline } \\
\text { scores, mean } \pm S D\end{array}$ & $\begin{array}{c}\text { Outcome } \\
\text { measure/Post- } \\
\text { treatment scores, } \\
\text { mean } \pm \text { SD }\end{array}$ \\
\hline & & $\begin{array}{l}\text { RBS-Self Injury } \\
\text { G1: } 1.75 \pm 2.01 \\
\text { G2: } 4.50 \pm 3.82 \\
\text { RBS-Compulsive } \\
\text { G1: } 5.50 \pm 3.90 \\
\text { G2: } 7.25 \pm 4.53 \\
\text { RBS-Ritualistic } \\
\text { G1: } 6.00 \pm 4.63 \\
\text { G2: } 5.88 \pm 4.91 \\
\text { RBS-Sameness } \\
\text { G1: } 8.08 \pm 5.62 \\
\text { G2: } 9.38 \pm 6.14 \\
\text { RBS-Restricted } \\
\text { G1: } 3.92 \pm 3.23 \\
\text { G2: } 4.13 \pm 2.75 \\
\text { SRS-Receptive } \\
\text { G1: } 11.92 \pm 2.64 \\
\text { G2: } 16.38 \pm 2.62 \\
\text { SRS-Cognitive } \\
\text { G1: } 19.50 \pm 2.88 \\
\text { G2: } 22.75 \pm 6.04 \\
\text { SRS-Expressive } \\
\text { G1: } 34.33 \pm 8.14 \\
\text { G2: } 39.88 \pm 11.68 \\
\text { SRS-Motivation } \\
\text { G1: } 13.00 \pm 4.69 \\
\text { G2: } 19.13 \pm 4.26 \\
\text { SRS-Preoccupations } \\
\text { G1: } 16.33 \pm 6.88 \\
\text { G2: } 22.50 \pm 7.67 \\
\text { EVT } \\
\text { G1: } 74.0 \pm 16.34 \\
\text { G2: } 47.5 \pm 24.74\end{array}$ & $\begin{array}{l}\text { RBS-Self injury } \\
\text { G1: }-0.83 \pm 1.53 \\
\text { G2: }-2.88 \pm 4.39 \\
\text { RBS-Compulsive } \\
\text { G1: }-2.42 \pm 3.55 \\
\text { G2: }-3.50 \pm 3.38 \\
\text { RBS-Ritualistic } \\
\text { G1: }-1.75 \pm 4.58 \\
\text { G2: }-2.00 \pm 3.02 \\
\text { RBS-Sameness } \\
\text { G1: }-4.67 \pm 6.05 \\
\text { G2: }-2.50 \pm 3.21 \\
\\
\text { RBS-Restricted } \\
\text { G1: }-1.58 \pm 2.50 \\
\text { G2: }-1.13 \pm 2.64 \\
\text { SRS-Receptive } \\
\text { G1: } 0.33 \pm 1.78 \\
\text { G2: }-2.25 \pm 3.06 \\
\text { SRS-Cognitive } \\
\text { G1: }-2.50 \pm 5.39 \\
\text { G2: }-2.13 \pm 5.74 \\
\text { SRS-Expressive } \\
\text { G1: }-4.17 \pm 7.31 \\
\text { G2: }-7.25 \pm 8.55 \\
\text { SRS-Motivation } \\
\text { G1: }-3.83 \pm 4.55 \\
\text { G2: }-5.75 \pm 4.89 \\
\text { SRS-Preoccupations } \\
\text { G1: }-3.33 \pm 7.43 \\
\text { G2: }-5.75 \pm 4.89 \\
\text { EVT } \\
\text { G1: }-2.14 \pm 10.12 \\
\text { G2: } 0 \pm 6.42 \\
\text { (a) }\end{array}$ \\
\hline $\begin{array}{l}\text { Hardan et al., } 2012^{89} \\
\text { RCT } \\
\text { G1: N-acetylcysteine (NAC) (900 } \\
\text { mg up to } 3 \text { times/day), 15/134 } \\
\text { G2: Placebo, 18/15 }\end{array}$ & $\begin{array}{l}\text { Age } \\
\text { G1: } 7.0 \pm 2.1 \\
\text { G2: } 7.2 \pm 2.2 \\
\text { IQ } \\
\text { NR }\end{array}$ & $\begin{array}{l}\text { ABC Irritability } \\
\text { G1: } 16.9 \pm 7.9 \\
\text { G2: } 14.8 \pm 9.6 \\
\\
\text { ABC Lethargy } \\
\text { G1: } 15.2 \pm 9.5 \\
\text { G2: } 12.1 \pm 7.8\end{array}$ & $\begin{array}{l}\text { EOT } \\
\text { ABC Irritability } \\
\text { G1: } 7.2 \pm 5.7 \\
\text { G2: } 13.1 \pm 9.9 \\
p<.001 \\
\text { ABC Lethargy }\end{array}$ \\
\hline
\end{tabular}




\begin{tabular}{|c|c|c|c|}
\hline $\begin{array}{c}\text { Author, Year } \\
\text { Study Design } \\
\text { Groups (dose), N enrollment I } \\
\mathrm{N} \text { final }\end{array}$ & $\begin{array}{c}\text { Mean age, } \\
\text { years } \pm \text { SD } \\
\text { Mean IQ } \pm S D\end{array}$ & $\begin{array}{c}\text { Outcome } \\
\text { measure/Baseline } \\
\text { scores, mean } \pm S D\end{array}$ & $\begin{array}{c}\text { Outcome } \\
\text { measure/Post- } \\
\text { treatment Scores, } \\
\text { mean } \pm \text { SD }\end{array}$ \\
\hline $\begin{array}{l}12 \text { weeks/EOT } \\
\text { Low RoB }\end{array}$ & & $\begin{array}{l}\text { ABC Stereotypy } \\
\text { G1: } 9.1 \pm 5.5 \\
\text { G2: } 8.9 \pm 6.5 \\
\text { ABC Hyperactivity } \\
\text { G1: } 23.4 \pm 9.0 \\
\text { G2: } 23.8 \pm 9.3 \\
\text { ABC Inappropriate } \\
\text { Speech } \\
\text { G1: } 4.9 \pm 3.2 \\
\text { G2: } 4.1 \pm 3.7 \\
\text { RBS-R Stereotypies } \\
\text { G1: } 6.7 \pm 3.8 \\
\text { G2: } 8.1 \pm 5.3 \\
\text { RBS Self-Injurious } \\
\text { Behavior } \\
\text { G1: } 3.9 \pm 4.4 \\
\text { G2: } 3.4 \pm 3.8 \\
\text { RBS Compulsions } \\
\text { G1: } 4.7 \pm 3.7 \\
\text { G2: } 5.8 \pm 4.8 \\
\text { RBS Rituals } \\
\text { G1: } 5.3 \pm 3.7 \\
\text { G2: } 6.6 \pm 4.5 \\
\text { RBS Sameness } \\
\text { G1: } 7.8 \pm 7.2 \\
\text { G2: } 9.2 \pm 8.1 \\
\text { RBS Restricted } \\
\text { G1: } 4.7 \pm 3.4 \\
\text { G2: } 5.2 \pm 3.7 \\
\text { SRS Total } \\
\text { G1: } 111.9 \pm 28.3 \\
\text { G2: } 104.7 \pm 28.1 \\
\text { SRS Social Awareness } \\
\text { G1: } 12.7 \pm 3.4 \\
\text { G2: } 13.5 \pm 3.7 \\
\text { SRS Social Cognition } \\
\text { G1: } 21.9 \pm 6.3 \\
\text { G2: } 21.2 \pm 5.8 \\
\text { (a) }\end{array}$ & $\begin{array}{l}\text { G1: } 11 \pm 9.4 \\
\text { G2: } 8.3 \pm 7.7 \\
\text { p=.ns } \\
\text { ABC Stereotypy } \\
\text { G1: } 5.6 \pm 5.7 \\
\text { G2: } 8.0 \pm 7.0 \\
\text { p=ns } \\
\text { ABC Hyperactivity } \\
\text { G1: } 12.4 \pm 11.4 \\
\text { G2: } 21.0 \pm 11.5 \\
\text { p=ns } \\
\text { ABC Inappropriate } \\
\text { Speech } \\
\text { G1: } 2.5 \pm 2.6 \\
\text { G2: } 3.6 \pm 3.6 \\
\text { p=ns } \\
\text { RBS Stereotypies } \\
\text { G1: } 4.6 \pm 3.4 \\
\text { G2: } 6.9 \pm 5.2 \\
\text { p= } .014 \\
\text { RBS Self Injurious } \\
\text { Behavior } \\
\text { G1: } 2.2 \pm 2.3 \\
\text { G2: } 3.0 \pm 3.6 \\
\text { p=ns } \\
\text { RBS Compulsions } \\
\text { G1: } 2.5 \pm 2.1 \\
\text { G2: } 5.2 \pm 5.0 \\
\text { p=ns } \\
\text { RBS Rituals } \\
\text { G1: } 4.3 \pm 3.4 \\
\text { G2: } 5.6 \pm 4.9 \\
\text { p=ns } \\
\text { RBS Sameness } \\
\text { G1: } 5.3 \pm 4.7 \\
\text { G2: } 7.9 \pm 6.2 \\
\text { p=ns } \\
\text { RBS Restricted } \\
\text { G1: } 3.5 \pm 2.3 \\
\text { G2: } 4.8 \pm 3.6 \\
\text { p=ns }\end{array}$ \\
\hline
\end{tabular}




\begin{tabular}{|c|c|c|c|}
\hline $\begin{array}{c}\text { Author, Year } \\
\text { Study Design } \\
\text { Groups (dose), N enrollment I } \\
\mathrm{N} \text { final } \\
\\
\text { Treatment duration/Follow-up } \\
\text { timepoint post-treatment } \\
\text { Risk of Bias }\end{array}$ & $\begin{array}{c}\text { Mean age, } \\
\text { years } \pm \text { SD } \\
\text { Mean IQ } \pm S D\end{array}$ & $\begin{array}{c}\text { Outcome } \\
\text { measure/Baseline } \\
\text { scores, mean } \pm S D\end{array}$ & $\begin{array}{c}\text { Outcome } \\
\text { measure/Post- } \\
\text { treatment scores, } \\
\text { mean } \pm \text { SD }\end{array}$ \\
\hline & & $\begin{array}{l}\text { SRS Social } \\
\text { Communication } \\
\text { G1: } 39.6 \pm 11.3 \\
\text { G2: } 39.3 \pm 8.6 \\
\text { SRS Social Motivation } \\
\text { G1: } 16.6 \pm 6.3 \\
\text { G2: } 16.9 \pm 6.5 \\
\text { SRS Autism } \\
\text { Mannerisms } \\
\text { G1: } 21.7 \pm 5.6 \\
\text { G2: } 21.4 \pm 7.3 \\
\text { CGI Severity } \\
\text { G1: } 5.1 \pm 0.7 \\
\text { G2: } 5.3 \pm 0.8\end{array}$ & 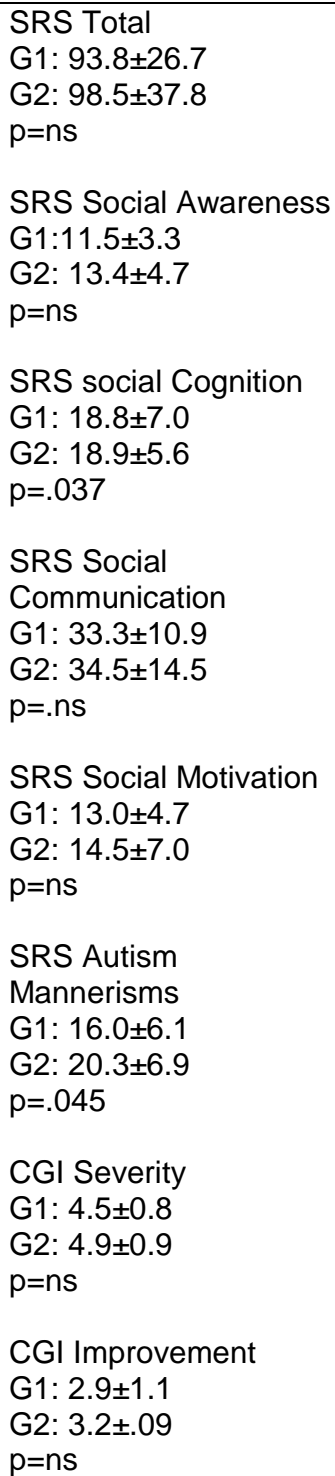 \\
\hline $\begin{array}{l}\text { Chez et al., } 2003^{90} \\
\text { RCT } \\
\text { G1: Donepezil hydrochloride only, / } \\
\text { G2: Placebo/ Donepezil } \\
\text { hydrochloride, / } \\
12 \text { wks/EOT } \\
\text { Moderate RoB }\end{array}$ & $\begin{array}{l}\text { Age } \\
\text { G1: } 6.8 \text { (2.1- } \\
\text { 9.9) } \\
\text { G2: } 6.9 \text { (4.1- } \\
\text { 10.3) } \\
\text { IQ } \\
\text { NR }\end{array}$ & $\begin{array}{l}\text { CARS } \\
\text { G1: } 34.7 \pm 7.7 \\
\text { G2: } 35.1 \pm 7.9 \\
\text { Expressive One-Word } \\
\text { Picture Vocabulary } \\
\text { Test (speech age in } \\
\text { months) } \\
\text { G1: } 35.7 \pm 27.8 \\
\text { G2: } 31.9 \pm 31.1\end{array}$ & $\begin{array}{l}\text { End of } 6 \text { wks } \\
\text { CARS } \\
\text { G1: } 33.3 \pm 8 \\
\text { G2: } 32.9 \pm 7.7 \\
\text { Expressive One-Word } \\
\text { Picture Vocabulary } \\
\text { Test (speech age in } \\
\text { months) } \\
\text { G1: } 43.3 \pm 27.2\end{array}$ \\
\hline
\end{tabular}




\begin{tabular}{|c|c|c|c|}
\hline $\begin{array}{c}\text { Author, Year } \\
\text { Study Design } \\
\text { Groups (dose), N enrollment I } \\
\mathrm{N} \text { final } \\
\\
\text { Treatment duration/Follow-up } \\
\text { timepoint post-treatment } \\
\text { Risk of Bias }\end{array}$ & $\begin{array}{c}\text { Mean age, } \\
\text { years } \pm \text { SD } \\
\text { Mean IQ } \pm S D\end{array}$ & $\begin{array}{c}\text { Outcome } \\
\text { measure/Baseline } \\
\text { scores, mean } \pm S D\end{array}$ & $\begin{array}{c}\text { Outcome } \\
\text { measure/Post- } \\
\text { treatment scores, } \\
\text { mean } \pm \text { SD }\end{array}$ \\
\hline & & $\begin{array}{l}\text { Receptive One-Word } \\
\text { Picture Vocabulary } \\
\text { Test (speech age in } \\
\text { months) } \\
\text { G1: } 38.8 \pm 23.5 \\
\text { G2: } 33.5 \pm 27.9\end{array}$ & $\begin{array}{l}\text { G2: } 33.8 \pm 32.9 \\
\text { Receptive One-Word } \\
\text { Picture Vocabulary } \\
\text { Test (speech age in } \\
\text { months) } \\
\text { G1: } 50.3 \pm 27 \\
\text { G2: } 39.83 \pm 27 \\
\text { EOT } \\
\text { CARS } \\
\text { G1: } 30.8 \pm 7.9 \\
\text { G2: } 30.9 \pm 9.1 \\
\text { G1 vs } G 2, p<0.05 \\
\text { Expressive One-Word } \\
\text { Picture Vocabulary } \\
\text { Test (speech age in } \\
\text { months) } \\
\text { G1: } 42.5 \pm 28.5 \\
\text { G2: } 40.9 \pm 38.8 \\
\text { G1 vs } G 2, p<0.05 \\
\text { Receptive One-Word } \\
\text { Picture Vocabulary } \\
\text { Test (speech age in } \\
\text { months) } \\
\text { G1: } 49.7 \pm 34.2 \\
\text { G2: } 87.37 \pm 14.2 \\
\text { G1 vs G2, p<0.05 }\end{array}$ \\
\hline $\begin{array}{l}\text { King et al., } 2001^{91} \\
\text { RCT } \\
\text { G1: Amantadine, 19/17 } \\
\text { G2: Placebo, 20/17 } \\
4 \text { wks/EOT } \\
\text { Moderate RoB }\end{array}$ & $\begin{array}{l}\text { Age } \\
5-19 \\
\text { IQ } \\
>35\end{array}$ & $\begin{array}{l}\text { ABC - Irritability } \\
\text { G1: } 19.1 \text { (3-38) } \\
\text { G2: } 18.7 \text { (3-33) } \\
\text { ABC - Hyperactivity } \\
\text { G1: } 29.4(16-42) \\
\text { G2: } 32.7 \text { (17-46) } \\
\text { CGI - Severity (Mild) } \\
\text { G1: } 0 \text { (0) } \\
\text { G2: } 1 \text { (5) } \\
\text { CGI - Severity } \\
\text { (Moderate) } \\
\text { G1: } 11 \text { (57.8) } \\
\text { G2: } 11 \text { (55) } \\
\text { CGI - Severity } \\
\text { (Severe) } \\
\text { G1: } 8 \text { (42.1) } \\
\text { G2: } 7 \text { (35) }\end{array}$ & $\begin{array}{l}\text { EOT } \\
\text { ABC - Response rate } \\
\text { (Reduction of at least } \\
\text { 25\% for irritability } \\
\text { and/or hyperactivity) } \\
\text { G1: } 7 \text { (37) } \\
\text { G2: } 9 \text { (47) } \\
\text { G1 vs G2, p=ns } \\
\\
\text { CGI - Improvement } \\
\text { (Marked Improvement) } \\
\text { G1: } 5 \text { (26) } \\
\text { G2: } 1 \text { (5) }\end{array}$ \\
\hline
\end{tabular}




\begin{tabular}{|c|c|c|c|}
\hline $\begin{array}{c}\text { Author, Year } \\
\text { Study Design } \\
\text { Groups (dose), N enrollment I } \\
\text { N final } \\
\\
\text { Treatment duration/Follow-up } \\
\text { timepoint post-treatment } \\
\text { Risk of Bias }\end{array}$ & $\begin{array}{l}\text { Mean age, } \\
\text { years } \pm \text { SD } \\
\text { Mean IQ } \pm S D\end{array}$ & $\begin{array}{l}\text { Outcome } \\
\text { measure/Baseline } \\
\text { scores, mean } \pm S D\end{array}$ & $\begin{array}{c}\text { Outcome } \\
\text { measure/Post- } \\
\text { treatment scores, } \\
\text { mean } \pm \text { SD }\end{array}$ \\
\hline $\begin{array}{l}\text { Duffy et al., } 2014^{92} \\
\text { Retrospective Cohort } \\
\text { G1: Steroid ( } 2 \mathrm{mg} / \mathrm{kg} / \text { day), } 20 / 20 \\
\text { G2: Control (non- steroid treated) } \\
\text { (NA), } 24 / 24 \\
\text { 4-14 months/EOT } \\
\text { High RoB }\end{array}$ & $\begin{array}{l}\text { Age } \\
\text { G1: } 3.909 \pm \\
1.248 \\
\text { G2: } 4.522 \pm \\
1.800 \\
\text { IQ } \\
\text { G1: NR } \\
\text { G2: NR }\end{array}$ & $\begin{array}{l}\text { EEG } \\
\text { NR } \\
\text { FMAER } \\
\text { NR }\end{array}$ & 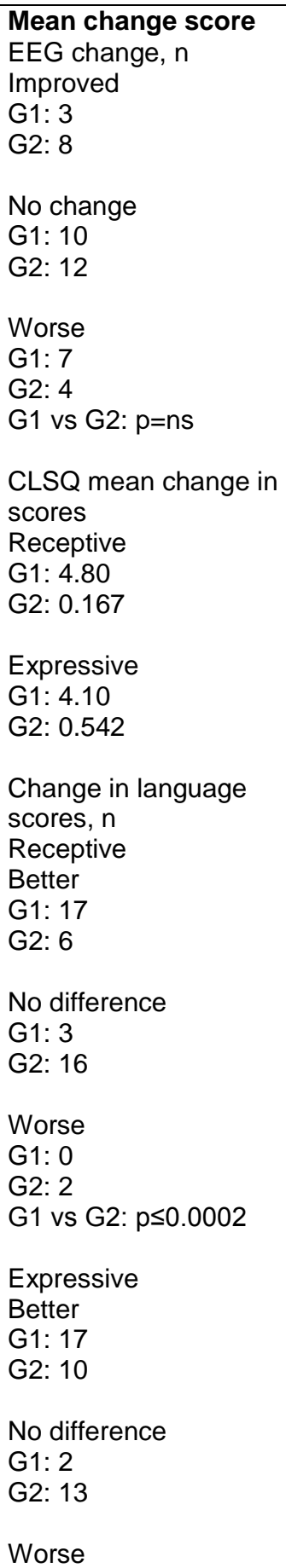 \\
\hline
\end{tabular}




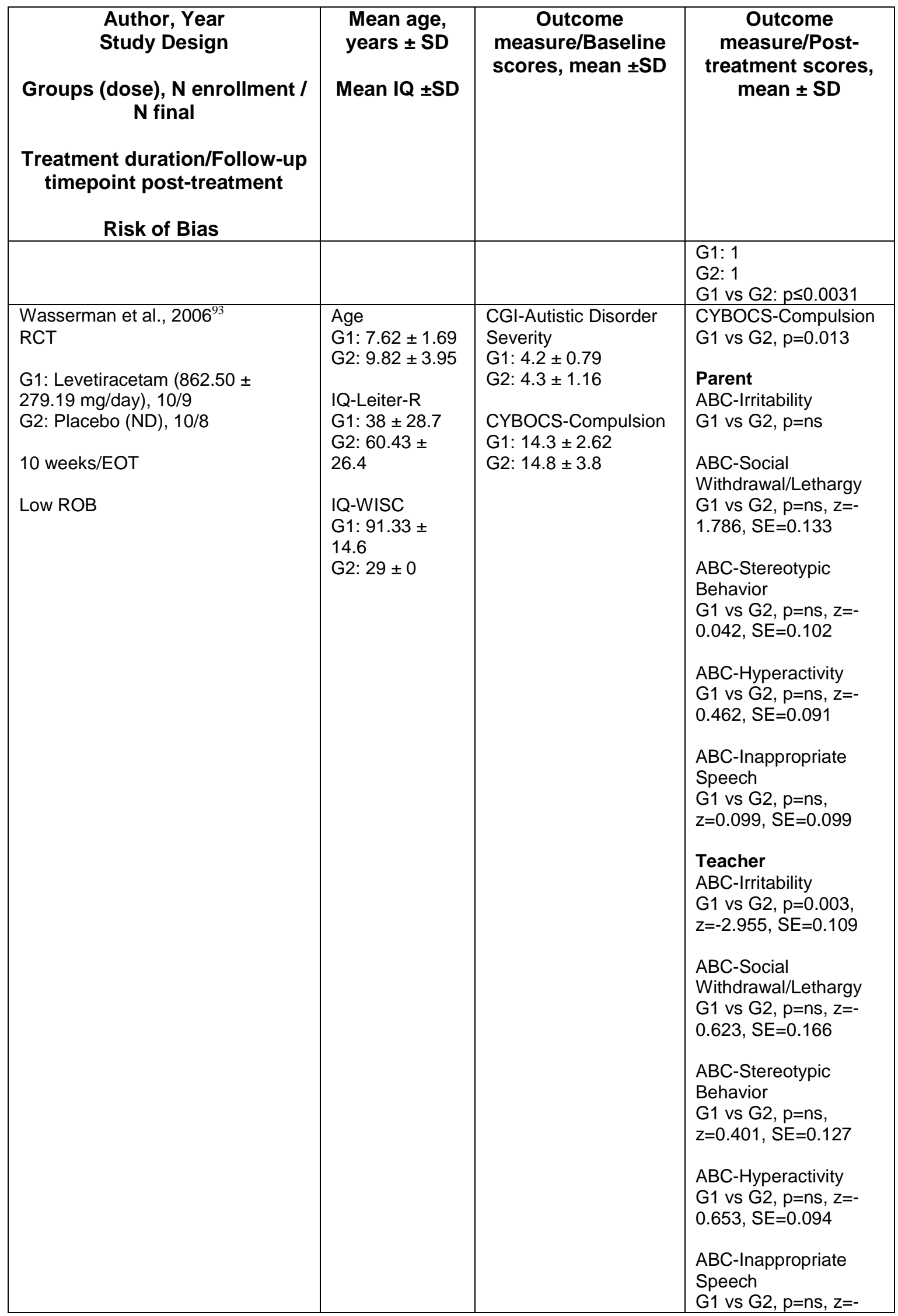




\begin{tabular}{|c|c|c|c|}
\hline $\begin{array}{c}\text { Author, Year } \\
\text { Study Design } \\
\text { Groups (dose), N enrollment I } \\
\mathrm{N} \text { final } \\
\\
\text { Treatment duration/Follow-up } \\
\text { timepoint post-treatment } \\
\text { Risk of Bias }\end{array}$ & $\begin{array}{c}\text { Mean age, } \\
\text { years } \pm \text { SD } \\
\text { Mean IQ } \pm S D\end{array}$ & $\begin{array}{c}\text { Outcome } \\
\text { measure/Baseline } \\
\text { scores, mean } \pm S D\end{array}$ & $\begin{array}{c}\text { Outcome } \\
\text { measure/Post- } \\
\text { treatment scores, } \\
\text { mean } \pm \text { SD }\end{array}$ \\
\hline & & & $0.268, \mathrm{SE}=0.105$ \\
\hline $\begin{array}{l}\text { Danfors et al., } 2005^{94} \\
\text { RCT } \\
\text { G1: Tetrahydrobiopterin/Placebo } \\
\text { (3mg/kg),12 } \\
\text { G2: Placebo/ Tetrahydrobiopterin } \\
\text { (ND), } 12 \\
12 \text { months/EOT } \\
\text { Moderate ROB }\end{array}$ & $\begin{array}{l}\text { Age } \\
4-7 \\
\text { IQ } \\
\text { NR }\end{array}$ & $\begin{array}{l}\text { CARS-Total Score } \\
\text { G1: } 35.4 \pm 2.6 \\
\text { G2: } 37.4 \pm 7.1 \\
\text { Social Interaction } \\
\text { G1: } 9 \pm 0.6 \\
\text { G2: } 10.8 \pm 1.6\end{array}$ & $\begin{array}{l}\text { Points Decreased } \\
\text { CARS-Total Score } \\
\text { G1: } 2.1 \pm 2.1 \\
\text { G2: } 2.1 \pm 4.3 \\
\text { G1 vs } G 2, p=n s \\
\text { Social Interaction } \\
\text { G1: } 1.6 \pm 1.1 \\
\text { G2: } 0.3 \pm 1.4 \\
\text { G1 vs } G 2, p=0.04\end{array}$ \\
\hline $\begin{array}{l}\text { Dean et al., } 2016^{95} \\
\text { RCT } \\
\text { G1: N-acetyl cysteine (500 } \\
\text { mg/day), } 48 / 34 \\
\text { G2: Placebo (ND),50 /37 } \\
6 \text { months/EOT } \\
\text { Moderate ROB }\end{array}$ & $\begin{array}{l}\text { Age } \\
\text { G1: } 78.4 \pm 21.7 \\
\text { G2: } 74.9 \pm 23.8 \\
\text { IQ } \\
\text { G1: } 74.3 \pm 15.6 \\
\text { G2: } 71.7 \pm 13.8\end{array}$ & $\begin{array}{l}\text { SRS-Total Score } \\
\text { G1: } 102.9 \pm 26.4 \\
\text { G2: } 99.7 \pm 24.8 \\
\text { CCC-General } \\
\text { Communication } \\
\text { G1: } 33.6 \pm 13.1 \\
\text { G2: } 34.7 \pm 14.6 \\
\text { CCC-Social Interaction } \\
\text { Deviance } \\
\text { G1: } 3.1 \pm 8.5 \\
\text { G2: } 3.3 \pm 6.7 \\
\text { Repetitive Behavior } \\
\text { Scale-Total Score } \\
\text { G1: } 28.5 \pm 19.4 \\
\text { G2: } 24.8 \pm 14.1 \\
\text { CGI-Severity } \\
\text { G1: } 4.1 \pm 1 \\
\text { G2: } 4.2 \pm 1 \\
\text { Developmental } \\
\text { Behavior Checklist- } \\
\text { Total Score } \\
\text { G1: } 57 \pm 23.2 \\
\text { G2: } 59 \pm 27.6\end{array}$ & $\begin{array}{l}\text { SRS-Total Score } \\
\text { G1: } 92.1 \pm 29.3 \\
\text { G2: } 88.7 \pm 27.7 \\
\text { G1 vs G2, p=ns } \\
\text { CCC-General } \\
\text { Communication } \\
\text { G1: } 34.7 \pm 11.9 \\
\text { G2: } 41.5 \pm 16.5 \\
\text { G1 vs G2, p=ns } \\
\text { CCC-Social Interaction } \\
\text { Deviance } \\
\text { Change Score } \\
\text { G1: } 0.05 \pm 8.4 \\
\text { G2: }-2.2 \pm 8.2 \\
\text { G1 vs } \mathrm{G} 2, p=n s \\
\text { Repetitive Behavior } \\
\text { Scale-Total Score } \\
\text { G1: } 24.3 \pm 24.3 \\
\text { G2: } 20.8 \pm 16.3 \\
\text { G1 vs G2, p=ns } \\
\text { CGI-Severity } \\
\text { G1: } 3.9 \pm 1 \\
\text { G2: } 3.6 \pm 1 \\
\text { G1 vs G2, p=ns } \\
\text { Developmental } \\
\text { Behavior Checklist- } \\
\text { Total Score } \\
\text { G1: } 43.9 \pm 25.2 \\
\text { G2: } 48.2 \pm 18 \\
\text { G1 vs } G 2, p=n s\end{array}$ \\
\hline $\begin{array}{l}\text { Nikvarz et al., } 2016^{96} \\
\text { RCT }\end{array}$ & $\begin{array}{l}\text { Age } \\
\text { G1: } 6.83 \pm 2.98\end{array}$ & $\begin{array}{l}\text { ABC-Irritability } \\
\text { G1: } 22.4 \pm 11.16\end{array}$ & $\begin{array}{l}\text { ABC-Irritability } \\
\text { G1: } 13.5 \pm 10.63\end{array}$ \\
\hline
\end{tabular}




\begin{tabular}{|c|c|c|c|}
\hline $\begin{array}{c}\text { Author, Year } \\
\text { Study Design } \\
\text { Groups (dose), N enrollment I } \\
\text { N final } \\
\text { Treatment duration/Follow-up } \\
\text { timepoint post-treatment } \\
\text { Risk of Bias }\end{array}$ & $\begin{array}{l}\text { Mean age, } \\
\text { years } \pm \text { SD } \\
\text { Mean IQ } \pm S D\end{array}$ & $\begin{array}{c}\text { Outcome } \\
\text { measure/Baseline } \\
\text { scores, mean } \pm S D\end{array}$ & $\begin{array}{c}\text { Outcome } \\
\text { measure/Post- } \\
\text { treatment scores, } \\
\text { mean } \pm \text { SD }\end{array}$ \\
\hline $\begin{array}{l}\text { G1: Memantine (up to } 20 \mathrm{mg} / \mathrm{day} \text { ), } \\
\text { 16/15 } \\
\text { G2: Risperidone (up to } 3 \mathrm{mg} / \mathrm{day} \text { ), } \\
\text { 18/15 } \\
8 \text { weeks/EOT } \\
\text { Moderate ROB }\end{array}$ & $\begin{array}{l}\text { G2: } 6.56 \pm 3.51 \\
\text { IQ } \\
\text { NR }\end{array}$ & $\begin{array}{l}\text { G2: } 21.26 \pm 6.85 \\
\text { ABC-Social } \\
\text { Withdrawal/Lethargy } \\
\text { G1: } 22.13 \pm 7.17 \\
\text { G2: } 17.53 \pm 9.66 \\
\text { ABC-Stereotypic } \\
\text { Behavior } \\
\text { G1: } 5.66 \pm 5.7 \\
\text { G2: } 5.66 \pm 5.97 \\
\text { ABC-Inappropriate } \\
\text { Speech } \\
\text { G1: } 6.27 \pm 4.4 \\
\text { G2: } 6 \pm 2.97 \\
\text { ABC-Hyperactivity } \\
\text { G1: } 30.4 \pm 9.47 \\
\text { G2: } 27.53 \pm 12.03 \\
\text { CARS-Total Score } \\
\text { G1: } 42.56 \pm 6 \\
\text { G2: } 40.16 \pm 5.52 \\
\text { CGI-Severity-Mildly III } \\
\text { G1: } 1 \text { (6.7) } \\
\text { G2: } 0 \text { (0) } \\
\text { CGI-Severity- } \\
\text { Moderately III } \\
\text { G1: } 1 \text { (6.7) } \\
\text { G2: } 3 \text { (20) } \\
\text { CGI-Severity-Markedly } \\
\text { III } \\
\text { G1: } 8 \text { (53.33) } \\
\text { G2: } 6 \text { (40) } \\
\text { CGI-Severity-Severely } \\
\text { III } \\
\text { G1: } 5 \text { (33.3) } \\
\text { G2: } 6 \text { (40) }\end{array}$ & 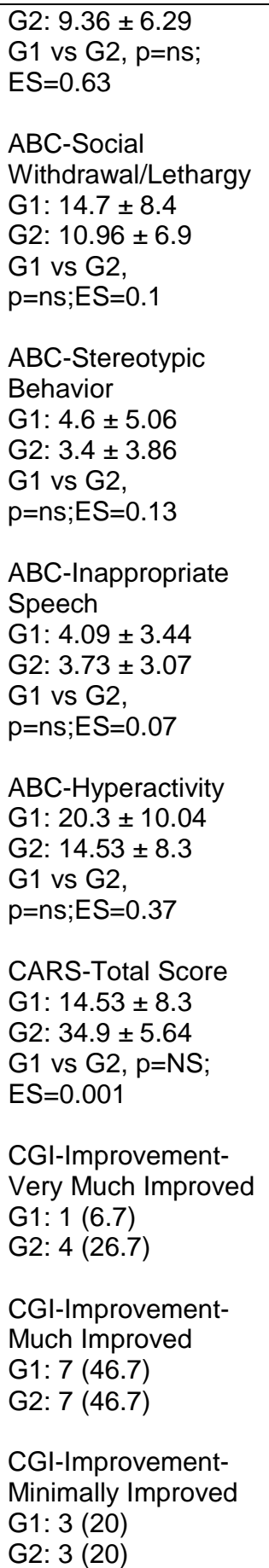 \\
\hline
\end{tabular}




\begin{tabular}{|c|c|c|c|}
\hline $\begin{array}{c}\text { Author, Year } \\
\text { Study Design } \\
\text { Groups (dose), N enrollment I } \\
\mathrm{N} \text { final } \\
\\
\text { Treatment duration/Follow-up } \\
\text { timepoint post-treatment } \\
\text { Risk of Bias }\end{array}$ & $\begin{array}{c}\text { Mean age, } \\
\text { years } \pm \text { SD } \\
\text { Mean IQ } \pm S D\end{array}$ & $\begin{array}{c}\text { Outcome } \\
\text { measure/Baseline } \\
\text { scores, mean } \pm S D\end{array}$ & $\begin{array}{c}\text { Outcome } \\
\text { measure/Post- } \\
\text { treatment scores, } \\
\text { mean } \pm \text { SD }\end{array}$ \\
\hline & & & $\begin{array}{l}\text { CGI-Improvement-No } \\
\text { Change } \\
\text { G1: } 4(26.7) \\
\text { G2: } 1(6.6) \\
\text { CGI change } \\
\text { G1 vs G2, p=ns }\end{array}$ \\
\hline $\begin{array}{l}\text { Aman et al., } 2016^{97} \\
\text { RCT } \\
\text { G1: Mementine (3-15 mg/day), } \\
\text { 60/52 } \\
\text { G2: Placebo (ND), 61/50 } \\
12 \text { weeks/OLE } 42 \text { weeks } \\
\text { Low ROB }\end{array}$ & $\begin{array}{l}\text { Age } \\
\text { G1: } 9 \pm 2.2 \\
\text { G2: } 8.9 \pm 2.2 \\
\text { IQ } \\
\text { G1: } 77.9 \pm 23.1 \\
\text { G2: } 75.7 \pm 19.4\end{array}$ & $\begin{array}{l}\text { SRS-Total Raw Score } \\
\text { G1: } 101.3 \pm 25.2 \\
\text { G2: } 100.2 \pm 21.4\end{array}$ & $\begin{array}{l}\text { 12 Weeks } \\
\text { SRS-Total Raw Score } \\
-7.2 \pm 6.6 \\
\mathbf{4 2} \text { weeks (OLE) } \\
\text { NR }\end{array}$ \\
\hline $\begin{array}{l}\text { Arnold et al., } 2006^{98} \\
\text { RCT } \\
\text { G1: Atomoxetine/Placebo }(1.4 \\
\text { mg/kg), } 16 \\
\text { G2: Placebo/Atomoxetine }(1.4 \\
\text { mg/kg), } 16 \\
6 \text { weeks/EOT } \\
\text { Moderate ROB }\end{array}$ & $\begin{array}{l}\text { Age } \\
9.26 \pm 2.93 \\
\text { IQ } \\
\text { NR }\end{array}$ & $\begin{array}{l}\text { ABC-Hyperactivity } \\
\text { G1: } 24.69 \pm 13.08 \\
\text { G2: } 22.5 \pm 12.87 \\
\text { ABC-Irritability } \\
\text { G1: } 16 \pm 9.28 \\
\text { G2: } 14.18 \pm 9.87 \\
\text { ABC-Social } \\
\text { Withdrawal/Lethargy } \\
\text { G1: } 8.69 \pm 9.24 \\
\text { G2: } 6.62 \pm 8.36 \\
\text { ABC-Stereotypic } \\
\text { Behavior } \\
\text { G1: } 7.37 \pm 6.2 \\
\text { G2: } 6.19 \pm 5.86 \\
\text { ABC-Inappropriate } \\
\text { Speech } \\
\text { G1: } 5.75 \pm 3.38 \\
\text { G2: } 4.68 \pm 2.41 \\
\text { CGI-Severity } \\
\text { G1: } 4.69 \pm 0.6 \\
\text { G2: } 4.69 \pm 0.6 \\
\text { Repetitive Behavior } \\
\text { Scale - Total Score } \\
\text { G1: } 53.12 \pm 22.2 \\
\text { G2: } 49.06 \pm 21.54\end{array}$ & $\begin{array}{l}\text { ABC-Hyperactivity } \\
\text { G1: } 19.31 \pm 13.42 \\
\text { G2: } 22.37 \pm 12.89 \\
\text { G1 vs G2, p=0.04; } \\
\text { ES=0.9 } \\
\text { ABC-Irritability } \\
\text { G1: } 13.06 \pm 9.28 \\
\text { G2: } 14.13 \pm 9.89 \\
\text { G1 vs G2, p=ns } \\
\text { ABC-Social } \\
\text { Withdrawal/Lethargy } \\
\text { G1: } 6.5 \pm 8 \\
\text { G2: } 7.43 \pm 9.64 \\
\text { G1 vs G2, p=0.01; } \\
\text { ES=1.18 } \\
\text { ABC-Stereotypic } \\
\text { Behavior } \\
\text { G1: } 4.69 \pm 5.84 \\
\text { G2: } 6.63 \pm 5.8 \\
\text { G1 vs G2, p=ns } \\
\text { ABC-Inappropriate } \\
\text { Speech } \\
\text { G1: } 4.87 \pm 2.85 \\
\text { G2: } 5.43 \pm 3.16 \\
\text { G1 vs G2, p=ns } \\
\text { Repetitive Behavior } \\
\text { Scale }- \text { Total Score }\end{array}$ \\
\hline
\end{tabular}




\begin{tabular}{|c|c|c|c|}
\hline $\begin{array}{c}\text { Author, Year } \\
\text { Study Design } \\
\text { Groups (dose), N enrollment I } \\
\text { N final } \\
\\
\text { Treatment duration/Follow-up } \\
\text { timepoint post-treatment } \\
\text { Risk of Bias }\end{array}$ & $\begin{array}{c}\text { Mean age, } \\
\text { years } \pm \text { SD } \\
\text { Mean IQ } \pm S D\end{array}$ & $\begin{array}{c}\text { Outcome } \\
\text { measure/Baseline } \\
\text { scores, mean } \pm S D\end{array}$ & $\begin{array}{c}\text { Outcome } \\
\text { measure/Post- } \\
\text { treatment scores, } \\
\text { mean } \pm \text { SD }\end{array}$ \\
\hline & & & $\begin{array}{l}\text { G1: } 43.5 \pm 23.94 \\
\text { G2: } 45 \pm 5.99 \\
\text { G1 vs G2, p=ns } \\
\text { CGI-Improvement } \\
\text { G1: } 9 \text { (56) } \\
\text { G2: } 4 \text { (25) } \\
\text { G1 vs G2, p=NR }\end{array}$ \\
\hline Buspirone & & & \\
\hline $\begin{array}{l}\text { Chugani et al., } 2016^{99} \\
\text { RCT } \\
\text { G1: Buspirone }(2.5 \mathrm{mg}), 54 / 46 \\
\text { G2: Buspirone }(5.0 \mathrm{mg}), 55 / 49 \\
\text { G3: Placebo (ND), 57/47 } \\
24 \text { weeks/EOT } \\
\text { RoB }\end{array}$ & $\begin{array}{l}\text { Age - } 2 \text { to < } 4 \\
\text { years } \\
\text { G1: } 25(46.3) \\
\text { G2: } 23(41.8) \\
\text { G3: } 25(43.9) \\
\text { Age - } 4 \text { to < } 6 \\
\text { years years } \\
\text { G1: } 29(53.7) \\
\text { G2: } 32(58.2) \\
\text { G3: } 32(56.1) \\
\text { IQ } \\
\text { NR }\end{array}$ & $\begin{array}{l}\text { ADOS-Composite } \\
\text { Total Score } \\
\text { G1: } 18.3 \pm 0.7 \\
\text { G2: } 18.4 \pm 0.6 \\
\text { G3: } 19.6 \pm 0.6 \\
\text { ADOS-Social Affect } \\
\text { Score } \\
\text { G1: } 13.9 \pm 0.5 \\
\text { G2: } 14.2 \pm 0.5 \\
\text { G3: } 15 \pm 0.5 \\
\text { ADOS-Restricted and } \\
\text { Repetitive } \\
\text { G1: } 4.4 \pm 0.3 \\
\text { G2: } 4.1 \pm 0.3 \\
\text { G3: } 4.7 \pm 0.3 \\
\text { VABS-Social Skills } \\
\text { G1: } 68.7 \pm 1.5 \\
\text { G2: } 71 \pm 1.5 \\
\text { G3: } 70.4 \pm 1.5 \\
\text { VABS-Communication } \\
\text { G1: } 65.5 \pm 2.2 \\
\text { G2: } 70.6 \pm 2.2 \\
\text { G3: } 68.5 \pm 2.2 \\
\text { ABC-Language } \\
\text { Deviance } \\
\text { G1: } 4.4 \pm 0.5 \\
\text { G2: } 3.7 \pm 0.5 \\
\text { G3: } 3.8 \pm 0.5 \\
\text { ABC-Anxiety } \\
\text { Composite } \\
\text { G1: } 0.536 \pm 0.1 \\
\text { G2: }-0.014 \pm 0.1 \\
\text { G3: } 58.6 \pm 3.8 \\
\text { ABC-Social Deviance }\end{array}$ & $\begin{array}{l}\text { ADOS-Composite } \\
\text { Total Score } \\
\text { G1: } 16.2 \pm 0.7 \\
\text { G2: } 17.9 \pm 0.7 \\
\text { G3: } 18.6 \pm 0.7 \\
\text { ADOS-Social Affect } \\
\text { Score } \\
\text { G1: } 13.3 \pm 0.6 \\
\text { G2: } 13.4 \pm 0.6 \\
\text { G3: } 14.1 \pm 0.6 \\
\text { ADOS-Restricted and } \\
\text { Repetitive } \\
\text { G1: } 3.5 \pm 0.03 \\
\text { G2: } 4.6 \pm 0.3 \\
\text { G3: } 4.4 \pm 0.3 \\
\text { VABS-Social Skills } \\
\text { G1: } 70.4 \pm 1.7 \\
\text { G2: } 72.3 \pm 1.7 \\
\text { G3: } 72.4 \pm 1.7 \\
\text { VABS-Communication } \\
\text { G1: } 68.2 \pm 2.3 \\
\text { G2: } 72.7 \pm 2.2 \\
\text { G3: } 69.7 \pm 2.2 \\
\text { ABC-Language } \\
\text { Deviance } \\
\text { G1: } 4.4 \pm 0.5 \\
\text { G2: } 3.7 \pm 0.5 \\
\text { G3: } 3.8 \pm 0.5 \\
\text { ABC-Anxiety } \\
\text { Composite } \\
\text { G1: } 0.118 \pm 0.1 \\
\text { G2: }-0.12 \pm 0.1 \\
\text { G3: }-0.242 \pm 0.1 \\
\text { ABC-Social Deviance }\end{array}$ \\
\hline
\end{tabular}




\begin{tabular}{|c|c|c|c|}
\hline $\begin{array}{c}\text { Author, Year } \\
\text { Study Design } \\
\text { Groups (dose), N enrollment I } \\
\mathrm{N} \text { final } \\
\\
\text { Treatment duration/Follow-up } \\
\text { timepoint post-treatment } \\
\text { Risk of Bias }\end{array}$ & $\begin{array}{c}\text { Mean age, } \\
\text { years } \pm \text { SD } \\
\text { Mean IQ } \pm S D\end{array}$ & $\begin{array}{c}\text { Outcome } \\
\text { measure/Baseline } \\
\text { scores, mean } \pm S D\end{array}$ & $\begin{array}{c}\text { Outcome } \\
\text { measure/Post- } \\
\text { treatment scores, } \\
\text { mean } \pm \text { SD }\end{array}$ \\
\hline & & $\begin{array}{l}\text { G1: } 15.3 \pm 1.2 \\
\text { G2: } 11.8 \pm 1.2 \\
\text { G3: } 14.2 \pm 1.2 \\
\text { Repetitive Behavior } \\
\text { Scale-Total Score } \\
\text { G1: } 46.9 \pm 3.5 \\
\text { G2: } 31.5 \pm 3.5 \\
\text { G3: } 36.7 \pm 3.5 \\
\text { CYBOCS-Total Score } \\
\text { G1: } 12.1 \pm 0.6 \\
\text { G2: } 11.5 \pm 0.6 \\
\text { G3: } 11.9 \pm 0.6 \\
\text { Sensory Processing } \\
\text { Measure-Total Score } \\
\text { G1: } 58.6 \pm 3.8 \\
\text { G2: } 67.2 \pm 3.7 \\
\text { G3: } 65.4 \pm 3.7\end{array}$ & $\begin{array}{l}\text { G1: } 12.1 \pm 1.1 \\
\text { G2: } 10.6 \pm 1.1 \\
\text { G3: } 11 \pm 1.1 \\
\text { Repetitive Behavior } \\
\text { Scale-Total Score } \\
\text { G1: } 38 \pm 3.3 \\
\text { G2: } 29.9 \pm 3.3 \\
\text { G3: } 34.2 \pm 3.2 \\
\text { CYBOCS-Total Score } \\
\text { G1: } 11.1 \pm 0.6 \\
\text { G2: } 10.7 \pm 0.6 \\
\text { G3: } 11.2 \pm 0.6 \\
\text { Sensory Processing } \\
\text { Measure-Total Score } \\
\text { G1: } 59.6 \pm 3.6 \\
\text { G2: } 68.1 \pm 3.5 \\
\text { G3: } 66.7 \pm 3.6\end{array}$ \\
\hline
\end{tabular}

ABC-Aberrant Behavior Checklist; CGI-Clinical Global Impression; VABS-Vineland Adaptive Behavior Scale; CYBOCS-Children's YaleBrown Obsessive Compulsive Scale; EOT-End of Treatment; SRS-Social Responsiveness Scale ; SDB-sleep-disordered breathing ; PLSPreschool Language Scale ; EOWPVT-Expressive One Word Vocabulary Test ; CSHQ- ; DS-duration of sleep ; TST-Total Sleep Time ; CARSChildhood Autism Rating Scale ; ADOS-Autism Diagnostic Observation Schedule ; ATEC-Autism Treatment Evaluation Checklist ; CGISClinical Global Impression-Severity ; SOD-sleep onset delay; CGAS- Children's Global Assessment Scale; SOL-sleep onset latency ; WASOwake after sleep onset ; SE-sleep efficiency; DBC-Developmental Behavior Checklist ; GHQ-General Health Questionnaire ; OSU- ; OACIS- ; OARS- ; RBS- ; EVT- ; EEG-electroencephalogram ; FMAER- frequency modulated auditory evoked response

\section{Harms}

Table F-7. Harms/adverse effects in studies of risperidone adjuncts

\begin{tabular}{|c|c|c|}
\hline Harm/Adverse Event & $\begin{array}{l}\text { N Studies Reporting Harm (\# } \\
\text { Participants With Harm/Total } \\
\text { Participants) }\end{array}$ & $\begin{array}{l}\text { Reported Rates Across } \\
\text { Studies }\end{array}$ \\
\hline \multicolumn{3}{|l|}{ Risperidone + piracetam } \\
\hline Appetite increase $^{73}$ & $1(7 / 20)$ & $35 \%$ \\
\hline Dry mouth ${ }^{73}$ & $1(4 / 20)$ & $20 \%$ \\
\hline EPS/impaired movement $^{73}$ & $1(6 / 20)$ & $30 \%$ \\
\hline Gastrointestinal problems $^{73}$ & $\frac{1(0 / 2)}{1(4 / 20)}$ & $20 \%$ \\
\hline Somnolence $^{73}$ & $1(23 / 20)$ & $\%$ \\
\hline \multicolumn{3}{|l|}{ Risperidone + amantadine } \\
\hline Appetite increase ${ }^{68}$ & $1(6 / 20)$ & $30 \%$ \\
\hline \multicolumn{3}{|l|}{ Risperidone + buspirone } \\
\hline Appetite Increase ${ }^{61}$ & $1(10 / 16)$ & $61.1 \%$ \\
\hline Somnolence ${ }^{61}$ & $1(3 / 16)$ & $18.75 \%$ \\
\hline \multicolumn{3}{|l|}{ Risperidone + celecoxib } \\
\hline Appetite Increase ${ }^{64}$ & $1(3 / 20)$ & $15 \%$ \\
\hline Dizziness ${ }^{64}$ & $\frac{1(2 / 20)}{1(20)}$ & $10 \%$ \\
\hline $\mathrm{GI}^{64}$ & $\begin{array}{l}1(\angle / 2) \\
1(5 / 20)\end{array}$ & $25 \%$ \\
\hline
\end{tabular}




\begin{tabular}{|c|c|c|}
\hline Harm/Adverse Event & $\begin{array}{l}\text { N Studies Reporting Harm (\# } \\
\text { Participants With Harm/Total } \\
\text { Participants) }\end{array}$ & $\begin{array}{l}\text { Reported Rates Across } \\
\text { Studies }\end{array}$ \\
\hline Insomnia ${ }^{64}$ & $1(2 / 20)$ & $10 \%$ \\
\hline Somnolence ${ }^{64}$ & $1(3 / 20)$ & $15 \%$ \\
\hline EPS/impaired movement ${ }^{64}$ & $1(10 / 20)$ & $50 \%$ \\
\hline \multicolumn{3}{|l|}{ Risperidone + Galantamine } \\
\hline Agitation/nervousness/restlessness ${ }^{67}$ & $1(4 / 20)$ & $20 \%$ \\
\hline Appetite Increase ${ }^{67}$ & $1(8 / 20)$ & $40 \%$ \\
\hline Somnolence $^{67}$ & $1(2 / 20)$ & $10 \%$ \\
\hline \multicolumn{3}{|l|}{ Risperidone + Ginkgo biloba } \\
\hline Agitation/nervousness/restlessness ${ }^{69}$ & $1(8 / 23)$ & \\
\hline Appetite decrease ${ }^{69}$ & $1(3 / 23)$ & $13 \%$ \\
\hline Appetite Increase ${ }^{69}$ & $1(6 / 23)$ & $26.1 \%$ \\
\hline EPS/impaired movement ${ }^{69}$ & $1(5 / 23)$ & $21.7 \%$ \\
\hline Gl symptoms ${ }^{69}$ & $1(8 / 23)$ & $34.8 \%$ \\
\hline Somnolence ${ }^{69}$ & $1(13 / 23)$ & $56.5 \%$ \\
\hline \multicolumn{3}{|l|}{ Risperidone + memantine } \\
\hline Appetite increase ${ }^{65}$ & $1(2 / 20)$ & $10 \%$ \\
\hline Dizziness ${ }^{65}$ & $1(3 / 20)$ & $15 \%$ \\
\hline $\mathrm{GI}^{65}$ & $1(5 / 20)$ & $25 \%$ \\
\hline Skin Changes ${ }^{65}$ & $1(2 / 20)$ & $10 \%$ \\
\hline Somnolence ${ }^{65}$ & $1(4 / 20)$ & $20 \%$ \\
\hline EPS/impaired movement ${ }^{65}$ & $1(6 / 20)$ & $30 \%$ \\
\hline \multicolumn{3}{|l|}{ Risperidone + N-acetylcysteine } \\
\hline Agitation/nervousness/restlessness ${ }^{63}$ & $1(6 / 17)$ & $35.3 \%$ \\
\hline Appetite decrease ${ }^{63}$ & $1(3 / 17)$ & $17.6 \%$ \\
\hline Appetite Increase ${ }^{63}$ & $1(5 / 17)$ & $29.4 \%$ \\
\hline Dry mouth ${ }^{62}$ & $1(2 / 20)$ & $10 \%$ \\
\hline EPS/impaired movement ${ }^{63}$ & $1(7 / 20)$ & $35 \%$ \\
\hline Gastrointestinal problems $^{62,63}$ & $2(23 / 37)$ & $5.9 \%-30 \%$ \\
\hline Headache $^{62}$ & $1(4 / 20)$ & $20 \%$ \\
\hline Somnolence ${ }^{63}$ & $1(9 / 20)$ & $45 \%$ \\
\hline \multicolumn{3}{|l|}{ Risperidone + Pentoxifylline } \\
\hline Agitation/nervousness/restlessness ${ }^{70}$ & $1(2 / 20)$ & $10 \%$ \\
\hline Appetite increase ${ }^{70}$ & $1(8 / 20)$ & $40 \%$ \\
\hline Dry mouth ${ }^{70}$ & $1(2 / 20)$ & $10 \%$ \\
\hline Gastrointestinal problems $^{70}$ & $1(6 / 20)$ & $30 \%$ \\
\hline Somnolence $^{70}$ & $1(9 / 20)$ & $45 \%$ \\
\hline Weight gain ${ }^{70}$ & $1(8 / 20)$ & $40 \%$ \\
\hline EPS/impaired movement ${ }^{70}$ & $1(7 / 20)$ & $25 \%$ \\
\hline \multicolumn{3}{|l|}{ Risperidone + Pioglitazone } \\
\hline Dizziness $^{60}$ & $1(2 / 20)$ & $20 \%$ \\
\hline Gastrointestinal problems $^{60}$ & $1(10 / 20)$ & $50 \%$ \\
\hline Headache ${ }^{60}$ & $1(3 / 20)$ & $15 \%$ \\
\hline \multicolumn{3}{|l|}{ Risperidone + Minocycline } \\
\hline Gastrointestinal problems $^{71}$ & $1(5 / 23)$ & $8.6 \%-13 \%$ \\
\hline Appetite increase $^{71}$ & $1(2 / 23)$ & $8.6 \%$ \\
\hline Dizziness $^{71}$ & $1(2 / 23)$ & $8.6 \%$ \\
\hline Headache $^{71}$ & $1(1 / 23)$ & $4.3 \%$ \\
\hline Insomnia ${ }^{71}$ & $1(2 / 23)$ & $8.6 \%$ \\
\hline Sedation $^{71}$ & $1(3 / 23)$ & $13 \%$ \\
\hline \multicolumn{3}{|l|}{ Risperidone + placebo } \\
\hline $\begin{array}{l}\text { Agitation/nervousness/restlessness }{ }^{63,66-} \\
70,73\end{array}$ & $7(18 / 138)$ & $4.2 \%-20.8 \%$ \\
\hline Appetite decrease ${ }^{61,63-65,67-70,72,73}$ & $10(19 / 196)$ & $5 \%-20 \%$ \\
\hline Appetite increase ${ }^{61,63-71,73}$ & $11(45 / 219)$ & $8.6 \%-41.7 \%$ \\
\hline Dizziness ${ }^{60,64,65,69,71,72}$ & $6(16 / 127)$ & $5 \%-20 \%$ \\
\hline Drooling/increased saliva ${ }^{66,68,69}$ & $3(10 / 64)$ & $5 \%-30 \%$ \\
\hline
\end{tabular}




\begin{tabular}{|c|c|c|}
\hline Harm/Adverse Event & $\begin{array}{l}\text { N Studies Reporting Harm (\# } \\
\text { Participants With Harm/Total } \\
\text { Participants) }\end{array}$ & $\begin{array}{l}\text { Reported Rates Across } \\
\text { Studies }\end{array}$ \\
\hline Dry mouth ${ }^{60-62,69,70,73}$ & $6(11 / 122)$ & $4.2 \%-15 \%$ \\
\hline EPS/impaired movement ${ }^{64-66,69,70,72,73}$ & $7(45 / 144)$ & $4.2 \%-45 \%$ \\
\hline Gastrointestinal problems ${ }^{60,62-73}$ & $13(58 / 241)$ & $4.3 \%-30 \%$ \\
\hline Headache ${ }^{60,62,71}$ & $3(6 / 63)$ & $4.3 \%-15 \%$ \\
\hline $\begin{array}{l}\text { Infection/fever/cold/congestion } \\
\text { symptoms }\end{array}$ & $1(5 / 24)$ & $20.8 \%$ \\
\hline Insomnia ${ }^{64,65,71,72}$ & $4(10 / 83)$ & $5 \%-25 \%$ \\
\hline Paresthesia $^{72}$ & $1(5 / 20)$ & $25 \%$ \\
\hline Sedation $^{71}$ & $1(4 / 23)$ & $17.4 \%$ \\
\hline Skin Changes ${ }^{63,65}$ & $2(2 / 34)$ & $5 \%-7.1 \%$ \\
\hline Somnolence ${ }^{63-67,69,70,72,73}$ & $9(59 / 178)$ & $5 \%-45 \%$ \\
\hline Weight Gain $^{70}$ & $1(7 / 20)$ & $35 \%$ \\
\hline \multicolumn{3}{|l|}{ Risperidone + Riluzole } \\
\hline Agitation/nervousness/restlessness ${ }^{66}$ & $1(7 / 20)$ & $35 \%$ \\
\hline Appetite decrease ${ }^{66}$ & $1(2 / 20)$ & $10 \%$ \\
\hline Appetite Increase ${ }^{66}$ & $1(12 / 20)$ & $60 \%$ \\
\hline Drooling/Increased Saliva ${ }^{66}$ & $1(6 / 20)$ & $60 \%$ \\
\hline EPS/Impaired movement ${ }^{66}$ & $1(4 / 20)$ & $10 \%$ \\
\hline Gastrointestinal problems $^{66}$ & $1(10 / 20)$ & $50 \%$ \\
\hline Somnolence $^{66}$ & $1(10 / 20)$ & $50 \%$ \\
\hline \multicolumn{3}{|l|}{ Risperidone + Topiramate } \\
\hline Appetite decrease $^{72}$ & $1(7 / 20)$ & $35 \%$ \\
\hline Dizziness $^{72}$ & $1(4 / 20)$ & $20 \%$ \\
\hline Gastrointestinal problems $^{72}$ & $1(6 / 20)$ & $30 \%$ \\
\hline Insomnia $^{72}$ & $1(5 / 20)$ & $25 \%$ \\
\hline Paresthesia $^{72}$ & $1(5 / 20)$ & $25 \%$ \\
\hline Somnolence $^{72}$ & $1(8 / 20)$ & $40 \%$ \\
\hline EPS/impaired movement $^{72}$ & $1(9 / 20)$ & $45 \%$ \\
\hline
\end{tabular}

*Harms reported by more than one participant. EPS=extrapyramidal symptoms; GI=gastrointestinal

Table F-8. Harms/adverse effects in studies of citalopram

\begin{tabular}{|c|c|c|}
\hline Harm/Adverse Event & $\begin{array}{l}\text { N Studies Reporting Harm } \\
\text { (\# Participants With } \\
\text { Harm/Total Participants) }\end{array}$ & $\begin{array}{l}\text { Reported Rates Across } \\
\text { Studies }\end{array}$ \\
\hline \multicolumn{3}{|l|}{ Citalopram hydrobromide } \\
\hline Agitation/Nervousness/Restlessness ${ }^{100,101}$ & $1(13 / 73)$ & $17.8 \%$ \\
\hline Anxiety ${ }^{100,101}$ & $1(8 / 73)$ & $11 \%$ \\
\hline Appetite increase $^{100,101}$ & $1(7 / 73)$ & $9.6 \%$ \\
\hline Appetite decrease ${ }^{100,101}$ & $1(11 / 73)$ & $15.1 \%$ \\
\hline Attention ${ }^{100,101}$ & $1(9 / 73)$ & $12.3 \%$ \\
\hline Challenging behavior ${ }^{100,101}$ & $1(64 / 73)$ & $87.7 \%$ \\
\hline Energy level changes ${ }^{100,}, 101$ & $1(28 / 73)$ & $38.4 \%$ \\
\hline Gl symptoms ${ }^{100,101}$ & $1(46 / 73)$ & $63 \%$ \\
\hline Headache $^{100,101}$ & $1(15 / 73)$ & $20.5 \%$ \\
\hline Hyperactivity ${ }^{100,101}$ & $1(9 / 73)$ & $12.3 \%$ \\
\hline $\begin{array}{l}\text { Infection/Fever/Cold/Congestion symptoms } \\
100,101\end{array}$ & $1(56 / 73)$ & $76.7 \%$ \\
\hline Insomnia ${ }^{100,101}$ & $1(28 / 73)$ & $38.4 \%$ \\
\hline Nightmares ${ }^{100,101}$ & $1(5 / 73)$ & $6.8 \%$ \\
\hline Repetitive behavior or language $^{100,101}$ & $1(8 / 73)$ & $11 \%$ \\
\hline Seizure ${ }^{100,101}$ & $1(2 / 73)$ & $2.7 \%$ \\
\hline Silliness ${ }^{100,101}$ & $1(9 / 73)$ & $12.3 \%$ \\
\hline Skin changes ${ }^{100,101}$ & $1(21 / 73)$ & $28.8 \%$ \\
\hline \multicolumn{3}{|l|}{ Placebo } \\
\hline Agitation/Nervousness/Restlessness ${ }^{100,101}$ & $1(7 / 76)$ & $9.2 \%$ \\
\hline
\end{tabular}




\begin{tabular}{|c|c|c|}
\hline Harm/Adverse Event & $\begin{array}{l}\text { N Studies Reporting Harm } \\
\text { (\# Participants With } \\
\text { Harm/Total Participants) }\end{array}$ & $\begin{array}{l}\text { Reported Rates Across } \\
\text { Studies }\end{array}$ \\
\hline Anxiety ${ }^{100,101}$ & $1(9 / 76)$ & $11.8 \%$ \\
\hline Appetite increase ${ }^{100,101}$ & $1(8 / 76)$ & $10.5 \%$ \\
\hline Appetite decrease $^{100,101}$ & $1(10 / 76)$ & $13.2 \%$ \\
\hline Attention $^{100,101}$ & $1(2 / 76)$ & $2.6 \%$ \\
\hline Challenging behavior ${ }^{100,101}$ & $1(44 / 76)$ & $57.9 \%$ \\
\hline Energy level changes ${ }^{100,101}$ & $1(15 / 76)$ & $19.7 \%$ \\
\hline $\mathrm{Gl}^{100,101}$ & $1(24 / 76)$ & $31.5 \%$ \\
\hline Headache ${ }^{100,101}$ & $1(10 / 76)$ & $13.2 \%$ \\
\hline Hyperactivity ${ }^{100,101}$ & $1(2 / 76)$ & $2.6 \%$ \\
\hline $\begin{array}{l}\text { Infection/Fever/Cold/Congestion symptoms } \\
100,101\end{array}$ & $1(42 / 76)$ & $55.3 \%$ \\
\hline Insomnia ${ }^{100,101}$ & $1(17 / 76)$ & $22.4 \%$ \\
\hline Silliness ${ }^{100,101}$ & $1(10 / 76)$ & $13.2 \%$ \\
\hline Skin changes ${ }^{100,101}$ & $1(9 / 76)$ & $11.8 \%$ \\
\hline Somnolence ${ }^{100,101}$ & $1(10 / 76)$ & $13.2 \%$ \\
\hline
\end{tabular}

${ }^{*}$ Harms reported by more than one participant. GI=gastrointestinal; EPS-extrapyramidal

Table F-9. Harms/adverse effects in studies of diet and nutritional supplements

\begin{tabular}{|c|c|c|}
\hline Harm/Adverse Event* & $\begin{array}{l}\text { N Studies Reporting Harm } \\
\text { (\# Participants With } \\
\text { Harm/Total Participants) }\end{array}$ & $\begin{array}{c}\text { Reported Rates Across } \\
\text { Studies }\end{array}$ \\
\hline \multicolumn{3}{|l|}{ Omega 3 fatty acids } \\
\hline Appetite decrease ${ }^{39}$ & $1(7 / 18)$ & $38.9 \%$ \\
\hline Bruising ${ }^{39}$ & $1(4 / 18)$ & $22.2 \%$ \\
\hline Challenging behaviors ${ }^{39}$ & $1(11 / 18)$ & $61.1 \%$ \\
\hline Epistaxis $^{41,45}$ & $2(2 / 43)$ & $3.4 \%-7.1 \%$ \\
\hline Eye/Vision changes ${ }^{39}$ & $1(3 / 18)$ & $16.7 \%$ \\
\hline Gl symptoms $^{39,41,45}$ & $3(28 / 61)$ & $3.4 \%-50 \%$ \\
\hline Hyperactivity ${ }^{39}$ & $1(3 / 18)$ & $16.7 \%$ \\
\hline $\begin{array}{l}\text { Infection/Fever/Cold/Congestion symptoms } \\
39,41,45\end{array}$ & $3(39 / 61)$ & $3.4 \%-22.2 \%$ \\
\hline Insomnia ${ }^{39}$ & $1(13 / 18)$ & $72.2 \%$ \\
\hline Lethargy ${ }^{39}$ & $1(4 / 18)$ & $22.2 \%$ \\
\hline Right leg internal rotation ${ }^{39}$ & $1(2 / 18)$ & $11.1 \%$ \\
\hline Self-stimulation ${ }^{41}$ & $\frac{1(L / 2)}{1(2 / 29)}$ & $6.9 \%$ \\
\hline Skin changes $39,41,45$ & $3(17 / 61)$ & $3.4 \%-55.6 \%$ \\
\hline \multicolumn{3}{|l|}{ DHA } \\
\hline Agitation/Nervousness/Restlessness ${ }^{42}$ & $1(2 / 24)$ & $8.3 \%$ \\
\hline \multicolumn{3}{|l|}{ Gluten-Dairy Free Diet } \\
\hline $\mathrm{Gl} \mathrm{symptoms}^{51}$ & $1(3 / 6)$ & $50 \%$ \\
\hline \multicolumn{3}{|l|}{ Gluten-Dairy Containing Diet } \\
\hline $\mathrm{Gl}^{51}$ & $1(3 / 6)$ & $50 \%$ \\
\hline \multicolumn{3}{|l|}{ Gluten-free, Casein-free Diet } \\
\hline $\mathrm{GI}^{59}$ & $1(4 / 8)$ & $12.5 \%-25 \%$ \\
\hline Appetite decrease ${ }^{59}$ & $1(3 / 8)$ & $37.5 \%$ \\
\hline \multicolumn{3}{|l|}{ Healthy, Low sugar Diet } \\
\hline $\mathrm{GI}^{59}$ & $1(2 / 14)$ & $7.1 \%$ \\
\hline Night wakings $^{59}$ & $1(3 / 14)$ & $7.1 \%$ \\
\hline \multicolumn{3}{|l|}{ Methyl B12 } \\
\hline Epistaxis $^{49}$ & $1(2 / 27)$ & $7 \%$ \\
\hline $\mathrm{GI}^{49}$ & $\frac{1(L / 27)}{1(1 / 27)}$ & $4 \%$ \\
\hline Hyperactivity $^{49}$ & $1(2 / 27)$ & $7 \%$ \\
\hline Inattention ${ }^{49}$ & $\frac{1(2 / 27)}{1(1 / 27)}$ & $4 \%$ \\
\hline Increased irritability ${ }^{49}$ & $1(1 / 27)$ & $4 \%$ \\
\hline
\end{tabular}




\begin{tabular}{|c|c|c|}
\hline Harm/Adverse Event* & $\begin{array}{c}\text { N Studies Reporting Harm } \\
\text { (\# Participants With } \\
\text { Harm/Total Participants) }\end{array}$ & $\begin{array}{c}\text { Reported Rates Across } \\
\text { Studies }\end{array}$ \\
\hline Infection/fever/cold/congestion symptoms ${ }^{49}$ & $1(6 / 27)$ & $4 \%-11 \%$ \\
\hline Insomnia ${ }^{49}$ & $1(1 / 27)$ & $4 \%$ \\
\hline Mouthing ${ }^{49}$ & $1(5 / 27)$ & $19 \%$ \\
\hline Musculoskeletal disorder ${ }^{49}$ & $1(1 / 27)$ & $4 \%$ \\
\hline Skin changes ${ }^{49}$ & $1(1 / 27)$ & $4 \%$ \\
\hline \multicolumn{3}{|l|}{ Peptizyde } \\
\hline Challenging behavior ${ }^{48}$ & $1(5 / 21)$ & $23.8 \%$ \\
\hline \multicolumn{3}{|l|}{ Placebo } \\
\hline Agitation/nervousness/restlessness ${ }^{93,94}$ & $2(4 / 16)$ & $10 \%-50 \%$ \\
\hline Appetite decrease ${ }^{39}$ & $1(3 / 19)$ & $15.8 \%$ \\
\hline Bruising ${ }^{39}$ & $1(2 / 19)$ & $10.5 \%$ \\
\hline Challenging behavior ${ }^{39,48,49}$ & $3(12 / 64)$ & $4 \%-21.1 \%$ \\
\hline Epistaxis $^{39}$ & $1(3 / 19)$ & $15.8 \%$ \\
\hline Eye/Vision changes ${ }^{39,41}$ & $2(2 / 47)$ & $3.6 \%-5.3 \%$ \\
\hline Gl symptoms $^{39,49,95}$ & $2(27 / 48)$ & $4 \%-47.4 \%$ \\
\hline Hyperactivity ${ }^{45,49}$ & $2(10 / 36)$ & $23.1 \%-30 \%$ \\
\hline Inattention $^{49}$ & $1(1 / 23)$ & $4 \%$ \\
\hline $\begin{array}{l}\text { Infection/Fever/Cold/Congestion } \\
\text { symptoms }\end{array}$ & $3(28 / 76)$ & $3.6 \%-26.3 \%$ \\
\hline Insomnia ${ }^{39,49}$ & $2(10 / 51)$ & $5.6 \%-36.8 \%$ \\
\hline Lethargy $^{39}$ & $1(2 / 19)$ & $10.5 \%$ \\
\hline Mouthing $^{49}$ & $1(1 / 23)$ & $4 \%$ \\
\hline Skin changes ${ }^{39,49,95}$ & $2(21 / 48)$ & $4 \%-47.4 \%$ \\
\hline Urinary changes $^{39,93}$ & $3(5 / 52)$ & $9 \%-10.5 \%$ \\
\hline
\end{tabular}

*Harms reported by more than one participant

${ }^{a}$ One study ${ }^{39}$ did not clearly report number of patients in each group that reported upper respiratory infections. This harm was not included in the "Infection/fever/cold/congestive symptoms" count in this table.

GI=gastrointestinal; EPS=extrapyramidal 
Table F-10. Harms/adverse effects in studies of other medical interventions

\begin{tabular}{|c|c|c|}
\hline Harm/Adverse Event & $\begin{array}{c}\text { N Studies Reporting Harm (\# } \\
\text { Participants With Harm/Total } \\
\text { Participants) }\end{array}$ & $\begin{array}{c}\text { Reported Rates Across } \\
\text { Studies }\end{array}$ \\
\hline \multicolumn{3}{|l|}{ Active tDCS stimulation } \\
\hline Skin changes ${ }^{83,84}$ & $1(3 / 10)$ & $30 \%$ \\
\hline \multicolumn{3}{|l|}{ Amantadine } \\
\hline Challenging behavior $^{91}$ & $1(2 / 19)$ & $10 \%$ \\
\hline Insomnia ${ }^{91}$ & $1(4 / 19)$ & $21 \%$ \\
\hline Somnolence $^{91}$ & $1(2 / 19)$ & $10 \%$ \\
\hline \multicolumn{3}{|l|}{ Bumetanide } \\
\hline Hyperactivity ${ }^{77}$ & $1(2 / 27)$ & $7.4 \%$ \\
\hline \multicolumn{3}{|l|}{ Busprinone (2.5 mg twice/day) } \\
\hline Abnormal behavior ${ }^{99}$ & $1(5 / 46)$ & $9 \%$ \\
\hline Affective disorder ${ }^{99}$ & $1(8 / 46)$ & $15 \%$ \\
\hline Agitation/nervousness/restlessness 99 & $1(7 / 46)$ & $13 \%$ \\
\hline Anxiety $^{99}$ & $1(6 / 46)$ & $11 \%$ \\
\hline Appetite decrease $^{99}$ & $1(17 / 46)$ & $31 \%$ \\
\hline Appetite increase $^{99}$ & $1(11 / 46)$ & $20 \%$ \\
\hline Challenging behavior ${ }^{99}$ & $1(33 / 46)$ & $71.7 \%$ \\
\hline Ear and labyrinth disorders ${ }^{99}$ & $1(7 / 46)$ & $13 \%$ \\
\hline Epistaxis $^{99}$ & $1(4 / 46)$ & $7 \%$ \\
\hline Eye disorders $^{99}$ & $1(7 / 46)$ & $13 \%$ \\
\hline Vomiting $^{99}$ & $1(20 / 46)$ & $37 \%$ \\
\hline Diarrhea $^{99}$ & $1(18 / 46)$ & $33 \%$ \\
\hline Constipation $^{99}$ & $1(5 / 46)$ & $9 \%$ \\
\hline Hyperactivity ${ }^{99}$ & $1(15 / 46)$ & $28 \%$ \\
\hline Immune system disorders ${ }^{99}$ & $1(5 / 46)$ & $9 \%$ \\
\hline Cough $^{99}$ & $1(25 / 46)$ & $46 \%$ \\
\hline Congestion $^{99}$ & $1(21 / 46)$ & $45.6 \%$ \\
\hline Rhinorrhea $^{99}$ & $1(15 / 46)$ & $28 \%$ \\
\hline Pyrexia $^{99}$ & $1(31 / 46)$ & $57 \%$ \\
\hline Ear Infection ${ }^{99}$ & $1(11 / 46)$ & $20 \%$ \\
\hline Upper respiratory tract infection ${ }^{99}$ & $1(6 / 46)$ & $11 \%$ \\
\hline Nasopharyngitis $^{99}$ & $1(6 / 46)$ & $11 \%$ \\
\hline Insomnia ${ }^{99}$ & $1(14 / 46)$ & $30 \%$ \\
\hline Musculoskeletal disorders $^{99}$ & $1(5 / 46)$ & $9 \%$ \\
\hline Skin changes $^{99}$ & $1(8 / 46)$ & $15 \%$ \\
\hline Sleep changes $^{99}$ & $1(14 / 46)$ & $26 \%$ \\
\hline Somnolence ${ }^{99}$ & $1(7 / 46)$ & $13 \%$ \\
\hline Urinary changes $^{99}$ & $1(6 / 46)$ & $11 \%$ \\
\hline \multicolumn{3}{|l|}{ Buspirone (5 mg twice/day) } \\
\hline Abnormal behavior $^{99}$ & $1(1 / 49)$ & $16 \%$ \\
\hline Affective disorder ${ }^{99}$ & $1(7 / 49)$ & $13 \%$ \\
\hline Agitation/nervousness/restlessness ${ }^{99}$ & $1(7 / 49)$ & $13 \%$ \\
\hline Anxiety $^{99}$ & $1(3 / 49)$ & $5 \%$ \\
\hline Appetite decrease ${ }^{99}$ & $1(11 / 49)$ & $20 \%$ \\
\hline Appetite increase $^{99}$ & $1(16 / 49)$ & $29 \%$ \\
\hline Challenging behavior ${ }^{99}$ & $1(29 / 49)$ & $59 \%$ \\
\hline Ear and labyrinth disorders ${ }^{99}$ & $1(3 / 49)$ & $5 \%$ \\
\hline Epistaxis $^{99}$ & $1(3 / 49)$ & $5 \%$ \\
\hline Eye disorders ${ }^{99}$ & $1(5 / 49)$ & $9 \%$ \\
\hline Vomiting $^{99}$ & $1(23 / 49)$ & $42 \%$ \\
\hline Diarrhea $^{99}$ & $1(22 / 49)$ & $40 \%$ \\
\hline Constipation $^{99}$ & $1(15 / 49)$ & $27 \%$ \\
\hline Hyperactivity 99 & $1(12 / 49)$ & $22 \%$ \\
\hline Immune system disorders ${ }^{99}$ & $1(3 / 49)$ & $5 \%$ \\
\hline Cough $^{99}$ & $1(23 / 49)$ & $42 \%$ \\
\hline Congestion $^{99}$ & $1(24 / 49)$ & $48.9 \%$ \\
\hline
\end{tabular}

\section{F-106}




\begin{tabular}{|c|c|c|}
\hline Harm/Adverse Event" & $\begin{array}{c}\text { N Studies Reporting Harm (\# } \\
\text { Participants With Harm/Total } \\
\text { Participants) }\end{array}$ & $\begin{array}{c}\text { Reported Rates Across } \\
\text { Studies }\end{array}$ \\
\hline Rhinorrhea $^{99}$ & $1(17 / 49)$ & $31 \%$ \\
\hline Pyrexia $^{99}$ & $1(24 / 49)$ & $44 \%$ \\
\hline Ear Infection ${ }^{99}$ & $1(6 / 49)$ & $11 \%$ \\
\hline Upper respiratory tract infection ${ }^{99}$ & $1(3 / 49)$ & $5 \%$ \\
\hline Nasopharyngitis $^{99}$ & $1(4 / 49)$ & $7 \%$ \\
\hline Insomnia ${ }^{99}$ & $1(23 / 49)$ & 46.9 \\
\hline Musculoskeletal disorders $^{99}$ & $1(2 / 49)$ & $4 \%$ \\
\hline Skin changes ${ }^{99}$ & $1(8 / 49)$ & $15 \%$ \\
\hline Sleep changes $^{99}$ & $1(19 / 49)$ & $35 \%$ \\
\hline Somnolence $^{99}$ & $1(6 / 49)$ & $11 \%$ \\
\hline Urinary changes $^{99}$ & $1(7 / 49)$ & $13 \%$ \\
\hline \multicolumn{3}{|l|}{ Divalproex sodium } \\
\hline Agitation/nervousness/restlessness ${ }^{75}$ & $1(2 / 16)$ & $12.5 \%$ \\
\hline $\begin{array}{l}\text { Infection/fever/cold/congestion } \\
\text { symptoms }\end{array}$ & $1(2 / 16)$ & $12.5 \%$ \\
\hline Insomnia ${ }^{75}$ & $1(2 / 16)$ & $12.5 \%$ \\
\hline Skin changes $^{75}$ & $1(2 / 16)$ & $12.5 \%$ \\
\hline Urinary changes $^{75}$ & $1(2 / 16)$ & $12.5 \%$ \\
\hline \multicolumn{3}{|l|}{ Donepezil hydrochloride } \\
\hline Challenging behavior ${ }^{90}$ & $1(5 / 17)$ & 29.4 \\
\hline \multicolumn{3}{|l|}{ Levetiracetam } \\
\hline Challenging behavior ${ }^{93}$ & $1(3 / 10)$ & $10 \%-20 \%$ \\
\hline \multicolumn{3}{|l|}{ Mecamylamine } \\
\hline Appetite decrease ${ }^{88}$ & $1(4 / 12)$ & $33.3 \%$ \\
\hline EPS/impaired movement ${ }^{88}$ & $1(2 / 12)$ & $16.7 \%$ \\
\hline Gl symptoms ${ }^{88}$ & $1(9 / 12)$ & $75 \%$ \\
\hline $\begin{array}{l}\text { Infection/fever/cold/congestion } \\
\text { symptoms }^{88}\end{array}$ & $1(9 / 12)$ & $75 \%$ \\
\hline Mental symptoms ${ }^{88}$ & $1(6 / 12)$ & $50 \%$ \\
\hline Somnolence $^{88}$ & $1(5 / 12)$ & $41.7 \%$ \\
\hline \multicolumn{3}{|l|}{ Melatonin } \\
\hline Appetite decrease ${ }^{82}$ & $1(2 / 6)$ & $35.3 \%$ \\
\hline Gl symptoms ${ }^{82}$ & $1(4 / 6)$ & $70.6 \%$ \\
\hline Somnolence $^{82}$ & $1(4 / 6)$ & $70.6 \%$ \\
\hline \multicolumn{3}{|l|}{ Memantine } \\
\hline Affective disorder ${ }^{97}$ & $1(2 / 60)$ & $3.3 \%$ \\
\hline Agitation/nervousness/restlessness ${ }^{97}$ & $1(4 / 60)$ & $6.7 \%$ \\
\hline Challenging behavior $^{97}$ & $1(10 / 60)$ & $8.3 \%$ \\
\hline Enuresis $^{97}$ & $1(2 / 60)$ & $3.3 \%$ \\
\hline Gl symptoms $^{97}$ & $1(11 / 60)$ & $1.7 \%-6.7 \%$ \\
\hline Headache $^{97}$ & $1(3 / 60)$ & $5 \%$ \\
\hline $\begin{array}{l}\text { Infection/fever/cold/congestion } \\
\text { symptoms }^{97}\end{array}$ & $1(30 / 60)$ & $1.7 \%-10 \%$ \\
\hline Insomnia ${ }^{97}$ & $1(5 / 60)$ & $1.7 \%-6.7 \%$ \\
\hline Self-injurous behavior ${ }^{97}$ & $1(2 / 60)$ & $3.3 \%$ \\
\hline \multicolumn{3}{|l|}{$\mathrm{N}$-acetylcysteine } \\
\hline Agitation/nervousness/restlessness ${ }^{89}$ & $1(3 / 14)$ & $21.4 \%$ \\
\hline Appetite decrease 89 & $1(2 / 14)$ & $14.3 \%$ \\
\hline Appetite increase $^{89}$ & $1(2 / 14)$ & $14.3 \%$ \\
\hline Asthma ${ }^{95}$ & $1(1 / 34)$ & $2.9 \%$ \\
\hline Cysts - Unspecified ${ }^{95}$ & $1(1 / 34)$ & $2.9 \%$ \\
\hline EPS/impaired movement ${ }^{89}$ & $1(3 / 14)$ & $21.4 \%$ \\
\hline Gl symptoms ${ }^{89,95}$ & $2(23 / 48)$ & $26.5 \% 100 \%$ \\
\hline $\begin{array}{l}\text { Infection/fever/cold/congestion } \\
\text { symptoms }{ }^{89,95}\end{array}$ & $2(20 / 48)$ & $2.9 \%-28.6 \%$ \\
\hline Skin changes ${ }^{95}$ & $1(2 / 34)$ & $5.9 \%$ \\
\hline
\end{tabular}




\begin{tabular}{|c|c|c|}
\hline Harm/Adverse Event" & $\begin{array}{l}\text { N Studies Reporting Harm (\# } \\
\text { Participants With Harm/Total } \\
\text { Participants) }\end{array}$ & $\begin{array}{c}\text { Reported Rates Across } \\
\text { Studies }\end{array}$ \\
\hline \multicolumn{3}{|l|}{ Tetrahydrobiopterin } \\
\hline Challenging behavior ${ }^{86}$ & $1(4 / 20)$ & $22 \%$ \\
\hline Hyperactivity $^{86}$ & $1(2 / 20)$ & $9 \%$ \\
\hline Insomnia ${ }^{86}$ & $1(2 / 20)$ & $9 \%$ \\
\hline Skin changes $^{86}$ & $1(2 / 20)$ & $9 \%$ \\
\hline Agitation/nervousness/restlessness ${ }^{94}$ & $1(3 / 5)$ & $60 \%$ \\
\hline Sleeping problems ${ }^{94}$ & $1(4 / 5)$ & $80 \%$ \\
\hline \multicolumn{3}{|l|}{ Steroid } \\
\hline Challenging behavior $^{92}$ & $1(10 / 20)$ & $50 \%$ \\
\hline Cushingoid appearance $^{92}$ & $1(18 / 20)$ & $90 \%$ \\
\hline Hypertension $^{92}$ & $1(2 / 20)$ & $10 \%$ \\
\hline Regression $^{92}$ & $1(2 / 20)$ & $10 \%$ \\
\hline Sleep changes $^{92}$ & $1(2 / 20)$ & $10 \%$ \\
\hline Weight gain $^{92}$ & $1(19 / 20)$ & $95 \%$ \\
\hline \multicolumn{3}{|l|}{ Hyperbaric oxygen } \\
\hline Barotrauma $^{78}$ & $1(11 / 29)$ & $37.9 \%$ \\
\hline \multicolumn{3}{|l|}{ Placebo } \\
\hline Abnormal behavior ${ }^{97,99}$ & $2(11 / 107)$ & $3.3 \%-16 \%$ \\
\hline Affective disorder $^{99}$ & $1(4 / 47)$ & $7 \%$ \\
\hline $\begin{array}{l}\text { Agitation/nervousness/restlessness }{ }^{75,89,} \\
93,94,97,99\end{array}$ & $6(56 / 117)$ & $1.6 \%-18 \%$ \\
\hline Anxiety $^{97,99}$ & $2(5 / 107)$ & $2 \%-6.6 \%$ \\
\hline Appetite decrease ${ }^{82,88,89,99}$ & $3(26 / 80)$ & $20 \%-62.5 \%$ \\
\hline Appetite increase $^{99}$ & $1(11 / 47)$ & $19 \%$ \\
\hline Barotrauma $^{78}$ & $1(3 / 29)$ & $10.3 \%$ \\
\hline Challenging behavior ${ }^{86,91,93,97,102}$ & $5(51 / 148)$ & $4.9 \%-39 \%$ \\
\hline Epistaxis $^{97,99}$ & $2(9 / 107)$ & $3.3 \%-12 \%$ \\
\hline EPS/impaired movement ${ }^{88,89}$ & $2(5 / 23)$ & $6.7 \%-25 \%$ \\
\hline GI symptoms ${ }^{80,82,86,88,89,92,97}$ & $7(45 / 141)$ & $1.6 \%-87.5 \%$ \\
\hline Vomiting $^{99}$ & $1(22 / 47)$ & $39 \%$ \\
\hline Diarrhea $^{99}$ & $1(20 / 47)$ & $35 \%$ \\
\hline Constipation $^{99}$ & $1(10 / 47)$ & $18 \%$ \\
\hline Headache $^{97}$ & $1(3 / 60)$ & $4.9 \%$ \\
\hline Hyperactivity $^{86,99}$ & $2(19 / 69)$ & $4 \%-32 \%$ \\
\hline $\begin{array}{l}\text { Infection/fever/cold/congestion } \\
\text { symptoms }^{75,88,89,97}\end{array}$ & $4(50 / 94)$ & $1.6 \%-75 \%$ \\
\hline Insomnia 86,93 & $2(5 / 32)$ & $10 \%-17 \%$ \\
\hline Cough $^{99}$ & $1(28 / 47)$ & $49 \%$ \\
\hline Congestion $^{99}$ & $1(27 / 47)$ & $57.4 \%$ \\
\hline Rhinorrhea $^{99}$ & $1(15 / 47)$ & $26 \%$ \\
\hline Pyrexia $^{99}$ & $1(29 / 47)$ & $51 \%$ \\
\hline Ear Infection ${ }^{99}$ & $1(8 / 47)$ & $14 \%$ \\
\hline Upper respiratory tract infection ${ }^{99}$ & $1(12 / 47)$ & $21 \%$ \\
\hline Nasopharyngitis $^{99}$ & $1(6 / 47)$ & $11 \%$ \\
\hline Insomnia $^{75,88,89,97,99}$ & $5(36 / 159)$ & $1.8 \%-34 \%$ \\
\hline Mental symptoms ${ }^{88}$ & $1(4 / 8)$ & $50 \%$ \\
\hline Musculoskeletal disorders ${ }^{99}$ & $1(5 / 47)$ & $9 \%$ \\
\hline Psychomotor hyperactivity $^{97}$ & $1(4 / 60)$ & $6.6 \%$ \\
\hline Repetitive behavior or language ${ }^{86}$ & $1(2 / 22)$ & $9 \%$ \\
\hline Seizure ${ }^{79,86}$ & $2(2 / 37)$ & $4.5 \%-6.7 \%$ \\
\hline Skin changes $^{77,80,99}$ & $3(11 / 100)$ & $3.7 \%-12 \%$ \\
\hline Sleep changes ${ }^{94,99}$ & $2(18 / 53)$ & $23 \%-83 \%$ \\
\hline Somnolence $75,82,88,99$ & $4(50 / 76)$ & $25 \%-81.3 \%$ \\
\hline Urinary changes $^{93,99}$ & $2(8 / 57)$ & $10 \%-12 \%$ \\
\hline
\end{tabular}

*Harms reported by more than one participant. GI=gastrointestinal; EPS=extrapyramidal 


\section{References}

1. Handen BL, Aman MG, Arnold LE, et al. Atomoxetine, Parent Training, and Their Combination in Children With Autism Spectrum Disorder and Attention-Deficit/Hyperactivity Disorder. J Am Acad Child Adolesc Psychiatry 2015 Nov;54(11):905-15. PMID: 26506581. 2. Smith T, Aman MG, Arnold LE, et al. Atomoxetine and Parent Training for Children With Autism and Attention-Deficit/Hyperactivity Disorder: A 24-Week Extension Study. J Am Acad Child Adolesc Psychiatry 2016 Oct;55(10):868-76.e2. PMID: 27663942.

3. Lamberti M, Siracusano R, Italiano D, et al. Head-to-Head Comparison of Aripiprazole and Risperidone in the Treatment of ADHD Symptoms in Children with Autistic Spectrum Disorder and ADHD: A Pilot, Open-Label, Randomized Controlled Study. Paediatr Drugs 2016 Aug;18(4):319-29. PMID: 27278054.

4. Aman M, Rettiganti M, Nagaraja HN, et al. Tolerability, Safety, and Benefits of Risperidone in Children and Adolescents with Autism: 21-Month Follow-up After 8-Week PlaceboControlled Trial. J Child Adolesc Psychopharmacol 2015 Aug;25(6):482-93. PMID: 26262903. 5. Scahill L, Sukhodolsky DG, Anderberg E, et al. Sensitivity of the modified Children's YaleBrown Obsessive Compulsive Scale to detect change: Results from two multi-site trials. Autism 2015 Apr 16PMID: 25882391.

6. Scahill L, Hallett V, Aman MG, et al. Brief Report: social disability in autism spectrum disorder: results from Research Units on Pediatric Psychopharmacology (RUPP) Autism Network trials. J Autism Dev Disord 2013 Mar;43(3):739-46. PMID: 23104617.

7. Wink LK, Early M, Schaefer T, et al. Body mass index change in autism spectrum disorders: comparison of treatment with risperidone and aripiprazole. J Child Adolesc Psychopharmacol 2014 Mar;24(2):78-82. PMID: 24564519.

8. Kent JM, Kushner S, Ning X, et al. Risperidone dosing in children and adolescents with autistic disorder: a double-blind, placebo-controlled study. J Autism Dev Disord 2013 Aug;43(8):1773-83. PMID: 23212807.

9. Kent JM, Hough D, Singh J, et al. An open-label extension study of the safety and efficacy of risperidone in children and adolescents with autistic disorder. J Child Adolesc Psychopharmacol 2013 Dec;23(10):676-86. PMID: 24350813.

10. Pandina GJ, Bossie CA, Youssef E, et al. Risperidone improves behavioral symptoms in children with autism in a randomized, double-blind, placebo-controlled trial. J Autism Dev Disord 2007 Feb;37(2):367-73. PMID: 17019624.

11. Williams SK, Scahill L, Vitiello B, et al. Risperidone and adaptive behavior in children with autism. J Am Acad Child Adolesc Psychiatry 2006 Apr;45(4):431-9. PMID: 16601648. 12. McDougle CJ, Scahill L, Aman MG, et al. Risperidone for the core symptom domains of autism: results from the study by the autism network of the research units on pediatric psychopharmacology. Am J Psychiatry 2005 Jun;162(6):1142-8. PMID: 15930063.

13. . Risperidone treatment of autistic disorder: longer-term benefits and blinded discontinuation after 6 months. Am J Psychiatry 2005 Jul;162(7):1361-9. PMID: 15994720.

14. Shea S, Turgay A, Carroll A, et al. Risperidone in the treatment of disruptive behavioral symptoms in children with autistic and other pervasive developmental disorders. Pediatrics 2004 Nov;114(5):e634-41. PMID: 15492353.

15. McCracken JT, McGough J, Shah B, et al. Risperidone in children with autism and serious behavioral problems. N Engl J Med 2002 Aug 1;347(5):314-21. PMID: 12151468.

16. Nagaraj R, Singhi P, Malhi P. Risperidone in children with autism: randomized, placebocontrolled, double-blind study. J Child Neurol 2006 Jun;21(6):450-5. PMID: 16948927. 
17. Miral S, Gencer O, Inal-Emiroglu FN, et al. Risperidone versus haloperidol in children and adolescents with AD : a randomized, controlled, double-blind trial. Eur Child Adolesc Psychiatry 2008 Feb;17(1):1-8. PMID: 18080171.

18. Troost PW, Lahuis BE, Steenhuis MP, et al. Long-term effects of risperidone in children with autism spectrum disorders: a placebo discontinuation study. J Am Acad Child Adolesc Psychiatry 2005 Nov;44(11):1137-44. PMID: 16239862.

19. Findling RL, Mankoski R, Timko K, et al. A randomized controlled trial investigating the safety and efficacy of aripiprazole in the long-term maintenance treatment of pediatric patients with irritability associated with autistic disorder. J Clin Psychiatry 2014 Jan;75(1):22-30. PMID: 24502859.

20. Ghanizadeh A, Sahraeizadeh A, Berk M. A head-to-head comparison of aripiprazole and risperidone for safety and treating autistic disorders, a randomized double blind clinical trial. Child Psychiatry Hum Dev 2014;45(2):185-92. PMID: 23801256.

21. Varni JW, Handen BL, Corey-Lisle PK, et al. Effect of aripiprazole 2 to $15 \mathrm{mg} / \mathrm{d}$ on healthrelated quality of life in the treatment of irritability associated with autistic disorder in children: a post hoc analysis of two controlled trials. Clin Ther 2012 Apr;34(4):980-92. PMID: 22444782. 22. Marcus RN, Owen R, Manos G, et al. Aripiprazole in the treatment of irritability in pediatric patients (aged 6-17 years) with autistic disorder: results from a 52-week, open-label study. J Child Adolesc Psychopharmacol 2011 Jun;21(3):229-36. PMID: 21663425.

23. Aman MG, Kasper W, Manos G, et al. Line-item analysis of the Aberrant Behavior Checklist: results from two studies of aripiprazole in the treatment of irritability associated with autistic disorder. J Child Adolesc Psychopharmacol 2010 Oct;20(5):415-22. PMID: 20973712. 24. Owen R, Sikich L, Marcus RN, et al. Aripiprazole in the treatment of irritability in children and adolescents with autistic disorder. Pediatrics 2009 Dec;124(6):1533-40. PMID: 19948625. 25. Marcus RN, Owen R, Kamen L, et al. A placebo-controlled, fixed-dose study of aripiprazole in children and adolescents with irritability associated with autistic disorder. J Am Acad Child Adolesc Psychiatry 2009 Nov;48(11):1110-9. PMID: 19797985.

26. Arnold LE, Vitiello B, McDougle C, et al. Parent-defined target symptoms respond to risperidone in RUPP autism study: customer approach to clinical trials. J Am Acad Child Adolesc Psychiatry 2003 Dec;42(12):1443-50. PMID: 14627879.

27. Martin A, Scahill L, Anderson GM, et al. Weight and leptin changes among risperidonetreated youths with autism: 6-month prospective data. Am J Psychiatry 2004 Jun;161(6):1125-7. PMID: 15169706.

28. Aman MG, Arnold LE, McDougle CJ, et al. Acute and long-term safety and tolerability of risperidone in children with autism. J Child Adolesc Psychopharmacol 2005 Dec;15(6):869-84. PMID: 16379507.

29. Aman MG, Hollway JA, McDougle CJ, et al. Cognitive effects of risperidone in children with autism and irritable behavior. J Child Adolesc Psychopharmacol 2008 Jun;18(3):227-36. PMID: 18582177.

30. Arnold LE, Farmer C, Kraemer HC, et al. Moderators, mediators, and other predictors of risperidone response in children with autistic disorder and irritability. J Child Adolesc Psychopharmacol 2010 Apr;20(2):83-93. PMID: 20415603.

31. Robb AS, Andersson C, Bellocchio EE, et al. Safety and tolerability of aripiprazole in the treatment of irritability associated with autistic disorder in pediatric subjects (6-17 years old):results from a pooled analysis of 2 studies. Prim Care Companion CNS Disord 2011;13(1)PMID: 21731831. 
32. Marcus RN, Owen R, Manos G, et al. Safety and tolerability of aripiprazole for irritability in pediatric patients with autistic disorder: a 52-week, open-label, multicenter study. J Clin Psychiatry 2011 Sep;72(9):1270-6. PMID: 21813076.

33. Posey DJ, Aman MG, McCracken JT, et al. Positive effects of methylphenidate on inattention and hyperactivity in pervasive developmental disorders: an analysis of secondary measures. Biol Psychiatry 2007 Feb 15;61(4):538-44. PMID: 17276750.

34. Jahromi LB, Kasari CL, McCracken JT, et al. Positive Effects of Methylphenidate on Social Communication and Self-Regulation in Children with Pervasive Developmental Disorders and Hyperactivity. Journal of Autism and Developmental Disorders 2009 Mar;39(3):395-404. PMID: EJ827753.

35. . Randomized, controlled, crossover trial of methylphenidate in pervasive developmental disorders with hyperactivity. Arch Gen Psychiatry 2005 Nov;62(11):1266-74. PMID: 16275814. 36. McCracken JT, Badashova KK, Posey DJ, et al. Positive effects of methylphenidate on hyperactivity are moderated by monoaminergic gene variants in children with autism spectrum disorders. Pharmacogenomics J 2014 Jun;14(3):295-302. PMID: 23856854.

37. Pearson DA, Santos CW, Aman MG, et al. Effects of extended release methylphenidate treatment on ratings of attention-deficit/hyperactivity disorder (ADHD) and associated behavior in children with autism spectrum disorders and ADHD symptoms. J Child Adolesc Psychopharmacol 2013 Jun;23(5):337-51. PMID: 23782128.

38. Sun C, Zou M, Zhao D, et al. Efficacy of Folic Acid Supplementation in Autistic Children Participating in Structured Teaching: An Open-Label Trial. Nutrients 2016;8(6)PMID: 27338456.

39. Mankad D, Dupuis A, Smile S, et al. A randomized, placebo controlled trial of omega-3 fatty acids in the treatment of young children with autism. Mol Autism 2015;6:18. PMID: 25798215. 40. Saad K, Eltayeb AA, Mohamad IL, et al. A Randomized, Placebo-controlled Trial of Digestive Enzymes in Children with Autism Spectrum Disorders. Clin Psychopharmacol Neurosci 2015 Aug 31;13(2):188-93. PMID: 26243847.

41. Bent S, Hendren RL, Zandi T, et al. Internet-based, randomized, controlled trial of omega-3 fatty acids for hyperactivity in autism. J Am Acad Child Adolesc Psychiatry 2014 Jun;53(6):65866. PMID: 24839884.

42. Voigt RG, Mellon MW, Katusic SK, et al. Dietary docosahexaenoic acid supplementation in children with autism. J Pediatr Gastroenterol Nutr 2014 Jun;58(6):715-22. PMID: 24345834. 43. Al-Ayadhi LY, Elamin NE. Camel Milk as a Potential Therapy as an Antioxidant in Autism Spectrum Disorder (ASD). Evid Based Complement Alternat Med 2013;2013:602834. PMID: 24069051.

44. Fahmy SF, El-hamamsy MH, Zaki OK, et al. L-Carnitine supplementation improves the behavioral symptoms in autistic children. Research in Autism Spectrum Disorders 2013;7(1):159-66. PMID: 1267033812; 2012-29769-018.

45. Bent S, Bertoglio K, Ashwood P, et al. A pilot randomized controlled trial of omega-3 fatty acids for autism spectrum disorder. J Autism Dev Disord 2011 May;41(5):545-54. PMID: 20683766.

46. Geier DA, Kern JK, Davis G, et al. A prospective double-blind, randomized clinical trial of levocarnitine to treat autism spectrum disorders. Med Sci Monit 2011 Jun;17(6):Pi15-23. PMID: 21629200. 
47. Bertoglio K, Jill James S, Deprey L, et al. Pilot study of the effect of methyl B12 treatment on behavioral and biomarker measures in children with autism. J Altern Complement Med 2010 May;16(5):555-60. PMID: 20804367.

48. Munasinghe SA, Oliff C, Finn J, et al. Digestive enzyme supplementation for autism spectrum disorders: a double-blind randomized controlled trial. J Autism Dev Disord 2010 Sep;40(9):1131-8. PMID: 20204691.

49. Hendren RL, James SJ, Widjaja F, et al. Randomized, Placebo-Controlled Trial of Methyl B12 for Children with Autism. J Child Adolesc Psychopharmacol 2016 Feb 18PMID: 26889605. 50. Hyman SL, Stewart PA, Foley J, et al. The Gluten-Free/Casein-Free Diet: A Double-Blind Challenge Trial in Children with Autism. J Autism Dev Disord 2015 Sep 5PMID: 26343026. 51. Navarro F, Pearson DA, Fatheree N, et al. Are 'leaky gut' and behavior associated with gluten and dairy containing diet in children with autism spectrum disorders? Nutr Neurosci 2015 May;18(4):177-85. PMID: 24564346.

52. Pusponegoro HD, Ismael S, Firmansyah A, et al. Gluten and casein supplementation does not increase symptoms in children with autism spectrum disorder. Acta Paediatr 2015 Nov;104(11):e500-e5. PMID: 26148018.

53. Whiteley P, Haracopos D, Knivsberg AM, et al. The ScanBrit randomised, controlled, singleblind study of a gluten- and casein-free dietary intervention for children with autism spectrum disorders. Nutr Neurosci 2010 Apr;13(2):87-100. PMID: 20406576.

54. Knivsberg AM, Reichelt KL, Hoien T, et al. A randomised, controlled study of dietary intervention in autistic syndromes. Nutr Neurosci 2002 Sep;5(4):251-61. PMID: 12168688.

55. Knivsberg A-M, Reichelt K-L, HÃ,ien T, et al. Effect of a Dietary Intervention on Autistic Behavior. Focus on Autism and Other Developmental Disabilities 2003 Win;18(4):247-56. 56. Harrison Elder J, Shankar M, Shuster J, et al. The Gluten-Free, Casein-Free Diet In Autism: Results of A Preliminary Double Blind Clinical Trial. Journal of Autism and Developmental Disorders 2006 Apr;36(3):413-20. PMID: 2006-07517-011.

57. Seung H, Rogalski Y, Shankar M, et al. The gluten- and casein-free diet and autism: Communication outcomes from a preliminary double-blind clinical trial. Journal of Medical Speech-Language Pathology 2007 Dec;15(4):337-45.

58. Ghalichi F, Ghaemmaghami J, Malek A, et al. Effect of gluten free diet on gastrointestinal and behavioral indices for children with autism spectrum disorders: a randomized clinical trial. World J Pediatr 2016 Jun 10PMID: 27286693.

59. Johnson CR, Handen BL, Zimmer M, et al. Effects of gluten free / casein free diet in young children with autism: A pilot study. Journal of Developmental and Physical Disabilities 2011 2015-12-09;23(3):213-25. PMID: 888750170; 2011-08445-004.

60. Ghaleiha A, Rasa SM, Nikoo M, et al. A pilot double-blind placebo-controlled trial of pioglitazone as adjunctive treatment to risperidone: Effects on aberrant behavior in children with autism. Psychiatry Res 2015 Sep 30;229(1-2):181-7. PMID: 26208985.

61. Ghanizadeh A, Ayoobzadehshirazi A. A randomized double-blind placebo-controlled clinical trial of adjuvant buspirone for irritability in autism. Pediatr Neurol 2015 Jan;52(1):77-81. PMID: 25451017.

62. Nikoo M, Radnia H, Farokhnia M, et al. N-acetylcysteine as an adjunctive therapy to risperidone for treatment of irritability in autism: a randomized, double-blind, placebo-controlled clinical trial of efficacy and safety. Clin Neuropharmacol 2015 Jan-Feb;38(1):11-7. PMID: 25580916. 
63. Ghanizadeh A, Moghimi-Sarani E. A randomized double blind placebo controlled clinical trial of N-Acetylcysteine added to risperidone for treating autistic disorders. BMC Psychiatry 2013;13:196. PMID: 23886027.

64. Asadabadi M, Mohammadi MR, Ghanizadeh A, et al. Celecoxib as adjunctive treatment to risperidone in children with autistic disorder: a randomized, double-blind, placebo-controlled trial. Psychopharmacology (Berl) 2013 Jan;225(1):51-9. PMID: 22782459.

65. Ghaleiha A, Asadabadi M, Mohammadi MR, et al. Memantine as adjunctive treatment to risperidone in children with autistic disorder: a randomized, double-blind, placebo-controlled trial. Int J Neuropsychopharmacol 2013 May;16(4):783-9. PMID: 22999292.

66. Ghaleiha A, Mohammadi E, Mohammadi MR, et al. Riluzole as an adjunctive therapy to risperidone for the treatment of irritability in children with autistic disorder: a double-blind, placebo-controlled, randomized trial. Paediatr Drugs 2013 Dec;15(6):505-14. PMID: 23821414. 67. Ghaleiha A, Ghyasvand M, Mohammadi MR, et al. Galantamine efficacy and tolerability as an augmentative therapy in autistic children: A randomized, double-blind, placebo-controlled trial. J Psychopharmacol 2013 Oct 15;28(7):677-85. PMID: 24132248.

68. Mohammadi MR, Yadegari N, Hassanzadeh E, et al. Double-blind, placebo-controlled trial of risperidone plus amantadine in children with autism: a 10-week randomized study. Clin Neuropharmacol 2013 Nov-Dec;36(6):179-84. PMID: 24201232.

69. Hasanzadeh E, Mohammadi MR, Ghanizadeh A, et al. A double-blind placebo controlled trial of Ginkgo biloba added to risperidone in patients with autistic disorders. Child Psychiatry Hum Dev 2012 Oct;43(5):674-82. PMID: 22392415.

70. Akhondzadeh S, Fallah J, Mohammadi MR, et al. Double-blind placebo-controlled trial of pentoxifylline added to risperidone: effects on aberrant behavior in children with autism. Prog Neuropsychopharmacol Biol Psychiatry 2010 Feb 1;34(1):32-6. PMID: 19772883.

71. Ghaleiha A, Alikhani R, Kazemi MR, et al. Minocycline as Adjunctive Treatment to Risperidone in Children with Autistic Disorder: A Randomized, Double-Blind PlaceboControlled Trial. J Child Adolesc Psychopharmacol 2016 Apr 29PMID: 27128958.

72. Rezaei V, Mohammadi MR, Ghanizadeh A, et al. Double-blind, placebo-controlled trial of risperidone plus topiramate in children with autistic disorder. Prog Neuropsychopharmacol Biol Psychiatry 2010 Oct 1;34(7):1269-72. PMID: 20637249.

73. Akhondzadeh S, Tajdar H, Mohammadi MR, et al. A double-blind placebo controlled trial of piracetam added to risperidone in patients with autistic disorder. Child Psychiatry Hum Dev 2008 Sep;39(3):237-45. PMID: 17929164.

74. Handen BL, Johnson CR, McAuliffe-Bellin S, et al. Safety and efficacy of donepezil in children and adolescents with autism: neuropsychological measures. J Child Adolesc

Psychopharmacol 2011 Feb;21(1):43-50. PMID: 21309696.

75. Hollander E, Chaplin W, Soorya L, et al. Divalproex sodium vs placebo for the treatment of irritability in children and adolescents with autism spectrum disorders.

Neuropsychopharmacology 2010 Mar;35(4):990-8. PMID: 20010551.

76. Du L, Shan L, Wang B, et al. A Pilot Study on the Combination of Applied Behavior Analysis and Bumetanide Treatment for Children with Autism. J Child Adolesc Psychopharmacol 2015 Sep;25(7):585-8. PMID: 26258842.

77. Lemonnier E, Degrez C, Phelep M, et al. A randomised controlled trial of bumetanide in the treatment of autism in children. Transl Psychiatry 2012;2:e202. PMID: 23233021. 
78. Sampanthavivat M, Singkhwa W, Chaiyakul T, et al. Hyperbaric oxygen in the treatment of childhood autism: a randomised controlled trial. Diving Hyperb Med 2012 Sep;42(3):128-33. PMID: 22987458.

79. Granpeesheh D, Tarbox J, Dixon DR, et al. Randomized trial of hyperbaric oxygen therapy for children with autism. Research in Autism Spectrum Disorders 2010 April 2010/June;4(2):268-75. PMID: 2010025300.

80. Rossignol DA, Rossignol LW, Smith S, et al. Hyperbaric treatment for children with autism: a multicenter, randomized, double-blind, controlled trial. BMC Pediatr 2009;9:21. PMID: 19284641.

81. Cortesi F, Giannotti F, Sebastiani T, et al. Controlled-release melatonin, singly and combined with cognitive behavioural therapy, for persistent insomnia in children with autism spectrum disorders: a randomized placebo-controlled trial. J Sleep Res 2012 Dec;21(6):700-9. PMID: 22616853.

82. Wright B, Sims D, Smart S, et al. Melatonin versus placebo in children with autism spectrum conditions and severe sleep problems not amenable to behaviour management strategies: a randomised controlled crossover trial. J Autism Dev Disord 2011 Feb;41(2):175-84. PMID: 20535539.

83. Amatachaya A, Auvichayapat N, Patjanasoontorn N, et al. Effect of anodal transcranial direct current stimulation on autism: a randomized double-blind crossover trial. Behav Neurol 2014;2014:173073. PMID: 25530675.

84. Amatachaya A, Jensen MP, Patjanasoontorn N, et al. The short-term effects of transcranial direct current stimulation on electroencephalography in children with autism: a randomized crossover controlled trial. Behav Neurol 2015;2015:928631. PMID: 25861158.

85. Dadds MR, MacDonald E, Cauchi A, et al. Nasal oxytocin for social deficits in childhood autism: a randomized controlled trial. J Autism Dev Disord 2014 Mar;44(3):521-31. PMID: 23888359.

86. Klaiman C, Huffman L, Masaki L, et al. Tetrahydrobiopterin as a treatment for autism spectrum disorders: a double-blind, placebo-controlled trial. J Child Adolesc Psychopharmacol 2013 Jun;23(5):320-8. PMID: 23782126.

87. Lv YT, Zhang Y, Liu M, et al. Transplantation of human cord blood mononuclear cells and umbilical cord-derived mesenchymal stem cells in autism. J Transl Med 2013;11:196. PMID: 23978163.

88. Arnold LE, Aman MG, Hollway J, et al. Placebo-controlled pilot trial of mecamylamine for treatment of autism spectrum disorders. J Child Adolesc Psychopharmacol 2012 Jun;22(3):198205. PMID: 22537359.

89. Hardan AY, Fung LK, Libove RA, et al. A randomized controlled pilot trial of oral Nacetylcysteine in children with autism. Biol Psychiatry 2012 Jun 1;71(11):956-61. PMID: 22342106.

90. Chez MG, Buchanan TM, Becker M, et al. Donepezil hydrochloride: A double-blind study in autistic children. Journal of Pediatric Neurology 2003 Oct-Dec;1(2):83-8. PMID: 2004-12732002.

91. King BH, Wright DM, Handen BL, et al. Double-blind, placebo-controlled study of amantadine hydrochloride in the treatment of children with autistic disorder. J Am Acad Child Adolesc Psychiatry 2001 Jun;40(6):658-65. PMID: 11392343. 
92. Duffy FH, Shankardass A, McAnulty GB, et al. Corticosteroid therapy in regressive autism: a retrospective study of effects on the Frequency Modulated Auditory Evoked Response (FMAER), language, and behavior. BMC Neurol 2014;14:70. PMID: 24885033.

93. Wasserman S, Iyengar R, Chaplin WF, et al. Levetiracetam versus placebo in childhood and adolescent autism: a double-blind placebo-controlled study. Int Clin Psychopharmacol 2006 Nov;21(6):363-7. PMID: 17012983.

94. Danfors T, von Knorring AL, Hartvig P, et al. Tetrahydrobiopterin in the treatment of children with autistic disorder: a double-blind placebo-controlled crossover study. J Clin Psychopharmacol 2005 Oct;25(5):485-9. PMID: 16160627.

95. Dean OM, Gray KM, Villagonzalo KA, et al. A randomised, double blind, placebo-controlled trial of a fixed dose of $\mathrm{N}$-acetyl cysteine in children with autistic disorder. Aust N Z J Psychiatry 2016 Jun 17PMID: 27316706.

96. Nikvarz N, Alaghband-Rad J, Tehrani-Doost M, et al. Comparing Efficacy and Side Effects of Memantine vs. Risperidone in the Treatment of Autistic Disorder. Pharmacopsychiatry 2016 Jun 14PMID: 27299475.

97. Aman MG, Findling RL, Hardan AY, et al. Safety and Efficacy of Memantine in Children with Autism: Randomized, Placebo-Controlled Study and Open-Label Extension. J Child Adolesc Psychopharmacol 2016 Mar 15PMID: 26978327.

98. Arnold LE, Aman MG, Cook AM, et al. Atomoxetine for hyperactivity in autism spectrum disorders: placebo-controlled crossover pilot trial. J Am Acad Child Adolesc Psychiatry 2006 Oct;45(10):1196-205. PMID: 17003665.

99. Chugani DC, Chugani HT, Wiznitzer M, et al. Efficacy of Low-Dose Buspirone for Restricted and Repetitive Behavior in Young Children with Autism Spectrum Disorder: A Randomized Trial. J Pediatr 2016 Mar;170:45-53.e1-4. PMID: 26746121.

100. King BH, Hollander E, Sikich L, et al. Lack of efficacy of citalopram in children with autism spectrum disorders and high levels of repetitive behavior: citalopram ineffective in children with autism. Arch Gen Psychiatry 2009 Jun;66(6):583-90. PMID: 19487623.

101. King BH, Dukes K, Donnelly CL, et al. Baseline factors predicting placebo response to treatment in children and adolescents with autism spectrum disorders: a multisite randomized clinical trial. JAMA Pediatr 2013 Nov;167(11):1045-52. PMID: 24061784.

102. Bryant CD, Yazdani N. RNA-binding proteins, neural development and the addictions. Genes Brain Behav 2016 Jan;15(1):169-86. PMID: 26643147. 